SLAC-R-593

April 2002

UC-414

\title{
Linac Coherent Light Source (LCLS) Conceptual Design Report
}

Published April 2002

Prepared for the Department of Energy under contract number DE-AC03-76SF00515 by Stanford Linear Accelerator Center, Stanford University; contract number W-7405-ENG-48 by Lawrence Livermore National Laboratory, University of California; and under contract number W-31-109ENG-38 by Argonne National Laboratory, University of Chicago. Printed in the United States of America 
This document, and the material and data contained therein, was developed under sponsorship of the United States Government. Neither the United States nor the Department of Energy, nor the Leland Stanford J unior University, nor their employees, nor their respective contractors, subcontractors, or their employees, makes any warranty, express or implied, or assumes any liability of responsibility for accuracy, completeness or usefulness of any information, apparatus, product or process disclosed, or represents that its use will not infringe privately owned rights. Mention of any product, its manufacturer, or suppliers shall not, nor is intended to imply approval, disapproval, or fitness for any particular use. A royalty-free, nonexclusive right to use and disseminate same for any purpose whatsoever, is expressly reserved to the United States and the University. 


\section{List of Authors and Contributors}

J. Arthur, Stanford Synchrotron Radiation Laboratory, Stanford, CA, USA

P. Anfinrud, National Institutes of Health, Bethesda, MD, USA

P. Audebert, Laboratoire pour l'Utilisation des Lasers, Paris, France

K. Bane, Stanford Linear Accelerator Center, Stanford, CA, USA

I. Ben-Zvi, Brookhaven National Laboratory, Upton, NY, USA

V. Bharadwaj, Stanford Linear Accelerator Center, Stanford, CA, USA

R. Bionta, Lawrence Livermore National Laboratory, Livermore, CA, USA

P. Bolton, Stanford Linear Accelerator Center, Stanford, CA, USA

M. Borland, Argonne National Laboratory, Argonne, IL, USA

P. H. Bucksbaum, University of Michigan, Ann Arbor, MI, USA

R. C. Cauble, Lawrence Livermore National Laboratory, Livermore, CA, USA

J. Clendenin, Stanford Linear Accelerator Center, Stanford, CA, USA

M. Cornacchia, Stanford Synchrotron Radiation Laboratory, Stanford, CA, USA

G. Decker, Argonne National Laboratory, Argonne, IL, USA

P. Den Hartog, Argonne National Laboratory, Argonne, IL, USA

S. Dierker, University of Michigan, Ann Arbor, MI, USA

D. Dowell, Stanford Linear Accelerator Center, Stanford, CA, USA

D. Dungan, Stanford Synchrotron Radiation Laboratory, Stanford, CA, USA

P. Emma, Stanford Linear Accelerator Center, Stanford, CA, USA

I. Evans, Stanford Synchrotron Radiation Laboratory, Stanford, CA, USA

G. Faigel, Research Institute for Solid State Physics, Budapest, Hungary

R. Falcone, University of California, Berkeley, CA, USA

W.M. Fawley, Lawrence Berkeley National Laboratory, Berkeley, CA, USA

M. Ferrario, Istituto Nazionale di Fisica Nucleare - Lab. Naz. di Frascati, Frascati, Italy

A.S. Fisher, Stanford Linear Accelerator Center, Stanford, CA, USA

R. R. Freeman, University of California, Davis, CA, USA

J. Frisch, Stanford Linear Accelerator Center, Stanford, CA, USA 
J. Galayda, Stanford Linear Accelerator Center, Stanford, CA, USA

J.-C. Gauthier, Laboratoire pour l'Utilisation des Lasers, Paris, France

S. Gierman, Stanford Linear Accelerator Center, Stanford, CA, USA

E. Gluskin, Argonne National Laboratory, Argonne, IL, USA

W. Graves, Brookhaven National Laboratory, Upton, NY, USA

J. Hajdu, Uppsala University, Uppsala, Sweden

J. Hastings, Stanford Synchrotron Radiation Laboratory, Stanford, CA, USA

K. Hodgson, Stanford Synchrotron Radiation Laboratory, Stanford, CA, USA

Z. Huang, Argonne National Laboratory, Argonne, IL, USA

R. Humphry, Stanford Linear Accelerator Center, Stanford, CA, USA

P. Ilinski, Argonne National Laboratory, Argonne, IL, USA

D. Imre, Brookhaven National Laboratory, Upton, NY, USA

C. Jacobsen, State University of New York at Stony Brook, Stony Brook, NY, USA

C.-C. Kao, Brookhaven National Laboratory, Upton, NY, USA

K. R. Kase, Stanford Linear Accelerator Center, Stanford, CA, USA

K.-J. Kim, Argonne National Laboratory, Argonne, IL, USA

R. Kirby, Stanford Linear Accelerator Center, Stanford, CA, USA

J. Kirz, State University of New York at Stony Brook, Stony Brook, NY, USA

L. Klaisner, Stanford Linear Accelerator Center, Stanford, CA, USA

P. Krejcik, Stanford Linear Accelerator Center, Stanford, CA, USA

K. Kulander, Lawrence Livermore National Laboratory, Livermore, CA, USA

O. L. Landen, Lawrence Livermore National Laboratory, Livermore, CA, USA

R. W. Lee, Lawrence Livermore National Laboratory, Livermore, CA, USA

C. Lewis, Queen's University Belfast, Belfast, Northern Ireland

C. Limborg, Stanford Synchrotron Radiation Laboratory, Stanford, CA, USA

E. I. Lindau, Stanford Syn. Rad. Lab., Stanford, CA, USA and Lund University, Sweden

A. Lumpkin, Argonne National Laboratory, Argonne, IL, USA

G. Materlik, HASYLAB at DESY, Hamburg, Germany

S. Mao, Stanford Linear Accelerator Center, Stanford, CA, USA

J. Miao, Stanford Synchrotron Radiation Laboratory, Stanford, CA, USA

LIST OF AUTHORS AND CONTRIBUTORS 
S. Mochrie, Yale University, New Haven, CT, USA

E. Moog, Argonne National Laboratory, Argonne, IL, USA

S. Milton, Argonne National Laboratory, Argonne, IL, USA

G. Mulhollan, Stanford Linear Accelerator Center, Stanford, CA, USA

K. Nelson, MIT, Cambridge, MA, USA

W. R. Nelson, Stanford Linear Accelerator Center, Stanford, CA, USA

R. Neutze, Uppsala University Uppsala, Sweden

A. Ng, University of British Columbia, British Columbia, Canada

D. Nguyen, Los Alamos National Laboratory, Los Alamos, NM, USA

H.-D. Nuhn, Stanford Synchrotron Radiation Laboratory, Stanford, CA, USA

D.T. Palmer, Stanford Linear Accelerator Center, Stanford, CA, USA

J.M. Paterson, Stanford Linear Accelerator Center, Stanford, CA, USA

C. Pellegrini, University of California at Los Angeles, CA, USA

S. Reiche, University of California at Los Angeles, CA, USA

M. Renner, Brookhaven National Laboratory, Upton, NY, USA

D. Riley, Queen's University Belfast, Belfast, Northern Ireland

C.V. Robinson, Oxford University, South Parks Road, Oxford, UK

S. H. Rokni, Stanford Linear Accelerator Center, Stanford, CA, USA

S. J. Rose, Rutherford Appleton Laboratory, Chilton, Oxon, UK

J. Rosenzweig, University of California at Los Angeles, CA, USA

R. Ruland, Stanford Linear Accelerator Center, Stanford, CA, USA

G. Ruocco, Universita' di L'Aquila, L'Aquila, Italy

D. Saenz, Stanford Linear Accelerator Center, Stanford, CA, USA

S. Sasaki, Argonne National Laboratory, Argonne, IL, USA

D. Sayre, State University of New York at Stony Brook, Stony Brook, NY, USA

J. Schmerge, Stanford Synchrotron Radiation Laboratory, Stanford, CA, USA

D. Schneider, Lawrence Livermore National Laboratory, Livermore, CA, USA

C. Schroeder, Stanford Synchrotron Radiation Laboratory, Stanford, CA, USA

L. Serafini, Istituto Nazionale di Fisica Nucleare - Sezione di Milano, Milan, Italy

F. Sette, European Synchrotron Radiation Facility, Grenoble, France 
S. Sinha, Argonne National Laboratory, Argonne, IL, USA

D. van der Spoel, Uppsala University, Uppsala, Sweden

B. Stephenson, Argonne National Laboratory, Argonne, IL, USA

G. Stupakov, Stanford Linear Accelerator Center, Stanford, CA, USA

M. Sutton, McGill University, Montreal, Quebec, Canada

A. Szöke, Lawrence Livermore National Laboratory, Livermore, CA, USA

R. Tatchyn, Stanford Synchrotron Radiation Laboratory, Stanford, CA, USA

A. Toor, Lawrence Livermore National Laboratory, Livermore, CA, USA

E. Trakhtenberg, Argonne National Laboratory, Argonne, IL, USA

I. Vasserman, Argonne National Laboratory, Argonne, IL, USA

N. Vinokurov, A Budker Institute for Nuclear Physics, Novosibirsk, Russia

X.J. Wang, Brookhaven National Laboratory, Upton, NY, USA

D. Waltz, Stanford Linear Accelerator Center, Stanford, CA, USA

J. S. Wark, Oxford University, Oxford, UK

E. Weckert, HASYLAB at DESY, Hamburg, Germany

Wilson-Squire Group, University of California, San Diego, San Diego, CA, USA

H. Winick, Stanford Synchrotron Radiation Laboratory, Stanford, CA, USA

M. Woodley, Stanford Linear Accelerator Center, Stanford, CA, USA

A. Wootton, Lawrence Livermore National Laboratory, Livermore, CA, USA

M. Wulff, European Synchrotron Radiation Laboratory, Grenoble, France

M. Xie, Lawrence Berkeley National Laboratory, Berkeley, CA, USA

R. Yotam, Stanford Synchrotron Radiation Laboratory, Stanford, CA, USA

L. Young, Argonne National Laboratory, Argonne, IL, USA

A. Zewail, California Institute of Technology, Pasadena, CA, USA 


\section{Preface}

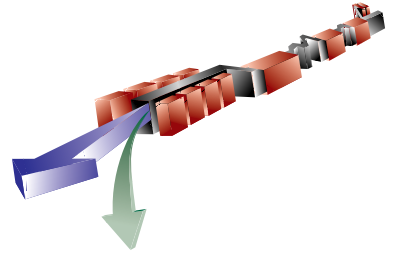

This Conceptual Design Report (CDR) describes the design of the LCLS. It will be updated to stay current with the developing design of the machine. This CDR begins as the baseline conceptual design and will evolve into an "as-built" manual for the completed FEL. The current released version of the CDR can be found on the LCLS web page,

\section{http://www-ssrl.slac.stanford.edu/lcls/.}

The Executive Summary, Chapter 1, gives an introduction to the LCLS project and describes the salient features of its design.

Chapter 2 is a stand-alone document that gives an overview of the LCLS. It describes the general parameters of the machine and the basic approaches to implementation.

The LCLS project does not include the implementation of specific scientific experiments. Nonetheless, significant work has been done on defining potential initial experiments to aid in assuring that the machine can meet the requirements of the experimental community. Chapter $\mathbf{3}$, Scientific Experiments, describes that work on potential experiments.

The chapter begins with a description of the unique characteristics of the LCLS radiation. Then it describes five experimental areas that can effectively use this radiation, 1) atomic physics, 2) plasma and warm dense matter, 3) structure of single particles and biomolecules, 4) femtochemistry, and 5) nanoscale dynamics in condense matter. In each of these fields, the basic scientific questions that can be addressed by the LCLS are described, the experimental requirements are defined, and appropriate initial experiments are defined.

Chapter 4, FEL Physics, describes the physics that underlies the LCLS design. It begins with a brief history, particularly of work on the Self Amplified Spontaneous Emission (SASE) principles. The SASE mode of operation is central to operation at the LCLS wavelengths and has been demonstrated recently at longer wavelengths.

Then the requirements for the electron beam are described. These are challenging and set the parameters for the generation, acceleration, manipulation, and transport of the electrons.

Finally, the characteristics of both the spontaneous and coherent radiation from the FEL are calculated. This provides important input to the design of the x-ray beam transport and to the design of the experiments. 
The basic parameters of the LCLS design are presented in Chapter 5, FEL Parameters and Performance. The design of the focusing system is laid-out. Sources of gain reduction and resulting tolerances are discussed and the electron beam tolerance goals are given. Then, the temporal structure of the $\mathrm{x}$-ray pulse is discussed. The start-to-end simulations are presented. The control of $\mathrm{x}$-ray power levels and an overview of the LCLS Commissioning are presented in this chapter.

The LCLS requires a high brightness electron beam with very low timing and intensity jitter. Chapter 6, Injector, describes the equipment required to generate this beam; the laser for the photocathode rf electron gun, the accelerator (L0), the matching section into the main linac and the beam diagnostic equipment. It describes beam simulations that have been used to optimize the design and give assurance of meeting the required parameters. These simulations are described along with the results. Experimental results from the Gun Test Facility at SLAC and other laboratories support the results of these simulations.

Chapter 7, Accelerator, describes the acceleration, manipulation, and transport of the electron beam from the Injector to the Undulator. The LCLS uses the last one third of the existing SLAC linac to accelerate the $150 \mathrm{MeV}$ beam from the injector to a final energy between $4.54 \mathrm{GeV}$ and $14.35 \mathrm{GeV}$. The linac will be modified to include two pulse compression chicanes, an $\mathrm{x}$-band accelerator to linearize the compression, a wiggler for Landau damping, new diagnostics including a pulse length monitor, and the transport line to the undulator.

These systems accelerate and compress the electron beam to produce very short pulses with very high currents while preserving the transverse emittance. This chapter describes the accelerator physics involved and the simulations that have been run. Also, it defines the tolerances required of the microwave and magnet systems required to meet the LCLS requirements.

The basis for the choice of parameters for the undulator line and a tolerance budget are derived in Chapter 8, Undulator. The choice of magnetic material and the requirements for measuring individual blocks are presented. Then the overall mechanical design of the undulator segments is described including the precision supports. The vacuum system design is discussed along with how the inner surface roughness requirements will be met. This roughness can be a source of disruptive wakefields. The electron and x-ray diagnostics at each gap between the undulator segments are described.

The tight requirements for keeping the electron beam and the FEL radiation collinear in the Undulator will be met with a beam-based alignment procedure. This procedure and the associated simulations are described.

Chapter 9, X-Ray Beam Transport and Diagnostics, describes the suite of X-ray transport and $\mathrm{X}$-ray diagnostic devices that are included in this construction project. This suite of devices will be used to commission the LCLS, to characterize the generated x-ray beam and to prove the key technologies required for doing experiments at the facility. 
Both specular (for the full spectral range) and crystal (for wavelengths shorter than $4.5 \AA$ ) optics will be employed. In the initial operation, it is expected that the high peak power and power density will prevent the utilization of the full FEL flux with conventional focusing and transport optics. On the other hand, there will be a unique opportunity to study the effect of high peak power density on materials and optical elements, thereby opening the path to the full exploitation of the radiation in the LCLS. Consequently, a system will be designed that allows intensity of the radiation to be varied from the level of current third-generation facilities up to the maximum LCLS intensity. This will be achieved by introducing a gas attenuation cell into the path of the FEL radiation. Further reduction factors can be obtained on the beam line optics and instrumentation by operating their crystal or specular optical elements at very low grazingincidence angles. These facilities are described in this chapter.

The LCLS takes advantage of the existing infrastructure at SLAC. Chapter 10, Conventional Facilities, describes the modifications required to existing buildings and utilities and the new construction that is required. An extension to the existing FFTB tunnel, two experimental halls and a tunnel connecting the two halls will be constructed.

The LCLS injector will be installed at sector 20 in the existing Off-Axis Injector Tunnel. This tunnel will require some modifications to bring it to current safety standards and to accommodate the specific requirements of the LCLS injector. A clean room will be constructed in the existing surface building for the gun laser.

The undulator is housed in the existing FFTB tunnel after the tunnel has been extended. Two new experimental halls will be built. The Near Hall will begin $40 \mathrm{~m}$ downstream of the undulator and the Far Hall will be constructed $322 \mathrm{~m}$ downstream the undulator, just outside of the PEP ring road. A tunnel that is $227 \mathrm{~m}$ long will connect the two halls. An office and laboratory structure will be constructed at grade on top of the far hall.

Chapter 11, Controls, describes the modification and extension of the existing control system at SLAC to meet the requirements of the LCLS. New systems that are added for the LCLS will be controlled using EPICS-based systems. The LCLS will be controlled from the existing Main Control Room.

The x-ray beam line controls have two major objectives. One objective is to provide control of the x-ray optical elements. The second objective is to provide sufficient data collection capability to allow for thorough testing of different components.

At SLAC the Beam Containment Systems (BCS), Machine Protection Systems (MPS) and Personnel Protection Systems (PPS) are included in the control system and are described in this chapter.

Chapter 12, Alignment, describes the procedures and methods used to position the LCLS components with their required accuracy. Most of the alignment requirements are well within the range of proven traditional alignment techniques. Alignment of the undulator section is the most 
demanding. The state-of-the-art equipment and procedures that are needed to meet the positioning requirements are described in this chapter.

Chapter 13, Environment, Safety and Health and Quality Assurance, describes the exiting programs at SLAC and their application to the LCLS project. A preliminary analysis of safety hazards is presented along with the planned mitigation.

The radiation concerns related to the LCLS fall into three distinct areas: radiation safety, radiation background in experiments, and machine protection. Chapter 14, Radiological Concerns, covers these concerns in the region downstream of the undulator, since the linac operation is within the existing safety envelop. The studies that are described in this chapter indicate that the radiation is quite manageable.

Chapter 15, Work Breakdown Structure, describes the work breakdown structure used of developing the costs and that will be used to manage the project. The chapter defines the scope of work of each element down to level 3.

Appendix A, Parameter Tables, provides and extensive list of the relative parameters and tolerances for the elements of the LCLS.

Appendix B, Control Points, list each piece of equipment along the LCLS beam line and then lists the associated control points.

Appendix C, Glossary, lists the acronyms used in this report and their definition. 


\section{Table of Contents}

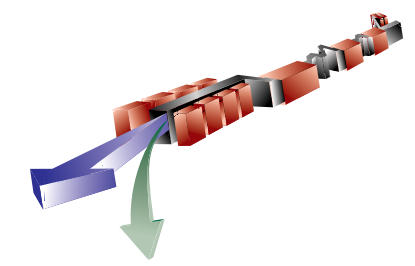

\section{Executive Summary}

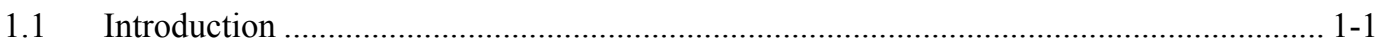

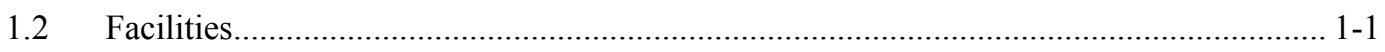

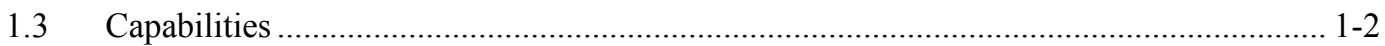

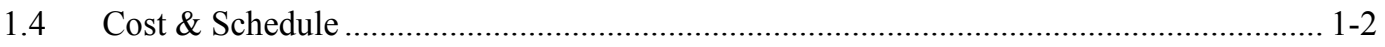

$1.5 \quad$ Acquisition Strategy ........................................................................................... 1-2

\section{Overview}

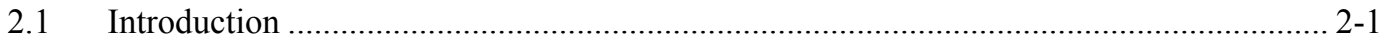

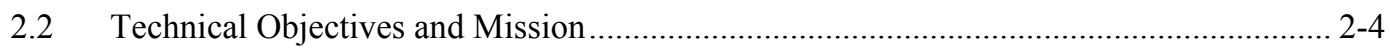

2.2.1 Design Goals …….................................................................................... 2-4

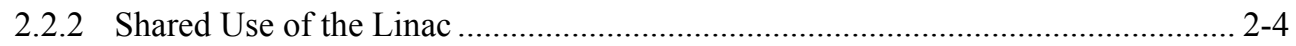

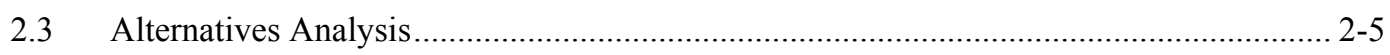

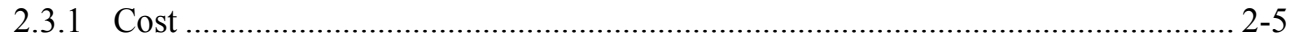

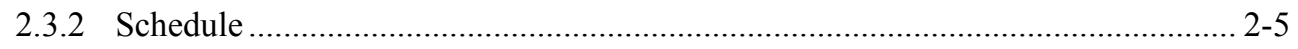

2.3.3 Technical ........................................................................................... 2 2-5

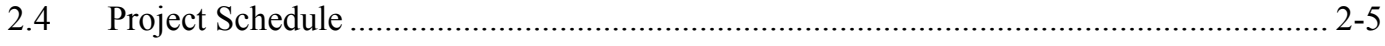

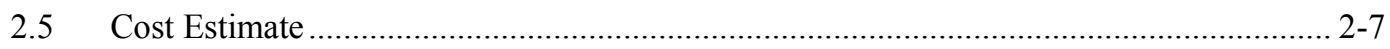

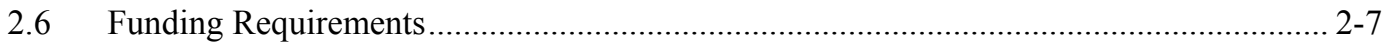

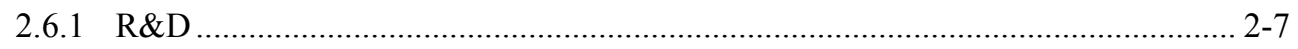

2.6.2 Project Engineering Design and Construction ................................................... 2-7

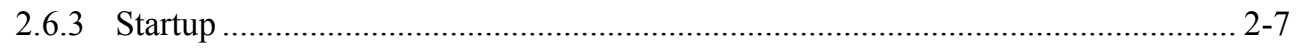

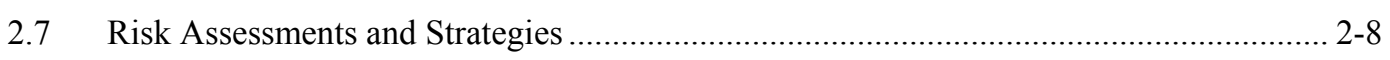

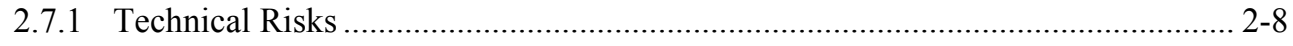

2.7.1.1 Performance of Photocathode Guns ................................................ 2-8

2.7.1.2 Acceleration and Compression .......................................................... 2-8

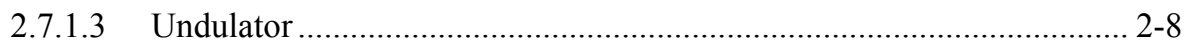

2.7.1.4 Wake Field Effects of the Undulator Vacuum Pipe............................... 2-9

2.7.1.5 SASE FEL Physics ...................................................................... 2-9

2.7.1.6 X-Ray Optics and Beam Handling ..................................................... 2-9

2.7.1.7 Conventional Facilities ..................................................................... 2-9

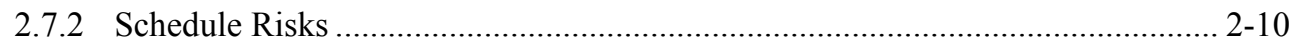

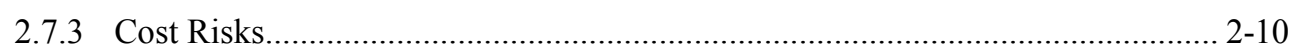

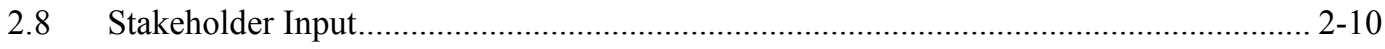

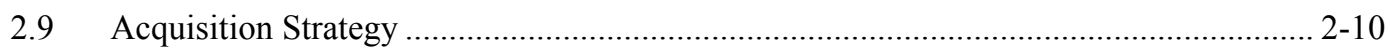


2.10 Design Alternatives

2.11 Principle of Operation ........................................................................................ 2-12

2.12 Overall Layout ................................................................................................ 2-13

2.13 Performance Characteristics ....................................................................... 2-14

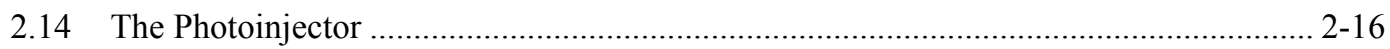

2.15 Compression and Acceleration ........................................................................... 2-16

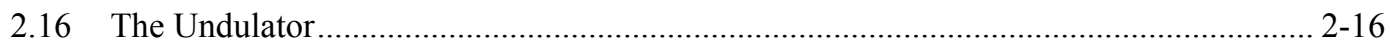

2.17 The X-Ray Optics and Experimental Areas ............................................................ 2-17

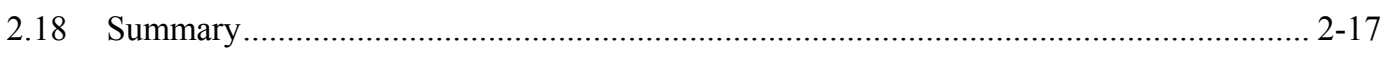

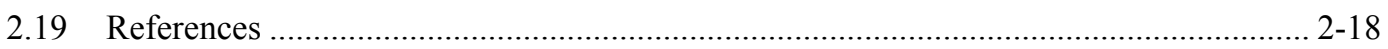

\section{Scientific Basis for Optical Systems}

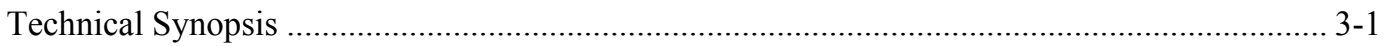

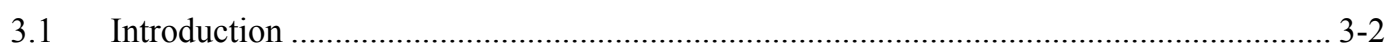

3.1.1 History of Scientific Interest in X-Ray Free Electron Lasers................................. 3-2

3.1.2 Unique Features of X-Ray FEL Radiation ....................................................... 3-3

3.1.3 The Role of the LCLS .............................................................................. 3-5

3.1.4 Science with X-Ray FELs ....................................................................... 3-6

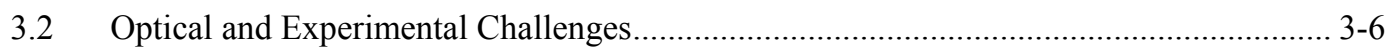

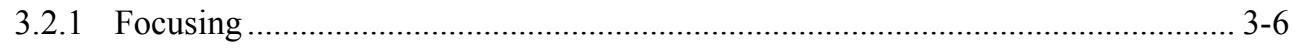

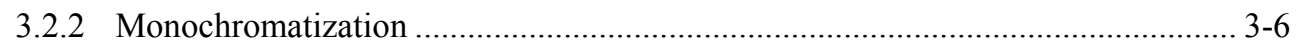

3.2.3 Harmonic Control.................................................................................... 3-7

3.2.4 Photon Pulse Manipulation ........................................................................... 3-7

3.2.5 Synchronization of an External Source (Laser Pump) ………............................. 3-8

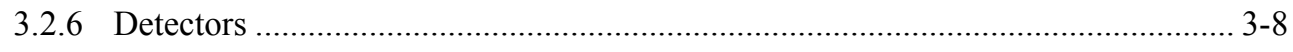

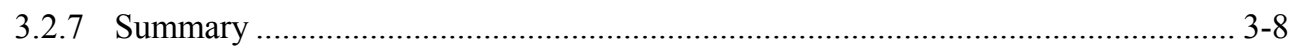

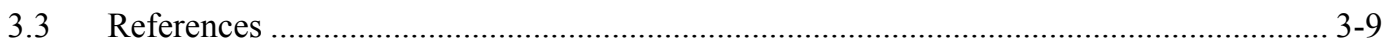

\section{FEL Physics}

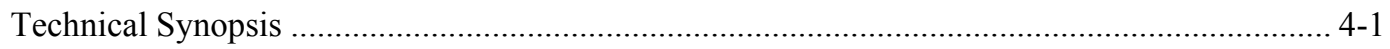

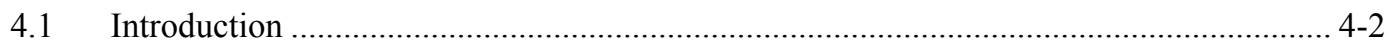

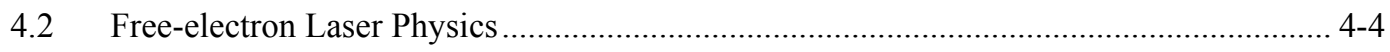

4.2.1 Coherent Undulator Radiation from a Single Electron ....................................... 4-4

4.2.2 Coherent Spontaneous Radiation from Many Electrons ......................................... 4-6

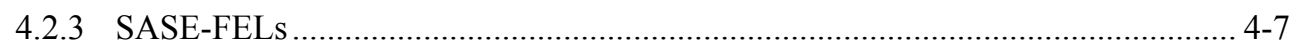

4.2.4 Slippage, Fluctuations and Time Structure ….................................................. 4-9

4.2.5 Nonlinear Harmonic Generation .................................................................. 4-9

4.2.6 Quantum Effects in Free-Electron Lasers .......................................................... 4-10

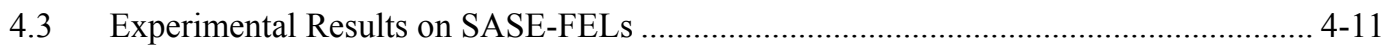

4.4 LCLS: An X-Ray SASE-FEL ........................................................................... 4- 4

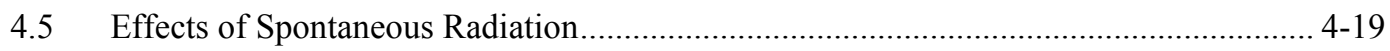

4.6 Undulator Wakefields .................................................................................................... 4-19 


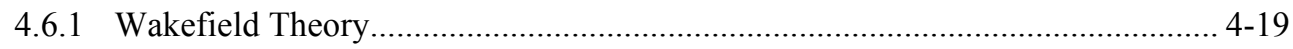

4.6.2 Wakefield Effects on the LCLS ........................................................................ 4-20

4.7 Emittance and Charge Control.......................................................................... 4-22

4.8 Control of X-Ray Pulse Length and Linewidth ........................................................... 4-23

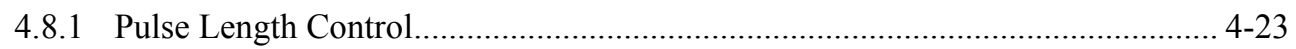

4.8.2 Linewidth Control ...................................................................................... 4-24

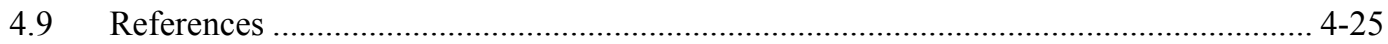

\section{$5 \quad$ FEL Parameters and Performance}

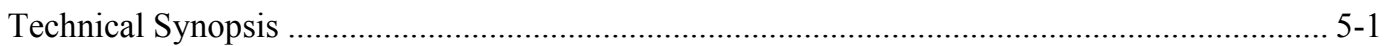

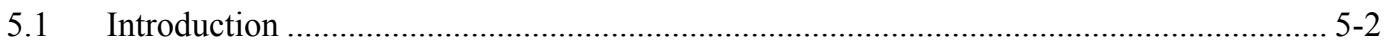

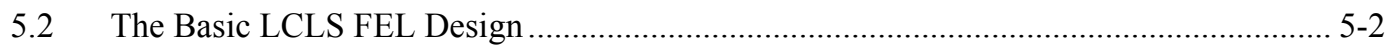

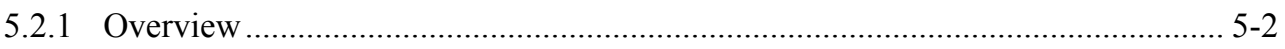

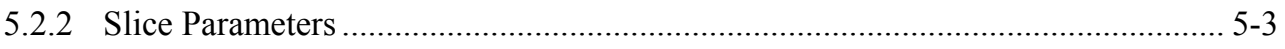

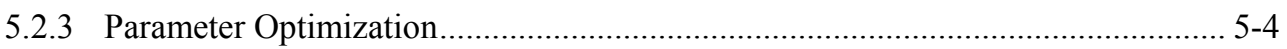

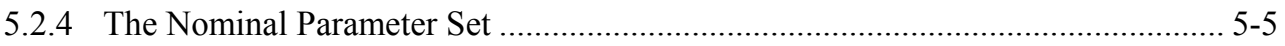

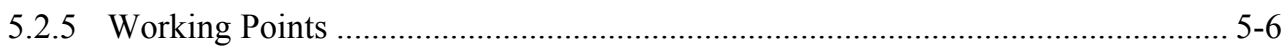

5.3 Electron Beam Focusing Along the Undulator............................................................ 5-7

5.3.1 Optimum Beam Size ……………............................................................... 5-7

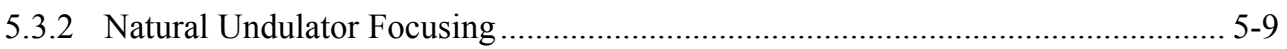

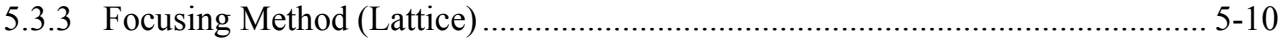

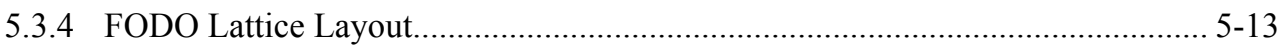

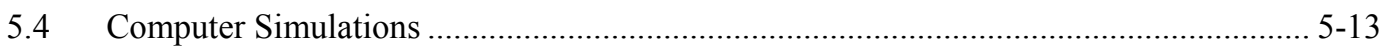

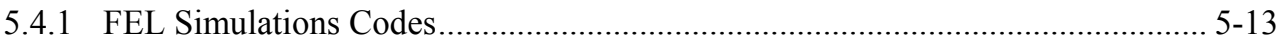

5.4.2 Start-To-End Simulations............................................................................... 5-14

5.4.2.1 Case I - Low Charge Limit ............................................................ 5-14

5.4.2.2 Case II - High Charge Limit ......................................................... 5-17

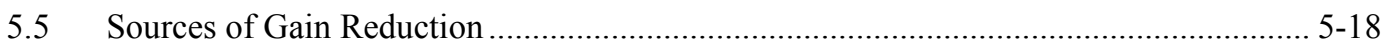

5.5.1 Undulator Trajectory Errors ………………............................................. 5-18

5.5.1.1 Undulator Steering and Corrector Description ................................... 5-18

5.5.1.2 Magnetic Field Errors ................................................................ 5-18

5.5.1.3 Undulator Trajectory Straightness Tolerances................................. 5-19

5.5.1.4 Steering Stations Separations........................................................... 5-19

5.5.1.5 Undulator Trajectory Matching Tolerances....................................... 5-19

5.5.2 Effects of the Emission of Spontaneous Radiation on Gain................................ 5-20

5.5.2.1 Average Energy Loss................................................................. 5-20

5.5.2.2 Energy Spread Increase ….......................................................... 5-21

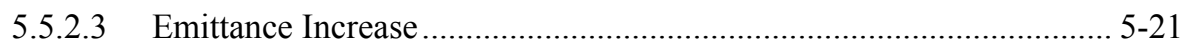

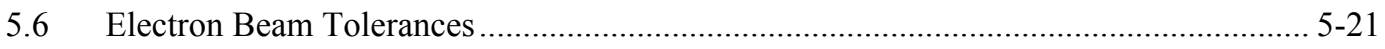

5.6.1 Electron Beam Tolerance Goals ...................................................................... 5-21

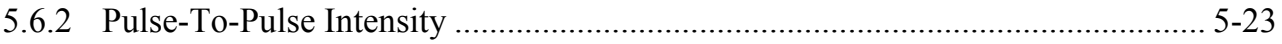

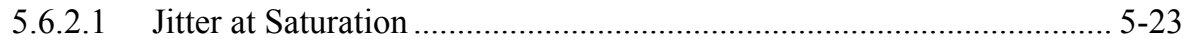

5.6.2.2 Jitter in the Exponential Gain Regime …………………………….... 5-25 


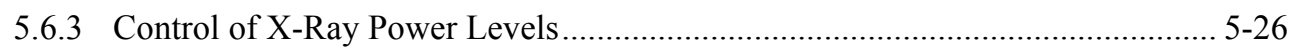

5.7 The Temporal Structure of the X-Ray Pulse ……………...................................... 5-27

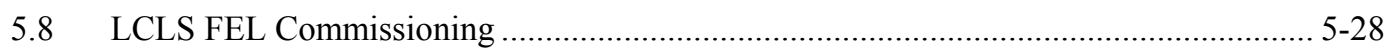

5.8.1 Procedural Aspects of the FEL Gain Commissioning ........................................ 5-28

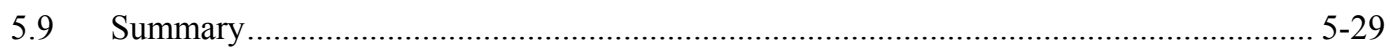

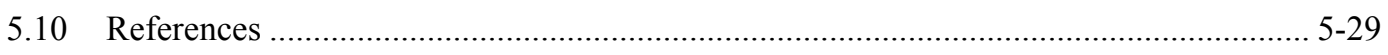

\section{Injector}

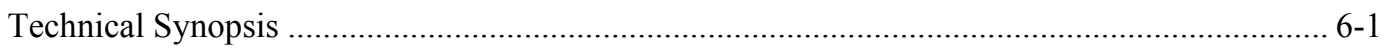

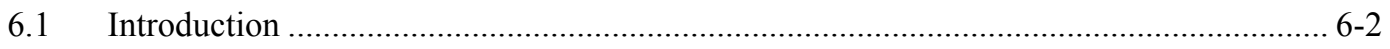

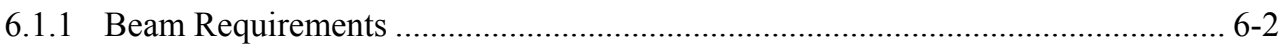

6.1.2 Emittance Compensation................................................................................ 6-4

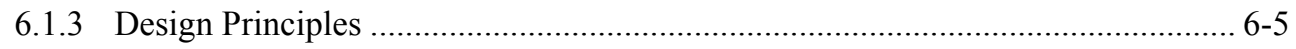

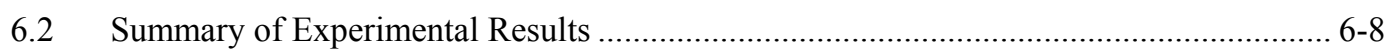

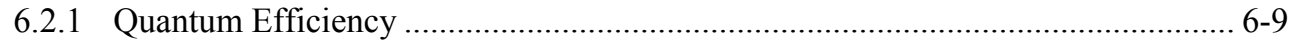

6.2.2 Transverse Emittance Measurements ........................................................... 6-10

6.2.2 Thermal Emittance …….............................................................................. 6-13

6.2.3 Longitudinal Emittance ................................................................................. 6-14

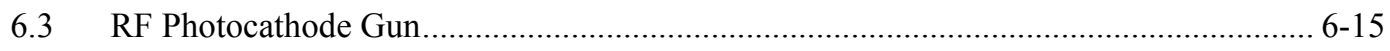

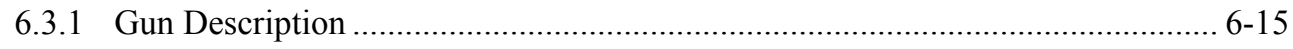

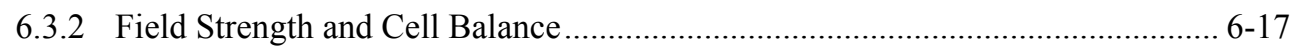

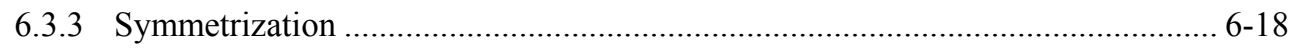

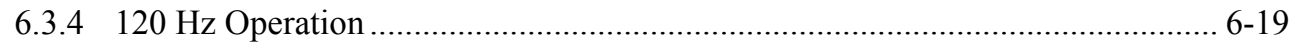

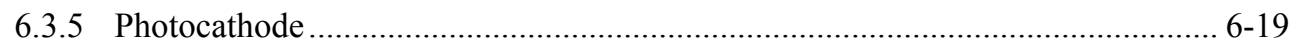

6.3.6 Emittance Compensating Solenoid ................................................................ 6-22

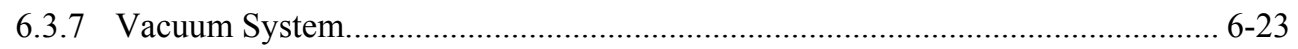

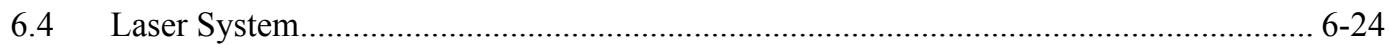

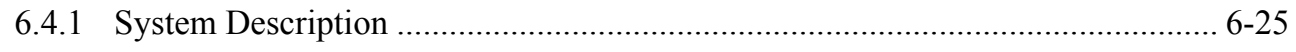

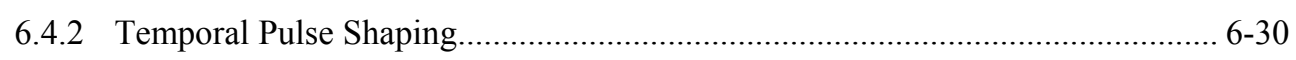

6.4.3 Fourier Relay Optics ................................................................................ 6-31

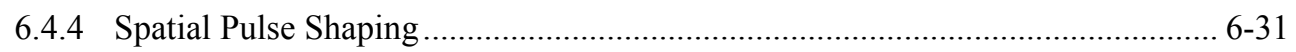

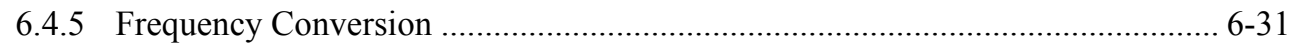

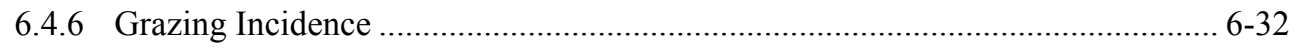

6.4.7 Stability of Laser Pulse .............................................................................. 6-33

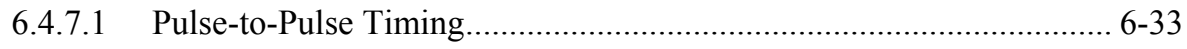

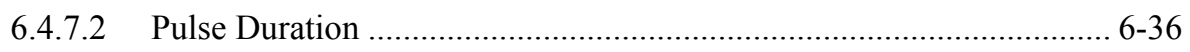

6.4.7.3 Optical Energy ............................................................................ 6-36

6.4.7.4 Spot Size and Position ............................................................. 6-37

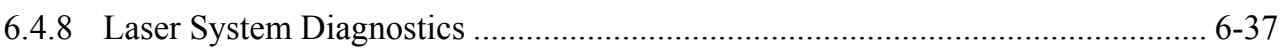

6.4.8.1 Cross-correlation Pulse Shape ……………....................................... 6-37

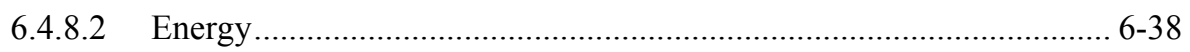

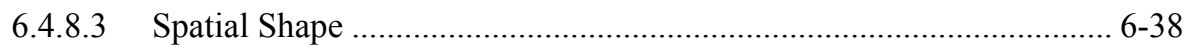

6.4.8.4 Stability of Spot Centroid ................................................................... 6-39 


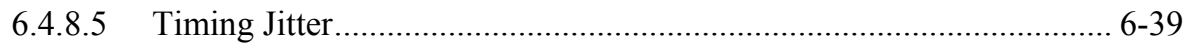

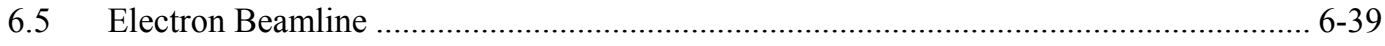

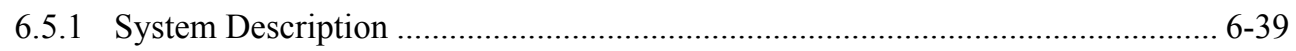

6.5.2 Standard Beamline Diagnostic Devices ………………………………........... 6-43

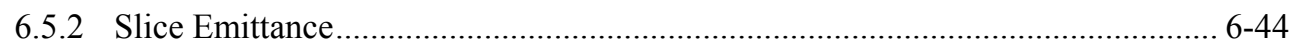

6.5.3 Temporal Pulse Shape .................................................................................. 6-45

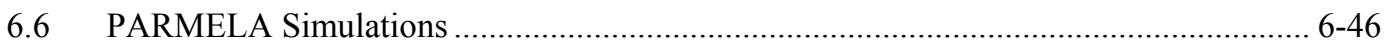

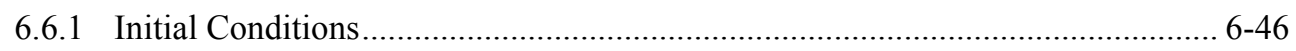

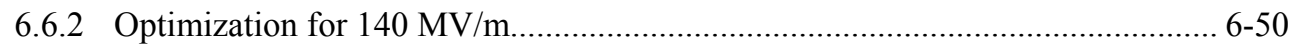

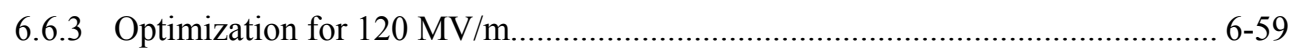

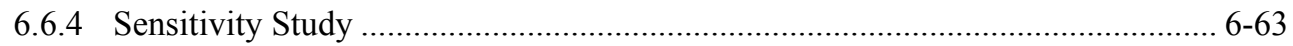

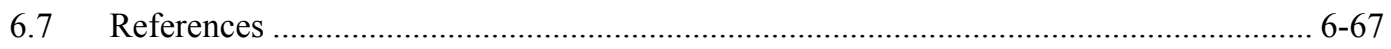

\section{Accelerator}

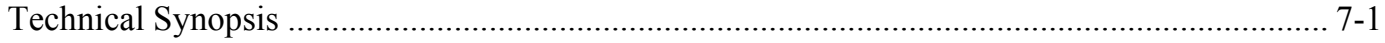

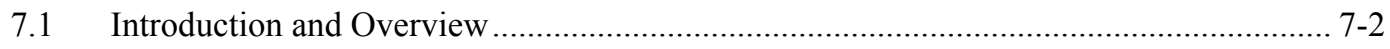

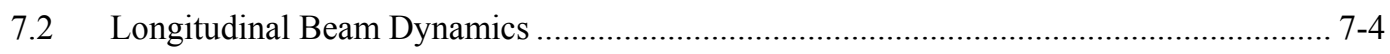

7.2.1 Bunch Compression Overview........................................................................... 7-4

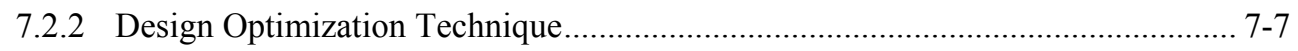

7.2.3 X-Band RF Compensation ............................................................................. 7-8

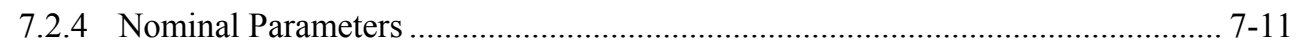

7.2.5 Longitudinal Tracking Summary …………….............................................. 7-13

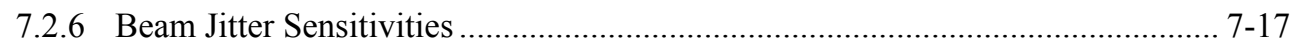

7.2.7 Energy Management and Overhead .............................................................. 7-21

7.2.8 Alternate Parameters ......................................................................................... 7-21

7.2.8.1 Variable Bunch Charge .................................................................... 7-21

7.2.8.2 Electron Chirp to Facilitate X-Ray Pulse Compression....................... 7-23

7.2.8.3 Long Wavelength SASE Radiation ……………............................. 7-25

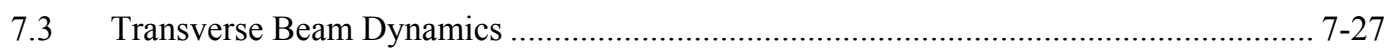

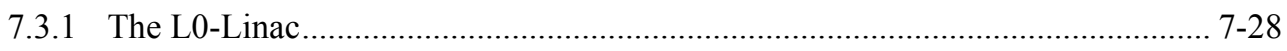

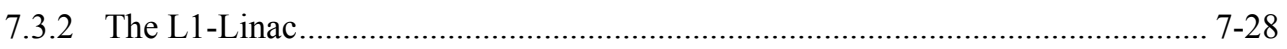

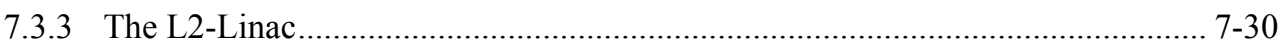

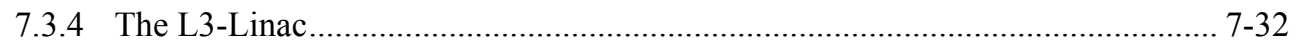

7.4 Electron Bunch Compressors …………………....................................... 7-34

7.4.1 First Bunch Compressor ............................................................................ 7-34

7.4.1.1 Overview and Parameters .................................................................. 7-35

7.4.1.2 Momentum Compaction .................................................................. 7-36

7.4.1.3 Coherent Synchrotron Radiation (CSR) ........................................... 7-37

7.4.1.4 Beam Size, Aperture, and Field Quality ............................................ 7-41

7.4.1.5 Tuning and Correction ................................................................... 7-42

7.4.2 Second Bunch Compressor ...................................................................... 7-43

7.4.2.1 Overview and Parameters ............................................................... 7-45

7.4.2.2 Momentum Compaction .............................................................. 7-47 
LCLS CONCEPTUAL DESIGN REPORT

7.4.2.3 Incoherent Synchrotron Radiation (ISR) ……………….................. 7-47

7.4.2.4 Coherent Synchrotron Radiation (CSR) ............................................. 7-48

7.4.2.5 Resistive Wall Longitudinal Wakefields in the Bends ......................... 7-51

7.4.2.6 Beam Size, Aperture and Field Quality ……………….................... 7-52

7.4.2.7 Tuning and Correction ................................................................... 7-53

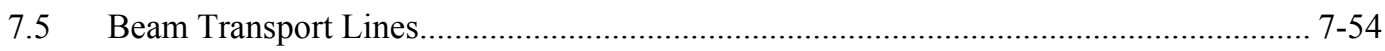

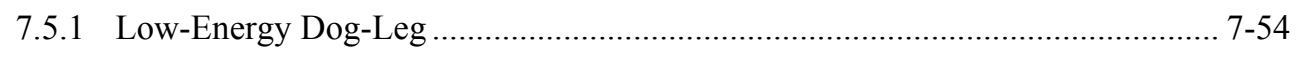

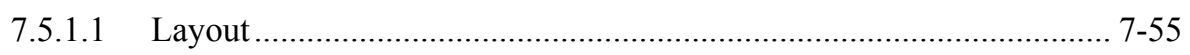

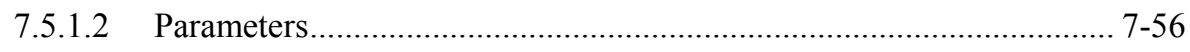

7.5.1.3 Coherent Synchrotron Radiation ..................................................... 7-57

7.5.1.4 Beam Size, Aperture, and Field Quality …….................................. 7-58

7.5.1.5 Tuning and Correction ...................................................................... 7-59

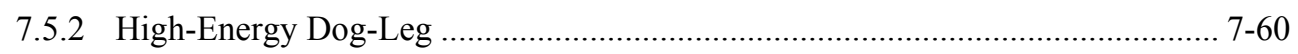

7.5.2.1 Parameters.................................................................................... 7-61

7.5.2.2 Coherent Synchrotron Radiation ................................................... 7-62

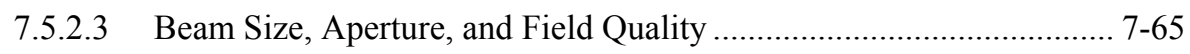

7.5.2.4 Tuning and Correction..................................................................... 7-66

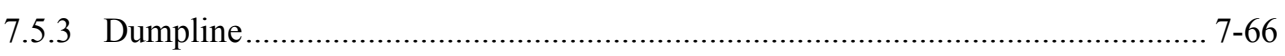

7.5.3.1 Beam Containment and Beam Dump.............................................. 7-68

7.5.3.2 Vacuum Chambers........................................................................... 7-69

7.6 Six-Dimensional Particle Tracking Studies ......................................................... 7-70

7.6.1 Electron Beam Evaluation........................................................................ 7-70

7.6.2 FEL Gain Estimation.............................................................................. 7- 74

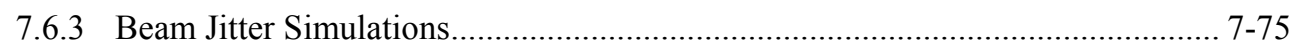

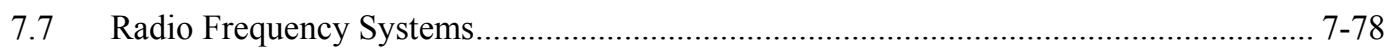

7.7.1 RF Distribution in the Injector and Linac .................................................... 7-79

7.7.2 Layout and Performance of the Present SLAC Linac RF .................................. 7-81

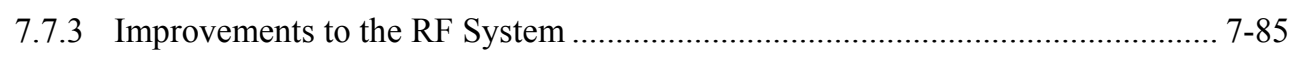

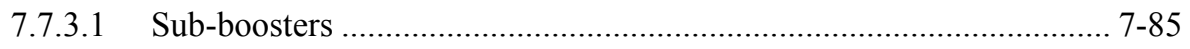

7.7.3.2 Phase and Amplitude Control Units ................................................. 7-85

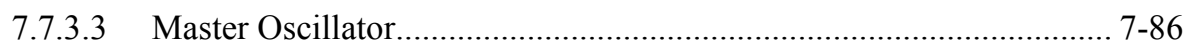

7.7.3.4 Timing System ......................................................................... 7-87

7.7.3.5 Synchronization Pulses for Experiments ........................................... 7-8

7.7.3.6 Beam Diagnostics ....................................................................... 7-88

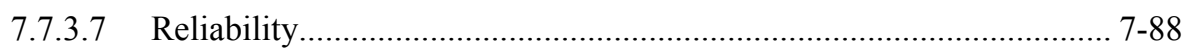

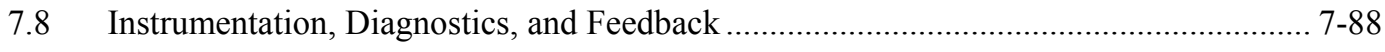

7.8.1 Transverse Emittance Diagnostics …………............................................... 7-89

7.8.1.1 ED0 Emittance Station.............................................................. 7-90

7.8.1.2 ED1 Emittance Station.............................................................. 7-91

7.8.1.3 L2-ED Emittance Station ............................................................ 7-91

7.8.1.4 L3-ED Emittance Station ............................................................. 7-91

7.8.1.5 ED2 Emittance Station.............................................................. 7-91

7.8.2 Bunch Length Diagnostics ........................................................................... 7-92

7.8.2.1 Transverse RF Deflector ............................................................... 7-92 
7.8.2.2 RF Zero-Phasing Technique ........................................................ 7-97

7.8.2.3 Electo-Optical Bunch Length Diagnostic .......................................... 7-98

7.8.3 Beam Energy Spread Diagnostics ......................................................... 7-100

7.8.3.1 DL1 Energy Spread Diagnostics.................................................. 7-100

7.8.3.2 BC1 Energy Spread Diagnostics..................................................... 7-100

7.8.3.3 BC2 Energy Spread Diagnostics................................................. 7-100

7.8.3.4 DL2 Energy Spread Diagnostics................................................... 7-100

7.8.4 Trajectory and Energy Monitors with Feedback Systems.................................. 7-101

7.8.4.1 Trajectory Feedback Systems ......................................................... 7-101

7.8.4.2 Energy Feedback Systems ................................................................ 7-101

7.9 The Wake Functions for the SLAC Linac ………................................................... 7-103

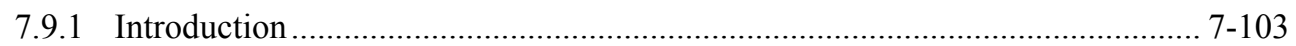

7.9.2 The Calculated Wakefields for the SLAC Linac............................................... 7-103

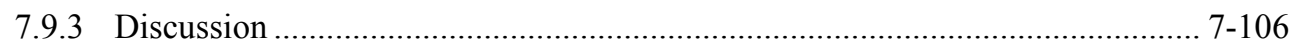

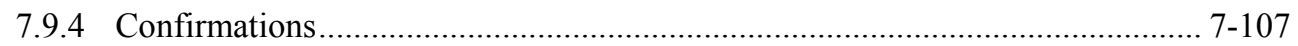

7.9.5 Resistive Wall Wakefields ........................................................................ 7-107

7.10 Parts List and Installation Issues.......................................................................... 7-109

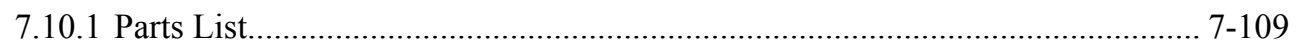

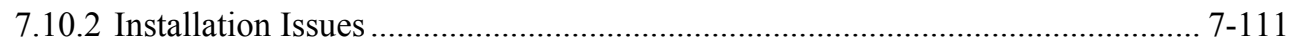



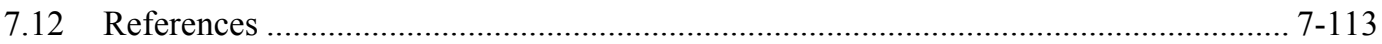

\section{Undulator}

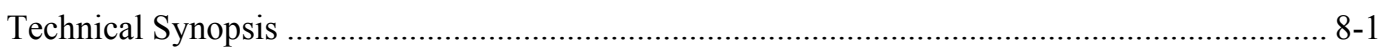

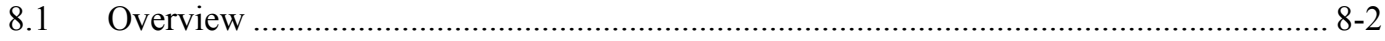

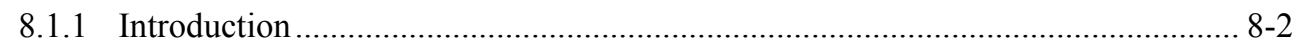

8.1.2 Undulator Line Design Summary ................................................................ 8-3

8.2 Theory and Tolerances for the Undulator................................................................ 8-4

8.2.1 Design of Undulator Segments ..................................................................... 8-4

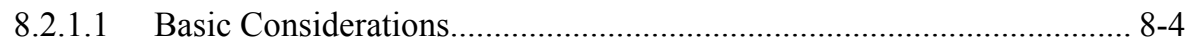

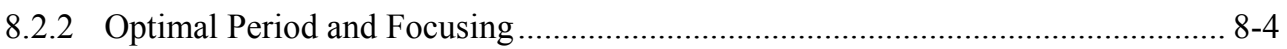

8.2.3 Irregularities and Imperfections …………….................................................... 8-7

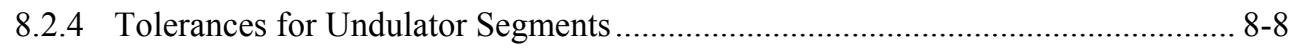

8.2.5 Derivation of the Tolerances for the X-Ray FEL ............................................... 8-9

8.2.5.1 Derivation of Basic Equations ……….............................................. 8-9

8.2.5.2 Trajectory Straightness ............................................................... 8-11

8.2.5.3 Phase Errors ............................................................................. 8-12

8.2.5.4 Resulting Power Gain Length Increase............................................... 8-14

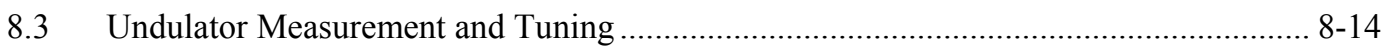

8.3.1 Requirements for the LCLS .................................................................... 8-14

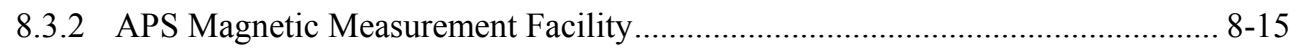

8.3.3 Requirements for Measurement Facility on LCLS Site .................................... 8-16

8.4 Measurement and Sorting of Magnet Blocks ........................................................ 8-16 
LCLS CONCEPTUAL DESIGN REPORT

8.4.1 Characterization of Magnet Blocks with Helmholtz Coils................................ 8-16

8.4.2 Characterization of Magnet Blocks with Half-Period Fixture ............................. 8-18

8.4.3 LCLS Prototype Undulator Segment ………............................................... 8-20

8.5 Magnetic Design for the Undulator …………....................................................... 8-20

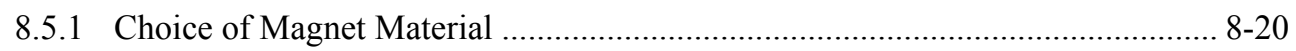

8.5.2 Undulator Magnetic Model .......................................................................... 8-22

8.5.2.1 Two-Dimensional Model ............................................................ 8-22

8.5.2.2 Three-Dimensional Model .............................................................. 8-24

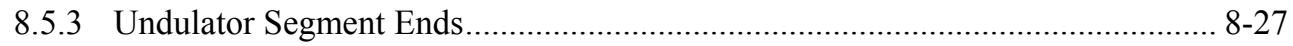

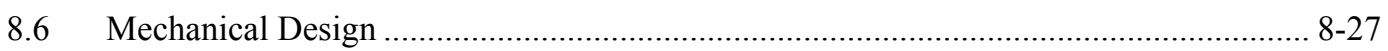

8.6.1 Design of Magnetic Structure ...................................................................... 8-27

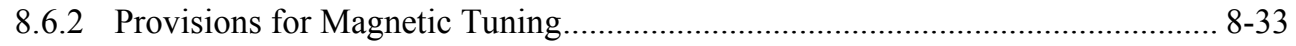

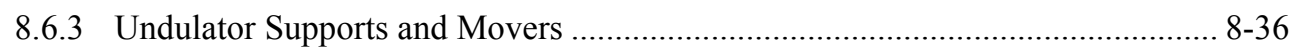

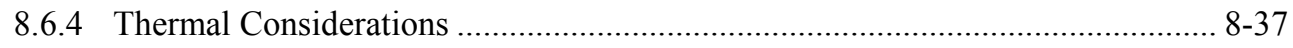

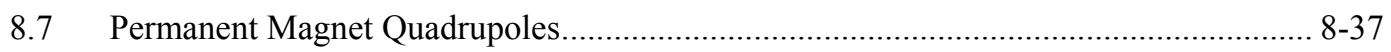

8.7.1 Quadrupole Mechanical Design ...................................................................... 8-38

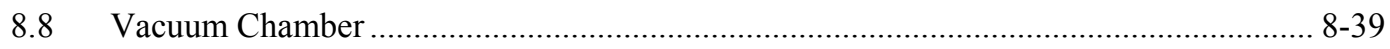

8.8.1 Electron Beam Collimation and Vacuum Chamber Design ............................... 8-40

8.8.1.1 Beam Parameters Used in These Calculations.................................... 8-40

8.8.1.2 Permanent Magnet Material.......................................................... 8-40

8.8.1.3 Undulator Vacuum Chamber .................................................... 8-42

8.8.1.4 Beam Strikes at the Entrance to the Vacuum Chamber ....................... 8-42

8.8.1.5 Beam Strikes Inside the Undulator ...................................................... 8-43

8.8.1.6 Adjustable Collimators to Protect Undulator and Vacuum Chamber .. 8-45

8.8.1.7 Fixed Aperture Protection Collimators .............................................. 8-46

8.8.1.8 Vacuum Chamber Surface Roughness................................................. 8-46

8.8.2 Vacuum System Requirements and Description ........................................... 8-47

8.8.3 Gas Load and Vacuum Pressure................................................................... 8-49

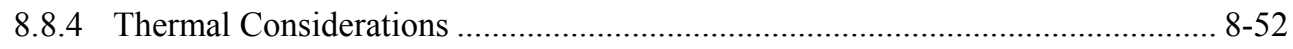

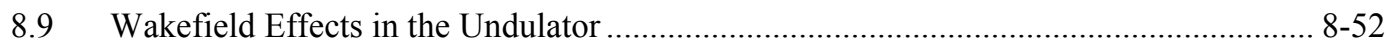

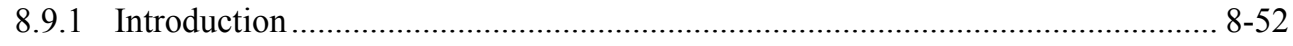

8.9.2 Wakefield Induced Beam Degradation ........................................................ 8-53

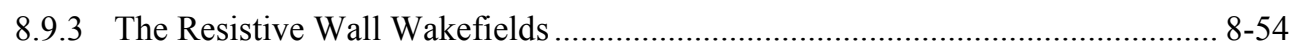

8.9.4 The Effect of Flange Gaps, Pumping Slots, and Bellows ................................... 8-56

8.9.5 The Effect of Wall Surface Roughness ............................................................. 8-57

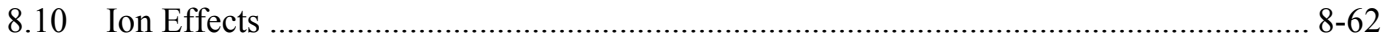

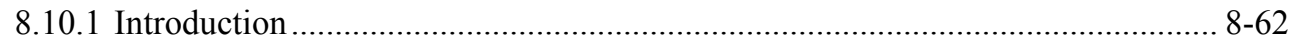

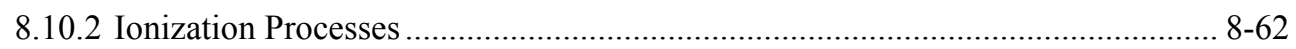

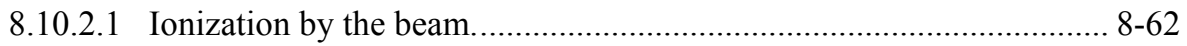

8.10.2.2 Ionization by incoherent synchrotron radiation. .................................. 8-62

8.10.2.3 Tunneling ionization in the coherent laser field. .................................. 8-63

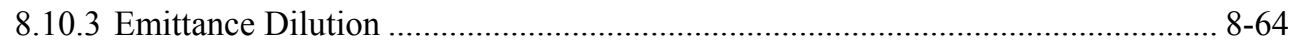

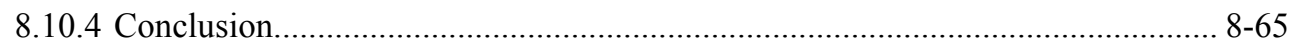

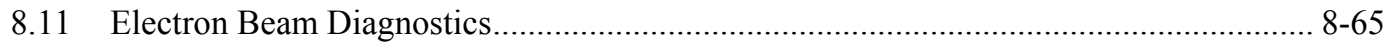

T A B L E OF CONTENTS 
8.11.1 Performance Requirements ...................................................................... 8-65

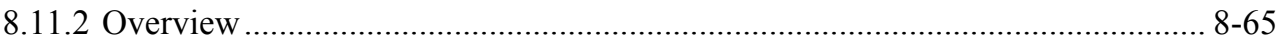

8.11.3 Undulator Beam Position Monitors............................................................... 8-66

8.11.4 Undulator Optical Transition Radiation (OTR) Electron-Beam Profile Monitors 8-68

8.11.5 Cherenkov Detectors .................................................................................. 8-71

8.11.6 Current Monitoring Toroids ............................................................................. 8-71

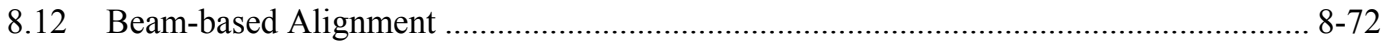

8.12.1 Undulator Beam-Based Alignment ............................................................... 8-72

8.12.1.1 Introduction..................................................................... 8-72

8.12.1.2 Simulation Results ................................................................... 8-75

8.12.1.3 Sensitivities .......................................................................... 8-86

8.12.1.4 Long Term Stability ................................................................. 8-87

8.12.1.5 Summary ............................................................................ 8-8

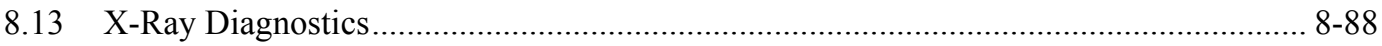

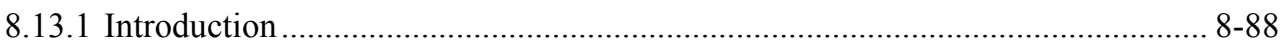

8.13.2 Undulator Cell Structure …........................................................................ 8-8

8.13.3 X-Ray Diagnostics Specifications and Experimental Set-up .......................... 8-89

8.13.4 On-Axis X-Ray Diagnostics....................................................................... 8-90

8.13.5 Off-Axis X-Ray Diagnostics .................................................................... 8-93

8.13.6 Effects of the Electron Beam Emittance ......................................................... 8-94

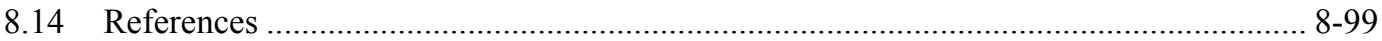

\section{$9 \quad$ X-Ray Beam Transport and Diagnostics}

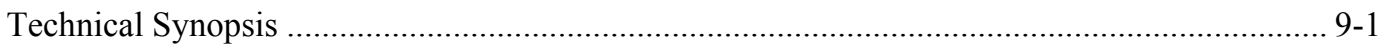

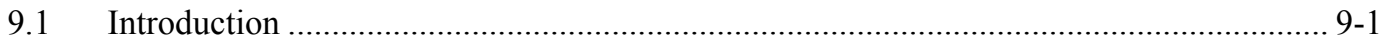

9.1.1 Objectives.............................................................................................. 9-1

9.1.2 General Considerations ............................................................................... 9-2

9.1.2.1 Beam Characteristics ................................................................. 9-2

9.1.2.2 Photon-Induced Damage................................................................. 9-3

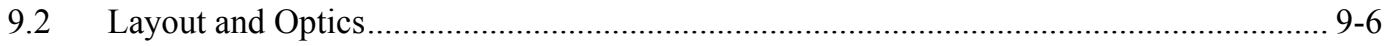

9.2.1 Experimental Halls .................................................................................. 9-6

9.2.2 Optical Enclosures ............................................................................... 9-7

9.2.2.1 Front End Enclosure ................................................................ 9-7

9.2.2.2 Hutch A1 ................................................................................ 9-14

9.2.2.3 Hutch A2 ........................................................................ 9-16

9.2.2.4 Hutch A4 .................................................................................. 9-20

9.2.2.5 Inter-Hall Transport ............................................................ 9-20

9.2.2.6 Hutch B1 ........................................................................... 9-20

9.2.2.7 Hutch B2 .......................................................................... 9-21

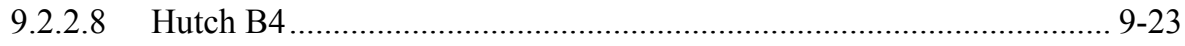

9.3 Mechanical and Vacuum .............................................................................. 9-23

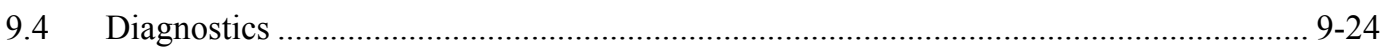

9.4.1 Diagnostics Layout ......................................................................................... 9-24 
LCLS CONCEPTUAL DESIGN REPORT

9.4.2 Facility Diagnostics Instruments ................................................................. 9-25

9.4.2.1 Direct Scintillation Imager............................................................. 9-25

9.4.2.2 Scattering Foil Imager .................................................................... 9-26

9.4.2.3 Micro-Strip Ion Chamber................................................................ 9-27

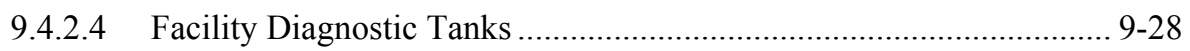

9.4.2.5 Ion Chamber Gas Mixing and Distribution System............................ 9-29

9.4.3 Commissioning Diagnostics ....................................................................... 9-30

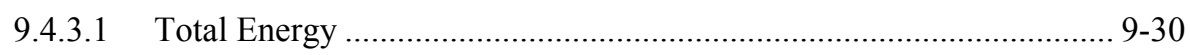

9.4.3.2 Pulse Length ................................................................................ 9-31

9.4.3.3 Photon Spectrum............................................................................. 9-32

9.4.3.4 Transverse Coherence.................................................................... 9-32

9.4.3.5 Spatial Shape and Centroid Location.................................................. 9-33

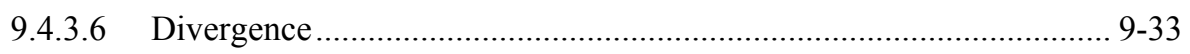

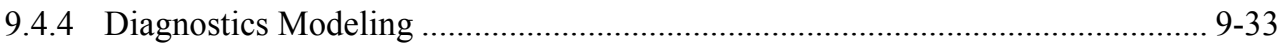

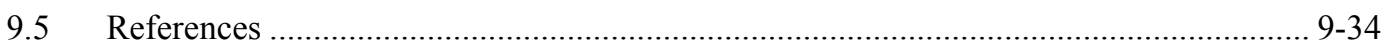

\section{Conventional Facilities}

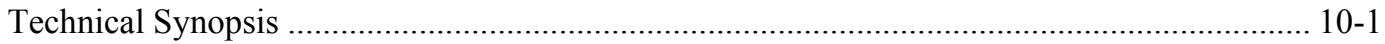

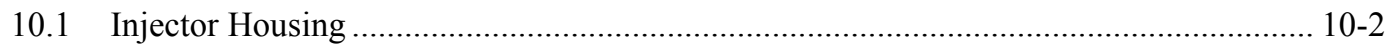

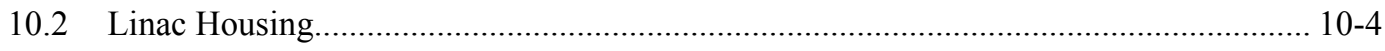

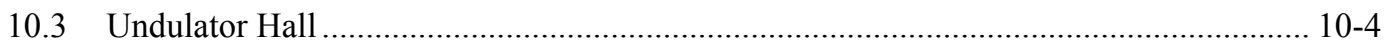

10.4 Experimental Halls ............................................................................................ 10-4

10.1.1 Near Experimental Hall................................................................................ 10-5

10.1.2 Far Experimental Hall ............................................................................... 10-7

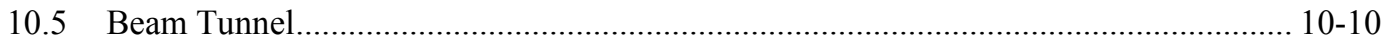

\section{Controls}

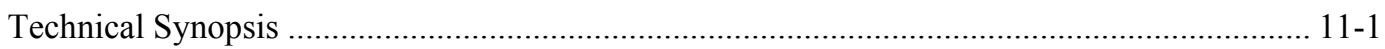

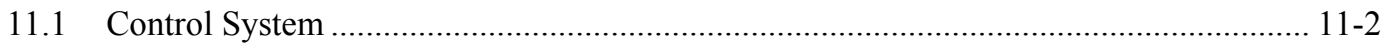

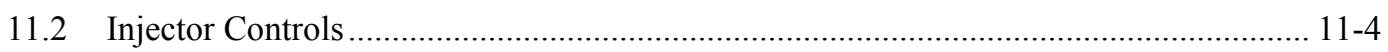

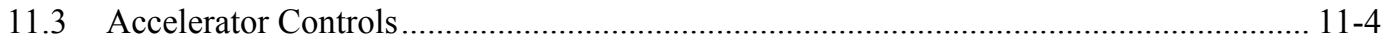

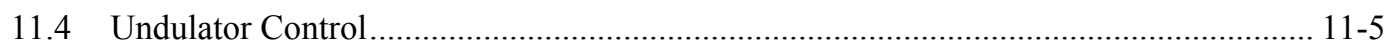

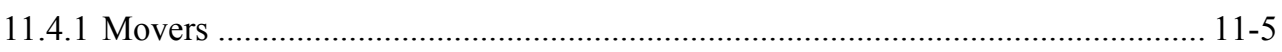

11.4.2 Undulator Diagnostics......................................................................... 11-5

11.5 X-Ray Beam Line Electronics and Controls................................................................

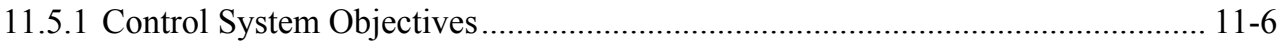

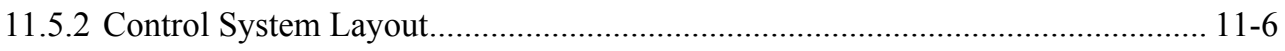

11.5.3 Motion Controls .................................................................................. 11-6

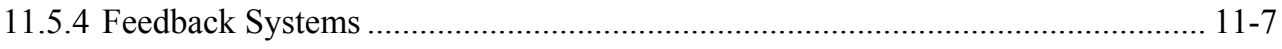

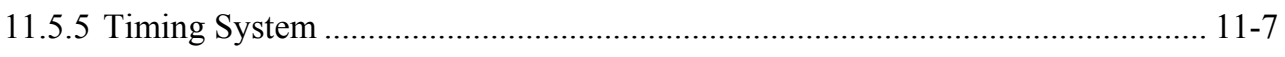



11.6.1 Radiation Safety Systems - Control System.................................................... 11-8

T A B L E OF CONTENTS 
11.6.2 Radiation Safety and Protection Systems Description ................................... 11-8

11.7 Machine Protection System .................................................................................... 11 1-10

\section{Alignment}

Technical Synopsis

12-1

12.1 Procedural Overview ...................................................................................... 12-1

12.2 LCLS Surveying Reference Frame................................................................... 12-2

12.2.1 Network Design Philosophy ..................................................................... 12-3

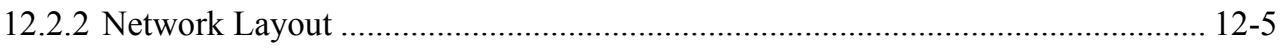

12.2.2.1 Injector Network ..................................................................... 12-5

12.2.2.2 Linac Network ........................................................................ 12-5

12.2.2.3 Undulator Hall Network ........................................................... 12-5

12.2.2.4 Transport Line/Experimental Area Networks................................... 12-8

12.2.3 Alignment Coordinate System ................................................................. 12-9

12.2.4 Tunnel Network Survey .............................................................................. 12-9

12.2.5 Surface Network................................................................................. 12-11

12.2.6 Data Analysis and Data-Flow ................................................................... 12-11

12.3 Layout Description Reference Frame .................................................................. 12-12

12.3.1 Lattice Coordinate System ................................................................... 12-12

12.3.2 Tolerance Lists .................................................................................. 12-12

12.3.3 Relationship Between Coordinate Systems .............................................. 12-12

12.4 Fiducializing LCLS Magnets ........................................................................ 12-13

12.5 Absolute Positioning of Components .................................................................... 12-13

12.5.1 Undulator Absolute Positioning ............................................................ 12-13

12.5.2 Injector, Transport Line and Experimental Area Absolute Positioning ............. 12-14

12.5.3 Quality Control..................................................................................... 12-14

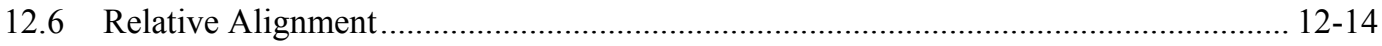

12.6.1 Relative Undulator Alignment .......................................................... 12-14

12.6.1.1 Introduction..................................................................... 12-14

12.6.1.2 Relative Quadrupole Positioning .................................................. 12-14

12.6.1.3 Undulator Alignment ........................................................ 12-15

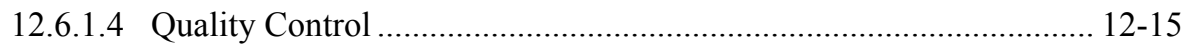

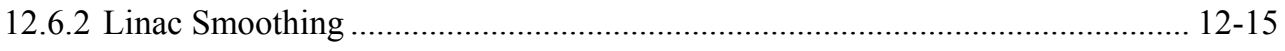

12.6.2.1 Purpose of Linac Smoothing...................................................... 12-15

12.6.2.2 Linac Straightness Measurement Procedure ................................... 12-15

12.6.3 Relative Alignment of Transport Line and Experimental Area Components .... 12-16

12.7 Undulator Monitoring System ........................................................................... 12-16

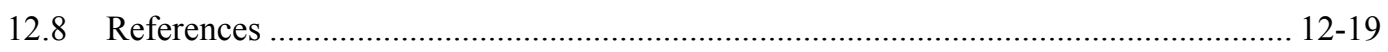

\section{Environment, Safety, and Health and QA}

13.1 Ionizing Radiation 


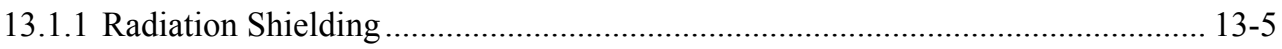

13.1.2 Personnel Protection System ...................................................................... 13-5

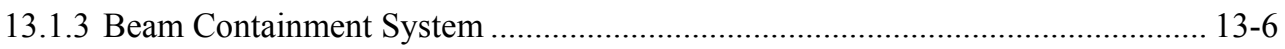

13.1.4 Radiation Safety Training ………............................................................... 13-6

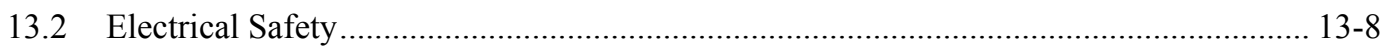

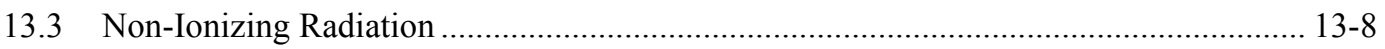

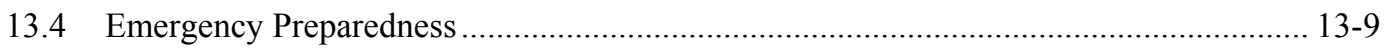

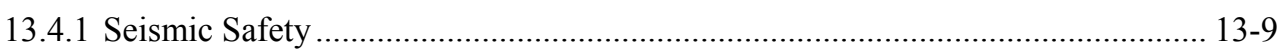

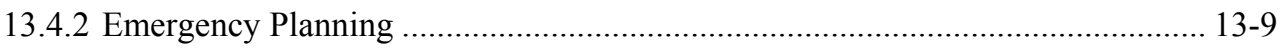

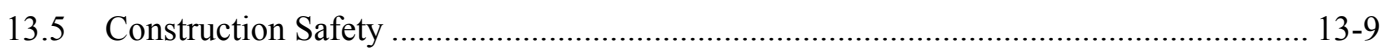

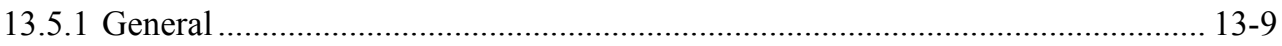

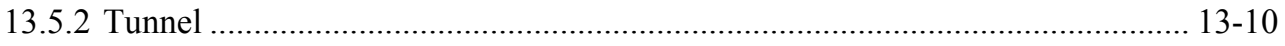

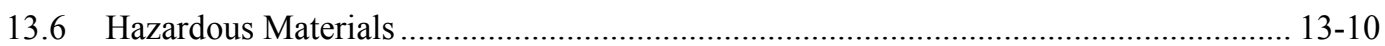

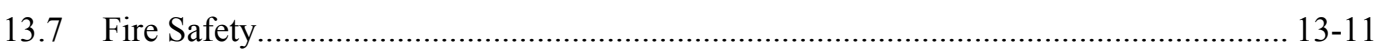

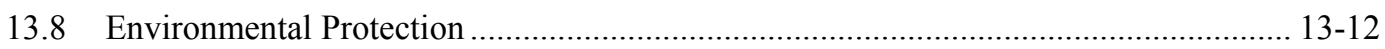

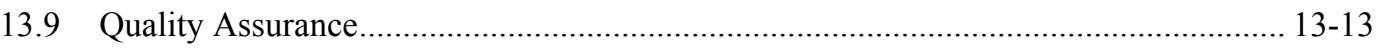

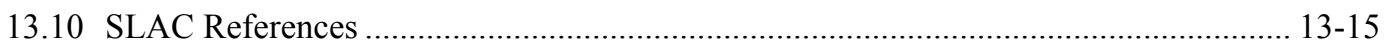

\section{Radiological Considerations}

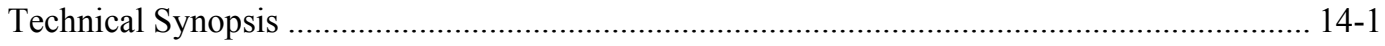

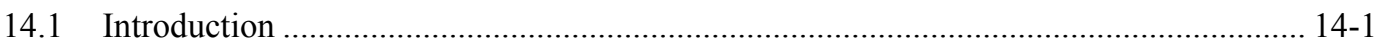

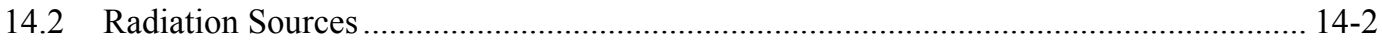

14.2.1 Beam Parameters .................................................................................... 14-2

14.2.2 Bremsstrahlung from Collimators ................................................................. 14-2

14.2.3 Bremsstrahlung from On-Axis Diagnostic X-Ray Stations ................................ 14-5

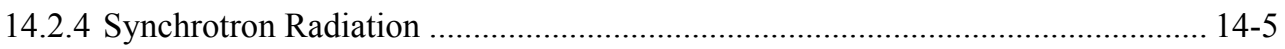

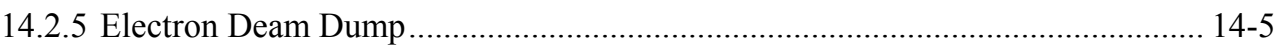

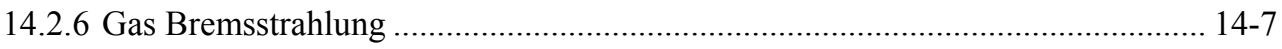

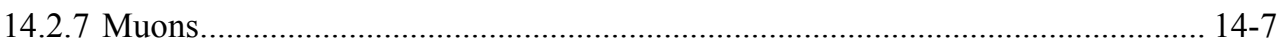

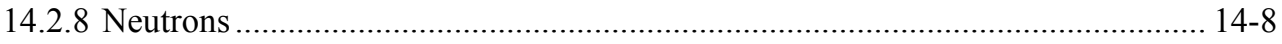

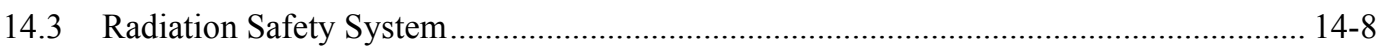

14.3.1 Minimum Shielding Requirements............................................................ 14-12

14.3.2 Beam Containment System ……………................................................... 14-13

14.3.3 Personnel Protection System (PPS) and Hutch Protection System (HPS) ......... 14-14

14.3.3.1 Stoppers .............................................................................. 14-15

14.3.3.2 Burn-Through Monitors.................................................................. 14-15

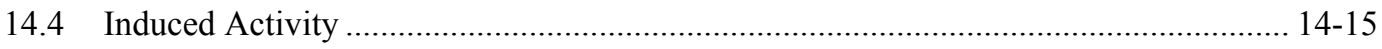

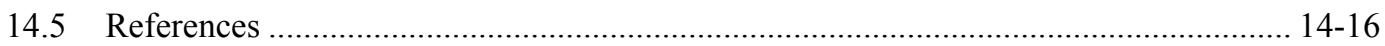

\section{Work Breakdown Structure}

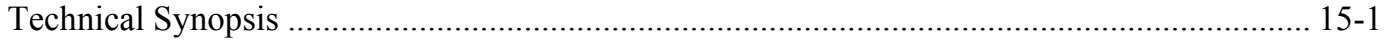

15.1 Level 3 Work Breakdown Structure …………….................................................. 15-1 


\section{A Parameter Tables}

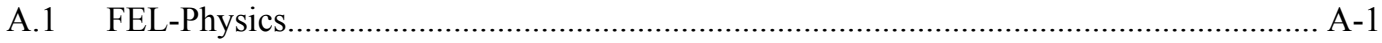

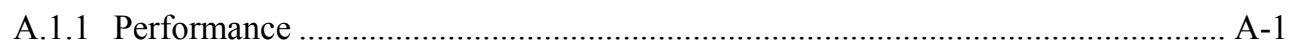

A.1.1.1 Electron Beam.......................................................................... A-1

A.1.1.2 Spontaneous Radiation .............................................................. A-1

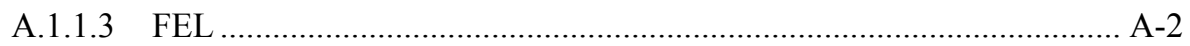

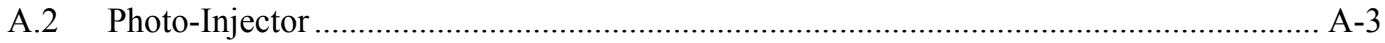

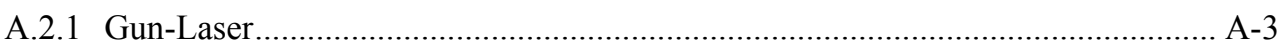

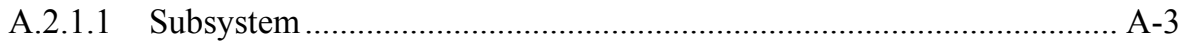

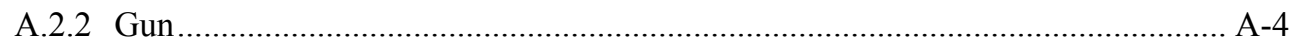

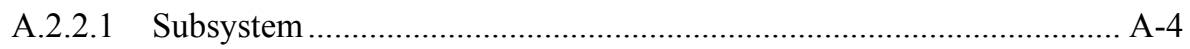

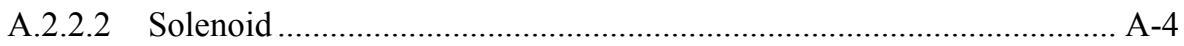

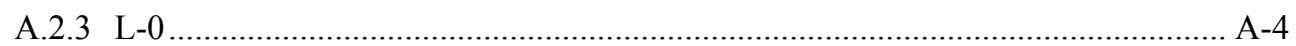

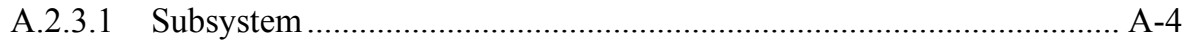

A.2.3.2 Electron Beam.................................................................................. A-5

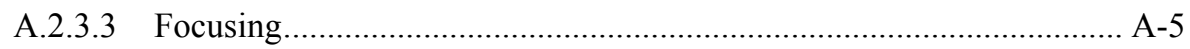

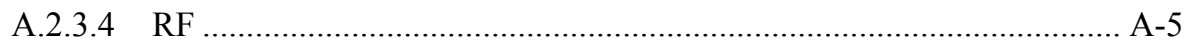

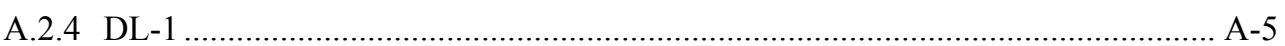

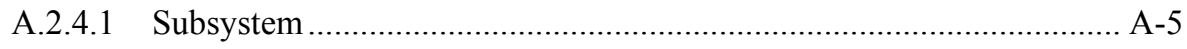

A.2.4.2 Electron Beam........................................................................... A-6

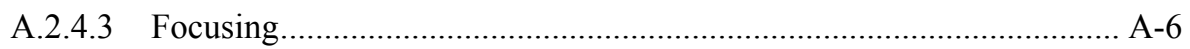

A.2.4.4 Quadrupole Magnets.......................................................................... A-6

A.2.4.5 Dipole Magnets............................................................................... A-6

A.2.4.6 Diagnostics ………..................................................................... A-6

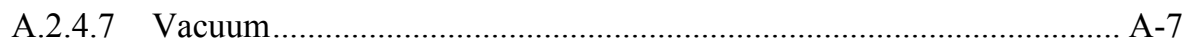

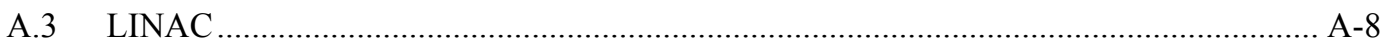

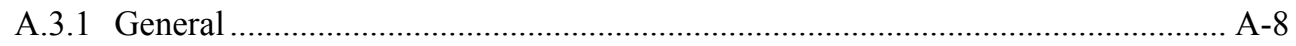

A.3.1.1 S-Band Accelerator Section........................................................... A-8

A.3.1.2 X-Band Accelerator Section …………............................................. A-8

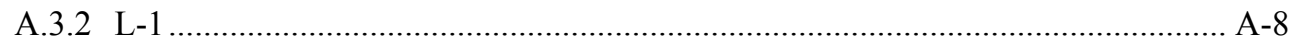

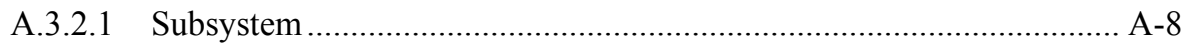

A.3.2.2 Electron Beam.................................................................................... A-9

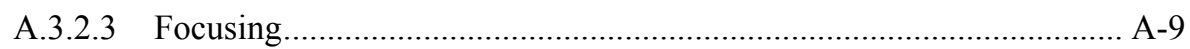

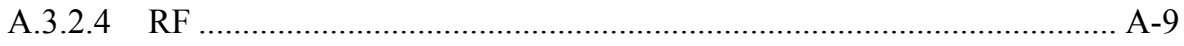

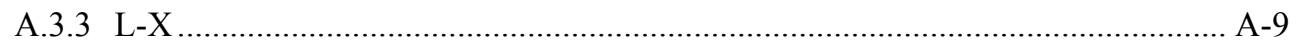

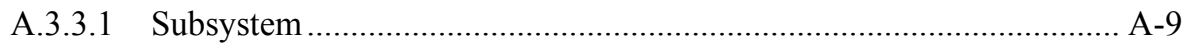

A.3.3.2 Electron Beam................................................................................ A-10

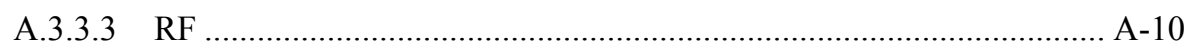

A.3.4 BC-1 …

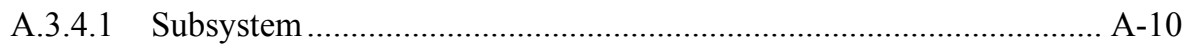

A.3.4.2 Electron Beam.................................................................................. A-11

A.3.4.3 Dipole Magnet …………................................................................ A-11 


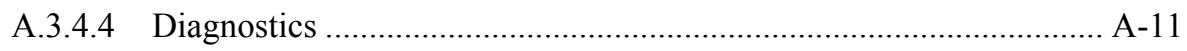

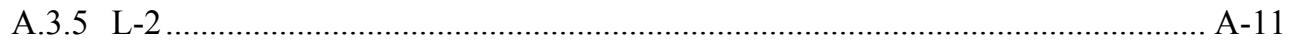

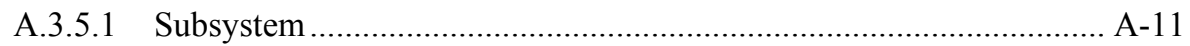

A.3.5.2 Electron Beam......................................................................... A-12

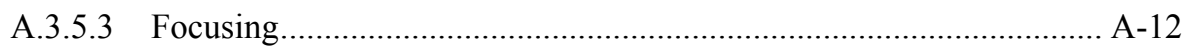

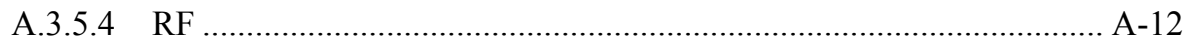

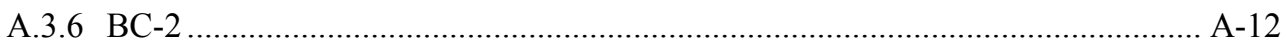

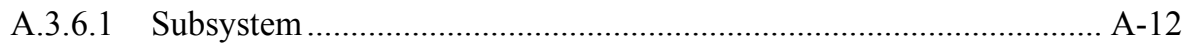

A.3.6.2 Electron Beam.......................................................................... A-13

A.3.6.3 Dipole Magnet ………....................................................................... A-13

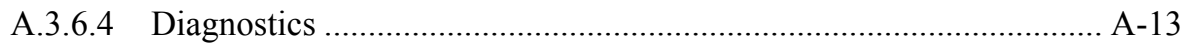

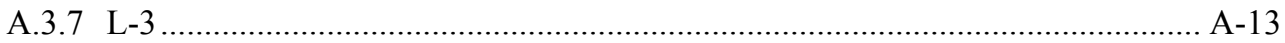

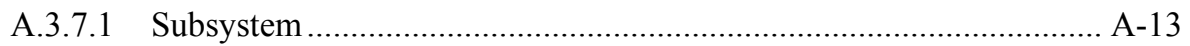

A.3.7.2 Electron Beam......................................................................... A-14

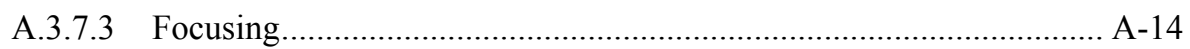

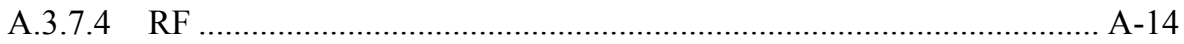

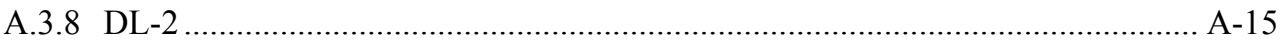

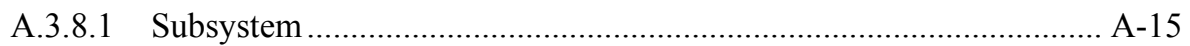

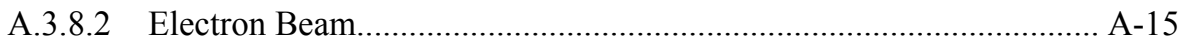

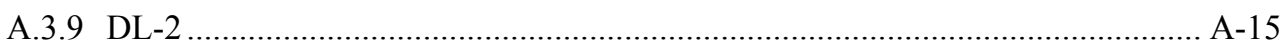

A.3.9.1 Quadrupole Magnet ..................................................................... A-15

A.3.9.2 Diagnostics ............................................................................. A-16

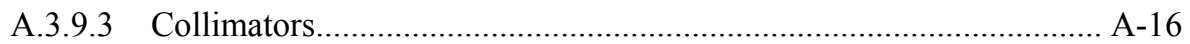

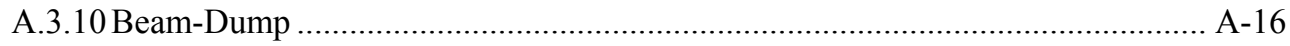

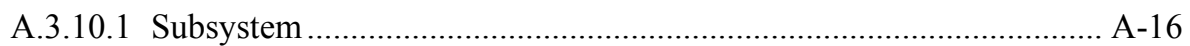

A.3.10.2 Electron Beam........................................................................ A-16

A.3.10.3 Dipole Magnets........................................................................... A-16

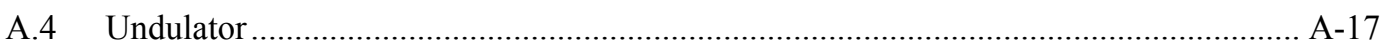

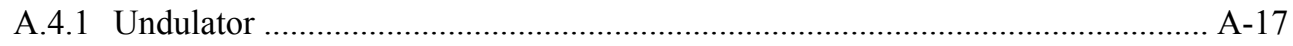

A.4.1.1 Undulator Magnet........................................................................ A-17

A.4.1.2 Electron Beam Optics ………........................................................ A-18

A.4.1.3 Electron Trajectory Correction ……................................................... A-19

A.4.1.4 Beam-Based-Alignment..................................................................... A-19

A.4.1.5 Electron Beam at Entrance........................................................... A-19

A.4.1.6 Electron Beam inside Undulator.......................................................... A-20

A.4.1.7 Vacuum System ...................................................................... A-20

A.4.1.8 Radiation Damage.................................................................... A-20

A.4.1.9 Temperature Stability.............................................................. A-20

A.4.1.10 Static Electron Beam Tolerances .................................................. A-21

A.4.1.11 Pulse-to-Pulse Electron Beam Tolerances ........................................... A-21

A.4.1.12 Undulator Tolerances.................................................................... A-22

A.4.1.13 Segment Alignment Tolerances ................................................... A-23

A.4.1.14 Quadrupole Alignment Tolerances .................................................... A-23

A.4.1.15 BPM Alignment Tolerances .......................................................... A-23 


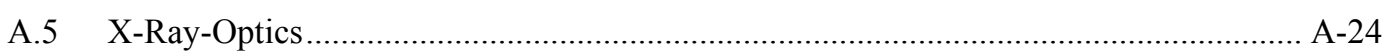

A.5.1 Radiation-Source ............................................................................... A-24

A.5.1.1 FEL Radiation.................................................................... A-24

A.5.1.2 Spontaneous Radiation ................................................... A-24

\section{B Control Points}

B.1 Injector Control Points....................................................................................

B.2 Accelerator Control Points - New Devices .............................................................. B-2

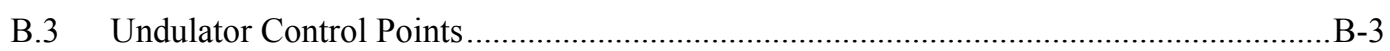

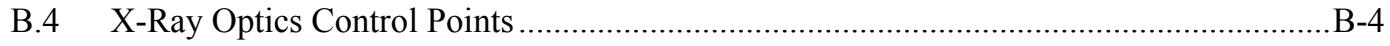

\section{Glossary}




\section{Executive Summary}

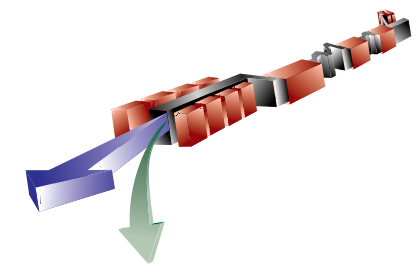

\subsection{Introduction}

The Stanford Linear Accelerator Center, in collaboration with Argonne National Laboratory, Brookhaven National Laboratory, Los Alamos National Laboratory, Lawrence Livermore National Laboratory, and the University of California at Los Angeles, have collaborated to create a conceptual design for a Free-Electron-Laser (FEL) R\&D facility operating in the wavelength range 1.5-15 A. This FEL, called the "Linac Coherent Light Source" (LCLS), utilizes the SLAC linac and produces sub-picosecond pulses of short wavelength x-rays with very high peak brightness and full transverse coherence.

The first two-thirds of the SLAC linac are used for injection into the PEP-II storage rings. The last one-third will be converted to a source of electrons for the LCLS. The electrons will be transported to the SLAC Final Focus Test Beam (FFTB) Facility, which will be extended to house a 122-m undulator system. In passing through the undulators, the electrons will be bunched by the force of their own synchrotron radiation to produce an intense, spatially coherent beam of $\mathrm{x}$-rays, tunable in energy from $0.8 \mathrm{keV}$ to $8 \mathrm{keV}$. The LCLS will include two experiment halls as well as x-ray optics and infrastructure necessary to make use of this x-ray beam for research in a variety of disciplines such as atomic physics, materials science, plasma physics and biosciences. This Conceptual Design Report, the authors believe, confirms the feasibility of constructing an xray FEL based on the SLAC linac.

\subsection{Facilities}

The facility is comprised of the following main elements:

1. A photoinjector and a short linac, where a bright electron beam is generated and accelerated to $150 \mathrm{MeV}$.

2. The main linear accelerator, consisting of the last one-third of the SLAC $3 \mathrm{~km} \mathrm{linac,}$ where the electron bunch is compressed and accelerated to $14.3 \mathrm{GeV}$.

3. The transport system to the undulator.

4. The undulator, where the electrons emit FEL and spontaneous radiation .

5. The undulator-to-experimental area transport line. 
6. Two experiment halls

7. X-ray optics for control of focus, intensity and spectral bandwidth

8. Basic infrastructure for future experiments

\subsection{Capabilities}

The LCLS is an x-ray source with unprecedented brightness and peak power. It will provide pulses of x-rays of duration $230 \mathrm{fs}$ or less, in an energy range $0.8-8 \mathrm{keV}$.

Table 1.1 Main performance characteristics of the Linac Coherent Light Source

\begin{tabular}{lcc}
\hline X-ray beam energy & $\mathbf{0 . 8} \mathbf{~ k e V}$ & $\mathbf{8 ~ k e V}$ \\
\hline FWHM x-ray pulse duration & & $230 \mathrm{fs}$ \\
\hline X-ray peak power & $10 \mathrm{GW}$ & \\
\hline Max. pulse repetition rate & & $\mathrm{GW}$ \\
\hline
\end{tabular}

In average brightness it will match or exceed existing storage ring-based sources. In peak brightness, it will surpass existing sources by a factor $10^{10}$.

\section{$1.4 \quad$ Cost \& Schedule}

The Total Estimated Cost of the LCLS is in the range \$165M-\$225M. The Total Project Cost is in the range $\$ 185 \mathrm{M}-\$ 245 \mathrm{M}$. A three-year construction schedule is proposed.

\subsection{Acquisition Strategy}

The lead contractor for acquisition of the Linac Coherent Light Source is Stanford University, which operates the Stanford Linear Accelerator Center. SLAC will collaborate with two national laboratories (Argonne National Laboratory and Lawrence Livermore National Laboratory) to construct the LCLS. 


\section{Overview}

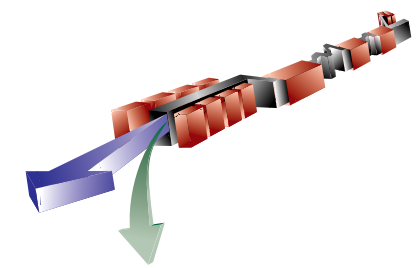

\subsection{Introduction}

The $\mathrm{x}$-ray research community has become accustomed to exponential increases in performance parameters of synchrotron light sources since the construction of the first dedicated facilities. Each stepwise increase in performance was initially perceived as revolutionary. Indeed, after their initial impact, the successive generations of x-ray sources have become indispensable tools for research in chemistry, materials science, biology and environmental sciences. The immediate and sustained nature of this impact was assessed in the 1984 Seitz-Eastman Report [1] to the National Research Council and, thirteen years later, in the Birgeneau/Shen Report [2] to the DOE Basic Energy Sciences Advisory Committee. The latter report states that:

"...the advent of synchrotron radiation sources over the last three decades...has led to a genuine scientific revolution."

In three decades, the average brightness of synchrotron sources has improved by about a factor $10^{10}$. This Conceptual Design Report proposes the construction of the Linac Coherent Light Source, the next major step in light source capability: an x-ray free-electron laser. In peak brightness, it will surpass existing sources by a factor of $10^{10}$. The Birgeneau/Shen Report [2] cited the scientific promise of an x-ray free electron laser, and recommended that DOE-BES allocate funds to $4^{\text {th }}$-generation source $R \& D$. In response to this recommendation, BESAC charged a subpanel chaired by Steven R. Leone to assess the scientific opportunities offered by new coherent light sources and to propose a research and development plan for novel coherent sources. The Leone Committee Report [3] stated that

"Given currently available knowledge and limited funding resources, the hard X-ray region (8-20 keV or higher) is identified as the most exciting potential area for innovative science. DOE should pursue the development of coherent source technology in the hard X-ray region as a priority. This technology will most likely take the form of a linac-based free electron laser device using self-amplified stimulated emission or some form of seeded stimulated emission."

At the time of the Leone Committee report, the Linac Coherent Light Source concept had been under development for nearly seven years by SLAC scientists, in collaboration with experts at UCLA, the Brookhaven National Laboratory, the Los Alamos National Laboratory, and the Lawrence Livermore National Laboratory. Argonne National Laboratory scientists joined the collaboration in 1999. The Leone Committee endorsed the multi-institutional nature and the 
mission of this collaboration. In response to the Leone Committee recommendations, DOE-BES has provided \$1.5M per year since 1999 for research and development of the LCLS concept.

The Leone Committee also stated that:

“... the scientific case for coherent hard x-ray sources is in the formative stages and appears extremely promising, but must be improved to attain a more compelling and rigorous set of experiments that can be achieved only if such a new coherent light source becomes available."

This recommendation was acted upon by the LCLS Scientific Advisory Committee, which took on the task of identifying and developing specific concepts for experiments at the LCLS. This committee, chaired by Gopal Shenoy and Jo Stöhr, created a report entitled "LCLS - The First Experiments" [4]. The report described six experiment plans, in diverse areas of science that exploited the extraordinary properties of the LCLS beam. Based on the BESAC review of this report, as well as on input gathered from the scientific community through workshops such as the May 2001 Basic Energy Sciences Workshop on Scientific Applications of Ultrashort, Intense, Coherent X-Rays, the DOE Office of Science approved Critical Decision 0, Approval of Mission Need, for the Linac Coherent Light Source, on 13 June 2001. Critical Decision 0 was the authorization for the creation of this Conceptual Design Report.

The First Experiments document provides three key insights into the scientific potentials of the LCLS. First, it is clear that, like existing synchrotron light sources, the LCLS will be a powerful tool for research spanning the physical and life sciences. The six examples were chosen to illustrate the breadth of opportunity:

- Atomic physics

- Plasma physics

- Structural studies on single particles and biomolecules

- Femtosecond chemistry

- Studies of nanoscale dynamics in condensed matter physics

- X-ray laser physics

Second, it is clear that the short duration of the LCLS pulse (230 fs and shorter) is of crucial importance to certain areas of science. The LCLS will provide the opportunity to observe atomic states and molecular structure on time scales characteristic of the processes of atomic transition, chemical bond formation and breaking, and transitions in condensed matter structures. With a sufficiently short pulse the LCLS can, in effect, function as a stroboscopic flash for freeze-frame photography of atomic, molecular and nanoscale structures as they evolve.

Third, it is clear that, as diverse as the scientific opportunities may be, it is possible to discern much commonality in the instrumentation requirements for LCLS experiments. It will be necessary to provide:

- Controlled attenuation 
- Filtering

- Monochromatization

- Focused beams

- Synchronization of the LCLS beam to a pump laser

- X-ray beam splitters with adjustable time delay

- $120 \mathrm{~Hz}$ x-ray detectors with large area and high angular resolution

For this reason, the scope of the LCLS Project also includes the development of the above listed prototypical capabilities and techniques, spanning the $0.8-8 \mathrm{keV}$ operating range of the facility. After characterizing the first pulses of SASE radiation from the LCLS, the "0th experiments" will be the performance characterization of optics and instrumentation developed as part of the Project. Chapter 3 of this report gives an overview of the proposals included in the First Experiments document and provides motivation for the selection of instrumentation to be included in the Project. Chapter 9 of this report describes the suite of x-ray diagnostics and prototypical instrumentation that will be included in the scope of the Project.

This Conceptual Design Report proposes to modify the SLAC Linac and associated facilities to create a Free-Electron Laser (FEL), the Linac Coherent Light Source (LCLS), capable of delivering coherent radiation of unprecedented characteristics at wavelengths as short as $1.5 \AA$. At its inception, the Stanford Synchrotron Radiation Laboratory shared the SPEAR Storage Ring as it was operated for high-energy physics experiments. Likewise, the LCLS will be integrated with the SLAC Two-Mile Accelerator, which will continue to support ongoing programs in particle physics and accelerator R\&D. The upstream 2/3 of the SLAC linac will be used concurrently for injection to the PEP-II B-Factory. The last one-third of the linac will be converted to a shared but independently operable 4-15 GeV electron linac. Construction of a dedicated linac for the LCLS would add about $\$ 300 \mathrm{M}$ to the Total Estimated Cost, more than doubling its price. SLAC management has pledged that $75 \%$ of the operating time of the last third of the linac will be available for operation of the LCLS.

The LCLS is based on the Self-Amplified Spontaneous Emission (SASE) principle, described in Chapter 4 of this report. Its design makes use of up-to-date technologies developed for the SLAC Linear Collider Project and the next generation of linear colliders, as well as the progress in the production of intense electron beams with radio-frequency photocathode guns. These advances in the creation, compression, transport and monitoring of bright electron beams make it possible to base the next (fourth) generation of synchrotron radiation sources on linear accelerators rather than on storage rings. 


\subsection{Technical Objectives and Mission}

\subsubsection{Design Goals}

The synchrotron radiation output of the LCLS is crucially dependent on the properties of the electron beam, which must be controlled throughout the acceleration process to ensure that the SASE process can be initiated and brought to saturation. However, it is possible to vary the electron beam characteristics in a linac-based light source over a much wider range than is the case for a storage ring. Thus, the properties of SASE radiation can be varied over a much wider range than in any given storage ring light source. In a linac-based source, there is much greater freedom to control bunch length, emittance, energy spread and peak current than in a storage ring.

In operation, the LCLS will explore the full range of its operating capabilities to produce $\mathrm{x}$ ray beams best suited to the needs of its community of users. However, to enable the coordinated planning of experiments for the LCLS, it is necessary to set well-defined parameters for its x-ray beams. A comprehensive list of design goals may be found in chapters 3 and 5 of this report. The prime performance characteristics of the SASE radiation are listed below in Table 2.1:

Table 2.1 Prime performance characteristics of SASE radiation.

\begin{tabular}{|c|c|c|}
\hline X-ray beam energy & $0.8 \mathrm{keV}$ & $8 \mathrm{keV}$ \\
\hline FWHM x-ray pulse duration & & \\
\hline X-ray peak power & $10 \mathrm{GW}$ & $8 \mathrm{GW}$ \\
\hline Max. pulse repetition rate & & \\
\hline
\end{tabular}

The LCLS is not limited to the range of pulse lengths and peak powers listed above. Chapter 4 describes the range of operating modes and performance characteristics that have been explored to date. It must be remembered that the power levels listed above are for the radiation produced in the SASE process. The LCLS beam will also produce copious spontaneous synchrotron radiation. Within the opening angle and bandwidth of the FEL radiation, the spontaneous radiation power is negligible. However, integrated over its full opening angle and spectral range, the peak spontaneous radiation power is $92 \mathrm{GW}$.

\subsubsection{Shared Use of the Linac}

- The LCLS will operate without interfering with injection to PEP-II. This requirement has no impact on the LCLS design.

- The LCLS will not prevent $50 \mathrm{GeV}$ operation of the linac. It must be possible to switch the linac from LCLS operations to $50 \mathrm{GeV}$ operations in 24 hours. 
- The LCLS operation will be compatible with transport of a $30 \mathrm{GeV}$ beam through the last $1 / 3$ of the linac, by rapid resetting of alternate operating parameters from the main control room.

- Up to $25 \%$ of the annual linac operating schedule may be dedicated to uses that preclude LCLS operation.

\subsection{Alternatives Analysis}

The purpose of an alternatives analysis is to choose the most efficient, cost effective path to the desired goal, a coherent $8 \mathrm{keV}$ x-ray beam. Evaluation of alternatives may be made in terms of the three components of a project baseline: technical performance, cost and schedule. The most compelling argument for construction of an $8 \mathrm{keV}$ x-ray laser based on the SLAC linac is the existence and availability of the SLAC linac itself, and the staff and infrastructure of the Stanford Linear Accelerator Center.

\subsubsection{Cost}

The SLAC site is the best choice among alternative sites for the LCLS because it makes use of a portion of the two mile linac as the source of a high-quality electron beam for the LCLS freeelectron laser. There is no other linac or synchrotron in the world capable of providing a $14 \mathrm{GeV}$ electron beam with properties suitable for the LCLS. Duplication of the SLAC linac facilities to be used for the LCLS would cost more than \$300M. Duplication of the core competencies and support staff necessary to operate the linac (required for other programs at SLAC) would incur significant additional annual expenditures beyond the operating cost of the LCLS.

\subsubsection{Schedule}

Early access to the extraordinary capabilities of the LCLS is extremely important in terms of the scientific opportunities that the facility will offer. Early access is equally important to planning the future of synchrotron radiation research over the next 20-30 years. The LCLS can produce first laser beams at the end of FY2007, at least five years before any other planned hard $\mathrm{x}$-ray lasers can be brought on line.

\subsubsection{Technical}

The SLAC linac technical performance is very well characterized. Risks associated with the operation of the linac itself are very low. Technical risks associated with undulators and beam lines are neither reduced nor increased by use of the SLAC linac for LCLS.

\subsection{Project Schedule}

The cost estimate is based on a three-year construction schedule, FY2005-2007. Major procurements for the undulator modules, injector and experiment halls can be placed as soon as 
construction funds are allocated if the Project Engineering Design funds are allocated as requested in FY2003-2004.

Major milestones for this project schedule are:

Table 2.2 Major project schedule milestones

\begin{tabular}{ll}
\hline Milestone & Date \\
\hline Project start & October 2004 \\
\hline Near Hall construction award & February 2005 \\
\hline Undulator first article received & January 2006 \\
\hline First beam from the injector to the main linac & June 2006 \\
\hline Near Hall beneficial occupancy, start installation & October 2006 \\
\hline Undulator delivery 50\% complete & October 2006 \\
\hline Far Hall beneficial occupancy, start installation & November 2006 \\
\hline First beam through bunch compressor 2 & April 2007 \\
\hline Start commissioning laser & May 2007 \\
\hline Undulator deliveries complete & June 2007 \\
\hline Project completion & September 2007 \\
\hline
\end{tabular}

The milestones quoted above are placed to provide approximately 3 months "float" in the schedule. The schedule "float" throughout is strongly dependent upon contract award dates in the first year of the project. The critical path for project completion is determined by the rate of delivery of undulators, assumed to be two per month. It is assumed that construction of both experiment halls can be awarded in the first year. However it should be kept in mind that, since the project begins with a two-year PED effort, complete bid packages can be released at the start of construction. Because of these PED funds and the fact that the LCLS project is a multilaboratory collaboration, funds can be committed rapidly from the very start of the project. With proper planning, commissioning of the FEL may begin before the last undulator is installed.

A four-year construction schedule has also been considered. The most attractive alternative is to build injector, linac, undulator systems and FFTB extension on a three-year schedule as outlined above. Commitment of funds to construction of the experiment halls would be delayed one year. FEL commissioning would continue through the fourth year of the project. After one year of FEL commissioning, reliability and stability of the laser will be well-understood, and commissioning of the x-ray beam lines may go more smoothly. 


\subsection{Cost Estimate}

The R\&D costs for the LCLS have been \$6M, prior to authorization of PED funds.

The estimated TEC range is $\$ 165 \mathrm{M}-\$ 225 \mathrm{M}$, and the TPC range is $\$ 185 \mathrm{M}-\$ 245 \mathrm{M}$.

\subsection{Funding Requirements}

\subsubsection{R\&D}

In the years F1999-2002, the abovementioned R\&D funds enabled the following activities:

- Experimental investigations of the SASE process

- Experimental investigation of rf gun performance

- RF photocathode gun design

- High quantum efficiency cathode fabrication

- Construction of a prototype LCLS undulator

- Theoretical investigations of the SASE process

- Theoretical and numerical calculations of the effects of coherent synchrotron radiation

- Computation of tolerances for magnet alignment, rf fields, magnet fields, etc.

- Numerical computation of damage thresholds for x-ray optics

- Experimental verification of computed damage thresholds of x-ray optics materials

- Tests of fabrication techniques for reflective and transmissive optics for the $\mathrm{x}$-ray beam

\subsubsection{Project Engineering Design and Construction}

Completion of the LCLS construction project in three years requires a nearly flat funding profile in the first two years. In the first year of the project, funds must be committed to both experiment halls, the undulator and the injector. This in turn requires $\$ 3 \mathrm{M}$ PED for the injector and a similar amount for the conventional construction in FY2004. A total of \$33.5M for PED was forecast in the supporting documentation for Critical Decision 0.

\subsubsection{Startup}

For either a three- or a four-year construction schedule, injector and linac commissioning begin in FY2006. Since FEL commissioning occurs late in FY2007, startup funding requirements are set to support linac commissioning activities for most of the year. 
On a four-year schedule, additional startup funds to support commissioning of the FEL and xray beam lines are required throughout FY2008. Though this increases the TPC, the increase is, for practical purposes, cancelled by the delay in the start of operating funds until FY2009.

\subsection{Risk Assessments and Strategies}

In the years since the start of R\&D funding for the LCLS, technical risks associated with the feasibility and success of an x-ray laser have been reduced considerably as a result of improved theoretical understanding of free-electron lasers along with several very successful and thorough experimental investigations of the SASE process. Several SASE FELs have demonstrated high gain and saturation at wavelengths ranging from $10.6 \mu \mathrm{m}$ to $90 \mathrm{~nm}$ and below. Recent results are presented in Chapter 4. A list of the major physics risk elements follows.

\subsubsection{Technical Risks}

\subsubsection{Performance of Photocathode Guns}

PARMELA results predict that slice emittances less than $1 \mathrm{~mm}$-rad will be produced by the LCLS gun. This prediction has been confirmed in computer simulations, performed by several groups using a wide variety of computational tools; the TESLA FEL design is based on achievement of slice emittance $0.8 \mathrm{~mm}-\mathrm{mrad}$, as predicted in simulations with HOMDYN, ASTRA and MAFIA. Achievement of LCLS design goals is based on achievement of a slice emittance of $1.2 \mathrm{~mm}-\mathrm{mrad}, 50 \%$ larger than predictions. Measurements of gun performance have been made at the Gun Test Facility (GTF) at SLAC, under conditions approaching those to be used in the LCLS. Agreement between emittances measured at the GTF and predictions of computer codes such as PARMELA has been very good. PARMELA results predict that, if matched to the LCLS today, the GTF gun would provide an electron beam at the entrance of the undulator that would reach saturation power near $1 \mathrm{GW}$ with a 140 -fs pulse duration.

\subsubsection{Acceleration and Compression}

It is necessary to accelerate electrons in the LCLS to $14.35 \mathrm{GeV}$ and, by means of dogleg and chicane bunch compressors, increase the peak current in the bunch to 3,400 A. This must be done while avoiding dilution of the beam emittance by Coherent Synchrotron Radiation (CSR) effects in the bend magnets of the compressors. Great progress has been made in theoretical, numerical and experimental investigation of CSR during the past year [5]. Based on this progress, the LCLS bunch compressor designs have been optimized to avoid microwave instability effects. A superconducting wiggler has been added to the LCLS design, upstream of the second bunch compressor. Computer codes, used to predict CSR effects on LCLS performance, have been benchmarked against codes written at three other laboratories. The agreement is good, with the LCLS codes providing slightly more pessimistic results than TraFiC4, the "particle-in-cell" code used to design the TESLA FEL. Start-to-end simulations of the LCLS predict that the LCLS will reach its design power output with a $1 \mathrm{nC}$ current pulse. 


\subsubsection{Undulator}

A prototype of the LCLS undulator module has been constructed and measured at Argonne National Laboratory. The field quality meets LCLS requirements and the measured peak field is $8 \%$ better than LCLS requirements. Prototype magnet movers have been built and are in testing. The prototype work carried out to date has significantly reduced the uncertainty and risk associated with undulator magnet field quality and stability.

\subsubsection{Wake Field Effects of the Undulator Vacuum Pipe}

Simulations indicate that performance goals for LCLS will be met if a smooth, 6-mm copper beam pipe is used in the undulator channel. Wake fields due to surface roughness and resistive wall impedance in the undulator beam pipe can have significant effects on the SASE process since it can cause a correlated energy spread to develop in the electron beam as it travels along the undulator. Direct measurements of candidate beam pipe material indicate that roughness effects are at acceptably small levels in commercially available tubes. Investigations of prototype chamber designs and fabrication techniques will be carried out in the coming year. Resistive wall effects in the 6-mm beam tube are expected to be important, and have been taken into account in start-to-end simulations and predictions of output power. Assuming best performance of the proven planar hybrid undulator design, the net effect of increasing the undulator gap and beam pipe diameter is not very significant; at increased gap, the improvement in longitudinal impedance is largely cancelled by the reduction in undulator peak field which results in increased gain length.

\subsubsection{SASE FEL Physics}

As described in Chapter 4, the theory of self-amplified spontaneous emission has been independently verified in experiments carried out at ANL and BNL by members of the LCLS Collaboration, as well as at the TESLA Test Facility. Theoretical predictions of gain, saturation, nonlinear harmonic generation, and temporal structure have been experimentally verified at wavelengths down to $98 \mathrm{~nm}$. Based on these results and on theoretical predictions, the LCLS can attain saturation over its full spectrum, for a range of achievable peak currents and electron pulse lengths. The LCLS undulator tunnel will be constructed about $30 \mathrm{~m}$ longer than the undulator line to make possible the addition of undulator segments and other hardware required for producing shorter or longer light pulses using seeding techniques.

\subsubsection{X-Ray Optics and Beam Handling}

Numerical simulations and experimental tests have shown that LCLS optical elements can be designed to handle the extraordinary peak power of the FEL. The choice of materials and of placement of optical elements is important, and low-Z materials will withstand the highest power densities encountered in the front hutch of the near Hall, as indicated in Table 9.4 (Chapter 9). Peak power densities are challenging but tractable in the Near Hall. In the Far Hall, power densities on the optics are more easily managed. 


\subsubsection{Conventional Facilities}

The ground stability of the Research Yard, where the undulator system and the Near Hall will be sited, has been carefully monitored and characterized since the Final Focus Test Beam program began in 1993. Experience with operation of the SLAC Linear Collider has demonstrated that the effects of ground vibrations and settling can be compensated by feedback control of the electron beam. The LCLS buildings are conventional in design and pose no special risks or challenges.

\subsubsection{Schedule Risks}

A three-year construction schedule is aggressive but achievable if sufficient PED funding is secured to have the critical and long-lead procurement packages ready for release as soon as construction begins. Schedule risk is also minimized by progressive commissioning of the injector and linac in advance of FEL commissioning. Procurement strategy for undulators will be carefully planned to minimize technical and schedule risk.

\subsubsection{Cost Risks}

The LCLS cost estimate has over $25 \%$ contingency, reasonable for this stage of planning. In the coming year, the cost estimate will be further refined.

\subsection{Stakeholder Input}

Throughout the planning process for the LCLS, every effort has been made to maintain and promote communication with the agencies responsible for science policy in the US, the prospective LCLS user community, and the management of SLAC itself. Since it was first conceived in 1992, the evolution of the LCLS design has been guided by input from the synchrotron science community. The Basic Energy Sciences Advisory Committee has carried out two formal assessments of the future of synchrotron radiation science in the US, the role of free electron lasers in general and the LCLS in particular. As already mentioned, the Birgeneau subpanel report and the Leone subpanel both supported the Critical Decision 0 finding that the LCLS fills a key mission need in Basic Energy Sciences research.

The LCLS R\&D effort has also been guided by two key advisory groups, the LCLS Science Advisory Committee and the LCLS Technical Advisory Committee. These committees were founded in 1999 to advise the SSRL and SLAC Technical Division Associate Directors on the LCLS scientific program and accelerator science/technology issues.

Since 1992, there have been 34 workshops, attended by members of the light source research community worldwide, which have addressed scientific opportunities and challenges of importance to the LCLS.

The SLAC Directorate and Faculty have been actively involved in planning the integration of LCLS operations and science with the rest of the SLAC Scientific Program. The main advisory 
body to the President of Stanford University, the SLAC Science Policy Committee, has received regular updates on LCLS activities and planning.

\subsection{Acquisition Strategy}

The lead contractor for acquisition of the Linac Coherent Light Source is Stanford University, which operates the Stanford Linear Accelerator Center. SLAC will collaborate with two national laboratories (Argonne National Laboratory and Lawrence Livermore National Laboratory) to construct the LCLS.

The SLAC site is the best choice among alternative sites for the LCLS, because it makes use of a portion of the Two Mile Linac as the source of a high-quality electron beam for the LCLS free-electron laser. There is no other linac or synchrotron in the world capable of providing a 14 $\mathrm{GeV}$ electron beam with properties suitable for the LCLS. Duplication of the SLAC linac facilities to be used for LCLS would cost more than \$300M. Duplication of the core competencies and support staff necessary to operate the linac (required for other programs at SLAC) would incur significant additional annual expenditures beyond the operating cost of the LCLS that would certainly exceed $\$ 30 \mathrm{M}$. The linac facilities will be shared between LCLS and other programs; however, $75 \%$ of the operations schedule of the linac will be available for LCLS.

A significant component of the LCLS budget as well as technical risk are associated with the undulator magnets that induce oscillatory motion of the electron beam as it passes through the magnets. Undulator magnets have been constructed for light sources and lasers at several laboratories around the world. The US DOE laboratory with the most recent and comprehensive experience in undulator design and construction is Argonne National Laboratory (APS). The APS is operated by the University of Chicago under contract with the Department of Energy. Since 1993, the APS has designed, procured and tested over 35 undulators and wiggler magnets, totaling over $75 \mathrm{~m}$ in length. This is to be compared with the required 33 undulators, totaling 112 $\mathrm{m}$, required for the LCLS.

The LCLS is a source of unprecedented peak x-ray power. The development of optical elements to collimate, focus and filter the beam poses unique challenges. Though it is impossible to create LCLS-like x-ray beams without actually building the LCLS, Lawrence Livermore National Laboratory (LLNL) has extensive related experience in development of precision highpower optics within its laser programs. LLNL has high-power laser facilities, which can be used for testing materials under conditions approximating the LCLS laser beam. Finally, LLNL has already developed computer simulation codes that can predict the effect of the LCLS beam on materials. For this reason, LLNL will manage the acquisition of x-ray beam handling systems to be put in the path of the x-ray beam. An alternative would be to re-develop this expertise at SLAC, incurring considerable delay and additional expense.

Brookhaven National Laboratory (BNL), the University of California-Los Angeles (UCLA), and Los Alamos National Laboratory (LANL) have contributed to the LCLS conceptual design. 
Milestone FEL experiments have been carried out at BNL by the LCLS Collaboration, and BNL has led the way in exploration of the capabilities of the 1.6-cell $\mathrm{rf}$ photocathode gun to be used in the LCLS. UCLA has provided theoretical support and innovation in design of the LCLS. LANL has provided research on photocathodes for RF guns. It is expected that these organizations will continue to play a key role in support of LCLS, and will participate in LCLS construction as necessary.

\subsection{Design Alternatives}

Although the fundamentals of the LCLS design have not changed since 1992, a wide range of alternatives has been evaluated in the course of preparing the design in this report. Placement of the injector linac within the main linac tunnel, and a newly excavated injector enclosure were considered at earlier stages of the design. The bunch compressor designs have been modified as understanding of CSR effects has improved. In the past year, the decision to increase the length of the FFTB tunnel was taken, to provide space for self-seeding systems that could be implemented as an upgrade to the LCLS to produce very short x-ray pulses from the laser. The most significant change in terms of cost has been the addition of the Far Hall, or Hall B, to the scope of the project. This was based on unanimous advice from the LCLS Science Advisory committee. The addition of the Far Hall significantly reduces technical risks associated with the design of high-power optics for the LCLS. As an ALARA measure, it was decided to move the front-end systems (gas attenuation cells, beam stoppers, etc) out of the Near Hall and into the more heavily shielded undulator tunnel. Significant effort has gone into the selection of x-ray beam handling systems, $x$-ray optics and x-ray diagnostics that can span the full spectrum of the LCLS. The technical challenges in optics design change character radically over the $0.15-1.5 \mathrm{~nm}$ range of the LCLS. Details of these and other design alternatives are mentioned in the body of the Conceptual Design Report, though of course emphasis is placed on description of the optimized design.

\subsection{Principle of Operation}

As described in Chapter 4, lasing action is achieved in an FEL when a high brightness electron beam interacts with an intense light beam while traveling through a periodic magnetic field. Under the right conditions, the longitudinal density of the electron beam becomes modulated at the wavelength of the light. When this occurs, electrons contained in a region shorter than an optical wavelength emit synchrotron radiation coherently; i.e., the intensity of the light emitted is proportional to the square of the number of electrons cooperating, rather than increasing only linearly with the number of electrons, as is the case with normal synchrotron radiation. The increasing light intensity interacting with the electron beam passing through the magnetic field enhances the bunch density modulation, further increasing the intensity of the light. The net result is an exponential increase of radiated power ultimately reaching about ten orders of magnitude above conventional undulator radiation. 
The main ingredients of an FEL are a high-energy electron beam with very high brightness (i.e., low emittance, high peak current, small energy spread) and a periodic transverse magnetic field, such as produced by an undulator magnet. Electrons bent in a magnetic field emit synchrotron radiation in a sharp forward cone along the instantaneous direction of motion of the electron, and hence the electric field of this light is predominantly transverse to the average electron beam direction. In most present FELs the light from many passes of the electron beam through the undulator is stored in an optical cavity formed by mirrors. Many of these FELs work in the IR range and some have been extended to the UV range. Extending these devices to shorter wavelengths poses increasing difficulties due primarily to the lack of good reflecting surfaces to form the optical cavity mirrors at these shorter wavelengths. It has recently become possible to consider another path to shorter wavelength, down to the Angstrom range. This new class of FEL achieves lasing in a single pass of a high brightness electron bunch through a long undulator by a process called Self-Amplified Spontaneous Emission (SASE). No mirrors are used. This is the process proposed for the LCLS.

The LCLS reaches the Angstrom range with this approach with a high energy $(14.3 \mathrm{GeV})$, high peak current $(3.4 \mathrm{kA})$, low normalized emittance (1.2 mm mrad), small energy spread $(0.02 \%)$ electron beam passing through a long $(121 \mathrm{~m})$ undulator magnet. The spontaneous radiation emitted in the first part of this long undulator, traveling along with the electrons, builds up as the bunch-density modulation begins to take place during a single pass, resulting in an exponential increase in the emitted light intensity until saturation is reached. Usually this occurs after about 10 exponential field gain lengths.

\subsection{Overall Layout}

Figure 2.1 shows the layout of the proposed facility. The PEP-II electron-positron collider uses the first $2 \mathrm{~km}$ of the Linear Accelerator as its injector. The last $1 \mathrm{~km}$ of the linac is used by the LCLS. 


\section{The LCLS \\ (Linac Coherent Light Source)}

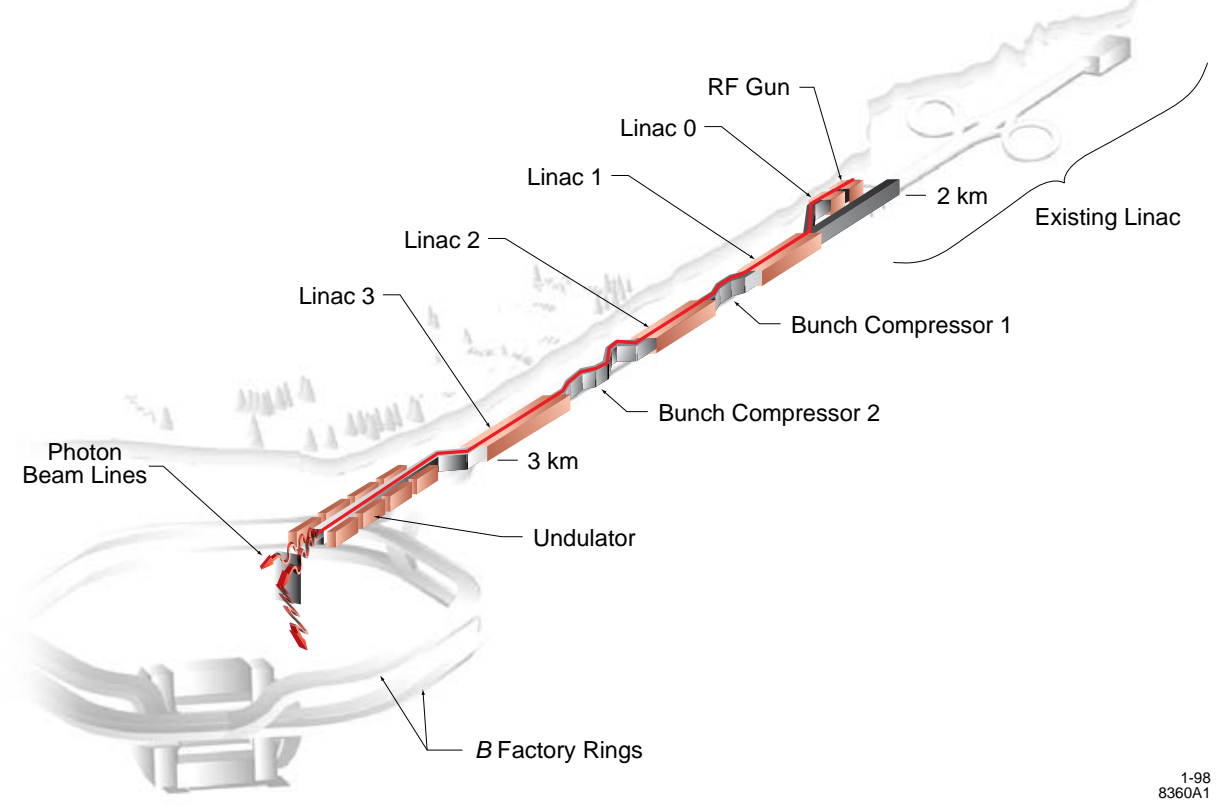

Figure 2.1 Layout of the Linac Coherent Light Source.

A new injector consisting of a gun and a short linac is used to inject an electron beam into the last kilometer of the SLAC linac. With the addition of two stages of magnetic bunch compression, the beam at the entrance to the undulator has an energy of $14.3 \mathrm{GeV}$, a peak current of 3,400 A, and a normalized emittance of $1.2 \mathrm{~mm}$-mrad. A transfer line takes the beam and matches it to the entrance of the undulator. The $121-\mathrm{m}$ long undulator will be installed in the tunnel that presently houses the Final Focus Test Beam Facility. After exiting the undulator, the electron beam is deflected onto a beam dump, while the photon beam enters the experimental areas.

The experimental areas are housed in two halls. The first hall, located just after the beam dump in the SLAC Research Yard, is a $30 \mathrm{~m} \times 55 \mathrm{~m}$ structure, which will contain two x-ray hutches for characterization of the beam and subsequently for experiments. The x-ray beam will pass through the Near Hall into a $227 \mathrm{~m}$ tunnel to the Far Hall, a $57 \mathrm{~m} \times 33 \mathrm{~m}$ structure with experiment facilities below grade and office/lab space on grade. This building will also include two x-ray hutches at the completion of the project.

\subsection{Performance Characteristics}

Figure 2.2 shows the peak and average brightness as a function of photon energy. The LCLS is designed to be tunable in the photon wavelength range $1.5-15 \AA$, corresponding to $4.5-14.3$ $\mathrm{GeV}$ electron energy. 

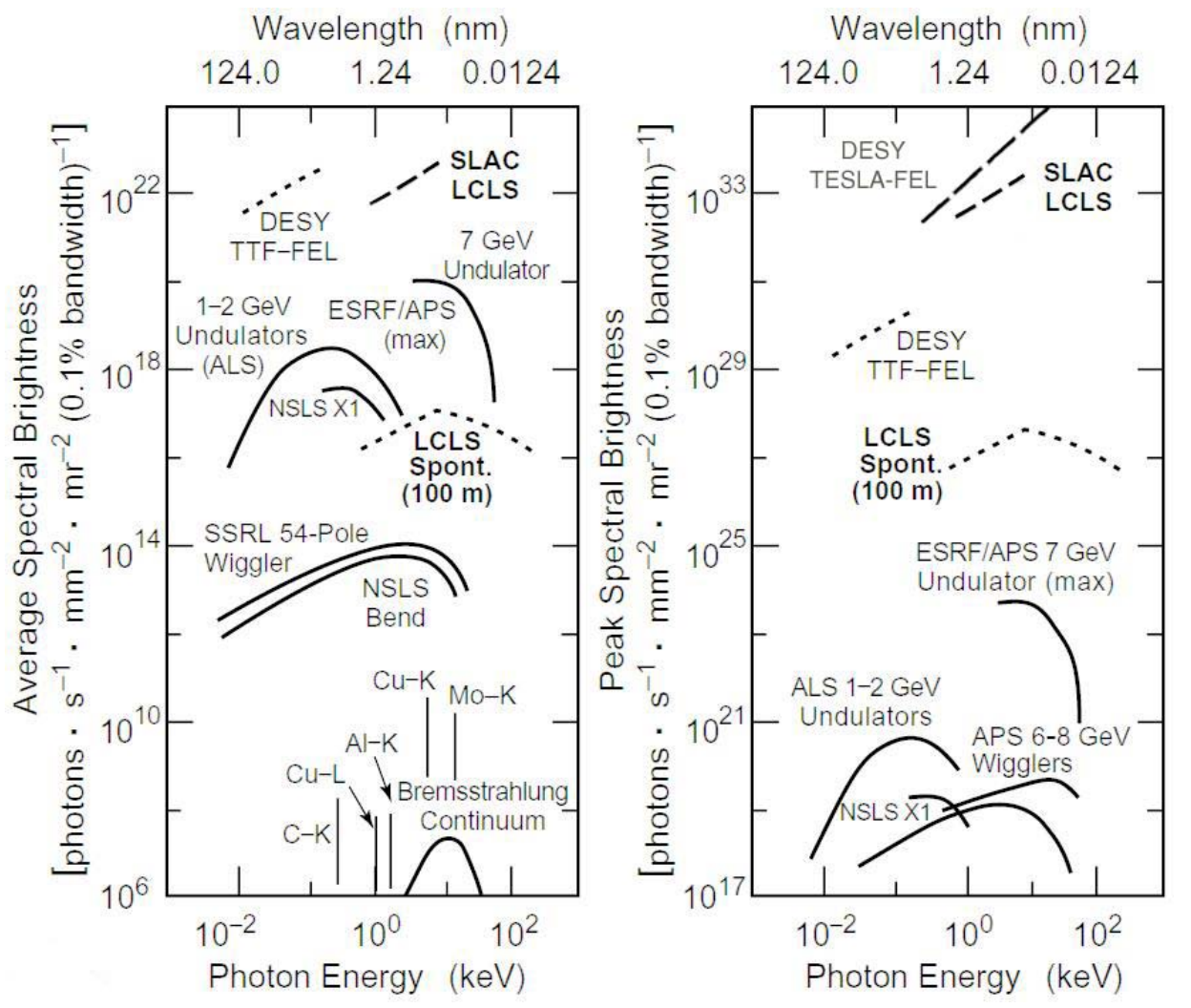

Figure 2.2 Average and peak brightness calculated for the LCLS and for other facilities operating or under construction. The data for the Average Spectral Brightness of the planned TESLA FEL facility is above the limit of the figure.

Table 2.3 lists some of the basic parameters of the LCLS electron beam, of the undulator, and of the FEL performance at the shortest operating photon wavelength.

Table 2.3 LCLS electron beam parameters.

\begin{tabular}{lccc}
\hline Parameters & Values & Units \\
\hline Electron beam energy & 4.54 & 14.35 & $\mathrm{GeV}$ \\
\hline Normalized rms slice emittance & 1.2 & 1.2 & $\mathrm{~mm} \mathrm{mrad}$ \\
\hline Peak current & 3,400 & 3,400 & $\mathrm{~A}$ \\
\hline Slice energy spread & 0.025 & 0.008 & $\%, \mathrm{rms}$ \\
\hline Projected energy spread & 0.20 & 0.06 & $\%, \mathrm{rms}$ \\
\hline RMS bunch length & 77 & 77 & $\mathrm{fs}$ \\
\hline Undulator period & 3 & $\mathrm{~cm}$ \\
\hline Number of undulator periods & 3,729 & \\
\hline Undulator magnetic length & 112.86 & $\mathrm{~m}$ \\
\hline
\end{tabular}


LCLS C O N C E P TUAL DESIG N REP O R T

\begin{tabular}{lccc}
\hline Undulator field & 1.325 & \\
\hline Undulator gap & 6 & Tesla \\
\hline Undulator parameter, $K$ & 3.7 & $\mathrm{~mm}$ \\
\hline FEL parameter, $\rho$ & $14.5 \times 10^{-4}$ & $5.0 \times 10^{-4}$ & \\
\hline Power gain length & 1.3 & 4.7 & $\mathrm{~m}$ \\
\hline Repetition rate & & & \\
\hline Saturation peak power & 19 & 120 & $\mathrm{~Hz}$ \\
\hline Peak brightness & & 8 & $\mathrm{GW}$ \\
\hline Average brightness & $5 \times 10^{31}-5 \times 10^{32}$ & $10^{32}-10^{33}$ & $\mathrm{Photons} /\left(\mathrm{s} \mathrm{mm} \mathrm{mrad}^{2}\right.$ \\
\hline
\end{tabular}

The curves for the presently operating third-generation facilities indicate that the projected peak brightness of the LCLS FEL radiation would be about ten orders of magnitude greater than currently achieved. Also note that the peak spontaneous emission alone (independent of the laser radiation) is four orders of magnitude greater than in present sources. This, coupled with subpicosecond pulse length, makes the LCLS a unique source not only of laser, but also of spontaneous radiation. This spontaneous radiation is also transversely coherent at wavelengths of $6 \AA$ and longer.

\subsection{The Photoinjector}

The design goal of radio-frequency photocathode guns currently under development at various laboratories is a $3 \mathrm{ps}(\mathrm{rms})$ long beam of $1 \mathrm{nC}$ charge with a normalized rms emittance of $1 \mathrm{~mm}$-mrad.

In a radio-frequency photocathode gun, electrons are emitted when a laser beam strikes the surface of a cathode [6]. The extracted electrons are accelerated rapidly to $7 \mathrm{MeV}$ by the field of a radio-frequency cavity. The rapid acceleration reduces the increase in beam emittance that would be caused by the space charge field. The variation of phase space distribution along the bunch, caused by the varying transverse space charge field along the bunch, is compensated with an appropriate solenoidal focusing field [7].

The laser will have a YAG-pumped Ti:sapphire amplifier operating at $780 \mathrm{~nm}$ that will be frequency tripled (3rd harmonic). Very restrictive conditions are required for the reproducibility of the laser energy and timing. Stable FEL operation requires a pulse-to-pulse energy jitter of better than $1 \%$ and a pulse-to-pulse phase stability of better than $0.5 \mathrm{ps}$ (rms). These tight tolerances are needed to ensure optimum compression conditions. 


\subsection{Compression and Acceleration}

The purpose of the compressors is to reduce the bunch length, thereby increasing the peak current to the 3,400 A required to saturate the LCLS. Accelerating the beam off the crest of the rf waveform in the linac creates an energy-phase correlation that can be used by a chicane to shorten the bunch by appropriate energy-path length dependence. It is preferable to utilize two, rather than one, chicane. This reduces the sensitivity of the final bunch length to the phase jitter in the photocathode laser timing [8]. The rms length of the bunch emitted from the cathode is $1 \mathrm{~mm}$ ( $3 \mathrm{ps}$ ). After compression, the bunch shortens to $0.02 \mathrm{~mm}$.

The choice of energies of the various compression stages is the result of an optimization that takes into account beam dynamics effects, the most relevant ones being the space charge forces in the early acceleration stage, the wakefields induced by the electromagnetic interaction of the beam with the linac structure [9], and the coherent synchrotron radiation emitted by a short bunch [10]. With all dynamic effects included, the simulations [11] indicate that the emittance dilution up to the entrance of the undulator will be less than $50 \%$.

From the linac exit a transport system carries the beam to the entrance of the undulator. This transport system includes a suite of diagnostics to characterize the electron beam.

\subsection{The Undulator}

Several candidate undulator types were evaluated, including pure permanent magnet helical devices, superconducting bifilar solenoids, and hybrid planar devices. Superconducting devices were ruled out because of their complexity and higher risk. A hybrid device has a stronger field, and, therefore, a shorter length, than a pure permanent magnet device. It also offers superior error control. The advantage of a pure permanent magnet system is that it allows superposition of focusing fields. Since the focusing quadrupoles can be placed in the interruptions and need not envelop the undulator, this property of pure permanent magnet undulators is not critical.

The other choice is between a planar and a helical undulator. Helical devices offer a shorter gain length to reach saturation, but are less understood than planar devices, particularly in terms of magnetic errors, a crucial factor in the SASE x-ray situation. Measurements of the magnetic field are also difficult. A planar hybrid undulator was chosen for this design for its superior control of magnetic errors and simplicity of construction and operation. The magnetic length of the undulator is $113.7 \mathrm{~m}$, its period is $3 \mathrm{~cm}$, and the pole-to-pole gap is $6 \mathrm{~mm}$.

\subsection{The X-Ray Optics and Experimental Areas}

After leaving the undulator, the electron beam, carrying an average power of $1.6 \mathrm{~kW}$, will be dumped into a shielding block by a sequence of downward-deflecting permanent magnets, while the FEL radiation will be transported downstream to the experimental areas. Anticipating the 
broad range of applications and associated beam requirements described in the "First Experiments" report, a corresponding range of transmissive, specular and crystal optics will be employed. A system of attenuators will allow the intensity of the tradition to be varied from the level of current third-generation facilities up to the maximum FEL output.

\subsection{Summary}

In summary, this Conceptual Design Report describes the design of an x-ray Free-Electron Laser operating on the single pass SASE principle. The FEL uses the unique capability of the SLAC linear accelerator to create an intense electron beam of low emittance, and a long undulator, to produce high brightness coherent radiation down to $1.5 \AA$. Theory and computations indicate that the peak brightness from such a device would be about ten orders of magnitude greater than currently achievable in third-generation synchrotron radiation sources. Such performance, coupled with the very short bunch length (230 fs FWHM) and full transverse coherence, would allow the exploration of new horizons in material science, structural biology, and other disciplines.

\subsection{References}

1 D.E. Eastman and F. Seitz, Major Facilities for Materials Research and Related Disciplines, Washington DC, National Academy Press, PB85-108550 (1984)

2 R. Birgeneau and Z. X. Shen, Report of the Basic Energy Sciences Committee Subpanel on D.O.E. Synchrotron Radiation Sources and Science, November 1997, p. 13

3 S.R. Leone, Report of the Basic Energy Sciences Advisory Committee Panel on Novel Coherent Light Sources, January 1999

4 G.K. Shenoy and J. Stohr, eds. LCLS - The First Experiments,

5 ICFA Beam Dynamics Mini-Workshop (Future Light Sources Sub-panel) Coherent Synchrotron and its impact on the beam dynamics of high brightness electron beams, January 14-18, 2002 at DESYZeuthen http://www.desy.de/csr/csr_workshop_2002/csr_workshop_2002_index.html

6 R.L. Sheffield, "Photocathode rf guns," in Physics of Particle Accelerators, AIP Vol. 184, pp.15001531, M. Month and M. Dienes, eds. (1992).

7 B. Carlsten, "New photoelectric injector design for the Los Alamos National Laboratory XUV accelerator," Nucl. Inst. and Meth. in Phys. Res., A285 (1989) 313. 
8 T.O. Raubenheimer, "Electron beam acceleration and compression for short wavelength FELs," Nucl. Instrum. Meth. in Phys. Res. A358, 40 (1995).

9 See, for example, A. Chao, "Physics of Coherent Beam Instabilities" in High Energy Accelerators, Ch. 2, J. Wiley \& Sons, eds., New-York, 1993.

10 Y.S. Derbenev et al., "Microbunch radiative head-tail interaction," DESY Report TESLA 95-05, Sept. 1995.

11 R. Assmann et al., "LIAR-A computer program for linear accelerator simulations," SLAC/AP-103, Oct. 1996. 


\section{Scientific Basis for
Optical Systems}

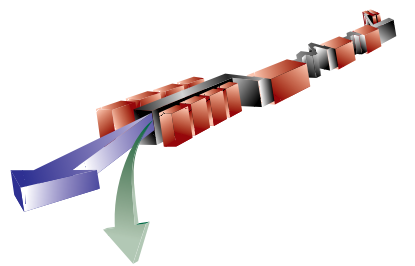

\section{TECHNICAL SYNOPSIS}

The LCLS Scientific Advisory Committee (SAC) has recommended experiments in five scientific disciplines for the initial operation of the LCLS. These experiments cover a variety of scientific disciplines: atomic physics, plasma physics, chemistry, biology and materials science. The $x$-ray optics and detectors needed to verify the LCLS capability to address these five disciplines will be constructed and installed as part of the LCLS project. The experiments are described in detail in the document "LCLS: The First Experiments" referenced earlier.

Two classes of experiments are proposed for the LCLS. The first class consists of experiments where the $x$-ray beam is used to probe the sample, as is done in most experiments at current synchrotron sources. In the second class, the LCLS beam is used to induce non-linear photoprocesses or create matter in extreme conditions. The same source can be used for both types of experiments by utilizing the six orders-of-magnitude change in photon flux density caused by focusing the LCLS beam, and by exploiting the strong dependence of the photo-absorption crosssection on photon energy and atomic number.

These experiments establish the basis for the designs of the x-ray optics to focus, monochromate, and manipulate the LCLS beam. In general, these designs are extensions of common practice at synchrotron sources today, but become demanding due to unprecedented peak powers, pulse lengths and coherence of the LCLS beam. These experiments also provide the requirements for a state of the art detector system for diffraction studies. Finally, the definition of the required synchronization of external lasers with the LCLS beam is derived from the experimental needs of these first five experiments. 


\subsection{Introduction}

\subsubsection{History of Scientific Interest in X-Ray Free Electron Lasers}

The last thirty years have witnessed an exponential increase in the capability of x-ray sources, and x-ray physics has seen an explosion of new techniques and applications. The key to this huge change has been the development of synchrotron radiation sources from high-energy electron storage rings. The scientific capabilities of synchrotron radiation $\mathrm{x}$-ray sources are reflected in the fact that in the US four such facilities are operated by DoE with a collective annual funding level of about \$200 million (FY2001). In 2001, 6500 scientists made use of these facilities for their research programs, which range from fundamental physics to materials science to biology and medicine to environmental science [1]. Now, another type of high-energy accelerator has the capability to drive an $\mathrm{x}$-ray source whose capabilities outshine those of a modern synchrotron source by nearly as much as the synchrotron does the 1960's laboratory source.

Advances in accelerator technology have been the driving force in the progress toward brighter synchrotron sources, with scientific applications developing in response to the availability of new sources. The rate of improvement in source capability has been tremendous: for thirty years x-ray source brightness has been increasing exponentially with a doubling time of about 10 months. A modern synchrotron radiation source is 11 orders of magnitude brighter than a 1960's laboratory x-ray source. Seldom, if ever, in history (perhaps only in the field of visible laser optics) has a scientific discipline seen its tools change so dramatically within the active life of a single generation of scientists. Such change makes it very difficult to predict the future. For example, no one foresaw the huge impact on biomedical research that has come in the last twenty years from synchrotron-based EXAFS and protein crystallography, even though those techniques had been developed many years previously using laboratory sources. The developing synchrotron source capability has made the techniques qualitatively and unexpectedly more powerful as scientific tools.

This history indicates that although it is very difficult to predict the eventual applications of the LCLS, a source that is more than 10 orders of magnitude brighter than today's synchrotron sources, it will make fundamental contributions to our understanding of the structure and dynamics of matter on the atomic scale. Over the past ten years there has been much consideration of the future development and applications of such new synchrotron radiation sources. A first workshop on "Fourth Generation Light Sources", at SLAC in 1992 [2], concentrated almost exclusively on accelerator technology rather than applications. This workshop served to alert the scientific community to the possibilities for x-ray FELs driven by linacs, including the SLAC linac. It is interesting to note that a workshop earlier in 1992 on "Applications of x-ray Lasers" [3] did not mention FEL sources at all; only chemical lasers were considered. The SLAC workshop directly stimulated the first workshops on scientific applications of x-ray FELs [4,5]. The next "Fourth Generation Light Sources" workshop, in 1996 at the ESRF [6], included sessions on both sources and applications. The discussions convinced nearly all the participants that linac based FELs would be the most effective machines for 
continuing to improve the performance of x-ray sources, and in particular, would provide the only viable route to a diffraction-limited hard $\mathrm{x}$-ray source. Subsequent workshops at DESY in 1996 [7,8] and APS in 1997 [9] have assumed that future fourth generation x-ray user facilities will be based on linac FELs, and have attempted to foresee the new science that these sources will bring. These workshops, as well as more than 20 others, have firmly established the scientific opportunities that LCLS provides.

\subsubsection{Unique Features of X-Ray FEL Radiation}

Intrinsic to the short-wavelength FEL process are several features, which give unique and useful attributes to the radiation that is produced. Because of the difficulty of creating an optical cavity at x-ray wavelengths, a high-gain, single-pass FEL design is used, relying on the process of self-amplified spontaneous emission (SASE). This implies a very short, high-energy electron pulse producing a similarly short but very intense FEL radiation pulse. The radiation has a relatively short longitudinal coherence length, but complete transverse coherence. In addition to the FEL radiation, the SASE process produces a spontaneous radiation spectrum rich in higher harmonics. Figure 3.1 shows a calculation of the LCLS radiation spectrum with FEL operation at $1.5 \AA$, and Table 3.1 gives some descriptive parameters for the beam. Compared with existing xray sources, the radiation has three truly unique aspects:

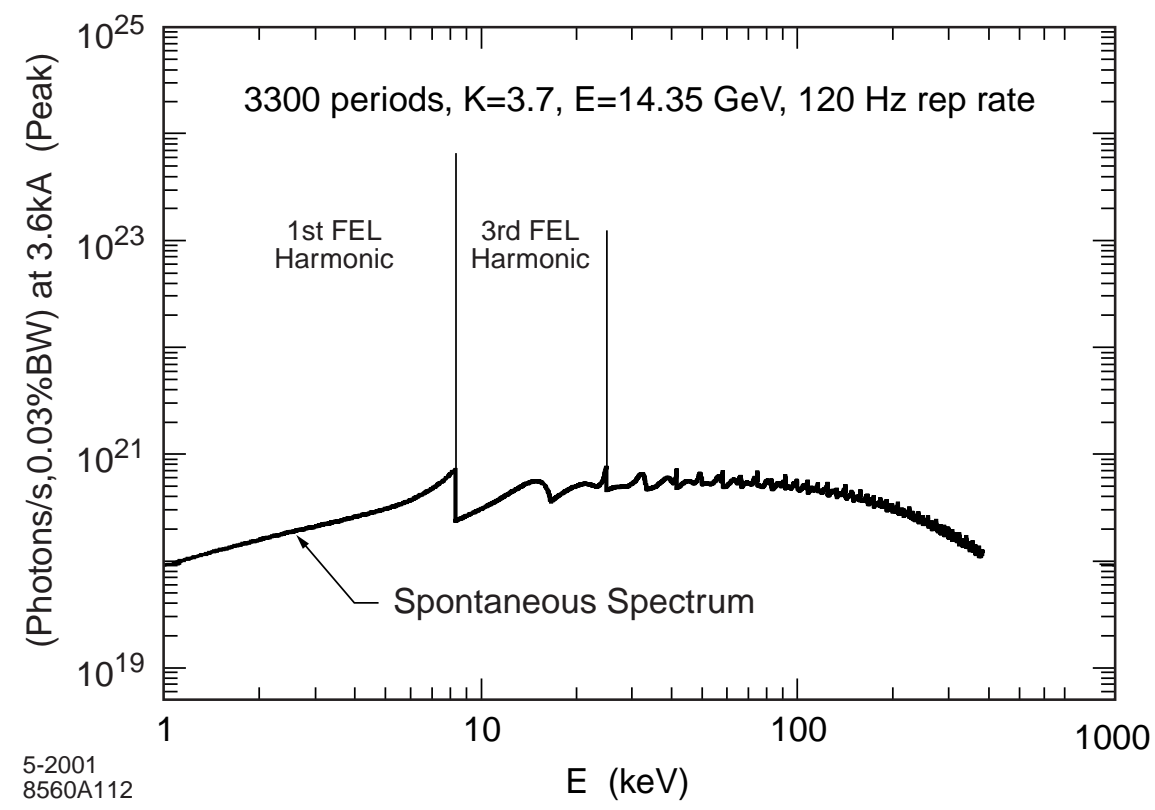

Figure 3.1 LCLS peak flux spectrum, with FEL radiation at $1.5 \AA$. The first harmonic FEL amplification is saturated. There is also some amplification of the third harmonic, but this is far from saturation, and is likely to be further reduced by magnet and beam errors.

The FEL peak intensity and peak brightness are both many orders of magnitude higher than can be produced by any other source (see Figure 3.2). Even the average brightness, though limited by the low repetition rate of the linac, is still orders of magnitude higher than the brightest synchrotron radiation. 
The sub-picosecond pulse length is orders of magnitude shorter than can be achieved with a synchrotron. There exist x-ray sources with comparable pulse lengths (for example, plasma sources and inverse Compton scattering sources), but they have very much lower brightness.

Table 3.1 Calculated characteristics of the LCLS radiation at the short wavelenth end of the operational range.

\begin{tabular}{lll}
\hline FEL wavelength & 1.5 & $\AA$ \\
\hline FEL bandwidth $(\Delta E / E)$ & 0.003 & \\
\hline Pulse duration (FWHM) & 230 & $\mathrm{fs}$ \\
\hline Pulse length (FWHM) & 69 & $\mu \mathrm{m}$ \\
\hline Peak coherent power & 8 & $\mathrm{GW}$ \\
\hline Peak coherent power density & $1.1 \times 10^{12}$ & $\mathrm{~W} / \mathrm{mm}^{2}$ \\
\hline FEL energy/pulse & 2.1 & $\mathrm{~mJ}$ \\
\hline Peak brightness & $1 \times 10^{33}$ & $\mathrm{flux} / \mathrm{mm}^{2} / \mathrm{mrad}^{2} / 0.1 \% \mathrm{BW}$ \\
\hline FEL photons/pulse & $1.1 \times 10^{12}$ & \\
\hline FEL photons/second & $1.3 \times 10^{14}$ & \\
\hline Degeneracy parameter & $10^{9}$ & \\
\hline Peak EM field (unfocused) & $2.5 \times 10^{10}$ & $\mathrm{~V} / \mathrm{m}$ \\
\hline Average FEL power & 0.25 & $\mathrm{~W}$ \\
\hline Average FEL brightness & $2.7 \times 10^{22}$ & $\mathrm{flux} / \mathrm{mm}^{2} / \mathrm{mrad}^{2} / 0.1 \% \mathrm{BW}$ \\
\hline Transverse size of FEL beam (FWHM) & 70 & $\mu \mathrm{m}$ \\
\hline Divergence of FEL beam (FWHM) & 1 & $\mu \mathrm{rad}$ \\
\hline Peak power of spontaneous radiation & 92 & $\mathrm{GW}$ \\
\hline
\end{tabular}

The FEL radiation has full transverse coherence (it is diffraction limited). In addition, the degeneracy parameter (photons per coherence volume in phase space) is many orders of magnitude greater than one. Only at the longest wavelengths can some synchrotron sources approach the diffraction limit, and no source has a degeneracy parameter much greater than one.

In addition to these features of the FEL radiation, the high-energy spontaneous radiation offers attractive characteristics. The spectrum of this radiation extends to nearly $1 \mathrm{MeV}$; above about $100 \mathrm{keV}$ it is far brighter than any synchrotron radiation. 

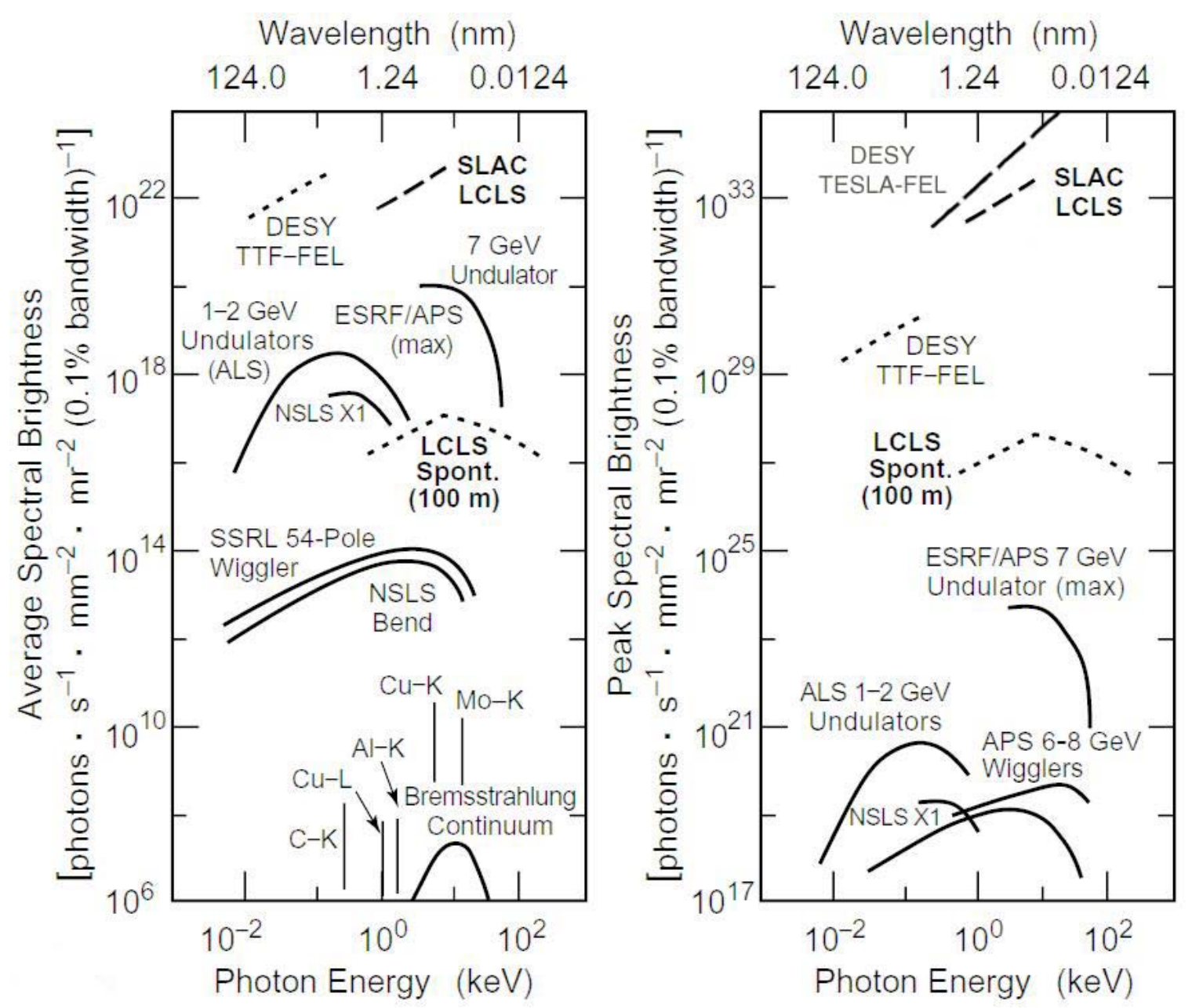

Figure 3.2 Average and peak brightness calculated for the LCLS and for other facilities operating or under construction. The data for the Average Spectral Brightness of the planned TESLA FEL facility are above the limit of the figure.

\subsubsection{The Role of the LCLS}

For the applications to be realized, much needs to be learned about the interaction between $\mathrm{x}$ ray FEL radiation and matter. But today's radiation sources cannot fully address this issue. A recent workshop [10] concluded that all existing laser and synchrotron sources fail by at least 3 orders of magnitude in frequency or power density to duplicate the conditions of an X-ray FEL. The basic interactions between atoms and electromagnetic fields with the strength of the FEL radiation are not well understood. It is not known exactly what kind of damage this radiation will cause in solid samples, or how best to moderate its intensity.

Therefore, the first scientific contribution of the LCLS will be to provide an understanding of the interactions between very intense, very high frequency electromagnetic radiation and matter. In the process of gaining this understanding, many technical issues must be confronted, such as fast, high-dynamic-range detectors, high peak power optics, and precise synchronization with external probes. It is very likely that as experience with the LCLS grows, further advances in 
accelerator science will lead to greater control over the FEL radiation. It may become possible to produce even shorter x-ray pulses, energy-chirped pulses, or pulses with special polarization states. All of these will lead to new applications.

\subsubsection{Science with X-Ray FELs}

As mentioned above, there have been many international workshops called to discuss the scientific applications of FEL x-ray sources. The need to develop the scientific case for an XFEL in order to move forward with its design and construction was emphasized by the Leone report on Novel Coherent Light Sources [11]. In response to this need the LCLS Scientific Advisory Committee (SAC) put considerable effort into defining a small set of particularly exciting experiments that could be carried out with LCLS. This information is presented in great detail in the report, "LCLS: The First Experiments" [12] and has been accepted as the basis for going forward with the LCLS by the Basic Energy Sciences Advisory Committee. Many of the techniques mentioned are already in use, or at least proof-of-principle experiments have been done. An attempt has been made to try to project the impact of an FEL source on the future importance of these techniques. It is certain that, once it is available, the FEL source will stimulate the development of completely new techniques, the importance of which is extremely difficult to predict. In the section below we describe the optics requirements derived from the "LCLS: The First Experiments" [12]. It is important to note that the majority of these systems are similar to those now found at all synchrotron radiation facilities. They provide the generic means of manipulating the photon beam.

\subsection{Optical and Experimental Challenges}

\subsubsection{Focusing}

The LCLS x-ray beam has typical dimensions of $100 \mu \mathrm{m}$ even with its extremely small divergence. In many applications this source dimension is more than sufficient and helps avoid sample damage issues for a wide variety of samples. In several experimental areas however there is the requirement to focus the beam to dimensions of order $0.1 \mu \mathrm{m}$ or smaller. In the case of atomic physics for example in studies of multiphoton excitation, getting to $0.1 \mu \mathrm{m}$ at the low energy end of the LCLS range $(0.8 \mathrm{keV})$ is critical. For the bio-imaging similar spot sizes are required at the high energy end of LCLS performance $(8.0 \mathrm{keV})$. Warm dense matter has less stringent requirements, $10 \mu \mathrm{m}$, but still requires focusing to achieve the needed energy density. Theses needs can be addressed with techniques developed at third generation synchrotron sources, refractive optics in the form of zone plates and reflective optics in the form of Kirkpatrick-Baez mirror systems. These methods will be carried over to the LCLS and demonstrated in the initial phase.

\subsubsection{Monochromatization}

The LCLS will have a natural bandwidth of $10^{-3}$ under normal conditions; however there is the possibility that the central wavelength may have pulse-to-pulse variations equal to its 
bandwidth. For some experiments this may not be a difficulty and the natural bandwidth is more than sufficient. However, for the warm dense matter experiments, when an external laser is used as the pump and the LCLS is used as the probe in Thomson scattering studies there will be a need to monochromatize the radiation. In the nanoscale dynamics experiment the bandwidth determines the longitudinal coherence length of the radiation and thus defines the coherent volume that is probed by the LCLS beam. To control the longitudinal coherence length the beam needs to be monochromatized as well. The techniques that are used routinely with both lab based $\mathrm{x}$-ray sources and synchrotron radiation, Bragg diffraction from perfect crystals, will work as well at LCLS. The issue for LCLS is the damage threshold for these optics. By far the most widely used material is Si and calculations show that there are no damage issues in the far hall and there may be no difficulties in the near hall. Monochromators will be developed for both situations and early studies will address directly damage issues for these critical systems.

\subsubsection{Harmonic Control}

The LCLS radiation is dominated by its fundamental wavelength, but the spectrum contains higher harmonics as well. In general, the experiments described in the Report, "LCLS: The First Experiments" [12] are not sensitive to harmonic contamination on the expected percent level. However in the case of the atomic physics studies of multiphoton excitation it is critical that the highest degree of harmonic rejection be achieved. When looking for processes where the energy required is the sum of the energy from several photons contamination from higher harmonics makes the experiment impossible. This contamination can also be important for scattering experiments where the counting is based on the deposited energy and detectors cannot discriminate between three photons of energy $E$ and a single photon of energy $3 E$ present due to harmonic contamination. For these applications it will be critical to eliminate the harmonic contamination in the incident beam. The standard method of mirror reflection, with the mirror angle chosen to reflect the fundamental and not the higher harmonics, will be evaluated for the low energy end of the LCLS operating range for use in the laser-matter interaction studies.

\subsubsection{Photon Pulse Manipulation}

The LCLS, as an XFEL source, will require the development of x-ray analogues of many tools routinely used in conventional laser experiments. In particular for the study of nanoscale dynamics using x-ray photon correlation spectroscopy pulse manipulation methods are required. For UV, visible and IR lasers optical techniques permit pulse splitting and delay as well as recombination. These tools are not easily realized for x-ray radiation and this is a challenge for the LCLS. There are designs that use Bragg reflection optics. The LCLS performance puts stringent demands on these methods as one tries to split and delay pulses over the range of fractions of a picosecond up to perhaps a nanosecond while preserving the transverse coherence. The ability to develop the x-ray analogues of conventional optical methods will be important for the life of LCLS as the science evolves to make full use of the coherence of the LCLS beam. 


\subsubsection{Synchronization of an External Source (Laser Pump)}

A wide range of experimental methods that have become routine in the ultrafast science community involve pump-probe techniques with the delay between pump and probe controlled to a small fraction of the pulse width. These techniques are inevitably based on a single laser source so that the setting and maintaining of the delay relies on mechanical stabilities that are no longer beyond the state of the art. The power of the LCLS as a probe for laser excited systems on the femtosecond time scale is unprecedented because LCLS can provide directly the atomic positions with Ángstrom resolution from diffraction studies. The difficulty is that the laser pump and x-ray probe must be synchronized with the same fractional precision as with femtosecond laser experiments. The present state of the art in synchrotron sources provides synchronization at perhaps the 1-ps level, a factor of 10-100 away from what LCLS will require. To address this critical need in femtochemistry, time resolved bio-imaging and other pump probe experiments one will first measure the jitter on the 10-100 fs time scale after phase locking the laser and x-ray beam. These techniques, developed to evaluate the jitter, will then be used as a means of tagging the data as it is acquired and providing the information to post process to derive the temporal dependences that are at the heart of the experiment.

\subsubsection{Detectors}

The power of the LCLS is that it will produce radiation at $8 \mathrm{keV}$, which is the standard for diffraction studies at atomic resolution. In synchrotrons around the world this power is already being exploited in the study of the structure of large macromolecules. The growth and success of these studies is based on the development of accurate, large-area, fast, 2-dimensional x-ray detectors. The technology for these conventional applications is now robust, but will not meet the demands for the LCLS. The bulk of the experiments using diffraction methods will require the acquisition of a full diffraction pattern from every LCLS pulse, an operating rate of $120 \mathrm{~Hz}$. This readout rate is unprecedented in the synchrotron radiation field. Furthermore, these detectors will require very large dynamic range for scattering experiments in the area of biomolecules with intensities approaching per pulse what one gets per second form synchrotron radiation sources, today. The spatial resolution required will also be at the state of the art, $25 \mu \mathrm{m}$. There are exciting developments that are just beginning to be applied to synchrotron radiation experiments that borrow from high energy physics. These involve technologies for vertex detectors in colliding beam experiments called pixel array detectors. These developments have the capabilities, in principle, to provide the parameters that one desires for the LCLS and recent results [13] seem to be very promising. This is an area that has received too little attention in the past and will be important to the success of the LCLS over its lifetime for certain classes of experiments.

\subsubsection{Summary}

The success of the LCLS experimental program rests on the development of x-ray optics and detectors that can manipulate the incident radiation and measure the scattered beam. They will require exquisite control and/or knowledge of the temporal relationship between external laser and the LCLS beam for a variety of pump probe experiments. The first experiments described in 
the Report, "LCLS: The First Experiments" [12] provide guidance as to which tools one develops first in the LCLS project. These developments don't end with the construction project completion. With the availability of first radiation one begins studying the interaction of the XFEL beam with matter and evaluating the performance of the optics that will be available. The developments during the life of the LCLS will be stimulated by, as well as stimulate, a rich variety of unique experimental methods that will add significantly to our understanding of the structure and dynamics of a wide range of physical systems.

\subsection{References}

1 For a complete review of DoE facilities see R. Birgeneau and Z.-X. Shen, co-chairs., "Report of the Basic Energy Sciences Advisory Committee, Synchrotron Radiation Light Sources Working Group" (1997)

2 M. Cornacchia and H. Winick, eds., "Workshop on Fourth Generation Light Sources", SSRL Report 92/02 (1992).

3 R. London, D. Mathews, and S. Suckewer, eds., "Applications of X-Ray Lasers", LLNL (1992).

4 W. Spicer, J. Arthur, and H. Winick, eds., "Workshop on Scientific Applications of Short Wavelength Coherent Light Sources", SLAC Report 414 (1992).

5 J. Arthur, G. Materlik, and H. Winick, eds., "Workshop on Scientific Applications of Coherent X-Rays", SLAC Report 437 (1994).

6 J.-L. Laclare, ed., "4th Generation Light Sources", ESRF (1996).

7 J. Schneider, ed. "X-Ray Free Electron Laser Applications", DESY (1996).

8 G. Materlik, ed., “A Superbrilliant X-Ray Laser Facility”, DESY (1997).

9 M. Knotek, J. Arthur, E. Johnson, and F. Dylla, eds., "Workshop on Scientific Opportunities for Fourth-Generation Light Sources", in preparation at APS.

10 R. Tatchyn, G. Materlik, A. Freund, and J. Arthur, eds., SLAC/DESY International Workshop on Interactions of Intense Sub-picosecond X-Rays with Matter, SLAC-WP-12 (1997).

11 S.R. Leone, Report of the Basic energy Sciences Advisory Panel on Novel Coherent Light Sources, (1999)

12 G.K. Shenoy and J. Stöhr, eds., "LCLS - The First Experiments", SSRL (2000).

13 A. G. MacPhee et.al., Science 295, 1261 (2002) 


\section{FEL Physics}

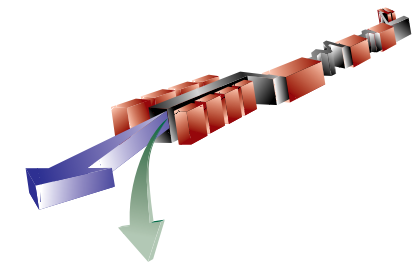

\section{TECHNICAL SYNOPSIS}

This chapter presents a review of the historical and technological developments of the Free Electron Laser that led to proposals to operate an FEL in the large gain regime, starting from the spontaneous radiation noise, without using an optical cavity. In this mode, called "SelfAmplified-Spontaneous-Emission" (SASE), lasing is produced in a single pass of an electron beam with high phase-space density through a long undulator, eliminating the need for optical cavities, which are difficult to build in the soft $x$-ray or $x$-ray spectral region.

A discussion of the spontaneous radiation produced in an undulator introduces the concepts and formulae for the radiation intensity, the number of photons produced per electron, brightness, and peak power. The spontaneous radiation is emitted incoherently, and its intensity increases linearly with the number of electrons. In an FEL, the intensity grows with the square of the number of electrons and the number of photons produced per electron is increased by many orders of magnitude. This is achieved by using the FEL collective instability, which produces microbunching of the electrons on the scale of the optical wavelength of the radiation. The microbunching and the radiation intensity grow exponentially. The inverse of the growth rate is called the FEL gain length.

Several conditions must be satisfied for the collective FEL instability to occur. A parameter of paramount importance is the electron density in phase space. The scaling laws for a SASEFEL, derived using these conditions, and the desire to minimize the undulator length define how the beam's 6-dimensional phase space density must increase as the radiation wavelength is decreased.

The time structure of the radiation pulse is determined by the electrons' slippage with respect to the radiation that they produce and by the fact that the FEL starts from the noise or fluctuations in the initial particle longitudinal distribution. The slippage in one gain length determines the cooperation length. The output radiation pulse comes in the form of spikes of random phase and amplitude and with a width of the order of the cooperation length.

In addition to the fundamental wavelength, the SASE process results in harmonics with significant intensity. The intensity of the third harmonic is about $1 \%$ of the intensity of the fundamental and extends the radiation wavelength range of the FEL to $0.5 \mathrm{~A}$.

These theoretical predications have been confirmed in a series of experiments. Large amplification of the spontaneous undulator radiation, reaching the saturation level, has been 
demonstrated in SASE FEL experiments in the infrared, visible, and UV spectral regions. Saturation has been observed at wavelengths as short as $98 \mathrm{~nm}$. The experimental results on the gain length, saturation and the intensity fluctuation distribution are in good agreement with the FEL collective instability theory.

The pulse length and linewidth of the x-ray pulses from the LCLS can be controlled and improved over the LCLS initial design, by manipulating the electron beam and/or the radiation pulse and with the use of multiple undulators. The possibility offered by the LCLS to study and develop these methods is important, and depends critically on the electron beam quality and the choice of undulator length and gap.

\subsection{Introduction}

Undulator radiation, particularly useful because of its small line width and high brightness, is being used in many synchrotron radiation sources built around the world and provides at present the brightest source of x-rays. For long undulators, the free-electron laser (FEL) collective instability gives the possibility of much larger x-ray intensity and brightness. The instability produces an exponential growth of the radiation intensity, together with a modulation of the electron density on the scale of the radiation wavelength. The radiation field initiating the instability is the spontaneous radiation field, or a combination of the spontaneous radiation field and an external field. In the first case the FEL is called a Self-Amplified Spontaneous Emission (SASE) FEL. If the external field is dominant it is called an FEL amplifier.

The FEL is a system consisting of a relativistic electron beam and a radiation field, interacting with each other while they propagate through an undulator. The undulator magnetic field generates an electron velocity component transverse to the direction of propagation. The transverse velocity couples the electron beam to the electric field component of the radiation field, thus producing an energy exchange between them. The coupling can lead to the formation of density modulations (structures), collective modes, in the electron beam, and to the generation of coherent electromagnetic radiation. The formation of structures also enhances the energy exchange. As a result, the transition from the original unstructured state of the electron beam to the collective state is an exponential process. This chapter discusses the physics of this process; some of the experimental results obtained recently, and the development of x-ray FELs.

The FEL is the result of many years of theoretical and experimental work on the generation of radiation from relativistic electron beams. The first generators of coherent electromagnetic (EM) radiation from electron beams were the microwave tubes. Their development received a strong impulse during World War II. Microwave tubes use slow wave structures, a fact that limits their operation mainly to long wavelengths, i.e., in the centimeter region. FELs were developed from the work on free electron beams. Motz [1] showed in 1951 that an electron beam propagating through an undulator magnet could be used to amplify radiation. The Ubitron, a microwave tube developed in 1960 by Philips [2], is quite similar to the FEL. Theoretical work on FELs was done in the 1960s and 1970s by Palmer [3], Robinson [4] and Csonka [5]. 
During the 1960s, the research on the generation of short wavelength coherent radiation turned mainly in the direction of atomic and molecular lasers, and optical resonators. While extremely successful in the infrared (IR), visible and UV, these lasers have limited tunability, and this line of development has limitations at shorter wavelengths. While soft x-ray lasers have been built at several laboratories, such as the University of Colorado, Princeton, and Livermore, their extension to the Angstrom region is problematic. The use of electron beams and FELs is an alternative when atomic and molecular lasers and microwave tubes cannot be used. Madey [6], in 1971, analyzed again the possibility of exchanging energy between free electrons and electromagnetic radiation in the small gain regime, using a quantum theoretical approach. He and his coworkers followed this work with successful experimental demonstration of a FEL amplifier [7], and an FEL oscillator [8] at $10 \mu \mathrm{m}$. This very important step led over the following years to a large interest in free-electron lasers, and to the successful construction and operation of many FEL oscillators, at wavelengths from the far IR to the near UV. These FEL oscillators operated in the small-signal gain regime, using the optical cavity as a feedback device starting from the spontaneous synchrotron radiation noise.

While the existence of an exponentially growing solution for the FEL equations has been studied by many authors [9], the first theory of a SASE FEL in the 1-dimensional (1-D) case, including the start from spontaneous radiation and saturation, was given in [10]. This theory describes all of the FEL physics, including saturation power and undulator saturation length, with one single quantity, the FEL parameter, $\rho$, a function of the electron beam density and energy, and of the undulator period and magnetic field. Further studies clarified the initiation process and its connection with spontaneous radiation [11]. The next important step was the extension of the theory to three dimensions (3-D) [12] to include diffraction effects and to show the existence of optical guiding. More recent work has extended the 3-D model to include also the initiation process [13] and the betatron motion [14].

The first proposal to use the FEL collective instability to produce infrared radiation using a single pass amplifier starting from noise was published by Kondratenko and Saldin in 1980 [15]. The first proposal to use the instability using a single pass amplifier starting from noise for a soft x-ray FEL was published by Murphy and Pellegrini in 1985 [16]. The choice of a single pass amplifier instead of an oscillator in the soft or hard x-ray region is motivated by the fact that optical cavities have large losses and that they are difficult to build and operate at these wavelengths.

An analysis of the scaling laws [17] for a single pass FEL, starting from noise, shows that the gain of a SASE-FEL depends on wavelength, and that to reach the soft or hard x-ray region one needs an electron beam with a large six dimensional phase-space density, a condition, which until recently, was difficult to satisfy.

The Murphy-Pellegrini proposal used a bypass in a storage ring to provide the electron beam. At that time, an electron storage ring was the accelerator delivering an electron beam with the highest phase space density. However, the limitations on emittance, peak current, and energy 
spread due to the storage ring collective effects, such as the microwave instability or the Touschek effect, limited the shortest FEL wavelength to about a few hundred Ångstroms.

The development of radio frequency photocathode electron guns [18], and the emittance compensation method [19] has changed this situation. At the same time the work on linear colliders has demonstrated that it is possible to accelerate and time compress electron beams without spoiling their brightness [20]. At a Workshop on Fourth Generation Light Sources held at SSRL in 1992 it was shown [21] that using these new developments one could build a 0.1 to $1 \mathrm{~nm}$ SASE FEL. This work led to further studies [22] and to two major proposals, LCLS at SLAC [23] and TESLA at DESY [24] for a $0.1 \mathrm{~nm}$ SASE-FEL, with peak power of the order of tens of GW, pulse length of about $230 \mathrm{fs}$ (FWHM) or shorter, full transverse coherence, and peak brightness about ten orders of magnitude larger than that of third generation synchrotron radiation sources.

While the theory of the SASE FEL has been developed starting in the 1980s, experimental results have been obtained only during the last few years, initially in the infrared to visible region of the spectrum, and more recently at wavelengths as short as $80 \mathrm{~nm}[25,26,27,28,29,30,31$, $32,33,34,35,36]$, the shortest wavelength reached by an FEL to date. The data agree well with the theoretical predictions on exponential growth and its dependence on electron beam parameters and on the intensity fluctuations. These results give us confidence that the present theory can be used to design an x-ray SASE FEL.

This chapter starts with a general overview of FEL physics in Section 4.2 and continues, in Section 4.3, with a review of the recent results of SASE experiments in the infrared, visible and ultra-violet wavelength-regime. Section 4.4 introduces the specific situation of the LCLS. The effects of the emission of ordinary undulator radiation on the FEL process is analyzed in Section 4.5. The effects of undulator vacuum chamber wakefields on the performance of the LCLS are assessed in Section 4.6. Section 4.7 discusses an option of emittance and charge control based and Section 4.8 evaluates options for x-ray pulse length and linewidth reduction that will be available as an upgrade of the present LCLS design.

\subsection{Free-electron Laser Physics}

\subsubsection{Coherent Undulator Radiation from a Single Electron}

The emission of radiation from relativistic electrons traveling through an undulator is reviewed. The reader is referred to other books or papers, as for instance reference [37], for a more general discussion. For a planar or helical undulator the radiation is emitted at the wavelength

$$
\lambda_{r}=\frac{\lambda_{u}}{2 \gamma^{2}}\left(1+a_{u}^{2}+\gamma^{2} \theta^{2}\right)
$$

where $\lambda_{u}$ is the undulator period, $m c^{2}$ the beam energy, $a_{u}=e<B_{u}{ }^{2}>^{1 / 2} c \lambda_{u} / 2 \pi m c^{2}$ is the undulator parameter, $B_{u}$ is the undulator transverse magnetic field, $\left\langle B_{u}{ }^{2}>^{1 / 2}\right.$ is the rms over one undulator period, and $\theta$ is the angle between the undulator axis and the direction at which the radiation is observed. Note, the relation between the frequently used undulator parameter, $K$, and $a_{u}$ is $K=a_{u}$ 
(for a helical undulator) and $K=a_{u} \sqrt{ } 2$ (for a planar undulator.) For reasons described in Chapter 8, a planar undulator was chosen for the LCLS. The rms width of the radiation line (bandwidth) on axis, $\theta=0$, is related to the number of undulator periods $N_{u}$ by

$$
\frac{\Delta \omega}{\omega}=\frac{1}{N_{u}} \text {. }
$$

The undulator is an extended linear source, but the coherent part of the radiation within the on-axis bandwidth Eq. (4.2) can be approximately described as an equivalent source at the undulator center, with rms divergence

$$
\sigma_{c}^{\prime}=\sqrt{\frac{\lambda_{r}}{\lambda_{u} N_{u}}}
$$

and an effective rms source radius ( diffraction limited)

$$
\sigma_{c}=\frac{1}{4 \pi} \sqrt{\lambda_{r} \lambda_{u} N_{u}}
$$

Notice that the product

$$
\sigma_{c} \sigma_{c}^{\prime}=\frac{\lambda_{r}}{4 \pi}
$$

is the minimum phase space area for a diffraction-limited photon beam.

The intensity of the radiation emitted on axis, at the wavelength given by Eq. (4.1) and its harmonics $n$, per unit frequency and solid angle is

$$
\frac{d^{2} I}{d \omega d \Omega}=\frac{N_{u}^{2} e^{2} \gamma^{2}}{c} \frac{a_{u}^{2}}{1+a_{u}^{2}} F_{n}\left(a_{u}\right),
$$

where

$$
\begin{aligned}
& F_{n}\left(a_{u}\right)=\left\{J_{(n-1) / 2}(\zeta)-J_{(n+1) / 2}(\zeta)\right\}^{2} n^{2}, \\
& \zeta=\frac{a_{u}{ }^{2}}{2\left(1+a_{u}{ }^{2}\right)},
\end{aligned}
$$

for a planar undulator. ( $n=1$ is the fundamental). For a helical undulator $F_{l}\left(a_{u}\right)=1$ for the fundamental and $F_{n}\left(a_{u}\right)=0$ for harmonics, i.e., $n>1$.

The coherent intensity is obtained by multiplying Eq. (4.6) by the solid angle corresponding to Eq. (4.3), $\Delta \Omega_{c}=\pi \sigma_{c}^{\prime 2}$ and the bandwidth given by Eq. (4.2). Dividing this intensity by the photon energy allows to rewrite the coherent intensity as the number of photons per electron, within the same solid angle and line width, as

$$
N_{p h}=\frac{\pi \alpha}{2} \frac{a_{u}^{2}}{\left(1+a_{u}^{2}\right)} F_{1}\left(a_{u}\right),
$$

where $\alpha$ is the fine structure constant. For a typical value $a_{u} \sim 1$, one obtains $N_{p h} \sim 10^{-2}$, showing that the undulator radiation process is rather inefficient. 


\subsubsection{Coherent Spontaneous Radiation from Many Electrons}

To avoid an increase in the radiation linewidth, angular spread, and transverse radius, the relative rms energy spread, $\sigma_{E} / E$, radius, $\sigma$, and angular divergence, $\sigma^{\prime}$, of the electron beam must be matched to the radiation linewidth $1 / N_{u}$, radius, $\sigma_{c}$, and angular divergence, $\sigma_{c}{ }^{\prime} . N_{u}$ is the number of undulator periods. This gives the conditions

$$
\begin{gathered}
\frac{\sigma_{E}}{E} \leq \frac{1}{N_{u}} \\
\sigma \leq \sigma_{c} \\
\sigma^{\prime} \leq \sigma_{c}{ }^{\prime} .
\end{gathered}
$$

The last two conditions can be written, using Eq. (4.5), as

$$
\varepsilon=\sigma \sigma^{\prime} \leq \frac{\lambda}{4 \pi}
$$

where $\varepsilon$ is the transverse beam emittance. The last condition can be seen as "phase space matching" of the electrons and photons.

The radiation generated from different electrons entering the undulator at different points in time $t_{o, k}$ (where $\mathrm{k}$ is an index of the electrons) along the bunch differs only by a phase factor. The electric field at frequency $\omega=2 \pi c / \lambda_{r}$ can be written as

$$
E_{k}=E_{0} e^{i \omega t_{o, k}}
$$

where $E_{0}$ is a common factor. The total electric field is then proportional to the bunching factor,

$$
B_{0}=\frac{1}{N_{e}} \sum_{k=1}^{N_{e}} e^{i \omega t_{0, k}},
$$

where $N_{e}$ is the number of electrons in the bunch. For a short undulator, i.e., short with respect to the FEL gain length defined in the next section, the bunching parameter does not appreciably change as the beam propagates through the undulator, but, as can be seen below, it can change in the case of a long undulator. In the "short undulator" case the total intensity is then

$$
I=I_{0}\left|B_{0}\right|^{2} N_{e}^{2} \text {. }
$$

Three cases can be considered:

a. Uniform beam current: This corresponds to a uniform distribution the electron phases with respect to radiation wave, giving $B_{0}=0$ and $I=0$;

b. Bunch length much shorter than the radiation wavelength: Then $\left|B_{0}\right|^{2} \sim 1$. In this case the radiation from all electrons has the same phase and the intensity is proportional to the square of the number of electrons and can be called coherent radiation;

c. In most cases, as for instance when the electron beam is generated by a thermionic cathode or by a photocathode, and when the bunch length is longer than the wavelength, the quantity $B_{0}$ is a random number, changing for each 
electron bunch that is produced. Averaging over many bunches one has $\left\langle B_{0}>=0\right.$, and $\left\langle\left|B_{0}\right|^{2}>\sim 1 / N_{e}\right.$.

This discussion assumes a classical picture of the electron beam and of the electromagnetic radiation. Quantum effects will be discussed later and are small in the LCLS case.

Considering now the emission of radiation by many electrons within the coherent solid angle and the line width defined before, in case $\mathrm{c}$, when there is no correlation between the field emitted by each one of them, the total number of photons emitted is simply

$$
N_{p h}=\frac{\pi \alpha}{2} N_{e} \frac{a_{u}^{2}}{1+a_{u}^{2}} F_{1}\left(a_{u}\right) .
$$

If case b would apply this number would be larger by another factor $N_{e}$, a very large enhancement.

\subsubsection{SASE-FELS}

In this section only the main results are considered and the reader is referred to the many papers already given as references for a more complete discussion.

The physical process on which an FEL is based is the emission of radiation from one relativistic electron propagating through an undulator. However collective effects can lead to interesting new situations when many electrons interact with the undulator and the radiation fields. Consider the emission of coherent radiation from $N_{e}$ electrons, that is the radiation at the wavelength $\lambda_{r}$, Eq.(4.1), within the coherent solid angle $\pi \sigma_{c}^{2}$, Eq. (4.3), and line width $\Delta \omega / \omega$, Eq. (4.2).

When there is no correlation between the fields generated by each electron, as in the case of spontaneous radiation, the total number of coherent photons emitted, given by Eq. (4.16), is about $1 \%$ of the number of electrons. If all electrons were within a radiation wavelength the number of photons would increase by a factor $N_{e}$. Even when this is not the case, and the electron distribution on the scale of $\lambda_{r}$ is initially random, the number of photons per electron is increased by the FEL collective instability, which produces an exponential growth of the intensity and of the bunching parameter

$$
B_{z}=\frac{1}{N_{e}} \sum_{k=1}^{N_{e}} e^{i \omega t_{z, k}},
$$

where $t_{z, k}=z_{k} / v_{k}$ is the time for electron $k$, moving with the longitudinal velocity $v_{\mathrm{k}}$, to reach the longitudinal position $z_{k}$. The growth saturates when the bunching parameter becomes of the order of one. For a long undulator the coherent intensity grows along the undulator as

$$
I \sim \frac{I_{0}}{9} \exp \left(z / L_{G}\right)
$$

where $L_{G}$ is the exponential growth rate, called the power gain length, and $I_{0}$ is the spontaneous coherent undulator radiation intensity for an undulator with a length $L_{G}$, and is proportional to the square of the initial value of the bunching factor, $\left|B_{0}\right|^{2}$. 
The instability growth rate, or gain length, is given in a 1-D model by [10]

$$
L_{G}=\frac{\lambda_{u}}{4 \sqrt{3} \pi \rho}
$$

where $\rho$ is the FEL parameter

$$
\rho=\left(\frac{a_{u}}{4 \gamma} \sqrt{F_{1}\left(a_{u}\right)} \frac{\Omega_{p}}{\omega_{u}}\right)^{2 / 3},
$$

$\omega_{u}=2 \pi c / \lambda_{u}$ is the undulator frequency, $\Omega_{p}=\left(4 \pi c^{2} r_{e} n_{e} / \gamma\right)^{1 / 2}$ is the beam plasma frequency, $n_{e}$ is the electron density, and $r_{e}$ is the classical electron radius. A similar exponential growth occurs if there is an initial input field that dominates any noise in the beam, i.e. amplified stimulated emission.

In the SASE case, saturation occurs after about 20 power gain lengths, and the radiated energy at saturation is about $E_{s a t}=\rho N_{e} E$ [10], where $E$ is the total kinetic energy of the electron beam. The number of photons per electron at saturation is then $N_{s a t}=\rho E / E_{p h}$, where $E_{p h}$ is the energy of a single photon. For an x-ray FEL with $E_{p h} \sim 10^{4} \mathrm{eV}, E \sim 15 \mathrm{GeV}, \rho \sim 0.5 \times 10^{-3}$ one obtains $N_{\text {sai }} \sim 10^{3}$, i.e., an increase of almost 5 orders of magnitude in the number of photons per electron.

The instability can develop only if the undulator length is much larger than the power gain length, and some other conditions are satisfied:

a. Beam emittance of the order of or smaller than the wavelength:

$$
\varepsilon \leq \frac{\lambda}{4 \pi}
$$

b. Beam relative energy spread smaller than the FEL parameter:

$$
\sigma_{E} / E<\rho
$$

c. Power gain length shorter than the radiation Rayleigh range:

$$
L_{G}<L_{R}
$$

where the Rayleigh range is defined as $L_{R}=2 \pi \sigma_{0}^{2} / \lambda_{r}$, and $\sigma_{0}$ is the radiation rms beam radius.

Condition a. says that the electron beam must match the transverse phase-space of the radiation. Condition $b$. limits the electron beam energy spread, and condition c. requires that more radiation be produced than is lost by diffraction. Conditions a. and c. depend on beam radius and radiation wavelength, and are not independent from each other. If they are satisfied, the 1-D model can be used with good approximation. If they are not satisfied the gain length is larger than the 1-D value, Eq. (4.19), as in the LCLS reference case where the emittance $\varepsilon \sim 3 \times(\lambda / 4 \pi)$. In these cases, it is convenient to introduce an effective FEL parameter, which includes three dimensional effects, defined as

$$
\rho_{\text {eff }}=\frac{\lambda_{u}}{4 \sqrt{3} \pi L_{G 3 D}},
$$


where $L_{G 3 D}$ is the three dimensional gain length obtained from numerical simulations that includes diffraction and emittance effects.

\subsubsection{Slippage, Fluctuations and Time Structure}

When propagating in vacuum, the radiation field is faster than the electron beam, and it moves forward, "slips", by one wavelength, $\lambda_{r}$, per undulator period. The slippage distance in one gain length defines the "cooperation length" [38]

$$
L_{c}=\frac{\lambda_{r}}{\lambda_{u}} L_{G}
$$

For a SASE FEL, the undulator radiation field at the frequency $\omega=2 \pi \lambda / c$, is proportional to $\boldsymbol{B}(\omega)$, the Fourier component of the initial bunching factor $B_{0}$ at $\omega$. The beam is generated either from a thermionic cathode or from a photocathode. The initial bunching and its Fourier component $\boldsymbol{B}(\omega)$, are random quantities. The initial value of $B_{0}$ is different for each beam section of length $\lambda_{r}$, and has a random distribution. The average values are then

$$
\langle\boldsymbol{B}(\omega)\rangle \sim\left\langle\boldsymbol{B}_{0}\right\rangle=0
$$

and

$$
\left\langle|\boldsymbol{B}(\omega)|^{2}\right\rangle \sim\left\langle\left|\boldsymbol{B}_{0}\right|^{2}\right\rangle=\frac{1}{N_{e}}
$$

As the electron beam and the radiation propagate through the undulator, the FEL interaction introduces a correlation on the scale length of $2 \pi L_{c}$, producing spikes in the radiation pulse and a random intensity distribution. The number of spikes is $[38,39] M=L_{B} /\left(2 \pi L_{\mathrm{c}}\right)$, where $L_{B}$ is the rms bunch length. The total energy probability distribution in the $\mathrm{x}$-ray pulse is a Gamma distribution function

$$
P(W)=M^{M} \frac{W^{M-1}}{<W>^{M} \Gamma(M)} \exp (-M W /<W>),
$$

where $<W>$ is the average energy of the $\mathrm{x}$-ray pulse. The standard deviation of this distribution is $1 / M^{1 / 2}$. The line width is approximately the same as for the spontaneous radiation, $\Delta \omega / \omega \sim 1 / N_{u}$.

\subsubsection{Nonlinear Harmonic Generation}

In a high-gain FEL strong bunching at the fundamental wavelength can drive substantial bunching and, for a planar undulator, significant emitted power at the harmonic frequencies [40]. This nonlinear harmonic generation occurs naturally in one long undulator for a SASE FEL with an initially uniform bunch, as well as in the second stage of a high-gain harmonic generation (HGHG) FEL [41] using a density-modulated bunch. Thus, such a harmonic generation mechanism may be utilized to reach shorter radiation wavelengths or to relax some stringent requirements on the electron beam quality for x-ray FELs.

A three-dimensional theory of harmonic generation in a high-gain FEL has been developed [42] using the coupled Maxwell-Klimontovich equations that include electron energy spread and 
emittance, radiation diffraction and guiding, and nonlinear harmonic interactions. In general, each harmonic field is a sum of a linear amplification term and a term driven by nonlinear harmonic interactions. After a certain stage of exponential growth, the dominant nonlinear term is determined by interactions of the lower nonlinear harmonics and the fundamental radiation.

As a result, parameters such as gain length, transverse profile, and temporal structure of the first few harmonics are eventually governed by those of the fundamental. For example, for SASE FELs, driven by the third power of the radiation field in the fundamental, the third nonlinear harmonic grows three times faster, is transversely coherent (with a smaller spot size), and has a more spiky temporal structure than the fundamental wavelength.

Using the LCLS parameters, the transverse profiles of the third nonlinear harmonic and the fundamental radiation are calculated in the exponential growth regime [42]. The third nonlinear harmonic (at $0.5 \AA$ ) is also transversely coherent but has a smaller spot size than the fundamental because of the nonlinear generation mechanism. From this analysis one obtains for the third harmonic power [42]

$$
\frac{P_{3}}{\rho P_{\text {beam }}} \approx 0.11 \times\left(\frac{P_{1}}{\rho P_{\text {beam }}}\right)^{3},
$$

where $P_{\text {beam }}$ is the total electron beam power, and $P_{1}$ is the fundamental radiation power.

The nonlinear harmonic radiation discussed here is generated when the fundamental frequency component of the FEL radiation bunches the electron beam strongly, producing Fourier components at higher harmonics. Thus, as long as the laser fundamental saturates after a certain length of undulator, the nonlinear harmonics are generated at certain levels, and are less sensitive to electron-beam parameters, undulator errors and wakefield effects than is true for other (linear) harmonic generation schemes. The most significant nonlinear harmonic generation occurs at the third harmonic, whose power approaches one percent of the fundamental power level near saturation. The even harmonics are also present due to the transverse gradient of the beam current. They normally have much lower power levels than their odd counterparts [42]. As discussed in another section, wakefields can reduce the power in the fundamental and one can expect a reduction also in the higher harmonics.

\subsubsection{Quantum Effects in Free-Electron Lasers}

The quantum theory of free-electron lasers has been studied by several authors, and a review of this work can be found in $[43,44]$. More recently, a many-electron quantum theory for a high gain SASE-FEL, like the LCLS, has been developed to look again at possible effects on the FEL start-up, and on the gain length [45].

Quantum effects in the FEL become important when the electron beam wave function exhibits degeneracy and the electrons can no longer be treated as distinguishable particles. The wave function will not be degenerate if the number of electrons in the beam is much less than the number of available states. This will be the case when the beam is sufficiently dilute such that the wave functions of the individual electrons do not overlap, i.e., $\varepsilon_{\perp}^{2} \varepsilon_{L}>N_{e} \lambda_{c}^{3}$, where $\varepsilon_{\perp}$ and $\varepsilon_{L}$ 
are the transverse and longitudinal emittance respectively, $N_{e}$ is the number of electrons, and $\lambda_{c}$ is the Compton wavelength. This condition is well satisfied for LCLS parameters.

Quantum corrections to the classical theory become important when the electron beam becomes sensitive to the recoil due to the emission or absorption of discrete photons. The recoil will be small provided the photon energy is much less than the energy emitted per electron at saturation $\hbar \omega<<E_{\text {beam }}$, where $\omega$ is the radiation frequency and $E_{\text {beam }}$ is the electron beam energy. The electron recoil due to discrete photon emission will result in an increased energy spread and therefore an increase in power gain length. The one-dimensional power gain length increases as $L_{G} / L_{\text {class }} \cong 1+\left(\hbar \omega / \rho E_{\text {beam }}\right)^{2} / 36$, for $\left(\hbar \omega / \rho E_{\text {beam }}\right)<1$, where $L_{\text {class }}$ is the power gain length predicted by classical theory. For the LCLS case $\rho E_{\text {beam }} / \hbar \omega \cong 816$, and the corrections are negligible.

Quantum fluctuations in the position and momentum of the electron beam will also act as effective bunching (i.e., noise) in the electron beam resulting in enhanced start-up of the SASE process. The effective bunching due to the quantum fluctuations will be of the order of $\left(\hbar \omega / \rho E_{\text {beam }}\right)$ compared to the classical shot noise and therefore will be small for LCLS parameters.

\subsection{Experimental Results on SASE-FELs}

Until the mid 1990s, very large gain in the SASE mode had been observed only at millimeter wavelengths [25]. After that the experimental studies of SASE FEL physics intensified. The first gain in the infrared wavelength regime at values between 2 to 10 has been observed at Orsay [26] and UCLA [27] and gain in the visible spectral region at Brookhaven [28]. Larger gain in the infrared has also been observed at Los Alamos [29], and gain as large as $3 \times 10^{5}$ at $12 \mu \mathrm{m}$ has been measured by a UCLA-LANL-RRCKI-SSRL collaboration [30].

The intensity distribution function has been measured for spontaneous undulator radiation, with no amplification and long bunches [46] and, more recently, for amplified radiation and a short bunch length [27, 30].

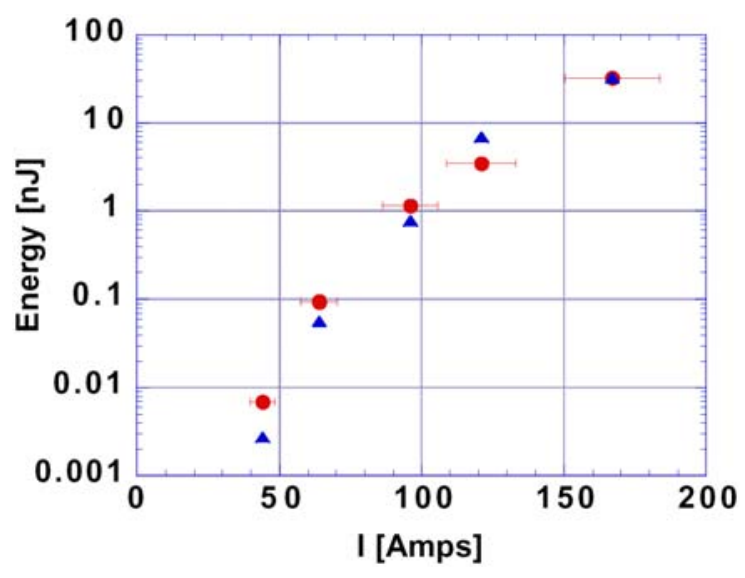


Figure 4.1 Measured values of the mean FEL intensity (circles) versus beam current, compared with a Ginger simulation (triangles) for the UCLA-LANL-RRCKI-SSRL $12 \mu \mathrm{m}$ SASE-FEL [30].

Results from the UCLA-LANL-RRCKI-SSRL experiment are shown in Figure 4.1 and Figure 4.2. An increase in output intensity by about $10^{4}$ occurs when changing the electron charge by a factor of seven and the peak current by a factor of four as shown in Figure 4.1. The change of bunch radius, energy spread, and length with charge makes it difficult to have a simple analytical model to evaluate the gain. The experimental data and the theory have been compared using the simulation code GINGER [47], and the measured values of electron parameters. The results, plotted in Figure 4.1, agree with the data within experimental errors. At $2.2 \mathrm{nC}$ the measured gain was $3 \times 10^{5}$, the largest measured, until recently, in the infrared. The intensity fluctuations, shown in Figure 4.2, are well described by a Gamma distribution function (Eq. (4.28)) with the $M$ parameter evaluated from the experimental data in agreement with the theory. The results have also been analyzed independently in reference [48], and again have been found to be in agreement with the theory.

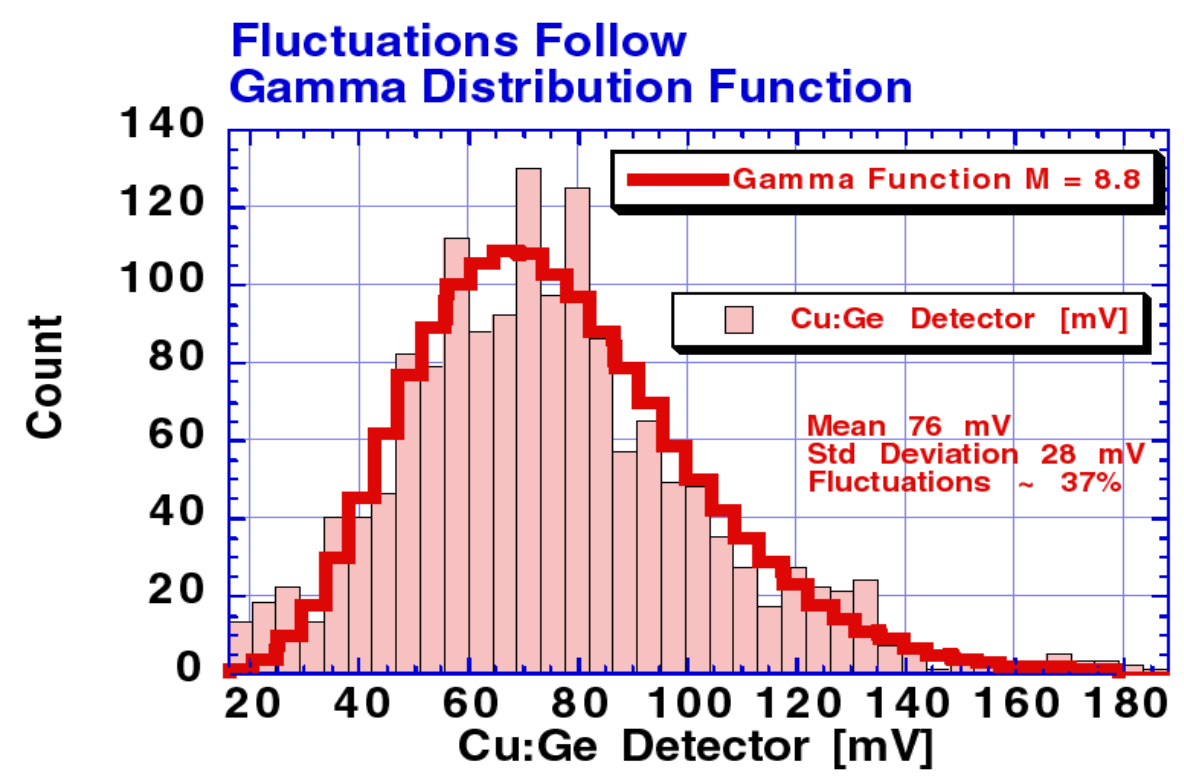

Figure 4.2 Intensity distribution over many events for the same experiment. The experimental data are fitted with a Gamma function distribution [30].

More recently, a BNL group [31] has demonstrated high gain harmonic generation, seeding the FEL with external laser radiation at a wavelength of $10.6 \mu \mathrm{m}$ producing FEL output radiation at a wavelength of $5.3 \mu \mathrm{m}$, with an intensity $2 \times 10^{7}$ times larger than spontaneous radiation. 
The TESLA Test Facility (TTF) SASE FEL at DESY [34], using the superconducting linac of, up to an energy of $310 \mathrm{MeV}$, and a $15-\mathrm{m}$ long undulator. The group has reached saturation at a wavelength of $98 \mathrm{~nm}$ (see Figure 4.3).

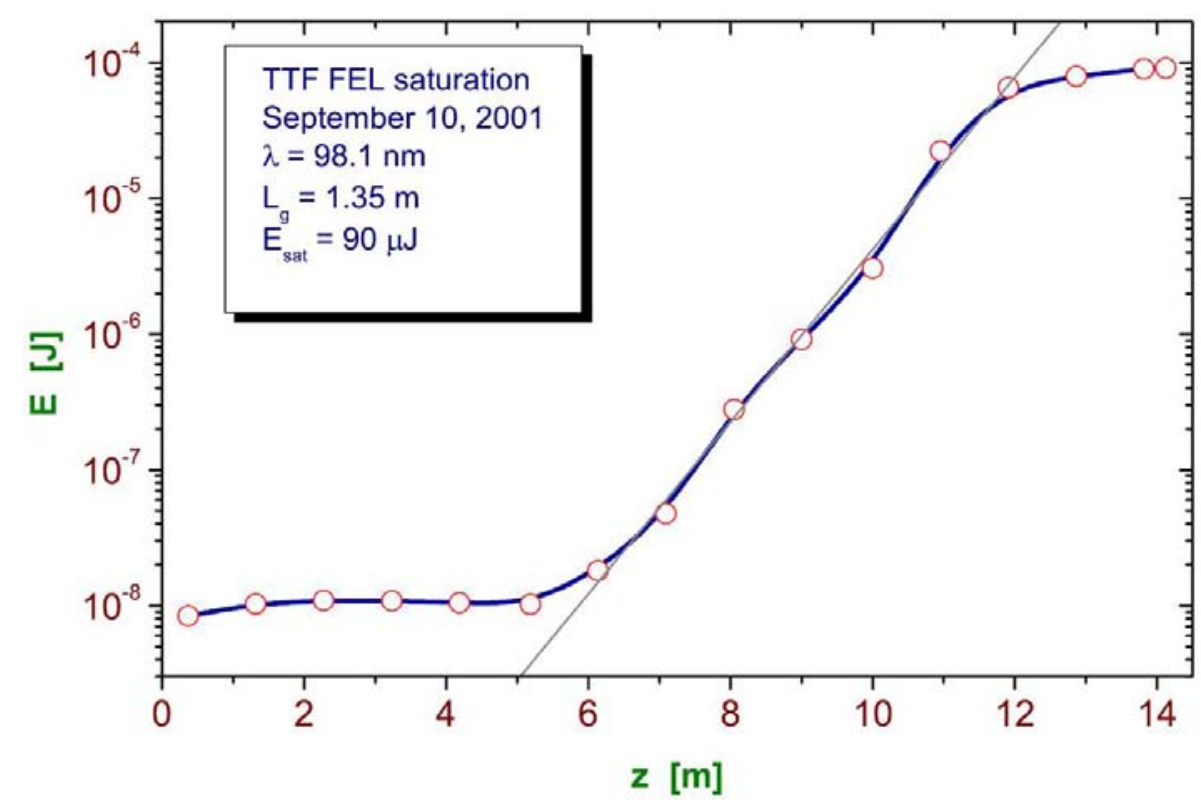

Figure 4.3 The TTF SASE-FEL achieves saturation at a wavelength of $98.1 \mathrm{~nm}$ on September 10, 2001. 
The LEUTL SASE FEL experiment at the APS [33, 35] using the APS injector linac with an energy range of 220-444 MeV, and a 21.6-m long undulator, has reached saturaton at $530 \mathrm{~nm}$, $385 \mathrm{~nm}$ and $130 \mathrm{~nm}$. Figure 4.4. shows the saturation at $530 \mathrm{~nm}(\mathrm{~A})$ and $385 \mathrm{~nm}(\mathrm{C})$ as well as output power reduction through the intentional reduction of peak current (B). The solid lines in the figure are simulations using the experimentally measured beam properties. The agreement is very good. The same good agreement was achieved in an absolute comparison of the measured data using measured beam properties to the simulated absolute energy results.

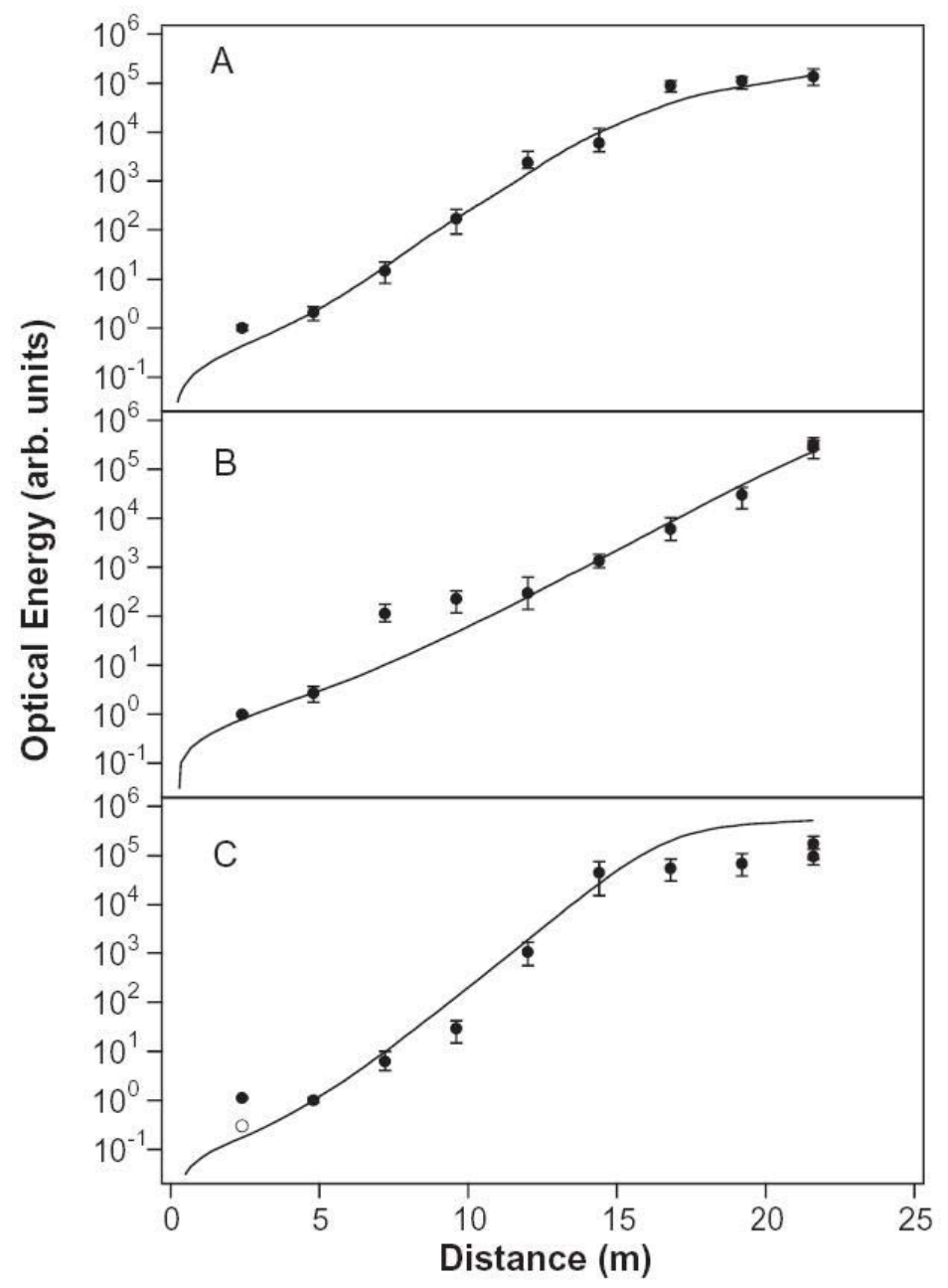

Figure 4.4 Exponential growth and saturation at $530 \mathrm{~nm}$ (case A) and $358 \mathrm{~nm}$ (case C), in the LEUTL experiment. Case $B$ shows the reduction in gain obtained by reducing the beam current [35]. 
The VISA experiment, a BNL-LLNL-SLAC-UCLA collaboration, has obtained a gain of about $2 \times 10^{8}$ and reached saturation at a radiation wavelength of $840 \mathrm{~nm}$ in 3.6-3.8 m using a 4-m long undulator with distributed strong focusing quadrupoles [36]. The properties of SASE radiation, as a function of distance through the undulator magnet have been measured and analyzed. For the first time, observation and analysis of the statistical intensity fluctuations of SASE radiation at saturation was studied, and compared to the data obtained during exponential growth. These results are compared to the start-to-end numerical model of the experiment, which follows the electron beam dynamics from photocathode emission, through acceleration, transport and the undulator. This combination of experimental results and start-to-end simulations resulted in a comprehensive description of the underlying beam dynamics and FEL process. Additional results based on VISA at saturation include measurements of FEL harmonic radiation and electron beam microbunching. The measured energy of the radiation as function of position along the undulator and the prediction from the numerical model are shown in Figure 4.5.

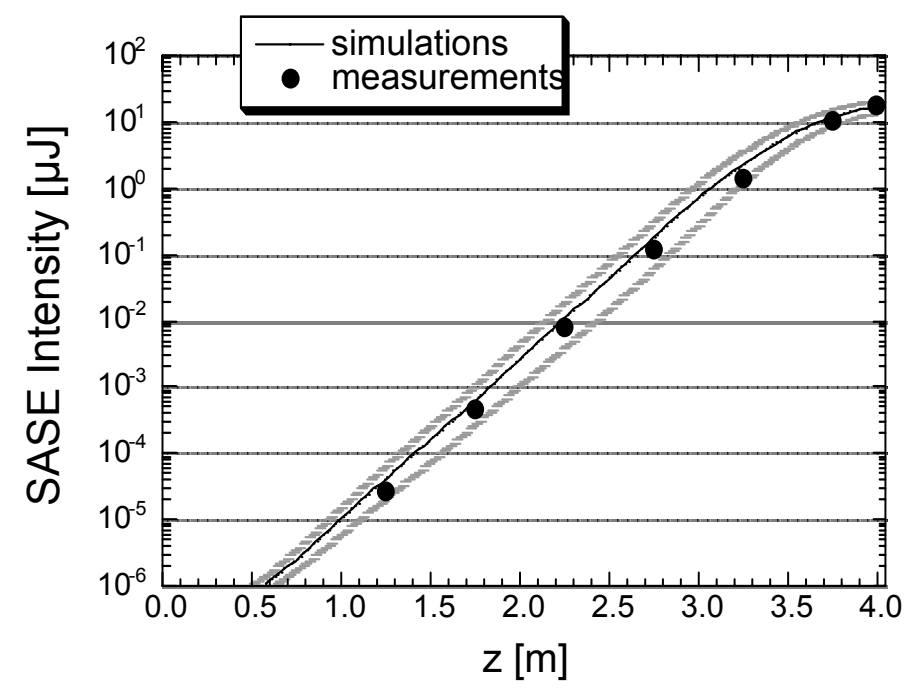

Figure 4.5 Exponential growth and saturation in the VISA experiment at $840 \mathrm{~nm}$, in a 4-m long undulator [36]. The outer curves are the predictions for the 1-sigma limits of the intensity fluctuations.

Similar experimental programs on SASE-FELs are being prepared at Spring8 in Japan, at BESSY-II in Berlin, in China and at other laboratories.

\subsection{LCLS: An X-Ray SASE-FEL}

The first proposal for a SASE-FEL using the SLAC linac was made in 1992 [21]. The initial design was developed by a study group until 1996 after which a design group prepared the LCLS Design Study Report [23] that was published in 1998.

A short summary of the main LCLS parameters is given in Table 4.1. A full list can be found in Appendix A. The components of the LCLS and its operational principals are described in full detail in this CDR. 
The LCLS will generate coherent radiation at a fundamental wavelength between $15 \AA$ and $1.5 \AA$. A strong third harmonic component is also produced as discussed above. The LCLS undulator also generates incoherent radiation, which, at the highest electron energy of $14.3 \mathrm{GeV}$, has a spectrum extending to about $500 \mathrm{keV}$ and a peak power density of $10^{13} \mathrm{~W} / \mathrm{cm}^{2}$, on-axis. The peak coherent power density of the first harmonic is about $2 \times 10^{14} \mathrm{~W} / \mathrm{cm}^{2}$, and the peak electric field is about $4 \times 10^{10} \mathrm{~V} / \mathrm{m}$.

The FEL saturation length, the saturation power, and the alignment tolerances depend significantly on the electron beam parameters, and the effect of wakefields in the undulator vacuum pipe, as is discussed in detail in the next sections. Assuming that the electron beam has the parameters given in Table 4.1, in particular a 1.2- $\mu \mathrm{m}-\mathrm{rad}$ normalized RMS emittance, the SASE amplification process can be simulated.

The simulations are done using the 3D time-dependent code GENESIS 1.3, which includes the effect of quantum fluctuation and wakefields [49]. As can be seen in the next sections, wakefields and quantum fluctuation from the incoherent spontaneous radiation have an effect on the amplification process of a SASE FEL. The GENESIS 1.3 code has been successfully benchmarked with various other FEL codes in the steady-state regime [50], and recently with GINGER for time-dependent simulation. In addition, the simulation of the UCLA/LANL/RRCKI/SLAC experiment [30] shows good agreement with the experimental data [51].

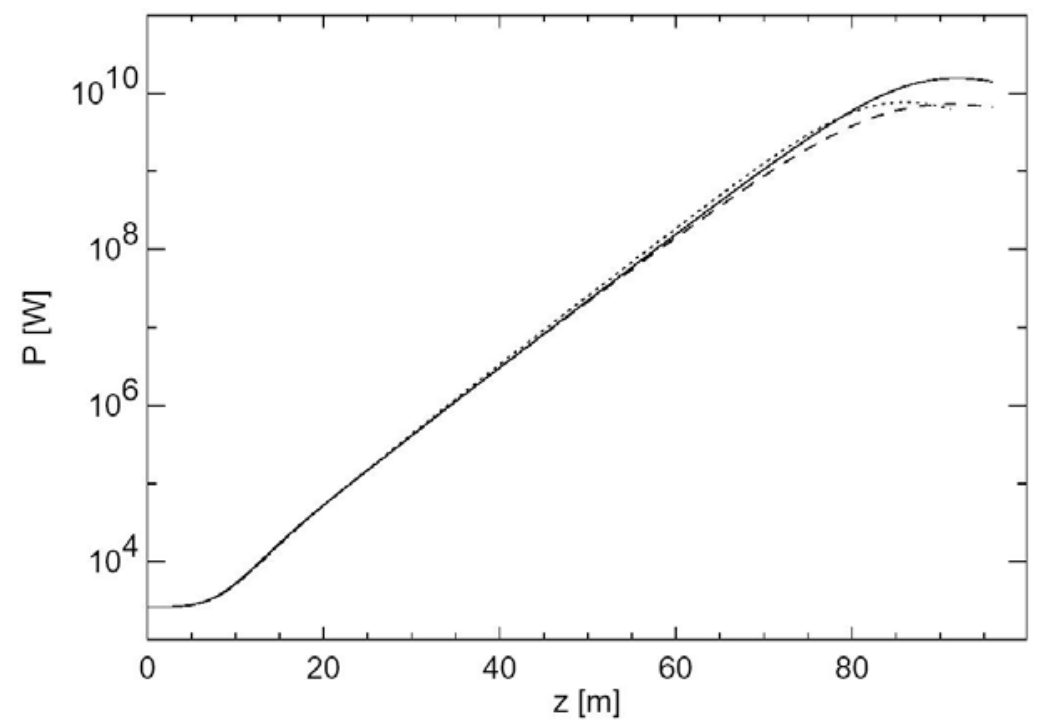

Figure 4.6 Power vs. undulator length for the LCLS case - normalized emittance $1.2 \mu \mathrm{m}$ rad, peak current $3.4 \mathrm{kA}$. Solid curve: no wakefields; dotted curve: long roughness bump case; dashed curve: short roughness bump case. The saturation length is about $90 \mathrm{~m}$, and the saturation power levels for the three cases are: $15 \mathrm{GW}, 8 \mathrm{GW}$ and $7 \mathrm{GW}$.

In Figure 4.6, power as a function of undulator length is plotted and shows a power gain length of $4.8 \mathrm{~m}$ and a saturation length of about $92 \mathrm{~m}$ as predicted by GENESIS. The solid curve represents the case where there are no undulator errors, misalignments, and no undulator wakefields. The effect of wakefields is given by the dashed and dotted curves and will be 
discussed in the next sections. The effects of errors and misalignments will be discussed in other chapters.

Table 4.1 LCLS electron beam, undulator, and FEL parameters. The electron beam parameters are valid at the undulator entrance.

\begin{tabular}{|l|l|l|}
\hline LCLS Eletron Beam Parameters @ $\mathbf{1 . 5} \AA$ & Value & Unit \\
\hline Electron energy & 14.35 & $\mathrm{GeV}$ \\
\hline Peak current & 3.4 & $\mathrm{kA}$ \\
\hline Normalized RMS slice emittance & 1.2 & $\mathrm{um} \mathrm{rad}$ \\
\hline RMS slice energy spread & $1 \times 10^{-4}$ & \\
\hline RMS bunch length & 77 & $\mathrm{fs}$ \\
\hline LCLS undulator parameters & & \\
\hline Undulator period & 3 & $\mathrm{~cm}$ \\
\hline Saturation length (including breaks) & 92 & $\mathrm{~m}$ \\
\hline Peak undulator field & 1.32 & $\mathrm{~T}$ \\
\hline Undulator parameter, $K$ & 3.711 & \\
\hline Undulator gap & 6 & $\mathrm{~mm}$ \\
\hline LCLS FEL parameters & & \\
\hline Radiation wavelength & 0.15 & $\AA$ \\
\hline FEL parameter. $O$ & $5 \times 10^{-4}$ & \\
\hline Power gain length & 4.8 & $\mathrm{~m}$ \\
\hline Effective FEL parameter. $O_{\text {aff }}$ & $2.93 \times 10^{-4}$ & \\
\hline Pulses repetition rate & 120 & $\mathrm{~Hz}$ \\
\hline Peak coherent power & 8 & $\mathrm{GW}$ \\
\hline Peak brightness & $0.8 \times 10^{33}$ & $*$ \\
\hline Average brightness & $4 \times 10^{22}$ & $*$ \\
\hline Cooperation length & 25 & $\mathrm{~nm}$ \\
\hline Intrinsic RMS intensity fluctuation & 6 & $\%$ \\
\hline Number of spikes & 270 & \\
\hline RMS line-width & $12 \times 10^{-4}$ & \\
\hline Total synchrotron radiation energy loss & $1.8 \times 10^{-3}$ & \\
\hline RMS Energy spread due to synchrotron radiation emission & $2 \times 10^{-4}$ & \\
\hline & & \\
\hline
\end{tabular}

* photon/(s mm $\left.\operatorname{mrad}^{2} 0.1 \% \mathrm{BW}\right)$

Since a SASE FEL starts from a random noise signal, SASE simulations require many runs with different values for the initial electron positions to reproduce the intensity distribution and obtain mean values for intensity and bunching factor. However, if only mean values are of interest, the amount of CPU time can be significantly reduced by approximating the SASE FEL with an FEL amplifier. The input power level of this equivalent amplifier has been estimated in [38], and this estimate has been used in the simulations. The predicted angular and spectral 
distributions of the radiation at the undulator exit are shown in Figure 4.7 and Figure 4.8, for the same case.

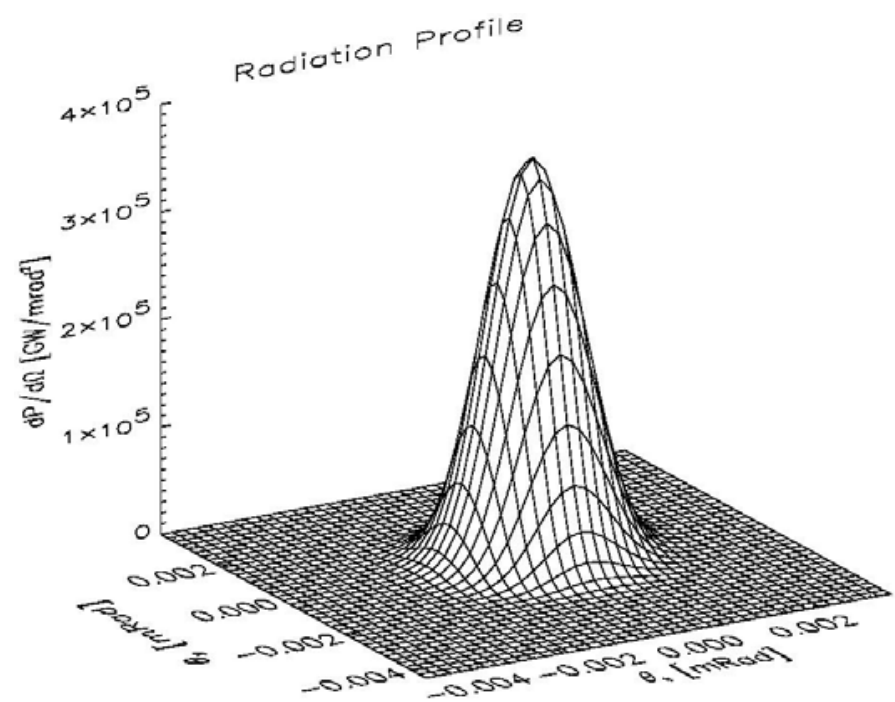

Figure 4.7 Angular distribution of the radiation at the undulator exit, showing a single Gaussian mode, with an rms aperture of about $2 \mu \mathrm{rad}$.

Filtering and focusing the radiation and transporting it to the experimental areas is a challenge. A normal incidence mirror at $100 \mathrm{~m}$ would see an energy flux density of about $1 \mathrm{~J} / \mathrm{cm}^{2}$, about $1 \mathrm{eV}$ /atom. The large power density of the LCLS x-ray pulses will push the optical elements and instrumentation into a new strong field regime, but also offers new opportunities for scientific research. The development of x-ray optics to handle these large power densities is described in Chapter 9 of this CDR.

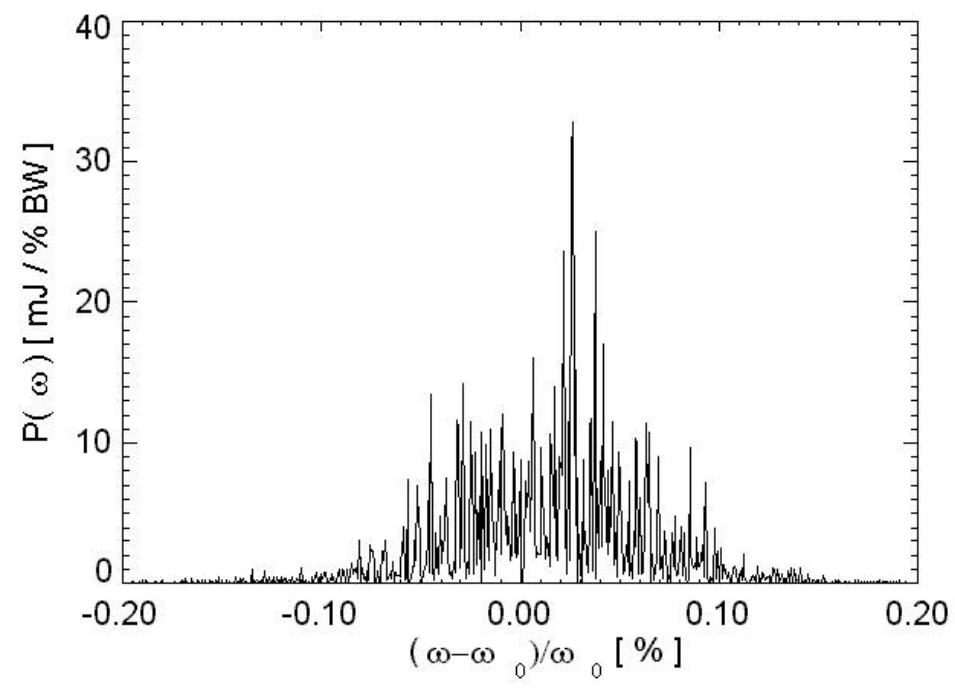

Figure 4.8 Spectral power distribution at undulator exit. 


\subsection{Effects of Spontaneous Radiation}

The emission of spontaneous radiation by electrons in the undulator decreases the average electron energy by $W_{e R}$, and increases the relative energy spread to $\sigma_{\gamma R}$ [52]. Both effects can reduce FEL gain if the conditions $W_{e R} / E_{\text {beam }}<\rho_{\text {eff }}$ and $\sigma_{\gamma R}<<\rho_{\text {eff }}$ are not satisfied. The two quantities $W_{e R}$ and $\sigma_{\gamma R}$ have been evaluated for the LCLS case and the results are $W_{e R} / E_{\text {beam }}$ $\approx 1.8 \times 10^{-3}$ and $\sigma_{\gamma R} \approx 1.5 \times 10^{-4}$. When compared to the effective FEL bandwidth, $\rho_{\text {eff }} \approx 5 \times 10^{-4}$, both effects have to be considered. The average energy loss will be different for different electron beam energies but it can be compensated for by micro-tapering the undulator. A large FEL parameter reduces the effect of the energy spread.

\subsection{Undulator Wakefields}

\subsubsection{Wakefield Theory}

There are three major sources of wakefields within the undulator vacuum chamber, i.e., resistive wall, geometric, and surface roughness wakefields, which are described in detail in Chapter 8. The rest of this section summarizes the basics of undulator chamber wakefields to support the simulation results shown in the next section.

For an x-ray FEL, with a large-peak-current electron beam and a long undulator, the wakefields in the undulator vacuum pipe can have an important effect on the lasing process, reducing the output power and changing the temporal structure of the $\mathrm{x}$-ray pulse. The effect of wakefields becomes noticeable as gain reduction if the condition that the variation in energy be small compared to the gain bandwidth, i.e., $(\Delta E / E)_{\text {wake }}<\rho_{\text {eff }}$, is satisfied. In the LCLS case, this gives the condition $W_{z}<30 \mathrm{kV} / \mathrm{m}$ for the longitudinal wakefield amplitude $W_{z}$. For every slice in the bunch, for which this condition is fulfilled will be somewhat affected by wakefields. Once the condition is fulfilled the effect growths with the wake-function amplitude.

In ultra relativistic approximation, the resistive longitudinal wakefield [53] from a single electron is

$$
W_{z}(s)=-\frac{4 c Z_{0}}{\pi a^{2}}\left(\frac{1}{3} e^{-s / s_{0}} \cos \frac{\sqrt{3} s}{s_{0}}-\frac{\sqrt{2}}{\pi} \int_{0}^{\infty} \frac{x^{2} e^{-x^{2} s / s_{0}}}{x^{6}+8} d x\right)
$$

for $s>0$ and $W_{z}(s)=0$ for $s<0$, where $s$ measures the longitudinal position of the test particle with respect to the particle generating the field, $Z_{0}$ is the vacuum impedance, $s_{0}=\left(2 a^{2} / Z_{0} \sigma\right)^{1 / 3}$, $\sigma$ is the conductivity of the material, and $a$ is the pipe radius. For a copper-plated vacuum chamber with a radius of $2.5 \mathrm{~mm} s_{0}=8.3 \mu \mathrm{m}$ and thus comparable with the LCLS rms bunch length of $23 \mu \mathrm{m}$.

Geometric wakefields arise if the aperture of the vacuum chamber varies along the undulator. Contributions of bellows, vacuum pump ports, and flanges result in wakefields, which are 
typically much smaller than the ones generated by the wall resistance. Thus, they are neglected in the following discussion on the effects of wakefields on the FEL performance.

The first model of surface roughness wakefields [54], which describes the surface as a distribution of bumps covering a smooth surface, results in a severe tolerance on the level of roughness. Based on measurements with an Atomic Force Microscope [55] typical surfaces [56, 57] resemble a smooth surface where the peak-to-valley height, $h$, is much smaller than the spacing, $p$, between crests. For a possible prototype of the LCLS undulator vacuum chamber, the RMS height might be $h=100 \mathrm{~nm}$ while $p$ may exceed tens or even hundreds of microns. A refined model [58], using the small-angle approximation, yields a much more relaxed tolerance on the acceptable roughness.

Both models rely on the condition that the bunch length is much larger than the size of the bumps, which is violated for the short LCLS bunches. Another model [59], valid for the LCLS case and based on a sinusoidal corrugation of the surface with an amplitude $h$ and a wavenumber $k=2 \pi / p$ with $h k<<1$, yields the single-particle wakefield

$$
W_{z}(s)=\frac{c Z_{0} e^{2}}{2 \sqrt{\pi}} \frac{h^{2} k^{3 / 2}}{a} \frac{\partial}{\partial s} \frac{1}{\sqrt{s}}\left[\cos \left(\frac{k s}{2}\right)+\sin \left(\frac{k s}{2}\right)\right] .
$$

Comparison shows that the two previous models overestimate the amplitude of wakefields when applied to short bunches.

A. Novokhatski and A. Mosnier pointed out [60] that a periodic, rectangular corrugation also allows synchronous modes (slowed down waveguide modes, which couple to the electron beam.) The wake potential is

$$
W_{z}(s)=-2 \kappa \cos \left(k_{0} s\right)
$$

where $\kappa=c Z_{0} e^{2} / 2 \pi a^{2}$ is the loss factor, $k_{0}=\sqrt{2 p / h a g}$ is the wave number, $p$ is the period, $h$ is the height, and $g$ is the length of the corrugation [61]. Similar results are obtained for a sinusoidal corrugation with an amplitude, $h$, and a period, $p=2 \pi / k$. If the aspect ratio between height and length of the corrugation becomes small, correction factors have to be applied to the loss factor and the wave number [61, 62], which scale in the limit $h \Rightarrow 0$ as $k_{0}=0$ and $Z=c Z_{o} e^{2} / 16 \pi a^{2} r^{4}$ with $r=h \sqrt{a k^{3}} / 2$. For the LCLS with a beam pipe radius of $a=2.5 \mathrm{~mm}$, a corrugation amplitude of $h=100 \mathrm{~nm}$ and a corrugation period, $p=100 \mu \mathrm{m}$, the correction factor reduces the estimated loss factor, $\kappa$, by $10^{5}$, which eliminates any impact on the FEL performance by the synchronous mode.

\subsubsection{Wakefield Effects on the LCLS}

For the discussion of the impact of wakefields on LCLS performance, the resistive wall wakefields, Eq. (4.30), the roughness wakefields, Eq. (4.31), and the synchronous mode for a sinusoidal corrugation of the vacuum pipe, Eq. (4.32), are used. Two cases of roughness wakefields are considered $p / h=50$ and 500 , both with $h=100 \mathrm{~nm}$. The resulting wake potentials are shown in Figure 4.9. The amplitudes of the roughness wakes drop by more than two orders of magnitude towards the longer periodicity and become negligible compared to the resistive wall 
wakefield. Atomic Force Microscope measurements [63] of surface roughness gives results in agreement with the second case, $p / \delta=500$

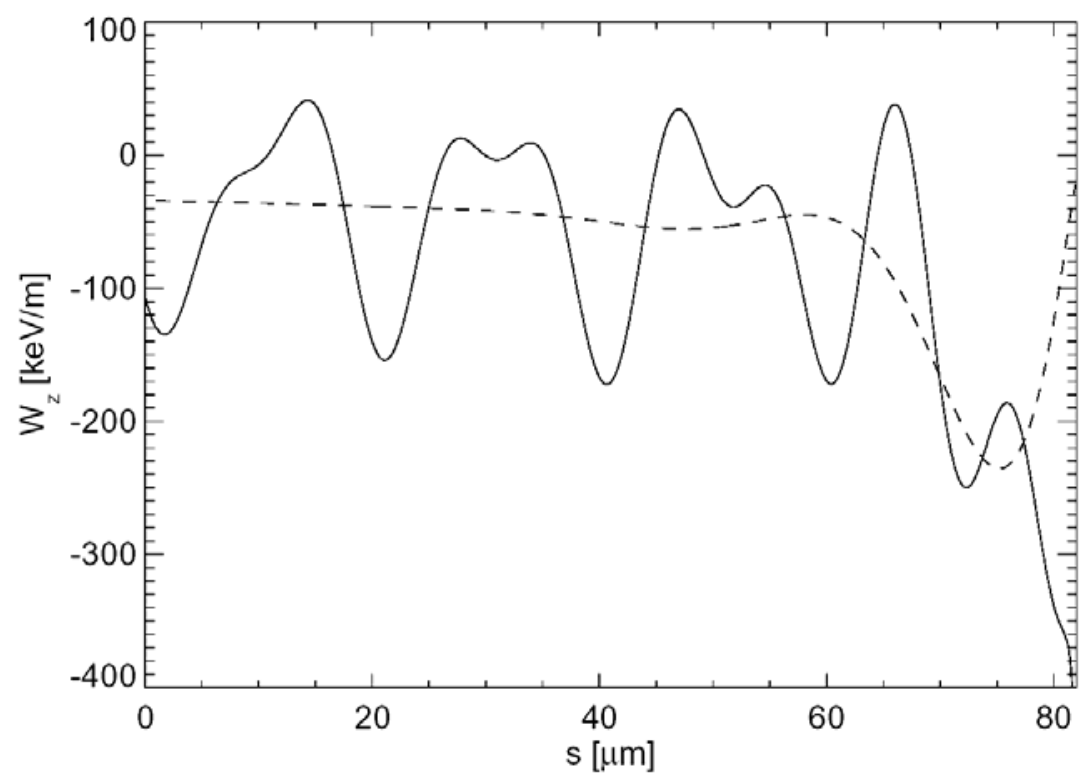

Figure 4.9 LCLS undulator wake potential including the resistive and roughness wakefields. The roughness wakefields include both the inductive model Eq. (4.31) and the synchronous mode Eq. (4.32), assuming a $1 \mathrm{nC}$ electron bunch, a bump height of $100 \mathrm{~nm}$, and a bump length of $5 \mu \mathrm{m}$ (solid line) or $50 \mu \mathrm{m}$ (dashed line). The resistive wall wakefield dominates the dashed curve. The head of the bunch is at the right hand side of the graph.

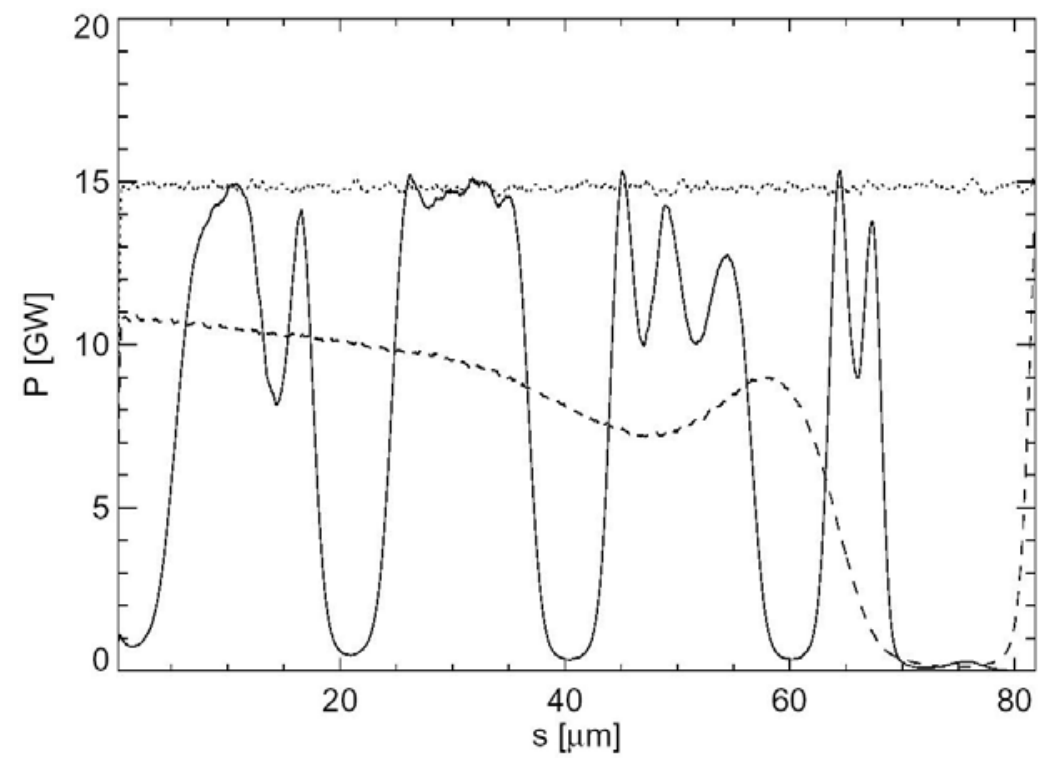

Figure 4.10 Effect of wakefields on the temporal radiation power profile at the undulator exit: Dotted line: no wakefields; dashed line: "long bump case"; solid line: short "bump case". The head of the bunch is at the right hand side of the graph. The simulation includes micro-tapering to offset the energy loss from spontaneous radiation. 
The effect of wakefields on the LCLS reference case, evaluated with the simplified model just discussed, is shown in Figure 4.6. As one can see from this figure, in the absence of wakefields the peak power is $18 \mathrm{GW}$. This is reduced to $10 \mathrm{GW}$ for the case of "long bumps", and to $6 \mathrm{GW}$ for the case of "short bumps". The mechanism for this loss of output power is shown in Figure 4.10: only the electrons with a small energy loss from wakefields radiate within the gain bandwidth and contribute to the output intensity. The energy loss is small where the total wake potential is close to zero. As a result multiple spikes arise in the power profile for the short bump case (Figure 4.10), because the wake potential has multiple zero crossings along the bunch (see Figure 4.9). It is also interesting to notice that the gain length is not directly changed by the wakefields (see Figure 4.6). It is only in the last part of the undulator that the power is reduced with respect to the zero wakefield case.

\subsection{Emittance and Charge Control}

As described in the previous sections, the phase-space density, i.e., the transverse and longitudinal emittances, of the electron beam is of critical importance for an x-ray FEL. One method of controlling these properties is to use the photocathode gun laser intensity, spot size and phase to change the charge and minimize the emittance as a function of charge $[64,65,66]$. The scaling laws for transverse emittance and rms bunch length are:

$$
\begin{gathered}
\varepsilon=\left(a Q^{2 / 3}+b Q^{4 / 3}+c Q^{8 / 3}\right)^{1 / 2}, \\
\sigma_{L}=d Q^{1 / 3}
\end{gathered}
$$

where $Q$ is the charge and the three terms in the equation for emittance represent the thermal, space charge and time dependent radio frequency plus chromatic focusing effects respectively. For charges smaller than about $2 \mathrm{nC}$ only the first two terms are important. The values of the coefficients $a, b, c$, and $d$ depend on the choice of the working point - RF voltage, shape of the laser pulse, and other parameters - of the injector.

Using the Ferrario working point [67], the values of the coefficients are $\mathrm{a}=0.076(\mu \mathrm{m} \mathrm{rad})^{2} /(\mathrm{nC})^{2 / 3}, b=0.18(\mu \mathrm{m} \mathrm{rad})^{2} /(\mathrm{nC})^{4 / 3}, d=4.8 \times 10^{-4} \mathrm{~m} /(\mathrm{nC})^{1 / 3}$. The thermal and space charge terms in the emittance are about equal for $Q=0.25 \mathrm{nC}$. The beam brightness, defined as the current over the product of the horizontal and vertical emittances, scales as

$$
B=\frac{1}{a+b Q^{2 / 3}} 2.5 \times 10^{11} \frac{\mathrm{C}^{2 / 3}}{\mathrm{~m} \mathrm{~s}} .
$$

To explore the operational range, we have chosen 2 cases, $0.25 \mathrm{nC}$ and $1 \mathrm{nC}$ in charge. At $0.25 \mathrm{nC}$, the brightness is close to the best possible value. The emittance and bunch length values are at $0.25 \mathrm{nC}: \varepsilon \sim 0.24 \mu \mathrm{m}-\mathrm{rad}$ and $\sigma L \sim 0.3 \mathrm{~mm}$, and at $1 \mathrm{nC}: \varepsilon \sim 0.5 \mu \mathrm{m}-\mathrm{rad}$, and $\sigma L \sim 0.48 \mathrm{~mm}$. Photocathode gun simulations at the Ferrario working point, using the Parmela code and including thermal effects, have been done recently for these two cases. The simulations give an emittance of $0.3 \mu \mathrm{m}$ rad at $0.25 \mathrm{nC}$ and $0.6 \mu \mathrm{m}$-rad at $1 \mathrm{nC}$. These results will be discussed in more detail in the Chapter 4 in the section about start-to-end simulations of the full LCLS system. 


\subsection{Control of X-Ray Pulse Length and Linewidth}

An FEL has a rather large bandwidth,

$$
\frac{\Delta \lambda}{\lambda}=\frac{\lambda}{2 \pi L_{c}}
$$

where the cooperation length $\mathrm{L}_{\mathrm{c}}$ is given by Eq. (4.25). When starting from noise, i.e., in SASE mode, this large gain bandwidth produces spikes in the radiation output as discussed before. Typically for an x-ray SASE FEL the number of spikes is large, about 250 in the LCLS case, hence the pulse length is about 250 times longer than the spike-separation, and the linewidth is about 250 times larger than the Fourier transform limited value for the total bunch length.

Given these physical properties of an FEL, it is in principle possible to reduce the bunch length to that of a single spike, about 1 fs, with a corresponding line width given by Eq. (4.36) of the order of $5 \times 10^{-4}$. At the other end, it is also possible to eliminate the spikes and produce a single pulse with a length of the order of the bunch length and a line-width of about $2 \times 10^{-6}$. Adding the capability of producing $x$-ray pulses with these characteristics would greatly add to the LCLS usefulness. For this reason both options have actively been explored. The present state of this work can be found in [68] and [69]. Some of the elements of this work are summarized here.

\subsubsection{Pulse Length Control}

One option to reduce the LCLS pulse length is to reduce the electron bunch pulse length by operating the LCLS compression system in a different configuration. The LCLS has two compression stages. In the reference design, these compressors are used to reduce the electron pulse length from about $10 \mathrm{ps}$ at the electron gun exit to $230 \mathrm{fs}$ at the linac exit. Reference [68] contains a discussion of the possibility of using the two compressors to reduce the electron pulse length to a smaller value. Reducing the pulse length in the compressor to about $10 \mathrm{fs}$ produces very large coherent synchrotron radiation effects. To mitigate these effects, the electron bunch charge has to be reduced to $0.2 \mathrm{nC}$. Even with the reduced charge the evaluation of the effect of Coherent Synchrotron Radiation (CSR) is open to questions. The current distribution in the bunch is non-uniform, and the energy spread at the linac exit is large, about $0.07 \%$, which is large compared to the LCLS FEL parameter. Such a large energy spread can increase the gain length, and the SASE process for this beam needs to be studied in more detail.

The other method to produce a short bunch is to introduce an energy chirping in the electron bunch [70], thus producing a chirped x-ray pulse and then use optical systems to slice or compress the radiation pulse. To avoid an increase of FEL gain length, the energy chirping of the electrons must be such that the central frequency variation per spike is a fraction of the spike linewidth. One of the cases studied in [68] shows that it might be possible to obtain a total electron energy chirping of $2 \%$, corresponding to a total line width of the radiation of $4 \%$ and a relative frequency change per spike of $8 \times 10^{-5}$, about $1 / 6$ of the spike width. 
A chirped x-ray radiation pulse can be sent through a dispersive optical system, followed by another optical system, which selects one "slice" of the pulse. Systems based on Fresnel zone optics or multilayers have been proposed to slice the x-ray pulse. Limitations in the optical systems, differences in optical path lengths — which become important for femtosecond-long pulses - and diffraction effects, limit the obtainable pulse length to the range of $10 \mathrm{fs}$ or larger. A pulse length of about 10 fs corresponds to a selection of about 10 slices out of the 250 of the incoming pulse. The intensity is thus reduced to $4 \%$ of the total intensity, and the intensity fluctuations become as large as $30 \%$.

A proposed alternative is to use a double reflecting grating system [71], or a reflecting grating-mirror array system to compress the pulse. Again temporal and diffraction limitation would limit the pulse length to $10 \mathrm{fs}$ or longer. The double grating system has the disadvantage of requiring a longitudinal separation between the two gratings of about $100 \mathrm{~m}$, and of low transmission efficiency. The reflecting grating-mirror array system would eliminate these problems and could produce radiation intensity comparable with the input intensity, and with the same level of intensity fluctuation of the standard LCLS case, about 7\%.

A more recent proposal is to use a chirped electron beam, propagating through a first undulator followed by a monochromator, providing a short pulse seed signal for a second undulator [72]. The system can produce $10 \mathrm{fs}$ to $20 \mathrm{fs}$ long pulses, with the same peak power as the LCLS.

The developments of the schemes to reduce the pulse length will require in-depth experimental studies of the electron beam acceleration and compression, of the production and transport before and through the undulator of a beam with a large energy spread, and of the slicing and/or compressing optical systems.

\subsubsection{Linewidth Control}

Two methods have been studied to reduce the linewidth of a SASE x-ray FEL. One is to follow the High Gain Harmonic Generation (HGHG) scheme with the fresh bunch technique [73, 74]. The other method, the Two-Stage FEL [75], consists of two undulators with an x-ray monochromator located between them. The first undulator operates in the linear high gain regime starting from shot noise in the electron beam. After the first undulator, the output radiation passes through the x-ray monochromator, which reduces the linewidth to the desired value, smaller than the FEL bandwidth. After this monochromator, the intensity fluctuations are $100 \%$. The monochromatization of the radiation is performed at a relatively low level of radiation power, which will reduce damage to the conventional monochromator x-ray optical elements. At the entrance of the second undulator, the monochromatic x-ray beam is then combined with an electron beam and amplified up to the saturation level. The radiation power at the entrance of the second undulator is dominant over the shot noise power, so that the input signal bandwidth is small with respect to the FEL amplifier bandwidth.

The realization of this two-stage FEL scheme for the LCLS requires two undulators 55 and $60 \mathrm{~m}$ in length. The output power at the end of the first undulator is $100 \mathrm{MW}$ (which is about 100 
times less than the saturation power) with the spectral bandwidth is $5 \times 10^{-4}$. Here, an electron beam with an emittance of $1.1 \mu \mathrm{m}-\mathrm{rad}$ and a peak current of $3.4 \mathrm{kA}$ is assumed. The monochromator selects a band, which is wider than the Fourier transform limited bandwidth of approximately $1.5 \times 10^{-6}$ but smaller than the gain bandwidth. The intensity fluctuation after this monochromator is close to $100 \%$. The total power transmission through the monochromator will be determined by the reflection coefficient of the elements of the monochromator and the ratio of the bandwidth of the monochromator to the bandwidth of the SASE FEL radiation after the first undulator. The reflection coefficient is expected to be in the range of $30 \%-50 \%$. The monochromator can be considered a linear filter, and therefore, the power distribution after the monochromator will remain a negative exponential distribution. The mean value of the radiation power after the monochromator will be about $100 \mathrm{~kW}$. This is the input radiation power at the entrance of the second undulator and is much greater than the shot noise power. After the monochromator, the radiation pulse can be combined at the entrance of the second undulator with either a new electron bunch or the original electron bunch passed through a bypass (which will remove the electron micro bunching produced in the first undulator). With a mean input radiation power of $100 \mathrm{~kW}$, the second stage would consist of an undulator about $60 \mathrm{~m}$ in length. This will allow the FEL process to reach saturation and reduce the intensity fluctuations at the output of the second undulator to less than $10 \%$. In the reference LCLS case the intensity fluctuation is $6 \%$, as shown in Table 4.1. The total undulator length needed or this scheme is about $115 \mathrm{~m}$, to which the space needed for the monochromator must be added.

The Two-Stage FEL scheme is compatible with the baseline design of the LCLS presented in this CDR. Its implementation requires the movement of central undulator segments to the beginning of the undulator line, where extra space has been reserved. The total available space for installing the device is about $156 \mathrm{~m}$.

\subsection{References}

1 H. Motz, J. Appl. Phys., 22, 527 (1951); H. Motz, W. Thon and R. N. Whitehurst, J. Appl. Phys. 24, 826 (1953); H. Motz and M. Nakamura, Ann. Phys. 7, 84 (1959).

2 R.M. Philips, IRE Trans. Electron Devices, 7, 231 (1960).

3 R.V. Palmer, J. Appl. Phys., $\underline{\mathbf{4 3}}, 3014$ (1972).

4 K.W. Robinson, Nucl. Instr. and Meth, $\underline{\mathbf{A 2 3 9}}, 111$ (1985).

$5 \quad$ P. Csonka, Part. Acc. $\underline{\mathbf{8}}, 225$ (1978).

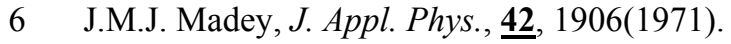

7 L.R. Elias, W.M. Fairbank, J.M.J. Madey, H.A. Schwettman and T.I. Smith, Phys. Rev. Lett., $\underline{\mathbf{3 6}}, 717$ (1976).

8 D.A.G. Deacon, L.R. Elias, J.M.J. Madey, G. J. Ramian, H.A. Schwettman and T.I. Smith, Phys. Rev. Lett., $\underline{\mathbf{3 8}}, 892$ (1977). 
9 N.M. Kroll, Phys. Quantum Electron. 5, 115(1978); P. Sprangle and R.A. Smith, NRL Memo-Report 4033 (1979); A. M. Kondratenko and E.L. Saldin, Dokl. Aka. NAUK SSSR, 249,843 (1979); D. B. McDermott and T. C. Marshall, Phys. Quantum Electron. 7, 509 (1980); A. Gover and P. Sprangle, IEEE J. Quantum Electron. QE-17, 1196 (1981); G. Dattoli et al., IEEE J. Quantum Electron. QE17. 1371 (1981); R. Bonifacio, C. Pellegrini, and L. Narducci, Opt. Commun., 50, 373 (1984); J.B. Murphy, C. Pellegrini and R. Bonifacio, Opt. Comm. 53, 197 (1985); J. Gea-Banacloche, G.T. Moore and M. Scully, SPIE 453, 393 (1984); P. Sprangle, C.M. Tang and C.W. Roberson, Nucl .Instr. and Meth, A239, 1 (1985); E. Jerby and A. Gover, IEEE J. Quantum Electron. QE-21, 1041 (1985); R. Bonifacio, F Casagrande and C. Pellegrini, Opt. Comm. 61, 55 (1987).

10 R. Bonifacio, C. Pellegrini, and L. Narducci, Opt. Commun., 50, 373 (1984).

11 K.-J. Kim, Nucl. Instr. and Meth, A250, 396(1986); J.-M. Wang and L.-H. Yu, Nucl Instr. and Meth, $\underline{\mathbf{A 2 5 0}}, 484$ (1986).

12 G.T. Moore, Nucl.Instr. and Meth, $\underline{\mathbf{A 2 3 9}}, 19$ (1985); E. T. Scharlemann, A. M. Sessler, and J. S. Wurtele, Phys. Rev. Lett. 프, 1925 (1985); M. Xie and D.A.G. Deacon, Nucl. Instr. and Meth, $\underline{\mathbf{A 2 5 0}}$, 426 (1986); K.-J. Kim, Phys. Rev. Lett. 57, 1871 (1986); L.-H. Yu, S. Krinsky and R. Gluckstern, Phys. Rev. Lett. $\underline{\mathbf{6 4}}, 3011$ (1990); Z. Huang and K.-J. Kim, Nucl. Instr. and Meth, $\underline{\mathbf{A 4 7 5}}, 59$ (2001).

13 S. Krinsky and L.-H. Yu, Phys. Rev. A $\underline{35}, 3406$ (1987).

14 M. Xie, Nucl. Instr. and Meth, $\underline{\mathbf{A 4 4 5}}, 59$ (2000); M. Xie, Nucl.Instr. and Meth, $\underline{\mathbf{A 4 4 5}}, 67$ (2000); M. Xie, in Proc. Intern.FEL-2000 Conf., Duke University (2000); Z. Huang and K.-J. Kim, Phys. Rev. E62, 7295 (2000).

15 A.M. Kondratenko and E. L. Saldin, Part. Acc. 10, 207 (1980); Ya. S. Derbenev, A.M. Kondratenko, and E.L. Saldin, Nucl. Instr. and Meth, $\underline{\mathbf{A 1 9 3}}, 415$ (1982).

16 J.B. Murphy and C. Pellegrini, Nucl. Instr. and Meth, $\underline{\mathbf{A 2 3 7}}, 159$ (1985); J. B. Murphy and C. Pellegrini, J. Opt. Soc. Am. B2, 259 (1985); K.-J. Kim et al., Nucl. Instr. and Meth, $\underline{\mathbf{A 2 3 9}}, 54$ (1985).

17 C. Pellegrini, "Progress Toward a Soft X-ray FEL", Nucl. Instr. and Meth, $\underline{\mathbf{A 2 7 2}}, 364$ (1988).

18 J.S. Fraser, R.L. Sheffield, and E.R. Gray, "A new high brightness electron injector for free-electron lasers driven by RF linacs," Nucl.Instr and Meth, $\underline{\mathbf{A 2 5 0}}$, pp. 71-76, 1986.

19 B. E. Carlsten Nucl.Instr and Meth, A285, 313-319 (1989); X. Qiu, K. Batchelor, I. Ben-Zvi, and X-J. Wang, "Demonstration of Emittance Compensation through the Measurement of Slice Emittance of a 10 ps Electron Pulse", Phys. Rev. Lett. 76, 3723-3726 (1996).

20 K. Bane, "Wakefield Effects in a Linear Collider", AIP Conf. Proc., vol. 153, p. 971 (1987); J. Seeman et al, "Summary of Emittance Control in the SLC Linac", US Particle Accelerator Conference, IEEE Conf. Proc. 91CH3038-7, p. 2064 (1991); J. Seeman et al, "Multibunch Energy and Spectrum Control in the SLC High Energy Linac", US Particle Accelerator Conference, IEEE Conf. Proc. 91CH3038-7, p. 3210 (1991); T. Raubenheimer, "The Generation and Acceleration of Low Emittance Flat Beams for Future Linear Colliders", SLAC-Report 387 (1991).

21 C. Pellegrini, "A 4 to $0.1 \mathrm{~nm}$ FEL based on the SLAC Linac", in Proc. of the Workshop on IV Generation Light Sources, M.Cornacchia and H. Winick eds., SSRL-SLAC Report 92/02, p. 364 (1992).

22 C. Pellegrini et al., "The SLAC Soft X-ray High Power FEL", Nucl. Instr. and Meth, $\mathbf{\mathbf { A 3 4 1 }}, 326$ (1994); G. Travish et al., "Parametric Study of an X-ray FEL", Nucl. Instr. and Meth, $\underline{\mathbf{A 3 5 8}}, 60$ (1995).

23 LCLS Design Study Report, Report SLAC-R-521, (1998).

24 J. Rossbach et al., Nucl. Instr. and Meth, $\underline{\mathbf{A 3 7 5}}, 269$ (1996). 
25 T. Orzechowski et al., Phys. Rev. Lett. 푸, 889 (1985); D. Kirkpatrick, Nucl. Instr. and Meth, $\underline{\mathbf{A 2 8 5}}$, 43 (1989); J. Gardelle, J. Labrouch and J.L. Rullier, Phys. Rev. Lett. $\mathbf{7 6}, 4532$ (1996).

26 R. Prazeres et al., Phys. Rev. Lett. 프, 2124 (1997).

27 M.Hogan et al., "Measurements of High Gain and Intensity Fluctuations in a SASE free-electron laser", Phys. Rev. Lett. 으, 289 (1998).

28 M. Babzien et al., "Observation of self-amplified spontaneous emission in the near-infrared and visible wavelengths", Phys. Rev. E $\underline{\text { 57, }}$ 6093-6100 (1998).

29 D. C. Nguyen et al., "Self Amplified Spontaneous Emission Driven by a High-Brightness Electron Beam", Phys. Rev. Lett. , 1 1, 810-13 (1998).

30 M. Hogan et al., "Measurements of Gain larger than $10^{5}$ in a $12 \mu \mathrm{m}$ SASE-FEL", Phys. Rev. Lett. $\underline{\mathbf{8 1}}$, 4867-4870 (1998).

31 L.-H. Yu et al, " First Lasing of a High-Gain Harmonic Generation Free-electron Laser Experiment", Nucl. Instr. and Meth, $\underline{\mathbf{A 4 4 5}}, 301$ (1999), and Proc. Intern.FEL-2000 Conf., Duke University (2000).

32 A. Tremaine et al., "Initial Gain Measurements of a $800 \mathrm{~nm}$ SASE-FEL, VISA", in Proc Intern.FEL2000 Conf., Duke University (2000);

33 S. V. Milton et al., "Observation of Self-Amplified Spontaneous Emission and Exponential Growth at 530nm," Phys. Rev. Lett., 85, 988-991 (2000), and Proc. Intern.FEL-2000 Conf., Duke University (2000).

34 J. Rossbach et al., "Observation of Self amplified Spontaneous Emission in the wavelength range from $80 \mathrm{~nm}$ to $180 \mathrm{~nm}$ at the TESLA test facility FEL at DESY", in Proc. Intern.FEL-2000 Conf., Duke University (2000); J. Andruszkow et al., Phys. Rev. Lett. 85, 3825-3829 (2000).

35 S. Milton and al. Science Express, May 17, (2001).

36 A. Tremaine et al., "Saturation Measurements of an $800 \mathrm{~nm}$ SASE FEL," in Proc. of the 23th International Free Electron Laser Conference Darmstadt, Germany, (2001).

37 J. B. Murphy and C. Pellegrini, "Introduction to the Physics of Free-Electron Lasers," in Laser Handbook, Vol. 6, W.B. Colson, C. Pellegrini, and A. Renieri eds., North Holland, (1990); R. Bonifacio et al., La Rivista del Nuovo Cimento, Vol. 13, N. 9, (1990).

38 R. Bonifacio, et al, "Spectrum, Temporal Structure, and Fluctuations in a High Gain Free-electron Laser Starting from Noise," Phys. Rev. Lett., $\underline{73}, 70$ (1994).

39 E.L. Saldin, E.A. Schneidmiller, and M.V. Yurkov, "Statistical properties of Radiation from VUV and X-ray Free Electron Laser", DESY rep. TESLA-free-electron laser 97-02, (1997).

40 R. Bonifacio, L. De Salvo and P. Pierini, Nucl. Instr.and Meth, A293, 627 (1990); H.P. Freund, S.G. Biedron and S.V. Milton, IEEE J. Quantum Electron., QE-36, 275 (2000); Nucl. Instr.and Meth, A445, 53 (2000); M. Xie, Nucl. Instr and Meth, A475, 51 (2001).

41 L.H. Yu, Phys. Rev. $\underline{\mathbf{A 4 4}}, 5178$ (1991).

42 Z. Huang and K.-J. Kim, Phys. Rev. $\underline{\mathbf{E} 62}$, November, 2000; Nucl. Instr.and Meth, $\underline{\mathbf{A 4 7 5}}, 112$ (2001).

43 R. Bonifacio, "On the classical and quantum feature of self amplified spontaneous emission", in Towards X-ray Free Electron Lasers, R. Bonifacio and W. A. Barletta eds, AIP Conf. Proc. 413, p. 15 (1997).

44 K.-J. Kim, "Temporal and transverse coherence of self amplified spontaneous emission", in Towards X-ray Free Electron Lasers, R. Bonifacio and W. A. Barletta eds, AIP Conf. Proc. 413, p. 3 (1997). 
45 C. Schroeder, C. Pellegrini, and P. Chen, "Quantum Fluctuations in Free-Electron Lasers", incourse of publication in Proc. Of the $2^{\text {nd }}$ Conf. on Quantum Aspects of Beam Physics, Capri (2000). M.C. Teich, T. Tanabe, T.C. Marshall and J. Galayda, Phys. Rev. Lett. 모, 3393 (1990).

W.M. Fawley, LBNL Report LBNL-49625 (2002).

E. L. Saldin, E. A. Schneidmiller, M. V. Yurkov, "Numerical simulation of the UCLA-LANLRRCKI-SLAC experiment on a high gain SASE FEL”, Nucl. Instr. and Meth, $\underline{\mathbf{A 4 2 9}}, 197-201$ (1999).

S. Reiche, Nucl. Instr. and Meth, $\underline{\mathbf{A 4 2 9}}, 243$ (1999).

S.G. Biedron et al., Nucl. Instr. and Meth, A445 (2000) 110.

S. Reiche, DESY-Print, DESY-THESIS-2000-012 (2000)

E.L. Saldin et al., Nucl. Instr. and Meth, $\underline{\mathbf{A 3 8 1}}, 545$ (1996).

K. Bane, SLAC report AP-87 (1991).

C.-K. Ng, Phys. Rev. D42, 1819 (1990); K. Bane, C.-K. Ng, and A. Chao, "Estimate of the impedance due to wall surface roughness," SLAC-PUB-7514, (1997).

G.L. Stupakov et al., "Effects of beam tube roughness on a X-Ray free-electron laser performance," Phys. Rev. ST 2, 60701/1-60701/6 (1999).

D.J. Whitehouse, Handbook of Surface Metrology (IOP Publishing, 1994)

K.J. Stout, Atlas of Machined Surfaces (Chapman and Hall, 1990).

G. V. Stupakov, Phys. Rev. ST Accel. Beams 1, 064401 (1998).

G.V. Stupakov, "Surface Roughness Impedance." AIP Conference Proceedings 581, p 141-152, ISBN 0-7354-0022-9, ISSN 0094-243X (2000).

A.V. Novokhatskii and A. Mosnier, Proc. 1997 Particle Accelerator Conf., 1661-1663 (1997).

K.L. Bane and A.V. Novokhatskii, SLAC, Tech. Rep. SLAC-AP-117 (1999).

G. V. Stupakov, in T. Roser and S. Y. Zhang, eds., Workshop on Instabilities of High Intensity Hadron Beams in Rings (American Institute of Physics, New York, 1999), no. 496 in AIP Conference Proceedings, pp. 341-350.

G. Stupakov, R.E. Thomson, D. Walz, and R. Carr, "Effects of Beam Tube Roughness on X-Ray Free Electron Laser Performance," Phys. Rev. ST Accel. Beams, 2(6), 060701/1-60701/6 (1999).

J. B. Rosenzweig and E. Colby, in AIP Conf. Proc., vol.335, p. 724 (1995).

J. B. Rosenzweig et al., "Optimal Scaled Photoinjector Designs for FEL Applications", in Proc. IEEE Part. Acc. Conf., p. 2045 (1999).

J. B. Rosenzweig, private communication.

M. Ferrario et al, New Design Study and Related Experimental Program for the LCLS RF Photoinjector," LCLS-TN-00-9 (2000).

V. Bharadwaj et al. "Ultrashort Light Pulses in the LCLS", LCLS-TN-00-08, SLAC (2000).

"LCLS: the first experiments", Report of the LCLS Scientific Advisory Committee, SLAC (2000).

P. Emma, "Chirping the LCLS Electron Beam." LCLS-TN-00-6, (2000).

C. Pellegrini, ":High Power femtosecond pulses from an X-ray SASE-FEL", Nucl. Instr. and Meth, $\underline{\mathbf{A 4 4 5}}, 124-127$ (2000). 
72 C. Schroeder et al., "Chirped-Beam Two-Stage SASE-FEL for High Power Femtosecond X-ray Pulse Generation", in Proc. of the 2001 Particle accelerator Conf., Chicago 2001, in course of publication.

73 I. Ben-Zvi, K. M. Yang and L.-H. Yu, Phys. Rev. $\underline{\mathbf{A 3 1 8}}, 726$ (1992)

74 L.-H. Yu et al., in Proc. of the $22^{\text {nd }}$ Intern. FEL Conf., Duke University (2000)

75 J. Feldhaus, E. L. Saldin, J. R. Schneider, E. A. Schneidmiller, and M. V. Yurkov, "Possible application of X-ray optical elements for reducing the spectral bandwidth of an X-ray SASE FEL", Opt. Commun. 140, 341-352 (1997); E. L. Saldin, E. A. Schneidmiller, Y. V. Shvyd'ko, and M.V. Yurkov, "X-ray FEL with a meV Bandwidth", in Proceedings of the $22^{\text {nd }}$ International Free Electron Laser Conference, Durham, 2000 (Elsevier, Amsterdam, in press); E. L. Saldin, E. A. Schneidmiller, and M. V. Yurkov, "Optimization of a seeding option for the VUV free electron laser at DESY", Nucl. Instr. and Meth, $\underline{\mathbf{A 4 4 5}}, 178-182$ (2000). 


\section{FEL Parameters and Performance}

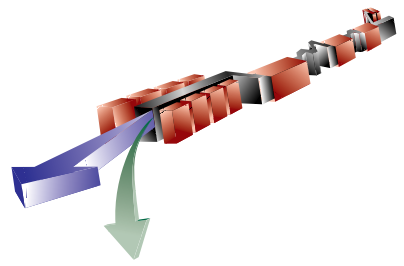

\section{TECHNICAL SYNOPSIS}

The FEL parameter optimization and performance characterizations that are described in Chapter 5 are based on three-dimensional theory and computer models. The investigation led to a selection of the best parameters and to a study of the sensitivity to changes in values of accelerator components and beam characteristics and to unavoidable imperfections in the settings of the beam characteristics, magnetic and mechanical components and electron beam monitoring. The focusing of the electron beam plays an important role in the production of the FEL radiation. The LCLS undulator optics has been optimized in terms of its focusing lattice and strength. The electron optics consists of FODO cells; with cell lengths between $7.3 \mathrm{~m}$ and $7.5 \mathrm{~m}$. Focusing is obtained by placing permanent magnet quadrupoles in the breaks between the undulator sections. The correction of the electron orbit is obtained by a small lateral displacement of the quadrupoles. Simulations indicate that the FEL radiation saturates at a length of $\sim 90 \mathrm{~m}$. The proposed LCLS undulator has a magnetic length of $121 \mathrm{~m}$, since it is a requirement that the FEL operate in the saturation regime. This fact not only gives the maximum output power, but also reduces the pulse-to-pulse fluctuations of the radiation.

Complete simulations of the LCLS, starting from the photocathode, and continuing through injector, linac, and undulator are an important help for understanding all of these effects and their impact on LCLS operation. The simulations, reported in this chapter, include thermal, rf, and space charge effects in the injector system, and wakefields and CSR in the linac-compressor system. The results of the simulations, presented for two cases, i.e., $1 \mathrm{nC}$ and $0.25 \mathrm{nC}$ bunch charge, show that under idealized conditions the beam emittance is small, about 0.5 and $0.3 \mu \mathrm{m}$ rad, respectively. Even with this small beam emittance, the wakefield effects in the linac and compressors reduce the LCLS output power and produce a transverse displacement and frequency chirp along the bunch.

The possibility of changing (i.e., lowering) the output power was investigated. This may be desirable if the peak power on the sample is excessive and if required for experimental purposes. The reduction in power, by either reducing the electron current or by increasing the beam emittance, is accompanied by an increase in fluctuations of the output power due to fluctuations in the beam characteristics from pulse-to-pulse, since the FEL no longer operates in the saturation regime. For this reason, the best way to reduce the output power is by placing an FEL absorption cell in the path of the radiation, as discussed in Chapter 9.

During commissioning both the electron beam and the $x$-ray radiation will be intensively characterized. Special x-ray commissioning diagnostics will be used, as described in Chapter 9. 


\subsection{Introduction}

The SASE process will produce pulses of coherent FEL x-ray radiation in the LCLS undulator with a harmonic spectrum that is adjustable over a large wavelength range. The operational wavelength is controlled by changing the energy of the electrons as described in Chapter 4 (Equation 4.1). The LCLS linac is designed to accelerator electrons to a final energy that is adjustable within the operational range between $4.54 \mathrm{GeV}$ and $14.35 \mathrm{GeV}$. The FEL wavelength is proportional to the inverse of the square of the electron energy. The electron energy can be changed between $4.54 \mathrm{GeV}$ and $14.35 \mathrm{GeV}$. The low energy limit corresponds to a wavelength of $15 \AA$ for the fundamental and $5 \AA$ for the third harmonic. The high-energy limit corresponds to a wavelength of $1.5 \AA$ for the fundamental and $0.5 \AA$ for the third harmonic. In addition to the coherent FEL radiation harmonics there will be a continuous spectrum of ordinary, incoherent undulator radiation, although much more intense than from ordinary insertion devices due to the high energy of the electron beam and the great length of the undulator.

The undulator consists of 33 individual undulator segments that are separated from each other by about 20 - to 40 -cm-long breaks, to provide space for focusing, steering, diagnostics and vacuum components. The lengths of these breaks are designed so that the $\mathrm{x}$-ray pulse and the electron beam slip with respect to each either by one or by two optical wavelengths, thus keeping the electrons in phase with the radiation. As described in Chapter 8, the first three breaks are individually adjusted to minimize the overall saturation length.

FEL theory predicts that the SASE process will saturate at about $90 \mathrm{~m}$ after the entrance to the undulator for the proposed baseline parameter set. This length includes the breaks between undulator segments. The tolerance budget for the undulator and the electron beam parameters has been set to limit the increase in saturation length to 1 field gain length, or about $10 \mathrm{~m}$. The total length of the LCLS undulator is $121 \mathrm{~m}$ for operational contingency.

The basic FEL parameters of the LCLS are discussed in Section 5.2. The design of the focusing system is discussed in Section 5.3. Computer simulations are described in Section 5.4. Sources of gain reduction and resulting tolerances are discussed in Section 5.5. Electron beam tolerances are discussed in Section 5.6. Section 5.7 discusses the temporal structure of the x-ray pulse. Section 5.8 gives an overview of the LCLS Commissioning

\subsection{The Basic LCLS FEL Design}

\subsubsection{Overview}

The basic parameters used to describe the FEL process include, for the electron beam, electron energy, $E$, normalized emittance, $\varepsilon_{n}$, peak current, $I_{p k}$, and relative rms energy spread

$\sigma_{E} / E$, and for the undulator, type, period, $\lambda_{U}$, gap, $g$, peak field, $B_{U}$, and average beta-function, $<\beta_{x, y}>$. From these parameters follow the undulator parameter, $K$, and the fundamental 
wavelength FEL radiation wavelength, $\lambda_{r}$. The nominal values for these parameters are listed in Table 5.2, their choice is discussed in the following sections.

\subsubsection{Slice Parameters}

As described in Chapter 4, the FEL instability comes from an interaction between the bunch electrons and the electromagnetic wave that is generated by those electrons and that is traveling with the electrons. At a given point in the process, the interaction is local on the scale of the optical wavelength. Interactions between different parts of the bunch occur due to slippage, i.e. due to the fact the for every undulator period traveled by the radiation, the electron beam falls behind by one optical wavelength. Thus the electrons in a given wavelength section interact with the radiation generated by electrons traveling at locations further towards the head of the bunch. This interaction is therefore limited to electrons that are not further apart in the bunch than the total slippage distance, $L_{\text {slip }}=N_{u} \lambda_{r}$, that corresponds to the total passage of the electron beam through the undulator, where $N_{U}$ is the total number of undulator periods. At a given position in the undulator during the exponential gain process, radiation that has been produced when the bunch was more than a power gain length before that position can practically be neglected compared to the more recently produced radiation amplitudes. The term cooperation length as the slippage length over one power gain length has been introduced to name the distance within the bunch over which there is strong interaction between bunch electrons through the electromagnetic radiation produced and acted upon by the bunch electrons. The FEL process is thus determined locally within a longitudinal slice of the electron bunch that has a thickness or length of the order of a cooperation length.

The FEL dynamics in one slice is not affected by the electron distribution in another slice if the two slices are significantly further apart than one cooperation length. If spatially separated slices have different electron energies they will just generate radiation of different wavelengths, the energy difference does not act as energy spread for the FEL process; only the energy distribution of the particles within a slice is relevant. Similar statements can be made for the emittances of the slices, the slices' relative transverse positions and their peak currents. Often, those parameters change along the electron bunch. If the bunch is much longer than the cooperation length, as is the case for the LCLS, it is important to distinguish between slice parameters and projected parameters. The projected parameters that are obtained after projecting the bunch to the same plane will give unrealistically pessimistic results when used to predict FEL performance. Reasonable performance predictions have to be done using projections over the width of a slice, only. The performance will be a function of the slice's position along the bunch. Whenever the terms emittance, energy spread and peak current are used in this report to characterize the FEL process they always stand for the terms slice emittance, slice energy spread and slice peak current. The terms projected emittance, projected energy spread, and average peak current are relevant because they name quantities that are more easily accessible to measurement and they affect the overall brightness of the x-ray pulses. For x-ray FELs such as the LCLS, the cooperation length is much shorter than the bunch length and presently too short to serve as a basis to measure slice parameters. Therefore the slice length if often increased to $5 \%$ to $10 \%$ of

FEL PARAMETERS AN D PERFORMANCE 5-3 
the bunch length, a value that is more accessible to diagnostics. Applying tolerances set for cooperation lengths slices to $10 \%$ of bunch length slices is a conservative approach.

The term slice emittance is not only used to characterize the electron bunch inside the undulator but also through injector and linac. This makes sense because electromagnetic fields, produced by space charge, chamber impedance, and coherent synchrotron radiation, create a dependence of the transverse position of the beam centroid on its longitudinal position within the bunch while the local electron density remains unaffected. Computer simulations show that slice emittance is not strongly affected during the acceleration and bunch compression processes in the linac.

Also used in this report is the attribute, nominal, such as nominal projected emittance or nominal slice emittance, to specify goal values for the parameters.

\subsubsection{Parameter Optimization}

The design of the LCLS FEL configuration has been greatly simplify by the use of Equations (5.1) and (5.2) given in [1], a parameterization of the results of 3-D FEL theory developed between 1985 and 1995 [2] [3][4]:

$$
\frac{L_{G, 1 D}}{L_{G}}=\frac{1}{1+\eta}
$$

where the parameter $\eta$ is

$$
\begin{aligned}
\eta= & a_{1} \eta_{d}^{a_{2}}+a_{3} \eta_{\varepsilon}^{a_{4}}+a_{5} \eta_{\gamma}^{a_{6}}+a_{7} \eta_{\varepsilon}^{a_{8}} \eta_{\gamma}^{a_{9}}+ \\
& a_{10} \eta_{d}^{a_{11}} \eta_{\gamma}^{a_{12}}+a_{13} \eta_{d}^{a_{14}} \eta_{\varepsilon}^{a_{15}}+a_{16} \eta_{d}^{a_{17}} \eta_{\varepsilon}^{a_{18}} \eta_{\gamma}^{a_{19}}
\end{aligned}
$$

$L_{G}$ and $L_{G, 1 D}$ are the 3-D and 1-D gain length, respectively. The 19 fit coefficients, $a_{i}$, are shown in Table 5.1. The results of Equations (5.1) and (5.2) have been checked against 3-D simulation codes and are in excellent agreement.

Table 5.1 Coefficients for Equation (5.2).

\begin{tabular}{llllllllll}
\hline$a_{1}$ & $a_{2}$ & $a_{3}$ & $a_{4}$ & $a_{5}$ & $a_{6}$ & $a_{7}$ & $a_{8}$ & $a_{9}$ & $a_{10}$ \\
0.45 & 0.57 & 0.55 & 1.6 & 3 & 2 & 0.35 & 2.9 & 2.4 & 51 \\
\hline$a_{11}$ & $a_{12}$ & $a_{13}$ & $a_{14}$ & $a_{15}$ & $a_{16}$ & $a_{17}$ & $a_{18}$ & $a_{19}$ & \\
0.95 & 3 & 5.4 & 0.7 & 1.9 & 1140 & 2.2 & 2.9 & 3.2 & \\
\hline
\end{tabular}

The scaling parameters express the deviation from the 1-D condition due to diffraction, $\eta_{d}$, emittance, $\eta_{\varepsilon}$ and energy spread, $\eta_{\gamma}$ :

$$
\eta_{d}=\frac{L_{G, 1 D}}{2 L_{R}} ; \quad \eta_{\varepsilon}=\frac{L_{G, 1 D}}{\beta_{x, y}} \frac{4 \pi \varepsilon_{n}}{\lambda_{r} \gamma} ; \quad \eta_{\gamma}=\frac{4 \pi L_{G, 1 D}}{\lambda_{u}} \frac{\sigma_{E}}{E} .
$$

Using the 1-D gain length

$$
L_{G, 1 D}=\frac{\lambda_{u}}{4 \pi \sqrt{3} \rho}
$$


and the total peak beam power

$$
P_{b, p k}=I_{p k} \gamma m c^{2} / e
$$

the peak power at saturation, $P_{\text {sat }}$, and the undulator length, $L_{s a t}$, needed for saturation can be approximated by

$$
P_{s a t} \approx 1.6 \rho\left(\frac{L_{G, 1 D}}{L_{G}}\right)^{2} P_{b, p k}
$$

and

$$
L_{s a t} \approx L_{G} \ln \left(\frac{P_{s a t}}{\rho E e \Delta \omega}\right)
$$

respectively. These equations use the FEL parameter, $\rho$, the bandwidth, $\Delta \omega$,

$$
\Delta \omega=\frac{2 \sigma_{\gamma} / \gamma}{\lambda_{r}} c
$$

the speed of light, $c$, the electron charge, $e$, and the relative electron energy spread, $\sigma_{E} / E$. The parameter optimization is constraint by practical limits for a number of parameters, including the undulator gap, $g \geq 6 \mathrm{~mm}, E<14.5 \mathrm{GeV}, I_{p k} \leq 3400 \mathrm{~A}, \sigma_{E} / E \geq 0.0001$ and $L_{u}<121 \mathrm{~m}$.

\subsubsection{The Nominal Parameter Set}

The LCLS is based on a planar hybrid undulator; this choice is discussed and justified in Chapter 8. Within the above constraints, the optimum operating parameters (Table 5.2) can be found using of Equations (5.1) and (5.2). A complete list of the LCLS parameters is given in

\begin{tabular}{|c|c|c|c|}
\hline \multirow{2}{*}{$\begin{array}{c}\text { Parameter } \\
E\end{array}$} & \multicolumn{2}{|c|}{ Values } & \multirow{2}{*}{$\frac{\text { Unit }}{\mathrm{GeV}}$} \\
\hline & 4.54 & 14.35 & \\
\hline$\varepsilon_{n}$ & \multicolumn{2}{|c|}{1.2} & $\mu \mathrm{m}-\mathrm{rad}$ \\
\hline$I_{p k}$ & \multicolumn{2}{|c|}{3400} & A \\
\hline RMS slice energy spread $\sigma_{E} / E$ & 0.025 & 0.008 & $\%$ \\
\hline Undulator Type & \multicolumn{2}{|c|}{ Planar Halbach HybridUndulator } & \\
\hline$\lambda_{u}$ & \multicolumn{2}{|c|}{0.03} & $\mathrm{~m}$ \\
\hline$g$ & \multicolumn{2}{|c|}{6} & $\mathrm{~mm}$ \\
\hline$B_{w}$ & \multicolumn{2}{|c|}{1.32} & $\mathrm{~T}$ \\
\hline K & \multicolumn{2}{|c|}{3.71} & \\
\hline$<\beta_{, x y}>$ & 18 & 7.3 & $\mathrm{~m}$ \\
\hline$\lambda_{r}$ & 15 & 1.5 & $\AA$ \\
\hline
\end{tabular}
Appendix A. These parameter tables are set up for the case that, except for the energy, the electron beam parameters are the same. Other parameter configurations are also available.

Table 5.2 Basic LCLS parameters at limits of operational wavelength range. 


\subsubsection{Working Points}

This report uses sets of parameters. The term "working point" is used for nominal parameter sets at each wavelength. Figure 5.1, Figure 5.2, and Figure 5.3 show the relation between the working points and the operational parameter space area at three different points within the operational range.
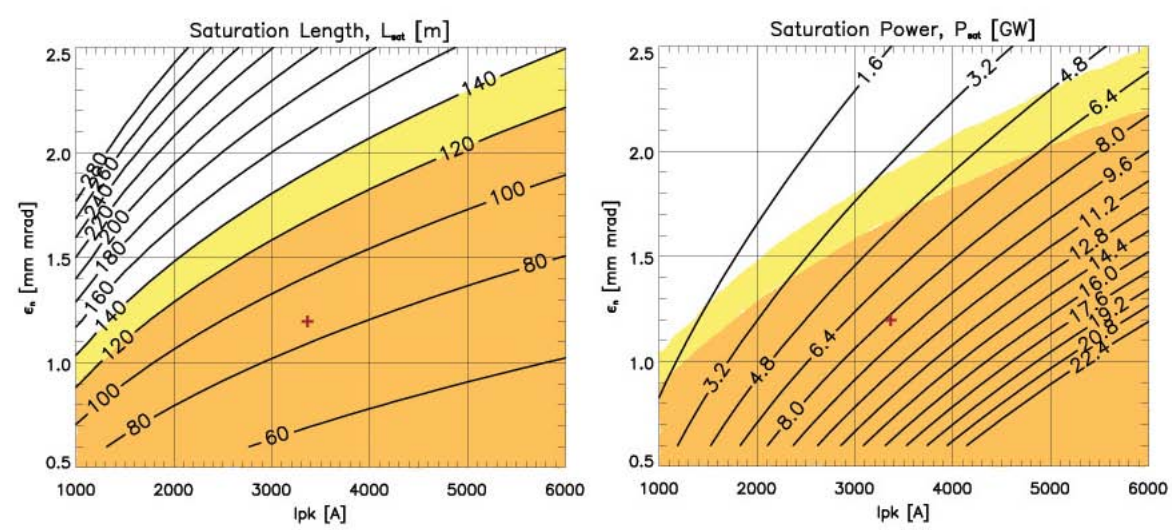

$\lambda_{r}=0.15 \mathrm{~nm}$
$\mathrm{E}_{0}=14.35 \mathrm{GeV}$
$\mathrm{dE}=1.47 \mathrm{MeV}$
$\beta_{\text {ext }}=18.00 \mathrm{~m}$
$\lambda_{u}=3.0 \mathrm{~cm}$
$\mathrm{a}_{w}=2.624$
$\mathrm{~B}_{u}=1.32 \mathrm{~T}$
gap $=6.00 \mathrm{~mm}$

Linear Undulator

Hybrid NdFeB

Halbach Type

Figure 5.1 Contour diagram of the saturation length, $L_{\text {sat }}$, and saturation power, $P_{\text {sat, }}$ as a function of normalized emittance, $\varepsilon_{n}$, and peak current, $I_{p k}$ for at the $1.5 \AA$ end of the LCLS operational range of the spectrum. The darker background color marks the parameter regime (peak current, normalized emittance) that will lead to saturation before the end of the 121-m long undulator. The parameter regime marked with lighter background shading will lead to saturation just after the end of undulator. The cross inside the dark background area marks the nominal working point. It is the center of an LCLS operational phase space volume. At the shortest wavelength, the nominal working point $\left(\varepsilon_{\mathrm{n}}=1.2 \mu \mathrm{m}-\mathrm{rad}, \mathrm{I}_{\mathrm{pk}}=3400 \mathrm{~A}\right)$ is expected to correspond to a saturation length of about $90 \mathrm{~m}$, well before the end of the undulator. The change of saturation power over the operational phase space volume is about a factor 2, i.e., small compared to the total energy gained from the FEL process.
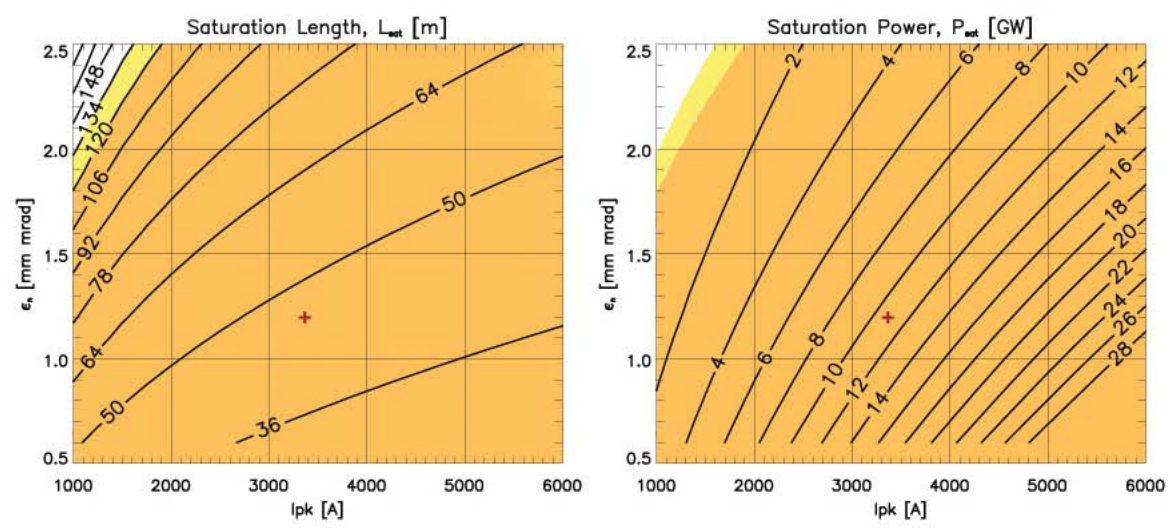

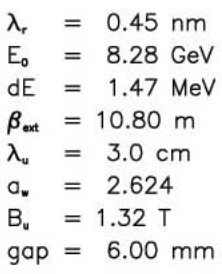

Linear Undulator

Hybrid NdFeB

Halbach Type

Figure 5.2 Contour diagram similar to Figure $\mathbf{5 . 1}$ but at the longer wavelength of the $4.5 \AA$. The nominal operation point corresponds to a saturation length of less than $50 \mathrm{~m}$, well before the center of the undulator. Much larger values of the normalized emittance and smaller values of the peak current will still keep the saturation point before the end of the undulator. 

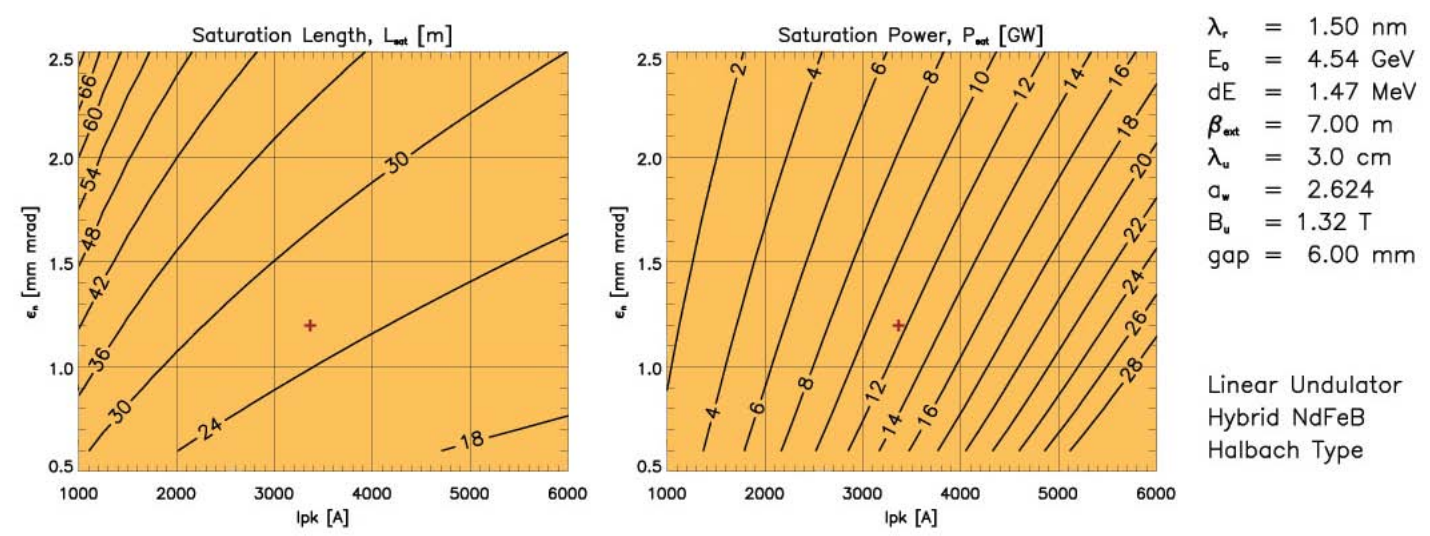

Figure 5.3 Contour diagram similar to Figure $\mathbf{5 . 1}$ and Figure $\mathbf{5 . 2}$ but at $15 \AA$, the long wavelength end of the operational range. The nominal operation point corresponds to a saturation length of about $25 \mathrm{~m}$. At this long wavelength the FEL process will saturate before the end of the undulator for a large parameter area.

The figures also show how peak current can be traded against normalized emittance when keeping the saturation length constant.

\subsection{Electron Beam Focusing Along the Undulator}

The criteria that let to the selection of the average $\beta$-function, the quadrupole strength and the cell spacing were established by first determining the optimum of the average $\beta$-function, and, after that, the maximum tolerable amplitude of the modulation of the $\beta$-function.

\subsubsection{Optimum Beam Size}

As the electron beam is transported through the LCLS undulator, transverse focusing is applied to keep the beam size, $\sigma_{x, y}$, approximately constant at

$$
\sigma_{x, y}=\sqrt{\beta_{x, y} \frac{\varepsilon_{n}}{\gamma}}
$$

In $1 D$ FEL theory the beam size affects the FEL parameter, $\rho$, via the bunch electron density

$$
n_{e}=\frac{I_{p k}}{2 \pi \sigma_{x} \sigma_{y} e c},
$$

resulting in a smaller gain length for a smaller beam size. 3-D effects, especially diffraction, will eventually lead to a decrease in FEL performance when the beam size becomes too small. Figure 5.4 and Figure 5.5 show relative FEL saturation power and saturation length as a function of the average $\beta$-function in the undulator for $1.5 \AA$ and $15 \AA$. 
LCLS CONCEPTUAL DESIG N R E P O R T

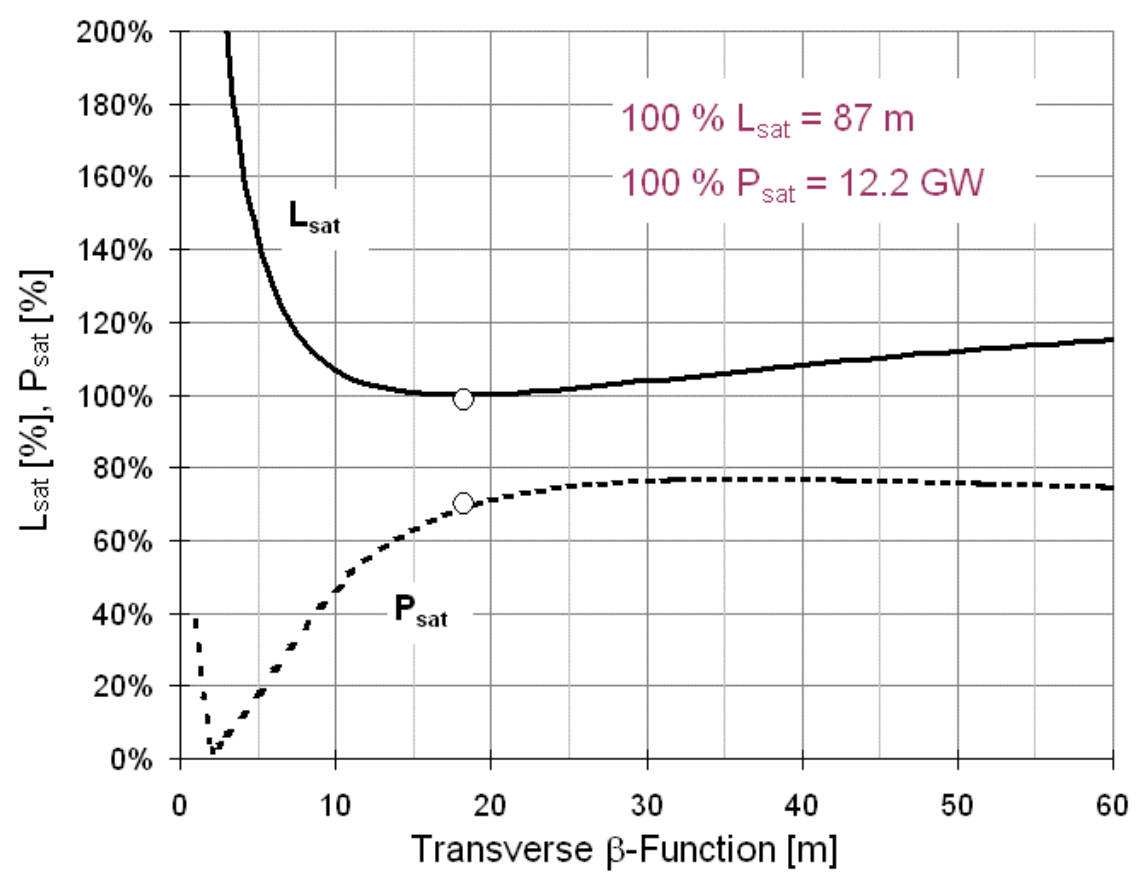

Figure 5.4 Power at saturation, $P_{\text {sat, }}$ and saturation length, $L_{\text {sat }}$, as a percentage of $12.2 \mathrm{GW}$ and $87 \mathrm{~m}$, respectively, as a function of the average $\beta$-function at a radiation wavelength of $1.5 \AA(14.35 \mathrm{GeV})$. The circles indicate the LCLS operating point.

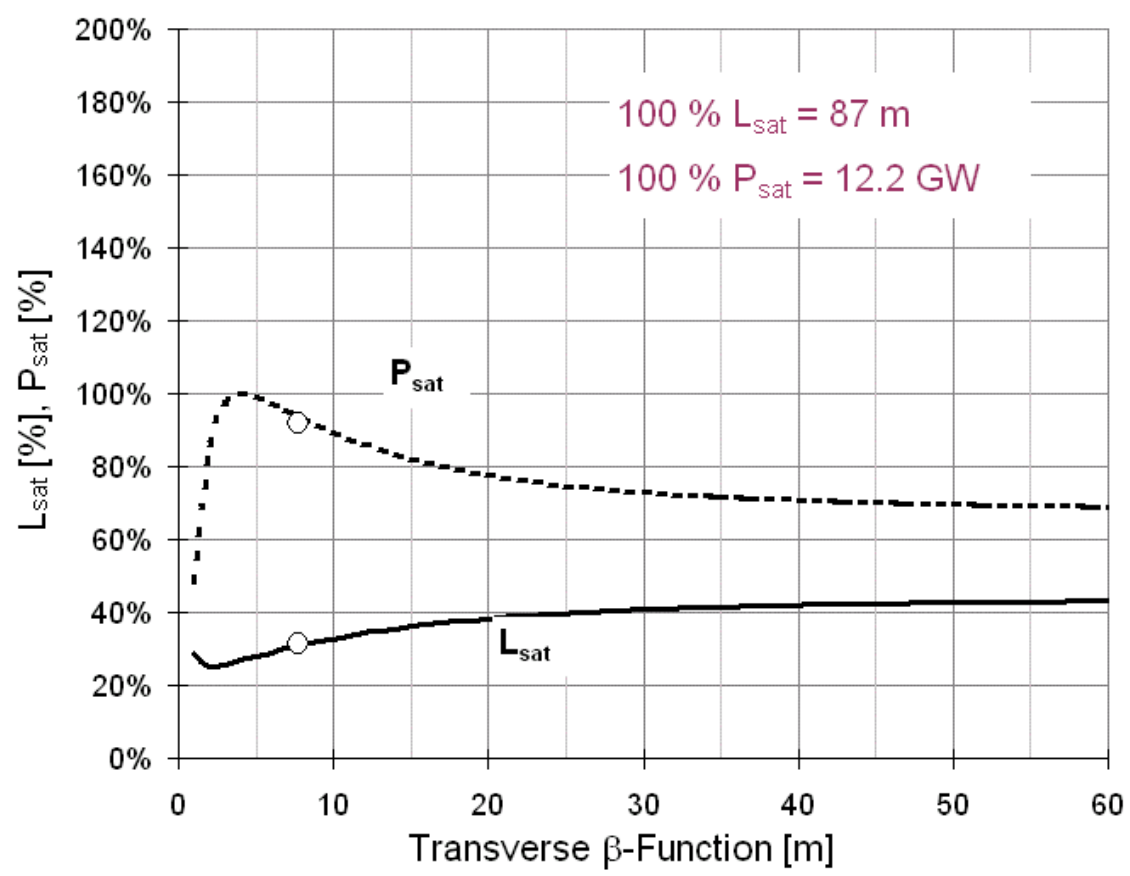

Figure 5.5 Power at saturation, $P_{\text {sat }}$, and saturation length, $L_{\text {sat, }}$, as a percentage of $12.2 \mathrm{GW}$ and $87 \mathrm{~m}$, respectively, as a function of the average $\beta$-function at a radiation wavelength of $15 \AA$ (4.54 GeV). The circles indicate the LCLS operating point. 
At every energy in the proposed range between $4.54 \mathrm{GeV}(15 \AA)$ and $14.35 \mathrm{GeV}(1.5 \AA)$, the minimum saturation length and the maximum saturation power occur at different values of the average $\beta$-function. The saturation length at $15 \mathrm{GeV}$ determines the length of the undulator. It is important at that energy to choose the $\beta$-function related to the minimum saturation length. This minimum occurs at a $\beta$-function of $18 \mathrm{~m}$ as is shown in Figure 5.4. This value was chosen for the $14.35 \mathrm{GeV}$ end of the LCLS operations range.

The average $\beta$-function value at which minimum saturation length occurs decreases with energy to reach about $2.1 \mathrm{~m}$ at $4.54 \mathrm{GeV}$. The average $\beta$-function value at which maximum saturation power occurs decreases with energy as well and reaches about $5 \mathrm{~m}$ at $4.54 \mathrm{GeV}$. As discussed below, the $\beta$-function value chosen for the LCLS at $4.54 \mathrm{GeV}$ is $7 \mathrm{~m}$, which can be reached from the high-energy value with constant gradient focusing. At this $\beta$-function value the saturation power is close to its maximum, which is desirable. At $4.54 \mathrm{GeV}$ saturation will occur during the first quarter of the undulator.

The following section discusses the need for and the choice of a quadrupole focusing lattice to generate the required average $\beta$-function.

\subsubsection{Natural Undulator Focusing}

In the ideal case the beam size along the undulator should be constant. Constant beam size focusing can, in principal, be achieved by using a modification to natural undulator focusing. It turns out that natural undulator focusing is too week to achieve the average $\beta$-function values required for the LCLS.

Natural focusing of a planar undulator exists in the plane perpendicular to the wiggle motion only (in this report, called x-plane, since the undulator, as shown in Chapter 8, has a vertical field). The focusing strength can be expressed by specifying the "natural" beta-function of the focusing system that is intrinsic to an undulator made of parallel poles

$$
\beta_{x}^{\text {nat }}=\sqrt{2} \gamma / k_{U} K
$$

By appropriately shaping the pole faces, half the focusing can be directed into the wiggle plane. This type of constant focusing in both planes is called ted-pole focusing [10] or sextupole focusing.

$$
\beta_{x, y}^{T P}=\sqrt{2} \beta_{x}^{\text {nat }}
$$

The amount of focusing that can be obtained this way is often smaller than required for optimum FEL performance, especially for high energy and short wavelength applications. For the LCLS the $\beta$-function from sextupole focusing alone would be $70 \mathrm{~m}$ at $14.35 \mathrm{GeV}$ and $22 \mathrm{~m}$ at $4.54 \mathrm{GeV}$, which would increase the saturation length and thus the length of the undulator by $22 \%$ at $14.35 \mathrm{GeV}$.

FEL P A R A METERS AN D PERFORMANCE 5-9 


\subsubsection{Focusing Method (Lattice)}

A focusing system stronger than that given by sextupole focusing can be obtained with external quadrupole fields. Possible lattice choices are two (FODO), three (Triplet), or more quadrupole magnets per unit cell. A FODO lattice was selected based on simplicity of design and on the cost-related desire to keep the number of magnets and associated instrumentation small. A Triplet lattice was studied and rejected because it establishes extremely tight alignment tolerances for the central quadrupole, which that could not be met.

Quadrupole focusing introduces an oscillation in the longitudinal phase of the electrons with respect to the ponderomotive potential well, while natural focusing maintains a constant phase [10]. This phase modulation could lead to de-trapping of particles and thus reduce FEL efficiency. Yu et al. [5], point out that such a reduction in gain can indeed occur for tapered wigglers, in which most of the output power is provided by trapped electrons, but that the same effect can actually be beneficial in the exponential gain regime. Here, the reduction of the dependence of the longitudinal velocity on betatron oscillation amplitudes in the case of alternating-gradient focusing tends to offset the effect of longitudinal velocity modulation.

The main betatron-oscillation of period, $2 \pi / \beta$ is modulated due to the beam envelope modulations caused by the change in $\beta$-function along the quadrupole lattice. The period of these modulations is equal to the length of a lattice cell. The optimum value for the LCLS $\beta$-function requires that the lattice cell need to be much shorter than the betatron-oscillation period, therefore, these modulations afflict large transverse angles to the outer beam electrons, resulting in a spread of the phases of the electrons with respect to the ponderomotive potential. For LCLS parameters this de-phasing has an affect on gain and is included in the simulations.

The choice of the FODO cell length is generally driven by a compromise between a reduction in envelope modulation amplitude

$$
\Delta \beta_{x, y} / \beta_{x, y}=\frac{1}{2}\left(\beta_{x, y, \max }-\beta_{x, y, \min }\right) / \beta_{x, y},
$$

which favors smaller cell lengths and a beam steering argument which favors longer cell lengths. The cell length needs to be smaller than the desired average $\beta$-function value. For the LCLS, a cell length of about $7.3 \mathrm{~m}$ was chosen (the FODO lattice consists of a number of different cell lengths as discussed below) using the considerations described above for the high-energy (i.e., short wavelength) end of the operational range where it is most important. This cell length is too long for the other low energy (long wavelength) end of the operational range, where is limits the minimum achievable average $\beta$-function to the value of the FODO cell spacing, about $7.3 \mathrm{~m}$. As can be seen from Figure 5.5, the impact of the increased $\beta$-function at lower energies is small.

The integrated quadrupole-strength needed to achieve the average $\beta$-function of $18 \mathrm{~m}$ at $14.35 \mathrm{GeV}$ is

$$
\int_{0}^{L_{Q_{F}}} \frac{d B_{Q_{F}}}{d r} d s=5.36 \mathrm{~T}
$$




$$
\int_{0}^{L_{Q_{D}}} \frac{d B_{Q_{D}}}{d r} d s=-5.30 \mathrm{~T},
$$

for the focusing and the defocusing quadrupoles, respectively. With the quadrupole length of $5 \mathrm{~cm}$, as described in Chapter 8, the quadrupole gradients will than be

$$
\begin{gathered}
\frac{d B_{Q_{F}}}{d r}=107.1 \mathrm{Tm} \\
\frac{d B_{Q_{D}}}{d r}=-105.9 \mathrm{Tm}
\end{gathered}
$$

The difference in the two gradients comes from the fact that, as explained above, the undulator segments provide additional, natural focusing but only in the vertical plane. Without the undulator segments the two gradients would be $\pm 109.4 \mathrm{Tm}$. The gradients, given in Equations (5.16) and (5.17), are adequate to achieve the average $\beta$-function amplitude of $7.3 \mathrm{~m}$ at $4.54 \mathrm{GeV}$, as well. As explained in Chapter 8, permanent magnet quadrupoles will be used. Figure 5.6 and Figure 5.7 show the average $\beta$-function over the full operational range. The bold line shows the actual values of the average $\beta$-function, limited towards lower energies (or longer wavelength) by the FODO cell length. The dotted line shows the values that the quadrupoles could in principal achieve and the dot-dash line shows the values needed for shortest saturation length.

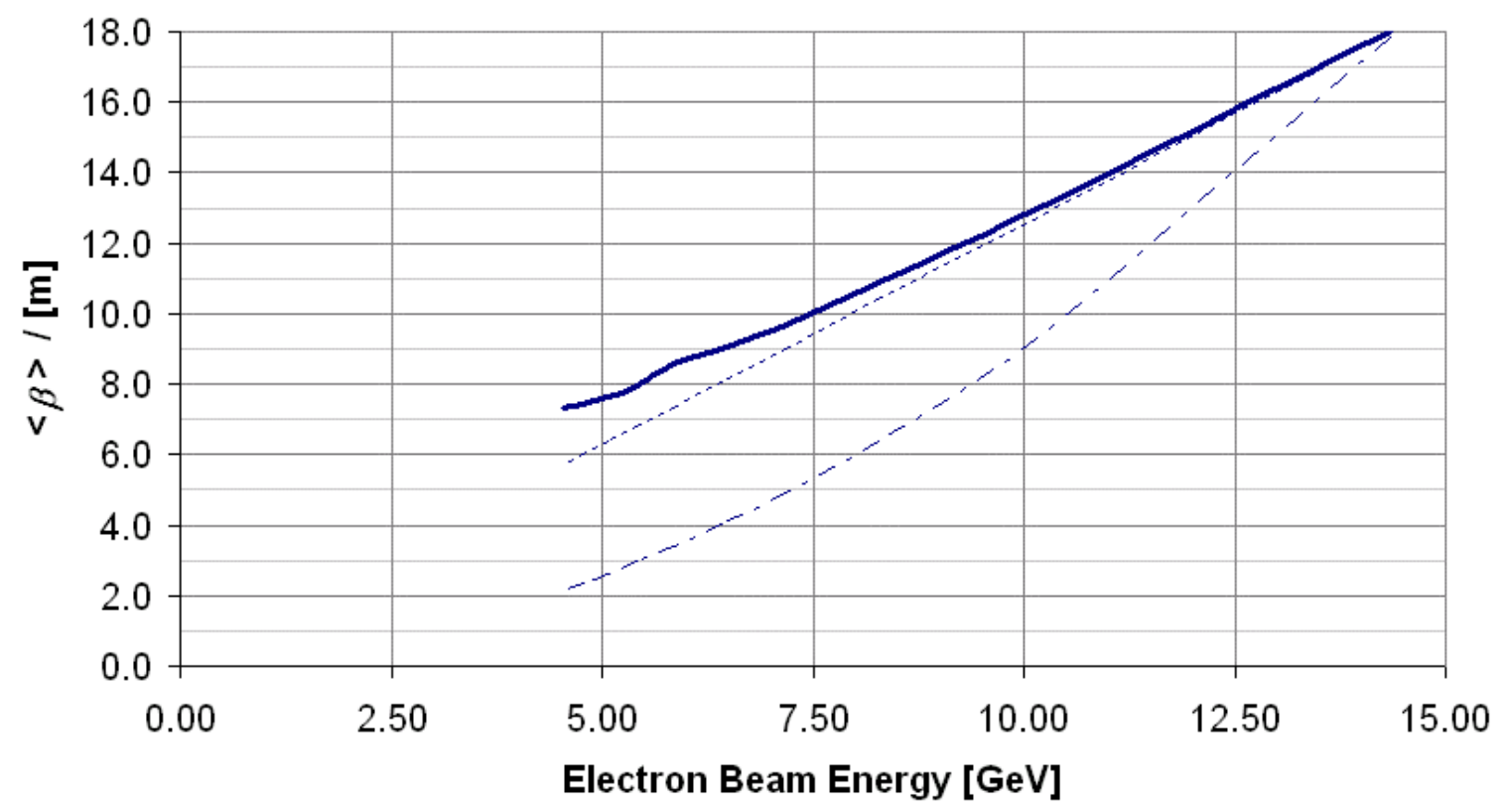

Figure 5.6 Average of the horizontal and vertical $\beta$-functions as a function of beam energy using permanent magnet quadrupoles and matching the electron beam focusing into the undulator (solid line). As the $\beta$-function amplitude comes close to the length of the FODO cell its dependence on energy deviates from linear (dotted line) by a small amount. The optimum $\beta$-function that gives the shortest saturation length (dash-dot line) has a similar dependence but increases faster with energy. The actual $\beta$-function stays sufficiently close to the optimum as can be seen from Figure 5.4 and Figure 5.5. 


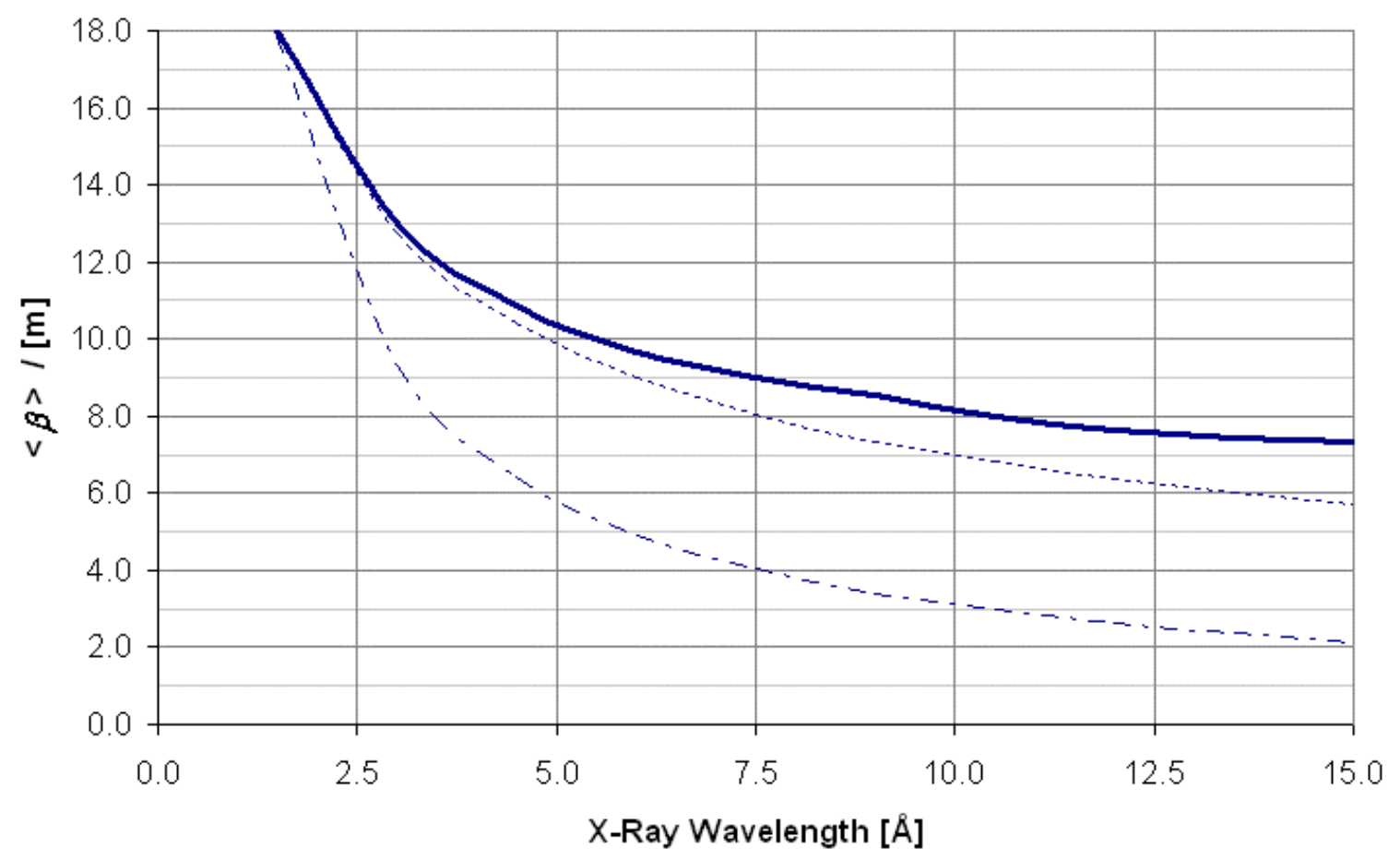

Figure 5.7 Same as Figure 5.6, except that the dependence to $x$-ray wavelength instead of energy is shown.

The horizontal and vertical $\beta$-functions, along the entire LCLS undulator, are shown in Figure 5.8 and Figure 5.9 for $14.35 \mathrm{GeV}$ and $4.54 \mathrm{GeV}$, respectively.

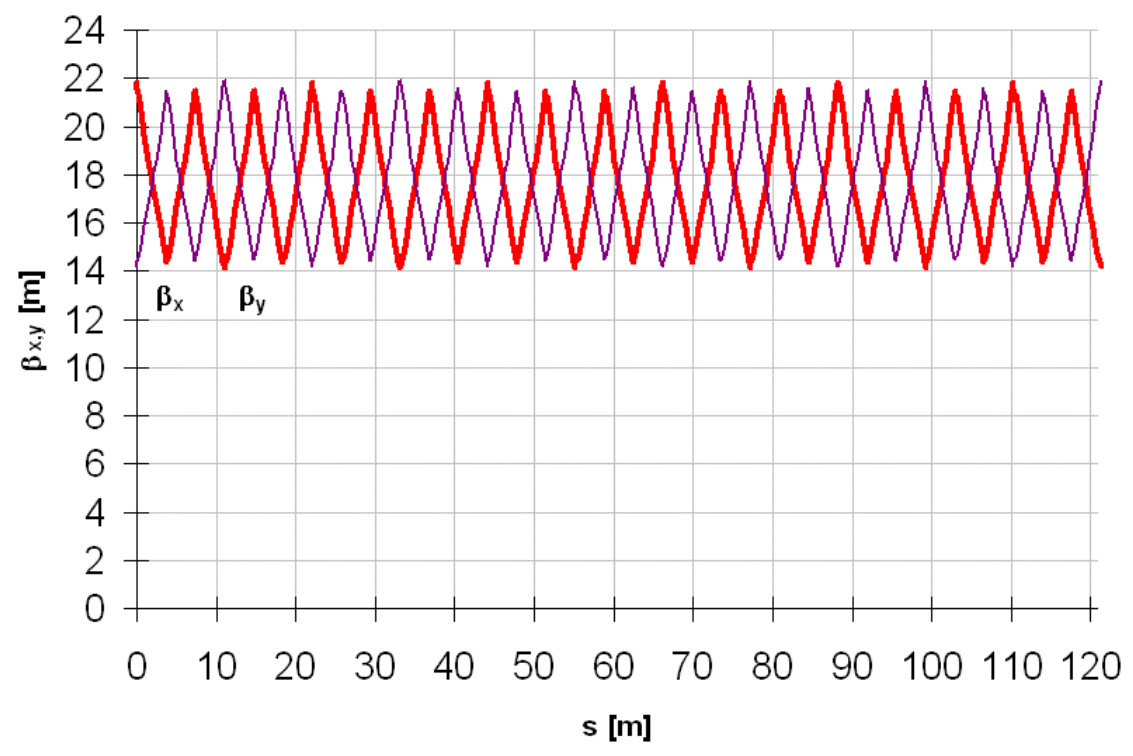

Figure 5.8 Horizontal and vertical $\beta$-functions in the LCLS at the high-energy limit of $14.35 \mathrm{GeV}$. 


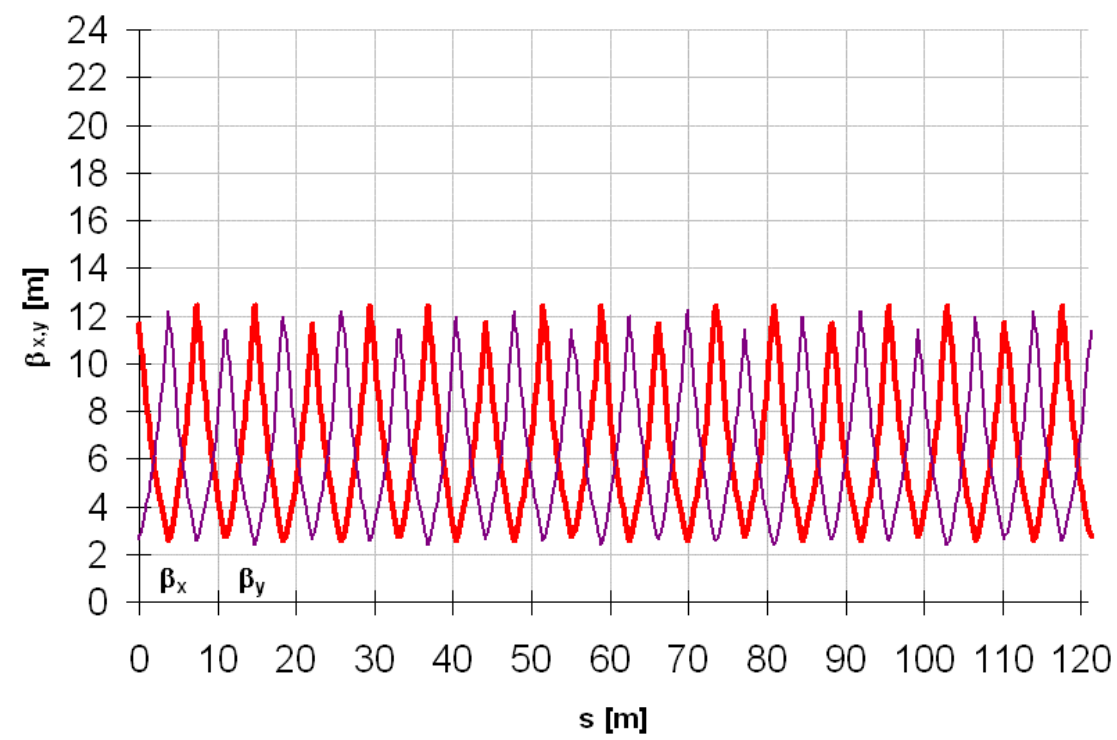

Figure 5.9 Horizontal and vertical $\beta$-functions in the LCLS at the low-energy limit of $4.54 \mathrm{GeV}$.

Both sets of $\beta$-functions are generated with the same set of quadrupole strengths; only the matching optics into the undulator has been changed.

\subsubsection{FODO Lattice Layout}

As described in Chapter 8, the LCLS undulator has a total of 33 undulator segments, separated by short breaks. The breaks are used to house the FODO lattice quadrupoles and for other purposes. Not all breaks are of the same length. Five different break lengths are used. Consequently, there is a number of different FODO cell lengths, as well. Except for the beginning of the undulator there is a regular pattern of two short and one long break length. The three breaklength period and the two-quadrupole periods $\left(\mathrm{Q}_{\mathrm{F}}, \mathrm{Q}_{\mathrm{D}}\right)$ generate a superperiod of 6 quadruples (and 6 undulator segments) or three FODO cells of lengths $7.311 \mathrm{~m}, 7.311 \mathrm{~m}$, and $7.428 \mathrm{~m}$. The last 5.5 superperiods are of that structure. The very first three break lengths have been optimized for reduced saturation length and are therefore different from the rest. Consequently the lengths of the three FODO cells of the first "superperiod" are $7.463,7.5145 \mathrm{~m}$, and $7.311 \mathrm{~m}$. The superperiod structure is reflected in the beating the $\beta$-functions in Figure 5.8 and Figure 5.9.

\subsection{Computer Simulations}

\subsubsection{FEL Simulations Codes}

Although a three-dimensional theory has been developed and allows the study of the effect of parameters like energy spread, emittance, and diffraction, the effects of magnet errors, misalignment, wakefields and realistic electron distributions can not be treated analytically, yet. For this reason, after one has used 3-D theory to search and optimize the basic parameters of an FEL, the most important tools for a subsequent and more precise study and optimization are the 
computer simulation codes [6,7]. Simulations for this design report used the 3-D codes GENESIS 1.3 [8], GINGER [9] (both time dependent), FRED-3D [10] (magnet error analysis, beam position control), as well as the linear code RON [11,12] (magnet tolerances). The codes been extensively cross checked [13] with each other as well as with experimental results from the LEUTL [14] and VISA [15] experiments.

\subsubsection{Start-To-End Simulations}

The overall system performance has been studied using start-to-end simulations [16]. The beam is transported from the injector through the linac and the undulator using the computer codes, PARMELA (Injector), ELEGANT (Linac) and GENESIS 1.3 (Undulator). The PARMELA code includes space charge, rf, and thermal emittance effects.

Two cases have been considered. One has a charge of $0.25 \mathrm{nC}$, and a bunch compression set to produce a peak current of about $1.5 \mathrm{kA}$. In this case the charge has been chosen using the scaling arguments discussed in Chapter 4, to provide the optimum beam emittance and brightness. The other case has a charge of $1 \mathrm{nC}$ and a peak current at the LCLS reference case.

\subsubsection{Case I - Low Charge Limit}

In the first case, the normalized emittance is about $0.3 \mu \mathrm{m}$-rad. The results, at the linac exit, are shown in Figure 5.10 and Figure 5.11. The first figure gives a "slice" description of the beam, showing various quantities along the longitudinal bunch coordinate.
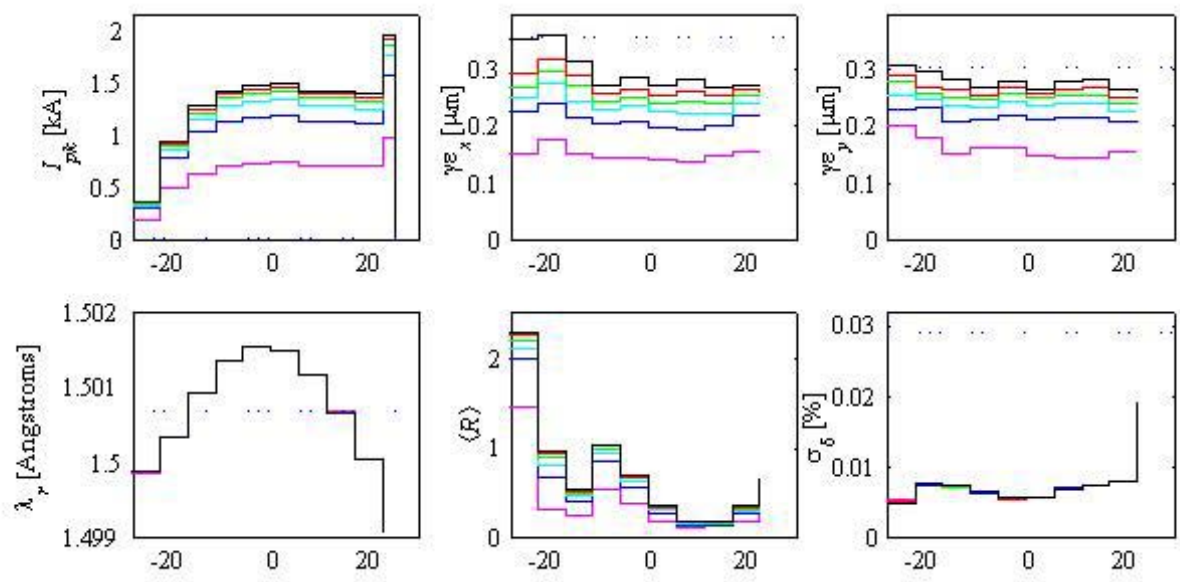

Figure 5.10 Peak current, horizontal and vertical normalized rms slice emittances, equivalent resonant wavelength, Courant-Snyder invariant, and rms slice energy spread along the electron bunch at the undulator entrance. The horizontal axis gives Iongitudinal position along the bunch in micrometer.

The $<R>$ parameter describes the displacements of the transverse centroid of the electron distribution along the bunch. It is defined per slice, using the Courant-Snyder invariant, as:

$$
\langle R\rangle=\sqrt{\frac{x^{2}+\left(x \alpha_{x}+x^{\prime} \beta_{x}\right)^{2}}{\varepsilon_{x} \beta_{x}}+\frac{y^{2}+\left(y \alpha_{y}+y^{\prime} \beta_{y}\right)^{2}}{\varepsilon_{y} \beta_{y}}}
$$


where $\beta_{x}, \beta_{y}, \alpha_{x}$, and $\alpha_{y}$ are the nominal projected beta and alpha functions per plane, and $\varepsilon_{x}$, and $\varepsilon_{y}$ are the rms emittances per plane. With the given definition, $<R>$ takes on the value of one for a horizontal or vertical displacement of amplitude equal to 1 sigma, as for instance in the case when $x=\left(\beta_{x} \varepsilon_{x}\right)^{1 / 2}, x^{\prime}=0, y=0, y^{\prime}=0$. Transverse displacements can be due to effects such as coherent synchrotron radiation produced in the linac compressors.

An examination of Figure 5.10 shows that the emittance is around $0.3 \mu \mathrm{m}-\mathrm{rad}$, and the corresponding current is about $1.5 \mathrm{kA}$ for most of the bunch. It is also interesting to notice that the electron energy distribution along the bunch produces a wavelength variation of about $0.1 \%$, larger than the expected x-ray SASE linewidth. The graph of $<R>$ shows that the compression process produces a transverse displacement of the electrons along the bunch of the order of $1 \sigma$. This displacement has an effect on the gain, and also gives a larger x-ray spot size at the undulator exit. This has been accounted for in the brightness estimate.
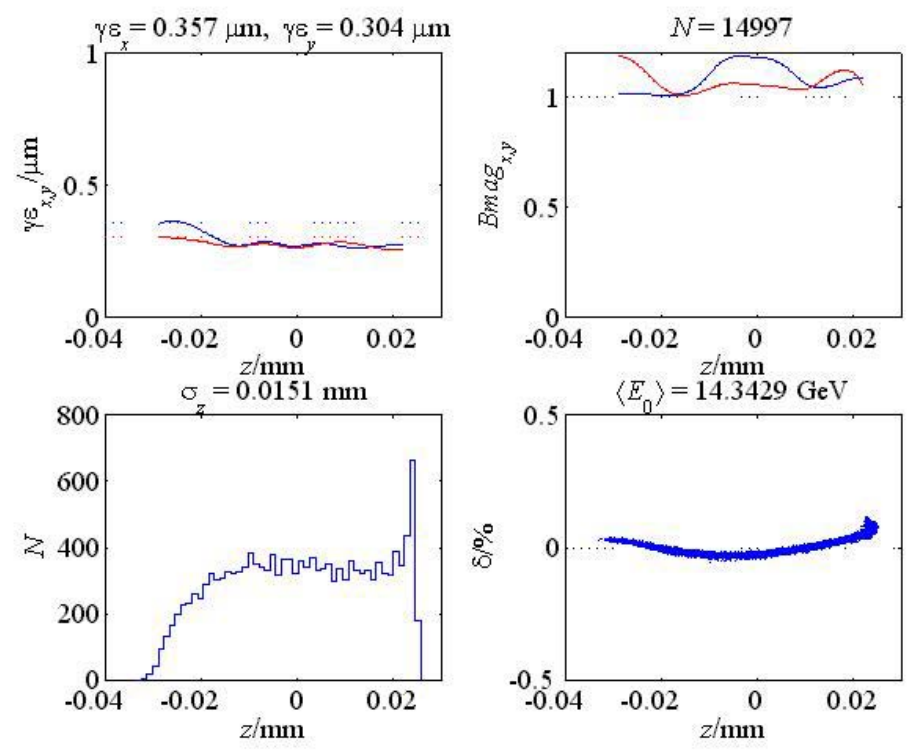

Figure 5.11 Electron beam characteristics at the linac exit for the $0.25-\mathrm{nC}$ case.

Figure 5.11 shows the longitudinal dependence of a set of beam parameters. The quantity $B_{m a g}$ describes the local variation of the individual slice phase space ellipses with respect to the projected phase space ellipse. A value of 1 corresponds to a full overlap.

The results of propagating the beam through the undulator is shown in Figure 5.12 and compared with the case of an "ideal beam" having uniform longitudinal distribution and a Gaussian transverse distribution. As one can see, the real effects, introduced by beam dynamics in the injector-linac-compressor systems, result in a loss of output power. The power in the reference case is $16.6 \mathrm{GW}$, and in the start-to-end case is $12.1 \mathrm{GW}$. This calculation does not include undulator wakefields, which have been estimated in Section 4.5.2. Note that there is no change in saturation length. 


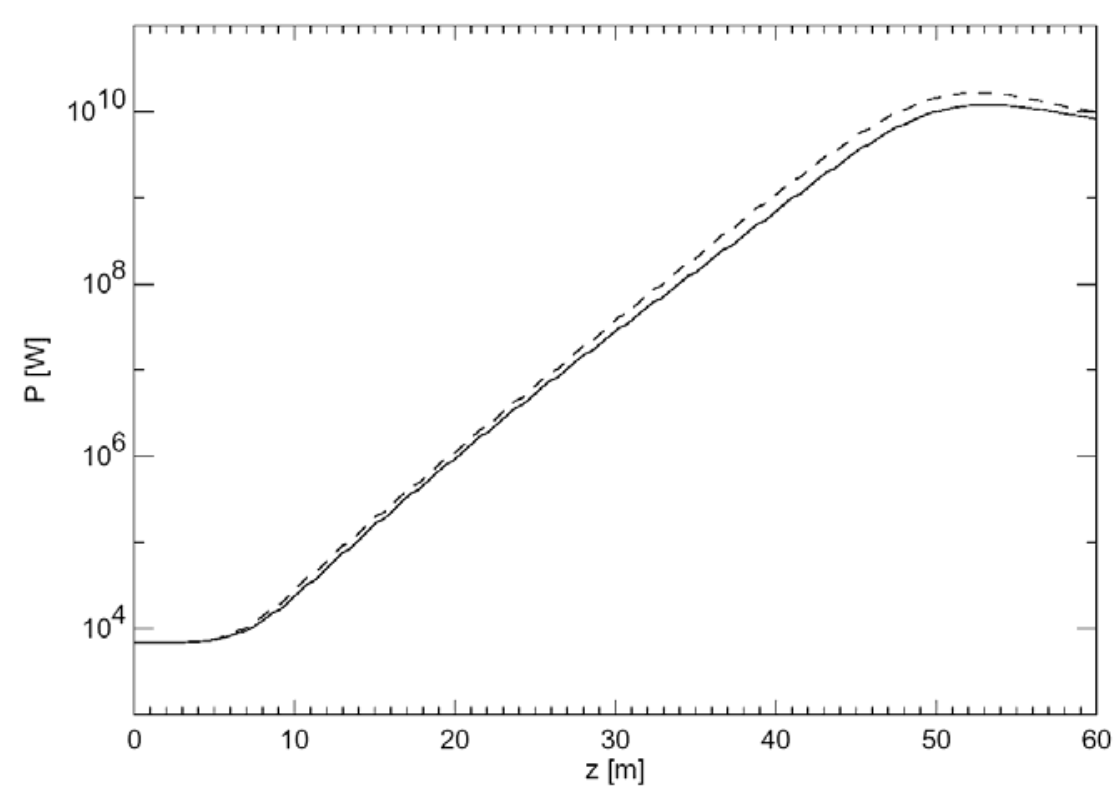

Figure 5.12 Power vs. undulator length for a 0.25 - $\mathrm{nC}$ case, with an emittance of $0.3 \mu \mathrm{m}$-rad and a peak current of $1.5 \mathrm{kA}$. No undulator wakefields have been included. The dashed line assumes a constant value of emittance and current along the bunch. The effect of using the longitudinal and transverse phase-space distribution produced in the gun-linaccompressor system (solid line) is a reduction in output saturation power.

The distribution of power along the bunch obtained in the start-to-end simulation is shown in Figure 5.13. The transverse displacement along the bunch and other effects produce a nonuniform power distribution.

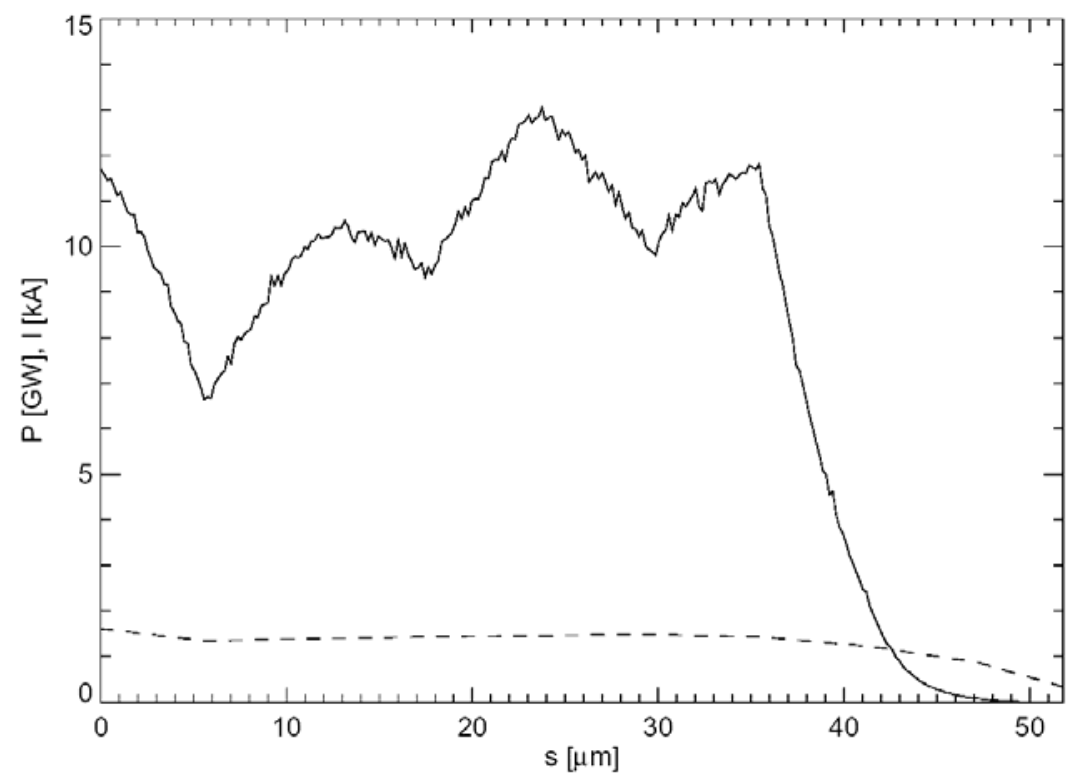

Figure 5.13 Power distribution at saturation (solid) along the electron bunch for the start-to-end simulations for a charge of $0.25 \mathrm{nC}$. The dashed curve is the current profile in kiloAmperes. The head of the bunch is to the right. 


\subsubsection{Case II - High Charge Limit}

The case of $1 \mathrm{nC}$ is shown in Figure 5.14, Figure 5.15 and Figure 5.16. The first of these figures shows the characteristics of the electron bunch at the undulator entrance, the second the FEL evolution along the undulator, and the third the power distribution along the bunch.
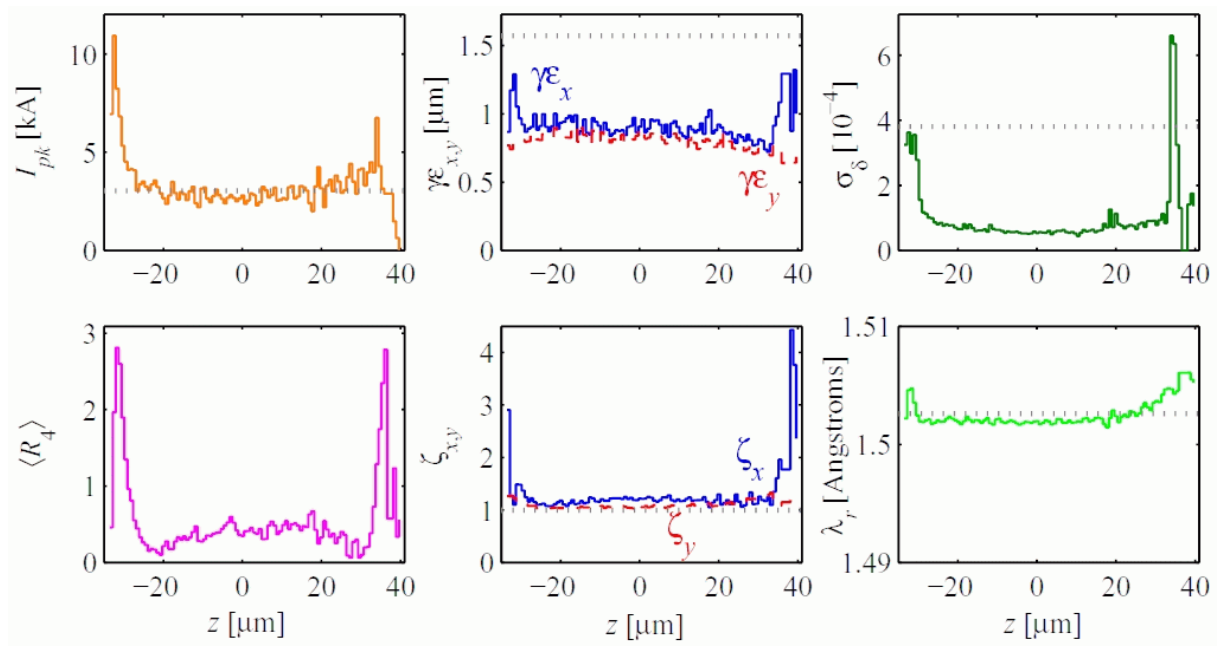

Figure 5.14 Electron beam characteristics at the linac exit for the $1 \mathrm{nC}$ case. The horizontal axis gives longitudinal position along the bunch in $\mu \mathrm{m}$.

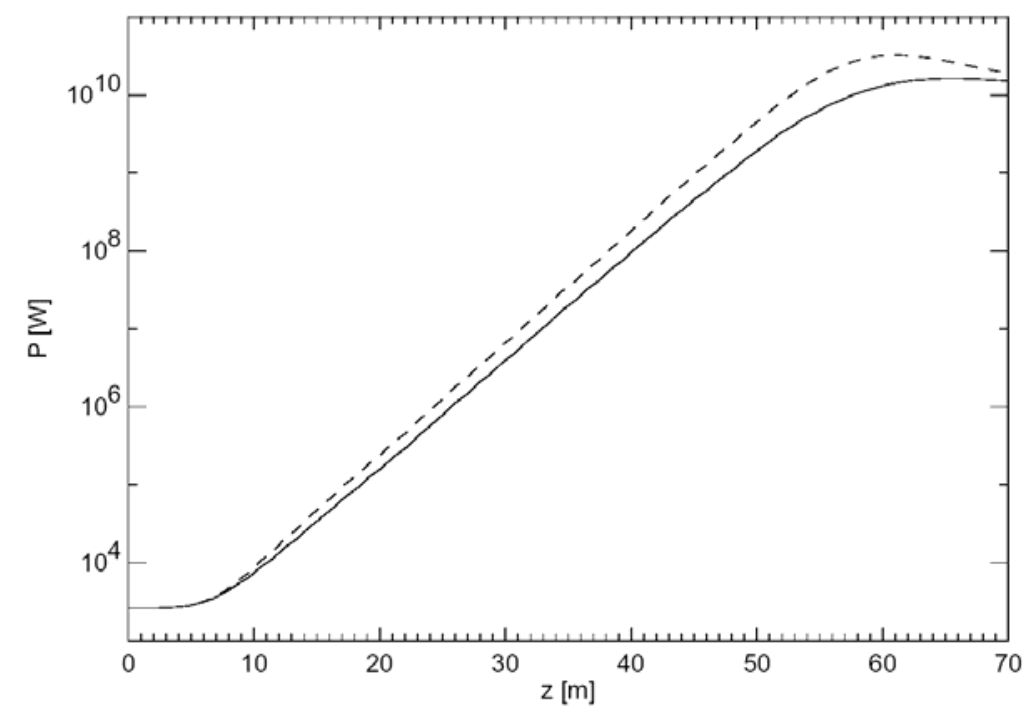

Figure 5.15 The dashed curve is the power vs. undulator length, in meters, for the "ideal" 1-nC case, with an emittance of $0.5-\mu \mathrm{m} \mathrm{rad}$, and a peak current of $3.4 \mathrm{kA}$. The solid curve is the start-to-end 1-nC case. The saturation power is $32 \mathrm{GW}$ in the ideal case and $16 \mathrm{GW}$ in the start-to-end case. No undulator wakefields have been included. 


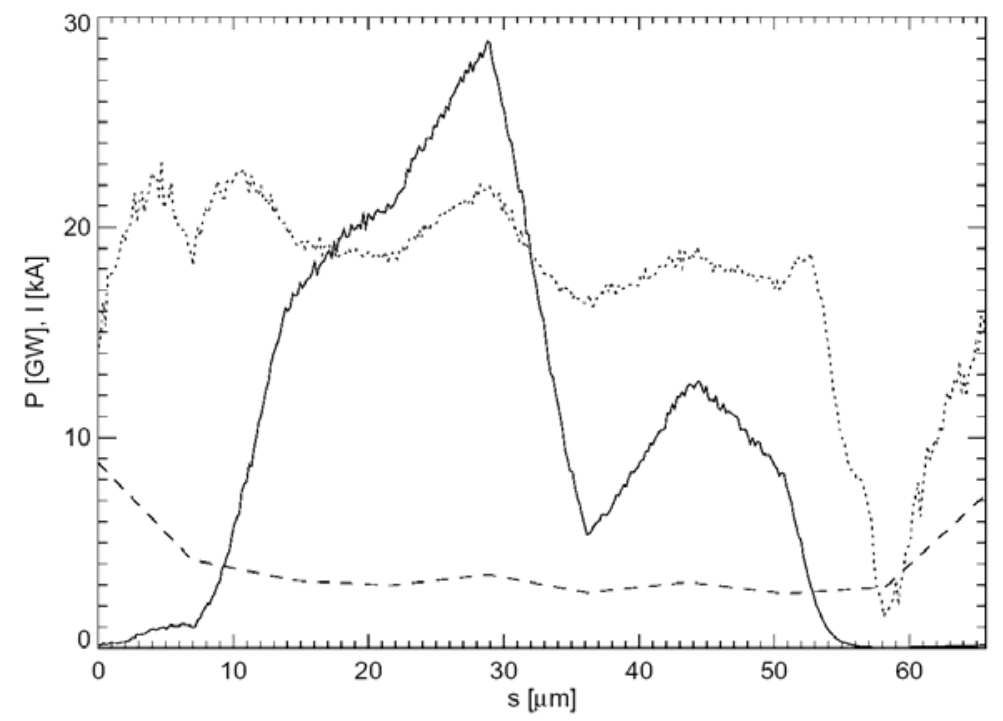

Figure 5.16 Power distribution along the bunch at saturation (solid line), and at a distance $100 \mathrm{~m}$ after saturation (dotted line). The dashed line depicts the current profile in units of kA. The head of the bunch is to the right.

\subsection{Sources of Gain Reduction}

\subsubsection{Undulator Trajectory Errors}

This section discusses the sensitivity of LCLS FEL performance on imperfections of the electron orbit in the undulator.

\subsubsection{Undulator Steering and Corrector Description}

As shown in detail in Chapter 8, the undulator is designed as a planar NdFeB hybrid structure with a period of $3 \mathrm{~cm}$ and a full gap height of $6 \mathrm{~mm} .113$ undulator periods form a 3.375-m long segment (Spacing between the centers of the first and last pole). Segments are separated by breaks that accommodate electron beam position monitors as well as 5 -cm long permanent magnet quadrupoles with a gradient of about $107 \mathrm{~T} / \mathrm{m}$. The quadrupoles are used for two purposes, electron beam focusing and steering. Steering is achieved by adjusting the quadrupoles' $x$ and $y$ positions with stepper-motor based systems that allow a total movement of $0.5 \mathrm{~mm}$ with a step size of $1 \mu \mathrm{m}$. The undulator is built from 33 segments resulting in a total length of about $121 \mathrm{~m}$, of which about $112.86 \mathrm{~m}$ is magnet length.

\subsubsection{Magnetic Field Errors}

The sources of magnetic field errors in the LCLS undulator are from misaligned quadrupoles, undulator pole errors, the earth field, and other stray fields. The misalignment of the quadrupoles has been strongly reduced by design. As described above, their transverse position can be remotely adjusted and is used for beam steering. The finite resolution of the movers is compensated by auxillary horizontal and vertical steering magnets. The effect of the earth field is small and will be corrected by beam-based-alignment. Stray fields will be avoided or minimized 
by design. The potentially most significant sources of FEL performance reduction come from errors in the on-axis magnetic field of the undulator and from dipole components of transversely misaligned quadrupoles. As shown in Chapter $\mathbf{8}$ these magnet errors can be strongly reduced by state-of-the-art sorting and shimming techniques.

\subsubsection{Undulator Trajectory Straightness Tolerances}

Radiation produced from the electron distribution emerges collinear to the electron beam path. In an ideal undulator, the electron beam executes transverse wiggle oscillations in the periodic magnetic field of the undulator along a straight line, causing the electron beam and the radiation pulse to travel on average on the same path with optimum transverse overlap. The electrons' wiggle motion reduce their z-velocity just enough to move exactly one optical wavelength for each undulator period traveled, effectively keeping the two components in phase. Both aspects are necessary for optimum gain. Field errors can cause the electron beam to deviate from the ideal trajectory, which reduces the overlap between the electron distribution and the radiation. It also moves the phases of the electrons with respect to the ponderomotive potential well. Based on computer simulations a transverse displacement by about $1 \mathrm{rms}$ beam radius or a phase slip of 18 degrees of optical wavelength per power gain length both cause the saturation length to increase by one power gain length. While the beam radius does not depend very strongly on the radiation wavelength, the phase slip for a given trajectory is inversely proportional to the wavelength. Thus, while, at long wavelengths, the overlap aspect often dominates the tolerance, in contrast, at x-ray wavelengths, the phase slip dominates the tolerance. The 18 degrees per power gain length is reached for the LCLS at $1.5 \AA$ with an rms trajectory amplitude of about $2 \mu \mathrm{m}$. Although this absolute accuracy seems difficult to achieve, it will be shown in Section 8.12 that it is obtainable with a beam-based alignment technique.

\subsubsection{Steering Stations Separations}

After the application of the beam-based alignment procedure the transverse position of the electron BPMs will be calibrated using the straight beam. Between two applications of the procedure the beam trajectory will be corrected by adjusting the transverse position of the quadrupoles based on the readings of the calibrated electron BPMs, setting tolerances for the resolution of the BPMs and the relative spacing of the steering stations, i.e., the combination of steerer quadrupole and BPM. Limited BPM resolution will force the beam on a zigzag trajectory between steering stations. If the steering stations are spaced too closely the effect gets amplified. On the other hand, if the steering stations are spaced too far apart, the undulator pole errors are not sufficiently corrected. There is an optimum for the separation of steering stations [17], which depends on the BPM resolution and the pole error. The smaller the pole errors the shallower is the optimum. As described in Chapter 8, the steering station separation for the LCLS has been chosen around the optimum.

\subsubsection{Undulator Trajectory Matching Tolerances}

The match of the electron trajectory at the entrance and end of each undulator section is done by making the strength of the end poles in the sequence $1 / 4,3 / 4,1$, and -1 times the strength the

FEL PARAMETERS AN D PER F O M A N C E 5-19 
regular pole (see Chapter 8, Section 8.5.3). The "matching sections," which are off resonance, do not contribute to lasing but will add a small phase shift, which reduces the space of the actual separation by a few centimeters. Estimates based on beam size arguments indicate that position and angle errors of about $5 \mu \mathrm{m}$ and $1 \mu \mathrm{rad}$, respectively, should not affect FEL performance. These tolerances can be achieved with state-of-the-art instrumentation (see Chapter 7).

\subsubsection{Effects of the Emission of Spontaneous Radiation on Gain}

Due to the rather large value of the undulator parameter, $K$, synchrotron radiation from the electron beam in the FEL undulator not only occurs at the resonant frequency and its harmonics but over a wide continuous spectrum of frequencies. As long as micro bunching can be neglected, the total peak synchrotron power radiated by a bunch is given by [18]

$$
\hat{P}_{\text {spont }}=Z_{o} I_{p k} e \frac{2 \pi c}{6 \lambda_{u}} \gamma^{2} K^{2} N_{u}=0.663 \cdot 10^{-15} \cdot \frac{s}{(T m)^{3}} \cdot(E / e)^{2} B_{u}^{2} L_{u} I_{p k}
$$

The power from spontaneous radiation grows linearly along the undulator up to $\hat{P}_{\text {spont }}=96 \mathrm{GW}$ after $120 \mathrm{~m}$ at $1.5 \AA$. This is more than ten times as much as can be expected for the fundamental peak of the coherent FEL radiation. While this large amount of incoherent radiation by itself makes the LCLS the brightest x-ray source available, it is undesirable when the LCLS is to be tuned for FEL lasing. Not only can it cause problems for the x-ray optics, but it also reduces the average electron energy, increases the incoherent energy spread and the emittance, and adds extra heat load to components that might be installed along the undulator for diagnostics and beam filtering purposes.

\subsubsection{Average Energy Loss}

The average energy loss $\Delta<\gamma>$ from spontaneous synchrotron radiation for each electron is

$$
\Delta\langle\gamma\rangle=-\frac{\hat{P}_{\text {spont }}}{I_{p k}} \frac{e}{m c^{2}}=-\frac{1}{3} \gamma^{2} K^{2} k_{u}^{2} L_{u}
$$

where $k_{u}=2 \pi / \lambda_{u}$, which causes the electrons to move away from the resonance. The resonant frequency of the radiation can be kept constant by reducing the magnetic field along the undulator (micro-tapering). The amount of field taper required is

$$
\Delta B_{u} / B_{u}=\frac{1+K^{2} / 2}{K^{2} / 2} \Delta\langle\gamma\rangle /\langle\gamma\rangle
$$

Table 5.3 Reduction in average energy and required amplitude of micro-tapering, $\Delta \mathrm{B}_{u} / \mathrm{B}_{u}$, due to

\begin{tabular}{|c|c|c|c|c|c|}
\hline$\lambda_{r}$ & $I_{p k}$ & $\Delta<p$ & $\langle\beta\rangle$ & $\Delta<p>|<p\rangle$ & $\Delta B_{u} / B_{u}$ \\
\hline $1.5 \AA$ & $3400 \mathrm{~A}$ & -46 & 28077 & $-1.64 \times 10^{-3}$ & $-1.9 \times 10^{-3}$ \\
\hline $15 \AA$ & $3400 \mathrm{~A}$ & -1.38 & 8879 & $-1.56 \times 10^{-4}$ & $-1.8 \times 10^{-4}$ \\
\hline
\end{tabular}
random photo-emission process in spontaneous undulator radiation in the LCLS for the two limits of the operational wavelength range. 
The loss in average beam energy, $\Delta<\gamma>\mid<\gamma>$, at the $1.5-\AA(14.35 \mathrm{GeV})$ end of the operational range is large enough to move the particles outside the FEL gain-bandwidth. Micro-tapering of the undulator segments will be required. The actual required change in magnetic field is very small. It is not necessary to taper the individual undulator segments, but the average field of each segment needs to be a bit smaller than the preceding segments. The required field taper at the high energy end of the operational range will be a bit too large for the low energy end of the range, where it will cause a small reduction in gain unless the taper is adjustable.

\subsubsection{Energy Spread Increase}

The statistical nature of the synchrotron radiation process increases the incoherent energy spread of the electrons by [19]:

$$
d<\Delta \gamma^{2}>=\frac{14}{15} \frac{\lambda_{c}}{2 \pi} r_{e} \gamma^{4} k_{u}^{3} K^{2} F(K) L_{u}
$$

where $F(K) \approx 0.6 K$ for $K>>1$ and for a planar undulator. $\lambda_{c}$ is the Compton wavelength $\left(\lambda_{c} / 2 \pi \approx 3.862 \times 10^{-13}\right)$. The amplitudes of the effect are shown in Table 5.4. The largest influence on FEL performance for the LCLS occurs at the high-energy end.

Table 5.4 Influence of Compton wavelength on FEL performance.

\begin{tabular}{ccc}
\hline$\gamma$ & $\boldsymbol{L}_{w}$ & $\sqrt{d<\Delta \gamma^{2}>}$ \\
\hline 28082 & $100 \mathrm{~m}$ & 6.5 \\
\hline 8880 & $80 \mathrm{~m}$ & 0.36 \\
\hline
\end{tabular}

There, the energy spread increase due to incoherent synchrotron radiation will reach the level of the initial rms energy spread which is $\sigma_{\gamma}=2.88$. Simulations with the code GENESIS 1.3 show no reduction in performance.

\subsubsection{Emittance Increase}

Spontaneous synchrotron radiation can cause an increase in rms beam emittance if the radiation occurs at a location with a finite dispersion function [20]. The dispersion function originates in the undulator, is of the order of the wiggle amplitude $(\sim 1 \mu \mathrm{m})$, and has a negligible effect on the emittance.

\subsection{Electron Beam Tolerances}

\subsubsection{Electron Beam Tolerance Goals}

This CDR uses a number of goal parameters for emittance and energy spread both as electron bunch slice quantities and projected quantities as defined in Section 5.2.2. These numbers are larger than those predicted by computer simulations. Establishing parameter goals decouples the design processes of the various FEL subsystems. The numbers are listed in Table $\mathbf{5 . 5}$ at two points in the FEL line, after the Injector and at the entrance to the undulator. The exception is the Projected Energy Spread, which is not relevant before the entrance to the undulator. 
Table 5.5 Goals for basic LCLS Beam Parameters for a beam charge of $1 \mathrm{nC}$. The numbers at the Undulator Entrance are for a beam energy of 14.35 GeV.

\begin{tabular}{lll}
\hline Parameter & Location & LCLS Goal Value \\
\hline Slice Emittance & Injector $(@ 150 \mathrm{MeV})$ & $1.0 \mathrm{~mm}$ mrad (RMS) \\
\hline Projected Emittance & Undulator Entrance & $1.2 \mathrm{~mm} \mathrm{mrad}(\mathrm{RMS})$ \\
\hline & Injector $(@ 150 \mathrm{MeV})$ & $1.2 \mathrm{~mm} \mathrm{mrad}(\mathrm{RMS})$ \\
\hline Slice Energy Spread & Undulator Entrance & $1.5 \mathrm{~mm} \mathrm{mrad}(\mathrm{RMS})$ \\
\hline & Injector $(@ 150 \mathrm{MeV})$ & $0.01 \%(\mathrm{RMS})$ \\
\hline Projected Energy Spread & Undulator Entrance & $0.01 \%(\mathrm{RMS})$ \\
\hline
\end{tabular}

In particular for the Slice Energy Spread, the goals have been limited to a level that is believed to be measurable even though simulations indicate that smaller levels could be achieved. A summary of Measurement Accuracy Goals and Precision Goals is given in Table $\mathbf{5 . 6}$ and

\section{Table 5.7.}

Table 5.6 Electron Beam Measurement Accuracy Goals for Absolute LCLS Parameters.

\begin{tabular}{llll}
\hline Parameter & Location & Parameter Range & Relative Accuracy \\
\hline Bunch Charge & Inj., DL2 and Dump & $0.1-1.0 \mathrm{nC}$ & $1 \%$ \\
\hline Bunch Length & After BC2 $(\sim 5 \mathrm{GeV})$ & $20-40 \mu \mathrm{m}$ & $10 \%$ \\
\hline Projected Rel. Energy Spread & DL2 $(14.35 \mathrm{GeV})$ & $0.02-0.1 \%$ & $20 \%$ \\
\hline 'Slice' Rel. Energy Spread & DL2 $(14.35 \mathrm{GeV})$ & $0.01 \%$ or larger & $30 \%$ \\
\hline Projected Emittance & Inj., BC1, BC2, DL2 & $0.3-3 \mathrm{~mm} \mathrm{mrad}$ & $20 \%$ \\
\hline 'Slice' Emittance & After BC2 & $0.2-2 \mathrm{~mm} \mathrm{mrad}$ & $30 \%$ \\
\hline Electron Beam Energy & DL2 or Dump & $4.5-15 \mathrm{GeV}$ & $2 \%$ \\
\hline
\end{tabular}

Table 5.7 Shot-to-Shot Precision Goals for LCLS Beam Parameters.

\begin{tabular}{llll}
\hline Parameter & Location & Parameter Range & Relative Precision \\
\hline Bunch Charge & Inj., DL2 and Dump & $0.1-1.0 \mathrm{nC}$ & $\sim 0.1 \%$ \\
\hline Bunch Length & After BC2 $(\sim 5 \mathrm{GeV})$ & $20-40 \mu \mathrm{m}$ & $\sim 5 \%$ \\
\hline Projected Rel. Energy Spread & DL2 $(14.35 \mathrm{GeV})$ & $0.02-0.1 \%$ & $\sim 5 \%$ \\
\hline 'Slice' Rel. Energy Spread & DL2 $(14.35 \mathrm{GeV})$ & $0.01 \%$ or larger & $\sim 10 \%$ \\
\hline Projected Emittance & Inj., BC1, BC2, DL2 & $0.3-3 \mathrm{~mm} \mathrm{mrad}$ & $\sim 10 \%$ \\
\hline 'Slice' Emittance & After BC2 & $0.2-2 \mathrm{~mm} \mathrm{mrad}$ & $\sim 15 \%$ \\
\hline Electron Beam Energy & DL2 or Dump & $4.5-15 \mathrm{GeV}$ & $7 \times 10^{-3} \%$ \\
\hline
\end{tabular}




\begin{tabular}{llll}
\hline Parameter & Location & Parameter Range & Relative Precision \\
\hline Pulse Arrival Time & Undulator & - & $50 \mathrm{fs}$ \\
\hline
\end{tabular}

\subsubsection{Pulse-To-Pulse Intensity}

In addition to the intensity fluctuations produced by the statistical nature of the SASE process, about $6 \%$ for the LCLS case as described in Chapter 4, there will be intensity jitter in the x-ray radiation due to intensity jitter of electron beam parameters, i.e. random changes from shot to shot of electron beam charge, current, emittance, energy spread. Charge fluctuations induce correlated changes in the other beam parameters, like emittance and current. However, fluctuations in bunch length and energy spread, not correlated to the charge, are also induced by jitter in the laser pulse arrival time with respect to the linac-rf, and by changes in the longitudinal and transverse charge distribution. These can be produced by changes in the laser pulse profile at the photo-cathode, and by changes in the laser centroid, or by the photoemission process. The effect of changes in the beam parameters affects the radiation intensity in one way if the FEL reaches saturation, and in a stronger way if saturation is not reached.

\subsubsection{Jitter at Saturation}

Table 5.8 and Table 5.9 show the sensitivities of the saturation power and saturation length to various FEL parameters at $1.5 \AA(14.35 \mathrm{GeV})$. They are given in the forms

$$
\frac{\Delta P_{\text {sat }} / P_{\text {sat }}}{\Delta \varepsilon_{n} / \varepsilon_{n}}=-1.5
$$

and

$$
\frac{\Delta L_{s a t} / L_{s a t}}{\Delta \varepsilon_{n} / \varepsilon_{n}}=0.8
$$

which means that increasing the normalized emittance by $10 \%$, i.e., $. \Delta \varepsilon_{n} / \varepsilon_{n}=0.1$, will reduce the saturation power by $15 \%$, i.e., $\Delta P_{\text {sat }} / P_{\text {sat }}=-0.15$.

The last three table rows are of relevance for jitter considerations. Notice the strong sensitivities of the saturation length to peak current and normalized emittance. The first four table rows are relevant for FEL design considerations. One can see that system fluctuations can be easily larger than SASE fluctuations.

Measuring the gain length, whose value depends on the system fluctuations but not on the SASE fluctuations, will give direct information on the effect of system fluctuations on the FEL. Using this information and making statistically significant intensity measurements for welldefined set of beam parameters, one will be able to separate the system and SASE fluctuations and monitor the intensity at each shot. 
Table 5.8 LCLS sensitivities to input parameters at $1.5 \AA$.

\begin{tabular}{|c|c|}
\hline$\frac{\Delta P_{\text {sat }} / P_{\text {sat }}}{\Delta \lambda_{\gamma} / \lambda_{\gamma}}=0.56 \quad\left(\right.$ variable $\left.\gamma_{r}\right)$ & $\frac{\Delta L_{s a t} / L_{s a t}}{\Delta \lambda_{\gamma} / \lambda_{\gamma}}=-0.6 \quad$ (variable $\left.\gamma_{r}\right)$ \\
\hline$\frac{\Delta P_{\text {sat }} / P_{\text {sat }}}{\Delta \lambda_{u} / \lambda_{u}}=3.6 \quad$ (variable $\left.\gamma_{r}\right)$ & $\frac{\Delta L_{\text {sat }} / L_{\text {sat }}}{\Delta \lambda_{u} / \lambda_{u}}=0.3 \quad$ (variable $\left.\gamma_{r}\right)$ \\
\hline$\frac{\Delta P_{\text {sat }} / P_{\text {sat }}}{\Delta \beta_{x, y} / \beta_{x, y}}=0.4$ & $\frac{\Delta L_{s a t} / L_{s a t}}{\Delta \beta_{x, y} / \beta_{x, y}}=0.0$ \\
\hline$\frac{\Delta P_{\text {sat }} / P_{\text {sat }}}{\Delta \gamma / \gamma}=1.9 \quad$ (variable gap) & $\frac{\Delta L_{\text {sat }} / L_{\text {sat }}}{\Delta \gamma / \gamma}=-0.3 \quad$ (variable gap) \\
\hline$\frac{\Delta P_{\text {sat }} / P_{\text {sat }}}{\Delta \sigma_{\gamma} / \sigma_{\gamma}}=-0.1$ & $\frac{\Delta L_{\text {sat }} / L_{\text {sat }}}{\Delta \sigma_{\gamma} / \sigma_{\gamma}}=0.0$ \\
\hline$\frac{\Delta P_{\text {sat }} / P_{\text {sat }}}{\Delta \varepsilon_{n} / \varepsilon_{n}}=-1.5$ & $\frac{\Delta L_{\text {sat }} / L_{\text {sat }}}{\Delta \varepsilon_{n} / \varepsilon_{n}}=0.8$ \\
\hline$\frac{\Delta P_{s a t} / P_{s a t}}{\Delta I_{p l} / I_{p k}}=1.8$ & $\frac{\Delta L_{s a t} / L_{s a t}}{\Delta I_{p l} / I_{p k}}=-0.5$ \\
\hline
\end{tabular}

Table 5.9 LCLS sensitivities to input parameters at $15 \AA$.

\begin{tabular}{lll}
\hline$\frac{\Delta P_{\text {sat }} / P_{\text {sat }}}{\Delta \lambda_{\gamma} / \lambda_{\gamma}}=0.0 \quad\left(\right.$ variable $\left.\gamma_{r}\right)$ & $\frac{\Delta L_{\text {sat }} / L_{\text {sat }}}{\Delta \lambda_{\gamma} / \lambda_{\gamma}}=-0.4 \quad$ (variable $\left.\gamma_{r}\right)$ \\
\hline$\frac{\Delta P_{\text {sat }} / P_{\text {sat }}}{\Delta \lambda_{u} / \lambda_{u}}=2.8 \quad\left(\right.$ variable $\left.\gamma_{r}\right)$ & $\frac{\Delta L_{\text {sat }} / L_{\text {sat }}}{\Delta \lambda_{u} / \lambda_{u}}=0.6 \quad$ (variable $\left.\gamma_{r}\right)$ \\
\hline$\frac{\Delta P_{\text {sat }} / P_{\text {sat }}}{\Delta \beta_{x, y} / \beta_{x, y}}=-0.2$ & $\frac{\Delta L_{\text {sat }} / L_{\text {sat }}}{\Delta \beta_{x, y} / \beta_{x, y}}=-0.2$ \\
\hline$\frac{\Delta P_{\text {sat }} / P_{\text {sat }}}{\Delta \gamma / \gamma}=1.2$ & (variable gap) & $\frac{\Delta L_{\text {sat }} / L_{\text {sat }}}{\Delta \gamma / \gamma}=0.0 \quad$ (variable gap) \\
\hline$\frac{\Delta P_{\text {sat }} / P_{\text {sat }}}{\Delta \sigma_{\gamma} / \sigma_{\gamma}}=-0.2$ & $\frac{\Delta L_{\text {sat }} / L_{\text {sat }}}{\Delta \sigma_{\gamma} / \sigma_{\gamma}}=0.0$ & \\
\hline$\frac{\Delta P_{\text {sat }} / P_{\text {sat }}}{\Delta \varepsilon_{n} / \varepsilon_{n}}=-1.5$ & $\frac{\Delta L_{\text {sat }} / L_{\text {sat }}}{\Delta \varepsilon_{n} / \varepsilon_{n}}=0.8$ & \\
\hline
\end{tabular}




$$
\frac{\Delta P_{s a t} / P_{s a t}}{\Delta I_{p l} / I_{p k}}=1.8 \quad \frac{\Delta L_{s a t} / L_{s a t}}{\Delta I_{p l} / I_{p k}}=-0.5
$$

\subsubsection{Jitter in the Exponential Gain Regime}

The undulator has been designed to be not significantly longer than the expected saturation length at the goal parameters. At the highest energy electron beam energy (corresponding to the shortest radiation wavelength) a deviation from the goal parameters, especially a reduction in peak current and an increase in electron beam emittance, will move the saturation point beyond the end of the undulator. The FEL output will then be determined by the exponential gain regime, resulting in much higher pulse-to-pulse variations.

According to 1-D FEL theory, the derivative of the peak power with respect to the peak current in the exponential gain regime.

$$
\frac{d P_{s a t}}{d I_{p k}}=\left(1+\frac{z}{3 L_{G}}\right) \frac{P_{s a t}}{I_{p k}}
$$

The sensitivities as defined in the previous section can be calculated to

$$
\frac{\Delta P_{z} / P_{z}}{\Delta I_{p k} / I_{p k}}=1+\frac{1}{3} \frac{z}{L_{G}}
$$

which can be a large increase compared to the saturation point, which gives

$$
\frac{\Delta P_{s a t} / P_{s a t}}{\Delta I_{p k} / I_{p k}}=\frac{4}{3}
$$

when making the equivalent calculations. In general, in the exponential gain regime, the sensitivity to fluctuations in peak current increases, as do the sensitivities to fluctuations in normalized emittance and energy spread. The relative sensitivities as obtained from GINGER simulations at $1.5 \AA$ are shown in Table 5.10.

Table 5.10 Sensitivities of LCLS performance to electron beam parameters at the end of the $121 \mathrm{~m}$ long undulator in the exponential gain regime (at $z / L_{G} \approx 11.3$ for $I_{p k}=1500 \mathrm{~A}$ ) and at saturation $\left(I_{p k}==3400 \mathrm{~A}\right)$.

\begin{tabular}{ll}
\hline Sensitivity at $\mathbf{I}=\mathbf{3 4 0 0} \mathbf{A}$ & Sensitivity at $\mathbf{I}=\mathbf{1 5 0 0} \mathbf{A}$ \\
\hline Saturation & Exponential Gain Regime \\
\hline$\frac{\Delta P / P}{\Delta I_{p k} / I_{p k}}=1.8$ & $\frac{\Delta P / P}{\Delta I_{p k} / I_{p k}}=6.4$ \\
\hline$\frac{\Delta P / P}{\Delta \varepsilon_{n} / \varepsilon_{n}}=-1.5$ & $\frac{\Delta P / P}{\Delta \varepsilon_{n} / \varepsilon_{n}}=-9.3$ \\
\hline
\end{tabular}




$$
\frac{\Delta P / P}{\Delta \sigma_{\gamma} / \sigma_{\gamma}}=0.2 \quad \frac{\Delta P / P}{\Delta \sigma_{\gamma} / \sigma_{\gamma}}=-1.8
$$

The numbers in Table 5.10 that are based on simulations are larger than those predicted by the 1-D formula, i.e., 1.3 compared to 1.8 at saturation and 4.8 compared to 6.4 at $z / L_{G} \approx 11.3$ for the sensitivity of peak electron beam current on peak x-ray radiation power. With the expected pulse-to-pulse jitter of the electron beam that as provided by the linac, i.e., $\Delta I_{p k} / I_{p k} \approx 10-20 \%$, $\Delta \varepsilon_{n} / \varepsilon_{n} \approx 5 \%, \Delta \sigma_{\gamma} / \sigma_{\gamma}<10^{-5}$, the x-ray power jitter will be large when operating at saturation but will be unacceptable when operating in the exponential gain regime. The peak current therefore may not be a suitable variable for controlling FEL output power. The implications of Table 5.10 are that the output power at saturation is expected to fluctuate by $20-36 \%$, due mostly to fluctuations in peak current. This fluctuation adds (quadratically) to the natural fluctuations of the SASE process (about 6\%; see Chapter 4).

\subsubsection{Control of X-Ray Power Levels}

For applications that use the x-rays, produced by the FEL, it is important that the output power levels be controllable (see Chapter 9). The feasibility of changing the output power by varying the peak current was studied.

By changing peak current, either by reducing the amount of charge per pulse or by increasing the pulse length, one can control (reduce) the FEL production over many orders of magnitude. Unfortunately, as the explained in the previous section this action not only reduces the saturation power, but it also increases the saturation length, which undesirably increases pulse-to-pulse jitter.

The conclusion is that the method is not promising. For this reason, a gas absorption cell after the FEL undulator will be used for this purpose. The device is described in detail in Chapter 9. 


\subsection{The Temporal Structure of the X-Ray Pulse}

Figure 5.17 shows the simulation results obtained with the time-dependent computer code GINGER. Self-Amplified Spontaneous Emission [21,22] relies on longitudinal electron density fluctuations (shot-noise bunching). Regions where the initial bunching is larger produce more radiation, thus accelerating the lasing process. Due to slippage during the transport through the undulator, those regions will expand to build spikes on the scale of $2 \pi L_{c}=4 \pi L_{G} \lambda_{r} / \lambda_{u}$ [23] as described in Chapter 4. The time-dependent simulations clearly show this phenomenon. For the LCLS, the spike structure length is of the order of $0.3 \mu \mathrm{m}$ at $1.5 \AA$ wavelength and $5 \mu \mathrm{m}$ at $15 \AA$.

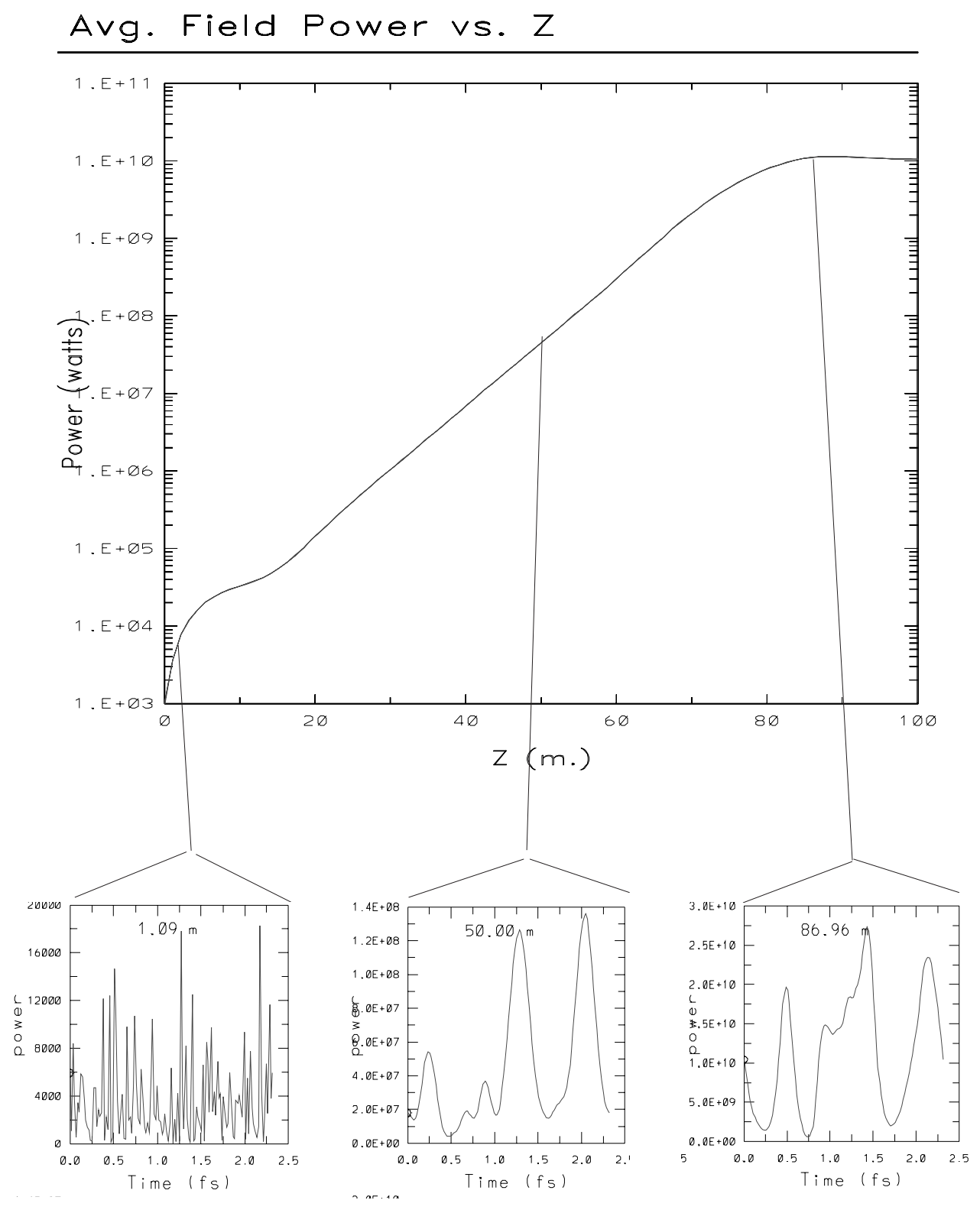

Figure 5.17 FEL output power pattern along the bunch for different position along the gain process. 


\subsection{LCLS FEL Commissioning}

\subsubsection{Procedural Aspects of the FEL Gain Commissioning}

The commissioning phase of the LCLS will include the following steps:

a. Measurement of the electron beam properties at the undulator entrance as a function of charge and compression.

b. Propagation of the electron beam through the undulator, alignment of the beam and measurement of its transverse distribution at a sufficient number of stations.

c. Measurement of the x-ray radiation intensity, line width, and angular distribution as a function of electron beam parameters to determine FEL gain, intensity fluctuations, spectral and coherence properties and compare them to the theoretical expectations. These measurements can be done at the undulator exit and at several stations along the undulator.

During commissioning and operation, it will be important to monitor the electron beam and $\mathrm{x}$-ray characteristics at each pulse. This is necessary in order to separate the FEL intensity fluctuations due to the SASE start-up from noise - of the order of about $6 \%$ for the reference LCLS case - from those due to system fluctuations in the drive laser- electron source-linac system, which can be much larger.

After commissioning is complete, during the LCLS operation, there will still be a need to monitor the electron beam and x-ray characteristic on a pulse-by-pulse basis to provide reference information to the user experiments for data reduction. If this reference information is based on electron beam parameters, the precision will be limited to that of the FEL intensity fluctuation. Monitoring pulse-by-pulse x-ray intensity directly will provide reference information with a higher level of precision.

The electron beam and radiation quantities to be measured for the commission of the LCLS and for a comparison of the FEL properties with theory are:

a. Electron bunch charge

b. Electron bunch center of mass position along the undulator

c. Electron bunch transverse distribution throughout the undulator (as a function of charge)

d. Electron bunch longitudinal distribution as well as integrated and slice energy spread (as a function of charge)

e. X-ray intensity within a defined solid angle and line width as function of electron bunch charge (These measurements can be done at the undulator exit and at several stations along the undulator.) 


\subsection{Summary}

The operating parameters have been optimized by an analysis a three-dimensional algorithm and by computer simulations. The results of the study are that the FEL design objectives are reachable with a 121-m long undulator, and with the beam characteristics given in Chapter 2,

Table 2.4-1, and in Appendix A (parameter list). A study of the effect of the electron beam optics on the FEL performance led to the choice of the FODO lattice cell length and the quadrupole strength. The sensitivity of the FEL performance to the main undulator and electron beam parameters was studied, and from this, tolerances for the pole-to-pole magnetic field variations and for the electron beam characteristics were derived.

\subsection{References}

1 M. Xie, "Design Optimization for an X-ray Free Electron Laser Driven by SLAC Linac," LBL Preprint No-36038, 1995, also, IEEE Proceedings for Pac95, No. 95CH3584, 183, 1996.

2 K.-J. Kim, "Three-dimensional analysis of coherent amplification and self-amplified spontaneous emission in free electron lasers," Phys. Rev. Lett., 57, p.1871, 1986.

3 Y.H. Chin, K.-J. Kim, M. Xie, "Three-Dimensional Free Electron Laser Theory Including Betatron Oscillations," LBL-32329, May 1992 49pp and Phys. Rev, A46, 6662 (1992).

4 L.H. Yu, S. Krinsky, R.L. Gluckstern, "Calculation of Universal Function for Free-Electron Laser Gain," Phys. Rev. Lett. 64, 3011 (1990).

5 L.H. Yu, S. Krinsky, R.L. Gluckstern, J.B.J. van Zeijts, "Effect of wiggler errors on free-electronlaser gain.” Phys. Rev. A45, 1163, 1992

6 H.-D. Nuhn, "Overview of SASE Free-Electron Laser Simulations Codes." in Proc. International Society for Optical Engineering, Free Electron Laser Challenges II, San Jose, CA, Jan 23-27, 1999, (SPIE v. 3614) pp. 119-130.

7 Proceedings of the X-Ray FEL Theory and Simulation Codes Workshop. H.-D. Nuhn and C. Pellegrini (edts.), SLAC, September 23-24, 1999.LCLS-TN-00-1. SLAC-WP-17. (2000).

$8 \quad$ S. Reiche, Nucl. Instr.. Meth. $\underline{\mathbf{A 4 2 9}}, 243$ (1999).

9 W.M. Fawley, Report LBNL-49625 (2002).

10 E.T. Scharlemann, "Wiggle plane focusing in linear undulators," J. Appl. Phys., 58(6), pp. 21542161, 1985.

11 R.J. Dejus, O.A. Shevchenko, and N.A. Vinokurov, Nucl. Instr.. Meth. A 429 (1999) 225.

12 R.J. Dejus, O.A. Shevchenko, and N.A. Vinokurov, Nucl. Instr.. Meth. A 445 (2000) 19.

13 S.G. Biedron, Y.C. Chae, R.J. Dejus, B. Faatz, H.P. Freund, S.V. Milton, H.-D. Nuhn, and S. Reiche, "The APS SASE FEL: Modeling and Code Comparison," in Proceedings of the 1999 Particle Accelerator Conference (PAC99), New York City, NY, USA, 1999. Nucl. Instrum. Meth. A 445, pp. $110-115$ (2000).

14 S. V. Milton et al., "Observation of Self-Amplified Spontaneous Emission and Exponential Growth at 530nm", Phys. Rev. Lett., 85, 988-991 (2000), and Proc. Intern.FEL-2000 Conf., Duke University (2000). 
15 A. Tremaine et al., "Initial Gain Measurements of a $800 \mathrm{~nm}$ SASE-FEL, VISA", in Proc. Intern.FEL2000 Conf., Duke University (2000);

16 M.D. Borland, Y.-C. Chae, J.W. Lewellen, S.V. Milton, R.Soliday, V. Bhradwaj, P. Emma, P. Krejcik, H.-D. Nuhn, W.M. Fawley, "Start-to-End Simulation of SASE FELs from the Gun through the Undulator," in Proceedings of the 23th International Free Electron Laser Conference and 8th Annual FEL User Workshop, Darmstadt, Germany, 20-24 Aug 2001.

17 P. Emma, "Electron Phase Slip in an Undulator with Dipole Field and BPM Errors," LCLS-TN-0014, (October 3, 2000).

18 K.-J. Kim, "Characterization of Synchrotron Radiation," in X-RAY DATA BOOKLET, K. Kirz et. al, ed., April 1986, LBNL PUB-490 Rev.

19 S. Reiche, in Proceedings of the 1997 Workshop, "Towards X-Ray Free Electron Lasers," at Lake Garda, Italy, 1997.

20 P.Emma and R.Brinkmann, "Emittance Dilution Through Coherent Energy Spread Generation in Bending Systems," in Proceedings of the 1997 Particle Accelerator Conference (PAC97), Vancouver, B.C, Canada, SLAC-PUB-7554. May 12-16, 1997.

21 R.Bonifacio, C.Pellegrini, and L.Narducci, "Collective instabilities and high gain regime in a free electron laser," Opt. Commun., 50(6), 1985.

22 J.Murphy and C.Pellegrini, "Generation of high intensity coherent radiation in the soft x-ray and VUV regions," J. Opt. Soc. Am. B2, 259, 1985.

23 R. Bonifacio et. al., "Spectrum, Temporal Structure, and Fluctuations in a High-Gain Free-Electron Laser Starting from Noise," Phys. Rev. Lett., 73(1), p.70, 1994. 


\section{Injector}

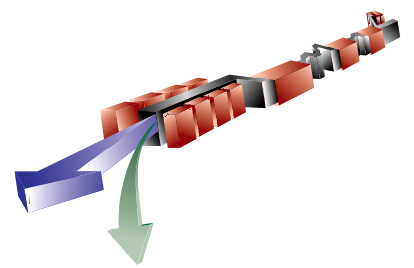

\section{TECHNICAL SYNOPSIS}

The injector for the LCLS is required to produce a single 150-MeV bunch of charge $1.0 \mathrm{nC}$ and $100 \mathrm{~A}$ peak current at a repetition rate of $120 \mathrm{~Hz}$ with a normalized rms transverse emittance of $1.0 \mu \mathrm{m}$. The required emittance is about a factor of 2 lower than has been achieved to date. The design employs a solenoidal field near the cathode of a specially designed rf photocathode gun that allows the initial emittance growth due to space charge to be almost completely compensated by the end of the booster linac. Following the booster linac, the geometric emittance simply damps linearly with energy. PARMELA simulations show that this design will produce the desired normalized emittance.

In addition to low emittance, there are two additional electron-beam requirements that pose a challenge: the timing and intensity jitters must have an rms value of $\leq 0.9 \mathrm{ps}$ and $\leq 2 \%$ respectively. For an rf photoinjector, these parameters are determined principally by the laser system. Commercial laser oscillators are available with a timing stability of 0.5 ps. The laser system described here uses feedback loops to maintain this stability in the amplification and pulse shaping stages. The desired laser-pulse energy tolerance is achieved by stabilizing the pumping laser for the amplifiers and by operating the second amplifier in saturation. RF systems with a phase stability of 0.5 ps are already routine for the SLAC linac.

Although additional $R \& D$ is in progress to ensure the performance of the photoinjector as planned, confidence in the present design is based on the performance of existing systems and projected improvement based on multi-particle code simulations. Simulations using these same codes match the measured performance of rf photoinjectors operating near the emittance level desired. Laser systems have been employed in high energy physics experiments with timing stability - with respect to the accelerated electron beam - that is close to the value required.

The injector is divided geographically between the electron source-consisting of an rf gun and laser system - the booster linac, and the Matching Section. However, to produce the minimal transverse emittance at high energy, the photoinjector must be treated as one unit. 


\subsection{Introduction}

Unlike light sources based on storage rings for which the beam properties of the stored beam due to synchrotron radiation are almost entirely decoupled from the properties of the injector beam, the performance of a linac-based Free Electron Laser (FEL) is directly dependent on the quality of the electron drive beam as produced by the injector and preserved by the main linac. As discussed in Chapter 4, FEL Physics, the optimum phase-space matching between the FEL electrons and photons is achieved if the electron beam emittance is $<\lambda / 4 \pi$, where $\lambda$ is the wavelength of the FEL radiation. A somewhat higher emittance can be accommodated by increasing the length of the undulator. The proposed Linac Coherent Light Source (LCLS) is an $\mathrm{X}$-ray FEL that will use the final third of the SLAC 3-km linac for the electron drive beam. The performance of the LCLS in the 1.5- $\AA$ regime is predicated on the availability of a 1-nC, 100-A beam at the $150-\mathrm{MeV}$ point with normalized rms transverse emittance of $1 \mu \mathrm{m}$. With this emittance, an undulator length of $\sim 100 \mathrm{~m}$ is required. An experimental program is underway at the Gun Test Facility (GTF) at SLAC to demonstrate a high-brightness beam meeting the LCLS requirements [1]. The GTF experiment uses a 1.6-cell S-band rf gun developed jointly with BNL and UCLA [2]. The gun exit is surrounded by a solenoid. After a short drift there is a standard SLAC 3-m accelerating section. At BNL using a similar configuration, a transverse normalized rms emittance of $2.4 \mu \mathrm{m}$ for a $0.9 \mathrm{nC}$ pulse with 10-ps FWHM Gaussian pulse length has been measured [3]. Simulations indicate that a factor of 2 decrease in emittance is expected if the temporal pulse shape is uniform rather than Gaussian. Unfortunately, all experiments to date have used the natural temporal pulse shape of the source laser, which generally approximates a Gaussian distribution.

Earlier simulation studies using the multi-particle code PARMELA predicted a transverse normalized emittance of $1 \mu \mathrm{m}$ - thermal emittance not included - for the LCLS photoinjector if a uniform (or even a truncated Gaussian) temporal charge distribution were used [4] as well as a uniform transverse distribution. While this result technically meets the LCLS requirements, it leaves no headroom for errors or practical difficulties. Consequently, simulation studies have continued with the goal of finding a photoinjector design for the LCLS that predicts a transverse emittance of no more than $0.8 \mu \mathrm{m}$ with the thermal emittance included.

Following the introduction and survey of experimental results, the chapter is divided into 4 major sections. The $\mathrm{rf}$ photocathode gun is described in some detail although a complete conceptual design does not yet exist. This is followed by a thorough explication of the Ti:sapphire drive laser system and then the proposed injector layout with instrumentation indicated. The final section is devoted to simulations.

\subsubsection{Beam Requirements}

The LCLS injector is required to produce a single bunch with nominal charge, $Q$, of $1 \mathrm{nC}$ at a repetition rate of $120 \mathrm{~Hz}$. After acceleration to $150 \mathrm{MeV}$, the bunch is required to have a peak current of at least $100 \mathrm{~A}$ and an integrated normalized rms emittance of $\leq 1 \mu \mathrm{m}$. These parameters 
correspond to those predicted for an optimized S-band photoinjector using emittance compensation and assuming an electron bunch with initial uniform spatial and temporal charge distributions with $\sigma_{\mathrm{r}}$ and $\sigma_{\mathrm{z}}$ of $0.71 \mathrm{~mm} \mathrm{rms}$ and $2.9 \mathrm{ps} \mathrm{rms}$ respectively. The photoinjector is actually designed to allow optimized performance with bunch charge as low as $0.2 \mathrm{nC}$. Given an optimized injector design for $1 \mathrm{nC}$, as the charge is lowered the emittance will remain optimized if the charge density is held constant by scaling each dimension of the bunch as $Q^{1 / 3}$. Thus bunches with $\sigma_{z}$ as short as 2 ps may be needed. Then, if the effects of thermal emittance are ignored, the optimized integrated emittance is expected to scale as $Q^{2 / 3}$ [5].

A low energy spread at $150 \mathrm{MeV}$ is also required. An acceptable value of $\sigma_{\gamma} / \gamma_{o} \leq 0.1 \%$ will be achieved for the integrated bunch by adjusting rf phases in the booster linac. An electron bunch can be analyzed in terms of axial slices. Unless otherwise specified, the thickness of a slice is some minor fraction of the total longitudinal width of the bunch. While the slice energy spread is important, it cannot be readily adjusted. A value of $\sigma_{\gamma}^{\text {slice }} / \gamma_{o} \leq 0.02 \%$ at $150 \mathrm{MeV}$ is desired.

In addition to low emittance and energy spread, there are two additional challenging requirements that are derived from the sensitivity of the current and energy jitters in the undulator to the charge and timing jitters at the gun. The first of these challenges concerns charge jitter at the gun. Simulations indicate that the rms peak current jitter in the undulator-where rms values are measured over a few seconds with the LCLS operating at $120 \mathrm{~Hz}$-will be $12 \%$ for an rms charge jitter at the gun of $6 \%$. (See Table 7.4.) However, when all sources of current jitter are taken into account, the rms charge jitter at the gun must be reduced to the order of about $2 \%$ to maintain the $12 \% \mathrm{rms}$ peak current jitter at the undulator. (See Table 7.5.) Since the goal is to keep the rms peak current jitter in the undulator under $12 \%$, a criterion of $\leq 2 \%$ has been adopted for the rms charge jitter for the LCLS photoinjector gun. Section 6.4, Laser System, describes how this tolerance will be achieved.

The second challenge concerns the timing jitter of the electron bunch with respect to the rf driving the gun and linac. The timing jitter affects both the peak current and energy in the undulator. To keep the rms peak current jitter in the undulator below $12 \%$, the rms timing jitter need only be less than $4.0 \mathrm{ps}$. However, the rms timing jitter must be less than $1.4 \mathrm{ps}$ to maintain the rms energy jitter in the undulator below the desired $0.1 \%$ (Table 7.4). But again, when the various sources of energy jitter are considered together, the rms timing jitter at the gun must be reduced to the order of 0.9 ps (Table 7.5). In Section 6.4, Laser System, it is shown that a value of $\leq 0.5 \mathrm{ps}$ for the rms timing jitter of the electron bunch with respect to the rf driving the gun and linac should be achievable. Since accomplishing this goal will slightly relieve the charge jitter criterion at the gun, this lower value of timing jitter has been adopted as the criterion for the injector laser system.

These beam requirements are summarized in Table 6.1 below. 
Table 6.1 Summary of Beam Requirements at End of LO.

\begin{tabular}{ll}
\hline Parameter & Value \\
\hline Charge per e bunch, $Q_{0}$ & $1 \mathrm{nC}$ \\
\hline Repetition Rate & $120 \mathrm{~Hz}$ \\
\hline Energy & $150 \mathrm{MeV}$ \\
\hline Peak current & $100 \mathrm{~A}$ \\
\hline Normalized projected transverse emittance, $\varepsilon_{n, r m s}$ & $\leq 1 \mu \mathrm{m}$ \\
\hline Integrated energy spread, $\sigma_{\gamma} / \gamma_{0}$ & $\leq 0.1 \% \mathrm{rms}$ \\
\hline Slice energy spread, $\sigma_{\gamma}^{\text {slice } / \gamma_{0}}$ & $\leq 0.02 \% \mathrm{rms}$ \\
\hline Timing jitter with respect to rf, $\Delta t_{0}$ & $\leq 0.9 \mathrm{ps} \mathrm{rms}$ \\
\hline Charge jitter, $\Delta Q / Q_{0}$ & $\leq 2.0 \% \mathrm{rms}$ \\
\hline Bunch length jitter, $\Delta l_{0} / l_{0}$ & $\leq 5 \% \mathrm{rms}$ \\
\hline
\end{tabular}

\subsubsection{Emittance Compensation}

The theory of linear emittance compensation in a high brightness rf photoinjector is well established [6,7]. It has been shown that the optimization of an rf photo-injector corresponds to accelerating and propagating the beam through the device as close as possible to two beam equilibria: a laminar Brillouin flow in drifts, and the so-called Invariant Envelope (IE) in accelerating sections. The IE is a generalization of Brillouin flow for an accelerated beam when rf focusing effects and spot-size adiabatic damping are taken into account. In the space-charge dominated regime, i.e., when the space-charge collective force is largely dominant over the emittance pressure, the bunch behaves as a laminar flow and can be represented in a simple model as a set of slices, each one described by an envelope equation that includes the local slice space-charge field. Mismatches between the space-charge correlated forces and the external focusing gradient produce slice envelope oscillations whose plasma frequencies can be computed from the model. The result is that plasma frequencies are independent of the slice currents to first order, i. e., all the slices have the same plasma frequency although the slice current affects the amplitude of each oscillation. This frequency independence results in reversible normalized emittance oscillations: a minimum emittance is correlated with an integral number of plasma oscillations for which the slices are periodically aligned in transverse phase space, and accelerating the beam through the IE damps these oscillations as the square root of the beam 
energy. Provided the oscillations are properly tuned, the normalized emittance reduces to a steady state minimum at the injector exit.

As a consequence of such a theory, the definition of the injector region has to be extended up to an energy high enough to exceed the laminar regime. The beam then enters the so-called emittance-dominated regime, where trajectory crossovers dominate over space-charge oscillations, and, in an ideal accelerator, the total normalized emittance remains constant.

The laminar regime extends up to an energy, $\gamma$, given by [7]:

$$
\gamma=\sqrt{\frac{2}{3}} \frac{\hat{I}}{I_{0} \varepsilon_{t h} \gamma^{\prime}}
$$

where $\varepsilon_{t h}$ is the thermal emittance, $I_{o}=17 \mathrm{kA}$ is the Alfvén current, $\hat{I}$ the peak current. The derivative of $\gamma$ with respect to $z$ is given by $\gamma^{\prime}=e E_{a c c} / m_{e} c^{2}$, where $E_{a c c}$ is the accelerating field.

With the expected LCLS parameters: $\hat{I}=100 \mathrm{~A}, E_{a c c}=25 \mathrm{MV} / \mathrm{m}$ and an estimated $\varepsilon_{\text {th }}$ of $0.3 \mu \mathrm{m}$ for a $\mathrm{Cu}$ cathode with $\mathrm{UV}$ excitation [8], the transition occurs at about $150 \mathrm{MeV}$. For this reason the emittance compensation process of the LCLS injector has to be optimized up to the exit of the booster linac, before injecting the beam in the main linac.

It follows from the discussion above that the basic point in the design of a photoinjector is to match properly the beam from the gun to the booster. This is done for a space-charge dominated beam falling within the paraxial limit if the following IE conditions [7] are met: first that the derivative, $\sigma^{\prime}$, of the rms transverse spot size,) $\sigma$, with respect to the independent variable $z$ is such that

$$
\sigma^{\prime}=0,
$$

implying a laminar waist governs at the booster entrance; and second that

$$
\gamma^{\prime}=\frac{2}{\sigma} \sqrt{\frac{\hat{I}}{2 I_{0} \gamma}},
$$

which gives the matched accelerating field for a traveling wave (TW) structure.

To optimize the photoinjector design, the semi-analytical code HOMDYN [9] was chosen to investigate the booster matching condition, taking advantage of the fast running capability of the code to explore a wide range of parameters. The next section describes how HOMDYN was used to find the optimal conceptual design for the injector, while the more tedious but also more detailed physical optimization using the tracking code PARMELA is described in Section 6.6, PARMELA Simulations.

\subsubsection{Design Principles}

The discussion here assumes the 1.6-cell S-band $\mathrm{rf}$ gun developed jointly with BNL and UCLA [2] surrounded by a solenoid just after the gun exit. After a drift, the gun is followed by two standard SLAC 3-m traveling wave (TW) accelerating sections. As discussed in reference 
[10], for a 1-nC uniform-charge distribution and a 10-ps long bunch with 1-mm hard-edge radius, good emittance performance and high peak current at the exit of the gun can be obtained with a peak field on the cathode of about $140 \mathrm{MV} / \mathrm{m}$, an injection phase (with respect to the rf zero crossing) on the order of $35^{\circ}$, and a moderate solenoid field strength of about $3.0 \mathrm{kG}$. Earlier design studies for the LCLS injector made use of a low-gradient booster as was then the standard [4]. In the following analysis, these parameters, which in this context will be called the standard or old working point, are taken as the starting condition for a new parameter-space search.

When observing envelope and emittance behavior while scanning the gun solenoid field strength using HOMDYN, an interesting feature can be seen that appears to be a very effective new working point for a split (gun separate from booster) rf photoinjector [11]. By increasing the solenoid strength, the emittance evolution shows a double minimum behavior in the drift region following the gun. For a unique value of the solenoid strength ( $3.1 \mathrm{kG}$ in this case) the envelope waist occurs where the emittance has its relative maximum ( $z \approx 1.5 \mathrm{~m}$ in this case) as shown by the red (bold) lines in Figs. 6.1 and 6.2. The solenoid value that produces this unique coincidence of waist and emittance relative maximum is a key constituent of the new working point.

The performance using the new working point relies on this feature of the emittance oscillation. If the booster entrance is located at $\mathrm{z} \approx 1.5 \mathrm{~m}$, where the beam laminar waist occurs, and if simultaneously the other IE matching condition is satisfied, i.e., Eq. (6-3), the second emittance minimum can be shifted to higher energy and frozen at a lower level, taking advantage of the additional emittance compensation occurring in the booster.

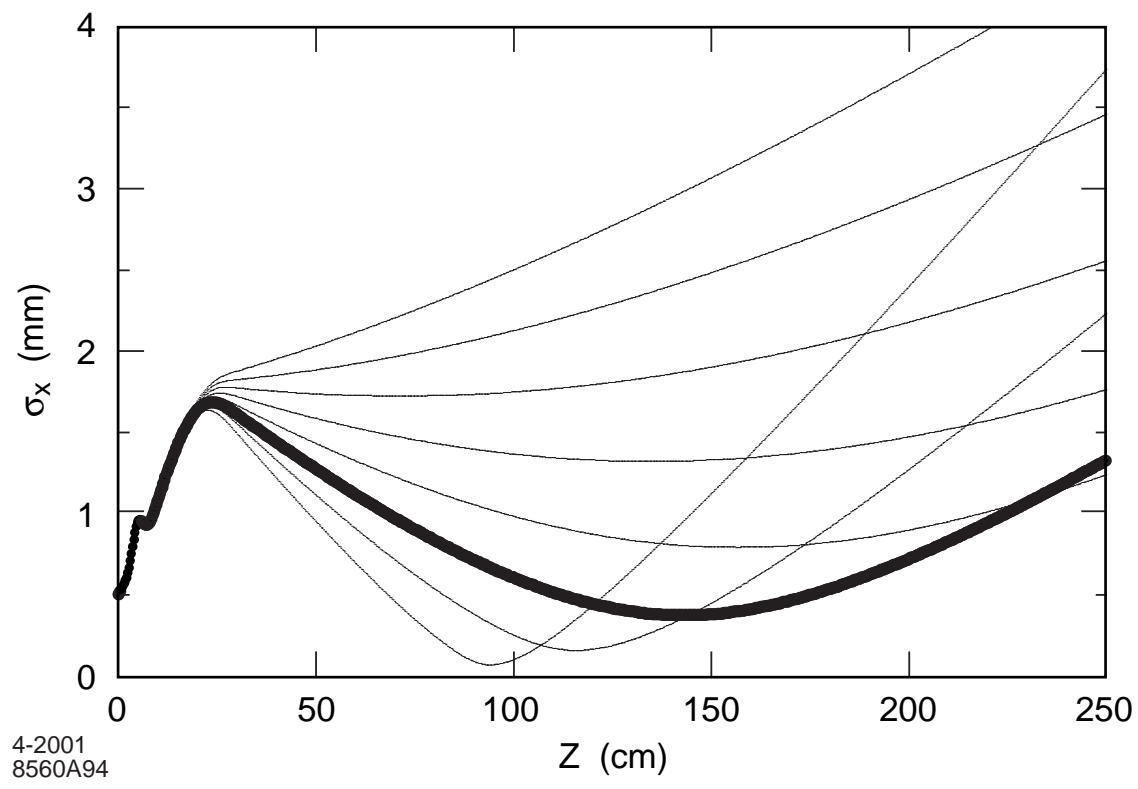

Figure 6.1 Beam envelope versus z. Each curve is for a different solenoid strength, i.e., values between 0.26 and $0.33 \mathrm{~T}$ in equal increments. 


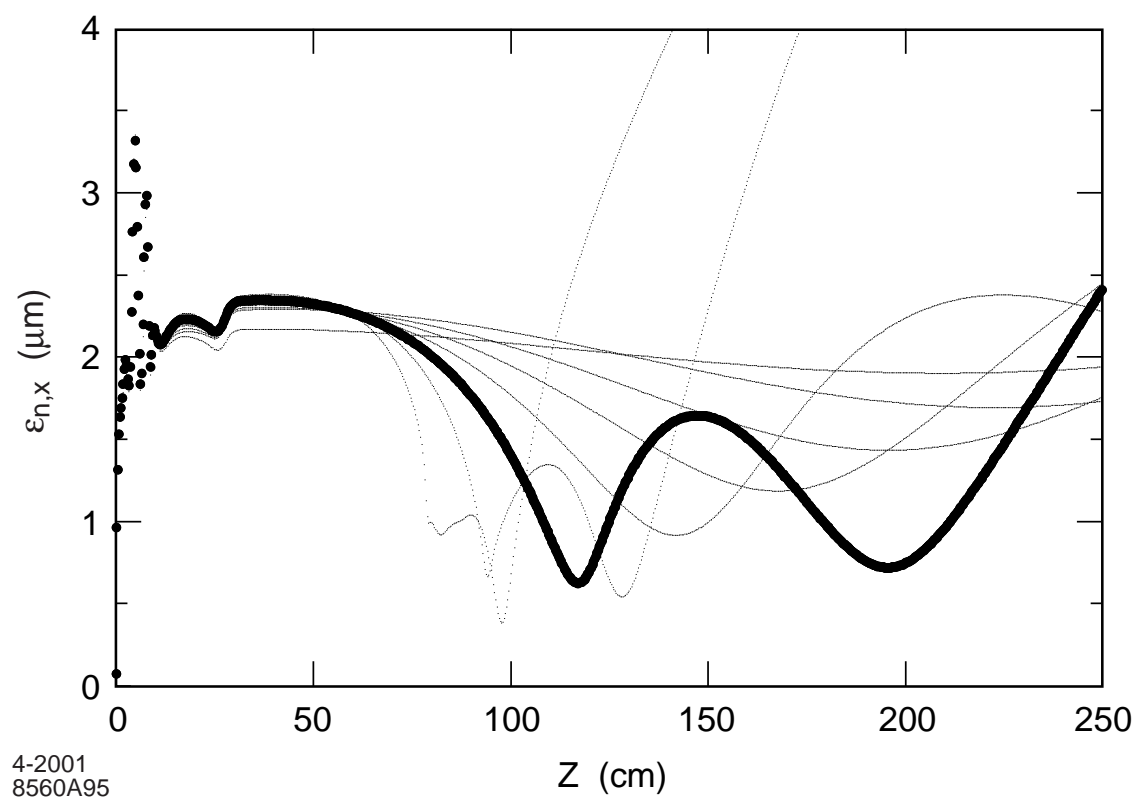

Figure 6.2 Beam emittance versus z. Each curve is for a different solenoid strength, i.e., values between 0.26 and $0.33 \mathrm{~T}$ in equal increments.

Since the minimum rms spot size in Figure 6.1 for the conditions of the new working point is $\sigma=0.41 \mathrm{~mm}$, the current averaged over the slices is $96 \mathrm{~A}$, and the average slice energy is 6.4 $\mathrm{MeV}$, the matched accelerating gradient of the traveling wave (TW) booster is required to be 35 $\mathrm{MV} / \mathrm{m}$. Two SLAC 3-m accelerating structures are required to drive the beam out of the space charge dominated regime, resulting in an energy of $216 \mathrm{MeV}$ in an 8-m long injector line (assuming a $0.5-\mathrm{m}$ long drift in between the two structures and not including the gun structure upstream of the cathode). As expected, the second emittance minimum, which is $0.5 \mu \mathrm{m}$, now occurs downstream of the booster structures, at $\mathrm{z}=10 \mathrm{~m}$. (This location will be taken as a reference position to quote emittance at the injector exit).

Despite the good emittance resulting from this design, the necessary gradient to match the beam to the booster exceeds the limit of reliable performance by available SLAC 3-m structures. One solution is to shift the solenoid location downstream and set the solenoid strength so as to recover the new working point conditions. By doing so the resulting spot size at the waist is bigger and thus, from Eq. (6.3), a lower matched gradient is required.

A lower gradient solution can also be achieved by increasing the focusing properties of the booster [12]. This can be done by means of standing wave (SW) structures or equivalently by a long solenoid around the first TW structure [11]. The second solution is chosen for the LCLS design to simplify the rf system. Setting the desired accelerating field of the TW sections to 26 $\mathrm{MV} / \mathrm{m}$ and scanning the long solenoid strength, a very good working point is indicated for a longitudinal field of $B_{z}=800 \mathrm{G}$. A beam dynamics simulation using HOMDYN indicates a very low emittance value, of $0.2 \mu \mathrm{m}$ (thermal emittance not included) at $160 \mathrm{MeV}$ as shown in Figure 6.3, while at the same time the other relevant LCLS requirements are very nearly fulfilled. For example, the peak current of $95 \mathrm{~A}$ is only slightly below the desired value. The energy spread 
can be minimized by injection into the booster off-crest. However, in this case the optimized phase of $12^{\circ}$ (relative to the crest) results in a residual rms energy spread at the exit of the booster of $0.25 \%$, which is 2.5 times the required value. In any case the final optimization must be done with a multi-particle tracking code.

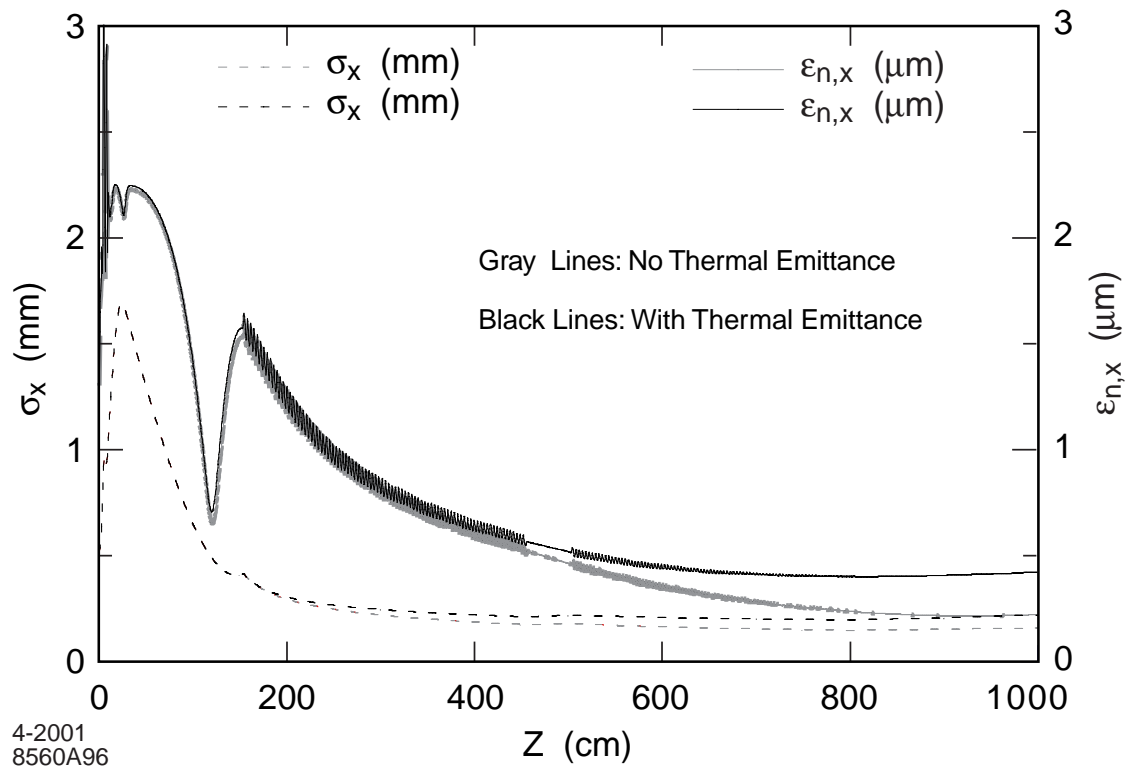

Figure 6.3 Beam envelope and emittance along the injector beamline, without thermal emittance (red/lower lines) and with thermal emittance (black/ upper lines).

In this design the total emittance is limited by the thermal emittance contribution. Figure 6.3 also shows the emittance evolution as computed by including a value of $\varepsilon_{t h}=0.3 \mu \mathrm{m}$, where the strength of the long solenoid has been reduced to $B_{z}=700 \mathrm{G}$ to recover an optimized design. The resulting total emittance is $0.4 \mu \mathrm{m}$.

\subsection{Summary of Experimental Results}

The design of the LCLS rf photocathode gun is based on the 1.6-cell low-emittance S-band rf photocathode gun (herein called the "prototype gun") that was designed by the BNL/SLAC/UCLA rf gun collaboration for x-ray FEL applications [2]. There are at least seven prototype guns in operation including guns at ANL, BNL, LLNL, SLAC, UCLA and the University of Tokyo. These guns all use the 1.6-cell symmetrized S-band design with several variations of mechanical contact and tuning of the cathode plate as well as modifications in the cooling capability due to different cooling channel locations. The design of the gun requires the emittance compensating solenoid to be located very close to the cathode. Several of the guns use a second solenoid behind the cathode to null the magnetic field at the cathode plane, while most use a steel flux return for the single solenoid to limit the field at the cathode to less than $5 \mathrm{G}$. Despite these variations, all of the guns are electrically identical and are all theoretically capable of producing essentially identical beams, assuming identical drive laser pulses are used. All the guns use metal cathodes, either $\mathrm{Cu}$ or $\mathrm{Mg}$. Several of the guns have been operated at peak electric 
fields exceeding $120 \mathrm{MV} / \mathrm{m}$ for limited periods, but to date no gun with a $\mathrm{Mg}$ insert has consistently operated at fields higher than $110 \mathrm{MV} / \mathrm{m}$.

\subsubsection{Quantum Efficiency}

While a metal cathode assures the ability to utilize the high field necessary to produce lowemittance beams, it also necessarily results in a cathode with relatively low quantum efficiency ${ }^{\mathrm{a}}$ since the metal reflects the vast majority of the incident photons. Depending on the cathode history, the QE for a $\mathrm{Cu}$ cathode can vary from a low of roughly $10^{-6}$ to a high of nearly $10^{-4}$. Recent measurements at UCLA on a clean-single crystal $\mathrm{Cu}(100)$ surface (chosen to minimize grain boundary scattering) resulted in a QE of $4.6 \times 10^{-5}$ with $90 \mathrm{MV} / \mathrm{m}$ peak field at the cathode and a $45^{\circ}$ emission phase (measured with respect to the zero field crossing) [13]. The QE for an identical cathode at SLAC was measured to be $3.6 \times 10^{-5}$ with an applied field of $100 \mathrm{MV} / \mathrm{m}$ at a $38^{\circ}$ emission phase [14]. A polycrystalline $\mathrm{Cu}$ cathode at SLAC with $100 \mathrm{MV} / \mathrm{m}$ peak field resulted in a QE of $6.1 \times 10^{-5}$ at $50^{\circ}$ emission phase as shown in Figure 6.4. Scaling these results to the LCLS operating parameters of $140 \mathrm{MV} / \mathrm{m}$ and $32^{\circ}$ phase, one expects a $\mathrm{QE}$ of greater than $7 \times 10^{-5}$. For the purposes of this report, a conservative value of QE of $1 \times 10^{-5}$ is assumed to be readily achievable.

If the cathode is not installed in a clean manner, surface contaminants can lead to significant reduction in the $\mathrm{QE}$ and also to spatial variation in $\mathrm{QE}$. Such variations have been measured at SLAC with a cathode that was not installed in a sufficiently controlled environment [1]. Figure 6.5 (A) shows the measured $\mathrm{QE}$ as a function of position across this cathode. Fluctuations greater than $50 \%$ were measured by scanning a laser beam $1 \mathrm{~mm} \times 0.3 \mathrm{~mm}$ across the cathode at grazing incidence. The average QE was $<1 \times 10^{-5}$. Subsequently the laser intensity was increased and scanned across the cathode again under the explosive emission regime [15]. There was a short term decrease in the QE spatial variation and significant improvement in the average QE as shown in Figure 6.5 (B), but at the expense of a significant increase in the micro-roughening of the cathode surface and the emitted dark current. The improvement in QE was only short lived (weeks) and eventually led to even larger spatial variations than initially measured. Subsequent cathodes installed in more stringently-controlled environments exhibited significantly higher QEs as shown in Figure 6.4 and less spatial variation in the QE as well. Only 10\% rms fluctuation in the QE over a $2.5 \times 2.5 \mathrm{~mm}$ area was measured at the GTF [14] after installing the cathode under a nitrogen environment. Thus for the LCLS gun, a load-lock will be implemented for installing cathodes to insure completely reproducible and controlled environments so that consistently high and relatively uniform QEs can be achieved.

\footnotetext{
${ }^{a}$ Quantum efficiency (QE) is herein defined as the number of electrons that escape to vacuum from the photocathode surface per incident (as opposed to absorbed) photon. A single photon wavelength is always assumed.
} 


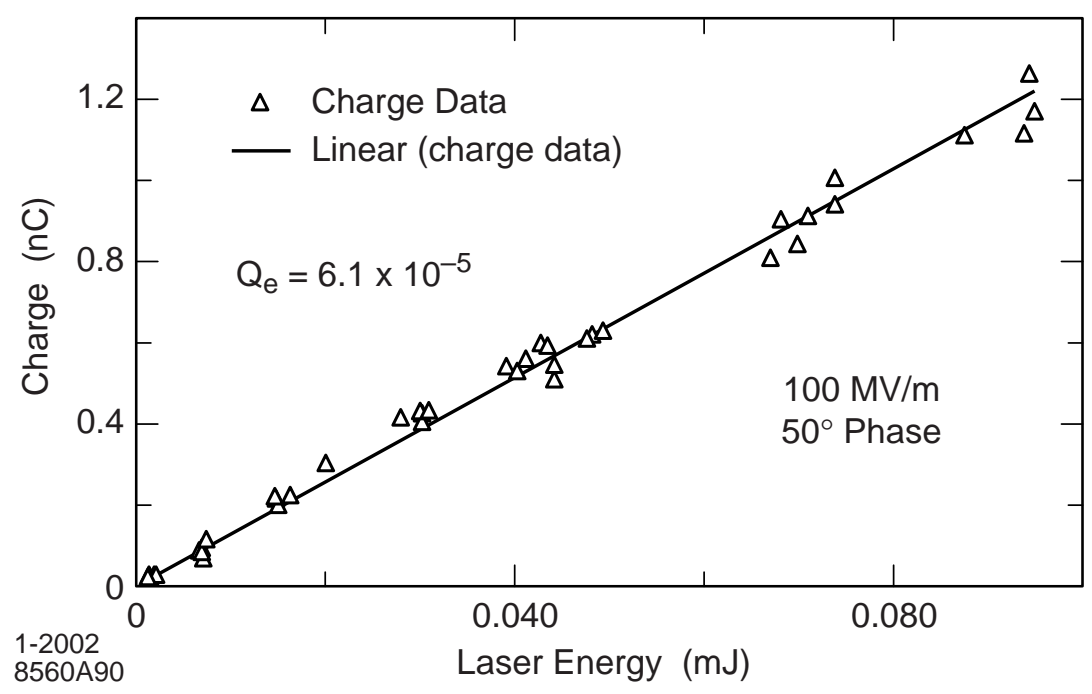

Figure 6.4 The measured charge extracted from a $\mathrm{Cu}$ photocathode as a function of laser energy with $100 \mathrm{MV} / \mathrm{m}$ peak rf field and $50^{\circ}$ laser injection phase.



(A)

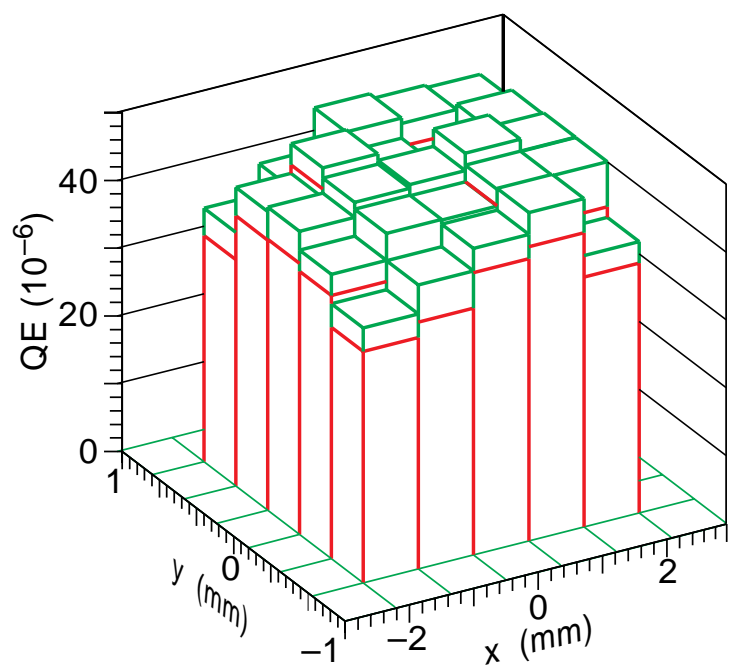

(B)

Figure 6.5 The measured quantum efficiency is plotted for a Cu cathode both before (A) and after (B) laser cleaning. The error in the QE values is indicated by the green slices at the top of each QE column.

\subsubsection{Transverse Emittance Measurements}

The history of the lowest emittance measurements for $1 \mathrm{nC}$ of charge is summarized Figure 6.6. The lowest emittances to date have been achieved with solenoidal emittance compensated photoinjectors with the lowest measured values near $2 \mu \mathrm{m}$ as described below. These measurements are consistent with the predictions of tracking codes like PARMELA if the physical parameters of the experiments are taken into account. PARMELA simulations also indicate that emittances on the order of $1 \mu \mathrm{m}$ should be achievable if the Gaussian temporal 
charge distribution used in these experiments were replaced with one that is uniform, i.e., flattop. In addition, simulations for more recent photoinjector designs (see for example Section 6.6. PARMELA Simulations), now predict an emittance significantly less than $1 \mu \mathrm{m}$.

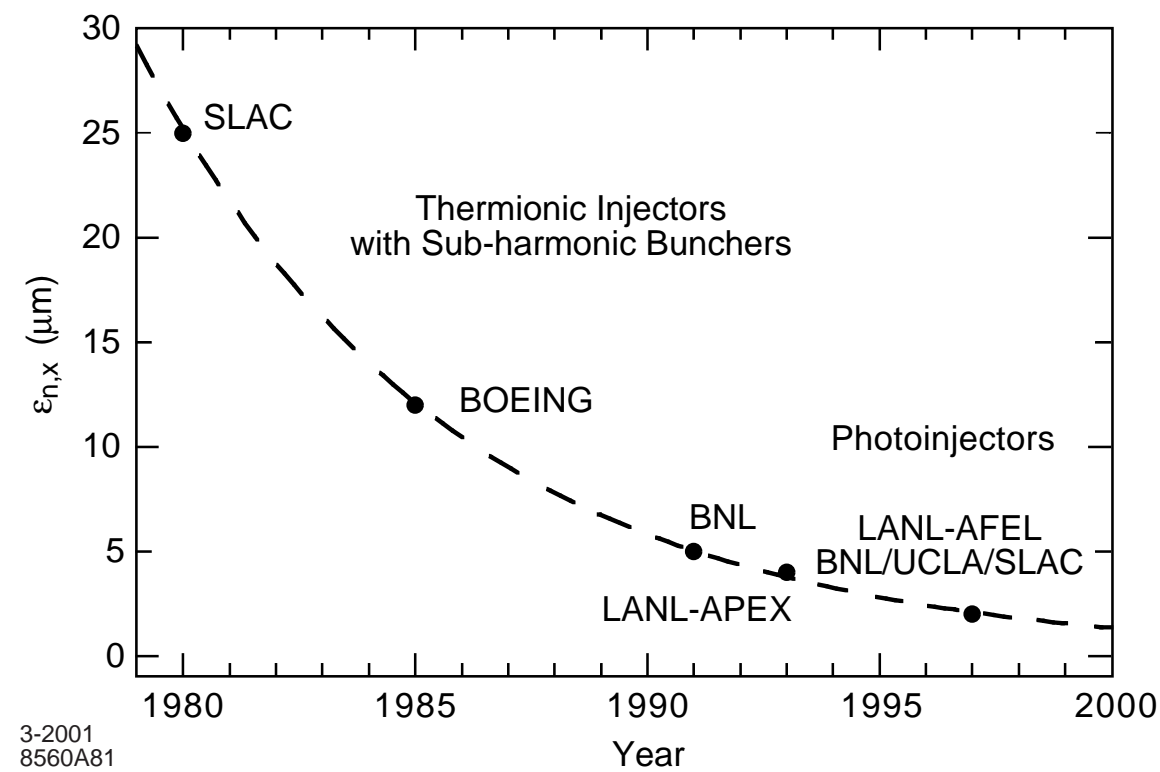

Figure 6.6 Normalized rms transverse emittance measured by the leading thermoionic (SLAC, BOEING) and rf photocathode injectors [16]. All data are for bunched beams with approximately $1 \mathrm{nC}$ of charge.

In the 1-nC regime, the lowest reported projected, normalized, rms emittance for an S-band gun is $2.4 \mu \mathrm{m}$ measured at BNL [3] using a two-screen emittance measurement technique with $0.9 \mathrm{nC}$ of charge. The emittance was measured using an approximately flat top transverse laser (clipped Gaussian) distribution and a 15-ps FWHM approximately Gaussian temporal distribution. However, the electron beam bunch length downstream of the booster accelerator was closer to 4 ps FWHM, which was attributed to rf compression in the gun. The beam was also measured using a quadrupole scan technique, which resulted in an emittance of $3.2 \mu \mathrm{m}$ with 0.8 $\mathrm{nC}$ of charge. The different results in the two measurement techniques were attributed to background subtraction errors in the quadrupole scan technique. A constant background obtained with a closed iris was used for background subtraction, while the real background varied with the electron beam image size due to image intensity changes during the quad scan. Since the beam size and, therefore, the intensity did not change during the two-screen measurement, the error is assumed to be less.

Temporal slice emittance measurements were also achieved on the same machine with roughly 1-ps resolution. By phasing the last booster accelerator section, a linear energy chirp (energy vs. time) on the beam was created and then spatially filtered in a dispersive section allowing only a narrow time slice through the slit. The resolution was limited by the minimum achievable electron beam size primarily due to the intrinsic uncorrelated energy spread in the beam and the jitter in the rf system. A two-screen emittance measurement setup was located 
downstream of the spatial filter. This type of slice measurement necessarily has some effect on the electron beam distribution since the linac phase is varied from the nominal operating parameters. BNL reports a slice emittance of $1.2 \mu \mathrm{m}$ for a 1-ps slice with $0.08 \mathrm{nC}$ and a full beam charge of $0.9 \mathrm{nC}$ with identical beam parameters as reported above [3]. The reported slice contains less than $10 \%$ of the total charge but covers roughly $25 \%$ of the electron beam pulse length.

At LANL, a 1.3-GHz integrated photoinjector (no drift space between gun and booster accelerator) has produced the lowest reported projected emittance to date from an L-band gun: $2.5 \mu \mathrm{m}$ with $1.1 \mathrm{nC}$ of charge [17]. The laser beam used had a clipped Gaussian transverse distribution (approximately 15\% variation across the beam) with 5.2-mm diameter and a 17-ps FWHM Gaussian temporal distribution. The electron beam bunch length was measured to be 15 ps FWHM using a streak camera. The measurements were performed with a $26 \mathrm{MV} / \mathrm{m}$ peak field on the cathode and an emission phase of $19^{\circ}$. This measurement used a streak camera with a $20 \mu \mathrm{m}$ slit to acquire time resolved images of quadrupole scans. The beam size was roughly $0.5 \mathrm{~mm} \mathrm{rms}$, and therefore this technique necessarily resulted in effectively analyzing single lines of the electron beam digitized image instead of full projections because of the presence of the streak camera input slit.

The streak camera used to acquire data for the projected emittance at LANL was also used for collecting slice emittance data. The slice emittance measurements were accomplished with approximately 7-ps resolution, which was limited by the streak camera. A more detailed description of the streak camera enhanced quadrupole scan technique is described in Section 6.5.3, Slice Emittance. With identical beam parameters as described above, the minimum measured slice emittance was $1.8 \mu \mathrm{m}$ for a 4-ps slice in the center of the bunch with approximately $0.3 \mathrm{nC}$ of charge. This slice contains almost $23 \%$ of the charge and also nearly $25 \%$ of the electron beam bunch length.

Measurements at lower charge, but close to the LCLS design current of $100 \mathrm{~A}$, have been made recently at the SLAC Gun Test Facility (GTF). Several measurements at the $200 \mathrm{pC}$ level with a 2 ps FWHM temporal Gaussian laser beam resulted in an emittance of $1.5 \mu \mathrm{m}$ in good agreement with PARMELA simulations [14]. These results indicate that emittances of approximately $1 \mu \mathrm{m}$ can be experimentally achieved at the $100 \mathrm{~A}$ level. Additional measurements are being made at the GTF with temporally flat laser profiles at the $1 \mathrm{nC}$ level.

As indicated in Section 6.1, Introduction, and earlier in this section, simulations show that the primary reason that emittances of $1 \mu \mathrm{m}$ have not yet been achieved is because no experiment to date has been conducted using a temporally flat top laser pulse to produce a temporally flat top electron bunch. While a measurement with a temporally flat top laser pulse is planned for the GTF in the near future, the degree of flatness that can actually be achieved is at present not well known. A simulation study with PARMELA is underway to determine the effect of a modest modulation (on the order of 20\%) would have on the emittance. The simulations also assume an azimuthally and radially independent electron beam, which is difficult to achieve experimentally. In fact the real beam depends on the convolution of the laser profile and the photocathode 
response. This can lead to significant variation in the space charge forces that cannot be properly compensated with solenoidal emittance compensation. A simulation study of the effect on the emittance of a spatially non-uniform but axially symmetric modulation of the charge distribution is also in progress.

All emittance experiments also suffer from shot to shot fluctuations and imperfect background subtraction, issues that are addressed for the LCLS design primarily in Section 6.4, Laser System.

In summary, although the emittance required for the LCLS has not yet been demonstrated with an rf photoinjector for a 1-nC beam, the experimental results are consistent with PARMELA simulations. PARMELA simulations also predict that if temporal as well as transverse pulse shaping is used, the LCLS emittance values should be achievable.

\subsubsection{Thermal Emittance}

One concern in the production of low emittance beams is the thermal emittance of the beam due to its excess energy as it leaves the cathode. The projected and slice emittances must be greater than or equal to the thermal emittance. The ideal method of determining $\varepsilon_{t h}$ is to measure the angle-resolved energy distribution for photoelectrons emitted from the cathode. Unfortunately no such measurement has been made for a $\mathrm{Cu}$ cathode to date. However, the emittance exiting the gun at very low charge levels where space charge is not significant has been conducted at BNL [18]. The beam size exiting the gun is measured as a function of the solenoidal focusing strength and the emittance is then fit to the measured data. The normalized rms thermal emittance has been measured to be $0.6 \mu \mathrm{m}$ for a $2-\mathrm{mm}$ diameter $\mathrm{Cu}$ cathode. The emittance contribution due to the finite pulse length of the electron beam has been estimated to be less than $5 \%$.

An alternate but indirect method of measuring $\varepsilon_{t h}$ has been suggested [19] and conducted at SLAC. For small changes in the work function of a metal, the quantum efficiency, $Q_{e}$, can be written as

$$
Q_{e} \approx Q_{e 0} e^{\frac{\Delta \Phi}{k T_{e}}},
$$

where $\Delta \Phi$ is the change in the work function, and $T_{e}$ is the effective temperature of the cathode, and $Q_{e 0}$ is the $\mathrm{QE}$ before the change. The change in the work function due to the Schottky effect is given by

$$
\Delta \Phi=\sqrt{\frac{e^{3} E_{p} \sin \Theta}{4 \pi \varepsilon_{0}}},
$$

where $E_{p}$ is the peak applied field and $\Theta$ is the emission phase. The Schottky effect can be thought of as altering the work function of the cathode material. Using Eqs. (6-4 and -5), a value of $T_{e}$ can be derived from the measured slope of $\ln (Q E)$ versus $\left(E_{p} \sin (\theta)\right)^{1 / 2}$. Then the corresponding value of the thermal emittance can be calculated from $T_{e}$ using the well-known relationship for uniform emission from a thermionic cathode of radius $r_{c}$ : 


$$
\varepsilon_{n, r m s, t h}=\frac{r_{c}}{2} \sqrt{\frac{k T_{e}}{m_{o} c^{2}}} .
$$

Using the data shown in Figure 6.7, the effective temperature is estimated to be $0.14 \mathrm{eV}$ resulting in a normalized, rms, thermal emittance of $0.3 \mu \mathrm{m}$ for a $2-\mathrm{mm}$ diameter cathode.

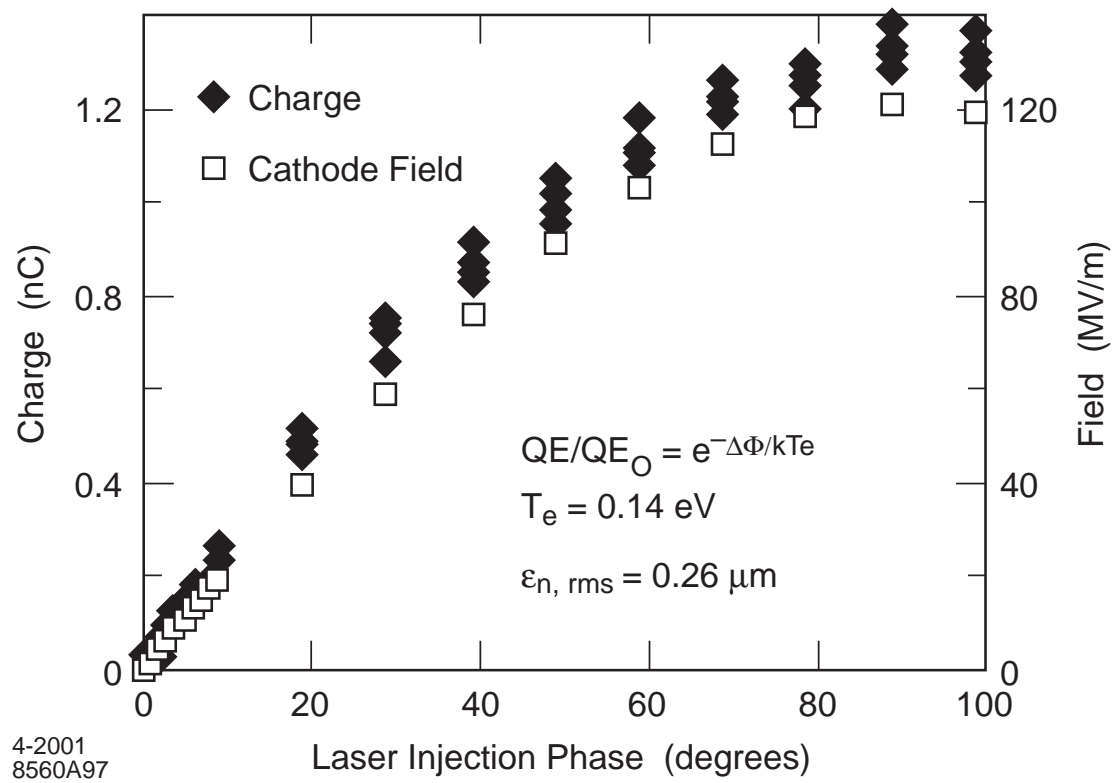

Figure 6.7 The measured charge extracted from a Cu photocathode with $\approx 190 \mu$ of laser energy and the corresponding rf field at the cathode are plotted as a function of injection phase.

\subsubsection{Longitudinal Emittance}

A significantly larger amount of work has been spent on measuring the transverse emittance as opposed to the longitudinal emittance. While both of the low emittance experiments at $1 \mathrm{nC}$ described above reported the electron beam pulse length, neither reported the energy spread. The BNL experiment, which utilized an energy chirp by intentionally misphasing a linac section, necessarily modified the longitudinal emittance of the beam, making simultaneous transverse and longitudinal emittance measurements under nominal operating parameters impossible. The LANL experiment utilizes an integrated photoinjector so that one can not individually optimize the phase of the gun and linac to simultaneously optimize the transverse and longitudinal emittances.

Work has begun on the prototype gun to systematically measure the longitudinal emittance. Preliminary results for a low charge beam of $0.15 \mathrm{nC}$ give $6.4 \mathrm{keV}$ for the uncorrelated, rms energy spread out of the gun. A detailed study of the uncorrelated longitudinal emittance of a 144-MHz rf photoinjector indicates that below the space charge limit, the uncorrelated longitudinal emittance and energy spread vary linearly with the surface charge density at the cathode [20]. Using this study's parameterization, the prototype gun experimental surface charge density would give $4 \mathrm{keV}, 40 \%$ lower than the observed $6.4 \mathrm{keV}$. Extrapolating to the LCLS charge of $1 \mathrm{nC}$, the low frequency gun study would predict $22 \mathrm{keV}$ for the uncorrelated rms energy spread out of the LCLS gun. The PARMELA simulation of the LCLS gun at 140 
$\mathrm{MV} / \mathrm{m}$ gives $8 \mathrm{keV}$. It is clear that further effort is needed to refine and understand these differences, however they are all close to the desired specification given in Table 6.1.

\subsection{RF Photocathode Gun}

The parameters for the LCLS rf photoinjector gun are listed in Table 6.2 below.

Table 6.2 LCLS RF Photoinjector Gun Parameters.

\begin{tabular}{|c|c|}
\hline Parameter & Value \\
\hline Cathode material & $\mathrm{Cu}$ (or possibly $\mathrm{Mg}$ ) \\
\hline Usable diameter of cathode & $12 \mathrm{~mm}$ \\
\hline Quantum efficiency & $>10^{-5}$ at $263 \mathrm{~nm}$ \\
\hline Nominal peak rf field & $140 \mathrm{MV} / \mathrm{m}$ \\
\hline Beam energy at gun exit ${ }^{\mathrm{a}}$ & $\sim 7.1 \mathrm{MeV}$ \\
\hline Energy spread (uncorrelated) at gun exit & $<0.2 \% \mathrm{rms}$ \\
\hline rf frequency & $2856 \mathrm{MHz}$ \\
\hline Bunch repetition rate & $120 \mathrm{~Hz}$ \\
\hline rf pulse duration & $\sim 3 \mu \mathrm{sec}$ \\
\hline rf peak power for $140 \mathrm{MV} / \mathrm{m}$ & $14 \mathrm{MW}$ \\
\hline Number of cells & 1.6 \\
\hline Length of gun & $0.168 \mathrm{~m}$ \\
\hline
\end{tabular}

a The beam energy is quoted for $140 \mathrm{MV} / \mathrm{m}$ peak field and emission phase of $32^{\circ}$ with respect to the rf zero crossing.

\subsubsection{Gun Description}

The LCLS gun design is anticipated to be a variation of the BNL/SLAC/UCLA 1.6-cell Sband rf photocathode gun [2], herein called the "prototype gun." The principal differences are the addition of a vacuum load-lock to better ensure cathode performance and improved cooling to allow operation at $120 \mathrm{~Hz}$. In addition, the rf power for the LCLS gun will utilize a dual feed to minimize field asymmetries. Each of these features will be discussed in more detail later. Unless specifically indicated otherwise, the gun discussed below is the prototype gun. 
The 1.6-cell $\mathrm{rf}$ prototype gun design is based on the earlier 1.5-cell gun developed at BNL [21]. To minimize emittance growth due to the $E_{z}$ component of the $\mathrm{TM}_{110}$ mode, the field amplitude in the gun was symmetrized. The original BNL zero-mode-suppressed side-coupling was replaced. The rf power for the prototype gun is symmetrically coupled into the full cell only, which does not suppress the zero mode. However, if the beam is extracted after several fill times of the zero mode, then the fields in the gun will be sufficiently close to the steady-state condition that the zero mode will have decayed to an insignificant level.

Since there is no direct $\mathrm{rf}$ coupling from the waveguide to the half cell, the cell-to-cell coupling was improved by increasing the iris size, which also increased the mode separation between the zero- and $\pi$-modes. To provide more rf focusing and decrease the peak field on the cell-to-cell iris, the half-cell length was slightly increased.

A cross section of the rf prototype gun is shown in Figure 6.8. The photocathode is located in the geometric center of the end plate of the half-cell. The end plate is removable to facilitate installation of cathode material other than $\mathrm{Cu}$ by implantation or by using an insert. As with the original BNL gun, the laser beam can be brought to the cathode either along the axis of the gun or at a grazing incidence through the $72^{\circ}$ side port.

(a)

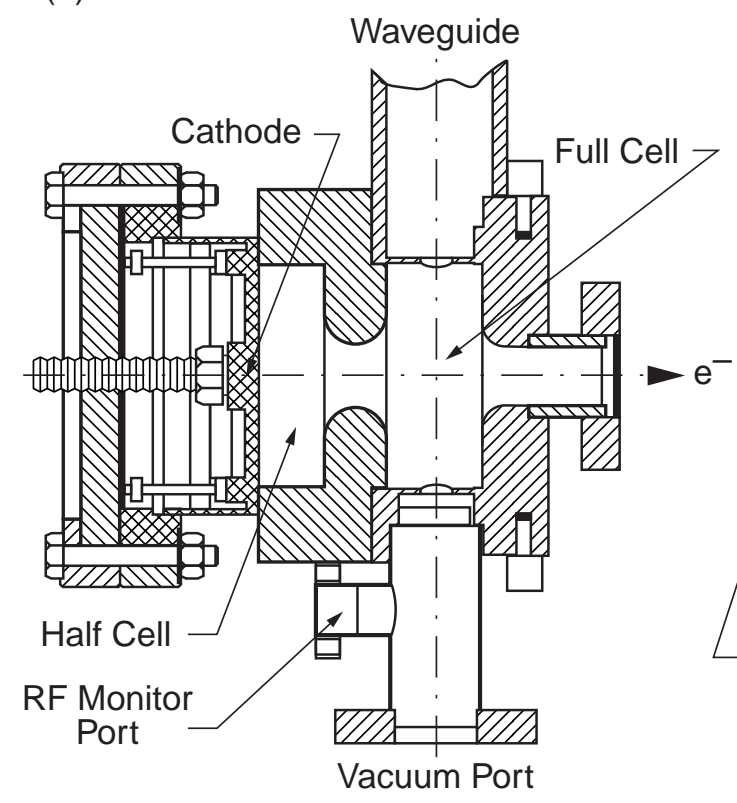

(b)

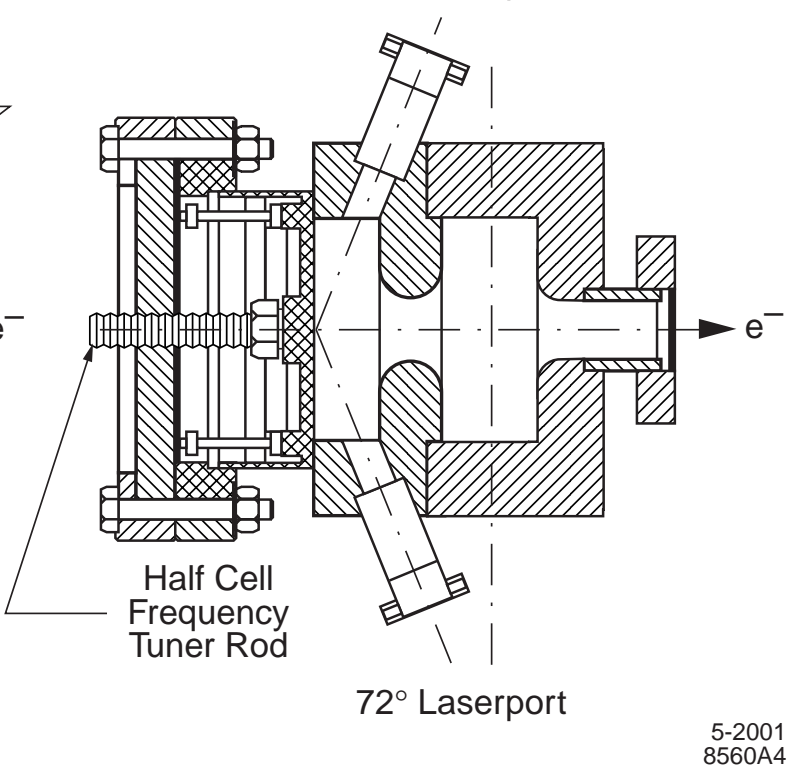

Figure 6.8

Cross section of rf prototype gun. (a) azimuthal orientation showing rf coupler and vacuum port; (b) orientation showing the off-axis laserports.

Electric field maps for the prototype gun were obtained with SUPERFISH. The $\pi$-mode fields are shown in Figure 6.9. 


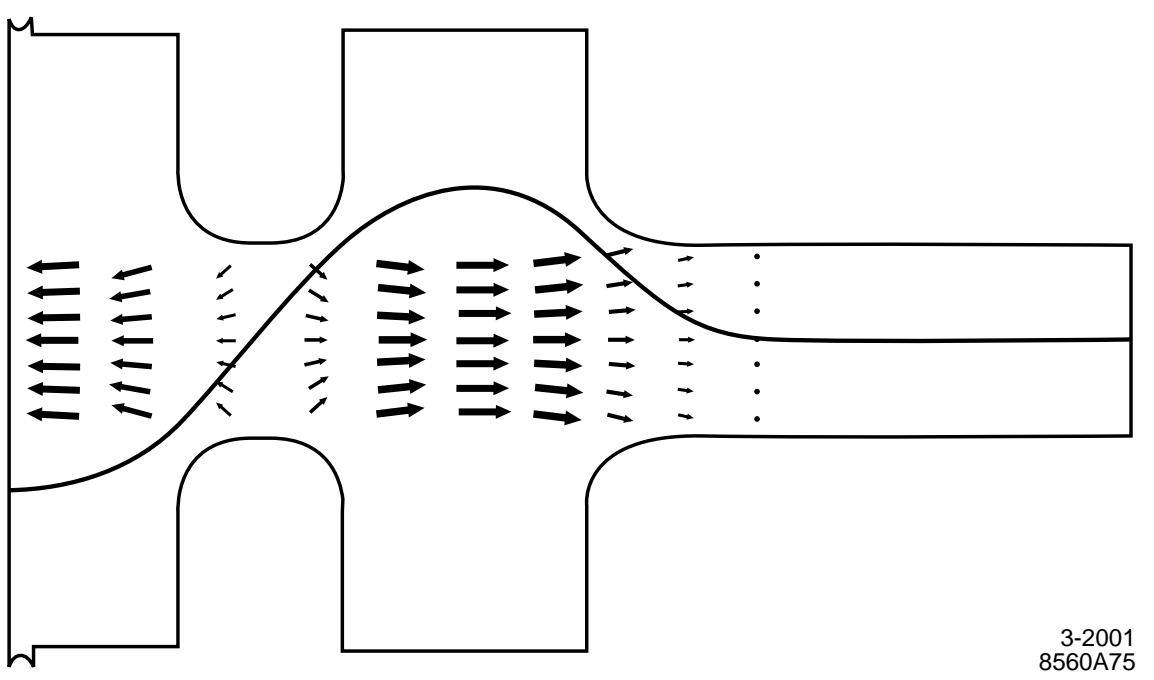

Figure 6.9 Field lines in the rf gun for $\pi$-mode obtained with SUPERFISH.

\subsubsection{Field Strength and Cell Balance}

Because of the deleterious effects of space charge on the beam emittance, it is important to accelerate the beam as rapidly as possible within the gun itself. Simulations indicate that the transverse emittance decreases as the field increases until about $140 \mathrm{MV} / \mathrm{m}$ on the cathode surface, at which point a local minimum in the emittance versus solenoidal and cathode field strengths has been observed using PARMELA. See Figure 6.10. 


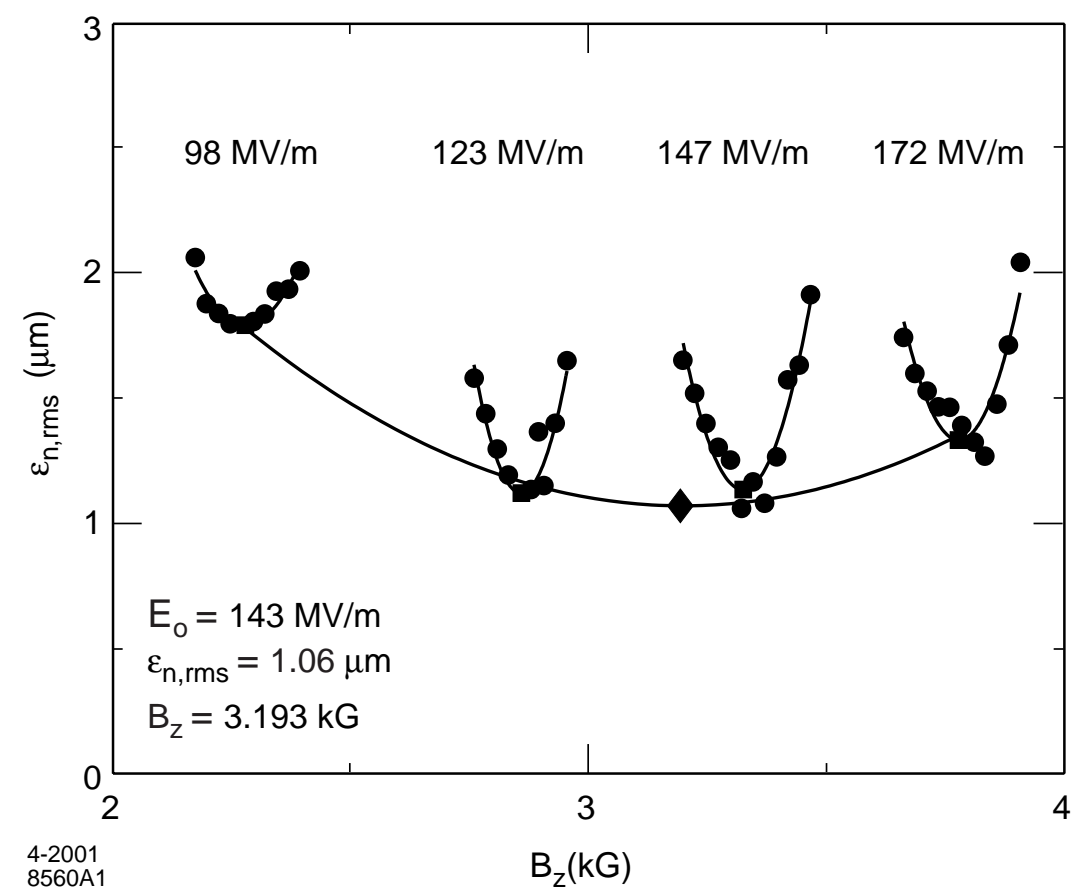

Figure 6.10

Emittance versus cathode field strength and peak solenoidal magnetic field.

Prototype guns have been operated for limited periods with fields up to $140 \mathrm{MV} / \mathrm{m}$. However, as the field is increased above $100 \mathrm{MV} / \mathrm{m}$, not only does the dark current typically increase rather dramatically (quickly exceeding the photocurrent), but the frequency and intensity of rf breakdowns also increases. RF breakdowns tend to leave pits in the cathode surface that lead to nonuniform emission. At the GTF, using a $\mathrm{Cu}$ cathode, $120 \mathrm{MV} / \mathrm{m}$ has been the typical operating field.

PARMELA simulations (See Section 6.6.4, Sensitivity Study) indicate the ratio of the field in the full cell to half cell should be close to unity. Unbalanced fields can lead to large correlated energy spreads exiting the gun (as designed into thermionic rf guns) and subsequent emittance growth. The LCLS gun will incorporate calibrated field probes in both cells to set and monitor the field ratio.

\subsubsection{Symmetrization}

The emittance growth due to multipole modes of $E_{z}$ in a gun cavity with a conventional asymmetric rf coupler (as for the earlier BNL 1.5-cell gun) is estimated to contribute $\sim 1 \mu \mathrm{m}$ to the transverse emittance [22]. This growth is dominated by the dipole mode and the contribution from the higher order modes is less than $0.1 \mu \mathrm{m}$. Therefore the higher order modes can be neglected. Symmetrization in the prototype gun is achieved by including a second identical "coupling" hole (which is also used for vacuum pumping) directly across from the rf waveguide coupling hole. This reduces the field amplitude dipole term by an order of magnitude over the unsymmetrized case. Further symmetrization of the dipole field phase can be achieved by utilizing a symmetric power feed instead of a single-sided feed. This is done with a magic tee 
along with two $\mathrm{H}$ bends that symmetrically feed $\mathrm{rf}$ power to the full cell of the LCLS 1.6-cell $\mathrm{rf}$ gun [23]. A diagnostics port in the magic tee will monitor rf power asymmetries. The coupling hole size will need to be reduced compared to the prototype gun to maintain the same rf coupling coefficient. Such a symmetric rf feed is planned for the LCLS gun.

\subsection{4 $120 \mathrm{~Hz}$ Operation}

The prototype gun was originally designed for low repetition rates, but it is estimated that it could be operated up to $40 \mathrm{~Hz}$. The stored energy in the cavity fields is $9.1 \mathrm{~J}$ for the LCLS design of $140 \mathrm{MV} / \mathrm{m}$. The LCLS gun requires $14 \mathrm{MW}$ of power from the klystron and has a filling time of approximately $670 \mathrm{~ns}$ assuming a $\mathrm{Q}_{0}$ of 12000 and a critically coupled cavity resulting in an average heat load of $3.8 \mathrm{~kW}$ at $120 \mathrm{~Hz}$. A slightly modified gun was designed and built by a $\mathrm{BNL} / \mathrm{KEK} / \mathrm{SHI}$ collaboration for operation up to $100 \mathrm{~Hz}$ [24]. Water cooling channels were added in the vicinity of the irises and satisfactory operation at $100 \mathrm{MV} / \mathrm{m}$ with a 4- $\mu$ s wide rf pulse at $50 \mathrm{~Hz}$ has been demonstrated at the University of Tokyo at Tokai [25]. At these operating conditions the gun is dissipating roughly $1 \mathrm{~kW}$ of power.

At $120 \mathrm{~Hz}$, the prototype gun with unmodified cooling channels would not operate at design specifications due to thermo-mechanical distortions. There are two possible solutions to this problem and some combination of the two will be adopted. The first solution, already utilized by the $\mathrm{BNL} / \mathrm{KEK} / \mathrm{SHI}$ collaboration, is to study the energy deposition in the $\mathrm{rf}$ gun and provide appropriate cooling at the optimal locations to minimize thermal gradients without compromising structural integrity [24]. One must also carefully consider the thermal distortions in the gun and not allow significant frequency shifts due to thermal expansion or introduction of higher order modes due to asymmetric cavity distortions.

Alternatively the thermal load can be reduced by a factor of three or more, i.e., to less than the 1-kW level already tested at Tokai, by properly shaping the rf pulse [26] and/or overcoupling the cavity to reduce the filling time. An overcoupled cavity with coupling coefficient of 1.5 instead of unity will dissipate $20 \%$ less power while only requiring $4 \%$ higher peak rf power to achieve an identical field in the gun. Using $30 \mathrm{MW}$ of rf power instead of $14 \mathrm{MW}$ to drive the gun, the rf field will build up to the desired value of $140 \mathrm{MV} / \mathrm{m}$ in only $750 \mathrm{~ns}$ instead of $2.8 \mu \mathrm{s}$. Once the desired accelerating voltage is reached, a fast (100 ns) rf attenuator on the klystron input could be used to stabilize the voltage at the desired value during beam extraction. The total $\mathrm{rf}$ pulse duration can thus be limited to about $1 \mu$ s with a corresponding reduction in the heat load of a more than a factor of three.

\subsubsection{Photocathode}

The choice of cathode material is a function of several restrictions including gun emittance, laser power at a given wavelength, longevity under rf processing or operation, and gun cavity construction. The use of a cathode plug or insert in an S-band gun has so far limited the cathode field to about $110 \mathrm{MV} / \mathrm{m}$ [27], whereas simulations indicate the transverse emittance drops with increasing field up to about $140 \mathrm{MV} / \mathrm{m}$. However, a load-lock coupled gun, which utilizes a back 
plane that is replaceable under vacuum, should eliminate this restriction. Such a system allows greater flexibility of cathode choice and easy upgrades as improved materials are realized.

A metal photocathode is chosen for the preliminary configuration for several reasons. Since the source is not required to produce a bunch train (multiple microbunches within each pulse), the lower QE of metal cathodes compared to alkali and semiconductor photocathodes is not a major concern. The QE for $\mathrm{Cu}$ illuminated with UV light at normal incidence depends on surface preparation, but a QE of $10^{-5}$ at $260 \mathrm{~nm}(4.77 \mathrm{eV})$ in a non-load-locked gun is achievable. See Section 6.2.1, Quantum Efficiency, and also references [28,29,30]. Much better QE is available from copper installed through a load-lock. This is illustrated by the data of Figure 6.11 for which a carefully prepared polycrystallline $\mathrm{Cu}$ photocathode was inserted into an ultra-vacuum surface analysis chamber using a load-lock (no baking) and the QE spectrum measured at low voltage [31]. The figure shows a QE of $2.3 \times 10^{-4}$ at $266 \mathrm{~nm}$. 


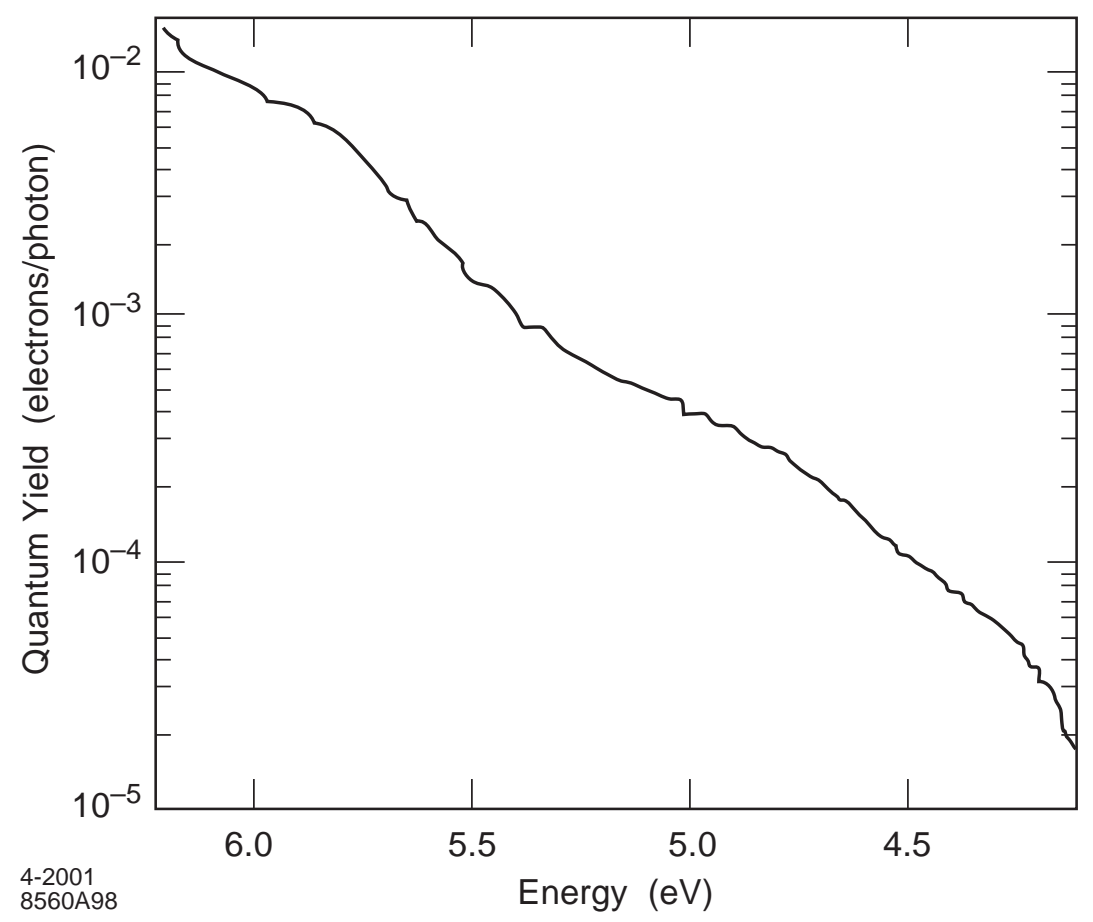

Figure 6.11 Copper QE as a function of quantum energy measured with low (22 V) dc bias with the surface untreated after installation in the analysis system using a load-lock. Photon wavelength in $\mathrm{nm}$ is equal to 1240 divided by the energy in $\mathrm{eV}$; i.e., at $4.8 \mathrm{eV}$ the wavelength is $260 \mathrm{~nm}$.

A gain in QE by a factor of two to four can be achieved by illuminating the cathode at a grazing angle This gain is primarily due to the increased absorption of $p$ - over $s$-polarized light [32], but its benefits are partially offset by the laser energy lost in shaping the pulse for grazing incidence.

At $260 \mathrm{~nm}$, an optical pulse of $500 \mu \mathrm{J}$ on the cathode is required to produce $1 \mathrm{nC}$ of charge when the $\mathrm{QE}$ is $10^{-5}$. A laser system to meet this requirement is relatively straightforward to design. (See Section 6.4, Laser System.)

At extremely high photon intensities, the metal surface will begin to disintegrate. Even with such disintegration, the $\mathrm{QE}$ of the cathode tends to remain high, presumably due to the enhancement of field emission along the surface disruptions. However, a large and undesirable increase in dark current accompanies such a surface [33]. The photon intensities planned for the operation of the LCLS source are well below this regime.

The principal advantages of metal cathodes are that they are easy to fabricate and that the entire end plate of the half cell can be formed in the standard manner of $\mathrm{Cu}$ rf cavities, permitting operation at the highest field values. The photoelectric response time of metal cathodes is on the sub-picosecond level, thus imposing no limitation on the desired temporal pulse shaping. 
The QE for a magnesium photocathode is about one order of magnitude higher than for $\mathrm{Cu}$ prepared in a similar manner [34,35]. The higher $\mathrm{QE}$ for $\mathrm{Mg}$ is attributed to its lower work function. For a given excitation energy, a lower work function implies a higher thermal emittance, which could be a limiting factor for a low emittance beam. However, if one considers threshold emission, i.e., emission in which the excitation energy is barely above the work function, the $\mathrm{QE}$ for $\mathrm{Mg}$ presumably would be roughly equal to that for $\mathrm{Cu}$. However, since the laser wavelength for $\mathrm{Mg}$ would be much longer than for $\mathrm{Cu}$, there would be a definite system advantage to $\mathrm{Mg}$. For this reason, and since the $\mathrm{QE}$ of $\mathrm{Mg}$ cathodes for threshold emission is potentially higher than for $\mathrm{Cu}$, the search for a way to use a $\mathrm{Mg}$ cathode with fields on the order of $120 \mathrm{MV} / \mathrm{m}$ - including how to fabricate a high rf-field removable plug-will continue. A loadlock system is particularly useful for Mg because of magnesium's extreme chemical reactivity to water vapor. A Mg plug can be embedded into a copper cathode plate, finish polished in a drynitrogen or argon glove box and transferred in-situ to the photocathode vacuum storage vessel for later transfer, under vacuum, into the gun.

\subsubsection{Emittance Compensating Solenoid}

For emittance compensation, a solenoid with precisely defined field symmetry and positioning will be used at the gun exit. The solenoid design for the prototype gun incorporates several pancake assemblies, each assembly including a pancake coil and steel flux straightener as well as alignment components. Conventional manufacturing techniques using molded coils do not accommodate the required flux straightener position accuracy, therefore the straighteners are positioned independently of the coil positioning. The physical length of the solenoid assembly is $22.5 \mathrm{~cm}$ including the flux return, while the pancakes themselves are $19.5 \mathrm{~cm}$. A map of the measured axial magnetic field is shown in Figure 6.12. The solenoid incorporates steel flux returns to reduce the field at the cathode to approximately 5 Gauss, negating the need for an identical bucking coil behind the cathode to null the field at the cathode plane. This solenoid design allows for a much simpler design of the load-lock system. 


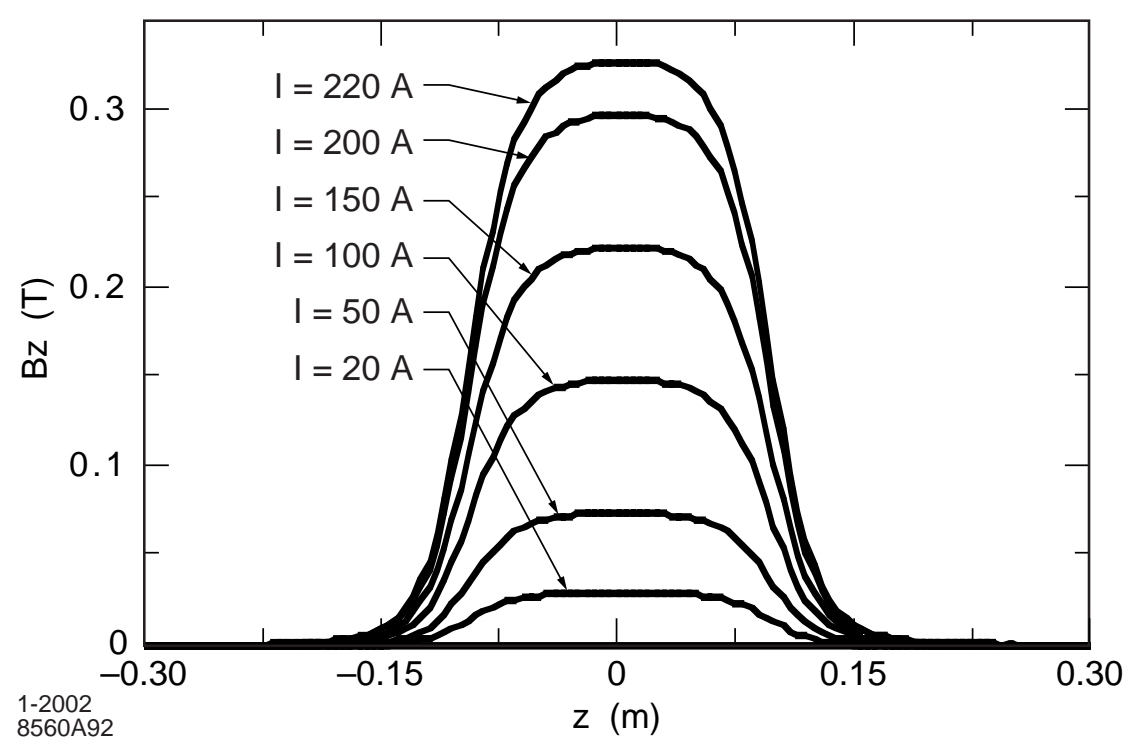

Figure 6.12 Measured axial magnetic field of the emittance compensation solenoid as a function of distance $z$ along the axis. In this figure, $z$ is referenced to the center of the solenoid. The peak value of $B_{2}$ increases as the solenoid current, I, increases.

\subsubsection{Vacuum System}

After brazing and before final tuning, the rf gun will undergo a $450^{\circ} \mathrm{C}$ vacuum bakeout in the SLAC Klystron Department's vacuum bakeout facility. This procedure removes excess hydrogen absorbed by the vacuum surfaces of the rf gun during brazing. Ion-NEG pumps (separate or combined) will be located in the rf waveguide near each of the two rf input couplers at the gun (the gun vacuum is separated by the rest of the $\mathrm{rf}$ waveguide by $\mathrm{rf}$ windows) and also in the beamline just downstream of the emittance compensating solenoid. Small 20 1/s ion pumps will be used in the waveguide, while a large $220 \mathrm{l} / \mathrm{s}$ ion pump will be in the beamline. Together these pumps should provide a pressure of $\leq 5 \times 10^{-10}$ Torr at the gun with the field gradient at its normal operating strength and the rf at $120 \mathrm{~Hz}$. The $220 \mathrm{l} / \mathrm{s}$ pump also maintains the vacuum in the diagnostic section following the gun.

A schematic of the proposed LCLS gun assembly is shown in Figure 6.13. A load-locked cathode storage and transport system is coupled to the rear of the gun. Through use of isolation gate valves, the load-lock has a number of advantages over a non-load-locked system:

1) Photocathodes are exchanged under vacuum without exposure to atmospheric (particularly the gun vault) environment. This eliminates the oxidation, dust accumulation, and carbon contamination onto the cathode surface that usually results from exchanging cathodes when opening the rear of the gun to ambient atmosphere.

2) The gun is not exposed to atmosphere in the cathode exchange procedure, therefore a bakeout of the gun itself is not required following the exchange.

3) Processing of the cathodes takes place ex-vault where the cathodes will be polished under inert atmosphere (in the case of $\mathrm{Cu}$ or $\mathrm{Mg}$ ), deposited in vacuum (in the case of $\mathrm{Cs}_{2} \mathrm{Te}$ ), 
or plasma-cleaned of carbon. In each instance, the processed cathode is subsequently moved in-situ to a cathode storage chamber, ready for attachment to the gun load-lock.

4) Through the use of multiple "transport/storage" vessels, any number of cathodes can be prepared and stored long-term, under vacuum, then installed rapidly as needed.

The load-lock/photocathode gun assembly is shown in Figure 6.13 with three sequential positions of a cathode plate: (1) loaded into the gun, (2) transported from the storage chamber to the gun transport chamber, and (3) in storage and attached to the gun load-lock.. Experience with the load-lock system of the SLAC Polarized Electron Gun shows that the entire process takes less than one shift (excluding any in-vacuo high-field processing of the new cathode, if needed).

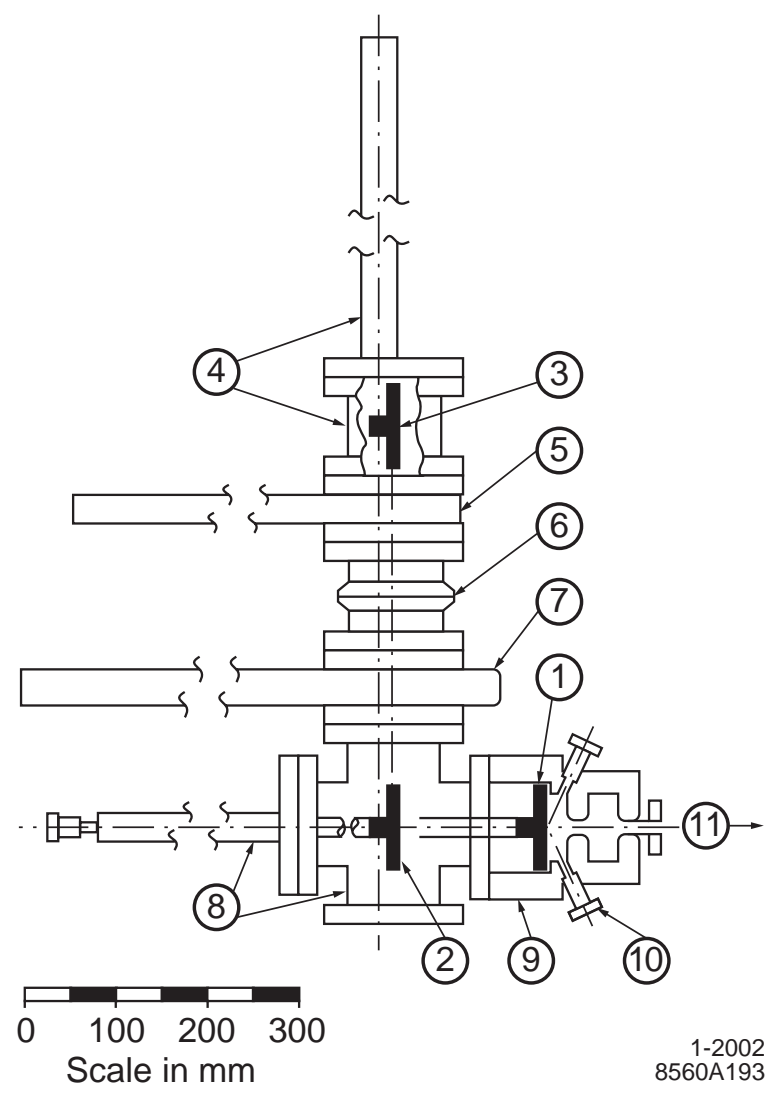

Figure 6.13 Schematic of photoinjector gun assembly showing vacuum transfer apparatus (load-lock) for cathode exchange. A photocathode (PC) plate is shown in it's three locations of the exchange process. The numbered components are: (1) PC plate in gun, (2) PC plate in transfer chamber, (3) PC plate in storage chamber, (4) storage chamber with transfer motion, (5) storage chamber gate valve, (6) quick-connect flange, (7) gate valve, (8) PC-exchange chamber with transfer motion, (9) rf photocathode gun, (10) laser light ports, (11) LCLS beamline.

\subsection{Laser System}

The laser system for the electron source is required to deliver a $500 \mu \mathrm{J}$ pulse of UV photons to the photocathode at a repetition rate of $120 \mathrm{~Hz}$. To meet the emittance requirements of the 
source, the laser pulse must have an adjustable pulse length and temporal shape, nominally a flat pulse 10-ps long, and a uniform transverse profile with an adjustable radius, nominally a hard edge at $1.0 \mathrm{~mm}$. Finally, stability is an important operational requirement, and, as discussed in Chapter 7, the timing stability in particular is crucial to meeting the energy stability requirements in the undulator. Table 6.3 summarizes the laser's design requirements.

\subsubsection{System Description}

The titanium-sapphire laser system of Figure 6.14 provides the ultraviolet light pulses for the rf gun. This system is first described briefly; subsequent sections then elaborate on various aspects of the design.

A CW, frequency-doubled, diode-pumped $\mathrm{Nd}: \mathrm{YVO}_{4}$ laser provides highly stable energy in the green $(532 \mathrm{~nm})$ to pump the $\mathrm{CW}$ mode-locked Ti:sapphire oscillator, which then delivers a stable, continuous train of 12-nJ, 100-fs FWHM pulses that repeat at $79.33 \mathrm{MHz}$. This frequency, the $36^{\text {th }}$ subharmonic of the linac's 2856-MHz rf, locks the timing of the laser pulses to the phase of the $\mathrm{rf}$ in the linac and $\mathrm{rf}$ gun. The wavelength is tuned to $780 \mathrm{~nm}$, near the peak for Ti:sapphire output. This frequency is tripled to $260 \mathrm{~nm}$ after amplification to provide a suitable wavelength for the photocathode of the gun. With some reservations about timing stability (see Section 6.4.7, Stability of Laser Pulse), oscillators of this type are commercially available (e.g., the SpectraPhysics $\pi$-mode ${ }^{\circledR}$ Tsunami). 
Table 6.3 Laser System Requirements.

\begin{tabular}{|c|c|}
\hline Parameter & Requirement \\
\hline Operating wavelength & 260-270 nm (for $\mathrm{Cu}$ cathodes) \\
\hline Pulse repetition rate & $120 \mathrm{~Hz}$ \\
\hline Number of micropulses per pulse & 1 \\
\hline Pulse energy on cathode ${ }^{a}$ & $>500 \mu \mathrm{J}$ \\
\hline Laser spot radius on cathode ${ }^{b}$ & $0.71 \mathrm{~mm} \mathrm{rms}$ \\
\hline Pulse rise time $(10-90 \%)$ & $0.5 \mathrm{ps}$ \\
\hline Pulse length & $2.9 \mathrm{ps} \mathrm{rms}$ \\
\hline Longitudinal pulse shape & Various, but nominally uniform \\
\hline Transverse pulse shape & Various, but nominally uniform \\
\hline Homogeneity on cathode & $10 \%$ ptp \\
\hline Optical energy jitter (in UV) & $\leq 2 \% \mathrm{rms}$ \\
\hline Laser-to-rf phase jitter & $\leq 0.5 \mathrm{ps} \mathrm{rms}$ \\
\hline Laser spot diameter jitter at cathode & $1 \%$ peak-to-peak \\
\hline Pointing jitter & $<1 \%$ of radius $\mathrm{rms}$ \\
\hline
\end{tabular}

a The design will be for $18 \mathrm{~mJ}$ of IR energy just after the amplifiers, resulting in at least $500 \mu \mathrm{J}$ available at the cathode. For a QE of $10^{-5}, 500 \mu \mathrm{J}$ of excitation light at $260 \mathrm{~nm}$ at the cathode will produce $1 \mathrm{nC}$ of charge.

b For a uniform, round, transverse cross section, the hard-edge radius, $r_{c}$, is related to the rms radius, $\sigma_{r}$, by $r_{c}=\sqrt{2} \sigma_{r}$. In this case, if $\sigma_{r}$ is $0.71 \mathrm{~mm}, r_{c}$ would be $1 \mathrm{~mm}$.

c For a uniform temporal cross section, the edge-to-edge pluse length, $l_{c}$, is related to the rms length, $\sigma_{t}$, by $l_{c}=\sqrt{12} \sigma_{t}$. In this case, if $\sigma_{t}$ is $2.9 \mathrm{ps}$, then $l_{c}$ would be $10 \mathrm{ps}$..

A Pockels cell and polarizer are used to gate single pulses at $120 \mathrm{~Hz}$ from the $79.33-\mathrm{MHz}$ pulse train. The selected pulses are then amplified by two Ti:sapphire crystals, both configured as 4-pass "bow-tie" amplifiers [36]. Both are pumped by a pair of Q-switched, doubled Nd:YAG lasers that fire in alternation, each producing a $60-\mathrm{Hz}$ train of 3 to $10-\mathrm{ns}$ pulses. Again, commercial lasers (such as Coherent's ${ }^{\circledR}$ Infinity or Spectra-Physics, ${ }^{\circledR}$ Quanta-Ray PRO) with an additional feedforward loop to decrease amplitude jitter will be used as discussed later in the 
section on stability. Fourier-relay optics (described below), beginning with a primary aperture between the two amplifiers and continuing to the final optics platform next to the gun, are used to maintain a good transverse mode while efficiently filling the pumped volume of the Ti:sapphire crystals.

In amplifiers for picosecond and especially sub-picosecond pulses, the peak power must be limited to avoid damage to optical components and nonlinearities. Chirped pulse amplification [37] is used to reduce the peak power in the amplifier. The large bandwidth of the Ti:sapphire oscillator, which enables it to produce the 0.5 -ps rise time required for the shaped pulse, also permits the pulse to be stretched to hundreds of picoseconds. In the dispersive region between a pair of gratings, different wavelengths take different optical paths. The resulting space, time, and wavelength correlations are then used to stretch the pulse. After amplification, the process can be reversed to compress the pulse to the original or any greater width. In addition, the oscillator's large bandwidth allows the pulse to be shaped in time by manipulating its Fourier transform under computer control (see below). Figure 6.14 includes the pulse shaper and stretcher after the oscillator and a compressor after the amplifier. An additional low-power compressor after the oscillator is used as a diagnostic for the pulse shaper. It compresses the pulses from the $89-\mathrm{MHz}$ train that are not selected by the Pockels-cell gate. A cross-correlator using a portion of the oscillator light can then probe the resulting pulse shape. 


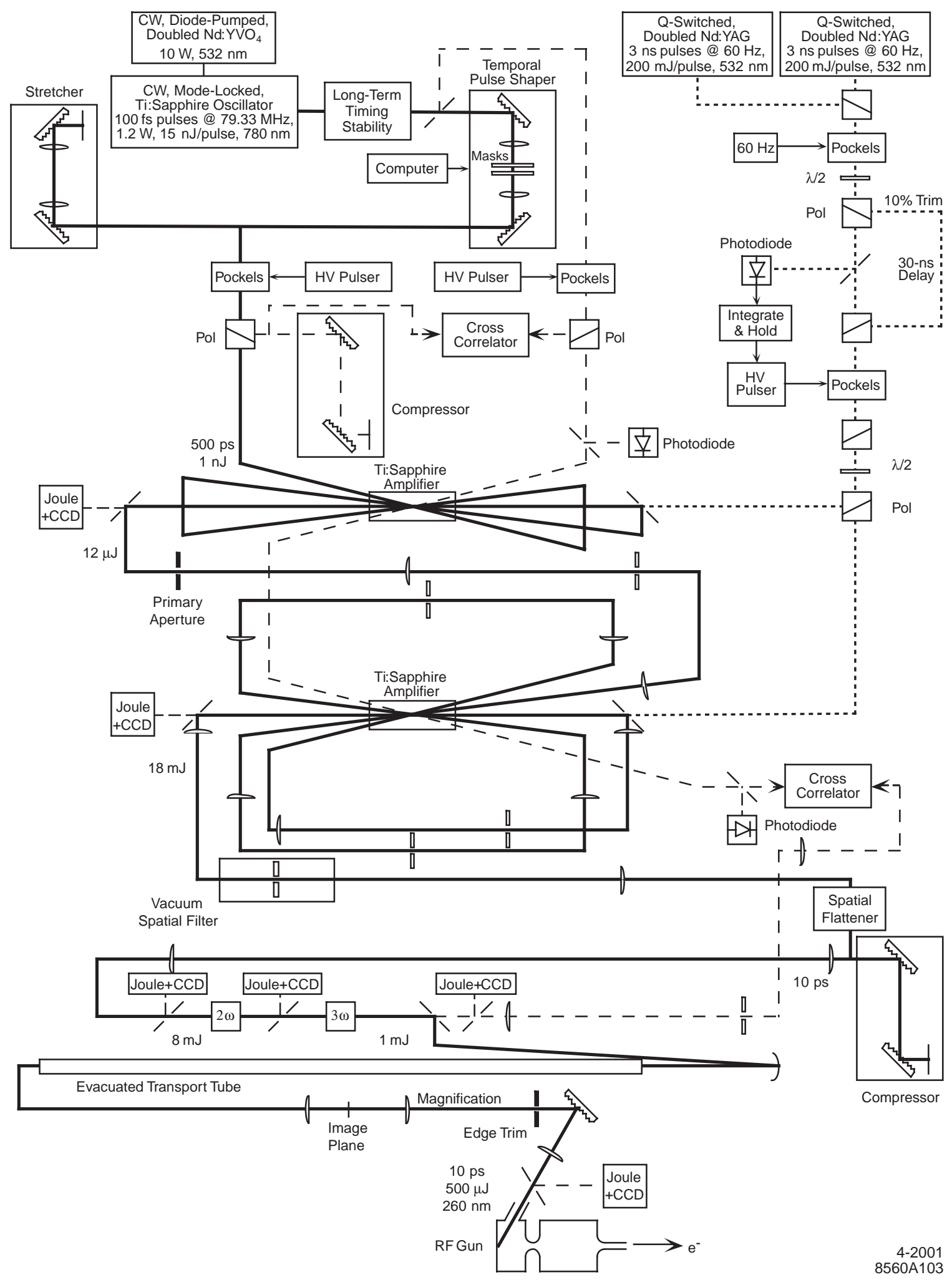

Figure 6.14 The drive laser for the rf photocathode electron gun for the LCLS. The thick lines show the main beam path, the widely spaced, dashed lines indicate diagnostic beams, and the closely spaced, dashed lines are pump beams. 
After the second amplifier, the transverse shape of the pulse is modified from Gaussian to uniform to better match the requirements for obtaining a low emittance from the gun. Next, two crystals triple the frequency of the light to a wavelength of $260 \mathrm{~nm}$. The flattened pulse also improves efficiency and uniformity in this harmonic-generation process.

Finally, the beam is transported through an evacuated tube to an optics platform next to the gun. Since the Fourier-relay image plane that follows the long transport tube has a spot size that is too small for the photocathode, the spot is magnified by imaging it onto a circular aperture that slightly trims the edge of the beam. This aperture is in turn imaged onto the photocathode, so that the illuminated region of the photocathode is precisely defined without jitter. The imaging includes compensation (discussed below) for the temporal and spatial distortion caused by grazing incidence on the photocathode.

The energy management of the laser system, also indicated in Figure 6.14, is as follows: transmission through the spatial flattener is $50-75 \%$ (with $\sim 100 \%$ a real possibility using the aspheric optics mentioned in Section 6.4.4, Spatial Pulse Shaping), through the compressor 50$65 \%$, through the frequency tripling stage $10-15 \% \%$, and through the optical transport to the gun $50 \%$ for an average overall IR to UV efficiency of $2.7 \%$. Consequently, starting with $18 \mathrm{~mJ}$ after the second amplifier, the required $500 \mu \mathrm{J}$ is delivered to the cathode.

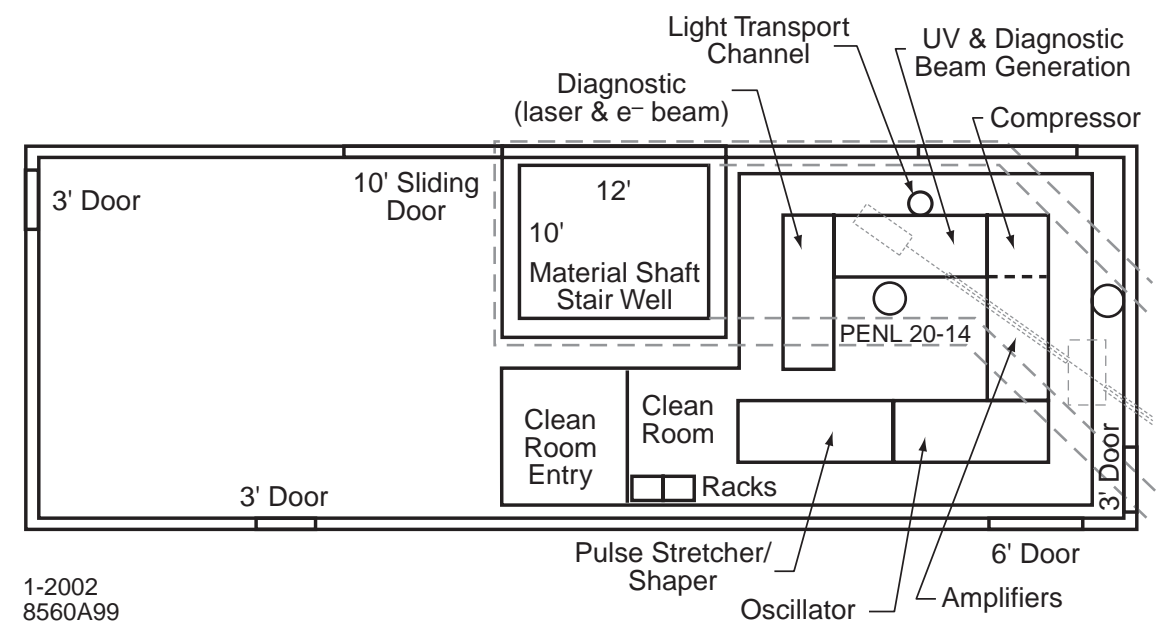

Figure 6.15 The surface building alongside the Klystron Gallery at Sector 20 showing the layout of the laser clean room. The injector vault below is dashed in.

The design actually calls for two complete laser systems. The second system will be a ready spare and also allow implementation of upgrades and conduction of short performance tests while the LCLS is operating with the first system. Many of the advanced features of the laser will need commissioning and no doubt will undergo improvements as time goes on. Meanwhile, light to the photocathode must be delivered to begin commissioning the rest of LCLS. The clean room for the 
laser, shown in Figure 6.15, will have optical tables for the two systems, so that one laser can deliver beam while the other is on standby, being repaired, or being used for further development. To provide for enhanced system reliability, the two lasers will be set up so that either will be able to send light to the photocathode in the vault below. An additional table provides space for shared diagnostics, like a streak camera.

\subsubsection{Temporal Pulse Shaping}

The spatial and temporal shape of the optical (and thus also the electron) pulse is nominally Gaussian. However simulations indicate that an emittance-compensated beam at the exit of the injector will have a lower transverse emittance if uniform temporal and spatial distributions of charge are extracted at the photocathode. As the beam makes its way through the linacs and compressors, simulations indicate that the bunch length at the entrance to the undulator is more sensitive to timing jitter when the beam starts with a uniform rather than a Gaussian temporal shape. To permit the injector pulse shape to be optimized for the lowest possible emittance, a short $\mathrm{X}$-band accelerating section is introduced just before $\mathrm{BC} 1$ to linearize the compression independently of the L0 pulse shape. (See Section 7.2.3, X-band RF Compensation.) However, since an experimental variation of the pulse shape will be needed to establish the final optimized configuration, the capability to shape (including a uniform shape) the temporal profile of the laser pulse is built into the system.

Temporal shaping of the optical pulse will be accomplished through the well-established technique of frequency-domain pulse shaping [38], which takes advantage of the large bandwidth of ultrafast laser pulses. The electric field of an ultra-short laser pulse with a Gaussian temporal envelope can be written as

$$
E(t)=E_{0} e^{j \omega_{0} t} e^{-(t / \sqrt{2} \tau)^{2}} .
$$

The pulse bandwidth is determined by the Fourier transform of this field, where the Fourier transform is

$$
E(\omega)=\frac{1}{\sqrt{2 \pi}} \int_{-\infty}^{+\infty} E(t) e^{-j \omega t} d t
$$

The power spectrum is then

$$
E^{2}(\omega)=E_{0}^{2} \tau^{2} e^{-\left(\omega_{0}-\omega\right)^{2} \tau^{2}}
$$

where $\tau$ is the $1 / e$ half width of the laser pulse intensity. For a Gaussian distribution of locked oscillator modes, the spectral full width at half maximum, $\Delta \omega$, is related to $\tau$ as $\Delta \omega \approx 1.67 / \tau$. Thus the shorter the pulse the larger is the spectral bandwidth.

The frequency spectrum is dispersed in space between a pair of diffraction gratings separated by a pair of lenses. Relay imaging between the gratings avoids introducing time dispersion in this section. Spatially resolved amplitude and phase masks at the dispersion plane modify the Fourier 
transform of the laser pulse and permit any pulse shape allowed by the bandwidth to be produced. In principle, one can make a square pulse with a rise time equal to the pulse duration of the original pulse. In Figure 6.14, the gratings and masks for pulse shaping are located between the oscillator and the first amplifier stage. This arrangement reduces the possibility of damage to the pulse shaping optics. The pulse shape will subsequently be modified by the gain properties of the amplifiers and the frequency conversion process. Thus, the Fourier transform produced by the masks and gratings must take these changes into account. To accomplish this, the amplitude and/or phase masks will be made with computer-addressable liquid-crystal optics [39]. Linear liquid-crystal arrays designed for this purpose have become commercially available [40]. Pulseshape measurements then allow feedback on the mask configuration, modifying it to produce the desired shape. Thus, the effects of frequency conversion and gain shaping will be readily taken into account, and it will be relatively simple to change the pulse shape if another is found to be advantageous.

\subsubsection{Fourier Relay Optics}

A technique known as Fourier relay imaging combines relay imaging, in which lenses form an image of an initial aperture at each pass through a subsequent amplifier or harmonicgeneration crystal, with the filtering of the beam's spatial Fourier transform. This approach can maintain a clean transverse mode and improve pointing stability, while also achieving better utilization of the pump energy. Initially, the oscillator beam is trimmed in an aperture. At each step a lens of focal length $f_{1}$ is placed a distance $f_{1}$ after one of the image planes. The Fourier transform is formed at the focus $f_{1}$ beyond the lens, where a pinhole removes higher spatial harmonics. A second lens with focal length $f_{2}$ then recollimates the beam (with magnification $\left.f_{2} / f_{1}\right)$ and forms the relay image at a distance $2\left(f_{1}+f_{2}\right)$ from the previous image plane. Similar imaging takes place at the harmonic-generation crystals, and finally an image of the aperture is relayed to the photocathode to define the area of photoemission. The relay lenses and pinholes are indicated in Figure 6.14.

\subsubsection{Spatial Pulse Shaping}

To shape the pulse in space, a position-dependent attenuation will be applied to the beam [41]. The demonstrated use of low loss aspheric refractive optics to flatten Gaussian profiles with $\sim 100 \%$ efficiency also shows promise [42]. Relay imaging must be used after the flattening, to limit diffraction. Since repeated filtering of the spatial Fourier transform would limit the steepness of a flat-top output pulse, Fourier filtering is not incorporated in the imaging after the spatial shaper. Also, flattening is generally lossy, and so the system must have sufficient gain. On the other hand, harmonic generation can be more efficient with a uniform intensity across the beam.

\subsubsection{Frequency Conversion}

The $780-\mathrm{nm}$ pulses will be frequency tripled to $260 \mathrm{~nm}$ in a pair of frequency conversion crystals using Type-II-Type-II tripling [43]. In this polarization-mismatch scheme, the beam is 
detuned from optimal conversion in the first crystal in order to allow efficient conversion to the third harmonic in the second. In Type II doubling, the beam is typically incident with a polarization angle of $45^{\circ}$ with respect to the e- and o-axes. For polarization mismatch, the beam is polarized at $35^{\circ}$ with respect to the o-axis, allowing approximately $50 \%$ conversion to the second harmonic. The unconverted fundamental beam is mixed with the converted beam in the second crystal. Frequency conversion efficiencies in excess of $50 \%$ to the third harmonic have been measured with picosecond 1- $\mu$ m laser pulses. A pair of $\mathrm{BBO}$ (beta- $\mathrm{BaB}_{2} \mathrm{O}_{4}$ ) crystals with phase matching angles of $42^{\circ}$ and $54^{\circ}$ respectively is proposed. The conservative energy estimates of Figure 6.14 assume a day-to-day tripling efficiency of $25 \%$, although twice this efficiency should be achievable.

In general, the intensity and wavelength dependence of frequency conversion can give rise to pulse distortion. However, frequency conversion will maintain the shape of our temporally and spatially uniform pulse (a cylindrical slug), and will allow the conversion efficiency to be optimized. The nonlinearity of the process can also sharpen the edges. On the other hand, an initial non-uniform shape will be distorted during conversion, and this must be accounted for in generating the input pulse shape. In addition, any structure on the pulse-ripples in time or space - can grow during the conversion process, again due to the nonlinearity [44]. This means that the constraints on the spatial and temporal uniformity before conversion will be more severe than those required at the photocathode.

\subsubsection{Grazing Incidence}

In the rf photocathode guns developed by the Brookhaven-SLAC-UCLA collaboration, the laser can be incident on the photocathode at either normal or near-grazing incidence $\left(72^{\circ}\right.$ from the normal). Measurements at SLAC [45] using grazing incidence, in which the UV light was changed from $p$-polarized (electric field nearly normal to the surface) to $s$-polarized ( $E$ parallel to the surface), have shown 5 to 6 times more photoemission for $p$. Part of this improvement, a factor of 2.5, is due to the lower reflectivity for $p$; the balance is attributed to the Schottky effect. Since the reflectivity is enhanced for normal incidence, the emission should be about half when compared to grazing incidence with $p$ polarization. Similar work at UCLA [46] showed the same effect, but with somewhat lower enhancement due perhaps to a lower rf field in the gun. An additional advantage of grazing incidence is that there is no need to insert a laser mirror directly downstream from the gun, right next to the electron-beam path, where it is a potential obstacle and a source of wakefields. The LCLS gun design allows either method.

However, grazing incidence introduces two geometric difficulties: a circular laser beam incident at a grazing angle illuminates an elliptical spot on the cathode. Also, if the spot is millimeters across, the side closer to the laser entry will emit picoseconds earlier than the other side. Corrections for both of these effects are needed to minimize emittance. The elliptical spot is made circular in the last relay of the beam in Figure 6.14, from the final image plane to the cathode. Here, the light reflects from a diffraction grating with a groove spacing and angle of 
incidence chosen to apply a compensating anamorphic magnification (different horizontally and vertically) and so illuminate a circular area.

A second constraint on the groove spacing and incidence angle provides a simultaneous correction of the time slew by adding a delay that varies across the beam (that is, by tilting the wavefront). By placing the grating in the beam near the final image plane, the time delay is correlated with the position across the cathode. Since gratings are lossy in the ultraviolet, care must be taken to make the beam size on the grating large enough to avoid damage.

\subsubsection{Stability of Laser Pulse}

\subsubsection{Pulse-to-Pulse Timing}

If the rms timing jitter of the electron bunch with respect to the rf driving the gun and linac is $1.4 \mathrm{ps}$ measured over a few seconds at $120 \mathrm{~Hz}$, then the energy of the beam in the undulator will vary by $0.1 \%$ rms. See Table 7.4. However, when all sources of energy jitter are taken into account, the rms timing jitter at L0 must be reduced to about 0.9 ps. See Table 7.5. A criterion of $\leq 0.5 \mathrm{ps}$ rms has been adopted for the LCLS photoinjector laser system as indicated in Table 6.3.

Almost all of the laser system's jitter originates in the oscillator. An rms jitter of $\leq 0.5 \mathrm{ps}$ has been measured on advanced commercial oscillators, such as the Spectra-Physics ${ }^{\circledR}$ Tsunami described earlier, or the Time-Bandwidth Products Nd:glass laser used at SLAC's Gun Test Facility. However, while 0.5-ps performance has been measured, the manufacturers have not made this their standard specification; considerable care is necessary to maintain such performance. These lasers use sealed housings, mechanical stabilization of the optical platform inside, and precise electronics to lock the cavity length to an external rf reference. Careful attention must also be paid to isolating the housing thermally, mechanically, and acoustically on the optical table.

In order to assure both short- and long-term stability, the laser system presented here has its timing stabilized twice. The arrangement is illustrated in Figure 6.16. The first technique is incorporated in the commercial oscillators that are being considered. A measurement is made of the laser oscillator's output phase with respect to rf from the accelerator's main rf drive line. The phase-error signal, which is first low-pass filtered and then amplified, drives a piezoelectric translation stage holding the end mirror of the laser oscillator. The oscillator incorporates a passive mode-locker (using a Kerr lens or Fabry-Perot saturable absorber), while the length of the oscillator cavity, initially set up to match a subharmonic of the accelerating frequency, is continuously adjusted to lock the phase of subsequent laser pulses to the rf. The bandwidth of the method is estimated to be in the kilohertz range.

Outside the oscillator cavity, the timing is then corrected for long-term drift. A prism is mounted on a piezo stage with a fast motor to provide an optical-trombone delay for the laser pulses. As shown in the figure, this delay is controlled by a similar phase-error to that used for the stage inside the oscillator, but using $2856 \mathrm{MHz}$ for greater sensitivity. It could additionally use 
the measured phase error of the pulses of electrons or ultraviolet light with respect to the rf, as shown in the figure. Both the piezo and motor are computer controlled. 


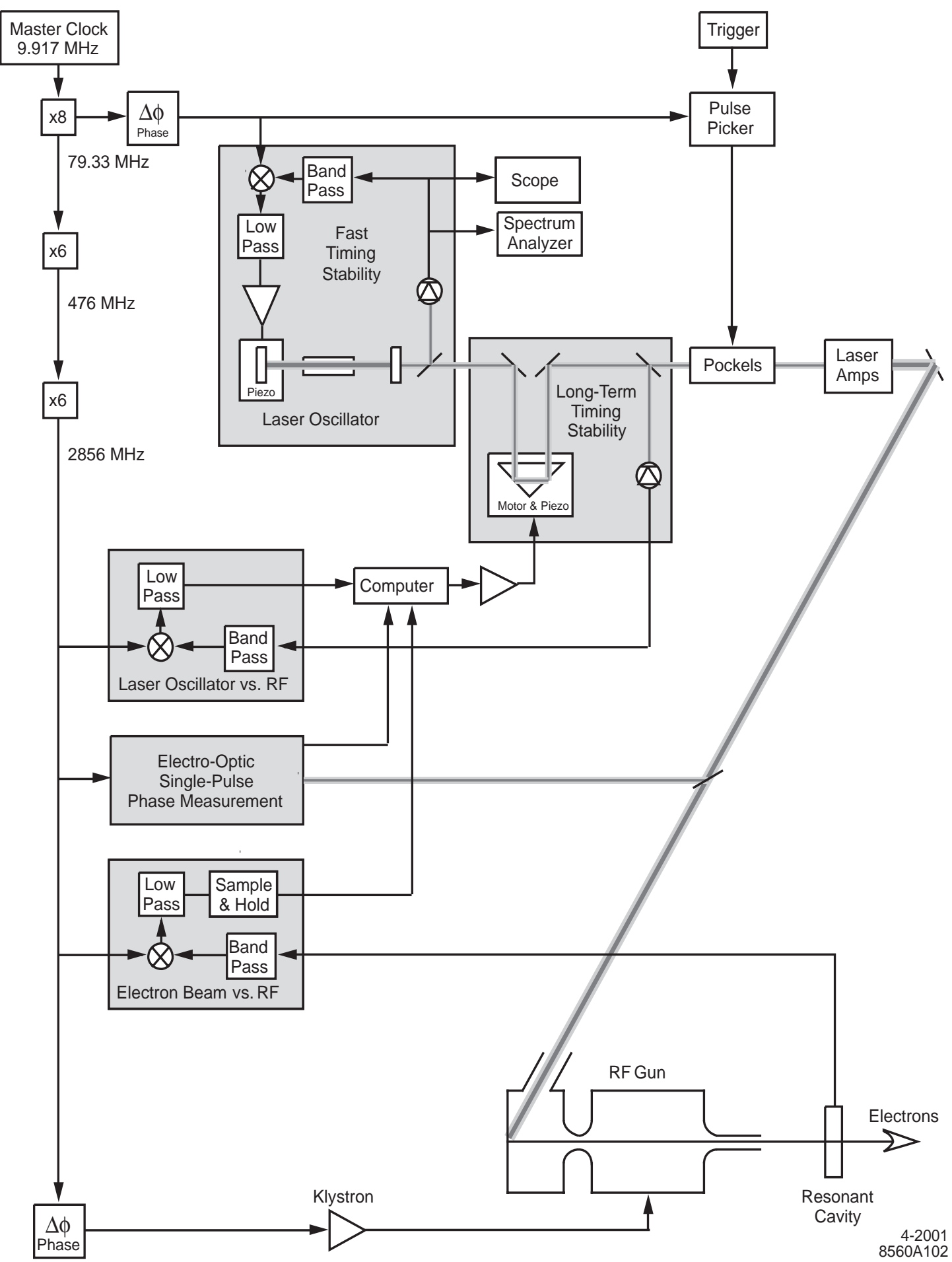

Figure 6.16 Timing stabilization schematic. 


\subsubsection{Pulse Duration}

Like timing jitter, the stability of the pulse duration is important for LCLS performance. The pulse duration should fluctuate by no more than the allowable timing jitter. A stable oscillator is again essential. If the bandwidth of the oscillator pulse is wider than that transmitted by the phase and amplitude masks used to shape the pulse in time, so that the masks are illuminated almost uniformly, then fluctuations in the oscillator width have little effect on the final pulse length or shape. The latter are determined only by the masks and the pulse compressor following the amplifiers.

\subsubsection{Optical Energy}

If the optical energy and thus the charge at the photocathode varies with an rms value of $6 \%$ over a period of a few seconds at $120 \mathrm{~Hz}$, the contribution to the peak charge jitter in the undulator will be at the LCLS limit of $12 \%$. See Table 7.4. However, when all sources of charge jitter are taken into account, the rms optical energy at the cathode is required to be $\leq 2 \%$ (see Table 7.5). Thus a criterion of $\leq 2 \% \mathrm{rms}$ (in the UV) has been adopted as indicated in Table 6.3. Harmonic generation compounds the difficulty of this criterion, since $2 \%$ jitter in the third harmonic requires $0.7 \%$ stability in the fundamental. It is difficult for a Pockels cell to trim the amplitude of a broadband pulse without affecting its temporal shape. Thus the UV energy at the gun is stabilized by using laser-diode pumping in the oscillator, by carefully controlling the beam mode and its pointing through the amplifiers through Fourier relay imaging, and by stabilizing the amplifier pumping with feedback.

The older generation of Ti:sapphire oscillators, both CW and mode locked, were pumped by green light from argon-ion lasers. In the newer generation, these have been replaced by diodepumped, frequency-doubled $\mathrm{Nd}: \mathrm{YVO}_{4}$ lasers, which have far lower noise. With $10 \mathrm{~W}$ of green, the pumps from both Spectra-Physics $\left({ }^{\circledR}\right.$ Millennia Xs) and Coherent $\left({ }^{\circledR}\right.$ Verdi-V10) have a rated noise (above $10 \mathrm{~Hz}$ ) below $0.04 \% \mathrm{rms}$. Most of this performance carries forward to the Ti:sapphire output, although there are some differences in how the manufacturers have tightened their specifications since moving to the new pumps. At the LCLS wavelength, an output power of at least $1.2 \mathrm{~W}$ with $\mathrm{rms}$ noise of $0.1 \%$ or less is expected.

To control the amplifier's pumping, the relatively long upper-state lifetime of Ti:sapphire (3.2 $\mu \mathrm{s}$, long compared to the few-nanosecond duration of the pump pulse) will be used to hold the total pump energy constant on every pulse. The pump beam has an rms jitter of about $2.2 \%$ (for the ${ }^{\circledR}$ Infinity delivering $200 \mathrm{~mJ}$ of green at $60 \mathrm{~Hz}$ ) to $3 \%$ (for the ${ }^{\circledR}$ Quanta-Ray, which can deliver more than $300 \mathrm{~mJ}$ and so provides some "headroom"). These pump lasers have maximum repetition rates of $100 \mathrm{~Hz}$. To obtain 120-Hz pumping for LCLS, we use a polarizer to merge the beams from two frequency-doubled Nd:YAG lasers operating in alternation at $60 \mathrm{~Hz}$ (Figure 6.41). One beam enters with vertical polarization, the other horizontal. After the polarizer, a Pockels cell pulsed at $60 \mathrm{~Hz}$ rotates the vertically polarized beam to create a $120-\mathrm{Hz}$ train with horizontal polarization. (This scheme has the additional feature that a failure of one pump cuts the repetition rate in half, rather than stopping LCLS completely.) The jitter will be corrected by picking off 10- 
$15 \%$ of the beam, enough to correct the jitter, which is then delayed by a 30 ns optical path. This delay gives time to adjust the high voltage on the Pockels-cell of Figure 6.14. Based on a measurement of the energy in the pump pulse, a portion (nominally half) of the delayed light is added back into the pump path, so that the pumping of the Ti:sapphire is trimmed for each pulse by up to $\pm 5 \%$. The pump has a narrow bandwidth, unlike the temporally shaped Ti:sapphire beam, and so it is easily trimmed by the Pockels cell. The full pump beam is not trimmed, because at $50 \%$ transmission a Pockels cell with a polarizer is in the linear part of the control range, while at $95 \%$ the curve is flat and nonlinear; a much larger voltage swing would be needed to effect the same change.

To correct for long-term drift in the UV pulse energy as monitored at the gun, a slower software feedback loop will adjust the set point in the faster feedback loop that stabilizes the amplifier pump energy.

\subsubsection{Spot Size and Position}

To carefully and reproducibly control the distribution of space charge in the gun for optimal emittance, the laser must maintain a $1 \%$ variation in the diameter of the laser spot on the photocathode with a centroid location that varies by no more than $1 \%$ of the diameter. Position stability will be achieved by trimming the edge of the beam with a circular aperture placed on the final relay-image plane before the gun; this aperture is then imaged onto the photocathode. A Gaussian beam could still have fluctuations in the position of its centroid within the aperture, but with the uniform pulse shape preferred for LCLS, pointing jitter does not cause any change in cathode illumination (as long as the full beam-trimming aperture is illuminated, despite the jitter).

\subsubsection{Laser System Diagnostics}

The laser system is designed with an integral diagnostic beam. (See Figure 6.14.) This beam is used to monitor the shot-to-shot amplifier gain and also is used for diagnosing the temporal shape of the UV pulse heading toward the photocathode. To obtain the diagnostic beam (narrowly spaced dashed line in the figure), a Pockels cell and polarizer gate a second oscillator pulse that follows the primary pulse by tens of nanoseconds. The diagnostic pulse makes only one pass through each of the amplifiers. A photodiode measures its energy at each stage to check the gain. The unstretched (100 fs) diagnostic pulse is then cross-correlated with the UV output pulse (3 $10 \mathrm{ps}$ ) to measure the pulse shape.

\subsubsection{Cross-correlation Pulse Shape}

The advantage of using a diagnostic beam for a cross-correlation measurement of the UV pulse is that the diagnostic beam retains the original 100-fs duration of the seed beam and so provides a comparable temporal resolution. Cross-correlation provides more information than an auto-correlation because the latter cannot distinguish temporal asymmetries. The diagnostic pulse will be chosen to arrive at a cross-correlator at the same time as a fraction of the primary pulse picked off by a beam splitter. There are a number of techniques for measuring the crosscorrelation of an infrared and UV pulse. It is anticipated that a single-shot polarization-gating 
cross-correlator [47] will be used. This will generate a third-order intensity cross-correlation of the 100-fs, 780-nm IR pulse and the 10-ps UV pulse. If the IR pulse is used as the gating pulse, the measured pulse shape is that of the UV pulse with a temporal resolution of approximately 100 fs. The two pulses are incident nearly collinearly on a nonlinear optic. The UV pulse to be detected is incident on a spatially resolving detector through crossed polarizers. In the absence of a gating pulse, no UV light is detected. Between the polarizers there is a Kerr medium, such as a thin piece of fused silica. When the gate pulse is incident on the Kerr medium, it acts as in instantaneous waveplate, which allows the portion of the UV pulse passing through the same space-time location to pass through the crossed polarizers and be detected. By choosing the crossing angle, detector and crystal size, and appropriate probe-beam energy, the pulse duration can be measured with a resolution approaching the 100-fs duration of the gate pulse. In addition, by following the cross-correlator with a spectrometer, the frequency resolution is improved. This FROG (frequency-resolved optical gating) technique [48] allows both the temporal and phase profiles of the beam to be determined.

The effect of the pulse shaping on the low-energy IR pulse will also be measured using crosscorrelation. In this case, all of the pulses (other than the pulse selected for amplification) from the 79.33-MHz train leaving the shaper and stretcher are selected. After recompression, their shape is measured in the cross-correlator shown in the low-energy area of Figure 6.14. The gating pulse comes from the diagnostic beam picked off before the shaper and stretcher. Again, all of the 79.33-MHz train is used except for the diagnostic pulse. Because both beams entering this crosscorrelator are trains (except for the 120 pulses per second selected by the Pockels cells), it can use a simpler swept time delay to scan the overlap of the pulse trains, rather than the single-shot approach of the output cross-correlator, where different time delays occur at different spatial locations.

\subsubsection{Energy}

The energy will be monitored using joulemeter probes in combination with calibrated pickoffs at several points in the system: after each amplifier stage, after each harmonic conversion, and just before the beam enters the rf gun's vacuum to strike the photocathode. These checks allow simple monitoring of amplifier and harmonic-generation efficiency. Photodiodes will be used at other points where the pulse energy will be low.

\subsubsection{Spatial Shape}

The spatial shape of the beam on the photocathode will be monitored by picking off a fraction of the beam near the window leading into the gun. A CCD (without the usual protective glass cover, since it would block the UV laser light), placed at the pick-off image plane and at the same angle to the beam as the cathode surface, would then image the beam spot. (The pick-off image plane is optically the same distance away as the cathode but physically located outside the highradiation area using an imaging fiber optic relay.) Typical CCDs are 4 to $9 \mathrm{~mm}$ wide, a good match for the spot needed on the cathode. For a grazing angle on the cathode, it is preferable to get a CCD on a printed circuit board rather than in a camera body since the body blocks the 
correct angle. Alternatively, the UV could be incident on a fluorescing surface at the correct angle, and a CCD camera could record the visible glow; however, the response may be somewhat less uniform than that of a direct hit on the CCD. Other CCDs check the beam's transverse mode after each amplifier and at each harmonic-generation step.

\subsubsection{Stability of Spot Centroid}

The same CCD at the gun will measure the stability of the spot centroid on the photocathode. A computer with a digital frame grabber will record the video image, calculate the centroid location, and keep statistics on its stability. For each laser pulse, the centroid will be calculated to better than one pixel, which is typically 8 to $13 \mu \mathrm{m}$, about $1 \%$ of the typical $1.0-\mathrm{mm}$ beam radius (hard edge). The mean and standard deviation can be calculated with even higher accuracy.

\subsubsection{Timing Jitter}

Most of the timing jitter is introduced in the laser oscillator. To measure it, some light is picked off with a fast photodiode just after the oscillator, as shown in Figure 6.16. Such diodes are available with rise times down to $7 \mathrm{ps}$. Time-domain measurements, using an equivalent-time sampling oscilloscope triggered by the rf of the gun, are limited to $2-3$ ps resolution. In the frequency domain, the same photodiode pulse can be the input to a spectrum analyzer. The timing jitter can be determined from the differences of this spectrum at high and low harmonics using well known techniques [49]. Finally one can mix this diode signal with rf (as already done for the piezo controlling the oscillator's end mirror). The phase error (DC) can then be measured with an ordinary oscilloscope, studied in a spectrum analyzer to identify possible noise sources with narrow frequencies, and ultimately recorded by the accelerator control system.

A measurement of the laser pulse jitter with respect to the arrival of the electron beam itself is also possible at a BPM or resonant cavity in the beam-line near the gun exit. In a similar fashion the timing jitter of the electron beam itself with the rf can be also estimated as shown in Figure 6.16.

\subsection{Electron Beamline}

\subsubsection{System Description}

The overall layout of the photoinjector system in the context of the injector vault and accelerator housing is shown in Figure 6.17. The electron beamline consists of the rf gun (see Section 6.3), Linac 0 (L0, also referred to as the booster accelerator), and the Matching Section. The gun is followed by an emittance compensating solenoid. Linac 0 consists of two SLAC-type 3-m S-band accelerator sections separated by a drift space. A solenoid is wrapped around the first section, L0-1. (See Section 6.1.3, Design Principles.) Linac 0 is followed by a Matching Section (MS) that brings the beam from the injector vault to Linac 1 (L1) in the accelerator housing. The optical design of the MS, also referred to as "dog-leg" 1 (DL1), is described in Section 7.5.1, Low-Energy Dog-Leg. The design accommodates various diagnostics that are discussed in the following subsections. 
Figure 6.17 also shows the new radiation shielding separating the injector vault from the linac housing. The injector personnel protection system is designed to allow access to the injector when the linac is operating (including $50-\mathrm{GeV}$ beams), but electron beams in the injector cannot be run when the main linac is in permitted access. (See Section 6.7, Radiation Protection Issues.) Turning off the first MS bend magnet allows independent operation of the injector and main linac. In this situation, a spectrometer dipole in the aisle just beyond the main linac bends the injector beam into a beam dump, while the main linac supplies beam to the Research Yard (End Station A, etc.).

A schematic layout (not to scale) showing only the principal beamline elements is shown in Figure 6.18. The diagnostics are discussed in Section 6.5.2, Standard Beamline Diagnostics. Because of the unique nature of the LCLS photoinjector, there is also the need for several stateof-the art diagnostics. These are described in Secs. 6.5.3, Slice Emittance, and 6.5.4, Temporal Pulse Shape.

The rf distribution system is also shown in Figure 6.18. UnSLEDed klystrons will be used to improve the phase and amplitude stability. The accelerating sections will operate at about 25 $\mathrm{MeV} / \mathrm{m}$ (see Section 6.6, PARMELA Simulations). The electron beam energy increment, $E$ in units of MeV, in a standard SLAC 3-m section installed in the 3-km linac, is

$$
E[M e V]=10 \sqrt{P[M W]},
$$

where $P$ is the klystron rf power at the klystron in units of MW. Thus the gun and each of the two 3-m sections require their own 5045 klystron. The $\mathrm{rf}$ deflector will also require a separate klystron. This arrangement will allow the rf phase and amplitude for the rf gun, the two sections, and the rf deflector to be controlled independently using low-power controls. 


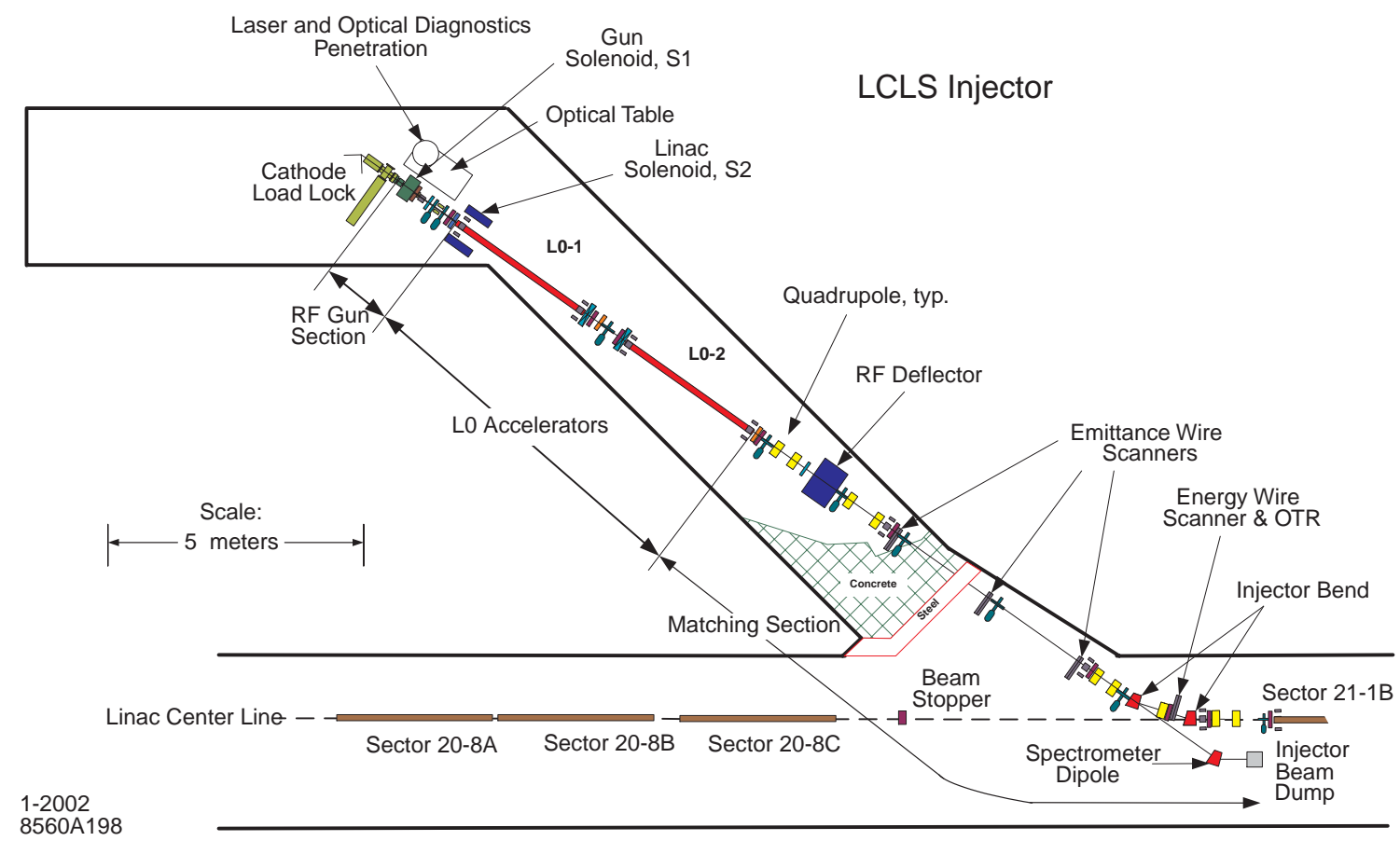

Figure 6.17 Scaled layout of the LCLS photoinjector in the Sector 20 off-axis injector vault of the SLAC 3-km linac. The rf gun and booster accelerator are at 350 to the main linac axis. This angle maximizes the inj ector length and gives access around the cathode load-lock.

Klystron 21-1 will not be needed for L1 since the corresponding accelerator sections will be permanently removed for Bunch Compressor 1 (BC1). The rf from this klystron will be rerouted to the rf gun. Klystrons 20-6, 20-7 and 20-8 are downstream of the positron source and thus are not used by PEP-II. During LCLS operation their rf will be rerouted to the $\mathrm{rf}$ deflector and to sections L0-1 and -2 respectively. Thus no new klystrons are needed for the injector.

The voltage for the modulators in Sectors 19 and 20 are presently controlled through the same variable voltage substation (VVS). The existing Personnel Protection System (PPS) works through this VVS. For the LCLS injector, a contactor could be added to each of the 3 modulators. These contactors could be used satisfy the PPS requirements for the LCLS injector vault in a manner similar to the linac injector vault at Sector 0 and the compressor klystrons for the Damping Rings. However, if part of Sector 20 is run on a time slot for PEP and the other part on a time slot for LCLS, there may be some jitter on LCLS modulators based on where PEP fires. An independent VVS for L0 will greatly reduce this jitter. An independent VVS negates the need for adding contactors to the existing VVS for Sectors 19 and 20. 

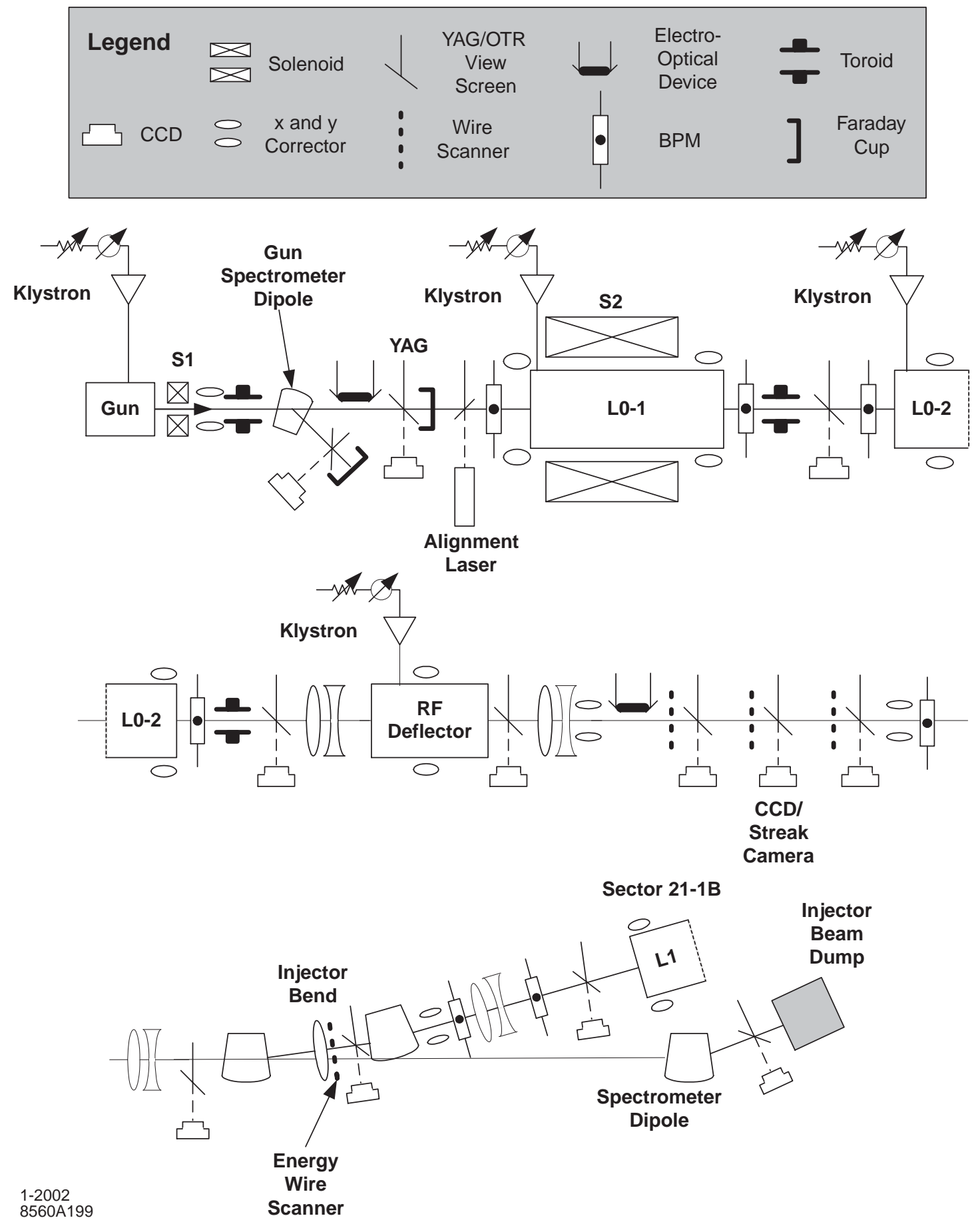

Figure 6.18 Schematic layout (not to scale) showing only the principal beamline elements, the location of diagnostics, and the rf distribution system. Not all BPMs are shown. The Matching Section (MS) begins at the end of the second accelerating section (L0-2). The photoinjector, which includes the MS, ends at the entrance to the Linac 1 (L1). 


\subsubsection{Standard Beamline Diagnostic Devices}

This section discusses diagnostic devices along the injector section of the beamline. The discussion follows the beam from low to high energy, and describes the key measurements to be made in the drifts spaces between accelerating structures. The devices are shown in Figure 6.18, which is schematic and has no consistent scale. For a scaled perspective of the space and distances discussed below, refer to Figure 6.17.

The photocathode gun is followed immediately by the gun solenoid S1. There is an approximate one-meter drift space between S1 and the linac section L0-1. The diagnostics in this area monitor gun performance and characterize the electron bunch launched from the photocathode. Bunch charge is measured with a toroid, and an electro-optic (EO) device measures the charge distribution in the time domain. (The EO device, an advanced diagnostic, is the topic of Section 6.5.4, Temporal Pulse Shape.) The charge distribution projected on the plane transverse to beam propagation is observed by inserting a YAG screen into the beam path and imaging the resultant optical profile with a CCD camera. A Faraday cup integrated into the design of the retractable screen allows a simultaneous measurement of bunch charge. Beam energy and energy spread are measured with a dipole spectrometer, which deflects the beam to a low-energy dump. The spectrometer is an important diagnostic for establishing proper $\mathrm{rf}$ gun performance and tuning. Transport devices in this section of the beamline include steering coils and BPMs. An alignment laser is used to establish a reference line for view screens along the downstream injector beamline.

The drift space between linac sections L0-1 and L0-2 is one-meter long. A toroid measures charge, and a view screen is available for observing the transverse profile of the bunch. Beam position monitors and steering coils at the exit/entrance of the linac sections are used for beam alignment.

The diagnostic and transport elements in the Matching Section, which begins at the exit of the L0-2 linac, are used to match the $150 \mathrm{MeV}$ injector beam through the bend and into the main linac, starting with L1. A properly matched beam has constraints on its transverse and longitudinal phase-space distributions. The transverse beam parameters, including the emittance, are obtained from beam profiles on the three upstream wire scanners. The wire scanner in the injector bend measures energy spread. The field strengths of the quadrupoles and the placement of the wire scanners are optimized for these measurements, which can be performed during normal beam operations. The longitudinal emittance and its more complicated $\Delta \mathrm{E}-\Delta \mathrm{t}$ phase-space distribution are determined using the rf deflector cavity, which imparts a time-dependent, vertical kick to the beam. After an extended drift, an image of the beam in the injector bend using the wire scanner or view screen, or alternatively in the injector dump line (see below) using the view screen, gives a direct representation of the longitudinal phase space density (energy and time on perpendicular axes). This information is key to optimizing the injector and understanding the performance of the downstream compressors. 
The Matching Section ends at the main linac section L1. The photoinjector can be operated independently of the main linac by turning off the injector bend and delivering the beam to the injector dump. This dump will be used when commissioning the injector, and for troubleshooting and injector studies. A spectrometer dipole on this alternative beam path allows full longitudinal beam analysis when running in this mode.

The rf deflecter allows the longitudinal and horizontal phase space, including the slice energy spread and slice horizontal emittance, to be measured at $150 \mathrm{MeV}$. See Section 7.8.2, Bunch Length Diagnostics. Because of their important effect on FEL performance, emittance and energy spread are also measured at several other locations along the path to the undulator. See Section 7.8.1, Tranverse Emittance Diagnostics, and Section 7.8.3, Beam Energy Spread Diagnostics, for further discussion.

Figure 6.18 also shows an electro-optic device and a streak camera in the Matching Section. These devices measure the temporal dependence of the charge distribution within the bunch. Slice emittance and EO diagnostics are the topics of the subsections that follow.

\subsubsection{Slice Emittance}

The wire-scanners described in Section 7.8.1, Transverse Emittance Diagnostics, are used to measure the projected transverse emittance of the full electron bunch. However, it is primarily the so-called "slice emittance," the (transverse) emittance of electrons in axial slices that are only a fraction of the full bunch length, that determines the performance of a SASE FEL. The slice scale of interest for a SASE FEL is the slippage length in the undulator, which for the LCLS is $0.5 \mu \mathrm{m}$ or $1 / 150^{\text {th }}$ of the FWHM length of the compressed bunch at the end of the linac. The length of an equivalent slice at the injector is about $100 \mathrm{fs}$. Multi-particle simulations of photoinjector beams indicate that within the bunch, the axial variation in the transverse space charge force causes a smooth, non-filamented dilution in phase space density, with concomitant full-bunch emittance growth relative to slice values [7]. The injector diagnostics will only be capable of measuring emittances for slices with lengths on the order of a couple picoseconds. Strategies for minimizing or reversing in the final beam the correlated emittance growth have received considerable attention, as in Section 6.1.2, Emittance Compensation

Two methods to measure the slice emittance will be available in the MS: one uses the rf deflector, the other a streak camera. As mentioned in Section 6.5.2, Standard Beamline Diagnostic Devices, the horizontal slice emittance can be measured using the rf deflector in combination with the well-established quadrupole scan procedure and straight-ahead wire scanner or view screen.

As demonstrated at LANL [17], a streak camera can be used in combination with a quadrupole scan to measure the slice emittance if the electron beam is sufficiently intense. A quad scan relies on a set of beamwidth measurements obtained as a quadrupole lens is scanned through a range of focal lengths, and yields the three parameters that characterize the region in phase space occupied by the beam. In a typical application, optical radiation emitted from a screen inserted in the beam path is imaged onto a light sensitive detector, and a beam width 
measurement is derived from the spatial dependence of the image intensity. Metal screens are typically prompt (subpicosecond) emitters of optical transition radiation (OTR), making them ideal light sources for preserving the axial structure (intensity) of the incident electron bunch within the emitted optical pulse. An apparatus or procedure capable of resolving both axial and transverse variations in OTR intensity enables a slice emittance measurement. Streak cameras with temporal resolution better than two picoseconds have been available for some time. In a slice emittance application, an image of a line segment on the OTR screen is made at the narrow $(20 \mu \mathrm{m})$ slit entrance to the streak camera. Preserved in the OTR pulse, the streak tube output displays on its horizontal axis the electron beam intensity along this line segment, and on its vertical axis the temporal dependence of this intensity. A charged coupled device (CCD) image of tube output is ideal for analyzing the beamwidth of different slices, which are represented by some number of adjacent pixel rows.

The feasibility of using a streak camera and OTR optical system as shown in Figure 6.18 as a backup for the rf deflector to measure the slice emittance at $150 \mathrm{MeV}$ is being studied.

\subsubsection{Temporal Pulse Shape}

Features of the photoinjector laser system described in Section 6.4, Laser System, that tailor it for reliable electron production also facilitate applications of the laser to novel electron beam diagnostics. Stable, unconverted laser light (infrared and visible) constitutes a diagnostic beam (probe) for applying electro-optic sampling techniques to the measurement of the temporal shape of the electron bunch. Temporal resolution of sampling measurements is determined by the duration of the probe pulse and its timing jitter relative to the UV pulse (which is used for photoelectron production). Nanosecond delay times can be set with subpicosecond stability for picosecond probe pulses. Probe pulses of millijoule energy are available.

The positive uniaxial crystals $\mathrm{LiNbO}_{3}$ and $\mathrm{LiTaO}_{3}$ are suitable candidates for electro-optic beam sampling. In the linear or Pockels regime, bias fields generated by the electron beam do not alter the crystal anisotropy. In previous work with $16-\mathrm{MeV}$ electrons, wakefield-induced phase retardation in $\mathrm{LiTaO}_{3}$ has been demonstrated with resolution of order $10^{-1}$ radians with wakefield sensitivity of order 1 radian-m/MV [50]. More recent work has demonstrated single-shot bunch shape measurements using a wavelength-chirped laser pulse [51].

The electron beam longitudinal distribution and bunch length will be monitored noninvasively using beam wakefield components as a Pockels-effect bias to induce accumulated phase retardation of a probe pulse as it propagates through the crystal. The wakefield-induced Pockels effect generates a linear response that is determined by wakefield dynamics. In a standard configuration using cross-polarized optics, a null signal is set for zero wakefield amplitude; i.e., when the probe waveform and beam wakefield are not coincident. Incident and transmitted probe pulses are transported to and from the crystal location by polarization-preserving optical fiber. In general, picosecond or nanosecond (i.e., uncompressed) probe durations can be used. In the picosecond case, signals can be scanned by varying the relative probe-beam timing. This 
scanning may not be necessary for the nanosecond case if a fast detector is available as discussed next.

Coincidence of the probe and beam wakefield timing generates a transmitted probe signal proportional to its accumulated retardation phase. This signal can be detected with fast diodes (tens of gigahertz bandwidths) and transient digitizers, or a streak camera, as well as with frequency-resolved optical-gating (FROG) [48]. FROG is an established ultrafast diagnostic, which measures the amplitude and phase history of the transmitted probe waveform with subpicosecond resolution. It is best suited for signals of short (picosecond) and ultrashort (subpicosecond) duration. Known electro-optic coefficients can also be used to estimate wakefield amplitudes from the probe signal.

The noninvasive feature of this diagnostic method affords the use of multiple sampling sites of known spacing for improved measurement of electron beam effects.

\subsection{PARMELA Simulations}

\subsubsection{Initial Conditions}

Emittance growth in the photoinjector from the rf gun through Linac 0 and the Matching Section has been studied using simulations based primarily on Version 3 of the LANLmaintained code PARMELA. The electric field map of the gun was obtained with SUPERFISH and directly used in PARMELA. SUPERFISH was also used to simulate the fields in the traveling-wave accelerating sections, and space harmonics were calculated for use in PARMELA. $\mathrm{RF}$ fields were assumed to be cylindrically symmetric. This is a reasonable assumption since, as discussed in Section 6.3.3, Symmetrization, the dipole rf fields which are normally dominant in an rf gun have been largely eliminated in the prototype gun [10]. Using a 3D map of fields generated with MAFIA, the UCLA version of PARMELA was used to verify that the higher order field components (i.e., quadrupole and higher) make a negligible contribution to the emittance growth in the gun. Work is in progress to study the effect of the dipole field phase component induced by single side rf power flow.

A comparison of PARMELA, both UCLS and LANL versions, with two PIC codes, Magic2D and Maxwell-T, was made to study the representation of image charges on the cathode. The codes agreed to within $20 \%$ on the transverse emittances and space-charge field strengths in the first picoseconds after emission. (Interestingly, both PIC codes estimated lower emittances than either version of PARMELA.)

A magnetic field map for the emittance compensating solenoid at the gun was produced using POISSON and passed to PARMELA. The field generated by POISSON is shown in Figure 6.19. It can be compared to the measured field shown in Figure 6.12. The magnetic field for the air core solenoid around the first accelerating section was modeled in PARMELA using single coils each with appropriate strength to represent the field. 


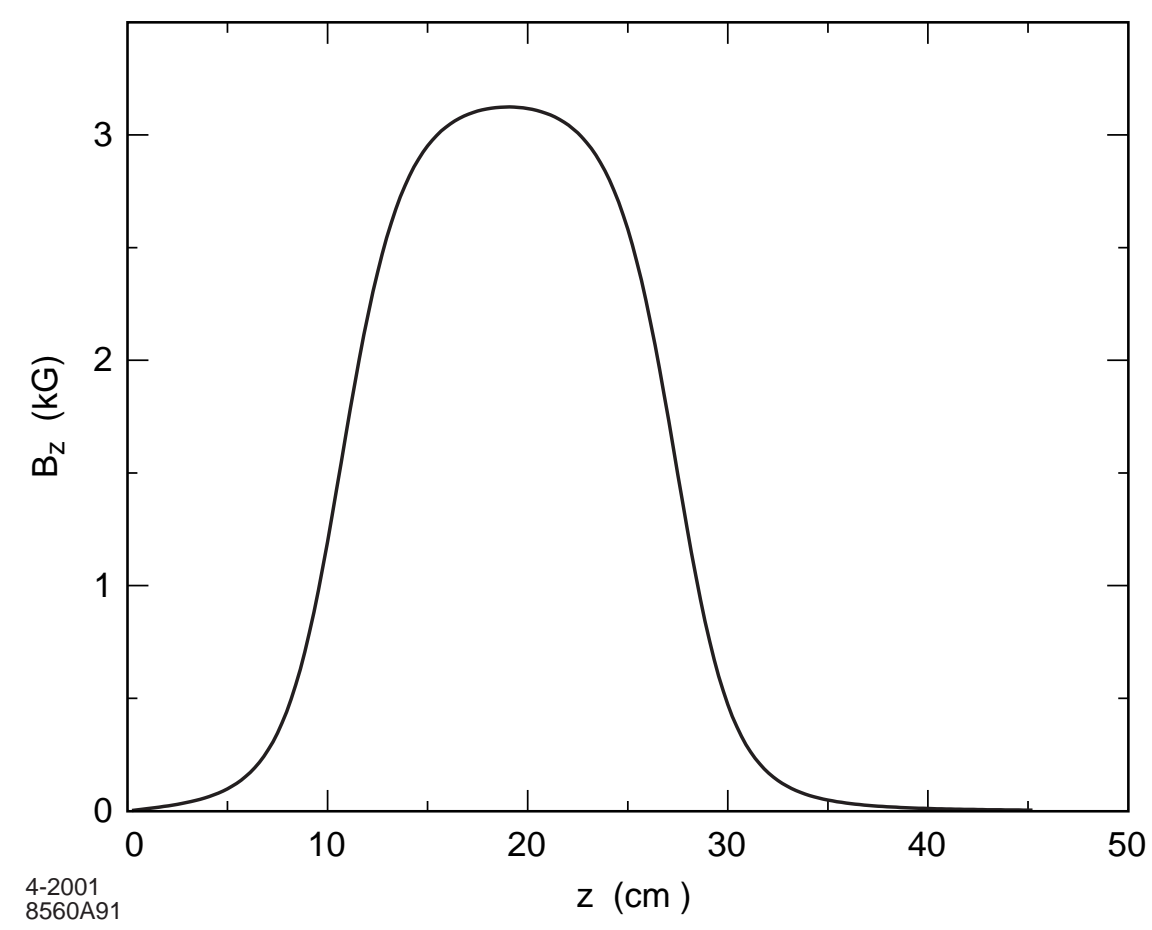

Figure 6.19 Axial magnetic field generated using POISSON of the emittance compensation solenoid as a function of distance $z$ along the axis for excitation current of $221 \mathrm{~A}$. The cathode surface is located at $z=0 \mathrm{~mm}$.

The multi-parameter space including charge, laser spot size, pulse length, solenoid field and accelerating gradient has been explored for tuning the 1.6-cell S-band rf gun beamline using a variety of simulation codes. (See Section 6.1.2, Emittance Compensation.) The overall result is that to produce a beam of the highest possible brightness, a 1-mm radius and 10-ps bunch length is about optimum for $1 \mathrm{nC}$ of charge if the peak rf field at the cathode is $140 \mathrm{MV} / \mathrm{m}$. Nearly identical results can be obtained using $120 \mathrm{MV} / \mathrm{m}$ if the radius is increased to $1.2 \mathrm{~mm}$. It is also clear that using spatial and temporal distributions that are uniform (flat top) rather than Gaussian will improve the resulting transverse emittance. As a practical matter, uniform temporal distributions can only be approximated. Therefore the PARMELA simulations discussed here have usually assumed rise times of $0.35 \mathrm{ps}$ or $0.7 \mathrm{ps}$, which are within the capability of the laser system described in Section 6.4, Laser System. For the PARMELA simulations, the initial temporal uniform distribution was generated by stacking 9(17) Gaussian distributions with an rms width of $0.35(0.7)^{\circ} \mathrm{S}$-band phase, each separated by $0.6(1.1)^{\circ}$ of S-band phase. The resulting temporal bunch shape is shown in Figure 6.20 for a rise time of 0.35 ps and in Figure 6.21 for 0.7 ps. A uniform spatial distribution is assumed for all the PARMELA simulations.

The basic layout of Linac 0 (L0) and the Matching Section (MS) are shown in Figure 6.17. The corresponding input parameters assumed for the PARMELA simulations are summarized in Table 6.4. A first series of simulations was done at $140 \mathrm{MV} / \mathrm{m}$ and injection phase of $32^{\circ}$ and is described in Section 6.6.2, Optimization for $140 \mathrm{MV} / \mathrm{m}$. However, the initial operation of the gun 
is likely to be at $120 \mathrm{MV} / \mathrm{m}$ to avoid $\mathrm{rf}$ breakdown. An optimization for $120 \mathrm{MV} / \mathrm{m}$ is presented in Section 6.6.3, Optimization for $120 \mathrm{MV} / \mathrm{m}$.

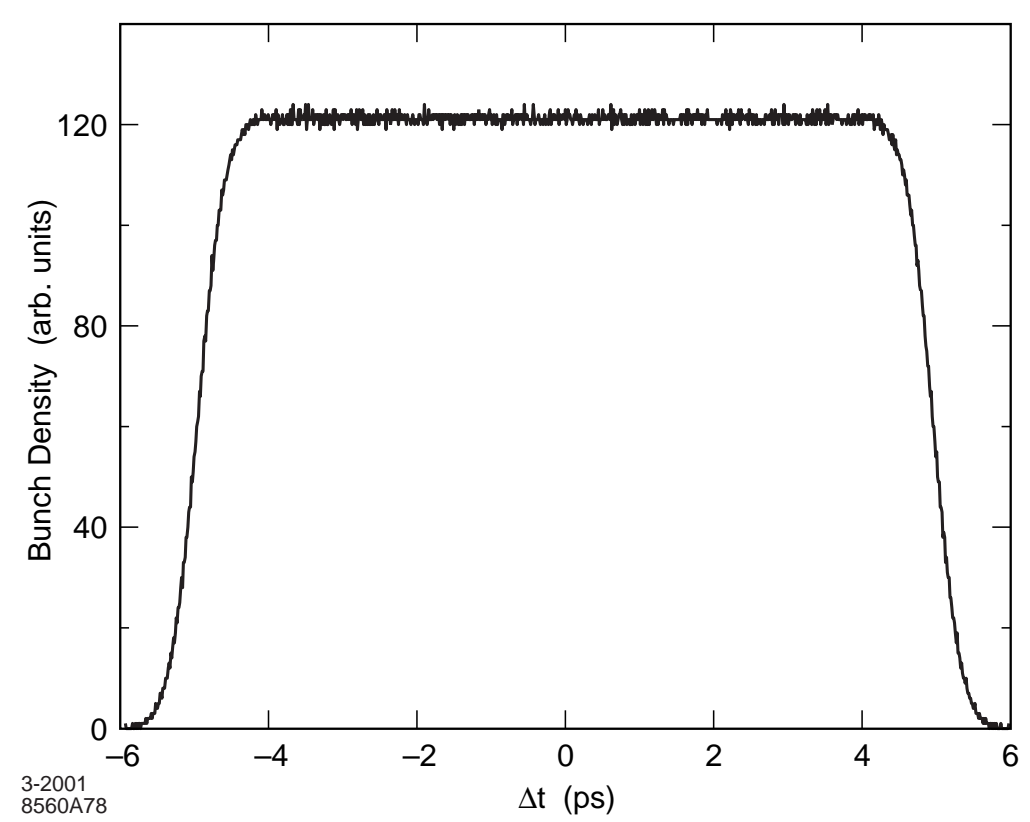

Figure 6.20 Temporal shape of electron pulse used as input for PARMELA simulations for the rf photoinjector. The rise time is about $0.35 \mathrm{ps}$.

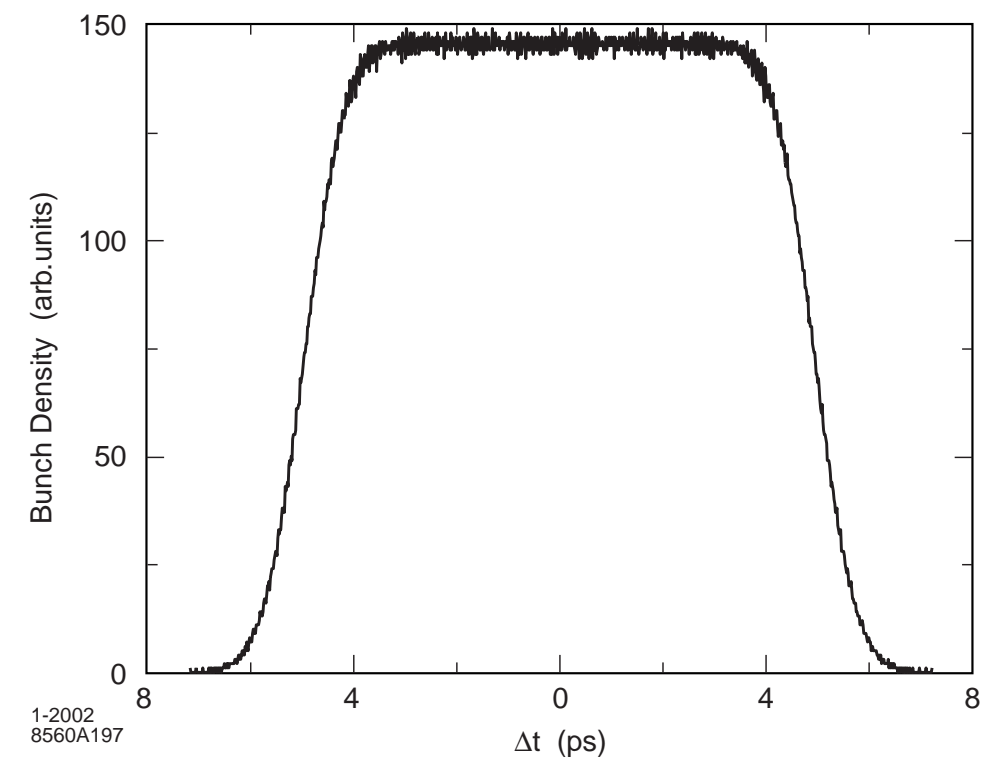

Figure 6.21 Temporal shape of electron pulse used as input for PARMELA simulations for the rf photoinj ector. The rise time is about 0.7 ps. 


\begin{tabular}{|c|c|c|}
\hline Parameter & Value at $140 \mathrm{MV} / \mathrm{m}$ & Value at $120 \mathrm{MV} / \mathrm{m}$ \\
\hline Bunch charge at cathode & $1.0 \mathrm{nC}$ & $1.0 \mathrm{nC}$ \\
\hline Longitudinal charge distribution at cathode & Uniform, rise time 0.35 and $0.7 \mathrm{ps}$ & Uniform, rise time 0.35 and $0.7 \mathrm{ps}$ \\
\hline Transverse charge distr. at cathode & Uniform & Uniform \\
\hline Bunch length at cathode & $2.9 \mathrm{ps} \mathrm{rms}$ & $2.9 \mathrm{ps} \mathrm{rms}$ \\
\hline Bunch radius at cathode & $1 \mathrm{~mm}$ hard edge & $1.2 \mathrm{~mm}$ hard edge \\
\hline Peak rf field at cathode & $140 \mathrm{MV} / \mathrm{m}$ & $120 \mathrm{MV} / \mathrm{m}$ \\
\hline Injection phase & $32^{\circ}$ & $27.3^{\circ}$ \\
\hline \multicolumn{3}{|l|}{ Emittance compensating solenoid: } \\
\hline Axial field & $3.15 \mathrm{kG}$ & $2.71 \mathrm{kG}$ \\
\hline Physical length & $22.5 \mathrm{~cm}$ & $22.5 \mathrm{~cm}$ \\
\hline $\begin{array}{l}\text { Location }{ }^{\mathrm{a}} \text { of peak field (also center of } \\
\text { physical solenoid) }\end{array}$ & $19.1 \mathrm{~cm}$ & $19.1 \mathrm{~cm}$ \\
\hline \multicolumn{3}{|l|}{ Booster accelerator: } \\
\hline Accelerating gradient $\mathrm{L} 0-1$ & $24.1 \mathrm{MV} / \mathrm{m}$ & $18 \mathrm{MV} / \mathrm{m}$ \\
\hline $\begin{array}{l}\text { Location }{ }^{\mathrm{a}} \text { of input coupler for first } \\
\text { Section }\end{array}$ & $1.4 \mathrm{~m}$ & $1.41 \mathrm{~m}$ \\
\hline Accelerating gradient $\mathrm{L} 0-2$ & $24.1 \mathrm{MV} / \mathrm{m}$ & $30.5 \mathrm{MV} / \mathrm{m}$ \\
\hline \multicolumn{3}{|l|}{ Linac focusing solenoid: } \\
\hline Axial field & $-1.75 \mathrm{kG}$ & $-0.75 \mathrm{kG}$ \\
\hline Physical length & $1.0 \mathrm{~m}$ & $0.80 \mathrm{~m}$ \\
\hline Location ${ }^{\mathrm{a}}$ of start of physical solenoid & $1.43 \mathrm{~m}$ & $1.40 \mathrm{~m}$ \\
\hline Location $^{\mathrm{a}}$ of exit of L0 & $7.9 \mathrm{~m}$ & $8.4 \mathrm{~m}$ \\
\hline Thermal emittance, $\varepsilon_{\mathrm{n}, \mathrm{th}}$ & $0.3 / 0.5 \mu \mathrm{m} \mathrm{rms}$ & $0.3 / 0.5 \mu \mathrm{m} \mathrm{rms}$ \\
\hline
\end{tabular}

a With respect to position of cathode. 
The emittance compensating solenoid is physically $22.5 \mathrm{~cm}$ long. For the simulations it is placed against the downstream edge of the rf gun, which results in the center of the solenoidal field being $19.1 \mathrm{~cm}$ from the cathode surface. The field map (Figure 6.19) indicates that for this configuration the field at the cathode is essentially zero without the use of a bucking coil.

\subsubsection{Optimization for $140 \mathrm{MV} / \mathrm{m}$}

Using only the gun, solenoid, and the immediately following drift space (i.e., no booster), a minimum value for the first emittance minimum along the drift was obtained by varying the solenoidal field and beam spot radius. A value of $B_{z}=3.15 \mathrm{kG}$ and hard-edge radius of $1 \mathrm{~mm}$ was found to be optimum. The emittance minimum very nearly coincides with that of the new working point described in Section 6.1.3, Design Principles. The slightly larger value of $B_{Z}$ here is consistent with the solenoid being displaced somewhat downstream because of the physical interference with the gun structure.

Next, including both the boosters (with an accelerating gradient of $24.1 \mathrm{MV} / \mathrm{m}$ ) and the second solenoid (S2), the emittance at the booster exit was minimized by varying the booster locations (keeping the drift distance between the two sections fixed at $0.5 \mathrm{~m}$. The results are summarized in Figure 6.22, which is really a compilation of 12 independent figures. The optimum position of the entrance to the first section was found to be $1.4 \mathrm{~m}$ from the cathode (the $\mathrm{S} 2$ field was $-1.5 \mathrm{kG}$ ). Note that as the booster is moved toward the position for the minimum emittance, the emittance decreases more gently and eventually monotonically, approximating the shape shown in Figure 6.3. Finally the emittance was minimized by varying the field and position of S2. An emittance minimum was found for a field of $-1.75 \mathrm{kG}$ and by positioning the start of the solenoid at $1.43 \mathrm{~m}$ with respect to the cathode. Finally, a thermal emittance of $0.3 \mu \mathrm{m}$ was added to the PARMELA deck [8]. Using these parameters and an input pulse rise time of $0.35 \mathrm{ps}$, a final emittance of $0.8 \mu \mathrm{m}$ was obtained for a $20 \mathrm{~K}$-particle run. See Figure 6.23. Using the same parameters but substituting a rise time of $0.7 \mathrm{ps}$ for the input pulse increased the emittance by about $10 \%$. 


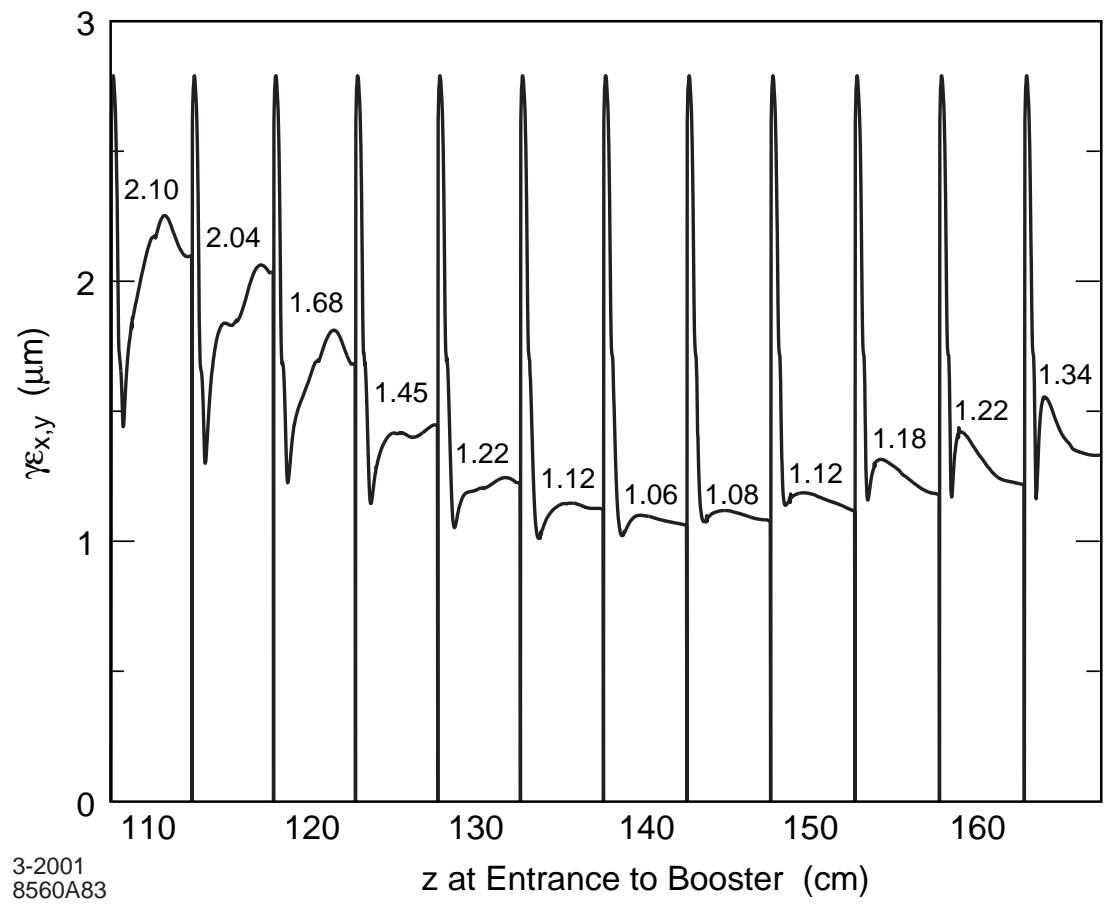

Figure 6.22 Emittance as a function of distance between cathode and booster-entrance is shown for 12 different positions of the booster. The minimum emittance at the booster-exit is indicated for each position. In each case the emittance compensating solenoid is set to $\mathrm{B}_{2}=3.15 \mathrm{kG}$.

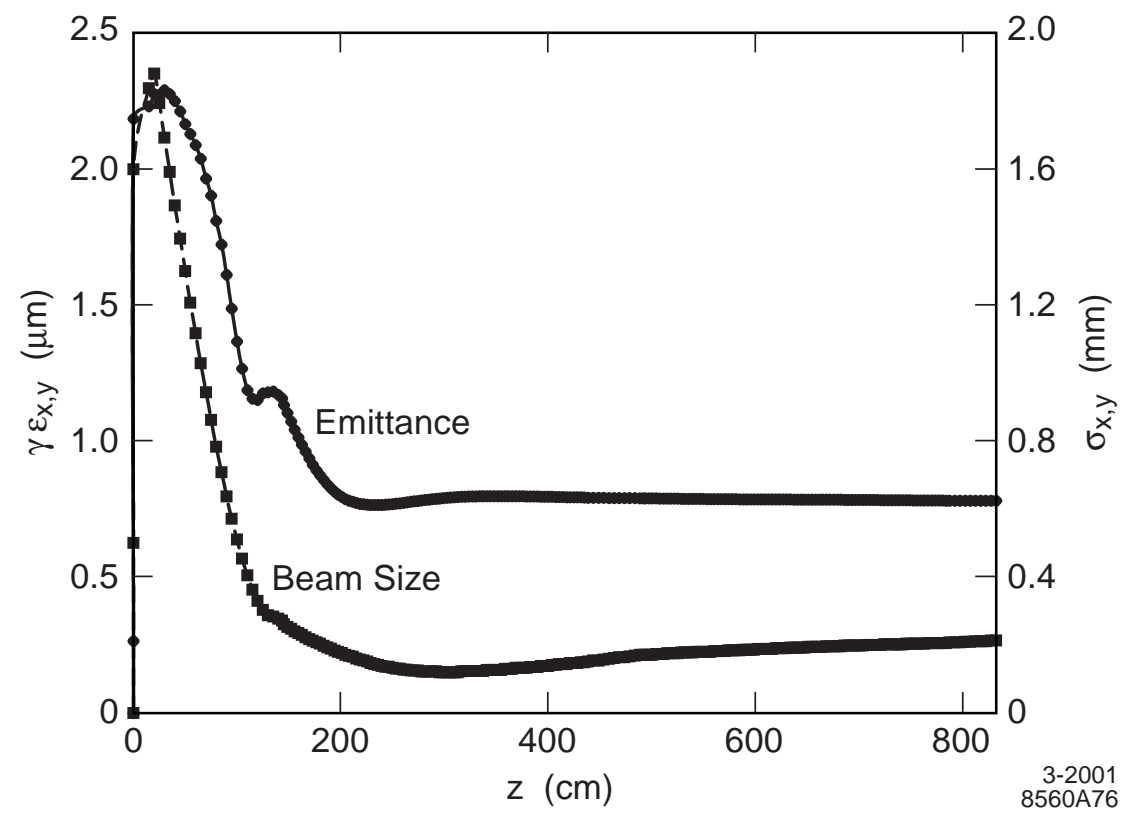

Figure 6.23 Transverse normalized rms emittance as a function of distance from the cathode for 20 $\mathrm{K}$ particles. A rise time of $0.35 \mathrm{ps}$ is assumed. A normalized rms thermal emittance of $0.3 \mu \mathrm{m}$ is included. 
The PARMELA simulation results are summarized in Table 6.5. As indicated therein, there is no significant improvement in the emittance generated by PARMELA as the number of particles tracked is increased beyond $20 \mathrm{~K}$. However, as needed by the algorithm computing the space charge for a 3D geometry, the number of particles was increased to $100 \mathrm{~K}$ to study the evolution of the beam in the Matching Section within which the beam becomes elliptical. The normalized transverse phase space at the exit of L0 for 100K particles is shown in Figure 6.24. The upper left plot is a normalized $x-y$ scatter plot, with $x_{n}$ and $y_{n}$ amplitudes in units of rms beam size. The normalized $x_{n}-x_{n}^{\prime}$ phase space is shown in the upper right with the rms emittance ellipse given by the circle of unity radius in the center. The normalized rms slice emittance in $x$ and $y$, as a function of axial distance along the bunch, is shown in the lower left. The projected value is shown by the horizontal dotted line. In the lower right, the beta-mismatch amplitude, $\zeta$, is shown as a function of $\Delta z$. The beta-mismatch amplitude between the slice Twiss parameters and the projected Twiss parameters is defined by Eq. (7.26) in Section 7.6.1, Electron Beam Evaluation. The mismatch is normalized such that $\zeta \geq 1$. A large variation in $\zeta$ within the bunch, which persists through the linac, may degrade the final FEL gain. 

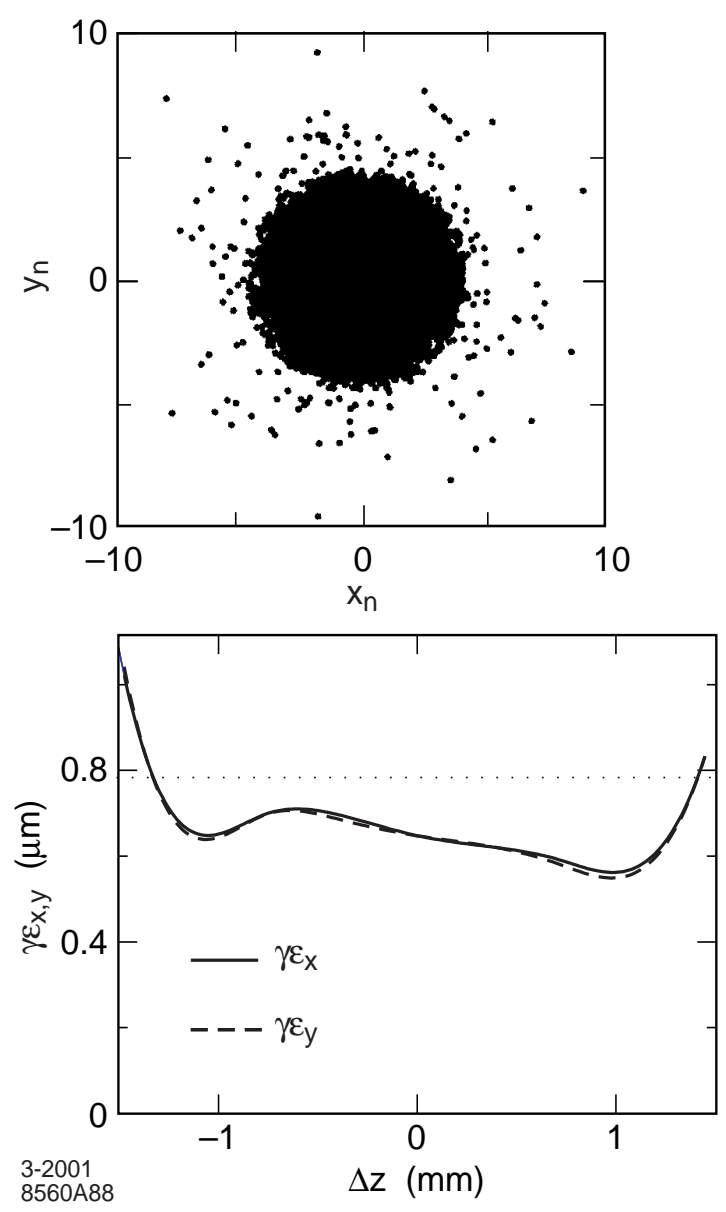
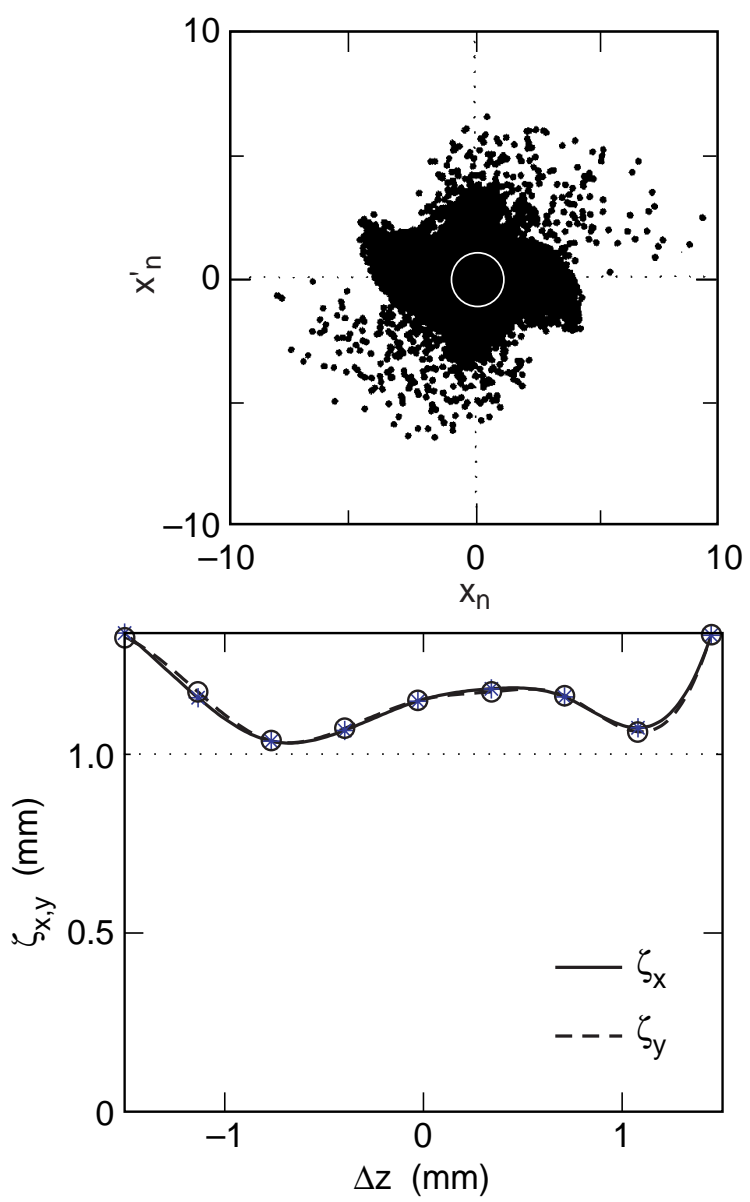

Figure 6.24

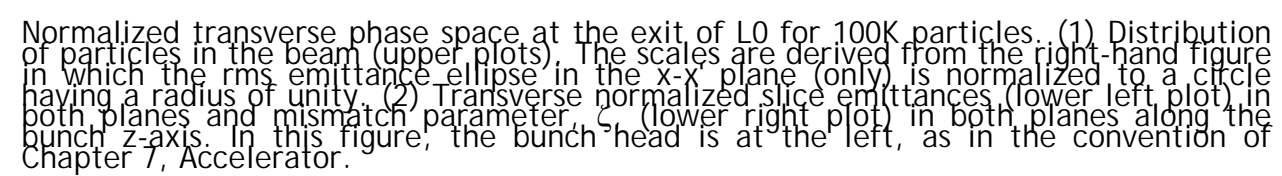

The phase space plots for a series of 9 slices identified in the lower right plot of Figure 6.24 are displayed in Figure 6.25. The blue/dark background in each case is the full projection in the $\mathrm{x}-\mathrm{x}^{\prime}$ plane, identical to the upper right plot of Figure 6.24. The red/light area represents the distribution in the $\mathrm{x}-\mathrm{x}^{\prime}$ plane of the particles in the particular slice. Note that the "halo" (particles outside the main core) occurs primarily in the first (head) and last (tail) slices. 


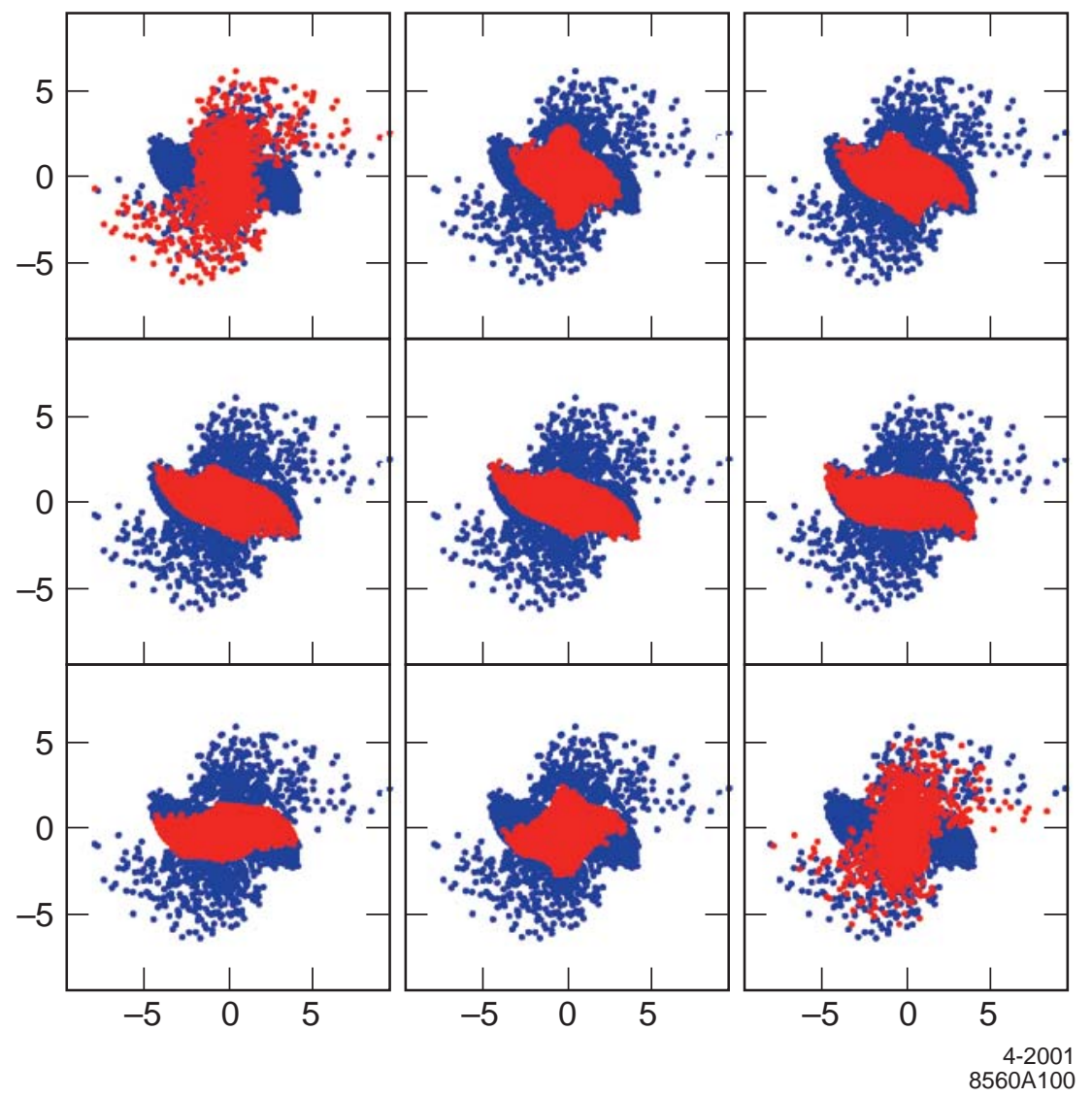

Figure 6.25 Transverse distribution in the $x-x^{\prime}$ plane of particles in a slice (red/light) at the exit of L0 for 100K particles shown against a background of the full projection (blue/dark). Scales are the same as for the upper right plot of Figure 6.24. In each plot read left to right, the location of the slice along the z-axis of the bunch can be identified by the corresponding asterisk in the lower right plot of Figure 6.24.

The longitudinal distribution of particles at the exit of L0 is shown in Figure 6.26. The energy as a function of axial position within the bunch is shown in the upper left with the corresponding particle distributions projected out from both planes shown in the upper right and lower left. The lower right distribution is the same as the lower left, but in terms of peak current instead of number of particles, and time instead of axial position.

The rms energy spread of the distribution shown in the upper right of Figure $\mathbf{6 . 2 6}$ is $\sigma_{\gamma} / \gamma_{o}=0.18 \%$. The L0-2 phase is set such that when the wakefields are included the total energy spread will be minimized. The effects of longitudinal wakefields in the booster are calculated using the 2D simulation code LiTrack (see Section 7.2.5, 2D Tracking Studies) with the PARMELA results at the exit of L0 as input. The rms energy spread in this case is reduced to $0.1 \%$. As indicated in Section 6.1.1, Beam Requirements, a low value of slice energy spread is also desired. The slice energy spread is plotted as a function of axial position in Figure 6.27. It can be seen that for the core of the distribution the slice energy spread is $\sigma_{\gamma}^{\text {slice }} / \gamma_{o}<0.005 \%$. 

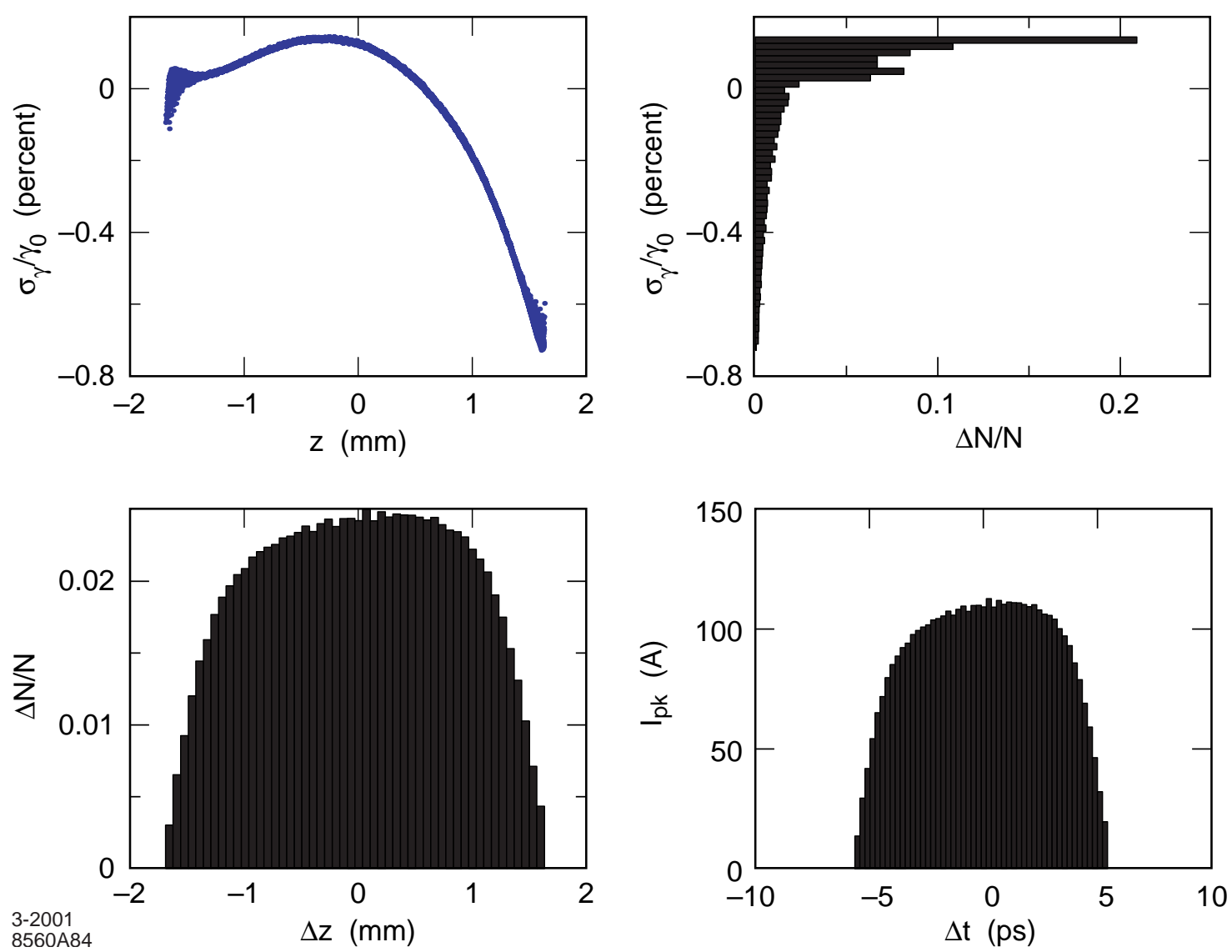

Figure 6.26

Longitudinal distribution of particles in the beam at the exit of $L 0$ for $100 \mathrm{~K}$ particles. In this figure, the bunch head is at the left, as in the convention of Chapter 7, Accelerator.

The Matching Section between L0 and L1 was designed using the simulation code MAD. A plot of the TWISS parameters as a function of axial distance along the beamline is shown in Figure 7.33. It is seen from the figure that the beta function gets very small, which could potentially result in undesirable emittance growth due to the high space charge density at this relatively low energy. To check for this possibility, the PARMELA simulation was extended through the MS. Since the beam size aspect ratio reaches 12, cylindrical symmetry cannot be assumed. The $100 \mathrm{~K}$ particle distribution shown in Figs. 6.24 to 26 was launched into the MS using Version 3 of PARMELA. The resulting particle distribution at the end of the MS (i.e., at the beginning of L1) is shown in Figs. 6.28 and 29 for. Note that although some small asymmetry between the $\mathrm{x}$ - and $\mathrm{y}$-emittances creeps in, there is no significant emittance growth.

The emittance values derived from the full distribution of particles are strongly influenced by the few particles outside the core. In Figure 6.30, the slice emittance along the bunch is displayed for various cuts in the transverse tails. A 5\% cut in the tails reduces the emittance for the central slices by about $15 \%$. The effect is even more dramatic when the brightness of each slice is plotted, as in Figure 6.31. 


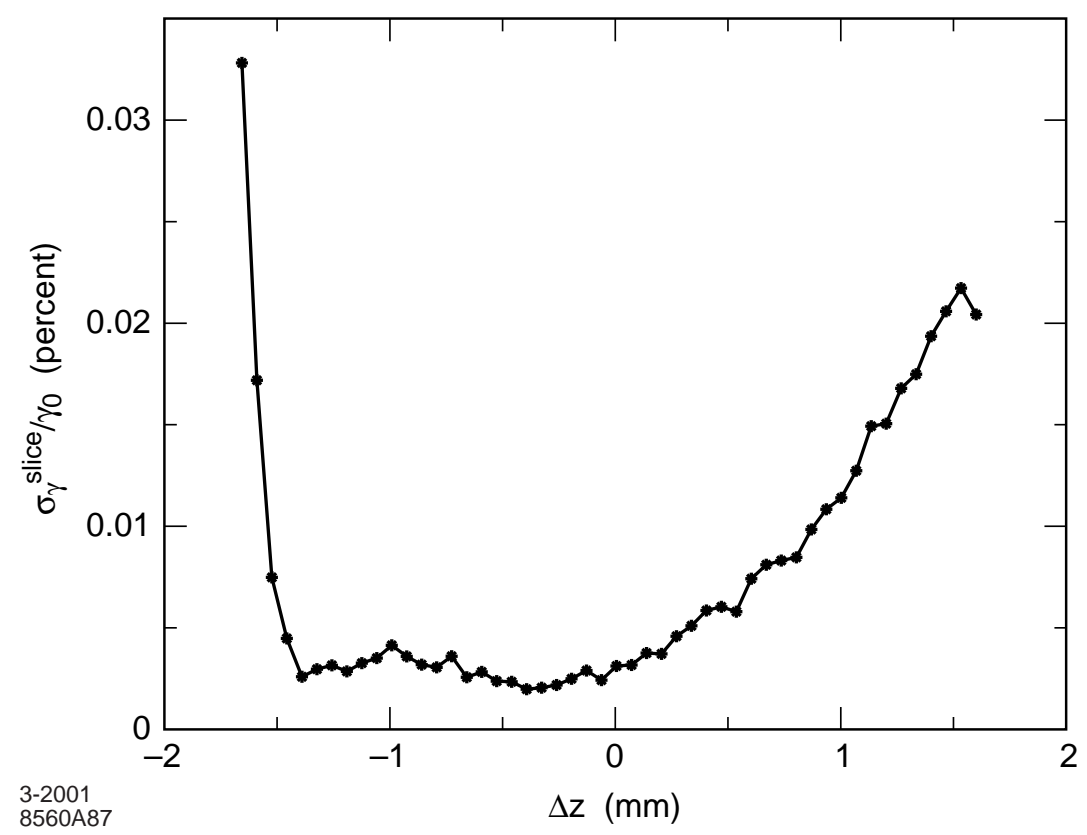

Figure 6.27 Slice energy spread at the exit of LO as a function of axial position. In this figure, the bunch head is at the left, as in the convention of Chapter 7, Accelerator.
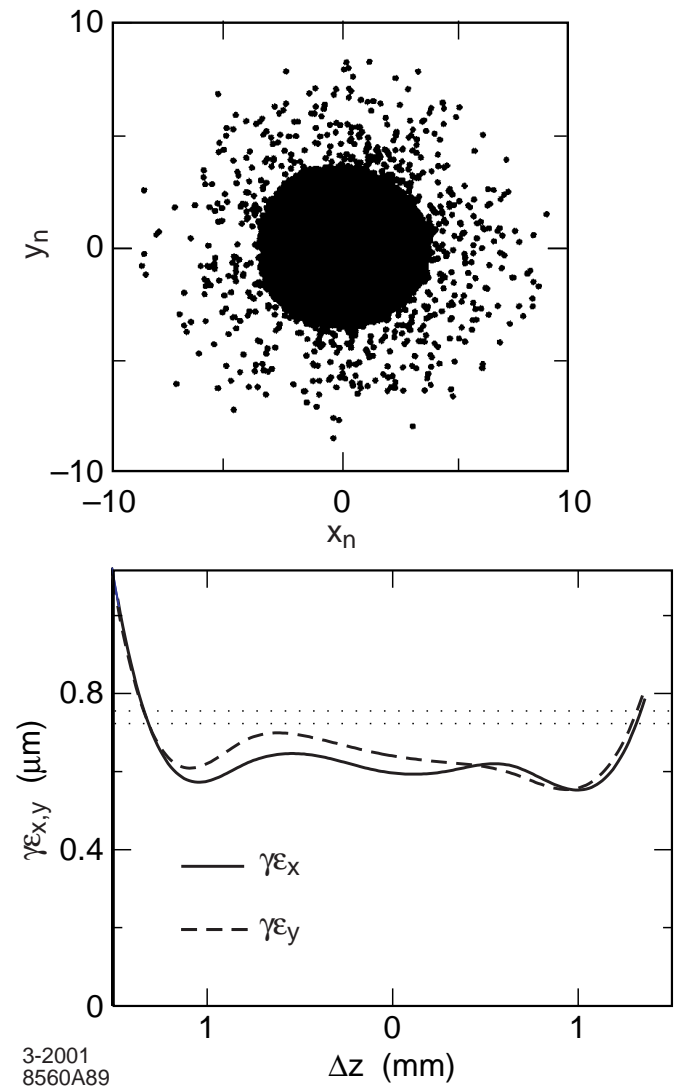
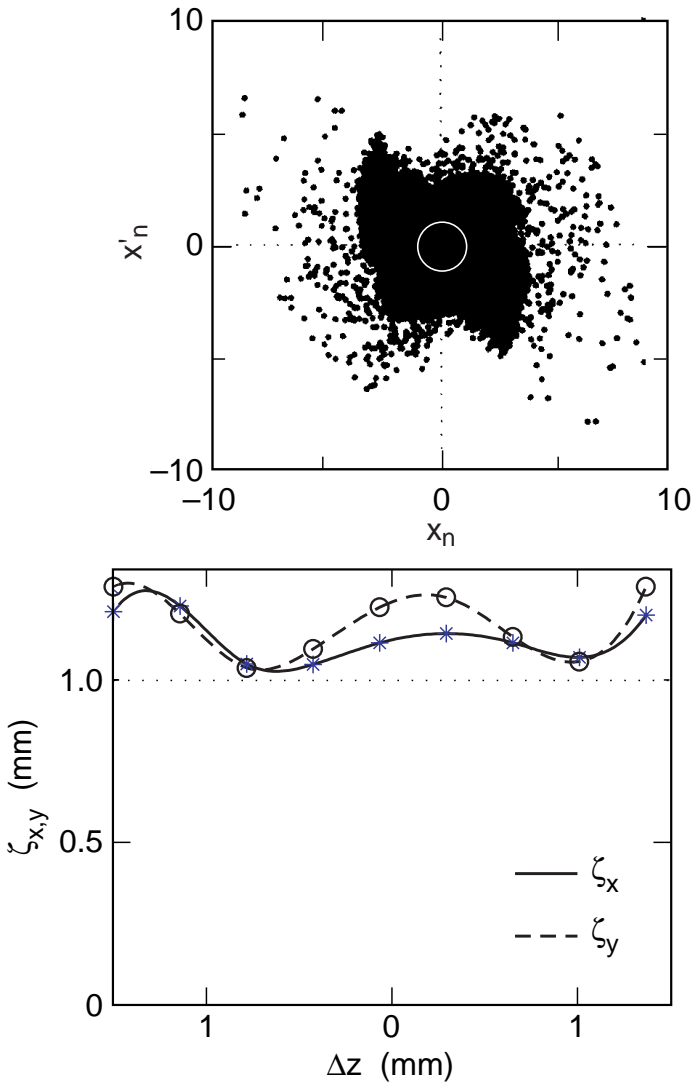

Figure 6.28 Transverse distribution of particles in the beam at the exit of MS. In this figure, the bunch head is at the left, as in the convention of Chapter 7, Accelerator. 

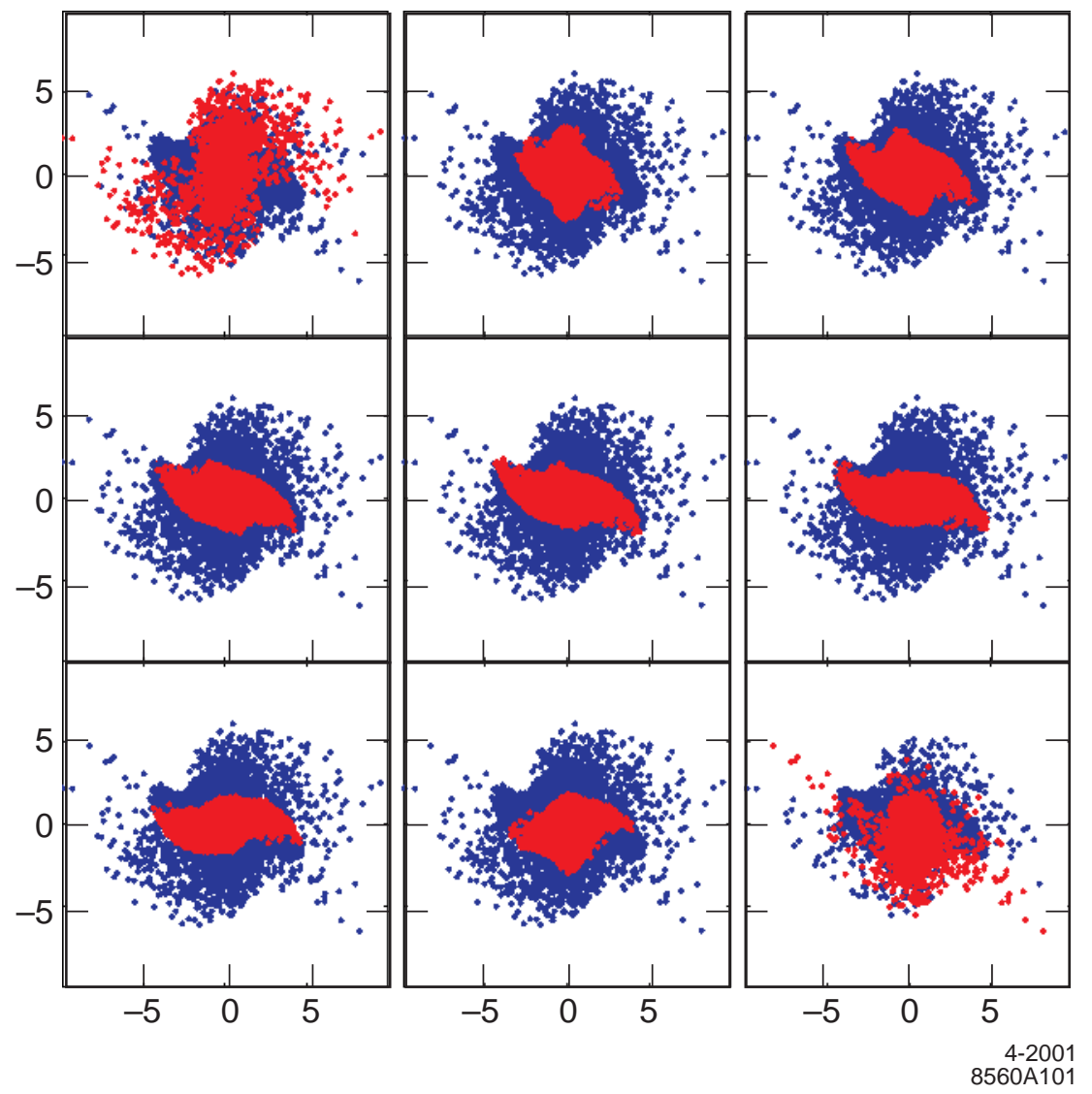

Figure 6.29 Slice emittance at the exit of MS.

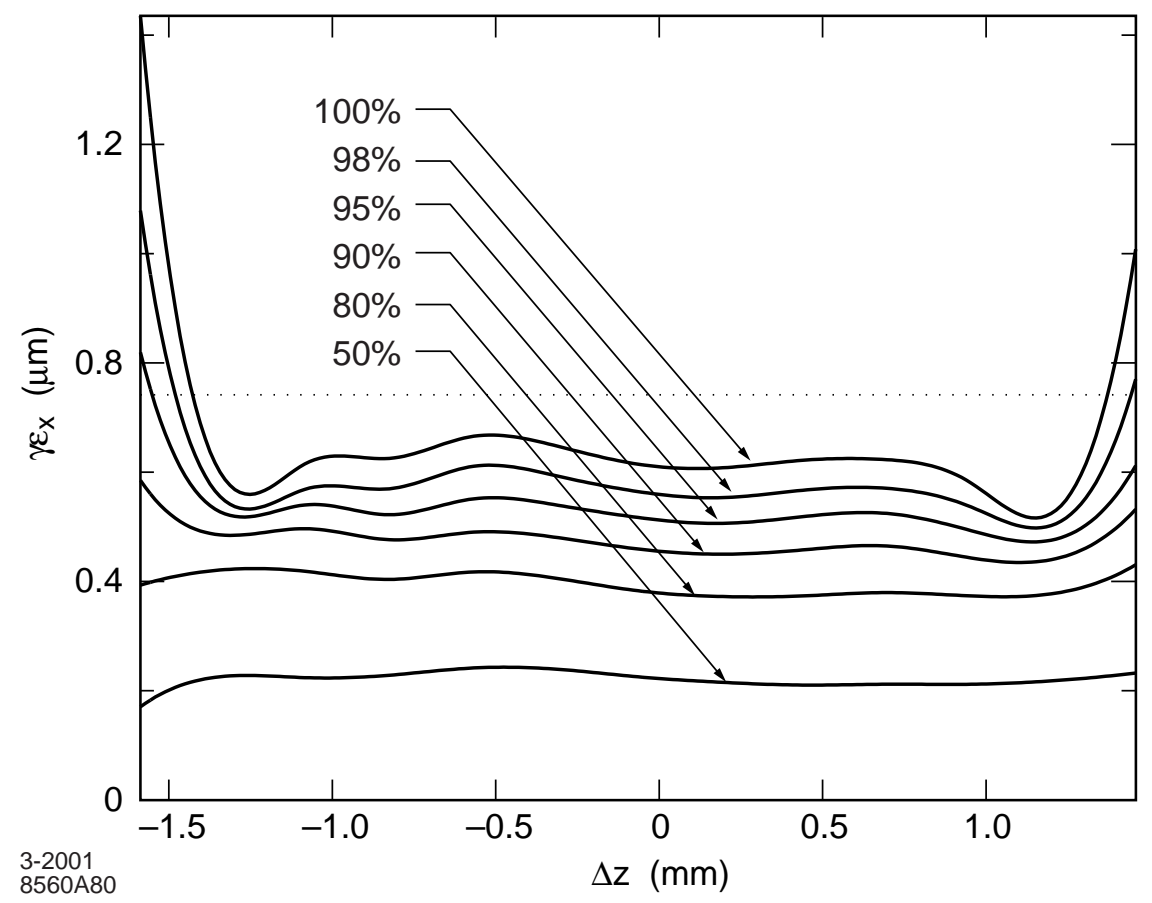

Figure 6.30 Slice emittance al ong bunch at end of MS for different cuts of transverse dimension. 


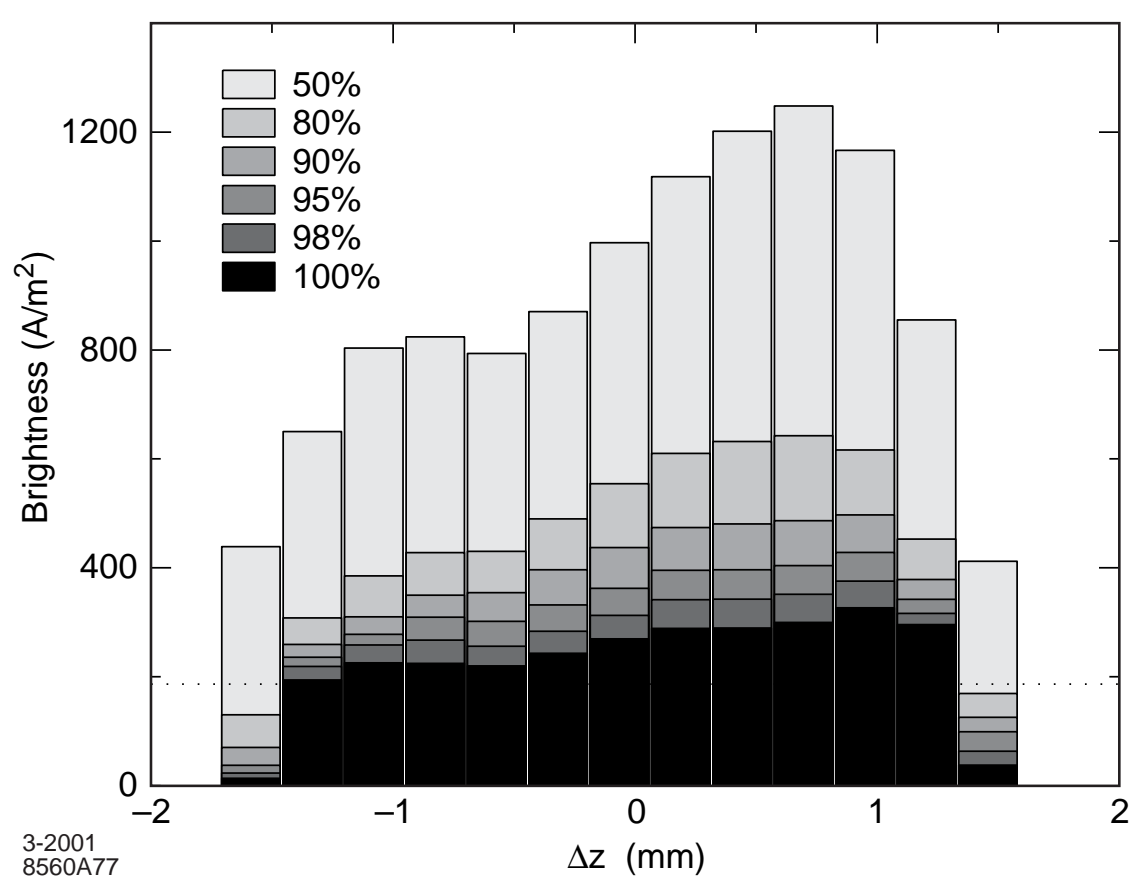

Figure 6.31 Brightness along bunch at end of MS for different cuts of transverse distribution.

Comparing the PARMELA output values shown here with the required values given in Table 6.1, it is seen that the emittance is comfortably below the required value. The variation of emittance with increase in rise time, decrease in number of particles tracked, and the effect of excluding thermal emittance are indicated. The energy spread - both projected and slice - are also within requirements. However, according to preliminary measurements performed at the Gun Test Facility (GTF), the correlated energy at the exit of the gun would be underestimated in the PARMELA simulations. Analytical work and PIC code simulations are in progress. While the rms bunch length doesn't change in either the L0 or MS, the temporal bunch shape becomes somewhat less uniform. Nonetheless, an examination of Figure 6.26, shows that for over about $65 \%$ of the bunch length the peak current is $>100 \mathrm{~A}$. 


\begin{tabular}{|c|c|}
\hline Parameter & Value \\
\hline Bunch charge & $1.0 \mathrm{nC}$ \\
\hline Booster phase & $2^{\circ}$ \\
\hline Bunch length & $2.9 \mathrm{ps} \mathrm{rms}$ \\
\hline Energy & $150 \mathrm{MeV}$ \\
\hline Integrated energy spread, $\sigma_{\gamma} / \gamma_{0}$ & $0.10 \% \mathrm{rms}$ \\
\hline Slice energy spread, $\sigma_{\gamma}^{\text {slice }} / \gamma_{0}$ & $0.005 \% \mathrm{rms}$ \\
\hline $\begin{array}{l}\text { Emittance at } 20 \mathrm{~cm} \text { from exit of } \mathrm{LO}-2 \text { for } 0.7 \text { ps rise time; } \\
\text { below } e_{t h} \text { in } \mu \mathrm{m} / \text { no. particles tracked: }\end{array}$ & $\begin{array}{l}\varepsilon_{\mathrm{n}, \mathrm{rms}}(\mu \mathrm{m}) ; \text { below for } \\
140 / 120 \mathrm{MV} / \mathrm{m}:\end{array}$ \\
\hline $0.3 / 10 \mathrm{~K}$ & $0.926 / 0.929$ \\
\hline $0.5 / 10 \mathrm{~K}$ & $0.952 / 0.956$ \\
\hline $0.3 / 100 \mathrm{~K}$ & $0.922 /$ \\
\hline $0.3 / 200 \mathrm{~K}$ & $/ 0.919$ \\
\hline $0.5 / 100 \mathrm{~K}$ & $0.948 /$ \\
\hline $0.5 / 200 \mathrm{~K}$ & $/ 0.958$ \\
\hline $\begin{array}{l}\text { Emittance at } 20 \mathrm{~cm} \text { from exit of } \mathrm{L0}-2 \text { for } 0.35 \mathrm{ps} \text { rise time; } \\
\text { below } \mathrm{e}_{\mathrm{th}} \text { in } \mu \mathrm{m} / \mathrm{no} \text {. particles tracked: }\end{array}$ & $\begin{array}{l}\varepsilon_{\mathrm{n}, \mathrm{rms}}(\mu \mathrm{m}) ; \text { below for } \\
140 \mathrm{MV} / \mathrm{m} / 120 \mathrm{MV} / \mathrm{m}:\end{array}$ \\
\hline $0.3 / 20 \mathrm{~K}$ & $0.80 /$ \\
\hline $0.3 / 100 \mathrm{~K}$ & $0.78 / 0.80$ \\
\hline
\end{tabular}

\subsubsection{Optimization for $120 \mathrm{MV} / \mathrm{m}$}

Typically S-band rf guns operate with peak rf fields of $120 \mathrm{MV} / \mathrm{m}$ or lower. Higher fields usually lead to excessive dark current or frequent rf breakdowns. Although a great deal of care will be taken to construct the LCLS gun so as to avoid these problems (the load-lock should help as well), the initial gun operation is anticipated to be at about $120 \mathrm{MV} / \mathrm{m}$. In this section, a reoptimization of the injector parameters is made for the case of $120 \mathrm{MV} / \mathrm{m}$ in order to determine more exactly the emittance achievable during initial operation. 
First HOMDYN was used to explore the transverse parameter space for a square pulse of 10 ps. The parameters that were examined included the gun solenoid (S1) field value, the injection phase, the linac position and gradient and the second solenoid (S2) field value. For a thermal emittance of $0.5 \mu \mathrm{m}$, the minimum normalized transverse emittance obtained was $0.6 \mu \mathrm{m}$ rms using an injection phase of $20^{\circ}, \mathrm{S} 1$ field of $2.71 \mathrm{kG}$, and a linac field of $24 \mathrm{MV} / \mathrm{m}(33 \mathrm{MV} / \mathrm{m}$ in HOMDYN units) with the entrance to L0-1 located $1.4 \mathrm{~m}$ from the cathode.

When the same parameters were used in PARMELA, including a square pulse of $10 \mathrm{ps}$, an emittance of $0.99 \mu \mathrm{m}$ was obtained. The higher emittance with PARMELA confirms that the parameter space near the working point as determined with HOMDYN must be further optimized with PARMELA. The parameter space was explored using PARMELA for a pulse with a finite rise time of $0.7 \mathrm{ps}$. (HOMDYN uses only absolutely square pulses.) The PARMELA optimization was initiated with parameter values obtained from the optimization with HOMDYN. The initial pulse profile is shown in Figure 6.21. It is built with 9 Gaussians of width 0.7 ps rms separated by $1.1 \mathrm{ps}$.

A first series of simulations was performed with a spot-size radius of $1 \mathrm{~mm}$. The best emittance was obtained by placing L0-1 $2.2 \mathrm{~m}$ away from the cathode. This solution was unsatisfactory as the same linac section is located $1.42 \mathrm{~m}$ from the cathode when parameters are optimized with the cathode peak rf field at $140 \mathrm{MV} / \mathrm{m}$.

To keep the distance cathode-to-Linac constant, the spot radius was increased from $1 \mathrm{~mm}$ to $1.2 \mathrm{~mm}$. This larger radius reduces the space charge effect without introducing excessive additional RF defocusing effects. Also, the field in L0-1 was decreased from $24.1 \mathrm{MV} / \mathrm{m}$ to $18 \mathrm{MV} / \mathrm{m}$ following the criteria presented in Eq. 6.3. This results in an energy at the exit of L0-1 of $58.25 \mathrm{MeV}$ instead of $78.18 \mathrm{MeV}$. By running L0-2 at $24.1 \mathrm{MV} / \mathrm{m}$ and $30.5 \mathrm{MV} / \mathrm{m}$, the energy at the end of the beamline is $130 \mathrm{MeV}$ and $150 \mathrm{MeV}$ respectively. No emittance growth in the Matching Section appears for either a $130 \mathrm{MeV}$ or $150 \mathrm{MeV}$ beam.

With the parameters presented in Table 6.4 for $10 \mathrm{~K}$ particles and $0.7 \mathrm{ps}$ rise time, the beam is smoothly converging along the beamline as presented in Figure 6.32. The projected emittance is $0.93 \mu \mathrm{m}$. 


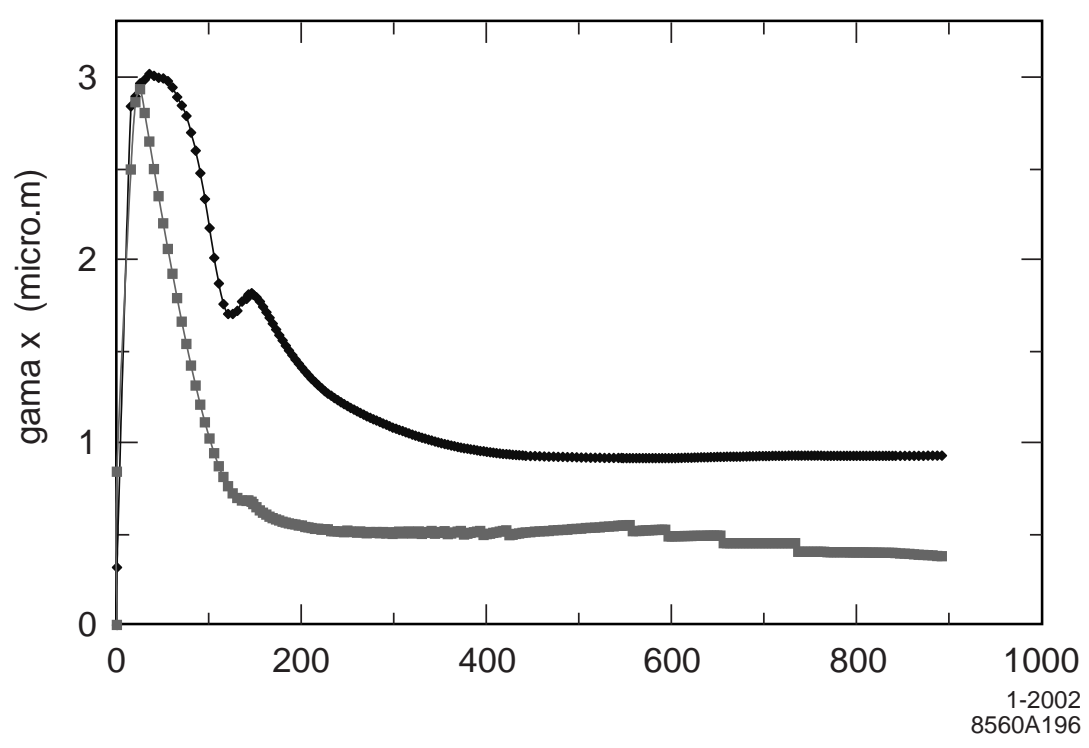

Figure 6.32 Transverse normalized rms emittance as a function of distance from the cathode for $10 \mathrm{~K}$ particles. A rise time of $0.7 \mathrm{ps}$ is assumed. A normalized rms thermal emittance of $0.3 \mu \mathrm{m}$ is included.

Figure 6.33 shows the transverse profile, horizontal phase space, slice emittance and mismatch parameters for 9 slices at the end of L0-2. When the bunch is cut into 99 slices, $97 \%$ of the particles are contained in slices, which have an emittance smaller than $1 \mathrm{~mm} . \mathrm{mrad}$, while $71 \%$ of the particles are contained in slices, which have an emittance smaller than $0.8 \mu \mathrm{m}$. By choosing to use 99 slices, each slice when transported to the undulator will have a length comparable to a slippage length. Figure 6.34 shows the longitudinal phase space distribution (upper left) and the projections on the upper right (energy) and lower left (time) at the end of L0-2. The L0-2 phase was chosen to minimize energy spread after including wakefield effects. Before including the wakefields the rms energy spread was $0.2 \%$ and after the inclusion of wakefields it became $0.06 \%$. 

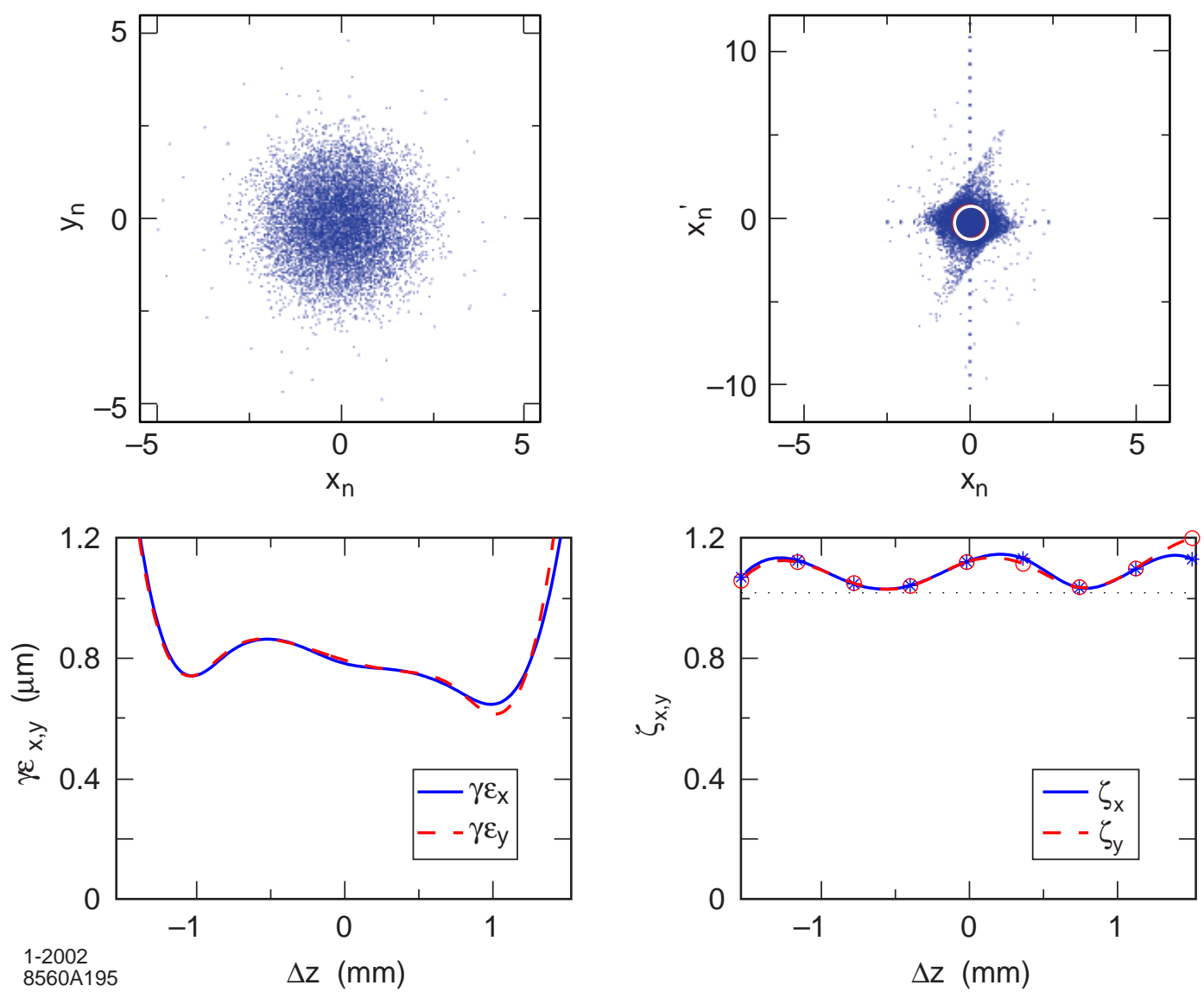

Figure 6.33 Longitudinal distribution of particles in the beam at the exit of L0-2. In this figure, the bunch head is at the left, as in the convention of Chapter 7, Accelerator. The peak rf field at the cathode is $120 \mathrm{MV} / \mathrm{m}$.

The optimization of the beamline for the gun run at $120 \mathrm{MV} / \mathrm{m}$ gives a projected emittance very similar to that obtained when optimizing the beamline for the gun run at $140 \mathrm{MV} / \mathrm{m}$. See Table 6.5. Similarly, for a rise time for the $10 \mathrm{ps}$ pulse of $0.7 \mathrm{ps}$, a projected emittance as small as $0.8 \mu \mathrm{m}$ has been computed with the gun run at $120 \mathrm{MV} / \mathrm{m}$. 

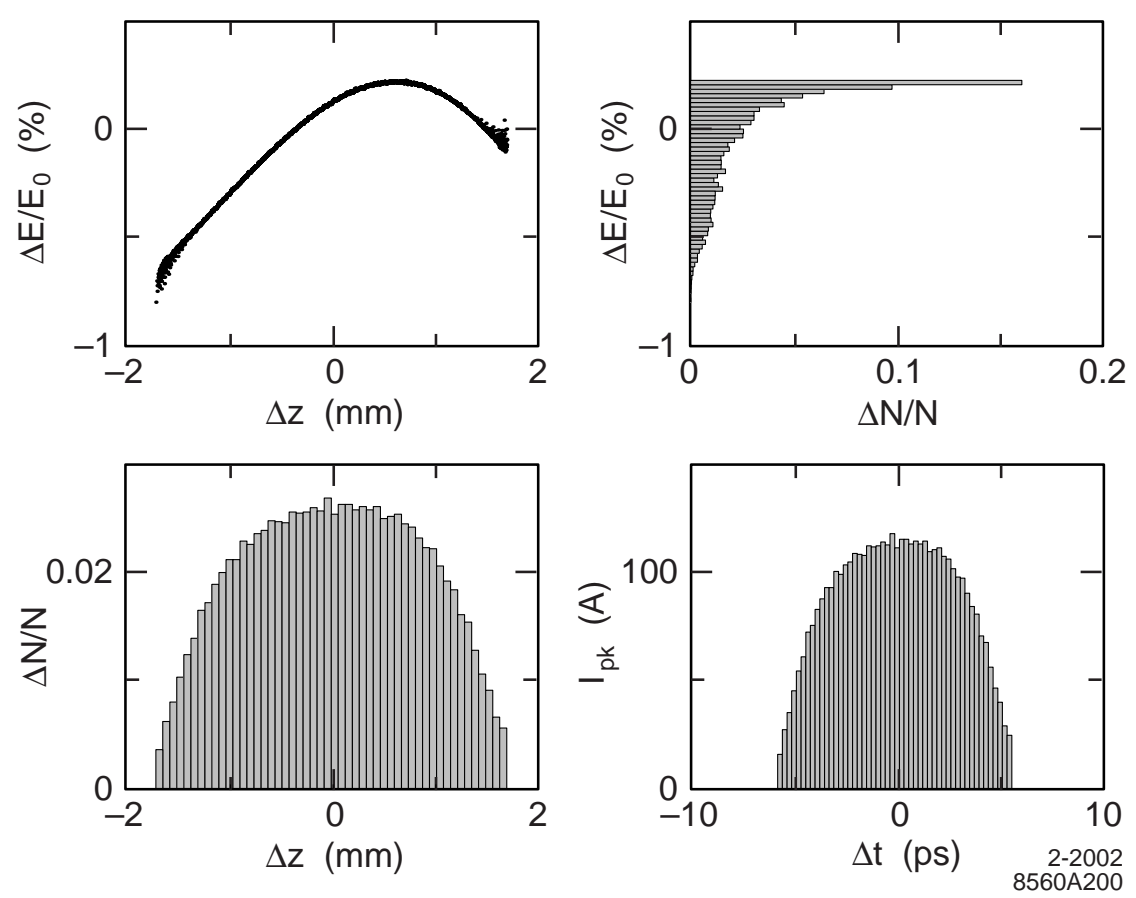

Figure 6.34 Longitudinal distribution of particles in the beam at the exit of L0-2. In this figure, the bunch head is at the left, as in the convention of Chapter 7, Accelerator. The peak rf field at the cathode is $120 \mathrm{MV} / \mathrm{m}$.

\subsubsection{Sensitivity Study}

To define levels of regulation on power supplies for the RF power feed and for the emittance compensation solenoid, and to specify requirements on the UV laser pulse stability and reproducibility, a sensitivity study was performed on the beamline optimized for a peak rf field at the cathode of $120 \mathrm{MV} / \mathrm{m}$. A single parameter was changed around its nominal value. The evolution of the emittance at the end of Linac 0-2 (L0-2) as a function of this parameter is shown in the 6 plots of Figure 6.35. To keep the projected emittance below $1 \mu \mathrm{m}$, the peak RF field amplitude should not vary by more than $0.5 \mathrm{MV} / \mathrm{m}$ around $120 \mathrm{MV} / \mathrm{m}$, the balance of the fields between the half cell and the full cell should not vary by more than $1 \%$ (ongoing studies indicate that $3 \%$ is probably more correct), and the laser injection phase should not vary by more than $4^{\circ}$ $\mathrm{S}$-Band. These are large variations compared to the much tighter tolerances that these systems normally meet. It is noted that a variation of only $0.4 \%$ on the solenoid field (S1) value increases the projected emittance from 0.93 to $1 \mu \mathrm{m}$. However, a tolerance of $0.1 \%$ or better will not difficult to achieve with the solenoid power supply.

A variation of $\pm 0.1 \mathrm{~mm}$ on the laser spot radius also gives a projected emittance close to 1 $\mu \mathrm{m}$. Finally an increase of $10 \%$ in charge still leaves the projected emittance just below $1 \mu \mathrm{m}$. 

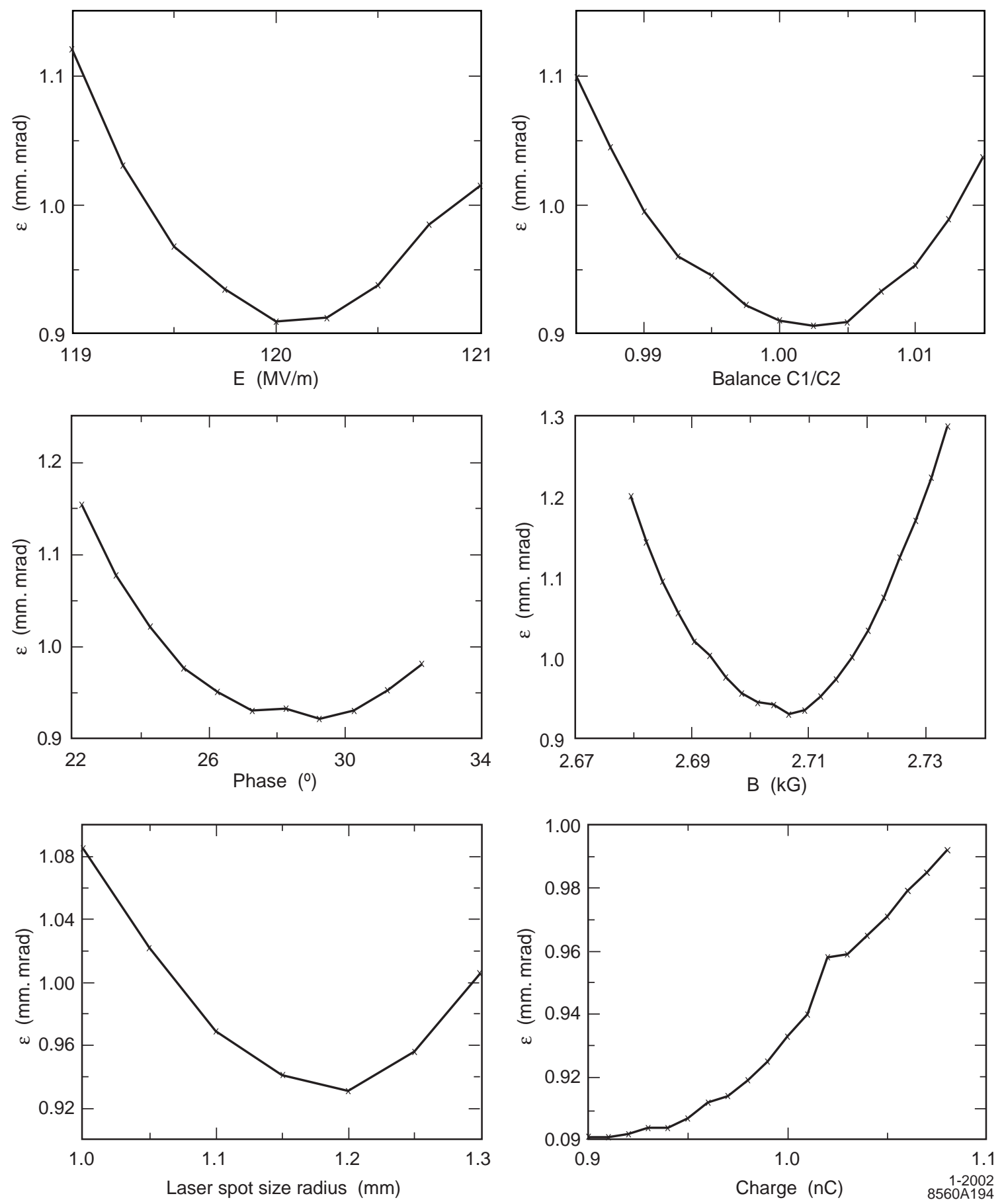

Figure 6.35 Emittance at the end of Linac 0-2 as a function of a single parameter with all other held at the value obtained when all parameters are optimized simultaneously.

The final tolerances on power supplies and on the laser beam properties will be slightly tighter than what is indicated above. All these variations combined together should give a deterioration of emittance such that on average the final projected emittance is on the order of the quadrature sum of the emittances obtained with single parameter variations. A study is underway 
to determine the full range of variations to be expected when the variations of each individual parameter are combined.

\subsection{References}

1 J.F. Schmerge et al., "Photocathode rf gun emittance measurements using variable length laser pulses," in SPIE 3614 (1999), p. 22.

2 D.T. Palmer et al., "Emittance studies of the BNL/SLAC/UCLA 1.6 cell photocathode rf gun," in Proc. of the 1997 Particle Accelerator Conf., p. 2687.

3 M. Babzien et al., "Observation of self-amplified spontaneous emission in the near-infrared and visible," Phys. Rev. E 57 (1998) 6093.

4 R. Alley et al, “The design for the LCLS rf photoinjector," Nucl. Instrum. and Meth. A 429 (1999) 324.

5 J. Rosenzweig and E. Colby, "Charge and wavelength scaling of rf photoinjector designs," in AIP Conf. Proc. 335 (1995), p. 724.

6 B. E. Carlsten, Nucl. Instrum. and Meth. A 285 (1989) 313.

7 L. Serafini and J. B. Rosenzweig, "Envelope analysis of intense relativistic quasilaminar beams in $\mathrm{rf}$ photoinjectors: a theory of emittance compensation," Phys. Rev. E 55 (1997) 7565.

8 J. Clendenin et al., "Reduction of thermal emittance of rf guns," Nucl. Instrum. and Meth. A 455 (2000) 198.

9 M. Ferrario, A. Mosnier, L. Serafini, F. Tazzioli and J. M. Tessier, "Multi-bunch energy spread induced by beam loading in a standing wave structure," Part. Acc. 52 (1996).

10 D. T. Palmer, “The next generation photoinjector," Ph. D. Thesis, Stanford University (1998).

11 M. Ferrario, J. E. Clendenin, D. T. Palmer, J. B. Rosenzweig and L. Serafini, "HOMDYN study for the LCLS rf photo-injector", in The Physics of High Brightness Beams, World Scienific (2000), p. 534.

12 J. Rosenzweig and L. Serafini, "Transverse particle motion in radio-frequency linear accelerators," Phys. Rev. E 49 (1994) 1599.

13 D.T. Palmer, S. Anderson and J.B. Rosenzweig, "Single Crystal Copper Photo-Cathode in the BNL/SLAC/UCLA 1.6 Cell RF Gun," in The Physics of High Brightness Beams, World Scienific (2000), p. 439.

14 J.F. Schmerge et al., "Transverse emittance measurements on an S-band rf gun," SLAC-PUB-8963 (2001), to be published in Nucl. Intrum. and Meth. A.

15 X.J. Wang et al., J. Appl. Phys. 72 (1992) 888.

16 P. G. O'Shea and L. Spentzouris, in AIP Conf. Proc. 472 (1999), p. 212.

17 S.M. Gierman, "Streak Camera Enhanced Quadrupole Scan Technique", in The Physics of High Brightness Beams, World Scienific (2000), p. 511.

18 W.S. Graves et al., "Measurement of thermal emittance for a copper photocathode," in Proc. of the 2001 Particle Accelerator Conf., p. 2227.

19 J.E. Clendenin and G.A. Mulhollan, "High quantum yield, low emittance electron sources," in Quantum Aspects of Beam Physics, Singapore, World Scientific (1999), p. 254.

20 D.H. Dowell, S. Joly and A. Loulergue, "Space charge limits of longitudinal emittance in RF photoinjectors," in AIP Conf. Proc. 398 (1997), p. 793.

21 K. Batchelor et al., in Proc. of the 2nd European Particle Accelerator Conf., (1990), p. 541. 
22 D.T. Palmer et al., in Proc. of the 1995 Particle Accelerator Conf. (1995), p. 982. In this reference it is pointed out that the dipole emittance term has been suppressed by an order of magnitude to the level of $0.1 \mu \mathrm{m}$, implying that in the unsymmetrized case the dipole emittance term would be $1 \mu \mathrm{m}$. See also B. Dwersteg et al., "rf gun design for the TESLA VUV free electron laser," Nucl. Instrum. and Meth. A 393 (1997) 93.

23 E.L. Ginzton, Microwave Measurements, McGraw-Hill (1957), p. 295.

24 X.-J. Wang et al., "Design studies for the LCLS $120 \mathrm{~Hz}$ rf gun," Informal Report BNL-67922 (Dec. 2000).

25 M. Uesaka (U. Tokyo) and M. Kando (JAERI Advanced Photon Research Center), private communication $(11 / 00)$.

26 J. Schmerge, "120 Hz RF gun operation," draft of internal SLAC technical report (6/00).

27 H. Braun et al., "Results from the CLIC Test Facility," in Proc. of the $5^{\text {th }}$ European Particle Accelerator Conf., (1996), p. 44.

28 T. Srinivasan-Rao et al., J. Appl. Phys. 69 (1991) 3291.

29 P. Davis et al., in Proc. of the 1993 Particle Accelerator Conf., p. 2976.

30 E. Chevallay et al., Nucl. Instrum. and Meth. A 340 (1994) 146.

31 G. Mulhollan, "Common Sense Copper and RF Guns," LCLS-TN-99-9 (July 1999).

32 D. Reis, Ph.D. Thesis, Univ. Rochester, Dept. of Physics and Astronomy, June 1999, UR-1573, pp. 130-131.

33 D. Reis et al., Nucl. Instrum. and Meth. A 429 (1999) 341.

34 D.T. Palmer, SLAC, private communication (1997).

35 X.-J. Wang et al., "FEL technologies R\&D and SASE gain enhancement observation at the BNL ATF," in Proc. of the 2000 European Particle Accelerator Conf., p. 779.

36 The gain per pass in Ti:sapphire amplifiers is sufficient to get from $1 \mathrm{~nJ}$ to a few $\mathrm{mJ}$ in eight (8) passes. For example, see S. Backus et al., Opt. Lett. 20 (1995) 2000.

35-1 J.A. Hoffnagle and C.M. Jefferson, Appl. Opt. 39 (2000) 5488.

37 P. Maine et al., IEEE J. Quantum Electron. QE-24 (1988) 398.

38 A.M. Weiner et al., J. Opt. Soc. B 5 (1988) 1563.

39 A.M. Weiner et al., Opt. Lett. 15 (1990) 326; A.M. Weiner, Rev. Sci. Instrum. 71 (2000) 1929.

40 ShapeShifter spatial light modulator, Meadowlark Optics, Frederick, CO.

41 B.M. Van Wonterghem et al., Proc. of the Conference on Laser Coherence Control, in SPIE 1870 (1993), p. 64.

42 J.A. Hoffnagle and C.M. Jefferson, Appl. Opt. 39 (2000) 5488.

43 R.S. Craxton, Opt. Commun. 34 (1980) 474.

44 For ultrafast laser pulses, the effects of group velocity walkoff and nonlinear phase effects must be considered when designing the conversion stage.

45 D.A. Reis, Ph.D. Thesis, Univ. Rochester, Dept. of Physics and Astronomy, June 1999, UR-1573, pp. 23 and 131.

46 P. Davis et al., Proc. 1993 Particle Accelerator Conf., p. 2976.

47 H.-S. Albrecht et al., Appl. Opt. 32 (1993) 6659.

48 D.J. Kane and R. Trebino, Opt. Lett. 18 (1993) 823. 
49 M.J.W. Rodwell et al., Opt. Lett. 11 (1986) 638; and M.J.W. Rodwell et al., IEEE J. Quantum Electronics 25 (1989) 817; also T. Kotseroglou et al., Nucl. Instrum. and Meth. A 383 (1996) 309.

50 M.J. Fitch et al., "Electro-optic measurement of the wake fields of $16 \mathrm{MeV}$ electron bunches," UR1585/FERMILAB-TM-2096 (1999).

[51] I. Wilke et al., "Single-Shot Electron-Beam Bunch Length Measurements," Phys. Rev. Lett. 88 (2002) 124801. 


\section{Accelerator}

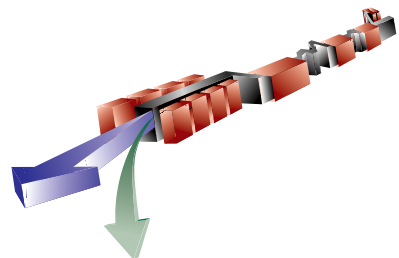

\section{TECHNICAL SYNOPSIS}

In order for the SASE FEL to operate in saturation, a high electron peak current with small transverse and longitudinal emittance is required. The LCLS nominally operates within a range of wavelengths from 15 to $1.5 \AA$. The most challenging parameters coincide with the 1.5- $A$ configuration with maximum peak current of $3.4 \mathrm{kA}$, transverse normalized 'slice' emittance of $1.2 \mu \mathrm{m}$, and top end energy of $14.35 \mathrm{GeV}$. Since the rf photocathode gun produces $1 \mathrm{nC}$ in a bunch length of $3 \mathrm{psec} r \mathrm{~ms}$, corresponding to a peak current of $100 \mathrm{~A}$, the bunch has to be compressed by a factor of 35 before it enters the undulator. The acceleration and compression is done in the final kilometer of the slightly modified SLAC S-band linac.

The bunch compressors consist of a series of magnetic chicanes, arranged and located such that non-linearities in the compression and acceleration process (longitudinal wakefields, $r f$ curvature, and second order momentum compaction) are approximately compensated. A short section of X-band rf is also used prior to the first compressor in order to linearize and stabilize the system. An optimal choice of linac design parameters both cancels the correlated energy spread after the final compression, and desensitizes the system to phase and charge variations. The electron energy at the first compressor is $250 \mathrm{MeV}$. This choice avoids space charge effects, while compressing the bunch early enough in the linac to ease the effects of transverse wakefields. In the first compressor, the bunch length is reduced from $\sim 1 \mathrm{~mm}$ to $0.2 \mathrm{~mm} \mathrm{rms}$. The second compressor produces a 22-um bunch. The energy of the second compressor, $4.54 \mathrm{GeV}$, is chosen as a balance between the conflicting requirements of longitudinal emittance dilution due to synchrotron radiation, and the need to cancel the final correlated energy spread. The design of the compressors is dominated by the need to reduce coherent synchrotron radiation (CSR) effects, which are most pronounced for short bunches. The most significant effect of CSR is to generate a correlated energy gradient along the bunch. By using a weak chicane and a large initial correlated energy spread, the effect of CSR on the transverse emittance can be reduced.

Simulations have been made which calculate emittance dilution in the linac due to transverse wakefields and anomalous momentum dispersion, each of which arises with component misalignments. These simulations include realistic correction techniques and successfully demonstrate the level of transverse emittance preservation required. Diagnostics, correction techniques, and feedback systems have also been incorporated into the design. Finally, the acceleration and compression systems are flexible enough to allow a high degree of operational variation in beam parameter choices, such as bunch charge, final beam energy, and an optional electron chirp, used for $x$-ray pulse compression. 


\subsection{Introduction and Overview}

In order to reach SASE saturation in a reasonable length undulator, a high electron peak current is required. For a radiation wavelength of $1.5 \AA$, an undulator period of $3 \mathrm{~cm}$, and an undulator parameter of $K \approx 3.7$, the desired electron beam should have a peak current of $3.4 \mathrm{kA}$, energy of $14.3 \mathrm{GeV}$, and transverse normalized rms 'slice' emittance of $\leq 1.2 \mu \mathrm{m}$. A longitudinal 'slice' of the electron beam is defined by the FEL slippage length $(\sim 0.5 \mu \mathrm{m}$ in this case). These values correspond to an rms bunch length of $22 \mu \mathrm{m}$ and charge of $1 \mathrm{nC}$, which is not possible in present rf photo-injectors due to space charge limitations. Therefore the bunch is accelerated and compressed in a series of linacs and magnetic chicanes. The accelerator must preserve the transverse emittance produced by the rf photo-injector. A slice emittance growth of $<20 \%$ and projected emittance growth of $50-100 \%$ are allotted to the accelerator. Extensive tracking studies indicate this level is achievable (see Section 7.6).

Energy spread requirements in the undulator at $14.3 \mathrm{GeV}$ are $<0.1 \%$ and $<0.02 \%$ for the rms coherent (correlated) and incoherent (uncorrelated) components, respectively. The nominal machine described here is designed to operate at a repetition rate of up to $120 \mathrm{~Hz}$ in a singlebunch mode with 1-nC of charge per bunch, and with the flexibility of running at significantly lower charge [1]. Future multi-bunch operations may also be possible [2], but are not described here. Figure 7.1 shows a schematic of the LCLS accelerator. The nominal beam energy, rms bunch length $\left(\sigma_{z}\right)$, and rms relative energy spread $\left(\sigma_{\delta}\right)$ are indicated at points along the accelerator, as are section parameters, such as length $(L)$, rf phase $\left(\varphi_{\mathrm{rf}}\right)$, and momentum compaction $\left(R_{56}\right)$.

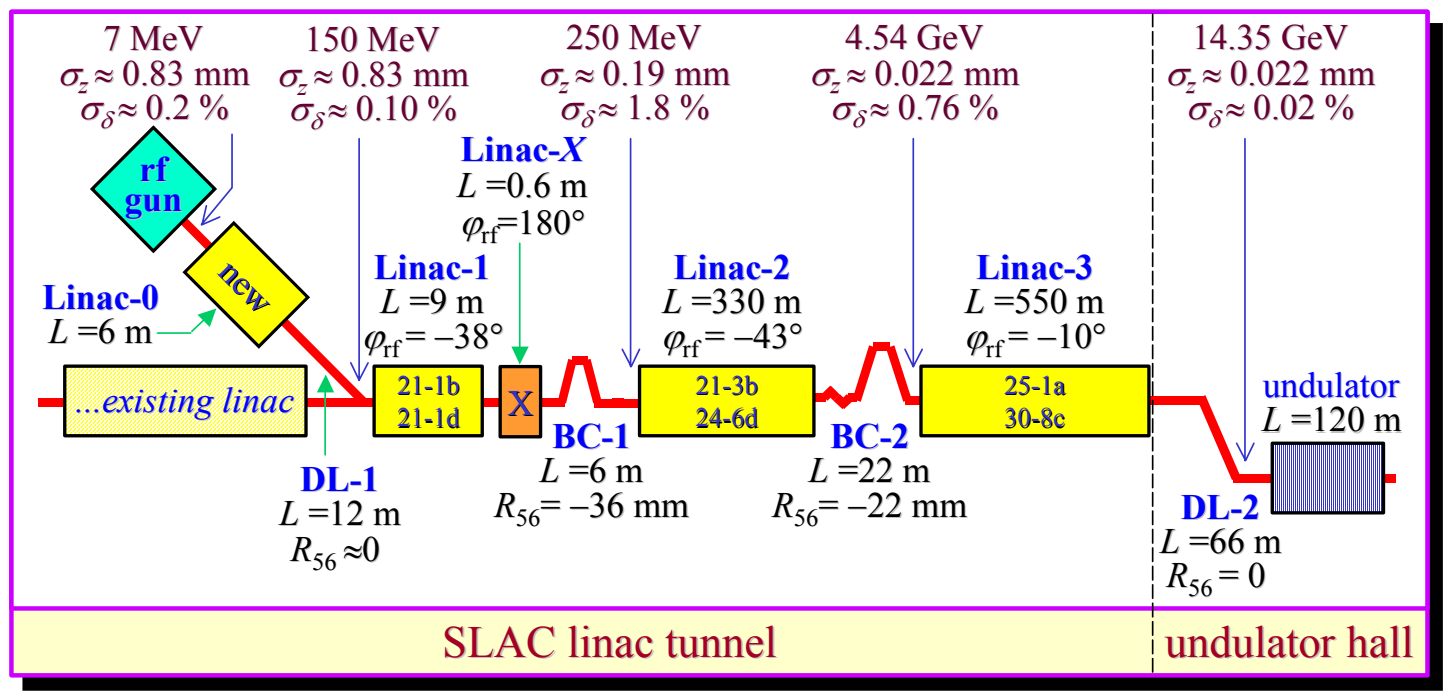

Figure 7.1 LCLS compression and acceleration schematic. The 'dog-legs' (DL-1 and DL-2) are simple transport lines and have no effect on bunch length, while the compressors are four-dipole chicanes. Acceleration crest is defined at $\varphi_{\mathrm{rf}}=0$.

The LCLS accelerator is composed of four S-band linac sections $\left(f_{\mathrm{rf}}=2.856 \mathrm{GHz}\right)$, one short $\mathrm{X}$-band section $\left(f_{\mathrm{rf}}=11.424 \mathrm{GHz}\right.$ ), and four separate bending sections. The first linac (Linac-0 or 
L0) is a new section installed in an existing off-axis enclosure adjacent to the beginning of sector21. L0 is composed of two 3-m RF structures and accelerates the $\sim 1-\mathrm{mm}$ long bunch from the rf gun to $150 \mathrm{MeV}$. The space-charge emittance compensation is also finalized in L0 (see Chapter 6). The transport line following L0 (low energy 'dog-leg' or DL1) provides energy and emittance diagnostics and includes a bending section for injection into the SLAC linac at a $35^{\circ}$ angle. Linac1 (L1) accelerates the bunch "off-crest" to $272 \mathrm{MeV}$ and generates a nearly linear energy correlation along the bunch to initiate the first stage of compression.

A short X-band rf section (LX), immediately following L1, is used to linearize the compression by removing the quadratic energy-time bunch correlation generated in L0 and L1. It operates at $180^{\circ}$ and decelerates the beam by $22 \mathrm{MeV}$ setting the $\mathrm{BC} 1$ compression energy at $250 \mathrm{MeV}$. The linearization allows strong compression in the first stage while maintaining a fairly uniform temporal distribution along the bunch. The first bunch compressor is a 4-dipole chicane $(\mathrm{BC} 1$, located at the start of linac sector-21), which shortens the bunch to $0.20 \mathrm{~mm} \mathrm{rms}$. Linac-2 (L2) then accelerates the bunch to $4.54 \mathrm{GeV}$, also at an off-crest phase, which maintains the desired linear energy correlation.

The second compressor is another 4-dipole chicane (BC2, at the end of linac sector-24) which compresses the bunch to its nominal final value of $22-\mu \mathrm{m}$ rms. The very high peak current generated by the compression in $\mathrm{BC} 2$ can drive a micro-bunching instability [3], [4], which can damage both the slice emittance and the slice energy spread. In order to damp these effects, a very strong one-period superconducting wiggler is placed just upstream of the $\mathrm{BC} 2$ chicane in order to increase the incoherent energy spread of the beam. An increase to $3 \times 10^{-5} \mathrm{rms}$ (from $3 \times 10^{-6}$ at $4.54 \mathrm{GeV}$ without wiggler) is sufficient to suppress the instability without adding too much energy spread for the 1.5- $\AA$ SASE FEL. This is described in more detail in Section 7.4.2.

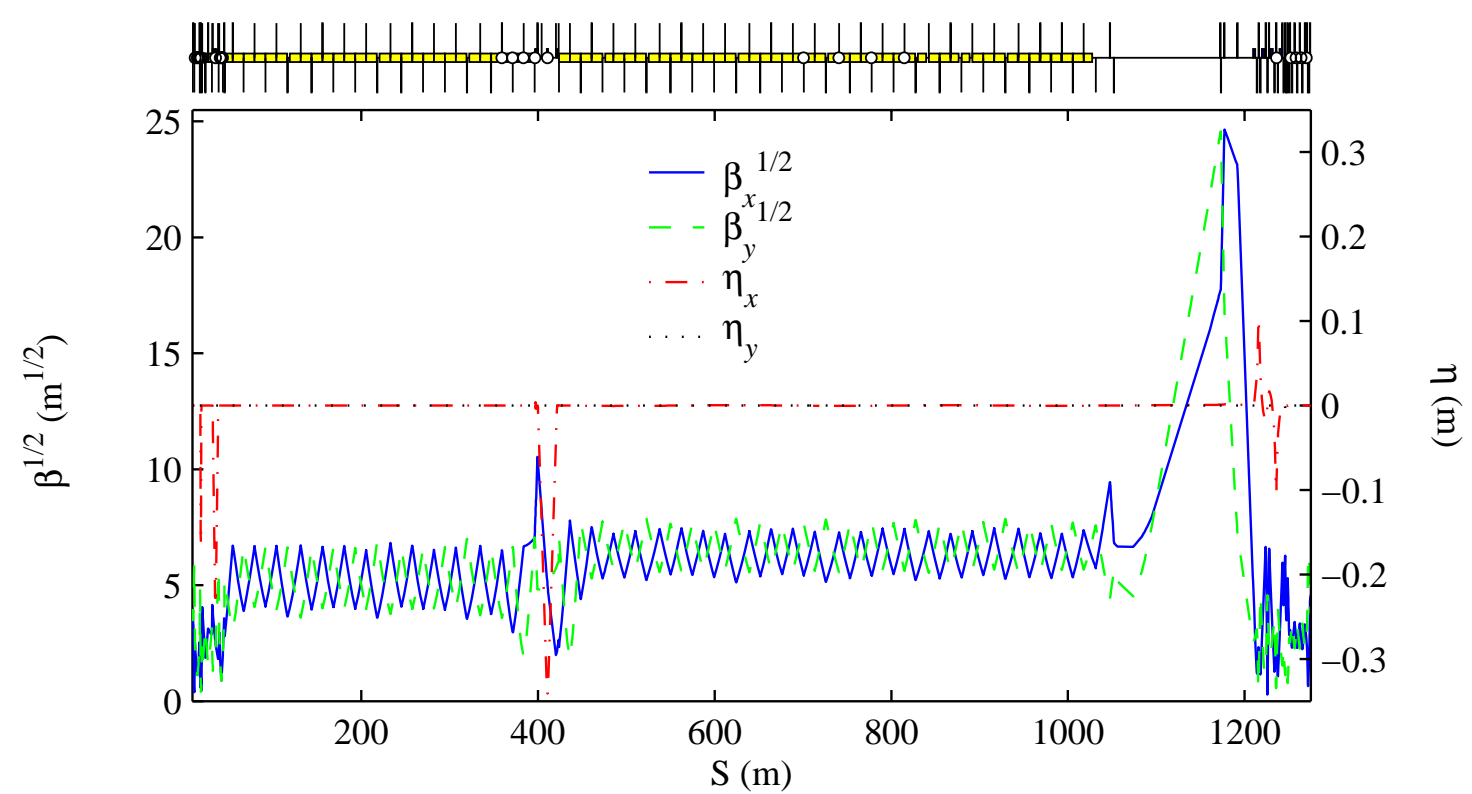

Figure 7.2 Twiss parameters for the LCLS accelerator from linac-0 exit to undulator entrance. The first compressor, $B C 1$, is at $S \approx 30 \mathrm{~m}$ and the second, $\mathrm{BC} 2$, is at $S \approx 400 \mathrm{~m}$. 
Finally, Linac-3 (L3) accelerates the beam, nearly on-crest, to $14.35 \mathrm{GeV}$ (a range of approximately $4.5-15 \mathrm{GeV}$ is available) and also cancels the linear correlated energy spread with the strong geometric longitudinal wakefield of the S-band rf-structures. The high-energy dog-leg bend system (DL2) is designed for energy and energy spread analysis, transverse emittance measurement, final beam transport, and matching into the undulator. It is also useful for collimating dark-current and any off-energy beam halo prior to the undulator.

Each system, its parameters, and design criteria, as well as beam dynamics in the LCLS accelerator are discussed in the following sections. Figure 7.2 shows the Twiss parameters for the 1266-meter LCLS accelerator from end of L0 injector to undulator entrance.

\subsection{Longitudinal Beam Dynamics}

To achieve the high peak current and small energy spread in the undulator, the bunch must be compressed in a series of magnetic chicanes. These are arranged and located such that nonlinearities in the compression and acceleration process (longitudinal wakefields, rf curvature, and second order momentum compaction) are mostly canceled. With a careful arrangement of parameters, the bunch compression process can be made more linear, which reduces the minimum bunch length achievable and helps to avoid very high peak current spikes in the temporal distributions, which may drive collective effects. An optimum choice of parameters compensates the correlated energy spread after final compression and desensitizes the system to phase and charge jitter. In addition, the compressors must be designed to mitigate the effects of synchrotron radiation so the transverse emittance is not diluted. A semi-analytic fast computer program has been written to choose the acceleration and compression parameters in order to provide for these concerns [5]. An accelerator design for a 1-nC bunch charge is described here, but studies have also shown that a very wide variety of machine configurations are possible (including a $0.25-\mathrm{nC}$ bunch charge scenario [6]) with simple, operational adjustments.

\subsubsection{Bunch Compression Overview}

The bunch is compressed by accelerating with an off-crest rf phase, thereby introducing a correlated energy spread along the bunch. This process is followed by a bending section, which has a linear path length dependence on particle energy. Temporarily ignoring wakefields, the final energy $\left(E_{f}\right)$ of a particle, which is at axial position $z_{i}$ with respect to bunch center, after nominal acceleration from $E_{i 0}$ to $E_{f 0}$ at an $\operatorname{rf}$ phase $\varphi_{0}$ (crest at $\varphi_{0}=0$ and $\varphi_{0} \neq \pi / 2$ ), is given by

$$
E_{f}=E_{i}+\frac{E_{f 0}-E_{i 0}}{\cos \varphi_{0}} \cos \left(\varphi_{0}+\Delta \varphi+2 \pi z_{i} / \lambda\right)
$$

with $\Delta \varphi$ as a potential $\mathrm{rf}$ phase error, $\lambda$ the $\mathrm{rf}$ wavelength, and $E_{i}$ the initial (not perfectly nominal) beam energy. Here, the convention is used that the leading 'head' of the bunch is in the direction $z<0$. The relative energy deviation after acceleration, $\delta_{f} \equiv\left(E_{f}-E_{f 0}\right) / E_{f 0}$, is then 


$$
\delta_{f} \approx \delta_{i}+\left(1-\frac{E_{i 0}}{E_{f 0}}\right)\left[\frac{\cos \left(\varphi_{0}+\Delta \varphi\right)-\left[2 \pi z_{i} / \lambda\right] \sin \left(\varphi_{0}+\Delta \varphi\right)}{\cos \varphi_{0}}-1\right] \text {, }
$$

where $\delta_{i} \equiv\left(E_{i}-E_{i 0}\right) / E_{f 0}$ represents small injection energy deviations scaled to the final energy. Wakefields are ignored here, and it is assumed that $\left|z_{i}\right|<<\lambda / 2 \pi$. From Eq. (7.2) the linear correlation constant, $k$, is defined as

$$
k\left(\varphi_{0}, \Delta \varphi\right) \equiv \frac{\partial \delta_{f}}{\partial z_{i}}=-\frac{2 \pi}{\lambda}\left(1-\frac{E_{i 0}}{E_{f 0}}\right) \frac{\sin \left(\varphi_{0}+\Delta \varphi\right)}{\cos \varphi_{0}} .
$$

After a bending section with path length dependence, $R_{56}$, where $z_{f}=z_{i}+R_{56} \delta_{f}$, the final bunch length, $\sigma_{z f}$, and energy spread, $\sigma_{\delta f}$, are functions of the initial bunch length, $\sigma_{z_{i}}$, and energy spread, $\sigma_{\delta_{i}}$, (assuming an uncorrelated initial bunch $\left\langle z_{i} \delta_{i}\right\rangle=0$ ):

$$
\begin{gathered}
\sigma_{z_{f}}=\sqrt{\left(1+k R_{56}\right)^{2} \sigma_{z_{i}}^{2}+R_{56}^{2} \sigma_{\delta_{i}}^{2}} \approx\left|1+k R_{56}\right| \sigma_{z_{i}}, \\
\sigma_{\delta_{f}}=\sqrt{k^{2} \sigma_{z_{i}}^{2}+\sigma_{\delta_{i}}^{2}} \approx|k| \sigma_{z_{i}} .
\end{gathered}
$$

The injection energy spread, scaled to the final energy, $\sigma_{\delta}$, is typically insignificant here. Minimum bunch compression is achieved for $R_{56}=-1 / k$, with under-compression at $\left|R_{56}\right|<1 /|k|$ and over-compression for $\left|R_{56}\right|>1 /|k|$. For a magnetic chicane, the $R_{56}$ value is negative (with bunch head at $z<0$ ), and compression is achieved with $k>0$ ( or $-\pi / 2<\varphi_{0}<0$; i.e., bunch head at a lower energy than bunch tail).

Since $k$ is a function of $\Delta \varphi$, the bunch length is sensitive to phase variations and injector timing jitter. For a single stage compressor, the final bunch length change, $\Delta \sigma_{z_{f}}$ for a phase change, $\Delta \varphi$, is given by [7]

$$
\frac{\Delta \sigma_{z_{f}}}{\sigma_{z_{f 0}}} \approx-\left(\frac{\sigma_{z_{i}}}{\sigma_{z_{f 0}}} \mp 1\right) \Delta \varphi \cot \varphi_{0}
$$

with under-compression expressed by the minus sign and over-compression by the plus sign. For $\varphi_{0}=20^{\circ}$ and a compression ratio of $\sigma_{z_{i}} / \sigma_{z f 0}=33$, phase jitter of only $0.1^{\circ}$ S-band ( $\left.0.1 \mathrm{psec}\right)$ results in final bunch length (or undulator peak current) jitter of $\sim 16 \%$. Figure 7.3 shows the required $R_{56}$, and the bunch length sensitivity to phase jitter, versus nominal rf phase, for a single stage compressor with compression ratios of both 33 and 3.3.

Clearly, a single stage compressor is too sensitive. Furthermore, the final bunch length of a single stage compressor is limited by non-linearities, such as $\mathrm{rf}$ curvature, which make compression from $\sim 1 \mathrm{~mm}$ to $22 \mu \mathrm{m}$ very difficult. However, a two-stage compression system brings about cancellations that can reduce this phase jitter sensitivity by more than an order of magnitude. This arrangement allows the first compressor to be located at relatively low energy so that the next linac accelerates a shorter bunch. The reduced transverse wakefield of the shorter bunch provides looser rf-structure alignment tolerances. Conversely, a single compression stage 
would need to be placed at a high enough beam energy so that space charge forces are not significant for a $22 \mu \mathrm{m}$ bunch. Such a single stage system would prolong the acceleration of the long bunch through more linac, which tightens alignment tolerances. For these reasons, a twostage compressor is used.
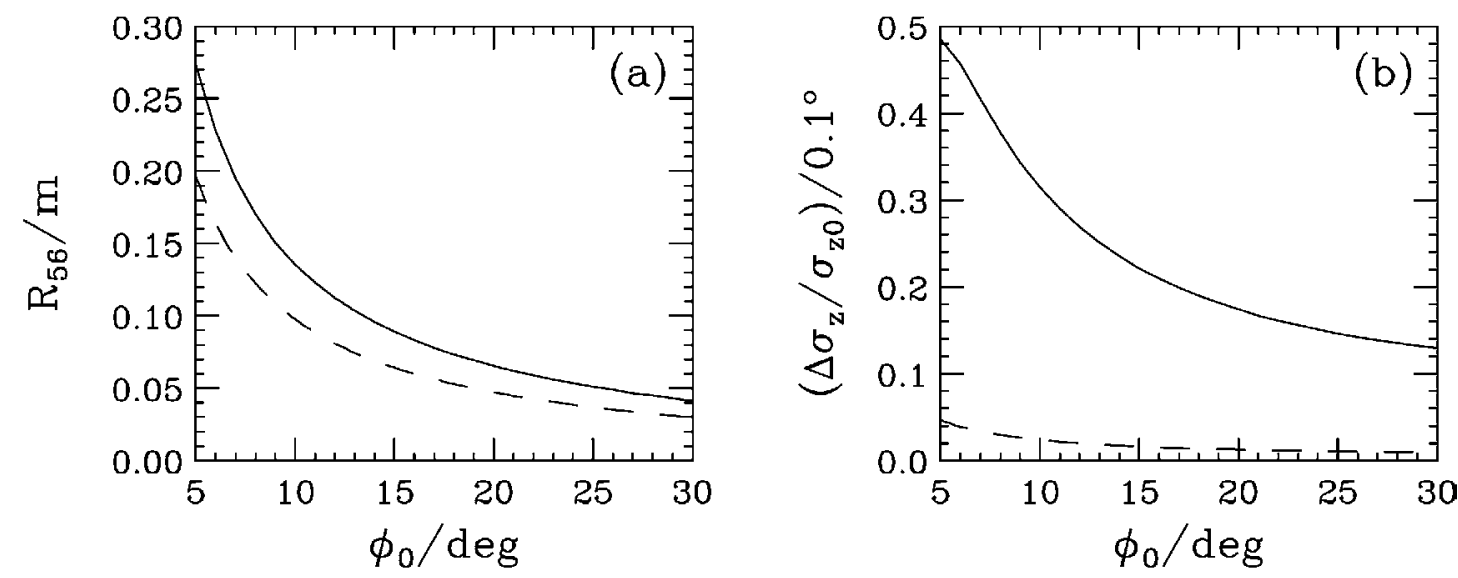

Figure 7.3 The required $R_{56}$ (a) and the relative bunch length change per $0.1^{\circ}(\mathrm{S}$-band) phase jitter (b) for a single stage compressor and linac which accelerates a 1 -mm bunch from 80 $\mathrm{MeV}$ to $250 \mathrm{MeV}$ at an rf phase of $\varphi_{0}$ for a final bunch length of both $30 \mu \mathrm{m}$ (solid) and $300 \mu \mathrm{m}$ (dashed).

The linear relations for a two-stage bunch compression system are similarly expressible. For a first linac which accelerates from $E_{0}$ to $E_{1}$ at $\mathrm{rf}$ phase $\varphi_{1}$ followed by a first compressor with $R_{56} \equiv \alpha_{1}$, then a second linac which accelerates from $E_{1}$ to $E_{2}$ at $\mathrm{rf}$ phase $\varphi_{2}$ followed by a second compressor with $R_{56} \equiv \alpha_{2}$, the final bunch length, $\sigma_{z_{2}}$, and energy spread, $\sigma_{\delta_{2}}$, are approximately

$$
\begin{gathered}
\sigma_{z_{2}} \approx \sqrt{\left[\left(1+\alpha_{1} k_{1}\right)\left(1+\alpha_{2} k_{2}\right)+\alpha_{2} k_{1} E_{1} / E_{2}\right]^{2} \sigma_{z_{0}}^{2}+\left[\alpha_{1}\left(1+\alpha_{2} k_{2}\right) E_{0} / E_{1}+\alpha_{2} E_{0} / E_{2}\right]^{2} \sigma_{\delta_{0}}^{2}}, \\
\sigma_{\delta_{2}} \approx \sqrt{\left[k_{2}\left(1+\alpha_{1} k_{1}\right)^{2}+k_{1} E_{1} / E_{2}\right]^{2} \sigma_{z_{0}}^{2}+\left[\alpha_{1} k_{2} E_{0} / E_{1}+E_{0} / E_{2}\right]^{2} \sigma_{\delta_{0}}^{2}} .
\end{gathered}
$$

In this case, $\sigma_{\delta_{0}}$ is the relative energy spread at injection to the first linac (at energy $E_{0}$ ) and $\sigma_{z_{0}}$ is the initial bunch length there. Note, $k_{1}$ is a function of the rf phase and initial and final energies of Linac-1 as in Eq. (7.3). The value for $k_{2}$, however, depends on the total phase error, which may have contributions from both Linac-1 and Linac-2:

$$
k_{2}=-\frac{2 \pi}{\lambda}\left(1-\frac{E_{1}}{E_{2}}\right) \frac{\sin \left(\varphi_{2}+\left(1+\alpha_{1} k_{1}\right) \Delta \varphi_{1}+\Delta \varphi_{2}\right)}{\cos \varphi_{2}} .
$$

It appears that the above relationships can be used to find a minimum final bunch length sensitivity to injection phase jitter, $\Delta \varphi_{1}$. In fact, longitudinal wakefields, rf curvature, and second order path length dependence, $T_{566}$, complicate these calculations sufficiently to invalidate this simple linear model. The above relationships can help one to understand sensitivities, but the 
optimal stability is best found with a complete computer simulation that includes the important non-linearities and wakefield effects.

\subsubsection{Design Optimization Technique}

In order to determine the best parameter set which provides the correct acceleration, compression, machine stability, and final energy spread, a fast computer program is used which semi-analytically models the longitudinal beam dynamics and minimizes a penalty function while varying the key system parameters [5]. The varied parameters are:

- $\quad 1^{\text {st }}$ and $2^{\text {nd }}$ compressor strengths $\left(R_{56-1}, R_{56-2}\right)$,

- $\quad$ RF phases of L1, L2, and L3 $\left(\varphi_{1}, \varphi_{2}, \varphi_{3}\right)$,

- Energy (or location) of $1^{\text {st }}$ and $2^{\text {nd }}$ compression stages $\left(E_{\mathrm{BC} 1}, E_{\mathrm{BC} 2}\right)$.

The penalty function, which is minimized, includes:

- The deviation of the final and intermediate bunch lengths from the desired ones, normalized to an allowable error (e.g., $22 \mu \mathrm{m} \pm 0.1 \mu \mathrm{m}$ rms at undulator, and $200 \mu \mathrm{m}$ $\pm 100 \mu \mathrm{m}$ after $\mathrm{BC} 1)$,

- The energy deviation at both compressors and at the undulator, with respect to the desired energy, each normalized to an allowable error (e.g., $14.35 \mathrm{GeV} \pm 0.02 \mathrm{GeV}$ at undulator, or $250,+250,-50 \mathrm{MeV}$ at $1^{\text {st }}$ compressor),

- The deviation of the final correlated energy spread at the undulator with respect to the desired energy spread, normalized to an allowable error (e.g., $0.01 \% \pm 0.002 \%$ this is a signed quantity allowing for a positive or negatively correlated energy chirp as a desired outcome),

- The four sensitivities of: 1) final relative energy error vs. gun-timing error, and 2) vs. bunch-charge error; 3) the final peak current vs. gun-timing error, and 4) vs. bunchcharge error, all normalized to an allowable error (e.g., $0 \pm 100 \mathrm{Amps} / \mathrm{psec}$ ).

The parameters are varied over a reasonable range by constraining the minimization scan. The parameters constrained are:

- The two $R_{56}$ values (e.g., $-10 \mathrm{~mm}$ to $-40 \mathrm{~mm}$ ),

- The mean rf accelerating gradient per linac section (e.g., $18 \mathrm{MV} / \mathrm{m}$ upper limit),

- The three rf phases per linac section (e.g., $-50^{\circ}$ to $+50^{\circ}$ ),

- The net active S-band linac length available to the LCLS beam ( 900 m).

The semi-analytic model includes longitudinal wakefields, non-linearities ( $T_{566}$ and sinusoidal rf), and the effects of errors (timing, phase, charge and energy). The model also includes the Xband rf section, its wakefield, and its four-fold frequency increase. The details of the model and its range of validity are reviewed in reference [5]. The final chosen parameters, which provide for optimum machine stability, as well as the undulator peak current and energy spread requirements 
are described below for the nominal case of a 1-nC bunch charge. Before final parameters are described, however, the motivations for the X-band rf section are described.

\subsubsection{X-Band RF Compensation}

A short X-band $\mathrm{rf}$ section $\left(f_{\mathrm{rf}}=11.424 \mathrm{GHz}\right)$ is included just prior to the $\mathrm{BC} 1$ chicane in order to better linearize the energy-time correlation along the bunch. A more linear correlation allows the bunch to be compressed to a much smaller length than would be possible without the X-band section. The 0.6-meter long X-band section is run at the decelerating crest phase $\left(180^{\circ}\right)$ so that second order energy-time correlations in the beam, which are induced in the S-band linac-0 and linac-1, can be removed. The net beam energy is only reduced by $22 \mathrm{MeV}$ (from $272 \mathrm{MeV}$ to $250 \mathrm{MeV}$ ) by the $\mathrm{X}$-band section, which is run at a relatively low gradient of $\sim 37 \mathrm{MV} / \mathrm{m}$. Without the X-band rf, the initial bunch length of $830 \mu \mathrm{m}$ can only be compressed to $\sim 400 \mu \mathrm{m}$ rms without a large spike appearing at the head of the temporal distribution. A large peak-current spike can drive severe beam instabilities due to coherent synchrotron radiation (CSR) in the chicanes, which can quickly destroy the horizontal emittance and slice energy spread. Since the results of the fast design optimization code (described above) indicate a first compression level of $200 \mu \mathrm{m}$ is desirable, the $\mathrm{X}$-band section is needed to allow this possibility.
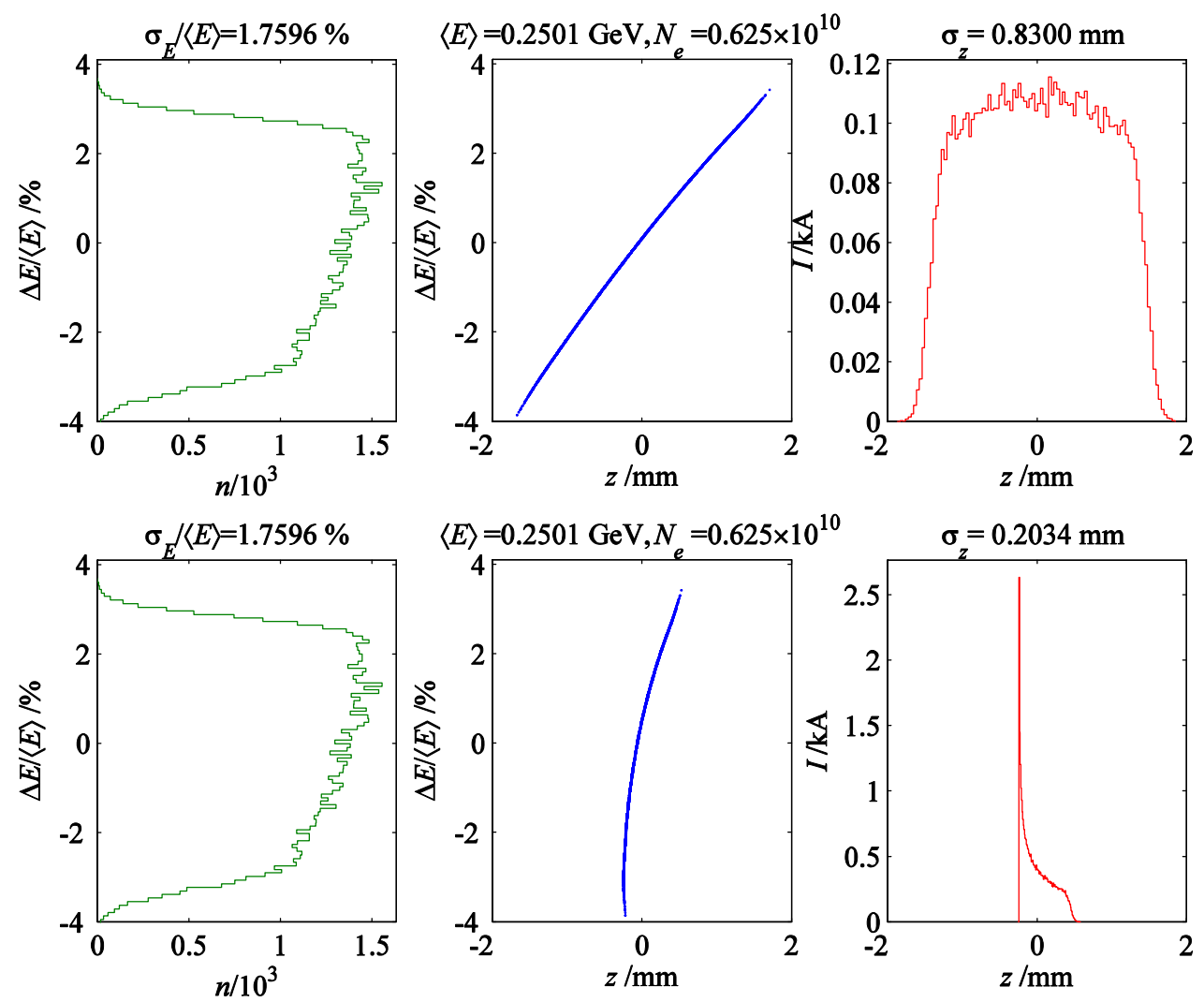

Figure 7.4 Simulated compression without X-band rf, where an 830- $\mu \mathrm{m}$ bunch is being compressed towards $200 \mu \mathrm{m}$ (top-row: after S-band rf, bottom-row: after chicane). The bunch begins to fold over itself causing an large undesirable peak current spike. 
Figure 7.4 shows a simulated compression without X-band $\mathrm{rf}$, where an $830-\mu \mathrm{m}$ bunch (top row of plots) is being compressed toward $200 \mu \mathrm{m}$ (bottom row of plots). The bunch begins to fold over itself after the chicane resulting in a peak current spike at the head of the bunch in excess of $2 \mathrm{kA}$. The effect is exaggerated by the $T_{566}$ of the chicane, which is always of a sign $\left(0<T_{566} \approx-3 R_{56} / 2\right)$ to amplify the S-band $\mathrm{rf}$ curvature (i.e., for an accelerating rf phase and a simple chicane without internal focusing optics).
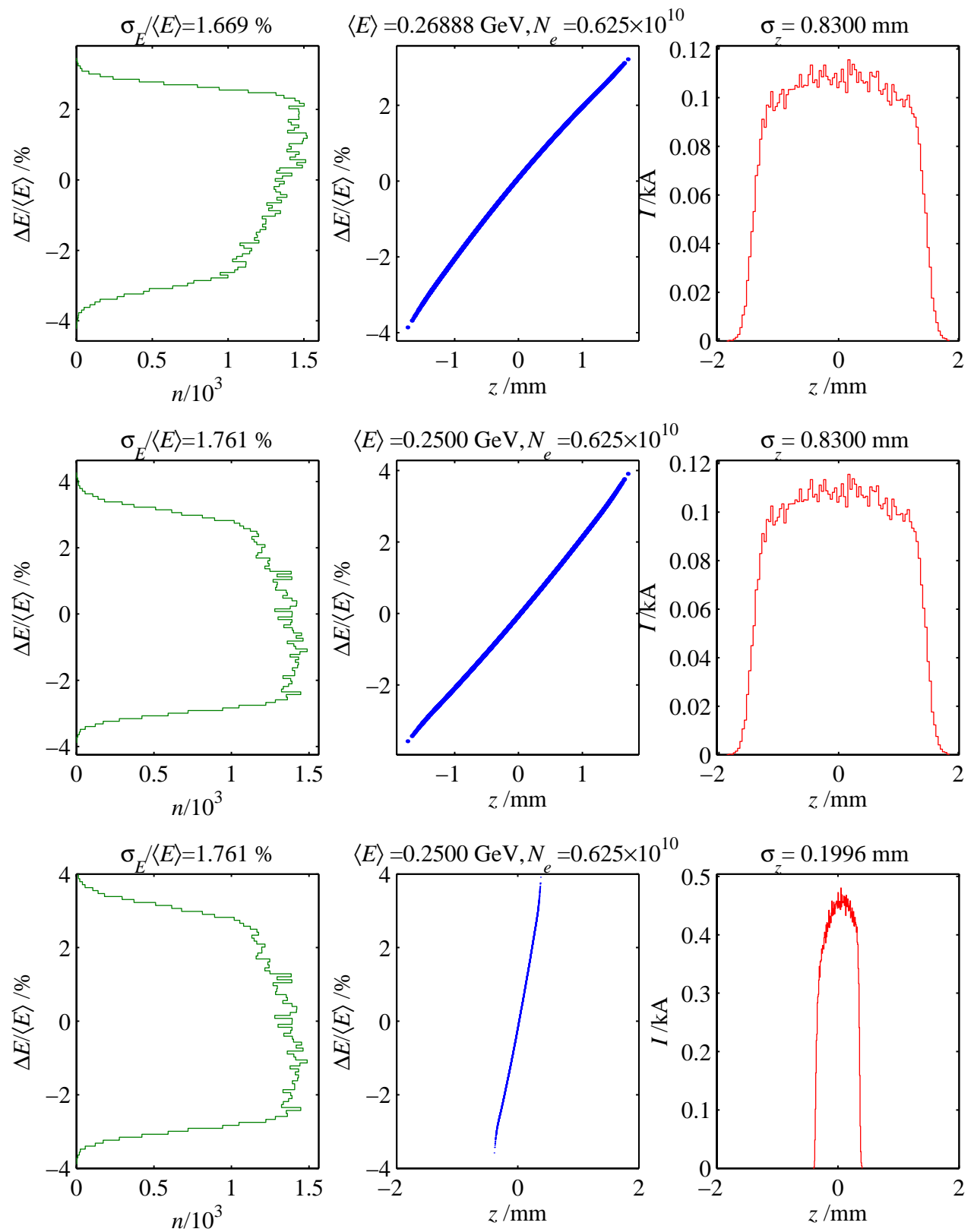

Figure 7.5 Simulated compression, now including X-band rf, where the same 830- $\mu$ m bunch is easily compressed to $200 \mu \mathrm{m}$, and the final temporal distribution is unaltered (top-row: after S-band rf, middle-row: after X-band rf, bottom-row: after chicane). In addition, the bunch can easily be compressed much further if desired. 
With the X-band rf included at a decelerating phase, and with the proper voltage (see below), the compression can be linearized such that a $200-\mu \mathrm{m}$ rms bunch length is easily achievable with no significant alteration in the temporal distribution. Figure 7.5 shows the simulation, starting from similar conditions as the top row of plots in Figure 7.4, but with some minor S-band phase and voltage adjustments (top row of Figure 7.5), and proceeding through the 20-MV X-band rf (middle row of Figure 7.5), and then through the chicane (bottom row of Figure 7.5). The final temporal distribution is nearly unaltered with the proper X-band rf compensation.

The X-band voltage, $V_{x}$, required to compensate the non-linear energy-time correlations induced in both the L0 and the L1 linacs, as well as that of the chicane, is given by [8]

$$
e V_{x}=\frac{E_{0}\left[1-\frac{1}{2 \pi^{2}} \frac{\lambda_{s}^{2} T_{566}}{R_{56}^{3}}\left(1-\sigma_{z} / \sigma_{z_{0}}\right)^{2}\right]-E_{i}}{\left(\lambda_{s} / \lambda_{x}\right)^{2}-1},
$$

where $\lambda_{s}$ and $\lambda_{x}$ are the S-band and X-band, respectively, $\mathrm{rf}$ wavelengths $(10.50 \mathrm{~cm}$ and $2.625 \mathrm{~cm}), \sigma_{z 0}$ and $\sigma_{z}$ are the initial and final bunch lengths, and $E_{i}$ and $E_{0}$ are the initial and final beam energies at gun and $\mathrm{BC} 1$ chicane, respectively. The net deceleration is small due to the large denominator in Eq. (7.8), which stems from the high harmonic number chosen $\left(\lambda_{s} / \lambda_{x}=4\right)$. For parameters $R_{56} \approx-35.9 \mathrm{~mm}, \quad T_{566} \approx-3 R_{56} / 2 \approx+53.9 \mathrm{~mm}, \quad \sigma_{z 0} \approx 830 \mu \mathrm{m}, \quad \sigma_{z} \approx 200 \mu \mathrm{m}$, $E_{i} \approx 7 \mathrm{MeV}$, and $E_{0} \approx 250 \mathrm{MeV}$, the X-band voltage which removes the $2^{\text {nd }}$-order energy-time correlation along the bunch after $\mathrm{BC} 1$ is $V_{x} \approx 22 \mathrm{MV}$. Tracking studies show that this voltage provides a more uniform temporal distribution after BC2 (see Figure 7.6 and Figure 7.7).

With the availability at SLAC of high-gradient X-band accelerating structures developed for the NLC project [9], this $4^{\text {th }}$ harmonic compensation strategy becomes a practical solution for the LCLS. A relatively low rf gradient of $37 \mathrm{MV} / \mathrm{m}$ allows a $0.6-$ meter long section to be used. The mean iris radius is $4.7 \mathrm{~mm}$, which is very large for the typical beam size of $100 \mu \mathrm{m}$ (see beta functions in Figure 7.14). The transverse wakefields of the $X$-band structure are strong, but the section is very short. The alignment tolerance of the short X-band structure is therefore $\sim 200 \mu \mathrm{m}$, which is not particularly challenging.

A similar X-band section is not required at the entrance to $\mathrm{BC} 2$, since the quadratic correlation in the bunch after the L2-linac is much less pronounced than that after L1, due to the shortened bunch in L2 with respect to the S-band rf.

In addition to linearizing the compression process, the X-band rf also makes the net compression less sensitive to $\mathrm{rf}$ gun-timing jitter. Since the net accelerating voltage, as a function of beam arrival time, is more linear when the X-band $\mathrm{rf}$ is included, the induced energy-time correlation along the bunch then becomes nearly independent of beam arrival time [8]. This loosens the rf gun-timing jitter sensitivity by nearly a factor of four with respect to that published in the LCLS Design Study Report [16] (see also Figure 7.8). The section will need a dedicated $\mathrm{X}$-band klystron, which is available at SLAC. The existing modulator at 21-2 will be used. 


\subsubsection{Nominal Parameters}

The nominal design parameters for the LCLS two-stage bunch compression system at a bunch charge of $1 \mathrm{nC}$ are summarized graphically in Figure 7.1 and numerically in Table 7.1 below.

A string of five linac sections and two chicane sections are used to compress an $830-\mu \mathrm{m}$ rms bunch at $150 \mathrm{MeV}$ to $22-\mu \mathrm{m}$ rms at $14.35 \mathrm{GeV}$. The final bunch is still under-compressed, so it is actually possible to compress it even further (to $<5 \mu \mathrm{m} \mathrm{rms}$ ). However, many severe challenges arise with a shorter bunch at $1 \mathrm{nC}$, such as coherent synchrotron radiation in BC2 (see Section 7.4.2) and resistive wall and surface roughness wakefields in the undulator vacuum chamber (see Chapter 8, "Wakefield Effects in the Undulator"). These effects can become intolerable for much shorter bunches, depending on the charge. An rms bunch length of $22 \mu \mathrm{m}$ at $1 \mathrm{nC}$ achieves the 3.4-kA peak current required and should allow management of the various micro-bunch limitations. A longer bunch is also operationally possible if the transverse "slice" emittance achieved is less than $1.2 \mu \mathrm{m}$ [e.g., $\sigma_{z} \approx 34 \mu \mathrm{m}$ at $\gamma \varepsilon \approx 1.0 \mu \mathrm{m}$ and $Q \approx 1 \mathrm{nC}$, see Eq. (7.11)].

Table 7.1 LCLS nominal compression and acceleration parameters per beamline section, for a 1-nC bunch charge. The phase of $-10^{\circ}$ in L3 helps to stabilize the beam energy.

\begin{tabular}{|c|c|c|c|c|c|c|c|c|}
\hline Beamline & $\begin{array}{c}\boldsymbol{E}_{\text {in }} \\
\mathrm{GeV}\end{array}$ & $\begin{array}{c}\boldsymbol{E}_{\text {out }} \\
\mathrm{GeV}\end{array}$ & $\begin{array}{c}\sigma_{z-\text { in }} \\
\mathrm{mm}\end{array}$ & $\begin{array}{c}\sigma_{z \text {-out }} \\
\mathrm{mm}\end{array}$ & $\begin{array}{c}\sigma_{\delta \text {-in }} \\
\%\end{array}$ & $\begin{array}{c}\sigma_{\mathcal{\delta} \text {-out }} \\
\%\end{array}$ & $\begin{array}{l}\varphi_{\mathbf{r f}} \\
\operatorname{deg}\end{array}$ & $\begin{array}{l}\boldsymbol{R}_{56} \\
\mathrm{~mm}\end{array}$ \\
\hline Linac-1 & 0.15 & 0.27 & 0.83 & 0.83 & 0.10 & 1.67 & -38.1 & - \\
\hline Linac- $X$ & 0.27 & 0.25 & 0.83 & 0.83 & 1.67 & 1.78 & 180 & - \\
\hline $\mathrm{BC} 1$ & 0.25 & 0.25 & 0.83 & 0.19 & 1.78 & 1.78 & - & -35.9 \\
\hline Linac-2 & 0.25 & 4.54 & 0.19 & 0.19 & 1.78 & 0.76 & -42.8 & - \\
\hline $\mathrm{BC} 2$ & 4.54 & 4.54 & 0.19 & 0.022 & 0.76 & 0.76 & - & -22.5 \\
\hline Linac-3 & 4.54 & 14.35 & 0.022 & 0.022 & 0.76 & 0.02 & -10.0 & - \\
\hline
\end{tabular}

For this ultra-relativistic beam, the bunch length cannot change through a linac (excluding L0), and the energy spread does not significantly change through the chicanes. Phase and current jitter tolerances are described in Section 7.2.6.

The first stage of compression is from $830 \mu \mathrm{m}$ to $190 \mu \mathrm{m}$. This level of compression is only possible by including the short X-band rf section (Linac- $X$ or LX) just prior to $\mathrm{BC} 1$. As described above, the X-band rf linearizes the compression. This is accomplished by running the X-band section at the decelerating rf crest $\left(180^{\circ}\right)$ at $22 \mathrm{MV}$. In this case, $\mathrm{L} 1$ accelerates to $272 \mathrm{MeV}$ and LX decelerates to $250 \mathrm{MeV}$. This choice, for the parameters of Table 7.1, also best minimizes final bunch length sensitivity to injector timing and charge jitter. Finally, the chosen value of $190 \mu \mathrm{m}$ also optimally scales the longitudinal wakefield in Linac-2 so that a cancellation is established between the Linac-2 wake and the small non-linearities ( $\mathrm{rf}$ curvature and $T_{566}$ ) of the Linac-1/BC1 pair. The parameters are not a unique solution but represent a workable set at $1 \mathrm{nC}$. Many other possible solutions are available, including parameter sets which optimize a bunch 
charge anywhere from $1 \mathrm{nC}$ to $0.1 \mathrm{nC}$, or optimize with an intentional electron chirp at the undulator. A qualitative summary of the effects of changes to the critical compression parameters is listed in Table 7.2. The two $R_{56}$ values are not considered free parameters here since their values depend on $\mathrm{rf}$ phases and other factors.

The lower limit on the choice for $\mathrm{BC} 1$ energy is set by space charge forces of the shorter bunch with $250 \mathrm{MeV}$ considered a safe energy. The upper limit is set by the desire to initially compress the bunch early in the linac to ease transverse wakefields. The chosen energy of $250 \mathrm{MeV}$ also desensitizes the system to injector timing jitter and is a practical solution for L1, which consists of one klystron powering three 3-meter S-band sections at an $\mathrm{rf}$ phase of $-38^{\circ}$ off crest at an average gradient of $17.5 \mathrm{MV} / \mathrm{m}$. The location (energy) of $\mathrm{BC} 2$ is set by the need to produce a very small energy spread at $14.3 \mathrm{GeV}$. This involves a balance between the longitudinal geometric wakefield in L3 (which scales with Linac-3 length) and the remaining $\delta-z$ correlation just after $\mathrm{BC} 2$. Other factors, including synchrotron radiation, are discussed in more detail in Section 7.4.1 (BC1) and Section 7.4.2 (BC2).

Table 7.2 Bunch compression parameter trade-offs: A qualitative summary of the effects of changes to the bunch compression parameters. Only limitations are noted. An "increase" of rf phase, $\varphi$, refers to moving farther off rf crest and $\sigma_{z 1}$ is the intermediate bunch length (after $\mathrm{BC} 1$, but before $\mathrm{BC} 2$ ).

\begin{tabular}{|c|c|c|}
\hline Parameter & Increase Parameter & Decrease Parameter \\
\hline$\sigma_{z_{1}} \approx 190 \mu \mathrm{m}$ & $\begin{array}{l}\text { - Insufficient L2 wake compensation for } \\
\text { L2/BC2 non-linearities. } \\
\text { - Requires stronger BC2 and more CSR. }\end{array}$ & $\begin{array}{l}\text { - CSR emittance growth increased in } \mathrm{BC} 1 \text {. } \\
\text { - Can increase jitter sensitivity. }\end{array}$ \\
\hline$\left|\varphi_{1}\right| \approx 38^{\circ}$ & $\begin{array}{l}\text { - Increased L1 energy spread. } \\
\text { - Inefficient acceleration. }\end{array}$ & $\begin{array}{l}\text { - Can increase jitter sensitivity. } \\
\text { - Increased } \mathrm{BC} 1 \text { strength and } \Delta \varepsilon \text { due to CSR. }\end{array}$ \\
\hline$\left|\varphi_{2}\right| \approx 43^{\circ}$ & $\begin{array}{l}\text { - Energy spread too large for } \\
\text { cancellation with L3 wake. } \\
\text { - Inefficient acceleration. }\end{array}$ & $\begin{array}{l}\text { - Energy spread too small—over-compensated } \\
\text { with L3 wake. } \\
\text { - Increased BC2 strength and CSR } \Delta \varepsilon \text {. }\end{array}$ \\
\hline$E_{1}=250 \mathrm{MeV}$ & $\begin{array}{l}\cdot \text { Longer L1 } \longrightarrow \text { stronger L1 transverse } \\
\text { wakes and increased L1 } \Delta \varepsilon \text {. } \\
\text { - Can increase jitter sensitivity. }\end{array}$ & $\begin{array}{l}\text { - Increase } \mathrm{BC} 1 \text { chicane strength and CSR } \Delta \varepsilon \text {. } \\
\text { - Increased space charge forces. }\end{array}$ \\
\hline$E_{2}=4.54 \mathrm{GeV}$ & $\begin{array}{l}\text { - Increase BC2 } \Delta \varepsilon \text { due to incoherent } \\
\text { synchrotron radiation, or lengthen BC2. } \\
\text { - Shorter L3— insufficient L3 wake for } \\
\text { L2 energy spread compensation. }\end{array}$ & $\begin{array}{l}\text { - Longer L3 } \rightarrow \text { L3 wake too large- over- } \\
\text { compensation of L2 energy spread. } \\
\text { - Shorter L2 } \longrightarrow \text { insufficient L2 wake } \\
\text { compensation for L2/BC2 non-linearities. }\end{array}$ \\
\hline
\end{tabular}

System optimization scans show that even higher BC2 energy (i.e., $>4.5 \mathrm{GeV}$ ) can further desensitize the final bunch length and final energy to gun timing and charge jitter. In this case, however, the $\mathrm{BC} 2$ chicane needs to be even stronger and longer, so a compromise has been made at $4.54 \mathrm{GeV}$, which is also consistent with operation at a $15-\AA$ FEL radiation wavelength, where the L3 linac RF is simply switched off. 


\subsubsection{Longitudinal Tracking Summary}

In this section the longitudinal geometric wakefield and other beam dynamics effects are applied in a $6 \mathrm{D}$ tracking simulation to graphically summarize the compression process through the accelerator. (A full 6D tracking summary is presented in Section 7.6.) A more detailed description of the wakefields for the SLAC S-band accelerating structures, as well as a justification for the use of the asymptotic wake, is given in Section 7.9. The tracking code used here is Elegant [10], which includes non-linearities such as $T_{566}, U_{5666}$ (the second and higherorder compression terms), longitudinal geometric wakefields of the rf-structures, resistive-wall longitudinal wakefields (where significant), the sinusoidal rf accelerating voltage, and the incoherent and coherent synchrotron radiation in the bends (CSR is a 1D line-charge model). The tracking proceeds from the output of the LCLS injector, at $150 \mathrm{MeV}$, to the undulator entrance at $14.3 \mathrm{GeV}$. The 6D input particle coordinates are from Parmela [11] after tracking through the LCLS injector to the end of $\mathrm{L} 0$ at $150 \mathrm{MeV}$ [12]. The tracking uses $2 \cdot 10^{5}$ macro-particles.

A more complete summary of the tracking is presented in Section 7.6. The Elegant simulations ignore space charge effects, since the compression process takes place at energies well above $150 \mathrm{MeV}$. The input particle coordinates from Parmela do, however, include the effects of space charge forces at energies below $150 \mathrm{MeV}$.

Figure 7.6 and Figure 7.7 show longitudinal phase space, energy distributions, and axial ( $z$ ) distributions at various points in the compression process. The input gun-laser pulse has a uniform temporal distribution with a 1-psec rise/fall-time and the RF gradient in the gun is $120 \mathrm{MV} / \mathrm{m}$. After acceleration to $150 \mathrm{MeV}$, the temporal distribution becomes slightly rounded, as shown in the figures, with a $0.83-\mathrm{mm}$ rms bunch length $(2.8-\mathrm{ps} \mathrm{rms}$, or 10.2-ps FWHM) and $0.11-\%$ rms projected relative energy spread at $150 \mathrm{MeV}$, with a bunch population of $6.25 \times 10^{9}$ $\mathrm{ppb}(1 \mathrm{nC})$. The rms incoherent energy spread is very small at just $3 \mathrm{keV}\left(2 \times 10^{-5}\right.$ of $\left.150 \mathrm{MeV}\right)$.

As Figure 7.6 and Figure 7.7 show, the compression process has been arranged so that many of the non-linearities, such as rf-curvature and wakefield effects, will be compensated, leaving a narrow energy profile at $14.3 \mathrm{GeV}$. The final rms bunch length is $22 \mu \mathrm{m}$ with $>3.0 \mathrm{kA}$ of peak beam current all along the bunch. Tails exist in the energy distribution (shown on $3^{\text {rd }}$ row of Figure 7.7). The core of the beam, however, has an rms energy spread of $\sim 0.01 \%$ with $\sim 80 \%$ of the particles contained within a $\pm 0.1 \%$ energy window. The energy tails $\left(\left|\Delta E / E_{0}\right|>0.1 \%\right)$, which comprise $20 \%$ of the beam, have been cut out of the bottom row of Figure $7.7(14.3 \mathrm{GeV})$ to show the core beam more clearly, while all particles are shown in the $3^{\text {rd }}$ row of Figure 7.7. Note, the incoherent component of the final energy spread at any particular slice of the bunch core ( $0.75 \mu \mathrm{m}$ slice $>$ FEL slippage length) is $0.008 \% \mathrm{rms}$, including the incoherent synchrotron radiation of the high-energy bends. The slight micro-bunching seen in the final temporal profile is a result of the CSR effects in the two compressor chicanes, and may be somewhat exaggerated here by possible overestimates of a 1D line-charge CSR model and also by the statistical noise levels of just $2 \times 10^{5}$ macro-particles. A superconducting one-period wiggler is added just before the $\mathrm{BC} 2$ chicane in order to add incoherent energy spread and damp this CSR-induced microbunching. This subject is covered in more detail in Section 7.4.2. 


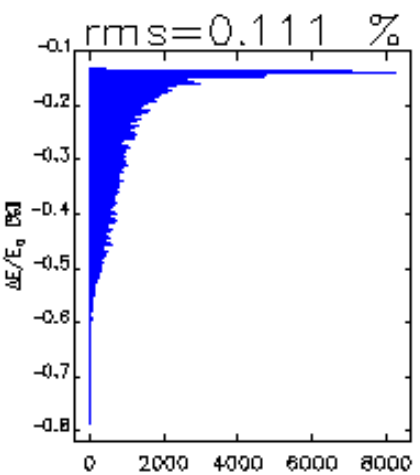

П
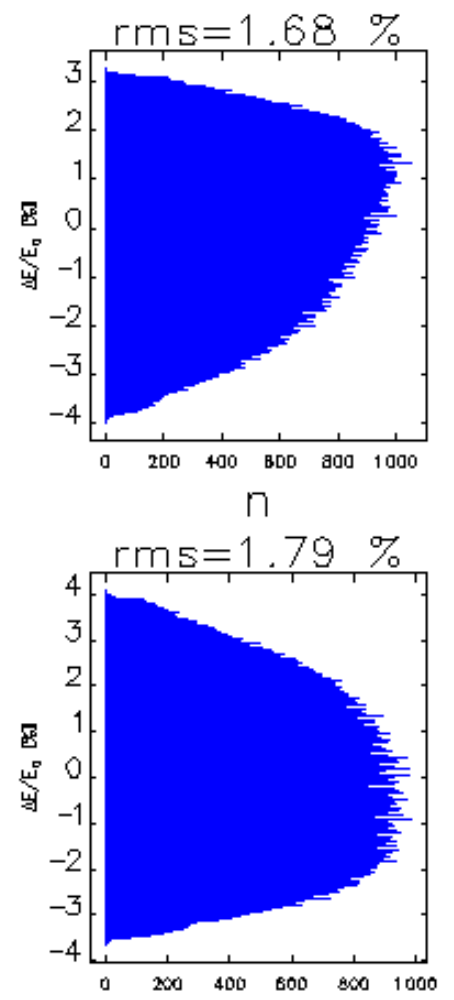

П

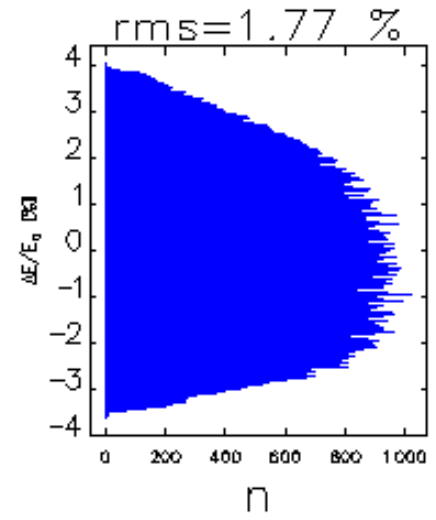

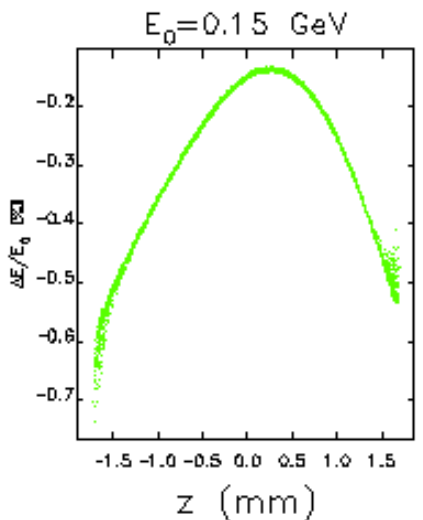

$z(\mathrm{~mm})$
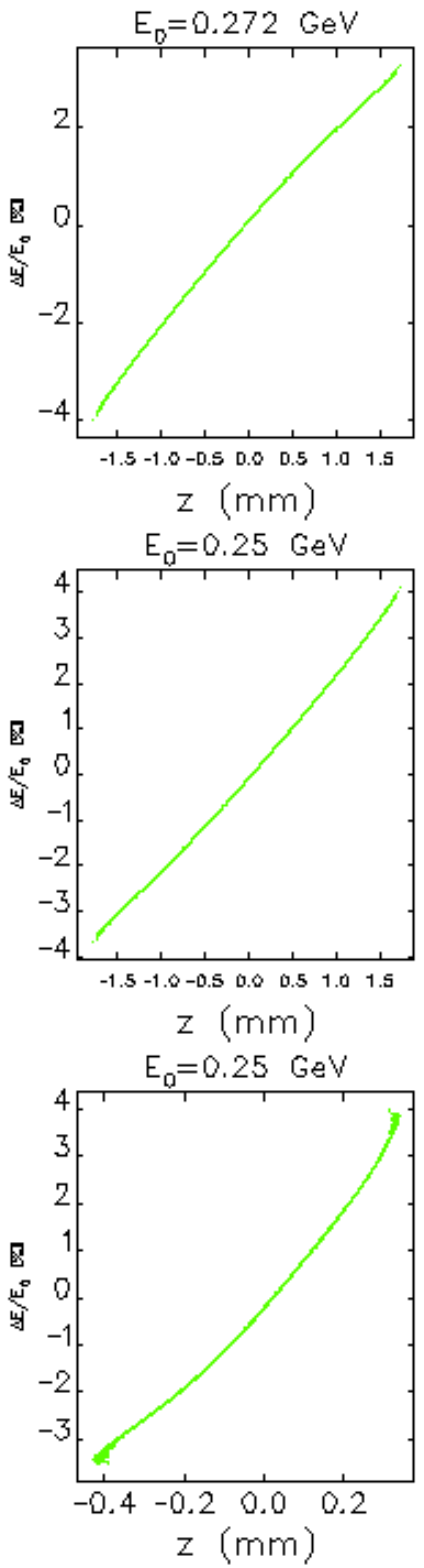
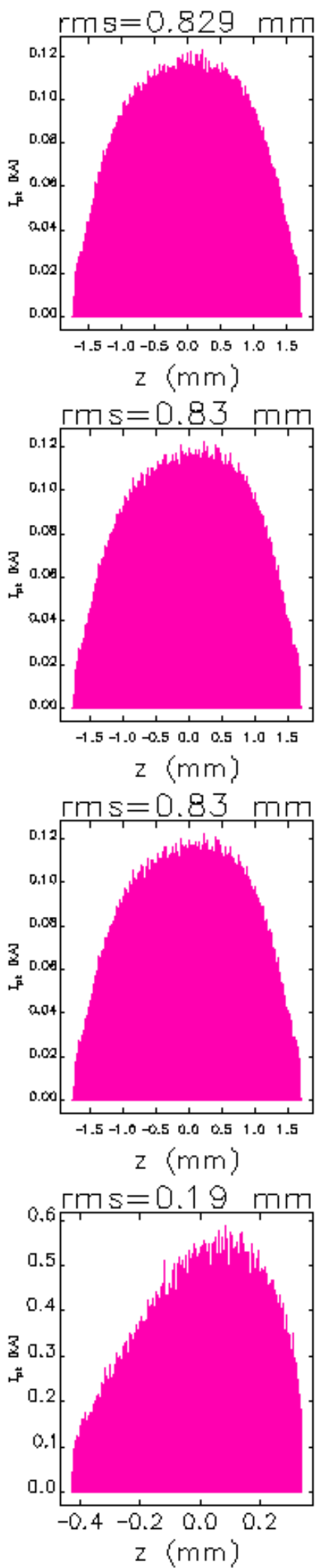

Figure 7.6 Energy (left column) and axial (right column) $e^{-}$distributions and longitudinal phase space (center column) after L0 at $150 \mathrm{MeV}$ (top row), after L1 at $272 \mathrm{MeV}$ (2nd row), after X-band at $250 \mathrm{MeV}$ (3rd row), after BC1 at $250 \mathrm{MeV}$ (4th row). Bunch head at left $(z<0)$. 

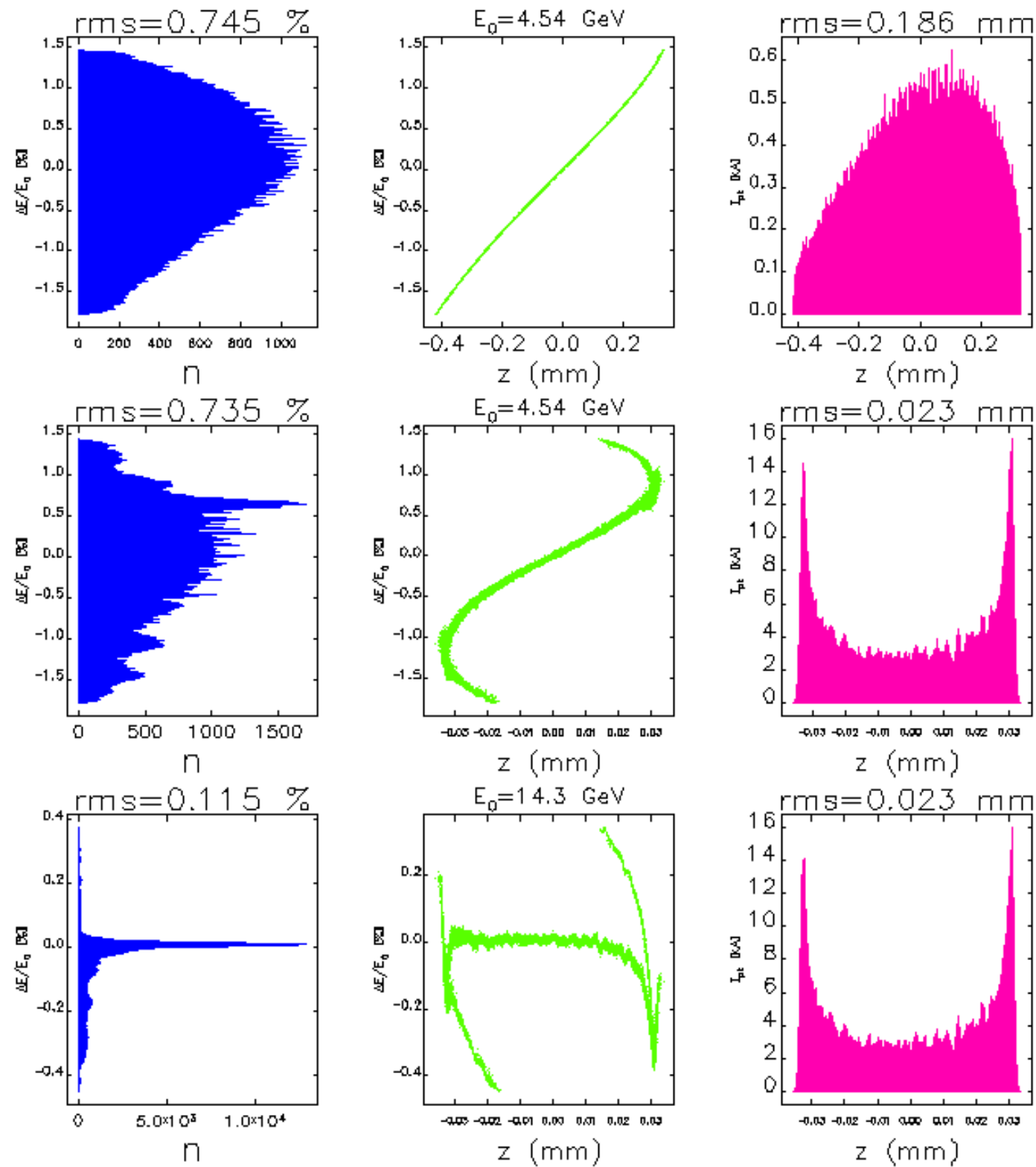

П
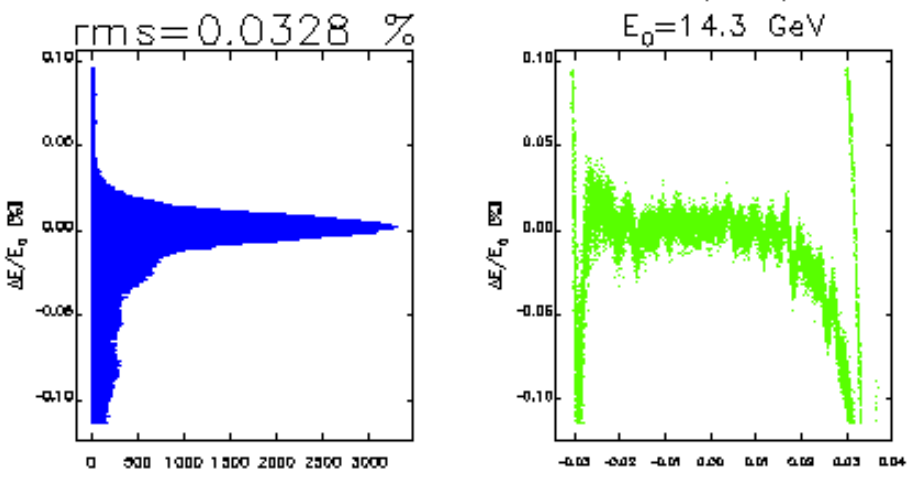

$z(\mathrm{~mm})$

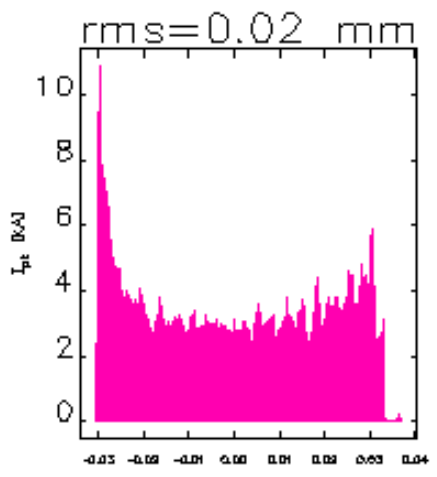

$z(m m)$

$z(m m)$

Figure 7.7 Energy (left column) and axial (right column) $e^{-}$distributions and longitudinal phase space (center column) after L2 at $4.54 \mathrm{GeV}$ (top row), after BC2 at $4.54 \mathrm{GeV}$ (2nd row), after L3 and DL2 at $14.35 \mathrm{GeV}$ (3rd row), and at undulator entrance, but after $20 \%$ of charge is cut from energy tails to show beam core (4th row). Bunch head at left $(z<0)$. 
The strong longitudinal wakefield in the L2-linac causes the extreme head and tail portions of the bunch, where the charge is lower, to have a slightly increased slope in the energy- $z$ correlation (see $3{ }^{\text {rd }}$-order curvature in top row of Figure 7.7, center plot). These head and tail sections are then over-compressed by the BC2 chicane (see $2^{\text {nd }}$ row of Figure 7.7, center plot) and lead to the large current spikes in the $z$-distribution of Figure 7.7 ( $2^{\text {nd }}, 3^{\text {rd }}$ and $4^{\text {th }}$ row of right-side plots). It is difficult to add higher $\mathrm{rf}$ harmonics to correct this level, since the energy is high, the bunch is very short, and the offending correlations are now $3^{\text {rd }}$ order, or higher. Finally, the longitudinal wakefield in L3 flattens most of the coherent energy spread into a tight core distribution shown in Figure 7.7 ( $3^{\text {rd }}$ and $4^{\text {th }}$ rows). This is an important cancellation which provides the narrow energy spread and depends on the strength of the longitudinal wakefield in L3, the bunch charge, the rf phase of L2, and the bunch length in L2 and L3. Here the projected energy spread is reduced to $<0.01 \% \mathrm{rms}$. In fact, a level of $<0.1 \% \mathrm{rms}$ is all that is required.

Calculations of the longitudinal wakefield for the micro-bunch in L3 are believed to be accurate to better than $10 \%$ (see Section 7.9). In order to allow for this potential error in the magnitude of the wakefield, the compression systems require a range over which they may be tuned. To demonstrate the tuning range required, a simulation has been run where the wakefield in L3 is arbitrarily scaled up by $20 \%$ (a factor of 1.2) and the compression systems were retuned. For this extreme case, the bunch length and energy spread at the end of L3 are completely recoverable to the conditions on the bottom row of Figure 7.7. The re-tuning produces new parameters, shown along with the nominal parameters in Table 7.3. This re-tuning works by increasing the $\mathrm{L} 2$ bunch length from $0.19 \mathrm{~mm}$ to $0.23 \mathrm{~mm}$ (moving L1 rf phase closer to crest), which increases the energy spread in $\mathrm{BC} 2$, and is then fully cancelled by the stronger L3-wake.

This simple test demonstrates the flexibility of the system and shows that a precise knowledge of the scale of the longitudinal micro-bunch wakefield is not absolutely necessary prior to construction. It does not, however, address how such a re-tune might be accomplished. Obviously, as in the case of machine commissioning, the beam and rf parameters need to be well measured and an empirical technique developed for tuning the system.

Table 7.3 Compression parameters before and after re-tuning. Changed parameters are in bold and are calculated with the L3 wakefield arbitrarily increased by $20 \%$. The nominal (or unchanged) parameters (repeated from Table 7.1) are in standard type.

\begin{tabular}{lcccccccc}
\hline Beamline & $\begin{array}{c}\boldsymbol{E}_{\text {in }} \\
\mathrm{GeV}\end{array}$ & $\begin{array}{c}\boldsymbol{E}_{\text {out }} \\
\mathrm{GeV}\end{array}$ & $\begin{array}{c}\sigma_{\boldsymbol{z} \text {-in }} \\
\mathrm{mm}\end{array}$ & $\begin{array}{c}\sigma_{\boldsymbol{z} \text {-out }} \\
\mathrm{mm}\end{array}$ & $\begin{array}{c}\sigma_{\mathcal{\delta} \text {-in }} \\
\%\end{array}$ & $\begin{array}{c}\sigma_{\mathcal{\delta} \text {-out }} \\
\%\end{array}$ & $\begin{array}{c}\left|\varphi_{\text {rf }}\right| \\
\text { deg }\end{array}$ & $\begin{array}{c}\left|\boldsymbol{R}_{\mathbf{5 6}}\right| \\
\mathrm{mm}\end{array}$ \\
\hline Linac-1 & 0.15 & 0.27 & 0.83 & 0.83 & 0.10 & $1.67 / \mathbf{1 . 4 6}$ & $38.1 / \mathbf{3 5 . 4}$ & - \\
\hline Linac- $X$ & 0.27 & 0.25 & 0.83 & 0.83 & $1.67 / \mathbf{1 . 4 6}$ & $1.78 / \mathbf{1 . 5 8}$ & 180 & - \\
\hline BC1 & 0.25 & 0.25 & 0.83 & $0.19 / \mathbf{0 . 2 3}$ & $1.78 / \mathbf{1 . 5 8}$ & $1.78 / \mathbf{1 . 5 8}$ & - & $35.9 / \mathbf{3 8 . 4}$ \\
\hline Linac-2 & 0.25 & 4.54 & $0.19 / \mathbf{0 . 2 3}$ & $0.19 / \mathbf{0 . 2 3}$ & $1.78 / \mathbf{1 . 5 8}$ & $0.76 / \mathbf{0 . 9 4}$ & $42.8 / \mathbf{4 2 . 2}$ & - \\
\hline BC2 & 4.54 & 4.54 & $0.19 / \mathbf{0 . 2 3}$ & 0.022 & $0.76 / \mathbf{0 . 9 4}$ & $0.76 / \mathbf{0 . 9 4}$ & - & $22.5 / \mathbf{2 2 . 3}$ \\
\hline Linac-3 & 4.54 & 14.35 & 0.022 & 0.022 & $0.76 / \mathbf{0 . 9 4}$ & 0.02 & 10.0 & - \\
\hline
\end{tabular}




\subsubsection{Beam Jitter Sensitivities}

Although it has been optimized, the two-stage compressor system is still sensitive to beam phase and bunch population variations (jitter). Figure 7.8 and Figure 7.9 show the final peak current $\left(I_{p k} \propto Q / \sigma_{z}\right)$, relative mean energy, $\left\langle\Delta E / E_{0}\right\rangle$, relative rms energy spread, $\left(\Delta E / E_{0}\right)_{\mathrm{rms}}$, and bunch arrival time variations, $\left\langle\Delta t_{f}\right\rangle$, versus both rf-gun timing, $\Delta t_{0}$, and relative charge variations, $\Delta Q / Q_{0}$, at the injector.

Table 7.4 lists sensitivities for rf phase, rf voltage, and chicane bend power supplies for the various systems. Each sensitivity causes a $+12 \%$ peak current increase or a $+0.1 \%$ relative electron energy increase. The photon beam will change by twice this amount. These sensitivities will be used to form a tolerance budget. The pulse-to-pulse rf phase sensitivities per linac are quite tight. However, the common mode phase sensitivity for the entire system is an order of magnitude looser. The gun timing jitter sensitivity and charge jitter sensitivity have been minimized by a careful choice of the linac acceleration and compression parameters (see Section 7.2.2).
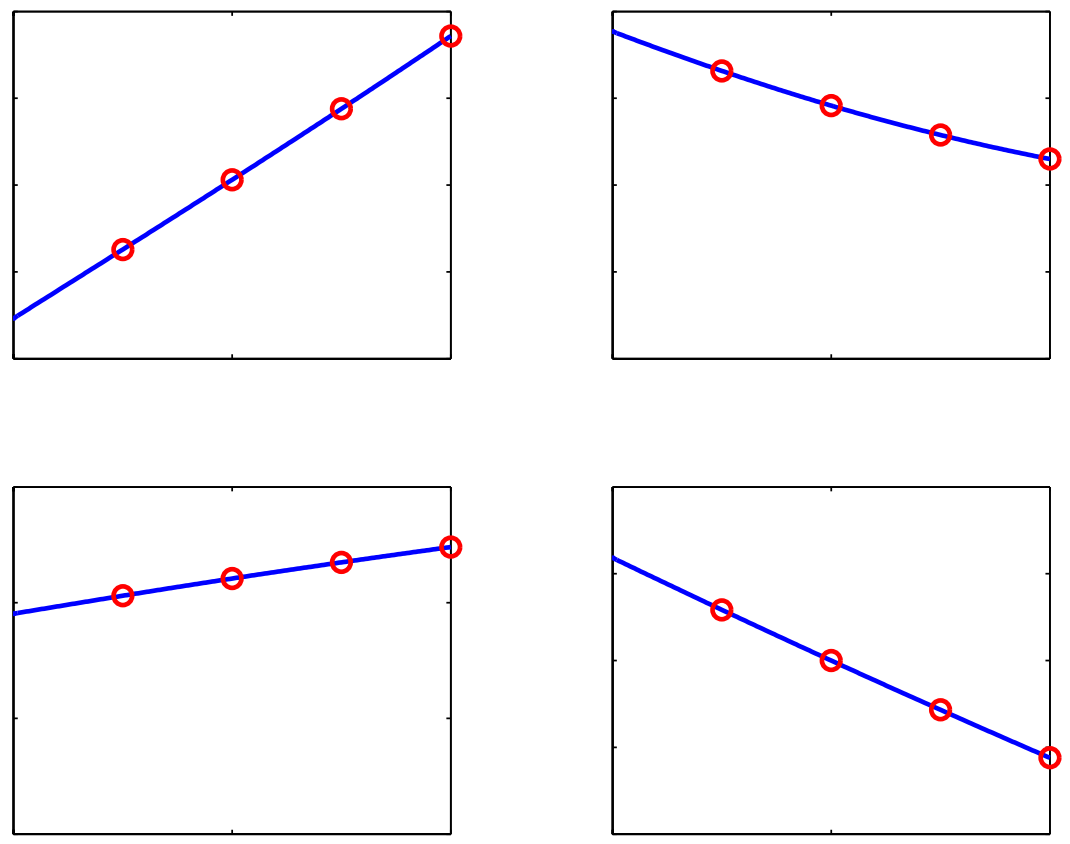

Figure 7.8 Beam energy, $\left\langle\Delta E / E_{0}\right\rangle ;$ rms bunch length, $\sigma_{z} ;$ rms energy spread, $\left(\Delta E / E_{0}\right)_{\mathrm{rms}}$; and undulator arrival time jitter, $\left\langle\Delta t_{f}\right\rangle$; all versus gun-timing jitter, $\Delta t_{0}$. A 1.8-ps timing jitter causes a $12 \%$ bunch length (or peak current) jitter. A 1.3-ps gun timing jitter causes a $0.1 \%$ relative electron beam energy jitter in the undulator. 

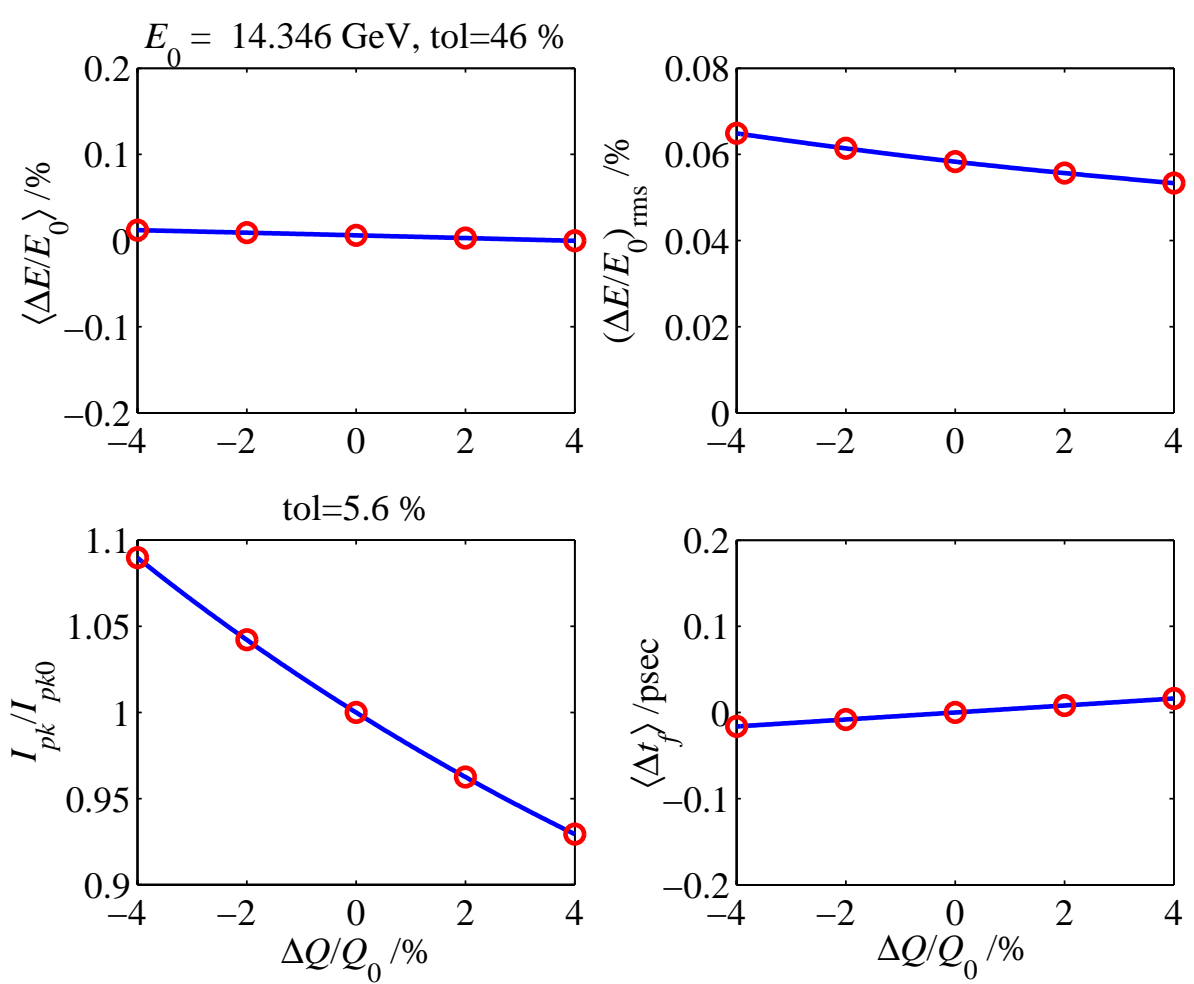

Figure 7.9 Same plots as Figure 7.8, but versus relative charge jitter, $\Delta Q / Q_{0}$, at the gun. A 5.6\% charge jitter causes a $12 \%$ peak current jitter. The beam energy is, for all practical purposes, insensitive to charge.

The sensitivities listed in Table $\mathbf{7 . 4}$ are used to generate a tolerance budget based on summing random, uncorrelated effects:

$$
\sqrt{\sum_{i=1}^{n}\left(\frac{p_{\text {tol }}}{p_{\text {sen }}}\right)_{i}^{2}}<1
$$

The tolerance to be chosen for each parameter is $p_{\text {tol }}$, and the weighting value taken from Table 7.4 for each sensitivity is $p_{\text {sen. }}$. For this budget, only the first fourteen $(n=14)$ sensitivities from Table 7.4 are applied. If $p_{\text {tol }}=p_{\text {sen }}$ for all $i$, then the summation produces $n^{1 / 2}$, or $\sqrt{ } 14$-times larger peak-current or beam energy jitter than the $12 \%$ and $0.1 \%$, respectively, used to calculate the above sensitivities. If the tolerances are chosen such that, $p_{\mathrm{tol}}<p_{\mathrm{sen}}$, for all $i$, a budget is formed where the less challenging tolerances (e.g., chicane bend power supplies) are pushed very much below their sensitivities, and the more challenging tolerances (e.g., L1 rf phase) are only slightly reduced. Table 7.5 lists two possible tolerance budgets. If the first budget (column 3, titled $\left.\left|\Delta I / I_{0}\right|<\mathbf{1 2 \%}\right)$ is used, the relative peak-current fluctuations in the undulator will be held to $<12 \% \mathrm{rms}$. If the second budget (column 4 , titled $\left|\left\langle\boldsymbol{\Delta} \boldsymbol{E} / \boldsymbol{E}_{\mathbf{0}}\right\rangle\right|<\mathbf{0 . 1 \%}$ ) is used, the final relative beam energy jitter in the undulator will be held to $<0.1 \% \mathrm{rms}$. If the smaller tolerance from each column is applied (bold-type), both performance requirements $\left(\left|\Delta I / I_{0}\right|<12 \%\right.$ and $\left|\left\langle\Delta E / E_{0}\right\rangle\right|<0.1 \%$ ) will simultaneously be met. 
The tolerances of Table 7.5 apply only for pulse-to-pulse jitter, which is too fast to be corrected with fast feedback systems. For variations slower than a few seconds, such as those induced by a temperature change, it is quite simple to hold the beam energy constant in each bend system by monitoring a few beam position monitors (see Section 7.8.4). It is also possible to hold the bunch length constant after each of the two compressors by employing a bunch length monitor (see Section 7.8.2) and feeding back on both rf phase and voltage upstream of the bends.

Table 7.4 Individual rms sensitivities $\left(p_{\text {sen }}\right)$ each cause a $+12 \%$ peak current change (column 3 ) or $+0.1 \%$ electron energy change at $14.35 \mathrm{GeV}$ (column 4). All sensitivities listed are approximately linear. These are not tolerances, but individual sensitivities used to form a tolerance budget.

\begin{tabular}{|c|c|c|c|c|}
\hline Parameter & Symbol & $\Delta I / I_{0}=+12 \%$ & $\left\langle\Delta E / E_{0}\right\rangle=+0.1 \%$ & Unit \\
\hline mean L0 rf phase & $\varphi_{0}$ & +0.67 & -3.6 & S-band deg \\
\hline mean L1 rf phase & $\varphi_{1}$ & $+0.16^{\dagger}$ & $-0.24^{\dagger}$ & S-band deg \\
\hline mean LX rf phase & $\varphi_{\mathrm{x}}$ & -1.1 & +12 & X-band deg \\
\hline mean L2 rf phase & $\varphi_{2}$ & $-0.22 \ddagger$ & $+0.36^{\ddagger}$ & S-band deg \\
\hline mean L3 rf phase & $\varphi_{3}$ & $>+20$ & +0.47 & S-band deg \\
\hline mean L0 rf voltage & $\Delta V_{0} / V_{0}$ & +0.29 & -0.33 & $\%$ \\
\hline mean L1 rf voltage & $\Delta V_{1} / V_{1}$ & +0.33 & -0.34 & $\%$ \\
\hline mean LX rf voltage & $\Delta V_{\mathrm{X}} / V_{\mathrm{x}}$ & -1.5 & +1.9 & $\%$ \\
\hline mean L2 rf voltage & $\Delta V_{2} / V_{2}$ & -1.0 & +0.64 & $\%$ \\
\hline mean L3 rf voltage & $\Delta V_{3} / V_{3}$ & $>+20$ & +0.15 & $\%$ \\
\hline Gun timing jitter & $\Delta t_{0}$ & -1.8 & +1.3 & psec \\
\hline Initial bunch charge & $\Delta Q / Q_{0}$ & -5.6 & -46 & $\%$ \\
\hline BC1 chicane & $\Delta B_{1} / B_{1}$ & -0.15 & +0.17 & $\%$ \\
\hline BC2 chicane & $\Delta B_{2} / B_{2}$ & +0.76 & +0.69 & $\%$ \\
\hline Initial bunch length & $\Delta \sigma_{z} / \sigma_{z_{0}}$ & +5.3 & -37.8 & $\%$ \\
\hline common L1/LX timing & $\varphi_{\mathrm{x}}=4 \varphi_{1}$ & +0.43 & -0.26 & S-band deg \\
\hline common net phase & $\varphi_{0}=\varphi_{1}=\varphi_{\mathrm{x}}=\varphi_{2}=\varphi_{3}$ & -4.0 & +1.4 & S-band deg \\
\hline
\end{tabular}

$\dagger$ The L1 phase sensitivities become $0.47^{\circ}$ and $1.6^{\circ}$ (left to right) if $\mathrm{BC} 1$ energy is held constant.

$\ddagger$ The L2 phase sensitivities become $0.36^{\circ}$ and $2.7^{\circ}$ (left to right) if $\mathrm{BC} 2$ energy is held constant.

The tolerances are quite tight for many of the parameters, especially those systems driven by a single klystron (e.g., L1). Since the rf tolerances represent a demand on the average phase or average voltage over the klystrons of that linac, multiple-klystron linacs such as L2 and L3 
actually have a per-klystron tolerance which is $\sqrt{ } 26$ and $\sqrt{ } 45$-times, respectively, looser than the tolerance listed. This means L2 has a per-klystron pulse-to-pulse jitter tolerance of $0.36^{\circ}$ and $0.36 \%$, and L3 has a per-klystron tolerance of $0.47^{\circ}$ and $0.34 \%$.

Table 7.5 Tolerance budget $\left(p_{\text {tol }}\right)$ for $<12 \%$ rms peak-current jitter (column 3 ) or $<0.1 \%$ rms final $e^{-}$ energy jitter (column 4). The tighter tolerance is in BOLD text and both criteria, $\left|\Delta / / I_{0}\right|<12 \%$ and $\left|\left\langle\Delta E / E_{0}\right\rangle\right|<0.1 \%$, are satisfied if the tighter tolerance is applied. The voltage and phase tolerances per klystron for L2 and L3 are $\sqrt{ } N_{k}$ larger.

\begin{tabular}{|c|c|c|c|c|}
\hline Parameter & Symbol & $\left|\Delta I / I_{0}\right|<12 \%$ & $\left|\left\langle\Delta E / E_{0}\right\rangle\right|<0.1 \%$ & Unit \\
\hline mean L0 rf phase (2 klystrons) & $\varphi_{0}$ & 0.10 & 0.10 & S-band deg \\
\hline mean L1 rf phase (1 klystron) & $\varphi_{1}$ & 0.10 & 0.10 & S-band deg \\
\hline mean LX rf phase (1 klystron) & $\varphi_{\mathrm{X}}$ & $\mathbf{0 . 3 0}$ & 0.8 & X-band deg \\
\hline mean L2 rf phase (28 klystrons) & $\varphi_{2}$ & $\mathbf{0 . 0 7}$ & 0.07 & S-band deg \\
\hline mean L3 rf phase (48 klystrons) & $\varphi_{3}$ & 1.0 & $\mathbf{0 . 0 7}$ & S-band deg \\
\hline mean L0 rf voltage (1-2 klystrons) & $\Delta V_{0} / V_{0}$ & 0.10 & 0.10 & $\%$ \\
\hline mean L1 rf voltage (1 klystron) & $\Delta V_{1} / V_{1}$ & 0.10 & 0.10 & $\%$ \\
\hline mean LX rf voltage (1 klystron) & $\Delta V_{\mathrm{x}} / V_{\mathrm{x}}$ & 0.25 & 0.25 & $\%$ \\
\hline mean L2 rf voltage (28 klystrons) & $\Delta V_{2} / V_{2}$ & 0.10 & $\mathbf{0 . 0 7}$ & $\%$ \\
\hline mean L3 rf voltage (48 klystrons) & $\Delta V_{3} / V_{3}$ & 1.0 & 0.05 & $\%$ \\
\hline BC1 chicane & $\Delta B_{1} / B_{1}$ & 0.02 & 0.02 & $\%$ \\
\hline $\mathrm{BC} 2$ chicane & $\Delta B_{2} / B_{2}$ & 0.05 & 0.05 & $\%$ \\
\hline Gun timing jitter & $\Delta t_{0}$ & 1.3 & 0.7 & psec \\
\hline Initial bunch charge & $\Delta Q / Q_{0}$ & 2.0 & 5.0 & $\%$ \\
\hline
\end{tabular}

A complete simulation of FEL performance in the presence of machine jitter is described in Section 7.6.3, where 6D particle tracking is performed repetitively to test the jitter tolerance budget above. The detailed simulation includes CSR and suggests lowering the gun timing jitter tolerance to $0.5 \mathrm{psec}$, rather than $0.7 \mathrm{psec}$ calculated above. Otherwise the budget is adequate.

The ability of the injector and the SLAC linac to achieve such pulse-to-pulse stability levels can be estimated by extrapolating from past SLC performance. With a beam energy feedback system installed at the end of the SLC linac, the remaining fast relative energy jitter was typically measured at $<0.04 \% \mathrm{rms}$ at $47 \mathrm{GeV}$ with a $120 \mathrm{~Hz}$ machine repetition rate. This level of energy error is induced by a phase error of $0.16^{\circ} \mathrm{rms}$ (S-band) in the SLC bunch compressor rf system (at $42 \mathrm{MV}$ ), which provides an upper limit on the performance of this single klystron's rms phase stability. The phase stability is actually significantly better than $0.16^{\circ}$ considering the additional effects of the remaining 210 klystrons and the typical $2 \%$ rms charge jitter (which by itself almost accounts for the $0.04 \%$ observed energy jitter). Measurements of the rf phase variations in the 
SLAC linac are also presented in Section 7.7.2, where rms phase variations of $<0.1^{\circ} \mathrm{S}$-band are seen over a period of 10-20 seconds, as described in [13]. Some improvements to RF stability will be made to the linac as described in Section 7.7.

Variations much larger than $0.1^{\circ} \mathrm{S}$-band have also been observed, but these levels are usually associated with changes over a much longer time scale, such as induced with day-night temperature and atmospheric pressure deviations. Energy and bunch-length feedback systems must address this long-term drift (see Section 7.8.4).

\subsubsection{Energy Management and Overhead}

Each linac section must operate with several spare klystrons to accommodate their inevitable failure rate (except L1 and LX, which each operate on just one klystron). Table 7.6 lists, for each LCLS linac section, the total number of klystrons potentially available, $N_{K}$, the number of klystrons held in reserve, $N_{R}$, the nominal rf phase, $|\varphi|_{i}$, the average energy gain per klystron, $\langle\Delta E\rangle_{K}$, the beam loading energy loss at $Q=1 \mathrm{nC}, \Delta E_{B}$, and the resulting maximum energy achievable, $E_{\max }$, (assuming an injection energy, $E_{i}$ ).

Table 7.6 Energy management parameters for the four main linac sections. The average energy gain is based on $19.3 \mathrm{MV} / \mathrm{m}$ and all LCLS-modified linac structure lengths.

\begin{tabular}{lcccccc}
\hline Linac & $N_{K}$ & $N_{R}$ & $|\varphi|_{i}[\mathrm{deg}]$ & $\begin{array}{l}\langle\Delta E\rangle_{K} \\
{[\mathrm{GeV}]}\end{array}$ & $\Delta E_{B}[\mathrm{MeV}]$ & $E_{i}[\mathrm{GeV}]$ \\
\hline L1 & 1 & 0 & 38.1 & 0.192 & $<1$ & 0.15 \\
\hline LX & 1 & 0 & 180 & -0.022 & $<1$ & 0.27 \\
\hline L2 & 28 & 2 & 42.8 & 0.230 & 28 & 0.25 \\
\hline L3 & 48 & 3 & 10.0 & 0.227 & 63 & 4.54 \\
\hline
\end{tabular}

For the average energy gain calculation, all existing linac structure lengths are used, allowing for the linac modifications which eliminate the acceleration of three klystrons and remove several other 3-meter sections (see Table 7.28). This will nullify 920-MeV of unloaded energy gain (with respect to the pre-LCLS linac). For future non-LCLS linac operation, this energy is not easily recovered; however, the maximum energy available is still within $\sim 1 \mathrm{GeV}$ of the pre-LCLS linac maximum energy of $\sim 50 \mathrm{GeV}$.

\subsubsection{Alternate Parameters}

\subsubsection{Variable Bunch Charge}

The nominal design parameters are described above for a 1-nC bunch, which produces SASE saturation with a $1.2-\mu \mathrm{m}$ normalized slice emittance and a 3.4-kA peak current. In fact, the LCLS accelerator is flexible enough to be operationally re-configured for a wide variety of beam parameters. In this case, the term operational means that the new parameter set is 'dialed-in' from the control room, as opposed to hardware modifications in the tunnel. This flexibility is 
demonstrated by studying the new machine parameters required over a wide range of bunch charge values.

The injector's beam emittance and bunch length is first estimated by applying scaling laws derived for a 1.6-cell S-band rf photo-cathode gun [14]:

$$
\begin{gathered}
\varepsilon_{N}[\mu \mathrm{m}] \approx 1.45 \cdot \sqrt{0.38 \cdot Q[\mathrm{nC}]^{4 / 3}+0.095 \cdot Q[\mathrm{nC}]^{8 / 3}+0.22 \cdot Q[\mathrm{nC}]^{2 / 3}} \\
\sigma_{z_{0}}[\mathrm{~mm}] \approx 0.83 \cdot Q[\mathrm{nC}]^{1 / 3}
\end{gathered}
$$

Here $Q$ is the bunch charge $(\mathrm{nC}), \varepsilon_{N}$ is the normalized rms emittance $(\mu \mathrm{m})$, and $\sigma_{z_{0}}$ is the initial rms electron bunch length $(\mathrm{mm})$ after the gun. The last term in the emittance relation represents a thermal emittance, which scales with the laser spot radius on the cathode. The bunch length scaling constant has been increased here $(0.63 \mathrm{~mm}$ in reference [14] becomes $0.83 \mathrm{~mm})$, to a less challenging level, in order to be consistent with the 1-nC nominal design described above.

The peak current required for SASE saturation at $\sim 87 \mathrm{~m}$, is given approximately by (see Chapter 5)

$$
I_{p k}[\mathrm{~A}] \approx 233 \cdot \varepsilon_{N}^{3}+1343 \cdot \varepsilon_{N}^{2}+834 \cdot \varepsilon_{N}+63
$$

where the slice energy spread is assumed to be $<0.02 \% \mathrm{rms}$, and the mean beta function in the undulator is $\sim 18 \mathrm{~m}$. At the nominal 1 -nC charge, the emittance is $\varepsilon_{N} \approx 1.2 \mu \mathrm{m}$, and the required peak current is $I_{p k} \approx 3400$ A.

For a reduced bunch charge, the emittance, initial bunch length, and required peak current are estimated from Eqs. (7.10) and (7.11). A fast optimization linac design code (described in Section 7.2.2) is then used to find a new set of acceleration and compression parameters which can provide SASE saturation at the same 87-meter point, and also optimize beam stability. Alternate scaling scenarios are also possible, but this simple example is used here as a demonstration of the configuration flexibility in the LCLS accelerator. Table 7.7 lists the various machine parameters and a few 'jitter' sensitivities (see Section 7.2.6) for six different values of the bunch charge ranging from 0.1 to $1.0 \mathrm{nC}$. In each case, full $2 \mathrm{D}$ tracking calculations are used to verify that the final energy $(14.35 \mathrm{GeV})$, peak current (listed), and energy spread $(<0.02 \% \mathrm{rms})$ are obtainable. Obtaining the smaller values of emittance with a lower charge needs experimental verification, and various beam diagnostics need to provide adequate resolution at the lower charge, but in any case, the linac design is seen to be quite flexible.

The extreme point at $0.1 \mathrm{nC}$ may be too low, but many of the other low-charge configurations are interesting since the jitter tolerances (last 3 rows) are generally looser. The smaller emittance with lower charge is not necessarily more difficult to preserve in the linac, since the reduced charge eases the effects of coherent synchrotron radiation in the chicanes and transverse wakefields in the linacs. In addition, the shorter initial bunch length allows weaker chicanes and lower values of correlated energy spread throughout the linac sections, both of which also ease emittance preservation issues. 
LCLS CON CEP TUAL DESIG N REP O R T

Table 7.7 Machine parameters for six different values of bunch charge and constant saturation length. The final projected relative energy spread is $<0.02 \% \mathrm{rms}$ in all cases.

\begin{tabular}{lccccccc}
\hline Bunch Charge $\rightarrow$ & $\mathbf{0 . 1}$ & $\mathbf{0 . 2}$ & $\mathbf{0 . 4}$ & $\mathbf{0 . 6}$ & $\mathbf{0 . 8}$ & $\mathbf{1 . 0}$ & $\mathbf{n C}$ \\
\hline Initial bunch length & 390 & 491 & 619 & 709 & 780 & 830 & $\mu \mathrm{m}$ \\
\hline Final bunch length & 8.3 & 14 & 21 & 23 & 23 & 22 & $\mu \mathrm{m}$ \\
\hline Norm. slice emittance & 0.54 & 0.60 & 0.74 & 0.89 & 1.04 & 1.20 & $\mu \mathrm{m}$ \\
\hline Peak current & 0.95 & 1.11 & 1.52 & 2.03 & 2.66 & 3.40 & $\mathrm{kA}$ \\
\hline L1 rf phase & -35.9 & -33.1 & -34.6 & -27.5 & -32.4 & -38.1 & deg-S \\
\hline L2 rf phase & -13.1 & -27.2 & -40.6 & -41.9 & -42.0 & -42.8 & deg-S \\
\hline L3 rf phase & -7.4 & -8.2 & -7.6 & -10.0 & -9.7 & -10 & deg-S \\
\hline$R_{56}$ of BC1 & -41.2 & -47.7 & -45.9 & -60.4 & -47.7 & -35.9 & $\mathrm{~mm}$ \\
\hline$R_{56}$ of BC2 & -63.6 & -33.1 & -20.6 & -22.5 & -23.1 & -22.5 & $\mathrm{~mm}$ \\
\hline energy spread in BC1 & 0.82 & 0.91 & 1.18 & 0.98 & 1.31 & 1.78 & $\%$ \\
\hline energy spread in BC2 & 0.08 & 0.14 & 0.29 & 0.43 & 0.58 & 0.76 & $\%$ \\
\hline bunch length after BC1 & 57 & 62 & 80 & 119 & 157 & 195 & $\mu \mathrm{m}$ \\
\hline Rms gun $\Delta t_{0} \rightarrow 12 \% \sigma_{z}$ & 1.3 & 5.8 & 11. & 4.7 & 6.5 & 4.0 & $\mathrm{psec}$ \\
\hline Rms gun $\Delta t_{0} \rightarrow 0.1 \% \Delta E / E_{0}$ & 5.8 & 3.8 & 2.8 & 3.4 & 2.0 & 1.4 & $\mathrm{psec}$ \\
\hline Rms $\Delta Q / Q \rightarrow 12 \% I_{p k}$ & 4.9 & 11. & - & 7.0 & - & 6.0 & $\%$ \\
\hline
\end{tabular}

\subsubsection{Electron Chirp to Facilitate X-Ray Pulse Compression}

The nominal final electron bunch length at $1 \mathrm{nC}$ is $22 \mu \mathrm{m}$ rms or $230 \mathrm{fsec}$ FWHM. If the entire electron bunch achieves SASE saturation, the x-ray pulse length will also be $230 \mathrm{fsec}$ FWHM. This may be too long for some experiments, so an option to reduce the photon pulse length to $\sim 50 \mathrm{fsec}$ is desirable.

Further reduction of the electron bunch length is possible with re-configuration of the compression parameters, but several limitations begin to appear when a significantly shorter electron bunch is used, such as increased horizontal emittance dilution due to CSR in the compressors, increased resistive-wall and roughness wakefields in the undulator, and increased peak-current jitter sensitivity to linac and injector variations. An alternate way in which to shorten the $\mathrm{x}$-ray pulse is to use the nominal electron bunch length, but provide a linear energy gradient (chirp) along the bunch so that the $\mathrm{x}$-ray pulse, which will then also be chirped, may be shortened using optical compression techniques.

An electron chirp can be added in several different ways. Unfortunately, the most intuitive way, shifting the L3-linac rf phase farther off acceleration crest, does not add a significant chirp due to the very short bunch length compared with the S-band rf wavelength. Another possibility involves increasing the $\mathrm{BC} 2$ chicane strength to 'over-compress' the bunch, such that the electron bunch length is again set at $22 \mu \mathrm{m}$, but the remaining energy-time correlation in the bunch has reversed sign, with respect to the nominal case (see nominal case in Figure 7.7). With over- 
compression, the L3 longitudinal wakefield will now add to the correlated energy spread, rather than canceling it. Thus, a large correlated electron energy spread (chirp) of 2\% FWHM can be generated at $14.35 \mathrm{GeV}$. The over-compression in the BC2 chicane, however, forces the bunch to pass through its minimum length (a few microns) inside the chicane, which may destroy the horizontal emittance with the potentially stronger CSR forces. This case requires a subtle calculation including the transverse beam dimensions and cannot, at present, be relied upon to accurately predict machine performance. Therefore, over-compression is presently viewed as a possible option needing experimental verification before it is seen as a realistic chirp strategy.

The most promising method of producing a significant chirp is to reduce the bunch charge from $1 \mathrm{nC}$ to $0.6 \mathrm{nC}$ and operationally re-configure the linac parameters. The reduced bunch charge makes the L3 wakefields weaker, which helps to leave some chirp in the electron beam after L3. The re-configuration is used to further amplify the chirp to $\sim 1 \%$ FWHM. This $1 \%$ electron chirp may then be used to compress the x-ray pulse by a factor of $\sim 5$, to a pulse length of $\sim 50$ fsec FWHM (see Chapter 9).

The reduced charge also allows the initial bunch length, prior to $\mathrm{BC} 1$, to be reduced from $0.83 \mathrm{~mm}$ to $0.71 \mathrm{~mm} \mathrm{rms}$, and the emittance to be slightly reduced from $1.2 \mu \mathrm{m}$ to $0.9 \mu \mathrm{m}$. This keeps SASE saturation at $\sim 87$ meters with a 2.1-kA peak current [see Eqs. (7.10) and (7.11)]. The machine parameters and sensitivities associated with this configuration are shown in Table 7.8 along side the nominal parameters. The final 'slice' energy spread in both cases is $<0.01 \% \mathrm{rms}$, but the gun-timing tolerance is, unfortunately, more challenging in the $0.6-\mathrm{nC}$ case. The $2 \mathrm{D}$ tracking output at $14.3 \mathrm{GeV}$ is shown in Figure 7.10, where the bunch length is $23 \mu \mathrm{m}$, but the correlated energy spread is large and linear at 1\% FWHM. Saturation should occur for the leading half of the bunch where the peak current exceeds $2.1 \mathrm{kA}$. Various other scenarios are also possible, especially if the required final chirp level is reduced.
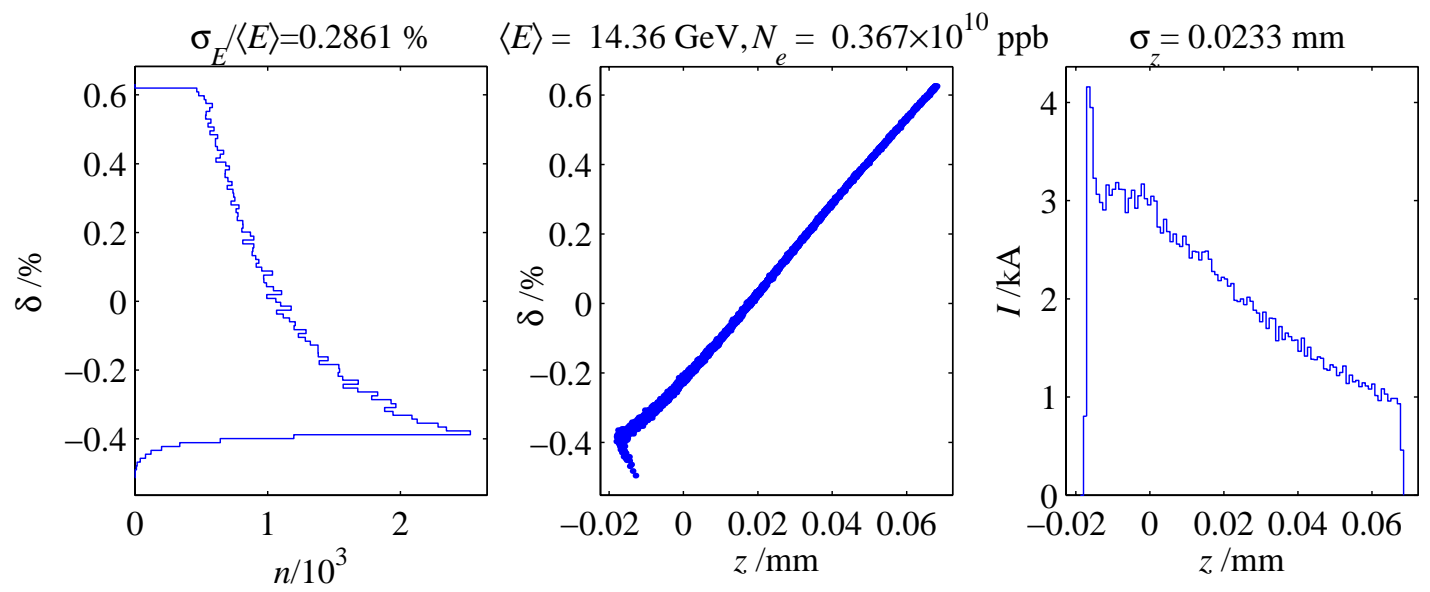

Figure 7.10 Chirped electron beam: energy (left) and axial (right) $e^{-}$distributions and longitudinal phase space (center column) at undulator entrance with a reduced charge of $0.6 \mathrm{nC}$ and a desired 1\% FWHM electron energy chirp. 
LCLS CON CEP TUAL DESIG N REP O R T

Table 7.8 Machine parameters for a 1\%-chirped electron bunch and a charge of $0.6 \mathrm{nC}$ juxtaposed against the nominal, 1-nC un-chirped parameters.

\begin{tabular}{|c|c|c|c|}
\hline Bunch Charge $\rightarrow$ & 0.6 nC (chirp) & 1 nC (nominal) & units \\
\hline Initial bunch length & 710 & 830 & $\mu \mathrm{m}$ \\
\hline Final rms bunch length & 23 & 22 & $\mu \mathrm{m}$ \\
\hline Norm. rms slice emittance & 0.9 & 1.2 & $\mu \mathrm{m}$ \\
\hline Peak current & 2.1 & 3.4 & $\mathrm{kA}$ \\
\hline X-band rf voltage & 5 & 22 & MV \\
\hline L1 rf phase & -22.8 & -38.1 & deg-S \\
\hline L2 rf phase & -41.3 & -42.8 & deg-S \\
\hline L3 rf phase & -10.0 & -10.0 & deg-S \\
\hline$R_{56}$ of $\mathrm{BC} 1$ & -60.1 & -35.9 & $\mathrm{~mm}$ \\
\hline$R_{56}$ of $\mathrm{BC} 2$ & -20.7 & -22.5 & $\mathrm{~mm}$ \\
\hline energy spread in $\mathrm{BC} 1(\mathrm{rms})$ & 0.69 & 1.78 & $\%$ \\
\hline energy spread in BC2 (rms) & 1.35 & 0.76 & $\%$ \\
\hline energy spread in undulator (FWHM) & 1.0 & 0.05 & $\%$ \\
\hline bunch length after BC1 (rms) & 300 & 195 & $\mu \mathrm{m}$ \\
\hline rms gun $\Delta t_{0} \rightarrow 12 \% \sigma_{z}$ & 0.50 & 4.0 & psec \\
\hline $\mathrm{rms}$ gun $\Delta t_{0} \rightarrow 0.1 \% \Delta E / E_{0}$ & 0.64 & 1.4 & psec \\
\hline $\operatorname{rms} \Delta Q / Q_{0} \rightarrow 12 \% I_{p k}$ & 17. & 6.0 & $\%$ \\
\hline
\end{tabular}

This scenario provides the ability to compress the x-ray pulse without adding significant technical risk to the preservation of the $6 \mathrm{D}$ electron phase space density. Other scenarios are also possible, including the full $1-\mathrm{nC}$ bunch charge and a negative chirp sign, an option which has been simulated in some detail but not presented here. The temporal distribution of the negatively chirped case is less uniform across the bunch and the energy-time correlation is less linear.

\subsubsection{Long Wavelength SASE Radiation}

The LCLS can also be configured for longer undulator radiation wavelengths in a continuum from $1.5 \AA$ up to a maximum of $\sim 15.0 \AA$. The $15-\AA$ limit of this range is easily arranged by switching off the rf acceleration in the L3-linac, re-scaling the bends and quadrupole magnets beyond $\mathrm{BC} 2$, and passing the $4.54-\mathrm{GeV}$ electron beam through the permanent magnet undulator.

At a longer wavelength, the SASE gain saturates in a much shorter distance than at $1.5 \AA$. This allows the electron beam parameters to be significantly relaxed at $15 \AA$. With the saturation length chosen in the very safe range of $\sim 60$ meters of undulator, the electron beam emittance can then be relaxed to $\gamma \varepsilon_{x, y} \approx 3 \mu \mathrm{m}$ and the peak current, with less compression, to $1.9 \mathrm{kA}$. With the full $1-\mathrm{nC}$ charge, the final bunch length is then $45 \mu \mathrm{m}$ rms. Other scenarios are possible, including lower charge, but the long $(45 \mu \mathrm{m})$ bunch relaxes rf phase and voltage jitter tolerances in the 
linac, so that the entire linac and injector configuration is technically much less challenging than that required at $1.5 \AA$.

The final absolute energy spread will decrease by a factor of two with the reduced compression (from $\sim 2.8 \mathrm{MeV}$ to $1.4 \mathrm{MeV}$ ), while the relative energy spread will increase with the reduced beam energy (from $0.02 \%$ to $0.03 \%$ ). The very small energy chirp along the electron bunch can be approximately maintained, even after the L $3 \mathrm{rf}$ is switched off.

The beta-match at the undulator entrance, due to its permanent magnet focusing, needs to be adjusted to produce $\sim 6$-meter average undulator beta functions, as opposed to the $\sim 18$-meter beta functions at $1.5 \AA(14.3 \mathrm{GeV})$. The re-match is accomplished using the four QM35-38 quadrupoles at undulator entrance (last four quads at right of Figure 7.36).

Table 7.9 lists the machine parameters for the $15-\AA$ case alongside the nominal $1.5-\AA$ case. Parameters not listed here are the same in the two configurations. The same injector beam, shown at top of Figure 7.6, is tracked in 2D through the re-configured LCLS accelerator with the L3linac rf switched off. The final longitudinal phase space at the undulator entrance, at $4.54 \mathrm{GeV}$, is shown in Figure 7.11. The temporal distribution is very flat with a nearly constant 2-kA peak current.

Table 7.9 Machine parameters for 15- $\AA$ SASE radiation with a saturation length of $\sim 60 \mathrm{~m}$ juxtaposed against the nominal $1.5-\AA$ configuration, both with $1-\mathrm{nC}$ of charge. Energy sensitivity is worse $(\times 3)$ at left, but also more tolerable $(\times 3)$ at $15 \AA$.

\begin{tabular}{lccr}
\hline Bunch Charge $\rightarrow$ & $\mathbf{1 5} \AA$ (long $\left.\lambda_{r}\right)$ & $\mathbf{1 . 5} \AA$ (nominal) & units \\
\hline Final rms bunch length & 45 & 22 & $\mu \mathrm{m}$ \\
\hline Norm. rms slice emittance & 3.0 & 1.2 & $\mu \mathrm{m}$ \\
\hline Peak current & 1.9 & 3.4 & $\mathrm{kA}$ \\
\hline L1 rf phase & -35.1 & -38.1 & deg-S \\
\hline L2 rf phase & -40.6 & -42.8 & deg-S \\
\hline$R_{56}$ of BC1 & -40.0 & -35.9 & $\mathrm{~mm}$ \\
\hline$R_{56}$ of BC2 & -21.7 & -22.5 & $\mathrm{~mm}$ \\
\hline energy spread in BC1 (rms) & 1.59 & 1.78 & $\%$ \\
\hline energy spread in BC2 (rms) & 0.72 & 0.76 & $\%$ \\
\hline energy spread in undulator $(\mathrm{rms})$ & 0.03 & 0.02 & $\% \mathrm{~m}$ \\
\hline bunch length after BC1 $(\mathrm{rms})$ & 200 & 195 & $\mathrm{psec}$ \\
\hline rms gun $\Delta t_{0} \rightarrow 12 \% \sigma_{z}$ & 2.3 & 4.0 & $\mathrm{psec}$ \\
\hline rms gun $\Delta t_{0} \rightarrow 0.1 \% \Delta E / E_{0}$ & 0.4 & 1.4 & $\%$ \\
\hline rms $\Delta Q / Q_{0} \rightarrow 12 \% I_{p k}$ & 11 & 6.0 &
\end{tabular}

This is just one possible long-wavelength configuration. Many long-wavelength scenarios are possible including reduced charge, increased peak current, and reduced emittance. This specific 
parameter set is described because of its potential as an LCLS startup configuration, with reduced technical challenges on all fronts: injector, linac, and undulator.
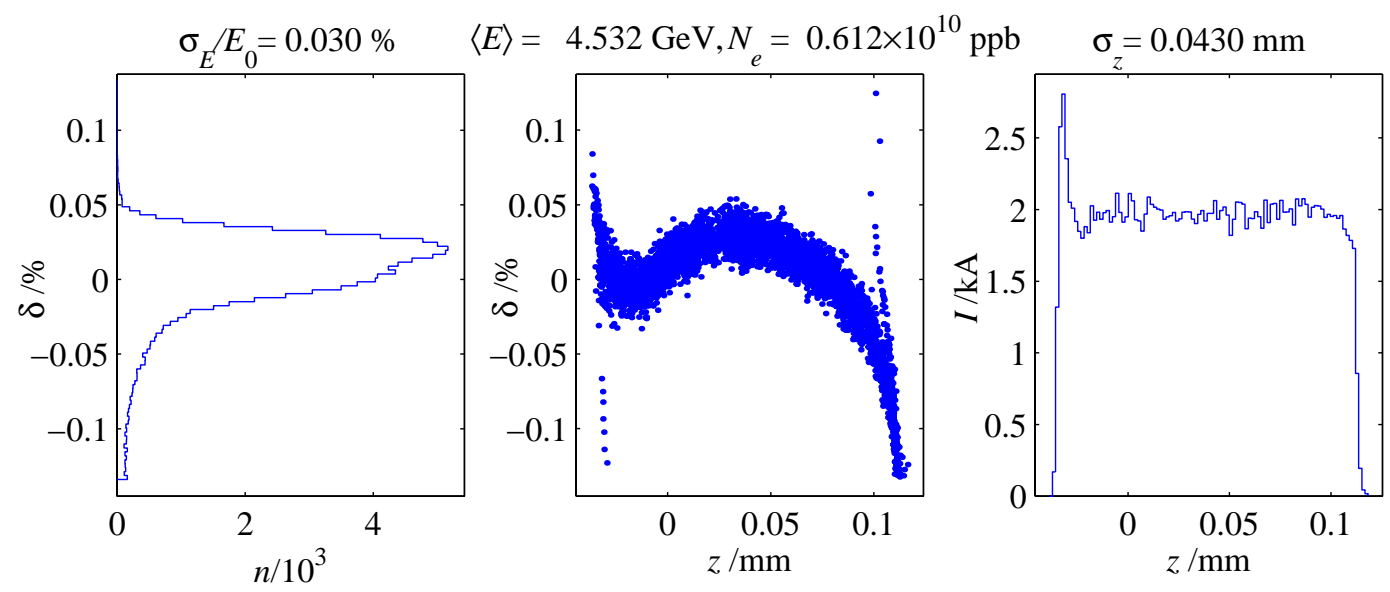

Figure 7.11 Longitudinal phase space of 4.54-GeV electron beam at undulator entrance after reoptimizing the LCLS accelerator to the $15-\AA$ configuration described above.

\subsection{Transverse Beam Dynamics}

The LCLS accelerator is composed of four separate S-band linac sections L0, L1, L2, and L3 (ignoring the very short $\mathrm{X}$-band section). Each of these linacs requires an individual lattice design in order to minimize emittance dilution due to transverse wakefields and momentum dispersion, both of which are generated through component misalignments. Each linac section has its own particular beam parameters, which motivate the optical design of that linac. For example, a large beam energy spread and short bunch length suggest weak focusing with large quadrupole spacing. Figure 7.12 shows the nominal rms energy spread and bunch length along the entire LCLS accelerator from the end of $\mathrm{L} 0$ at $150 \mathrm{MeV}$ to the entrance of the undulator at $14.35 \mathrm{GeV}$.

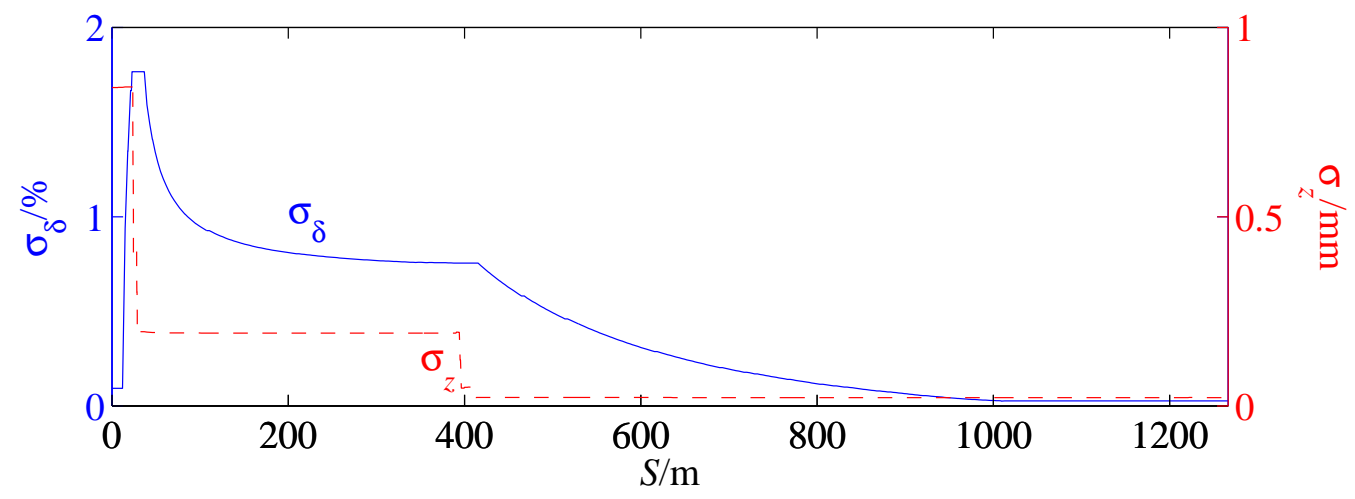

Figure 7.12 Nominal rms energy spread (solid-blue) and rms bunch length (dash-red) along the entire LCLS accelerator from L0-exit at $150 \mathrm{MeV}$ to undulator entrance at $14.35 \mathrm{GeV}$. 
Table 7.10 summarizes the four linacs and their various beam parameters. The final energy after L3 is variable from 4.5 to $15 \mathrm{GeV}$ through appropriate phasing and rf power. The rf phase angles of the various linacs as well as their lengths are chosen in a computer optimization which is described in Section 7.2.2.

Table 7.10 Beam parameters of the four separate S-band linac sections (plus X-band linac, LX).

\begin{tabular}{llccccc}
\hline Beam Parameter & Unit & L0 & L1 & LX & L2 & L3 \\
\hline Initial energy & $\mathrm{GeV}$ & 0.007 & 0.150 & 0.272 & 0.250 & 4.54 \\
\hline Final energy & $\mathrm{GeV}$ & 0.150 & 0.272 & 0.250 & 4.54 & $4.54-15$ \\
\hline Active linac length & $\mathrm{m}$ & 6 & 9 & 0.6 & 329 & 553 \\
\hline rf phase (crest at 0) & $\mathrm{deg}$ & -2 & -38.1 & 180 & -42.8 & -10 \\
\hline Initial rms energy spread & $\%$ & 0.20 & 0.10 & 1.64 & 1.78 & 0.76 \\
\hline Final rms energy spread & $\%$ & 0.10 & 1.64 & 1.78 & 0.76 & 0.02 \\
\hline rms bunch length & $\mathrm{mm}$ & 0.83 & 0.83 & 0.83 & 0.19 & 0.022 \\
\hline
\end{tabular}

\subsubsection{The L0-Linac}

A more complete description of the L0-linac is given in Chapter 6. Linac-0 provides the initial acceleration and transverse emittance compensation from the rf photocathode gun. It is a new beamline constructed at a $35^{\circ}$ angle with respect to the existing SLAC linac at the start of sector-21 and is situated in an existing, off-axis linac housing. This space was provided in the original SLAC-linac design, which was built with two off-axis injector enclosures at both the one-third and two-thirds locations along the linac (at the end of both sectors 10 and 20). Linac-0 is composed of two 3-meter acceleration sections, each powered by an separate klystron (20-7 and 20-8), and nominally accelerates the electron beam to $150 \mathrm{MeV}$. It is a space charge dominated system including solenoid focusing, but no quadrupole magnets prior to the $150-\mathrm{MeV}$ point. Following L0 is an adjustable matching section and a transverse emittance diagnostic section, ED0 (see Section 7.8.1). The achromatic bend system, DL1 (see Section 7.5.1), bends the beam $35^{\circ}$ onto the SLAC linac axis and into the L1-linac section, which starts at the 21-1b location. The two DL1 bends are located at the 21-1a location where space already exists with no 3-meter rf-section installed there.

The $35^{\circ}$ DL1 bend system provides energy and energy spread measurement capability prior to injection into the L1-linac. An energy stabilizing feedback system, a switchable beam dump, and a bunch length monitor will also be located here (see Secs. 7.5.1 and 7.8.4).

\subsubsection{The L1-Linac}

L1 initiates the compression process by accelerating from $150 \mathrm{MeV}$ to $272 \mathrm{MeV}$ off crest, thereby generating the necessary linear energy- $z$ correlation so the first chicane, BC1, will compress the bunch. The L1-linac is composed of three existing 3-meter rf structures 21-1b, 21$1 \mathrm{c}$, and 21-1d (the 21-1a section was never installed in anticipation of just such an intermediate 
injector). Because of the large off-crest $\mathrm{rf}$ phase angle and the relatively long bunch, the rms energy spread in L1 rapidly increases from $0.1 \%$ to $1.7 \%$. Therefore, dispersion generated by misaligned quadrupoles, and transverse wakefields generated by misaligned $\mathrm{rf}$ structures, are both potential sources of emittance dilution. At this energy, however, space charge forces are insignificant (see Chapter 6).

To choose the best focusing lattice for L1, several lattice designs have been simulated using Liar [15] and Elegant [10]. These computer programs calculate the transverse emittance dilution along a linac and include both longitudinal and transverse wakefields, random quadrupole, BPM, and rf-structure misalignments and the dispersion these generate. They also provide various trajectory correction algorithms.

Several different quadrupole spacing schemes were simulated (see Section 7.3.2 of reference[16]), with a simple 3-meter spacing settled upon. In order to insert quadrupole magnets and BPM-steering pairs at a 3-meter spacing, $18-\mathrm{cm}$ of waveguide will be cut off from the downstream ends of the first two L1 rf sections (21-1b and 21-1c). This same cut-off technique has been used in the past for various SLC modifications.

In order to find the best L1-linac focusing strength, the betatron phase advance per cell (there are only 1.5 cells) was varied from $15^{\circ}$ to $90^{\circ}$ in $15^{\circ}$ steps. The simulations are made with Liar and use $300-\mu \mathrm{m}$ rms random quadrupole, BPM, and rf-structure transverse misalignments (with gaussian distributions cut at $3-\sigma$ ). These are pessimistic conditions in order to optimize the lattice. The same 10 seeds were then run for each lattice, and one-to-one steering was applied at each BPM in both planes. A horizontal and vertical corrector, and a BPM which reads both $x$ and $y$, are used near each of the three L1 quadrupole magnets.

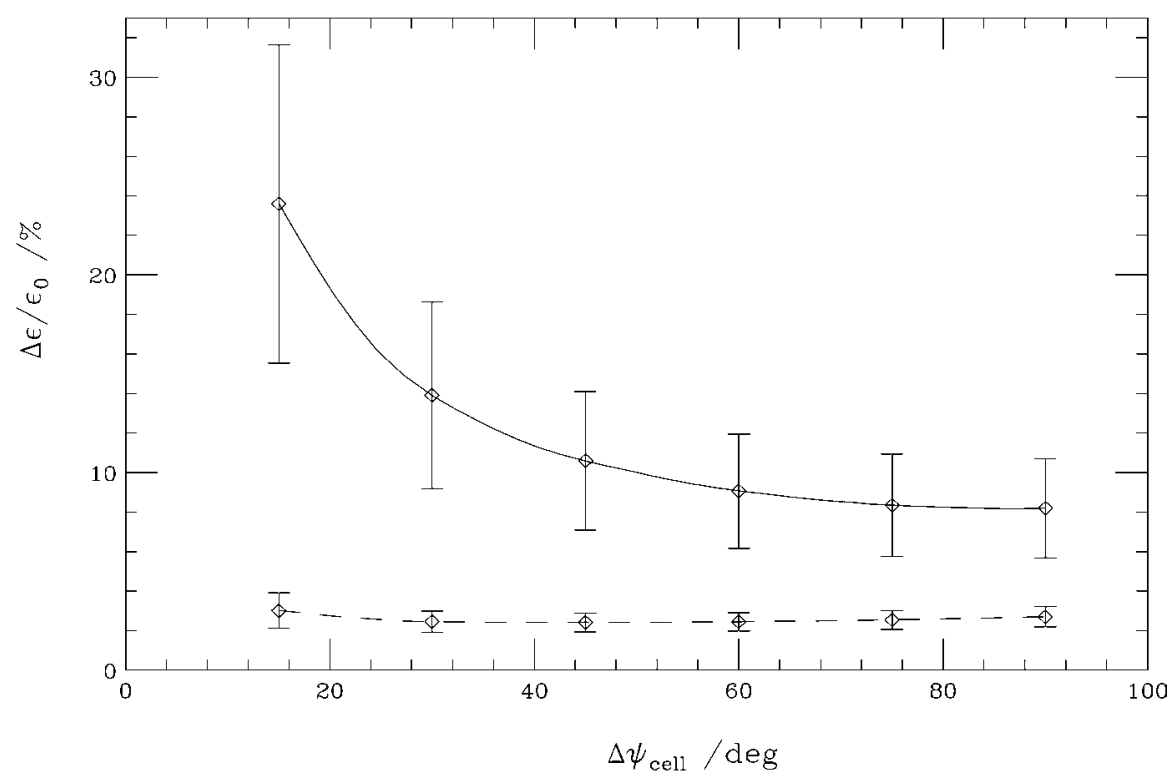

Figure 7.13 Horizontal relative emittance growth (from Liar) versus phase advance/cell for L1 lattice over 10 seeds (solid: wakes-ON, dash: wakes-OFF). Quadrupole, BPM, and rf-structure misalignments of $300 \mu \mathrm{m} \mathrm{rms}$ and one-to-one steering are applied. Vertical behavior (not shown) is similar. Error bars show spread over ten seeds. 


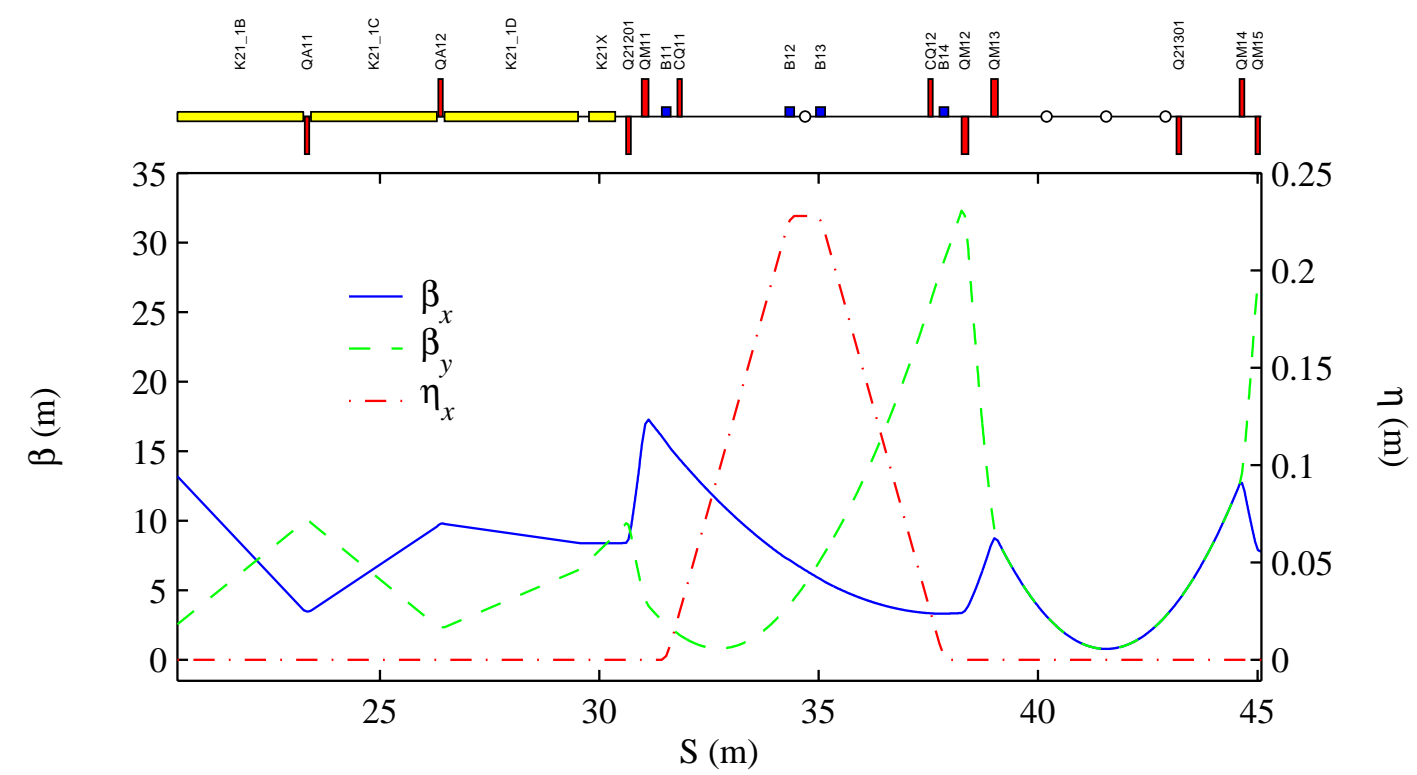

Figure 7.14 Dispersion and beta functions along $\mathrm{L} 1(S<30 \mathrm{~m})$ and through $B C 1$ chicane and ED1 emittance diagnostic section $(S>30 \mathrm{~m}$ ). L1 quad spacing is 3 -meters at $75 \%$ cell phase advance. X-band is if at $S \approx 30 \mathrm{~m}$. Small circles in top schematic indicate profile monitors.

In fact, beam-based alignment techniques in the SLC linac have been used to align quadrupoles and BPMs to $\sim 100 \mu \mathrm{m}$ rms [17]. Figure 7.13 shows horizontal emittance growth versus phase advance/cell with and without transverse wake effects. Error bars are the error on the mean over the 10 seeds. Beta functions and beamline layout are shown in Figure 7.14 for the lattice at the chosen $75 \%$ cell.

Included in Figure 7.14, and following L1, is the BC1 bunch compressor chicane (see Section 7.4.1). BC1 is followed by a transverse emittance diagnostic section (ED1, see Section 7.8.1), which is included in order to measure the transverse emittance immediately after $\mathrm{BC} 1$.

\subsubsection{The L2-Linac}

The energy spread is large (0.8-1.8\%) over the entire 350-meter length of L2, and the bunch is only partially compressed, making L2 the most problematic linac section with respect to transverse emittance dilution. The L2-linac begins at the 21-3b location and ends at 24-6d. The lattice choice for L2 was made using the computer codes Liar and Elegant and varying the phase advance per cell and the quadrupole spacing. Several spacing schemes were tested including (1) the existing 12-meter spacing, (2) a 6-meter quadrupole spacing over the full L2 length, and (3) a 6-meter spacing for the first 60 meters followed by a 12-meter spacing. Although the shorter quadrupole spacing in the first 60 meters reduced the wakefield induced emittance growth, the reduction was not large enough compared with the increased cost of modifications required. Furthermore, well tested and very effective emittance correction techniques are also possible and are described below. 


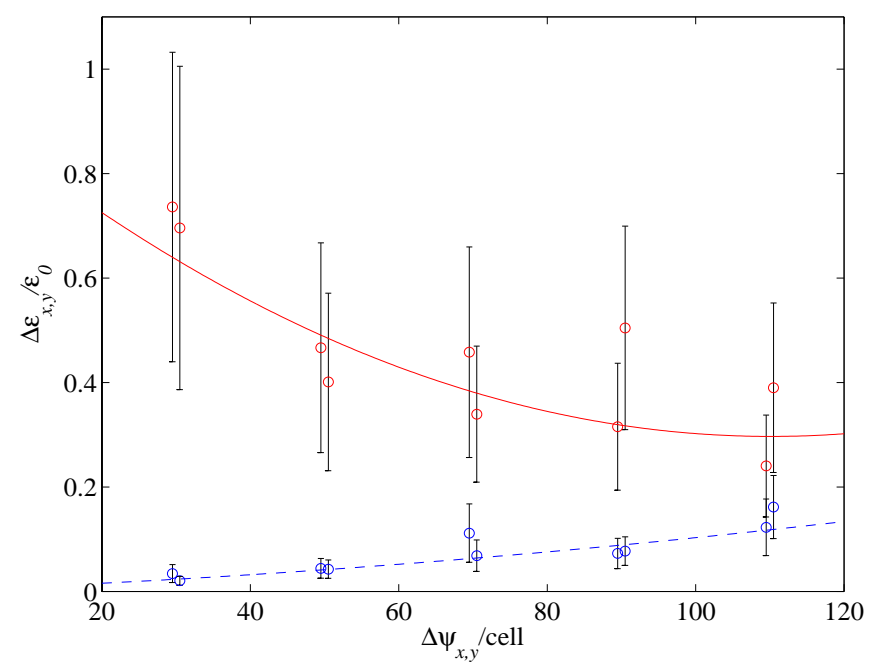

Figure 7.15 Relative mean emittance growth ( $x$ and $y$ ) versus phase advance/cell for L2 over 10 seeds (solid-red: wakes ON, dash-blue: wakes OFF). Points with slight left-offset are $x$ and right-offset are $y$. Quadrupole, BPM, and rf-structure misalignments of $300 \mu \mathrm{m} \mathrm{rms}$ are used and one-to-one steering (no 'bumps' applied). Error bars show statistical error on mean value over 10 seeds.

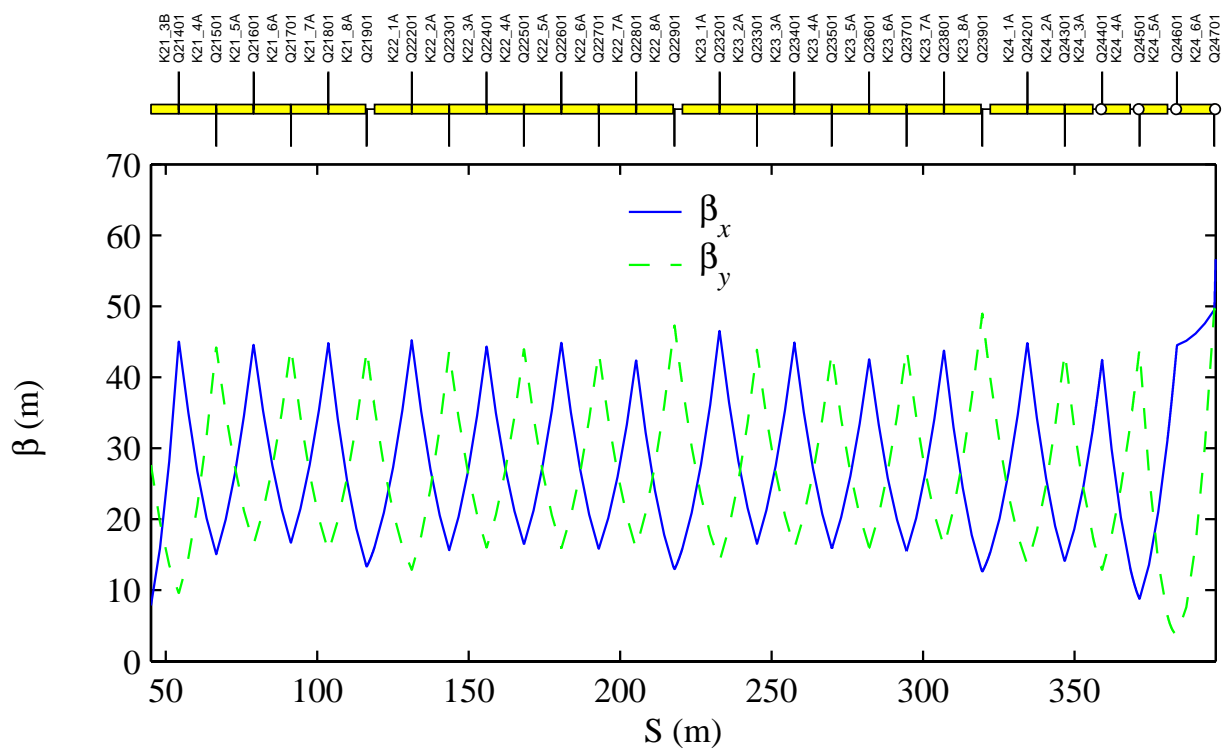

Figure 7.16 Beta functions along L2 at 55\%cell phase advance and 12-meter quadrupole spacing. Small circles in schematic at top indicate profile monitors (wire-scanners) for emittance measurement at the end of L2 (L2-ED).

Using Liar to study emittance correction schemes, it was found that even though the L2 emittance growth (not including CSR in the chicanes) can easily reach $50 \%$, localized trajectory 'bumps' can be used to restore the emittance to just 5-10\% dilution. Figure 7.15 shows emittance dilution, with trajectory corrections but no emittance corrections applied, versus phase advance per cell for the existing 12-meter quadrupole spacing over the length of L2. The minimum emittance growth occurs above $90 \%$ cell. The mean growth at $90 \%$ cell is $\sim 30 \%$ in each plane if no 
further correction is attempted. The choice of $55^{\circ}$ cell is described below. Although Figure 7.15 suggests a stronger lattice, the improvement is not significant with respect to the statistical spread shown by the error bars. The weaker lattice was chosen for reasons of convenience and in order to reduce the trajectory jitter caused by vibrating quadrupole magnets. A stronger lattice is easily installed simply by changing power supply settings.

Figure 7.16 shows the beta functions through the L2-linac at $55^{\circ}$ per cell and a 12 -meter quadrupole spacing. Four profile monitors are indicated at the end of L2 as small circles on the schematic. These are wire scanners used to measure the L2 output emittance as correction bumps are empirically optimized. This trajectory-based emittance correction scheme is similar to that used in the SLAC linac for SLC operations [18]. Simulations of trajectory-based emittance corrections were performed using Liar with an older design of the L2-linac in reference [16]. In this study the mean value of the emittance over 100 random misalignment seeds was reduced from $\sim 100 \%$ to $<10 \%$ in each plane.

No new magnets are needed in the L2-linac. The existing quadrupoles are used at their present locations. One new low current (25 A) bulk quadrupole power supply is installed in each of sectors 23 and 24 in order to achieve rms regulation tolerances of $<0.5 \%$ (not easily achieved with the existing 200-Ampere bulk supply per sector). In addition, the existing 20-ampere booster power supplies (one per quadrupole) will be used to adjust the focusing within a sector.

Quadrupole roll and gradient error tolerances are loose at $\sim 0.5^{\circ}$ and $\sim 1 \% \mathrm{rms}$, respectively ( $\Delta \varepsilon / \varepsilon_{0} \approx 2 \%$ total over 30 quadrupoles). Magnet transverse vibration tolerances are $\sim 1 \mu \mathrm{m} \mathrm{rms.}$ Tolerances on field harmonics (e.g., 12-pole) in the quadrupoles are extremely loose.

\subsubsection{The L3-Linac}

The L3-linac begins at 25-1a and ends at the end of sector-30 (30-8c). Eight 3-meter sections from 24-7a through 24-8d are removed to install the long BC2 bunch compressor chicane. Note that the section at 25-1c was removed years ago for the NPI gun, but might now be replaced to partially compensate for the 24-7 and 24-8 removals. The short bunch of $22 \mu \mathrm{m}$ in L3 effectively eliminates transverse wakefields as a source of emittance dilution, and the rms energy spread shrinks from $0.8 \%$ down to $<0.1 \%$ due to the strong longitudinal wakefield in L3. (Note, the rf phase in L3 is set at $-10^{\circ}$ for slightly improved energy stability, not to alter the beam energy spread.) In this case the dominant emittance dilution mechanism is due to momentum dispersion generated by quadrupole and BPM misalignments. This suggests a weak focusing lattice. Liar simulations were run for L3 using the existing SLAC linac 12-meter quadrupole spacing, but varying the phase advance per cell over the set $20^{\circ}, 30^{\circ}, 40^{\circ}, 60^{\circ}$ and $90^{\circ}$. Figure 7.17 shows the horizontal relative emittance growth versus phase advance per cell for L3 with and without transverse wakefield effects. This clearly demonstrates the weak transverse wakefield effect due to the extremely short bunch in L3. Large quadrupole, BPM, and rf-structure misalignments of $300-\mu \mathrm{m}$ rms were used as well as a one-to-one steering algorithm and the nominal undulator energy of $14.3 \mathrm{GeV}$. 
A phase advance per cell of $33^{\circ}$ is chosen for L3 since it is near the minimum emittance dilution. This particular phase $(33.5 \%$ cell horizontally) also allows the center of the last bend of the $\mathrm{BC} 2$ chicane to be separated by $2 n \pi$ in total horizontal betatron phase from the center of the first bend in the DL2 dogleg. In this way the correlated emittance growth effects of coherent synchrotron radiation in the two bend systems (BC2 and DL2) might be approximately cancelled, rather than added as they would with a $(2 n+1) \pi$ separation (see Section 7.4.2 and 7.5.2). This even- $\pi$ symmetry works because the first bend of DL2 bends in a 'left' direction whereas the first bend-pair of DL2 bends to the 'right'.

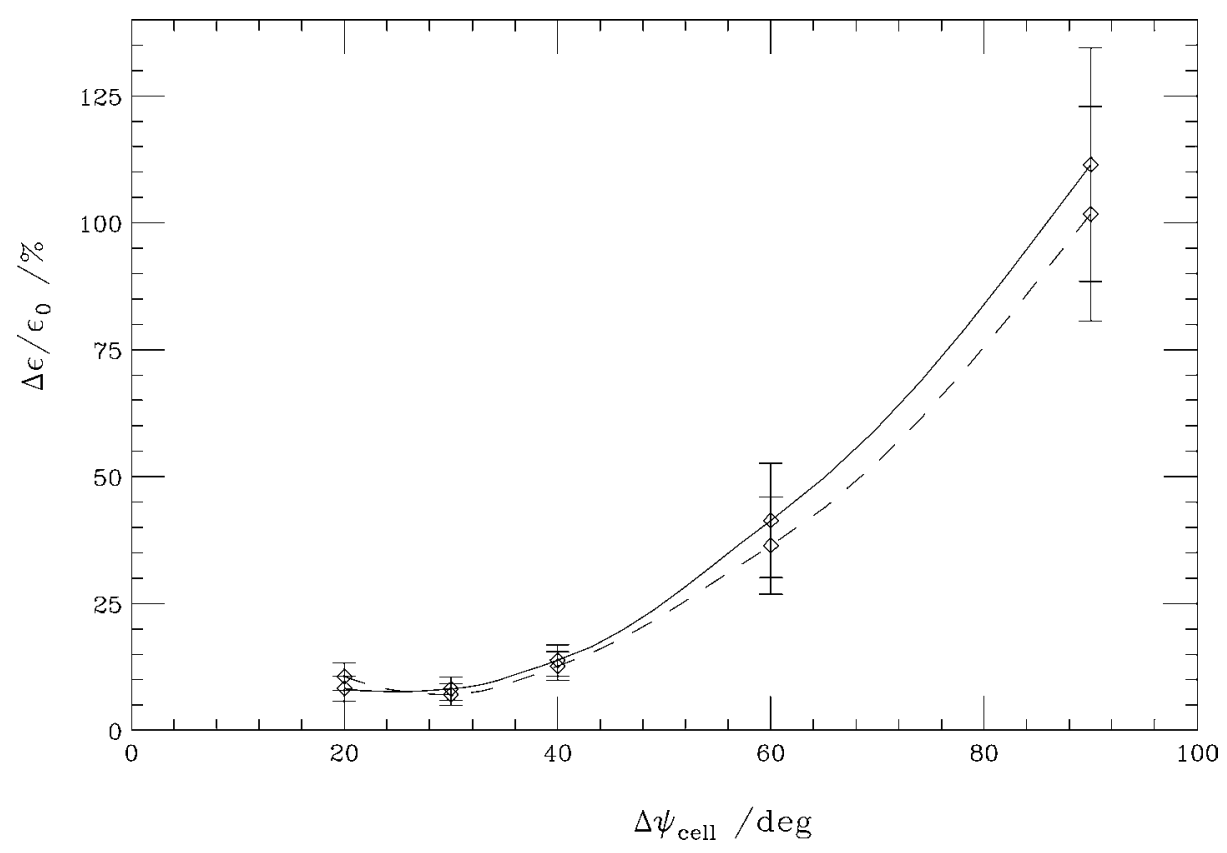

Figure 7.17 Mean relative emittance growth versus phase advance/cell for L3 and 12-meter quadrupole spacing over 10 seeds (solid: wakes ON, dash: wakes OFF). Quadrupole, $\mathrm{BPM}$, and rf-structure misalignments of $300 \mu \mathrm{m}$ rms were used as well as one-to-one steering (no bumps applied). The phase chosen is $33 \%$ cell.

The L3 phase advance per cell in the vertical plane $(33.0 \%$ cell) is chosen in order to set a $(2 n+1) \pi / 2$ vertical phase advance between a transverse rf deflecting structure in $25-5 \mathrm{a}$ (used for bunch length measurements - see Section 7.8.2) and the profile monitor PR31 in DL2. This allows the transverse $\mathrm{rf}$ to be used as a diagnostic to analyze the details of the final longitudinal phase space population just prior to the undulator.

The $33 \%$ cell phase advance also optimizes the phase advance separation of the existing sector-28 wire scanners for an improved $x$ and $y$ emittance resolution. The first and last wires will be relocated to set each wire-to-wire separation at 1.5 cells, or $45^{\circ}$ (see Section 7.8.1). Betafunctions and beamline layout are shown in Figure 7.18 for $33 \%$ cell. 


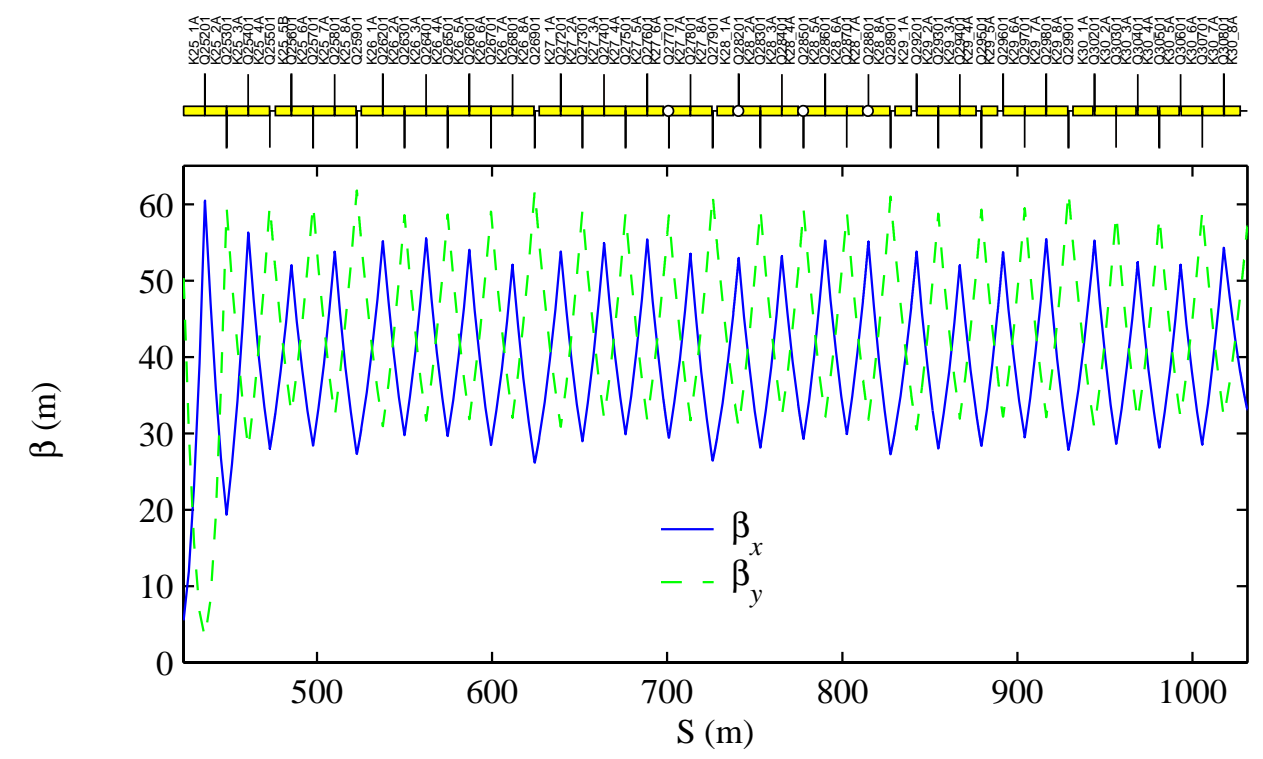

Figure 7.18 Beta functions along L3 at a phase advance of $33 \%$ cell. Four existing sector-28 wire scanners are indicated by small circles in schematic at top (L3-ED: first and last wires are relocated to optimize emittance resolution).

No new magnets are needed in the L3-linac, except those used for matching surrounding the BC2 chicane. One new low current (25 A) bulk quadrupole power supply is installed in each of sectors 25 through 29 in order to achieve rms regulation tolerances of $<0.5 \%$ (not easily achieved with the existing 200-Ampere bulk supply per sector). In addition, the existing 20-Ampere booster power supplies (one per quadrupole) will be used to adjust the focusing within a sector.

\subsection{Electron Bunch Compressors}

\subsubsection{First Bunch Compressor}

The first compression-stage, $\mathrm{BC} 1$, is a magnetic chicane designed to introduce the energy dependence of a particle's path length $\left(\Delta z=R_{56} \delta E / E_{0}\right)$ needed to compress a $830 \mu \mathrm{m}$ bunch to $200 \mu \mathrm{m}$. Several designs are possible, but the simplicity of a four-dipole magnetic chicane is attractive because it: (1) adds no net beamline bend angle or offset, (2) generates no chromaticity or high order dispersion (with rectangular bends) since it contains no quadrupole magnets, and (3) allows simple tuning of the momentum compaction, $R_{56}$, with a single power supply.

The short bunch demands a chicane design where coherent synchrotron radiation (CSR) generated within the bends does not significantly dilute the emittance. In addition, the chicane length should be minimized so that the number of removed linac accelerating sections is small. An optimized parameter set has been chosen with a $250-\mathrm{MeV}$ chicane motivated by synchrotron radiation effects and linac longitudinal beam dynamics simulations. This system and its adjacent diagnostics will replace four existing 3-meter rf sections in sector-21 (21-2a,b,c,d). A phosphor screen profile monitor, a BPM, and a horizontal beam collimator will be included at the center of the chicane. 


\subsubsection{Overview and Parameters}

The chicane design is set by the need to reduce transverse emittance dilution due to coherent synchrotron radiation (CSR) in the bends, which is most pronounced for short bunches [19], [20], [21], [22], [23]. Since the dominant component of the energy spread generated by CSR is correlated along the bunch, only the projected transverse emittance is altered. The emittance of the bunch slices is typically unchanged. The effect can be minimized by using a weak chicane and a large initial correlated energy spread. A symmetric double-chicane is also possible [16] and can be used to reduce the projected emittance growth. Unfortunately, the added bending for the double-chicane increases the possibility of a CSR-induced micro-bunching instability [3]. For this reason single chicanes are used in the present design.

Table 7.11 Parameters of $1^{\text {st }}$ bunch compressor chicane, BC1.

\begin{tabular}{|c|c|c|c|}
\hline Parameter & Symbol & Unit & Value \\
\hline Beam energy & $E$ & $\mathrm{GeV}$ & 0.250 \\
\hline Initial rms bunch length & $\sigma_{z_{i}}$ & $\mathrm{~mm}$ & 0.83 \\
\hline Final rms bunch length & $\sigma_{z_{f}}$ & $\mathrm{~mm}$ & 0.19 \\
\hline RMS total incoming relative energy spread (at $250 \mathrm{MeV}$ ) & $\sigma_{\delta}$ & $\%$ & 1.78 \\
\hline RMS uncorrelated relative energy spread (at $250 \mathrm{MeV}$ ) & $\sigma_{\delta_{u}}$ & $10^{-5}$ & 1 \\
\hline Momentum compaction & $R_{56}$ & $\mathrm{~mm}$ & -35.9 \\
\hline Second order momentum compaction & $T_{566}$ & $\mathrm{~mm}$ & +53.9 \\
\hline Total chicane length ( $1^{\text {st }}$ bend to last) & $L_{\text {total }}$ & $\mathrm{m}$ & 6.56 \\
\hline Floor length of each of four dipole magnets & $L_{B}$ & $\mathrm{~m}$ & 0.20 \\
\hline Floor length of drift between first two and last two dipoles & $\Delta L$ & $\mathrm{~m}$ & 2.60 \\
\hline Floor length of drift between center two dipoles & $\Delta L_{c}$ & $\mathrm{~m}$ & 0.50 \\
\hline Bend angle of each dipole & $\left|\theta_{B}\right|$ & $\operatorname{deg}$ & 4.62 \\
\hline Magnetic field of each dipole & $|B|$ & $\mathrm{kG}$ & 3.36 \\
\hline Maximum dispersion in chicane center ( $\approx$ beamline excursion $)$ & $\left|\eta_{\max }\right|$ & $\mathrm{m}$ & 0.229 \\
\hline Projected CSR emittance dilution $\left(\gamma \varepsilon_{0}=1 \mu \mathrm{m}\right)$ & $\Delta \varepsilon_{\mathrm{CSR}} / \varepsilon_{0}$ & $\%$ & 5 \\
\hline CSR-induced relative energy spread (at $250 \mathrm{MeV}$ ) & $\sigma_{\delta \mathrm{CSR}}$ & $\%$ & 0.029 \\
\hline CSR- induced relative energy loss (at $250 \mathrm{MeV}$ ) & $\delta_{\mathrm{CSR}}$ & $\%$ & -0.068 \\
\hline
\end{tabular}

Motivations and quantitative arguments for the choices of these parameters are described in the following sections. Results of longitudinal beam dynamics simulations have been used to set the final $\mathrm{BC} 1$ bunch length at $195 \mu \mathrm{m}$ rms. The bunch length is adjustable using the $R_{56}$ of $\mathrm{BC} 1$ and the rf phase of L1. The center two dipoles of the chicane will be placed on remotely movable 
horizontal stages in order to: 1) allow non-LCLS linac operation, 2) ease dipole field quality tolerances, and 3) allow a high-resolution BPM to be placed at the center of the chicane to provide energy feedback. With the dipoles switched off, the $\mathrm{BC} 1$ (and also the $\mathrm{BC} 2$ ) chicane can then be straightened out. The maximum horizontal beamline excursion at chicane center is equal to the maximum dispersion, $\left|\eta_{\max }\right|$, listed in the table. The excursion is toward the tunnel 'wall' (north) to keep the 'aisle' clear.

Figure 7.19 shows the dispersion and beta functions through the $\mathrm{BC} 1$ chicane, and Table 7.11 lists parameters for the chicane. Magnet locations are shown at the top of the figure. This plot and other calculations are made for a net $R_{56}$ of $-35.9 \mathrm{~mm}$.

\subsubsection{Momentum Compaction}

The momentum compaction $\left(R_{56}\right)$ of a chicane made up of rectangular bend magnets is negative (for bunch head at $z<0$ ). For ultra-relativistic electrons and small bend angles, the net $R_{56}$ of the chicane is given in Eq. (7.12) where the symbol definitions are taken from Table 7.11.

$$
R_{56} \equiv \frac{\partial z}{\partial \delta} \approx-2 \theta_{B}^{2}\left(\Delta L+\frac{2}{3} L_{B}\right)
$$

Free parameters are: bend lengths, angles, and drift lengths. The required $R_{56}$ is determined from the desired compression, the energy spread, and the rf phase of L1, which is chosen in the parameter optimization as described in Section 7.2.2. The second order momentum compaction ( $\left.T_{566}\right)$ of a rectangular-bend chicane (no quadrupole magnets) is $T_{566} \approx-3 R_{56} / 2$ [24].

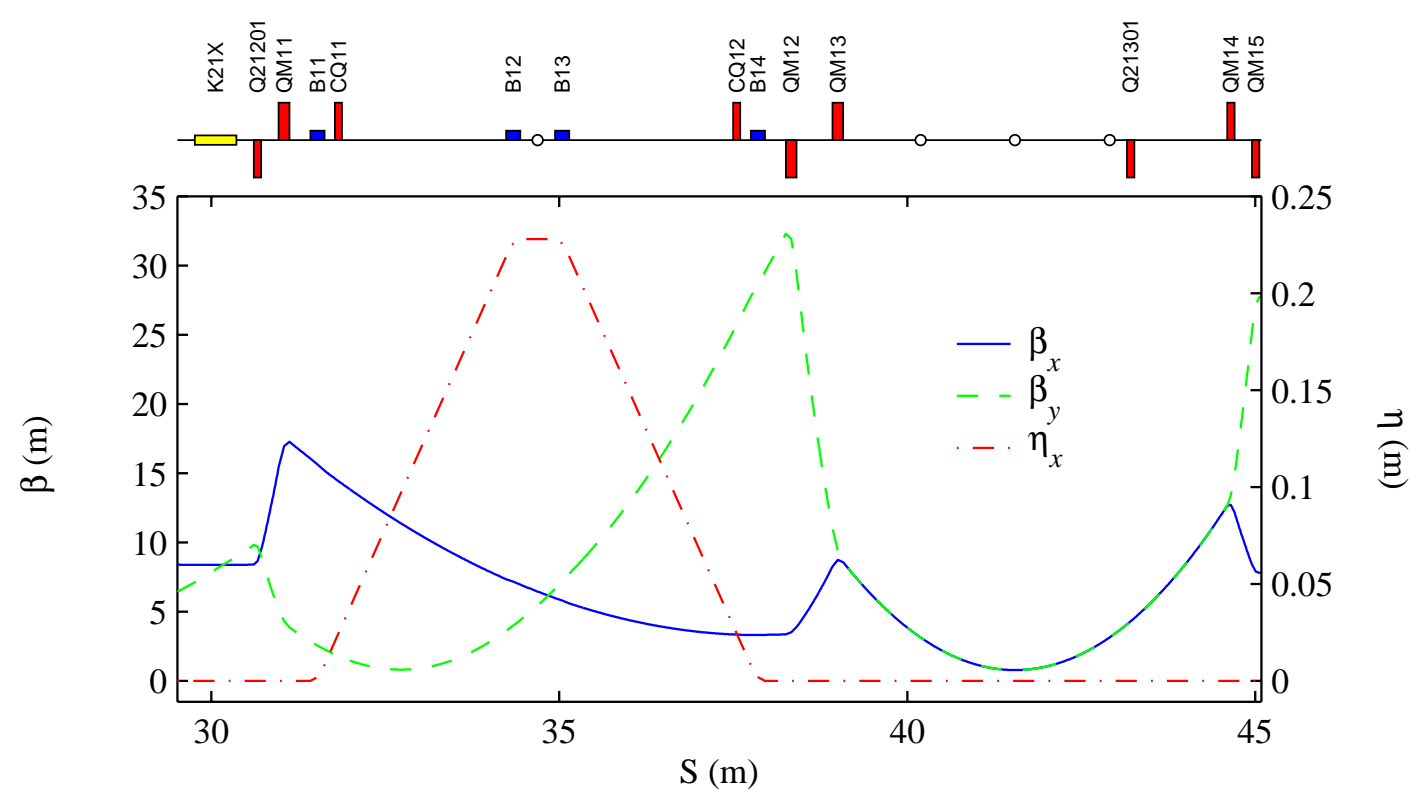

Figure 7.19 Dispersion and beta functions through $\mathrm{BC} 1$ chicane for $R_{56} \approx-35.9$. X-band if structure ('K21X' in yellow) is upstream of first chicane. Energy spread profile monitor is small circle in center of $1^{\text {st }}$ chicane. Two quads inside first chicane are for horizontal dispersion correction and are nominally off. 
A beam delay must also be accounted for in the rf phase of the following linac (L2 in this case) as the beam path length increases through a chicane. The phase delay is described in Eq. (7.13), where the rf phase lag is $\Delta \varphi$. The L2 $\mathrm{rf}$ phase needs to be delayed with respect to the chicane-off phase by $d \Delta \varphi / d R_{56} \approx \pi / \lambda \approx 1.72^{\circ} / \mathrm{mm}$ (or $61.5^{\circ}$ S-band with the nominal $R_{56}$ of $-35.9 \mathrm{~mm})$ :

$$
\Delta \varphi=\frac{2 \pi \Delta s}{\lambda}=\frac{4 \pi}{\lambda}\left[2 L_{B}\left(1-\frac{\theta_{B}}{\sin \theta_{B}}\right)+\Delta L\left(1-\frac{1}{\cos \theta_{B}}\right)\right] \approx \frac{\pi R_{56}}{\lambda} .
$$

\subsubsection{Coherent Synchrotron Radiation (CSR)}

\section{Introduction}

For very short bunches, the coherent component of synchrotron radiation can be significant and may dilute the horizontal emittance by generating energy spread in the dipoles. In this case, however, the energy spread is mostly correlated along the bunch and is not a random effect. For an rms bunch length, $\sigma_{z}$, dipole length, $L_{B}$, bend radius, $R\left(\approx L_{B} / \theta_{B}\right)$, and $N$ electrons per bunch, the CSR-induced rms relative energy spread per dipole for a gaussian bunch under steady-state conditions is [20]

$$
\sigma_{\delta} \approx 0.22 \frac{N r_{e} L_{B}}{\gamma R^{2 / 3} \sigma_{z}^{4 / 3}}
$$

where $r_{e}$ is the classical electron radius and $\gamma$ is the Lorentz energy factor. This is valid for a dipole magnet where radiation shielding (see Figure 7.20) of a conducting vacuum chamber is not significant; i.e., for a full vertical vacuum chamber height $h$ which satisfies [25]

$$
h>>\left(\pi \sigma_{z} \sqrt{R}\right)^{2 / 3} \equiv h_{c} .
$$

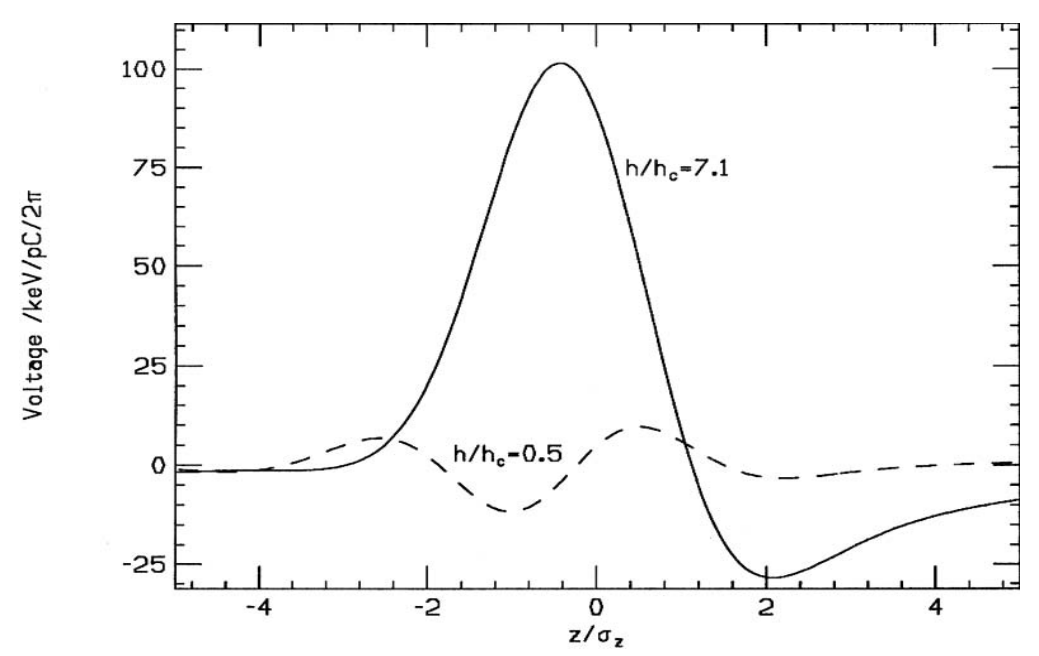

Figure 7.20 Coherent radiation steady-state wake per $2 \pi$ bend $\left(R=19.4 \mathrm{~m}, \sigma_{z}=30 \mu \mathrm{m}\right.$, $h_{c}=5.6 \mathrm{~mm}$ ) for both shielded (dash: $h / h_{c}=0.5$ ) and unshielded (solid: $h / h_{c}>1$ ) coherent radiation of a gaussian bunch. Here the bunch head is at $z>0$ and $V>0$ represents energy loss. 
Typically, the value of $h$ required to adequately shield the CSR effects is too small to allow an adequate beam aperture (in this case, for $R \approx 2.5 \mathrm{~m}, h<<10 \mathrm{~mm}$ will shield a $190-\mu \mathrm{m}$ bunch). The shielding will not, however, suppress coherent radiation stemming from current spikes with characteristic length $<<\sigma_{z}$. In addition, with very small apertures, geometric or resistive wakefields can also generate emittance dilution. For these reasons it is not planned to incorporate radiation shielding into the chamber design.

In non-steady-state conditions, as the bunch enters the magnet, the CSR fields go through a transient regime as the radiation from the tail of the bunch catches up with the head. The characteristic length required for this transient region is $L_{0}$, given by [20]

$$
L_{0} \equiv\left(24 \sigma_{z} R^{2}\right)^{1 / 3}
$$

After the length $L_{0}$, and for a gaussian bunch, the fields begin to take on the steady-state form as given in Eq. (7.14). The radiation generated within the bend can also catch-up to the bunch after it exits the bends. This bend-exit transient can be a very important contribution to the emittance growth and must also be included in the calculations.

Since the bunch length shortens through the chicane, the local energy spread induced at each dipole increases, with the final dipole generating the most significant energy spread (the bunch length is virtually constant in the first and last dipoles). The rms horizontal emittance after a single chicane can be written as

$$
\begin{gathered}
\Delta x=\int_{\text {bends }} R_{16}(s) \frac{d \delta}{d s} d s, \quad \Delta x^{\prime}=\int_{\text {bends }} R_{26}(s) \frac{d \delta}{d s} d s \\
\varepsilon^{2}=\varepsilon_{0}^{2}+\varepsilon_{0}\left[\beta\left\langle\Delta x^{\prime 2}\right\rangle+2 \alpha\left\langle\Delta x \Delta x^{\prime}\right\rangle+\left(1+\alpha^{2}\right)\left\langle\Delta x^{2}\right\rangle / \beta\right]+\left\langle\Delta x^{2}\right\rangle\left\langle\Delta x^{\prime 2}\right\rangle-\left\langle\Delta x \Delta x^{\prime}\right\rangle^{2} \\
=\varepsilon_{0}^{2}+2 \zeta \varepsilon_{0} \Delta \varepsilon+\Delta \varepsilon^{2} \geq\left(\varepsilon_{0}+\Delta \varepsilon\right)^{2}, \quad \zeta \geq 1 .
\end{gathered}
$$

Here $R_{16}(s)$ and $R_{26}(s)$ are the standard transfer matrix elements from point $s$ to end of the chicane, $\beta$ and $\alpha$ are the nominal Twiss parameters at end of the chicane, and $\varepsilon_{0}$ and $\varepsilon$ are the initial and final rms horizontal emittances. The change in centroid coordinates, $\Delta x$ and $\Delta x^{\prime}$, pertain to a single longitudinal bunch 'slice'. Their second moments (e.g., $\left\langle\Delta x^{2}\right\rangle$ ) are ensemble averages over the entire bunch, and $\zeta$ is the Courant-Snyder invariant [see Eq. (7.26)]. In ideal unshielded, steady-state conditions, where $\left\langle\Delta x^{2}\right\rangle\left\langle\Delta x^{\prime 2}\right\rangle \approx\left\langle\Delta x \Delta x^{\prime}\right\rangle^{2}$, the relative emittance growth for just the last bend of a single chicane, using Eq. (7.14) and Eq. (7.17), and a constant gaussian bunch length, $\sigma_{z}$, in that last bend magnet is

$$
\frac{\varepsilon}{\varepsilon_{0}} \approx \sqrt{1+\frac{(0.22)^{2}}{36} \frac{r_{e}^{2} N^{2}}{\gamma \varepsilon_{N} \beta}\left(\frac{|\theta|^{5} L_{B}}{\sigma_{z}^{4}}\right)^{2 / 3}\left[L_{B}^{2}\left(1+\alpha^{2}\right)+9 \beta^{2}+6 \alpha \beta L_{B}\right]}
$$

with $\varepsilon_{N}\left(\equiv \gamma \varepsilon_{0}\right)$ introduced to represent the initial normalized (invariant) emittance. This is typically an underestimate of the growth, since it does not include radiation effects in the rest of 
the chicane, which can be significant. It also shows the importance of matching $\beta$ and $\alpha$ to the phase space distortion effects of the radiation.

Calculations of the CSR-induced emittance dilution have been made using the TraFiC4 code [22] written at DESY and also using Elegant [10] (see below, "CSR Calculations with a Transient Model"), which result in a projected emittance growth of $\sim 5 \%$. The slice emittance is, however, almost unchanged ( $<<1 \%$ increase). Calculations using TraFiC4 were, however, based on an older chicane design and a gaussian beam [16], [26].

The $R_{56}$ of the $\mathrm{BC} 1$ chicane is also intended to be adjustable to allow for correction of the longitudinal phase space of the linac output beam and to provide control of the final bunch length. An $R_{56}$ tuning range of 0 to $-65 \mathrm{~mm}$ will allow a wide flexibility in the LCLS accelerator to provide for different machine configurations at variable bunch charge, etc.

\section{CSR Calculations with a Transient Model}

More complete calculations of the CSR effects on the bunch through $\mathrm{BC} 1$ have been made using TraFiC4, and also using Elegant. Both codes are time domain treatments that include field transients at entrance and exit of the bends. TraFiC4 is a 3D full-field treatment including $x-z$ correlations in the beam at high dispersion points, the space charge forces, and longitudinal as well as transverse forces. The bunch distribution used is, however, typically gaussian, although other possibilities exist. Elegant is a 1D line-charge model, which ignores the transverse extent of the beam when calculating the CSR wakefield. This limitation can generate an overestimate of the projected emittance growth, but it is not considered to be a large effect for the LCLS compressor chicanes. Elegant also only calculates the longitudinal CSR forces.

In Elegant, it is easily possible to use the actual temporal profile of the bunch, tracked from the injector, and to calculate the CSR-generated longitudinal 'wakefields' of this non-gaussian line-charge [27]. The bunch is binned into 500 slices and the CSR-wakefield is calculated using the results of reference [21], which predict the rate of energy change of a particle at point $z$ in the bunch:

$$
\begin{aligned}
\frac{d E(z, \phi)}{d(c t)}= & -\frac{2 r_{e} m c^{2}}{3^{1 / 3} R^{2 / 3}}\left\{\left(\frac{24}{R \phi^{3}}\right)^{1 / 3}\left[\lambda\left(z-R \phi^{3} / 24\right)-\lambda\left(z-R \phi^{3} / 6\right)\right]\right. \\
& \left.+\int_{z-R \phi^{3} / 24}^{z} \frac{d z^{\prime}}{\left(z-z^{\prime}\right)^{1 / 3}} \frac{d \lambda\left(z^{\prime}\right)}{d z^{\prime}}\right\} .
\end{aligned}
$$

The bend radius is $R$, the bend angle is $\phi$, the line-charge density distribution is $\lambda(z), r_{e}$ is the classical electron radius, $m c^{2}$ is the rest energy of the electron, and $\lambda\left(z-R \phi^{3} / \ldots\right)$ implies evaluation of $\lambda$ at $\left(z-R \phi^{3} / \ldots\right)$. This relation can be used to calculate the distribution of the rate of the energy change of the electrons as a function of bend angle $\phi$ when the bunch enters the bend magnet. The magnets are sliced 10-20 times and Eq. (7.19) is evaluated for each slice, allowing for a changing $\lambda(z)$, as shown in the plots of Figure 7.7. The energy change continues between 
magnets, where radiation in the previous bends can still alter the bunch. This is modeled in Elegant by using the bend-exit model outlined in [28].

Figure 7.21 shows the 'real' temporal distribution of the LCLS electron bunch in BC1, as it compresses, and the CSR-wakefield, from Eq. (7.19), within the third bend of chicane-1, using the temporal distribution taken from tracking upstream of $\mathrm{BC} 1$. The variable color traces represent the two functions sampled in each of ten points along the bend magnet.

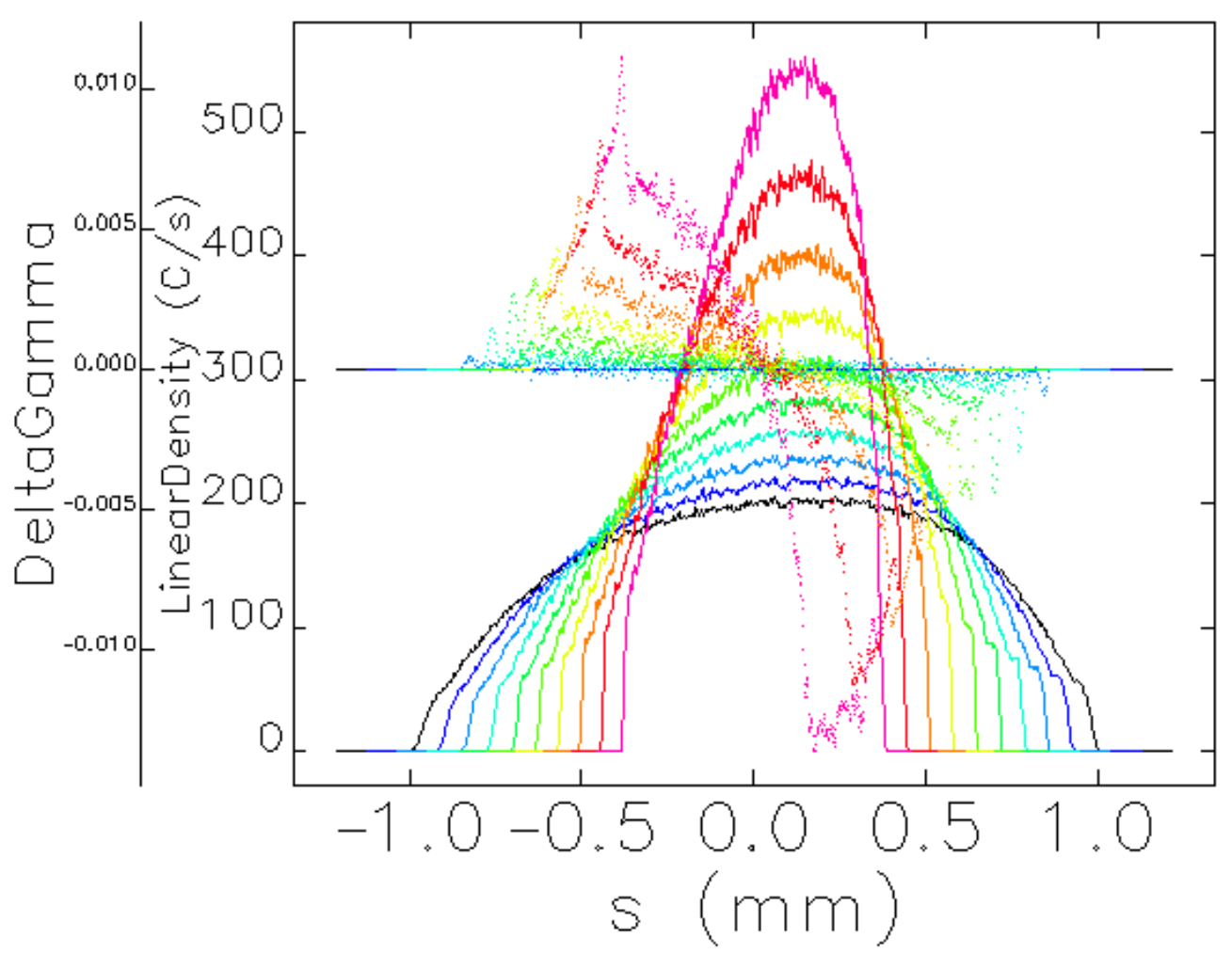

Figure 7.21 Temporal distribution of bunch (solid: "LinearDensity") and CSR-wakefield (dots: "DeltaGamma") within third bend of chicane in BC1. The variable color traces represent the two functions sampled in each of ten points along the bend magnet.

Figure 7.22 shows the position spread in the electron beam, $x$, without CSR (left) and with CSR (right), versus the axial bunch coordinate, $z$, plus their distribution projections, at the end of the BC1-chicane, using $2 \times 10^{5}$ tracked particles (similar plot for $x^{\prime}$-angle vs. $z$ is not shown). The temporal profile used for $\lambda(z)$ is the 'real' expected temporal distribution with its non-linear energy correlations, shown in the $3^{\text {rd }}$ row of Figure 7.6 and partially above in Figure 7.21, which are based on $6 \mathrm{D}$ tracking through the accelerator up to the entrance of the $\mathrm{BC} 1$ chicane. The initial bunch length is $830 \mu \mathrm{m} \mathrm{rms}$, the final bunch length is $195 \mu \mathrm{m} \mathrm{rms}$, the initial emittance is $1.0 \mu \mathrm{m}$, the charge is $1 \mathrm{nC}$, and the horizontal beta and alpha functions at first bend entrance are $\beta_{x} \approx 16.7 \mathrm{~m}$ and $\alpha_{x} \approx 2.0$ (see Figure 7.19).

The final emittance is increased by $5 \%$ (including all particles), while the slice emittance is unchanged. If the linear energy correlation is removed from the spatial, $x$, and angular, $x$, 
coordinates (i.e., by slightly altering the residual dispersion after the chicane using the small 'tweaker' quadrupole magnets included in the chicane - see CQ11 and CQ12 labels in Figure 7.19), the projected emittance growth is reduced to $1 \%$. This tweaker-quad correction has been successfully tested in simulations, but unfortunately couples energy jitter into horizontal position jitter. For this reason the tweaker-quads will probably only be used to correct dispersion errors.
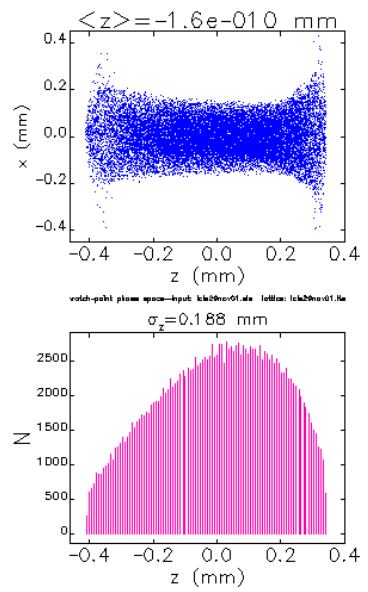

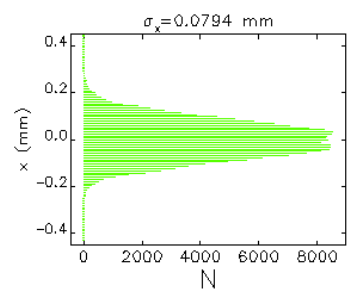

.
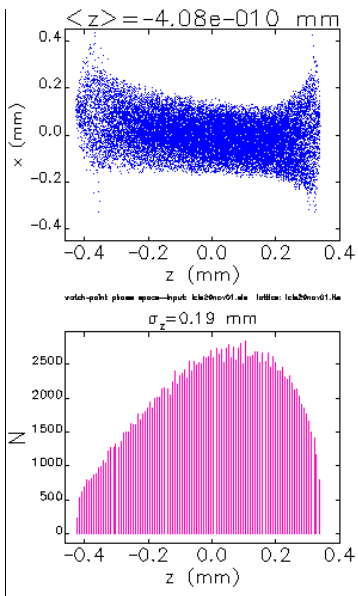

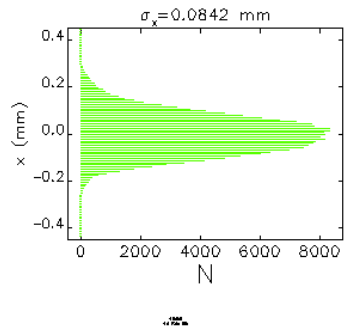

.

Figure 7.22 Horizontal position, $x$, without CSR (left) and with CSR (right), versus $z$, for LCLS bunch profile (i.e., tracked through upstream systems) after BC1. The projected emittance growth is $5 \%$.

The total energy loss due to CSR is $0.17 \mathrm{MeV}$ (or $0.068 \%$ ). The change in central trajectory produced by the energy loss in the bends has been corrected in the tracking by steering so that the electron beam does not pass off-center through quadrupoles. The mismatch effect on the horizontal beta and alpha functions at the end of the chicane is negligible.

\subsubsection{Beam Size, Aperture, and Field Quality}

From the parameters in Table 7.11 $\left(\eta_{\max }\right.$ and $\left.\sigma_{\delta}\right)$, the horizontal rms beam size in the center of the $\mathrm{BC} 1$ chicane is $4.1 \mathrm{~mm}$. A reasonable $R_{56}$ tuning range for $\mathrm{BC} 1$, allowing a wide range of flexibility, is 0 to $-65 \mathrm{~mm}$. The displacement of the center two dipoles is remotely controlled while varying the fields, and hence $R_{56}$. This also allows the chicane to be straightened out for non-LCLS operations (dipoles off).

Field quality tolerances for the four dipoles are listed in Table 7.12. Quadrupole field components $\left(b_{1} / b_{0}\right)$ are correctable, while sextupole $\left(b_{2} / b_{0}\right)$ and decapole $\left(b_{4} / b_{0}\right)$ components, without specialized correction magnets, are not. Magnet roll errors generate anomalous vertical dispersion, which should be correctable with vertical steering. Field quality tolerances on the center pair of dipoles are tight but achievable, especially in consideration of the empirical corrections built into the system (i.e., the tweaker quads).

A pair of adjustable collimator jaws will be included in the center of the chicane, just upstream of a profile monitor, which can be used to cut the horizontal (i.e., energy) tails. The collimator will be nominally open with a full horizontal gap of $\sim 8 \mathrm{~cm}$, but each jaw will be 
independently controlled in order to select any energy band. The collimator will be very useful in diagnosing tails in the electron beam. It is not envisioned that any significant collimation will be made during normal LCLS operations. Otherwise this may introduce an intolerably large pulseto-pulse charge jitter downstream of $\mathrm{BC} 1$.

\subsubsection{Tuning and Correction}

Dispersion errors (typically horizontal) are generated by quadrupole field components in the center dipoles of BC1 (see Table 7.12). A pair of small correction quadrupoles are included in the chicane (see Figure 7.19), with the first one (CQ11) placed near the end of B11 at $\eta_{x} \approx 24 \mathrm{~mm}$, $\beta_{x} \approx 14 \mathrm{~m}$, and the second (CQ12) just upstream of B14 ( $\left.\eta_{x} \approx 24 \mathrm{~mm}, \beta_{x} \approx 3.3 \mathrm{~m}\right)$. Two orthogonal linear combinations of these quadrupoles can then be used to correct emittance dilution due to dispersion errors over a large range. Since the dispersive beam size at these quadrupoles is $\eta_{x} \sigma_{\delta} \approx 430 \mu \mathrm{m}$, and the betatron beam size is much smaller at $\left(\beta_{x, y} \varepsilon_{x, y}\right)^{1 / 2}<170 \mu \mathrm{m}$, these quadrupoles have little effect on beta functions. Two orthogonal linear combinations of these quadrupoles can then be used to correct up to $\sim 250 \%$ horizontal emittance dilution due to dispersion errors. The specifications for these correction quadrupoles are given in Table 7.13 and their locations are shown in Figure 7.19.

The four dipoles are powered in series with one main power supply so that regulation errors have little effect (Table 7.4). The rms regulation tolerance for this main supply is $0.02 \%$. Separate trim coils will also be included in each BC1 main dipole so that compensation can be made for magnet-to-magnet construction variations in the dipole field strength. In addition, vertical dipole correctors will be included nearby to allow vertical steering. The alignment and field strength sensitivities for quadrupole magnets in the BC1 area are shown in Figure 7.23. Each sensitivity shown corresponds individually to a summed $x$ and $y$ emittance dilution of $\Delta \varepsilon_{x} / \varepsilon_{x 0}+\Delta \varepsilon_{y} / \varepsilon_{y 0}=2 \%$. Quadrupole field errors affect beam matching and the sensitivities here assume the mismatch has completely filamented and diluted the emittance (a conservative assumption).

Table 7.12 Dipole magnet tolerances for BC1. Field harmonics are evaluated on a 20-mm radius and each entry individually corresponds to a $2 \%$ emittance dilution.

\begin{tabular}{cccccc}
\hline Magnet & Quantity & Roll Angle $[\mathrm{mrad}]$ & $\left|\boldsymbol{b}_{\mathbf{1}} / \boldsymbol{b}_{\mathbf{0}}\right|[\%]$ & $\left|\boldsymbol{b}_{\mathbf{2}} / \boldsymbol{b}_{\mathbf{0}}\right|[\%]$ & $\left|\boldsymbol{b}_{\mathbf{4}} / \boldsymbol{b}_{\mathbf{0}}\right|[\%]$ \\
\hline B11 \&B14 & 1 each & 3.9 & 0.30 & 22. & 100 \\
\hline B12 \& B13 & 1 each & 2.6 & 0.02 & 0.07 & 0.28 \\
\hline
\end{tabular}

Table 7.13 Dispersion correction quadrupoles for BC1 chicane-1 for horizontal emittance correction of up to $250 \%$ (with $\Delta \varepsilon / \varepsilon \approx 1 \%$ step size control).

\begin{tabular}{ccccc}
\hline Maximum Pole-Tip Field $[\mathrm{kG}]$ & Quantity & Step Size $[\mathrm{kG}]$ & Pole Radius $[\mathrm{mm}]$ & Length $[\mathrm{m}]$ \\
\hline 0.5 & 2 & 0.05 & 50 & 0.05 \\
\hline
\end{tabular}




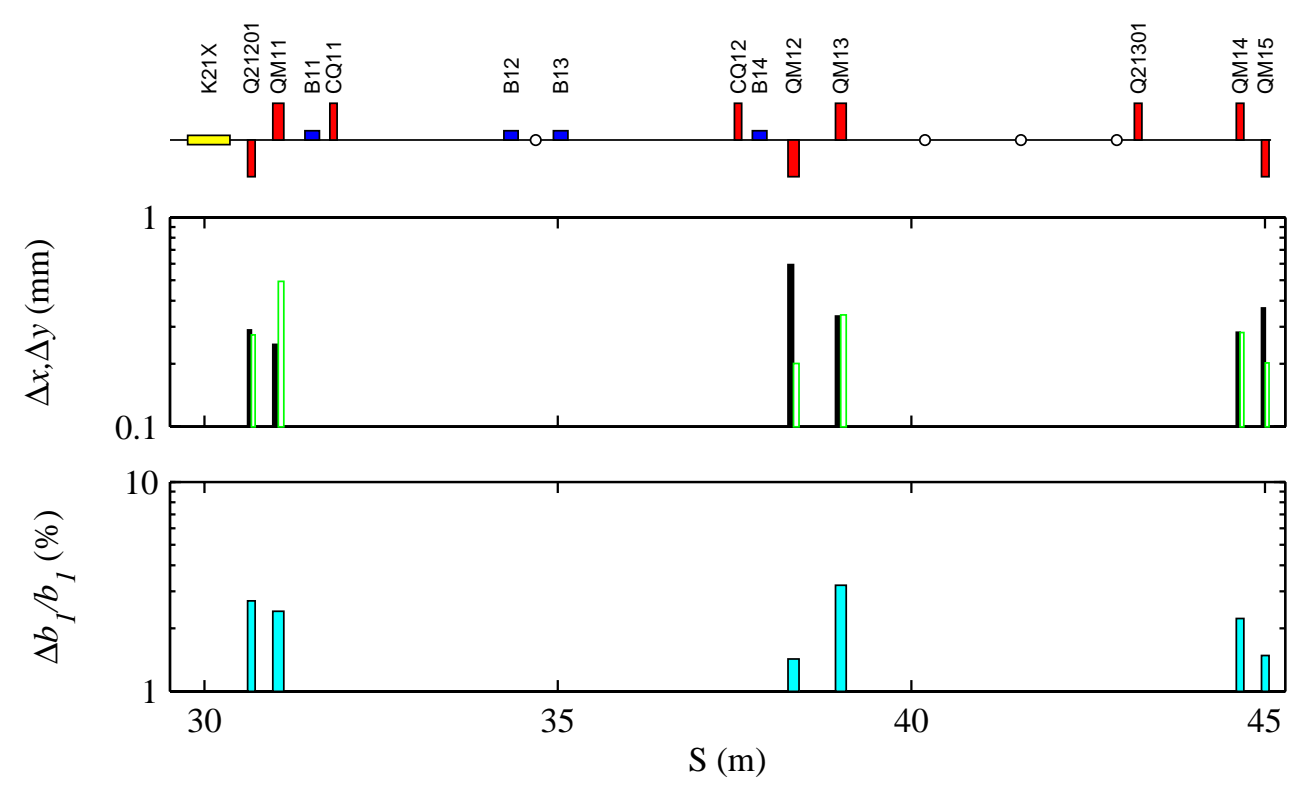

Figure 7.23 Alignment (top: black is $x$, and green/white is $y$ ) and gradient sensitivities (bottom) for BC1 quadrupoles. Each bar individually corresponds to a 'filamented' emittance dilution of $\Delta \varepsilon_{x} / \varepsilon_{x 0}+\Delta \varepsilon_{y} / \varepsilon_{y 0}=2 \%$.

An insertable tune-up dump will also be included after the $\mathrm{BC} 1$ emittance diagnostics (at $S \approx 44 \mathrm{~m}$ of Figure 7.19) in order to allow invasive tuning of the $\mathrm{BC} 1$ and upstream systems. The dump will need to handle a $1-\mathrm{nC}$ electron beam at $120 \mathrm{~Hz}$ and $250 \mathrm{MeV}$ or $30-\mathrm{W}$ of average power.

Finally, a profile monitor (phosphor screen) will be included in the center of the $\mathrm{BC} 1$ chicane where the dispersion is large $\left(\sigma_{x} \approx 4.1 \mathrm{~mm}\right)$. This device will allow measurement of the correlated energy spread and therefore will also reveal the temporal distribution of the bunch as it enters the $\mathrm{BC} 1$ chicane. A horizontal collimator just upstream of the profile monitor will be used to diagnose beam tails, and one BPM of $\leq 20-\mu \mathrm{m}$ resolution will be located in the center of the $\mathrm{BC} 1$ chicane. The BPM reading will provide a high-resolution relative energy measurement ( $\delta \approx 0.01 \%$ ), per beam pulse (see Section 7.8.4). The betatron component of the beam position will be small and also correctable by incorporating $\geq 2$ BPMs up or downstream of the chicane.

\subsubsection{Second Bunch Compressor}

Like the first compressor, the second compressor, $\mathrm{BC} 2$, is a four-dipole magnetic chicane. It is designed to compress a $\sim 200 \mu \mathrm{m}$ bunch to $\sim 20 \mu \mathrm{m}$. The high energy and short bunch demand a chicane design where both coherent and incoherent synchrotron radiation generated within the bends do not significantly dilute the horizontal or longitudinal emittance. In addition, the chicane length is minimized so that the number of removed linac accelerating sections is not too large. An optimized parameter set has been chosen with a $4.54-\mathrm{GeV}$ chicane motivated by synchrotron radiation effects and linac longitudinal beam dynamics simulations. This system will replace the eight existing 3-meter rf sections at the end of sector-24 (24-7a,b,c,d and 24-8a,b,c,d). A 
phosphor screen profile monitor, a BPM, and a horizontal beam collimator will be included at the chicane center.

In addition, a short one-period, superconducting wiggler will be located just upstream of the BC2 chicane. This wiggler is used to increase the intrinsic (uncorrelated) energy spread of the beam in order to damp CSR micro-bunching effects in the BC2 chicane [3]. The relative energy spread due to incoherent synchrotron radiation (ISR), $\sigma_{\delta_{I S R}}$ per bend magnet is given by

$$
\sigma_{\delta_{I S R}} \approx \frac{1}{L_{B}} \sqrt{\left(4.13 \times 10^{-11} \mathrm{~m}^{2} \cdot \mathrm{GeV}^{-5}\right) E^{5}\left|\theta_{B}^{3}\right|},
$$

where $L_{B}$ is the magnet length, $\theta_{B}$ is the bend angle, and $E$ is the beam energy $(4.54 \mathrm{GeV}$ in the wiggler). If we use two $7-\mathrm{cm}$ long coils sandwiching two $10-\mathrm{cm}$ long coils, with the field profile shown in Figure 7.24, then a 6-Tesla peak field increases the incoherent energy spread to $3 \times 10^{-5}$ (rather than $3 \times 10^{-6}$ without wiggler). This is enough to reduce the CSR-induced micro-bunching effect to tolerable levels (see below). The wiggler is then $\sim 60 \mathrm{~cm}$ in physical length and composed of superconducting magnets with a local liquid He cryogenics supply in or near sector24. This scenario has been used in the past to supply the superconducting spin-rotator solenoids presently installed in the SLAC linac at north damping ring entrance and exit.

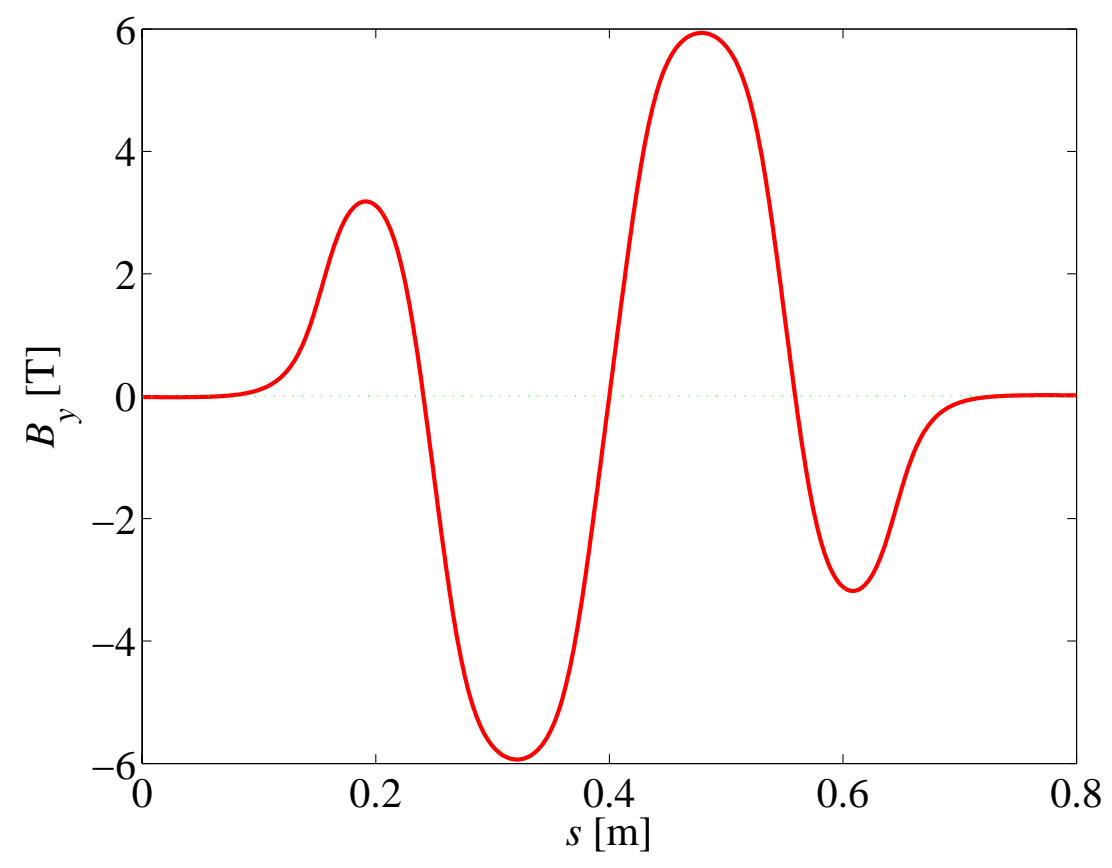

Figure 7.24 Magnetic field profile of one-period superconducting wiggler placed just upstream of BC2 chicane to increase incoherent energy spread to $3 \times 10^{-5} \mathrm{rms}$ at $4.54 \mathrm{GeV}$, which damps CSR micro-bunching.

The wiggler has almost no effect on bunch length, and CSR effects are small since the bunch length is $\sim 200 \mu \mathrm{m}$ long throughout the wiggler. The 'slice' horizontal emittance is, however, increased by the spontaneous radiation in the wiggler, where the dispersion is not zero. The beta 
function through this wiggler is held to $<75 \mathrm{~m}$, which generates a $4 \%$ 'slice' emittance growth. This can be further reduced if the beta function is made even smaller, and has not yet been fully optimized.

Figure 7.25 shows the final longitudinal phase space at the undulator entrance $(14.35 \mathrm{GeV})$ both with (top) and without (bottom) the wiggler switched on. Without the wiggler, the very small incoherent energy spread in $\mathrm{BC} 2\left(3 \times 10^{-6} \mathrm{rms}\right.$ at $\left.4.54 \mathrm{GeV}\right)$ and the effects of CSR cause a severe micro-bunching instability within $\mathrm{BC} 2$. Switching on the wiggler increases the incoherent energy spread in $\mathrm{BC} 2$ to $3 \times 10^{-5} \mathrm{rms}$, suppressing the instability. After acceleration to $14.3 \mathrm{GeV}$ and compression by a factor of $\sim 9$, this larger incoherent energy spread is only $8 \times 10^{-5}$ in the undulator, which is well below the safe level of $1 \times 10^{-4}$. The wiggler can also be switched off or adjusted to as much as $\sim 7.5 \mathrm{~T}$ maximum peak field. This effect is presently under intense study as it may effect many bunch compressor applications.

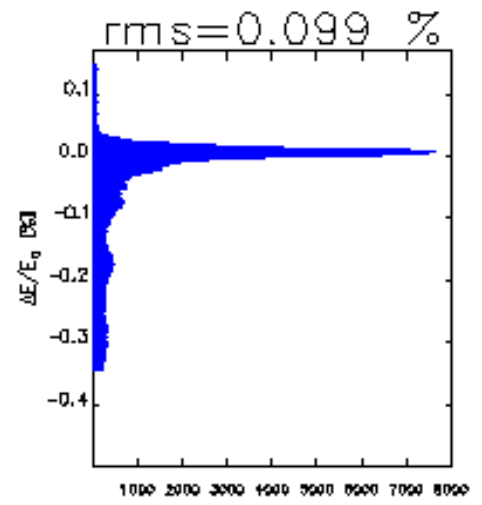

П

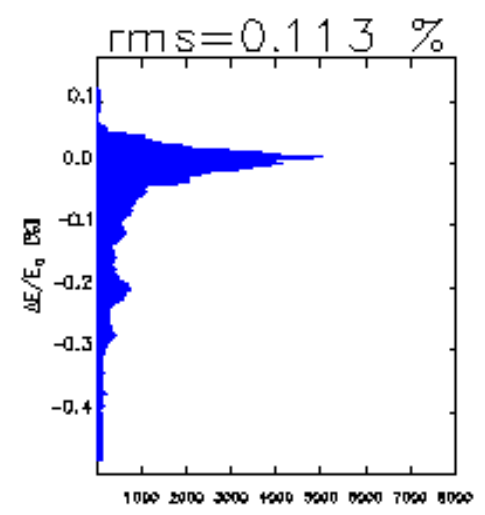

门
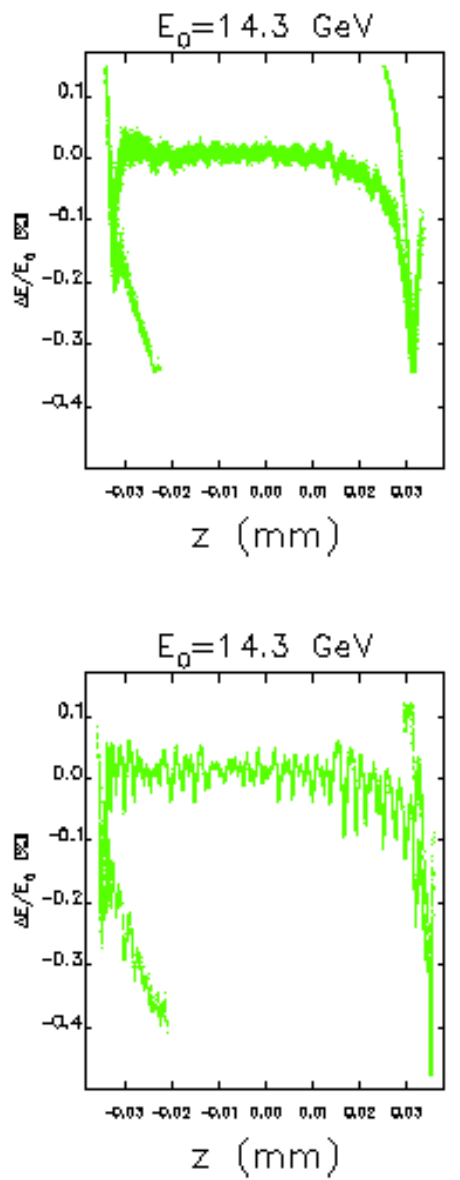
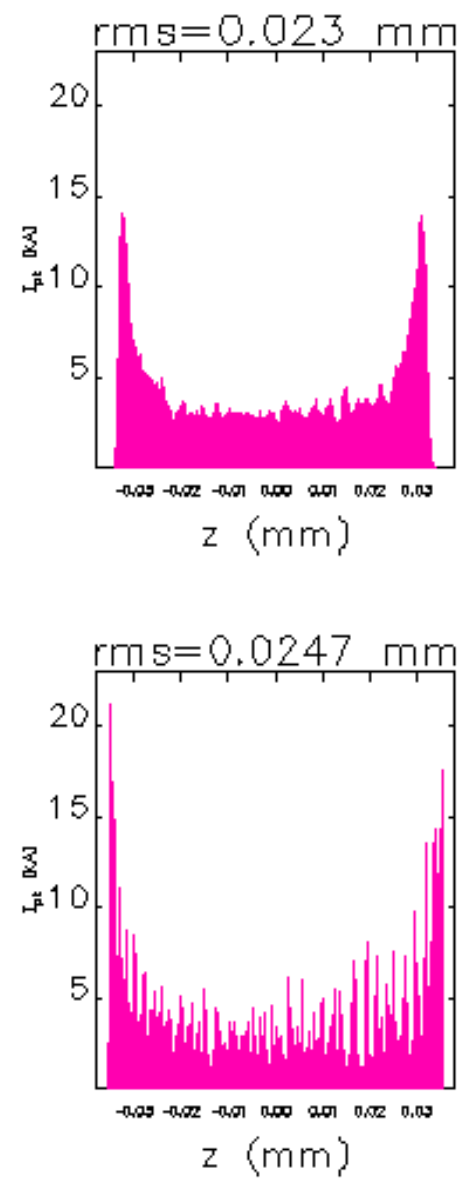

Figure 7.25 Final longitudinal phase space at undulator entrance (14.35 GeV) both with (top) and without (bottom) superconducting pre-BC2 wiggler switched on.

\subsubsection{Overview and Parameters}

Motivations and quantitative arguments for the choices of these parameters are described in the following sections. Results of longitudinal beam dynamics simulations have been used to set 
L C L S C O N C E P T U A L D E S I G N R E P O R T

the incoming BC2 bunch length at $195 \mu \mathrm{m}$ rms. The bunch length after BC2 is adjustable using the $R_{56}$ of $\mathrm{BC} 2$ and the rf phase of $\mathrm{L} 2$. The parameters of the $\mathrm{BC} 2$ chicane are listed in Table 7.14.

Table 7.14 Parameters of 2nd bunch compressor chicane, BC2.

\begin{tabular}{|c|c|c|c|}
\hline Parameter & Symbol & Unit & Value \\
\hline Beam energy & $E$ & $\mathrm{GeV}$ & 4.54 \\
\hline Initial rms bunch length & $\sigma_{z i}$ & $\mu \mathrm{m}$ & 195 \\
\hline Final rms bunch length & $\sigma_{z f}$ & $\mu \mathrm{m}$ & 22 \\
\hline RMS incoming relative energy spread (at $4.54 \mathrm{GeV}$ ) & $\sigma_{\delta}$ & $\%$ & 0.76 \\
\hline RMS uncorrelated relative energy spread (at $4.54 \mathrm{GeV}$, with wiggler) & $\sigma_{\delta_{u}}$ & $10^{-5}$ & 3 \\
\hline Net momentum compaction & $R_{56}$ & $\mathrm{~mm}$ & -22.5 \\
\hline Net second order momentum compaction & $T_{566}$ & $\mathrm{~mm}$ & +33.8 \\
\hline Total system length ( $1^{\text {st }}$ bend to last) & $L_{\text {total }}$ & $\mathrm{m}$ & 22.1 \\
\hline Floor length of each of four dipole magnets & $L_{B}$ & $\mathrm{~m}$ & 0.400 \\
\hline Floor length of drift between first two and last two dipoles & $\Delta L$ & $\mathrm{~m}$ & 10 \\
\hline Floor length of drift between center two dipoles & $\Delta L_{c}$ & $\mathrm{~m}$ & 0.50 \\
\hline Bend angle for each of four dipoles & $\left|\theta_{B}\right|$ & $\operatorname{deg}$ & 1.878 \\
\hline Magnetic field for each of four dipoles & $|B|$ & $\mathrm{kG}$ & 12.41 \\
\hline Maximum dispersion in chicane center $(\approx$ beamline excursion $)$ & $\left|\eta_{\max }\right|$ & $\mathrm{m}$ & 0.341 \\
\hline Slice emittance dilution due to ISR of chicane only (at $\gamma \varepsilon_{0}=1 \mu \mathrm{m}$ ) & $\Delta \varepsilon_{\mathrm{ISR}} / \varepsilon_{0}$ & $\%$ & 0.3 \\
\hline Projected emittance dilution due to CSR (includes all particles) & $\varepsilon_{x} / \varepsilon_{x 0}$ & - & 2.5 \\
\hline rms ISR relative energy spread of chicane only (at $4.54 \mathrm{GeV}$ ) & $\sigma_{\delta \mathrm{ISR}}$ & $10^{-6}$ & 8.4 \\
\hline rms CSR relative energy spread (at $4.54 \mathrm{GeV}$ ) & $\sigma_{\delta \mathrm{CSR}}$ & $\%$ & 0.053 \\
\hline CSR relative energy loss (at $4.54 \mathrm{GeV}$ ) & $\left\langle\delta_{\mathrm{CSR}}\right\rangle$ & $\%$ & 0.071 \\
\hline
\end{tabular}

A nominal rms final bunch length of $22 \mu \mathrm{m}$ is used throughout the following descriptions (unless otherwise noted). As in the case of $\mathrm{BC} 1$, the center two dipoles will be placed on remotely movable horizontal stages in order to: 1) allow non-LCLS linac operation, 2) to ease dipole field quality tolerances, and 3) to allow a high-resolution BPM to be placed at the center of the chicane. With the dipoles switched off, the BC2 (and the BC1) chicane can then be straightened out. The maximum horizontal beamline excursion at chicane center is equal to the maximum dispersion, $\left|\eta_{\max }\right|$, listed in the table. The excursion is toward the tunnel 'wall' (north) to keep the 'aisle' clear. Figure 7.26 shows the dispersion and beta functions through the $\mathrm{BC} 2$ chicane. Magnet locations are shown at the top of the figure. This plot, and other calculations, is made for a net $R_{56}$ of $-22.5 \mathrm{~mm}$. 


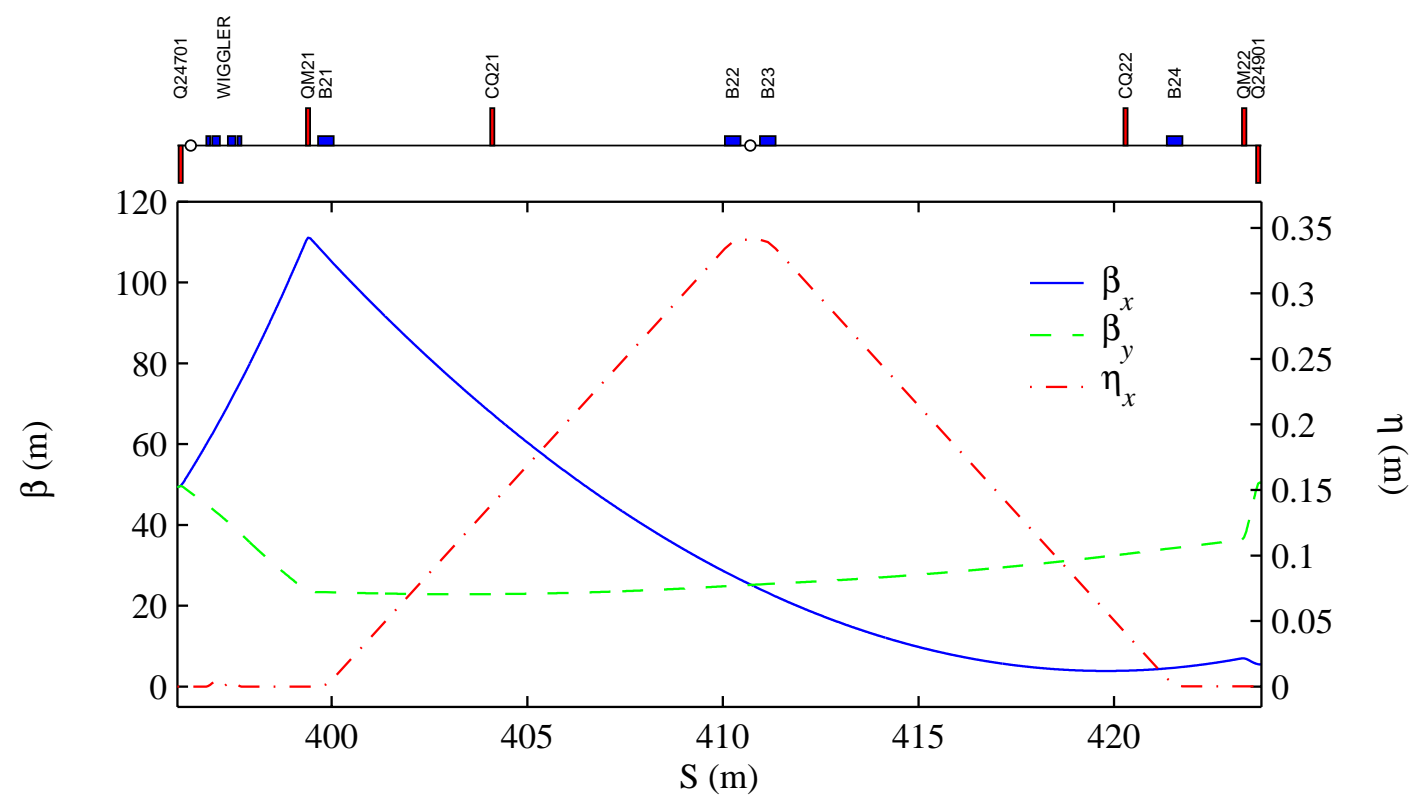

Figure 7.26 Dispersion and beta functions through $\mathrm{BC} 2$ chicane for $R_{56} \approx-22.5 \mathrm{~mm}$. The energy spread profile monitor is indicated by a small circle at the center of the chicane in schematic at top. The two quadrupoles inside the chicane are for dispersion correction and are nominally switched off. The superconducting wiggler is located at $S \approx 397 \mathrm{~m}$.

\subsubsection{Momentum Compaction}

The momentum compaction $\left(R_{56}\right)$ for a chicane is given by Eq. (7.12). The second order momentum compaction is $T_{566} \approx-3 R_{56} / 2$ (as in $\mathrm{BC} 1$ ). A beam delay as described in Eq. (7.13) is also necessary for the L3 $\mathrm{rf}$ phase. The L3 $\mathrm{rf}$ phase needs to be delayed, with respect to the chicane-off phase, by $d \varphi / d R_{56} \approx \pi / \lambda \approx 1.72^{\circ} / \mathrm{mm}$ (or $38.7^{\circ}$ with the nominal BC2 $R_{56}$ value of $-22.5 \mathrm{~mm})$.

\subsubsection{Incoherent Synchrotron Radiation (ISR)}

Horizontal emittance dilution will occur if significant energy spread is generated anywhere within the chicane (or the wiggler). Synchrotron radiation within the dipoles generates energy spread, which breaks the linear achromaticity of the chicane and therefore dilutes the horizontal emittance. Using a typical symmetric beta function through a single chicane with its maximum, $\beta_{\max }$, at start and end of the chicane and its minimum, $\beta_{\min }$, in the middle, and using symbols defined in Table 7.14, then

$$
\beta_{\max }=2 \beta_{\min }=4 L_{B}+2 \Delta L+\Delta L_{c} .
$$

The additive ISR emittance dilution [29] can then be approximated, for $\Delta \varepsilon / \varepsilon_{0}<<1$, by

$$
\Delta \gamma \varepsilon_{\mathrm{ISR}} \approx\left(8 \times 10^{-8} \mathrm{~m}^{2} \cdot \mathrm{GeV}^{-6}\right) \cdot E^{6}\left(\left|\theta_{B 1}\right|^{5}+\left|\theta_{B 2}\right|^{5}\right) \frac{4 \Delta L+6 L_{B}+\Delta L_{c}}{2 L_{B}^{2}} .
$$


The parameters of Table 7.14 are chosen such that the relative ISR emittance growth is $0.3 \%$ ('slice' emittance growth). This incoherent energy spread is generated through a random process and therefore cannot be corrected. Unlike most other dilution effects in the LCLS accelerator, here the 'slice' emittance is increased, and so the growth is held to a very small level. The energy in $\mathrm{BC} 1$ is too low for this effect to be important.

\subsubsection{Coherent Synchrotron Radiation (CSR)}

\section{Introduction}

The effects of CSR have been described in Section 7.4.1 pertaining to the $\mathrm{BC} 1$ design. The $\mathrm{BC} 2$ compression-stage is also a 4-dipole chicane and the bunch is linearly compressed from $195 \mu \mathrm{m}$ to $22 \mu \mathrm{m}$. Calculations of the CSR-induced emittance dilution have been made using the DESY TraFiC4 code [22] and Elegant [10] (see below, "CSR Calculations with a Transient Model"). The slice emittance is, however, almost unchanged.

Shielding is, however, not practical for $\mathrm{BC} 2$ since a full vacuum chamber height of $<3 \mathrm{~mm}$ would be required to significantly shield the shortest bunch. This presents an aperture restriction and may also generate geometric and/or resistive wakefields comparable to the CSR effects being shielded.

The $R_{56}$ of the $\mathrm{BC} 2$ chicane is also intended to be adjustable to allow for correction of the longitudinal phase space of the linac output beam and to provide control of the final bunch length. An $R_{56}$ tuning range of 0 to $-50 \mathrm{~mm}$ will allow a wide flexibility in the LCLS accelerator to provide for very different machine configurations at variable charge, etc.

\section{CSR Calculations with a Transient Model}

More complete calculations of the CSR effects on the bunch through BC2 have been made using TraFiC4 and also using Elegant. The details of the Elegant calculation are described in Section 7.4.1. Figure 7.27 shows the additional rms energy spread induced by CSR (top) and the normalized horizontal emittance (bottom) along the DL2 bend system ( $1^{\text {st }}$ bend to 5 meters after $4^{\text {th }}$ bend). The total accumulated CSR-wakefield energy gradient along the bunch generated within the BC2 chicane is plotted in Figure 7.28.

More detail is shown in Figure 7.29 which plots the temporal distribution of the bunch as it compresses and the evolving CSR-wakefield, from Eq. (7.19), within the third bend of the chicane using the temporal distribution taken from tracking upstream of $\mathrm{BC} 2$. The variable color traces represent the two functions sampled in each of twenty points along the bend magnet.

Figure 7.30 shows the angular spread in the electron beam, $x^{\prime}$, without CSR (left) and with CSR (right), versus the axial bunch coordinate, $z$, plus their distributions at the end of the BC2chicane using $2 \times 10^{5}$ tracked particles. The temporal distribution used here is the 'real' expected temporal distribution with non-linear correlations shown in the last row of Figure 7.6, which is based on $6 \mathrm{D}$ tracking through the entire LCLS up to the entrance of $\mathrm{BC} 2$ including the SCwiggler. The initial bunch length is $195 \mu \mathrm{m} \mathrm{rms}$, the final bunch length is $22 \mu \mathrm{m} \mathrm{rms}$, the charge 
is $1 \mathrm{nC}$, and the horizontal beta and alpha functions at first bend entrance are $\beta_{x} \approx 105 \mathrm{~m}$ and $\alpha_{x} \approx 5.0$ (see Figure 7.26).
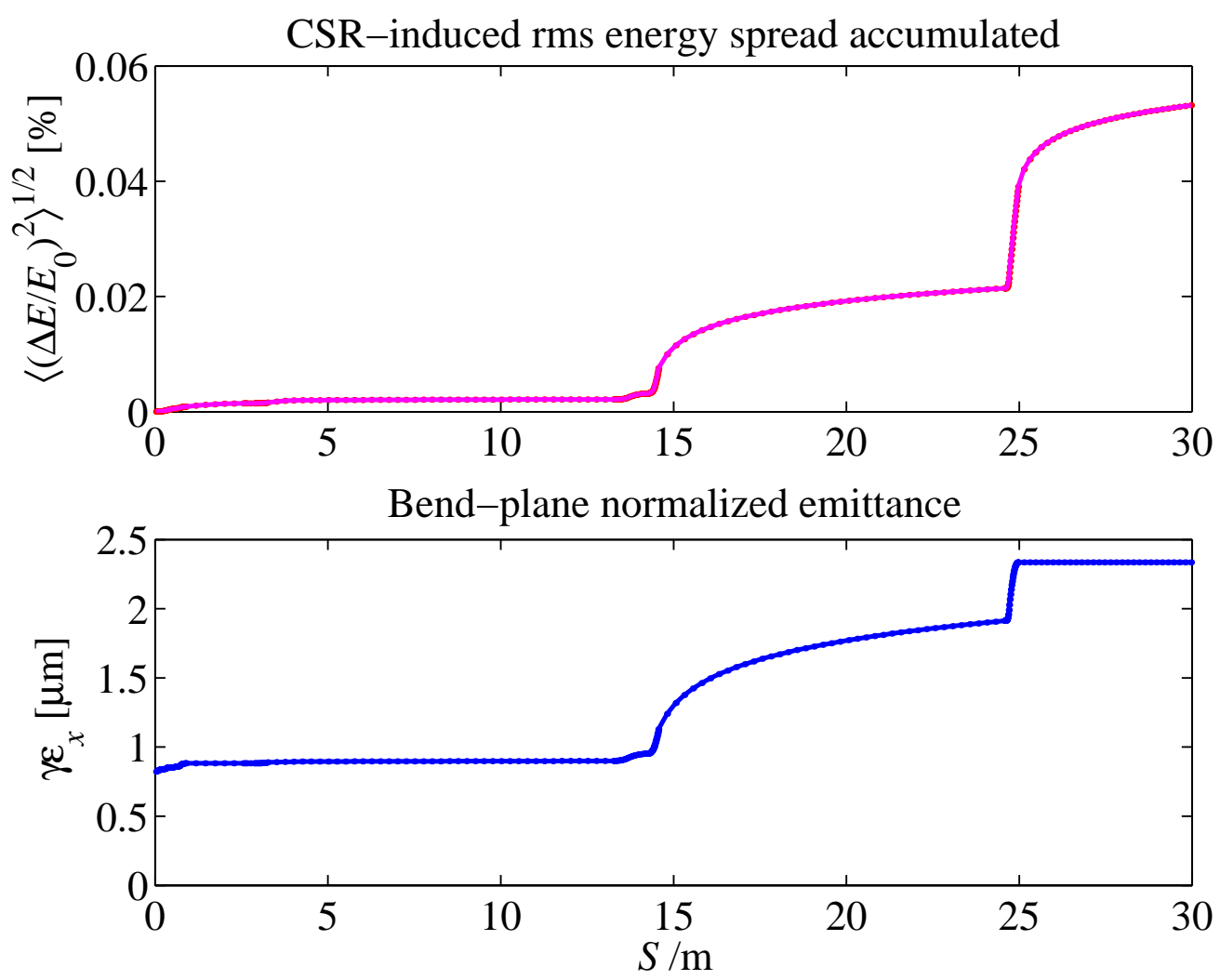

Figure 7.27 Additional rms energy spread induced by CSR (top) and the normalized horizontal emittance (bottom) along the BC2 chicane $\left(1^{\text {st }}\right.$ bend to 5 meters after $4^{\text {th }}$ bend).

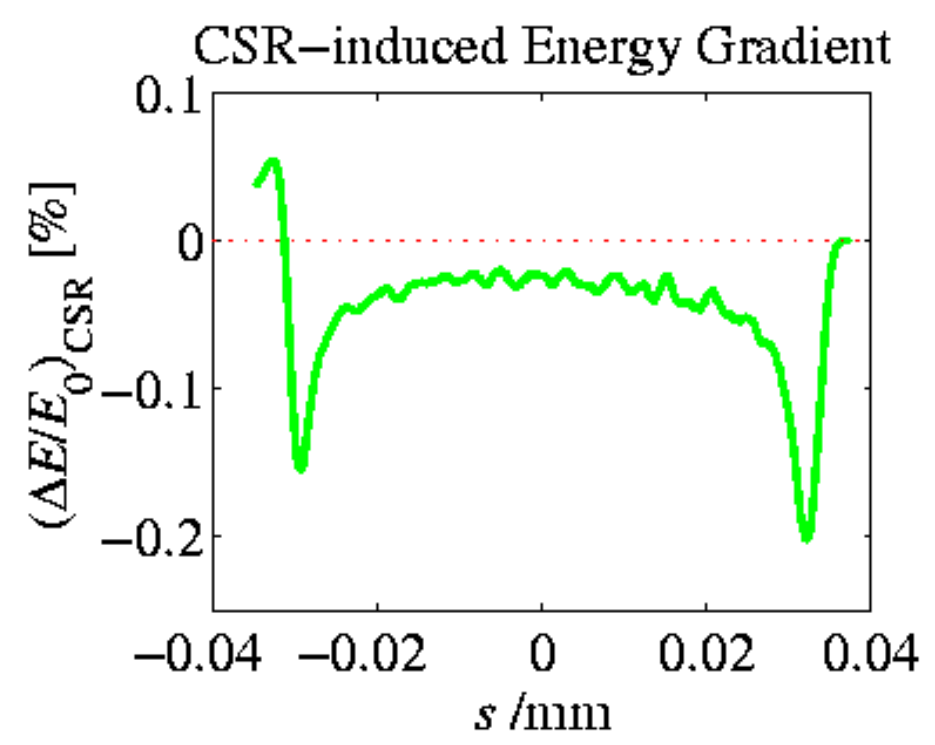

Figure 7.28 CSR-wakefield energy gradient along the bunch generated within the BC2 chicane. 


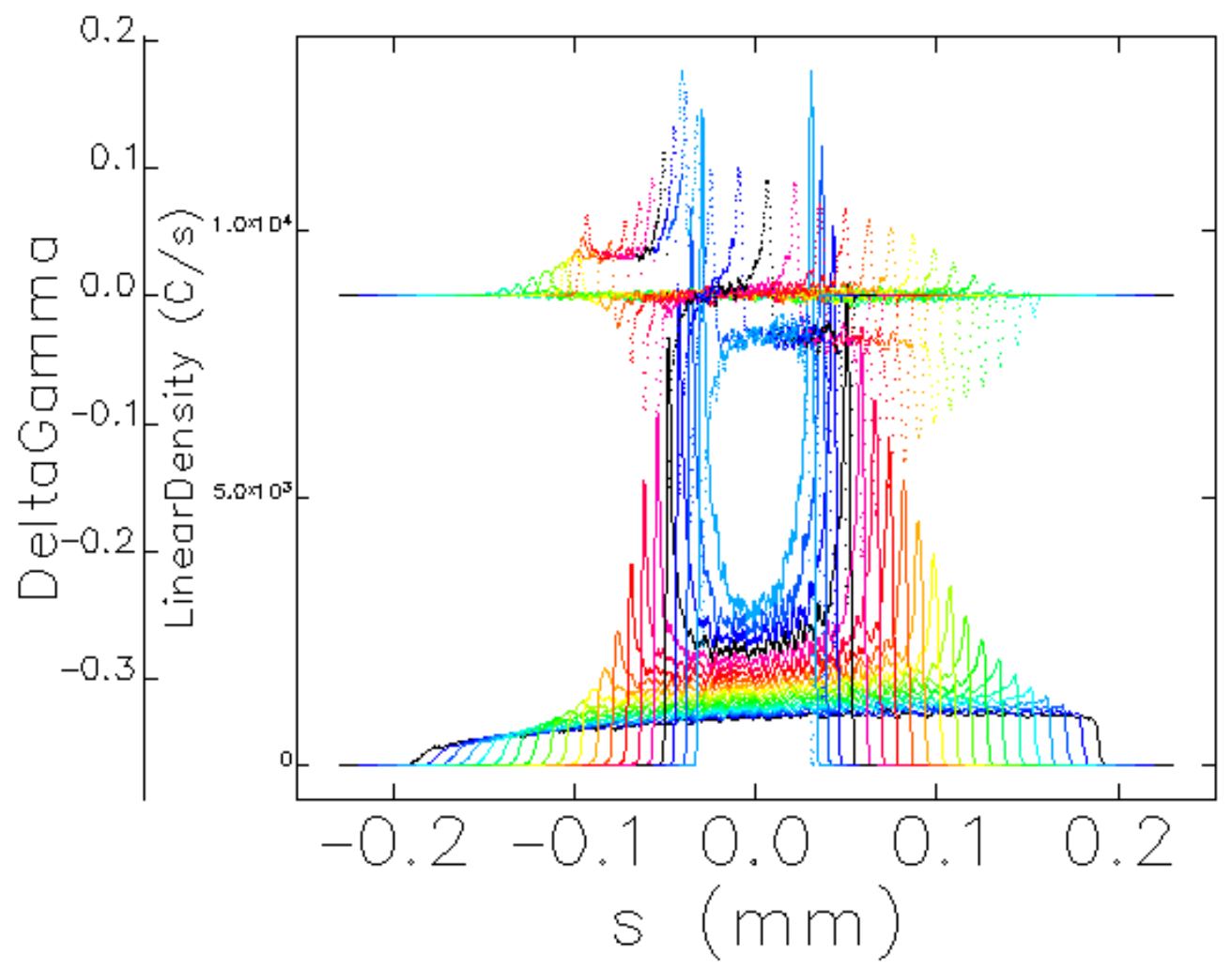

Figure 7.29 Temporal distribution of bunch (solid: "LinearDensity") and CSR-wakefield (dots: "DeltaGamma") within third bend of chicane in BC2. The variable color traces represent the two functions sampled in each of ten points along the bend magnet.
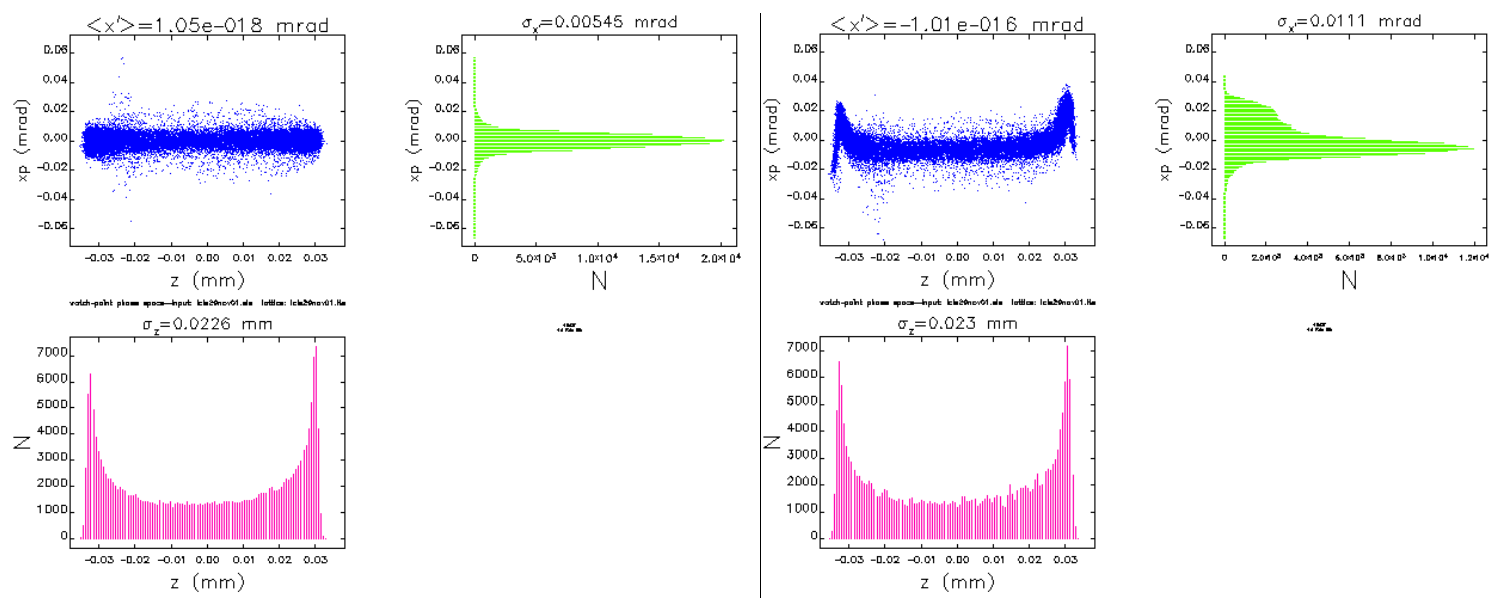

$\therefore$

Figure 7.30 Horizontal angle, $x^{\prime}$, without CSR (left) and with CSR (right), versus $z$, for LCLS bunch profile (i.e., tracked through upstream systems) after BC2. The projected emittance growth is 2.5-times larger (mostly is dominated by effects at bunch head and tail). The slice emittance is almost unchanged.

The final projected emittance is increased by a factor of 2.5 , but the slice emittance is nearly unchanged (except for the 7\% SC-wiggler effect). The large CSR increase is based on a 1D CSR 
model, which has been seen to somewhat overestimate the effect as compared to the 3D model. In addition, the projected emittance growth is due to slice transverse offsets, which dominantly occur at the extreme head and tail of the bunch due to the very high current spikes at these locations. The projected emittance growth calculated by integrating over only the central core of the beam with $75 \%$ of the particles is actually only $60 \%$, and this is also only the projected emittance, not the slice.

The total energy loss due to CSR is $-3.2 \mathrm{MeV}$ (or $-0.071 \%$ ). The change in central trajectory produced by the energy loss in the bends has been corrected in the tracking by steering so that the electron beam does not pass off-center through quadrupoles. The mismatch effect on the horizontal beta and alpha functions at the end of the chicane is significant ([i.e., $\zeta \approx 1.23$; see Eq. (7.26)]), but this is not a mismatch of the individual slices. It is a mismatch of the projected phase space, which should have a limited effect on the SASE FEL gain.

Finally, it is also possible to generate 'slice' emittance growth with the effects of CSR. The slice growth is generated when the longitudinal CSR wakefield has a significant transverse gradient over the width of the bunch [30]. These effects have been studied using TraFiC4 with a previous, stronger design for $\mathrm{BC} 2$, with $R_{56} \approx-30 \mathrm{~mm}$. The slice emittance growth seen in these calculations was too small to resolve numerically (i.e., $<1 \%$ ).

\section{Transverse CSR Forces}

A transverse force or "centripetal force" is described in [31] which originates from radiation of trailing particles and depends on the local charge density along the bunch. The maximum force takes place at the center of the bunch and its effect on transverse emittance is estimated in the reference. This estimate predicts an emittance growth of $<<1 \%$ for the worst case (last dipole of chicane-2 where bunch is shortest). In addition, calculations with a full-field model, which include the transverse forces, predict tolerable emittance growth (see below, "CSR Calculations with a Transient Model").

\subsubsection{Resistive Wall Longitudinal Wakefields in the Bends}

Longitudinal resistive wall wakefields in the $\mathrm{BC} 2$ vacuum chambers also induce energy spread, which may dilute the emittance. The rms relative energy spread generated in a cylindrical vacuum chamber of length $L$, radius $a$, and conductivity $\sigma$ is

$$
\sigma_{\delta_{\mathrm{RW}}} \approx 0.22 \frac{e^{2} c N L_{B}}{\pi^{2} a E \sigma_{z}^{3 / 2}} \sqrt{\frac{Z_{0}}{\sigma}},
$$

where $Z_{0}(\approx 377 \Omega)$ is the free space impedance. Note, Eq. (7.23) is for a long, gaussian bunch (see Section 7.9.5). For parameters of Table 7.14 using $1 \mathrm{nC}$, aluminum chambers $\left(\sigma \approx 3.6 \times 10^{7} \Omega^{-1} \mathrm{~m}^{-1}\right.$ ), and $a=15 \mathrm{~mm}$ through the final bend, where the bunch length is $22 \mu \mathrm{m}$, the rms energy spread generated by resistive wake is $\sim 1 \times 10^{-6}$, which is more than an order of magnitude smaller than the CSR energy spread generated in this final bend. Stainless steel, on the other hand, will generate five times this value and should probably be avoided. The other bends 
generate much less energy spread since the bunch is longer there. (The resistive-wall effects in $\mathrm{BC} 1$ are an order of magnitude smaller.)

\subsubsection{Beam Size, Aperture and Field Quality}

From the parameters in Table 7.14 $\left(\left|\eta_{\max }\right|\right.$ and $\left.\sigma_{\delta}\right)$, the horizontal $\mathrm{rms}$ beam size in the center of the $\mathrm{BC} 2$ chicane is $2.6 \mathrm{~mm}$. This sets some tolerances on the field quality of the center two dipoles (per chicane) since field harmonics may generate anomalous dispersion, which can dilute the transverse emittance. Without dipole magnet movers, the horizontal displacement of the beam within the center dipoles is dependent on the $R_{56}$ value chosen. For an $R_{56}$ tuning range of 0 to $-50 \mathrm{~mm}$, the horizontal dipole aperture required for the center two bends is $52 \mathrm{~cm}$. This is a large aperture dipole with tight field quality tolerances over most of the aperture. To relax this tolerance, and to allow the placement of a high resolution BPM in a small aperture in the center of the chicane, the center two dipoles are mounted on remotely movable stages to physically move the magnets as the bend angles are varied (precise synchronous control is not required). This locks the horizontal beam position with respect to the dipole aperture so that good field quality is only needed over an aperture of $3 \mathrm{~cm}\left(12 \sigma_{x}\right)$, rather than $52 \mathrm{~cm}$. It also allows the chicane to be straightened out for non-LCLS operation (dipoles off). This same retractable chicane design has been built and tested at the LEUTL facility at Argonne National Laboratory [32].

In addition, small correction quadrupoles are included in the chicane (CQ21 and CQ22 in Figure 7.26) to provide linear horizontal dispersion correction to compensate for errors. Field quality tolerances for the four dipoles are listed in Table 7.15. The tolerances on the two center pairs of dipoles are tight but achievable, especially in consideration of the empirical corrections built into the system.

Table 7.15 Dipole magnet tolerances for BC2 chicane (without empirical correction). Field harmonics are evaluated on a $20-\mathrm{mm}$ radius and each entry individually corresponds to a $2 \%$ emittance dilution.

\begin{tabular}{lccccc}
\hline Magnet & Quantity & Roll Angle $[\mathrm{mrad}]$ & $\left|\boldsymbol{b}_{\mathbf{1}} / \boldsymbol{b}_{\mathbf{0}}\right|[\%]$ & $\left|\boldsymbol{b}_{\mathbf{2}} / \boldsymbol{b}_{\mathbf{0}}\right|[\%]$ & $\left|\boldsymbol{b}_{\mathbf{4}} / \boldsymbol{b}_{\mathbf{0}}\right|[\%]$ \\
\hline B21 \& B24 & 1 each & 1.8 & 0.11 & 14 & 100 \\
\hline B22 \& B23 & 1 each & 1.7 & 0.01 & 0.05 & 0.5 \\
\hline
\end{tabular}

Quadrupole field components $\left(b_{1} / b_{0}\right)$ larger than these tolerances are correctable, while sextupole $\left(b_{2} / b_{0}\right)$ and decapole $\left(b_{4} / b_{0}\right)$ components, without specialized correction magnets, are not. Magnet roll errors generate anomalous vertical dispersion, which may be corrected with vertical steering or the later addition of small correction skew quadrupoles if necessary. The alignment and field strength sensitivities for quadrupole magnets in the BC2 area are shown in Figure 7.31. Each sensitivity shown corresponds individually to a filamented $x$ and $y$ emittance dilution of $\Delta \varepsilon_{x} / \varepsilon_{x 0}+\Delta \varepsilon_{y} / \varepsilon_{y 0}=2 \%$.

A pair of adjustable collimator jaws will be included in the center of the chicane, just upstream of the profile monitor, which can be used to cut the horizontal (i.e., energy) beam tails. 
The collimator will be nominally open with a full horizontal gap of $\sim 6 \mathrm{~cm}$, but each jaw will be independently controlled in order to select any energy band. The collimator will be very useful in diagnosing tails in the electron beam. It is not envisioned that any significant collimation will be made during normal LCLS operations. This might otherwise introduce an intolerably large pulseto-pulse charge jitter or wakefields within $\mathrm{BC} 2$.
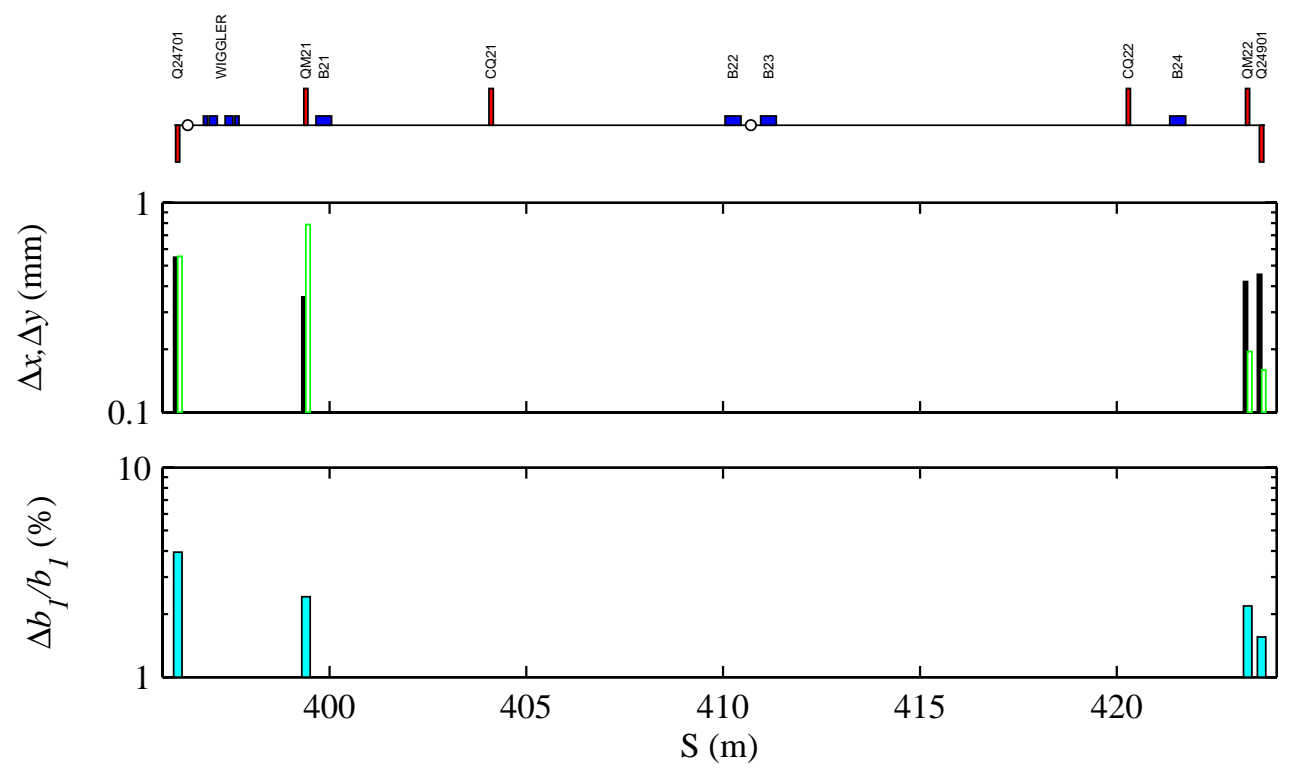

Figure 7.31 Alignment (top: black is $x$, and green/white is $y$ ) and gradient sensitivities (bottom) for $\mathrm{BC} 2$ quadrupoles. Each bar individually corresponds to a 'filamented' emittance dilution of $\Delta \varepsilon_{x} / \varepsilon_{x 0}+\Delta \varepsilon_{y} / \varepsilon_{y 0}=2 \%$

\subsubsection{Tuning and Correction}

Dispersion errors (typically in the horizontal plane) may be generated by small quadrupole field components in the center two dipoles of the chicane (see Table 7.15). A pair of small correction quadrupoles are included in the chicane with the first quadrupole (CQ21) placed near the end of $\mathrm{B} 21$ at $\eta_{x} \approx 139 \mathrm{~mm}, \beta_{x} \approx 65 \mathrm{~m}$, and the second (CQ22) just upstream of $\mathrm{B} 24$ ( $\eta_{x} \approx 41 \mathrm{~mm}, \beta_{x} \approx 4 \mathrm{~m}$ ). Two orthogonal linear combinations of these quadrupoles can then be used to correct up to $\sim 250 \%$ horizontal emittance dilution due to dispersion errors. Since the dispersive beam size at these two quadrupoles is $\eta_{x} \sigma_{\delta} \approx 1 \mathrm{~mm}$ and $310 \mu \mathrm{m}$, respectively and the betatron beam size is much smaller at $\left(\beta_{x, y} \varepsilon_{x, y}\right)^{1 / 2}<86 \mu \mathrm{m}$, these quadrupoles have little effect on the final beta functions. The specifications for these correction quadrupoles are given in Table 7.16 and their locations are shown in Figure 7.26.

The four main dipoles of the chicane will be powered in series with one power supply so that field regulation tolerances are met (see Table 7.4). The rms regulation tolerance for this main supply is $0.05 \%$. Trim coils will be included in each BC2 main dipole for compensation of magnet-to-magnet construction errors in the dipole field strengths. This will also allow some horizontal steering. In addition, vertical dipole correctors will be included nearby to facilitate vertical steering. 
Table 7.16 Dispersion correction quadrupoles for BC2 chicane for horizontal emittance correction of up to $250 \%$ (with $\Delta \varepsilon / \varepsilon \approx 1 \%$ step size control).

\begin{tabular}{ccccc}
\hline Maximum Pole-Tip Field $[\mathrm{kG}]$ & Quantity & Step Size $[\mathrm{kG}]$ & Pole Radius $[\mathrm{mm}]$ & Length $[\mathrm{m}]$ \\
\hline 2.2 & 2 & 0.14 & 50 & 0.05 \\
\hline
\end{tabular}

An insertable tune-up dump will also be included after BC2 in order to allow invasive tuning of the $\mathrm{BC} 2$ and upstream systems. The dump will need to handle a 1-nC beam at $120 \mathrm{~Hz}$ and $4.54 \mathrm{GeV}$, or $550 \mathrm{~W}$ of average power.

Finally, a phosphor screen profile monitor is included in the center of the BC2 chicane where the dispersion is large $\left(\sigma_{x} \approx 2.6 \mathrm{~mm}\right)$. This device allows measurement of the correlated energy spread and therefore also reveals the temporal distribution of the bunch as it enters the chicane. A horizontal collimator just upstream of the profile monitor will be used to diagnose beam tails, and one BPM of $\leq 40-\mu \mathrm{m}$ resolution will be located in the chicane center. The BPM reading provides a high-resolution relative energy measurement $\left(\delta \approx 1.2 \times 10^{-4}\right)$ per beam pulse (see Section 7.8.4).

\subsection{Beam Transport Lines}

This section discusses the two beam transport lines. The first is a low-energy bend system (DL1) used to transport the electrons from the off-axis injector into the main linac. The second is the high-energy dog-leg (DL2) used for L3-to-undulator transport, as well as energy and energy spread analysis. The DL2 beamline horizontally displaces the undulator axis from that of the main linac in order to protect the undulator from potential beam halo and dark current. In addition, a short vertical bending system (VB) removes the slight $\left(\sim 0.3^{\circ}\right)$ downward slope of the accelerator at the entrance to the undulator. This leveling-bend allows the experimental areas to be located closer to ground level.

\subsubsection{Low-Energy Dog-Leg}

The function of the low-energy 'dog-leg' (DL1) is to transport 150-MeV electrons from the new injector linac (L0) into the existing SLAC linac. While it is possible to design the dog-leg as a first bunch compression stage, this necessitates a large incoming correlated energy spread of 1 $2 \%$. In this case, the chromaticity of the quadrupole magnet within the dog-leg, required for a linear achromat, will generate large second order dispersion which needs sextupole compensation. Due to this, and also the need for easy $R_{56}$ tuning (not natural in a dog-leg), DL1 is designed as a simple transport line. Its design requirements are:

- Provide a horizontal beamline deflection of $35^{\circ}$ over a short distance,

- Should not alter the bunch length (i.e., should be nearly isochronous),

- Should introduce no significant transverse emittance dilution,

- Should provide a dispersive section for energy and energy spread measurement. 
A simple system that satisfies these conditions is composed of two dipole magnets of equal strength with a field lens located between them to produce a linear achromat. The dipoles are rectangular bends. A profile monitor based on optical transition radiation (OTR) [33] and a BPM at the high dispersion point will provide energy and energy spread measurements.

The momentum compaction, $R_{56}$, of such a system for ultra-relativistic electrons and small angles is

$$
R_{56} \approx \frac{1}{3} \theta_{B}^{2} L_{B}
$$

where $\theta_{B}$ and $L_{B}$ are the bend angle and length of each dipole, respectively. A 1.3-meter long beamline with two $17.5^{\circ}$ bends provides the required deflection and the $20-\mathrm{cm}$ long dipoles produce an $R_{56}$ of $+6.3 \mathrm{~mm}$ (opposite sign of a chicane). Therefore, an extreme electron which is off energy by $1 \%$ will move axially by only $60 \mu \mathrm{m}$, which is small compared to the $1-\mathrm{mm}$ rms bunch length. The effect of the second order momentum compaction, $T_{566}$, is even less. Note, the nominal incoming relative rms energy spread from L0 is actually $0.1 \% \mathrm{rms}$. The system is therefore, for all practical purposes, isochronous. Nevertheless, the non-zero $R_{56}$ value and the second order term of $T_{566} \approx 0.14 \mathrm{~m}$ has been taken into account throughout the design and stability optimization, and in the 2D and 6D particle tracking.

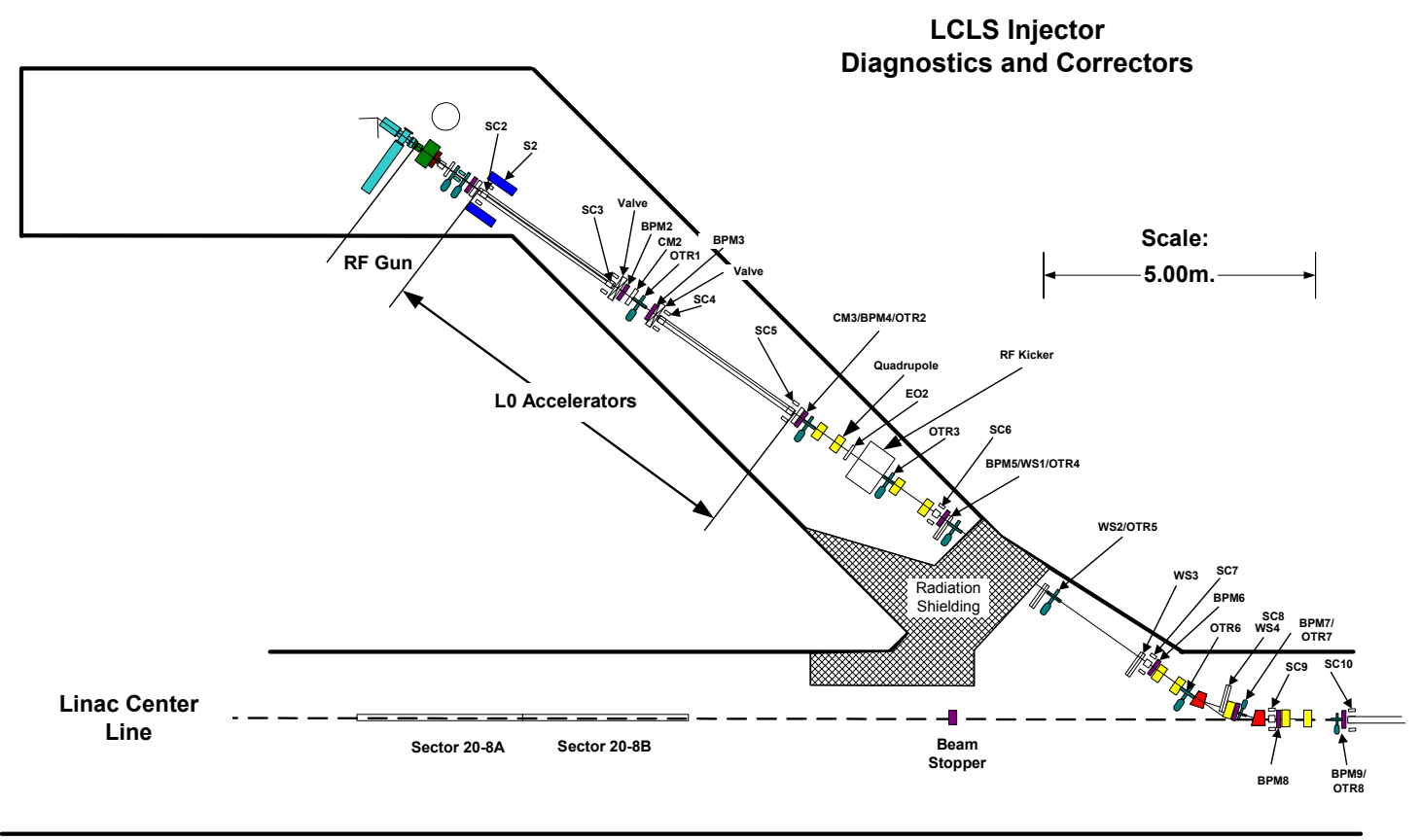

Figure 7.32 LCLS Injector tunnel layout. The L0-linac is composed of the two off-axis accelerator sections. The L1-linac starts with the $21-1 \mathrm{~B}$ section at right.

\subsubsection{Layout}

The LCLS injector will be housed in an existing off-axis injector tunnel provided by the original SLAC site design. This tunnel is located at the two-thirds point of the SLAC main linac 
at sector $21-1$ and is oriented at $45^{\circ}$ horizontally with respect to the axis of the main linac. The layout of the injector tunnel, with the proposed LCLS beamline installed, is shown in Figure 7.32. The gun, injector linac (L0, which is two 3-meter S-band rf sections), emittance diagnostic section (ED0), and $35^{\circ}$ bending system are shown, along with variable matching quadrupoles at the L1-linac entrance. Also shown in the figure are the shielding walls required to allow personnel access into the injector tunnel while the main linac is operating (but not the reverse situation).

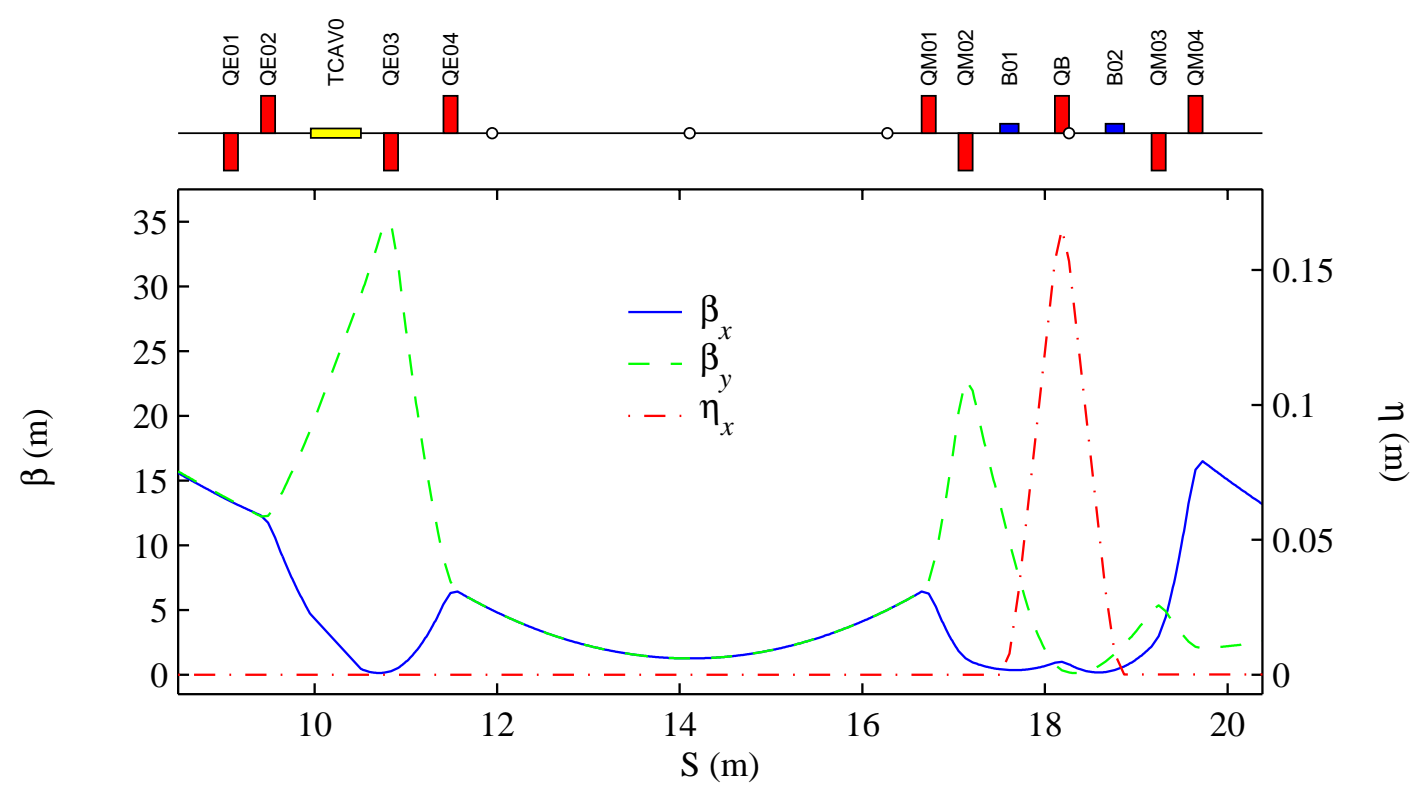

Figure 7.33 Dispersion and beta functions along 150-MeV low energy dog-leg (DL1). Profile monitors are indicated by small circles in top schematic. L0-linac ends at left side $(S \approx 8.7 \mathrm{~m})$.

\subsubsection{Parameters}

The main parameters of DL1 are summarized in Table 7.17. The dispersion and beta functions along the $150-\mathrm{MeV}$ beamline are shown in Figure 7.33. Space charge effects on emittance, energy spread, and bunch length are negligible at $150 \mathrm{MeV}$ through the full length of transport from the end of L0 to the start of L1 at 21-1b (see Chapter 6).

The four quadrupole magnets (QE01-04; each powered by an independent power supply) just after the L0-linac sections are used to adjust the beta-match into the adjacent 4.7-m drift section, where three profile monitors (OTR and/or wire scanners) are located (see Figure 7.32 and Figure 7.33). This constitutes the emittance measurement section (ED0) at $150 \mathrm{MeV}$ immediately after the L0-linac (see also Section 7.8.1). The horizontal and vertical beta functions are forced into the same parabolic sweep through the 4.7-m drift shown in Figure 7.33. The profile monitors (PR) are used to measure the $x$ and $y$ emittances, beta and alpha functions. The PRs are equally separated by 


$$
\Delta s=\frac{\alpha_{0} \gamma \sigma_{\min }^{2}}{\varepsilon_{N}},
$$

where $\alpha_{0}$ is the incoming alpha function $\left(\alpha \equiv-\beta^{\prime} / 2\right), \gamma$ is the beam energy in units of electron rest mass, $\sigma_{\min }$ is the rms beam size at the second PR (at the beam waist where the size is smallest), and $\varepsilon_{N}$ is the normalized emittance.

Table 7.17 Parameters of DL1 (the low energy 'dog-leg' beamline).

\begin{tabular}{lccc}
\hline Parameter & Symbol & Unit & Value \\
\hline Beam energy & $E$ & $\mathrm{MeV}$ & 150 \\
\hline Total horizontal deflection (sum of 2 bends) & $\theta$ & $\mathrm{deg}$ & 35 \\
\hline RMS bunch length & $\sigma_{z}$ & $\mathrm{~mm}$ & 0.83 \\
\hline RMS energy spread throughout beamline (at $150 \mathrm{MeV})$ & $\sigma_{\delta}$ & $\%$ & 0.10 \\
\hline Momentum compaction & $R_{56}$ & $\mathrm{~mm}$ & 6.3 \\
\hline Second order momentum compaction & $T_{566}$ & $\mathrm{~mm}$ & 140 \\
\hline Length of each of two dipole magnets & $L_{B}$ & $\mathrm{~m}$ & 0.20 \\
\hline Bend angle of each dipole & $\mid \theta_{B}$ & $\mathrm{deg}$ & 17.5 \\
\hline Magnetic field of each dipole & $|B|$ & $\mathrm{kG}$ & 7.64 \\
\hline Maximum horizontal dispersion & $|\eta|_{\mathrm{max}}$ & $\mathrm{m}$ & 0.165 \\
\hline Projected emittance dilution due to CSR (at $\left.\gamma \varepsilon_{0}=1 \mu \mathrm{m}\right)$ & $\Delta \varepsilon_{\mathrm{CSR}} / \varepsilon_{0}$ & $\%$ & 1 \\
\hline RMS CSR-induced relative energy spread $(\mathrm{at} 150 \mathrm{MeV})$ & $\sigma_{\delta \mathrm{CSR}}$ & $\%$ & 0.008 \\
\hline CSR-induced relative energy loss (at $150 \mathrm{MeV})$ & $\delta_{\mathrm{CSR}}$ & $\%$ & -0.02 \\
\hline
\end{tabular}

With an incoming alpha function at the first PR of $\alpha_{0}=\sqrt{3}$, a constant $60^{\circ}$ phase advance is set between each PR. This is the ideal configuration so that the emittance measurement precision is the least sensitive to mismatched incoming beams. The beta function at the waist is chosen based on the desired minimum beam size on the center monitor, $\sigma_{\min }$. For a $65-\mu \mathrm{m}$ rms beam size at the waist and $\varepsilon_{N}=1 \mu \mathrm{m}$ at $150 \mathrm{MeV}$, the beta function at the waist is then $\beta_{0}=1.25 \mathrm{~m}$, and the incoming beta function, for a $60^{\circ}$ phase advance, is four times this value, or 5 meters. Each PR is then separated by precisely $\Delta s=2.165$ meters. The system provides a robust emittance measurement with optimum precision, minimum beta-mismatch sensitivity, and nominal rms beam sizes (with $\varepsilon_{N}=1 \mu \mathrm{m}$ ) on the PRs of $130 \mu \mathrm{m}, 65 \mu \mathrm{m}$, and $130 \mu \mathrm{m}$, in order. A similar system is used just downstream of the first bunch compressor, $\mathrm{BC} 1$.

\subsubsection{Coherent Synchrotron Radiation}

The effects of CSR (see Section 7.4.1) have been studied for the DL1 bends using both Elegant and TraFiC4. The bunch length is fairly long here $(0.83 \mathrm{~mm} \mathrm{rms})$, but the bends are strong $(R \approx 0.65 \mathrm{~m})$. It is possible to completely shield the CSR with a $8.5-\mathrm{mm}$ full height conducting vacuum chamber. Without the shielding, CSR calculations using a transient model with Elegant predict an emittance growth of 1\%, with a similar result from TraFiC4 [34]. Figure 
7.34 shows the temporal distribution of the bunch and the CSR-wakefield, from Eq. (7.19), within the first bend of DL1, using the temporal distribution taken from upstream tracking.

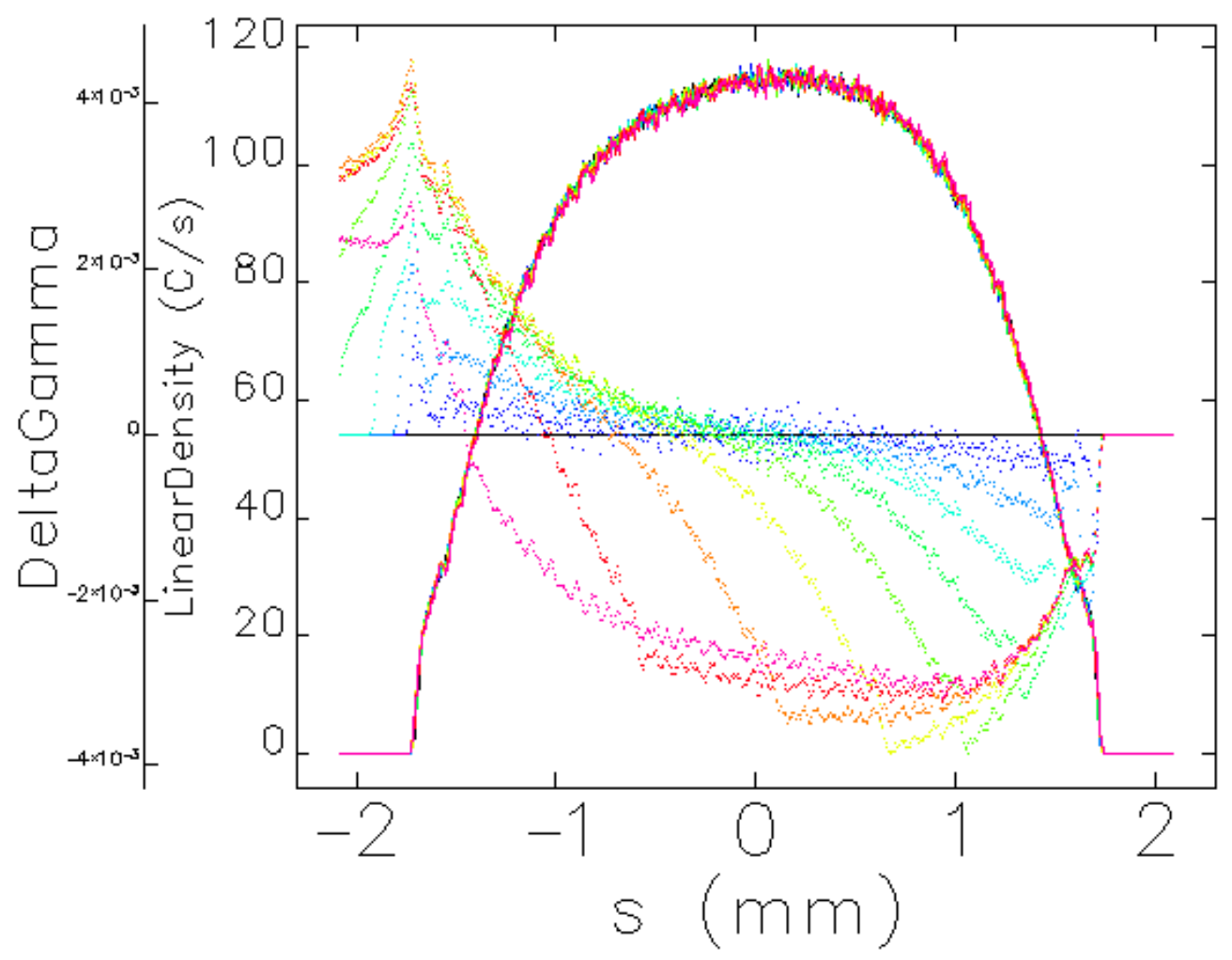

Figure 7.34 Constant temporal distribution of bunch (solid: "LinearDensity") and CSR-wakefield (dots: "DeltaGamma") within first bend of DL1. The variable color traces represent the two functions sampled in each of ten points along the bend magnet.

The variable color traces represent the two functions sampled in each of ten points along the bend magnet. The temporal distribution is nearly constant over the DL1 bend system due to the small energy spread and near-isochronicity of the beamline. The projected emittance growth due to CSR across DL1 bends is small at $\sim 1 \%$.

\subsubsection{Beam Size, Aperture, and Field Quality}

The horizontal beam sizes in DL1 reach peak values of $\sim 100 \mu \mathrm{m}$ and the sagitta in the bends is $7.6 \mathrm{~mm}$. A $2.5-\mathrm{cm}$ full aperture in the bends is, therefore, adequate. The large dispersion in the DL1 field lens quadrupole, QB, and the strong bends set the tolerances on field quality. Table 7.18 lists dipole field sensitivities in DL1.

Although the dipoles will be powered in series, their fields may differ slightly due to construction errors. These differences are correctable with trim coils or steering magnets. With the two bend magnets powered in series, the current regulation tolerance is not difficult to meet, at $0.04 \% \mathrm{rms}$. This tolerance is set by the path length error induced by a varying field and the need to keep this component of the final electron energy jitter (at $14.3 \mathrm{GeV}$ ) stable to $<<0.1 \%$ (see tolerance budget of Table 7.5). The horizontal trajectory oscillations induced by this level of 
regulation are insignificant $(<<1 \%$ of the beam size). Failure to meet the tolerances on the quadrupole content in the bends results in a beta function mismatch (since the dispersion is small in the bends). This mismatch can be compensated with the adjustable matching quadrupoles before and after DL1. The alignment and field strength sensitivities for quadrupole magnets in the DL1 area are shown in Figure 7.35. Each sensitivity shown corresponds individually to a filamented emittance dilution of $\Delta \varepsilon_{x} / \varepsilon_{x 0}+\Delta \varepsilon_{y} / \varepsilon_{y 0}=2 \%$.

Table 7.18 Dipole magnet tolerances for DL1. Field harmonics are evaluated on a 20-mm radius and each entry individually corresponds to a $2 \%$ emittance dilution.

\begin{tabular}{lccccc}
\hline Magnet & Quantity & Roll Angle [mrad] & $\left|\boldsymbol{b}_{\mathbf{1}} / \boldsymbol{b}_{\mathbf{0}}\right|[\%]$ & $\left|\boldsymbol{b}_{\mathbf{2}} / \boldsymbol{b}_{\mathbf{0}}\right|[\%]$ & $\left|\boldsymbol{b}_{\mathbf{4}} / \boldsymbol{b}_{\mathbf{0}}\right|[\%]$ \\
\hline B01 \& B02 & 1 each & 14 & 0.18 & 30 & 100 \\
\hline
\end{tabular}

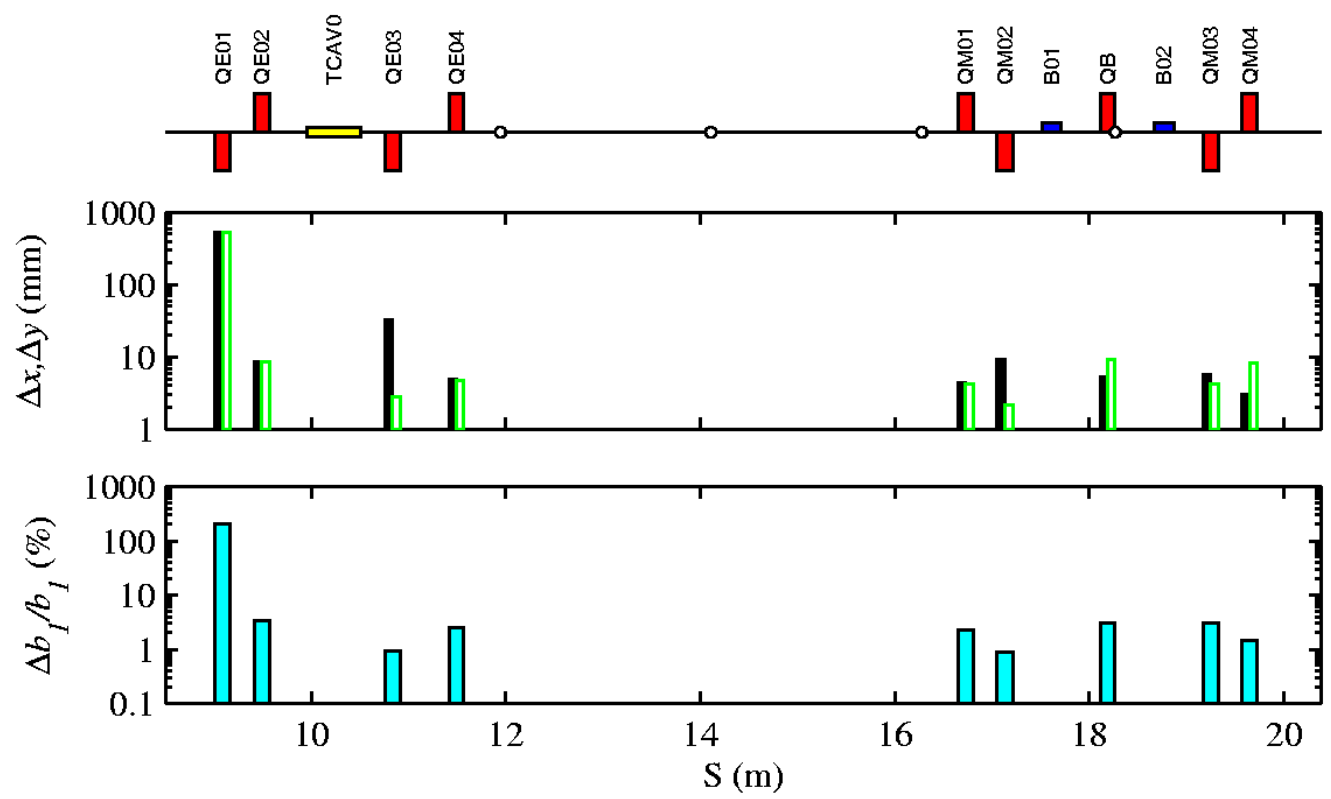

Figure 7.35 Alignment (top: black is $x$, and green/white is $y$ ) and strength sensitivities (bottom) for DL1 quadrupoles. Each bar individually corresponds to 'filamented' emittance dilution of $\Delta \varepsilon_{x} / \varepsilon_{x 0}+\Delta \varepsilon_{y} / \varepsilon_{y 0}=2 \%$.

\subsubsection{Tuning and Correction}

The DL1 system is quite insensitive to reasonable errors. The emittance and the beam matching can be measured in the diagnostic section following $\mathrm{BC} 1$ ( $\mathrm{BC} 1$ can be switched off if necessary). The field lens, "QB", is adjustable in strength and can be used to partially correct the dispersion if necessary. There are also four independently adjustable quadrupole magnets at the end of the L0-linac, and four more at the input to the L1-linac, any of which can be used to correct the matching in both planes. The beam energy will be held constant in DL1 by monitoring the BPM near the "QB" field lens and adjusting the L0 rf voltage with a feedback loop. The energy spread can be minimized by measuring the horizontal beam profile near "QB" and phasing the L0-linac (see fourth profile monitor in Figure 7.33). 
In addition, a 55-cm long S-band transverse RF deflecting structure will be located just after the L0-linac (see "RF Kicker" in Figure 7.32). This system will be used to 'streak' the bunch for bunch-length, slice emittance, and slice energy spread measurements using screens in the DL1 system. This diagnostic is described in more detail in Section 7.8.2.

An insertable tune-up dump will also be included after DL1 in order to allow invasive tuning of the DL1 and upstream systems. The dump will need to handle a 1-nC beam at $120 \mathrm{~Hz}$ and $150 \mathrm{MeV}$ or $18-\mathrm{W}$ of average power.

\subsubsection{High-Energy Dog-Leg}

The requirements for beam transport from the L3-linac to the LCLS undulator are fairly simple. The transport line must:

- Include bends to introduce precise energy and energy spread measurement capability without generating significant CSR or other emittance dilution effects,

- Include precise transverse emittance and matching diagnostics for final verification/tuning prior to undulator,

- Provide adjustable undulator-input beta-matching for the various beam energies (i.e., various radiation wavelengths) desired,

- Not alter the bunch length (must be nearly isochronous),

- Make use of the existing FFTB tunnel and its components wherever possible, as long as the performance of the transport line is not compromised,

- Adjust the vertical beamline angle to remove the $0.3^{\circ}$ downward linac angle so that experimental areas do not need to be located below ground level.

Energy and energy spread diagnostics are built into a four dipole horizontal inflector beamline (DL2) where the first bend is located just inside the beginning of the undulator hall, which previously housed the Final Focus Test Beam (FFTB). Primarily to meet the small energy spread measurement capability, a doublet of Chasman-Green [35] type cells is used. A cell consists of a dipole pair sandwiching a quadrupole triplet. The horizontal dispersion function in the center of each cell reaches a maximum, while the horizontal beta function converges towards a minimum. A horizontal OTR monitor here $\left(\eta_{x} \approx 50 \mathrm{~mm}, \beta_{x} \approx 1.6 \mathrm{~m}\right)$ is capable of measuring an rms energy spread of $0.03 \%$ at $14.3 \mathrm{GeV}$ with a nominal betatron beam size contribution of only $10 \%$ at $\gamma \varepsilon_{x} \approx 1 \mu \mathrm{m}$ (see Secs. 7.8.2 and 7.8.2.3).

The Chasman-Green type cells are advantageous since they introduce very little path length energy dependence and generate minimal emittance dilution due to synchrotron radiation. The net system forms a 4-dipole dog-leg (DL2) displacing the beamline horizontally toward the south by $0.45 \mathrm{~m}$. The net $R_{56}$ for the 4-dipole system is set to zero by allowing the dispersion function to reverse sign in half of the bends (see Figure 7.36).

Bends of $\theta_{B} \approx 0.65^{\circ}$ and $L_{B} \approx 2.62 \mathrm{~m}$ produce $R_{56} \approx 0$, and a second order term of $T_{566} \approx 73 \mathrm{~mm}$, which is, for a worst-case energy spread of $\sim 0.1 \%$, completely isochronous (i.e., 
$0.073-\mu \mathrm{m}$ axial position shift per $0.1-\%$ energy deviation). The emittance dilution due to incoherent synchrotron radiation at $14.3 \mathrm{GeV}$ is insignificant at $1 \%$.

In order to include a high-resolution relative energy spectrometer that is insensitive to variable incoming betatron oscillations, the centers of the Chasman-Green cells are separated by a unity optical transformer $(+\mathbf{I})$ in the horizontal plane. The signals from two BPMs, one placed at the maximum dispersion point in each cell, are then subtracted to eliminate all incoming betatron oscillations and to enhance the relative energy signal (the dispersion is of opposite sign in the two cells - see Figure 7.36). With two BPMs of $10-\mu \mathrm{m}$ resolution, a relative energy change of $8 \times 10^{-5}$ can be resolved per pulse. Such resolution will be used in an energy feedback system controlling the L3-linac rf (see Section 7.8.4).

Two vertical dipole magnets in DL2 remove the downward vertical angle imposed by the orientation of the SLAC linac. An upward net bend of $0.3^{\circ}$ is added after the last horizontal bend. This makes the undulator level with respect to gravity. The vertical bends are $0.4 \mathrm{~m}$ long and each bend $0.15^{\circ}$. They are separated by four quadrupole magnets to form a linear achromat (see Figure 7.36). These vertical bends are too weak to generate significant momentum compaction or synchrotron radiation (coherent or otherwise).

\subsubsection{Parameters}

DL2 follows the beam switchyard (BSY), which transports electrons from linac to undulator hall. The DL2 parameters are summarized in Table 7.19. Beta-functions and dispersion are shown in Figure 7.36. The energy spread measuring profile monitor is indicated at $S \approx 1236 \mathrm{~m}$.

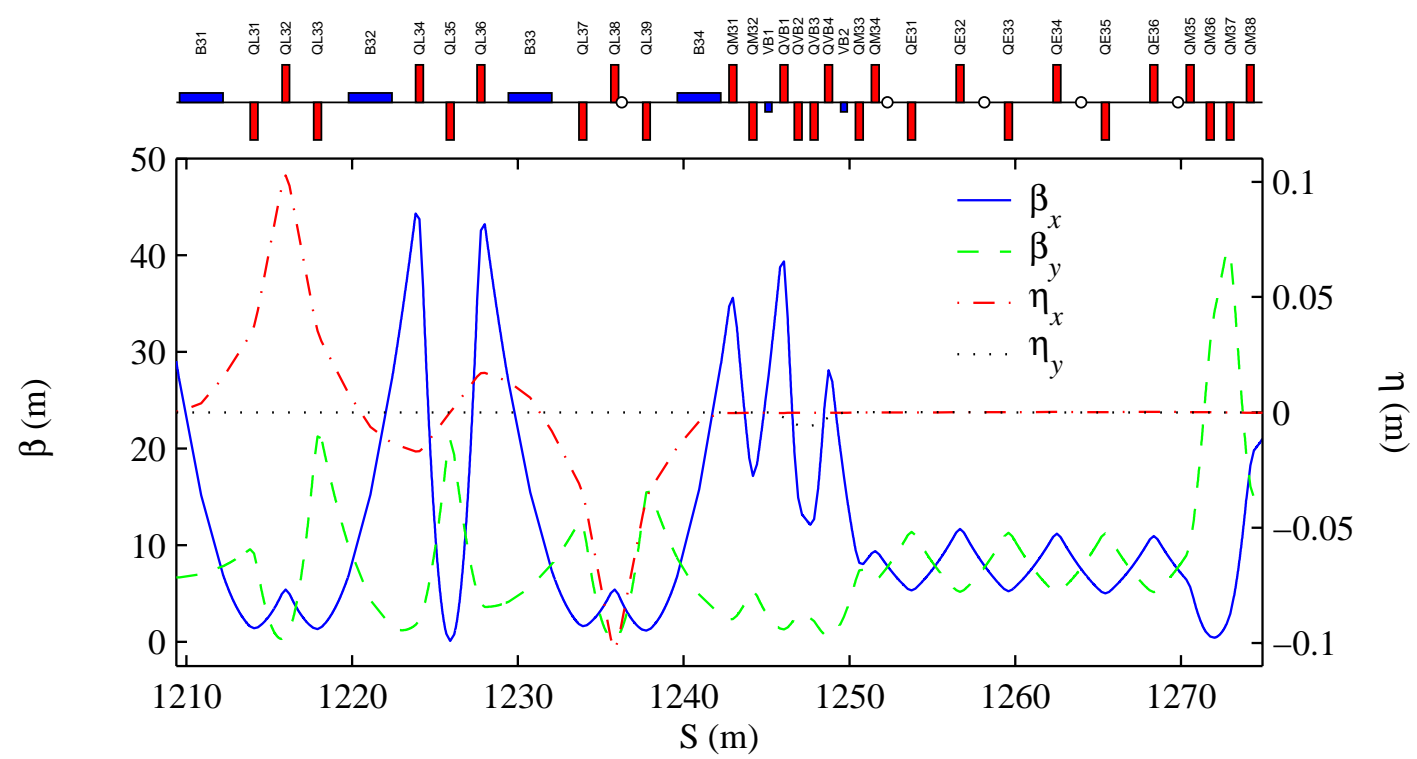

Figure 7.36 Dispersion and beta functions through DL2/ED2 beamline up to undulator entrance. Four-dipole dog-leg (DL2), 2-dipole vertical bend (VB), and final diagnostic section (ED2) are shown. Profile monitors are indicated by small circles in top schematic.

Table 7.19 Nominal parameters of high-energy dog-leg (DL2) beamline. 
LCLS CONCEPTUAL DESIG N REP O R T

\begin{tabular}{|c|c|c|c|}
\hline Parameter & Symbol & Unit & Value \\
\hline Nominal high-end beam energy & $E$ & $\mathrm{GeV}$ & 14.35 \\
\hline Total horizontal beamline inflection & $\Delta x$ & $\mathrm{~m}$ & 0.45 \\
\hline Total vertical beamline angle change (sets a level undulator) & $\Delta \theta_{y}$ & $\operatorname{deg}$ & 0.30 \\
\hline Nominal rms bunch length throughout dog-leg & $\sigma_{z}$ & $\mu \mathrm{m}$ & 22 \\
\hline RMS core relative energy spread $(14.35 \mathrm{GeV})$ & $\sigma_{\delta}$ & $\%$ & $<0.02$ \\
\hline RMS uncorrelated energy spread (14.35 GeV, with wiggler) & $\sigma_{\delta_{u}}$ & $10^{-4}$ & 8 \\
\hline Net momentum compaction & $R_{56}$ & $\mathrm{~mm}$ & 0 \\
\hline Net second order momentum compaction & $T_{566}$ & $\mathrm{~mm}$ & 5 \\
\hline Length of each of four horizontal dipole magnets & $L_{H}$ & $\mathrm{~m}$ & 2.62 \\
\hline Length of each of two vertical dipole magnets & $L_{V}$ & $\mathrm{~m}$ & 0.4 \\
\hline Bend angle of each of 4 horizontal dipoles & $\left|\theta_{H}\right|$ & $\operatorname{deg}$ & 0.65 \\
\hline Bend angle of each of 2 vertical dipoles & $\left|\theta_{V}\right|$ & $\operatorname{deg}$ & 0.15 \\
\hline Magnetic field of each horizontal dipole (at $14.35 \mathrm{GeV}$ ) & $\left|B_{H}\right|$ & $\mathrm{kG}$ & 2.07 \\
\hline Magnetic field of each vertical dipole (at $14.35 \mathrm{GeV}$ ) & $\left|B_{V}\right|$ & $\mathrm{kG}$ & 3.13 \\
\hline Maximum horizontal dispersion & $\left|\eta_{\max }\right|$ & $\mathrm{m}$ & 0.103 \\
\hline Emittance dilution due to ISR (at $\gamma \varepsilon_{0}=1 \mu \mathrm{m}$ ) & $\Delta \varepsilon_{\mathrm{ISR}} / \varepsilon_{0}$ & $\%$ & 0.8 \\
\hline Projected emittance dilution due to CSR (at $\gamma \varepsilon_{0}=1 \mu \mathrm{m}$ ) & $\Delta \varepsilon_{\mathrm{CSR}} / \varepsilon_{0}$ & $\%$ & 8 \\
\hline RMS ISR relative energy spread (at $14.35 \mathrm{GeV}$ ) & $\sigma_{\delta \mathrm{ISR}}$ & $10^{-4}$ & 0.05 \\
\hline RMS CSR relative energy spread (at $14.35 \mathrm{GeV}$ ) & $\sigma_{\delta \mathrm{CSR}}$ & $10^{-4}$ & 4.7 \\
\hline
\end{tabular}

\subsubsection{Coherent Synchrotron Radiation}

With constant bunch length over DL2, the optical symmetry is arranged to cancel the CSR horizontal emittance effect arising between bend pairs. The emittance growth is calculated using Elegant as well as TraFiC4 (see Section 7.4.1). The Elegant calculations, with a gaussian temporal distribution, result in an emittance growth of $1 \%$. The TraFiC4 code also predicts $1 \%$ for a gaussian temporal distribution. The growth predicted with Elegant and using the tracked temporal distribution of Figure 7.7, bottom row of plots, is $8 \%$ (including all particles, and for $\left.\gamma \varepsilon_{0}=1 \mu \mathrm{m}\right)$, but this is concentrated at the sharp current spikes of the bunch head and tail.

The emittance growth between pairs of bends is $40 \%$, which demonstrates the cancellation symmetry. Figure $\mathbf{7 . 3 7}$ shows the additional rms energy spread induced by CSR (top) and the normalized horizontal emittance (bottom) along the DL2 bend system ( $1^{\text {st }}$ bend to 3 meters after $4^{\text {th }}$ bend). To isolate the effects of the DL2 bends, this calculation tracks an LCLS $e^{-}$bunch distribution which has not been altered by CSR effects in $\mathrm{BC} 1$ or $\mathrm{BC} 2$. The full effect with CSR in all bends is examined in Section 7.6. 

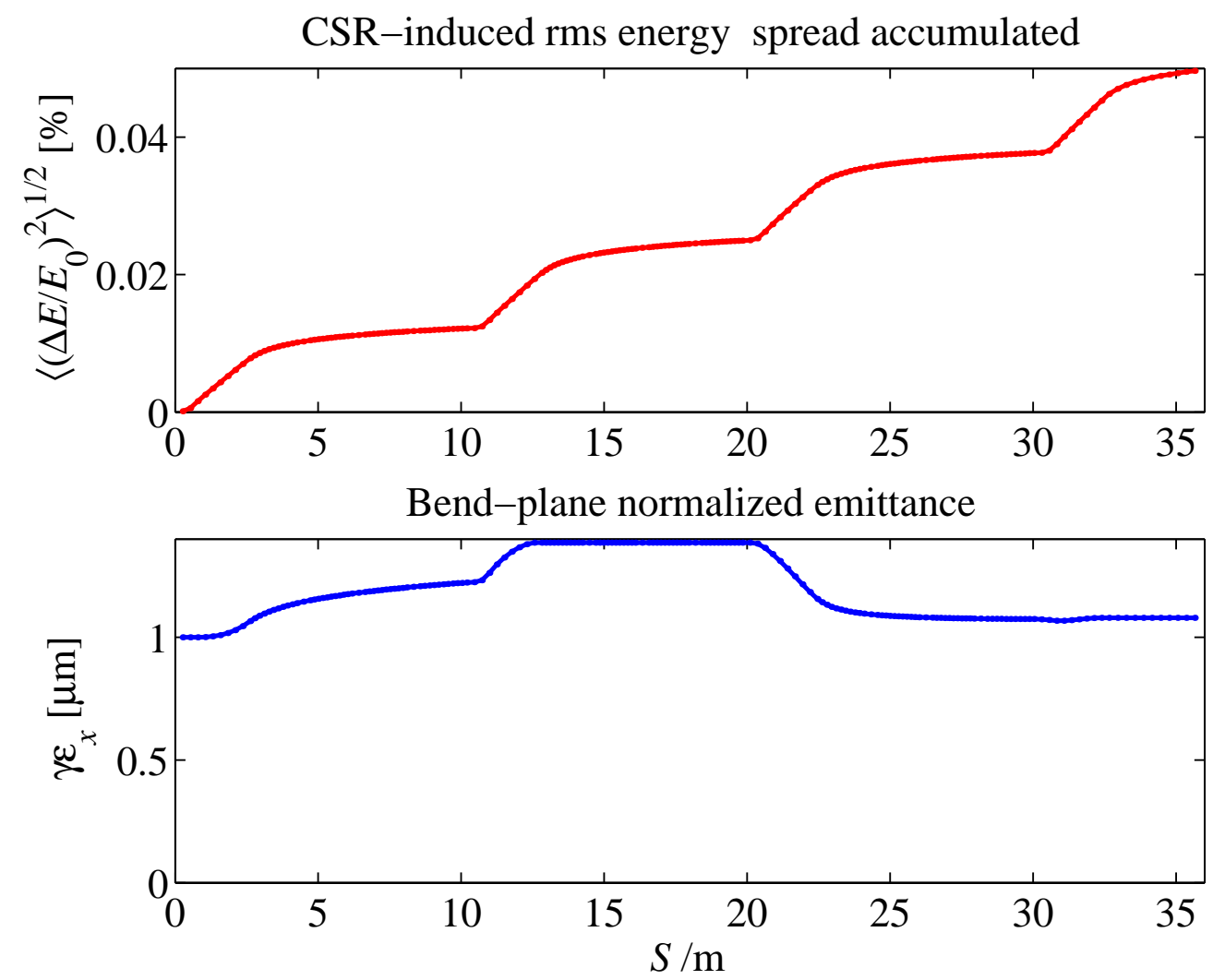

Figure 7.37 Additional rms energy spread induced by CSR (top) and the normalized horizontal emittance (bottom) along the DL2 bend system ( $1^{\text {st }}$ bend to 3 meters after $4^{\text {th }}$ bend).

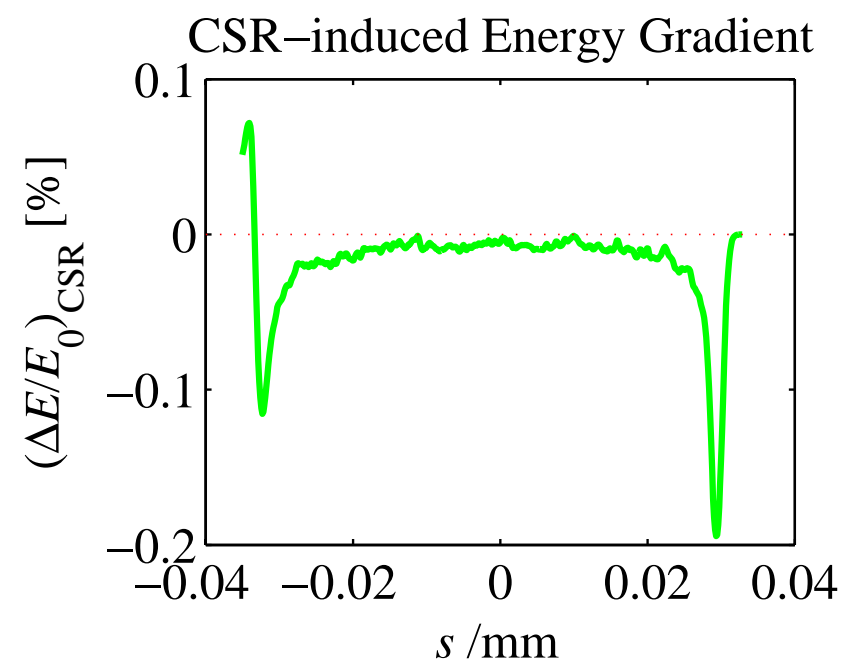

Figure 7.38 CSR-wakefield energy gradient along the bunch generated within DL2 bend system.

The total accumulated CSR-wakefield energy gradient along the bunch generated within the DL2 bend system is shown in Figure 7.38. The emittance growth and energy spread is concentrated at bunch head and tail and has little effect on beam parameters sliced on the scale of 
the FEL slippage length $(\sim 0.5 \mu \mathrm{m})$. The energy loss and rms energy spread induced by CSR are $5.5 \mathrm{MeV}$ and $0.047 \%$, respectively.

Figure 7.39 shows the temporal distribution of the bunch and the CSR-wakefield, from Eq. (7.19), within the first bend of DL2, using the temporal distribution taken from tracking upstream of DL2. The variable color traces represent the two functions sampled in each of ten points along the bend magnet. The temporal distribution is nearly constant over the DL2 bend system due to the small energy spread and isochronicity of the beamline. Figure $\mathbf{7 . 4 0}$ shows the horizontal beam position, $x$, without CSR (left) and with CSR in all LCLS bends (right), versus axial bunch coordinate, $z$, for the LCLS bunch profile (i.e., tracked through upstream systems).

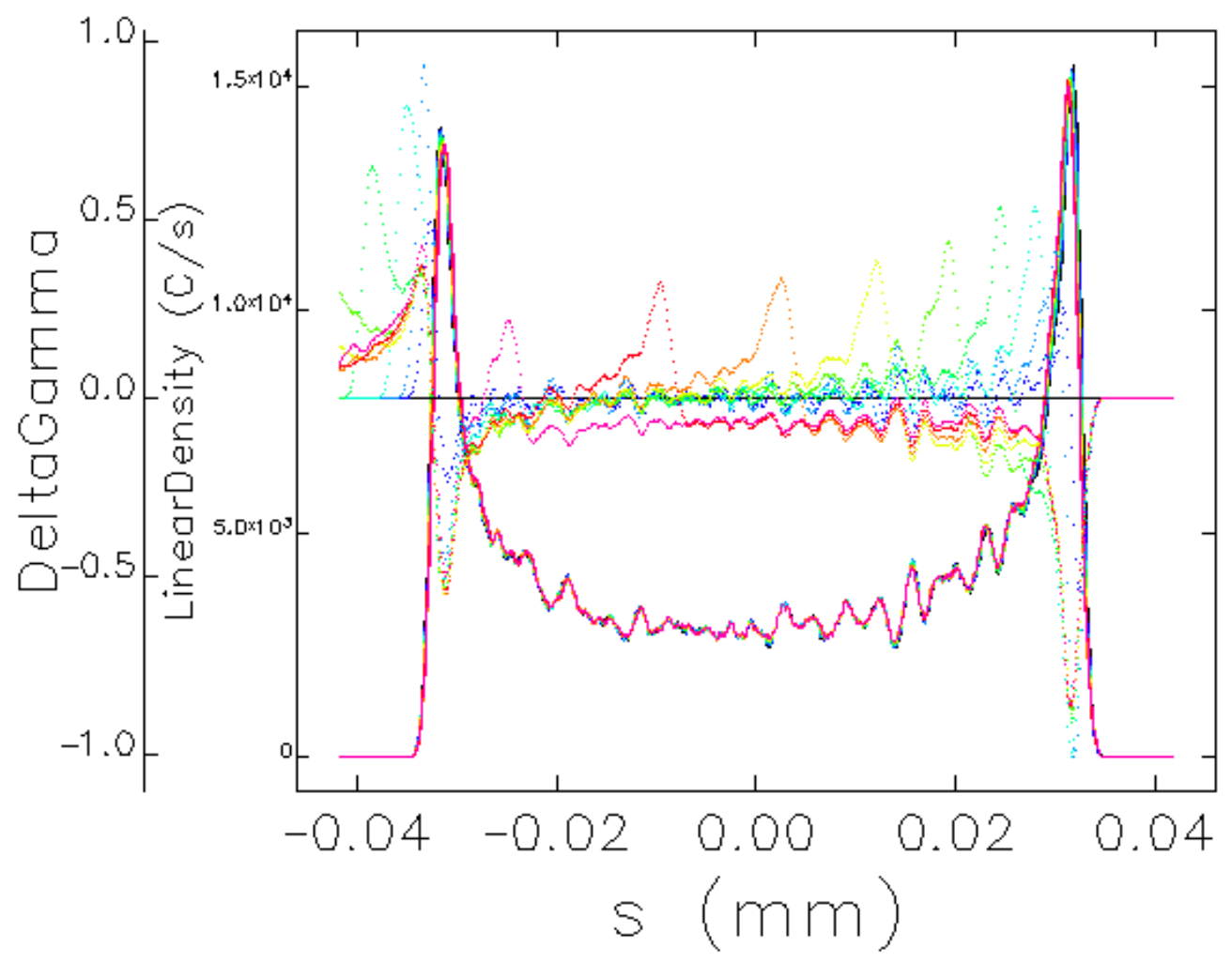

Figure 7.39 Constant temporal distribution of bunch (solid: "LinearDensity") and CSR-wakefield (dots: "DeltaGamma") within first bend of DL2. The variable color traces represent the two functions sampled in each of ten points along the bend magnet.

Since the $\mathrm{BC} 2$ chicane also contribute a horizontal emittance growth through correlations of $x$ and $x^{\prime}$ with $z$, these might be partially cancelled by 'bucking' the $\mathrm{BC} 2$ correlations against those of the DL2 bends. The last bend of BC2 bends to the 'left', while the first bend-pair in DL2 bends to the 'right', so a net horizontal betatron phase advance between these two of $\Delta \psi_{x}=2 n \pi$ provides the possibility of cancellation. The net phase is set by slight adjustments in the L3-linac phase advance per cell. The BC2/DL2 cancellation is, of course, not completely effective, but at least this arrangement is superior to an odd- $\pi$ phase advance, which would possibly amplify the emittance growth. 

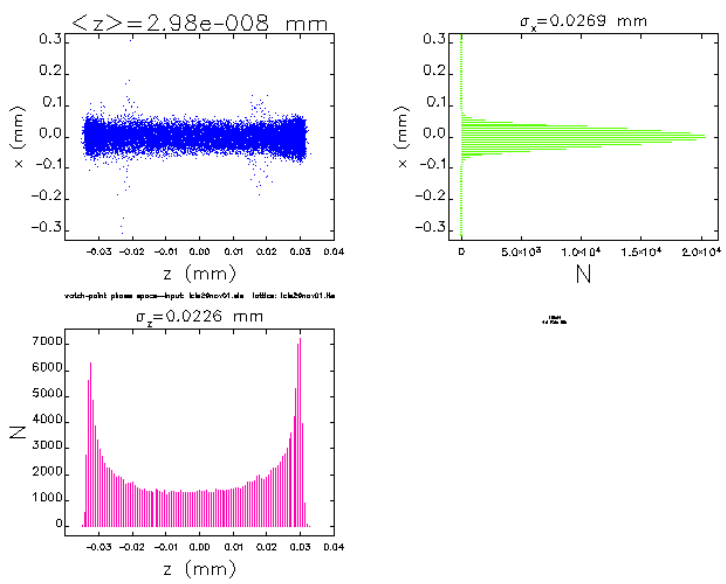
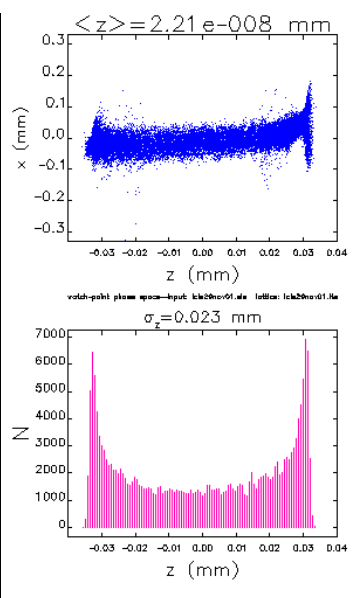

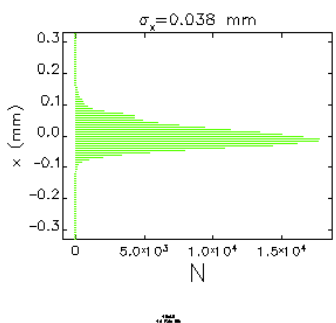

.

Figure 7.40 Horizontal position, $x$, without CSR (left) and with CSR (right), versus $z$, for the LCLS bunch profile (i.e., tracked through upstream systems) after DL2 bends. Projected emittance growth is 2.5 due to CSR in BC2, but dominated by bunch head and tail.

\subsubsection{Beam Size, Aperture, and Field Quality}

The beam size in DL2 reaches a peak value of $\sim 50 \mu \mathrm{m}$. A $2.5-\mathrm{cm}$ full aperture is, therefore, completely adequate. The large dispersion in the DL2 quadrupoles sets the tolerances on field quality and gradient errors. Table $\mathbf{7 . 2 0}$ lists dipole tolerances, while the alignment and field strength sensitivities for quadrupole magnets in the DL2 area are shown in Figure 7.41. Each sensitivity shown corresponds individually to a filamented $x$ and $y$ emittance dilution of $\Delta \varepsilon_{x} / \varepsilon_{x 0}+\Delta \varepsilon_{y} / \varepsilon_{y 0}=2 \%$.

Table 7.20 Dipole magnet tolerances for DL2 with an exaggerated $0.05 \%$ rms energy spread. Field harmonics are evaluated on a $20-\mathrm{mm}$ radius and each entry individually corresponds to a $2 \%$ emittance dilution.

\begin{tabular}{lcccc}
\hline Magnet & Quantity & Roll Angle [mrad] & $\left|\boldsymbol{b}_{\mathbf{1}} / \boldsymbol{b}_{\mathbf{0}}\right|[\%]$ & $\left|\boldsymbol{b}_{\mathbf{2}} / \boldsymbol{b}_{\mathbf{0}}\right|[\%]$ \\
\hline B31-B34 & 4 & 80 & 2.1 & 100 \\
\hline VB1 \& VB2 & 1 each & 170 & 5.4 & 100 \\
\hline
\end{tabular}

The most challenging of these sensitivities are the absolute gradient errors of some of the quadrupoles, $\left|\Delta b_{1} / b_{1}\right|<1.5 \%$. Although many of these magnets are powered in series, their gradients may differ slightly due to construction errors. A tolerance of $<1.5 \%$ is not trivially achievable. The effect on the beam, however, is the generation of linear dispersion or beta function mismatch in the undulator. This can easily be tuned-out empirically by adjusting the quadrupoles in DL2. Quadrupole alignment sensitivities are no tighter than $500 \mu \mathrm{m}$ and do not present a major challenge. Dipole field errors (not listed) are correctable with steering.

A pair of adjustable collimator jaws will be included in the center of the $1^{\text {st }}$ DL2 bend-pair, just upstream of the profile monitor, which can be used to cut beam energy tails. The collimator will be nominally open with a full horizontal gap of $\sim 2 \mathrm{~cm}$, but each jaw will be independently 
controlled in order to collimate potential energy tails. The collimator may be useful in diagnosing tails in the electron beam, or for masking beam halo, which generates background radiation in the undulator. It is not envisioned that any significant collimation will be made during normal LCLS operations.

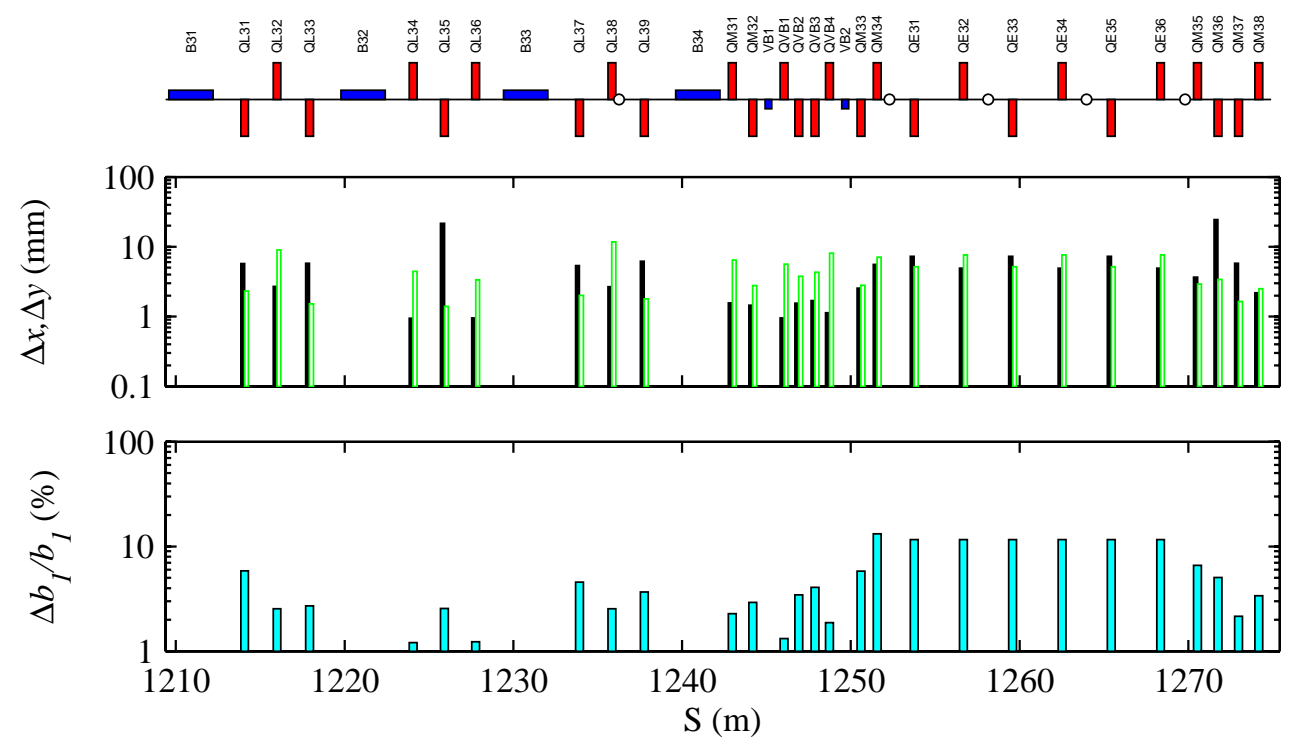

Figure 7.41 Alignment (top: black is $x$, and green/white is $y$ ) and strength sensitivities (bottom) for DL2 quadrupoles with an exaggerated $0.05 \%$ rms energy spread. Each bar individually corresponds to 'filamented' emittance dilution of $\Delta \varepsilon_{x} / \varepsilon_{x 0}+\Delta \varepsilon_{y} / \varepsilon_{y 0}=2 \%$.

\subsubsection{Tuning and Correction}

The dominant error, which will likely arise in DL2, is anomalous linear dispersion or beta mismatch. Quadrupole field strength errors are the most likely cause. The various quadrupoles on separate power supplies will be used in appropriate linear combinations to generate dispersion and matching correction control. The emittance and matching, just prior to undulator entrance, can be measured in the ED2 diagnostic section following DL2.

An insertable tune-up dump will also be included after DL2 in order to allow invasive tuning of the upstream systems. The dump will need to handle a $1-\mathrm{nC}$ beam at $120 \mathrm{~Hz}$ and $15 \mathrm{GeV}$, or $1.8-\mathrm{kW}$ of average power. A fast beam dump will also be located just downstream of the linac and well before the undulator in order to dump the electron beam during conditions of exceptionally poor beam quality. This will help to preserve the permanent magnet undulator fields, and to provide a 'veto' for unwanted pulses. This kicker is only fast enough to veto a 'second' bad pulse.

\subsubsection{Dumpline}

After leaving the undulator, the "spent" electron beam is separated from the x-ray beam by an array of five permanent magnet vertical dipoles flanked on either side by an electromagnet dipole. These magnets deflect the $e^{-}$beam downward into a beam dump. The reason for the permanent 
magnets is based on the SLAC Beam Containment System (BCS) philosophy, which discourages power supply excited electromagnets, which could fail. Five permanent magnets and two DC electromagnets are deemed sufficient to guarantee the safe delivery of any electron beam onto trajectories that eventually target in the acceptable region of the dump. The two DC electromagnets are to be operated in series from one unipolar power supply. This together with locked terminal covers at the magnets and the power supply end is sufficient to prevent upward deflection of any electron beam. A meter relay set at a predetermined excitation current value prevents under-deflection of the beam. Should either the meter relay fail or the power supply be set at a value lower than that required for the beam energy, the resulting trajectories would be as shown in Figure 7.42. A protection collimator (uncooled) backed by a burn-through monitor (BTM) guarantees beam containment. The particular combination of permanent magnets and DC electromagnets was chosen to minimize the distance from the end of the undulator to the beam dump, to allow operation over an energy range from $2 \mathrm{GeV}$ to $15 \mathrm{GeV}$ and to minimize the vertical range of all trajectories on the dump (see Figure 7.43). All magnets are C-type magnets, open to the bottom, to allow easy separation of the x-ray beam from the "spent" electron beam energies.

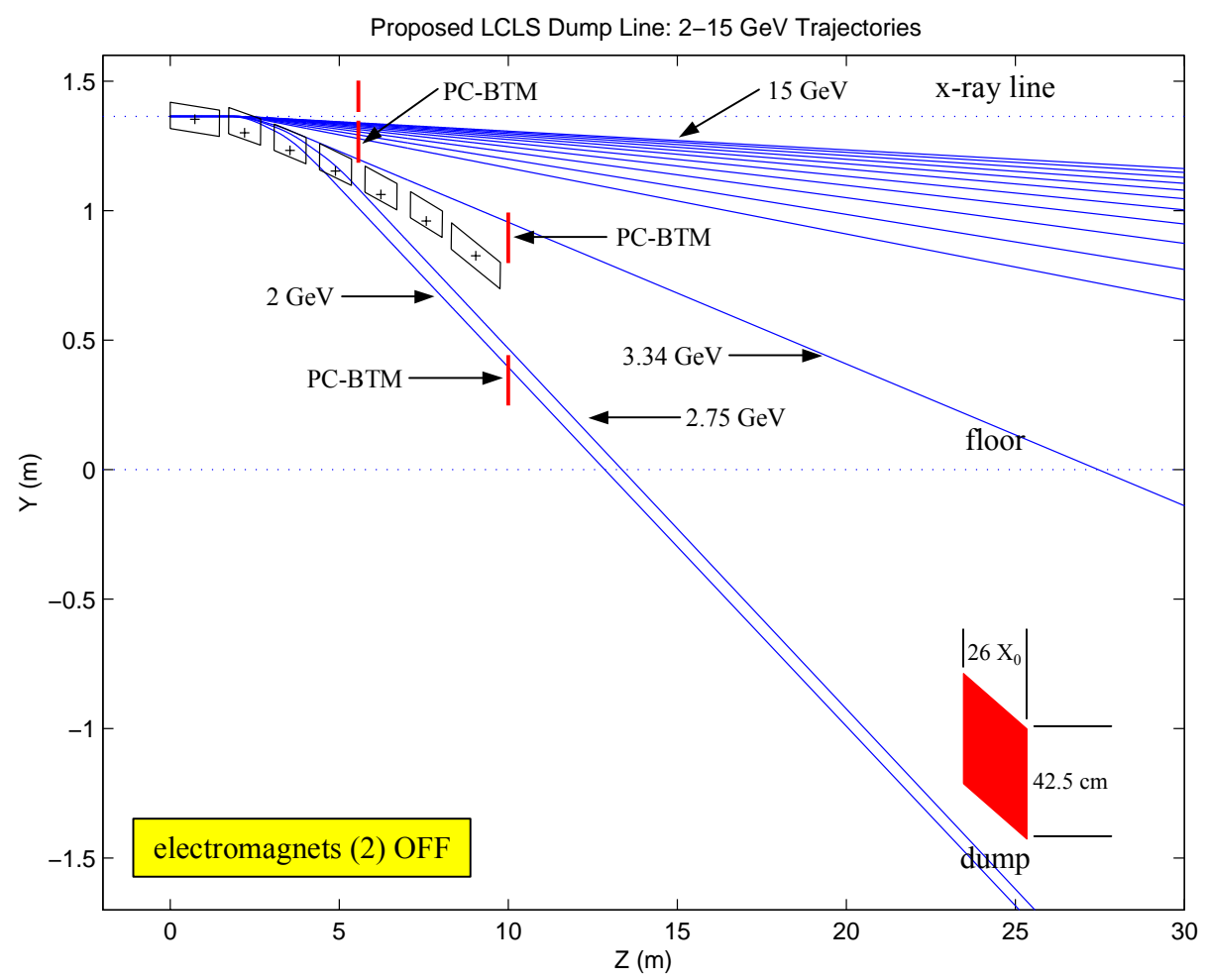

Figure 7.42 LCLS Dumpline 2-15 GeV trajectories: electromagnets off. The burn-through monitors (PC-BTM) cover all possible energy beams for the case with tripped electromagnets. The dump is the red block at lower right.

At $15 \mathrm{GeV}$, the deflection in each permanent magnet is $\theta \approx 0.484^{\circ}$ for a total bend angle of $2.421^{\circ}$ for five magnets. The electromagnets were originally designed and built for the SLAC SLC Linac-to-Arcs matching sections. They were measured to $880 \mathrm{~A}$ with significant saturation. It 
is suggested that $600 \mathrm{~A}$ should be an upper limit for LCLS. Below that level the excitation curve is linear. At $15 \mathrm{GeV}$, the deflection in each magnet is then $2.416^{\circ}$. Note, this is approximately the same as the value from all five permanent magnets. The total deflection from five permanent magnets and two electromagnets is then $7.254^{\circ}$.

Lower beam energies are deflected by larger angles and some of the trajectories leave the magnetic field before reaching the last magnet(s) in the array. For example, if $2 \mathrm{GeV}$ is arbitrarily selected as the lower limit, the deflection is $3.632^{\circ}$ and the beam will already leave the magnetic field at the end of the first permanent magnet and will not be subject to any of the other magnets' strengths. This effect of the lower energy trajectories "leaving early" and forming straight lines is actually advantageous in that it limits the required vertical size of the beam dump.

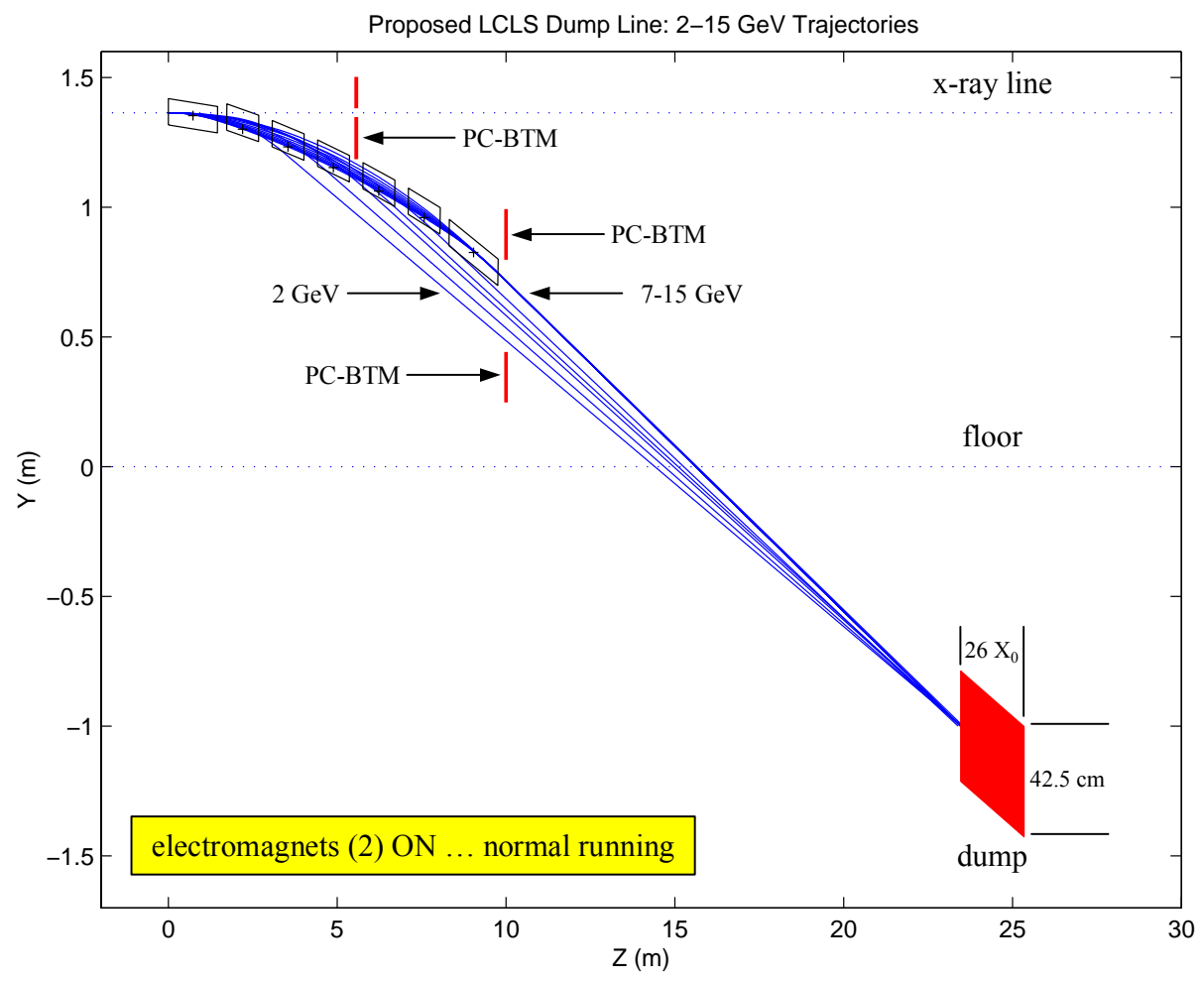

Figure 7.43 LCLS Dumpline 2-15 GeV trajectories: electromagnets on.

\subsubsection{Beam Containment and Beam Dump}

The BCS protection collimator/BTM will be a 3-section device. The first section will contain all electron beam energies above $\sim 3.3 \mathrm{GeV}$ for the case where the first electromagnet dipole is accidentally off. It is optimally located between permanent magnet dipoles \#3 and \#4 and will cover the vertical region from the x-ray beam down to an elevation just above the magnet poles. The second section will be located down-beam of the second electromagnet dipole and will cover the vertical region from approximately the "allowed" $2-\mathrm{GeV}$ trajectory down to an elevation, which contains at least the "unallowed" $2-\mathrm{GeV}$ trajectory. The third section is above the allowed trajectories at that location and covers those trajectories, which could not be collimated at the first 
location.

The collimator sections consist of 3-inch thick carbon steel slabs of appropriate transverse size which act as shower builders, followed by standard BTM's of comparable transverse size. If they are incorporated into the vacuum chambers, the slabs would be stainless steel.

As can readily be seen from the ray trace schematics, the particular arrangement of the electromagnet and permanent magnets results in a small region of beam impingement on the beam dump for all allowed trajectories. This allows in principle the use of the existing FFTB beam dump. It is a device, which was originally designed for the SLC extraction beam dump locations and has a maximum power absorption capacity of $100 \mathrm{~kW}$ for appropriate cooling water flow rate. Maximum beam energy rating is $70 \mathrm{GeV}$. The design is a $16-3 / 4$ inch $(425 \mathrm{~mm})$ diameter aluminum cylinder, peripherally water-cooled to minimize radiolysis and cooling water activation. Neither a hydrogen recombiner nor a radioactive water loop is required, i.e. the standard low-conductivity water system (LCW) is sufficient. The dump is 26 radiation-lengths $\left(X_{0}\right)$ long.

The beam dump is to be located below the research yard floor level to more easily shield it than is possible for the present FFTB dump. An elevation of $1 \mathrm{~m}$ below ground for the highest trajectory $(15 \mathrm{GeV})$ was selected. This puts the location of the front face of the dump some $24 \mathrm{~m}$ downbeam of the entrance to the first electromagnet dipole.

\subsubsection{Vacuum Chambers}

The magnet vacuum chambers are specific to the locations in the dump magnet array reflecting the multi-energy vertical beam stay clear requirements. All chambers are rectangular in cross-section.

The size of the first DC dipole chamber will be approximately the size of the magnet gap and pole width. The first three permanent magnets should share a single chamber to minimize flanges and bellows. The outside width of the chamber will be of the order of the magnet gap. The height will have to be large enough to include not only all the allowed trajectories from $15 \mathrm{GeV}$ down to $2 \mathrm{GeV}$, but also the unallowed trajectories when either the first electromagnet dipole is off, or when a low energy beam (lower energy than anticipated by the electromagnet current excitation setting) enters the magnetic array. The first protection collimator is built into the chamber and wall, but the BTM is external in air and attached to the chamber.

From this point on, the x-ray beam vacuum pipe is separate from the dump line. The following two permanent magnets also share another rectangular chamber consistent with the beam stay clear requirements of the remaining allowed and unallowed trajectories. The second electromagnet dipole has its own chamber with the second protection collimator built into its lower end wall. The BTM will again be external to the vacuum chamber in air.

Further design study is required to examine the technical feasibility and economic merits of having all five permanent magnet dipoles share one large rectangular chamber including the two protection collimators. Such a chamber is now installed in the six permanent magnets in the FFTB. The remaining vacuum chambers to the beam dump will be of circular cross-section for 
economic reasons.

\subsection{Six-Dimensional Particle Tracking Studies}

In this section the electron beam quality is evaluated by slicing the beam longitudinally after detailed 6D tracking through the injector, compressors, and main linac. The final electron beam density is used to estimate the FEL performance for the nominal LCLS undulator parameters (see also [36]).

\subsubsection{Electron Beam Evaluation}

The entire LCLS accelerator, from rf-gun to undulator entrance, has been tracked in six dimensions using Parmela for the injector, up to $150 \mathrm{MeV}$, and then tracking these same $2 \times 10^{5}$ macro-particles using Elegant [10] for the linac and compressors, up to undulator entrance at 14.35 GeV. The tracking calculations include the following effects:

- An estimated thermal emittance included at the cathode (see Chapter 6),

- Space charge forces up to $150 \mathrm{MeV}$ for the LCLS gun and injector,

- Longitudinal and transverse geometric wakefields of the S-band and X-band accelerating structures (transverse wakes are only applied past the L0-linac),

- Bunch compression including all linear and non-linear energy correlations induced in the linacs and compressors,

- Transverse misalignments (past L0) of BPMs, quadrupoles and all 3-meter accelerating structures (BPMs: $150 \mu \mathrm{m}$ rms, quadrupoles: $150 \mu \mathrm{m} \mathrm{rms}$, structures: $300 \mu \mathrm{m}$ rms; all gaussian distributions with $3-\sigma$ cuts),

- Trajectory correction (past L0) applied using existing (and planned) steering elements and misaligned BPMs,

- Coherent synchrotron radiation in all bends, with a transient field model integrated over the 'real' evolving non-gaussian temporal bunch distribution, and including radiation effects between and after bend magnets [27], [28],

- Incoherent synchrotron radiation effects in all bends, which adds slice emittance and slice energy spread,

- First and second-order lumped optics of each half-magnet (every magnet is split into two pieces),

- Resistive-wall longitudinal wakefields of the micro-bunch in several long sections of 1 -inch diameter stainless-steel and aluminum vacuum chambers which are located in the L3-linac and DL2 beamlines.

An example of a final steered trajectory and the related emittance growth over the entire LCLS is shown in Figure 7.44. The normalized rms projected emittance here does not include the effects of CSR (see below), and no 'emittance-bump' corrections [18] have been applied to 
minimize the emittance growth. The final projected emittance growth for this particular misalignment seed is $\Delta \varepsilon_{x} / \varepsilon_{x 0} \approx 215 \%$ and $\Delta \varepsilon_{y} / \varepsilon_{y 0} \approx 160 \%$, with $\gamma \varepsilon_{x 0, y 0} \approx 0.8 \mu \mathrm{m}$. This can be greatly improved by applying well-tested emittance correction techniques [16], [18]. Reference [16] describes a simulation in which 100 different random misalignment cases, with emittance growth of up to $300 \%$, were all corrected to $<10 \%$ using trajectory bumps. The slice emittance growth along the bunch length also never exceeds $\sim 10 \%$ in either plane. The large emittance spikes in the plot at right of Figure $\mathbf{7 . 4 4}$ occur at locations (e.g., chicanes) where the dispersion is very large and the projected emittance calculation is dominated by dispersion.

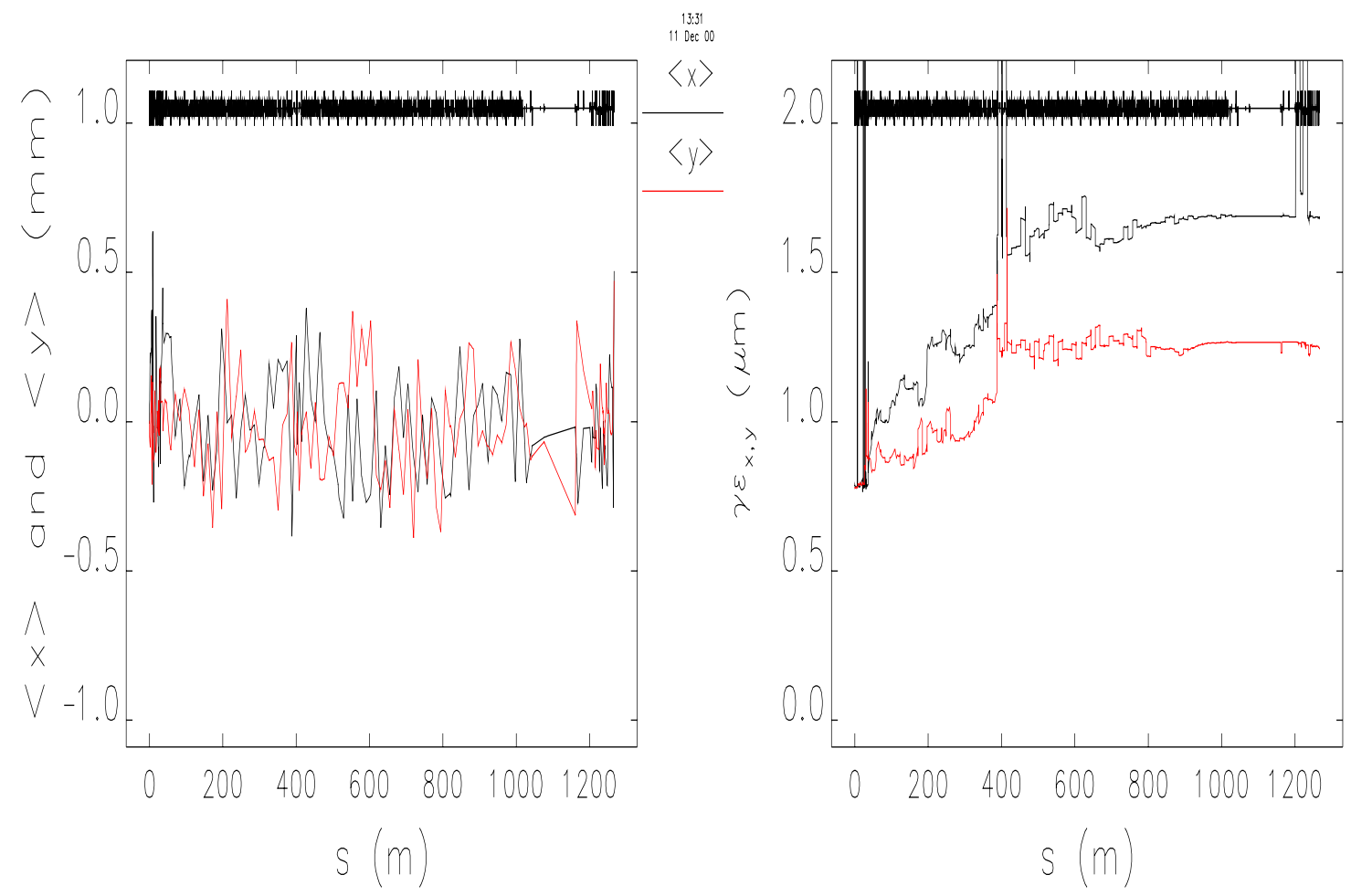

Figure 7.44 An example of $x$ (black) and $y$ (red) corrected trajectories (left), and projected emittance growth (right; without CSR), given BPM, quadrupole and rf-structure misalignments as described above, along the entire LCLS, from end of LO to entrance of undulator. The emittance can be corrected using trajectory bumps (not included here).

The projected emittance growth, now including ISR and CSR in every bend magnet, but for perfectly aligned components, is shown in Figure 7.45. The net projected horizontal emittance growth is $\sim 2.5$, dominated by effects at bunch head and tail, but the slice is still nearly unaffected (see below). The central trajectory has been corrected so that the CSR-generated energy loss in the bends does not cause large betatron oscillations through the linac sections. 


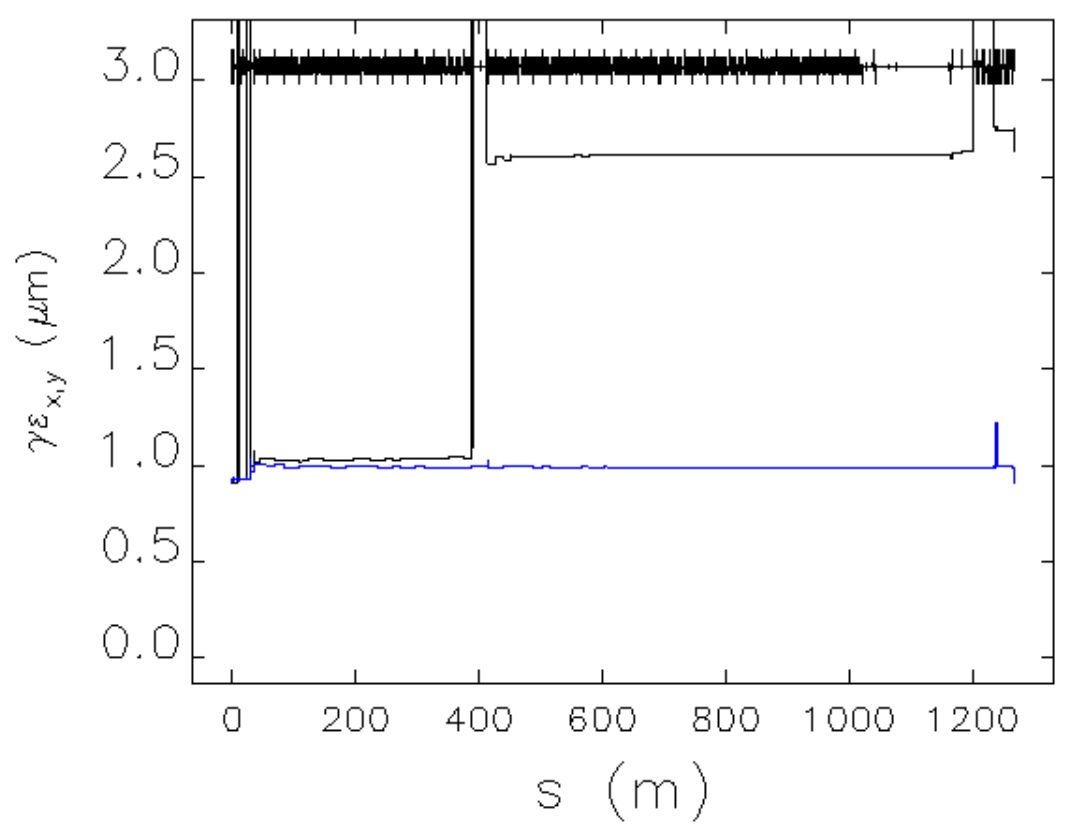

Figure 7.45 Normalized rms projected emittance along the LCLS accelerator, including CSR in every bend magnet, and for perfectly aligned components (black: $\gamma \varepsilon_{x}$, blue: $\gamma \varepsilon_{y}$ ).

The slice emittances, both before and after the accelerator for the case with CSR, is shown in Figure 7.46, where "Slice Number" refers to the bunch length coordinate (bunch head at slice-1). The dashed/red lines ( $x$ at left, $y$ at right) represent slice emittance at $150 \mathrm{MeV}$ (at L0-linac exit). The solid/blue lines ( $x$ at left, $y$ at right) represent slice emittances at $14.35 \mathrm{GeV}$ (at undulator entrance). The large emittance peaks at bunch head and tail at $150 \mathrm{MeV}$ contain very little charge and are washed into the core of the beam after compression and acceleration where they contribute only weakly to the emittance of the core.
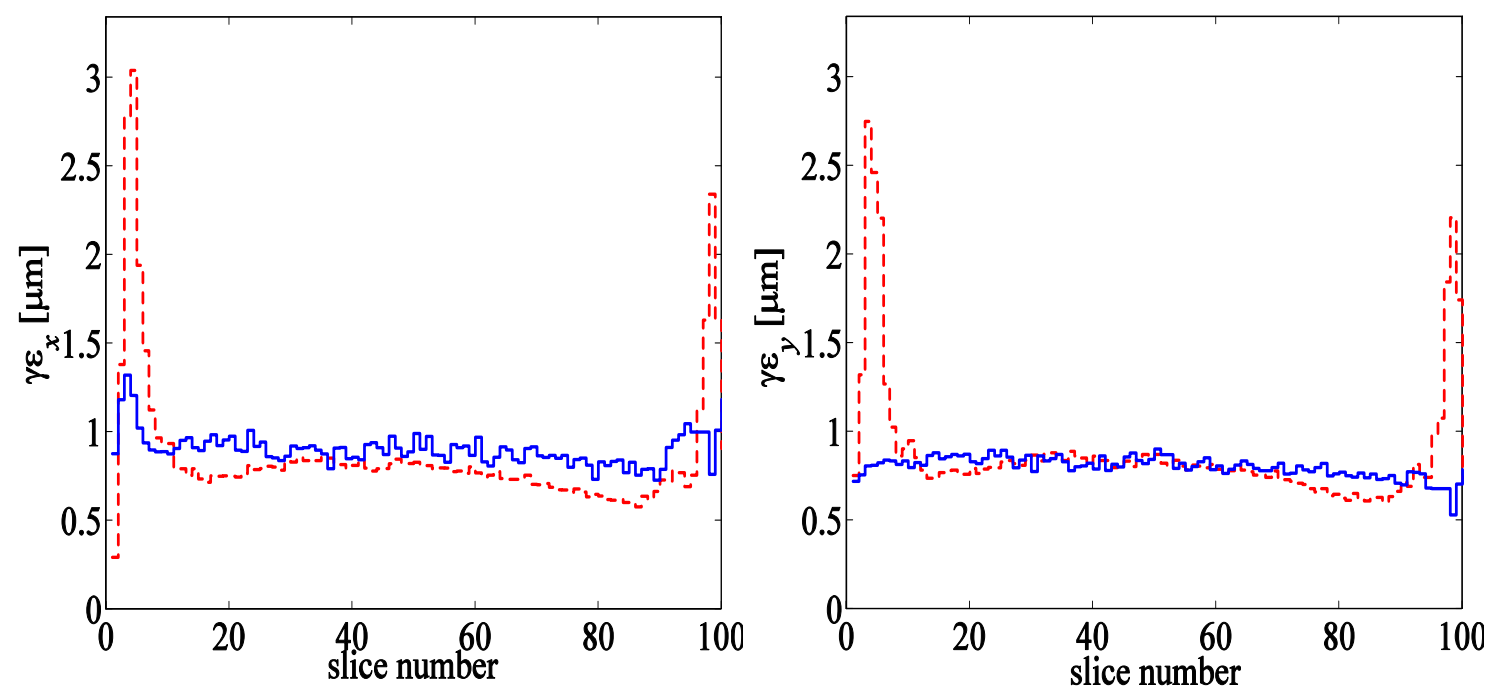

Figure 7.46 Slice emittances ( $x$ at left, $y$ at right) at $150 \mathrm{MeV}$ (dash/red) and $14.35 \mathrm{GeV}$ (solid/blue) after entire LCLS accelerator, including CSR. Bunch head is at slice number-1. 
The effects of CSR also change the final projected energy spread, but have very little effect on the bunch length. Figure 7.47 (left) shows the longitudinal phase space at $14.35 \mathrm{GeV}$ at entrance to undulator with CSR effects included in all bends. The rms projected energy spread is $0.1 \%$, including the large tails, but the energy spread of the core slices is $<0.01 \%$ rms. The spatial $x-y$ cross-section of the electron beam, including CSR, is shown at right of Figure 7.47. Some weak tails out to $10 \sigma$ are indicated, but the core of the beam is well concentrated. A similar plot (not shown) of $x^{\prime}$ versus $y^{\prime}$ shows smaller tails.
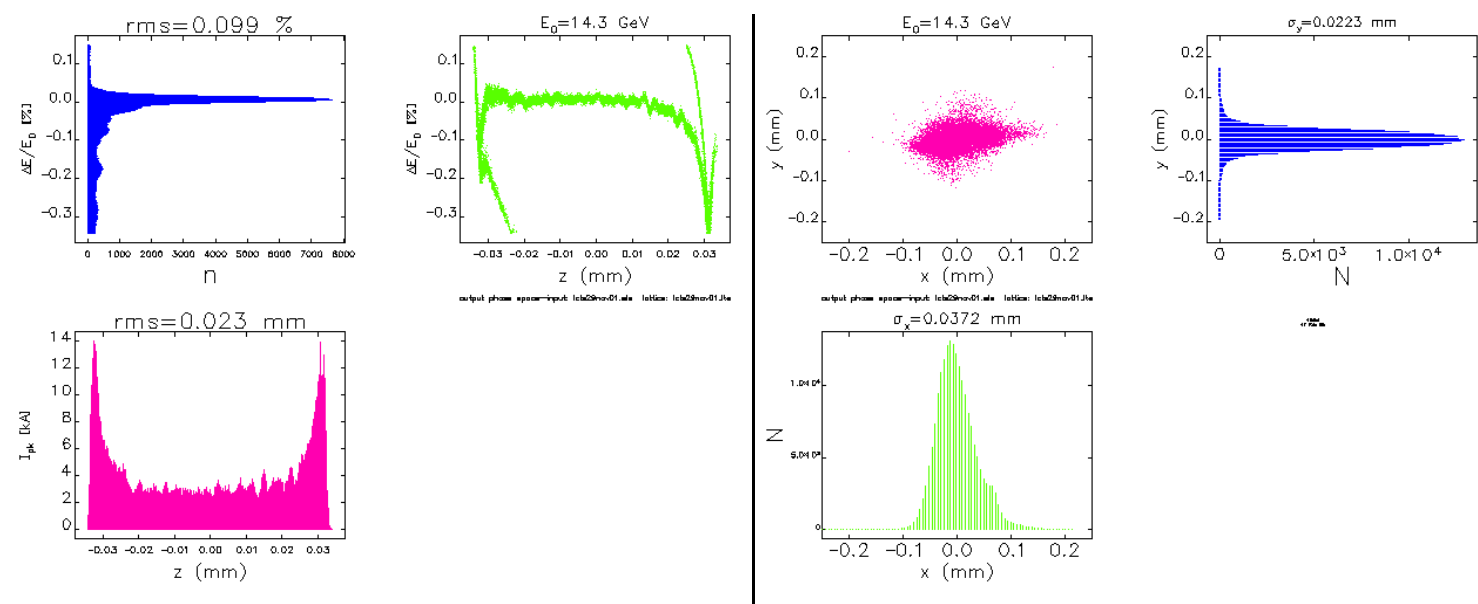

$=$

Figure 7.47 Longitudinal phase space (left) and spatial $x-y$ cross-section (right) of electron beam at undulator entrance at $14.35 \mathrm{GeV}$, including CSR but for perfect component alignment.

At the left of Figure 7.48 is the beta-mismatch amplitude per slice. This is defined in Eq. (7.26) where the subscripted parameters represent the Twiss parameters of the integrated bunch, while the non-subscripted parameters are the Twiss parameters of each slice. Precise empirical matching of the integrated bunch is possible using profile monitors and adjustments of the DC quadrupole magnets prior to the undulator, but the match of each slice may still vary over the bunch. In fact, matching the integrated bunch may not actually match any of the slices. The transverse RF deflecting structure can be used to help diagnose slice versus projected mismatch effects (see Section 7.8.2).

$$
\zeta \equiv \frac{1}{2}\left(\beta_{0} \gamma-2 \alpha_{0} \alpha+\gamma_{0} \beta\right) \geq 1
$$

As a simple example, this mismatch amplitude will take a value $\zeta=1.25$ for $\alpha_{0}=0$ and $\beta / \beta_{0}=2$ (or $\beta / \beta_{0}=0.5$ ). A mismatch level of $\zeta<1.5$ in the undulator is desirable [37].

The slice energy spread is also shown in Figure $\mathbf{7 . 4 8}$ at right. The core of the beam has an rms energy spread of $0.008 \%$. The extreme energy tails $\left(\left|\Delta E / E_{0}\right|>0.1 \%\right)$ have been cut out here ( $80 \%$ of the particles remain) in order to better reveal the slice energy spread, rather than the extreme tail effects. 

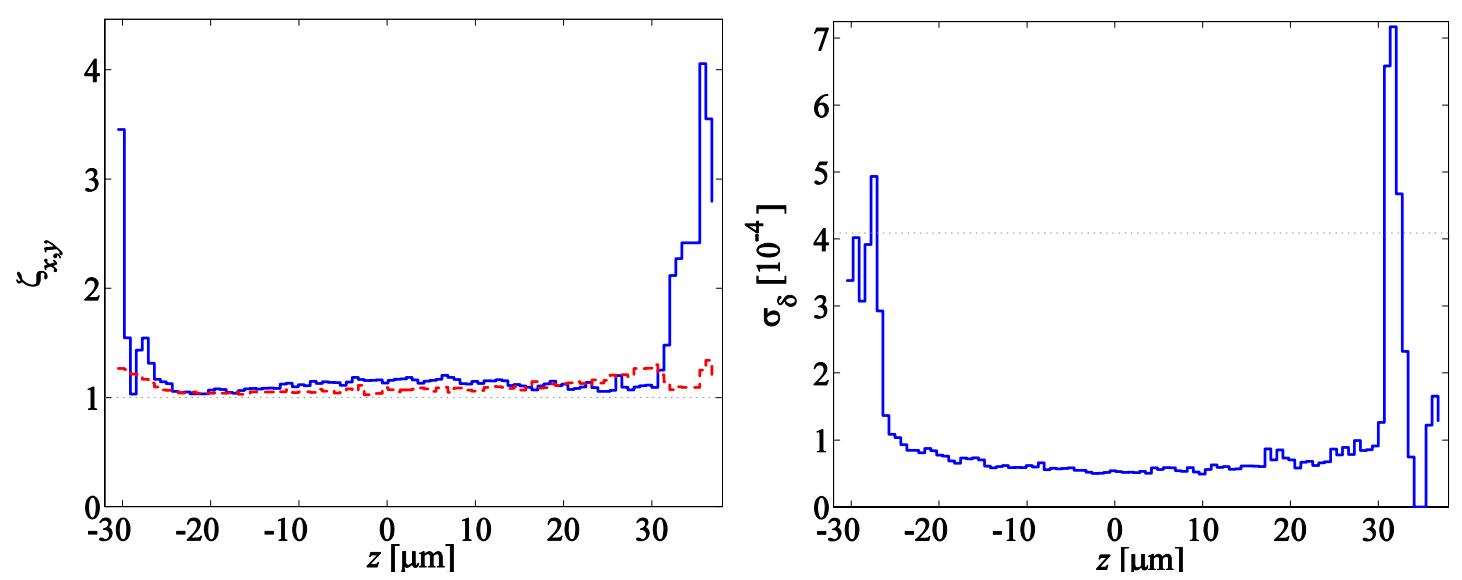

Figure 7.48 Beta-mismatch amplitude (left: $x$ solid/blue, $y$ dashed/red), and rms relative energy spread (right) and along the bunch at $14.35 \mathrm{GeV}$, including CSR effects for the $80 \%$ core.

\subsubsection{FEL Gain Estimation}

Finally, the FEL evaluation described by Ming Xie [38] has been applied to each slice of the bunch. The bunch is sliced 100 times which approximates the worst-case slippage length of the FEL at $1.5 \AA(0.7 \mu \mathrm{m})$. In this analysis, the electron emittance, peak current, energy, and energy spread of each slice is used to calculate the radiation wavelength, $\lambda_{r}$, the FEL-parameter, $\rho$, the 3D-power-gain-length, and the saturation power, for the nominal LCLS planar undulator with $K \approx 3.71, \lambda_{u} \approx 3 \mathrm{~cm},\left\langle\beta_{x, y}\right\rangle \approx 18 \mathrm{~m}$, and $E_{0} \approx 14.346 \mathrm{GeV}$. Figure 7.49 shows these 'slice' quantities all plotted against longitudinal position within the bunch. The curves show the parameters calculated over all particles, which are within an energy range $-0.1 \leq \Delta E / E_{0} \leq+0.1 \%$, which eliminates $20 \%$ of the particles with large energy tails, and therefore the calculations also use a reduced total bunch charge of $0.8 \mathrm{nC}$. Plots of $\gamma \varepsilon_{x, y}$ and $\zeta_{x, y}$ show $x$ (solid) and $y$ (dash).

The dotted horizontal lines in the plots represent the full bunch-integrated values, which are in fact irrelevant. Figure 7.49 shows a 3D power-gain-length of $3.35 \mathrm{~m}$ over the longitudinal core of the electron bunch, which would saturate in $\sim 67 \mathrm{~m}$. The dotted lines in the gain-length and power plots are the levels calculated if the bunch-integrated (projected) emittances and energyspread values are used.

This dimensionless 4D transverse centroid amplitude, $\left\langle R_{4}\right\rangle$, plotted in Figure 7.49, reduces to $\left\langle R_{4}\right\rangle=1$ in the simple instructive case of a one-sigma horizontal oscillation amplitude with $\alpha_{x}=0$, $x= \pm\left(\beta_{x} \varepsilon_{x}\right)^{1 / 2}$ and $x^{\prime}=y^{\prime}=0=y$. The effects of CSR in BC2 dominate the variation in $\left\langle R_{4}\right\rangle$, almost completely in the bend-plane (i.e., much more $x$ contribution than $y$ ). In addition, the slice mismatch parameter is shown in $x$ and $y, \zeta_{x, y}$ (see Eq. 7.26), to indicate the beta/alpha function variations along the bunch. The calculation of the gain-length and saturation power does not, however, include the mismatch variation over the slices, $\zeta_{x, y}$, or the transverse oscillation amplitudes of each slice, $\left\langle R_{4}\right\rangle$. These effects, along with undulator wakefields, misalignments, and pole errors, may further increase the gain-length beyond what is shown here. 

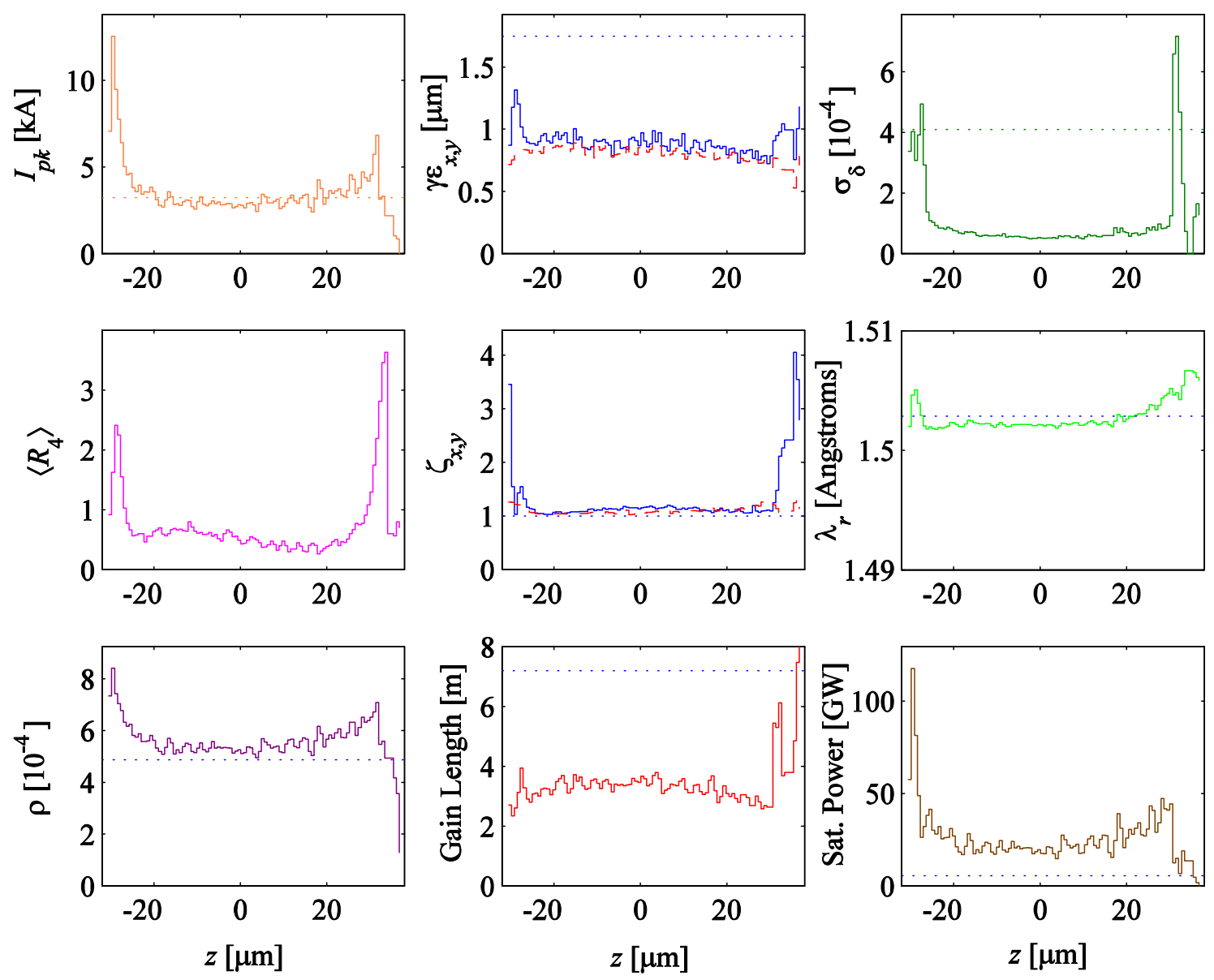

Figure 7.49 Beam and FEL evaluation of the sliced beam at $14.35 \mathrm{GeV}$ using $80 \%$ of beam core. The dotted lines represent the bunch-length-integrated values, which are irrelevant.

\subsubsection{Beam Jitter Simulations}

Full start-to-end jitter simulations have been performed using the tightest (bold) tolerances from Table 7.5 and repetitively tracking the entire system while varying machine parameters such as charge, gun timing, rf phases, and rf voltages, etc. The gun-laser timing jitter is reduced to $0.5 \mathrm{psec}$ here (as compared to $0.7 \mathrm{psec}$ in Table 7.5) to reduce the final energy jitter level from $0.1 \%$ to $0.06 \% \mathrm{rms}$. The simulations apply repeated $6 \mathrm{D}$ particle tracking, with $10^{5}$ macroparticles per tracked beam pulse, using the computer codes Parmela [11], Elegant [10], and Genesis [39]. No misalignments are added, so the effects of transverse wakes are not yet included here.

Parmela is used to simulate the photoinjector, ending at $150 \mathrm{MeV}$, because it includes space charge forces, which are important in the gun and L0-linac. Elegant is used for the remainder of the linac, ending at the entrance to the undulator. The macroparticle output of Parmela is used directly as input to Elegant, which is a 6-D tracking code that includes rf curvature effects, longitudinal and transverse wakefields of the accelerating structures, coherent synchrotron radiation, incoherent synchrotron radiation, and chromatic effects in quadrupoles and dipoles. Elegant ignores space-charge forces, which is acceptable because of the high beam energy beyond the $150-\mathrm{MeV}$ injector. 
Genesis is used for the FEL calculations. Ideally, Genesis would be used to perform full timedependent calculations for each simulated pulse. However, this would require $\sim 10^{7}$ macroparticles per pulse, and is not practical. Instead, the output of Elegant is cut into 136 longitudinal slices; chosen because it is near the number of slippage lengths in the bunch. Each slice is analyzed to obtain relevant first and second moments, i.e., energy, energy spread, centroids of particle position and angle, rms emittances, Twiss parameters, and beam current. Each slice is simulated independently in Genesis, under the implicit assumption that slices do not influence each other. The low- and high-energy tails of the beam are also removed, to avoid artificially inflating the rms energy spread.

Jitter is included in the Parmela and Elegant simulations using gaussian random numbers with a $\pm 3 \sigma$ cut-off. The variation in gun charge output, $Q$, is modeled as $Q=Q_{0}\left[1+(0.03) \cdot \Delta \varphi_{l}\right] \cdot\left[1+\Delta E_{l} / E_{l}\right] \cdot\left[1+\Delta V_{g} / V_{g}\right]$, where $\Delta \varphi_{l}$ is the laser phase error, $\Delta E_{l} / E_{l}$ is the relative laser energy error, and $\Delta V_{g} / V_{g}$ is the relative gun voltage error. The coefficient of $(0.03)$ is an empirical value obtained from experiments with a BNL-style gun at the Low Energy Undulator Test Line (LEUTL) at APS/ANL [40].

Table 7.21 Results of start-to-end jitter simulations using Parmela, Elegant, and Genesis.

\begin{tabular}{lccccc}
\hline Parameter & symbol & units & mean & rms & $\begin{array}{c}1 / 2 \text { quartile } \\
\text { range }\end{array}$ \\
\hline FEL 3D power gain length & $L_{g}$ & $\mathrm{~m}$ & 3.53 & 0.19 & 0.13 \\
\hline FEL output power & $P_{0}$ & $\mathrm{GW}$ & 6.8 & 1.6 & 1.0 \\
\hline Relative $e^{-}$energy error $\left(E_{0} \approx 14.346 \mathrm{GeV}\right)$ & $\Delta E / E_{0}$ & $\%$ & 0 & 0.06 & 0.04 \\
\hline Peak current & $I_{p k}$ & $\mathrm{kA}$ & 3.3 & 0.27 & 0.17 \\
\hline Bunch length (full-width of $80 \%$ core slices) & $\Delta t_{F W}$ & $\mathrm{fs}$ & 188 & 19 & 13 \\
\hline RMS $e^{-}$energy spread & $\sigma_{\delta}$ & $10 \%$ & 0.8 & 0.07 & 0.03 \\
\hline Horizontal normalized emittance & $\gamma \varepsilon_{x}$ & $\mu \mathrm{m}$ & 0.80 & 0.02 & 0.01 \\
\hline Vertical normalized emittance & $\gamma \varepsilon_{y}$ & $\mu \mathrm{m}$ & 0.70 & 0.01 & 0.01 \\
\hline Bunch arrival time & $\langle\Delta t\rangle$ & $\mathrm{fs}$ & 0 & 45 & 31 \\
\hline Horizontal centroid amplitude $(\%$ of beam size $)$ & $A_{x}$ & $\%$ & 84 & 8.0 & 0.4 \\
\hline Vertical centroid amplitude $(\%$ of beam size $)$ & $A_{y}$ & $\%$ & 8.0 & 0.6 & 4.3 \\
\hline
\end{tabular}

Table 7.21 lists the results of simulations with 227 different beam pulses (i.e., random seeds). The quantities for which statistics are shown are averaged or summed over the central $80 \%$ of the slices (the "core slices"), which excludes from analysis the ends of the bunch, which are heavily corrupted by CSR and can be neglected for FEL evaluation. The bunch length is the full length of the core slices. The horizontal centroid amplitude, $A_{x}$, for a slice is defined as $A_{x}{ }^{2}=\left[x^{2}+\left(\alpha_{x} x+\beta_{x} x^{\prime}\right)^{2}\right] /\left(\varepsilon_{x} \beta_{x}\right)$, where $x$ and $x^{\prime}$ are the position and angle of the centroid of the 
slice, $\alpha_{x}$ and $\beta_{x}$ are the projected Twiss parameters, and $\varepsilon_{x}$ is the projected geometric emittance. The vertical centroid amplitude, $A_{y}$, is defined in an analogous fashion.

In addition to mean values over the simulated pulses, the table also lists rms deviations and half the quartile ranges. Where meaningful, the rms deviations and quartile ranges are expressed as percentages of the corresponding mean values. The quartile range is the interval containing the central $50 \%$ of the samples. Unlike the rms deviation, a few outlier points do not affect it. (For a gaussian distribution, the half-quartile-range is $\sim 70 \%$ of the standard deviation.) From this observation one sees that the rms values for the energy spread, horizontal emittance, and horizontal centroid amplitude are all 'pulled' by outlier points.

Figure 7.51 shows gain length strongly correlated with current, energy spread, and horizontal centroid deviation, with the expected sign. For example, higher current produces shorter gain length. One also sees that $A_{x}$ is on average fairly large, which is surprising given that the trajectory and angle for the ideal beam are steered to zero. However, this is understandable since the steering correction is computed, as in practice, for the entire beam, rather than the core slices with energy tails removed.
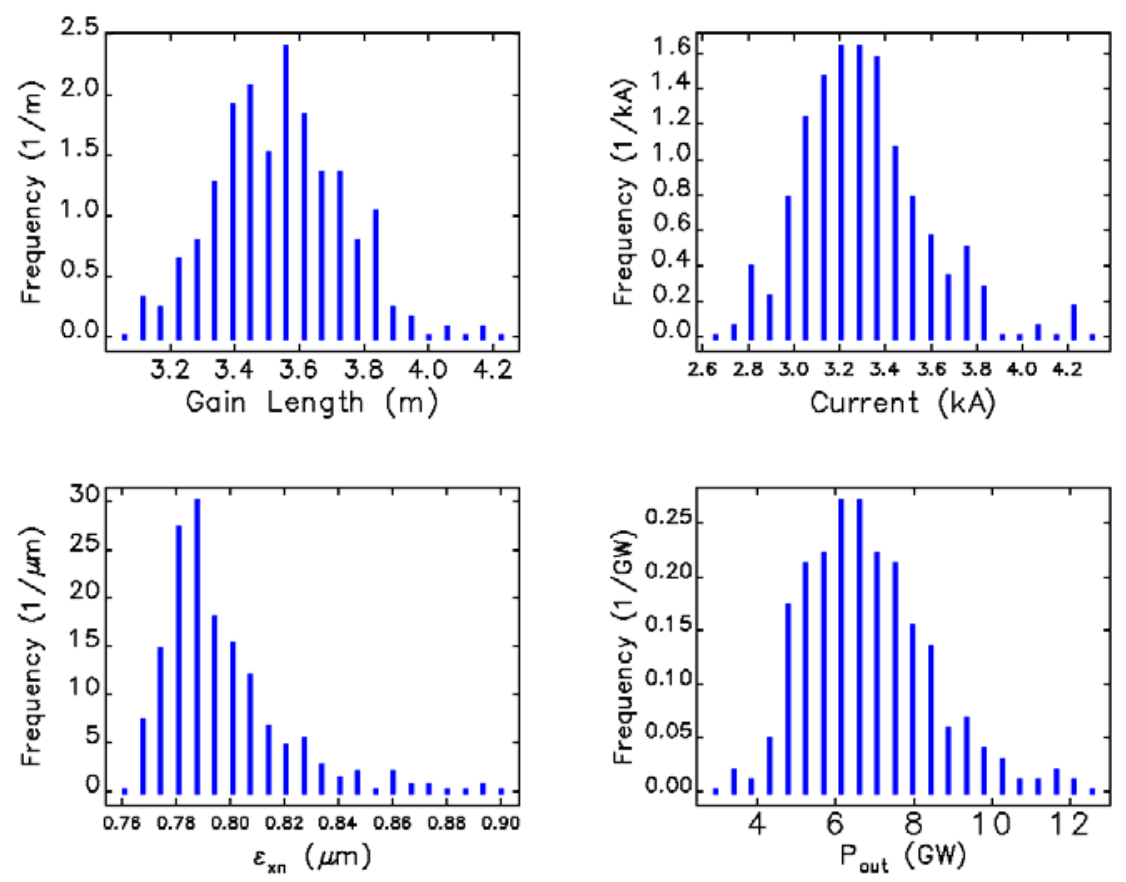

Figure 7.50 Distributions of core-slice-averaged values for the beam and FEL for 227 seeds.

Figure 7.51 shows scatter plots of the core-slice-averaged gain length and core-slice-averaged beam properties for the 227 random seeds (i.e., 227 varied beam pulses).

Computing correlation coefficients between the FEL properties and the jittered parameters indicates which parameters are most responsible for FEL output jitter. This analysis shows that $22 \%$ of FEL output power variation is due to gun-laser timing jitter, with another $19 \%$ due to L1 rf phase jitter. Similarly, $15-20 \%$ of the variation in the light wavelength is due to each of the 
quantities laser timing, L0 voltage, L1 phase, and L1 voltage. This illustrates the importance of start-to-end jitter simulation, given that all of these quantities are in the photoinjector or the first section of the linac.
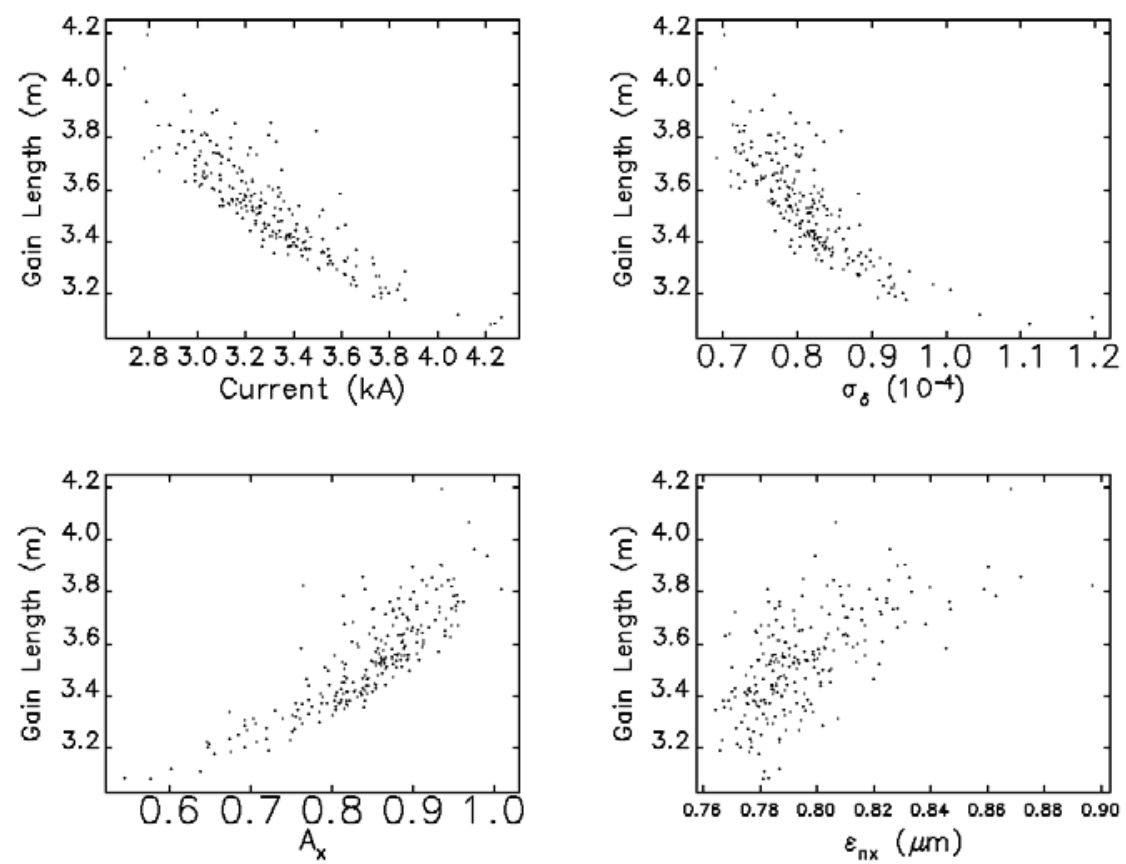

Figure 7.51 Scatter plots of the core-slice-averaged gain length and core-slice-averaged beam properties for the 227 random seeds (i.e., 227 varied beam pulses).

\subsection{Radio Frequency Systems}

The installed complement of S-band klystrons in the SLAC linac, from sector-21 through sector-30, is capable of accelerating the LCLS beam to the required energy of $14.35 \mathrm{GeV}$. This includes $6 \%$ overhead to allow for klystron failures and maintenance. In addition to setting the electron beam energy, and hence the optical wavelength of the LCLS FEL, precise control of rf phase and amplitude is also required to manipulate the longitudinal phase space of the beam to produce the desired short bunch at the end of the linac. Specifically, the linac $\mathrm{rf}$ is used to introduce energy-time correlations for bunch compression and to compensate for wakefields generated by the accelerating structures. This process is supplemented with an additional, higherharmonic X-band accelerating structure installed ahead of the first bunch compressor. The success of the bunch compression and wakefield compensation schemes requires very tight tolerances for phase and amplitude control of the linac, as described earlier in this chapter.

The jitter tolerance specifies the pulse-to-pulse variation that is acceptable in the linac phase and amplitude parameters and still maintains the desired peak current and energy at the entrance to the undulator. Pulse-to-pulse random variations cannot be corrected by feedback and therefore place upper limits on the phase and amplitude noise level of individual components such as klystrons. Longer-term drifts, ranging from several seconds to several hours, can be corrected by 
feedback systems. It is assumed that beam-based diagnostics of relative bunch length and relative energy will be developed with sufficient accuracy to provide feedback for the rf phase and amplitude to control variations slower than $\sim 0.5 \mathrm{~Hz}$.

Some modifications and improvements to the SLAC linac rf system are necessary to meet the tighter tolerances for LCLS operation. The changes must remain compatible with the other SLAC linac functions, chief among which is its role as the injector for the PEP II B-Factory. The rf controls and timing system must coexist with the PEP II injection cycles as well as allow the linac to be switched back to alternate beams for end-station experiments.

Although beam-based feedback will be the final mechanism to stabilize rf phase and amplitude, there are several reasons for keeping the low level rf distribution system as stable as possible. Some development work on feedback tuning algorithms is to be expected before subsystems can be cascaded together. Any extension in the duration over which the beam remains stable and within tolerance makes the task of tuning easier, both during the period of commissioning the accelerator and subsequent operation.

\subsubsection{RF Distribution in the Injector and Linac}

The major components of the rf system, starting with the gun laser, through the linac and bunch compressors, are shown schematically in Figure 7.52. The laser is included in this description since the laser oscillator mode-lock frequency and timing stability are critical to the layout of the low-level rf systems and the phase stability of the beam. Figure 7.52 shows that there is some rearrangement of the klystrons as a result of the LCLS installation in the SLAC linac, but no new S-band klystron stations, with their associated modulators need be added. This assumes that two of the present klystrons in sector-20, downstream of the positron production area used by PEP II, can be used to power the LCLS injector instead. The loss of a total of three klystrons, plus various section modifications as described in Table 7.28, still allows alternate beams to run through the linac for end-station experiments. The only impact is a slightly reduced $(\sim 2 \%)$ voltage overhead for such beams.

\section{INSTALLED KLYSTRON COMPLEMENT}

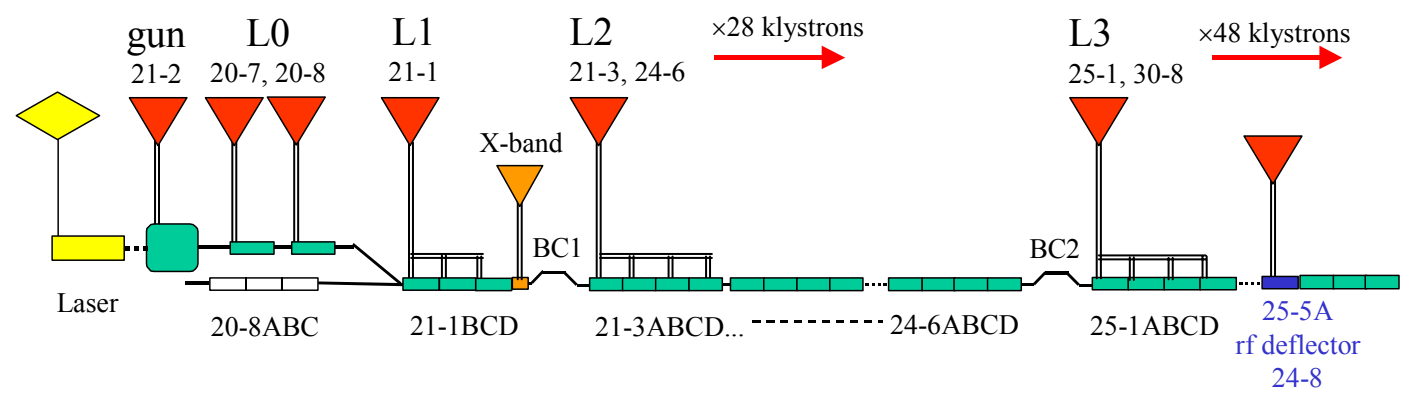

Figure 7.52 Allocation of major components of the LCLS rf system. 
The rf gun and the each of the two booster accelerating sections in the L0-linac are each powered by an individual klystron. This is to allow vernier control of the phase and amplitude of the individual sections, which is necessary for both diagnosing and optimizing the performance of the injector at different bunch charges. Individual klystrons also allow the phase and amplitude to be controlled at low power levels with existing technology where electronically controlled devices can provide the necessary fine resolution, pulse-to-pulse response, and reproducibility.

A standard SLAC S-band accelerating section is 3 meters long and normally the power from one klystron is divided equally over four 3-m sections. The L1-linac is made up of only three sections powered by one klystron. The first two sections are shortened by $20 \mathrm{~cm}$ to accommodate extra quadrupole/corrector/BPM packages, and the power is divided to give $50 \%$ in the first structure and $25 \%$ in the other two. The higher gradient in the first structure is slightly advantageous from a beam dynamics point of view.

Following L1, a short X-band rf section, operating at $11.424 \mathrm{GHz}$, provides $4^{\text {th }}$ harmonic correction to the energy gradient along the bunch before it passes through the first bunch compressor chicane. This section requires a modest power source to operate at $37 \mathrm{MV} / \mathrm{m}$ over a length of $0.6 \mathrm{~m}$ to generate the needed $22 \mathrm{MV}$ of X-band $\mathrm{rf}$.

The klystrons in the injector and L1 must operate unsaturated to provide for feedback control of the amplitude. A typical operating point would be $5 \%$ below the maximum power output of the klystron to allow enough overhead for feedback operation.

The L2 accelerating sections are powered by 26 klystrons plus 2 in standby as spares. The majority of these klystrons can be operated in saturation, with no amplitude control, and having global phase control. Two klystrons near the end of L2 will be operated unsaturated to provide for feedback control of the amplitude. Only one of these two klystrons will be in 'feedback' mode at any one time, with the other reserved as a spare, or as a standard saturated klystron. The feedback klystron will have its phase on-crest to decouple phase and amplitude control. The average phase of L2 will be controlled by feedback adjustment of the phase of the last full sector in L2 (sector-23). This provides a fine resolution control of the average phase, with only one of the four sectors varied, and yet provides adequate dynamic range. Using a sector at high energy will have the least impact on the relative energy profile and hence the focusing lattice in L2.

The L3 accelerating section is powered by 45 klystrons plus 3 klystrons in standby as spares. The majority of these klystrons can be operated in saturation, with no amplitude control, and having global phase control. Two klystrons near the end of L3 will also operate in unsaturated mode to provide for feedback control of the amplitude. The phase for the entire L3 linac will also be controlled by feedback using two or more sectors of L3.

An additional S-band klystron running unsaturated, with independent amplitude and phase control, will power the rf deflecting structure at the 25-5A location in the L3 linac. 


\subsubsection{Layout and Performance of the Present SLAC Linac RF}

The SLAC linac is divided into 30 sectors, of which the LCLS will utilize sectors 21 through 30. The rf distribution for two adjacent, nominal sectors is shown in Figure 7.53, showing how the rf power is derived for each sector and distributed to each of the eight klystrons in the sector. A 476-MHz master oscillator located in sector-0 of the linac transmits low-level power along a phase stabilized Main Drive Line (MDL).

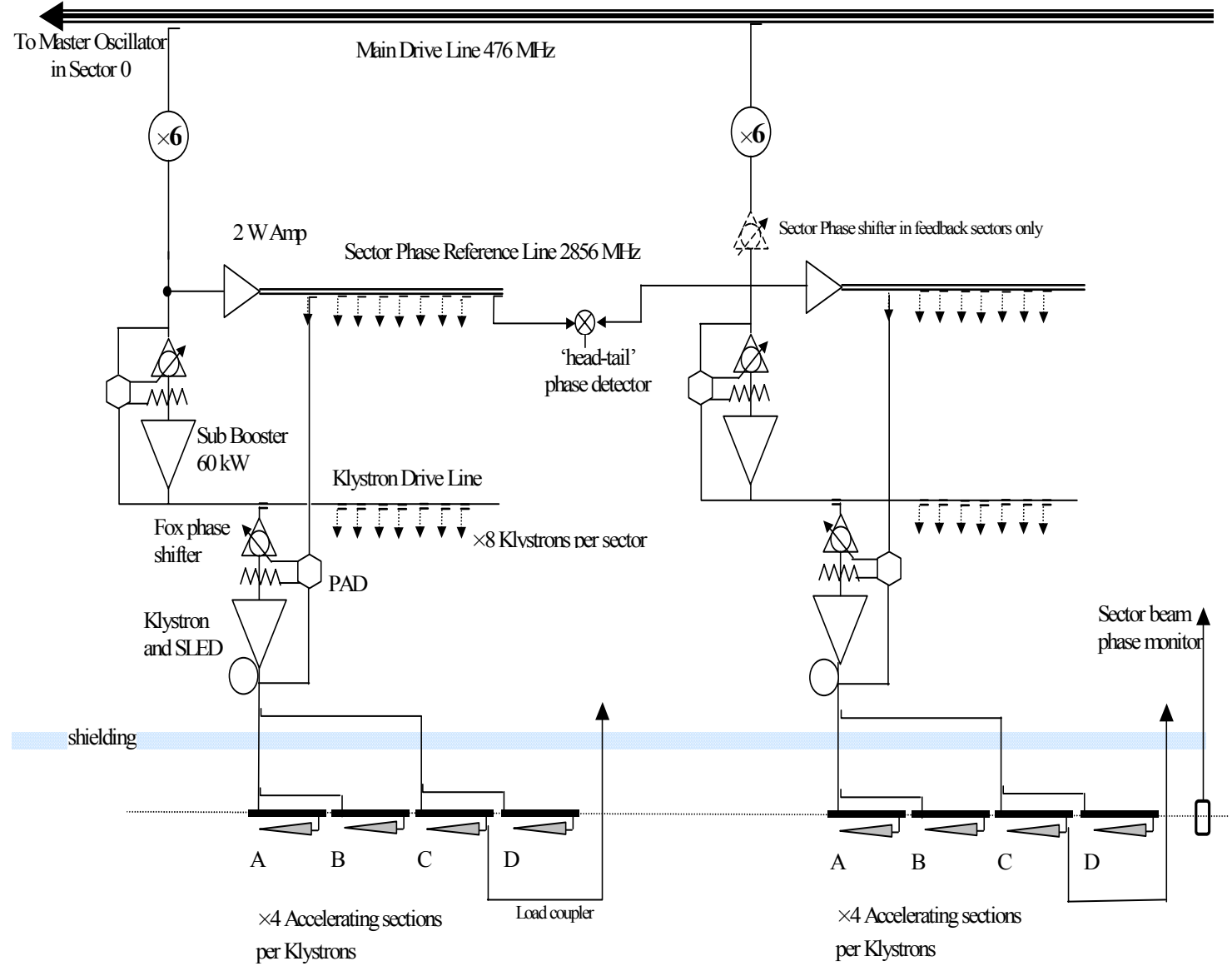

Figure 7.53 Schematic of two adjacent nominal sectors showing distribution of rf power to the klystrons.

An interferometer controls the overall phase length of the MDL to compensate for temperature related diurnal phase variations, an example of which is shown in Figure 7.54. At each sector boundary a $\times 6$-multiplier is coupled to the MDL and provides $2856-\mathrm{MHz}$ power for the sector phase reference line and the sub-booster driving 8 klystrons. The sector drive line and the Phase Reference Line (PRL) run the length of one sector and are temperature stabilized over most (but not all) of their length. A 'head-tail' phase detector monitors the phase error between adjacent sectors. Phase errors of the order of several degrees between adjacent sectors are typical in the present distribution system, as shown in Figure 7.55, and are the result of imperfect compensation of temperature discrepancies and other various sources. 


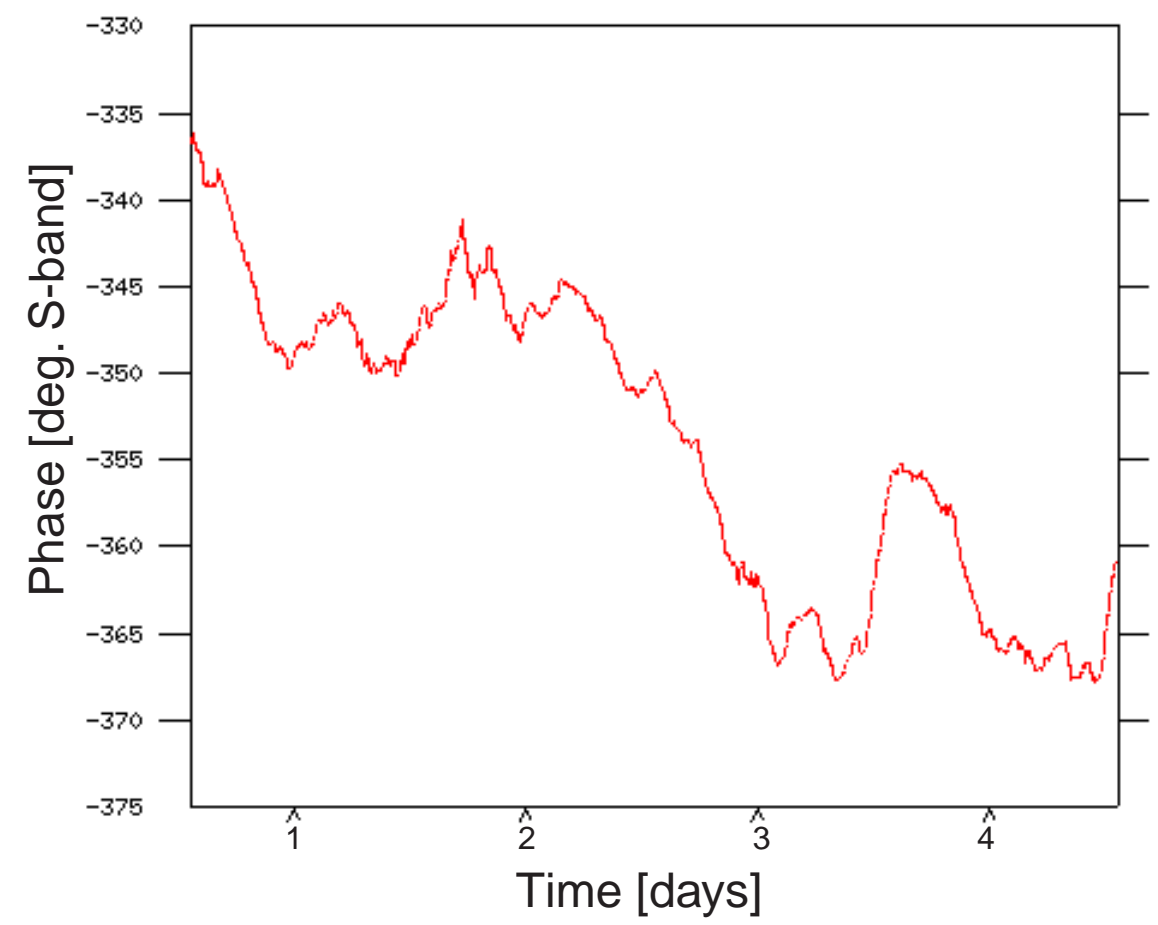

Figure 7.54 Measurement of phase variations seen along the linac main drive line over a period of several days.

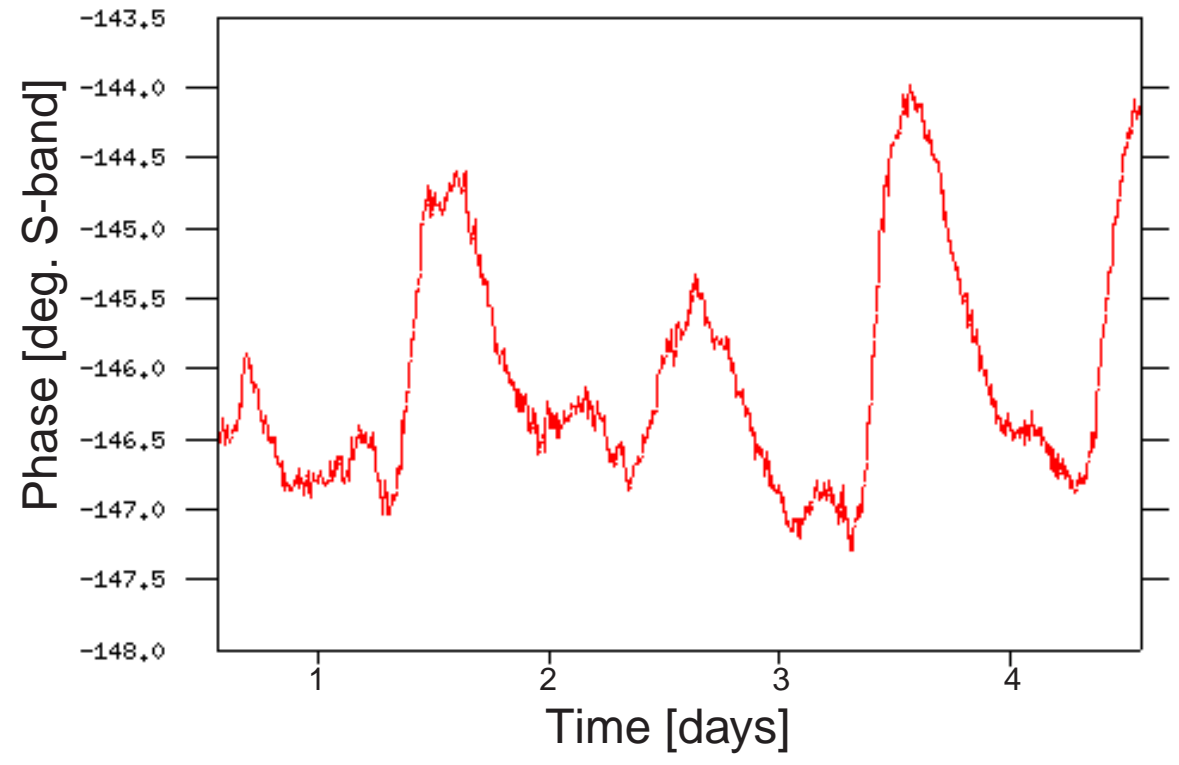

Figure 7.55 Measurement of the phase variations between two adjacent linac sectors over a period of several days.

The control loops for each klystron are shown in greater detail in Figure 7.56. The phase variation measured locally at individual klystrons is less pronounced than the errors for the sector drive line. 


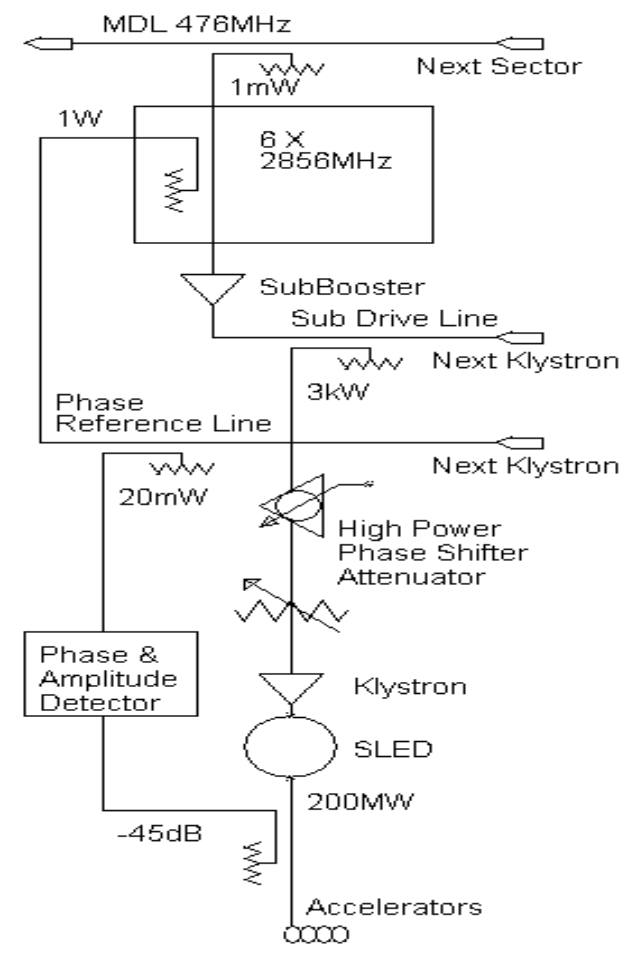

Figure 7.56 Drive power and control loops for a typical linac klystron.

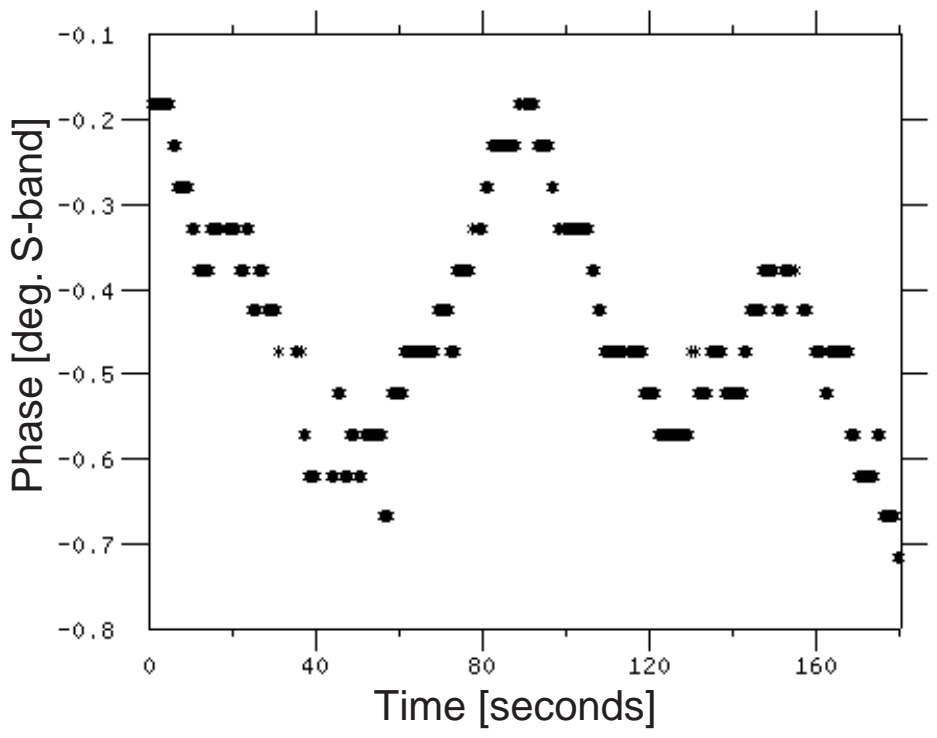

Figure 7.57 Phase variations measured at the PAD of a single klystron over a period of minutes. Each point is an average over 32 beam pulses.

Measurements at the Phase and Amplitude Detector (PAD), in Figure 7.57, show typically less than $1^{\circ} \mathrm{S}$-band $\mathrm{rms}$ phase variation over several minutes. On a 17 -second time scale, the pulse-to-pulse phase variation of a single klystron measured at its $\mathrm{PAD}$, shown in Figure 7.58, is stable to within $0.07^{\circ} \mathrm{rms}$. This data is typical of a sample of 73 operating klystrons that were 
scanned during a period when the outside temperature was stable. The pulse-to-pulse amplitude stability over 2 seconds is $0.06 \%$ rms, measured at the PAD, as shown in Figure 7.59.
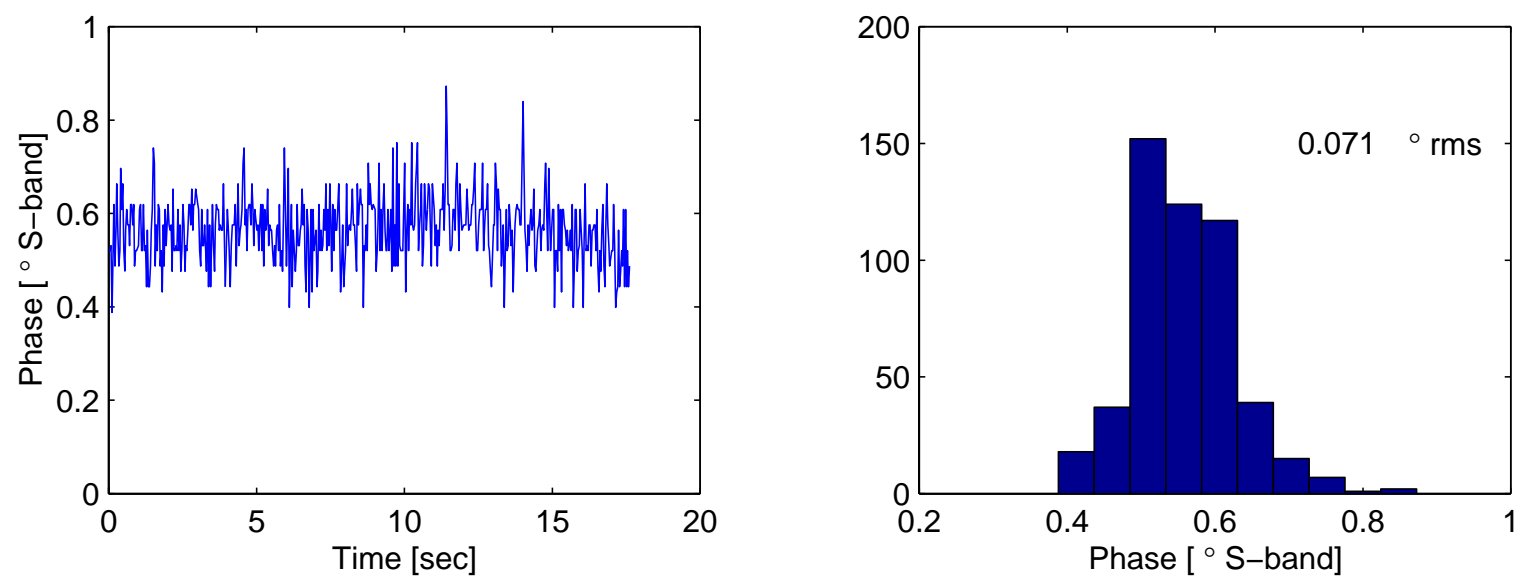

Figure 7.58 Pulse-to-pulse phase variations, and histogram, measured at PAD of a single klystron shows 0.07 -degree S-band rms variation over 17 seconds.

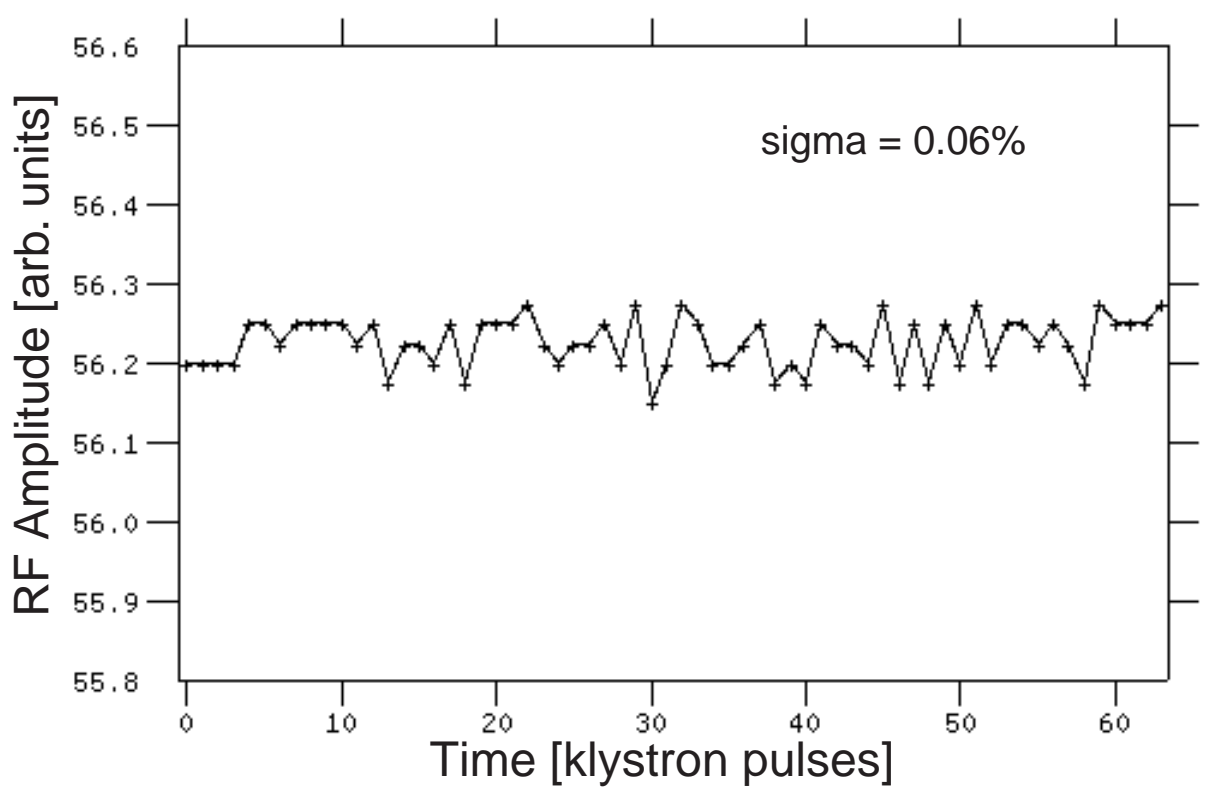

Figure 7.59 Pulse-to-pulse relative amplitude variations measured at the PAD of a single klystron shows $0.06 \%$ rms variation over 2 sec (horizontal axis is in $1 / 30$-sec ticks).

Analysis of the performance of the present linac shows that individual SLAC klystrons, when selected for superior stability, can meet LCLS pulse-to-pulse jitter tolerances over a short $(\sim 2 \mathrm{sec})$ time scale. Some improvements are planned for the rf distribution and control system. These will facilitate beam-based feedback control to be applied to the LCLS linac sections. Phase and amplitude control of individual klystrons will be implemented for parts of the linac as well as global sector controls. The phase gymnastics for PEP II injection need to be decoupled from the 
LCLS rf distribution while preserving compatibility with the present timing control system. These changes are described in the following section.

\subsubsection{Improvements to the RF System}

Improvements are being planned for the SLAC linac rf and control systems and will be implemented before LCLS commissioning. The requirements of the subsystems and individual components are reviewed in this section in order that the rf system as whole can function within the tolerance specifications for the LCLS, as opposed to the isolated performance of single klystrons. The reliability of the components is also considered here, since the operating criteria for acceptable noise or drift of components becomes much narrower for LCLS than it has been in the past.

\subsubsection{Sub-boosters}

The present linac uses one klystron sub-booster per sector to provide $60 \mathrm{~kW}$ of drive power divided into 8 klystrons. Low power phase control is therefore only done at the input side to the sub-booster klystron and hence changes the phase of all 8 klystrons. The high power mechanical phase shifters on the individual klystrons are only capable of coarser, $0.125^{\circ}$ steps. Also they were not designed for pulse-to-pulse operation, typically making only a few tens of phase corrections per day in present linac operation. For comparison, the low-power phase shifter at the input to the sub-booster klystron is electronically controlled and its resolution is within the required tolerances.

The three klystrons in the injector, the L1-linac klystron, and the four feedback control klystrons in the L2 and L3 linacs, as well as the special X-band and rf deflector klystrons, will require individual sub-boosters. This allows low-power phase shifters to be used at each individual klystron, on the input side to its sub-booster, to enable the necessary pulse-to-pulse fine resolution phase control.

The power requirement for individual sub-boosters can be met with solid-state amplifiers. These solid-state-sub-boosters (SSSBs) have the additional advantages of lower noise level and greater reliability than the present klystron sub-boosters.

\subsubsection{Phase and Amplitude Control Units}

Klystrons that are equipped with the new SSSBs and low-power phase shifters will require some revisions to their control systems, as shown in Figure 7.60. The existing Phase and Amplitude Detecting units (PADs) meet the LCLS specifications.

The Isolator-Phase-shifter-Attenuator chassis (IPA) contains the high-power mechanical phase shifter. The new SSSB will have an integral low power phase shifter and high power attenuator, both of which will be capable of pulse-to-pulse corrections. In these stations the IPA chassis will no longer be required. 


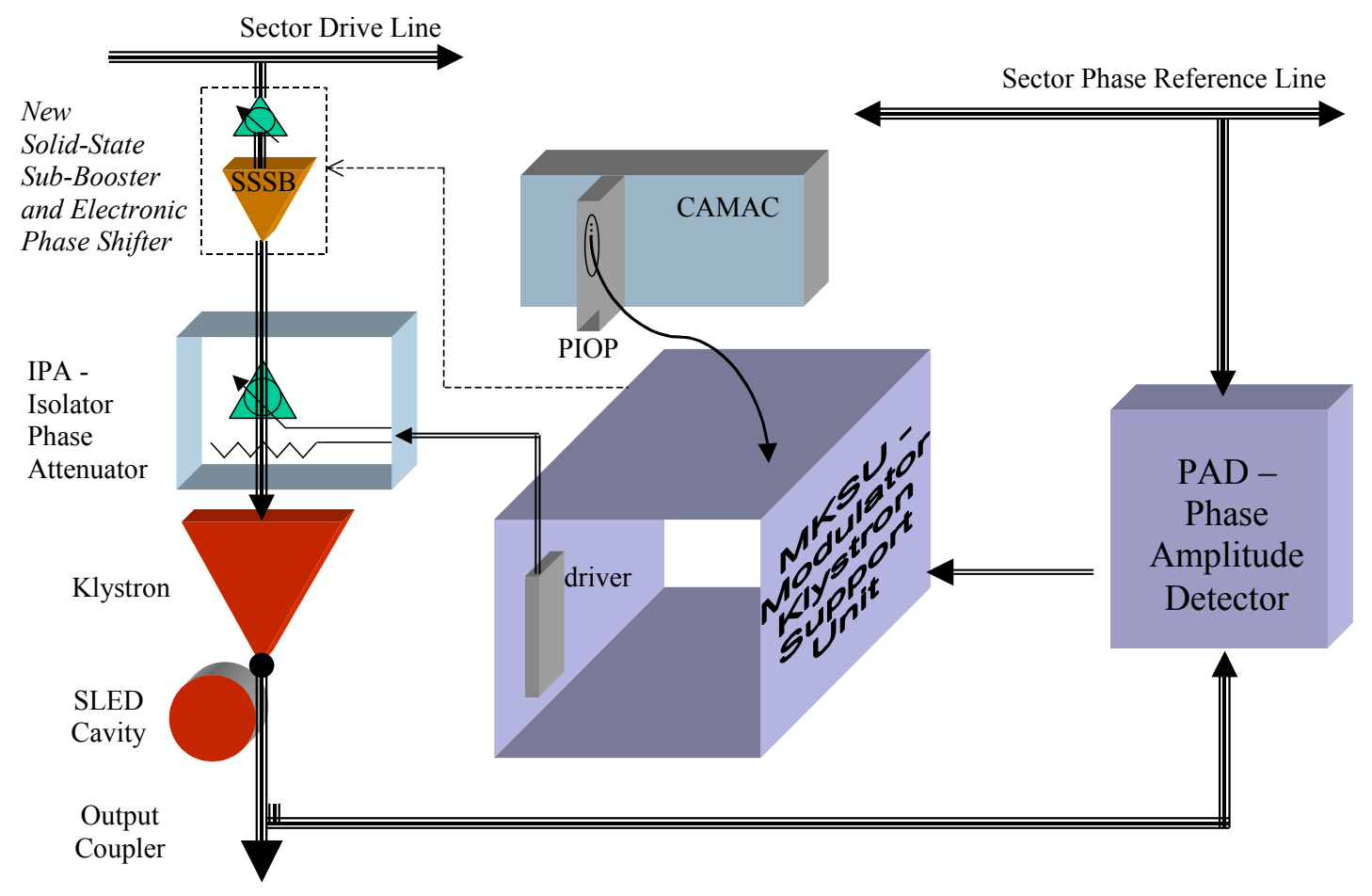

Figure 7.60 Control system components at each klystron station.

The Modulator-Klystron Support Unit (MKSU), which contains the drive hardware for the IPA, will need to be modified to accommodate the additional drivers for a low-power phase shifter and high power attenuator.

A new Parallel Input/Output Processor (PIOP) for the CAMAC control of the MKSU will be designed to accommodate the higher resolution and characteristics of the low-power phase shifter. The new PIOP will also incorporate software to allow for pulse-to-pulse feedback and more diagnostics. The existing PIOP is based on obsolete hardware and cannot be upgraded without extensive redesign work.

\subsubsection{Master Oscillator}

The present Main Drive Line (MDL) transmits $476 \mathrm{MHz}$ along the linac from sector-0 where it is derived from a master oscillator $\mathrm{VCO}$ at $8.5 \mathrm{-MHz}$. The $8.5-\mathrm{MHz}$ coincides with the revolution frequency of the damping rings. The frequency shifts on the MDL for the purposes of PEP II injection are not compatible with the fixed frequency, mode-locked laser of the LCLS photoinjector.

A second master oscillator will instead be housed at the LCLS injector, as shown in Figure 7.61. This new master oscillator will be phase locked to the MDL to allow for straight ahead beams being run down the LCLS portion of the linac for end station experiments. The master oscillator will be based on a crystal oscillator VCO in a temperature-stabilized oven. The crystal frequency will be chosen to be compatible with the mode-lock frequency of the laser and be a sub-harmonic of $2856 \mathrm{MHz}$. 


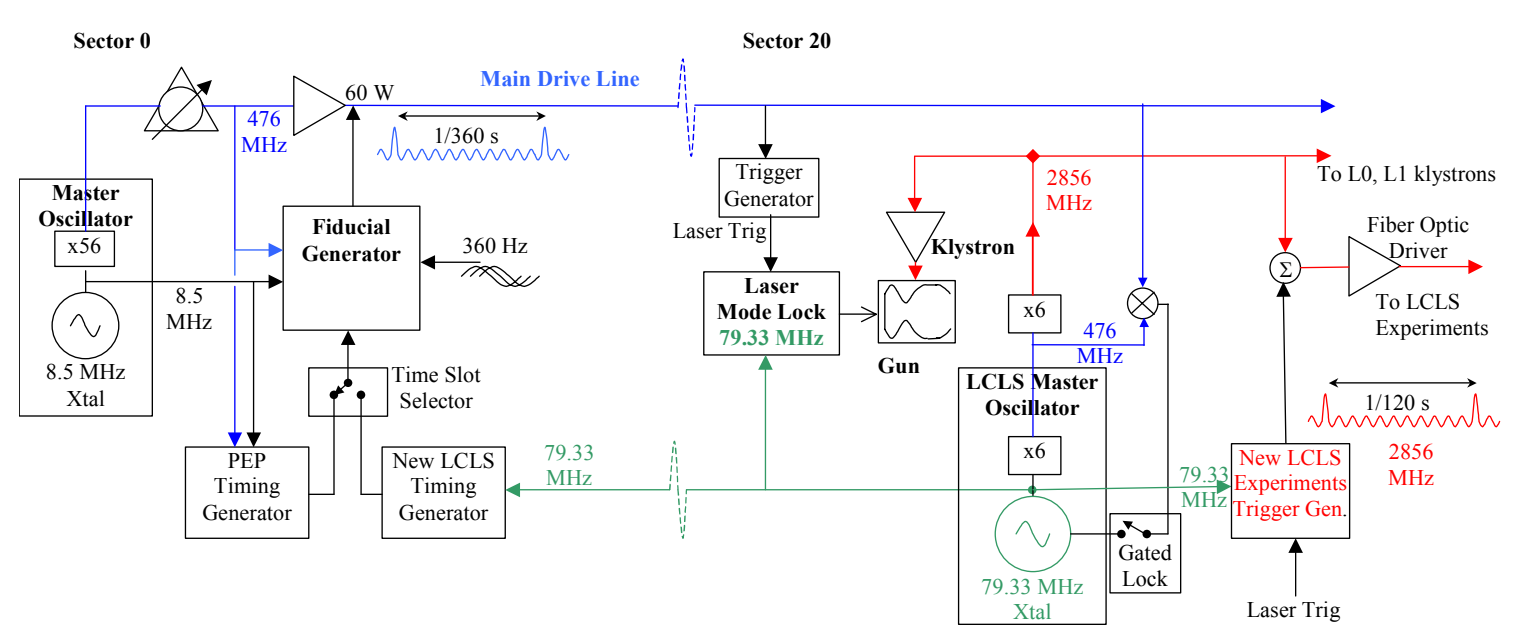

Figure 7.61 Timing and rf distribution in sector- 0 and sector-20 of the linac.

The mode-lock frequency of the laser should be as close as possible to $80 \mathrm{MHz}$, plus or minus one or two $\mathrm{MHz}$ deviation at most. This is dictated by the operating range of commercially available lasers that have the best stability properties at the desired wavelength and power for LCLS operation. The present conceptual design uses $79.33 \mathrm{MHz}$ VCO for the mode-lock frequency, which is the 36th sub-harmonic of $2856 \mathrm{MHz}$. For comparison, the damping ring 8.5$\mathrm{MHz}$ revolution frequency is the 336th sub-harmonic of $2856 \mathrm{MHz}$. The sector-0 master oscillator VCO and the LCLS VCO frequencies are therefore in the ratio 6:56.

For instrumentation and diagnostics associated with the laser it is convenient to have a phaselocked reference signal close in frequency to $10 \mathrm{MHz}$. The crystal for the VCO will be at $79.33 \mathrm{MHz}$ and a divide-by-eight module will supply the 9.916-MHz diagnostic reference signal for the laser.

The gun, the L0, and the L1 klystrons are all located close to the LCLS master oscillator and will use this stable 2856-MHz reference as their drive signal. These are the systems with the most stringent phase tolerances and so will share a single, local phase reference.

\subsubsection{Timing System}

The present linac timing system is based on $360-\mathrm{Hz}$ fiducials superimposed on the $476-\mathrm{MHz}$ MDL frequency. Its purpose is to synchronize the beam at the damping ring's $8.5-\mathrm{MHz}$ revolution frequency, and hence the $2856 \mathrm{MHz}$ in the linac, with the phase zero-crossing of the $360-\mathrm{Hz}$ power grid. This feature is to be preserved in the LCLS linac because of shared hardware with the PEP II system.

In order for the LCLS to operate, the fiducial generator must also supply pulses that are synchronized to the zero-crossing of the $79.33-\mathrm{MHz}$ laser mode-lock frequency. This requires a new 79.33-MHz connection from the LCLS VCO to the fiducial generator at the sector- 0 master oscillator.

The linac can operate at a maximum of $120-\mathrm{Hz}$ repetition rate, so there are 3 possible "time slots" for it to be synchronized to within the $360-\mathrm{Hz}$ line power cycle. The LCLS beam can 
therefore run on a different time slot from the PEP II beam. Beam codes in the timing system will allow PEP II beams to be read out on one time slot and LCLS beams on another. This will allow the same control system hardware, such as the microprocessors, BPMs, etc., to be shared between PEP II and LCLS.

\subsubsection{Synchronization Pulses for Experiments}

As indicated in Figure 7.61, a new 2856-MHz phase reference line is planned to provide the LCLS scientific experiments with synchronization pulses. It will take advantage of fiber optic technology to avoid attenuation over the longer distance. Furthermore, only a single fiber is required along the length of the linac, without multiple receivers or couplers enroute. A distribution system for the synchronization pulses is planned in the experimental halls.

\subsubsection{Beam Diagnostics}

Pulse-to-pulse measurements of relative bunch length at $1-10 \mathrm{~Hz}$ will be available for feedback control of the rf phase. These will be based on CSR detectors and/or cavity spectral power monitors. The bunch length monitors will be calibrated against the absolute bunch length measurement from the rf deflecting cavity and/or the zero-phasing technique (see Section 7.8.2).

Direct measurement of the beam phase with respect to the linac rf is desirable from the point of view of feedback control. However, the thermal sources of phase drift that need to be compensated in the rf distribution system are equally likely to disturb the phase measurement at the $0.1^{\circ} \mathrm{S}$-band level required here. A technique of measuring the phase of the beam-induced signal in the accelerating structures relative to the drive $\mathrm{rf}$ has been studied. One accelerating structure per klystron is typically equipped with an output coupler on its load, where such measurements can be made. Each sector is also equipped with an S-band beam phase monitor that can also provide some information of the average beam phase with respect to that sector. Both these techniques will be developed as options for phase monitoring and control. This may be a suitable technique for long-term phase control at the 1-deg level in the L3-linac where, with no next bunch compressor, there are no other phase diagnostics.

\subsubsection{Reliability}

Critical klystrons at the gun, L0 and L1 linacs need to be specifically chosen from the complement of SLAC klystrons in order to meet the stability requirements. This sorting technique is presently used in the existing SLAC linac for critical locations in the particle sources and bunch compressors.

\subsection{Instrumentation, Diagnostics, and Feedback}

Critical to the preservation of the transverse emittance and the generation of a low-energy spread micro-bunch are the precise measurement techniques and correction schemes used to initially commission and maintain the machine. The LCLS accelerator has many phase space diagnostics and correction schemes built into the design. The relevant beamlines and optics are specifically designed to enhance the performance of these critical diagnostics. 


\subsubsection{Transverse Emittance Diagnostics}

There are five different transverse emittance diagnostic stations distributed along the LCLS accelerator, of which four are new installations (the existing sector-28 station [41] will be slightly modified). In three of these cases the emittance measurement is accomplished with four consecutive profile monitors placed along the beamline with appropriate phase advance between monitors to optimize resolution. (Only three monitors are necessary, with four used to improve resolution and provide redundancy.) The two low-energy stations use three consecutive profile monitors over a drift section. These allow non-invasive emittance measurements to be made during normal machine operation, or can also be made using a 'quadrupole-scan' technique, taking advantage of the nominal beam waist on the center profile monitor. The emittance measurement stations and their parameters are summarized below in Table 7.22.

Table 7.22 Transverse emittance measurement stations along the LCLS $\left(\gamma \varepsilon_{x, y}=1 \mu \mathrm{m}\right)$.

\begin{tabular}{lcccccc}
\hline Location & $\begin{array}{c}\text { Station } \\
\text { Name }\end{array}$ & $\begin{array}{c}\text { Energy } \\
(\mathrm{GeV})\end{array}$ & $\begin{array}{c}\sigma_{\boldsymbol{x}} \\
(\mu \mathrm{m})\end{array}$ & $\begin{array}{c}\sigma_{\boldsymbol{y}} \\
(\mu \mathrm{m})\end{array}$ & $\begin{array}{c}\text { No. of Prof. } \\
\text { Monitors }\end{array}$ & Existing \\
\hline Following L0 & ED0 & 0.150 & $65-130$ & $65-130$ & 3 & No \\
\hline Following BC1 & ED1 & 0.250 & $40-80$ & $40-80$ & 3 & No \\
\hline At the end of Linac-2 & L2-ED & $4.1-4.5$ & $41-72$ & $42-70$ & 4 & No \\
\hline Sector-28 in Linac-3 & L3-ED & $9.1-10.9$ & $40-55$ & $39-57$ & 4 & Yes \\
\hline Prior to undulator & ED2 & 14.35 & 16 & 16 & 4 & No \\
\hline
\end{tabular}

The energy range of each diagnostic station listed in Table 7.22 (e.g., L2-ED) indicates accelerator sections separate the monitors. A range of beam sizes in the table represents the minimum and maximum over the several profile monitors. The ED0, ED1 and ED2 stations listed in Table 7.22 are dedicated, non-accelerating emittance diagnostic stations designed to produce reasonable sizes in $x$ and $y$ at all monitors. The betatron phase advance between profile monitors (PR) is set to the optimal value for a three or four-monitor station $\left(60^{\circ}\right.$ or $45^{\circ}$, respectively). These three sections are shown schematically in Figure 7.33 (ED0), Figure 7.19 (ED1), and Figure 7.36 (ED2), with small circles indicating profile monitor locations on the beamline schematics. In the case of ED0 and ED1, drift sections separate the monitors. In ED2, quadrupole doublets separate the monitors. In all cases there exists an upstream variable matching section which can be tuned in order to empirically match the beam. For three (four) monitors, a $60^{\circ}\left(45^{\circ}\right)$ separation minimizes emittance resolution sensitivity to incoming beta mismatch errors. Figure 7.62 shows the emittance measurement resolution for the four-PR systems ED2 (solid) versus the phase of an incoming beta-mismatch with a large amplitude of $\zeta=1.5$, as defined in Eq. (7.26). In this case, the subscripted parameters in Eq. (7.26) represent the ideal matched beam and the non-subscripted parameters are the perturbed beam. 


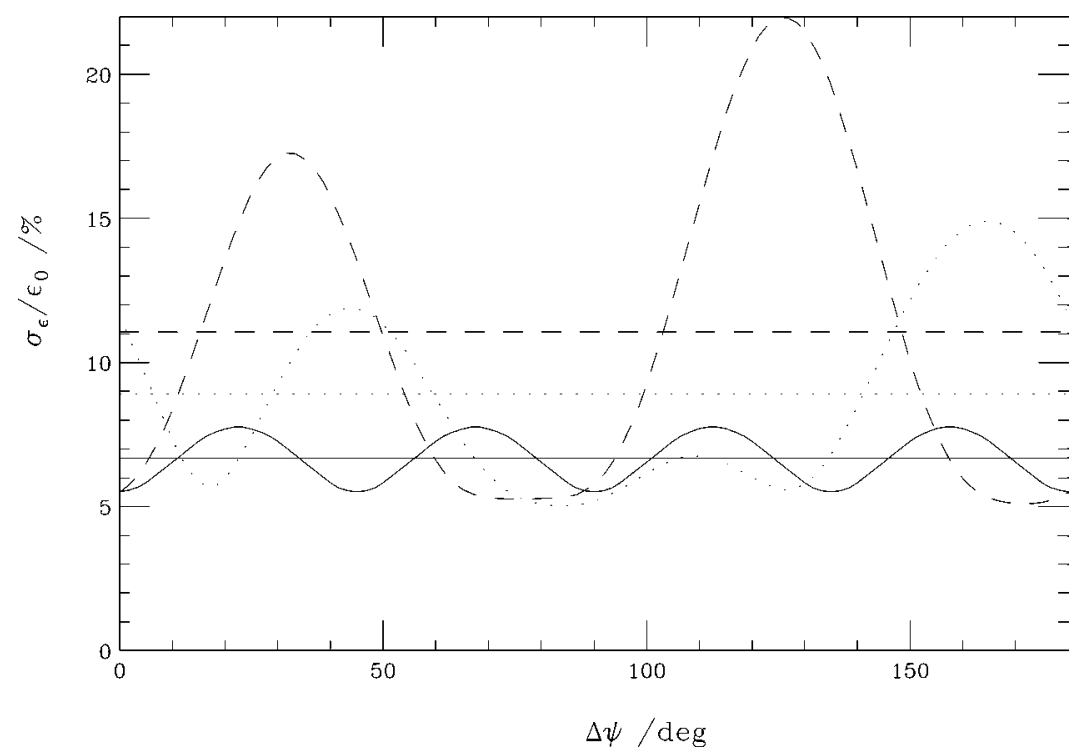

Figure 7.62 Emittance resolution versus beta-mismatch phase for a mismatch level of $\zeta=1.5$ and beam size resolution of $5 \%$. Resolution is shown for the ED2 sections (solid), the existing SLC sector-28 system (dash) and a modified sector-28 for SLC (dots). Flat lines are the average resolution over all possible beta-mismatch phases.

For comparison, the same resolution sensitivity to mismatch phase is plotted (dash) for the existing linac sector-28 system of the SLC. A modified SLC system is also shown (dots) - (see L3-ED description below). The relative beam size resolution used here is $5 \%$. The average emittance measurement resolution (for $\zeta=1.5$ ) for an optimally designed system $(6.7 \%)$ is nearly a factor of 2 better than that (11\%) of a system where profile monitors are a latter addition to an existing optical design (as in the case of the old SLC sector-28 system). The resolution at the worst-case phase is nearly three times better. The equal beam sizes at each of the four profile monitors, in the case of ED2, in both $x$ and $y$ may also help reduce systematic errors and will simplify the measurement interpretation (i.e., the beam is matched for ED2 when all profile monitors show equal beam sizes).

\subsubsection{ED0 Emittance Station}

The ED0 station (see schematic of Figure 7.32 or Figure 7.33) is used to confirm and optimize the emittance and matching from the injector. It is a resolution optimized three-monitor system (i.e., $60^{\circ}$ betatron phase advance separates each monitor). This choice is minimally sensitive to incoming beta function errors. For the nominal emittance of $\gamma \varepsilon=1 \mu \mathrm{m}$ at $150 \mathrm{MeV}$, the rms matched beam sizes are $130 \mu \mathrm{m}$ on the two outboard monitors, and $65 \mu \mathrm{m}$ on the middle monitor. The profile monitors are wire scanners. In addition, the center location, where a beam waist nominally exists, will also include an OTR monitor [33]. This will allow single shot measurements of beam size and also permit 'quad-scans' to be done at this location. The 'quadscan' is accomplished by varying an upstream quadrupole magnet's gradient and recording the beam size at the various readings. This has the resolution advantage of allowing many more than three beam size measurements to be used in the emittance calculation. It has the disadvantage of 
disturbing nominal operations during the 'quad-scan'. The two methods are complementary, and the 3-PR system envisioned allows either method to be used.

\subsubsection{ED1 Emittance Station}

The ED1 station (Figure 7.19) is placed directly after BC1. It is crucial for the measurement and empirical correction of dispersion errors generated in $\mathrm{BC} 1$. The $\mathrm{BC} 1$ chicane can also be switched off to help isolate the different errors of BC1, L1, and DL1. Like ED0, ED1 is a dedicated three-monitor (wire-scanners) emittance measurement section. At $250 \mathrm{MeV}$ the rms matched beam sizes are $80 \mu \mathrm{m}$ on the two outboard monitors, and $40 \mu \mathrm{m}$ on the middle monitor. As in the case of ED0, three wire scanners will be used, and an OTR monitor will also be located at the center location where a waist nominally exists.

\subsubsection{L2-ED Emittance Station}

The L2-linac is the most sensitive to orbit variation (note large emittance growth in Figure 7.15) and therefore can be expected to require frequent, perhaps daily, emittance optimization. The L2-ED station is placed at the end of L2 (Figure 7.16). It is a space-constrained, four-profilemonitor (wire-scanners) station with non-optimal phase advance and an expected emittance resolution of $\sim 10 \%$. This section will be used to empirically minimize the wakefield emittance dilution of L2. Due to space constraints, it is, at present, the least optimized system.

\subsubsection{L3-ED Emittance Station}

The L3-ED station (Figure 7.18) is composed of four existing sector-28 profile monitors (wire scanners). The linac optics, however, change somewhat in this area for LCLS operation and therefore a small modification to the existing scanner locations is called for. The first sector-28 scanner will be moved upstream by one cell ( 24 meters) and the last scanner will be moved downstream by one cell. The LCLS optics then produce an average of $54^{\circ}$ per plane between scanners, which is nearly optimal. In fact, the statistical resolution of the SLC configuration is also marginally improved (Figure 7.62: dots). The rms beam sizes are not identical at each monitor; however, the phase advance between scanners provides nearly optimal statistical measurement resolution. The L3-ED station will be used primarily to guide BC2 dispersion corrections. Emittance dilution occurring within L3 (see Figure 7.17) is expected to be very small due to the short bunch and small energy spread there.

\subsubsection{ED2 Emittance Station}

A final emittance measurement section (Figure 7.36) is included just upstream of the undulator entrance. This section will be used to make precise adjustments to the final horizontal and vertical beta functions (using quadrupoles QM33-36 of Figure 7.36) and to confirm and optimize the final emittance immediately before the undulator. This emittance measurement section is also used to diagnose potential emittance dilution arising in DL2 through dispersion errors or CSR. Like ED0 and ED1, ED2 is a dedicated emittance measurement section, but with a $16-\mu \mathrm{m}$ beam size at each monitor in both planes at $14.3 \mathrm{GeV}$. Four wire-scanners (and redundant OTR monitors at one of these locations) will be used for the profile monitors. 


\subsubsection{Bunch Length Diagnostics}

The peak current delivered to the LCLS undulator is a critical parameter. It is determined by both the charge and the final bunch length. To setup the compression, the bunch length needs to be measured before and after $\mathrm{BC} 1$, and after $\mathrm{BC} 2$. In addition, once the bunch compressors are set up, a bunch length feedback system will be required for stabilization of the compression. These feedback systems have not yet been fully designed.

The bunch lengths of interest are approximately 1000, 200, and $20 \mu \mathrm{m} \mathrm{rms}(10,2$, and 0.2 psec full width, respectively). Measuring 10-psec accurately using a streak camera is fairly standard. The 2-psec measurement is more challenging and probably not reliable. Direct measurement of the final 0.2-psec bunch is quite a different issue. Bunch length monitors [42] designed to use coherent synchrotron radiation (CSR) have demonstrated fast, non-invasive measurements in the LCLS regime. They, however, provide a relative bunch length measurement. Absolute bunch length requires an understanding of the frequency spectrum of the radiation, the various component attenuation functions, and the CSR process.

\subsubsection{Transverse RF Deflector}

A very promising technique to measure the micro-bunch after $\mathrm{BC} 2$ is to use a transverse rf deflecting cavity. This idea has been used in the past [43], [44] and has been suggested again recently [45]. The high frequency time variation of the deflecting field is used to 'pitch' or 'yaw' the electron bunch, while the resulting transverse beam width is measured on a simple profile monitor (OTR). This is a reliable, single-shot measure of the absolute bunch length. The technique is completely analogous to a streak camera, but with much better potential resolution. Detailed studies have been made of this technique [46], including wakefield and chromatic effects, and recent beam measurements have also been made [47]. As an additional benefit, 2.44meter long S-band rf deflecting structures are immediately available at SLAC, where they were fabricated and tested in the early 1960's [48]. A cut-away view of the S-band traveling-wave rfdeflector is shown in Figure 7.63.

The bunch length, $\sigma_{z}$, can be calculated from knowledge of the deflecting voltage, $V_{0}$, the rf wavelength, $\lambda_{\mathrm{rf}}$, and the beam energy at the screen, $E_{s}$.

$$
\sigma_{z} \approx \frac{\lambda_{\mathrm{rf}}}{2 \pi} \frac{E_{S}}{\left|e V_{0} \sin \Delta \psi \cos \varphi\right|} \sqrt{\frac{\left(\sigma_{y}^{2}-\sigma_{y_{0}}^{2}\right)}{\beta_{d} \beta_{S}}}
$$

Included here is the product of $\left(\beta_{d} \beta_{s}\right)^{1 / 2} \sin (\Delta \psi)$, which is the (measurable) vertical transfer matrix element from angle-to-position and deflector-to-screen. Finally, $\varphi$ is the $\mathrm{rf}$ phase of the deflector ( $\varphi=0$ at zero-crossing) and $\sigma_{y}$ and $\sigma_{y 0}$ are the measured vertical beam sizes with rf-on and rf-off, respectively. The voltage of the deflector is easily calibrated using simple BPM measurements as a function of RF phase. Table 7.23 lists the parameter values associated with this bunch length measurement after BC2. The rf deflecting structure will be placed downstream of the BC2 chicane at $5.4 \mathrm{GeV}$ at the 25-5a location (at $S \approx 475 \mathrm{~m}$ in Figure 7.18) where an 
existing 3-meter accelerating structure will need to be removed. The screen will be located at the 25-902 location (at $S \approx 520 \mathrm{~m}$ in Figure 7.18) where space is available.

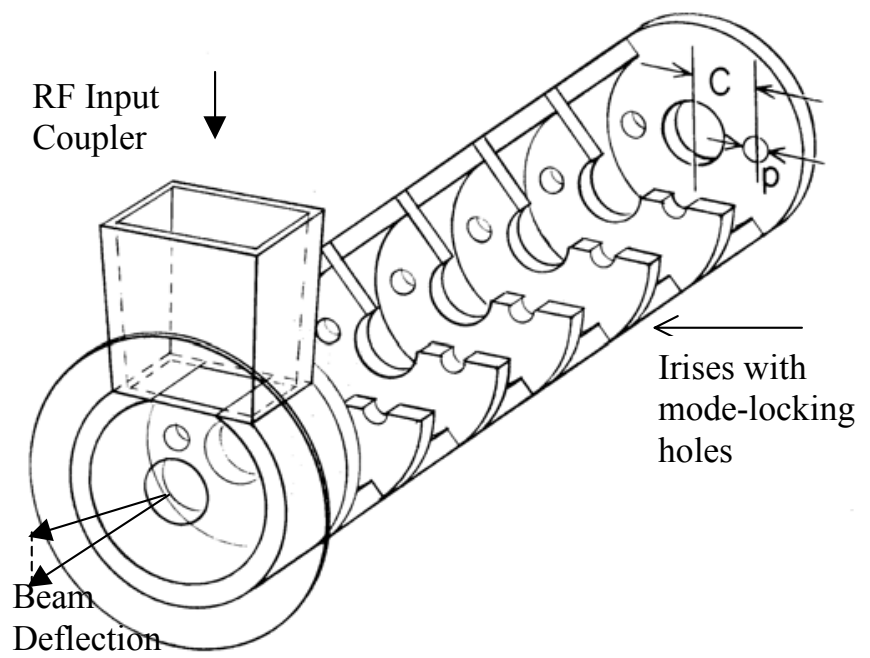

Figure 7.63 Schematic of a SLAC S-band transverse deflecting structure. The kick is vertical here.

Table 7.23 Parameters for bunch length measurement using a 2.44-meter long S-band rf deflecting structure at the 25-5a location and a screen at the 25-902 location.

\begin{tabular}{lccc}
\hline Parameter & symbol & value & unit \\
\hline rf deflector voltage & $V_{0}$ & 20 & $\mathrm{MV}$ \\
\hline Peak input power & $P_{0}$ & 25 & $\mathrm{MW}$ \\
\hline rf deflector phase $\left(\right.$ crest at $\left.90^{\circ}\right)$ & $\Phi$ & 0 & $\mathrm{deg}$ \\
\hline Nominal beam size at screen & $\sigma_{y_{0}}$ & 76 & $\mu \mathrm{m}$ \\
\hline Beam size with deflector on (two-phase mean) & $\sigma_{y}$ & 282 & $\mu \mathrm{m}$ \\
\hline Beta function at rf deflector & $\beta_{d}$ & 56 & $\mathrm{~m}$ \\
\hline Beta function at screen & $\beta_{s}$ & 60 & $\mathrm{~m}$ \\
\hline Betatron phase from deflector to screen & $\Delta \psi$ & 65 & $\mathrm{deg}$ \\
\hline Normalized rms emittance & $\varepsilon_{N}$ & 1 & $\mu \mathrm{m}$ \\
\hline Beam energy at deflector & $E_{d}$ & 5.4 & $\mathrm{GeV}$ \\
\hline Beam energy at screen & $E_{s}$ & 6.2 & $\mathrm{GeV}$ \\
\hline RMS bunch length & $\sigma_{z}$ & 22 & $\mu \mathrm{m}$ \\
\hline
\end{tabular}

The transverse $\mathrm{rf}$ deflector will be powered in a pulse-stealing scheme where the $\mathrm{rf}$ is switched on at $\sim 1 \mathrm{~Hz}$, while the machine operates at $120 \mathrm{~Hz}$. An off-axis screen is used to intercept the 'streaked' electron beam, while a pulsed opposite-plane kicker (or an rf phase at non-zero crossing) is used to knock that bunch over and into the screen. The rf 'streak' will be applied vertically ('pitch') so that beam correlations in the $x$ - $t$ plane, caused by CSR forces in the 
BC2 chicane, might be easily diagnosed. A simultaneous horizontal kicker will be used to move the beam horizontally to the off-axis screen. This keeps transverse wakefields from adding to the beam 'pitch', which would occur if a non-zero deflector phase were used. In addition to being used for bunch length measurements, the 'streaked' beam on the profile monitor also allows the possibility of doing 'slice' emittance measurements [49] in the horizontal plane.

Figure 7.64 shows the effect of a $20-\mathrm{MV}$ vertical deflector. The parameters are those given in Table 7.23. The figure shows three plots in two panels (deflector OFF at left and ON at right). At upper left of each panel is the bunch population in $y-z$ space. At upper right and lower left are the vertical $(y)$ and temporal ( $z$ ) projected distributions, respectively (note $22-\mu \mathrm{m}$ rms bunch length). The particle tracking is performed using Elegant and includes wakefields, second-order optical effects of momentum deviations, and the sinusoidal time variation of all $\mathrm{rf}$ fields. In this case the 'streak' is in the vertical plane, while a centroid kick is applied horizontally (not visible in these plots). With the 'streak' vertical, but the centroid kick horizontal (using a simple kicker just downstream of the rf deflector), no large transverse wakefields are generated vertically between the deflector and screen.
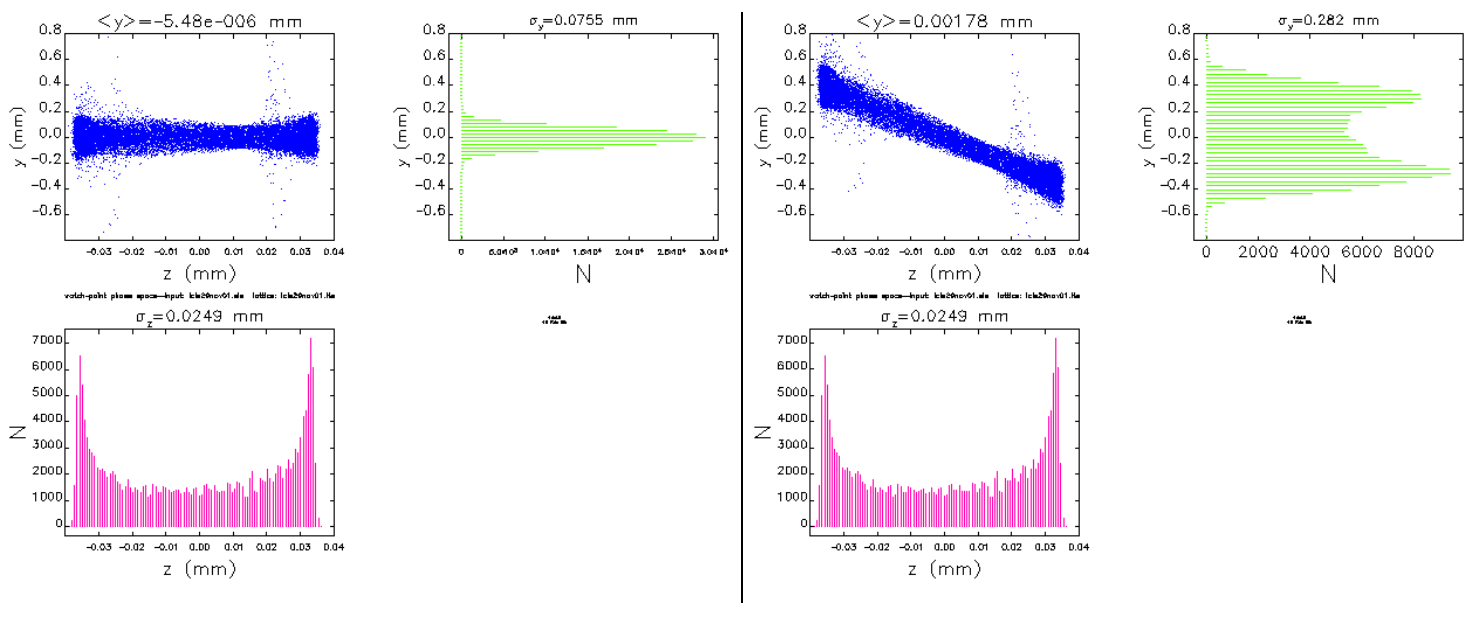

Figure 7.64 Vertical vs. longitudinal space at 25-902 screen with rf deflector OFF (left) and ON (right) showing 'streaked' beam. The rms vertical beam size on the screen is nominally $\sigma \mathrm{y} 0 \approx 76 \mu \mathrm{m}$ but increases to $\sigma \mathrm{y} \approx 282 \mu \mathrm{m}$ with rf deflector ON (bunch head at $\mathrm{z}<0$ ).

The effects of transverse wakefields upstream of the rf-deflector can introduce an initial $y$-z beam correlation ('pitch'), which, for very large levels, might bias the bunch length measurement result. This initial correlation can be mitigated by setting the rf-deflector at each rf zero-crossing point (i.e., 0 and $\pi$ ) in sequence. The three-beam size measurements $(\varphi=0, \varphi=\pi$, and $V=0)$ are then used in parabola-fit (much like a 'quad-scan' emittance measurement) and the systematic error due to the wakefield will be cancelled. In addition, the initial $y-z$ tilt is measured and might be used to determine upstream trajectory changes necessary to cancel this wakefield (see ref. [47] for real examples of this technique).

As shown in Figure 7.65, the temporal structure of the horizontal emittance can also be viewed using the vertical deflector. This will help to diagnose the effects of CSR in the BC2 
chicane where $x-z$ correlations ('yaw') in the beam are the dominant mechanism for projected emittance growth. The CSR effects of all bends are included in this figure.
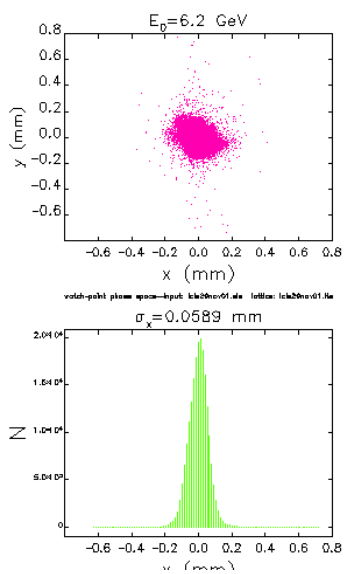

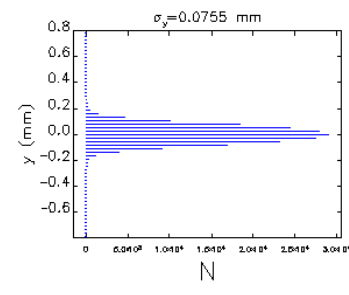

ins-
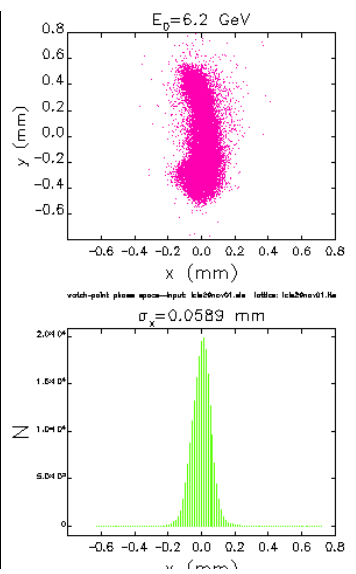

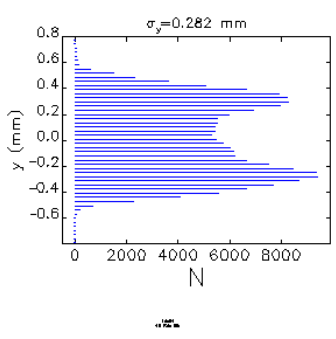

$\ldots$

Figure 7.65 Vertical vs. horizontal position on 25-902 screen for V0 $=0$ (left) and V0 $=20 \mathrm{MV}$ (right). The rms vertical beam size on the screen is $76 \mu \mathrm{m}$ (left) and $282 \mu \mathrm{m}$ (right). In this case, CSR is included for DL1, BC1, and BC2 upstream of the deflector, which generates the variable $x-y$ correlations along the bunch seen at right.

The rf deflector can also be used to make a direct measurement of the detailed electron bunch population in longitudinal phase space. Another OTR screen (PR31) is installed downstream of the deflector, at a location of large horizontal momentum dispersion where the horizontal beta function is small enough so that the horizontal beam extent across the screen represents dominantly the momentum spread in the beam. By switching on the transverse $\mathrm{rf}$ as a vertical deflector and allowing the 'pitched' bunch to propagate to this new screen, the vertical extent across the screen then represents the time axis along the bunch, while the horizontal is the energy axis. This requires a stable rf phase $\left(|\Delta \varphi|<0.2^{\circ}\right)$ at zero-crossing (i.e., $\varphi=0$ or $\varphi=\pi$ ) so that little trajectory centroid kick is induced over the potentially long distance to the new screen. This also requires the vertical phase advance between deflector and dispersion screen to be approximately $\pi / 2+n \pi$, which has been arranged in the design of the L3-linac optics. This is an infrequent technique, which requires the beam to be dumped after intercepting the screen.

Figure 7.66 shows a simulation of this process through the LCLS, where the deflector is set at $20 \mathrm{MV}$ with no centroid kick, and the screen is located 760 meters downstream at a location (at $S \approx 1218 \mathrm{~m}$ in Figure 7.36) with momentum dispersion $\eta_{x} \approx 50 \mathrm{~mm}$ and beta functions $\beta_{x} \approx 1.6 \mathrm{~m}$, and $\beta_{y} \approx 14.8 \mathrm{~m}$. The beta functions are still large enough to wash out some of the temporal and momentum resolution, but the image on the profile monitor (at right) is still a good representation of the real bunch population in longitudinal phase. Using this arrangement, the slice energy spread, and its temporal structure, can be measured to a resolution of $0.02 \%$ if the horizontal screen image is resolved to $10 \mu \mathrm{m}$. 


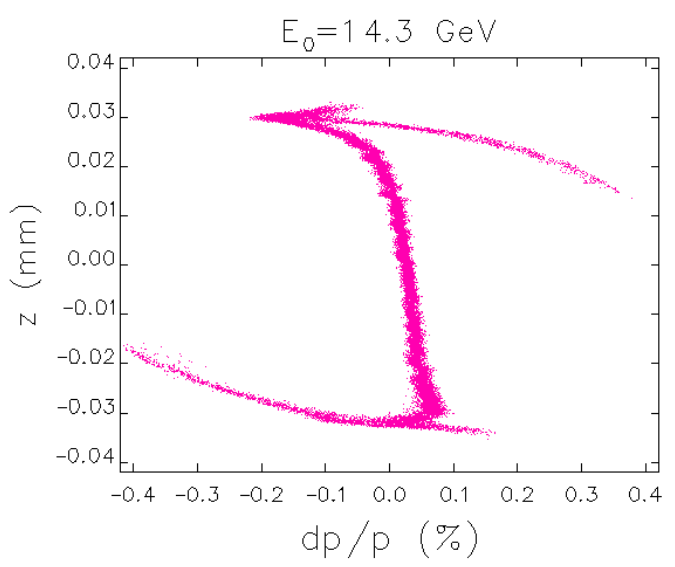

watch-point phase space--input |cls29nov01.ele lattice: |cls29nov01. Ite

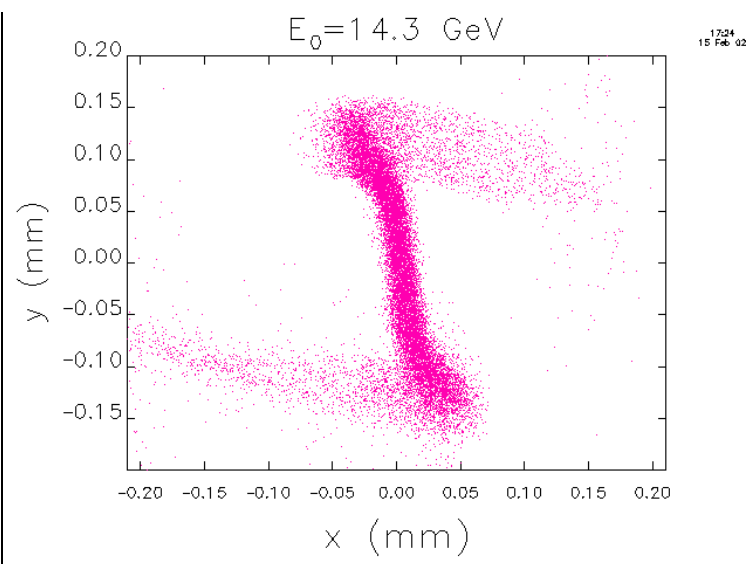

watch-point phase space--input Icls29nov01,ele lattice: Icls29nov01. Ite

Figure 7.66 Longitudinal phase space (left) $[\sigma z \approx 22 \mu \mathrm{m}]$ and its PR31 screen reconstruction (right) at $S \approx 1218 \mathrm{~m}$ in Figure 7.36 at $14.3 \mathrm{GeV}(\sigma \mathrm{y} \approx 90 \mu \mathrm{m}, \sigma \mathrm{x} \approx 40 \mu \mathrm{m})$, with if deflector at $20 \mathrm{MV}$ and no centroid kick (bunch head a bottom: $\mathrm{z}<0, \mathrm{y}<0$ ).

In addition, a shorter RF deflector will also be located just after L0 at $150 \mathrm{MeV}$ (see Figure 7.32: "RF Kicker" and Figure 7.33: "TCAV0"). This structure is just 55-cm long and presently installed in the SLAC linac where it has been unused for many years. A location just after L0 allows it to measure both slice emittance and slice energy spread. With 1-MV applied (1.3 MW), the OTR screen between the DL1 dipoles $\left(\eta_{x} \approx 150 \mathrm{~mm}\right)$ is used to directly measure longitudinal phase space, including slice energy spread, absolute bunch length, and temporal pulse shape. This deflector will be powered by the existing 20-5 klystron. Figure 7.67 shows a simulation with deflector off and on. The $x-y$ screen image with deflector on (right) should be compared to the longitudinal phase space plot at top of Figure 7.6.
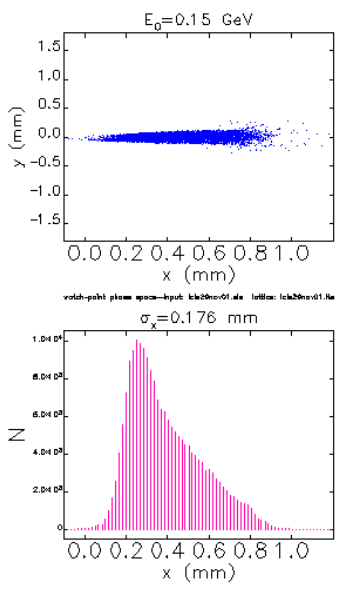

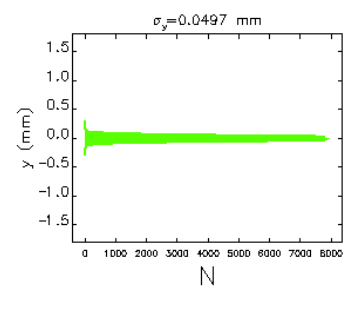

$\therefore$
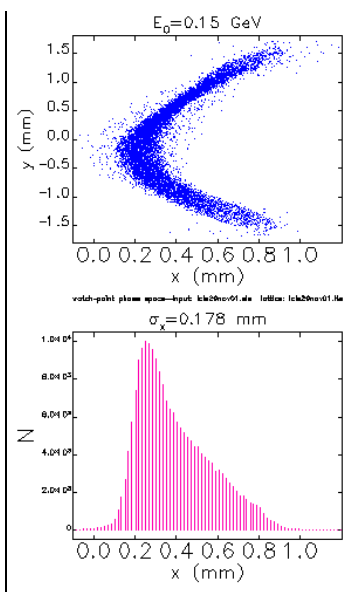

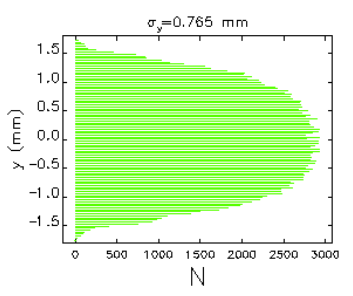

$\therefore$

Figure 7.67 Simulated profile monitor between DL1 bends with 55-cm rf deflector OFF (left) and with voltage set to $1 \mathrm{MV}$ (right). The plot at right is a direct measurement of the longitudinal phase space, including slice energy spread and absolute bunch length.

Since the bunch can be 'streaked' in time vertically across a profile monitor, it is also possible to measure the horizontal slice emittance. This is easily accomplished by observing the 
beam on the center OTR profile monitor of the ED0 emittance diagnostic section (at $S \approx 14.1 \mathrm{~m}$ in Figure 7.33). With a beam waist $\left(\alpha_{x}=\alpha_{y}=0\right)$ nominally located at this monitor, a quadrupole gradient scan can be done while measuring the sliced horizontal beam size. A simulation of this 'streak' effect is shown in Figure 7.68, for nominal quadrupole settings. In addition to slice emittance, the horizontal Twiss parameter variations along the bunch length can also be measured. Of course, the absolute bunch length and temporal profile can also be measured here.
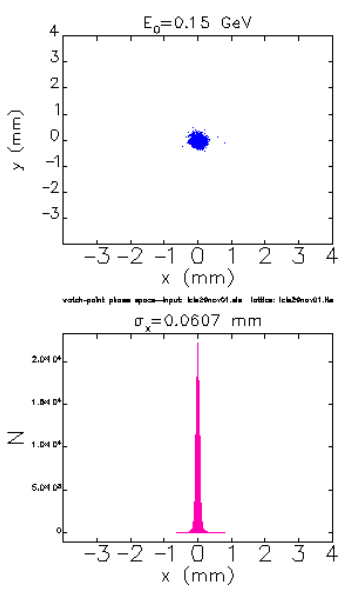

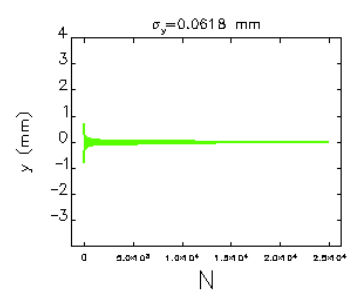

$\therefore$
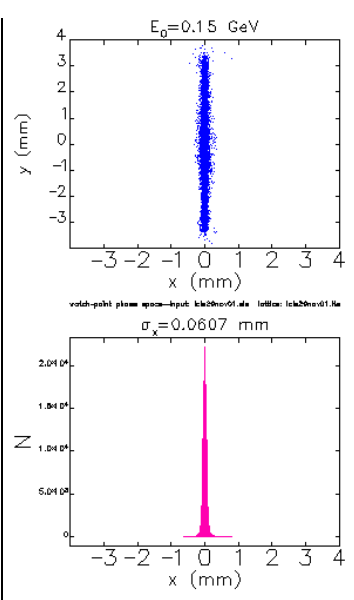

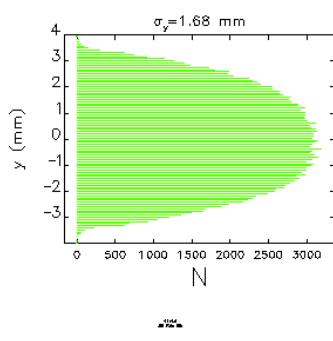

$\approx$

Figure 7.68 Simulated profile monitor in middle of ED0 emittance diagnostic section with 55-cm rf deflector OFF (left) and with voltage set to $1 \mathrm{MV}$ (right). The deflector 'streaks' the beam vertically such that the horizontal slice emittance can easily be measured.

\subsubsection{RF Zero-Phasing Technique}

Beyond the rf-deflectors, CSR detectors will be used as relative bunch length monitors, calibrated using a streak camera for the 10-psec range, and calibrated using the rf-deflector, or a "zero-phasing technique" [50] for the 2-psec and 0.2-psec ranges.

The zero-phasing technique is another way to measure the micro-bunch in an absolute sense, by employing an accelerating rf system at zero-crossing angle to generate correlated energy spread. The transverse beam size is measured in a dispersive region to extract the bunch length. In the LCLS, the entire L3-linac is used as the rf system. The measurement is invasive and performed infrequently to calibrate or crosscheck the CSR detectors. The micro-bunch is transported to the energy spread measuring profile monitor in DL2 (PR31) with a $+90^{\circ}$ L3 rf phase (i.e., zero crossing producing no acceleration in L3). The measurement is then repeated at a $-90^{\circ} \mathrm{rf}$ phase. The bunch length is then given by the average of the two energy-spread values.

$$
\sigma_{z} \approx \frac{\lambda}{4 \pi} \frac{E_{i}}{E_{f}-E_{i}}\left(\sigma_{\delta_{1}}+\sigma_{\delta_{2}}\right)
$$

Here $\lambda$ is the rf wavelength ( $105 \mathrm{~mm}), E_{i}$ is the L3 injection energy $(4.54 \mathrm{GeV}), E_{f}$ is the L3 final energy obtained with the L3 $\mathrm{rf}$ phase at crest-phase $(14.6 \mathrm{GeV})$, and $\sigma_{\delta_{1}}$ and $\sigma_{\delta_{2}}$ are the two energy spread measurements at $+90^{\circ}$ and $-90^{\circ}$. 
This method is simulated in Figure 7.69, including CSR and wakefields, and demonstrates an absolute bunch length measurement with an accuracy of a few percent. In all cases shown the beam is not accelerated in L3-linac, for a final beam energy of $4.54 \mathrm{GeV}$. The $75-\mu \mathrm{m}$ FWHM bunch length generates FWHM energy spread values of $1.64 \%$ (at $\varphi=-90^{\circ}$, left) and $0.333 \%$ (at $\varphi=+90^{\circ}$, right). When this is used in Eq. (7.28), it reproduces the 75- $\mu \mathrm{m}$ FWHM bunch length to within a few percent. Statistical resolution is dependent on the profile monitor used and should be 5-10\%. The horizontal FWHM beam size at the DL2 energy spread profile monitor (PR31; $\eta_{x} \approx 50 \mathrm{~mm}$ ) associated with the three cases is shown at bottom of Figure 7.69. Relative bunch length monitors are then calibrated from this, or the rf-deflector measurements. With BC2 switched off, the bunch length after $\mathrm{BC} 1$ can also be measured with either of these techniques.
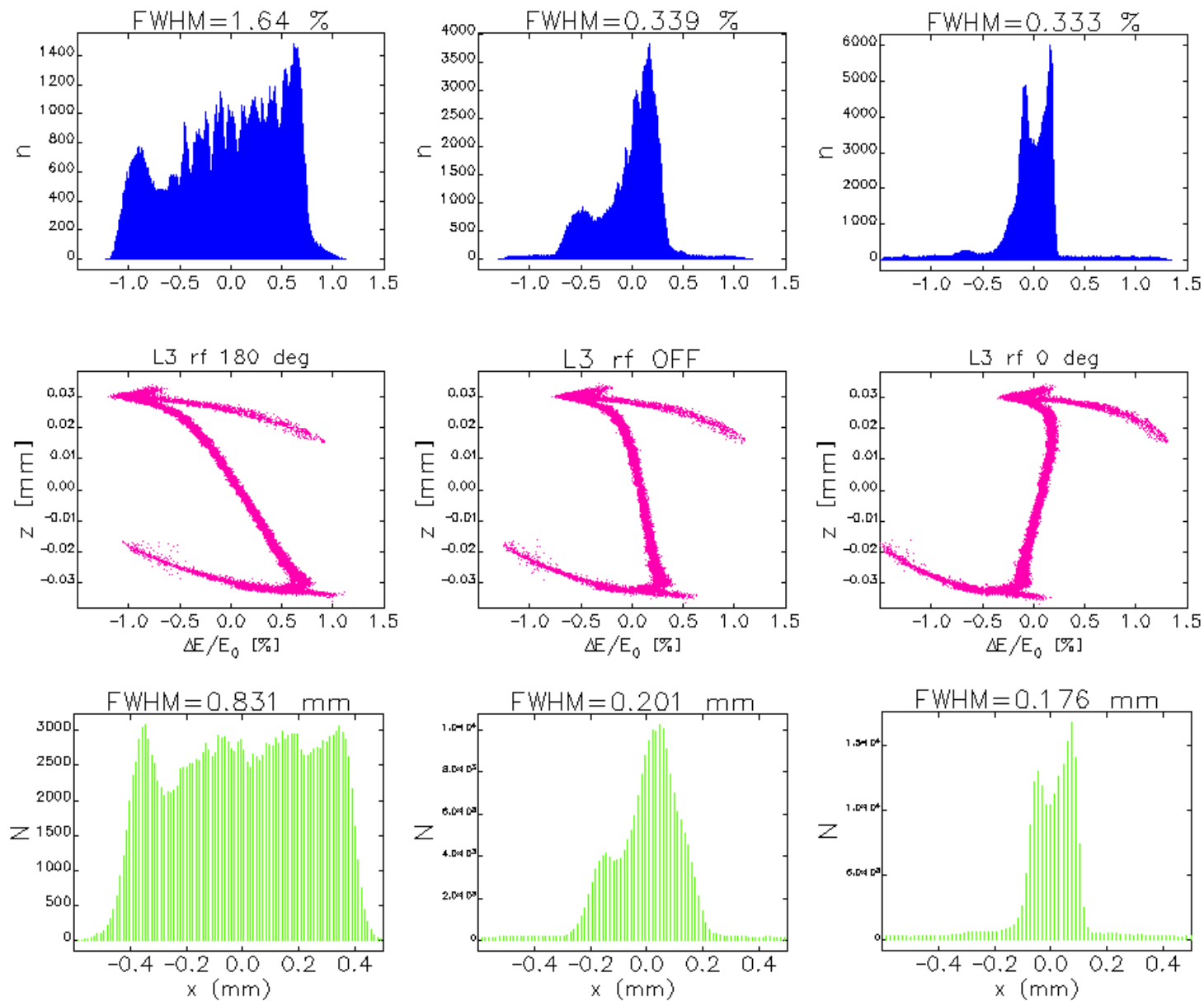

Figure 7.69 Simulated 'zero-phasing' technique for bunch length measurement. Top row is energy distribution after DL2 at $4.54 \mathrm{GeV}$ for $-90^{\circ}$ (left), rf-off (center), and $+90^{\circ}$ (right) in L3. Middle row is longitudinal phase space, and bottom row is horizontal beam profile.

\subsubsection{Electo-Optical Bunch Length Diagnostic}

An electro-optical (EO) device will also be employed as a bunch length and bunch arrivaltime diagnostic [51]. The concept, as illustrated in Figure 7.70, shows a laser pulse with a chirped waveform co-propagating with the electron beam. The active element is a thin $(\sim 100 \mu \mathrm{m})$ electro-optic crystal such as $\mathrm{LiNbO}_{3}$ through which the pulse propagates. The transmission of 
portions of this laser probe is determined according to the dynamic phase retardation induced by the electric field from the electron bunch. Any transmission attributed to intrinsic (static) phase retardation (i.e., without an electron bunch) is cancelled using a pair of polarizers with a biasing optical compensator. Polarizers are positioned up- and down-stream of the electro-optic crystal and are cross-polarized relative to each other. The electric field of the electron bunch modulates the transmitted intensity of the laser pulse in the following way. The short-lived bunch field induces a birefringence that generates phase retardation in the laser pulse. Consequently a polarization component orthogonal to that of the incident pulse can be transmitted to a spectrometer during a 'gated' time interval. The temporal structure of the bunch-induced modulation can come from knowledge of the initial wavelength chirp on the laser waveform. A spectrometer, in this case part of a Frequency Resolved Optical Gating (FROG) device, is used to measure which part of the band has been 'gated' by the electron bunch.

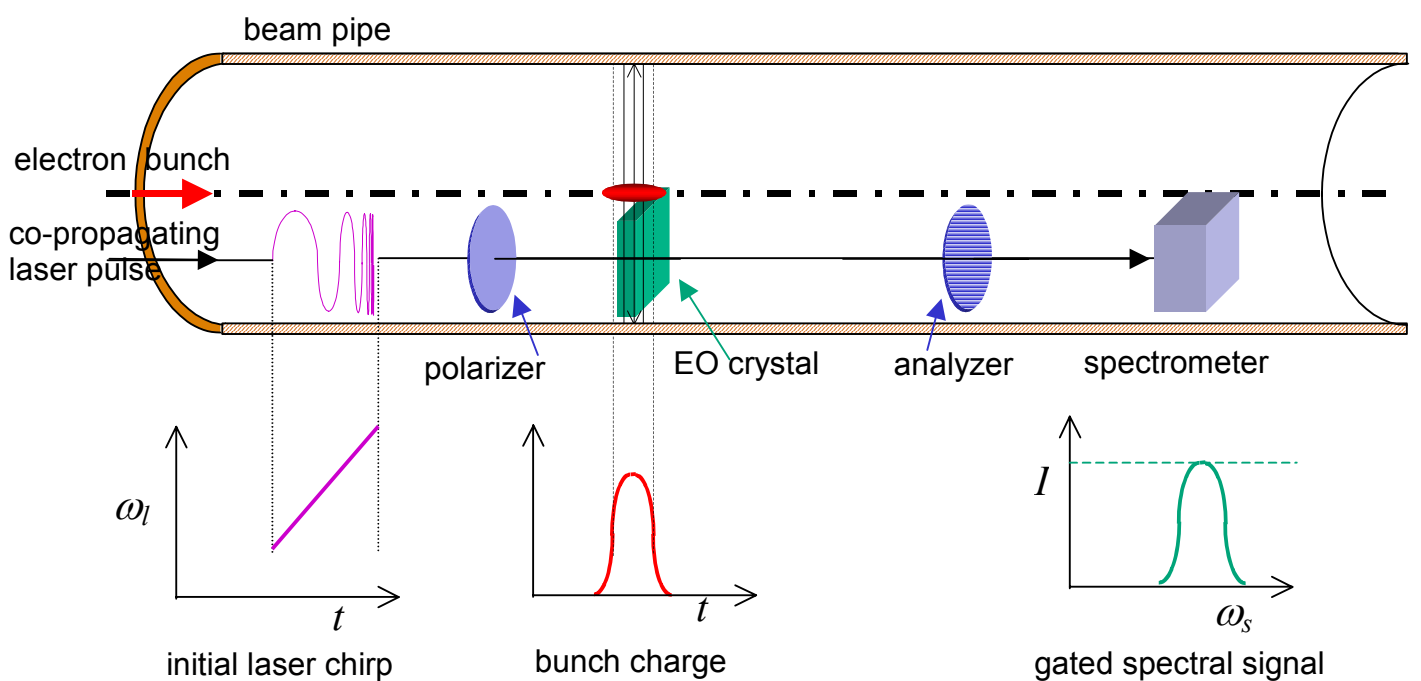

Figure 7.70. The principal of measurement of the electron bunch length by modulation of a chirped laser pulse in an electro-optic crystal by the electric field of the bunch. In practice only the EO crystal is in vacuum. All other optical components are located outside a window.

In order for the FROG to adequately record transmission of a single laser pulse from a single electron bunch a photon flux of $10 \mu \mathrm{J}$ is required in the gated spectral signal. This translates into a peak power requirement of $\sim 200 \mathrm{MW}$. The chirped laser waveform is stretched to a 10-ps duration (thereby allowing up to $10 \mathrm{ps}$ of relative timing jitter between laser and beam). The high peak power and proportionately wide laser bandwidth requirements can be met with a Ti:Sapphire laser oscillator seeding a regenerative amplifier. The extent of the waveform chirp is limited by the pulse bandwidth which can exceed $10 \mathrm{~nm}$. At this high peak power, care is required to remain below the fluence damage threshold near the $1-\mathrm{J} \cdot \mathrm{cm}^{-2}$ level in the optical materials. The co-propagating geometry is chosen since a transverse light propagation geometry would require focusing the laser light to micron level spot sizes in the EO crystal in order to preserve temporal resolution. This would exceed damage thresholds by several orders of magnitude. One constraint on the co-propagating geometry is the relative slippage between the 
photons slowed down in the crystal and the electric field of the bunch propagating at a velocity equal to $c$. A thin crystal with a low refractive index is therefore appropriate. The extremely high electric field strengths of several $\mathrm{GV} / \mathrm{m}$ from the $15-\mathrm{GeV}$, 1-nC LCLS bunch will afford use of EO crystals with $100-\mu \mathrm{m}$ thickness and relatively low electro-optic coefficients. For comparison quartz has an EO coefficient as low as $0.2 \times 10^{-12} \mathrm{~V} / \mathrm{m}$ with a refractive index of $n=1.5$ compared to a more usual EO material such as $\mathrm{LiNbO}_{3}$ with a coefficient of $30 \times 10^{-12} \mathrm{~V} / \mathrm{m}$ and $n=2.3$. It is envisaged that such an electro-optic measurement station will be installed in the DL2 beamline to measure the electron bunch length characteristics before it enters the undulator.

\subsubsection{Beam Energy Spread Diagnostics}

\subsubsection{DL1 Energy Spread Diagnostics}

The energy spread measurement in DL1 is made with a single profile monitor (OTR monitor) located between the two dipoles of DL1 at the point where the horizontal dispersion function reaches a value of $\eta_{x} \approx-153 \mathrm{~mm}$ with a horizontal beta function of $\beta_{x} \approx 0.79 \mathrm{~m}$ (at $S \approx 18.5 \mathrm{~m}$ in Figure 7.33). For the nominal emittance and nominal energy spread of $\sigma_{\delta} \approx 0.10 \%$ at $150 \mathrm{MeV}$, the betatron beam size is $52 \mu \mathrm{m}$, but the dispersive size is $153 \mu \mathrm{m}$. This produces a systematic energy spread measurement error of $6 \%$. The statistical error depends on the profile monitor and should be $5-10 \%$.

\subsubsection{BC1 Energy Spread Diagnostics}

The energy-spread measurement in $\mathrm{BC} 1$ is made with a profile monitor (phosphor) located in the center of the chicane at a point where the horizontal dispersion function is $\eta_{x} \approx 228 \mathrm{~mm}$ and the horizontal beta function converges towards a minimum of $\beta_{x} \approx 6.5 \mathrm{~m}$ (at $S \approx 34.8 \mathrm{~m}$ in Figure 7.19). For the nominal emittance and nominal energy spread of $\sigma_{\delta} \approx 1.78 \%$ at $250 \mathrm{MeV}$, the betatron beam size is $115 \mu \mathrm{m}$, but the dispersive size is $4.1 \mathrm{~mm}$. This produces no systematic error in the energy-spread measurement. The collimator jaws in $\mathrm{BC} 1$, upstream of the profile monitor, can be used to select energy bands for diagnostic purposes.

\subsubsection{BC2 Energy Spread Diagnostics}

The energy-spread measurement in $\mathrm{BC} 2$ is also made with a single profile monitor (phosphor) located in the center of the first chicane at a point where the horizontal dispersion function is $\eta_{x} \approx 341 \mathrm{~mm}$ and the beta function converges towards a minimum of $\beta_{x} \approx 25 \mathrm{~m}$ (at $S \approx 411 \mathrm{~m}$ in Figure 7.26). For the nominal emittance and nominal energy spread of $\sigma_{\delta} \approx 0.76 \%$ at $4.54 \mathrm{GeV}$, the betatron beam size is $53 \mu \mathrm{m}$, but the dispersive size is $2.6 \mathrm{~mm}$. As in the case of $\mathrm{BC} 1$, this produces no systematic error in the energy-spread measurement. As in $\mathrm{BC} 1$, the collimator jaws in $\mathrm{BC} 2$, upstream of the profile monitor, can also be used for diagnostic purposes.

\subsubsection{DL2 Energy Spread Diagnostics}

The energy spread measurement in DL2 is made with a profile monitor (a retractable OTR monitor) located where the dispersion function is $\eta_{x} \approx 91 \mathrm{~mm}$ and the beta function converges towards a minimum of $\beta_{x} \approx 4.0 \mathrm{~m}$ (at $S \approx 1236 \mathrm{~m}$ in Figure 7.36). For the nominal emittance and a core rms energy spread of $\sigma_{\delta} \approx 0.03 \%$, the betatron beam size is $12 \mu \mathrm{m}$, but the dispersive size 
is $27 \mu \mathrm{m}$. The total beam size is then $30 \mu \mathrm{m}$, and a horizontal profile monitor produces an energy spread measurement accuracy of $10 \%$ at $14.35 \mathrm{GeV}$. Of course, the betatron beam size can always be subtracted in quadrature if the emittance and beta function are known.

\subsubsection{Trajectory and Energy Monitors with Feedback Systems}

\subsubsection{Trajectory Feedback Systems}

Trajectory feedback systems will be placed at the entrance of the L2-linac, the L3-linac and at the undulator entrance. As at the SLC [52], these systems will each be composed of approximately ten BPMs which record both $x$ and $y$ positions, preceded by a set of two horizontal and two vertical fast dipole corrector magnets controlled by a microprocessor based cascaded feedback system. The cascade algorithm isolates each feedback system such that the systems do not all respond to the same trajectory changes.

In order to control transverse orbit variations to better than $1 / 10^{\text {th }}$ of the beam size, the individual one-pulse BPM resolution for the three 10-BPM feedback systems described above needs to be $50 \mu \mathrm{m}, 20 \mu \mathrm{m}$ and $10 \mu \mathrm{m}$ rms, respectively (decreasing with increasing energy). Trajectory variations which occur at frequencies below $\sim 10 \mathrm{~Hz}$ will be stabilized. Faster variations cannot be damped significantly and will need to be identified at the source. Additional trajectory control systems (e.g., in the L0-linac) are addressed in Chapter 6.

Transverse vibrations of quadrupole magnets will generate orbit variations, which if fast enough will not be damped by feedback systems. Tolerance calculations in L2 (strongest quadrupole gradients) indicate that uncorrelated random vibrations of all $28 \mathrm{~L} 2$ quadrupoles at the level of $400 \mathrm{~nm} \mathrm{rms}$ will generate orbit centroid fluctuations in the undulator, which are $6 \%$ of the beam size there $(6 \%$ of $30 \mu \mathrm{m})$. Measurements in the SLC indicate that existing linac magnet vibrations are $<250 \mathrm{~nm}$ [53] with the highest frequency content at $59 \mathrm{~Hz}$ and $10 \mathrm{~Hz}$ driven by cooling water. These observed vibrations are small enough to limit undulator orbit centroid jitter to below the goal of $<20 \%$ of nominal beam size. At lower energies in $\mathrm{L} 1$ and $\mathrm{BC} 1$, most of the new quadrupole magnets are air-cooled.

\subsubsection{Energy Feedback Systems}

Energy feedback systems will be placed in each bending region (DL1, BC1, BC2, and DL2). A single BPM or group of BPMs placed at a high dispersion point will be used to determine energy variations, and upstream rf will be used to stabilize the energy. A group of klystron phases will be used to control the energy while maintaining the correlated energy spread such that bunch compression in each system is also held constant. Similar systems, but without the bunch length control aspect, have been successfully tested at the SLC [54].

\section{DL1 Feedback}

The DL1 Energy Feedback System consists of at least 3 BPMs - two placed near the QM02 and QM04 quadrupoles, and a third near QB (see Figure 7.33). With this simple system energy variations can be distinguished from betatron oscillations using a linear combination of the three BPMs. The large dispersion at the QB BPM $(153 \mathrm{~mm})$ produces a relative energy resolution at 
$150 \mathrm{MeV}$ of $\delta<1.5 \times 10^{-4}$ with a $20-\mu$ m resolution BPM, which is almost ten times better than needed (see Table 7.5). The energy can be maintained by controlling the output power of one of the two L0 klystrons, which would need to run in an unsaturated configuration. The fast, pulse-topulse voltage stability of this unsaturated klystron will, however, still need to be $<0.1 \% \mathrm{rms}$.

\section{BC1 Feedback}

The BC1 Energy Feedback System consists of one BPM-one placed at the center of the chicane (see Figure 7.19). The large nominal dispersion in the chicane $(228 \mathrm{~mm})$ should produce a relative energy resolution of $\delta \approx 9 \times 10^{-5}$ with a $20-\mu \mathrm{m}$ resolution BPMs, which is four times better than needed (see Table 7.5). To allow voltage control, the single klystron powering L1 will not be operated in saturation. The fast, pulse-to-pulse voltage stability of this unsaturated klystron will, however, still need to be $<0.1 \%$ rms. Similarly, the rf phase of L1 will be controlled with a feedback system which maintains a relative bunch length measurement (based on CSR or a resonant cavity) after BC1. Fast energy stabilization using the BPMs and L1 rf voltage at $<10 \mathrm{~Hz}$ opens the L1 phase tolerance by a factor of three compared with that required with no energy stabilization (i.e., $\sim 0.3^{\circ}$ rather than $0.1^{\circ} \mathrm{rms}$ - see footnotes of Table 7.4). Therefore, the bunch length monitor will provide rf phase feedback but at a much slower rate of $<1 \mathrm{~Hz}$.

\section{BC2 Feedback}

The BC2 Energy Feedback System is identical to that of BC1 and consists of one BPM at the center of the $\mathrm{BC} 2$ chicane. The large nominal dispersion in the chicane $(341 \mathrm{~mm})$ should produce a relative energy resolution of $\delta \approx 1.2 \times 10^{-4}$ with a $40-\mu \mathrm{m}$ resolution BPMs, which is five times better than the minimum required. To maintain the energy in $\mathrm{BC} 2$, one of the 28 klystrons near the end of L2 will be nominally set at $1 / 2$-voltage ( $1 / 4$-power) and at a crest phase. This voltagecontrolled klystron will be at the high-energy end of L2, at 24-5 or 24-6, to reduce the effects on the focusing strength. With respect to the nominal parameters described in Table 7.1, the phasing of the remaining 27 powered klystrons needs to be adjusted from $-42.8^{\circ}$ to $-43.3^{\circ}$ and the rf voltage per klystron raised by $2 \%$. The power level of this one klystron will be controlled by the BPM-based energy feedback system at a rate of $<10 \mathrm{~Hz}$. The dynamic range for one klystron with $\pm 120 \mathrm{MeV}$ adjustability is then $\pm 2.6 \%$ of beam energy at BC2. Larger, manual changes will be implemented by switching on or off spare klystrons. The measured bunch length is held constant by adjusting the rf phase of the last sector or two (23-24) at a slower rate $\sim 1 \mathrm{~Hz}$. A full study of this feedback system has not yet been performed.

\section{DL2 Feedback}

The DL2 Energy Feedback System consists of 2 BPMs - one placed at QL32 and another at QL38 (see Figure 7.36). Since the $2 \times 2$ transfer matrix between these quadrupoles is $+\mathbf{I}$, differencing these two $x$ BPM readings will cancel any betatron signal leaving only the energy signal. The large nominal dispersion at the two BPMs $(103 \mathrm{~mm})$ produces a relative energy resolution of $\delta \approx 7 \times 10^{-5}$ with two $10-\mu \mathrm{m}$ resolution BPMs. This high-resolution relative energy signal may also be useful to the downstream x-ray experiments in order to monitor the fluctuations in the x-ray wavelength. The final bunch length is insensitive to L3 $\mathrm{rf}$ phase or voltage, so only the beam energy needs to be well controlled here. Two klystrons in sector-30 at 
$1 / 2$-voltage ( $1 / 4$-power) and crest-phase can be used to control the beam energy in DL2. A dynamic range of $\pm 1.7 \%$ is then possible. Larger, manual changes can be implemented by switching on or off spare klystrons. The rf phase then only needs to be held constant to a level of a few degrees. This might be done by occasional phase scanning using the BPMs in DL2 to check the beam phase.

In all cases above, the energy resolution is more than adequate to drive feedback systems for stabilization of the compression systems (see Table 7.5). The remaining critical items, which need further study, are the resolution and time characteristics of the CSR- or cavity-based bunch length monitors.

\subsection{The Wake Functions for the SLAC Linac}

\subsubsection{Introduction}

Obtaining wake functions for the SLAC linac structure that are sufficiently accurate to be used in beam dynamics studies for bunches as short as $20 \mu \mathrm{m}$ (rms) is not an easy task. It requires an accurate knowledge of the impedance of the structure over a large frequency range, which is difficult to obtain both by means of frequency-domain and time-domain calculations. Direct timedomain integration, using a computer program such as the MAFIA module T2 [55], needs a very large mesh and hence prohibitive amounts of computer time. Even then, errors will tend to accumulate in the results. The approach actually employed, uses a frequency-domain calculation applied to a simplified model of the linac structure, an approach that also has its difficulties. The wake functions obtained are the wakefields excited by a point charge, as a function of distance behind that charge. By performing a convolution over the bunch, the wakefields left by a bunch of arbitrary shape can be obtained.

A SLAC structure is $3 \mathrm{~m}$ long; it consists of 84 cells. It is a constant gradient structure, and both the cavity radius and the iris radius gradually become smaller (the change in iris radius is $0.5 \% /$ cell) along the length of each structure. In our calculations each SLAC constant gradient structure is broken into five pieces. Each piece is represented by a periodic model with an average iris radius, and the rounded iris profiles are replaced by rectangular ones. The wakefields for the five models are obtained and then averaged to obtain wake functions to represent an entire structure. Questions as to the accuracy and applicability of the calculated wake functions concern primarily:

- The accuracy of the periodic calculations themselves,

- Transient effects and effects at the ends of a structure,

- The fact that the irises vary in the real structure,

- The effects of resistivity/roughness of the iris surface. 


\subsubsection{The Calculated Wakefields for the SLAC Linac}

The wakefields for the SLAC linac structure were first calculated many years ago [56]. The methods and results presented here are essentially the same, though there are some changes in the details (see, for details, where these methods are applied to the NLC X-band structure [57] and the SBLC S-band structure [58]). To obtain the wakefields for each of the representative periodic models, several hundred mode frequencies and loss factors are obtained using computer programs that employ mode-matching techniques. The accuracy of these calculations is not easy to assess. The density of modes obtained is one consistency check, and good agreement is found.

The high-frequency contribution to the impedances is obtained employing the so-called "Sessler-Vainsteyn optical resonator model" [57,59]. It is a simple model that combines the Poynting flux at the iris due to the beam with the diffraction due to light at the edges of a periodic array of circular mirrors. It yields the expected high-frequency dependence of the impedance; e.g., the real part of the longitudinal impedance varies with frequency as $\omega^{-3 / 2}$. Figure 7.71 shows the real part of the longitudinal impedance, averaged over frequency bins, for the geometry of cell 45 ( $a$ in the plot is the iris radius; $11.6 \mathrm{~mm}$ ). The dashed curve is the Sessler-Vainsteyn prediction. At the higher frequencies the agreement is seen to be quite good.

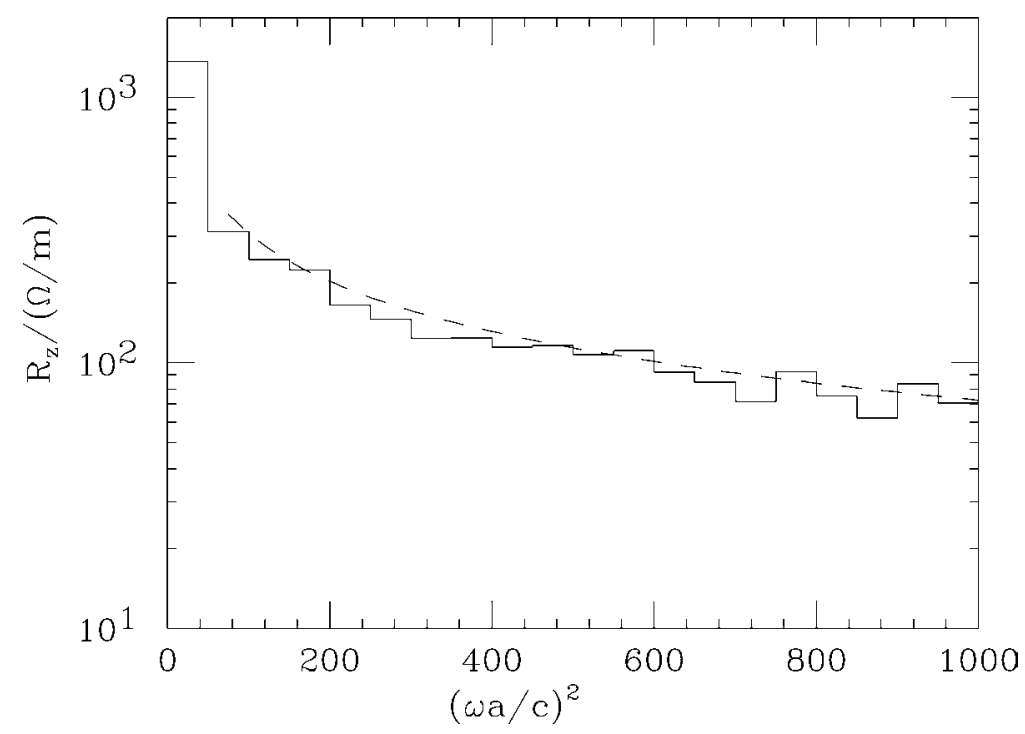

Figure 7.71 The real part of the longitudinal impedance, averaged over frequency bins, for the geometry of cell 45 . The Sessler-Vainsteyn prediction is given by the dashed curve.

As a dipole example, the real part of the longitudinal dipole impedance, averaged over frequency bins, for the geometry of cell 45 is plotted in Figure 7.72, with the dashed curve again giving the Sessler-Vainsteyn prediction. There appears to be a $15 \%$ systematic difference between the two results at high frequencies, probably due to the approximate nature of the Sessler-Vainsteyn model. The agreement, however, is acceptable for our requirements. 


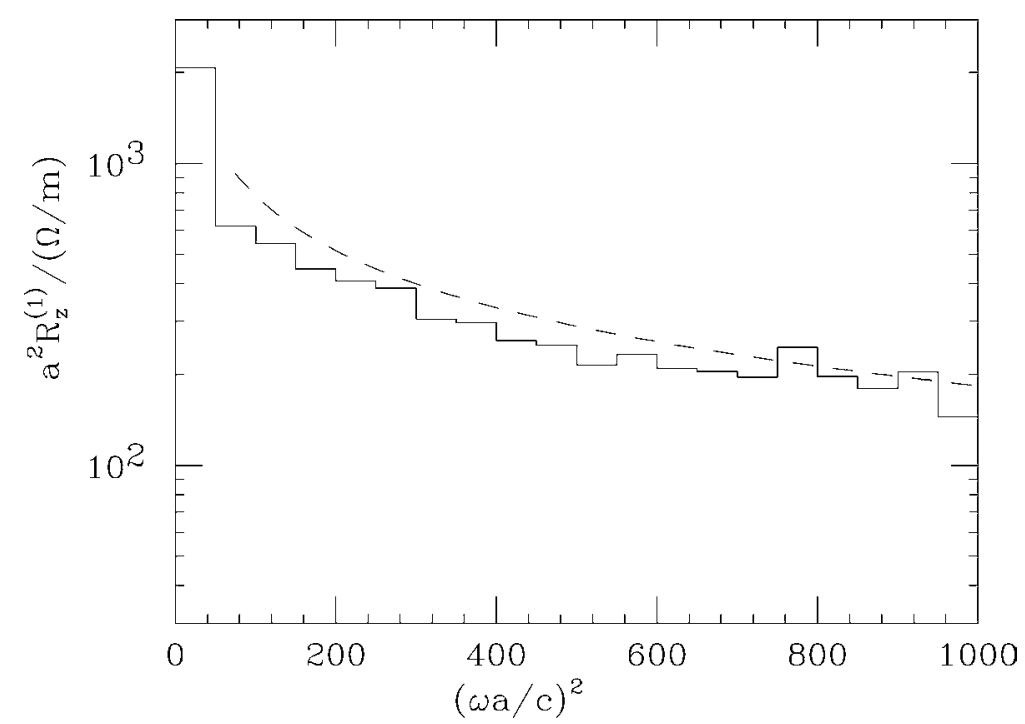

Figure 7.72 The real part of the longitudinal dipole impedance, averaged over frequency bins, for geometry of cell 45 . The Sessler-Vainsteyn prediction is given by dashed curve.

The longitudinal wakefield of the 5 representative cells - cells 1, 23, 45, 65, and 84-is given in Figure 7.73, and the average (with the end cells weighted by one half), representing the whole structure, is given by the dashes.

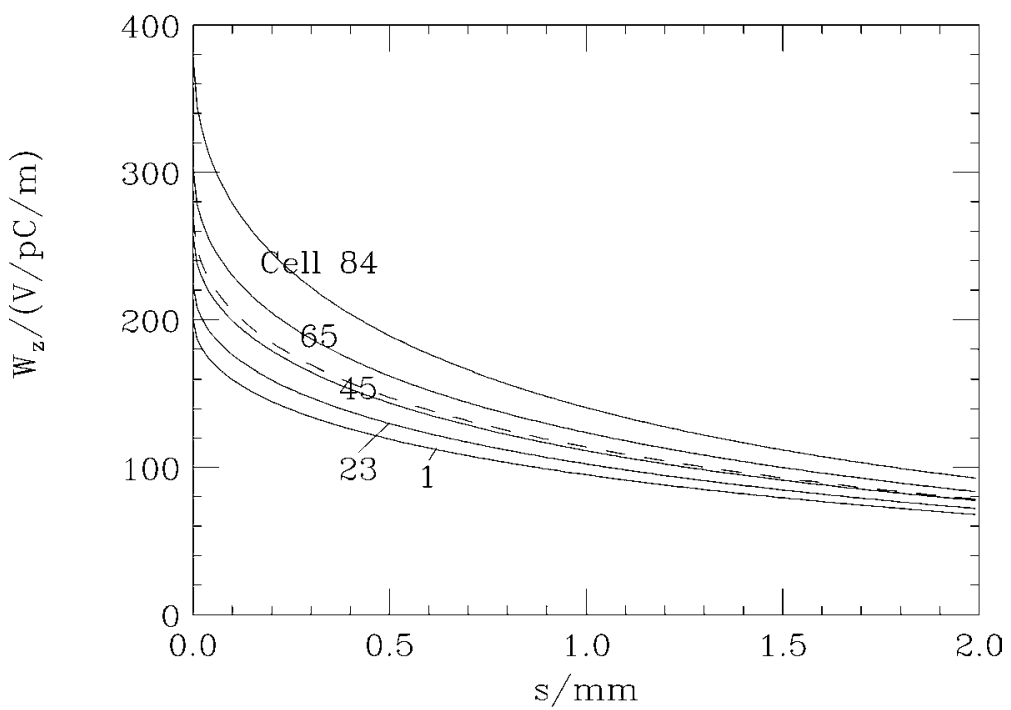

Figure 7.73 The longitudinal wakefield of representative cells in the SLAC structure (solid curves). The average represents the whole structure (the dashes).

One important consistency check is that for each model structure, the longitudinal wakefield at the origin must satisfy [60]

$$
W_{\|}(0)=\frac{Z_{0} c}{\pi a^{2}}
$$


with $Z_{0} \approx 377 \Omega$ and $a$ the iris radius. In all cases the computed values are low, but in no case by more than $4 \%$. Similarly, the transverse wakefield of the representative cells is given in Figure 7.74, and the average, again representing the whole structure, is given by the dashes. The transverse wakefield of the representative cells must satisfy Eq. (7.30) [57]. In all cases the agreement is within $1-2 \%$.

$$
\frac{d}{d s} W_{\perp}(0)=\frac{2 Z_{0} c}{\pi a^{4}}
$$

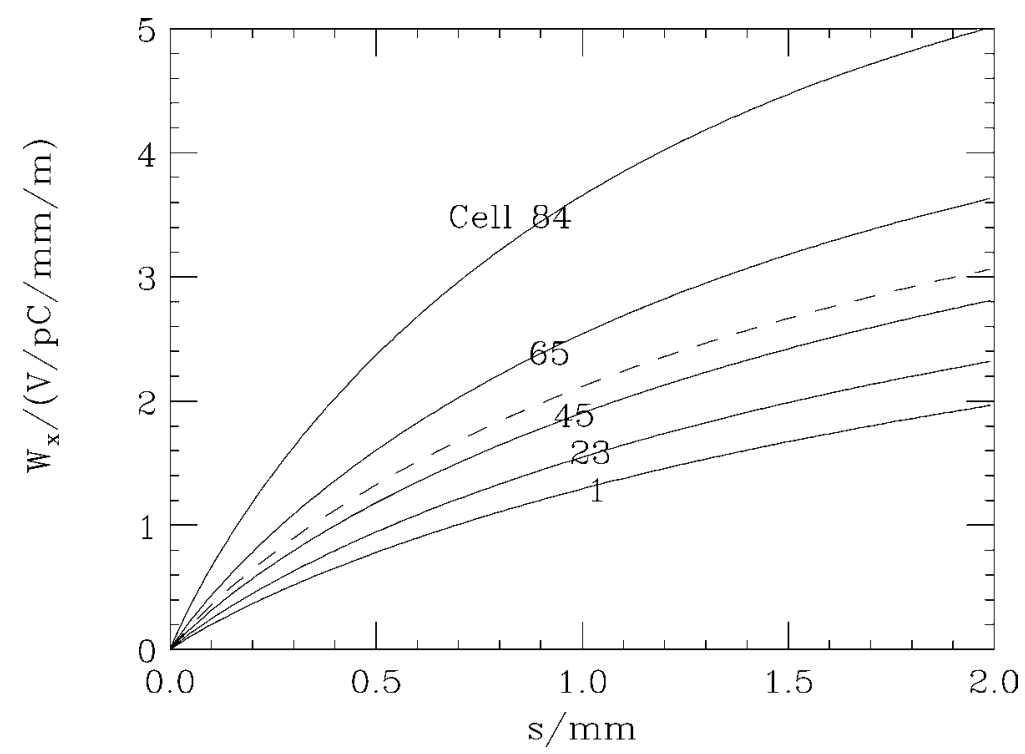

Figure 7.74 The transverse (dipole) wakefield of representative cells in the SLAC structure (solid curves). The average represents the whole structure (the dashes).

\subsubsection{Discussion}

The calculated wakefields are asymptotic in that they apply only after the beam has traversed a critical number of cells $N_{\text {crit. }}$. For a gaussian bunch, for the total loss obtained using only the asymptotic wake functions to be within a few percent of the loss when transient effects are also included, requires that [58]

$$
N_{\text {crit }}=\frac{\alpha a^{2}}{p \sigma_{z}}
$$

with $\alpha \geq 0.5, p$ the structure cell length, and $\sigma_{z}$ the bunch length. The transient region is largest for short bunches. In the LCLS the bunch is shortest after the second bunch compressor where $\sigma_{z}$ $\approx 22 \mu \mathrm{m}$. Taking, in addition, $a=1 \mathrm{~cm}$ and $p=3.5 \mathrm{~cm}$, then $N_{\text {crit }} \approx 70$, which represents about $80 \%$ of the length of one structure or $0.5 \%$ of the length of the third linac. In most of the SLAC linac the 3-m structures are combined in groups of four, with nearly no extra space in between them; the groups of four are separated by about $20-\mathrm{cm}$ of beam tube, and every $100 \mathrm{~m}$ there is an extra $4 \mathrm{~m}$ of beam tube. With this arrangement, after the end of the structures, the wakefields generated by the beam do not have a chance to return to their initial conditions, except partially at 
the $3 \%$ of the structures that follow the $4 \mathrm{~m}$ gaps. Therefore, using the asymptotic wakefields to represent the entire SLAC linac, and ignoring the transient effects, should result in very little error even for a $20-\mu \mathrm{m}$ bunch.

There are three other effects that can be important for short bunches: (1) The so-called "catch-up distance" effect: If the head of the beam generates a wakefield at a certain position in the linac, due to causality the tail does not feel the effect until a distance $\sim 0.5 a^{2} / \sigma_{z}$, which in this case is $2.5 \mathrm{~m}$, later. Since this is a small fraction of the 550-meters of accelerating structure after the second compressor, this effect should not be important. (2) For $\sigma_{z} \leq a / \gamma$, which in this case is $0.3 \mu \mathrm{m}$, the impedance drops dramatically. Since the minimum bunch length is $20 \mu \mathrm{m}$, this effect should also not be important for the LCLS. (3) The effect of the resistivity of the iris surface is shown, in the following section, not to be a significant effect for the LCLS accelerator.

Finally, it should be noted that the above estimates all assume that the bunch is gaussian, which it is not. The real bunch shape is rectangular with spikes at the edges of the distribution (see bottom of Figure 7.7). The Fourier transform of such a bunch shape reaches to higher frequencies than the gaussian approximation, and therefore the short bunch effects will become somewhat more pronounced than estimated above. However, even with this consideration, the calculated wake functions will accurately represent the wakefield effects in the linac for the LCLS project.

\subsubsection{Confirmations}

There have been confirmations, both theoretical and by measurement, of the calculated SLAC wake functions and, more recently, of the similarly calculated wake functions for the NLC and the DESY-SBLC linac. All of the measurements, however, have been done for bunch lengths significantly larger than the $20-\mu \mathrm{m}$ of interest here. As to theoretical comparisons, the calculated SLAC wake functions have been confirmed, for gaussian bunches down to $\sigma_{z} \approx 0.5 \mathrm{~mm}$, using the time domain program TBCI [61]. A time domain program exists that is able to obtain accurate results for short bunches in accelerating structures [62]. For a 100- $\mu \mathrm{m}$ bunch in the NLC structure, the results of this program, as well as the results of an independent frequency domain program [63], agree with our frequency domain results to within a few percent.

As to confirmation by measurement in the SLC linac, the total wakefield-induced energy loss [64] and more recently the wakefield-induced voltage of a bunch [65], [66] have been shown to agree quite well, for bunch lengths down to $0.5 \mathrm{~mm}$. Also, in the ASSET test facility, the short range transverse wakefield of a $0.5-\mathrm{mm}$ bunch in the NLC structure has been measured, and the results agree quite well with the calculated results [67].

\subsubsection{Resistive Wall Wakefields}

In addition to geometric wakefields, the micro-bunch beyond $\mathrm{BC} 2$ experiences a longitudinal resistive wall (RW) wakefield which introduces a small coherent energy spread along the bunch [68], [70]. For a bunch which is much longer then the characteristic length, $s_{0}$, 


$$
s_{0} \equiv\left(\frac{2 a^{2}}{Z_{0} \sigma}\right)^{1 / 3}
$$

the rms relative energy spread induced in a smooth cylindrical chamber of radius, $a$, and conductivity, $\sigma$, is

$$
\sigma_{\delta_{\mathrm{RW}}} \approx(0.22) \frac{e^{2} c N L}{\pi^{2} a E \sigma_{z}^{3 / 2}} \sqrt{\frac{Z_{0}}{\sigma}}
$$

This is for a gaussian bunch, which is long compared to $s_{0}$. To more accurately estimate the RW energy spread generated after $\mathrm{BC} 2$, the beamline is broken into two discrete sections of significantly small radius (Table 7.24). Remaining sections have much larger radii $(40-400 \mathrm{~mm})$ and are ignored here. The short iris surfaces of the copper accelerating structures in L3 contribute no significant component to the resistive wall wakefields [69].

Table 7.24 The two main beamline sections that transport the micro-bunch and contribute to a resistive wall wake energy spread generated between BC2 and undulator entrance.

\begin{tabular}{lccccc}
\hline Beamline Section & Material & Conductivity & Radius & Length & $\boldsymbol{s}_{\mathbf{0}}$ \\
& & $\Omega^{-1}-\mathrm{m}^{-1}$ & $\mathrm{~mm}$ & $\mathrm{~m}$ & $\mu \mathrm{m}$ \\
\hline Linac-3 non-accelerating chambers & Stainless Steel & $0.14 \times 10^{7}$ & 12.7 & 76 & 85 \\
\hline Linac-to-undulator beamline & Aluminum & $3.6 \times 10^{7}$ & 12.7 & 106 & 29 \\
\hline
\end{tabular}

The 76-meters of 1-inch diameter stainless steel are distributed along Linac-3 as quadrupole/BPM chambers, and other short non-accelerating sections including 22 meters beyond sector-30 in the beam switchyard, before the aperture significantly increases. The aluminum sections are new chambers for the DL2/ED2 beamline leading up to the undulator entrance, which follow the large radius beam switchyard. The replacement of the existing 100 meters of stainless chamber, which is presently used in the undulator hall, with aluminum removes an effect which would otherwise increase the coherent energy spread by $0.05 \% \mathrm{rms}$, with an energy gradient which is nearly linear across the bunch. The associated transverse resistive-wall wakefields are insignificant for reasonable electron trajectories.

For these sections, the bunch $(22 \mu \mathrm{m})$ is shorter than $s_{0}$, so the RW energy spread of the short-bunch $\left(\sigma_{z} / s_{0}<1\right)$ is calculated using the point-charge wake function [70],

$$
E_{z}(s)=-\frac{4 q c Z_{0}}{\pi a^{2}}\left(\frac{1}{3} e^{-s / s_{0}} \cos \frac{\sqrt{3} s}{s_{0}}-\frac{\sqrt{2}}{\pi} \int_{0}^{\infty} \frac{d x x^{2} e^{-x^{2} s / s_{0}}}{x^{6}+8}\right),
$$

which is convoluted with the bunch distribution similar to that at bottom of Figure 7.7. This estimate ignores the frequency dependence of the conductivity, an effect which is quite small. The results for each of the two sections of Table 7.24 are shown in Figure 7.75. 


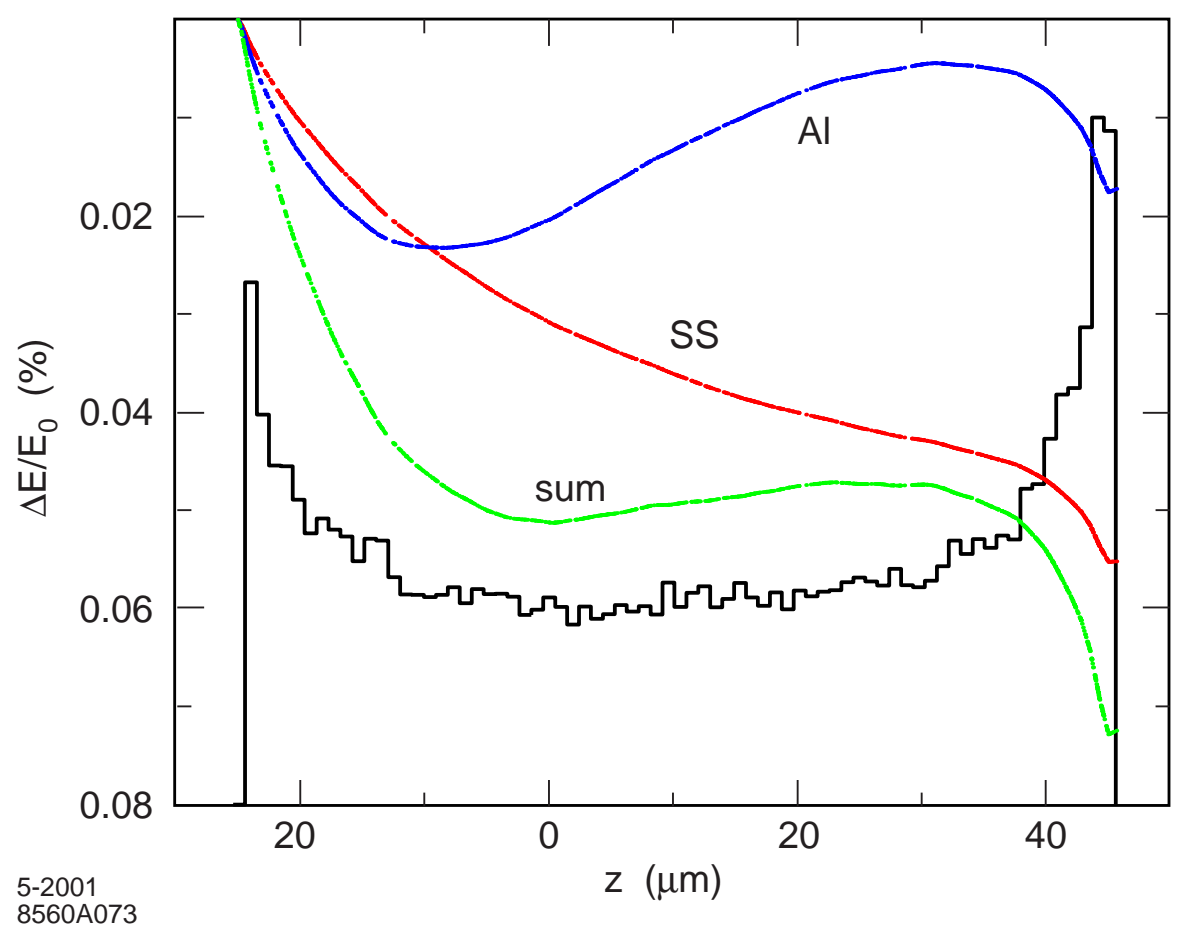

Figure 7.75 Resistive wake of 1-nC bunch after convolution with the temporal bunch distribution (solid/black, arbitrary vertical scale) at bottom of Figure 7.7. The contributions, and their sum, are labeled.

For this bunch distribution (shown on arbitrary vertical scale as solid curve in the figure), the total rms RW energy spread generated by both sections is $0.015 \%$ (with respect to the mean), which is well below the maximum chirp tolerance $(\sim 0.1 \% \mathrm{rms})$. If the linear component of the energy spread is removed (by a small rf phase change in L2), this is reduced to $0.009 \%$. This energy spread is, of course, a gradient along the bunch and has almost no effect on the slice energy spread. The longitudinal resistive-wall wakefields prior to the undulator are therefore, very small, but are included in all of the $2 \mathrm{D}$ and $6 \mathrm{D}$ tracking.

\subsection{Parts List and Installation Issues}

\subsubsection{Parts List}

A list of LCLS magnet power supplies is given in Table 7.25 with each supply designated as new, existing, or recycled (re-used from a previous SLAC installation).

A list of new, existing, and recycled BPMs (beam position monitors) located throughout the LCLS accelerator is given in Table 7.26. Just 19 new BPM stripline monitors are needed in the tunnel, the rest are recycled from the FFTB or are already located in the existing beamline. The several new BPMs needed in the L0-linac are not included here (see Chapter 6). The required rms BPM resolution at a minimum bunch charge of $0.2 \mathrm{nC}$ is also estimated in the table. Most LCLS BPMs, with the exception of chicane locations, are suitable with a chamber ID of $\sim 2.5 \mathrm{~cm}$.

Table 7.25 Power supply and magnet list for the LCLS accelerator (not including injector). 
LCLS CONCEPTUAL DESIG N REP O R T

\begin{tabular}{|c|c|c|c|c|c|c|c|c|}
\hline $\begin{array}{l}\text { Magnet } \\
\text { String } \\
\text { Location }\end{array}$ & $\begin{array}{l}\text { Existing, } \\
\text { New or } \\
\text { Recycled }\end{array}$ & $\begin{array}{c}\text { No. of } \\
\text { Power } \\
\text { Supplies }\end{array}$ & $\begin{array}{c}\text { Magnets } \\
\text { per } \\
\text { Supply }\end{array}$ & $\begin{array}{l}\text { Uni- } \\
\text { or Bi- } \\
\text { polar }\end{array}$ & $\begin{array}{c}\text { Magnet } \\
\text { Type }\end{array}$ & $\begin{array}{c}\text { Max. } \\
\text { Current } \\
{[\mathrm{A}]}\end{array}$ & $\begin{array}{c}\text { Max. } \\
\text { Voltage } \\
\text { [V] }\end{array}$ & $\begin{array}{c}\text { Est. Reg. } \\
\text { Tolerance } \\
{[\%]}\end{array}$ \\
\hline DL1 & $\mathrm{N}$ & 1 & 2 & $\mathrm{U}$ & $5 \mathrm{D} 7.1$ & 450 & 10 & 0.02 \\
\hline $\mathrm{BC} 1$ & $\mathrm{~N}$ & 1 & 4 & $\mathrm{U}$ & $5 \mathrm{D} 7.1$ & 400 & 20 & 0.02 \\
\hline $\mathrm{BC} 2$ & $\mathrm{~N}$ & 1 & 4 & $\mathrm{U}$ & 1D15.7 & $\sim 1000$ & $?$ & 0.05 \\
\hline DL2 & $\mathrm{N}$ & 1 & 4 & $\mathrm{U}$ & 4D102.36T & 375 & 40 & 0.02 \\
\hline DL2 & $\mathrm{N}$ & 1 & 2 & $\mathrm{U}$ & VB & $?$ & $?$ & 0.05 \\
\hline $\mathrm{DL} 1 / \mathrm{BC} 1$ & $\mathrm{~N}$ & 12 & 1 & B & 1Q5.6 & 12 & 36 & 0.1 \\
\hline $\mathrm{L} 1 / \mathrm{BC} 1$ & $\mathrm{~N}$ & 4 & 1 & $\mathrm{~B}$ & QA & 8 & 5 & 0.1 \\
\hline $\mathrm{L} 2 / \mathrm{L} 3$ & $\mathrm{E}$ & 69 & 1 & $\mathrm{U}$ & QE & 20 & $?$ & 0.1 \\
\hline $\mathrm{BC} 1$ & $\mathrm{~N}$ & 3 & 1 & $\mathrm{U}$ & QE & 25 & 2 & 0.1 \\
\hline L2/L3 & $\mathrm{N}$ & 6 & 8 & $\mathrm{U}$ & QE & 25 & $?$ & 0.1 \\
\hline L2 & $\mathrm{N}$ & 1 & 5 & $\mathrm{U}$ & QE & 25 & $?$ & 0.1 \\
\hline $\mathrm{BC} 2$ & $\mathrm{~N}$ & 4 & 1 & $\mathrm{U}$ & QE & 200 & 20 & 0.1 \\
\hline $\mathrm{BC} 2$ & $\mathrm{~N}$ & 2 & 1 & $\mathrm{~B}$ & $\mathrm{QE}$ & 12 & 1 & 0.1 \\
\hline BSY & $\mathrm{E}$ & 1 & 3 & $\mathrm{U}$ & $50 \mathrm{Q} 1,2,3$ & 310 & $?$ & 0.1 \\
\hline BSY & $\mathrm{E}$ & 1 & 1 & $\mathrm{U}$ & QSM & 9.8 & $?$ & 0.1 \\
\hline DL2 & $\mathrm{E}$ & 3 & 1 & $\mathrm{U}$ & $0.91 \mathrm{Q} 17.7$ & 240 & $?$ & 0.05 \\
\hline DL2 & $\mathrm{R}$ & 2 & 4 & $\mathrm{U}$ & $0.91 \mathrm{Q} 17.7$ & 250 & 120 & 0.05 \\
\hline DL2 & $\mathrm{R}$ & 2 & 2 & $\mathrm{U}$ & $0.91 \mathrm{Q} 17.7$ & 250 & 60 & 0.05 \\
\hline DL2 & $\mathrm{R}$ & 9 & 1 & $\mathrm{U}$ & $0.91 \mathrm{Q} 17.7$ & 250 & 30 & 0.05 \\
\hline DL2 & $\mathrm{R}$ & 1 & 6 & $\mathrm{U}$ & $0.91 \mathrm{Q} 17.7$ & 100 & 80 & 0.05 \\
\hline
\end{tabular}

A list of LCLS profile monitors is given in Table 7.27. This list does not include monitors placed upstream of the end of the L0-linac. The profile monitors are wire-scanners, OTR monitors (or both in a few cases), or phosphor screens. Wire-scanners allow non-invasive, but integrated pulse measurements, while screens allow single shot measurements, which are destructive. The phosphor screens are used where the beam size is large and diffuse in BC1 and $\mathrm{BC} 2$. The profile monitors are used to measure transverse emittance $(\varepsilon)$, energy spread $(\delta)$, or bunch length $\left(\sigma_{z}\right)$, as indicated in the table. The rms transverse beam size listed, $\sigma_{x, y}$, is the $x(\approx y)$ size in the case of emittance measurements, the $x$ size in the case of energy spread, and the $y$ size in the case of bunch length. Beam size measurement resolution of $\leq 10 \%$ is required with a charge level as low as $0.2 \mathrm{nC}$ per bunch. 
L C L S C O N C E P T U A L D E S I G N R E P O R T

Table 7.26 BPM list (not including injector or L0). Estimated rms resolution is based on a minimum operable bunch charge of $0.2 \mathrm{nC}$. Just 19 new BPM strip-lines are required.

\begin{tabular}{|c|c|c|c|c|}
\hline Accelerator Area & $\begin{array}{c}\text { Location Range, } S \\
{[\mathrm{~m}]}\end{array}$ & $\begin{array}{c}\text { Existing, New or } \\
\text { Recycled }\end{array}$ & Quantity & $\begin{array}{l}\text { Est. Resolution } \\
{[\mu \mathrm{m}]}\end{array}$ \\
\hline DL1/ED0 & $0-21$ & $\mathbf{N}$ & 11 & 25 \\
\hline L1-linac & $21-31$ & $\mathbf{N}$ & 2 & 20 \\
\hline L1-linac & 30 & $\mathrm{E}$ & 1 & 20 \\
\hline $\mathrm{BC} 1$ & $31-46$ & $\mathbf{N}$ & 5 & 20 \\
\hline $\mathrm{BC} 1 / \mathrm{ED} 1$ & 43 & $\mathrm{E}$ & 1 & 20 \\
\hline L2-linac & $46-397$ & $\mathrm{E}$ & 28 & 10 \\
\hline $\mathrm{BC} 2$ & $397-423$ & $\mathbf{N}$ & 1 & 10 \\
\hline L3-linac/BSY & $423-1200$ & $\mathrm{E}$ & 58 & 10 \\
\hline DL2/ED2 & $1200-1280$ & $\mathrm{R}$ & 28 & 5 \\
\hline
\end{tabular}

Table 7.27 Profile monitor list with nominal rms beam sizes for $\gamma \varepsilon=1 \mu \mathrm{m}$ (not including injector system).

\begin{tabular}{|c|c|c|c|c|c|c|}
\hline Accelerator Area & $\begin{array}{l}\text { Location, } \\
S,[\mathrm{~m}]\end{array}$ & $\begin{array}{l}\text { Monitor } \\
\text { Type }\end{array}$ & $\begin{array}{l}\text { Measures } \\
\varepsilon, \delta \text { or } \sigma_{z}\end{array}$ & Quantity & $\begin{array}{c}\text { Energy } \\
{[\mathrm{GeV}]}\end{array}$ & $\begin{array}{c}\text { Beam Size, } \\
\sigma_{x, y}[\mu \mathrm{m}]\end{array}$ \\
\hline DL1/ED0 & $10-15$ & wire/OTR & $\varepsilon$ & 3 & 0.15 & $65-130$ \\
\hline DL1 & 16.5 & OTR & $\delta$ & 1 & 0.15 & 135 \\
\hline $\mathrm{BC} 1$ & 32.6 & Phos. & $\delta$ & 1 & 0.25 & 1740 \\
\hline $\mathrm{BC} 1 / \mathrm{ED} 1$ & 39.8 & Wire & $\varepsilon$ & 3 & 0.25 & $40-80$ \\
\hline L2-linac & $359-397$ & Wire & $\varepsilon$ & 4 & $4.1-4.5$ & $41-72$ \\
\hline $\mathrm{BC} 2$ & 404 & Phos. & $\delta$ & 1 & 4.5 & 1560 \\
\hline L2-linac & 524 & OTR & $\sigma_{z}$ & 1 & $4.5-6.2$ & 250 \\
\hline L3-linac & $701-815$ & Wire & $\varepsilon$ & 4 & $4.5-11$ & $39-57$ \\
\hline DL2 & 1218 & OTR & $\delta$-slice & 1 & $4.5-15$ & 20 \\
\hline DL2 & 1237 & OTR & $\delta$ & 1 & $4.5-15$ & 38 \\
\hline DL2/ED2 & $1252-1270$ & wire/OTR & $\varepsilon$ & 4 & $4.5-15$ & 17 \\
\hline
\end{tabular}

\subsubsection{Installation Issues}

Some of the necessary rf modifications to the SLAC linac are listed in Table 7.28. Some accelerating structures must be removed in order to locate compressor chicanes and matching sections. And in one case, a new 3-meter structure can be added back to the linac (the extinct NPI gun located at 25-1c). 
L C L S C O N C E P T U A L D E S I G N R E P O R T

Table 7.28 Summary of modified, removed or added S-band if sections for the LCLS design. The energy loss quoted here assumes that the rf power inputs are re-configured.

\begin{tabular}{lccc}
\hline Linac section & reason for modification & net $\Delta L$ removed/added & net energy loss \\
{$[\mathbf{m}]$} & -0.35 & -8 \\
\hline $21-1 \mathrm{~b}, \mathrm{c}$ & shorten sections for L1 quads & -12.2 & -235 \\
\hline $21-2 \mathrm{a}, \mathrm{b}, \mathrm{c}, \mathrm{d}$ & remove for BC1 chicane and X-band & -3.0 & -34 \\
\hline $21-3 \mathrm{a}$ & remove for ED1 emittance diagnostic & -9.1 & -103 \\
\hline $24-3 \mathrm{~d}, 4 \mathrm{~d}, 5 \mathrm{~d}$ & remove to add L2-ED profile monitors & -12.2 & -235 \\
\hline $24-7 \mathrm{a}, \mathrm{b}, \mathrm{c}, \mathrm{d}$ & remove for BC2 chicane & -12.2 & +34 \\
\hline $24-8 \mathrm{a}, \mathrm{b}, \mathrm{c}, \mathrm{d}$ & remove for BC2 chicane & +3.0 & -34 \\
\hline $25-1 \mathrm{c}$ & replace missing 3-m section (NPI) & -3.0 & -34 \\
\hline $25-5 \mathrm{a}$ & remove to add transverse rf deflector & -3.0 & $-\mathbf{8 8 5}$ \\
\hline $27-6 \mathrm{~d}$ & remove to add L3-ED profile monitors & $-\mathbf{5 2 . 1}$ & \\
\hline total = & & - &
\end{tabular}

\subsection{Operational Issues}

The LCLS will operate at $120 \mathrm{~Hz}$ concurrent with PEP-II B-Factory operations. An electron beam can be accelerated through the entire SLAC linac to $50 \mathrm{GeV}$ by switching off and straightening the bunch compressor chicanes, or by providing adequate aperture in the chicane bends. This second option, however, compromises the ability to provide a high-resolution BPM in the center of the chicane, which is a critical requirement for the bunch length and energy feedback systems.

With the chicanes switched off, the DL1 bends are also switched off, as are the other dedicated LCLS focusing magnets in the $\mathrm{L} 1$ and $\mathrm{BC} 1$ area. This re-configuration will require a switching time on the order of a few minutes at best. The possibility of fast, pulse-to-pulse switching between LCLS and 30-GeV electrons in the end-station can probably only be realized by building a by-pass beamline from sector- 21 to sector-30, as is done in the PEP-II injection scenario.

A fast-pulsed kicker in the beam switchyard, just downstream of the linac and well before the undulator, should be installed in order to dump the electron beam during conditions of exceptionally poor beam quality. This will help to preserve the permanent magnet undulator fields, and to provide a 'veto' for unwanted pulses. It will also allow more invasive tuning with the electron beam passing through the bulk of the accelerator. The kicker will, of course, only be fast enough to dump the next pulse(s), after detection of a poor beam-quality trigger. Beam collimators just upstream of the undulator will provide protection from the first poor-quality pulse. Such poor quality conditions can be produced by klystron trips (especially in L1 or L2), the firing of the transverse rf deflector in sector-25, or any number of previously determined 
beam quality conditions. Such a kicker magnet, its power supply, and the beam dump are all presently available by recycling one of the SLC "single-beam-dumper" systems located in the beam switchyard.

\subsection{References}

1 P. Emma, "LCLS Accelerator Parameters and Tolerances for Low Charge Operations", LCLS-TN-9903, May 1999.

2 V. Bharadwaj, et al, "LCLS II Design", LCLS-TN-00-11, October 1998.

3 S. Heifets, S. Krinsky, G. Stupakov, "CSR Instability in a Bunch Compressor", SLAC-PUB-9165, March 2002.

4 E. L. Saldin, E. A. Schneidmiller, M. V. Yurkov, "Longitudinal Phase Space Distortions in Magnetic Bunch Compressors", FEL-2001 Conf., Darmstadt, Germany, DESY-01-129 (2001).

5 P. Emma, "Automated Optimization of Accelerator Parameters for Peak FEL Performance", to be published as an LCLS technical note.

6 P. Emma, P. Krejcik, C. Pellegrini, S. Reiche, J. Rosenzweig, Proceedings of the 2001 Particle Accelerator Conference, Chicago, IL, 2001.

7 K. L. Bane et al., "Electron Transport of a Linac Coherent Light Source (LCLS) Using the SLAC Linac," Proceedings of the 1993 Part. Accel. Conf., Washington, DC, 1993.

8 P. Emma, "X-Band RF Harmonic Compensation for Linear Bunch Compression in the LCLS", LCLSTN-01-01, January 2001.

9 “Zeroth-Order Design Report for the Next Linear Collider", SLAC Report 474, May 1996.

10 M. Borland, "Elegant: A Flexible SDDS-Compliant Code for Accelerator Simulation", ICAP-2000, Darmstadt, Germany, September 2000.

11 L. M. Young, J. H. Billen, "Parmela", LA-UR-96-1835, Rev, January 8, 2000.

12 C. Limborg et al., "New Optimization for the LCLS Photo-Injector", to be published at EPAC-2002, Paris, France, June 2002.

13 R. Akre, et al., "Measurements on SLAC Linac RF System for LCLS Operation", Proceedings of the 2001 Part. Accel. Conf., Chicago, IL, 2001.

14 J.B. Rosenzweig, and E. Colby, Proceedings of the Conference on Advanced Acceleration Concepts, AIP vol. 335, p. 724 (1995).

15 R. Assmann et al., "LIAR - A New Program for the Modeling and Simulation of Linear Accelerators with High Gradients and Small Emittances," 18th International Linac Conference, Geneva, Switzerland, August, 1996.

16 "LCLS Design Study Report", SLAC-R-521, April 1998.

17 C.E. Adolphsen et al., "Beam-Based Alignment Technique for the SLC Linac," Proceedings of the 1989 Part. Accel. Conf., Chicago, IL, 1989.

18 J.T. Seeman et al., "The Introduction of Trajectory Oscillations to Reduce Emittance Growth in the SLC Linac," 15th International Conf. on High Energy Accelerators, Hamburg, Germany, July 1992.

19 L.V. Iogansen, M.S. Rabinovich, Sov. Phys. JETP, vol.37(10), 1960, p. 83.

20 Ya. S. Derbenev, J. Rossbach, E. L. Saldin, V. D. Shiltsev, "Microbunch Radiative Tail-Head Interaction", TESLA-FEL 95-05, DESY, Sep. 1995. 
21 E. L. Saldin, E. A. Schneidmiller, M. V. Yurkov, "On the Coherent Radiation of an Electron Bunch Moving in an Arc of a Circle", TESLA-FEL 96-14, Nov. 1996.

22 M. Dohlus, A. Kabel, T. Limberg, "Wakefields of a Bunch on a General Trajectory Due to Coherent Synchrotron Radiation", Proceedings of the $19^{\text {th }}$ International Free Electron Laser Conference, Beijing, China, Aug. 18-21, 1997.

23 R. Li, C. L. Bohn, J. J. Bisognano, "Analysis on the Steady-State Coherent Synchrotron Radiation with Strong Shielding", Proceedings of the 1997 Part. Accel. Conf., Vancouver, BC, Canada, 1997.

24 F. Zimmermann, "Longitudinal Single Bunch Dynamics and Synchrotron Radiation Effects in the Bunch Compressor," NLC-Note-3, SLAC, October 1994.

25 R. L. Warnock, "Shielded Synchrotron Radiation and Its Effect on Very Short Bunches," SLAC-PUB5375, November 1990.

26 T. Limberg, "Emittance Growth in the LCLS due to Coherent Synchrotron Radiation," SLAC internal note, Oct. 1997.

27 M. Borland, "A Simple Method for Simulation of Coherent Synchrotron Radiation in a Tracking Code", ICAP-2000, Darmstadt, Germany, September 2000.

28 G. Stupakov, P. Emma, "CSR Wake for a Short Magnet in Ultrarelativistic Limit", submitted to EPAC-2002, Paris, France, June 3-7, 2002.

29 T. O. Raubenheimer et al., "Chicane and Wiggler Based Bunch Compressors for Future Linear Colliders," SLAC-PUB-6119, May 1993.

30 M. Dohlus, A. Kabel, T. Limberg, "Uncorrelated Emittance Growth in the TTF-FEL Bunch Compression Sections Due to Coherent Synchrotron Radiation and Space Charge Effects", Proceedings of the 2000 European Particle Accelerator Conference, Vienna, Austria, 2000.

31 Ya. S. Derbenev, V.D. Shiltsev, "Transverse Effects of Microbunch Radiative Interaction," SLACPUB-7181, Fermilab-TM-1974, May 1996.

32 M. Borland, "Bunch Compressor Design for the APS Linac", unpublished ANL note.

33 S. Anderson, et al, "OTR Monitor for the ATF Extraction", KEK/ATF internal report, ATF-01-08, June, 2001.

34 Andreas Kabel, private communication.

35 R. Chasman, K. Green, E. Rowe, IEEE Trans., NS-22, 1765 (1975).

36 M. Borland et al., "Start-to-End Simulation of Self-Amplified Spontaneous Emission Free-Electron Lasers from the Gun through the Undulator," Proc. of FEL2001, Darmstadt, Germany, August 2001.

37 H.-D. Nuhn, to be published as an LCLS technical note.

38 Ming Xie, "Design Optimization for an X-ray Free Electron Laser Driven by SLAC Linac", Proceedings of the 1995 Part. Accel. Conf., Dallas, TX, 1995.

39 S. Reiche, NIM A 429 (1999) 242.

40 J. Lewellen, private communication.

41 M.C. Ross et al., "Wire Scanners for Beam Size and Emittance Measurements at the SLC," Proceedings of the 1991 Part. Accel. Conf., San Francisco, CA, 1991.

42 D.X. Wang, "Electron Beam Instrumentation Techniques Using Coherent Radiation," Proceedings of the 1997 Part. Accel. Conf., Vancouver, BC, Canada, 1997.

43 G. A. Loew, O. H. Altenmueller, "Design and Applications of R.F. Deflecting Structures at SLAC", PUB-135, Aug. 1965. 
44 R.H. Miller, R.F. Koontz, D.D. Tsang, "The SLAC Injector", IEEE Trans. Nucl. Sci., June 1965, p804-8.

45 X.-J. Wang, "Producing and Measuring Small Electron Bunches", Proceedings of the 1999 Particle Accelerator Conference, New York, NY, March 1999.

46 P. Emma, J. Frisch, and P. Krejcik, "A Transverse RF Deflecting Structure for Bunch Length and Phase Space Diagnostics", LCLS-TN-00-12, August 2000.

47 P. Emma, et al., "Bunch Length Measurements Using a Transverse RF Deflecting Structure in the SLAC Linac", to be published in the Proceedings of the 2002 European Particle Accelerator Conference, Paris, France, June 3-7, 2002.

48 O. H. Altenmueller, R. R. Larsen, and G. A. Loew, "Investigations of Traveling-Wave Separators for the Stanford Two-Mile Linear Accelerator", The Review of Scientific Instruments, Vol. 35, Number 4, April 1964.

49 I. Ben-Zvi, J. X. Qui, X. J. Wang, "Picosecond-Resolution 'Slice' Emittance Measurement of Electron-Bunches", Proceedings of the 1997 Particle Accelerator Conference, Vancouver, BC, Canada, May 1997.

50 D.X. Wang, "Measuring Longitudinal Distribution and Bunch Length of Femtosecond Bunches with RF Zero-Phasing Method," Proceedings of the 1997 Part. Accel. Conf., Vancouver, BC, Canada, 1997.

51 P. Bolton, et al., LCLS technical note to be published.

52 T. Himel et al., "Adaptive Cascaded Beam-Based Feedback at the SLC," Proceedings of the 1993 Part. Accel. Conf., Washington, DC, 1993.

53 J. L. Turner et al., "Vibration Studies of the Stanford Linear Accelerator," Proceedings of the 1995 Part. Accel. Conf., Dallas, TX, 1995.

54 G.S. Abrams et al., "Fast Energy and Energy Spectrum Feedback in the SLC Linac," Proceedings of the 1987 Part. Accel. Conf., Washington, DC, 1987.

55 The MAFIA collaboration, User Guide, CST GmbH, Darmstadt, Germany.

56 K. Bane, P. Wilson, Proceedings of the 11th Int. Conf. on High Energy Accelerators, CERN (Birkhuser Verlag, Basel, 1980), p. 592.

57 K. Bane, SLAC-NLC-Note 9, February 1995, and in "Zeroth-order Design Report for the Next Linear Collider," SLAC Report 474, p. 367, May 1996.

58 K.L.F. Bane, M. Timm, T. Weiland, "The Short Range Wake Fields in the SBLC Linac", Proceedings of the 1997 Part. Accel. Conf., pp. 515, Vancouver, BC, Canada, 1997.

59 E. Keil, Nucl. Instr. Meth. 100, 419 (1972).

60 R. Gluckstern, Phys. Rev D, 29, 2780 (1989).

61 K.L. Bane, T. Weiland, "Verification of the SLC Wake Potentials," SLAC/AP-001, Jan. 1983.

62 A. Novokhatskii, A. Mosnier, "Short Bunch Wake Potentials for a Chain of TESLA Cavities," DAPNIA-SEA-96-08, Nov. 1996.

63 K.L.F. Bane, et al., "Calculations of the Short Range Longitudinal Wakefields in the NLC Linac", Proceedings of the 1998 European Particle Accelerator Conference, June 22-26, 1998.

64 K.L. Bane et al., "Measurements of the Longitudinal Phase Space in the SLC Linac," SLAC-PUB5255, May 1990.

65 K.L. Bane et al., "Measurement of the Longitudinal Wakefield and the Bunch Shape in the SLAC Linac," SLAC-PUB-7536, May 1997. 
66 K.L.F. Bane, et al., "Obtaining the Bunch Shape in a Linac from Beam Spectrum Measurements", Proceedings of the 1999 Part. Accel. Conf., New York, NY, USA, 1999.

67 C. Adolphsen, et al., "Measurement of Wake Field Suppression in a Damped and Detuned X Band Accelerator Structure", SLAC-PUB-7519, May 1997. 4pp, Submitted to Phys. Rev. Lett.

68 O. Napoly, O. Henry, "The Resistive-Pipe Wake Potentials for Short Bunches," Particle Accelerators, Vol. 35, pp. 235-247, 1991.

69 G. Stupakov, private communication, 2000.

70 K.L.F. Bane, M. Sands, "The Short-Range Resistive Wall Wakefields," Contributed to Micro Bunches: A Workshop on the Production, Measurement and Applications of Short Bunches of Electrons and Positrons in Linacs and Storage Rings, Upton, New York, September 28-30, 1995. 


\section{Undulator}

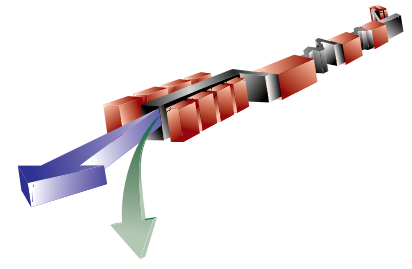

\section{TECHNICAL SYNOPSIS}

The LCLS Undulator is made up of 33 individual undulator segments. Each undulator segment will be a permanent-magnet planar hybrid device with a period length of $30 \mathrm{~mm}$ and a fixed gap of nominally $6 \mathrm{~mm}$. The actual gap will be adjusted as necessary to yield an effective $K$ of 3.71. Each undulator segment is $3.42 \mathrm{~m}$ long, with 226 poles per jaw. The poles will be made of vanadium permendur and the magnets of a grade of $N d F e B$ with a high intrinsic coercivity for better resistance to radiation-induced demagnetization. The electron beam will be focused by a separated function FODO lattice, using permanent-magnet quadrupoles placed between the undulator segments. These focusing or defocusing lenses will share the drift spaces between the undulator segments with electron beam position monitors and steering magnets. The drift space after every third undulator segment will be longer to provide space for $x$-ray diagnostics. Although the standard short drift space will be $187 \mathrm{~mm}$ long and the standard long one $421 \mathrm{~mm}$ long, the lengths of the first three drift spaces will be modified slightly to reduce the overall saturation length. The electron beam trajectory is required to be straight to within a few microns over a distance of $\sim 10 \mathrm{~m}$ to achieve adequate overlap of the electron and photon beams. It is shown that this specification, although presently beyond state-of-the-art mechanically, can be achieved with beam-based techniques.

A detailed magnetic design for the undulator segments is given, and a mechanical scheme for holding the magnets and poles with the necessary rigidity is presented. Provision has been made for magnetic tuning of the undulator segments by allowing small, few-period long adjustments to the magnetic gap, and by providing for side shims. In addition, adjustment of the gap at the ends of the undulator segments will be possible on a sub-micron level in order to adjust the phasing between the undulator segments. Tolerances have been developed that will set the magnetic tuning requirements for the individual undulator segments.

The smoothness requirement for the inside surface of the vacuum chamber is analyzed, and found to be achievable. Ions produced by passing electron or $x$-ray beams can have a deleterious effect on the electron bunch, but, at the design vacuum level, the effect of the ion production is not significant.

Diagnostics for the electron beam will include beam position monitors and Cherenkov detectors after each undulator segment, optical transition radiation imaging after every $3^{\text {rd }}$ undulator segment, and wire scanners and current monitors at the ends of the undulator line. 


\subsection{Overview}

\subsubsection{Introduction}

In a single pass FEL (free electron laser) operating in the SASE (self-amplified spontaneous emission) regime, exponential gain of the coherent radiation intensity and saturation after about twenty power gain lengths are predicted by theory (see Chapter 4). An FEL operating at saturation will have a more stable radiation output. Therefore, a goal in the design of the undulator line is to allow saturation to be reached while minimizing the required undulator length. Minimizing the undulator length helped guide many of the parameter choices for the undulator line and was also used in allocating error tolerances. Sufficient diagnostics must be included between undulator segments to effectively and conveniently monitor the electron and x-ray beams along the length of the undulator line. These diagnostics will be used as an aid in electron beam tuning, to identify problems, to monitor the intensity gain in the x-ray beam, and to confirm that saturation has been achieved.

The basis for choice of parameters for the undulator line is explained in Section 8.2. A tolerance budget for trajectory straightness through the undulator segments, phase errors, and positioning errors is also given in that section. Section $\mathbf{8 . 3}$ gives requirements for the magnetic measurement of the undulator segments. Considerations to be used in the measurement and sorting of the magnet blocks for the undulator segments are given in Section 8.4. Considerations in the choice of the grade of $\mathrm{NdFeB}$ magnets are explained in Section 8.5.1, and a magnetic design is given in Section 8.5.2, along with results from the magnetic modeling calculations. Some considerations of the end tuning for the undulator segments are given in Section 8.5.3. Section 8.6 shows the mechanical design for the undulator segments, including the scheme for holding the magnets and poles (see Section 8.6.1), the provisions included for magnetic tuning, both through the main part of the undulator segment and through the ends (see Section 8.6.2), the supports for the undulator segments that also provide for overall position adjustment (see Section 8.6.3), and the impact of temperature changes on the undulator segments (see Section 8.6.4). The design for the permanent magnet quadrupoles is given in Section 8.7. The effect of missteering of the electron beam so that it strikes the vacuum chamber and subsequently the undulator segments, and the possibility of using collimators are considered in Section 8.8.1, along with possibilities for reducing the roughness of the inside of the vacuum chamber. A description of the vacuum system is in Section 8.8.2, pumping and outgassing considerations are given in Section 8.8.3, and thermal considerations are in Section 8.8.4. Section 8.9 discusses wakefield sources in the undulator vacuum chamber. Section 8.9.5 considers the effect of the roughness of the inside surface of the vacuum chamber on the wakefields and concludes that the smoothness requirements can be met. Section 8.10 considers the means by which ions could be produced and the effect that those ions could have on the beam, concluding that the necessary vacuum is readily achievable. The electron beam diagnostics include pickup electrode beam position monitors, Cherenkov detectors, optical transition radiation imagers, wire scanners, and current monitors, as described in Section 8.11. Section 8.12 describes the beam-based alignment scheme, including 
the results of simulations. Finally, Section 8.13 describes the x-ray diagnostics that will be located after every third undulator segment.

\subsubsection{Undulator Line Design Summary}

The Linac Coherent Light Source (LCLS) undulator line will consist of 33 undulator segments separated by breaks of various lengths. The undulator segments are 3.4-m-long permanent-magnet planar hybrid arrays with a period of $30 \mathrm{~mm}$ and a magnetic gap of $6 \mathrm{~mm}$. The maximum outside dimension of the vacuum chamber is $5.6 \mathrm{~mm}$. Focusing quadrupoles, in a FODO lattice, and electron beam diagnostics will be located in the breaks between undulator segments. Every third break will be longer in order to also accommodate x-ray diagnostics. Thus, taking the alternating focusing and defocusing quadrupoles into account, the 'super-period' length before the undulator line repeats itself is six undulator segments. The first three break lengths will be different from those in the main part of the undulator line, however, because small modifications there help reduce the overall undulator length needed for saturation.

Other options for the undulator segments were considered, such as bifilar helical electromagnetic devices using either superconducting DC coils or warm pulsed coils. These were rejected because they were shown to be costly, complicated, difficult to hold to mechanical tolerances, and difficult to provide with steering corrections. Also, access to the beam pipe would be impaired because it would be completely surrounded by the undulator segments. A significant amount of R\&D would be required to make a superconducting magnet design work. In contrast, planar hybrid technology is well established, and the requirements for the undulator segments, while demanding, have been met in existing devices. These arguments led to the choice of the planar hybrid design.

The electron beam beta function and the undulator period were selected to minimize the saturation length. The FEL simulation code RON $[1,2]$ has been used to optimize parameters such as the length of the undulator segments and the break lengths between them. Tolerances for individual undulator segments have also been determined.

The quadrupole focusing magnets are made using permanent magnets. No provision is planned for adjusting the strength of the quadrupole, so magnetic tuning and adjustment of the integrated quadrupole gradient will be done during the manufacture of the quadrupole, before it is installed. The strength of the quadrupoles will not be adjusted over the proposed operating range of the LCLS electron beam energy. Instead, the beam parameters at the entrance to the undulator line will be adjusted for proper matching.

In addition to focusing, the quadrupole magnets between the undulator segments serve two other functions: they will be used in the initial alignment to establish a straight-line trajectory and they will be moved mechanically on vertical and horizontal slides to correct the trajectory to approximate a straight line. 


\subsection{Theory and Tolerances for the Undulator}

\subsubsection{Design of Undulator Segments}

\subsubsection{Basic Considerations}

One possibility for creating an x-ray FEL is to use the SASE scheme. This scheme involves only two elements: an undulator and the electron beam propagating through the undulator. The electron beam is unstable in that it bunches at the wavelength of the fundamental harmonic of the spontaneous undulator radiation. When the bunching is small, the system is linear, so the Fourier harmonics of the beam current at this frequency grow exponentially with distance traveled through the undulator. The power gain length is the characteristic length where the squared magnitude of the fundamental Fourier harmonic increases by a factor of $e$. At some distance from the beginning of the undulator, the electron beam has become significantly bunched and there is no further growth; this distance is the saturation length. The coherent undulator radiation produced by the bunched beam is the output of the FEL. An advantage of this FEL scheme is the absence of mirrors, which are a serious problem for x-ray wavelengths. A disadvantage is that the radiation spectrum is relatively wide. From the point of view of building such a device, the main problems are obtaining a high-current low-emittance low-energy-spread electron beam to keep the saturation length within reasonable limits (i.e., not much over 100 meters) and to meet the tight tolerances for field errors, misalignments and steering errors of the undulator.

Typically the saturation length is about 20 times the power gain length. For an FEL that is barely (or not) long enough to saturate, nearly all the output light comes from the end of the undulator line. Most of the line, therefore, is devoted to bunching the electron beam by linearly amplifying the initial electron density fluctuations. Therefore, the goal in optimizing this part of the undulator line is to minimize the power gain length.

\subsubsection{Optimal Period and Focusing}

The main parameters of the LCLS project are listed in Table 8.1 and in Appendix A. In Figure 8.1 and Figure 8.2 the dependence of the saturation length on the undulator period and matched beta function for the planar permanent magnet undulator is shown. This dependence was obtained using the formulas of Halbach [3] and Ref. [4] and takes into account both the energy spread due to quantum excitation and the undulator "filling factor" (the fraction of the undulator occupied by undulator segments rather than by the breaks between them). 
LCLS CONCEPTUAL DESIGN REP O R T

Table 8.1 Parameters of the LCLS project.

\begin{tabular}{ll}
\hline Parameter & Value \\
\hline Radiation wavelength & $1.5 \AA$ \\
\hline Beam energy & $14.35 \mathrm{GeV}$ \\
\hline Normalized slice emittance & $1.2 \mathrm{~mm}-\mathrm{mrad}$ \\
\hline Beam peak current & $3.4 \mathrm{kA}$ \\
\hline Slice energy spread (standard deviation) & $1.4 \mathrm{MeV}$ \\
\hline Focusing & FODO \\
\hline Undulator period & $30 \mathrm{~mm}$ \\
\hline Undulator parameter K & 3.71 \\
\hline Undulator effective peak on-axis field & $1.3250 \mathrm{Tesla}$ \\
\hline Nominal magnetic gap & $6 \mathrm{~mm}$ \\
\hline Undulator segment length & $3.420 \mathrm{~m}$ \\
\hline Break length (short) & $0.187 \mathrm{~m}$ \\
\hline Break length (long) & $0.421 \mathrm{~m}$ \\
\hline Supercell length (6 undulator segments) & $22.110 \mathrm{~m}$ \\
\hline Number of undulator segments & 33 \\
\hline
\end{tabular}

Figure 8.1 and Figure 8.2 show that the design values of $3 \mathrm{~cm}$ for the undulator period and $18 \mathrm{~m}$ for the beta function are close to optimal at the $14.35 \mathrm{GeV}$ energy-end of the operational range. For lower energy spread and emittance, the optimal undulator period decreases.

The calculation above assumes a planar permanent magnet undulator. Calculations were also done for a superconducting helical undulator. For a period of $2.4 \mathrm{~cm}$, a field of $1.3 \mathrm{~T}$, and with other parameters the same as for the planar permanent magnet option, the saturation length is about $50 \mathrm{~m}$. Although this saturation length is shorter than for a planar undulator, there remain some as yet untested aspects to the mechanical design of a superconducting helical device. Since planar permanent magnet undulator segments are an established technology, they will be used for the LCLS project. 


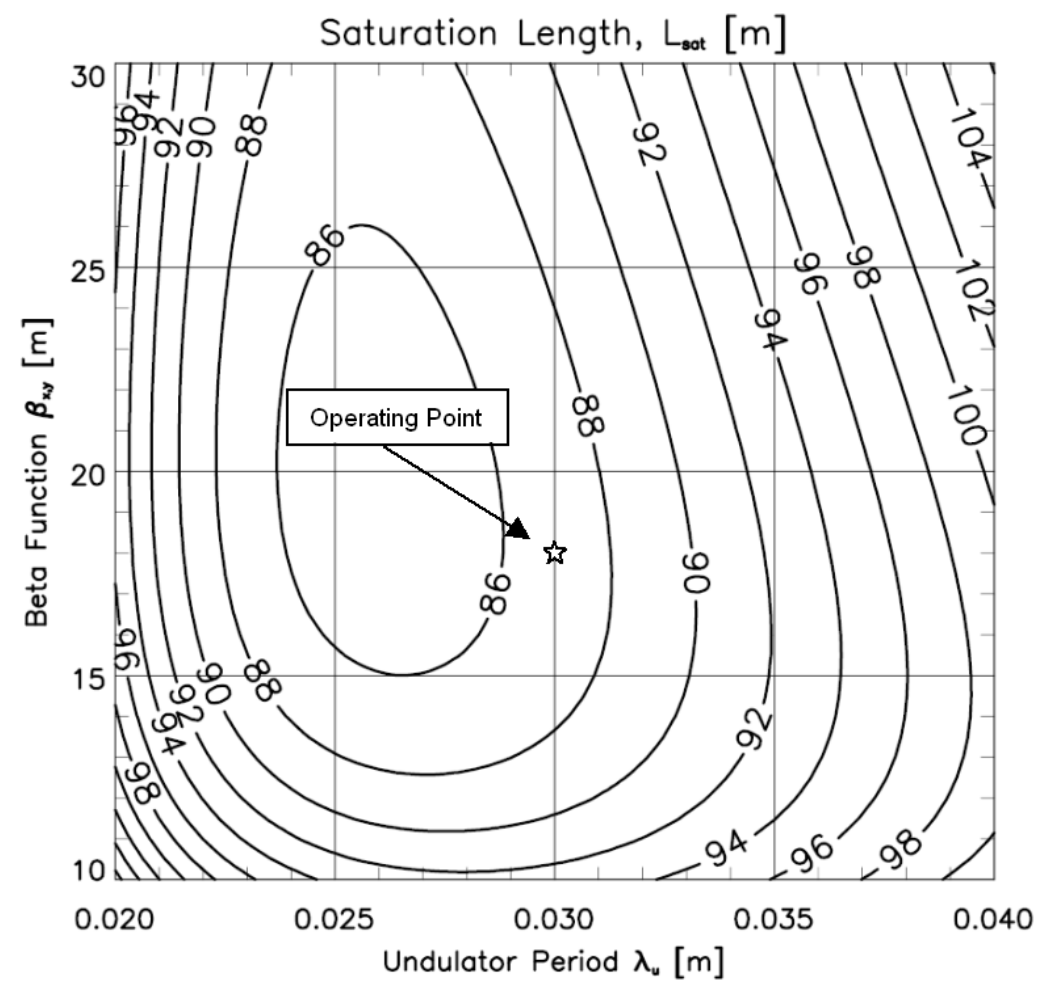

Figure 8.1 Contours of constant saturation length. While the wavelength of the light produced is always $1.5 \AA$, the magnetic field strength in the undulator is adjusted with the undulator period length according to Halbach's formula [3], and the energy of the electron beam is changed as needed to preserve the wavelength of the light. All numbers are in meters.

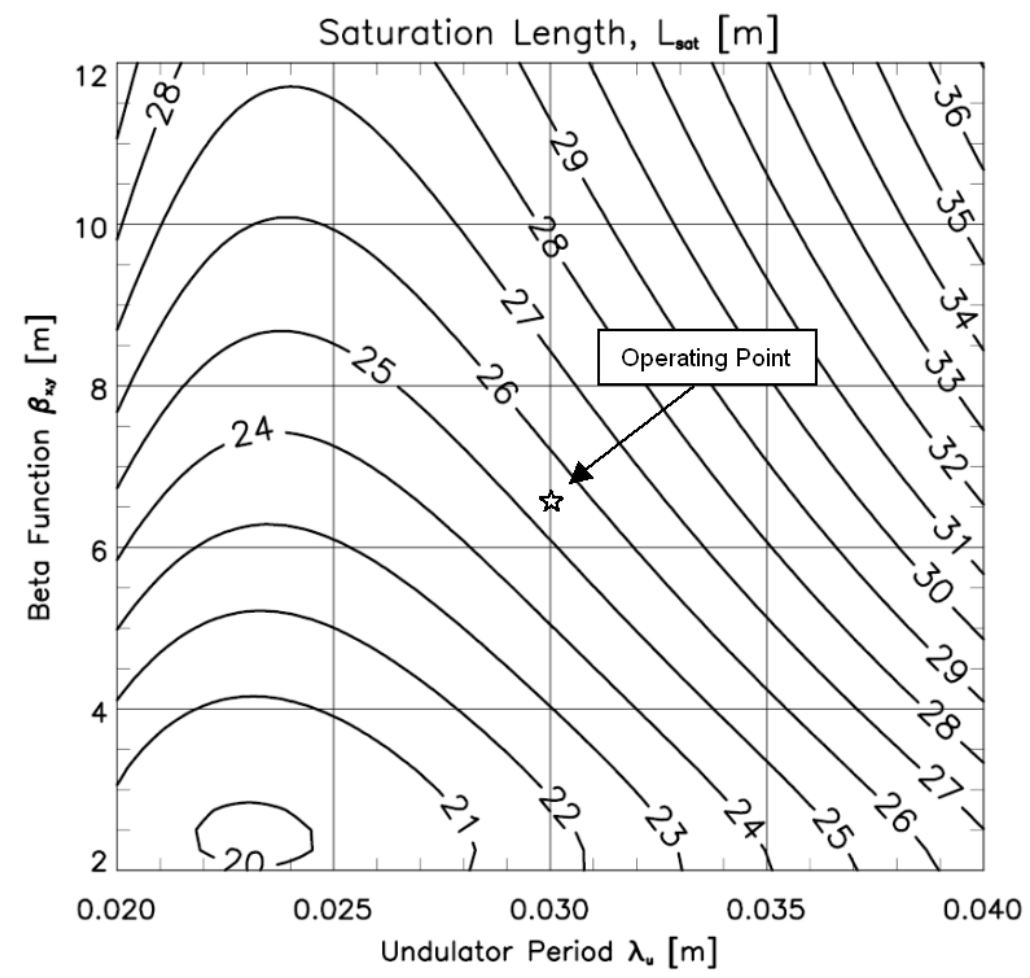

Figure 8.2 Same as Figure $\mathbf{8 . 1}$ expect that the wavelength is $15 \AA$. 


\subsubsection{Irregularities and Imperfections}

The linear theory of high gain is well developed now (see, for example, [5]). Nevertheless, the design of a real magnetic system for a short-wavelength high-gain FEL requires consideration of an inhomogeneous magnetic system with focusing quadrupoles inserted into the breaks between undulator segments. Field, steering, and alignment errors must be considered. The linear time-independent code RON $[1,2]$ was written for the optimization of such magnetic systems. It has been cross-checked with 3D codes such as GINGER and GENESIS and was used successfully for the design optimization of the Argonne FEL [6]. This code has now also been used for the optimization of the LCLS undulator line.

The simplest way to provide proper focusing is to use a FODO lattice, and this choice has been made for the LCLS project. The magnetic system of the undulator will consist of undulator segments with breaks between the segments where quadrupoles and beam position monitors will be installed. After every third undulator segment, the break will be longer so that photon diagnostics can be installed as well. This layout is geometrically similar to the existing APS FEL except that the photon diagnostics are only after every third undulator segment. Another lattice based on quadrupole triplets between undulator segments was considered and rejected because of very tight tolerances for the relative alignment of the three-quadrupole centers.

The following parameter choices were made, based on the results of RON [1,2] calculations:

- The optimal undulator segment length was found to be near $3.4 \mathrm{~m}$. For shorter lengths, the "filling factor" is less, making the effective power gain length longer. (This assumes that the break length is kept at about $0.2 \mathrm{~m}$, which is required for the quadrupoles.) For longer lengths, the power gain length at a beam energy of $4.5 \mathrm{GeV}$ increases due to the larger variation of the beta function within the undulator. The longer lengths are also more difficult mechanically, given the demanding tolerances.

- The optimal average value for the beta function was found to be $18 \mathrm{~m}$. The focal lengths of the quadrupoles are chosen accordingly.

- The break lengths between undulator segments were optimized by calculating the corrections to the "resonance" break length due to the effect of finite emittance and diffraction.

- An option that included magnetic bunchers between the undulator segments was considered and optimized. No significant improvement was found, so no magnetic bunchers are included in the undulator line design.

- The effect of the residual trajectory errors after simulated beam-based alignment [7] was calculated for the optimized undulator. The increase of the saturation length was found to be less than $5 \mathrm{~m}$. 
- The effect of the spread of deflection parameters $K$ in different undulator segments was simulated. This way the corresponding tolerances were found.

\subsubsection{Tolerances for Undulator Segments}

The aim of our optimization is to minimize the power gain length and consequently the saturation length. There are tens of significant parameters in the system, and a deviation in any of these parameters will increase the power gain length. A tolerance budget was worked out for the various parameters so that the overall power gain length increase does not exceed $3 \%$, which corresponds to a 4-m increase in saturation length. Tolerances were set assuming simultaneous worst cases for all parameters. The overall tolerances for the undulator line were used to determine tolerances for a single undulator segment.

The following requirements for the undulator segment field errors were developed. (The derivation is described in the next section.)

- The trajectory walk-off from a straight line must not exceed 2 microns in one segment. The beam-based alignment technique will minimize deviations in the transverse beam coordinates near the beam position monitors (BPMs) between the undulator segments, so the trajectory walk-offs $x(z)$ and $y(z)$ with zero initial (at the upstream BPM) and final (at the downstream BPM) coordinates have to be specified:

$$
\begin{aligned}
& x(z)=\frac{1}{\gamma} \int_{0}^{z} I_{1 x}\left(z^{\prime}\right) d z^{\prime}, y(z)=\frac{1}{\gamma} \int_{0}^{z} I_{1 y}\left(z^{\prime}\right) d z^{\prime} \\
& I_{1 x}(z)=\frac{e}{m c^{2}}\left[\int_{0}^{z} B_{x}\left(z^{\prime}\right) d z^{\prime}-\frac{1}{L} \int_{0}^{L} \int_{0}^{z^{\prime}} B_{x}\left(z^{\prime \prime}\right) d z^{\prime \prime} d z^{\prime}\right] \\
& I_{1 y}(z)=\frac{e}{m c^{2}}\left[\int_{0}^{z} B_{y}\left(z^{\prime}\right) d z^{\prime}-\frac{1}{L} \int_{0}^{L} \int_{0}^{z^{\prime}} B_{y}\left(z^{\prime \prime}\right) d z^{\prime \prime} d z^{\prime}\right]
\end{aligned}
$$

where $\gamma$ is the relativistic factor, $e$ and $m$ are electron charge and mass, $c$ is the velocity of light, $B_{x}$ and $B_{y}$ are the measured transverse components of magnetic field, and $L$ is the cell length (the distance between BPMs). The 2-micron deviations in both the $x$ and $\mathrm{y}$ directions give an increase in the power gain length of less than $0.2 \%$, and can be achieved with present magnetic measurement and tuning techniques.

- The reduction in spectral intensity of the zero-angle radiation must not exceed $4 \%$. The spectral intensity of the zero-angle radiation is

$$
\frac{e^{2} k^{2}|A|^{2}}{2 \pi c \gamma^{2}}
$$


where $k$ is the fundamental harmonic wavevector of the undulator radiation, and

$$
A=\int_{0}^{L} I_{1 y}(z) e^{-i \frac{k}{2 \gamma^{2}}\left[z+\int_{0}^{z} I_{1 x}^{2}\left(z^{\prime}\right) d z^{\prime}+\int_{0}^{z} I_{1 y}^{2}\left(z^{\prime}\right) d z^{\prime}\right]} d z
$$

The "reduction" is as compared with an ideal undulator, but in practice the comparison can be with the best undulator, i.e., the one which gives the highest value of $|A|$. A $4 \%$ intensity reduction corresponds to an increase in the power gain length by $1.1 \%$.

- The calculated electron phase deviation from the design value must be less than $10^{\circ}$ in one segment. This phase is simply the electron-wave slippage:

$$
\varphi=\frac{k}{2 \gamma^{2}}\left[L+\int_{0}^{L} I_{1 x}^{2}(z) d z+\int_{0}^{L} I_{1 y}^{2}(z) d z\right]
$$

and the "design value" is an integer multiple of $2 \pi$. A $10^{\circ}$ phase error causes an increase in power gain length of $1.7 \%$.

- The undulator median plane must be defined (and after that aligned) with an accuracy better than 50 microns vertically. If the beam is off-axis vertically by 50 microns, the beam will see a stronger undulator field, resulting in about $10^{\circ}$ of additional phase slippage.

Implicit in these tolerances is the need for the magnetic field strength to be uniform along the length of the undulator line. If the magnetic field in one undulator segment is wrong by $\Delta \mathrm{B} / \mathrm{B}=$ $1.5 \times 10^{-4}$, the resulting phase error will be $10^{\circ}$. This tolerance agrees with the result from independent simulations performed using the code RON [2] to vary the strength of one undulator segment. Then the change in the overall gain became significant when segment-to-segment variations reached $\Delta \mathrm{B} / \mathrm{B}=1.3 \times 10^{-4}$. This translates into an error in the magnetic field strength of $1.7 \mathrm{G}$, or an error in the undulator segment magnetic gap by $1.2 \mu \mathrm{m}$.

\subsubsection{Derivation of the Tolerances for the X-Ray FEL}

The following is based on a simple picture of acceleration (or deceleration) of the electron by the given radiation eigenmode.

\subsubsection{Derivation of Basic Equations}

According to the Floquet theorem, the wave field eigenmode in the periodic amplifying system can be represented as:

$$
E_{x}=\mathfrak{R}\left[u(x, y, z) e^{p z} e^{i k(z-c t)}\right]
$$


where $u(x, y, z+L)=u(x, y, z), L$ is the system period, $k$ is the design value of the undulator radiation fundamental harmonic wavevector, and $p$ is the complex growth rate. Then the electron longitudinal motion equations are:

$$
\begin{aligned}
& \frac{d E}{d z}=e E_{x} \frac{d x}{d z} \\
& \frac{d t}{d z}=\frac{1}{v_{z}}
\end{aligned}
$$

where $e$ and $E=\gamma m c^{2}$ are the electron charge and energy, $t$ is the moment of time when the electron passes the longitudinal coordinate $z$, and the electron velocity $v_{z}$ can be expressed through the electron energy and angles:

$$
\frac{1}{v_{z}} \approx \frac{1}{c}\left[1+\frac{1}{2 \gamma^{2}}+\frac{1}{2}\left(\frac{d x}{d z}\right)^{2}+\frac{1}{2}\left(\frac{d y}{d z}\right)^{2}\right]
$$

The angles $d x / d z=\alpha$ and $d y / d z$ can be calculated from the measured magnetic field $\boldsymbol{B}$ using the trajectory equations:

$$
\begin{aligned}
& \frac{d}{d z}\left(\frac{d x}{d z}\right)=-\frac{e}{\gamma m c^{2}} B_{y}(z) \\
& \frac{d}{d z}\left(\frac{d y}{d z}\right)=\frac{e}{\gamma m c^{2}} B_{x}(z)
\end{aligned}
$$

The LCLS undulator line includes beam position monitors and angle steering at the section ends. For ideal steering, the trajectory displacement at the segment ends is zero for the equilibrium (beam centroid) trajectory. Therefore, for the undulator specification, the solution of Eq. (8.10) with $x(0)=x(L)=y(0)=y(L)=0$ is chosen. It is convenient to introduce the corresponding "corrected" dimensionless first field integrals $I_{1 \mathrm{x}}=-\gamma d x / d z$ and $I_{1 y}=\gamma d y / d z$ :

$$
\begin{aligned}
& I_{1 x}(z)=\frac{e}{m c^{2}}\left[\int_{0}^{z} B_{x}\left(z^{\prime}\right) d z^{\prime}-\frac{1}{L} \int_{0}^{z} \int_{0}^{z^{\prime}} B_{x}\left(z^{\prime \prime}\right) d z^{\prime \prime} d z^{\prime}\right] \\
& I_{1 y}(z)=\frac{e}{m c^{2}}\left[\int_{0}^{z} B_{y}\left(z^{\prime}\right) d z^{\prime}-\frac{1}{L} \int_{0}^{z} \int_{0}^{z^{\prime}} B_{y}\left(z^{\prime \prime}\right) d z^{\prime \prime} d z^{\prime}\right]
\end{aligned}
$$

The maximum particle energy variation 


$$
\Delta E=e \int_{0}^{z} E_{x}\left[x, y, z^{\prime}, t\left(z^{\prime}\right)\right] \frac{d x}{d z}\left(z^{\prime}\right) d z^{\prime}
$$

is proportional to

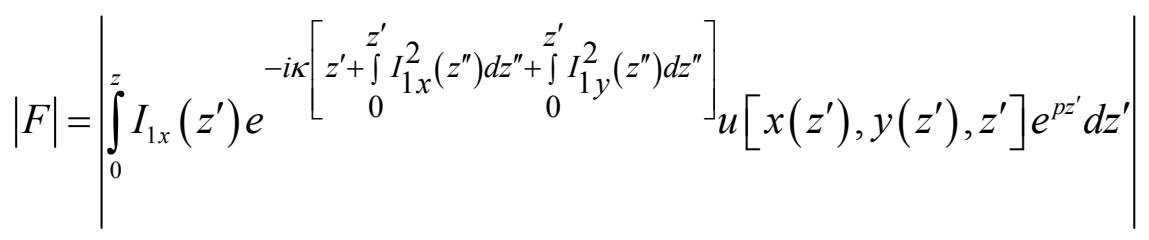

where $\kappa=k /\left(2 \gamma^{2}\right)$. For the LCLS the breaks between undulator segments are relatively short and produce a phase shift of an integer multiple of the x-ray wavelength. Therefore it is close to the homogeneous undulator case, described analytically in Ref. [8]. Then the fundamental eigenmode is close to the Gaussian beam with almost flat wavefronts

$$
u \propto e^{-\frac{x^{2}+y^{2}}{2 \sigma_{r}^{2}}}
$$

The relative reduction of the maximum energy gain caused by different field imperfections is calculated using Eq. (8.14) and $F$ is expanded near the "ideal" state, by different kinds of "imperfections".

\subsubsection{Trajectory Straightness}

Using Eq. (8.15) at $z=N L$ at the end of the $N^{\text {th }}$ undulator segment, one can expand $F$ in $x$ and $y$ :

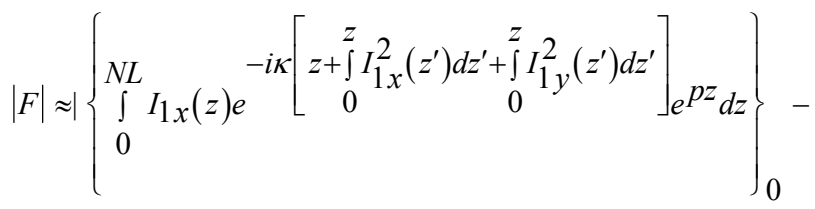

$$
\begin{aligned}
& \left.\frac{1}{2 \sigma_{r}^{2}} \int_{0}^{N L} I_{1 x}(z) e^{-i \kappa\left[z+\int_{0}^{z} I_{1 x}^{2}\left(z^{\prime}\right) d z^{\prime}+\int_{0}^{z} I_{1 y}^{2}\left(z^{\prime}\right) d z^{\prime}\right.}\right]_{e} p z\left[x^{2}(z)+y^{2}(z)\right] d z
\end{aligned}
$$

For the "ideal" case (the curly brackets in Eq. (8.16)), the slow part of the expression under the integral is almost constant. Therefore, Eq. (8.16) leads to

$$
\frac{F-F_{0}}{F_{0}} \approx-\frac{\left\langle x^{2}\right\rangle+\left\langle y^{2}\right\rangle}{2 \sigma_{r}^{2}},
$$


where the averaging takes place over the length $N L$, and can be replaced by averaging over each undulator segment, i.e.,

$$
\begin{aligned}
& \left\langle x^{2}\right\rangle=\frac{1}{L \gamma^{2}} \int_{0}^{L}\left[\int_{0}^{z} I_{1 x}\left(z^{\prime}\right) d z^{\prime}\right]^{2} d z, \\
& \left\langle y^{2}\right\rangle=\frac{1}{L \gamma^{2}} \int_{0}^{L}\left[\int_{0}^{z} I_{1 y}\left(z^{\prime}\right) d z^{\prime}\right]^{2} d z .
\end{aligned}
$$

\subsubsection{Phase Errors}

Now suppose that $\langle x\rangle=y=0$ (so $u=1$ ) and $F$ can be represented as the sum of contributions of $N$ undulator segments:

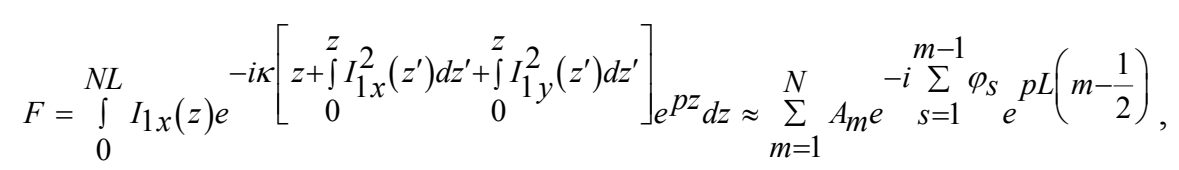

where

$$
\begin{aligned}
& A_{m}=\int_{0}^{L} I_{1 x}(z) e^{-i k\left[z+\int_{0}^{z} I_{1 x}^{2}\left(z^{\prime}\right) d z^{\prime}+\int_{0}^{z} I_{1 y}^{2}\left(z^{\prime}\right) d z^{\prime}\right]} d z
\end{aligned}
$$

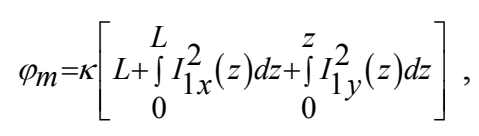

and the first field integrals in Eqs. 8.2-14 and 8.2-15 are calculated for the undulator segment number $m$. $A$ is the dimensionless amplitude of the spontaneous undulator radiation in the forward direction for one undulator segment. For the "ideal" case all $A_{m}=A_{0}$ are equal and $\varphi_{m}-\operatorname{Im}(p L)=$ $2 \pi q$ ( $q$ is an integer). Then

$$
F_{0}=A_{0} \frac{e^{\Re(p L N)}-1}{e^{\Re(p L)}-1} e^{\frac{1}{2} p L}
$$

For ideal phasing, $\varphi_{m}=2 \pi q-\operatorname{Im}(p L)$ (one can do it by the proper adjustment of the field at the undulator segment ends), and Eq. (8.19) gives: 


$$
\begin{aligned}
|F|^{2} & =\sum_{m=1}^{N} \sum_{m=1}^{N} A_{m} A_{n}^{*} e^{\Re(p)(m+n-1) L} \\
& \approx \sum_{m=1}^{N} \sum_{n=1}^{N}\left[\left|A_{0}\right|^{2}+A_{0}\left(A_{n}^{*}-A_{0}^{*}\right)+A_{0}^{*}\left(A_{m}-A_{0}\right)\right] e^{\Re(p)(m+n-1) L} \\
& \approx\left|F_{0}\right|^{2}+2\left|A_{0}\right|^{2} \operatorname{Re}\left[\frac{e^{\Re(p L N)}-1}{\mathfrak{R}(p L)}-1 \sum_{n=1}^{N} \frac{A_{n}-A_{0}}{A_{0}} e^{\Re(p) L n}\right] \\
& =\left|F_{0}\right|^{2}\left(1+2\left\langle\mathfrak{R}\left(\frac{A_{m}-A_{0}}{A_{0}}\right)\right\rangle\right)
\end{aligned}
$$

For $A_{m}=A_{0}$ but non-ideal phases,

$$
\begin{aligned}
|F|^{2} & =\left|A_{0}\right|^{2} \sum_{m=1}^{N} \sum_{n=1}^{N} e^{-i \sum_{s=1}^{m-1} \varphi_{S}+i \sum_{s=1}^{n-1} \varphi_{S}} e^{\Re(p)(m+n-1) L} \\
& \approx\left|F_{0}\right|^{2}-\left|A_{0}\right|^{2} \sum_{m=1}^{N} \sum_{n=1}^{m-1}\left(\sum_{s=n}^{m-1} \varphi_{S}\right)^{2} e^{\Re(p)(m+n-1) L}
\end{aligned}
$$

For the average case of uncorrelated undulator phase advances, one can estimate the sum in Eq. (8.24) as:

$$
|F|^{2} \approx\left|F_{0}\right|^{2}\left[1-\frac{1}{2 \sinh (\mathfrak{R}(p L))}\left\langle\varphi_{m}^{2}\right\rangle\right]
$$

But, for the worst case it gives:

$$
\left(|F|^{2}\right)_{\min } \approx\left|F_{0}\right|^{2}\left[1-\frac{1}{2 \sinh (\mathfrak{R}(p L)) \tanh (\mathfrak{R}(p L) / 2)}\left(\varphi_{m}^{2}\right)_{\max }\right] \text {. }
$$

Let $\varphi_{m}$ be distributed homogeneously over the interval $\left(-\left|\varphi_{m}\right| \max ,\left|\varphi_{m}\right|_{\max }\right)$. Then $\left\langle\varphi_{m}{ }^{2}\right\rangle=\left(\left|\varphi_{m}\right| \max \right)^{2} / 3$, and, with $92 \%$ probability

$$
\left(\left|\varphi_{m}\right|_{\max }^{2}\right) /[2 \sinh (\Re(p L))]
$$

is the upper limit for the correction in Eq. (8.24). 


\subsubsection{Resulting Power Gain Length Increase.}

Gathering the results of Eqs. (8.17), (8.23), and (8.24),

$$
\frac{|F|-\left|F_{0}\right|}{\left|F_{0}\right|}>-\frac{\left\langle x^{2}\right\rangle+\left\langle y^{2}\right\rangle}{2 \sigma_{r}^{2}}+\left\langle\mathfrak{R}\left(\frac{A_{m}-A_{0}}{A_{0}}\right)\right\rangle-\frac{L_{g}}{2 L}\left|\varphi_{m}\right|_{\max }^{2},
$$

where $L_{g}=1 /[2 \operatorname{Re}(p)]$ is the power gain length.

This reduction of the wave-electron interaction efficiency can be expressed as an effective reduction of the undulator parameter $K$ for the ideal magnetic system:

$$
\frac{K-K_{0}}{K_{0}}>-\frac{\left\langle x^{2}\right\rangle+\left\langle y^{2}\right\rangle}{2 \sigma_{r}^{2}}+\left\langle\operatorname{Re} \frac{A_{m}-A_{0}}{A_{0}}\right\rangle-\frac{L_{g}}{2 L}\left|\varphi_{m}\right|_{\max }^{2} .
$$

Now one can use analytical theory [8] or computer code to calculate the corresponding increase of the power gain length. For the LCLS magnetic system, a 3\% increase in power gain length corresponds to a $5.4 \%$ reduction in $K$. Taking $\sigma_{r}^{2} \approx \varepsilon \beta$ [8], one can make the tolerance budget.

\subsection{Undulator Measurement and Tuning}

\subsubsection{Requirements for the LCLS}

The main tolerance requirements for alignment and field quality within a section of the undulator line that includes an undulator segment and a quadrupole are given in Table 8.2.

Table 8.2 Alignment tolerances for undulator segment

\begin{tabular}{ll}
\hline Alignment Tolerance & Value \\
\hline Horizontal and vertical trajectory excursion & $2 \mu \mathrm{m}$ \\
\hline "Radiation amplitude" deviation (see Eq., (8.5)) & $2 \%$ \\
\hline Phase slip between two undulator segments & 10 degrees \\
\hline Vertical positioning error & $50 \mu \mathrm{m}$ \\
\hline
\end{tabular}

These tolerances correspond to approximately $3 \%$ growth of the power gain length. They are demanding, but achievable. They have already been met by the undulator segments that were tuned magnetically for installation in the APS FEL. Those undulator segments have a period length of $33 \mathrm{~mm}$, close enough to the $30 \mathrm{~mm}$ period length of the LCLS undulator segments that the tuning techniques developed for the APS undulator segments should transfer directly. 


\subsubsection{APS Magnetic Measurement Facility}

In order to meet these requirements, the APS magnetic measurement facility is equipped with a 6-m-long granite bench for Hall probe and moving coil measurements and a flipping coil system for first and second field integral measurements.

The magnetic measurement capabilities are as follows: Hall probe measurements can measure the field strength (i.e. effective K-value) within an accuracy of $10^{-4}$ with proper averaging. Measurements of the first and second field integrals with a flipping coil system are reproducible with accuracies of $5 \mathrm{G}-\mathrm{cm}$ and $1000 \mathrm{G}-\mathrm{cm}^{2}$, corresponding to $0.1 \mu \mathrm{rad}$ and $0.2 \mu \mathrm{m}$, respectively, for an electron beam energy of $14.3 \mathrm{GeV}$. Measurements of phase slippage over the length of the device are reproducible to 0.5 degrees in phase.

Therefore, the present state-of-the-art measurement methods meet the accuracy requirements of the LCLS undulator line. For some measurements and adjustments, however, special attention is required to achieve the required accuracy. For example, horizontal field measurements in the presence of a strong vertical field are extremely difficult due to the planar Hall effect. The Hall voltage is given in the following equation:

$$
V_{h}=V_{0}+R_{h} B_{y} I_{e}-P B_{t}^{2} I_{e} \sin (2 \phi)
$$

The last term represents the planar Hall effect, where $\phi$ is the angle between the component of the magnetic field that lies in the plane of the Hall probe and the direction of the current in the plane of the Hall probe. If there is a field component parallel to the probe plane, some additional Hall voltage may appear depending on the direction of the field component in the measurement. This planar Hall voltage is usually very small, but can become significant when $B_{t}$ is much larger than $B_{y}$. Thus, measurements of the transverse component of the undulator field are made more complicated by the planar Hall effect.

Two different Hall probes have been tested for their ability to measure the transverse field. Measurements with each probe were compared to integrated measurements made with a stretched coil. The tests showed that it was impossible to match the reference data for both first and second field integrals simultaneously using the Bell probe. This could be due to imperfect alignment of the poles in the beam direction, but if it is, it would be because the probe had more demanding alignment requirements than the undulator application. In contrast, the Sentron probe measurements could be made to match the reference data after careful alignment of probe and undulator segment in the vertical direction. One cannot rely on Hall probe measurements alone, however, because each probe has a different sensitivity to angle and position errors. Calibration by some other means is always needed.

The other crucial adjustments are the phase tuning within an undulator segment and the adjustment of the phasing between undulator segments. Phase tuning within an undulator segment can be done by shimming or by mechanical local adjustments to the undulator gap. Phasing between undulator segments is affected by the physical separation between undulator segments 
and by the phasing through the adjacent end sections of the two undulator segments. The physical break length is of course set when the undulator line is constructed. The phasing through the end sections can be adjusted by applying phase shims near the ends of the undulator segment or by introducing a small gap change at the end of the undulator segment. The latter technique has the advantage that the phasing can be adjusted during the actual operation of the FEL, using the FEL output as feedback. It would allow phase error accumulation to be avoided through the whole beamline.

\subsubsection{Requirements for Measurement Facility on LCLS Site}

The existing measurement facility at APS has adequate resolution and reproducibility for the measurement and tuning of undulator segments for the LCLS project. However, the shipment of tuned undulator segments from Argonne to SLAC may affect the magnetic quality of the undulator segments so they no longer meet the demanding magnetic field quality and stability requirements. For example, a gap change of just over a micrometer may introduce $10^{\circ}$ phase slippage. To meet these demanding magnetic requirements, very rigid and reliable construction of the device is necessary. Also, the final tuning of phase slippage should be done by phase tuning at the ends of undulator segments after delivery of the devices to the LCLS site. It is necessary to have the same undulator measurement systems at SLAC as at APS. (A Helmholtz-coil system to measure individual magnet blocks is probably not necessary, however.) In addition, a pulsed wire system for finding the center of a quadrupole within the required tolerance $(<50 \mu \mathrm{m})$ will be acquired.

\subsection{Measurement and Sorting of Magnet Blocks}

\subsubsection{Characterization of Magnet Blocks with Helmholtz Coils}

The requirements for the LCLS undulator are demanding. To help ensure that these requirements can be met, tight tolerances must be imposed on the magnetic properties of the permanent magnet blocks to be used in the undulator segments. In addition to requiring that the magnets be strong (a remanence $\mathrm{B}_{\mathrm{r}}$ of $1.2 \mathrm{~T}$ is specified for the $\mathrm{NdFeB}$ permanent magnet blocks), they are also required to be uniform. Each individual magnet block is required to have a total magnetic moment that is within $\pm 1 \%$ of the average total moment, where the average is taken over the entire population of magnet blocks. The direction of the total magnetic moment vector is also required to lie within $2^{\circ}$ of the mechanical axis of the magnet block.

These requirements are checked by measurements made using a system of Helmholtz coils. The vendor measures the magnet blocks when they are manufactured, and the blocks must be measured again by the manufacturer of the undulator magnetic structure. Those measurements are then used in sorting algorithms in order to minimize the effect of errors.

The Helmholtz coil measurement system uses a servomotor to rotate the magnet that is held at the center between a pair of coils. A fast 16-bit ADC board is used to measure the signal from the coils on the fly as the magnet is rotated. Typically 2000 points per turn are recorded. A digital integration in software gives the flux of the magnet block at each point during the rotation. 
This gives the orientation of the magnetic moment in one plane; to determine the angle in the perpendicular plane requires that the magnet block be turned by $90^{\circ}$ and the measurement repeated. This by $90^{\circ}$ repositioning are done manually. The data file for each magnet block is analyzed to determine the total magnetic moment and its projections along the mechanical axes of the block.

A set of 500 magnets has been received for use in building a prototype LCLS undulator segment. Their field uniformity exceeds the specification. The vendor's Helmholtz coil measurements for those blocks show that the total moment on each magnet block lies within $\pm 0.5 \%$ of the average total moment of all the blocks. The magnet blocks have effectively no transverse ( $\mathrm{x}$ ) component to their magnetic moment. They have a systematic vertical (y) component to the magnetic moment, however, and the angle of magnetization is $1.0 \pm 0.3^{\circ}$ from the mechanical axis of the magnet. The magnets are symmetric so that they can be installed in the prototype in either of two orientations, i.e., with the vertical component of the magnetic moment up or down. This will allow the direction of the vertical magnetic moment to be chosen when the magnetic structure is assembled.

Some of the magnets have also been measured with the APS Helmholtz coil system. These measurements show excellent agreement with the vendor's measurements. The rms difference was $\pm 0.06 \%$ for the first 35 magnet blocks to be measured.

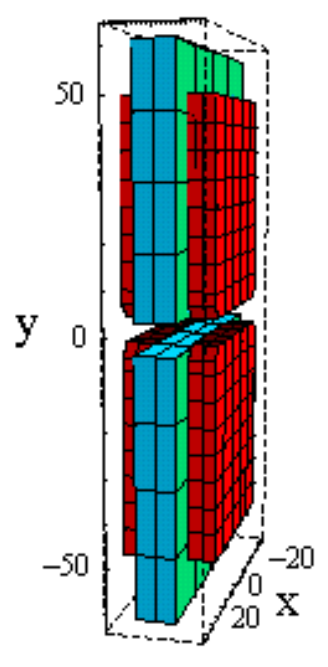

$\mathrm{Z}$

Figure 8.3 Schematic drawing of the half-period measurement fixture. A pole (shown in red) is on either side of the central magnet (shown in blue), on both top and bottom.

\subsubsection{Characterization of Magnet Blocks with Half-Period Fixture}

The LCLS magnets were measured using a specially designed fixture based on the halfperiod-model of the device. This fixture consists of 4 poles and two magnets as shown in Figure 8.3. 
The magnet installed in the bottom part of the fixture remains in place and only the top magnet is changed. A 2-axis Hall probe and a moving coil were used to measure the field integral. Hall probe measurements of the vertical field integral for each of the magnets, are shown in Figure 8.4. The direction of the vertical component of the magnet moment makes a systematic difference for nearly all of the magnet blocks. The magnet in the bottom of the fixture has positive $\mathrm{M}_{\mathrm{y}}$. When magnets of opposite sign of $\mathrm{M}_{\mathrm{y}}$ are paired, the integrated field is smaller, and, in fact, is very close to the earth's field contribution of $-15 \mathrm{G}-\mathrm{cm}$. This difference can also be seen in Figure 8.5, where the field integral measurements were repeated with the vertical moment of the top magnet reversed for some of the magnet blocks. The vertical moment of the magnet block makes a difference in the field integral that is nearly systematic, but a few of the magnet blocks give a different result. It probably results from a different distribution of magnet moment within the block, and cannot be determined by the Helmholtz coil measurements of the total moment.

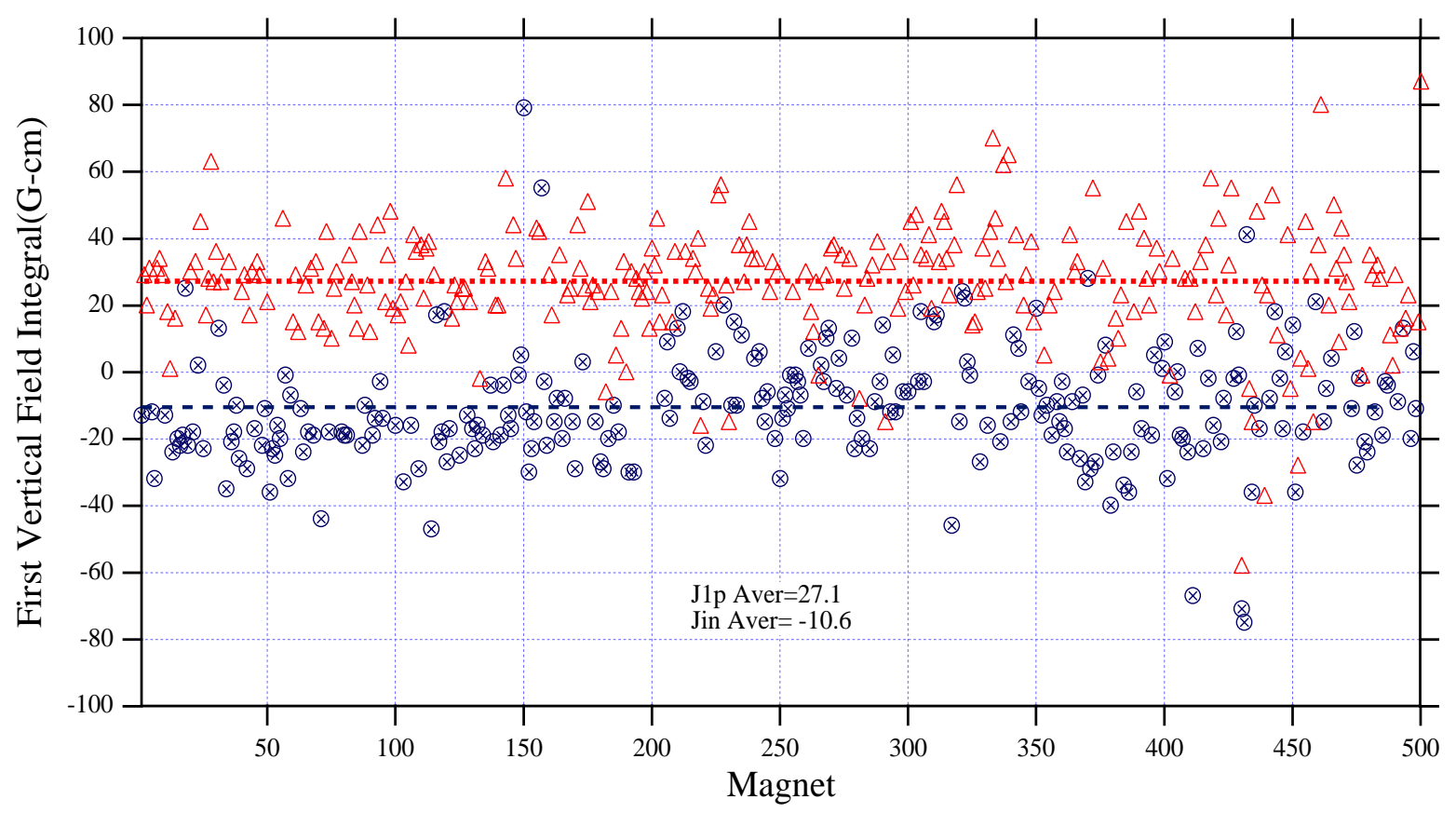

Figure 8.4 Integral of the vertical field through the half-period fixture, for different top magnets. The red triangles are for magnets with positive $M_{y}$ (the same as the bottom magnet); the blue circles are for negative $M_{y}$ (opposite from the bottom magnet). The lines show the average value for the similarly shaped points 


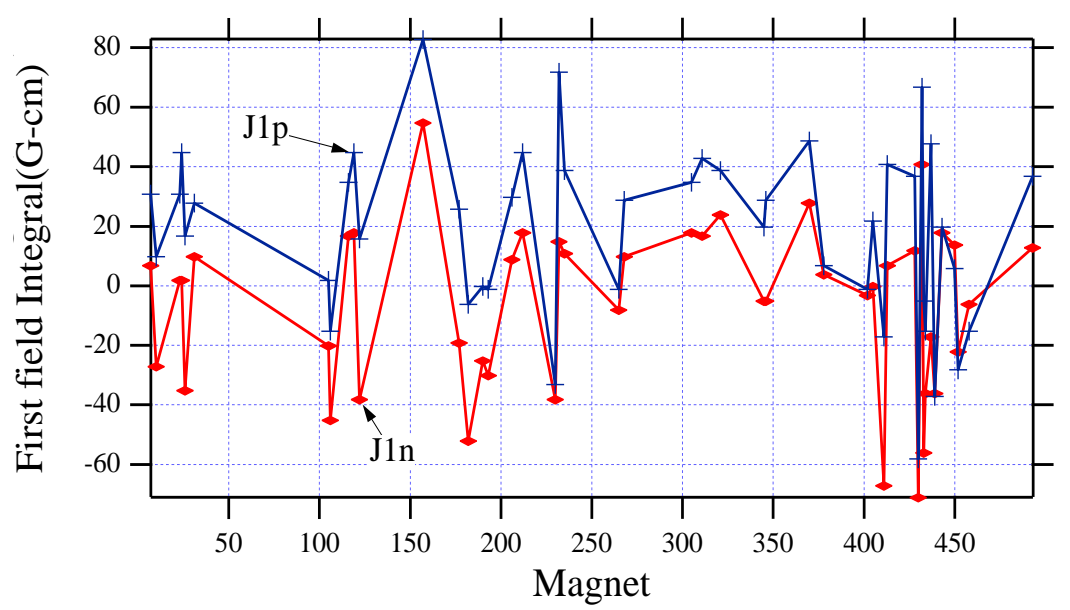

Figure 8.5 First vertical field integral through the half-period fixture when the same magnet block is oriented with its vertical moment positive (blue + ) and negative (red -) for a few of the magnets. The positive orientation usually gives a higher integral, but not always.

Another characteristic of the assembled magnetic structure that cannot be predicted from the Helmholtz coil measurements is the phase variation. This can be seen in Figure 8.6. The vertical component of the field in the fixture has been measured for both orientations of the vertical moment of the magnet block (the direction of the main component of the block's moment is the same). The difference between these two measurements is plotted. The plot also includes the result of a calculation that assumes a uniform distribution of magnetization. The difference seen between the measurement and calculation can affect the contribution to the phase error from the magnet block.

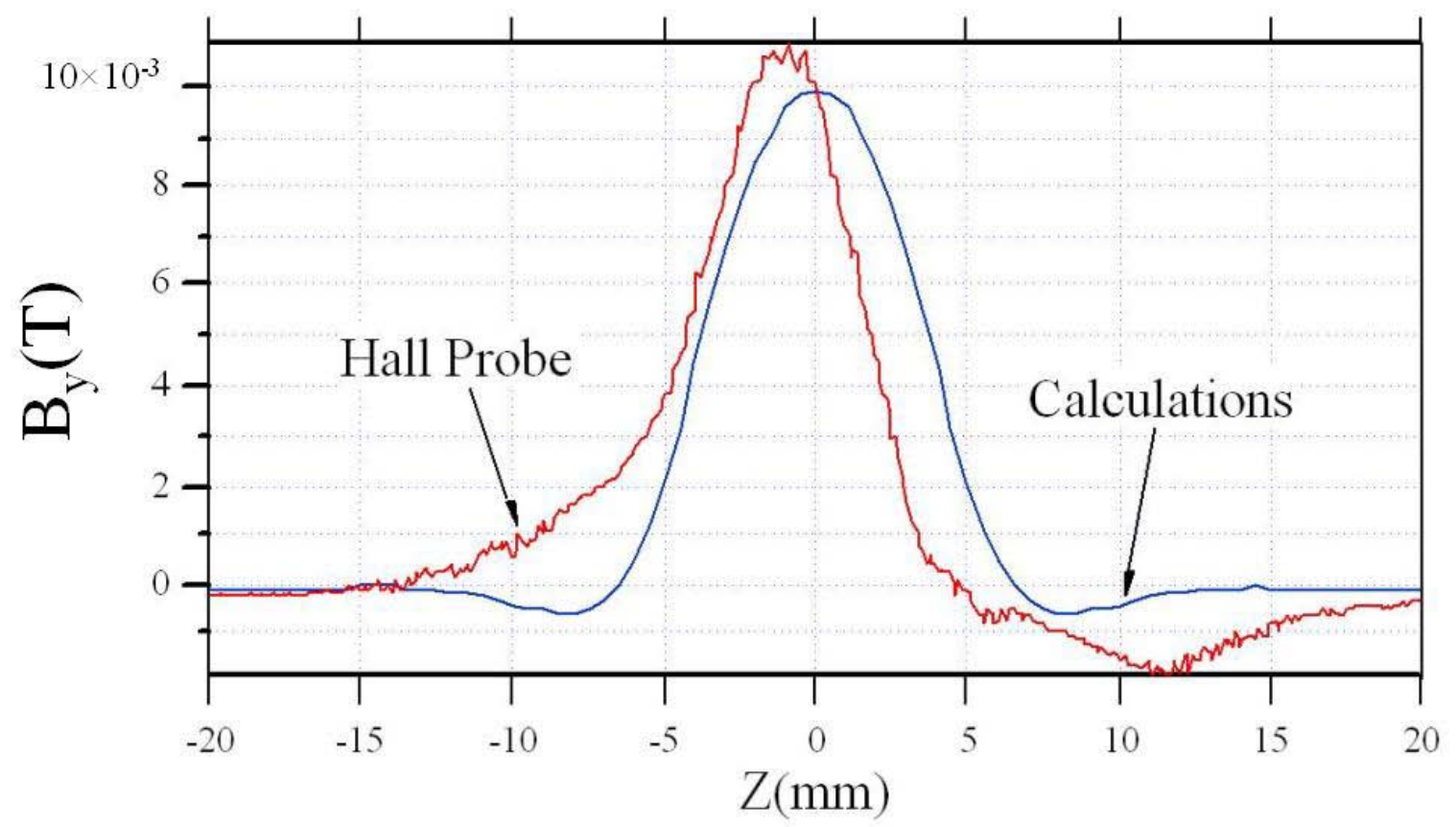

Figure 8.6 Difference in vertical on-axis field when a magnet block is rotated to change the vertical component of the field, plotted vs. $z$. The measured effect may differ from the calculation if the distribution of the vertical magnetization in the magnet block is not uniform; this can affect the phase errors. 


\subsubsection{LCLS Prototype Undulator Segment}

A prototype undulator segment for the LCLS is under construction. Measurements of the magnet blocks using both the Helmholtz coils and the half-period fixture will be used to sort and orient the magnet blocks for installation in the magnetic structure. The best criteria to use are still being investigated.

\subsection{Magnetic Design for the Undulator}

A standard planar hybrid undulator, with a period of $30 \mathrm{~mm}$ and an effective $\mathrm{K}$ of 3.71 (effective B of 1.3250 Tesla) has been designed for the LCLS. The magnetic gap of each undulator segment will be set to whatever is needed to achieve the field strength; it is expected to be near $6 \mathrm{~mm}$.

\subsubsection{Choice of Magnet Material}

The possibility of radiation damage to the magnets and ways of reducing the risk of radiation damage to the magnets is one of the first things to consider in the design of an undulator magnetic structure. Radiation exposure has been found to be a danger to undulator magnets. At the European Synchrotron Radiation Facility (ESRF), an insertion device was damaged when its magnets were demagnetized locally, adjacent to the electron beam [9,10]. At the Advanced Photon Source (APS), the magnets in the insertion devices in one sector were found to be slightly demagnetized after a run in which the dose to that sector was unusually high. The dose was beyond the range of measurement of the dosimeters being used, and the dosimetry techniques are being modified for the future. In order to avoid (or delay) future damage, more attention is being paid to maintaining a high injection efficiency and consideration is being given to other possibilities for reducing the dose to the insertion devices in that sector. Research is ongoing to investigate what levels and types of radiation exposure are hazardous to magnets. Although much is still unknown about this, a reasonable assessment can be made about the potential risks to LCLS undulator magnets based on what is known. The conclusion is that the possibility of radiation damage to the undulator magnets cannot be ignored. Initial operating experience with the APS FEL showed that the dose received by the undulator magnetic structures is comparable to the dose received by the undulator segments installed in the APS storage ring. In addition, the radiation exposure of the LCLS magnets is potentially more damaging than the radiation exposure of storage ring magnets, because of the higher energy of the LCLS electron beam. Magnets can tolerate high levels of low-energy radiation (hundreds of mega-rads of 1-MeV Co radiation) without demagnetizing, but higher energy radiation may cause demagnetization at lower doses [11]. Thus, the 14.35-GeV electron beam of the LCLS is more capable of producing potentially damaging radiation than the $7-\mathrm{GeV}$ beam of the APS or the $6 \mathrm{GeV}$ beam of ESRF.

The magnetic design of the undulator structure will also affect the radiation hardness of the undulator magnetic material. This is because the likelihood of radiation-induced demagnetization has been found to increase when the demagnetizing field experienced by the magnet block is stronger [12]. Part of the analysis that is done during the magnetic design process is to examine 
the localized demagnetizing field experienced by different parts of the magnet blocks, over the full range of intended gaps. A normal goal of the design process is to maximize the on-axis field of the undulator segment at the smallest gap while ensuring that the demagnetizing field seen by the magnets is not excessive at small gaps, nor at any larger gaps - after all, the undulator segment must be assembled one jaw at a time (corresponding to a very large gap) before the jaws are brought close together. The maximum allowable demagnetizing field is determined by the grade of the magnet material being used and what that grade of material can tolerate without permanent demagnetization. Additional margin in the maximum allowable field should be included for temperature effects, because permanent demagnetization will occur at a weaker demagnetizing field if the magnet is at a higher temperature, and neither an air-conditioning failure nor transport in an enclosed truck on a hot day should put undulator segments at risk. Temperature dependence information is available from the magnet manufacturer in the form of B$\mathrm{H}$ curves at different temperatures. Similarly, additional margin should be allowed for radiation exposure because exposure is more likely to result in demagnetization if the demagnetization field seen by the magnet is closer to the field at which permanent demagnetization occurs in the absence of radiation.

One way of reducing the demagnetization risk of the magnets is in the choice of the grade of magnet material. Different grades of $\mathrm{NdFeB}$ magnet material were considered and material (N39SH) meeting LCLS needs is commercially available. The remanence of this material is 1.23 to 1.29 Tesla and the intrinsic coercivity $\mathrm{iH}_{\mathrm{c}}$ is a minimum of $21 \mathrm{kOe}$. This grade of magnet has a high remanent field but it was mainly chosen because of its particularly high intrinsic coercivity. The high coercivity correlates with a better resistance of the magnet material to radiation-induced demagnetization, and with this grade of magnet the high coercivity can be obtained without sacrificing magnetic remanence as compared to the older $\mathrm{N} 38 \mathrm{H}$ grade of $\mathrm{NdFeB}$ that has been used in many insertion devices, including most of those at the APS.

Table 8.3 Predicted values for the undulator magnetic model.

\begin{tabular}{ll}
\hline Parameter & Value \\
\hline Period length & $30 \mathrm{~mm}$ \\
\hline Gap & $6 \mathrm{~mm}$ \\
\hline Peak field on axis ${ }^{1}$ & 1.390 Tesla \\
\hline Effective field on axis $^{2}$ & 1.348 Tesla \\
\hline Effective K & 3.776 \\
\hline Force per pole & $258 \mathrm{~N}$ \\
\hline
\end{tabular}

1 The peak field is the maximum measured field amplitude.

2 The measured field amplitude is not exactly sinusoidal. The effective field is the amplitude of an equivalent sinusoidal field that would produce the same first harmonic energy as the measured field. 

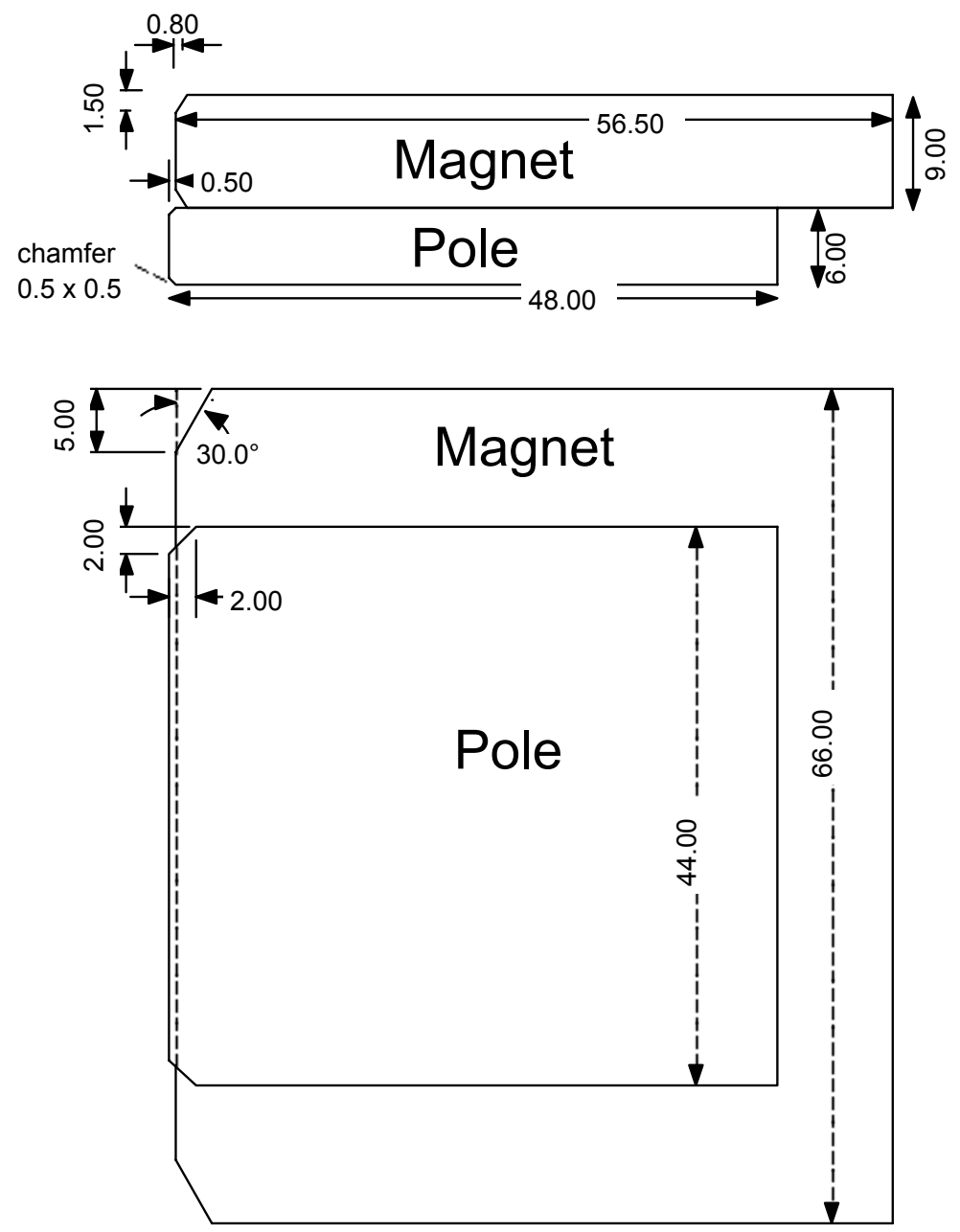

Figure 8.7 Magnetic design for the undulator magnetic structure. Minor changes to this can made for mechanical convenience without affecting magnetic performance.

\subsubsection{Undulator Magnetic Model}

The magnetic design of an undulator segment is shown in Figure 8.7. The design was developed using the magnetic calculation codes Opera-2D and Opera-3D, by Vector Fields. In the calculations, a remanent field of 1.2 Tesla is used for the magnet material. This is slightly lower than advertised by the vendor, but it has worked well for modeling APS undulator segments in the past. The B-H curve that is used for the vanadium permendur of the pole is one that also has worked well previously. The magnetic characteristics of the model as predicted using the 3dimensional code are shown in Table 8.3. Note that there is a small margin as compared to the specification of $\mathrm{K}_{\mathrm{eff}}=3.71$ at $6 \mathrm{~mm}$ gap. (The gap of the device will be adjusted during magnetic tuning to make the final $\mathrm{K}_{\text {eff }}$ be the design value of 3.71.) 


\subsubsection{Two-Dimensional Model}

In the magnetic design process, two-dimensional modeling is used for the initial scoping of the problem. The relative thicknesses (parallel to the beam direction) of the magnet and pole are determined, along with the heights of the magnet and pole. The details of the chamfering at the tips of the magnet and pole are also determined. These parameters are set so that the effective magnetic field on axis is a maximum, while ensuring that the demagnetizing field on the magnet does not become excessive. In the 2D model, the demagnetizing field is worst at open gap. Figure 8.8 shows the demagnetizing field throughout the magnet. The worst is predicted to be $12.713 \mathrm{kOe}$, in the region of the magnet chamfer. This is about 1.11 times the magnet coercivity $\mathrm{bH}_{\mathrm{c}}$ of $11.4 \mathrm{kOe}$. The typical magnet specification that has been used in the past required that the magnet not demagnetize below $1.2 \times \mathrm{H}_{\mathrm{c}}$, so this allows ample margin. The margin is even greater for the high-coercivity magnet grade that will be used.

The other feature of the magnetic model that is checked in the 2D calculations is the value of $\mu$ in the magnet at closed gap. Figure 8.9 shows the calculated value of the permeability $\mu$ in the pole where it is 90 or below, i.e., where the pole is nearing saturation, for a gap of $6 \mathrm{~mm}$. The $\mu$ in the pole is shown to be 90 or higher everywhere except in the chamfered corner, and the minimum $\mu$ that extends across the pole is 90 . Although there may be some small redistribution of the flux due to the saturation near the pole chamfer, the central region of the pole still serves well as a flux conduit.

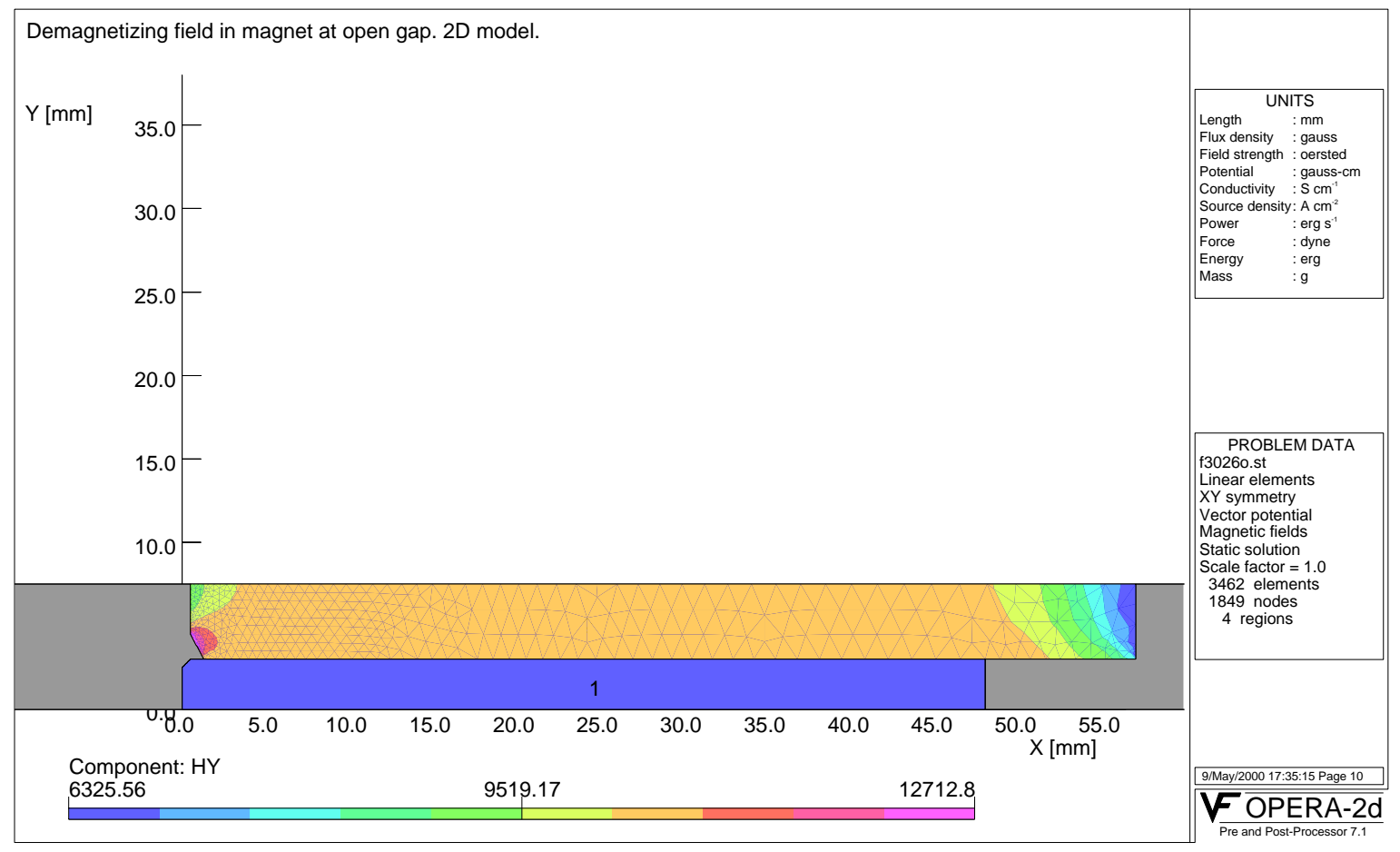

Figure 8.8 A quarter-period model of the magnet structure showing the demagnetizing field in the magnet, in the 2-D model, at open gap. The particle beam would travel up the page on the left, in what is labeled here as the ' $y$ ' direction. The half-pole is on the bottom here, and the half-magnet on top. 


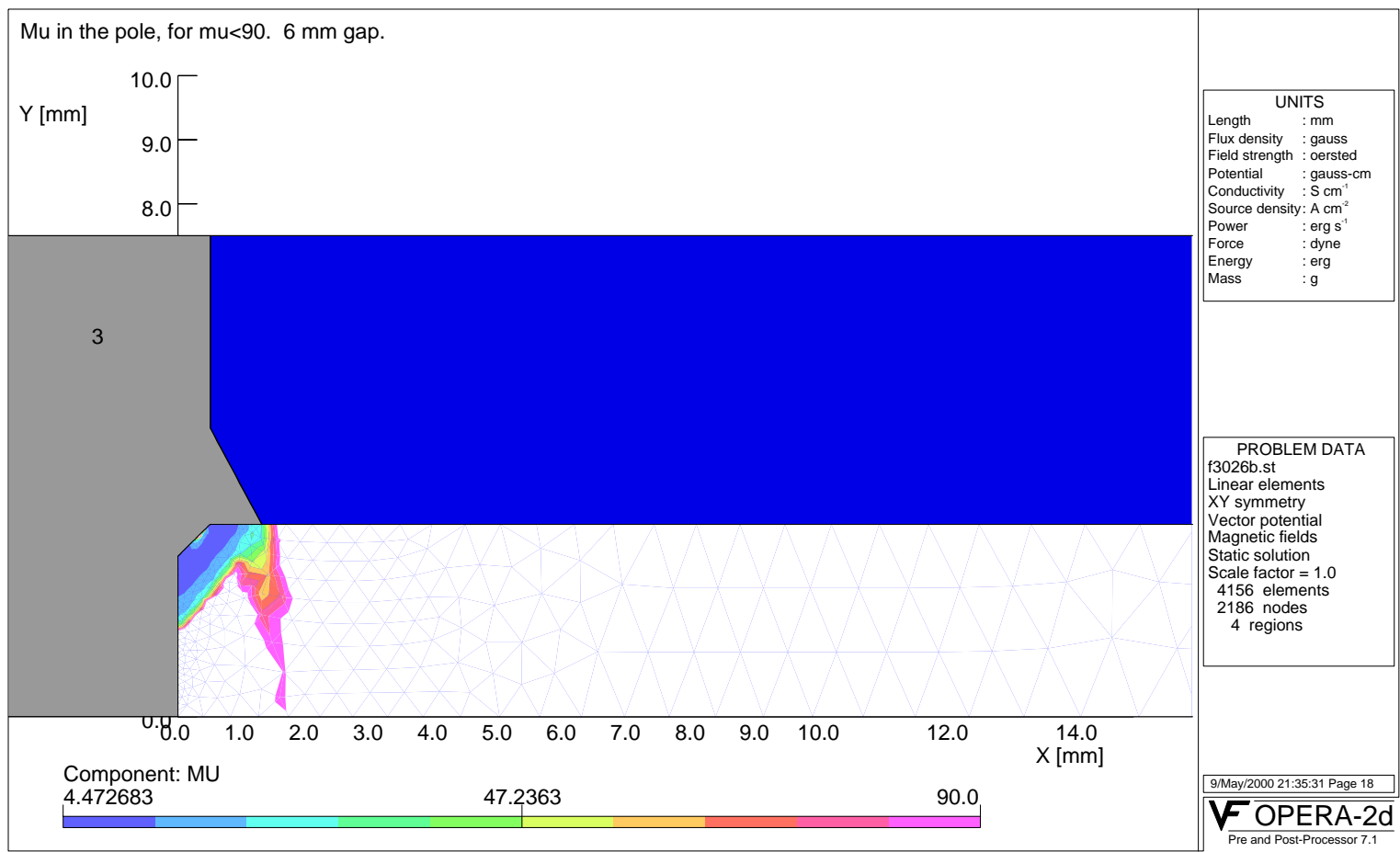

Figure 8.9

Partial quarter-period model of the magnetic structure, showing the areas of the pole where $\mu$ is below 90 at minimum gap $(6 \mathrm{~mm})$. In the white areas, $\mu$ is above 90 . Although the pole is saturating in the region near the chamfer, as shown by the low $\mu$, most of the pole, even at the tip, is comfortably far from saturation.

\subsubsection{Three-Dimensional Model}

The undulator magnetic design is also examined using three-dimensional calculations, particularly to determine the peak field on axis and to examine the distribution of the demagnetizing field within the magnet. Figure 8.10 shows the calculated demagnetizing field in the magnet in a plane immediately adjacent to the pole, at the nominal gap of $6 \mathrm{~mm}$. The magnet extends farther transversely than the pole; the demagnetizing field is worst at the transverse edge of the pole and next to the gap. It is also of interest to view the demagnetizing field in the plane perpendicular to this, at the edge of the pole where, in this view, the field is strongest. This is shown in Figure 8.11. The strongest demagnetizing field is about 1.53 Tesla, leaving a good margin to the $21 \mathrm{kOe}$ intrinsic coercivity of the $\mathrm{N} 39 \mathrm{SH}$ magnet material grade. The peak demagnetizing field calculated at open gap in the 3D model is about the same as at minimum gap.

Another characteristic of the model that is checked is the transverse uniformity of the on-axis field. The pole can easily be wide enough that the transverse alignment of the undulator is not critical. Figure 8.12 shows the transverse roll off in the peak on-axis field. An error of $\Delta B / B=$ $1.3 \times 10^{-4}$ is reached at a transverse position of $\pm 2.9 \mathrm{~mm}$. 


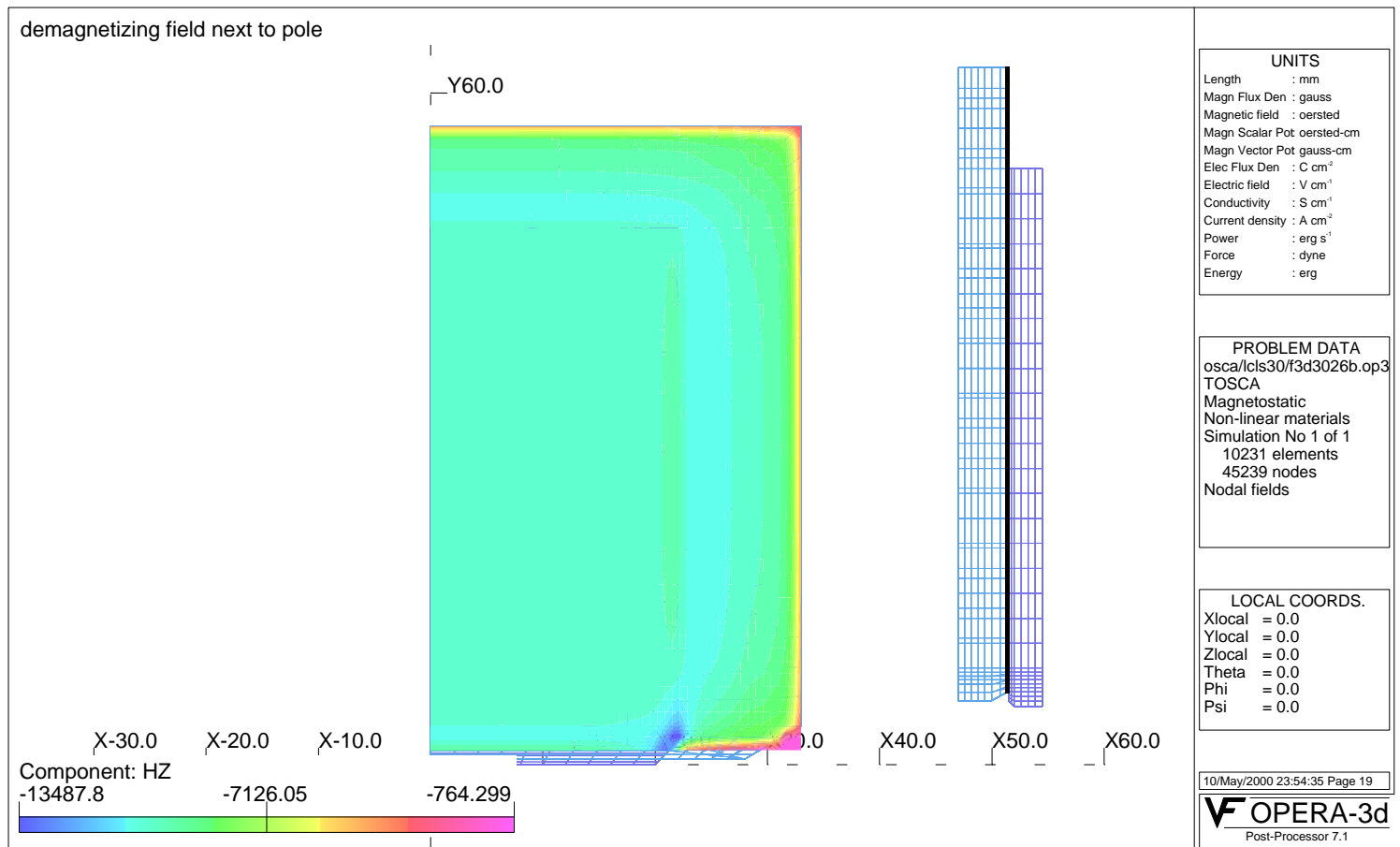

Figure 8.10 Three-dimensional model calculation showing the demagnetizing field in the magnet at minimum gap, in a plane immediately adjacent to the pole. The inset at right is a side view of the model; the black line shows the plane where the demagnetizing field was calculated.

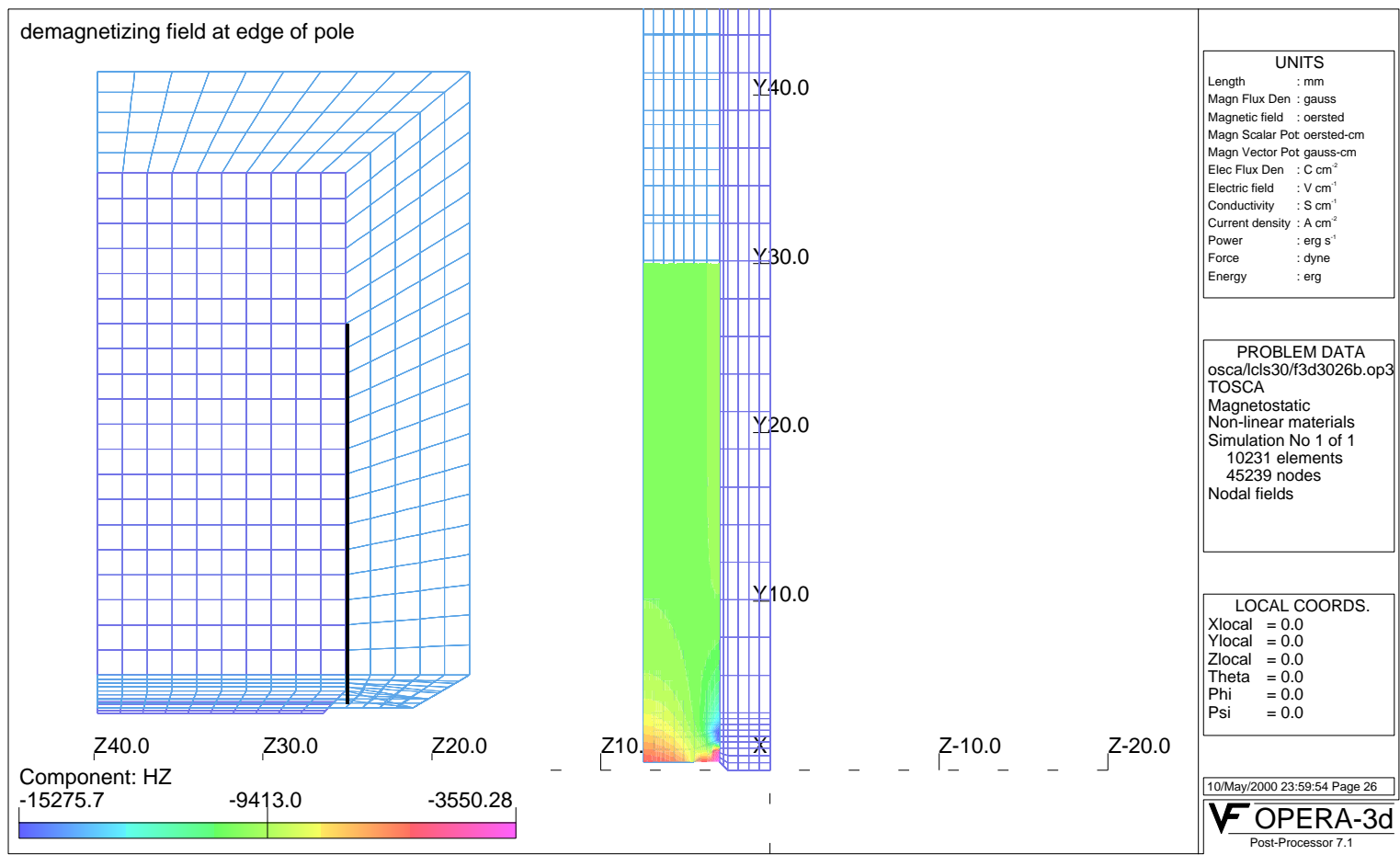

Figure 8.11 Side view of the demagnetizing field in the magnet. The black line in the inset at left (not to scale) shows the plane at the edge of the pole where the demagnetizing field was calculated. The worst demagnetizing field is adjacent to the pole and just above 
the chamfer.

Once the magnetic gap of the undulator is determined, the maximum size for the vacuum chamber is also set. The difference between undulator gap and vacuum chamber outside dimension must include allowances for mechanical variations of $0.20 \mathrm{~mm}$, an allowance for the difference between the magnetic model calculation and reality of $0.12 \mathrm{~mm}$ (this is based on the measured variation in the APS standard undulator segments A), and an allowance for uncertainty in the magnet material remanent field of $0.08 \mathrm{~mm}$. This adds up to a total of $0.40 \mathrm{~mm}$. Thus, if the nominal magnetic gap of the undulator is to be $6 \mathrm{~mm}$, the maximum external dimension of the vacuum chamber is $5.6 \mathrm{~mm}$. Although building a prototype undulator segment may allow some relaxation in this difference, it will not be large.

Some options were considered for ways to increase the minimum gap of the undulator. The magnets and poles could be wedged, but this only increased the field by about $3 \%$. It would allow an increase in the gap by about $0.3 \mathrm{~mm}$, but wedging the magnets and poles is an expensive alternative. The small increase in undulator gap was not deemed to be worthwhile. Wedging the magnet would also mean that the magnet blocks would not be symmetric top-to-bottom. If the magnet blocks are symmetric, the option exists to turn them over if, for instance, reversing the vertical component of the block's magnetic moment would improve the overall undulator magnetic field.

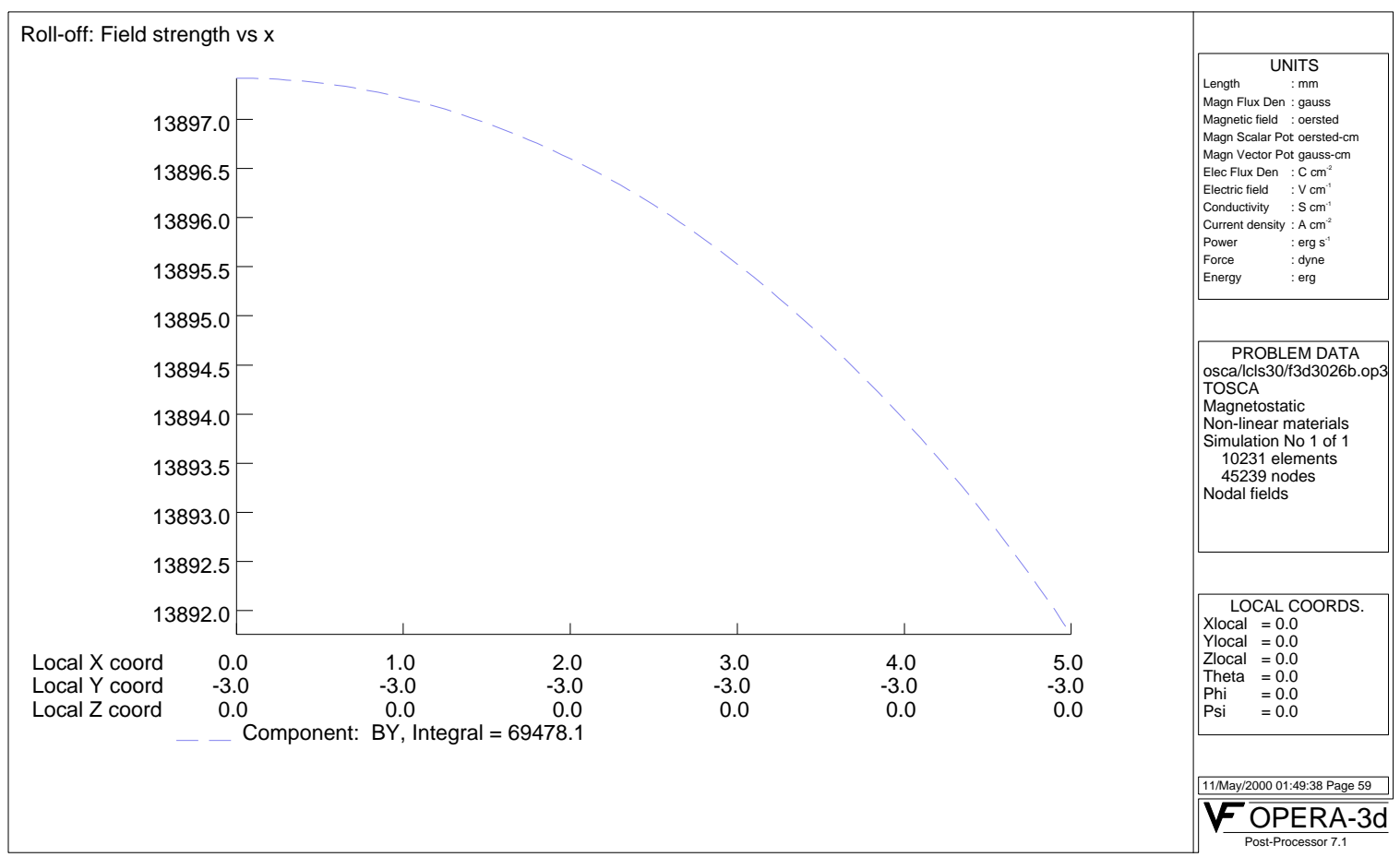

Figure 8.12 On-axis field strength under the pole vs. transverse position. A difference in field strength by $\Delta B / B=1.3 \times 10^{-4}$ occurs at transverse positions of $\pm 2.9 \mathrm{~mm}$. 


\subsubsection{Undulator Segment Ends}

It is critical that the trajectory of the electrons be straight through the undulator line. The undulator will be tuned magnetically to keep the trajectory straight through the undulator. Attention must be paid to the design of the ends, as well as their implementation, in order to avoid a trajectory kick or offset. Ideally, the undulator segments will have neither kick nor offset. This can be achieved in the design by making the strengths of the end poles in the sequence $1 / 4,-3 / 4$, $1,-1, \ldots$ because that end configuration gives zero angle and zero displacement to the beam trajectory. In the first approximation, this is accomplished by making the last magnet only half as strong as the rest of the magnets. The magnet will be made weaker by baking it to increasingly higher temperatures, until the desired strength is reached.

There will also be a magnetic shield at each end of the undulator segment to limit how far the end field extends. This shield will be in place when the undulator segment is tuned, so its effect will be included in the magnetic measurements.

Proper phasing between undulator segments also demands proper tuning of the undulator segment ends. The magnetic phasing must match the physical distance between undulator segments. End phase tuning techniques were developed for the APS FEL that could tune the phasing by $\pm 38^{\circ}$; these techniques will be applied to the LCLS undulator segments. It is, of course, preferable to start with a mechanical break distance that matches the phasing achieved with no tuning of the undulator segment ends. The original calculations for different break distances assumed that the ends of the undulator segments have sharp magnetic cutoffs. In reality, of course, the ends aren't sharp, so those break distances need to be adjusted before final dimensioned plans are made. The necessary correction has been estimated by scaling from the $3.3-\mathrm{cm}$ undulator segments of the APS FEL. The correction changes the regular short and long break lengths from 231 and $463 \mathrm{~mm}$, respectively, for sharp segment ends, to 187 and $421 \mathrm{~mm}$. This is not a negligible correction, especially when accumulated over many undulator segments. The proper corrections for the real undulator will be determined once the prototype undulator segment is assembled and measured.

\subsection{Mechanical Design}

\subsubsection{Design of Magnetic Structure}

The 3.4 meter long LCLS undulator segment has a fixed 6-mm pole gap. The design approach, developed for the LCLS, will allow extremely precise tolerances to be achieved. These tolerances are mandatory for the proper operation of this device. A cross-section of the undulator segment housing and pole structure is detailed in Figure 8.13. Figure 8.14 is an enlarged crosssectional view of the magnets and their holders. The tolerances for the device are listed in Table 8.4. 
Table 8.4 LCLS undulator segment tolerance specification ( $X$ is the undulation plane, $Y$ is the vertical plane and $\mathrm{Z}$ is along the beam direction)

\begin{tabular}{|c|c|}
\hline Parameter & Tolerance \\
\hline Pole gap tolerance & $\pm 0.006 \mathrm{~mm}$ \\
\hline Neighbor pole gap difference (selective assembly) & $\pm 0.003 \mathrm{~mm}$ \\
\hline \multirow[t]{2}{*}{ between neighboring poles } & $\pm 0.050 \mathrm{~mm}$ \\
\hline & $\pm 0.050 \mathrm{~mm}$ \\
\hline Pole thickness & $-0.05 \mathrm{~mm}$ \\
\hline Pole transverse displacement (top and bottom) & $\pm 0.20 \mathrm{~mm}$ \\
\hline Pole displacement in " $Z$ " direction (top and bottom) & $\pm 0.10 \mathrm{~mm}$ \\
\hline Pole face parallelism (top and bottom) & $<0.1 \mathrm{~mm}$ \\
\hline (angle may open outside only) & $<1.75 \mathrm{mrad}$ \\
\hline Pole gap rotation around "Z" Axes over the whole length & $<5.25 \mathrm{mrad}$ \\
\hline $\begin{array}{l}\text { Undulator segment sag due to its weight in the vertical (y) } \\
\text { direction }\end{array}$ & $<0.002 \mathrm{~mm}$ \\
\hline Undulator gap adjustment (possible) & $\pm 0.005 \mathrm{~mm}$ \\
\hline (adjustment resolution) & $0.001 \mathrm{~mm}$ \\
\hline Undulator segment end motion (top and bottom) & $0.080 \mathrm{~mm} *$ \\
\hline (end motion resolution) & $<0.001 \mathrm{~mm}$ \\
\hline \multicolumn{2}{|l|}{ Supporting pillar alignment: } \\
\hline "X" direction & $\pm 2.0 \mathrm{~mm}$ \\
\hline "Y" direction & $\pm 0.5 \mathrm{~mm}$ \\
\hline “Z” direction & $\pm 2.0 \mathrm{~mm}$ \\
\hline Pitch, roll, \& yaw of the pillars & $0.75 \mathrm{mrad}$ \\
\hline $\begin{array}{l}\text { Undulator segment vertical and horizontal remote } \\
\text { alignment: }\end{array}$ & $\pm 3.0 \mathrm{~mm}$ \\
\hline Travel range & $<0.002 \mathrm{~mm} *$ \\
\hline \multicolumn{2}{|l|}{ Resolution } \\
\hline \multicolumn{2}{|l|}{ Drive system installation accuracy on top of the pillars: } \\
\hline "X" position accuracy & $\pm 0.5 \mathrm{~mm}$ \\
\hline "Y" position accuracy & $\pm 0.05 \mathrm{~mm}$ \\
\hline “Z” position accuracy & $\pm 1.0 \mathrm{~mm}$ \\
\hline
\end{tabular}

* actual requirements shall be determined during prototyping phase. 


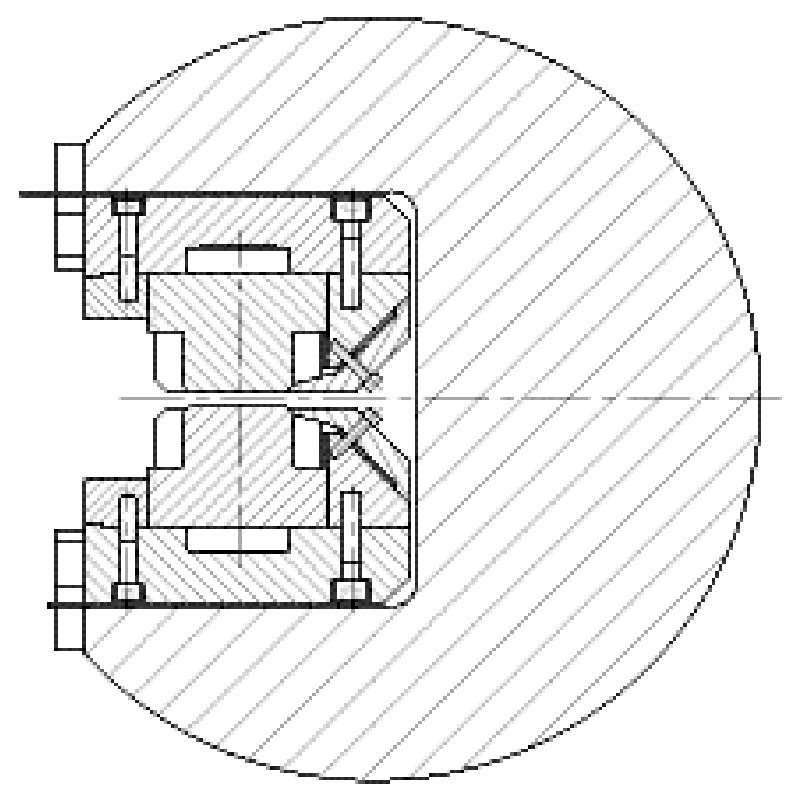

Figure 8.13 Cross-section of the undulator segment and strongback

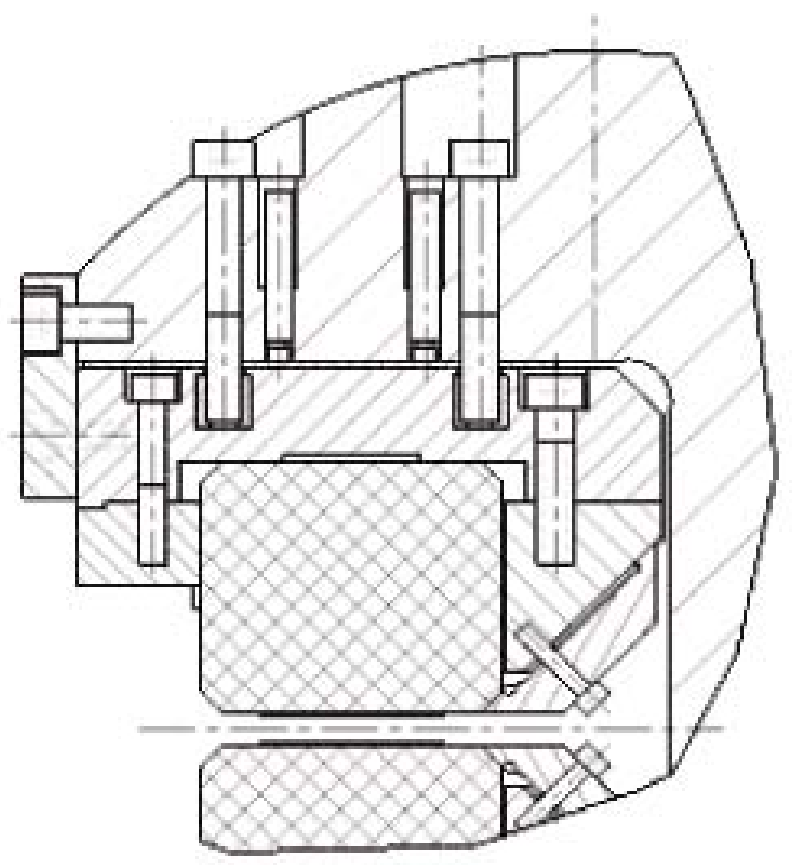

Figure 8.14 Enlarged view of the undulator segment

Parameters for the prototype undulator segment are listed in Table 8.5. The poles of the prototype undulator segments are located in precisely machined grooves located in the two sidebars. In order to achieve the maximum peak field, the magnets extend beyond the edges of the poles in three directions - on both sides, and in the direction away from the magnetic gap. There is no space on the gap side of the pole to clamp the pole into place, so the poles have 
nonmagnetic "ears" added to their sides for clamping. The "ears" are made of titanium alloy and are ground and heat-treated along with the poles (see Figure 8.15). Titanium alloy was chosen because it possesses nearly the same thermal expansion as the pole material (i.e. vanadium permendur alloy). Figure 8.16, Figure 8.17 and Figure 8.18 show several pictures of a 9 pole, 5 period LCLS undulator segment model. It is obviously much shorter than the actual undulator segment, but clearly conveys the concepts used in the design.
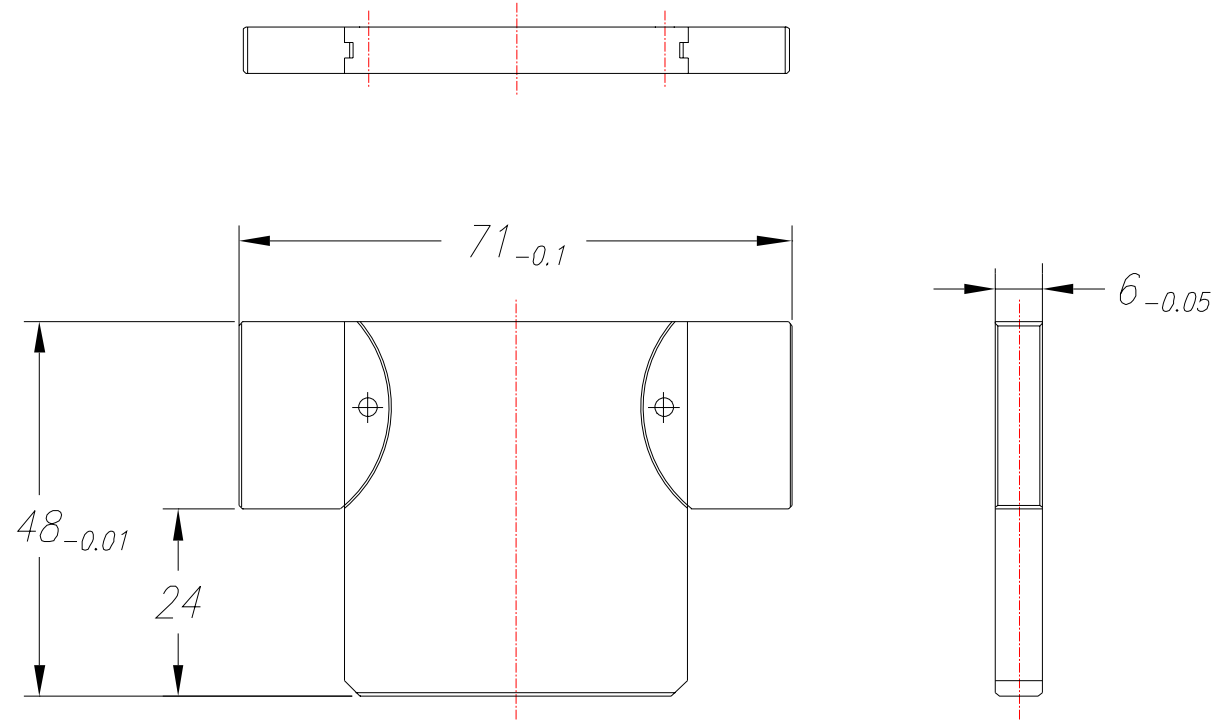

Figure 8.15 Titanium 'ears' are attached to the vanadium permendur poles so the poles can be clamped in place.

Table 8.5 Parameters for the LCLS prototype undulator segment:

\begin{tabular}{ll}
\hline Parameter & Value \\
\hline Pole Gap (nominal) & $6 \mathrm{~mm}$ \\
\hline Period & $30 \mathrm{~mm}$ \\
\hline Pole Thickness & $6 \mathrm{~mm}$ \\
\hline Magnet Thickness & $9 \mathrm{~mm}$ \\
\hline Effective Field $^{3}$ & 1.325 Tesla \\
\hline Effective K Value & 3.71 \\
\hline Outside Dimensions & $3400 \times 305 \times 450 \mathrm{~mm}$ \\
\hline Weight & $1100 \mathrm{~kg}$. \\
\hline
\end{tabular}

3 Note that the calculated effective field specified in Table 8.3 is a bit larger than the goal value given here. As stated in the text, the gap will be adjusted to make the final field equal to the goal value. 
The magnetic structure is designed in such a way that only one clamp is required to hold each pole and each magnet, thus allowing one side of the structure (the back side in Figure 8.18) to remain open for the insertion of side shims.

The undulator segment core is made from a solid 3.4-m long titanium bar, which has a diameter of approximately $305 \mathrm{~mm}$. A precise window machined along the entire length of the bar is used to locate the top and bottom magnetic jaws. The feasibility of this technique will be proven through manufacturing and testing of the prototype as unknown variables in the machining process such as material relaxation could cause twisting, bowing or warping. Titanium was chosen for the core material due to its low specific weight and low thermal expansion coefficient. The low specific weight will lighten the structure and thus decrease potential deflection of the undulator segment between supports; deflections of only a few microns are anticipated. Titanium's low thermal expansion coefficient will minimize thermally induced deflections caused by variations in the tunnel temperature. Temperature stability is very important to keep the magnetic field from changing significantly. The base plate, with its slots to hold the magnets and poles of the magnetic structure, is made of aluminum in order to partially compensate for the influences of temperature fluctuations, which can change the gap distances between poles (see Figure 8.13).

The prototype undulator segment has a total of 226 poles per jaw, and 225 magnets. The length of the magnetic array proper, including all poles and magnets, is $3381 \mathrm{~mm}$. There is some space allowed at each end before the magnetic shield. The magnetic shields are $5 \mathrm{~mm}$ thick at each end, and the overall length of the undulator segment, including shields, is $3410 \mathrm{~mm}$. Once the bolt heads are added in, the total mechanical length of the segment is $3422 \mathrm{~mm}$. The Ti bar by itself is $3400 \mathrm{~mm}$ long.

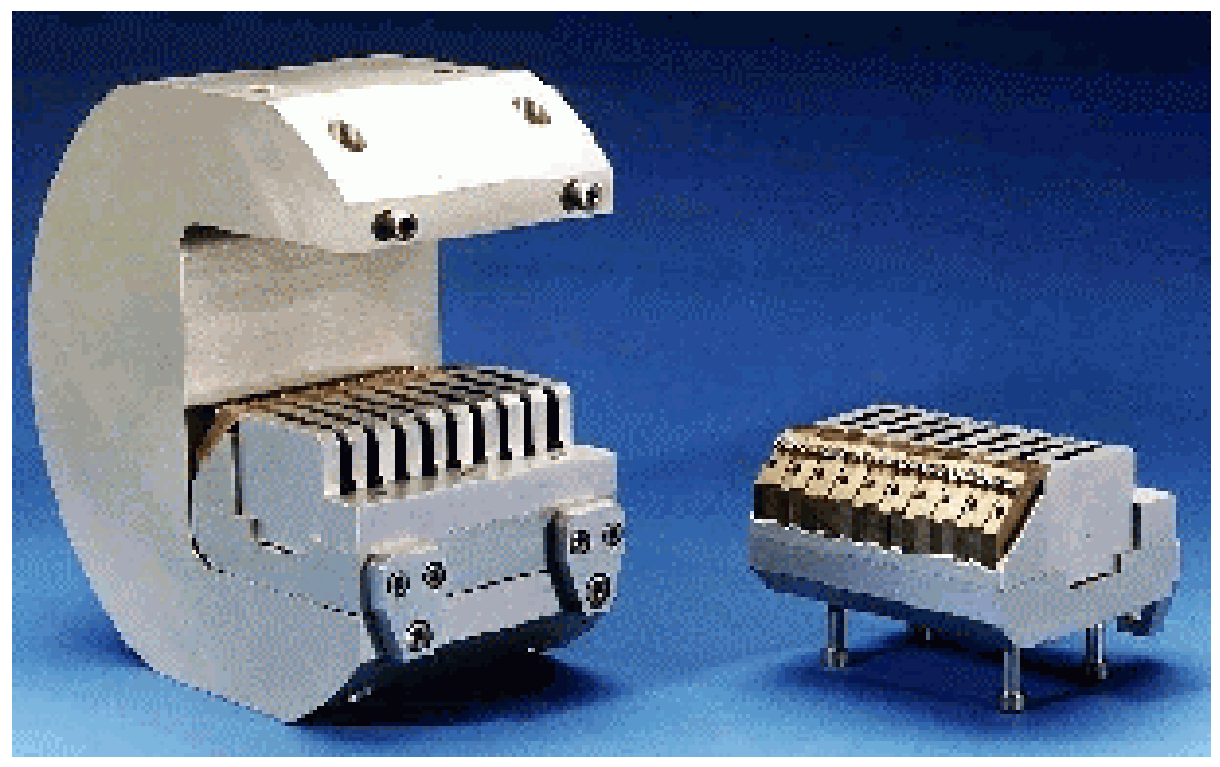

Figure 8.16

View of the short model of an undulator segment with upper jaw removed from the assembly 


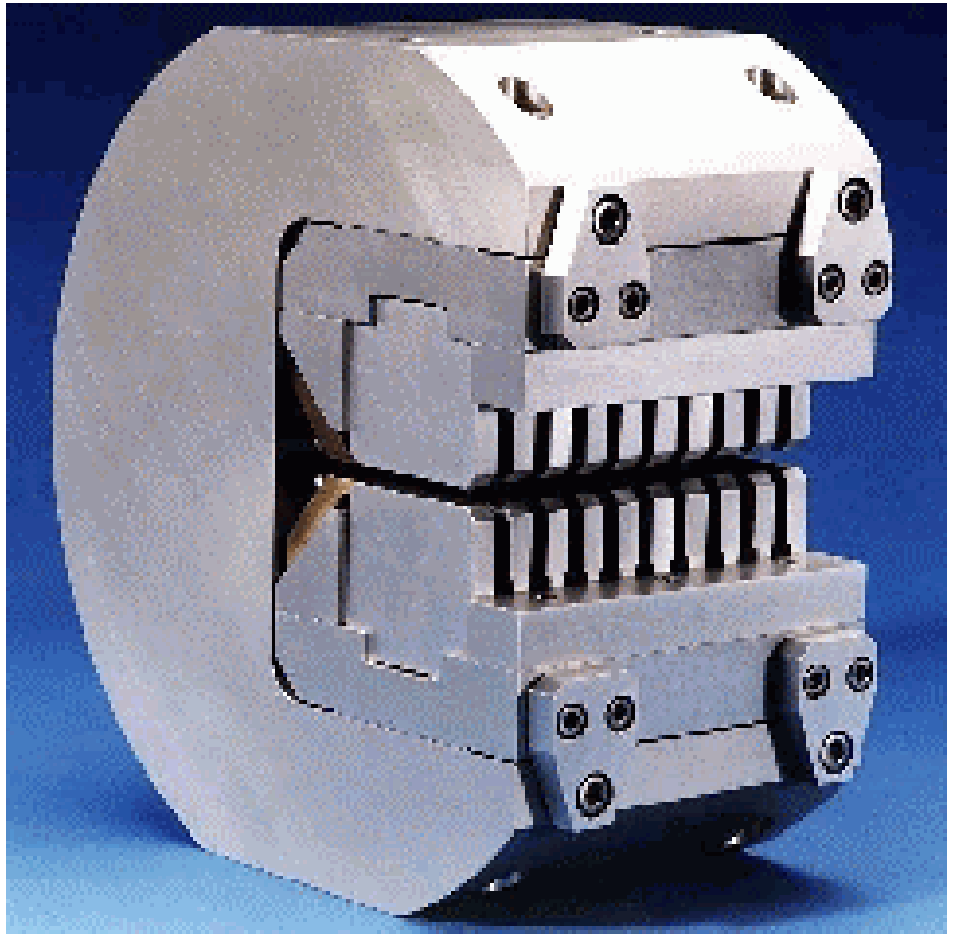

Figure 8.17

View of the short model (9 poles, 10 magnets) of an undulator segment, fully assembled. The C-shape housing will be made from titanium.

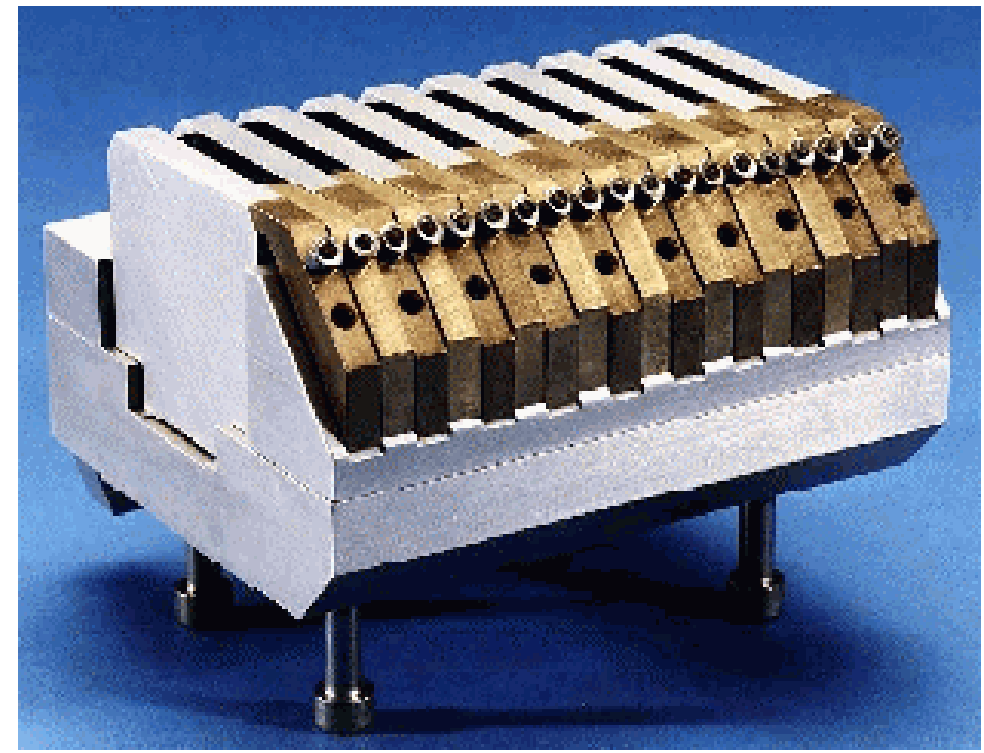

Figure 8.18 Upper jaw of the short model. Nine (dark) pole pieces are between the 10 magnets.

The attractive magnetic force generated by a $6-\mathrm{mm}$ gap with a $30-\mathrm{mm}$ undulator period is $17.7 \mathrm{~N} / \mathrm{mm}$ length or $60,000 \mathrm{~N}$ for the entire structure. A cross-section of the titanium bar was modeled using ANSYS code. With an applied force of $17.7 \mathrm{~N} / \mathrm{mm}$ length, the titanium bar has a deflection in the "Y" direction relative to the beam axis on the order of 7 microns per side. This was verified with several model variations of increasing complexity. Figure 8.19 shows the 
deflection in the "Y" direction for one of the model cases where the force was distributed along a line corresponding to the location of the magnetic jaws; the maximum deflection is $7.19 \mu \mathrm{m}$.

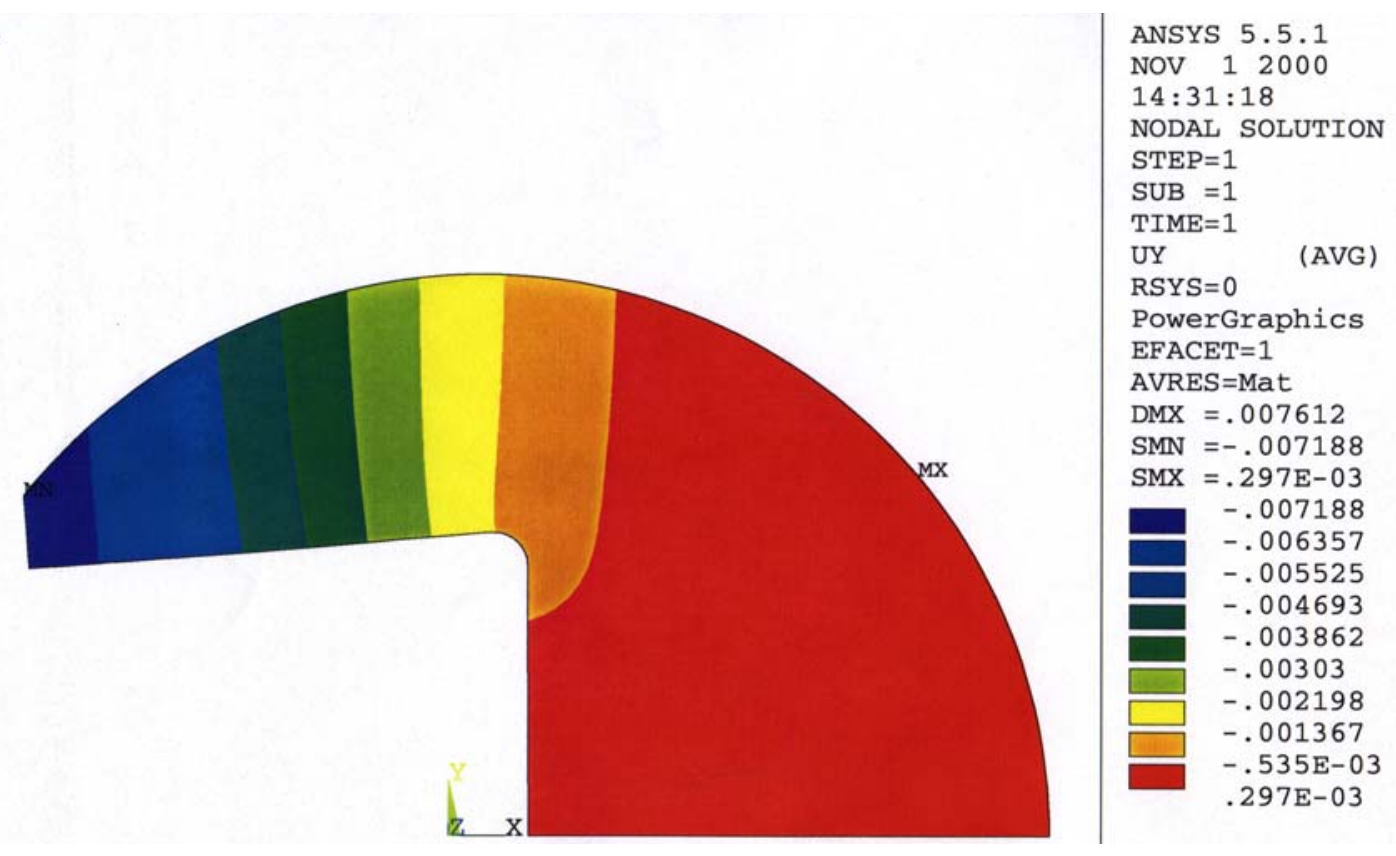

Figure 8.19 Calculated deflection of the titanium bar due to the magnetic forces.

\subsubsection{Provisions for Magnetic Tuning}

The magnets and poles of each jaw are assembled onto a base plate of aluminum, as mentioned above. The base plate for each jaw consists of five blocks placed end to end with three long blocks approximately 1-m long in the middle and a shorter block at each end. The design allows precision shims of various thicknesses to be placed between the core and jaw blocks in order to achieve precision tuning of the undulator segment. A series of these shims in 2-micron increments is being made for this purpose by precisely nickel-plating brass shims. There is also a design provision to achieve the same results using "push-pull" screws.

The magnetic structure can also be magnetically tuned using low carbon steel screws, referred to as side shims, which can be screwed in towards the poles in order to divert some of the magnetic flux and decrease the field under the pole. These side shims can be installed anywhere along the length of the undulator segment as required for precise tuning. The shim block assembly is shown in Figure 8.20.

The two end blocks of the base plate for each jaw can also be bent slightly, up to 80 microns, in order to change the gap at the end of the undulator segment. While this provides another means of tuning the undulator field strength, its primary function is for adjusting the phasing between the undulator segments. For this purpose, four PZT (Lead Zirconate Titanate) translators are housed inside holes within the titanium core as shown in Figure 8.21. The resolution of these translators is on the sub-micron level allowing very precise tuning of the undulator taper. 

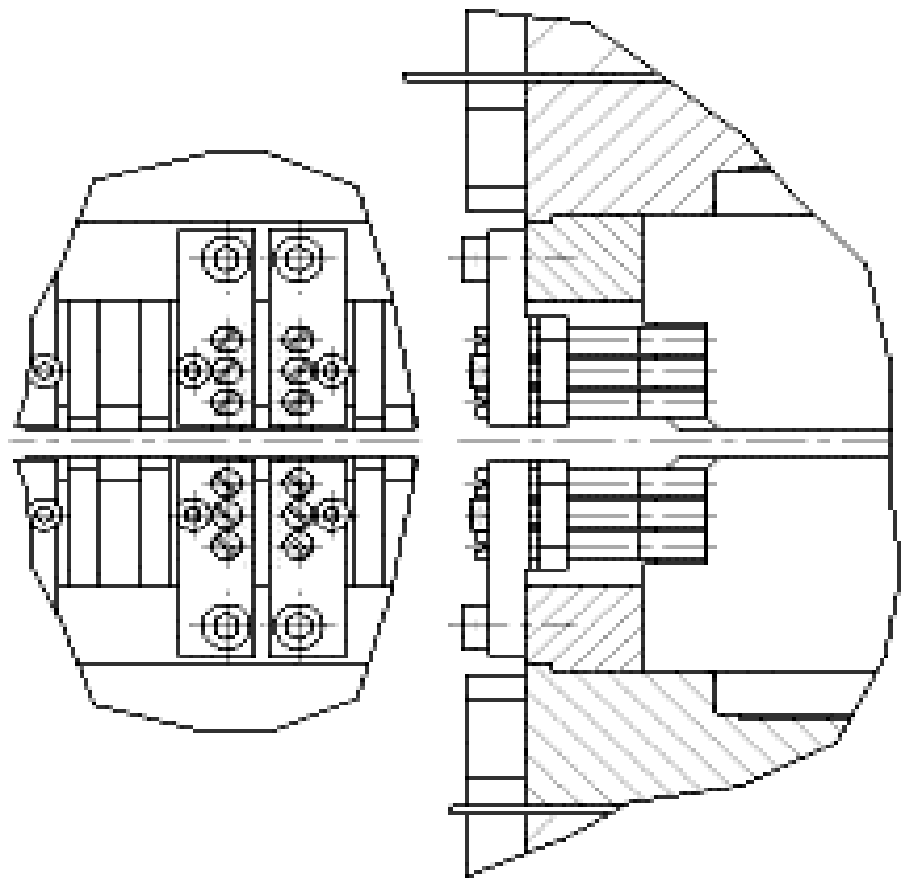

Figure 8.20 Low-carbon steel screws can be installed as necessary and positioned at varying

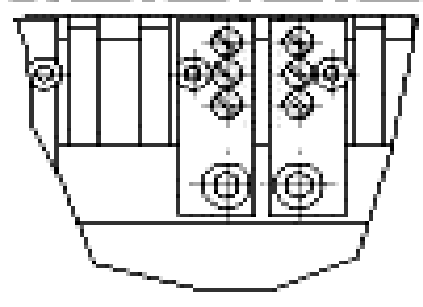
distances from the poles in order to adjust the undulator field locally.

In conclusion, the following summarizes all of the design provisions that were made in order to achieve the high magnetic performance required for undulator segments. It is worth noting that the magnetic tolerance specifications for these segments are nearly an order of magnitude more stringent than those of the standard APS undulator "A".

- Precise tuning and measurement capabilities are required in order to make a precision magnetic device. For this reason, the space between poles is completely open (pole and magnet clamps are located only inside the device).

- For a very small fixed pole gap it becomes exceedingly complicated to use standard shims on top of the magnets to precisely tune the magnetic structure. Alternate provisions were made so that "push-pull" screws or a series of 2 micron increment shims can be used for tuning, greatly simplifying the process.

- Side shims can be used for final tuning.

- All components of the magnetic structure such as the poles, magnets, sidebars, and base plate have very precise mechanical tolerances.

- Along the length of the undulator segment, the magnetic structure is divided into five sections. The three middle sections can be tuned manually and the end sections can be slightly bent from a remote location with sub-micron accuracy in order to taper the 
undulator segment.

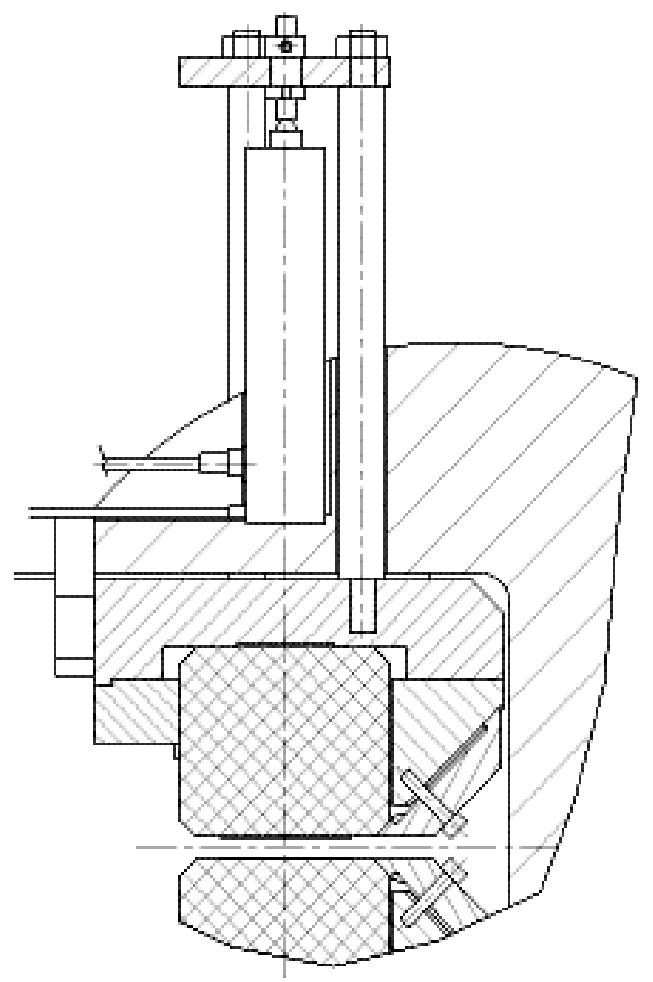

Figure 8.21 PZT translator located at the end of the undulator segment to adjust the magnetic gap of the end section. This adj usts the phasing between undulator segments.
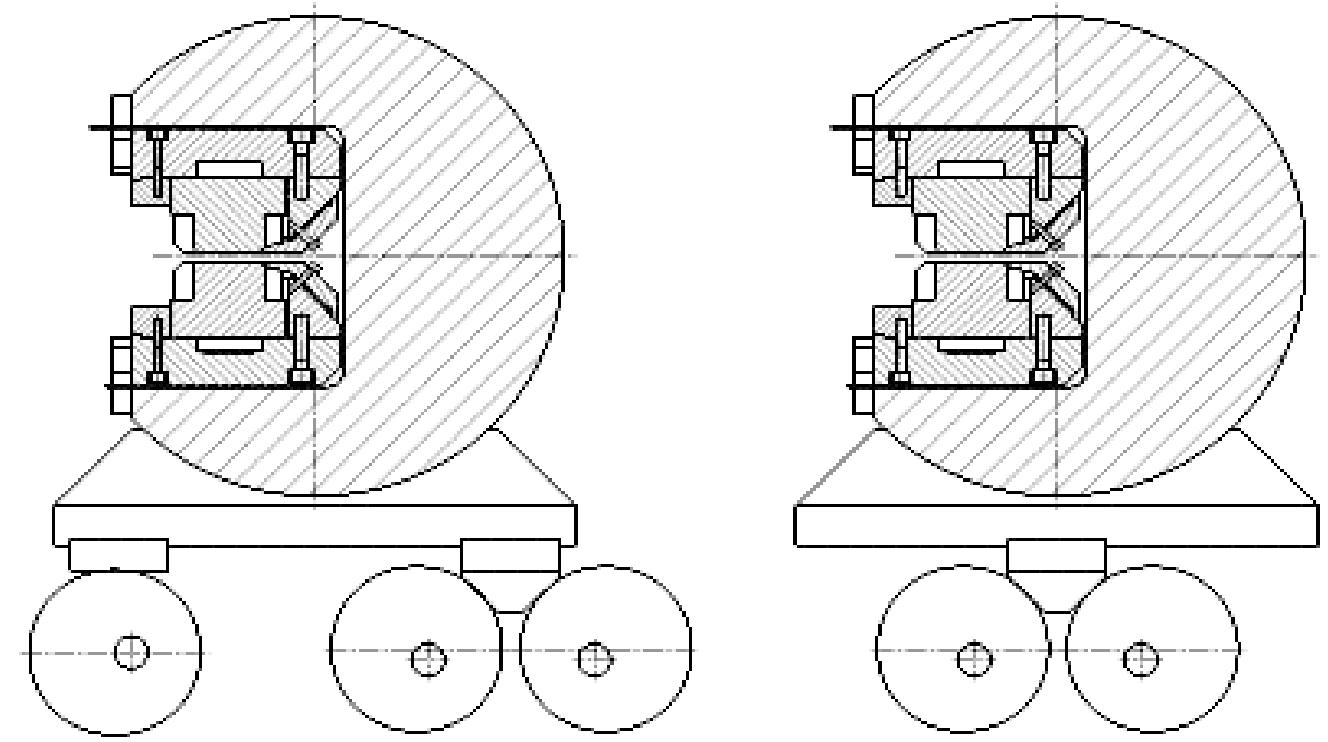

Figure 8.22 Schematic View of the undulator segment and movers showing three of the cams on one end of the segment and two on the other end.

- To ensure that the tight tolerances are met, each magnet and pole will be certified and a selective assembly procedure will be employed. The assembly will be performed in 
an area where the temperature stability is better than $\pm 1^{\circ} \mathrm{C}$.

- The core of the undulator segment is made from a solid titanium bar, to insure that long-term dimensional stability is maintained. Additionally, the titanium core shall be heat treated after both the rough and final machining processes in order to stress relieve the core.

\subsubsection{Undulator Supports and Movers}

Each undulator segment is supported on two pillars equipped with camshaft drive systems. The mounting scheme for the two ends of the undulator segment is shown in Figure 8.22. It is a three-point support. The cams that provide the support are eccentric, with an eccentricity of 3 $\mathrm{mm}$. The support that uses a single cam can adjust the height only. The double-cam supports can adjust the height or the lateral position. By using the three support point adjustments 5 degrees of freedom in position adjustment are possible. There are no plans for adjustment of the $\mathrm{z}$ position.
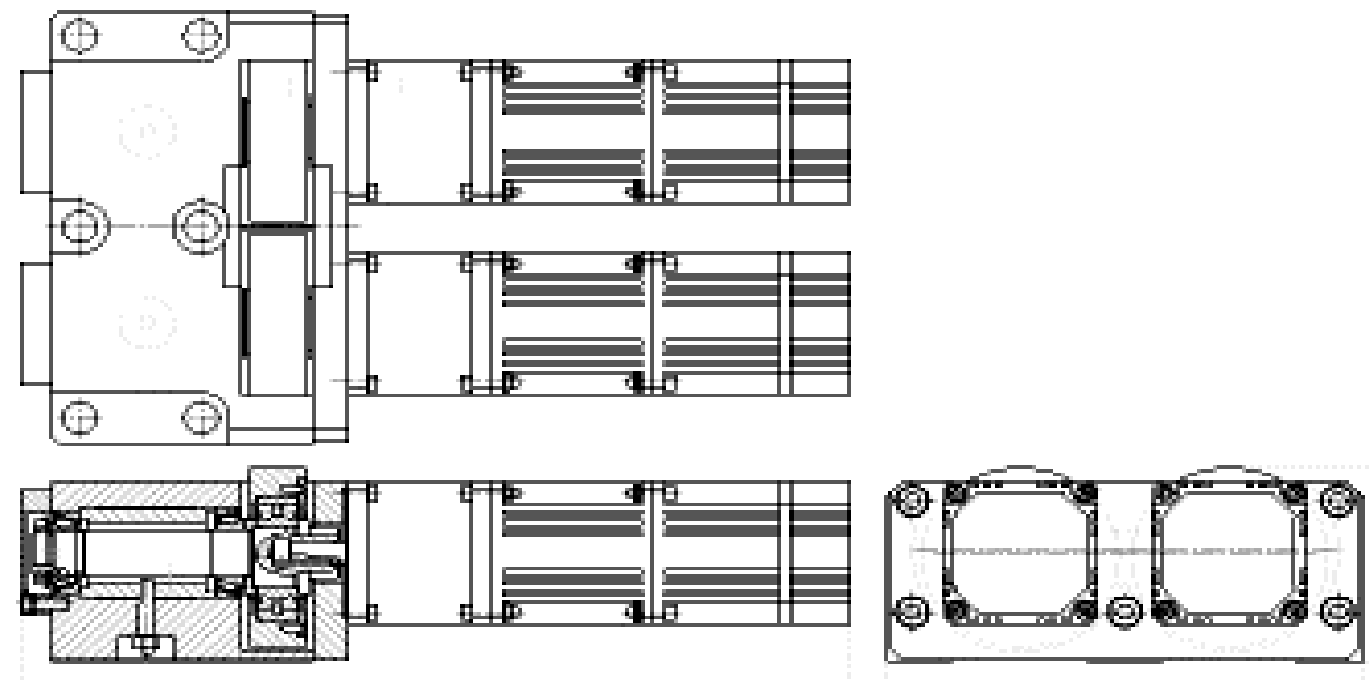

Figure 8.23 Eccentric cam mover system to adjust both height and lateral position.

Theoretically, the resolution of the mover system is better than one micron. In reality, the friction between the camshaft rings and undulator segment supports, tolerance inside of the roller bearings, etc. will determine the actual repeatability. This is expected to be well under the required tolerance.

Two different designs have been made for these units, one with only one camshaft and the other with dual camshafts. These systems are shown in Figure 8.23 and Figure 8.24.

The piers are monolithic structures that will support the ends of each girder. The piers will also support components located between the girders. The piers will be made of Anocast ${ }^{\circledR}$ material. Its properties are more uniformly controlled and better known than concrete, and SLAC 
has had positive experience with it and less satisfactory experiences with concrete for support structures.

To eliminate the diurnal temperature cycles associated with the ground, it is only necessary for the piers to extend 1-2 feet below the concrete floor [13]. Given that the piers should be set into the sandstone ground structure, the piers will extend about $60 \mathrm{~cm}$ below the floor level or more as required to reach the sandstone. The piers will be isolated from the concrete floor.
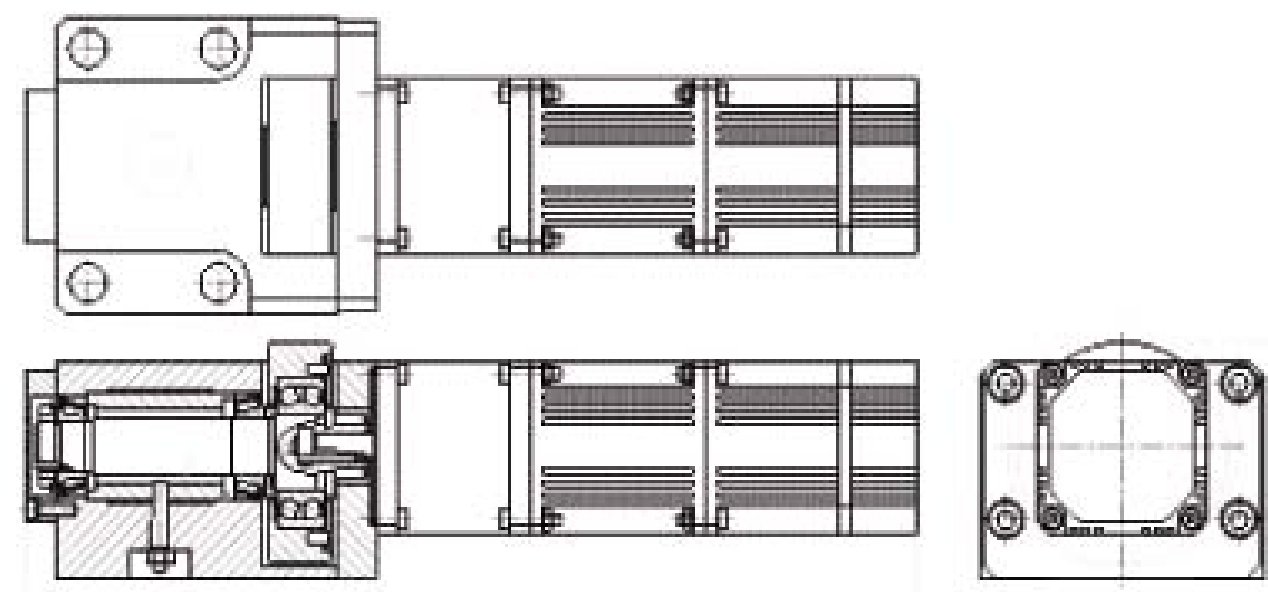

Figure 8.24

Eccentric cam alignment system to support and position one end of the undulator segment.

\subsubsection{Thermal Considerations}

Due to the low thermal expansion of titanium, it is estimated that if the tunnel temperature changes by $\pm 0.5^{\circ} \mathrm{C}$, the resulting length change of the undulator segment will be on the order of only $\pm 15 \mu \mathrm{m}$. This change would not have a significant impact on the output wavelength of the undulator. The temperature variation in the strength of the undulator magnetic field due to the reduction in the remanent field of the permanent magnets at higher temperatures would have an effect, however. The remanence of the $\mathrm{NdFeB}$ magnets decreases by about $0.1 \%$ per ${ }^{\circ} \mathrm{C}$. This results in a decrease in the on-axis undulator field strength of about $0.054 \%$ per ${ }^{\circ} \mathrm{C}$. To compensate for this effect, the magnetic gap can be reduced due to differential thermal expansion as the temperature increases. Magnetic calculations show that to compensate for a $1^{\circ} \mathrm{C}$ temperature rise, the decrease in pole gap needs to be on the order of $8.6 \times 10^{-4}$ of the gap. To accomplish this, an aluminum plate was placed between the titanium core of the undulator segment and the magnet/pole assemblies. The predicted decrease in pole gap for the model is approximately $8.26 \times 10^{-4}$, or almost the ideal value.

\subsection{Permanent Magnet Quadrupoles}

The FODO lattice of the undulator line incorporates permanent magnet quadrupoles. No provision is planned for adjusting the strength of the quadrupole, so magnetic tuning and 
adjustment of the integrated quadrupole gradient will be done during the manufacture of the quadrupole, before it is installed.

The optical strength of the quadrupoles was one of the parameters that was optimized in order to minimize the power gain length. The value chosen is $0.112 \mathrm{~m}^{-1}$ for a beam of $14.35 \mathrm{GeV}$, but the minimum is very flat and a variation of up to $10 \%$ would not significantly affect the power gain length. When the LCLS is run at an energy other than $14.35 \mathrm{GeV}$ (e.g., at $4.5 \mathrm{GeV}$ ), the strength of the quadrupoles will not be adjusted. At $14.35 \mathrm{GeV}$ the averaged beta function is about $18 \mathrm{~m}$, whereas at $4.5 \mathrm{GeV}$ it is closer to $7 \mathrm{~m}$.

Each quadrupole magnet is installed on slides so it can be moved, remotely, in both horizontal and vertical directions. This enables the quadrupoles to also serve as steerers. They will be used, along with the separate electromagnetic steerers, in the beam-based alignment procedure.

In the simulations that were run, the quadrupoles were placed in the middle of the break section. In the final mechanical design, the quads will be displaced to allow for optimal use of the break. Therefore, the final position of the quadrupoles will not be known until the mechanical design for the inter-undulator diagnostics is completed. However their relative separation will not change.

\subsubsection{Quadrupole Mechanical Design}

The space between undulator segments is very limited. The quadrupole design is very compact; the length is only $50 \mathrm{~mm}$ with an aperture of only $11.3 \mathrm{~mm}$. The quadrupole assembly is shown in Figure 8.25.
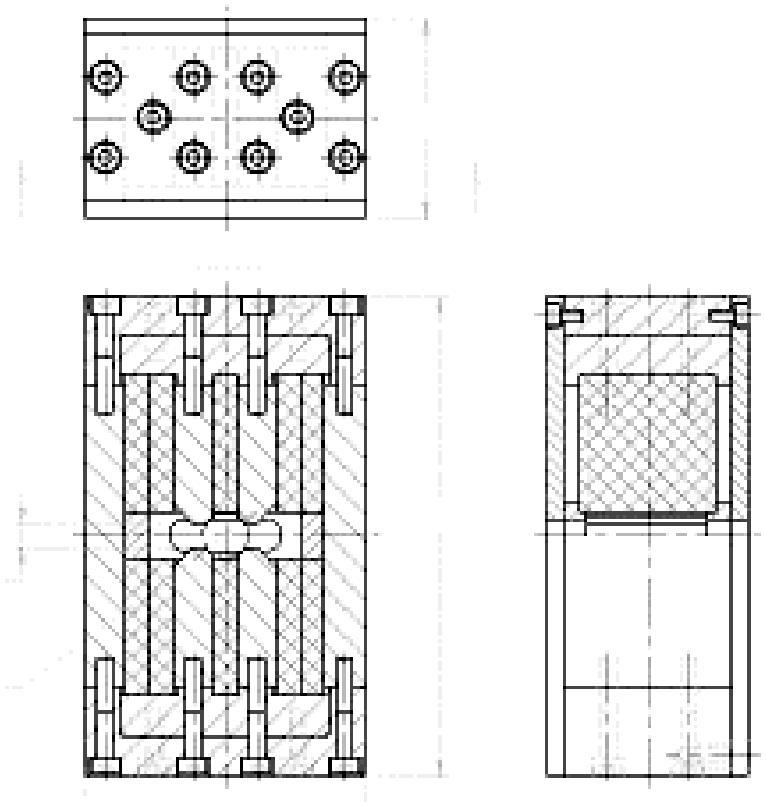

Figure 8.25 Sketch of the permanent magnet quadrupole. Ten identical permanent magnet blocks are used. 
The quadrupole is designed in such a way that top and bottom halves of the assembly are symmetrical about the beam center. The outer housing of the assembly is fabricated from low carbon steel, thus providing a complete magnetic shield on all six sides. The top and bottom housing sections are fitted with aluminum inserts used to define the location of the magnets and poles. These sections, once fitted with the aluminum inserts, are precisely machined together side by side to ensure that the holders and inserts end up in the same plane. Similarly, the two carbon steel side shields are clamped together and precisely ground, thus creating two identical halves. As a result of this machining approach, the assembly, once bolted together, precisely defines the $\mathrm{X}$ and $\mathrm{Y}$-dimensions (the Z-dimension is the beam direction). These in turn determine the gaps for the poles and magnets.

In the center of the four poles are the two central magnets. On the outside of each pole are pairs of magnets located side by side. Each of the magnets is slightly larger than the neighboring poles and overlaps the poles on three sides. All of the magnets in the assembly are the same size; thus, the outer magnet pairs are twice the thickness of the inner magnets.

Each end of the quadrupole assembly is fitted with a shield that has a 20 -mm wide groove that allows insertion of a Hall probe for magnetic measurement purposes. The two side shields each contain a pair of precise holes in the median plane that are used to house standard tooling balls for alignment of the assembly in the beam line.

The quadrupoles are supported by the same Anocast ${ }^{\circledR}$ piers as the BPM modules but otherwise not coupled to them. Their transverse position (horizontal and vertical) can is remotely controllable by $\mathrm{x}, \mathrm{y}$-slides. The quadrupoles have a bore of $11.5 \mathrm{~mm}$ and thus provide plenty of aperture for the $6 \mathrm{~mm}$ OD vacuum chamber to fit without interference. There will be horizontal and vertical corrector coils connected to each quadrupole.

\subsection{Vacuum Chamber}

The undulator vacuum chamber is 120 meters long and must fit into the 6-mm undulator gap. The chamber will be segmented in the same way as the undulator with a diagnostics section between each undulator segment. It will be pumped by ion pumps at each diagnostics chamber. The diagnostics chambers need to be designed with a relatively smooth bore to minimize wakefields.

The proposed chamber through the undulator segments is formed from a stainless steel tube with copper plating on the inside to reduced the resistivity as seen by the beam. The following analysis shows that such a chamber can meet the LCLS requirements and withstand missteering events. It cannot withstand continuous missteering into the wall for extended periods of time. The Machine Protection System will prevent this. 


\subsubsection{Electron Beam Collimation and Vacuum Chamber Design}

This section analyzes the vacuum chamber and magnetic material with regard to the need for protection from accidental electron beam losses and discusses the vacuum chamber surface roughness limits.

\subsubsection{Beam Parameters Used in These Calculations}

It is of vital importance to fully understand the consequences of exposure to the undulator to the primary electron beam. Of greatest concern is the response of the permanent magnet material and that of the undulator vacuum chamber to such events. The electron beam parameters (at injection into the undulator) and geometric dimensions that were used in the analysis are listed in Table 8.6.

Table 8.6 Parameters used in calculations of the effect of beam losses in the undulator.

\begin{tabular}{ll}
\hline Parameter & Value \\
\hline Beam Energy & $\mathrm{E}_{\mathrm{o}}=5-15 \mathrm{GeV}$ \\
\hline Bunch Charge & $\mathrm{Q}=1 \mathrm{nC}\left(\mathrm{N}=0.625 \times 10^{10} \mathrm{e}^{-} / \mathrm{bunch}\right)$ \\
\hline Repetition Rate & Pulse Repetition Rate, $\mathrm{PRR} \leq 120 \mathrm{~Hz}$ \\
\hline Average Power & $\mathrm{P}_{\mathrm{av}} \leq 1.8 \mathrm{~kW}$ \\
\hline Beam Transverse Size & $\sigma \sim 32 \mu \mathrm{m}$ \\
\hline Magnet Gap & $\mathrm{g}=6.0 \mathrm{~mm}$ \\
\hline Vacuum Chamber & $\mathrm{OD}=6.0 \mathrm{~mm}, \mathrm{ID}=5.0 \mathrm{~mm}$ \\
\hline Wall thickness & $\mathrm{t}=0.50 \mathrm{~mm}$ \\
\hline
\end{tabular}

\subsubsection{Permanent Magnet Material}

The material used for this analysis is neodymium-iron-boron (NdFeB) (2-14-1) and its material properties are listed Table $\mathbf{8 . 7}$

Table 8.7 Material Properties of neodymium-iron-boron (NdFeB) (2-14-1)

\begin{tabular}{ll}
\hline Property & Value \\
\hline Modulus of Elasticity & $\mathrm{E}=1.5 \times 10^{11} \mathrm{~N} / \mathrm{m}^{2}\left(21.74 \times 10^{6} \mathrm{psi}\right)$ \\
\hline Poisson Ratio & $v=0.3$ \\
\hline Tensile Strength & $\sigma_{\mathrm{UT}}=80 \mathrm{~N} / \mathrm{mm}^{2}(11,600 \mathrm{psi})$ \\
\hline Coefficient of Thermal Expansion & $\alpha_{\|}=3.4 \times 10^{-6} /{ }^{\circ} \mathrm{C}$ \\
& $\alpha_{\perp}=-4.8 \times 10^{-6} /{ }^{\circ} \mathrm{C}$ \\
& $\mathrm{E} \alpha_{\|}=5.1 \times 10^{5}\left(74 \mathrm{psi} /{ }^{\circ} \mathrm{C}\right)$ \\
\hline Specific Gravity & $\mathrm{E} \alpha_{\perp}=7.2 \times 10^{5}\left(104 \mathrm{psi} /{ }^{\circ} \mathrm{C}\right)$ \\
\hline Specific Heat & $\rho=7.4$ to $7.5 \mathrm{~g} / \mathrm{cm}^{3}$ \\
\hline
\end{tabular}


Based on composition values and atomic weights of the constituents, the effective values of radiation length, atomic number, and material critical energy of the material are: $X_{o}=11.54$ $\mathrm{g} / \mathrm{cm}^{2} ; X_{\delta} / \rho \sim 1.55 \mathrm{~cm} ; Z \sim 29 ; \varepsilon_{o} \sim 17.3 \mathrm{MeV}$ respectively. The minimum ionization loss is $\mathrm{dE} / \mathrm{dx}=\varepsilon_{o} / X_{o} \sim 1.5 \mathrm{MeV} /\left(\mathrm{g} / \mathrm{cm}^{2}\right)$. Note: these values very closely match those of copper.

For a square hit up front with no shower multiplicity (i.e., $\Pi\left(e^{-}\right)=1$ ), the normalized power deposition is $P^{\prime} \sim 1.35 \mathrm{~W} / \mathrm{cm}$. For the highest envisioned incident beam energy of $15 \mathrm{GeV}$, shower maximum of the electromagnetic cascade occurs at a depth of $T_{\max } \sim 5.8 \mathrm{~cm} X_{o} \equiv 9.0 \mathrm{~cm}$, and the maximum shower multiplicity is $\Pi_{\max }^{\left(e^{-}\right)} \sim 106$ [14]. Consequently, the maximum normalized power deposition is $P_{\max }^{\prime}=P^{\prime} \Pi_{\max }^{\left(e^{-}\right)} \sim 145 \mathrm{~W} / \mathrm{cm}$.

First, the exposure at the undulator entrance is estimated where the assumed Gaussian distributed beam has a predicted transverse size of $\sigma=32 \mu \mathrm{m}$. Assuming a uniform particle distribution inside $0<r \leqslant \sigma$, a heat source term is defined as $S=C P^{\prime} / A_{b} \sim 16.5 \times 10^{3} \mathrm{~W} / \mathrm{cm}^{3}$, where $\mathrm{C} \sim 0.4$, and $A_{b}=\sigma^{2} \pi \sim 32.2 \times 10^{-6} \mathrm{~cm}^{2}$. For a specific heat capacity of $\rho c=3.43$ $\mathrm{Ws} /\left(\mathrm{cm}^{3 \circ} \mathrm{C}\right)$, the temperature rise per pulse $(\mathrm{RF}-$ bunch) for a pulse repetition rate $(\mathrm{PRR})=120 \mathrm{~Hz}$ is

$$
\Delta T=\frac{S}{\rho c P R R} \approx 40^{\circ} \mathrm{C} / \text { pulse }
$$

The consequential thermal stresses are proportional to the product of the coefficient of thermal expansion $\alpha$ and the modulus of elasticity $E$ :

$$
\sigma_{t h} \propto E \alpha \Delta T
$$

Numerically $\sigma_{t h}$ is $\sim 2.83 \times 10^{7} \mathrm{~N} / \mathrm{m}^{2}$ (4100 psi or $\sim 0.35 \sigma_{U T}$ ) for a fully restrained body. Since this is near a surface, actual stresses are somewhat lower. This should not present any structural challenge to the magnetic material, even for repeated exposures. At $T_{\max }$ the effective transverse beam size increases to $\sigma_{\text {eff }} \sim 220 \mu \mathrm{m}$ (from Monte Carlo simulations for copper and scaling.). Using $\Pi_{\max }^{\left(e^{-}\right)}=106$, the heat source term is $S \sim 37 \mathrm{~kW} / \mathrm{cm}^{3}$, and the resulting single pulse temperature rise is $\Delta T \sim 90^{\circ} \mathrm{C} /$ pulse.

Somewhat higher temperatures are actually observed short of $T_{\max }$, at a depth of $\sim 3.5$ to $4 X_{o}$ for $E_{o}=15 \mathrm{GeV}$, since the expanding transverse shower has not yet caught up with the rapidly increasing shower multiplicity. At $4 X_{o}, \sigma_{\text {eff }}$ is $\sim 130 \mu \mathrm{m}$ and $\Pi\left(e^{-}\right)$is $\sim 75$. The resulting effective heat source term is $S_{4 X_{o}} \sim 73 \mathrm{~kW} / \mathrm{cm}^{3}$, for which the temperature rise per pulse is

$$
\Delta T \sim 175^{\circ} \mathrm{C} / \mathrm{pulse}
$$

and the consequential thermal stress rise is of the order of $\sigma_{t h}$ is $\sim 1.24 \times 10^{8} \mathrm{~N} / \mathrm{m}^{2} \quad(18,000 \mathrm{psi}$ or $\left.\sim 1.6 \sigma_{U T}\right)$. 
The permanent magnet material must therefore be protected from direct hits by the electron beam. The material is a powder-metallurgical product and is quite brittle, i.e., has very low ductility, and exposure to one pulse might fracture it. Independent of structural concerns, temperature changes of this magnitude would cause permanent changes in the remanent magnetic field, which are not acceptable.

Since the magnetic material has atomic properties very similar to copper, neither copper nor materials with equal or higher atomic number are suitable as primary collimator materials. The primary material must be protected by a low-Z material like titanium.

\subsubsection{Undulator Vacuum Chamber}

There are two distinctly different beam exposure scenarios for the undulator vacuum chamber. The first is direct $\mathrm{e}^{-}$-beam exposure at the entrance to the undulator with the momentum vector approximately parallel to the undulator and vacuum chamber axis (this assumes that no collimator is in place). The second exposure scenario results from excessive beam deflection inside the undulator resulting in the beam impinging at shallow angles onto the vacuum chamber. Selection of an appropriate material for the vacuum chamber involves tradeoffs between physics performance, survival during direct primary beam exposure, and ease of manufacture, and thus economics. Physics performance dictates a chamber material of low electrical resistivity, at least on the inside surface, to keep the resistive wall wake function at acceptable levels. Materials like copper and aluminum are good choices.

Long-term survival against direct hits by the e-beam requires a low-Z material with good strength and endurance characteristics. Titanium and some of its alloys, as well as some aluminum alloys, are good choices. Since the undulator and its vacuum chamber are $\sim 100 \mathrm{~m}$ long, the chamber needs to be built in segments (anticipated modular length $\sim 3.4 \mathrm{~m}$ ) and joined by vacuum flanges and bellows. The materials mentioned above are technically feasible to use, but they also present fabrication, installation, and economic challenges. Copper, aluminum, titanium, and stainless steel were evaluated for possible use as vacuum chamber material. Stainless steel is the final choice, and an analysis of its response to the two exposure scenarios is presented below. Cost effective manufacturing, ease of installation, and maintenance for ultra-high vacuum make stainless steel a first choice, but at the expense of high electrical resistivity. This handicap can be compensated by surface coating with a low resistivity material.

\subsubsection{Beam Strikes at the Entrance to the Vacuum Chamber}

Using the minimum ionization loss and no shower multiplicity $\left(\Pi^{\left(e^{-}\right)}=1\right)$, the power deposition at normal incidence to the chamber is

$$
\begin{gathered}
P^{\prime}=(-\rho d E / d x) N \times 1.6 \times 10^{-19} P R R= \\
11.6 \times 10^{6} \times 0.625 \times 10^{10} \times 1.6 \times 10^{-19} \times 120=1.4 \mathrm{~W} / \mathrm{cm}
\end{gathered}
$$

using $\mathrm{dE} / \mathrm{dx}$ for iron to approximate stainless steel. 
Next, again assuming a Gaussian beam intensity distribution with $\sigma \sim 32 \mu \mathrm{m}$, and also assuming uniform intensity within the radial interval $0<r \leq \sigma$, the heat source term becomes $S$ $\sim 17 \mathrm{~kW} / \mathrm{cm}^{3}$. The temperature rise per single rf-bunch follows as $\Delta T=17 \times 10^{3} /(4 \times 120) \sim 35^{\circ} \mathrm{C}$. The resulting thermal stresses are $\sigma_{t h} \sim 8.63 \times 10^{7} \mathrm{~N} / \mathrm{m}^{2}$ (12,500 psi) for a fully restrained body. Thin-walled tubing and beam exposure near the surface (inside or outside) will remove some of these restraints and thereby reduce the magnitude of these stresses at the expense of increased elastic strain. The endurance limit for the type of stainless steel used for vacuum chamber tubing (300 Series) is $\sigma_{\text {End }} \sim 1.73 \times 10^{8} \mathrm{~N} / \mathrm{m}^{2}(25,000 \mathrm{psi})$, and there would be no problem for this level of beam exposure. The yield strength $\sigma_{y} \sim \sigma_{t h}$ is $\sim 2.07 \times 10^{8} \mathrm{~N} / \mathrm{m}^{2}(30,000$ psi) is also significantly above the exposure stress, and no plastic, permanent deformations would occur.

\subsubsection{Beam Strikes Inside the Undulator}

Once inside the undulator, the electron beam can experience additional deflections. Based on alignment considerations for both the undulator and the quadrupoles, it is desirable to have a dynamic range of $\pm 500 \mu \mathrm{m}$ at each magnet mover. For an assumed maximum quadrupole gradient of $105 \mathrm{~T} / \mathrm{m}$ and an effective magnetic length of $l_{\text {eff }}=5 \mathrm{~cm}$, the maximum kick angle becomes $50 \mu \mathrm{rad}$. The present design value of the center-to-center module length is $3.4 \mathrm{~m}$. The deflection at the end of one modular section is therefore $170 \mu \mathrm{m}$. The vacuum chamber inside diameter is $5.0 \mathrm{~mm}$. It can readily be shown that five consecutive maximum kicks will amount to a deflection of $\sim 2.6 \mathrm{~mm}$ and the beam could strike the chamber wall near the end of undulator segment 5 with a maximum angle of $\theta \sim 300 \mu \mathrm{rad}$ (see Figure 8.26). The shortest longitudinal distance, $l_{i}$, over which the $2 \sigma$ core of the incident Gaussian distribution could strike the vacuum chamber is then $l_{i}=2 \sigma / \theta=21.3 \mathrm{~cm} \equiv 13 X_{o}$ for stainless steel with $1 X_{o} \sim 1.66 \mathrm{~cm}$. Similarly, the shortest distance of the momentum vector traversing the vacuum chamber wall is $l_{t}=t / \theta=167$ $\mathrm{cm} \equiv 100 X_{o}$. This means that every conceivable $\mathrm{e}^{-}$energy envisioned for the undulator reaches the peak of the electromagnetic cascade inside the vacuum chamber wall, and also, with the exception of particles scattered out of the wall in the transverse direction, the chamber wall is almost a complete absorber of the cascade. Examining the region of shower maximum where the normalized power deposition varies little $(d P / d T \sim 0)$ and using the longitudinal interval of $T_{\max } \pm$ $1 X_{o} \equiv 4.5$ to $6.5 X_{o} \equiv 3.3 \mathrm{~cm}$, Monte Carlo simulations using the EGS code show that $\sim 0.23 P_{a v}$ is absorbed in this region. The volume element defined by $2 \sigma$ and $\pm 1 X_{o}$ is a "skewed" ellipsoid, and after folding in a double convoluted Gaussian, the expected power deposition is $P_{4.5-6.5 \mathrm{X}_{\mathrm{o}}}$ $0.23 C P_{a v}=0.23 \times 0.4 \times 1.8 \times 10^{3} \sim 165 \mathrm{~W}$. Let $\Delta \sigma$ be the average transverse increase in $\sigma$ at the depth location of the ellipsoid; then the two axes are $\left(l_{i}+2 \Delta \sigma \sim l_{i} \sim 21.5 \mathrm{~cm}\right)$ and $(2 \sigma+2 \Delta \sigma \sim$ $0.27 \mathrm{~cm})$. The effective volume of the ellipsoid is then $\mathrm{V}=A_{\text {eff }} h \sim\left[\left(l_{i}+2 \Delta \sigma\right)(2 \sigma+2 \Delta \sigma) \pi / 4\right]$ $2 X_{o} \sim[21.5 \times 0.27 \pi / 4] 2 \times 1.66 \times 300 \times 10^{-6} \sim 4.54 \times 10^{-3} \mathrm{~cm}^{3}$.

Before arriving at a heat source term $S$, allowance has to be made for transverse leakage of shower particles out of the chamber wall. Monte Carlo calculations of a beam impinging in the center of a thin-walled stainless steel tube of similar wall thickness $(1.27 \mathrm{~mm})$ with the momentum vector parallel to the tube axis have been made. These resulted in volumetric power deposition values approximately a factor of 3.5 lower than those found for a semi-infinite 
medium of the same material ( $E_{o}$ was $50 \mathrm{GeV}$ and $\sigma=100 \mu \mathrm{m}$ for the tube and $200 \mu \mathrm{m}$ for the semi-infinite medium). Then $P_{4.5-6.5 \mathrm{X}_{\mathrm{o}}} \sim 40 \mathrm{~W}$. The heat source term for assumed uniformly distributed heat sources is then

$$
S_{\text {eff }}=P_{\text {eff }} / V \sim 9 \mathrm{~kW} / \mathrm{cm}^{3}
$$

and the temperature rise per pulse for stainless steel with $\rho c=4 \mathrm{Ws} /\left(\mathrm{cm}^{3}{ }^{\circ} \mathrm{C}\right)$ is

$$
\Delta T_{\text {pulse }}=S_{\text {eff }} /(\rho c P R R) \approx 20^{\circ} \mathrm{C}
$$

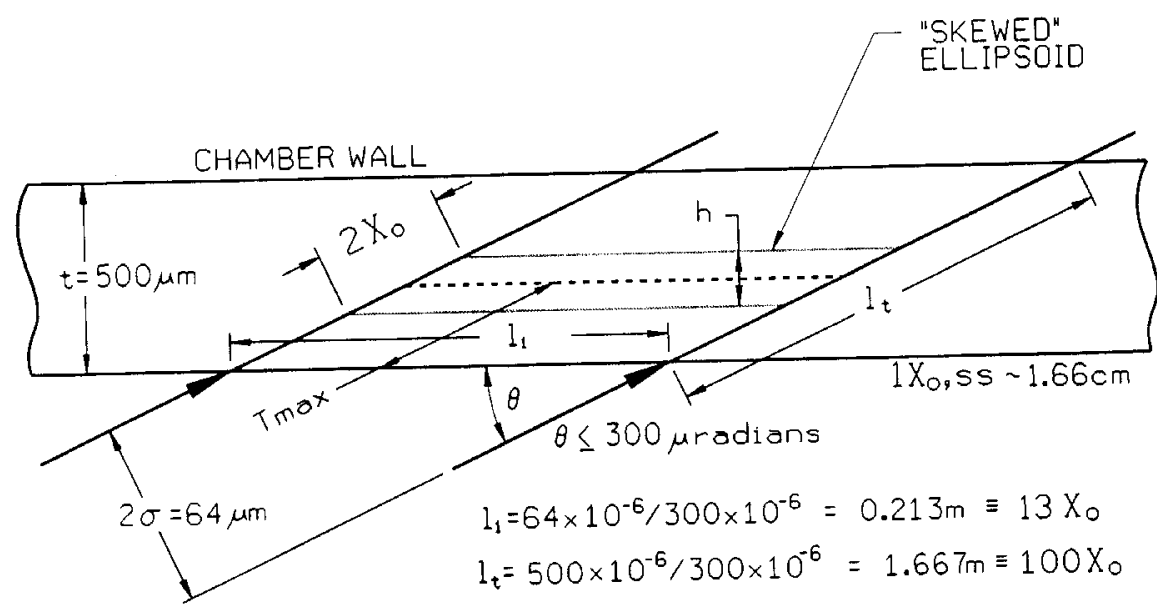

Figure 8.26 Model of accidental exposure of vacuum chamber to electron beam.

The consequential thermal stress spike in a fully restrained body would be about $\sigma_{t h} \sim 4.55 \times$ $10^{7} \mathrm{~N} / \mathrm{m}^{2}$ (6600 psi). Since a thin-walled tube is not very restrained, actual stresses would be even lower. Both pulse temperature rise and thermal stress are very modest; and one could comfortably manage even $2 \mathrm{nC}$ per pulse.

Next the steady state conditions are evaluated for when the beam is accidentally "parked" on the chamber for a long time. The effective power is converted into a heat load per unit length based on the distance of impingement, $l_{i}$. Then $P_{\text {eff }}^{\prime}=P_{\text {eff }} l_{\mathrm{i}}=40 / 21.5 \sim 2 \mathrm{~W} / \mathrm{cm}$. Assume a hypothetical heat sink at $\pi=180^{\circ}$ from the line source. Then the azimuthal conduction heat flux in the tube is $q_{c}^{\prime \prime}=P_{\text {eff }}^{\prime} / 2 t=2 /(2 x 0.050) \sim 20 \mathrm{~W} / \mathrm{cm}^{2}$. Ignoring natural convection and thermal radiation losses for the moment, the maximum temperature difference over $\pi$, i.e., $\delta=r \pi=0.864$ $\mathrm{cm}$ and for a thermal conductivity of $k=0.165 \mathrm{~W} /\left(\mathrm{cm}^{2}{ }^{\circ} \mathrm{C} / \mathrm{cm}\right)$ is $\Delta T=q_{c}^{\prime \prime} \delta / k=20 \times 0.864 / 0.165$ $\sim 105{ }^{\circ} \mathrm{C}$. This difference is modest, and to first approximation, a uniform temperature can be assumed for heat rejection by thermal radiation over the entire surface of the longitudinal section of chamber under consideration. The radiation heat flux is then $q_{r}^{\prime \prime}=P_{\text {eff }} / \pi \mathrm{d}=2 /(0.06 \pi)$ $\sim 1.06 \mathrm{~W} / \mathrm{cm}^{2}$. For stainless steel tubing, the thermal emissivity is $\varepsilon \sim 0.1$ of black body. Solving the Stefan-Boltzmann equation for the surface temperature of the tubing, for black body radiation 
to an ambient temperature $T_{a} \sim 300 \mathrm{~K} \equiv 27^{\circ} \mathrm{C}$, one finds $\mathrm{T}_{\mathrm{s}}=\left(q_{r}^{\prime \prime} / \varepsilon \sigma+T_{a}^{4}\right)^{1 / 4}=1345 \mathrm{~K}=$ $1072^{\circ} \mathrm{C}$.

Even if one factored in a small reduction due to the contribution of natural convection, steady state temperatures of this magnitude are too high for the stainless steel chamber and for the adjacent permanent magnet material. The MPS system will detect such an errant beam condition and rate-limit the beam

In addition to stainless steel, three other potential magnet vacuum chamber materials were analyzed. Aluminum was found to comfortably withstand the consequences of single rf-bunch hits; but for continuous exposure to a $1.8 \mathrm{~kW}$ beam, the steady state temperature exceeds the melting point. OFE copper was found to be able to take individual bunch hits but was marginal for repeated exposure at the same location; i.e., single bunch thermal stresses were modestly above the endurance limit. Continuous beam exposure resulted in steady state temperatures near the melting point, and this material is not suitable for a vacuum chamber. Early detection of errant beam would remove this handicap for both copper and aluminum. A proposed ceramic $\left(\mathrm{Al}_{2} \mathrm{O}_{3}\right)$ vacuum chamber concept has also been analyzed. Single pulse temperature and stress rises were modest, and the steady state temperature was well within the capability of this material. When compared to stainless steel, neither of these materials was cost effective; and the ceramic chamber presented additional engineering challenges.

In summary, the proposed stainless steel vacuum chamber can comfortably accept missteered beam pulses inside the undulator and will not suffer any damage for $\sigma \geq 32 \mu \mathrm{m}$ and $P_{a v}=1.8 \mathrm{~kW}$. However, a continuous beam exposure must be detected and beam delivery terminated before significant temperature increases in the chamber and adjacent magnetic material occur.

\subsubsection{Adjustable Collimators to Protect Undulator and Vacuum Chamber}

The analysis of various collimator concepts resulted in selection of a jaw design with multiple materials. For many reasons, such as fabrication, water-cooling, compactness, etc., it is still highly desirable to use copper as the primary power absorber material. For fully annealed OFE copper to withstand the exposure to a very large number of pulses, the cyclic thermal stresses should not exceed 3.45 to $4.15 \times 10^{7} \mathrm{~N} / \mathrm{m}^{2}$ (5000 to $6000 \mathrm{psi}$ ). Working backwards, the effective transverse beam size for an assumed Gaussian distributed beam should be $\sigma \gtrsim 50 \mu \mathrm{m}$ at the beam entrance face of a copper collimator jaw where $\Pi\left(e^{-}\right)=1$. But the transverse beam size at that location is only $\sigma \sim 38 \mu \mathrm{m}$. To guarantee long term survival of the copper, the transverse beam size must be increased. Using a spoiler of a lower $\mathrm{Z}$ material with appropriate mechanical properties is a simple and passive method of achieving this goal. In the past a titanium alloy (Ti$6 \mathrm{~A} l-4 \mathrm{~V}$ ) has been successfully used for this purpose. Modeling with the Monte Carlo code EGS resulted in a minimum spoiler thickness required of $\sim 0.3 X_{o}$. This will protect the front part of the copper near the interface with the titanium. However, the region of the highest power density and therefore temperature rise for the beam energies of interest and for copper is at a depth of $\sim 3 X_{o}$. Since there is significant shower multiplicity to that depth without a commensurate transverse spread of the beam, the minimum size of the beam needs to increase to $\sigma \sim 160 \mu \mathrm{m}$ at the front 
face of the jaw. A spoiler of thickness $\sim 1 X_{o}(\equiv 3.77 \mathrm{~cm})$ will do the job. The shower multiplicity in the titanium alloy at that depth is $\Pi^{\left(e^{-}\right)} \sim 6$. The effective heat source term is $S_{1 X_{o}} \sim$ $4500 \mathrm{~W} / \mathrm{cm}^{3}$, the resulting single pulse temperature rise for the standard beam parameters is $\Delta T_{\text {pulse }} \sim 11^{\circ} \mathrm{C}$, and the consequential thermal stress rise is $\sigma_{t h} \sim 2.14 \times 10^{7} \mathrm{~N} / \mathrm{m}^{2}$ (3100) psi $<\sigma_{\text {End }}$. At a depth of $3 X_{o}$ (i.e., $1 X_{o} \mathrm{Ti}+2 X_{o} \mathrm{Cu}$ ), $\Pi^{\left(e^{-}\right)}$is $\sim 45$ and $\sigma_{\text {eff }}$ is $\sim 310 \mu \mathrm{m}$. Then the heat source term becomes $S_{3 X_{o}} \sim 9 \mathrm{~kW} / \mathrm{cm}^{3}, \Delta T_{\text {pulse }} \sim 22^{\circ} \mathrm{C}$, and $\sigma_{\text {th }} \sim 4.28 \times 10^{7} \mathrm{~N} / \mathrm{m}^{2} \quad(6200 \mathrm{psi})$ $\sim \sigma_{E n d}$. This is acceptable for long term operation. The spoiler thickness can be increased even more, but it would be at the expense of more power deposition in the titanium jaw, which has poor thermal conductivity. The Ti-slab can be mechanically attached directly to the entrance face of the copper block.

The steady state power deposition into $1 X_{o}$ of Ti is $\mathrm{P} \sim 6 \mathrm{~W}$ and can be conducted into the copper. The copper block needs to be water-cooled. To completely attenuate and absorb a $15 \mathrm{GeV}$ cascade shower, a total jaw length of $\sim 30 X_{o}$ is recommended. However, most of the power has been absorbed after $\sim 15 X_{o}$, and one could switch to a higher $Z$ material at that depth and keep the assembly compact. The recommendation is to have a jaw composed of $1 X_{o} \equiv 3.77$ $\mathrm{cm}$ Ti-6Al-4V, followed by $14 X_{o} \mathrm{Cu} \equiv 20.23 \mathrm{~cm}$, and completed with $15 X_{o} \mathrm{~W} \equiv 5.16 \mathrm{~cm}$ for a total length of $30 X_{o} \equiv 27.2 \mathrm{~cm} \equiv 10.7 \mathrm{inch}$. Here W stands for preferably W-26Re or a freemachining tungsten composite with good ductility. The transverse size should be of the order of 8 $\mathrm{cm} \times 8 \mathrm{~cm}$. Only the copper section of the jaw would be water-cooled. A flow rate of $\sim 0.5$ to 1 gpm is recommended. Such jaws could be mounted to standard SLAC collimator designs with only minor modifications.

\subsubsection{Fixed Aperture Protection Collimators}

Since the undulator represents such a large investment and is the centerpiece of the whole LCLS, it is prudent to back up the adjustable collimators with an additional fixed aperture protection collimator just ahead of the entrance to the undulator. Such a collimator could be made of copper or even tungsten (if space were at a premium), would not have to be water-cooled, and would be a sacrificial device since exposure to primary beam would be an accidental occurrence. The aperture of the collimator would need to be less than the inside diameter of the vacuum chamber (ID $=5 \mathrm{~mm})$, and its length should be 25 to $30 X_{o}(\sim 36$ to $44 \mathrm{~cm})$ with sufficient transverse size to shadow all of the undulator structure. Its presence would also reduce exposure of the undulator to scattered radiation from the many possible sources in the e-beam transport system.

Additional fixed aperture collimators need to be placed in the transport system at strategic locations for equipment protection reasons. They should be patterned after the FFTB protection collimator design and can be either copper if $\sigma \gtrsim 50 \mu \mathrm{m}$ or must have a titanium insert (like Ti$6 \mathrm{Al}-4 \mathrm{~V})$ in locations where $\sigma<50 \mu \mathrm{m}$.

\subsubsection{Vacuum Chamber Surface Roughness}

Recent computer modeling [15] (Section 8.9.5) gave indications that the surface roughness on the inside of the undulator vacuum chamber may have deleterious effects on beam quality. 
Two effects, namely the geometric wall wakefield and the resistive wall wakefield, can negatively influence beam performance. The $6 \mathrm{~mm}$ OD $\times 5 \mathrm{~mm}$ ID stainless steel tubing needs to have a small surface roughness. Various roughness-reducing procedures have been investigated. The semiconductor industry has an ongoing need for ultra pure gas transport systems, and much R\&D effort was invested in recent years to satisfy these demands. Present state-of-the-art technology identified fully austenitic stainless steel Type 316-L with very stringent chemical composition limits as a successful starting material. It is extruded into seamless tubing with special high quality dies, to great straightness, and under very clean conditions. The tubing is then cleaned and electropolished in lengths up to $6 \mathrm{~m}$. Tubing with a surface roughness of $R_{a} \sim 125 \mathrm{~nm}$ (rms) is readily available off the shelf and for modest cost. Flat surfaces are readily electropolished to $R_{a}$ $\sim 50 \mathrm{~nm}$, and such a value might also be achievable inside a tube with more R\&D effort. Another quantity in surface roughness evaluation is the total peak-to-valley depth of roughness, $R_{t}$ or $R_{\max }$, which varies from 4 to 12 times the $R_{a}$ value.

An R\&D program is underway at SLAC/SSRL to use such high quality industrial tubing as starting material and then improve the surface quality to $\mathrm{R}_{\mathrm{a}}<100 \mathrm{~nm}$ (rms) by further electropolishing. The best results to date from small samples (12 to $15 \mathrm{~mm}$ long $\times 5$ to $6 \mathrm{~mm}$ wide) cut out at random locations from $0.5 \mathrm{~m}$ long tubing sections showed $\mathrm{R}_{\mathrm{a}}$ values from a best of $10 \mathrm{~nm}$ to the $50 \mathrm{~nm}(\mathrm{rms})$ range. The azimuthal values were always somewhat lower than the longitudinal ones. The measurements were made with an atomic force microscope and distances traversed with the stylus were $\sim 55 \mu \mathrm{m}$ azimuthally and $\sim 70 \mu \mathrm{m}$ longitudinally. It appears that the surface quality of the commercial products can be significantly improved to achieve values of surface roughness that will not negatively impact the performance of the LCLS undulator. Electro-polishing $3.4 \mathrm{~m}$ long tubing sections will, however, present additional challenges.

Since stainless steel has a high electrical resistivity, the resistive wall wake is increased. To reduce this effect, a thin layer of copper ( $>$ skin depth) is deposited onto the highly electropolished surface using a thin nickel substrate for better adhesion. This process is then followed by further electro-polishing the copper surface. Best values achieved to date are $\mathrm{R}_{\mathrm{a}} \sim 120 \mathrm{~nm}$ (rms), but further refinements in the process are possible and should make the surface inside the undulator vacuum chamber smooth enough that it does not materially degrade the electron beam.

\subsubsection{Vacuum System Requirements and Description}

The vacuum system for the undulator must have the following attributes to provide a good environment for the electron and photon beams:

- The vacuum system components must provide low impedance to avoid producing beam instabilities and minimize higher-order mode heating.

- Adequate pumping must be provided to maintain a low beamline pressure $\left(<10^{-7}\right.$ Torr average).

- The beamline must provide an adequate aperture for the electron and photon beams. 
The LCLS beam pipe is a $6 \mathrm{~mm}$ outside diameter, $0.5 \mathrm{~mm}$ thick wall stainless steel (type 316L) tube. The interior surface of the tube will be plated with approximately $0.01 \mathrm{~mm}$ of OFE copper to minimize ohmic heating from the image current induced by the electron beam. The beam pipe is constructed in segments that are $3.42 \mathrm{~m}$ long and which correspond to the undulator magnet segments. The beam pipe segments are constructed from type 316-L stainless steel to minimize the magnetization of the heat-affected zone at the welds. After welding, each beam pipe section will be annealed to completely de-magnetize the welds. Vacuum processing of the vacuum tubes section will consist of baking at $200^{\circ} \mathrm{C}$ for a minimum of 4 hours.

Between undulator segments there are short gaps of 18.7 and long gaps of $42.1 \mathrm{~cm}$ every third segment where vacuum pumping, beam steering, and diagnostic components are located. A typical segment gap is shown in Figure 8.27.

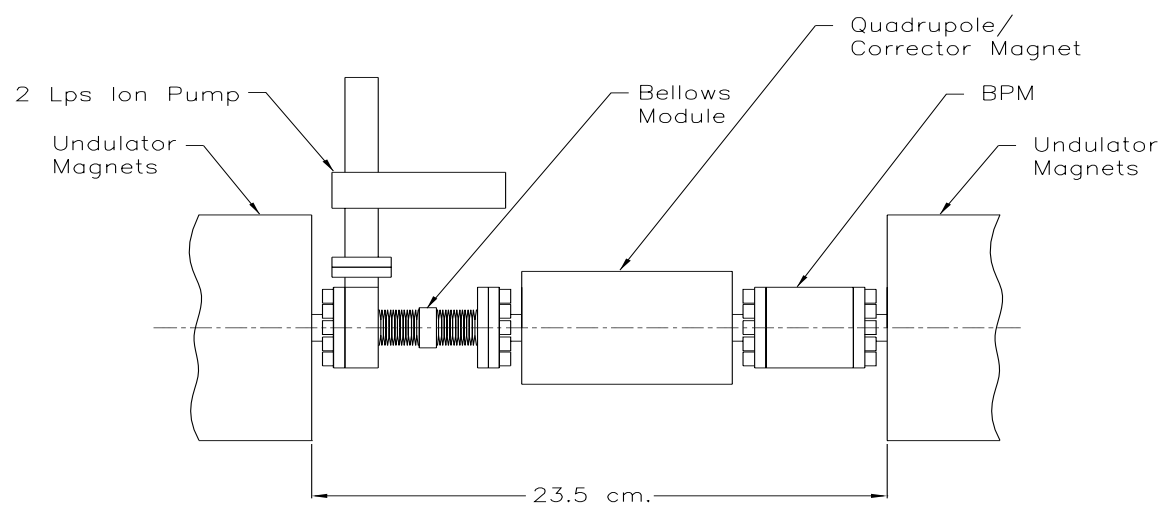

Figure 8.27 Undulator segment gap with vacuum and magnet components.

Between each undulator segment, there is an $\mathrm{rf}$ shielded bellows module. The purpose of the bellows is to provide flexibility for ease of installation of adjacent segments of the beam pipe and to account for any minor length changes due to temperature fluctuations. A cross-section of the LCLS bellows module is shown in Figure 8.28. Since space is tight, vacuum pumping of the beam pipe is accomplished within the bellows module. A $21 / \mathrm{s}$ noble diode ion pump is mounted on one of the bellows end flanges. Pumping occurs through ten 1-mm diameter holes in the beam pipe. Conductance losses through the module decrease the actual pumping speed to approximately 1 l/s.

The sliding rf fingers within the bellows are fabricated from a thin wall type 316-L stainless steel tube. The ends of the tube have $0.15 \mathrm{~mm}$ slits, which allow the tube to flex radially and provide spring force to maintain contact with the adjacent tubes. The tube slits are plated with $0.01 \mathrm{~mm}$ thick silver. The contacting surfaces of the adjacent tubes are plated with $0.01 \mathrm{~mm}$ thick rhodium. The combination of the relatively soft silver and the hard rhodium provide a sliding surface with both good lubricity and good electrical conductivity.

The flanges in the vacuum system are raised face type using a "VAT" style seal. The VAT seal is a commercially available, silver-plated, soft copper gasket. The gasket has a raised bead 
around the inside diameter of the beam tube, which is crushed between the flanges. The raised bead acts as the RF seal across the flanged joint.

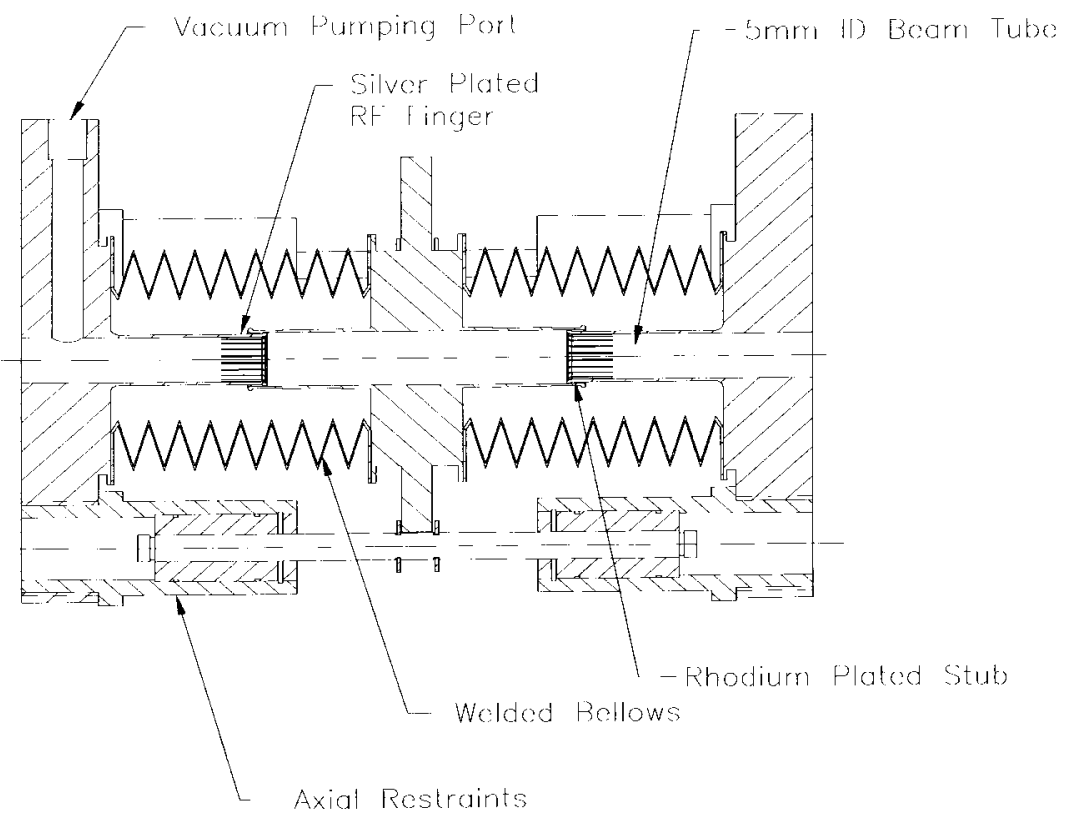

Figure 8.28 Bellows module cross-section.

\subsubsection{Gas Load and Vacuum Pressure}

In general, accelerator beam pipes are conductance-limited. In the case of the undulator vacuum system, this problem is extreme. It turns out that the amount of pumping at $3.42 \mathrm{~m}$ intervals has little effect on the average system pressure. This can be demonstrated with a simple calculation. Assuming a constant gas load along the length of the beam pipe and considering the symmetry of the vacuum system, the average beam pipe pressure is calculated analytically using the following equation:

$$
P_{a v}=\left(q \frac{p L}{C}\right)\left(\frac{C}{S}+\frac{1}{3}\right)
$$

where $\quad P_{a v}$, average pressure, Torr

$q, \mathrm{H}_{2}$ gas load $=1 \times 10^{-11}$ Torr-liter $/ \mathrm{sec}-\mathrm{cm}^{2}$

$p$, circumference $=\pi(5 \mathrm{~mm})=1.57 \mathrm{~cm}$

$L$, half-length of segment $=171 \mathrm{~cm}$

$\mathrm{C}$, beam pipe conductance $=0.0269$ liter $/ \mathrm{sec}$

$S$, pumping speed 
Assuming a pumping speed of $1 \mathrm{l} / \mathrm{s}$, the average pressure is $3.59 \times 10^{-8}$ Torr. If the pumping speed is increased to $10 \mathrm{l} / \mathrm{s}$, the average pressure decreases to $3.34 \times 10^{-8}$ Torr (only a $7.6 \%$ improvement). From this, it can be seen that minimizing the gas load has a much greater effect on beam pipe pressure than does pumping speed. The gas load within the undulator vacuum system comes from two processes, thermal desorption and photo-desorption. Thermal desorption is common to all types of vacuum systems; it is the heat-stimulated release of gas constituents adsorbed on the walls of the system. Photo-desorption is the outgassing that occurs due to synchrotron radiation hitting the walls of the beam pipe and desorbing gas molecules. Good thermal desorption data exists for UHV processed copper plated stainless steel from the PEP-II project $[16,17]$. Typically, $q_{t}=5 \times 10^{-13}$ Torr-liter $/ \mathrm{sec}-\mathrm{sq} \mathrm{cm}\left(@ \mathrm{~T}=20^{\circ} \mathrm{C}\right)$ after a $200^{\circ} \mathrm{C}$ bake for 4 hours. Since the undulator vacuum system is expected to operate at $20^{\circ} \mathrm{C}$, this value will be used.

Photodesorption is a little harder to estimate. The undulator produces $90 \mathrm{GW}$ of total peak power. Photon flux is estimated using $100 \mathrm{GW}$ of power hitting the walls of the vacuum system using the following formula:

$$
N_{\gamma}=\frac{P_{S R} t f\left(6.242 \times 10^{15} \mathrm{keV} / \mathrm{J}\right)}{E_{\text {photon }}}
$$

where

$$
\begin{aligned}
& N_{\nsim} \text {, photon flux }=\text { photons } / \mathrm{sec} \\
& P_{S R}, \text { synchrotron radiation power }=100 \times 10^{9} \text { Watts } \\
& t \text {, pulse length }=100 \times 10^{-15} \mathrm{sec} \\
& f \text {, pulse frequency }=120 \mathrm{sec}^{-1} \\
& E_{\text {photon }}, \text { average photon energy }=200 \mathrm{keV} / \text { photon }
\end{aligned}
$$

$N_{\gamma}$ for the undulator is $3.74 \times 10^{13}$ photons $/ \mathrm{sec}$. The distribution of the spontaneous photon flux is assumed to increase linearly along the length of the vacuum system as shown in Figure 8.29. 


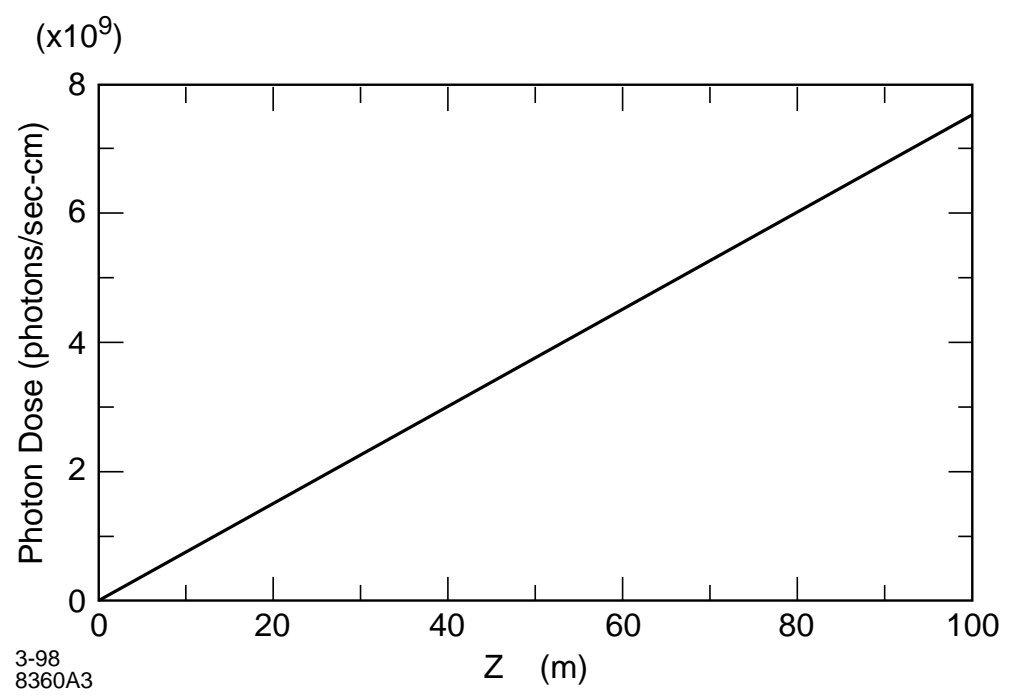

Figure 8.29 Undulator photon flux profile.

The photo-desorption gas load is calculated as follows:

$$
q_{p}=N_{\gamma} \eta\left(2.83 \times 10^{-20} \text { Torr }-1 / \text { molecule }\right)
$$

where

$q_{p}$, photo-desorption gas load $=$ Torr-liter $/ \mathrm{sec}$

$\eta$, photo-desorption rate $=$ molecules $/$ photon

Photo-desorption of copper and stainless steel beam tubes were investigated in the past. Brookhaven National Laboratory conducted studies for the PEP-II Project, determining the values of $\eta$ with respect to flux [18]. The photon flux for the undulator vacuum system is in fact quite low, so, realistically, photon scrubbing will not occur during the lifetime of the machine. From the Brookhaven results, it has been determined that an $\eta=5 \times 10^{-3}$ molecules/photon is appropriate for design purposes. Using this value, the photo-desorption profile is calculated and is shown along with the calculated thermal desorption profile in Figure 8.30.

Using the desorption profiles, a vacuum pressure profile for the 100-m long undulator vacuum system is calculated using VACCALC [19], a pipeline pressure computer code. The entire undulator beam pipe is modeled using discrete pipeline segments. Each segment is defined by its length (m), conductance (1/s), gas load (nTorr-1/s), and pumping speed (1/s). All values for conductance, gas load and pumping speed are calculated for "hydrogen." Figure 8.31 shows the pressure profile along the length of the undulator vacuum system, with its average pressure being $1.06 \times 10^{-9}$ Torr ( 1 nTorr). This is well below the design requirement of $10^{-7}$ Torr. 




Figure 8.30 Undulator thermal and photo-desorption profiles.

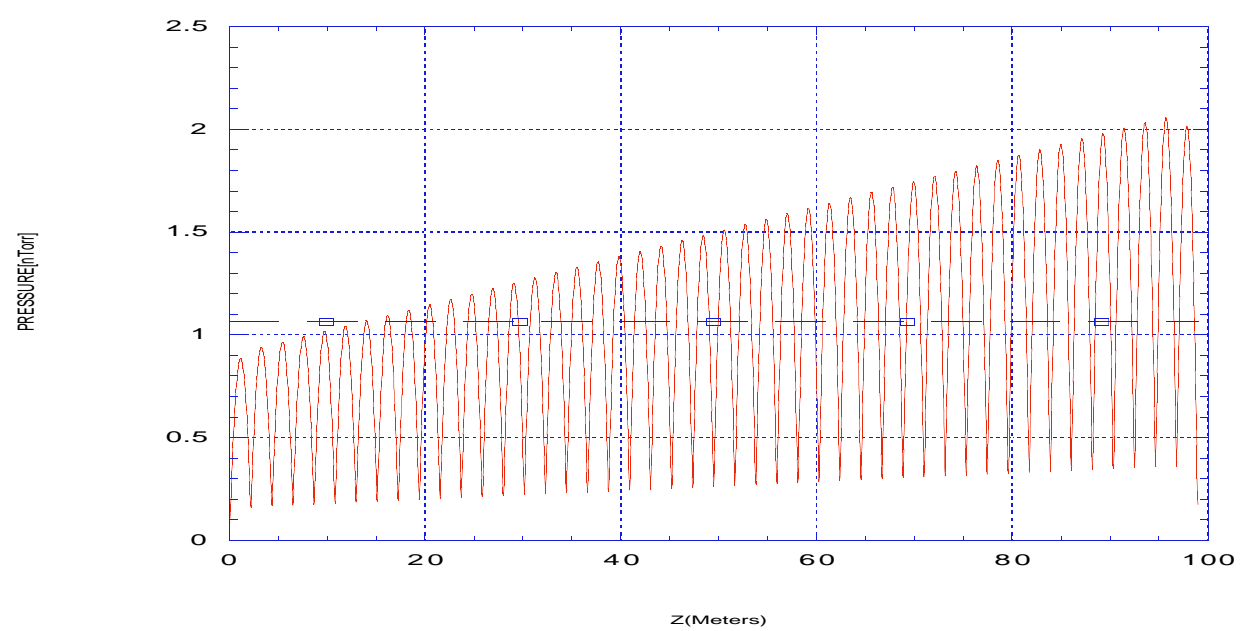

Figure 8.31 LCLS vacuum pressure profile.

\subsubsection{Thermal Considerations}

As described above, $100 \mathrm{GW}$ peak of synchrotron radiation power strikes the walls of the beam pipe in the worst case scenario. However, the length of the photon pulse is $70 \times 10^{-15} \mathrm{sec} \mathrm{rms}$ and the frequency of the pulse is $120 \mathrm{~Hz}$. Therefore, the total power absorbed by the entire $121 \mathrm{~m}$ of beam pipe is 1.2 Watts. With such a low level of incident power, there is no need to actively cool the vacuum chamber. 


\subsection{Wakefield Effects in the Undulator}

\subsubsection{Introduction}

When the electron beam moves through the undulator it will excite longitudinal and transverse wakefields due to the resistance and the discontinuities in the beam tube wall. Let us assume that the wall geometry is cylindrically symmetric. Then the longitudinal (monopole) wakefield will generate an energy loss and an increase in energy spread independent of the beam orbit, and the transverse (dipole) wakefield will generate an emittance growth that does depend on the orbit. It is, however, important to recognize that the forces due to the wakefields are correlated with longitudinal position. Assuming the bunch is composed of many slices at different longitudinal positions, the wakefields affect only the centroid values of the slices - i.e., the average energy and the average position of the slices in, respectively, the longitudinal and the transverse case. The distributions of the slices about their centroids are not affected.

The critical issues concerning the electron beam with respect to wakefield effects in the undulator are:

- The absolute value of the maximum relative energy deviation of a bunch "slice" (slippage length: $\sim 0.5 \mu \mathrm{m}$ ) with respect to the mean of the whole bunch generated over the length of the undulator at $14.3 \mathrm{GeV}$ should be less than $\sim 0.1 \%$. This tolerance is derived from GINGER simulations.

- The dilution of the "projected" emittance (emittance projected over the entire bunch) should not exceed $\sim 10 \%$.

- The mean energy loss over the undulator, including radiation losses, will determine the necessary taper of the magnetic fields of the undulator dipoles.

Since undulator wakefields have very little effect on the "slice" energy spread and the "slice" emittance, these tolerances are not considered here.

In this report the longitudinal and transverse wakefield effects on the LCLS beam during its time in the undulator are estimated to see how well these conditions are satisfied. Note that the beam dynamics and wakefield concepts that are presented are thoroughly discussed, with equations in [20].

\subsubsection{Wakefield Induced Beam Degradation}

In the longitudinal case, the wake function for a Gaussian bunch, from which the average wake (also known as the loss factor) $\left\langle W_{z}\right\rangle$ and the rms deviation of the wake with respect to the mean, $\left(W_{z}\right)_{r m s}$ (the units are $\left.\mathrm{V} / \mathrm{C} / \mathrm{m}\right)$ are derived, is first obtained. Then the wakefield induced energy loss is given by

$$
\langle\delta\rangle=-\frac{e^{2} N L\left\langle W_{z}\right\rangle}{E},
$$


with $N$ the number of particles in the bunch, $L$ the length of travel, and $E$ the beam energy. The rms energy spread, with respect to the mean, becomes

$$
\sigma_{\delta}=\frac{e^{2} N L\left(W_{z}\right)_{r m s}}{E}
$$

In the transverse case, the average of the transverse wakefield of a Gaussian bunch $\left\langle W_{x}\right\rangle$ (here in units of $\mathrm{V} / \mathrm{C} / \mathrm{m}^{2}$ ) is first obtained. The focusing lattice in the undulator is a FODO type with a phase advance of $13 \%$ cell at $14.3 \mathrm{GeV}$, and therefore a smooth focusing analysis is appropriate. First, to study the effect of injection jitter, the wake function is applied to obtain the projected emittance growth due to a betatron oscillation in the undulator. The offset at the end of the undulator, averaged over the bunch, can be written as

$$
\langle x\rangle=x_{0} e^{i L / \beta}(1-i v),
$$

with $x_{0}$ the initial offset, $\beta$ the beta function, and $v$ the strength parameter (as long as it is small). The strength parameter is given by

$$
v=\frac{e^{2} N L\left\langle W_{x}\right\rangle \beta}{2 E}
$$

The relative emittance growth, if it is small, is then given by

$$
\frac{\Delta \varepsilon}{\varepsilon}=\frac{x_{0}^{2} v^{2} \gamma}{2 \varepsilon_{N} \beta}
$$

with $\gamma$ the energy factor and $\varepsilon_{N}$ the normalized emittance. If the beam tube wall is not perfectly straight and aligned then even if the beam does not undergo betatron oscillations the projected emittance can grow. However, unlike in the case of injection jitter, since this error is a static error some correction can usually be performed to reduce the effect. The misalignment errors are divided into two types, those that are uncorrelated to each other and those that are correlated. In the former category suppose the wakefield generating object is composed of $M_{p}$ equal, randomly misaligned pieces. Then the emittance growth will again be approximately given by Eq. (8.44), but with $x_{0}$ replaced by the rms misalignment of the pieces divided by the factor $M_{p}{ }^{1 / 2}$. For correlated errors, the largest effect, for a given misalignment amplitude, is when the misalignment varies as $\cos (z / \beta)$, and the results are the same as given in Eq. (8.44), but with $x_{0}$ representing the misalignment amplitude. If the oscillation frequency of the misalignments differs from the betatron wave number by $\Delta k$ then the effect on emittance decreases according to $\operatorname{sinc}^{2}(\Delta k L)$.

The above effects are due to a resonance term in the equation of motion. But there is normally also a bounded, non-resonance term. For example, consider the case where the beam's trajectory follows the magnetic focusing axis in the undulator, but this axis is not aligned with the axis of the impedance generating objects. In this case the projected emittance of the beam will grow and then again decrease within the undulator. Even though the final emittance growth in the LCLS undulator due to this effect is small, there may be a tolerance for bunch emittance growth anywhere within the undulator. In such a case this effect will set a tolerance for the alignment of the wakefield generating axis with respect to the magnetic axis. For this example, the maximum 
emittance growth is given by Eq. (8.44), with $x_{0}$ the distance between the two axes and with $v$ $=2 e^{2} N\left\langle W_{x}\right\rangle \beta^{2} / E$.

In the calculations below, the following bunch and machine properties are considered: total charge of $1 \mathrm{nC}$, rms bunch length $\sigma_{z}=20 \mu \mathrm{m}$, normalized emittance $\varepsilon_{N}=1.0 \mathrm{~mm}$-mrad (the undiluted emittance is used for a conservative estimate), and energy $E=14.3 \mathrm{GeV}$. The length of the undulator is $L=121 \mathrm{~m}$ and the average focusing beta function is $\beta=18 \mathrm{~m}$. Note that in reality the bunch shape is not Gaussian, and to obtain a more accurate result one would need to use the real bunch shape in the calculations. The results given in this report should serve as reasonable estimates. The generators of wakefields considered are the wall resistance of the beam tube, the flange gaps, the pumping slots, and the bellows. The effect of the beam position monitors (BPMs) is discussed in Section 8.11.3. Finally, the effects of wall surface roughness are considered.

\subsubsection{The Resistive Wall Wakefields}

The beam tube inside the undulator is made of stainless steel plated with copper. It is of circular cross-section and has a radius of $a=2.5 \mathrm{~mm}$. The normal formulas for the resistive wall wakefields are valid only if $\sigma_{z} / s_{0}>1$, with the characteristic distance

$$
s_{0}=\left(\frac{2 a^{2}}{Z_{0} \sigma}\right)^{1 / 3}
$$

where $Z_{0}=377 \Omega$ and $\sigma$ is the conductivity of the metal. For stainless steel (SS) $\sigma=$ $1.7 \times 10^{6} \Omega^{-1}-\mathrm{m}^{-1}$, for copper $(\mathrm{Cu})$ it is $60 \times 10^{6} \Omega^{-1}-\mathrm{m}^{-1}$. Therefore, for the beam tube with SS $s_{0}$ $=27 \mu \mathrm{m}$, with $\mathrm{Cu} s_{0}=8 \mu \mathrm{m}$. Thus, in either case, since $\sigma_{z} \sim 20 \mu \mathrm{m}$, the normal formulas should be valid. The average of the longitudinal wakefield of a Gaussian bunch is given by

$$
\left\langle W_{z}\right\rangle \approx \frac{\Gamma(3 / 4)}{4 \sqrt{2} \pi^{2}} \frac{c}{a \sigma_{z}^{3 / 2}}\left(\frac{Z_{0}}{\sigma}\right)^{1 / 2}, \quad \sigma_{z} / s_{0}>1
$$

with $\Gamma(3 / 4) \approx 1.23$, and the rms is given by $\left(W_{z}\right)_{r m s} \approx(1.02)\left\langle W_{z}\right\rangle$. Combined with Eq. (8.40)this gives $\langle\delta\rangle=0.34 \%$ for stainless steel and $0.06 \%$ for copper, and similar results for the induced rms energy spread. The effects of the more realistic, non-Gaussian bunch distribution is calculated using the point-charge wake function [21],

$$
W_{z}(s)=-\frac{4 c Z_{0}}{\pi a^{2}}\left(\frac{1}{3} e^{-s / s_{0}} \cos \frac{\sqrt{3} s}{s_{0}}-\frac{\sqrt{2}}{\pi} \int_{0}^{\infty} \frac{x^{2} e^{-x^{2} s / s_{0}}}{x^{6}+8} d x\right)
$$

generalized to the case where, $s_{0}$, is larger than or similar to the bunch length. Here, $a$, is the radius and, $\sigma$, the conductivity of the vacuum chamber. Here the resistive-wall loss and spread are both smaller than that of the Gaussian case examined above.

For the transverse case 


$$
\left\langle W_{x}\right\rangle \approx \frac{\Gamma(1 / 4)}{2 \sqrt{2} \pi^{2}} \frac{c}{a^{3} \sigma_{z}^{1 / 2}}\left(\frac{Z_{0}}{\sigma}\right)^{1 / 2}, \sigma_{z} / s_{0}>1
$$

with $\Gamma(1 / 4) \approx 3.63$. Combined with Eq. (8.43) it is seen that $v$ is 0.58 and 0.10 for the SS and $\mathrm{Cu}$ cases, respectively. For an extreme $100-\mu \mathrm{m}$ oscillation (e.g. random pulse-to-pulse jitter which is not correctable) the emittance growth is $260 \%$ for SS and $8 \%$ in the case of $\mathrm{Cu}$.

As to the effects of static errors, it is noted first that the beam tube is composed of 33 equal pieces. With uncorrelated, random misalignments with an rms of $100 \mu \mathrm{m}$, the emittance growth will be a factor of 33 less than given above. Or, conversely, the misalignment tolerance for $10 \%$ emittance growth, assuming copper is used, is $800 \mu \mathrm{m}$. For a correlated, cosine variation of misalignments of amplitude $100 \mu \mathrm{m}$ the emittance growth is approximated by the above jitter results multiplied by $\operatorname{sinc}^{2}(\Delta k L)$, where $\Delta k$ is the deviation from the betatron wave number of the wall oscillation wave number. Finally, if the static emittance growth has to be kept to less than $10 \%$ anywhere within the undulator, the axis of the beam tube must be aligned to the axis of the quads to within $200 \mu \mathrm{m}$ in the case of copper.

\subsubsection{The Effect of Flange Gaps, Pumping Slots, and Bellows}

The flange gaps are small cavities with a gap of $g=0.25 \mathrm{~mm}$; over every $3.42-\mathrm{m}$ section there are 4 , or a total of $M=132$ objects in the entire undulator. For the flange gaps, since $\sigma_{z} / a$ $<<1$, the diffraction wakefield model applies [22]:

$$
\left\langle W_{z}\right\rangle \approx \frac{\Gamma(1 / 4)}{4 \pi^{5 / 2}} \frac{Z_{0} c M}{a L} \sqrt{\frac{g}{\sigma_{z}}}, \quad \sigma_{z} / a<<1
$$

with $\Gamma(1 / 4) \approx 3.63$, and $\left(W_{z}\right)_{\text {rms }} \approx(0.40)\left\langle W_{z}\right\rangle$ gives the average effect of all the flange gaps. In the transverse case

$$
\left\langle W_{x}\right\rangle \approx(4.63) \frac{Z_{0} c M}{\pi^{3} a^{3} L} \sqrt{g \sigma_{z}}, \sigma_{z} / a<<1 .
$$

Substituting for the parameters of the flange gaps, $\langle\delta\rangle=0.012 \%$ and $\sigma_{\delta}=0.005 \%$, the emittance growth due to a $100 \mu \mathrm{m}$ oscillation is very small, $0.08 \%$.

The pumping slots consist of ten, longitudinally arranged ovals in each $3.42 \mathrm{~m}$ section, or a total of $M=330$ slots. For each slot the width $w=1 \mathrm{~mm}$ and length $g=5 \mathrm{~mm}$. Usually in accelerators pumping slots are inductive. In the LCLS case, however, since $\pi \sigma_{z}$ is small compared to the slot width, energy will radiate into the slot. The diffraction formulas of the previous paragraph, multiplied by an azimuthal filling factor $w / 2 \pi a(=0.064)$, can be used to estimate the wakefields. The results are that $\langle\delta\rangle=0.008 \%, \sigma_{\delta}=0.004 \%$, and the emittance growth due to a $100 \mu \mathrm{m}$ oscillation is $0.04 \%$. There is one bellows in every $3.42 \mathrm{~m}$ section, giving a total of 33 . The bellows are shielded, so that the wake effects should be negligible, and will be taken to be zero.

The results of the above calculations, as well as the effects of the BPMs (see Section 8.12), are summarized in Table 8.8. Note that a stainless steel surface (top row) is clearly not acceptable. It is further noted that, with copper, the resistive wall wakefield still dominates the 
impedance, though in this case, the rms energy spread is nearly a factor of two below the maximum tolerable deviation per slice. Finally, another effect that will add to these values is that of incoherent synchrotron radiation within in the undulator, which will produce a relative energy loss of $0.16 \%$ for a $14.3 \mathrm{GeV}$ electron beam. Combining this with the loss factors $(\mathrm{Cu})$ of results in a total loss of $\sim 0.3 \%$ which is approximately the undulator field taper required. These results are for a Gaussian bunch distribution. The effects of the non-Gaussian distribution are examined in Chapter 4.

Table 8.8 The total longitudinal and transverse wakefield effects, for a Gaussian axial distribution, due to the various types of objects in the LCLS undulator. Given are the average energy loss, $\langle\delta\rangle$, the rms energy spread, $\sigma_{\delta}$ and the relative correlated emittance growth, $\Delta \varepsilon / \varepsilon_{0}$, of a $100 \mu \mathrm{m}$ betatron oscillation.

\begin{tabular}{lccc}
\hline Type of Objects & $\langle\boldsymbol{\delta}\rangle / \%$ & $\boldsymbol{\sigma}_{\boldsymbol{\delta}} / \%$ & $\boldsymbol{\Delta} \varepsilon_{\mathbf{\varepsilon}} / \boldsymbol{\%}$ \\
\hline Resistive Wall (SS) & 0.340 & 0.350 & 260 \\
\hline Resistive Wall (Cu) & 0.060 & 0.060 & 8 \\
\hline Flange Gaps & 0.008 & 0.003 & 0.08 \\
\hline Pumping Slots & 0.006 & 0.002 & 0.06 \\
\hline BPMs & 0.019 & 0.007 & 0.007 \\
\hline
\end{tabular}

\subsubsection{The Effect of Wall Surface Roughness}

In the first model of wakefields [23] the roughness was simulated by a collection of bumps of a given shape randomly distributed over a smooth surface. If the bump dimensions are small compared to the bunch length, the impedance in this model is purely inductive. For such simple shapes of the bumps as hemispheres or cubes, the model predicts relatively large impedance and results in severe tolerances on the level of roughness. A more realistic model of roughness effects was developed in Ref. [24]. In this model, the rough surface is considered as a terrain with a slowly varying slope. As was shown in direct measurements of the surface roughness with Atomic Force Microscope [25], this representation of the roughness is adequate for the real surface of the prototype pipe for the LCLS undulator. In the limit when the bunch length is larger than the correlation length of the roughness, the impedance in this model is also inductive, 
however the tolerance on the rms height of the surface roughness are much looser than predicted in [23].

In another approach [26], the roughness wakefield was associated with the excitation of a resonant mode whose phase velocity is equal to the speed of light. The existence of such modes in a round pipe with periodically corrugated walls with rectangular shape of the corrugation was studied theoretically in Ref. [27]. In the case when the typical depth of the wall perturbations is comparable to the period, it was shown that the loss factor of such modes reaches the theoretically maximal value for the resonant wakefield. However, as was shown in [28], when the height of the periodic wall corrugations becomes smaller than the period, the loss factor for the mode rapidly decreases.

A naive idea of a rough surface as a microscopic mountain country with sharp peaks and deep canyons does not correspond to reality. A metal surface with a good finish more resembles water surface of a swimming pool in quiet weather. Pictures of scanned surfaces for different types of machining can be found in surface metrology books [29,30]. Most of them are characterized by a typical peak-to-valley height $h$ of the roughness that is much smaller than the spacing between the crests $g$. The aspect ratio $g / h$ can easily exceed a hundred for smooth surfaces. For illustration, Figure 8.32 shows the profile of a surface of a metal pipe measured in Ref. [25]. This pipe is considered as a possible prototype for the vacuum chamber of the LCLS undulator. The rms height of the roughness for this surface is about $100 \mathrm{~nm}$, and the transverse size $g$, as is seen from the picture, can exceed tens or even a hundred of microns.

The small ratio $h / g$ implies a small angle $\Theta$ between the tangent to the surface and the horizontal plane. Using the smallness of this parameter it is possible to develop a perturbation theory of electromagnetic interaction of the beam with the surface based on the so-called smallangle approximation [24]. This approach extends the earlier treatments [31,32] of an axisymmetric periodic perturbation of the boundary. It also agrees with the more general results of Ref. [33] valid for nonperiodic axisymmetric boundary perturbations.

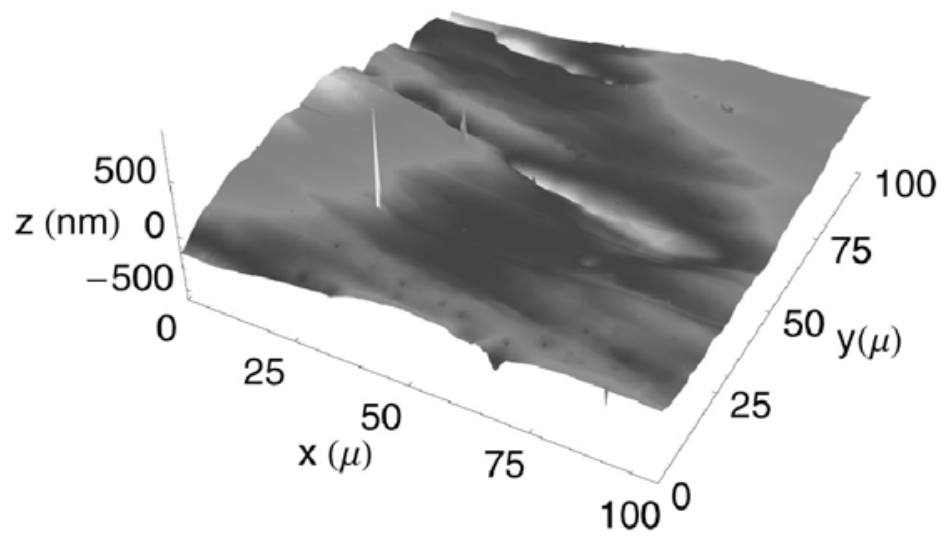

Figure 8.32 A sample surface profile measured with Atomic Force Microscope in Ref. [29]. Note the different scales in the vertical and horizontal directions. 
As follows from Refs. [31,32], for a periodically corrugated wall with the wavelength $\lambda_{0}$ much smaller than the pipe radius $b$, there exist synchronous modes in the pipe which propagate with phase velocity equal to the speed of light. The wavelength of these modes is below $2 \lambda_{0}$, so that only a short bunch of length $\sigma_{z} \leq 2 \lambda_{0}$ can efficiently excite these modes. If, on the other hand, the bunch length is larger than $\lambda_{0}$, the excitation of these modes will be relatively weak. In the roughness problem the parameter $g$ plays the role of $\lambda_{0}$, and two different regimes are expected depending on whether $\sigma_{z}$ is larger or smaller than $\mathrm{g}$.

In the regime where $\sigma_{z}>g$ one expects an inductive impedance because the beam does not lose energy by excitation of synchronous modes. However, the interaction between the head and the tail of the bunch can cause energy variation along the bunch and may interfere with the lasing.

Let $h(x, z)$ denote the local height of the rough surface as a function of coordinate $x$ in azimuthal direction, and coordinate $z$ along the axis of the pipe. The requirement $h \ll g$ can alternatively be expressed as $\Theta \approx|\nabla h| \ll 1$. The treatment of Ref. [24] was additionally limited by the assumption that the bunch length is larger than the typical size of the roughness bumps, $\lambda \sim \sigma_{z} \gg g$. It was found that in this limit the impedance is purely inductive, and the inductance $\mathfrak{L}$ per unit length of the pipe is given by the following formula:

$$
\mathfrak{L}=\frac{Z_{0}}{2 \pi c b} \int_{-\infty}^{\infty} \frac{\kappa_{z}^{2}}{\sqrt{\kappa_{\vartheta}^{2}+\kappa_{z}^{2}}} S\left(\kappa_{z}, \kappa_{\vartheta}\right) d \kappa_{z} d \kappa_{\vartheta},
$$

where $Z_{0}=4 \pi / \mathrm{c}=377 \mathrm{Ohm}$, and $S\left(\kappa_{z}, \kappa_{\vartheta}\right)$ is the spectrum of the surface profile as a function of wave numbers $k_{z}$ and $k_{\vartheta}$ in the longitudinal and azimuthal directions, respectively. The spectral density $S$ can be defined as a square of the absolute value of Fourier transform of $h$,

$$
S\left(\kappa_{z}, \kappa_{\vartheta}\right)=\frac{1}{(2 \pi)^{2} A}\left|\int_{A} h(z, x) e^{-i \kappa_{z} z-i \kappa \vartheta x} d z d x\right|^{2},
$$

where the integration goes over the surface of a sample of area $A$. It is assumed that the sample area is large enough so that the characteristic size, $\sqrt{A}$, is much smaller than the correlation length, $g$, of the roughness.

In Ref. [34] a comparison was done between the small-angle approximation and a previous model of roughness, developed in [23]. It was shown that in the region of mutual applicability both models give the results, which, within a numerical factor, agree with each other.

A detailed study of the surface roughness for a prototype of the LCLS undulator pipe using the Atomic Force Microscope was done in Ref. [25]. A high quality Type 316-L stainless steel tubing from the VALEX Corporation with an outer diameter of $6.35 \mathrm{~mm}$ and a wall thickness of $0.89 \mathrm{~mm}$ with the best commercial finish, A5, was used for the measurements. The samples to be analyzed were cut from this tubing using an electrical discharge wire cutting process, to eliminate damage from mechanical processing. The samples were subsequently cleaned chemically to remove particles adhering to the surface from the cutting process, which used a brass wire. 
The measured profiles were Fourier-transformed and the inductance $\mathfrak{L}$ per unit length of the pipe was calculated using Eq. (8.51). Because this inductance is inversely proportional to the pipe radius $b$, a convenient quantity is the product $\mathfrak{L} b$, which does not depend on the pipe radius and characterizes the intrinsic properties of the surface. The computed value of this product was found to be between $3 \times 10^{-4} \mathrm{pH}$ and $5 \times 10^{-4} \mathrm{pH}$.

These values should be compared with the impedance budget for the LCLS beam. For the nominal parameters of the LCLS: beam charge $1 \mathrm{nC}, \sigma_{z}=20 \mu \mathrm{m}$, undulator length $112 \mathrm{~m}$, and final beam energy $E=14.3 \mathrm{GeV}$, one finds that the requirement that the relative energy spread $\delta E_{\text {rms }} / E$ generated by the wake be less than $0.05 \%$ is met for $\mathfrak{L}<1.6 \mathrm{pH} / \mathrm{m}$. For the vacuum pipe radius $b=2.5 \mathrm{~mm}$ the tolerance on the product $\mathfrak{L} b$ is $(\mathfrak{L} b)_{\mathrm{tol}}=4 \times 10^{-3} \mathrm{pH}$. The measured value of the impedance is seen to be almost an order of magnitude smaller than the tolerance.

It should be emphasized here that the above results are based on two assumptions that are not completely fulfilled for the LCLS. First, a Gaussian beam distribution was assumed. As detailed simulations show [58], for the LCLS the bunch shape more resembles a rectangular than a Gaussian shape. Second, Eq. (8.51) used for the calculation of the inductance, was derived in the limit $\sigma_{z} \gg g$, which, as roughness measurements indicate, is not satisfied.

The theory for the case $\sigma_{z}<g$, which is more pertinent to the measurements, was developed in [14]. In this theory the roughness was treated as a sinusoidal wall corrugation with the amplitude $h_{0}$ and the period $2 \pi / \kappa$. The amplitude $h_{0}$ of the corrugation is assumed much smaller than the period, $h_{0} \kappa \ll 1$, which is a requirement of the small-angle approximation. Such a corrugation qualitatively simulates a rough surface with parameter $g \sim \kappa^{-1}$ and the rms height of the bumps of the order of $h_{0}$.

This theory shows, that when using the long-bunch approximation $\left(\sigma_{z} \kappa \gg 1\right)$ in the regime where $\sigma_{z} \kappa<1$, the wake is overestimated by a factor of $\left(\sigma_{z} \kappa\right)^{-1 / 2} \sim\left(g / \sigma_{z}\right)^{1 / 2}$. For this reason, the result of Ref. [25] should be considered as an upper boundary for the roughness impedance.

Using the result of Ref. [35], the wake for a rectangular bunch shape, $\rho(s)=1 / l_{z}$ for $0<s<l_{z}$ can be calculated. The parameters used in the calculation are: beam charge $1 \mathrm{nC}, h_{0}=.28 \mu \mathrm{m}$ (corresponding to the rms roughness of $0.2 \mu \mathrm{m}$ ), $g=2 \pi / \kappa=100 \mu \mathrm{m}, L=112 \mathrm{~m}, \mathrm{E}=14.3 \mathrm{GeV}, b$ $=2.5 \mathrm{~mm}$. The average energy loss for the distribution shown in Figure 8.33 is $4.5 \times 10^{-5}$ and the rms energy spread is $2 \times 10^{-5}$. In addition to the mechanism of the wake generation described above involving interaction with short-wavelength waves, $\lambda \leq g$, there is another contribution to the wake, which was first pointed out by A. Novokhatski and A. Mosnier [26]. It comes from a relatively low-frequency synchronous mode with $\lambda \gg g$. 


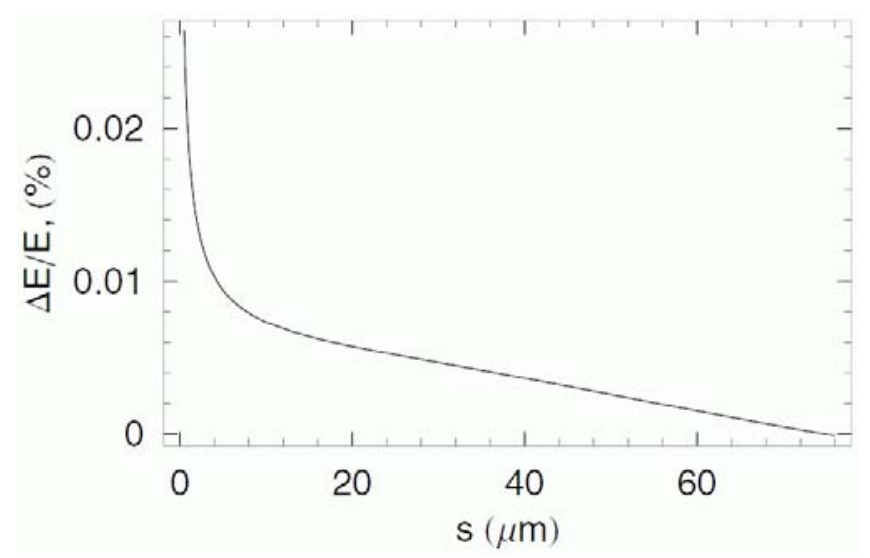

Figure 8.33 The relative energy loss of the LCLS beam at the end of the undulator as a function of position within the bunch.

The properties of the synchronous mode in the case of rectangular corrugation of the wall were studied in Ref. [27]. In this paper, the wall roughness was modeled by axisymmetric periodic steps on the surface of height $\delta$, width $g$, and period $p$. All three parameters were assumed much smaller than the pipe radius $b$. The model gives for the frequency $\omega_{0}$ of the mode

$$
\omega_{0}=c \sqrt{\frac{2 p}{\delta b g}},
$$

and for the longitudinal wakefunction of the point charge

$$
w(s)=\frac{Z_{0} c}{\pi b^{2}} \cos \left(\omega_{0} s / c\right)
$$

Surprisingly, the amplitude of the wake in this approximation does not depend on the roughness properties at all. These results however are valid if $k p \ll 1$. Eq. (8.53) shows that when $\delta$ becomes very small, the parameter $k$ increases and eventually $k p$ becomes comparable to unity. Hence, this model becomes invalid in the limit $\delta \rightarrow 0$. The results of computer simulations that confirm the predictions of this model can be found in Refs. [36,37].

To take into account the effect of the shallowness of the roughness a different model was developed in Ref. [28]. In this model the roughness was treated as a sinusoidal perturbation of the wall with $h_{0} \kappa \ll 1$. It was found that, indeed, under certain conditions, a low-frequency synchronous mode with $\lambda \kappa \gg 1$ can propagate in this system. The longitudinal wake generated by this mode is given by

$$
w(s)=\frac{2 Z_{0} c}{\pi b^{2}} U \cos \left(\omega_{0} s / c\right)
$$

where the dimensionless factor $U$ and the frequency of the mode $\omega_{0}$ depend on the parameter $r \equiv h_{0} \sqrt{b \kappa^{3}} / 2$. The plot of these two functions is shown in Figure 8.34. In the limit $h_{0} \rightarrow 0$ the frequency $\omega_{0}$ approaches $\kappa c / 2$, and $U \approx r^{4} / 32$. For large values of $r, \omega_{0} \approx 2 c / h \sqrt{b \kappa}$ and $U \rightarrow 1 / 2$. 
To estimate the wake, realistic parameters of roughness, i.e. $h_{0}=0.28 \mu \mathrm{m}$ (corresponding to the rms roughness of $0.2 \mu \mathrm{m}$ ), $g=2 \pi / \kappa=100 \mu \mathrm{m}$, and $b=2.5 \mathrm{~mm}$, are used. This gives a value of $r \equiv h_{0} \sqrt{b \kappa^{3}} / 2=0.11$. The corresponding loss-factor parameter is

$$
U \approx 4.5 \cdot 10^{-6},
$$

which indicates that the effect of the wake in this regime will be negligibly small.

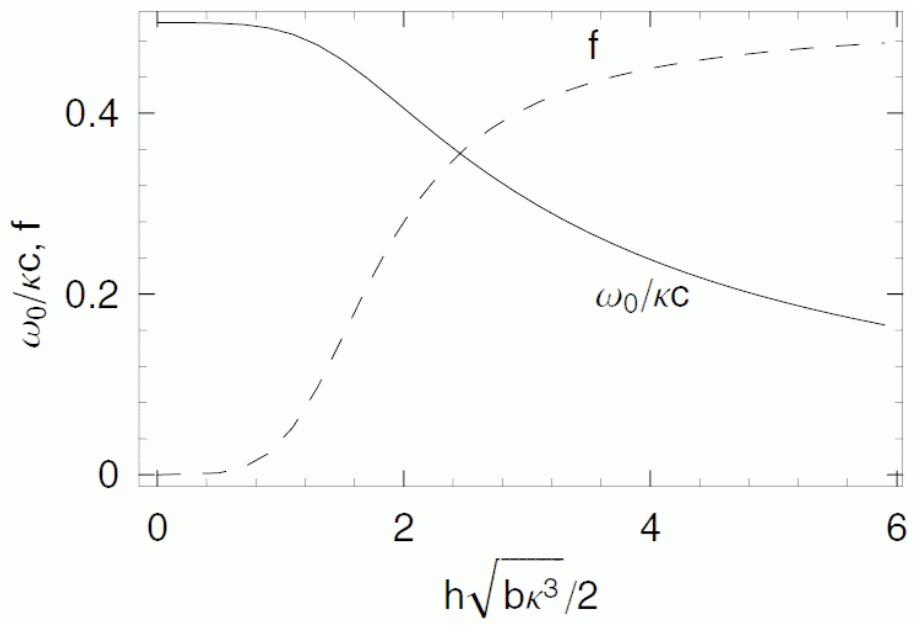

Figure 8.34 Synchronous mode dispersion relation.

It is important to emphasize here that the wakefield generated by the roughness is very sensitive to the geometry of the surface profile. The models which do not take into account the large aspect ratio of the real roughness - the ratio of the characteristic size along the surface (correlation length) and the typical height of the bumps - tend to overestimate the impedance and lead to very tight tolerances for the surface smoothness. The latest models that include the large aspect ratio into consideration predict much smaller impedance which is below the tolerable level for the LCLS undulator, if the typical height $\sim 100 \mathrm{~nm}$ and $g \sim 100 \mu \mathrm{m}$. The surface measurements [25] show that roughness with such characteristics can be achieved in a pipe with a good surface finish.

\subsection{Ion Effects}

In this section the number of ions generated during a bunch passage in the $121 \mathrm{~m}$ long undulator line of the LCLS x-ray FEL is calculated, emittance dilution caused by these ions is discussed, and the acceptable vacuum pressure is estimated.

\subsubsection{Introduction}

This section investigates ion production by the beam and by the synchrotron-radiation photons during a bunch passage in the LCLS undulator [38], and three different mechanisms of emittance dilution induced by these ions. The acceptable vacuum pressure for FEL operation is estimated from the calculated emittance growth. 


\subsubsection{Ionization Processes}

There are three conceivable mechanisms by which ions can be created:

\subsubsection{Ionization by the beam.}

A typical ionization cross-section for a $15 \mathrm{GeV}$ electron beam and carbon monoxide or nitrogen gas is of the order of 2 Mbarn (the ionization cross section for hydrogen molecules would be approximately 10 times smaller). The 2-Mbarn cross section translates into an ion line density of about

$$
e_{i o n}\left[\mathrm{~m}^{-1}\right] \approx 5 N_{b} p[\mathrm{nTorr}]
$$

at the end of the bunch, or 320 ions per meter for a pressure of $10 \mathrm{nTorr}$ and $\mathrm{N}_{\mathrm{b}}=6.310^{9}$ electrons per bunch.

\subsubsection{Ionization by incoherent synchrotron radiation.}

The ionization cross-section of $8-\mathrm{keV}$ photons for typical elements is about 100 barn [39]. Even though the number of photons at $1.5 \AA$ is three orders of magnitude higher than the number of electrons, this cross section is so much smaller than the collision-ionization cross section that the photoionization at Angstrom wavelengths can be neglected in comparison.

In addition to the photons emitted at the first (and higher) FEL harmonic wavelengths, a broad spontaneous photon spectrum extends to much lower energies, where the photoionization cross section is considerably higher. Below about $100 \mathrm{eV}$ the photoionization cross section becomes comparable to, and may even exceed by up to a factor of 5 , the cross section for collisional ionization.

From Figure 6 in Ref. [38], illustrating the spontaneous photon spectrum, and from Figure 8.35, showing its low-energy part, it is estimated that, at the end of the undulator, there are about $6 \times 10^{10}$ photons per bunch with energies below $1 \mathrm{keV}$, and fewer than $5 \times 10^{9}$ photons whose energy is below $100 \mathrm{eV}$. Thus, the number of low-energetic photons is about equal to the number of electrons in the bunch. With an rms opening angle of 10-20 $\mu \mathrm{rad}$ for the spontaneous radiation (and an even wider opening angle at low photon energies), the photoionization processes occur on average far away from the beam orbit. Therefore, considering the small number of low-energy photons, the ions are assumed to be produced by photo-ionization form a diffuse halo, whose effect on the beam is negligible compared with that of the much denser ion cloud produced by collisional ionization inside the beam. 


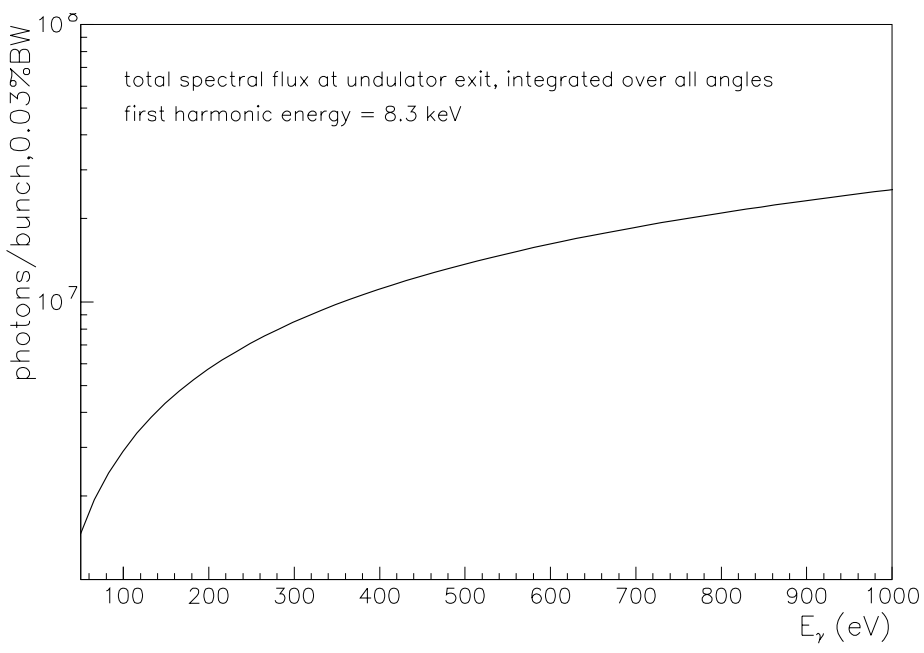

Figure 8.35 Number of photons per $0.03 \%$ frequency interval and per bunch passage at the end of the LCLS undulator; the spontaneous flux increases linearly along the undulator.

\subsubsection{Tunneling ionization in the coherent laser field.}

Up to frequencies of the order

$$
\omega_{t}=c e E / \sqrt{2 m_{e} c^{2} I}
$$

the tunnel effect is determined simply by the instantaneous value of the electric field [40,41]. In Eq. (8.58), the parameter $\mathrm{E}$ is the electric field, $\mathrm{m}_{\mathrm{e}}$ the electron mass, and $\mathrm{I}$ the ionization potential. The peak electric field of the laser pulse can be roughly estimated from the equation

$$
\hat{E} \approx\left(\frac{2 N_{\gamma} h v}{(2 \pi)^{3 / 2} \varepsilon_{0} \sigma_{x} \sigma_{y} \sigma_{z}}\right)^{1 / 2}
$$

and is found to be about $85 \mathrm{GV} / \mathrm{m}$. Somewhat arbitrarily using $I \approx 20 \mathrm{eV}$, the threshold frequency is $\omega_{t} \approx 10^{16} \mathrm{~s}^{-1}$, which is very low compared to the FEL frequency $\omega \approx 10^{19} \mathrm{~s}^{-1}$. This means that the standard formula for static tunneling ionization does not apply here. To determine if the coherence of the FEL $x$-rays is important, the photon density is calculated as

$$
n_{\gamma} \approx \frac{N_{\gamma}}{(2 \pi)^{3 / 2} \sigma_{x} \sigma_{y} \sigma_{z}} \approx 7.2 \times 10^{24} \mathrm{~m}^{-3} \text {, }
$$

which implies that in a sphere with a radius equal to the Bohr radius $\mathrm{a}_{0}\left(\mathrm{a}_{0} \approx 0.5 \AA\right)$ on average there are only $1.2 \times 10^{-6}$ photons at any given time during the pulse. It is thus legitimate to consider the photons as incoherent [42], in which case, as seen under point 2, their contribution to the ionization is insignificant. 


\subsubsection{Emittance Dilution}

Ions could dilute the bunch emittance in various ways: first, the ions induce a tune shift across the bunch which could lead to filamentation and to an effective increase in the transverse emittance; second, the electrons or, third, the ions generated by the bunch head can excite the bunch tail and cause a beam break-up instability.

Pessimistically assuming that all electrons originating in the ionization process are dispersed and lost before the end of the bunch (using this assumption, which is not fulfilled for the LCLS, the actual tune shift will be overestimated), one can estimate the ion-induced shift in betatron phase advance between head and tail of the bunch at the end of the undulator:

$$
\Delta \psi_{\beta_{x y}} \approx \frac{\beta_{x, y} r_{e} \lambda_{i o n} L_{u}}{\gamma \sigma_{x, y}\left(\sigma_{x}+\sigma_{y}\right)}
$$

Using an ion line density $\lambda_{\text {ion }}$, as expected for collisional ionization, Eq. (8.57), the phase shift is $\Delta \psi_{x, y} \approx 4 \times 10^{-6}$ rad for $1 \mathrm{nTorr}$ and $4 \times 10^{-4}$ rad for $100 \mathrm{nTorr}$. Significant emittance growth due to filamentation would be expected only for an average pressure exceeding $100 \mu$ Torr, for which the phase shift approaches $1 \mathrm{rad}$.

Since, different from the situation in most other accelerators, the bunch length in the LCLS is same order than the transverse beam size, the electrons do not escape from the bunch during its passage, but the electrons generated by the head will still affect the trailing particles. The resulting emittance growth can be estimated from a first-order perturbation expansion, in analogy to the treatment in [43]:

$$
\Delta \varepsilon_{y} \approx \frac{\pi^{2} N_{b} \lambda_{i o n}^{2} r_{e}^{3} \sigma_{z} L_{u}^{2} \hat{y}^{2} \beta_{y}}{54 \sqrt{2 \pi} \gamma^{2} \sigma_{y}^{3}\left(\sigma_{x}+\sigma_{y}\right)^{3}}
$$

where $\hat{y}$ describes the amplitude of an initial vertical perturbation of the form $y_{b}{ }^{0}(s, z)=$ $\hat{y} \cos (s / \beta+\phi) \sinh \left(\omega_{i} z+\theta\right)$ with $\omega_{i} \equiv\left[4 N_{b} r_{e} / 3 \sqrt{2 \pi} \sigma_{x} \sigma_{y}\left(\sigma_{x}+\sigma_{y}\right)\right]^{1 / 2}$, where $s$ is the longitudinal position along the beam line, and $z$ denotes the longitudinal position of a particle with respect to the bunch center. Inserting numbers, $\omega_{i} \sigma \approx 0.3$. Exactly the same expression with the subindices $x$ and $y$ interchanged applies to the horizontal case, and, by symmetry, it yields the same emittance growth. Inserting numbers and assuming an ion density as in Eq. (8.57), Eq. (8.62) is rewritten as

$$
\Delta\left(\gamma \varepsilon_{y}\right)[\mathrm{m}] \approx 4 \times 10^{-19}\left(\frac{\hat{y}}{\sigma_{y}}\right)^{2}(p[\text { nTorr }])^{2} .
$$

For a huge perturbation, $\hat{y} \approx 10 \sigma_{y}$, one finds that the emittance growth becomes significant when the pressure approaches $10^{-4}$ Torr, which is three orders of magnitude higher than the anticipated operating pressure. 


\subsubsection{Conclusion}

In this section, the number of ions generated during a bunch passage in the LCLS undulator and their effect on the beam emittance has been established. The ionization of the residual gas due to the coherent x-rays and, from the viewpoint of beam dynamics, also the ionization due to the lower-energetic spontaneous photons were found to be insignificant compared with the collisional ionization by the beam. There is not a tight tolerance on the vacuum pressure in the LCLS undulator. Even a pressure as high as $1 \mu$ Torr would still appear to be perfectly acceptable.

\subsection{Electron Beam Diagnostics}

\subsubsection{Performance Requirements}

For proper operation of the LCLS free electron laser, the electron beam trajectory through the undulator segment must deviate from straightness by no more than 2 microns rms over approximately a 10 -meter field gain length. The goal for beam trajectory measurement stability is required to be better than 1 micron rms for at least an 8 hour time period. Imaging diagnostics must be able to resolve the transverse beam size as small as 30 microns rms. These requirements are to be met using $0.1-1.0 \mathrm{nC}$ of charge per pulse with a repetition rate as high as $120 \mathrm{~Hz}$. The vacuum chamber has a full aperture of $5 \mathrm{~mm}$.

\subsubsection{Overview}

The LCLS undulator will consist of an array of thirty-three 3.4-meter-long undulator segments separated by short breaks containing diagnostics and permanent magnet focusing elements. The thirty-three segments are arranged in sets of three, forming eleven half-cells, with somewhat longer breaks between half-cells to allow for additional diagnostics. Approximately 15 $\mathrm{cm}$ will be available for electron beam diagnostics at each transition section.

Shown in Table 8.9 is a summary of the electron beam diagnostics to be used in the undulator segment. A total of 48 beam position monitors (BPMs) are required between the undulator segments and at the extreme upstream and downstream ends of the undulator. Two BPMs will be used in the breaks between half-cells. Optical transition radiation imaging diagnostics will be provided in these longer breaks at every third diagnostic station, in addition to being located at three successive locations upstream of the undulator for a three-screen emittance measurement. A complementary measure of emittance will be provided by a set of four wire scanners located upstream of the undulator. Working in combination with a set of 33 Cherenkov detectors, the wire scanners will provide a very precise measure of the beam size averaged over many pulses. In addition these wire scanners will assist in defining the average incoming electron beam trajectory (position and angle).

The Cherenkov detectors are sensitive to hard gamma and high energy electrons but not the $\mathrm{x}$-rays generated in the FEL process. Located at all diagnostics stations, they detect electron beam losses generated by the wire scanners for emittance measurement, or as a result of beam 
missteering. A current monitoring toroid will be placed at the entry and exit of the entire array, providing information on beam intensity and transfer efficiency.

Table 8.9 Undulator electron beam diagnostics

\begin{tabular}{lcll}
\hline Type & Quantity Location \\
\hline Beam position monitor & 48 & All stations \\
\hline Optical transition radiation (OTR) imaging diagnostic & 13 & Every third station \\
\hline Wire scanners & 4 & Upstream of Undulator segments \\
\hline Cherenkov Detectors & 33 & All stations \\
\hline Current Monitors & 2 & Upstream and downstream of undulator \\
\hline
\end{tabular}

\subsubsection{Undulator Beam Position Monitors}

Conventional button-type or stripline-type pickup electrodes combined with similarly conventional electronics, e.g. amplitude to phase conversion, can provide sub-micron single shot beam position measurement resolution when using small beam apertures. Two micron rms resolution with a $4 \mathrm{~mm}$ aperture has been demonstrated at the Advanced Photon Source (APS) low energy undulator test line (LEUTL) [44]. Because the sensitivity of conventional button- or stripline-type beam position monitors (BPMs) scales with vacuum chamber aperture [45], electronics stability need only be better than about one part in a thousand in the determination of "difference over sum" to achieve micron-scale measurement stability with a 5-mm aperture.

While position monitoring resolution at the micron scale is straight forward, even for single shot diagnostics, absolute accuracy becomes extremely difficult below the 50-micron scale, owing to fabrication and assembly tolerances, and survey and alignment limitations. Separate Anocast ${ }^{\circledR}$ piers that provide better than 1-micron stability support the modules. The positions of the BPM modules are locally adjustable for alignment purposes.

Because absolute accuracy is difficult, it will be important to make use of beam-based alignment in the determination of position monitor offsets relative to nearby focusing elements. It is expected that this determination can be made at the 2-micron level or better using a procedure expected to take a few of hours of machine time.

In order to assure the long-term stability of position measurement, the combination of standard button-style pickup electrodes with a cavity-based pickup in a compact assembly is envisaged (Figure 8.36). The strengths of the cavity-based position monitor are that it has a very stable and reproducible electrical center, very high resolution (10s of $\mathrm{nm}$ ) with very small amounts of beam [46], and that it can be very accurately machined, down to the micron-level [47]. Disadvantages of the cavity BPM technique are the need for a reference phase signal for determining the sign of the position measurement, in addition to possible problems associated with wakefield effects on the beam. Because a higher order mode conveys the position information, the rejection of the fundamental mode frequency in the electronics places a fundamental limitation on position determination, although this limitation is expected to be at or 
below the 1-micron level. The raw position signal from a cavity BPM is proportional to beam charge, so that a normalization procedure will be necessary to extract position information.

\section{Compact RF Beam Position Diagnostic}

Conceptual Design (Cross section)

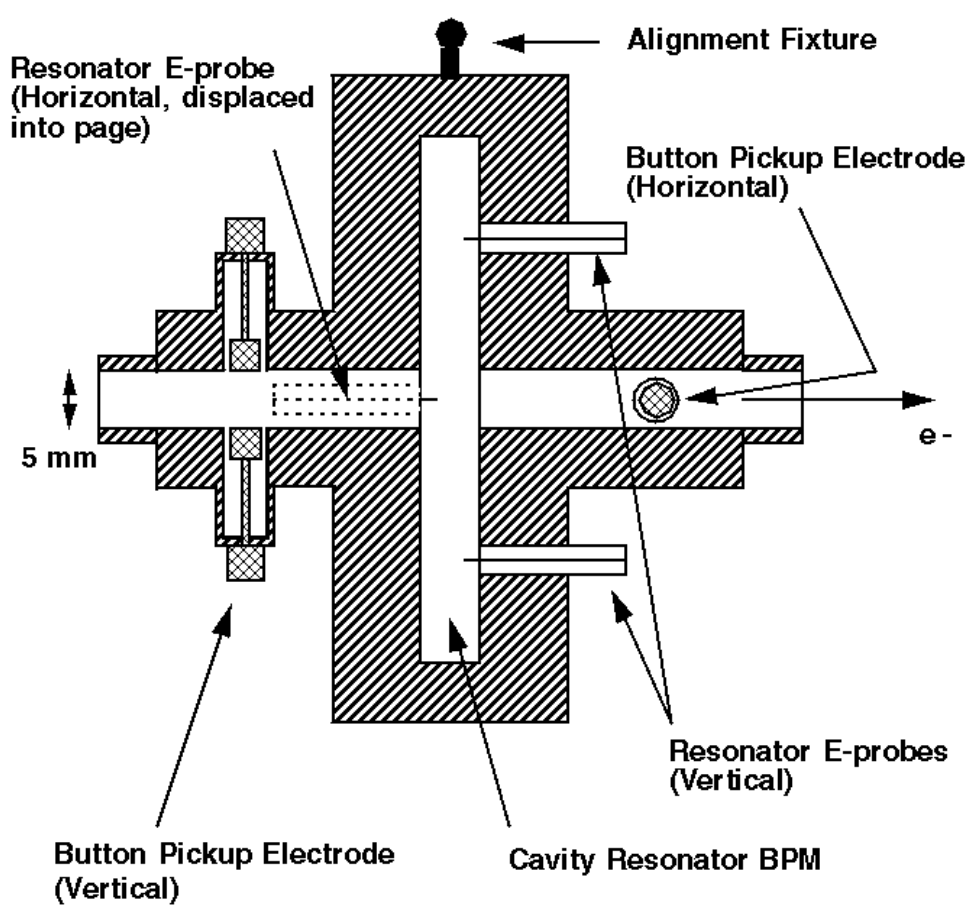

Figure 8.36 Conceptual design for a combined button- and cavity-type position monitor

Recent research at Stanford, supporting the NLC project, has produced an X-band cavityBPM design, which solves the fundamental mode problem by a clever arrangement of the output waveguides [48]. In this design, a second cavity is also integrated in the design with its fundamental mode frequency set to match the position sensitive cavity's mode frequency. This second cavity can then be used to extract the phase (sign) information, in addition to providing charge normalization.

The incorporation of conventional button- and cavity- type pickup electrodes in the same assembly affords several advantages over either system alone. It is relatively simple to calculate the sensitivity (e.g. volts per micron) of a conventional button-type position monitor. The analogous sensitivity for the cavity monitor, though potentially much higher, depends on such things as cavity Q, waveguide coupling efficiency, etc., and as such may not be as easily determined a priori. A cross-calibration of the two with beam immediately resolves any uncertainties in the determination of length scales. Similarly, the cavity monitor has a very easily determined and highly accurate electrical center, reproducible at the sub-micron level, while conventional electronics have always performed poorly in this regard. By steering to the cavity null and recording the readbacks from the conventional electronics attached to the buttons, uncertainty in electrical center stability of the conventional electronics is significantly reduced. 
Using this "belt and suspenders" approach will increase confidence in the electron beam trajectory determination, in addition to simplifying the electronics for the cavity, since no microwave phase determinations will be needed to determine the sign of the position.

A fundamental limitation on the ability to align the beam along the undulator segments will be mechanical stability of components arising from thermal drift, ground settling, etc. Even with careful design, 10 micron-scale component position drifts over 24 to 48 hour periods should be expected. While a careful initial alignment $(<50$ microns absolute) is important, the key to success will be to have a robust beam-based alignment algorithm in place for the relative determination of magnet and diagnostic centroids.

\subsubsection{Undulator Optical Transition Radiation (OTR) Electron-Beam Profile Monitors}

It will be critical to the ultimate understanding of the LCLS SASE physics for the electronbeam transverse size and profile to be monitored between the undulator segments. The transverse size of $\sigma_{x, y}=30 \mu \mathrm{m}$ for a $14.5 \mathrm{GeV}$ beam involving a $1 \mathrm{nC}$ bunch is a challenge. It is also required that the bremsstrahlung radiation generated by the electron beam's interaction with the radiation converter be minimized. Based on experiences gained at several laboratories, the use of optical transition radiation (OTR) converter screens/foils made of low-z, ultra thin foils as a minimally intercepting technique is planned. Such thin foils should generate lower levels of bremsstrahlung than the crystals proposed for $\mathrm{x}$-ray beam diagnostics.

Several successful examples of OTR experiments can be cited. Beam sizes of $30 \mu \mathrm{m}(\sigma)$ for a $600-\mathrm{MeV}$ beam at APS using an aluminum mirror surface as converter [49] have been observed. In addition, preliminary images at $7-\mathrm{GeV}$ in a transport line at APS have been obtained. At Jefferson Lab's CEBAF, 0.8- $\mu \mathrm{m}$ thin aluminum foils have been used with a 4-GeV, $200 \mu \mathrm{A}$ beam for beam sizes of $50 \mu \mathrm{m}(\sigma)$ [50]. Experiments have also been done at SLAC with a 30$\mathrm{GeV}$ beam as reported at PAC '99 [51]. More recently in the APS SASE FEL project, OTR imaging from a flat mirror as well as a $6-\mu \mathrm{m}$ thick foil have been used between undulator segments for the $217-\mathrm{MeV}$ beam. Total system resolution has been limited by the camera optics employed, not the OTR mechanism.

Some brief background on the OTR mechanism is in order [52]. Optical transition radiation is generated at the boundary of media with different dielectric constants, such as vacuum and a metal, as the charged particle transits the boundary. It is a surface phenomenon and therefore has no volume effect as do scintillators or known saturation effects. One might simplistically describe this process by the formation of an image charge in the foil as the charged particle approaches the boundary. As the charge reaches the boundary, the charge and its image basically act as a collapsing dipole, and a burst of broadband radiation is emitted. As shown in Figure 8.37 there is both forward OTR and backward OTR relative to the e-beam direction. If the foil surface is inclined at $45^{\circ}$ to the beam direction, then the backward OTR is emitted in an annular cone around the angle of specular reflection, $90^{\circ}$ in this case. Although the azimuthal intensity peak is at $1 / \gamma$, where $\gamma$ is the Lorentz factor, OTR is emitted into large angles as well. Most of the total

U N D U L A T O R 8-69 
power is at angles greater than $1 / \gamma$. For this reason, the OTR is not diffraction-limited at $1 / \gamma$, but limited only by the aperture of the collecting optics for a given wavelength. Figure 8.38 shows a schematic of the OTR angular distribution features at $40 \mathrm{MeV}$. For a wide-aperture optical system, sub-10 $\mu \mathrm{m}$ resolution is expected. At APS, B. Yang has recently tested in the lab a camera-lens package with sub-10 $\mu \mathrm{m}$ resolution for the chicane beam size monitor.

\section{OPTICAL TRANSITION RADIATION PATTERNS}

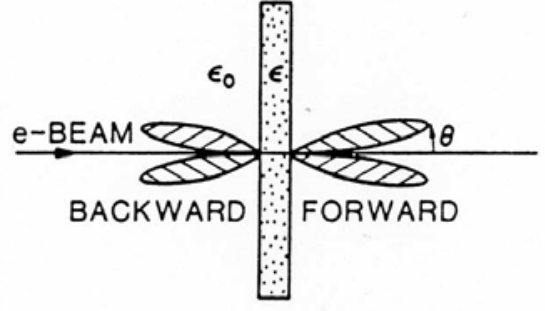

a.) NORMAL INCIDENCE

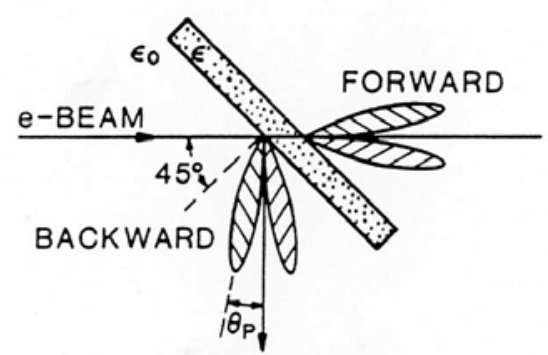

b.) OBLIQUE INCIDENCE $\left(\phi=45^{\circ}\right)$

CHERENKOV RADIATION PATTERN $\left(\theta \sim 46^{\circ}\right)$

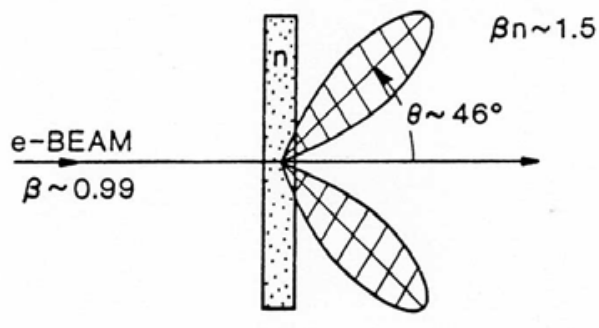

Figure 8.37 A schematic of forward OTR and backward OTR generation for interfaces normal to the beam (left) and at $45^{\circ}$ to the beam (right). The dielectric constants for vacuum $\left(\varepsilon_{0}\right)$ and the medium $(\varepsilon)$ are indicated.

In addition to the beam profile function, one may take advantage of the laser-like angular distribution of OTR to measure e-beam angle. As has been done in previous experiments in the 1980 's, the camera-lens system can be set so the sensor is at the focal plane of the lens. In this configuration far-field or angular distribution information is obtainable as schematically illustrated in Figure 8.39. Since in this case $1 / \gamma \approx 35 \mu \mathrm{rad}$, the optics and distances would have to be chosen appropriately. Alternatively, at a $10-\mathrm{m}$ distance the angular divergence contribution from OTR would give a projected spot size of $\sim 700 \mu \mathrm{m}$ with no lens. The centroid of such an image is related to beam direction and angle. An angle sensitivity of several micro-radians should be possible. 


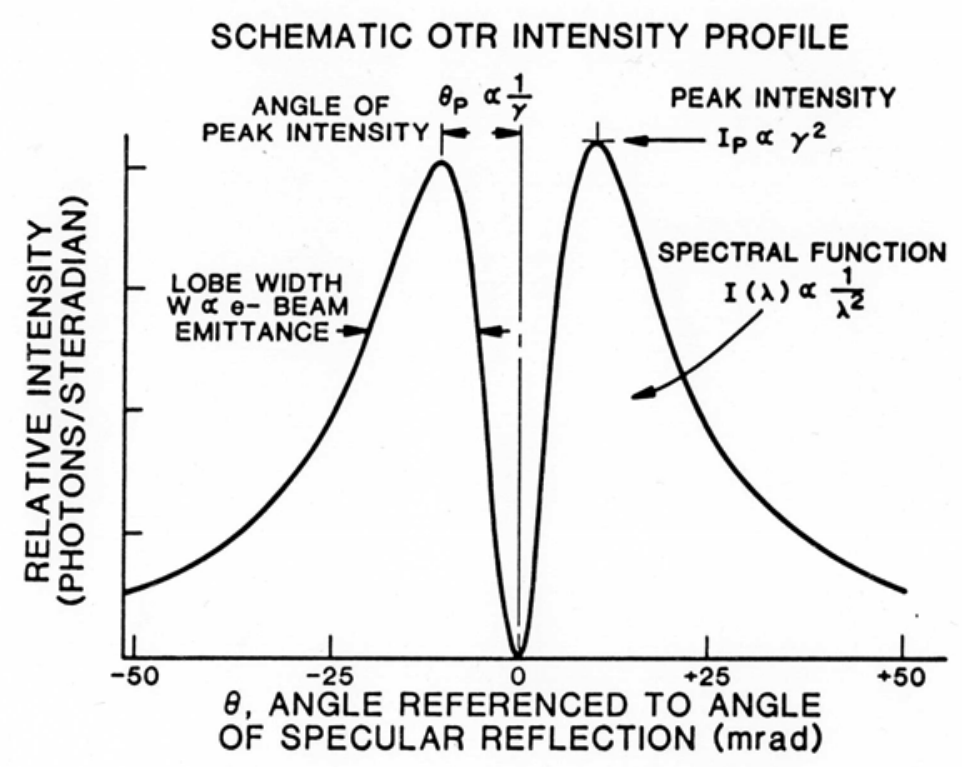

Figure 8.38 A schematic of the OTR intensity profile in the angular distribution pattern. The angle $\theta$ is referenced to the angle of specular reflection and the peak is at $1 / \gamma$.

Some tests on foil survivability are needed. An engineering design for stretching the thin foil across an aperture of few-mm diameter is also needed. In addition, another foil that is opaque to visible synchrotron radiation will be installed upstream of the $45^{\circ}$ foil. Extensions of OTR techniques to $\mathrm{x}$-ray transition radiation (XTR) or diffraction radiation (DR) techniques may provide some trades on spatial resolution and nonintercepting features, respectively. A schematic of the diagnostics station is shown in Figure 8.54. 


\section{OPTICAL RAY DIAGRAM FOR OTR IMAGING}

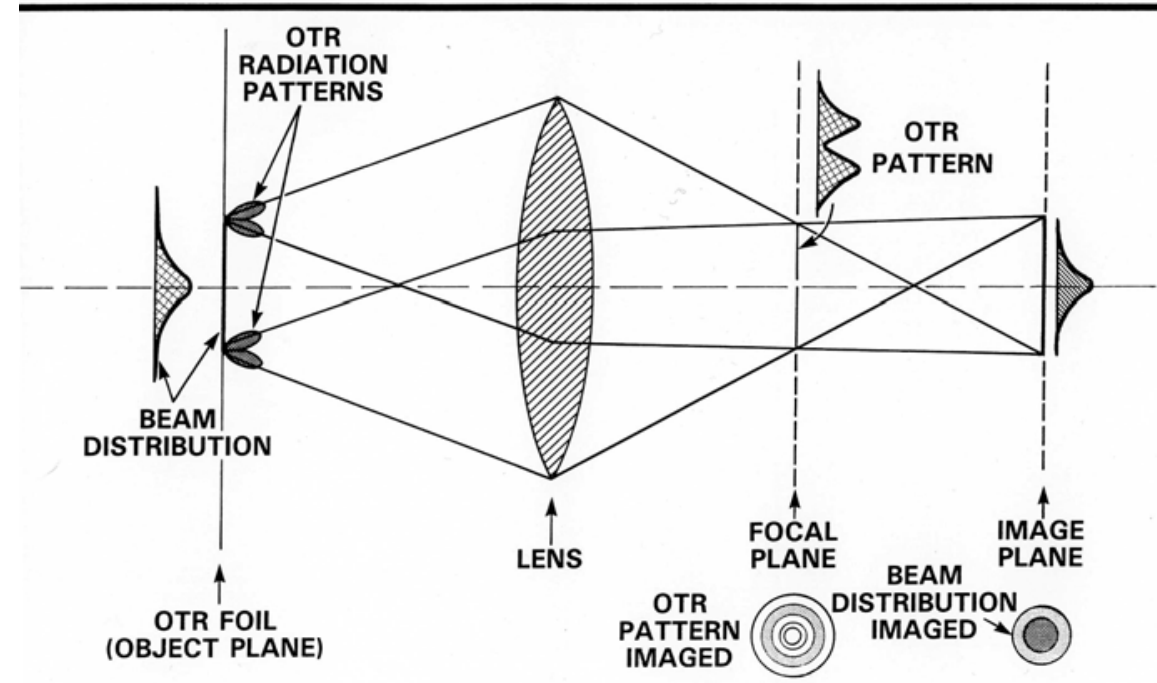

Figure 8.39 An optical ray diagram for imaging of OTR when the sensor is positioned at the focal plane and image plane. Such imaging provides the OTR angular distribution pattern or the beam profile, respectively.

\subsubsection{Cherenkov Detectors}

A series of Cherenkov detectors will be placed at all diagnostics stations between undulator segments. These devices are similar to those used at the PEP-II project as beam loss monitors [53]. These devices are quite simple conceptually, involving a small piece of fused silica glass, which emits Cherenkov radiation when traversed by high energy charged particles. A miniature photomultiplier tube is used to amplify these small pulses, which are then transmitted to data acquisition electronics, e.g. a gated integrator followed by a digitizer. To prevent FEL x- rays from corrupting the bremsstrahlung gamma ray signal, the fused silica pellet is embedded in a lead-lined housing such that only very high energy photons can penetrate. Refinements to the PEP-II mechanical design and data acquisition have been carried out recently at the APS [54].

\subsubsection{Current Monitoring Toroids}

High quality pulsed current monitoring toroids are commercially available, together with front end electronics, if so desired. It is important to measure the charge entering and exiting the undulator to provide information for normalization of data sets and to tune for maximum transport efficiency. It should be straightforward to monitor a nominal $1 \mathrm{nC}$ pulse with a few percent accuracy shot to shot. Data acquisition is provided. 


\subsection{Beam-based Alignment}

\subsubsection{Undulator Beam-Based Alignment}

The electron trajectory within the LCLS undulator needs to be straight to a high degree of accuracy so that the 1.5 - $\AA$ radiation grows efficiently over each gain length. For the LCLS, this condition requires a trajectory straightness of a few microns over a $\sim 10$-meter length. This level is very difficult to achieve with component survey alignment techniques. For this reason, the final alignment will rely on empirical beam-based alignment, which makes use of beam position monitor (BPM) readings as a function of large, deliberate variations of the electron energy. The BPM measurements at various energies are analyzed and then converted to 1) quadrupole magnet transverse position offsets, 2) BPM readback offset corrections and 3) adjustments of the incoming beam position and angle at the undulator entrance (initial launch conditions). The alignment procedure is repeated three times in succession for the initial machine startup, and then one pass of the procedure is reapplied approximately once per month, as necessary. Between these infrequent applications, a fine steering technique will be used for daily trajectory control, and a fast feedback system will maintain the trajectory over the time scale of a few pulses. This section primarily discusses the most involved alignment procedure, which is applied during the initial machine commissioning period. The effects of various errors are included in a full simulation of the alignment procedure.

\subsubsection{Introduction}

The readback $\mathrm{m}_{\mathrm{i}}$ of the $i^{\text {th }} \mathrm{BPM}$, which measures the centroid of the transverse position of the electron bunch at location $s_{i}$ along the beamline, can be written as

$$
m_{i}=\sum_{j=1}^{i} \theta_{j} C_{i j}-b_{i}
$$

where $\theta_{j}$ is the kick angle at point $j(<i)$ due to a transversely misaligned quadrupole magnet or undulator pole field error upstream of BPM- $i, C_{i j}$ is the transfer coefficient which maps a beam angle at point $j$ to a position at point $i$, and $b_{i}$ is the readback offset (mechanical misalignment and/or electrical bias) of the $i^{\text {th }}$ BPM. This is described graphically in Figure 8.40 where the BPMs are shown as circles. The kick angles in the figure are represented as dipoles, however they are completely equivalent to either quadrupole magnets with transverse displacements and/or field strength errors of the undulator poles. The quadrupole focusing within the undulator is not explicitly shown in the figure, but it is represented mathematically in the transfer coefficients, $C_{i j}$. The initial launch conditions are ignored for now (more on this below).

Since the kick angles $\theta_{j}$ are inversely proportional to beam momentum $p$, whereas the BPM offsets $b_{i}$ are independent of momentum, variations of the beam energy (momentum) can be used to measure both parameters simultaneously. This is clear by substituting a dipole field error, $\Delta B_{j}$, (equivalent to a quadrupole misalignment) for $\theta_{j}$ and explicitly showing the momentum dependence of Eq. (8.64).

U N D U L A T O R 8-73 


$$
m_{i k}=\frac{1}{p_{k}} \sum_{j=1}^{i} e \Delta B_{j} \ell C_{i j}\left(p_{k}\right)-b_{i}
$$

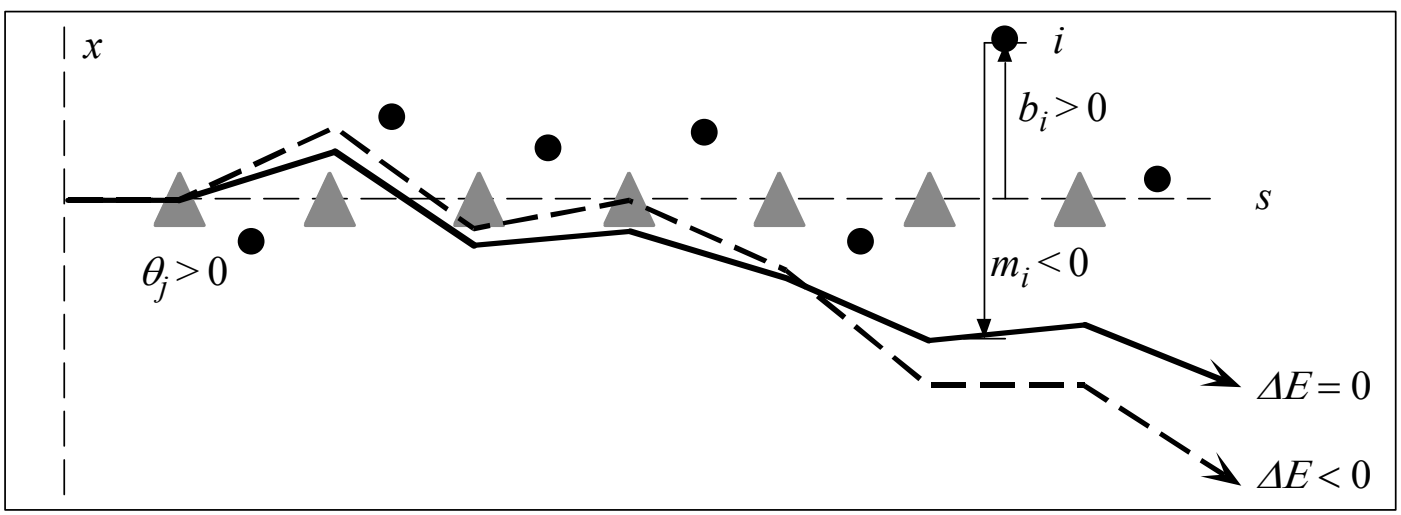

Figure 8.40

Schematic of electron trajectory for nominal beam energy $(\Delta E=0)$ and for a lower energy $(\Delta E<0)$. Undulator-induced oscillations of central traj ectory not shown.

Here $\ell$ is the dipole length, $e$ is the electron charge, and the subscript $k$ on momentum and BPM readback is introduced to indicate the different values of beam momentum. The transfer coefficients, $C_{i j}\left(p_{k}\right)$, also include a momentum dependence, except in the case of no focusing. As a simple example, this no-focusing case is graphically represented in Figure $\mathbf{8 . 4 1}$ as a linear dependence of $m_{i}$ on $1 / p$ plus an offset, $b_{i}$. The measurement then reduces to a line-fit where the slope is equal to the summation term in Eq. (8.65) and the offset is equal to $-b_{i}$. The general case, including quadrupole focusing, is similar but does not appear as the simple line-fit shown.

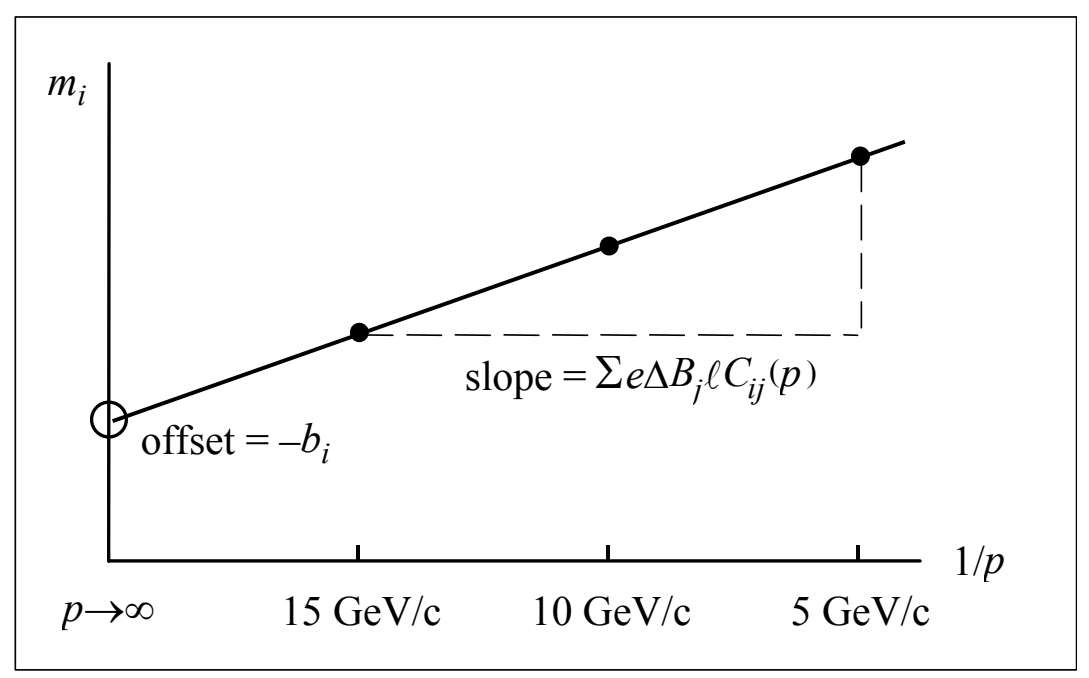

Figure 8.41 Graphical representation of Eq. (8.65). The BPM readback is linear with $1 / p$, has a slope equal to the summation term, and an offset equal to $-b_{i}$. The plot is linear only if the $C_{i j}$ elements are momentum-independent (e.g., no focusing).

The solutions are more effectively obtained in a linear fit using all BPMs and energies simultaneously. A matrix expression for this linear system is given in Eq. (8.66). Here the elements $P_{i j}(k) \equiv e C_{i j} / p_{k}$ are the scaled momentum-dependent transfer coefficients which map the 
$j^{\text {th }}$ kick to the $i^{\text {th }}$ BPM. The equation, as written here, also indicates $N$ BPMs, $N$ kicks and two different momenta $(k=1,2)$.

$$
\left\lfloor\begin{array}{c}
m_{11} \\
m_{21} \\
\vdots \\
m_{N 1} \\
m_{12} \\
m_{22} \\
\vdots \\
m_{N 2}
\end{array}\right\rfloor=\left[\begin{array}{cccccccc}
-1 & 0 & \cdots & 0 & P_{11}(1) & 0 & \cdots & 0 \\
0 & -1 & \cdots & 0 & P_{21}(1) & P_{22}(1) & \cdots & 0 \\
\vdots & \vdots & \ddots & \vdots & \vdots & \vdots & \ddots & \vdots \\
0 & 0 & \cdots & -1 & P_{N 1}(1) & P_{N 2}(1) & \cdots & P_{N N}(1) \\
-1 & 0 & \cdots & 0 & P_{11}(2) & 0 & \cdots & 0 \\
0 & -1 & \cdots & 0 & P_{21}(2) & P_{22}(2) & \cdots & 0 \\
\vdots & \vdots & \ddots & \vdots & \vdots & \vdots & \ddots & \vdots \\
0 & 0 & \cdots & -1 & P_{N 1}(2) & P_{N 2}(2) & \cdots & P_{N N}(2)
\end{array}\right\rfloor \cdot\left\lfloor\begin{array}{c}
b_{1} \\
b_{2} \\
\vdots \\
b_{N} \\
\Delta B_{1} \ell \\
\Delta B_{2} \ell \\
\vdots \\
\Delta B_{N} \ell
\end{array}\right\rfloor
$$

There are a very large number of undulator poles along the undulator (3762) and therefore too many to determine, so the BPM data are fitted to quadrupole magnet misalignments and BPM offsets only. Therefore, any BPM readback sensitivity to energy change will be identified as upstream quadrupole offsets, and the determined quadrupole misalignments (in the wiggle-plane) will necessarily be biased in order to best cancel the net dipole error (i.e., real localized pole errors plus quadrupole misalignment). The quality of this cancellation is examined in the simulation section described below. The non-wiggle plane has no dipoles and therefore the determined quadrupole positions in this plane will not be biased (unless pole roll errors or other stray magnetic fields exist). This is a significant advantage for the energy scan technique because all bend fields, without explicit knowledge of their source, are approximately canceled by biasing the final quadrupole positions in order to best remove the trajectory's sensitivity to energy variations.

To explicitly write Eq. (8.66) in terms of quadrupole misalignments, $\Delta B_{j} \ell$ is replaced with the quadrupole magnet misalignment $\Delta x_{j}$, and $P_{i j}(k)$ is replaced with

$$
P_{i j}(k) \rightarrow\left[1-Q_{11}^{j}(k)\right] R_{11}^{j i}(k)-Q_{21}^{j}(k) R_{12}^{j i}(k) .
$$

Where $Q^{j}{ }_{11}(k)$ and $Q^{j}{ }_{21}(k)$ are the thick-lens transfer matrix elements across the $j^{\text {th }}$ quadrupole magnet evaluated at the $k^{\text {th }}$ momentum, and $R_{1}{ }^{j i}(k)$ and $R_{12}{ }^{j i}(k)\left(=C_{i j}\right)$ are the position-to-position and angle-to-position, respectively, transfer matrix elements from the exit of the $j^{\text {th }}$ quadrupole to the $i^{\text {th }}$ BPM, also evaluated at the $k^{\text {th }}$ momentum. Note, a thin lens quadrupole of pole-tip field $B$, radius $r$, and length $\ell$ has a focal length $f=r p / B \ell$ e. Then the right side of Eq. (8.67) reduces to $C_{i j}(k) / f$, where the minus sign indicates that a horizontally focusing quadrupole $(1 / f<0)$ displaced in the positive direction $(\Delta x>0)$ will kick the beam in the positive direction.

In practice, the linear system of Eq. (8.66) is solved by imposing 'soft-constraints' on the solutions to stabilize the system. The inclusion of the soft-constraints is equivalent to including the additional known information that the quadrupole and BPM offsets are zero to within a reasonable scale (e.g., $\sim 1 \mathrm{~mm}$ ). The constraints are not hard limits but rather weight the fit error 
$\left(\chi^{2}\right)$ such that the solutions do not wander out to large values over long distances (see Ref. [55]).

Eq. (8.68) shows the standard least-squares minimization where additional 'soft-constraints' are included for the BPM and the quadrupole offsets (last two terms) [56]:

$$
\chi^{2}=\sum_{i, k}\left\{\frac{1}{\sigma_{m_{i}}}\left(m_{i k}-\left[\sum_{j=1}^{i} P_{i j}(k) \Delta x_{j}-b_{i}\right]\right)\right\}^{2}+\sum_{i=1}^{N}\left\{\frac{\Delta x_{i}}{\sigma_{x}}\right\}^{2}+\sum_{i=1}^{N}\left\{\frac{b_{i}}{\sigma_{b}}\right\}^{2}
$$

The BPM and quadrupole offsets are weighted by their respective soft-constraints, $\sigma_{x}$ and $\sigma_{b}$ $(\approx 1 \mathrm{~mm}$ is used in the initial simulations to follow), and the fit deviations are weighted by the measurement error of the BPM reading, $\sigma_{m_{i}}$ (i.e., the estimated BPM resolution-in this case $\sim 1 \mu \mathrm{m} \mathrm{rms}$ ). The solutions are then found in the usual way where the gradient of $\chi^{2}$ with respect to the solutions, $\Delta x_{i}$ and $b_{i}$, is set to zero and the resulting linear system is solved.

Ignoring the initial launch conditions of the beam at the entrance to the undulator in Eq. (8.66) has the effect of defining the reference line for the determined BPM and quadrupole misalignments $\left(b_{i}\right.$ and $\left.\Delta x_{j}\right)$ as the incoming position and angle of the beam. At a point when Eq. (8.66) has been solved and the $N$ values of $b$ and $\Delta x$ are available, a best line fit (initial position and angle) is extracted from these data and the launch conditions are accordingly adjusted with steering dipoles prior to the undulator so that the quadrupole and BPM offset corrections will not systematically follow these initial erroneous launch conditions. This also limits the size of the quadrupole and BPM offset corrections applied. On the next application of the procedure, the launch conditions will be much closer to the axis defined by the initial BPM and quadrupole positions (the initial tunnel survey).

The advantage to scanning the beam energy, compared with scanning quadrupole field strengths, is that the energy-scanned BPM data is sensitive to all bending fields whether due to quadrupole misalignments, undulator pole errors or other stray fields such as the earth's field. By using this field sensitive data, the solutions obtained provide the best minimization of all bending by biasing the quadrupole positions slightly offset with respect to the beam centroid. The net effect is to produce minimal erroneous bending within the undulator, even without explicit knowledge of the fields. Given undulator pole field errors and other stray fields, this produces a much straighter trajectory than is obtainable by varying quadrupole field strengths.

\subsubsection{Simulation Results}

Simulations have been run for the entire beam-based alignment algorithm, from initial rough steering through final precision alignment. The simulations use 32 quadrupoles and 32 BPMs within the undulator, where the quadrupoles are separated by $3.68 \mathrm{~m}$ (center-to-center) and have a field gradient of $107 \mathrm{~T} / \mathrm{m}$ and length of $5 \mathrm{~cm}$. One high-resolution BPM is located at the upstream face of each quadrupole, and each quadrupole is mounted on a mechanical mover. The weak natural field gradients of a real undulator segment are not included in this simulation, but a large energy error is included. 
A conservative set of statistical and systematic errors is included in the simulations as summarized in Table 8.10. The undulator poles are assumed to have random uncorrelated relative field strength errors of $0.04 \% \mathrm{rms}$. In fact, shimming techniques used to correct the pole fields will introduce pole-to-pole field error correlations, which are more forgiving than uncorrelated errors. The $0.04 \%$ random uncorrelated error used here (in the wiggle-plane only) is therefore considered as something of a worst case. Sensitivities of the final results to the size of the input errors are studied in the next section.

Table 8.10 List of errors used in simulation of beam-based alignment procedure. All random errors have gaussian distributions.

\begin{tabular}{llll}
\hline Description & Value & Unit & Comments \\
\hline BPM rms resolution & 1 & $\mu \mathrm{m}$ & net resolution - not necessarily single pulse \\
\hline BPM offsets (uncorrelated) & 50 & $\mu \mathrm{m}$ & rms BPM-to-BPM survey and/or electrical offsets \\
\hline BPM offsets (correlated) & 300 & $\mu \mathrm{m}$ & rms BPM 'random walk' over length of undulator \\
\hline BPM mean calibration errors & 10 & $\%$ & mean calibration error over all BPMs \\
\hline BPM rms calibration errors & 3 & $\%$ & rms calibration error spread over all BPMs \\
\hline quad. offsets (uncorrelated) & 50 & $\mu \mathrm{m}$ & rms quad-to-quad trans. alignment survey errors \\
\hline quad. offsets (correlated) & 300 & $\mu \mathrm{m}$ & rms quad 'random walk' over length of undulator \\
\hline mean beam energy error & 2.0 & $\%$ & mean error of electron beam energy over the scan \\
\hline rms beam energy error & 0.5 & $\%$ & rms error of electron beam energy over the scan \\
\hline quad. mean gradient errors & 0.3 & $\%$ & mean gradient error over all quadrupoles \\
\hline quad. rms gradient errors & 0.3 & $\%$ & rms gradient error spread over all quadrupoles \\
\hline undulator pole errors & 0.04 & $\%$ & rms uncorrelated field errors over all poles \\
\hline mover mean calibration errors & 5 & $\%$ & mean calibration error over all magnet movers \\
\hline mover rms calibration errors & 3 & $\%$ & rms calibration error spread over all magnet movers \\
\hline incoming trajectory bias & 10 & $\sigma$ & initial orbit pos. and ang. in beam size units (static) \\
\hline incoming orbit jitter & $0-0.1$ & $\sigma$ & rms orbit variation in beam size units (dynamic) \\
\hline
\end{tabular}

A beam energy error simulates the imprecise knowledge of the electron energy during the scan. A $2 \%$ average error (constant over the scan) and a $0.5-\%$ rms error (variable over the scan) are included. In the table, the 'correlated' BPM and quadrupole offsets (misalignments) define a random walk where the expectation value of the square of the misalignment, $\left\langle\Delta x^{2}\right\rangle$, is related linearly to its distance from the undulator entrance [57]. This treatment approximates the long undulator survey 'wander' error arising over 120 meters. In this case, an rms level of $100 \mu \mathrm{m}$ per 10 meter length, or $\sim 300 \mu \mathrm{m}$ over 100 meters is included. The BPMs and quadrupoles both follow the same random walk plus an additional $50 \mu \mathrm{m}$ rms 'uncorrelated' component applied to both BPMs and quadrupoles separately. Two adjacent elements are therefore misaligned with 
respect to each other by $\sqrt{ } 2 \times 50 \mu \mathrm{m} \approx 70 \mu \mathrm{m} \mathrm{rms}$. Over longer distances the relative misalignment increases.

The 1- $\mu \mathrm{m}$ BPM resolution listed is the net rms measurement resolution over many pulses. Averaging can also be used to get better resolution (i.e. 100 pulses are saved at each energy, the BPM single-pulse resolution can be closer to $10 \mu \mathrm{m})$. The BPM offsets must, however, be constant to a level of $\sim 1 \mu \mathrm{m}$ over the few hour period during which the energy is being scanned. This implies, for example, adequate temperature stability for the BPM electronics, etc. The various 'calibration' errors in the table imply that the BPMs (or magnet movers) are misscaled so that, for example, an actual displacement of $100 \mu \mathrm{m}$ will read back as $110 \mu \mathrm{m}$. The 'incoming trajectory bias' is a static (constant) beam launch error which is ten times that of the rms beam size in both position and angle $(\sim 10 \times 30 \mu \mathrm{m}$ and $\sim 10 \times 1.5 \mu \mathrm{rad})$. The 'incoming orbit jitter' is a randomly varying launch position and angle error, which occurs during the energy-scan data acquisition. The simulation shown here includes no orbit jitter, but in fact the results are insensitive up to a $10 \% \mathrm{rms}$ launch jitter. The jitter can actually be reduced even further in practice, to a level of a few percent, by acquiring $\sim 100$ orbits and using the 8-10 pre-undulator BPMs to select only those orbits which produce a constant mean trajectory launch. In addition, the variable trajectory can be included as additional fit parameters; an option which was found to be unnecessary in these simulations.

A mover reproducibility error has also been studied [58], which provides a small random mechanical error on the final position of the quadrupole magnet mover. This effect has been ignored here since small dipole steering coils will be used to augment the magnet movers and provide a very fine vernier steering control. The quadrupole magnet movers are then controlled to a level of a few microns, and the steering coils are used for smaller corrections. Without the steering coils the magnet movers would require a mechanical reproducibility precision of $<1 \mu \mathrm{m}$.

The undulator alignment procedure for initial machine startup is outlined in Table 8.11. The machine time required for each step is only a rough estimate. Practical experience and computer automation may significantly reduce these time estimates. The initial step specifies reducing the strength of the $2^{\text {nd }}$ bunch compressor chicane in order to increase the bunch length in the undulator to $\sim 150 \mu \mathrm{m} \mathrm{rms}$. Although not studied in detail, this should alleviate transverse resistive wall wakefields as a source of systematic error, which may otherwise be significant when large trajectory excursions occur during the alignment procedure. Geometric wakefields increase with a longer bunch, but should still be quite weak with a $150-\mu \mathrm{m}$ bunch length.

Figure 8.42 shows the specific quadrupole and BPM misalignments used in this simulation with respect to a line defined by the linac beamline axis (as are all figures in this section unless otherwise noted). In this plot only, the initial BPM offsets are shown (blue diamonds). In plots to follow, the actual readbacks of the BPMs, including noise and calibration errors, are shown (green circles). 
Table 8.11 Beam-based undulator alignment procedure. Beam energy is $14.3 \mathrm{GeV}$ unless otherwise noted, as in step-3.

\begin{tabular}{ll}
\hline Step \# & Description \\
\hline 0 & $\begin{array}{l}\text { Adjust the } 2^{\text {nd }} \text { bunch compressor chicane for a } \sim 150 \mu \mathrm{m} \text { rms electron bunch length to } \\
\text { minimize transverse wakefields in the undulator }\end{array}$ \\
\hline 1 & Adjust the launch using best position and angle fit to $1^{\text {st }}$ six undulator BPMs \\
\hline 2 & $\begin{array}{l}\text { Apply weighted steering to reduce (not zero) simultaneously both the absolute BPM } \\
\text { readings }(\div 50 \mu \mathrm{m}) \text { and the applied magnet mover changes }(\div 50 \mu \mathrm{m})\end{array}$ \\
\hline 3 & $\begin{array}{l}\text { Save } \sim 100 \text { sets of BPM readings for each of } 5,10 \& 14.3 \mathrm{GeV} \text { beam energies while } \\
\text { scaling upstream linac magnets to the new energy each time }\end{array}$ \\
\hline 5 & $\begin{array}{l}\text { Run BPM data through analysis program to determine BPM and quadrupole offsets } \\
\text { (select from data sets to minimize orbit jitter) }\end{array}$ \\
\hline 6 & $\begin{array}{l}\text { Adjust launch position and angle to remove determined linear component of BPM and } \\
\text { quadrupole offsets }\end{array}$ \\
\hline 7 & $\begin{array}{l}\text { Fine steer offset-corrected BPM readings to approximately zero using a minimum } \\
\text { number of magnet movers and steering coils }\end{array}$ \\
\hline 8 & \begin{tabular}{l} 
Repeat steps 3-7 until peak BPM readings at 5 GeV are $<15 \mu \mathrm{m}$ \\
\hline
\end{tabular}
\end{tabular}
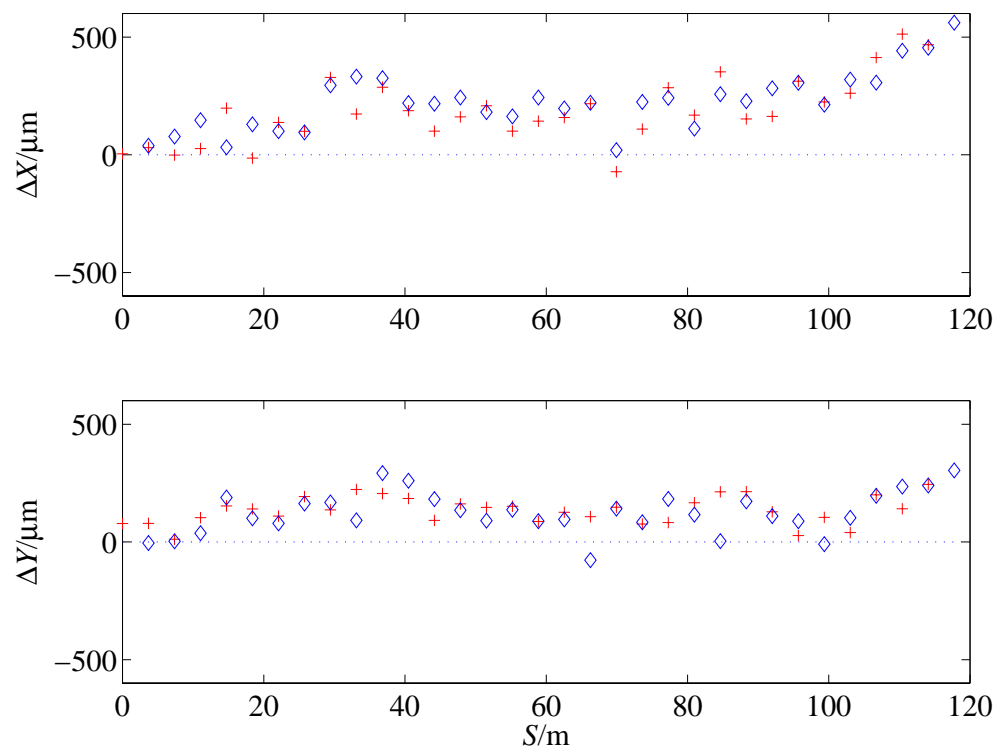

Figure 8.42 Quadrupole (red-cross) and BPM (blue-diamond) initial misalignments used in the simulation, with respect to the line defined by the linac beamline axis.

The resultant, un-steered horizontal and vertical beam trajectory through the undulator is shown in Figure 8.43 (prior to step-1, Table 8.11). The true orbit (blue solid line) is shown, as in 
plots to follow, every $1.5 \mathrm{~cm}$ along the undulator, at the center of every pole of $\sim 8000$ poles. The BPM readbacks (green circles) are, in practice, the only known (measured) quantities. This trajectory is an example of the first beam pulse in the newly installed undulator.

The first correction (step-1, Table 8.11) is to rough-steer the incoming trajectory based on the first six BPM readbacks in the undulator, which are used in a best fit to an incoming betatron oscillation (see black dashed line in first 20 meters of Figure 8.43). Table 8.12 shows the initial launch conditions for this simulation both before and after the step-1, Table 8.11, rough correction. At this rough stage, direct use of the first few BPM readbacks, before their offsets are corrected, is still very effective in removing large incoming launch errors.
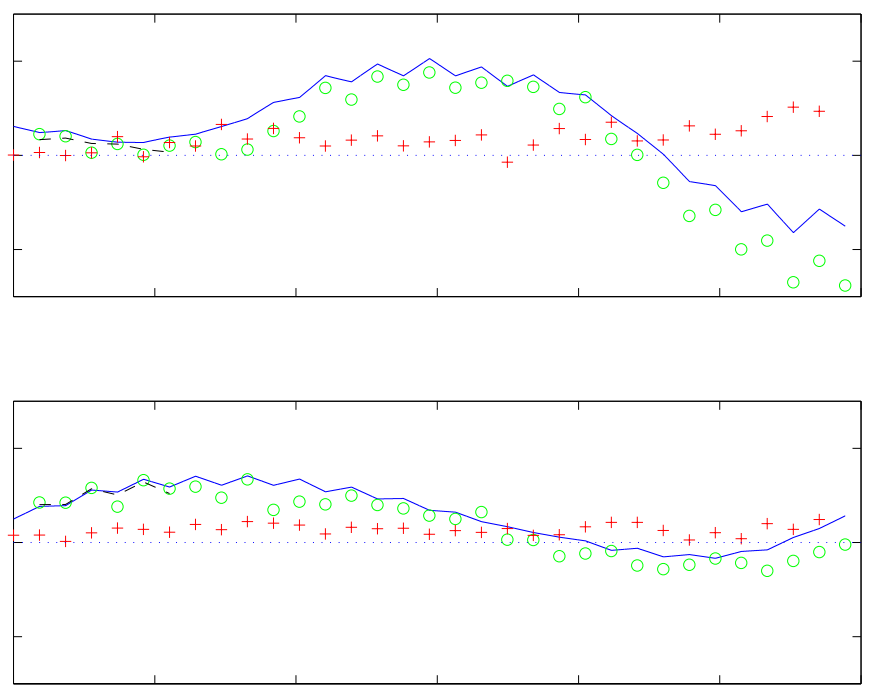

Figure 8.43 Simulated first beam trajectory (blue-solid) through the newly installed undulator, including incoming trajectory bias of $\sim 300 \mu \mathrm{m}$ and $\sim 15 \mu \mathrm{rad}$. Also shown are BPM readbacks (green-circle), quadrupole positions (red-cross), and the fitted initial launch using the readbacks of the first six BPMs (dash-first 20 meters only).

The launch conditions are significantly improved in this case, reducing the trajectory amplitude and, given systematic errors such as BPM calibration and quadrupole field gradient errors, improving the speed of convergence of the algorithm. A complete correction is not possible at this stage since the BPMs used in the launch fit may include large offsets, and the misaligned quadrupoles between the BPMs may kick the trajectory off of a free betatron oscillation. Note, the quality of this correction is dependent on the specific set of misalignments (random seed). The random seed shown here is fairly typical. The quality of this correction impacts only the speed of convergence. 
LCLS CONCEPTUAL DESIGN REP O R T

Table 8.12

Initial launch conditions at undulator entrance before and after step-1, Table 8.11 correction.

\begin{tabular}{ccc}
\hline Launch parameter & Before Step-1, Table 8.11 & After Step-1, Table 8.11 \\
\hline$\langle\mathrm{x}\rangle$ & $310 \mu \mathrm{m}$ & $14 \mu \mathrm{m}$ \\
\hline$\left\langle\mathrm{x}^{\prime}\right\rangle$ & $14 \mu \mathrm{rad}$ & $3.5 \mu \mathrm{rad}$ \\
\hline$\langle\mathrm{y}\rangle$ & $250 \mu \mathrm{m}$ & $13 \mu \mathrm{m}$ \\
\hline$\left\langle\mathrm{y}^{\prime}\right\rangle$ & $17 \mu \mathrm{rad}$ & $-1.7 \mu \mathrm{rad}$ \\
\hline
\end{tabular}

Step-2, Table 8.11, involves a weighted steering procedure using the undulator quadrupole magnet movers where the absolute readings of the BPMs are minimized with respect to a $50 \mu \mathrm{m}$ weighting and the applied magnet mover changes are simultaneously minimized with respect to a $50 \mu \mathrm{m}$ weighting. Without this weighting the magnet movers can move by up to $\sim 1 \mathrm{~mm}$ in order to exactly zero each BPM reading which, due to the large BPM offsets, is an unnecessary and undesirable steering accuracy at this early stage. With the weighting included, the movers change by $\sim 50 \mu \mathrm{m}$, which is sufficient to correct the large trajectory deviations of Figure 8.43. Figure 8.44 shows the trajectory after the step- 2 weighted steering is applied. This step simply improves the trajectory before the energy is changed.
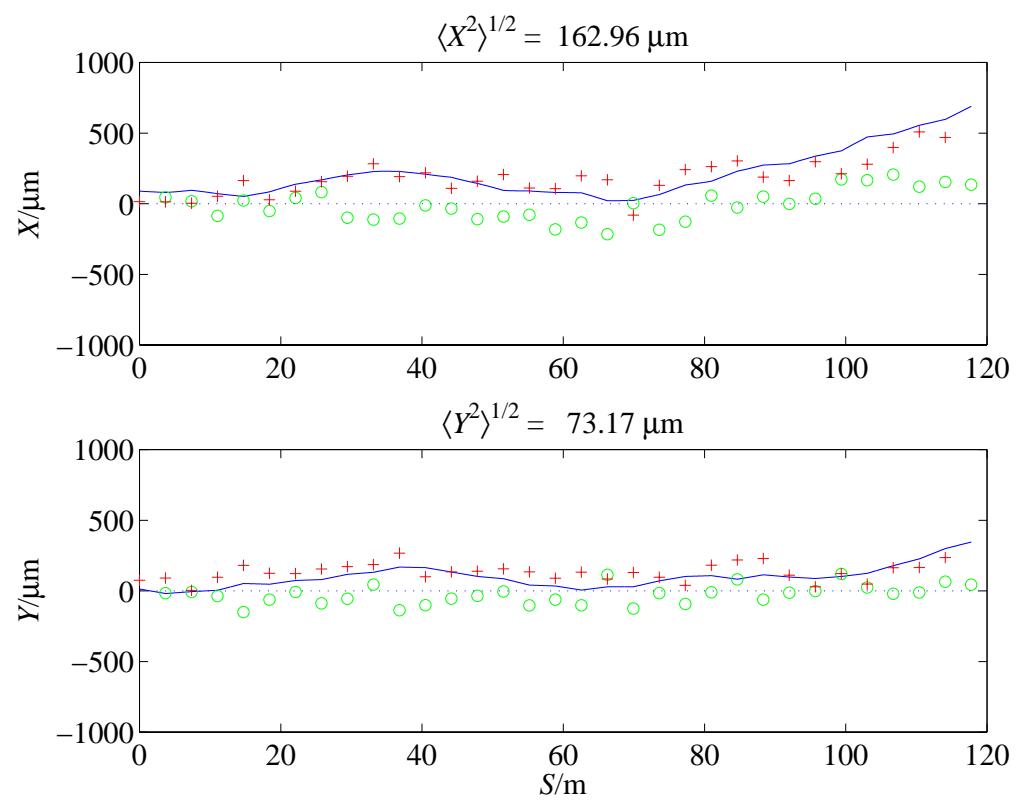

Figure 8.44 Trajectory (blue-solid) at $14.3 \mathrm{GeV}$ after application of step-2, Table 8.11, weighted steering. BPM readbacks (green-circle) and applied magnet mover changes are both minimized with respect to a $50 \mu \mathrm{m}$ weighting. The post-steering quadrupole positions (red-cross) are also shown. These BPM readings are saved for analysis.

These 14.3-GeV BPM data are saved and the energy is then lowered to $10 \mathrm{GeV}$ by switching off half of the klystrons in linac-3. The fields of the magnets upstream of the undulator are scaled to the new energy and any beam position differences upstream of the undulator, with respect to 
the $14.3-\mathrm{GeV}$ orbit, are manually corrected, if necessary, until the launch position at $10 \mathrm{GeV}$ is within $\pm 3 \mu \mathrm{m}$ of that at $14.3 \mathrm{GeV}$. Note, any beam angle difference originating at the undulator entrance will not be detectable with the BPMs upstream of the undulator. However, this angle will eventually be properly incorporated into a correction of the transverse position of the first undulator quadrupole. Figure 8.45 shows the new trajectory at $10 \mathrm{GeV}$, while Figure 8.46 shows a third trajectory at $5 \mathrm{GeV}$ (most of the linac-3 klystrons switched off). No changes are made to the undulator components during the energy scan. Only the pre-undulator trajectory is adjusted, if necessary, to maintain a constant beam position at the undulator entrance.
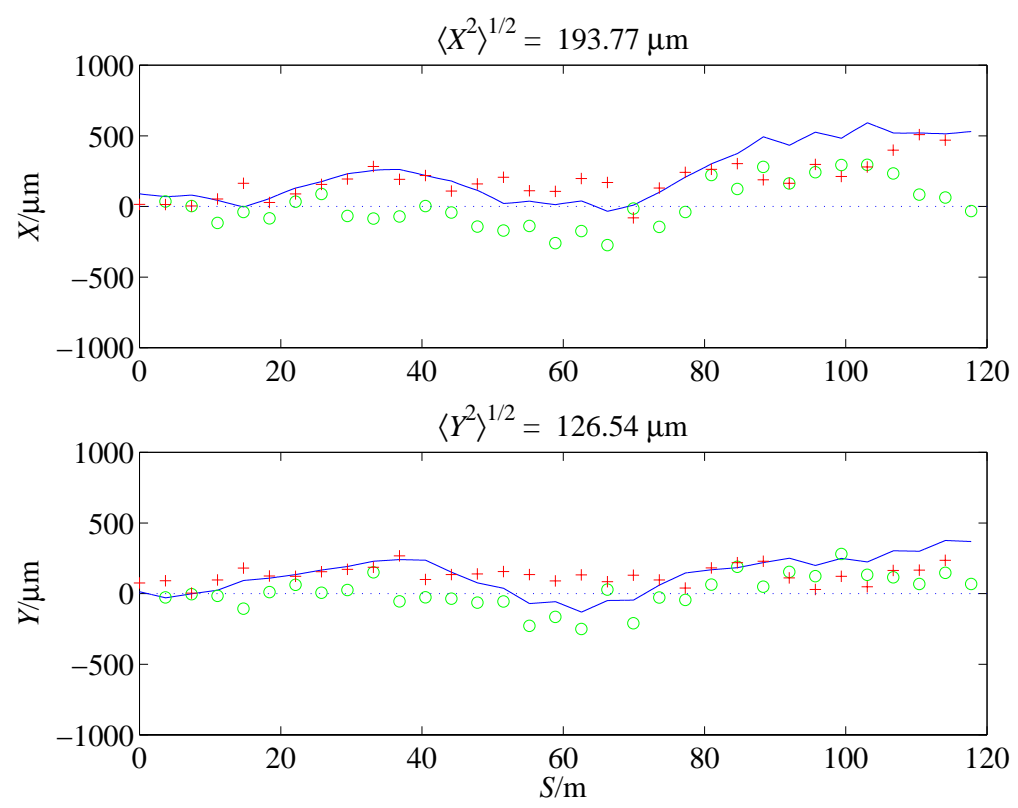

Figure 8.45 Trajectory (blue-solid) at $10 \mathrm{GeV}$. BPM readbacks (green-circle) and quadrupole positions (red-cross) are also shown. These BPM readings are saved for analysis.

Since the fields of the magnets upstream of the undulator will be scaled to the lower energies, the beta functions at the undulator entrance will be constant during the procedure. Since the undulator focusing is accomplished with permanent magnet quadrupoles, a betatron-mismatch, with respect to the energy dependent periodic beta functions of the undulator FODO lattice, will propagate through the undulator at the lower beam energies. In the worst case $(5 \mathrm{GeV})$ the beam size will beat at twice the betatron frequency reaching a peak rms size of $\sim 80 \mu \mathrm{m}$ as compared to the $50 \mu \mathrm{m}$ beam size of the $5-\mathrm{GeV}$ periodic beta functions. This worst-case beat in beam size should have no significant effect on the alignment procedure, which utilizes trajectory centroid information only.

With the 5, 10 and 14.3-GeV BPM readback data saved, the analysis program is run, which calculates BPM offsets, and quadrupole magnet positions with respect to the step-1-corrected incoming beam position and angle. Figure 8.47 shows the calculated quadrupole offsets as well as the true offsets (used in the simulation). The fine structure of the calculated offsets agrees well with the true offsets. The values differ, however by a straight line which is due to both 1) the 
step-1-corrected launch bias, and 2) the correlated component of the BPM and quadrupole offset errors (the random walk-off effect of the initial survey). A line is then fit to the calculated BPM and quadrupole offsets. The slope and offset of the best line fit is used to readjust the initial launch position and angle at the undulator entrance.
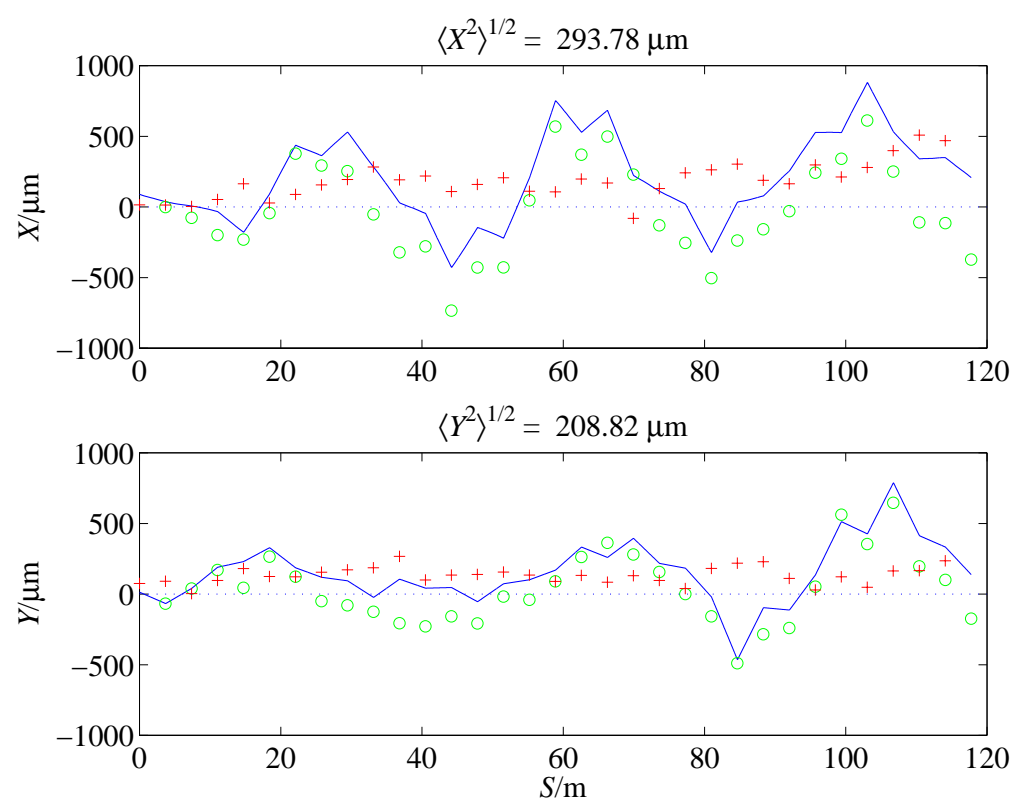

Figure 8.46 Trajectory (blue-solid) at $5 \mathrm{GeV}$. BPM readbacks (green-circle) and quadrupole positions (red-cross) are also shown. These BPM readings are saved for analysis.

Figure 8.48 shows the new electron trajectory after the launch conditions, the quadrupole magnet movers and the BPM offsets are corrected. The BPM offsets and the mover corrections applied are the differences between the best line fit and the data (error bars). In addition, a final steering using a minimum number of steering coils is applied to remove any remaining detectable betatron oscillation (based on BPM readback). At each stage in the simulation, the real magnet mover limitations (calibration errors) and BPM errors (calibration and resolution) are incorporated.

The linear component, which remains in the Figure 8.48 trajectory is due to the correlated quadrupole and BPM offsets (random walk of initial tunnel survey). Since these offsets are due dominantly to the difference between the line defined by the linac beamline axis and the slightly different line established by the undulator beamline, the alignment procedure inevitably launches the electrons straight down the undulator vacuum chamber, which presumably follows these correlated tunnel survey errors. The true trajectory shown in Figure 8.48 is then actually the most desired trajectory, where a slight change in beam 

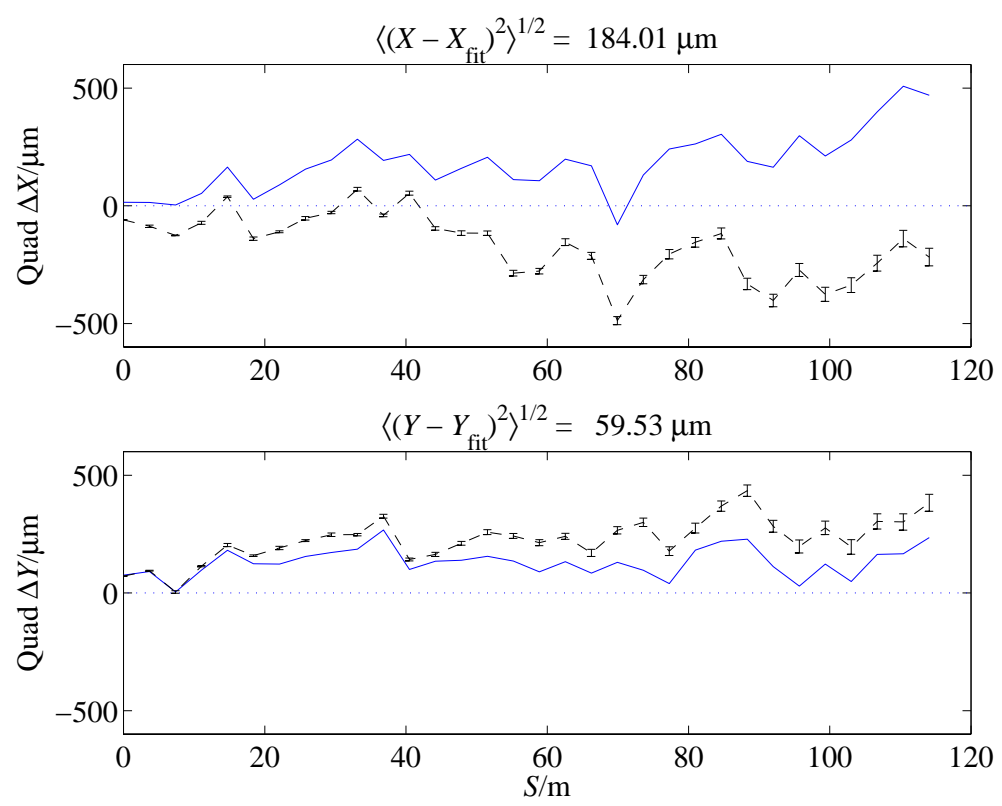

Figure 8.47 True (blue-solid) and calculated (black-dash) quadrupole positions. The BPM offsets are similar but not shown here. The fine structure is reproduced well.
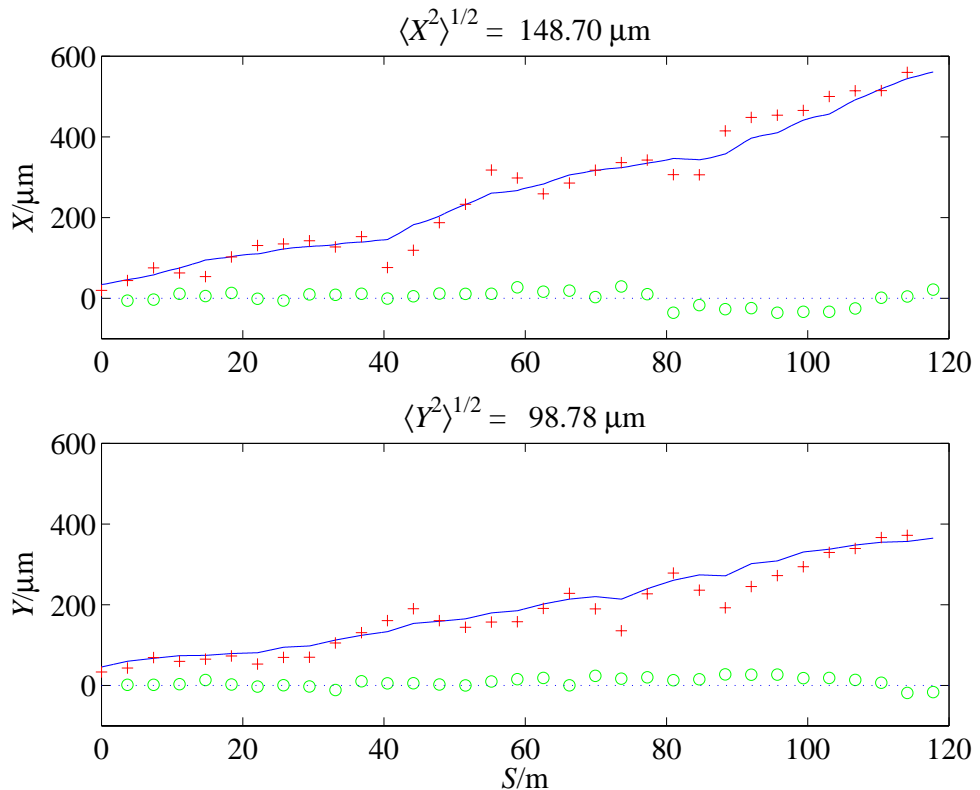

Figure 8.48 Trajectory (blue-solid) at $14.3 \mathrm{GeV}$ after one iteration of steps 1-7. BPM readbacks (green-circle) and quadrupole positions (red-cross) are also shown. The trajectory is fairly straight but does not necessarily follow the line established by the linac axis. This is due to the correlated BPM and quadrupole misalignments (random walk of the initial tunnel survey).

position and angle at the undulator entrance is used to launch the electron beam down the center of the undulator, and the beam follows along near the center of the quadrupoles. The tiny 
dispersion generated by this slight position and angle change is completely negligible, especially in consideration of an rms relative energy spread of $<0.1 \%$.

Figure 8.49 shows the same data as in Figure 8.48 except that the linear component of the true orbit has been removed in order to show more clearly the straightness of the trajectory. Figure 8.50 shows this linear-corrected view of the final trajectories after a second iteration of steps 3-7 is applied, and Figure 8.51 shows this view after a third iteration. Each plot lists the rms of the real trajectory, $\left\langle\Delta X^{2}\right\rangle^{1 / 2}$, the rms of the BPM readings, $\left\langle\Delta X_{B P M}{ }^{2}\right\rangle^{1 / 2}$, and the rms quadrupole alignment, $\left\langle\Delta X_{Q U A D}{ }^{2}\right\rangle^{1 / 2}$. The rms of the electron trajectory over the length of the undulator with respect to a straight line achieves a value of $<8 \mu \mathrm{m}$ after the $2^{\text {nd }}$ iteration and $<3 \mu \mathrm{m}$ after the $3^{\text {rd }}$ iteration. The BPM readbacks achieve an rms level of $1 \mu \mathrm{m}$, while the quadrupoles are aligned to $11 \mu \mathrm{m}$ and $7 \mu \mathrm{m}$ in $x$ and $y$ respectively. The $x$ alignment is worse since the positions of the magnets are slightly biased in order to best correct the net dipole field, including undulator pole errors.
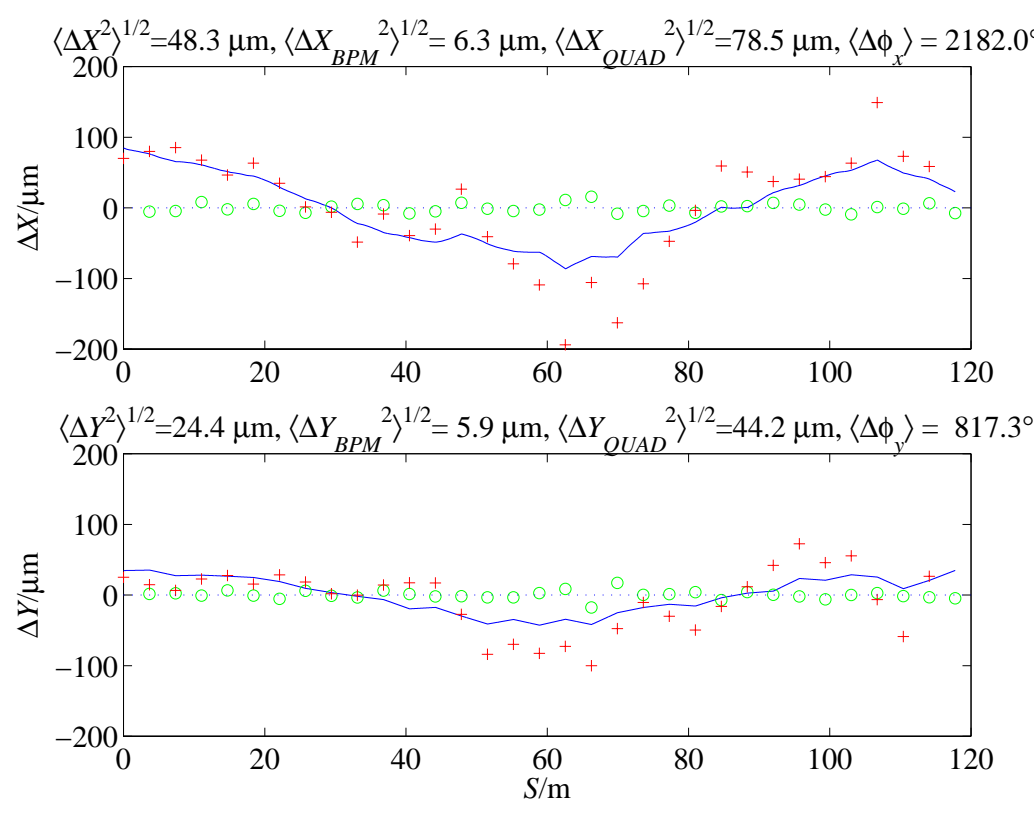

Figure 8.49 Trajectory (blue-solid) at $14.3 \mathrm{GeV}$, with linear component removed, after one iteration of steps 1-7. BPM readbacks (green-circle) and quadrupole positions (red-cross) are also shown. The quadrupole positions are shown in the same linear-corrected coordinates of the true orbit. The beam trajectories with respect to a straight line over the undulator length (for this first pass correction) have rms values of $x_{r m s}=48 \mu \mathrm{m}, y_{\mathrm{rms}}=24 \mu \mathrm{m}$ and peak values of $x_{p k}=86 \mu \mathrm{m}, y_{p k}=43 \mu \mathrm{m}$. 

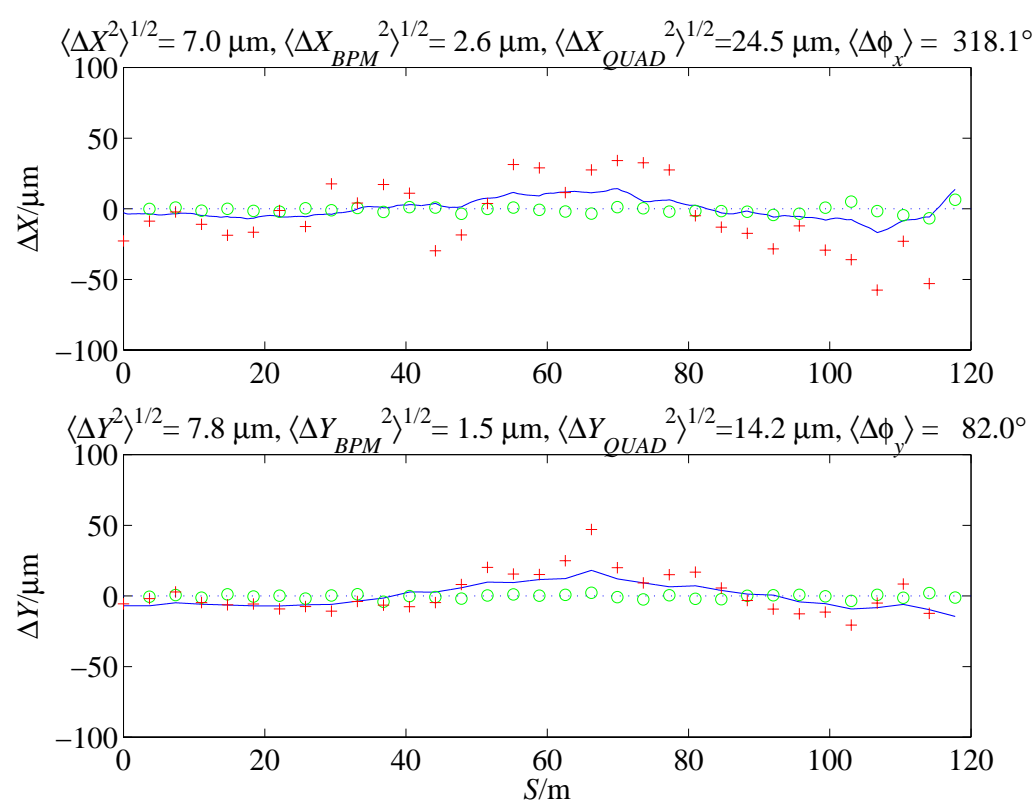

Figure 8.50 After two iterations of steps 3-7 $\left(x_{\mathrm{rms}}=7.0 \mu \mathrm{m}, \mathrm{y}_{\mathrm{rms}}=7.8 \mu \mathrm{m}\right.$ and peak values of $\left.\mathrm{x}_{\mathrm{pk}}=17 \mu \mathrm{m}, \mathrm{y}_{\mathrm{pk}}=18 \mu \mathrm{m}\right)$. A new scale is used here.

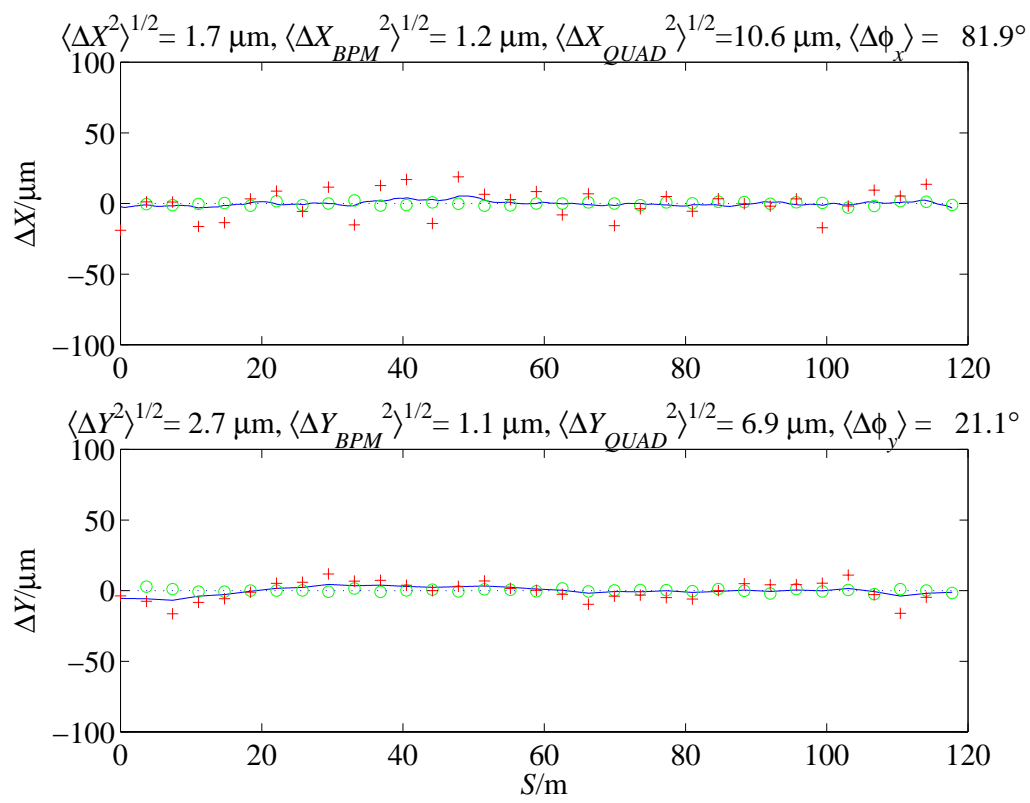

Figure 8.51 After three iterations of steps 3-7 $\left(x_{\mathrm{rms}}=1.7 \mu \mathrm{m}, \mathrm{y}_{\mathrm{rms}}=2.7 \mu \mathrm{m}\right.$ and peak values of $\left.\mathrm{x}_{\mathrm{pk}}=5.3 \mu \mathrm{m}, \mathrm{y}_{\mathrm{pk}}=6.8 \mu \mathrm{m}\right)$.

The raw BPM readbacks also give a clear indication as to whether the procedure is converging and when to terminate the process. As the alignment is improved, the BPM readbacks will respond less and less to energy variations. With the energy lowered to $5 \mathrm{GeV}$ the BPM readings should change, with respect to the readbacks at $14.3 \mathrm{GeV}$, by $<15 \mu \mathrm{m}$ when the procedure has converged to the level shown here. In these simulations at $5 \mathrm{GeV}$, the peak 
readings change by $<150 \mu \mathrm{m}$ after the second pass and by $<15 \mu \mathrm{m}$ after the third pass. In practice, this should provide a clear convergence signal.

To further evaluate the quality of the final trajectory obtained, the trajectory simulation data are used to calculate the total path length of the electrons over the length of the undulator. The electron path length is compared with the path length of a 1.5 - $\AA$-wavelength x-ray beam that follows a perfectly straight path down the center of the undulator. A phase slip error is then calculated for each plane, $\left\langle\Delta \phi_{x}\right\rangle$ and $\left\langle\Delta \phi_{y}\right\rangle$, with the total phase error as the sum. The results are shown on each trajectory plot for each iteration pass. The final trajectory after the third correction reduces the phase error to $\left\langle\Delta \phi_{x}\right\rangle \approx 82^{\circ}$ and $\left\langle\Delta \phi_{y}\right\rangle \approx 21^{\circ}$, for a total phase error of $\left\langle\Delta \phi_{x}\right\rangle+$ $\left\langle\Delta \phi_{y}\right\rangle \approx 103^{\circ}$. The horizontal phase error is larger than the vertical due to the undulator pole errors. FEL simulations using Ginger indicate that a total phase error of $\sim 400^{\circ}$ increases the saturation length by one power gain length. Finally, studies with the FEL code RON, and using the precise trajectories, which result from these simulations, also indicate a saturation length increase of less than one power gain length for the resulting simulated trajectory.

Figure 8.52 shows the final quadrupole magnet mover settings after the three iterations have been completed. The final alignment correction requires magnet mover settings with an rms value of $\sim 100 \mu \mathrm{m}$ and maximum excursions of $\sim 350 \mu \mathrm{m}$.
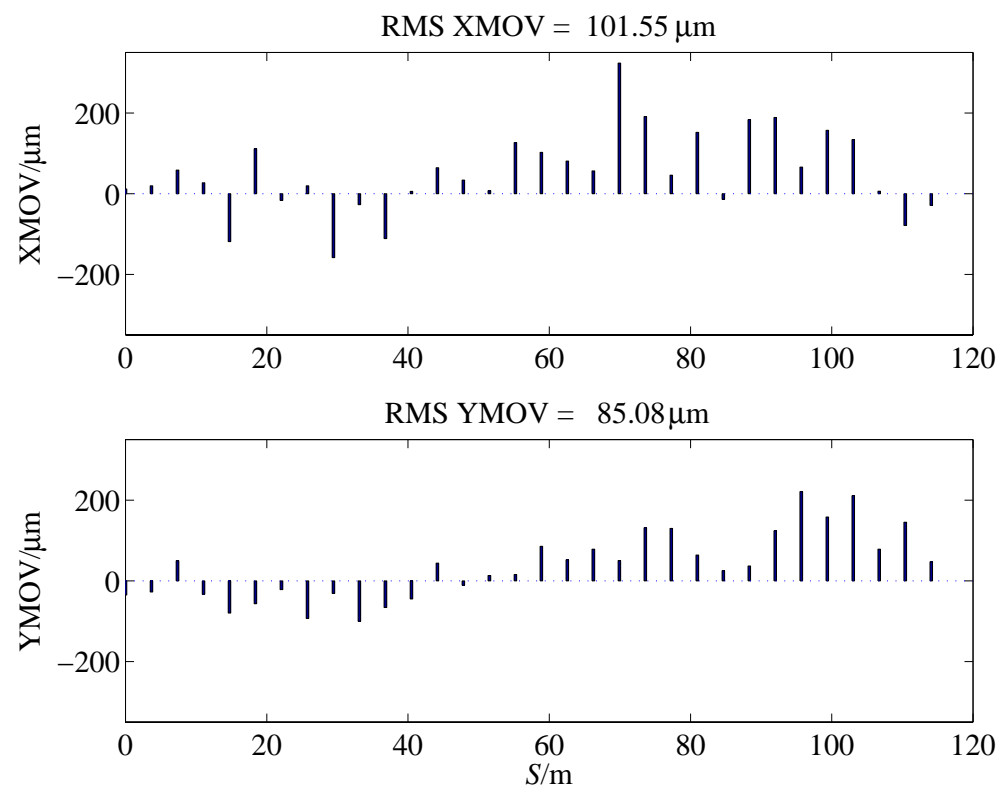

Figure 8.52 Final quadrupole magnet mover settings after three iterations of the alignment procedure. The movers are initially set to zero prior to the alignment procedure and the final rms values shown are: $x_{r m s}=102 \mu \mathrm{m}$ and $y_{r m s}=85 \mu \mathrm{m}$.

\subsubsection{Sensitivities}

The sensitivities of the final rms trajectory to the simulation input errors are summarized below in Table 8.13. A new simulation is run using the input errors listed in Table 8.10, except that for each run a different individual error is approximately doubled in value. The first row 
summarizes the simulation already described, and every following row represents a new simulation where the noted error has been increased with respect to Table 8.10.

Table 8.13 Trajectory and phase error sensitivities to simulation input errors. All errors are those of Table 8.10 unless noted, in which case that error has been approximately doubled in value. The random seed used is unchanged for purposes of comparison.

\begin{tabular}{lcccc}
\hline Increased input error & \multicolumn{2}{c}{$\mathbf{x}_{\mathbf{r m s}} / \mathbf{y}_{\mathbf{r m s}}[\boldsymbol{\mu m}]$} & \multicolumn{2}{c}{$\left\langle\Delta \phi_{\mathbf{x}}\right\rangle /\left\langle\Delta \phi_{\mathbf{y}}\right\rangle[\mathbf{d e g}]$} \\
\hline & after 2 ${ }^{\text {nd }}$ pass & after ${ }^{\text {rd }}$ pass & after $2^{\text {nd }}$ pass & after $3^{\text {rd }}$ pass \\
\hline (using all errors of Table 8.10) & $7.0 / 7.8$ & $1.7 / 2.7$ & $318 / 82$ & $82 / 21$ \\
\hline 2- $\mu \mathrm{m}$ BPM resolution & $5.6 / 7.3$ & $2.5 / 5.2$ & $351 / 89$ & $117 / 67$ \\
\hline $0.1-\%$ rms undulator pole errors & $8.0 / 7.8$ & $2.0 / 2.7$ & $544 / 82$ & $350 / 21$ \\
\hline $100-\mu m$ uncorrelated BPM \& quad offsets & $11 / 14$ & $2.3 / 3.2$ & $721 / 232$ & $112 / 29$ \\
\hline $0.6-\%$ quad. gradient errors (mean \& rms) & $6.5 / 8.9$ & $1.5 / 2.7$ & $229 / 104$ & $74 / 22$ \\
\hline
\end{tabular}

All errors are returned to their original values for each new run, except for the noted error. As the table demonstrates in most cases, three iterations of the alignment procedure render the algorithm fairly insensitive to the precise set of beamline errors. A doubled BPM resolution and (independently) a more-than-doubled set of pole field errors have a noticeable impact, but the rms trajectory is still $\leq 5 \mu \mathrm{m}$ and the total phase error is $<400^{\circ}$.

\subsubsection{Long Term Stability}

After beam-based alignment has achieved a straight trajectory, ground motion effects in the FFTB tunnel can slowly alter the undulator alignment. Betatron oscillations over full or partial sections of the undulator can be removed by occasional small steering corrections. Higher frequency trajectory disturbances (e.g., from BPM to BPM), however, are much more damaging and difficult to remove. When the trajectory is distorted sufficiently to impact the FEL gain, and small isolated steering corrections are no longer helpful, one single iteration of the beam-based alignment procedure will need to be repeated.

An estimate of the long term stability of alignment can be made by applying the "ATL-law" [59], which estimates the effects of diffusive ground motion. The variance, $\sigma^{2}$, of the relative transverse alignment between two points separated by a distance, $L$, is described by

$$
\sigma^{2}=A \cdot T \cdot L
$$

where $T$ is the time since the two points were initially aligned, and $A$ is a site-specific constant, usually specified in units of $\mu \mathrm{m}^{2} / \mathrm{m} / \mathrm{s}$. Measurements of the coefficient $A$ have been made at many locations on the globe, including in the actual FFTB tunnel at SLAC where the LCLS undulator will reside. A value of $A \approx 5 \times 10^{-7} \mu \mathrm{m}^{2} / \mathrm{m} / \mathrm{s}$ has been measured in the FFTB tunnel [60] and is used here to estimate the time required for which beam-based alignment must be repeated. The distance $L$ is taken as the BPM-to-BPM spacing, since these short scale trajectory distortions cause the largest electron-to-photon phase errors, and misalignments over distances greater than, 
or of the order of one power gain length have much less impact. With $L=3.68 \mathrm{~m}$ and $A=5 \times 10^{-7} \mu \mathrm{m}^{2} / \mathrm{m} / \mathrm{s}$, a 30 -day period will produce a relative misalignment of $2 \mu \mathrm{m}$ rms between BPMs. While steering corrections will control the build-up of the trajectory amplitude caused by this slow drift, the phase errors induced by localized, short scale misalignments will eventually reduce the FEL gain. An average closed trajectory bump of $2 \mu \mathrm{m}$ amplitude $(=\Delta x=\Delta y)$ at each of $N=32$ BPMs in both planes, at a radiation wavelength of $\lambda_{r}=1.5 \AA$, will produce a phase error of

$$
\Delta \phi=\frac{2 \pi N}{L \lambda_{r}}\left(\Delta x^{2}+\Delta y^{2}\right)
$$

which amounts to $170^{\circ}$ over the undulator. From this rough model it is estimated that a single iteration of the beam-based alignment procedure $(\sim 2.5$-hour procedure $)$ will be required once per month.

\subsubsection{Summary}

An electron trajectory of $<3 \mu \mathrm{m}$ rms, with respect to a straight line over the length of the LCLS undulator, is achievable using energy scanned beam-based alignment. The electron-tophoton phase error, at $1.5 \AA$, over the full undulator length can be reduced to $\sim 100^{\circ}$, which is completely adequate. Prior to the alignment procedure, initial BPM and quadrupole uncorrelated misalignment levels of $100 \mu \mathrm{m}$ rms, plus $300 \mu \mathrm{m}$ rms correlated component are correctable. Effects such as BPM and magnet mover calibration errors, quadrupole field errors, energy errors, and undulator pole field errors have been included in a detailed simulation which demonstrates this level can be achieved realistically with reasonable confidence. Only one random seed has been presented here as an example, but several seeds have been run with similar success. An estimated time of 8-12 hrs is required to accomplish the initial beam-based alignment. Based on ground motion studies in the FFTB tunnel, one 2.5-hour iteration of the procedure will be required approximately once per month to maintain the alignment.

\subsection{X-Ray Diagnostics}

\subsubsection{Introduction}

The x-ray diagnostics consists of tools for measurement and analysis of the spectral and spatial characteristics of spontaneous and SASE radiation along the undulator line. The main goal is to measure the absolute flux of x-rays as a function of the distance along the undulator line. Furthermore, the diagnostics permits verification of the overlapping of radiation cones from different undulator segments. 


\subsubsection{Undulator Cell Structure}

The LCLS undulator line is designed as a set of standard cells shown schematically in Figure 8.53. Each half-cell consists of six 3.42-m long undulator segments with two $187 \mathrm{~mm}$ breaks and one $421 \mathrm{~mm}$ break between undulator segments. The short breaks are filled with the focusing or defocusing lens, electron BPMs and steering coils. The last break includes those same components and additional x-ray diagnostics.

The length of the undulator has been optimized based on the requirement of operating the LCLS with electron beam energies over the range of $4.5 \mathrm{GeV}$ to $14.5 \mathrm{GeV}$. Also, it has been shown independently that the chosen cell length corresponds to the optimum position of the BPMs [61].
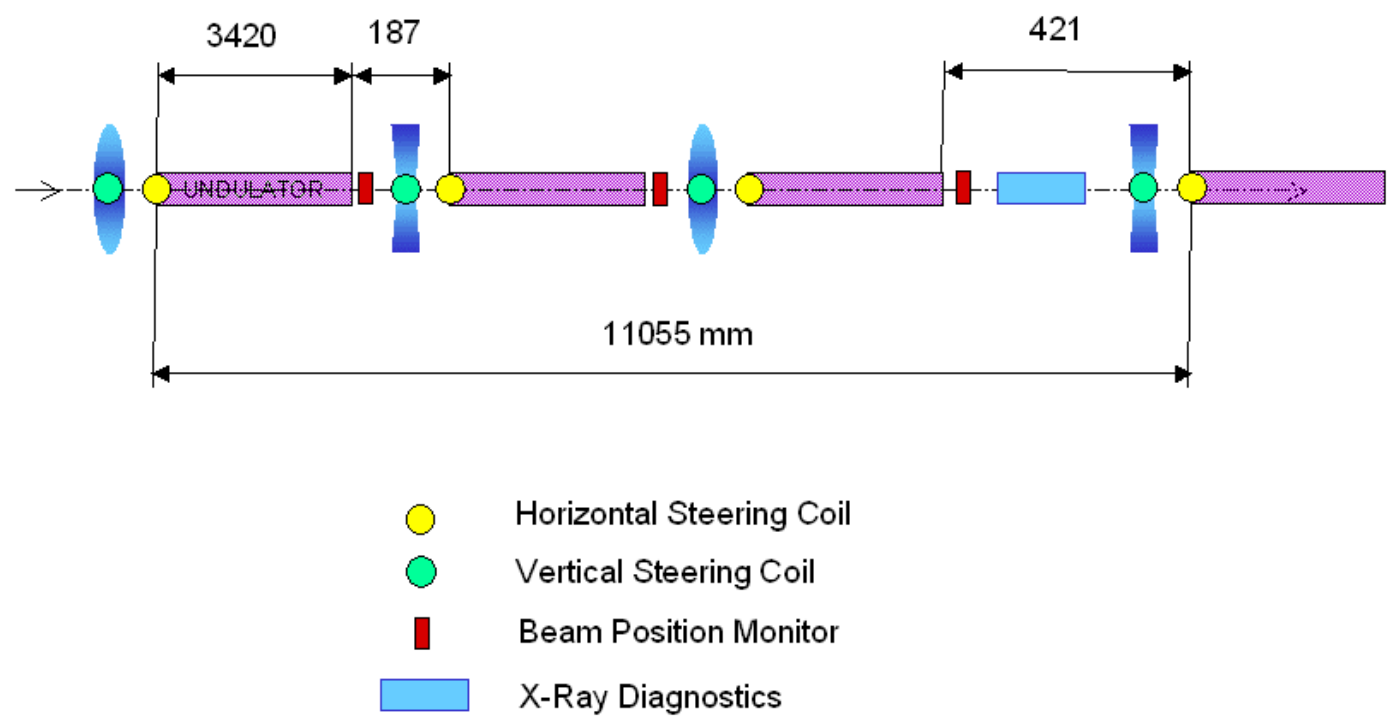

Figure 8.53 Cell structure of the LCLS undulator

\subsubsection{X-Ray Diagnostics Specifications and Experimental Set-up}

In order to utilize the x-ray diagnostics efficiently and meaningfully, the diagnostics should satisfy the following requirements:

- one shot sensitivity for all types of spectral, flux and spatial measurements;

- several microradian angular resolution;

- accuracy of the absolute flux measurement better than $10 \%$; and

- $\quad$ many 4 orders of magnitude in dynamic range.

An experimental set-up that meets all the above requirements is shown in Figure 8.54. It consists of a $200-\mu$ m-thick diamond (111) crystal monochromator, x-ray CCD cameras and a PIN diode [62]. The monochromator has a bandpass of $10^{-4}$ and can analyze $\mathrm{x}$-ray radiation in the

\footnotetext{
4 For the LEUTL FEL attenuation of the radiation by as much as 9 orders of magnitude was necessary and has been achieved.
} 
energy range from 4 to $9 \mathrm{keV}$. The combination of the monochromator and the CCD camera as an area detector is used for the spectral and spatial (angular) measurements, whereas the combination of the monochromator with the PIN diode provides absolute flux measurements along the undulator line. The OTR part of the diagnostics is described in Section 8.11.4.

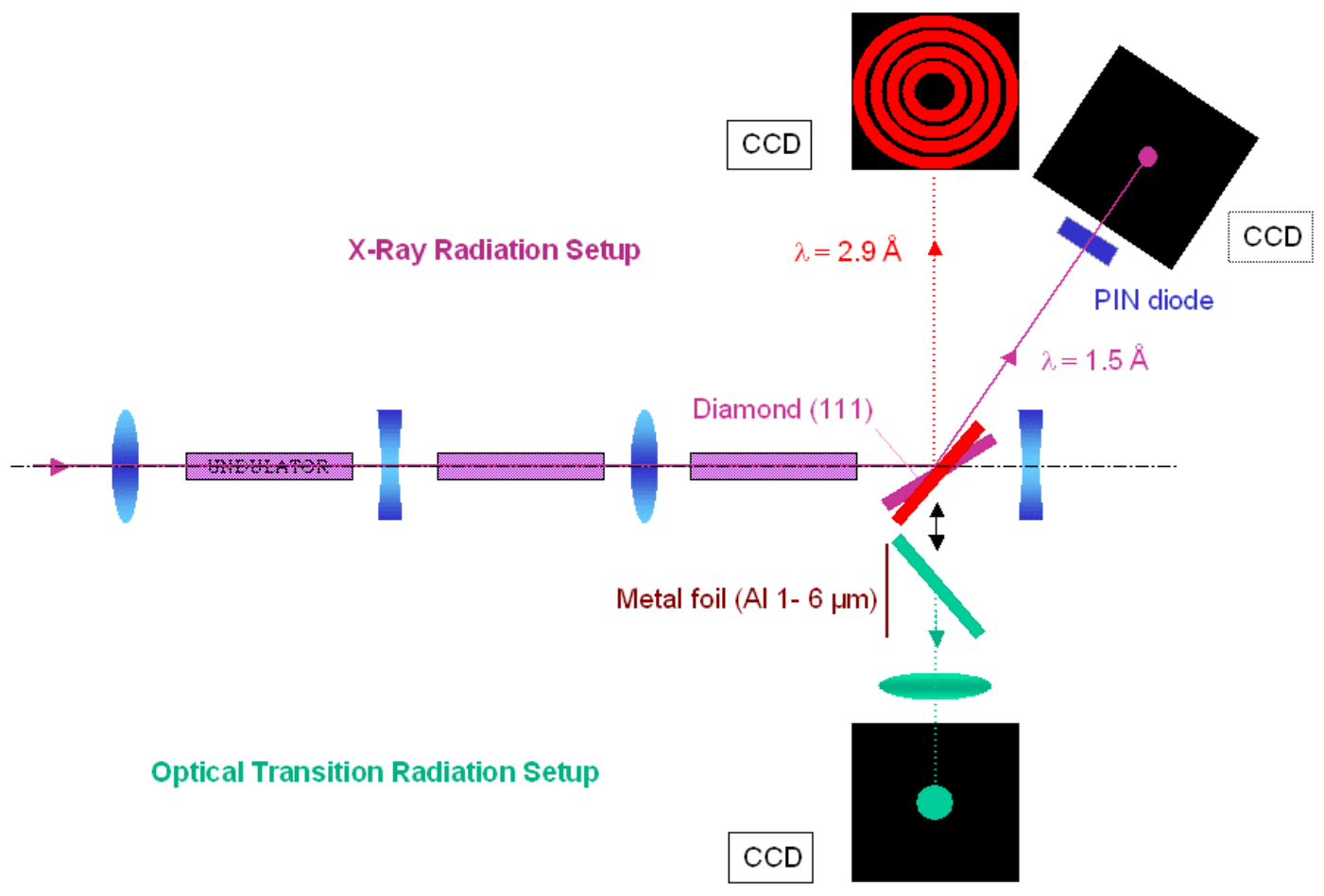

Figure 8.54 X-Ray/ OTR Diagnostics setup

\subsubsection{On-Axis X-Ray Diagnostics}

The purpose of the on-axis x-ray diagnostics is to measure in absolute units the increase in the spectral flux along the undulator line and to provide information about the spatial distribution of radiation. In order to analyze the performance of the on-axis x-ray diagnostics, a set of calculations has been carried out using the program SRW [63] for near and far field cases.

For on-axis x-ray diagnostics, the monochromator is set at a Bragg angle of 21.35 degrees which selects the fundamental undulator harmonic at the energy of $8.27 \mathrm{keV}$. Calculations of the spectral flux generated by the undulator cell and transmitted through the monochromator yield $5 \cdot 10^{6}$ photons/shot, or $1.6 \mathrm{nC}$ charge registered by the silicon PIN diode. A cooled silicon PIN diode could detect a single x-ray photon. The dynamic range of the PIN diode covers ten orders of magnitude without any additional filtering.

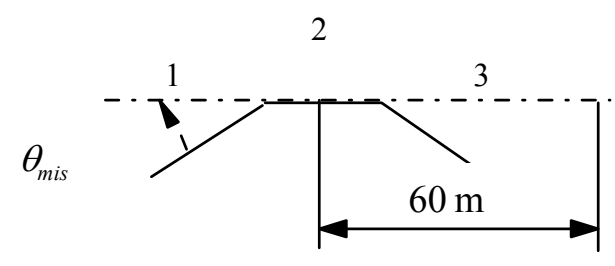


Figure 8.55

Undulator trajectories in the cell.

The x-ray diagnostics setup will provide absolute measurements (within 10\%) of spectral flux after each undulator cell. The growth rate could be derived from the measured flux at successive diagnostics stations. The growth rate of the flux is a valuable means of evaluating and studying the development of the SASE process.

The spatial flux distribution for one undulator cell is the superposition of radiation from three undulator segments. In order to evaluate the sensitivity of the x-ray diagnostics to the angular misalignment of undulator segments, e-beam trajectories in the first and third undulator segments in the cell are given a missteering angle $\theta_{\text {mis }}$ (Figure 8.55). A series of calculations of spontaneous radiation for a cell have been performed for a nominal electron beam emittance of $0.05 \mathrm{~nm} \cdot \mathrm{rad}$ at a photon energy of $8.29 \mathrm{keV}$ and at a distance of $60 \mathrm{~m}$ from the undulator cell. This $8.29 \mathrm{keV}$ energy is detuned slightly, towards higher energy, from the fundamental energy of $8.27 \mathrm{keV}$, in order to reduce the angular divergence of the undulator radiation. The calculated spatial intensity distribution is shown in Figure $\mathbf{8 . 5 6}$ for a missteering angle of $4 \mu \mathrm{rad}$, and calculated horizontal profiles are shown in Figure $\mathbf{8 . 5 7}$ for missteering angles of 2, 3, and $4 \mu \mathrm{rad}$. The figures show that a missteering of $4 \mu \mathrm{rad}$ can be clearly discerned if the diagnostics station is far enough from the undulator cell.

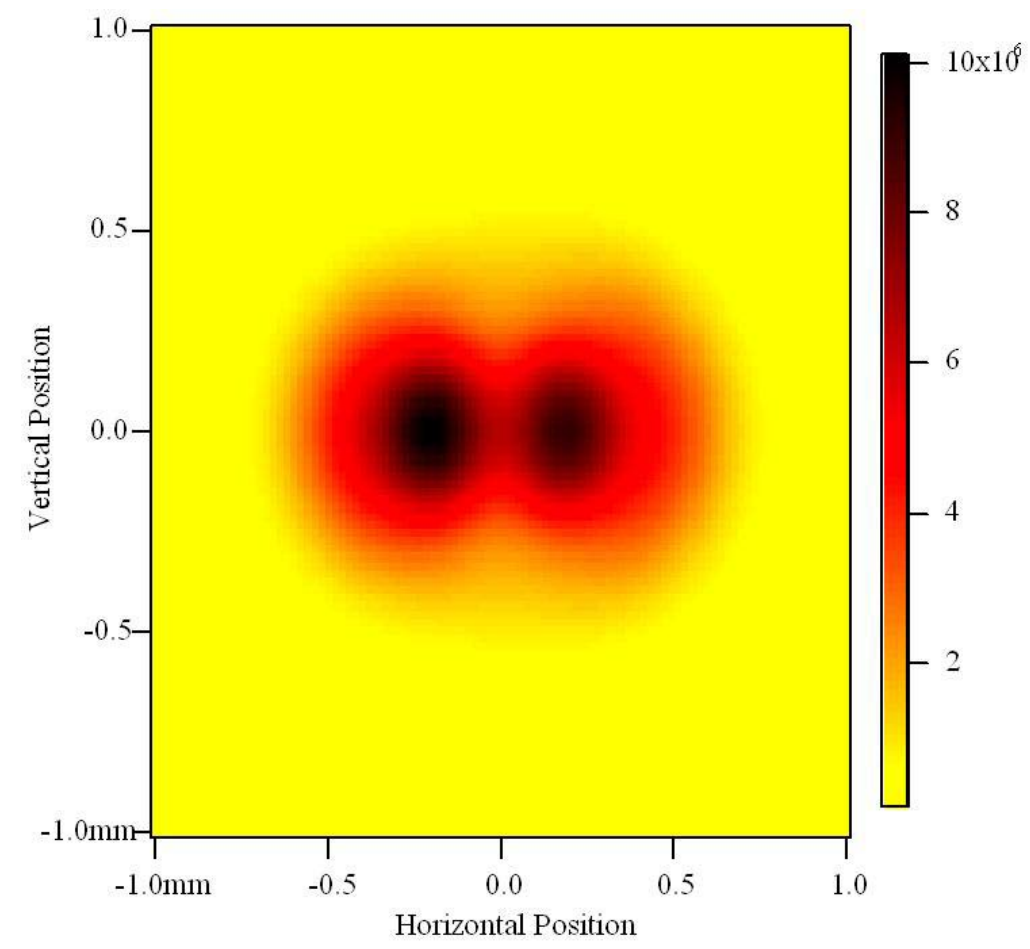

Figure 8.56 Calculated spatial distribution of the undulator radiation from a three-undulator cell with a missteering $\theta_{\text {mis }}=4 \mu \mathrm{rad}$, an electron beam emittance of $0.05 \mathrm{~nm} \cdot \mathrm{rad}$, and a photon energy of $8.29 \mathrm{keV}$, at $60 \mathrm{~m}$ from the undulator segments. 
Measurements of the spatial distribution of radiation generated in each undulator cell will complement the electron beam-based alignment, but will not substitute for it. x-ray diagnostics will be especially useful in the first steps of the beam-based alignment procedure.

Knowing the calculated flux distribution and the efficiency of the x-ray diagnostics, one can estimate the required sensitivity of the system at $60 \mathrm{~m}$, which was found to be $5 \cdot 10^{2}$ electrons/pixel/shot for a $7 \times 7 \mu \mathrm{m}^{2}$ pixel CCD. That is substantially above the CCD noise level. Appropriate filtering in front of the CCD will keep the flux in the last diagnostics stations within the dynamic range (about $10^{5}$ e per pixel) of the detector.

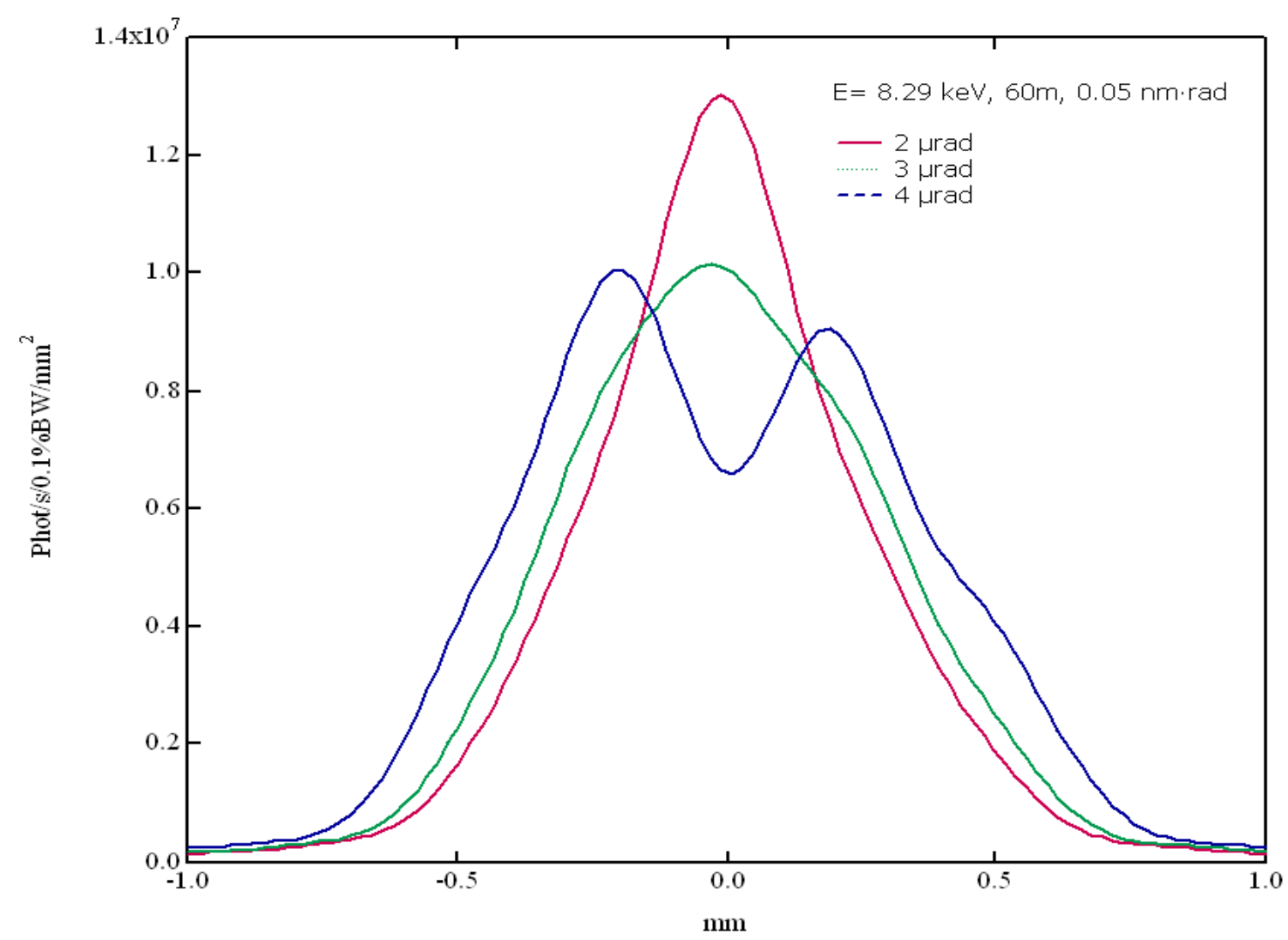

Figure 8.57 Horizontal profiles of the undulator radiation for missteering angles $\theta_{\text {mis }}$ of 2,3 and 4 rad.

When the on-axis diagnostics stations are in use, the electron beam will always be hitting the diamond crystal in one of the diagnostics stations. The electron beam energy loss in a $200-\mu \mathrm{m}-$ thick diamond crystal is equal to $0.25 \mathrm{MeV} /$ particle and independent of whether the beam energy is 4.5 or $14.5 \mathrm{GeV}$. As a result, a $1 \mathrm{nC}$ electron beam deposits an average power of $30 \mathrm{~mW}$ for a $120 \mathrm{~Hz}$ repetition rate. Finite element analysis shows that the most simple cooling design (clamped crystal, no coolant) will lead to a $0.06 \mu \mathrm{rad}$ slope error on the crystal, which is negligibly small compared with the $10 \mu \mathrm{rad}$ width of the crystal rocking curve. After exiting the crystal, the electron beam will have an angular spread of $40 \mu \mathrm{rad}(\mathrm{rms})$. 
Recent experimental data taken at the FFTB facility at SLAC show that a diamond crystal can withstand a high energy, highly focused electron beam without any visually observed damage [64].

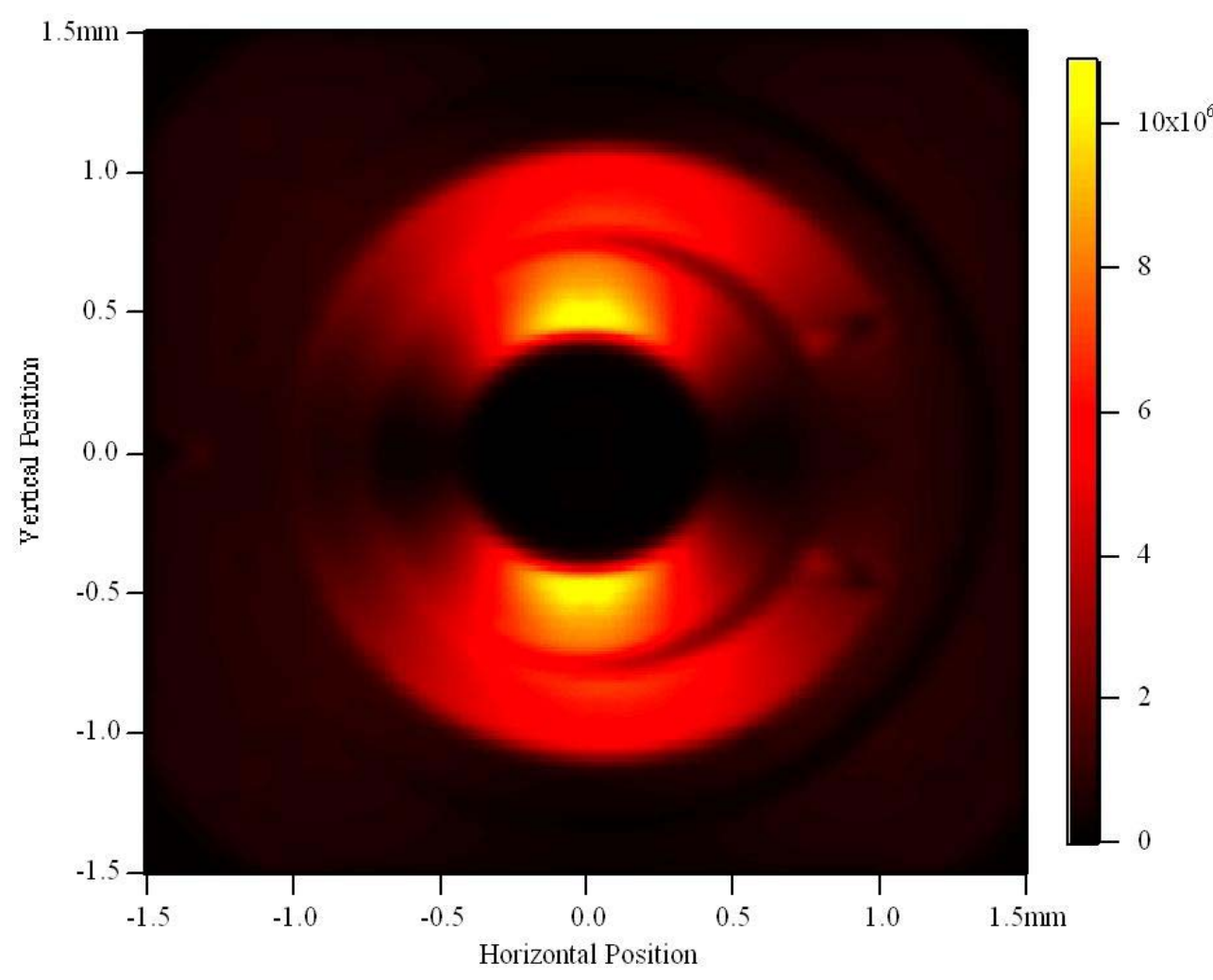

Figure 8.58 Calculated spatial distribution of the red-shifted radiation (at $4.25 \mathrm{keV}$ ) from two undulator segments, at $6 \mathrm{~m}$ from the undulator segments, and with a missteering angle $\theta_{\text {mis }}$ of $10 \mu \mathrm{rad}$ between the undulator segments.

\subsubsection{Off-Axis X-Ray Diagnostics}

The off-axis, "red-shifted" x-ray diagnostics is complementary to the on-axis diagnostics. The crystal monochromator in this case can have a small hole to let the electron beam go through unperturbed. This allows radiation to be observed from each undulator cell without any trajectory distortion. A similar technique was successfully implemented for the LEUTL diagnostics, where a mirror was used instead of the crystal.

For the "red-shifted" radiation at $4.25 \mathrm{keV}$, the crystal monochromator has a Bragg angle of 45 degrees and the angle between the CCD camera and the radiation is 90 degrees. The calculated spatial distribution of the undulator radiation at a distance of $6 \mathrm{~m}$ from two undulator segments is shown in Figure 8.58, and the horizontal profile in Figure 8.59. An intentional angular misalignment of $\theta_{\text {mis }}=10 \mu \mathrm{rad}$ has been introduced in the trajectory between the two undulator segments. 


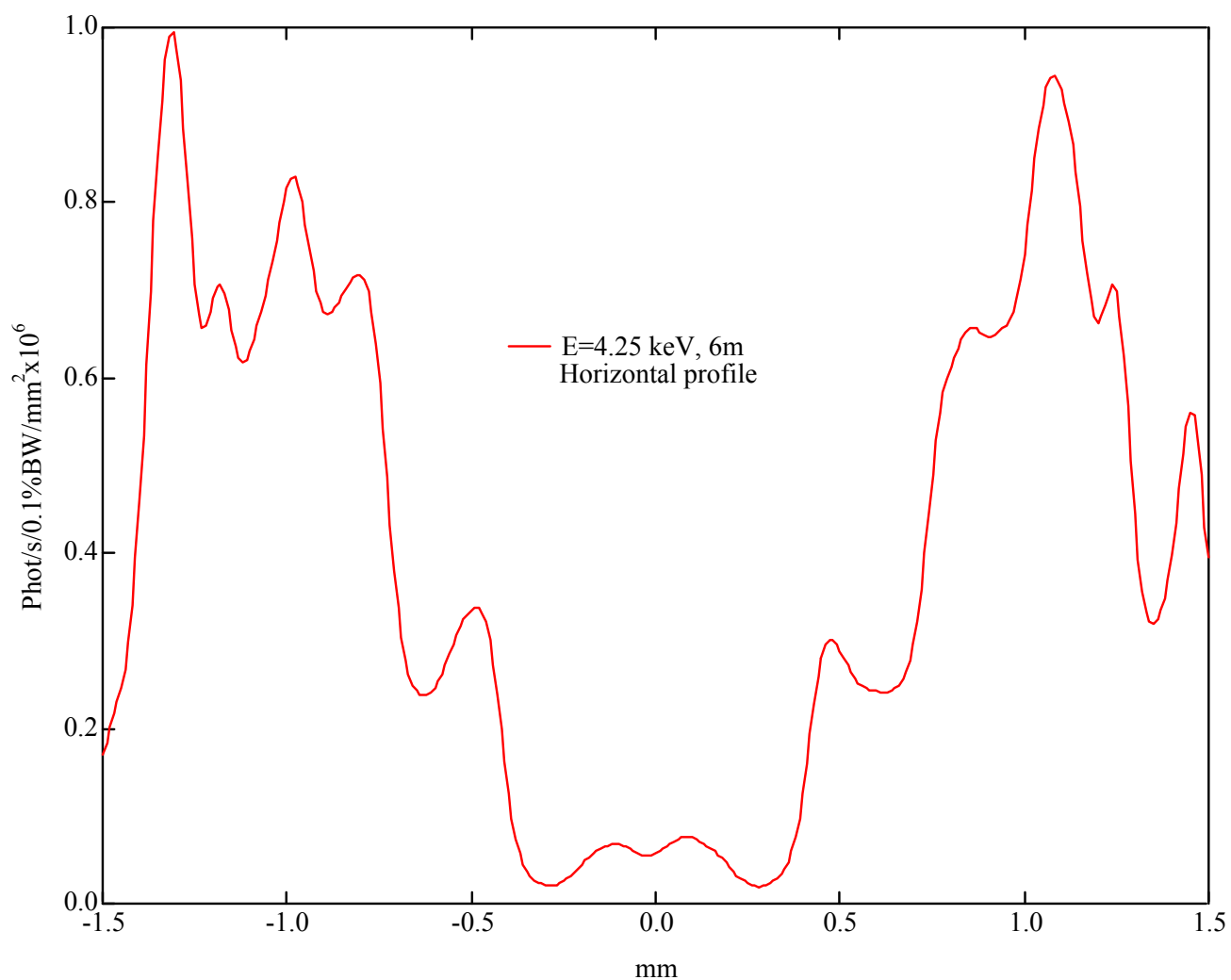

Figure 8.59 Calculated horizontal profile of the red-shifted radiation (at $4.25 \mathrm{keV}$ ) from two undulator segments, at $6 \mathrm{~m}$ from the undulator segments, and with a missteering angle $\theta_{\text {mis }}$ of $10 \mu \mathrm{rad}$ between the undulator segments.

Calculation of the detection efficiency shows that the CCD camera will get $4 \cdot 10^{2} \mathrm{e}^{-} /$pixel per shot for the $20 \times 20 \mu \mathrm{m}^{2}$ size pixel, which is again substantially above the CCD noise level.

\subsubsection{Effects of the Electron Beam Emittance}

The influence of the beam emittance on the spatial distribution of the radiation has been studied. Figure 8.60 shows the calculated horizontal profiles of undulator radiation from two undulator segments with a missteering angle of $4 \mu \mathrm{rad}$ between the undulator segments, for different values of the electron beam emittance.

Calculations were also done of the spatial intensity distribution and horizontal profile from a three-segment cell at $60 \mathrm{~m}$ from the undulator segments, with a missteering $\theta_{\text {mis }}$ of $10 \mu \mathrm{rad}$, and at a photon energy of $8.3 \mathrm{keV}$ (detuned from the $8.27 \mathrm{keV}$ fundamental to higher energy). The spatial intensity distribution for an electron beam emittance of $0.05 \mathrm{~nm}-\mathrm{rad}$ is shown in Figure 8.61, and the horizontal profiles for three different beam emittances are shown in Figure 8.62. Differences between the $0.025,0.05$, and $0.1 \mathrm{~nm}$-rad emittances are clearly observable. 


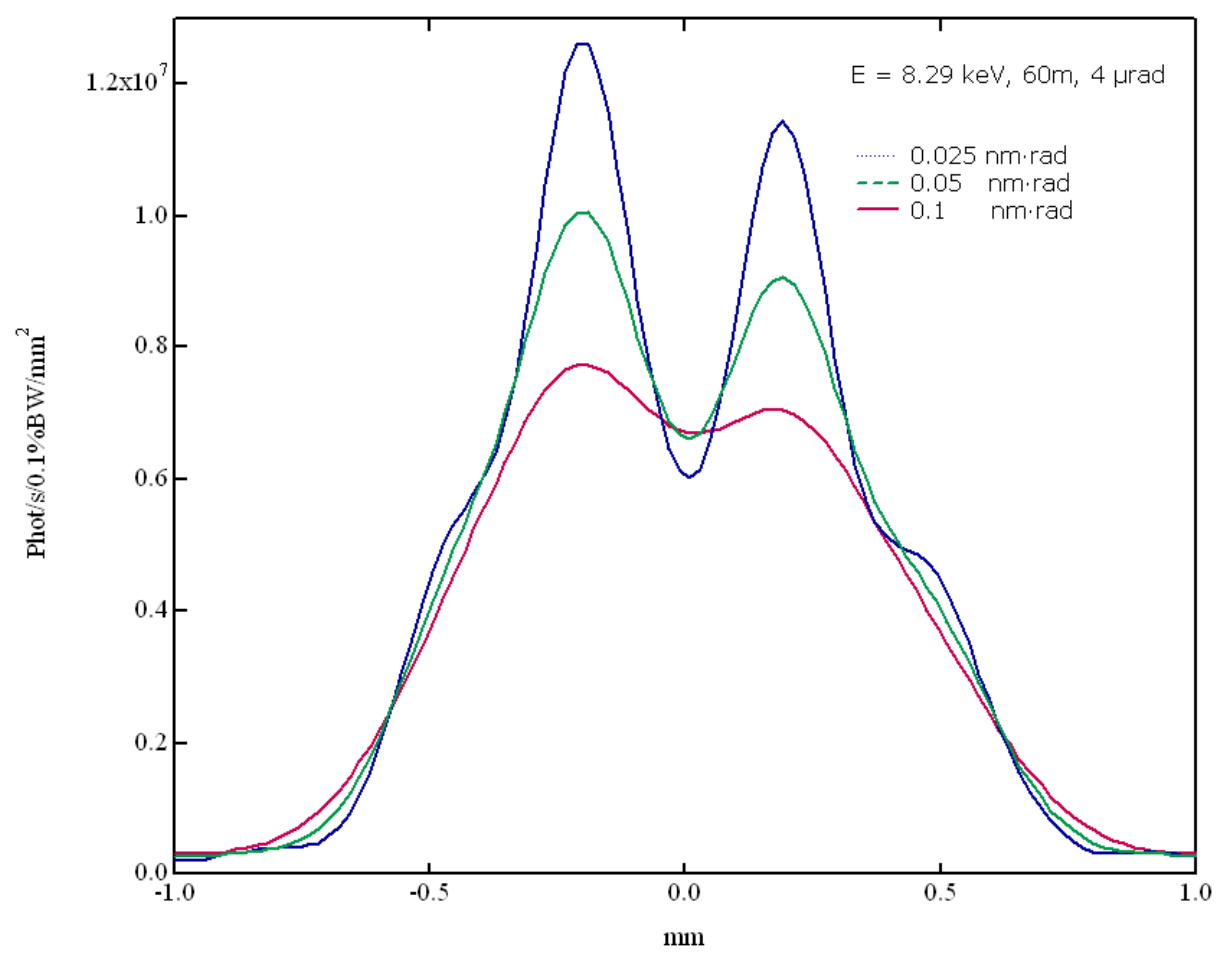

Figure 8.60 Calculated horizontal profile of the on-axis $(8.29 \mathrm{keV})$ undulator radiation from two undulator segments with a missteering angle $\theta_{\text {mis }}$ of $4 \mu \mathrm{rad}$ between the undulator segments, at $60 \mathrm{~m}$ from the undulator segments. The different curves are for different electron beam emittances.

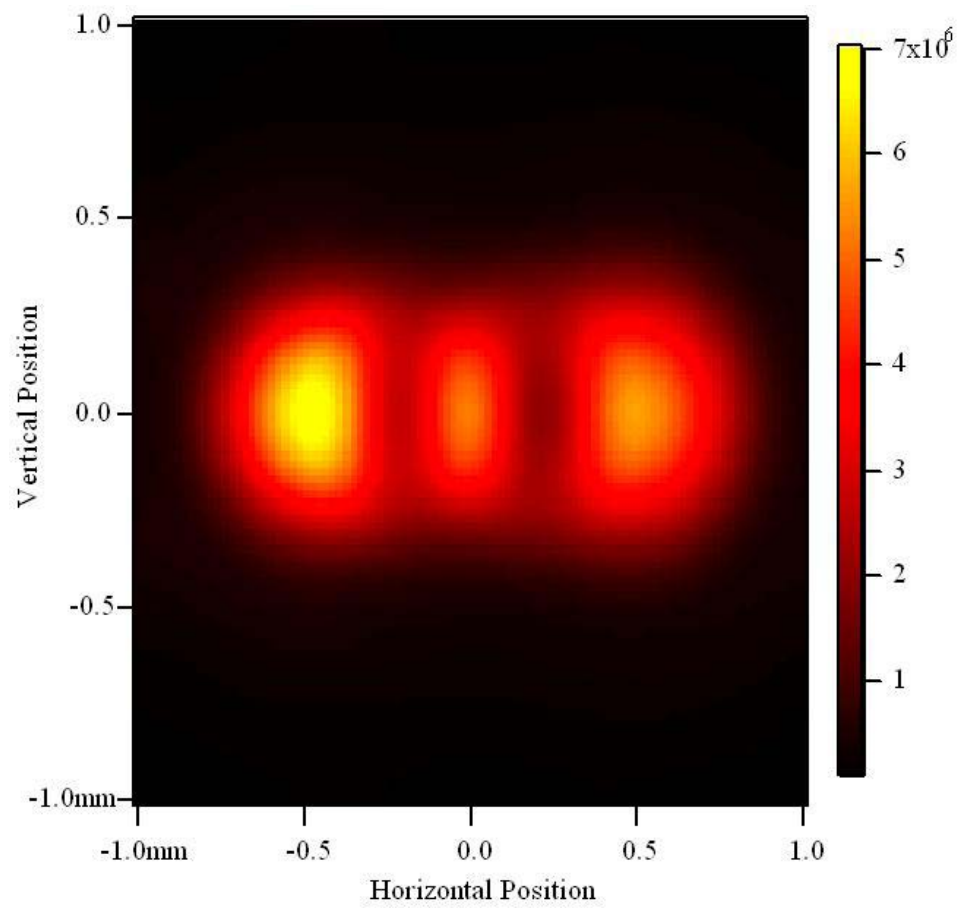

Figure 8.61 Calculated spatial distribution of the undulator radiation from a three-undulator cell with a missteering angle $\theta_{\text {mis }}$ of $10 \mu \mathrm{rad}$, at $8.3 \mathrm{keV}$ (slightly detuned from the $8.27 \mathrm{keV}$ fundamental to higher energy), and with an electron beam emittance of $0.05 \mathrm{~nm}$-rad, at $60 \mathrm{~m}$ from the undulator segments. 


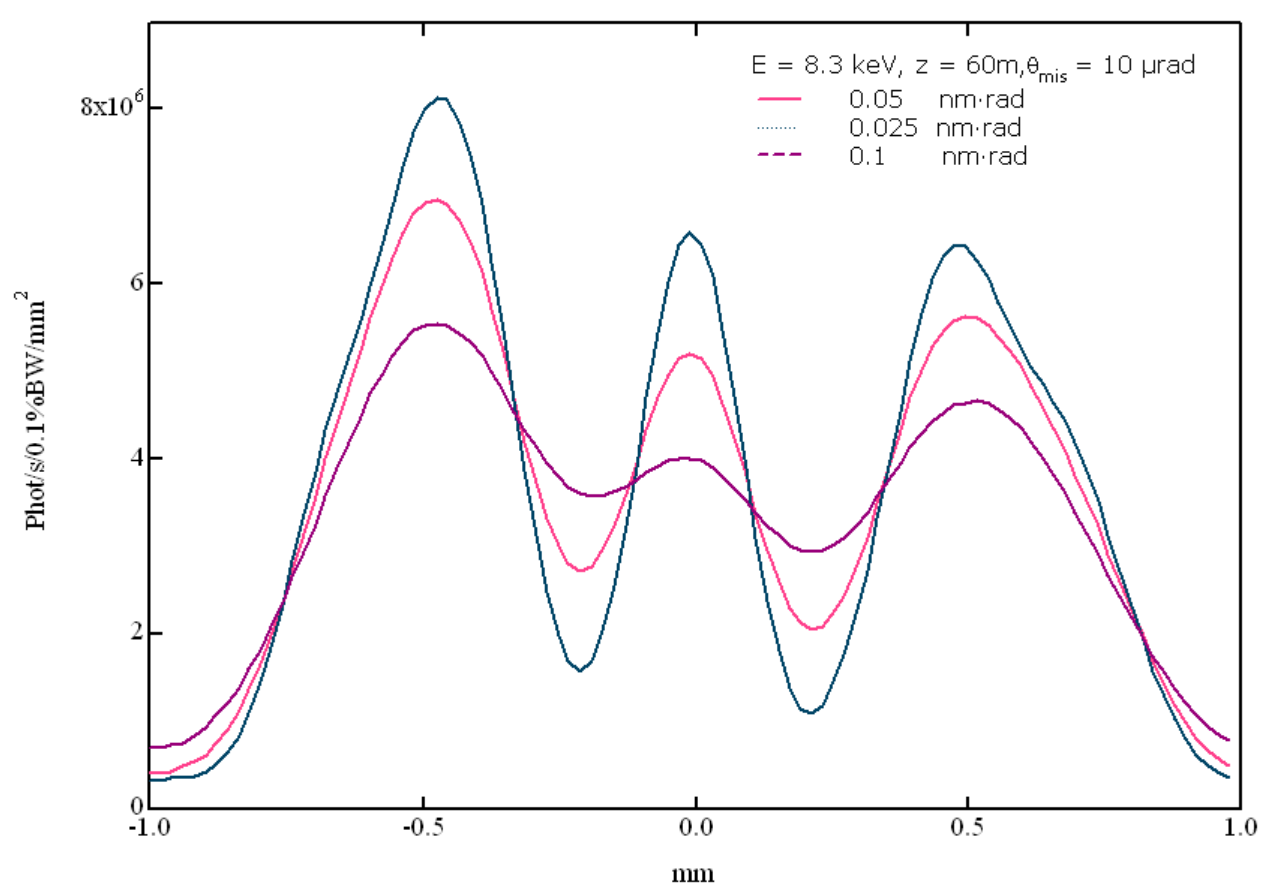

Figure 8.62 Calculated horizontal profiles of the undulator radiation from a three-segment cell with a missteering angle $\theta_{\text {mis }}$ of $10 \mu \mathrm{rad}$, at $8.3 \mathrm{keV}$ (slightly detuned from the $8.27 \mathrm{keV}$ fundamental to higher energy), at $60 \mathrm{~m}$ from the undulator segments. Curves are shown for three different electron beam emittances: $0.05,0.025$, and $0.1 \mathrm{~nm}$-rad.

\subsection{References}

1 R.J. Dejus, O.A. Shevchenko, and N.A. Vinokurov, Nucl. Instrum. Methods A 429 (1999) 225.

2 R.J. Dejus, O.A. Shevchenko, and N.A. Vinokurov, Nucl. Instrum. Methods A 445 (2000) 19.

3 K. Halbach, J. de Physique 44, Colloque C1, supplement to \#2 (1983), C1.

4 M. Xie, IEEE Proceedings of the 1995 Particle Accelerator Conference, (IEEE, 1996), p. 183.

$5 \quad$ K.-J. Kim and M. Xie, Nucl. Instrum. Methods A 331 (1993) p. 359.

6 E. Gluskin, C. Benson, R.. Dejus, P.K. Den Hartog, B.N. Deriy, O.A. Makarov, S.V. Milton, E.R. Moog, V.I. Ogurtsov, E.M. Trakhtenberg, K.E. Robinson, I.B. Vasserman, N.A. Vinokurov, and S. Xu, Nucl. Instrum. Methods A 429 (1999) p. 358.

$7 \quad$ P. Emma, R. Carr, and H.-D. Nuhn, Nucl. Instrum. Methods A 429 (1999) p. 407.

$8 \quad$ M. Xie, Nucl. Instrum. Methods A 445 (2000) p. 59.

9 J. Chavanne, P. Elleaume, and P. Van Vaerenbergh, "Partial Demagnetization of ID6 and Dose Measurements on Certain IDs," ESRF Machine Technical Note 1-1996/ID, (Jan. 1996).

10 P. Elleaume, personal communication.

11 T. Ikeda and S. Okuda, Nucl. Instrum. Meth. Phys. Res. A407 (1998) 439.

12 R.D. Brown and J.R. Cost, J. Appl. Phys. 63, 3537 (1988) and IEEE Trans. Magn. 25, 3117 (1989).

13 Eckhart and Drake, "Analysis of Heat and Mass Transfer"

14 B. Rossi, High Energy Particles 
15 K. Bane and P. Wilson, Proceedings of the 11th Int. Conf. on High Energy Accelerators, CERN (Birkhuser Verlag, Basel, 1980), p. 592.

16 A. Berman, "Vacuum Engineering, Calculations, Formulas, and Solved Exercises," Academic Press (1992).

17 D. Behne, "Outgassing Test Results from PEP-II Standard Straight Section Vacuum Chambers," (1997).

18 C. Forester, et al., "Photon Stimulated Desorption (PSD) Measurements of Welded Copper Beam Chambers for the PEP-II Asymetric B-Factory," 4th European Particle Accelerator Conference (1994).

19 M. Sullivan, "A Method for Calculating Pressure Profiles in Vacuum Pipes," PEP-II AP Note No. 694 (1994).

20 A. Chao, "Physics of Collective Beam Instabilities in High Energy Accelerators," John Wiley (1993).

21 K.L.F. Bane, M. Sands, "The Short-Range Resistive Wall Wakefields," Contributed to Micro Bunches: A Workshop on the Production, Measurement and Applications of Short Bunches of Electrons and Positrons in Linacs and Storage Rings, Upton, New York, September 28-30, 1995.

22 K. Bane and M. Sands, "Wakefields of Very Short Bunches in an Accelerating Cavity," Particle Accelerators, Vol. 25, pp. 73-05 (1990).

23 K. L. F. Bane, C. K. Ng, and A. W. Chao, Estimate of the impedance due to wall surface roughness, Report SLAC-PUB-7514, SLAC (1997).

24 G. V. Stupakov, Phys. Rev. ST Accel. Beams 1, 064401 (1998).

25 G. Stupakov, R. E. Thomson, D. Walz, and R. Carr, Phys. Rev. ST Accel. Beams 2, 060701 (1999).

26 A. Novokhatski and A. Mosnier, in Proceedings of the 1997 Particle Accelerator Conference (IEEE, Piscataway, NJ, 1997), pp. 1661-1663.

27 K. L. F. Bane and A. Novokhatskii, The Resonator impedance model of surface roughness applied to the LCLS parameters, Tech. Rep. SLAC-AP-117, SLAC (March 1999).

28 G. V. Stupakov, in T. Roser and S. Y. Zhang, eds., Workshop on Instabilities of High Intensity Hadron Beams in Rings (American Institute of Physics, New York, 1999), no. 496 in AIP Conference Proceedings, pp. 341-350.

29 D. J. Whitehouse, Handbook of Surface Metrology (IOP Publishing, 1994).

30 K. J. Stout, Atlas of Machined Surfaces (Chapman and Hall, 1990).

31 Chatard-Moulin and A. Papiernik, IEEE Trans. Nucl. Sci. 26, 3523 (1979).

32 S. Krinsky, in W. S. Newman, ed., Proc. International Conference on High-Energy Accelerators, Geneva, 1980, CERN, European Lab. for Particle Physics (Birkhäuser Verlag, Basel, Switzerland, 1980), p. 576.

33 R. L. Warnock, An Integro-Algebraic Equation for High Frequency Wake Fields in a Tube with Smoothly Varying Radius, Report SLAC-PUB-6038, SLAC (1993).

34 K. L. F. Bane and G. V. Stupakov, Wake of a rough beam wall surface, Tech. Rep. SLAC-PUB8023, SLAC (December 1998), presented at International Computational Accelerator Physics Conference (ICAP 98), Monterey, CA, 14-18 Sep 1998.

35 G. V. Stupakov, in Physics of, and Science with, the X-ray Free-Electron Laser (2000).

36 M. Dohlus, H. Schlarb, R. Wanzenberg, R. Lorenz, and T. Kamps, Estimation of longitudinal wakefield effects in the TESLA-TTF FEL undulator beam pipe and diagnostic section, Tech. Rep. DESY-TESLA-FEL-98-02, Deutsches Elektronen-Synchrotron, Hamburg, Germany (March 1998). 
37 A. Novokhatsky, M. Timm, and T. Weiland, Single bunch energy spread in the TESLA cryomodule, Tech. Rep. DESY-TESLA-99-16, Deutsches Elektronen-Synchrotron, Hamburg, Germany (March 1999).

38 R. Tatchyn, et al., "Research and Development Toward a 4.5-1.5 ^ Linac Coherent Light Source (LCLS) at SLAC," SLAC-PUB-95-6994 (1995).

39 D.E. Cullen et al., "Tables and Graphs of Photon-Interaction Cross Sections from $10 \mathrm{eV}$ to $100 \mathrm{GeV}$ Derived from the LLNL Evaluated Photon Data Library (EPDL)," Lawrence Livermore National Laboratory, UCRL-504000-Vol.6-Rev.4-Pt.A (1989).

40 L.V. Keldysh, "Ionization in the field of a strong electromagnetic wave," Soviet Physics JETP, Vol. 20, no. 5 (1965).

41 T.O. Raubenheimer, private communication (1997).

42 P. Chen, private communication (1997).

43 T.O. Raubenheimer and F. Zimmermann, "A Fast Beam-Ion Instability in Linear Accelerators and Storage Rings," Physical Review E, Vol. 52, 5, pp. 5487-5498 and SLAC-PUB-6740 (1995).

44 S.V. Milton, E. Gluskin, N.D. Arnold, C. Benson, W. Berg, S.G. Biedron, M. Borland, Y.-C. Chae, R.J. Dejus, P.K. Den Hartog, B. Deriy, M. Erdmann, Y.I. Eidelman, M.W. Hahne, Z. Huang, K.-J. Kim, J.W. Lewellen, Y. Li, A.H. Lumpkin, O. Makarov, E.R. Moog, A. Nassiri, V. Sajaev, R. Soliday, B.J. Tieman, E.M. Trakhtenberg, G. Travish, I.B. Vasserman, N.A. Vinokurov, G. Wiemerslage, and B.X. Yang, "Exponential Gain and Saturation of a Self-Amplified Spontaneous Emission Free-Electron Laser", Science 292, 2037 (2001). Also published in Sciencexpress online at www.sciencexpress.org on 17 May 2001 as 10.1126/science.1059955.

45 S. Kim, "Calculating BPM Coefficients with Green's Reciprocation Theorem", Advanced Photon Source light source note LS-278, March 1999, URL

$$
\text { http://www.aps.anl.gov/techpub/1snotes/1s278/1s278.ps. }
$$

46 R. Lorenz, "Cavity Beam Position Monitors", proceedings of the 1998 Beam Instrumentation Workshop, Stanford, USA, URL

http://www.slac.stanford.edu/pubs/confproc/biw98/lorenz1.pdf.

47 R.O. Hettel et al., "Investigation of Beam Alignment Monitor Technologies for the LCLS FEL Undulator", proceedings of the 1998 Beam Instrumentation Workshop, Stanford, USA, URL:

http://www.slac.stanford.edu/pubs/confproc/biw98/hettel.pdf.

48 S. Smith, SLAC, private communication.

49 A.H. Lumpkin et al., "Optical Techniques for Electron-Beam Characterizations on the APS SASE FEL Project", Nucl. Instr. And Meth. A429, 336 (1999).

50 J.-C. Denard et al., "Experimental Diagnostics Using Optical Transition Radiation at CEBAF", Proc. of the 1994 Beam Instrumentation Workshop, AIP Conf. Proc. \# 333, 224 (1995).

51 P. Catravas et al., "Beam Profile Measurement at $30 \mathrm{GeV}$ using Optical Transition Radiation", Proc. of the 1999 Particle Accelerator Conference, Vol. 3, 2111 (1999).

52 L. Wartski et al., IEEE Trans. Nucl. Sci., Vol NS-22, June 1975, p.15.

53 A. Fisher, "Instrumentation and Diagnostics for PEP-II", Proceedings of the 1998 Beam Instrumentation Workshop, Stanford, CA, (1998), URL:

http://www.slac.stanford.edu/pubs/confproc/biw98/fisher1.pdf .

54 A. Pietryla, "Design of the Cerenkov Radiation Detection System for the Advanced Photon Source Storage Ring", PAC01, June 18-22, 2001, Chicago, IL, 
http://pacwebserver.fnal.gov/papers/Tuesday/AM_Poster/TPAH304.pdf

55 P. Emma, "Beam Based Alignment of Sector-1 of the SLC Linac", SLAC-PUB-5787, March 1992.

56 This technique was suggested by C. Adolphsen.

57 This misalignment component was suggested by C. Adolphsen.

58 LCLS Design Study Report, SLAC-R-521 and UC-414, Rev. Dec. 1998.

59 V. Shiltsev, "Space-Time Ground Diffusion: The ATL Law for Accelerators", 4th International Workshop on Accelerator Alignment (IWAA95), Tsukuba, Japan, 14-17 Nov. 1995.

60 R. Assmann, et. al., "Beamline Stability Measurements with a Stretched Wire System in the FFTB", 18th International Linac Conference (Linac 96), Geneva, Switzerland, 26-30 Aug. 1996.

61 P. Emma, "Electron Phase Slip in an Undulator with Dipole Field and BPM Errors", Proceedings of the $18^{\text {th }}$ ICFA Beam Dynamics Workshop on Future Light Sources, "Physics of and Science with the X-Ray Free-Electron Laser", held 10-15 Sept. 2000, Arcidosso, Italy.

62 The diagnostics set up for the LCLS undulator line is, in principle, quite similar to that for the Low Energy Undulator Test Line (LEUTL) at the Advanced Photon Source [see E. Gluskin, et.al., "The magnetic and diagnostics systems for the APS SASE FEL", Nucl. Instrum. Methods A 429 (1999), pp.358-364]. The LEUTL diagnostics were developed for visible light, however, whereas the LCLS diagnostics are for X-rays.

63 O. Chubar and P. Elleaume, "Accurate and Efficient Computation of Synchrotron Radiation in the Near Field Region", Proc. of the 1998 European Particle Accelerator Conference EPAC98, 22-26 June 1998, p. 1177-1179.

64 W.-K.Lee, R. Iverson, P. Krejcik, D. McCormick, Summary of the diamond test with SLAC focused beam, October 2000. 


\section{X-Ray Beam
Transport and
Diagnostics}

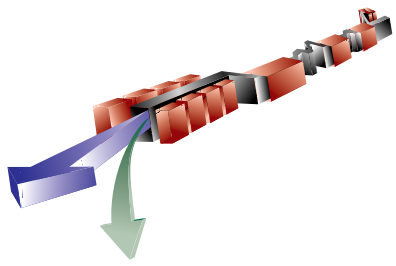

\section{TECHNICAL SYNOPSIS}

The photon beam transport system will deliver the LCLS radiation to the experiments. It is scientifically desirable to perform certain types of experiments relatively close to the x-ray source (the undulator), while others are better carried out at a distance of several hundred meters from the source. Therefore a long beamline is planned, with experimental areas grouped in two experimental halls, beginning about $50 \mathrm{~m}$ and about $400 \mathrm{~m}$ from the source. The beamline passing from the source through these halls will transport the FEL beam in high vacuum to the experimental apparatus. Several experimental stations will be built in these halls, and there is room for future expansion. However, only one experiment will be active at a time, and beam stops will be inserted to shield the areas downstream from the active experiment.

The $x$-ray optics system has the job of filtering the intensity, spectral, and spatial characteristics of the FEL beam as needed for the experiments. Most of the techniques that will be applied to perform the filtering (slits, absorbers, mirrors, monochromators) are commonly used at synchrotron sources. However, the LCLS presents special concerns due to the very high peak power density in the FEL beam. The LCLS optics system has been conservatively designed to perform under conditions of extreme peak power. An experimental program for studying highpower effects is planned.

The beam transport and optics systems will be used together to enable an extensive array of diagnostics measurements on the FEL $x$-ray pulses. The diagnostics instruments will be used to characterize the FEL beam during the initial commissioning of LCLS, and also to monitor the performance of LCLS during experimental operation.

\subsection{Introduction}

\subsubsection{Objectives}

The x-ray beam transport system comprises the photon beamline components between the undulator and the User experiments. The design is based on the User and FEL physics requirements for the $\mathrm{x}$-ray optics and x-ray diagnostics, as well as the facility requirements (i.e., the facility protocols and guidelines). The User and FEL physics requirements are discussed in Chapter 3.

The chapter begins with a presentation of general considerations for the design of optical elements and beam transport. Section 9.2 then gives a detailed discussion of the beamline layout and the optical components included. Section 9.3 describes the mechanical support and vacuum 
techniques to be used throughout the beamline. Section 9.4 presents the plans for beam diagnostics that will be used for initial studies of the FEL process, and for beam characterization during User experiments.

\subsubsection{General Considerations}

\subsubsection{Beam Characteristics}

In translating the User and facility requirements to hardware, the attributes of the FEL x-ray output must be considered. Detailed properties of both the coherent and spontaneous radiation have been calculated. The characteristics, that are most relevant for the beam transport and optics design (including power density on the optical elements), are shown in Table 9.1 for locations approximating the front of Hall A, and Hall B.

Table 9.1 Characteristics of the FEL $x$-ray beam

\begin{tabular}{|c|c|c|c|c|}
\hline \multirow[t]{2}{*}{ FEL photon energy } & \multicolumn{2}{|c|}{$0.828 \mathrm{keV}$ (4.54 GeV electrons) } & \multicolumn{2}{|c|}{ 8.27 keV (14.35 GeV electrons) } \\
\hline & FEL fundamental & Spontaneous & FEL fundamental & Spontaneous \\
\hline Energy per pulse (mJ) & 3 & 1.4 & 2.5 & 22 \\
\hline Peak power (GW) & 11 & 4.9 & 9 & 81 \\
\hline Photons/pulse & $23 \times 10^{12}$ & & $1.9 \times 10^{12}$ & \\
\hline Divergence ( $\mu$ rad FWHM) & 9 & 780 & 1 & 250 \\
\hline $\begin{array}{l}\text { Spot size at } 50 \mathrm{~m} \\
\text { Hall A }(\mu \mathrm{m} \text { FWHM })\end{array}$ & 610 & $\begin{array}{l}\text { Limited by } \\
\text { apertures }\end{array}$ & 130 & $\begin{array}{l}\text { Limited by } \\
\text { apertures }\end{array}$ \\
\hline $\begin{array}{l}\text { Spot size at } 400 \mathrm{~m} \\
\text { Hall B ( } \mu \mathrm{m} \text { FWHM) }\end{array}$ & 4400 & & 570 & \\
\hline $\begin{array}{l}\text { Peak energy density at } 50 \mathrm{~m} \\
\text { Hall } \mathrm{A}\left(\mathrm{J} \mathrm{cm}^{-2}\right)\end{array}$ & 0.59 & & 11.9 & \\
\hline $\begin{array}{l}\text { Peak energy density at } 400 \mathrm{~m} \\
\text { Hall B }\left(\mathrm{J} \mathrm{cm}^{-2}\right)\end{array}$ & 0.01 & & 0.57 & \\
\hline
\end{tabular}

Due to its comparatively large divergence, most of the spontaneous radiation will intersect the walls of the undulator beam pipe or the first fixed mask of the optical system, and will not be transmitted into the experimental Halls. The on-axis spontaneous radiation that will reach the Halls consists mostly of odd harmonics of the undulator fundamental, with a spectral flux about five orders of magnitude below that of the FEL fundamental [1].

One of the principal design goals of the LCLS optics system is to contain the main photon beam entirely within the beamline vacuum pipe under all conditions. Because of its small divergence, this goal is not difficult to achieve without limiting the passage of the FEL beam. The spontaneous radiation must be limited, and this will be achieved by placing apertures (fixed masks) at the entrance points of the experimental Halls. 


\subsubsection{Photon-Induced Damage}

Only the coherent light poses a problem; the spontaneous emission is divergent and will be reduced by upstream apertures. The spontaneous radiation is also mostly at larger energies than the fundamental, and is not strongly absorbed in optical components.

\section{Normal Incidence}

A material exposed to the LCLS FEL radiation at normal incidence will experience an unprecedented peak x-ray power density. X-ray absorption and damage mechanisms under these conditions have never been explored experimentally, and may exhibit nonlinear effects. One of the goals of initial LCLS research will be to study these effects. However, the nonlinear effects are expected to be much weaker than those encountered in the visible region of the spectrum [2]. Therefore, for the purpose of estimating damage to optical materials, it is not unreasonable to use linear extrapolations of known absorption and melting properties. Table 9.2 shows linearextrapolation calculations for different materials at the location of Hutch A2, near the front of experimental hall A, for the worst case FEL energy of $827 \mathrm{eV}$ where absorption is largest, and also for an energy of $8270 \mathrm{eV}$ [3]. Dose rates given here are for normal incidence, calculated from photo-ionization cross sections, with the photon beam areal density calculated for a propagated Gaussian beam.

Comparing the predicted dose and the dose required to melt, one finds that $\mathrm{Li}, \mathrm{Be}$, and possibly B and C can be safely used in the unattenuated FEL beam at the location of Hutch A2 throughout the energy range of LCLS (although these latter materials approach 0.5 of the melt limit at the low-energy end of the range). At the higher energies, Si can possibly be used also.

Table 9.2 Normal-incidence peak energy dose and damage to materials in Hutch A2.

\begin{tabular}{|c|c|c|c|}
\hline \multirow[t]{2}{*}{ Material } & \multirow[t]{2}{*}{ Melt (eV/atom) } & \multicolumn{2}{|c|}{ Dose (eV/atom) } \\
\hline & & $827 \mathrm{eV}$ & $8270 \mathrm{eV}$ \\
\hline $\mathrm{Li}$ & 0.1 & 0.02 & 0.0005 \\
\hline $\mathrm{Be}$ & 0.3 & 0.08 & 0.001 \\
\hline B & 0.5 & 0.2 & 0.003 \\
\hline C (graphite) & 0.9 & 0.4 & 0.007 \\
\hline $\mathrm{Al}$ & 0.2 & 0.4 & 0.2 \\
\hline $\mathrm{Si}$ & 0.4 & 0.6 & 0.2 \\
\hline $\mathrm{Cu}$ & 0.3 & 1.1 & 0.4 \\
\hline
\end{tabular}

\section{Grazing Incidence}

Calculations for grazing incidence mirrors include the effect of energy density dilution through the angle (the footprint area increases), reflectivity, and deposition throughout an e- 
folding depth for the photons. The results demonstrate an interplay between atomic number, incidence angle, and photon energy. The absorbed energy density is [4]:

$$
\eta_{A}(\mathrm{eV} / \text { atom })=E_{\text {Pulse }}\left(\frac{\theta_{i}}{D_{w}^{2}}\right)\left(\frac{1-R}{\delta_{p} \rho}\right)
$$

Here $E_{P u l s e}$ is the energy of the FEL pulse in $\mathrm{eV}, D_{\mathcal{W}}$ is the beam diameter at the optic, $\delta_{p}$ is the $1 /$ e penetration depth of the light into the material in a direction normal to the surface, $\rho$ is the atomic density of the material, and $\mathrm{R}$ is the reflection coefficient. Following conventional analysis [2,4], we show $\eta_{A}$ vs. $\theta_{i}$ in Figure 9.1 for three candidate reflecting materials: Au (high$\mathrm{Z}$ ), Ni (medium-Z), and Be (low-Z) located at the front of Hall A. Three representative energies characteristic of the LCLS's coherent fundamental and 3rd harmonic are shown $(900 \mathrm{eV}, 8600$ $\mathrm{eV}$, and $30000 \mathrm{eV}$ ). Selecting $\eta_{A} \leq 0.01$ (a criterion suggested by earlier experimental work at SSRL [5] and safe with respect to melt), and an incidence angle of $0.5 \mathrm{mrad}$, we may safely use a $\mathrm{Ni}$ - or $\mathrm{Au}$-coated mirror for low energies $(1 \mathrm{keV})$ and very high energies $(30 \mathrm{keV})$, and a Becoated mirror for intermediate energies $(8 \mathrm{keV})$.

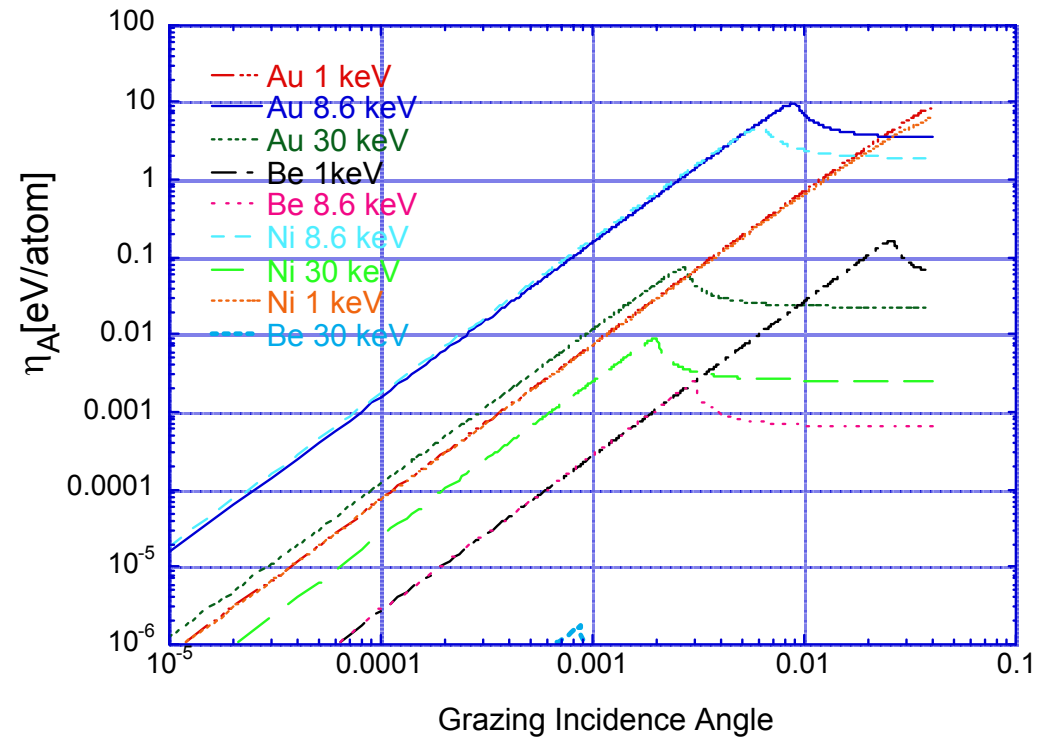

Figure 9.1 Peak power energy loading of candidate LCLS mirror materials vs. (TE) grazing incidence angle and LCLS energy.

\section{Diffraction}

The reflection bandwidth of a perfect crystal is much smaller than the bandwidth of the FEL beam. This means that nearly all of the FEL beam will not be diffracted but will pass into the crystal, and therefore in estimating high-power effects one can neglect the diffraction process entirely and treat the reflecting crystal as a pure absorber. Unless grazing incidence geometry is 
used, the angle of incidence on the crystal will not have a large effect on the power density, and so the calculations for normal incidence shown in Table 9.2 can be used to estimate the probability of damage to the diffracting crystal. These calculations show that $\mathrm{Be}$ and diamond crystals should be safe to use as monochromators in Hall A. Si might also be considered at the high-energy end of the LCLS range.

Table 9.3 summarizes the possible materials that can be used if there is no FEL radiation attenuation. In Hall $\mathrm{B}$ the increased spot size reduces the energy density by a factor $\sim 15$, and more standard materials are usable at all photon energies.

Table 9.3 Summary of suitable materials for optical components, without any FEL radiation attenuation

\begin{tabular}{|c|c|c|c|c|}
\hline & \multicolumn{2}{|c|}{ Hall A } & \multicolumn{2}{|c|}{ Hall B } \\
\hline & $0.8 \mathrm{keV}$ & $8 \mathrm{keV}$ & $0.8 \mathrm{keV}$ & $8 \mathrm{keV}$ \\
\hline Transmission & $\begin{array}{c}\mathrm{Li}, \mathrm{Be}, \\
\text { possibly } \mathrm{B}, \mathrm{C}\end{array}$ & $\begin{array}{l}\mathrm{Li}, \mathrm{Be}, \mathrm{B}, \mathrm{C} \\
\text { possibly } \mathrm{Si}\end{array}$ & Anything & Anything \\
\hline Grazing incidence & $\begin{array}{c}\text { Anything (extremely } \\
\text { grazing) }\end{array}$ & $\mathrm{Be}$ & Anything & Anything \\
\hline Crystal diffraction & $\begin{array}{l}\text { No good crystal } \\
\text { candidates }\end{array}$ & $\begin{array}{c}\mathrm{Be}, \mathrm{B}, \mathrm{C} \\
\text { possibly } \mathrm{Si}\end{array}$ & Anything & Anything \\
\hline Multilayers & $\begin{array}{l}\text { All low to moderate } \\
\text { Z }\end{array}$ & $\begin{array}{l}\text { All low to moderate } \\
\text { Z }\end{array}$ & Anything & Anything \\
\hline
\end{tabular}

Continued R\&D into x-ray photon-material interactions and damage is imperative to test the calculations shown above. Not all known physics has yet been included in the modeling described above. More importantly, the remarkable photon densities are expected to instigate new processes, which are the specific topic of proposed atomic physics experiments. The effects of the intensity spikes within a single FEL pulse, with characteristic spike width less than $1 \mathrm{fs}$ and intensity up to 5 times the nominal value, are unknown.

\section{Absorbers and Attenuators}

Gas, liquid or metal attenuators (see Section 9.2.2.1) will be constructed to reduce the FEL beam intensity, both as an experimental control, and to avoid damage to optical components and diagnostics. The gas attenuator [6] can be used for initial studies of scattering of the LCLS pulses by absorbing media, to answer some of the physics questions mentioned above. The chamber design includes ports for line-of-sight fluorescence detection, as well for the introduction of external magnetic and electric fields. Due to its location inside the Front End Enclosure, provisions for a detector shielding enclosure have been included. 


\subsection{Layout and Optics}

\subsubsection{Experimental Halls}

Two experimental halls are planned, one close to the undulator exit (Hall A, starting about 50 $\mathrm{m}$ from the undulator end) and one considerably farther downstream (Hall B, starting about 400 $\mathrm{m}$ from the undulator end) (see Figure 9.2). The total experimental floor area will allow the installation of several experimental stations; the hall locations are determined by local access roads and topography. Optics in Hall B will experience a reduced power density that should allow a wide range of materials to be used for samples and optical elements. Hall A will be useful for those experiments requiring maximum power density.

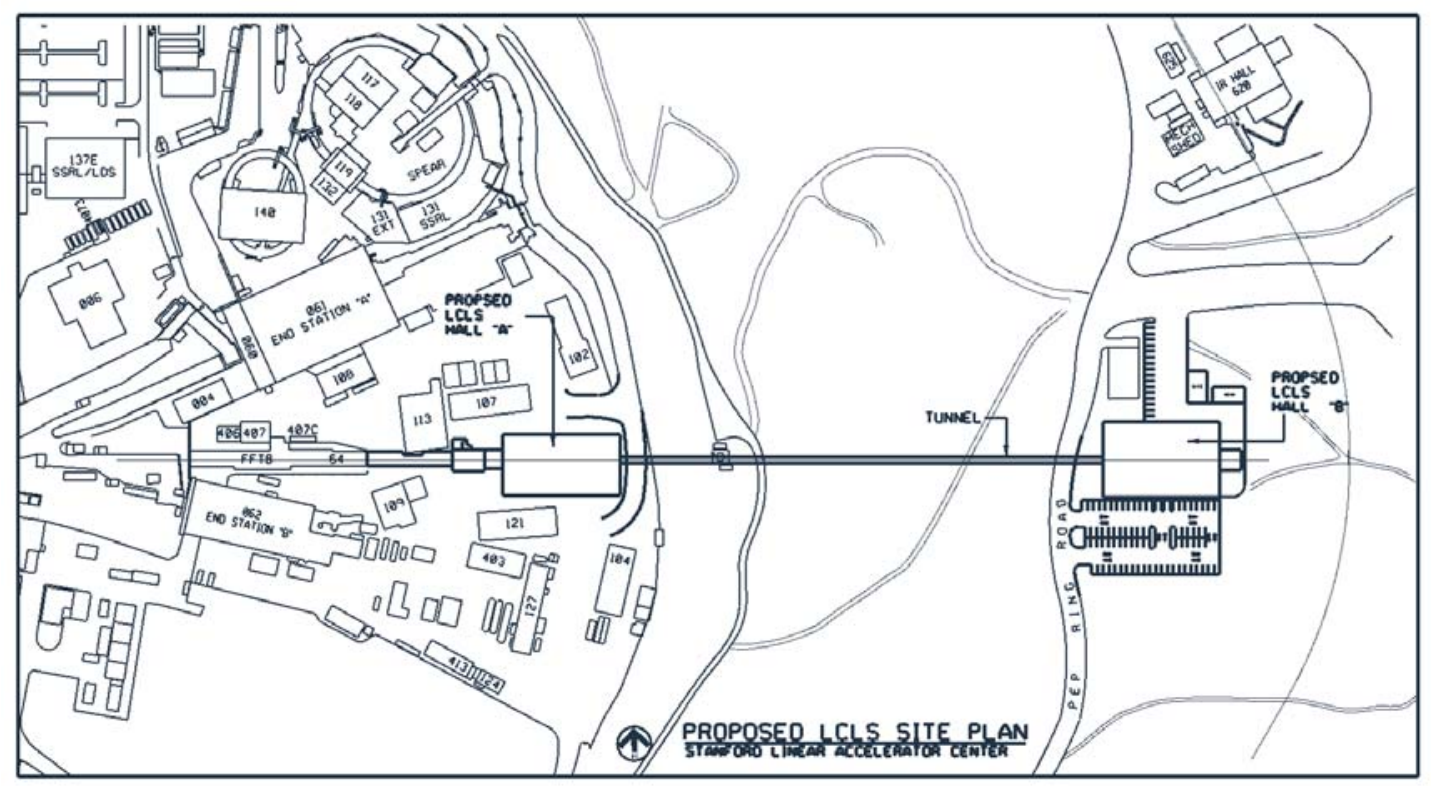

Figure 9.2 LCLS site plan showing experimental halls.

An additional reason for a near hall (Hall A) involves the transmission of the spontaneous synchrotron radiation (SR) to experiments. Close to the undulator, a few-mm aperture should transmit a usable fraction of this spectrum. Transporting the same SR cone to the far hall would require an unworkably large vacuum aperture.

The large flight distance to Hall B will place some stringent requirements on beam pointing accuracy (the specification of beam wander in the LCLS design study report is $\sim 10 \%$ of the beam diameter, independent of path length). However, similar stringent stability requirements must implicitly be met for reliable SASE FEL operation - the angular acceptance for SASE saturation through the undulator is on the same order as the beam divergence, so any beam angle excursions 
larger than this value would probably quench any coherent output (a similar constraint applies to the position of the beam axis). To achieve this level of accuracy, it may be necessary to stabilize the system against slow drifts with an active monitor and feedback system.

The remainder of Section 9.2 describes the optical layout and optical elements in detail.

\subsubsection{Optical Enclosures}

\subsubsection{Front End Enclosure}

Elements in the Front End Enclosure (see Figure 9.3) include fixed masks, a fast valve, vertical and horizontal slits ( 2 of each), a gas attenuator, a variable-thickness solid attenuator, and a beam stop including a burn-through monitor.

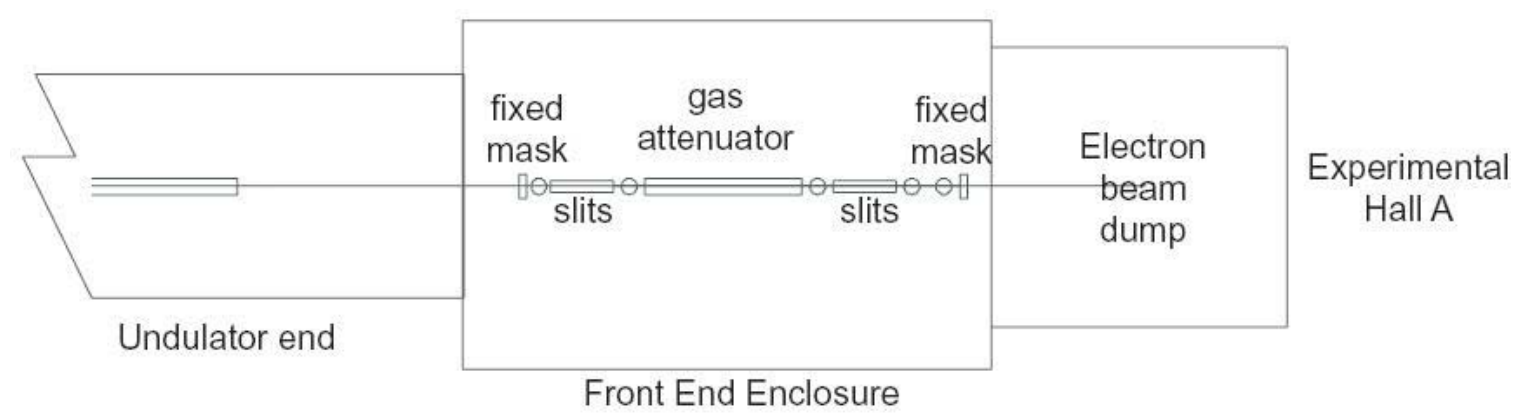

Figure 9.3 Layout of the Front End Enclosure

\section{Fixed Mask}

The very first element of the photon transport system is a fixed mask, located $9 \mathrm{~m}$ downstream from the undulator, where it will not be hit by the deflected electron beam. The purpose of this mask, and a similar one located at $28 \mathrm{~m}$ from the undulator, is to insure that all radiation allowed downstream is confined within a very small angular region. This in turn will insure that all radiation in the first experimental hall stays within the beam pipe. Separated by 19 $\mathrm{m}$ and each having an aperture with diameter $4.5 \mathrm{~mm}$, the fixed masks limit the transmitted angular range to $240 \mu \mathrm{rad}$ (FWHM). At the back end of Hall A, this transmitted angular range would have a diameter of $16 \mathrm{~mm}$, well-contained within the beam pipe.

The aperture diameter of the fixed masks, $4.5 \mathrm{~mm}$, is much larger than the diameter of the coherent FEL beam (note that the masks are nearly as large as the beam pipe through the undulator). Within the rather limited mis-steering range that will support FEL amplification, there is no possibility that the coherent radiation will strike the fixed masks. Thus, their purpose is only to intercept the wings of the spontaneous radiation. The peak-power densities at the masks will not be problematic, and they can be made from standard metal x-ray absorbers. Average power levels on these masks will be negligible, though water-cooling will be included. 
Fast Valve

Provision is made for a fast $(<0.1 \mathrm{sec})$ vacuum valve, to protect the upstream vacuum system in the event of vacuum failure in the experimental area. The sensors that trigger this valve will be interlocked with the linac controls, so that the valve will not be subjected to FEL radiation.

\section{Vertical Slit and Horizontal Slit}

The x-ray beam entering the x-ray optics system consists of an intense coherent FEL line with an FWHM angular divergence of about $1 \mu \mathrm{rad}(9 \mu \mathrm{rad})$ for an electron energy of $15 \mathrm{GeV}$ (5 $\mathrm{GeV}$ ), surrounded by a broad spontaneous distribution with an FWHM angular width of about $250 \mu \mathrm{rad}(780 \mu \mathrm{rad})$. For particular experimental applications, the spontaneous radiation can constitute a noise source and will need to be removed. These considerations have led to the introduction of the two-slit-pair system shown in Figure 9.4. Each slit assembly consists of a two movable jaws defining an adjustable horizontal aperture, and two movable jaws defining an adjustable vertical aperture. The first slit assembly is located just upstream of the absorption cell so that low energy spontaneous radiation can be filtered out for scattering experiments located at the cell. The second slit-pair, located about $15 \mathrm{~m}$ farther downstream, can also be used as an independent aperture, or combined with the first slit-pair to provide an angular collimator with an extremely small acceptance, providing a broad range of spectral-angular filtering options, including the delivery of quasi-monochromatic beams. An additional function of the slits (when operated in a collimator mode) will be to protect downstream optics such as mirrors from excessive peak power damage due to beam jitter.

Because the slit assemblies are located close to the FEL source, the peak power density needs to be considered. It is not intended for the slits to actually intercept the FEL beam, but in order to effectively cut out the spontaneous radiation background the slit jaws must come very close to the FEL beam. Two slightly different concepts for the slit jaws are under consideration. One concept treats the slit jaw as a grazing-incidence mirror, reflecting unwanted radiation out of the main beam path, and into a downstream mask. This slit jaw would be best coated with a highlypolished layer of high- $Z$ material. At grazing incidence, this material could survive the spontaneous radiation and the wings of the FEL radiation. The other concept treats the slit jaw as a pure absorber at normal incidence. If made of Be it could withstand the spontaneous radiation and the wings of the FEL radiation. Further analysis of the expected radiation pattern will help determine which concept is better.

Either concept requires a long slit jaw with precision motion control. We propose to use a modified version of an existing SLC collimator design as presently employed in the SLAC beam switchyard for collimator C-0 and momentum slit SL-2 [7], with new jaws. The jaws will be water-cooled for optimal dimensional stability during operation. The jaws are remotely adjustable by means of stepper motors and can be differentially adjusted to control $d_{u v}, d_{d v}, d_{u h}$, and $d_{d h}$ (see Fig. 9.4), as well as the average vertical and horizontal midplanes of the slits. A maximal incidence-angle range of about $0-1.5 \mathrm{mrad}$ is envisaged and the minimum aperture size will be variable from 0 to $>1 \mathrm{~cm}$.

9-8 - R A Y B E A M T R A N S P O R T A N D D D A G N O S TIC C 


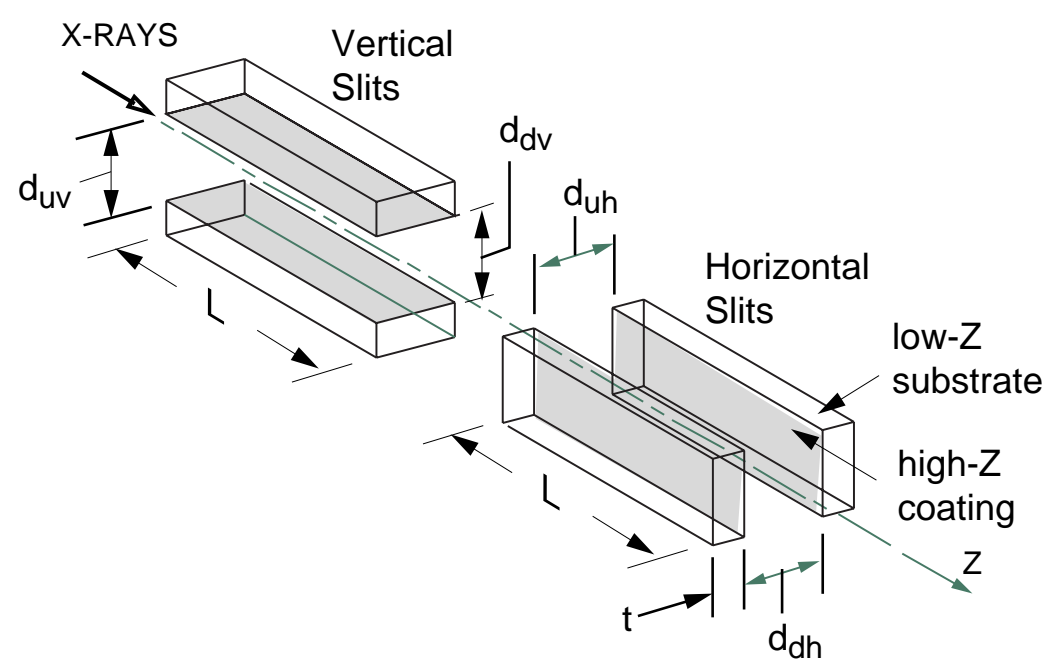

Figure 9.4 The LCLS $x$-ray slit configuration. Gap dimensions $d_{u v}, d_{d v}, d_{u h}, d_{d h}$ are independently adjustable.

The total footprint of the spontaneous radiation at both slit locations will be significantly larger than the upstream slit apertures $d_{u v}$ and $d_{u h}$. This means that not only can one or more of the slits absorb most of the spontaneous x-ray power during operation, but also that most of it will impact the jaws' upstream facets at normal or near-normal incidence. At the locations of the slits, the spontaneous peak power density at normal incidence can attain off-axis values that are only three orders of magnitude below that of the coherent line, which brings the peak power densities anticipated for the jaws to levels at which little or no experimental data exists. Similar peak power levels in the high-Z reflecting material (assuming $\sim 99 \%$ reflectivity) can be expected for scenarios where the LCLS coherent line impacts the jaw surface, due to jitter or for other reasons.

Although there is some evidence of survival of mirrors exposed to very high specific power densities from alternative sources, the processes that take place in the temporal and spectral regimes of the LCLS [8] are still very poorly understood and more experimental and theoretical studies will be needed.

\section{Attenuator/Absorber}

Controlled attenuation of the coherent pulses of the LCLS could be accomplished by passage through a gas, solid, or liquid. Over the long-wavelength range of the LCLS fundamental ( $800 \mathrm{eV}$ to about $4 \mathrm{keV}$ ), it is unlikely that any solid absorber (except perhaps one made of pure lithium) would survive undamaged in the Front End Enclosure (see Figure 9.5). For shorter wavelengths, absorption cross sections are lower, and a solid absorber made of light elements is practical. 


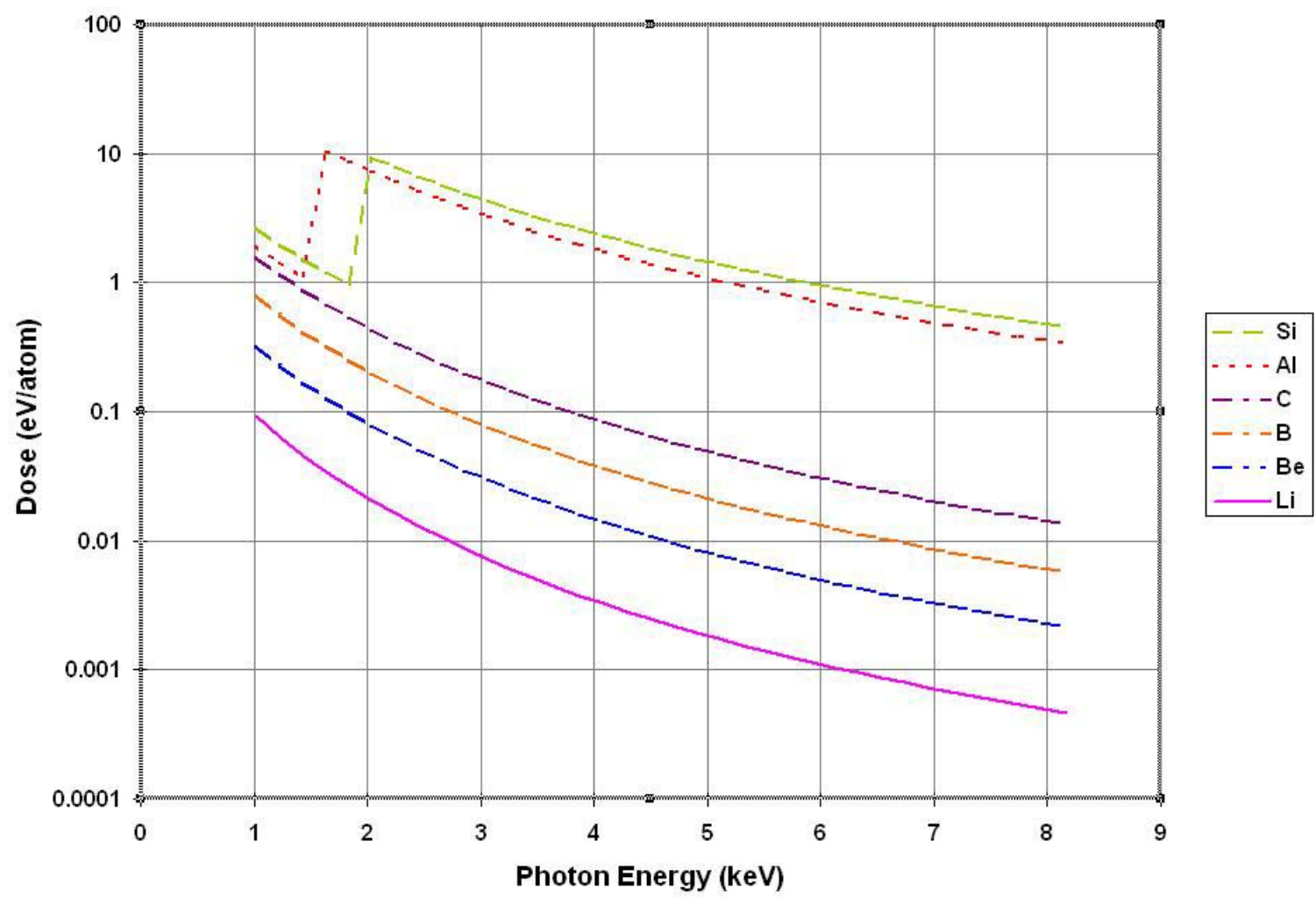

Figure 9.5 Energy dose absorbed from one shot of LCLS for different absorber materials, at a location in the Front End Enclosure.

Therefore, in addition to conventional solid absorbers, LCLS plans to use a gas absorber cell, using high-pressure puff valves to introduce the absorbing gas into the path of the coherent FEL photons (see Figure 9.6) [5]. The axial dimensions of the chamber and the number of valve nozzles must be adequate to allow a sufficient thickness of the gas to provide two or more orders of magnitude of attenuation over the $800-4000 \mathrm{eV}$ range. The combined axial and transverse dimensions are determined by the requirement of maintaining an average vessel pressure of $<0.0075$ Torr, corresponding to the Knudsen-through-molecular flow regimes [9]. This pressure, which is sufficiently low to be reduced to $<10^{-6}$ Torr by the differential pumping sections bracketing the chamber, will be determined primarily by:

1) the average volume of gas introduced into the chamber per puff;

2) its average pressure;

3) the axial conductance out of the gas cell;

4) the chamber volume;

5) the puff valve repetition rate; and

6) the capacity of the primary pump(s) connected directly to the chamber. 


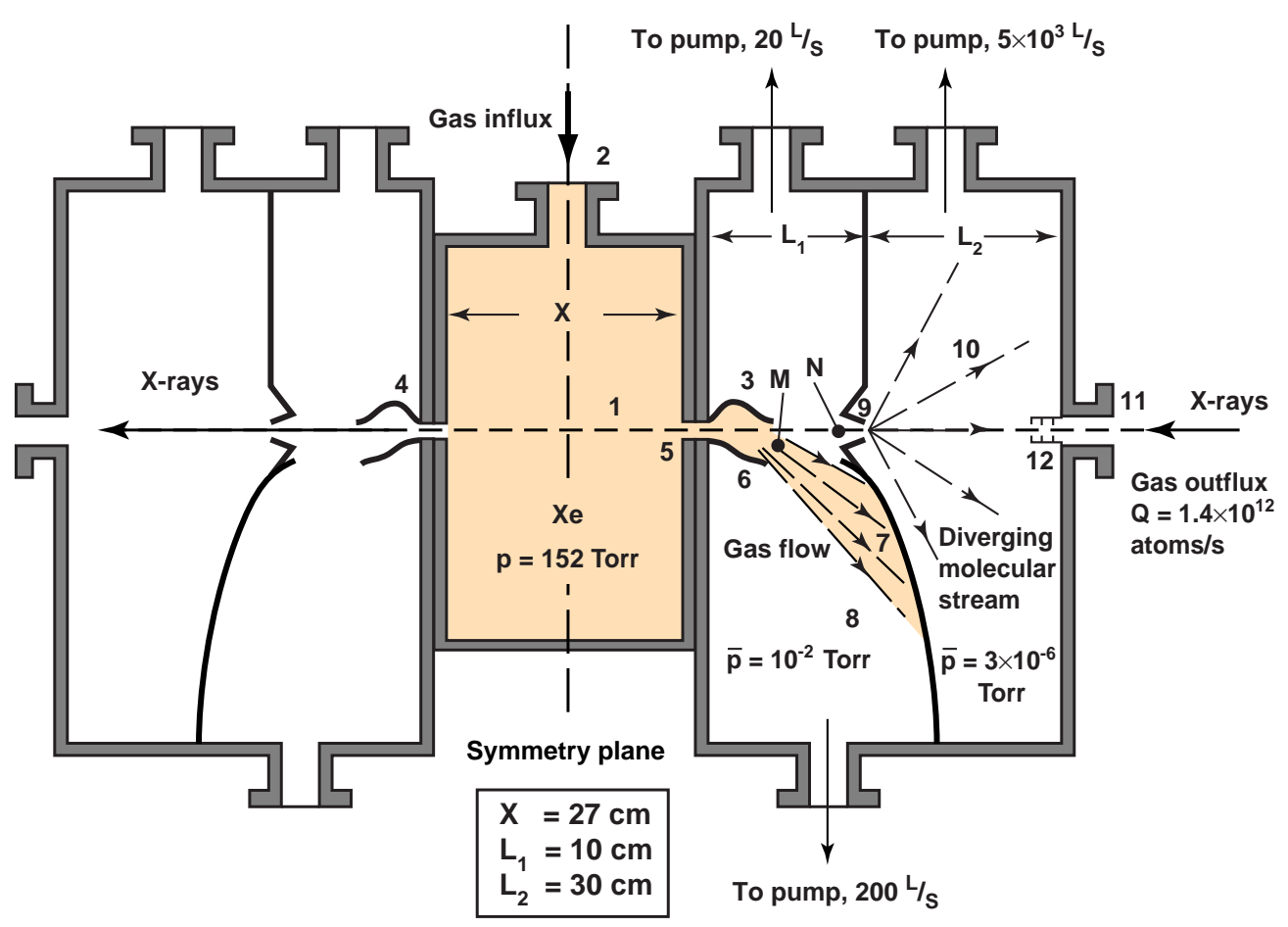

Figure 9.6 The conceptual design for the gas cell attenuator

The operation of the gas cell in the weak-field (linear) regime using xenon as an absorber has been calculated for reference. In Figure 9.7 the absolute attenuation of x-rays through xenon for four given pressure, $t_{\mathrm{g}}$, [Torr-cm] products is plotted from 800 to $25000 \mathrm{eV}$. The curves indicate that a 2000 Torr Xe gas jet with $\mathrm{t}_{\mathrm{g}}=1 \mathrm{~cm}$ would provide at least two orders of magnitude of attenuation over the low-energy range of LCLS $(800-4000 \mathrm{eV})$. Note that this absorption calculation assumes that the absorption mechanisms are essentially uni-molecular and linear. With suitable design and a sufficiently low repetition (pulse) rate the loading of the vacuum system by the required amount of gas should be maintainable at acceptable levels. 
Weak-Field Attenuation Curves for Xenon vs. (Pressure x Distance)

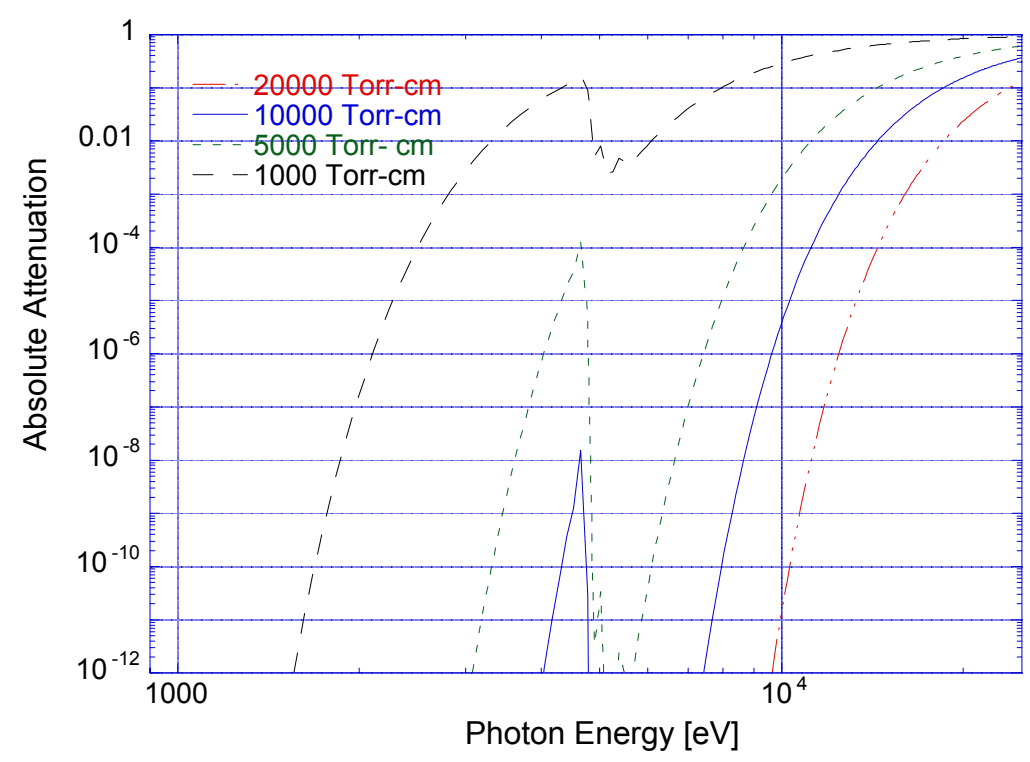

Figure 9.7 Weak-field attenuation curves for xenon.

The absorption cell can also be used for initial studies of scattering of the LCLS pulses by absorbing media. The chamber design includes ports for line of sight fluorescence detection, as well for the introduction of external magnetic and electric fields. Due to its location inside the FFTB tunnel, provisions for a detector shielding enclosure have been included.

While the absorption of xenon in the linear regime can be calculated, some corrections may be required for the actual LCLS pulses, whose intensity and degeneracy parameters lie well outside the regime of weak-field interactions. Fundamental questions remain about the effects of nonlinear scattering and absorption processes on the temporal shape and the longitudinal and transverse coherence of the pulses.

\section{Solid Attenuator}

At energies above $4 \mathrm{keV}$, solid attenuators become practical. Figure 9.5 above shows that the absorbed dose in $\mathrm{B}$ and $\mathrm{C}$ has a reasonably safe value of less than $0.1 \mathrm{eV} /$ atom for FEL energies above $4 \mathrm{keV}$. This suggests that $\mathrm{B}_{4} \mathrm{C}$ would make a good absorber material. Table 9.4 shows the $\mathrm{B}_{4} \mathrm{C}$ thicknesses needed to vary the attenuation linearly from 0.1 to 1 and logarithmically from $10^{-1}$ to $10^{-10}$, for an x-ray energy of $8 \mathrm{keV}$. 
L C L S C O N C E P T U A L D E S I G N R E P O R T

Table 9.4 Thicknesses of boron carbide required for attenuation of $8 \mathrm{keV} x$-rays

\begin{tabular}{|c|c|c|c|}
\hline \multicolumn{2}{|c|}{ Linear attenuator } & \multicolumn{2}{c|}{ Logarithmic attenuator } \\
\hline Desired attenuation & $\mathbf{B}_{\mathbf{4}} \mathbf{C}$ thickness $(\mathbf{m m})$ & Desired attenuation & $\mathbf{B}_{\mathbf{4}} \mathbf{C}$ thickness (mm) \\
\hline 1 & 0 & $10^{-1}$ & 4.1 \\
\hline 0.9 & 0.2 & $10^{-2}$ & 8.1 \\
\hline 0.8 & 0.4 & $10^{-3}$ & 12.2 \\
\hline 0.7 & 0.6 & $10^{-4}$ & 16.3 \\
\hline 0.6 & 0.9 & $10^{-5}$ & 20.4 \\
\hline 0.5 & 1.2 & $10^{-6}$ & 24.4 \\
\hline 0.4 & 1.6 & $10^{-7}$ & 28.5 \\
\hline 0.3 & 2.1 & $10^{-8}$ & 32.6 \\
\hline 0.2 & 2.8 & $10^{-9}$ & 36.7 \\
\hline 0.1 & 4.1 & $10^{-10}$ & 40.7 \\
\hline
\end{tabular}

Log attenuator

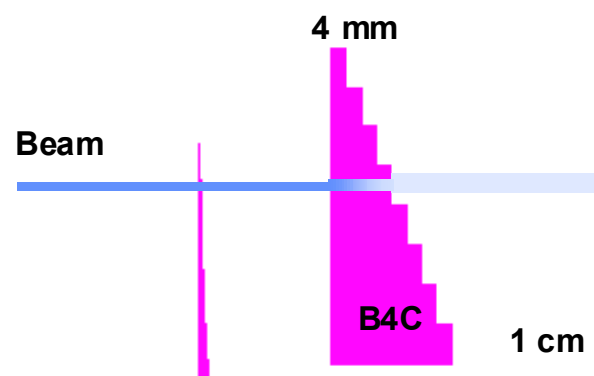

$32.6 \mathrm{~mm}$

$4 \mathrm{~mm}$

Linear attenuator

Figure 9.8 A linear/ log attenuator system

The attenuators will be fashioned from single plates of $\mathrm{B}_{4} \mathrm{C}$ milled in a staircase pattern to the thicknesses specified in Table 9.4 as shown in Figure 9.8. The linear and logarithmic attenuators will be mounted on separate translation stages allowing all combinations of linear and logarithmic attenuation to be applied. 
The attenuator translation stages will provide motion in the $\mathrm{X}$ and $\mathrm{Y}$ directions with a precision of $<1 \mathrm{~mm}$.

\section{Diagnostics Tanks}

Space is available downstream from the gas and solid attenuators for beam diagnostic measurements such as pulse intensity and pulse shape. The diagnostics will monitor the operation of the attenuators. See Section 9.4.2.

\section{Beam Stop with Burn-Through Detector}

At the downstream end of the Front End Enclosure there is an insertable beam stop. This device consists of an upstream beryllium section to reduce the peak power of the FEL beam, and downstream copper and heavy metal sections to absorb the full spectrum of the LCLS. The beam stop includes an integral burn-through detector, which, in case the beryllium section fails to insert, will protect the radiation absorbers and shut down the LCLS. The radiation absorbers are duplicated with separate control systems so that the risk of a radiation accident is negligible.

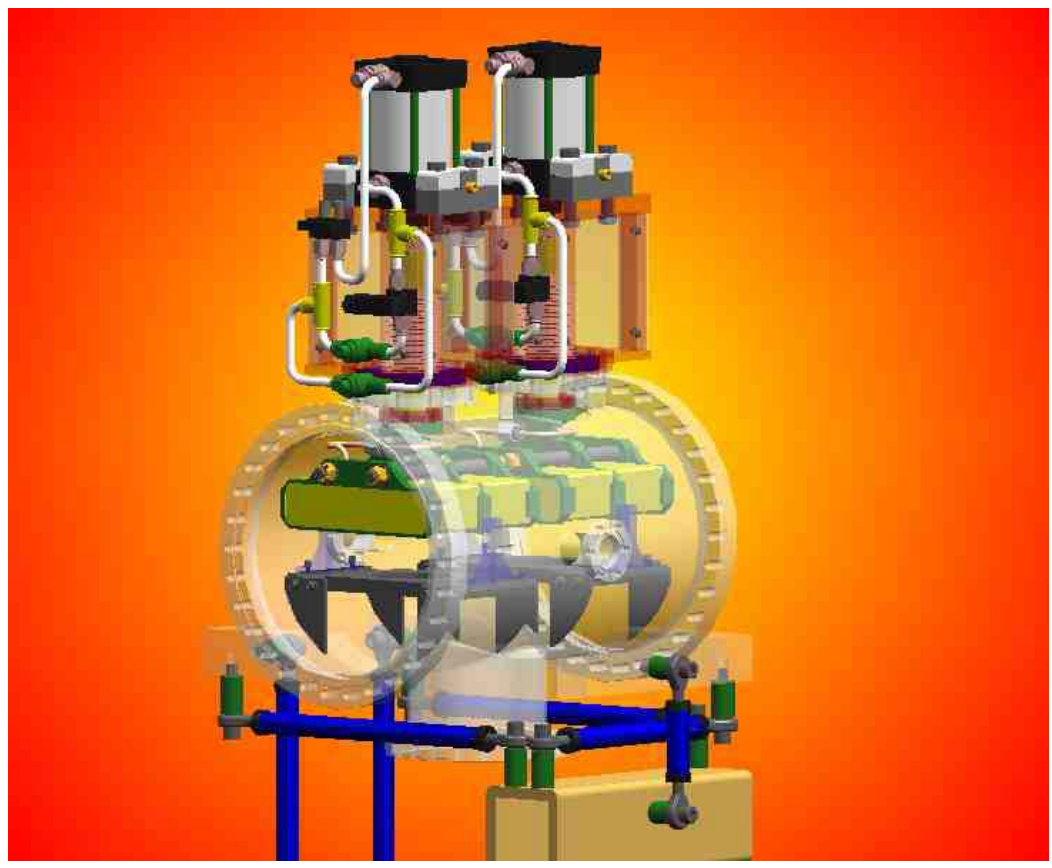

Figure 9.9 Concept of the insertable beam stop with burn-through detector.

\subsubsection{Hutch A1}

The first hutch in Hall A (Figure 9.10) will contain optical elements which condition the $\mathrm{x}$ ray beam for the Hall A experiments. Only one such element will be included in the initial LCLS, though space is made available for future optics. Hall A is intended primarily for high-intensity experiments, using the full bandwidth of the coherent FEL beam. 


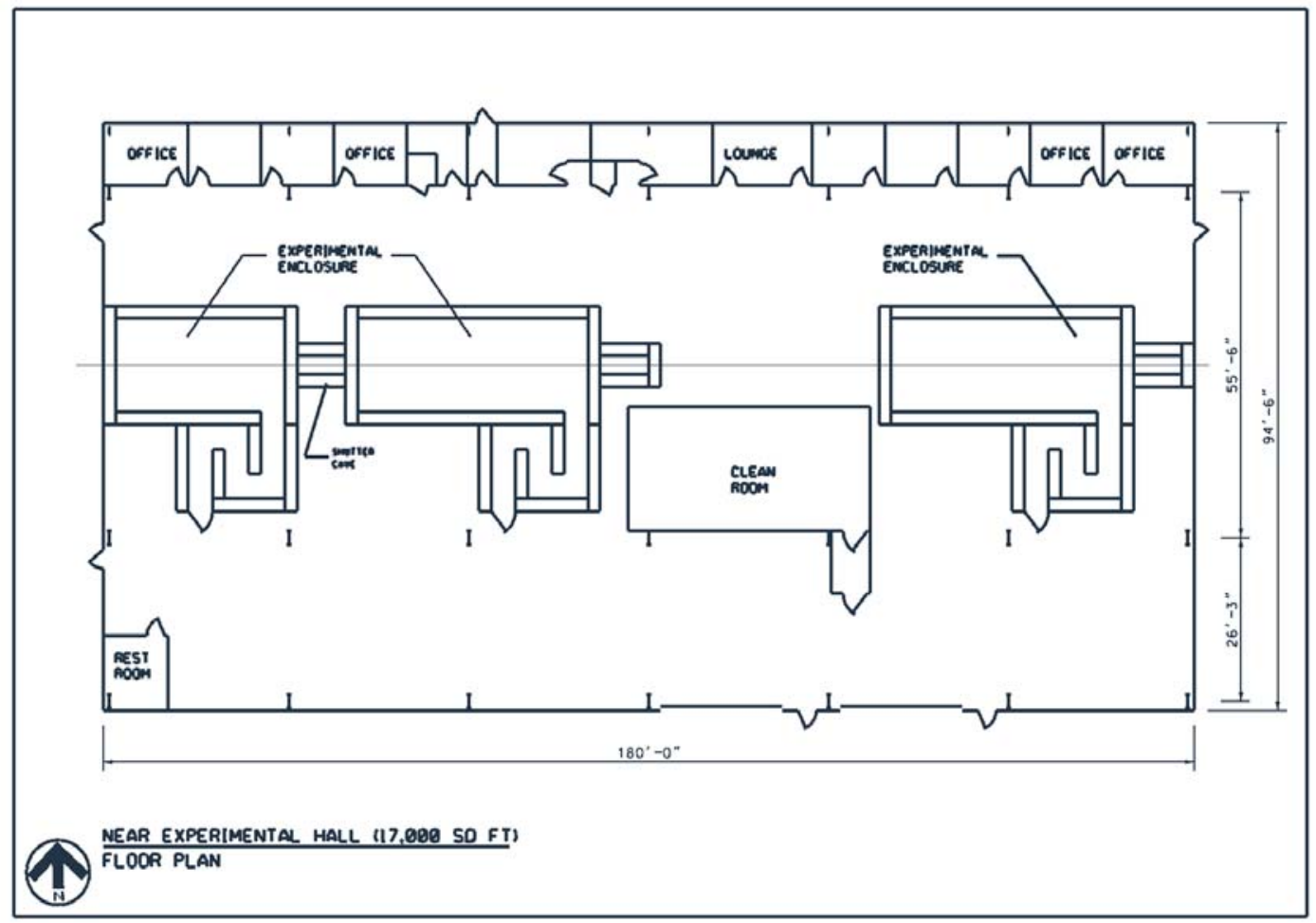

Figure 9.10 Layout of Experimental Hall A

\section{Dual-Mirror Harmonic Rejection System}

Some experiments (in particular, atomic physics experiments) need a very clean spectrum without higher harmonics. With a small aperture upstream, the LCLS spectrum contains only odd harmonics. The strongest higher harmonic, the third, is expected to experience some FEL amplification, but its intensity will be about two orders of magnitude below that of the fundamental. A pair of grazing-incidence mirrors can add several more orders of magnitude to that ratio.

The separation of the $3^{\text {rd }}$ harmonic contaminant requires a grazing incidence mirror system with graze angle above the critical angle for the $3^{\text {rd }}$ harmonic while lower than the critical angle for the fundamental component. It is possible to trade total reflectance of the primary radiation for suppression of the $3^{\text {rd }}$ harmonic. By increasing the angle of incidence closer to the critical angle for the fundamental, greater suppression of the $3^{\text {rd }}$ harmonic is possible; but at the price of reduced reflectivity in the fundamental. Reflectivity of the $3^{\text {rd }}$ harmonic will be on order a few percent. Because of this modest rejection capability, and to simplify the beamline geometry, a two-mirror system is the appropriate choice.

The harmonic separator mirrors are parallel to one another; and the second mirror has angle adjustment available to fine-tune the direction of the outgoing beam. The slope error in the mirrors must be kept below a fraction of the natural beam divergence and leads to severe, though technically achievable, figure constraints. The opto-mechanical tolerances are given in Table 9.5. 
Table 9.5 Mirror requirements

\begin{tabular}{|c|c|c|}
\hline Parameter & Requirement & Specification \\
\hline Slope Error & $\begin{array}{l}\text { Negligible contribution to beam } \\
\text { divergence. }\end{array}$ & $\begin{array}{l}\text { Better than } 0.5 \mu \text { rad slope error, or } \\
5 \mathrm{~nm} \text { over typical } 10 \mathrm{~mm} \text { ripple } \\
\text { wavelength. } \\
\text { Flatness to } 1 / 200 \text { wave RMS, } 1 / 40 \\
\text { wave P-V }\end{array}$ \\
\hline Surface roughness & Scattering losses below $10 \%$ & Surface finish $<20 \mathrm{~nm}$ RMS. \\
\hline Size & $\begin{array}{l}\text { Intercept beam over angular range of } \\
0.7 \text { to } 1.5 \text { degrees }\end{array}$ & $50 \mathrm{~mm}$ total length. \\
\hline Angular positioning & $\begin{array}{l}\text { Allow tradeoff between } 3^{\text {rd }} \text { harmonic } \\
\text { suppression and fundamental } \\
\text { efficiency }\end{array}$ & $\begin{array}{l}\text { Mirrors free to rotate from } 0 \text { to } \\
2 \text { degrees both slaved and } \\
\text { independently. Rotation error } \\
\text { throughout this range must be } \\
<1 \mu \mathrm{rad} \text {. }\end{array}$ \\
\hline Lateral positioning & $\begin{array}{l}\text { Illuminate repeatable areas on } \\
\text { mirrors. Ability to operate beamline } \\
\text { without order separator. }\end{array}$ & $\begin{array}{l}\text { Translate mirrors completely out of } \\
\text { beam and reposition them to better } \\
\text { than } 10 \mu \mathrm{m} \text { tolerance. }\end{array}$ \\
\hline
\end{tabular}

The flatness constraint will be met by means of iterative polishing. Grain structure in $\mathrm{Be}$ is considered to be a near insurmountable barrier to achieving both the surface finish and the final figure requirement. Meeting these requirements in $\mathrm{Si}$ is, though non-trivial, well within the capability of existing commercial vendors. A hybrid optic material, e.g., sputtered $\mathrm{Be}$ on a $\mathrm{Si}$ substrate, is a possibility although the thermal loads on the Si are negligible. A detailed thermal study will be needed to confirm initial calculations that the several degree rise in temperature on the mirror surface expected during operation will not increase figure error. The mismatch between the coefficient of thermal expansion for $\mathrm{Be}$ and $\mathrm{Si}$ ( $\mathrm{Be}$ is 3 times higher) may effectively bar the use of a (non-cooled) hybrid material.

Flatness must be maintained once the mirrors are mounted without inducing any stress into the optic. Angular motion that meets these specifications will be achieved with a Picomotor driven flexure using an approach proven successful in the LLNL EUVL effort [10].

\section{Spools, Chambers, and Beam Stop}

Hutch A1 contains a diagnostics chamber for diagnostics associated with adjustment of the mirrors. It also contains several spool pieces, which may in future be replaced by additional mirror systems and monochromators. At the back end of Hutch A1 is an insertable beam stop with integral burn-through detector.

\subsubsection{Hutch A2}

Hutch A2 will house LCLS experiments. It will also contain beam-conditioning optics, which need to be close to the experiments, in particular, focusing systems with short focal length. 


\section{Kirkpatrick-Baez Focusing System}

One effective technique for focusing x-rays to sub-micron spot diameter uses total external reflection mirrors in the Kirkpatrick-Baez, (KB), geometry (see Figure 9.11). To achieve small spot size the KB mirrors must exactly hold a precise elliptical geometry. Using bending fixtures to apply a precise bending moment to each end of a mirror, a near perfect figure can be obtained from a previously figured flat. Sub-micron spot size has been demonstrated from a system of this type [11] and ray tracing results indicate that beams with cross sections of less than $0.04 \mu^{2}$ (gains in excess of $10^{5}$ ) are achievable.

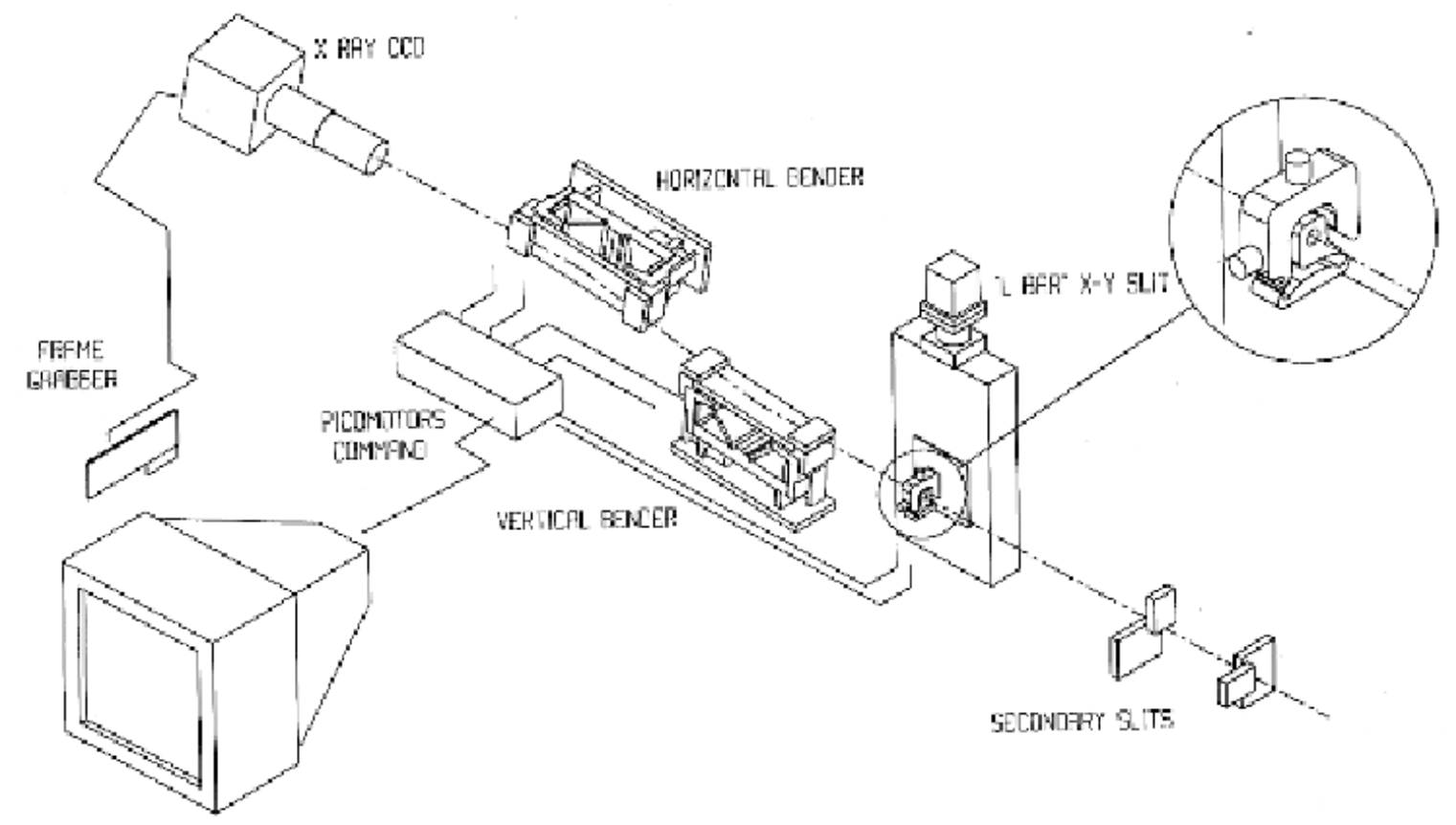

Figure 9.11 Schematic of the Kirkpatrick-Baez system

Improvement on current results requires improvement in the mirror figure; specifically it requires better conformance to the ideal elliptical profile. Our approach will be to simplify the typical bending arrangement by applying a bending moment to one end of the mirror. The mirror cross-sectional thickness will vary as a function of length along the mirror. Analytic optimization of the thickness profile allows us to accurately predict the deformation of the mirror under the applied bending moment and precisely control the resultant figure.

A significant advantage of the $\mathrm{KB}$ approach is that the mirror substrates must only meet the (exacting) specifications for the flat mirrors used for the order separator. Angular and bending moment adjustments for the mounts also have similar specifications. It has recently been demonstrated [10] that deposition of thin films on the surface of can be used to allow control of figure at the nm level. 


\section{Refractive Focusing System}

Another promising system for focusing the FEL beam involves refractive optics. It is straight forward to show that low-Z refractive optics can withstand the full power loading of the $8 \mathrm{keV}$ FEL and therefore can be used with confidence for applications desiring to achieve the highest power levels in the focal spot. A low-Z refractive focusing optic for the LCLS will consist of one or more "blazed phase plates", which are the most general form of refractive optics. The lenses will be made by replicating diamond-turned forms in $\mathrm{C}$ and/or $\mathrm{Li}$.

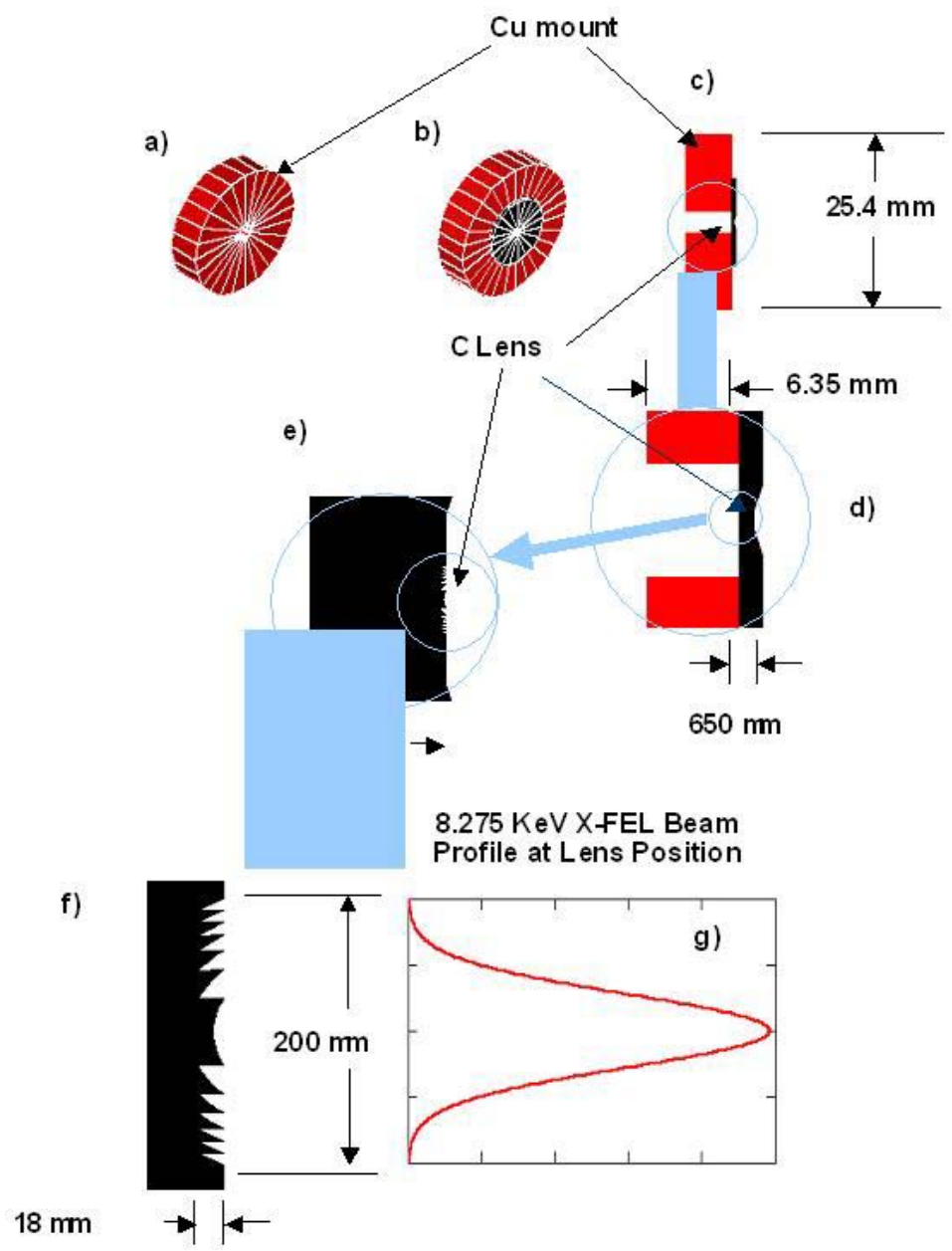

Figure 9.12 Refractive focusing optics for LCLS

Figure 9.12 shows details of a single lens design. The lens is carved into the face of a $\mathrm{C}$ (graphite) disk and mounted over a hole drilled through a $25.4 \mathrm{~mm}$ diameter $\mathrm{Cu}$ mount. The graphite disk is 650 microns thick except in the center where it thins down to 400 microns. The active portion of the lens is $200 \mu \mathrm{m}$ in diameter and consists of 6 concentric grooves machined to a maximum depth of $18.8 \mu \mathrm{m}$. The plot of the LCLS beam profile at the lens, Figure 9.12g, shows that the $200-\mu \mathrm{m}$ lens diameter nicely captures most of the beam.

The shape of the grooves is determined by calculating, at the position of the lens, the phase change necessary to convert the diverging Gaussian FEL beam into a converging Gaussian 
waveform whose waist is at the sample position. The radial phase profile was converted to a depth profile by multiplying by the optical constant for graphite, which is $18.8 \mu \mathrm{m} / 2 \pi$ radians phase change (with respect to vacuum) at $8.275 \mathrm{keV}$.

Several of these lenses will be stacked into single machined mount to achieve shorter focal lengths.

\section{Apertures}

Apertures must be used to eliminate the halo of stray radiation surrounding the focal spot. Survivability is an issue in the design of the apertures in Hutch A2. The basic concept is to utilize a laminate consisting of $4 \mathrm{~mm}$ of $\mathrm{B}_{4} \mathrm{C}, 150$ microns of $\mathrm{Al}$, and 200 microns of Ta. This laminate has sufficient absorption to block x-rays up to the 3rd harmonic. Furthermore the $\mathrm{B}_{4} \mathrm{C}$ attenuates the direct FEL beam enough to prevent damage to the Al, which further attenuates the beam enough to prevent damage to the Ta.

A series of holes having diameters from $1 \mathrm{~mm}$ down to 100 microns will be drilled through the laminate, which will then be mounted on a movable stage that provides both rotation and translation of the laminate. A second, fixed, laminate having a single $1 \mathrm{~mm}$ diameter hole keeps light from passing through all but a single hole in the movable laminate (Figure 9.13). The ability to rotate the laminate is necessary because of the large aspect ratio of the holes. Using a downstream intensity monitor, and starting with the largest diameter hole, the movable laminate will be rotated into a position that maximizes the signal. The laminate will be shifted to the next smaller diameter hole and rotated again to achieve highest intensity downstream. This process will be repeated with successively smaller holes until the hole of the desired diameter is positioned and aligned.

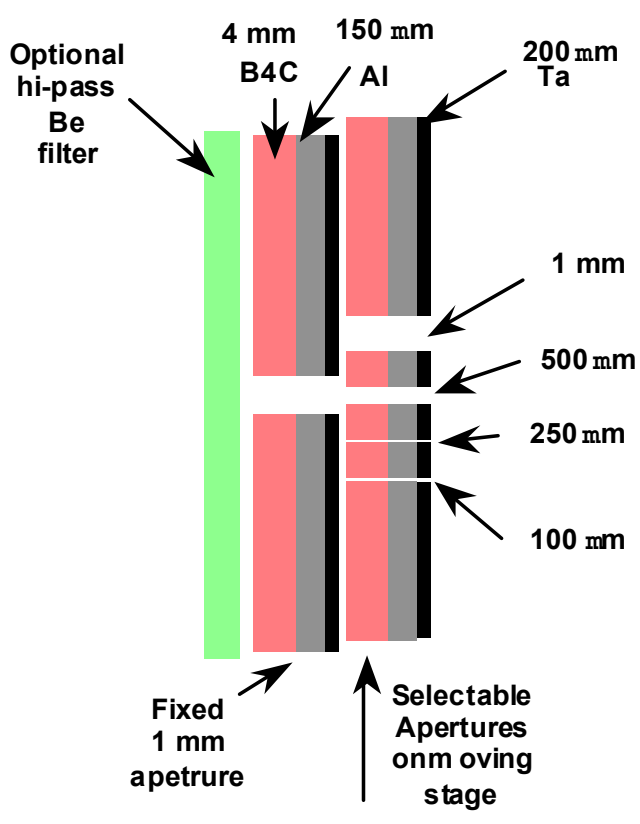

Figure 9.13 Apertures for the FEL x-ray beam 
The stages used to position the apertures will have positioning precision $<10 \mu \mathrm{m}$ and angular precision of $<1 \mathrm{mrad}$.

\section{Attenuator}

Some experiments require a local attenuator for calibration and to prevent damage to sensitive components during alignment. The local attenuator will have a design very similar to the solid attenuator located upstream in the Front End Enclosure (see Section 9.2.2.1).

\section{Beam Intensity Monitors}

Beam intensity monitors are required to measure the absolute flux incident on the samples and the amount of flux transmitted through the samples. These monitors will be of the ion chamber type described in the facility diagnostics section (Section 9.4.2.3).

\section{Sample Chamber}

The sample chamber in Hutch A2 will be instrumented for studies required to characterize the interaction between the FEL pulse and matter. In addition to sample holders and photon spectrometers, it will include electron and ion time-of-flight spectrometers.

Beam Stop

At the back end of Hutch A2 is an insertable beam stop with integral burn-through detector.

\subsubsection{Hutch A4}

Hutch A4 will initially be used for commissioning diagnostics, which will be housed in a diagnostics tank. See Section 9.4.2.

\section{Fixed Mask and Beam Stop}

At the back end of Hutch A4 is an insertable beam stop with integral burn-through detector. Behind the beam stop is a fixed mask with $4.5 \mathrm{~mm}$ diameter aperture, identical to the fixed masks in the Front End Enclosure. As with those masks, its purpose is to cut the divergence of the spontaneous radiation, so that all transmitted radiation remains within the beam pipe. The coherent FEL radiation cannot strike this mask, and so peak power is not a concern.

\subsubsection{Inter-Hall Transport}

A beam pipe connects the two main halls through a tunnel. It is about $250 \mathrm{~m}$ long. Access will be available along the length of the tunnel. In the center of the tunnel, a diagnostics tank will permit beam intensity and position measurements.

\subsubsection{Hutch B1}

The first hutch in Hall B will contain optical elements, which condition the x-ray beam for the Hall B experiments (see Figure 9.14). Only one such element will be included in the initial LCLS, though space is made available for future optics. Hall B is intended primarily for experiments, which prefer to be far from the source, in order to reduce the peak intensity or to allow focusing to a minimum spot size. 


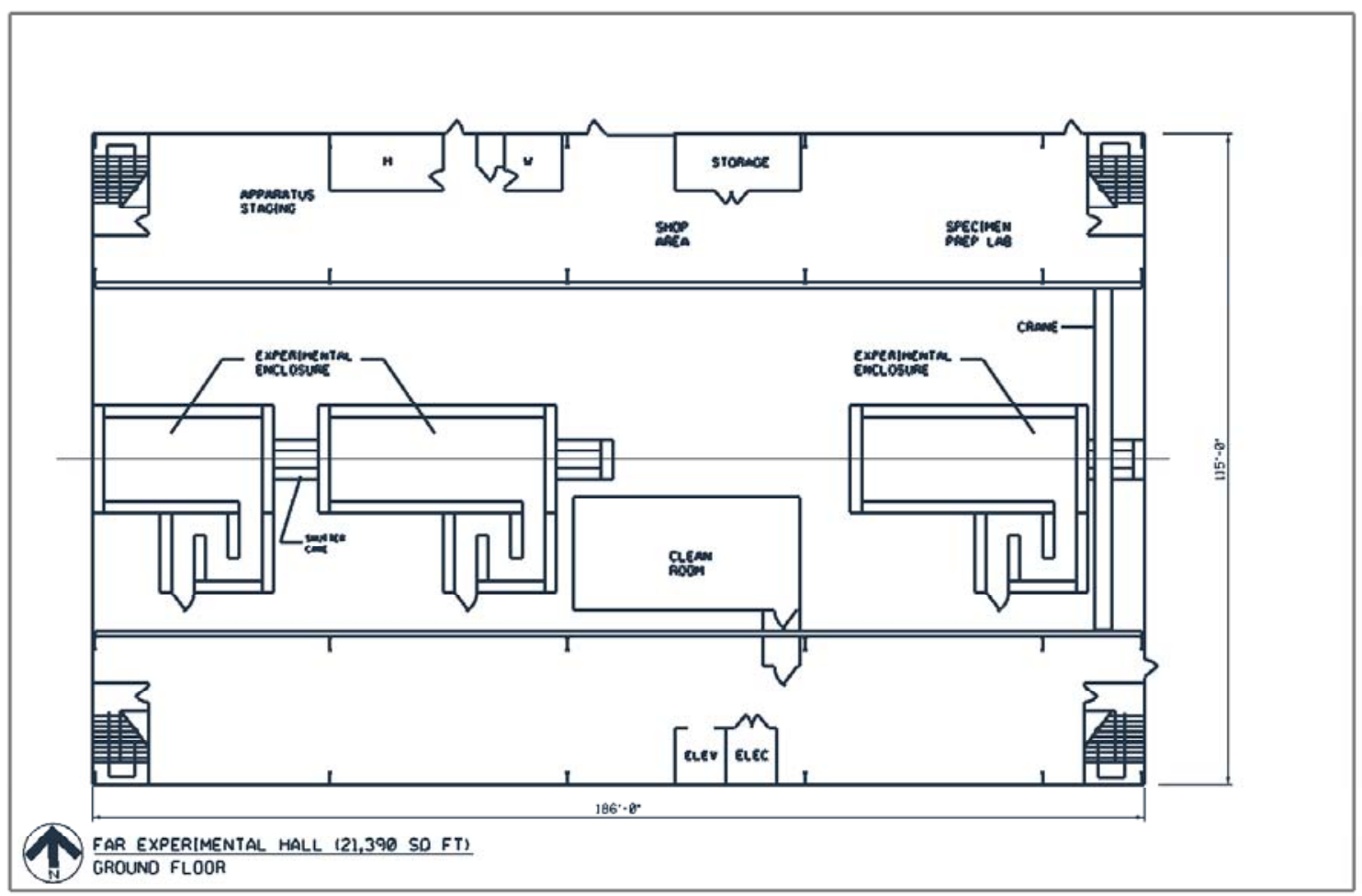

Figure 9.14 Experimental Hall B

\section{Monochromator}

Some Hall B experiments will require a bandwidth narrower than the intrinsic bandwidth of the FEL. In Hall B, standard monochromator crystals such as silicon or diamond should not suffer any damage due to the peak power, and so standard crystal monochromator designs can be used [12].

\section{Spools, Chambers, and Beam Stop}

Hutch B1 contains a diagnostics chamber for diagnostics associated with adjustment of the monochromator. It also contains several spool pieces, which may in future be replaced by additional mirror systems and monochromators. At the back end of Hutch B1 is an insertable beam stop with integral burn-through detector.

\subsubsection{Hutch B2}

Hutch B2 will house LCLS experiments. It will also contain beam-conditioning optics, which need to be close to the experiments, in particular, focusing systems with short focal length. Hutch B2 will also contain an optics tank with an x-ray pulse splitter and delay system, producing from each FEL pulse a pair of $x$-ray pulses with adjustable sub-ns delay. 


\section{Pulse Split/Delay}

This system will use crystal diffraction to split the FEL pulse, direct the two x-ray pulses around unequal path lengths, and bring them back onto the primary beam path with a time delay between them. Figure 9.15 shows the proposed scheme. The beam-splitting is accomplished by a very thin $(10 \mu \mathrm{m})$ silicon crystal. The radiation within the bandwidth for Bragg diffraction from this crystal is reflected with high efficiency $(80 \%)$, whereas the radiation outside the Bragg bandwidth is efficiently transmitted $(75 \%$ at $8 \mathrm{keV})$. By orienting the crystals in the two beam paths to reflect slightly different $x$-ray energies, the pulse is effectively split and sent around two separate paths. A simple translation can then be used to change the relative path lengths, and thus the pulse delay. The overall efficiency of the system for each path is about $30 \%$ at $8 \mathrm{keV}$. The bandwidth $(\delta \mathrm{E} / \mathrm{E})$ for each crystal reflection is about $2.5 \times 10^{-5}$, so two pulse energies can easily be selected from the LCLS bandwidth of about $10^{-3}$.

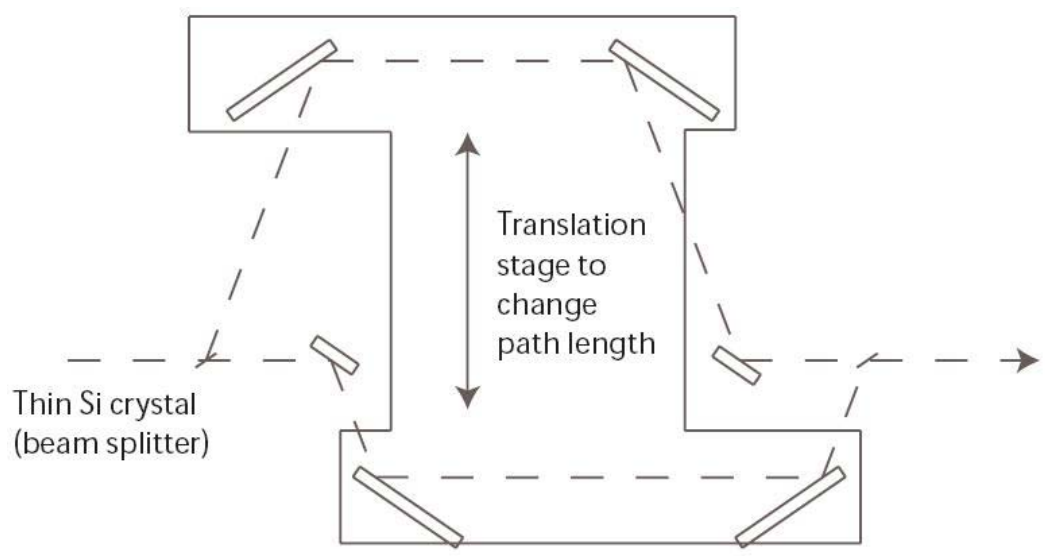

Thick Si crystal reflectors on translation stage

Figure 9.15 Pulse split and delay technique. Delay values of several hundred picoseconds can be achieved, with accuracy of a few femtoseconds.

\section{Focusing System}

Focusing in Hutch B2 will use a Kirkpatrick-Baez mirror system identical to that used in Hutch A2. If only one such mirror system is available initially, it can be transported and installed in either Hutch A2 or Hutch B2 as needed.

\section{Apertures}

The apertures used in Hutch B2 will be very similar to those used in Hutch A2.

\section{Attenuator}

Some experiments require a local attenuator for calibration and to prevent damage to sensitive components during alignment. The local attenuator will have a design very similar to the solid attenuator located in the Front End Enclosure. 


\section{Beam Intensity Monitors}

Beam intensity monitors are required to measure the absolute flux incident on the samples and the amount of flux transmitted through the samples. These monitors will be of the ion chamber type described in the facility diagnostics section (Section 9.4.2.3).

\section{Sample Chamber}

The sample chamber in Hutch B2 will be instrumented for development of sub-picosecond time-resolved experiments, such as laser pump/x-ray probe and $\mathrm{x}$-ray pump/x-ray probe experiments. It will include a goniometer for holding crystal samples, and windows for laser and scattered x-ray beams.

\section{Beam Stop}

At the back end of Hutch B2 is an insertable beam stop with integral burn-through detector.

\subsubsection{Hutch B4}

Hutch B4 may be used for future facility diagnostics.

\section{Beam Stop}

At the back end of Hutch B4 is a fixed (not insertable) beam stop. Power levels at this point will not damage materials such as copper, and so no burn-through monitor is required.

\subsection{Mechanical and Vacuum}

The beam transport mechanical and vacuum system contains approximately 400 meters of vacuum beam pipe and is maintained at $10^{-7}$ Torr by approximately 70 ion pumps. The basic design of a section of beam pipe is shown in Figure 9.16. These sections are repeated through the halls and tunnel, except in places where the pipe is replaced by one of the tanks or other instruments in the beam line.

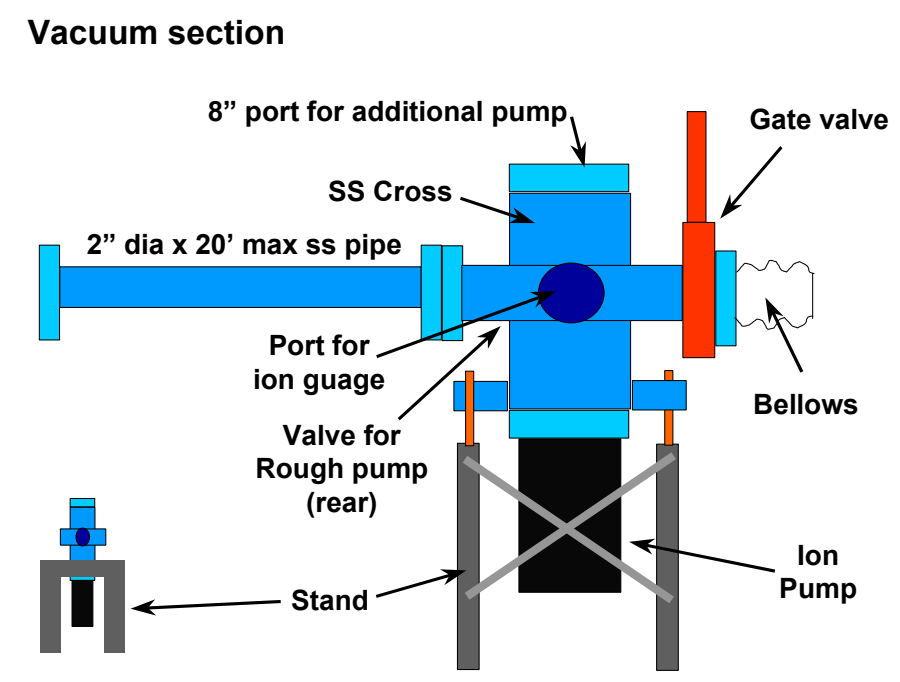

Figure 9.16 Typical section of vacuum beam pipe 
The pipes are 2" stainless steel electroplated inside and out and connected with metal sealed gaskets and welded $45 / 8$ " Conflat flanges. The maximum pipe length is 20 feet. The pumping section consists of a stainless-steel cross with 8 " flanges top and bottom to accommodate the ion pumps. Each ion pump has its own power supply. Additional 4 5/8" ports on the beam left and right accommodate ion gauges and a valve for rough pumping. The section terminates with an isolation valve and a bellows for alignment. The isolation valves are all metal gate valves such as manufactured by VAT. The stands are plasma-cut plates with cross bracing for earthquake protection.

The isolation valves around some of the tanks contain integrated welded Be windows, in order to allow $\mathrm{x}$-ray experiments to take place in rough vacuum or in air, if desired. The windows are Brush-Wellman pinhole-free S-65 polished Be disks $46 \mathrm{~mm}$ in diameter and 250 microns thick.

Some LCLS optics tanks (e.g., the mirror tanks) require ultra-high vacuum conditions. Other tanks (e.g., the gas attenuator) require pressures much higher than $10^{-7}$ Torr. These special tanks will be isolated from the main vacuum system by differential pumping sections. There are commercial differential pumping systems, which can do this job [13].

\subsection{Diagnostics}

\subsubsection{Diagnostics Layout}

The diagnostics are located in "diagnostics tanks" distributed along the beam line as shown in Figure 9.17. The diagnostics fall into two categories: 1) facility/monitoring diagnostics, and 2) commissioning diagnostics.

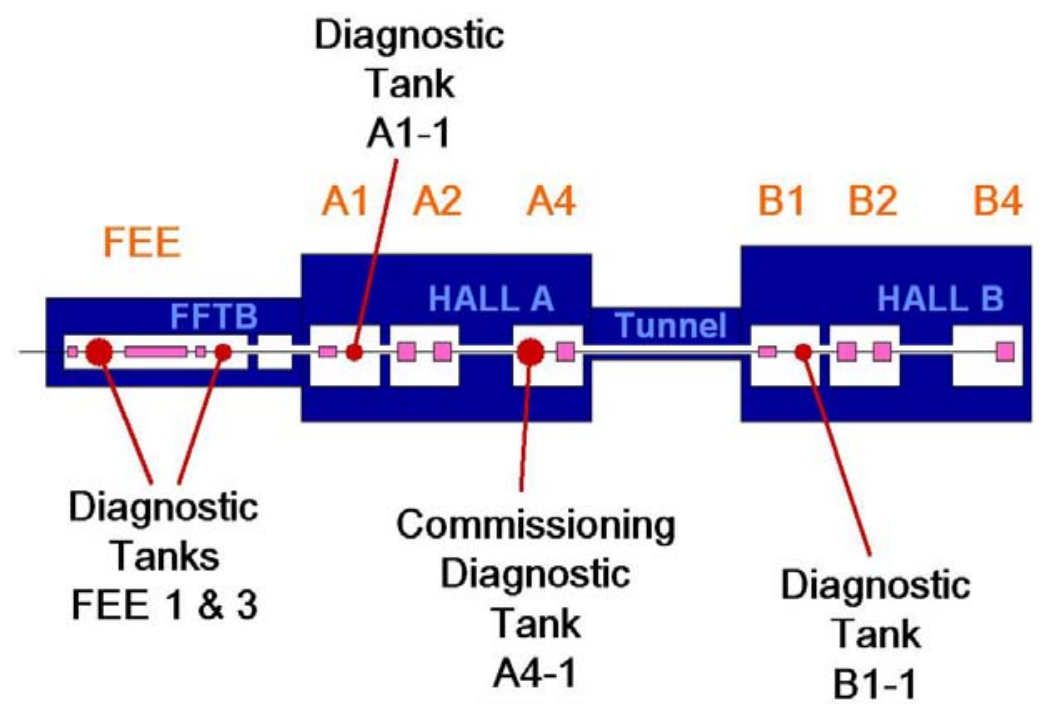

Figure 9.17 Tank locations for LCLS x-ray diagnostics

The facility diagnostics mainly provide pulse-to-pulse information about the beam energy, spatial shape, and centroid, as it is transported through the beam transport system to the 
experimental halls. Because of the fluctuating nature of the SASE FEL, it is critical to monitor these beam parameters on a pulse-by-pulse basis. This information is used to 1) provide feedback on the FEL performance, 2) aid in adjusting, monitoring, and setting facility optical systems (slits, attenuators, monochromator), and 3) provide energy, shape, and centroid information to users.

These diagnostics are located after the slits and attenuators in the Front End Enclosure, after the mirrors in the upstream end of Hall A, after the monochromator in the upstream and of Hall B, and at the very end of Hall B. They are intended to be "non-intrusive" if possible, allowing most of the beam to pass through without substantial modification. Avoiding some type of modification (coherence, intensity, etc.) may be difficult, especially at the lowest FEL photon energy.

The commissioning diagnostics are intended to measure the basic FEL performance parameters during commissioning and may be "intrusive". The goals of the commissioning diagnostics are to measure

1. Total pulse energy;

2. Pulse length;

3. Photon energy spectrum;

4. Transverse coherence;

5. Spatial shape and centroid location; and

6. Divergence.

The pulse energy, pulse length, spectrum and transverse coherence measurements will be performed in the "commissioning diagnostics tank" in Hutch A4. The divergence and shape measurements will be made by the "facility diagnostics" distributed along the beam line.

Characterizing the performance of LCLS will require pulse-by-pulse measurements of total energy, pulse length, spectrum, divergence, spatial shape, and transverse coherence. The concepts presented here are extensions of proven techniques that have worked well at synchrotron sources. However, further development will be needed to properly adapt them to the LCLS. Section 9.4.4 describes the first steps that will be taken along this development path.

\subsubsection{Facility Diagnostics Instruments}

Each facility diagnostics tanks contains one or more of the following systems:

\subsubsection{Direct Scintillation Imager}

The Direct Scintillation Imager (Figure 9.18) is an insertable, high-resolution scintillator viewed by a CCD camera for measuring spatial distributions and for alignment and focusing of optical elements. Traditional instruments have used phosphorus screens to convert x-rays to visible light that can be recorded by a CCD. Even with a microscope objective to magnify the screen, the spatial resolution is limited by the spatial resolution of the phosphorus that is typically 
in the range of 10 to 50 microns. Such resolutions are of marginal utility to the LCLS, which has a beam diameter at $8 \mathrm{keV}$ of 100 microns. Recently, workers at the ESRF synchrotron facility have used thin-film single-crystal scintillators for $\mathrm{x}$-rays, achieving $0.8-\mu \mathrm{m}$ resolution. The scintillator is a 5 micron thick Ce doped YAG crystal on a 100 micron YAG substrate. Other crystals such as LSO are likely to work as well.

The gated-intensified CCD camera can be read out at $120 \mathrm{~Hz}$, providing pulse-to-pulse information.

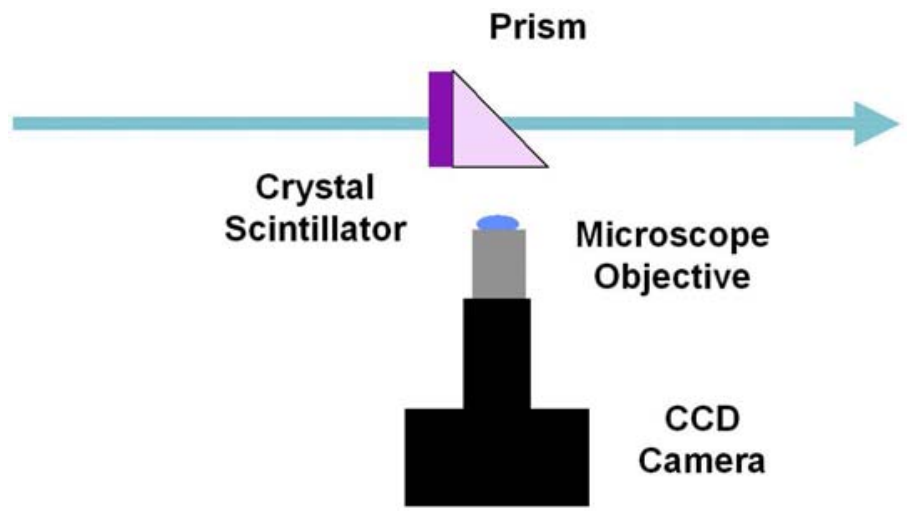

Figure 9.18 Direct Scintillation Imager

The Direct Scintillation Imager is intrusive - it blocks the beam. Its wide field of view will allow viewing of the spontaneous radiation pattern. In addition, its scintillator is susceptible to damage at the full FEL intensities.

\subsubsection{Scattering Foil Imager}

The Scattering Foil Imager (Figure 9.19) overcomes the FEL damage problems of the Direct Scintillation Imager by utilizing a thin foil of a low-Z material such as Be to act as a beam splitter to partially reflect a portion of the beam onto the YAG imaging camera which remains out of the beam. The reflected intensity can be adjusted by changing the angle of incidence. A reflectivity of $10^{-4}$ can be obtained with an incident angle of $1^{\circ}$ at $8 \mathrm{keV}$ and an incident angle of $>2^{\circ}$ at 0.8 $\mathrm{KeV}$. Further analysis of this concept is needed to assess the effects of background radiation on the crystal due to Compton scattering of the FEL beam by the Be foil, and of fluorescence from an oxide layer on the foil surface. 


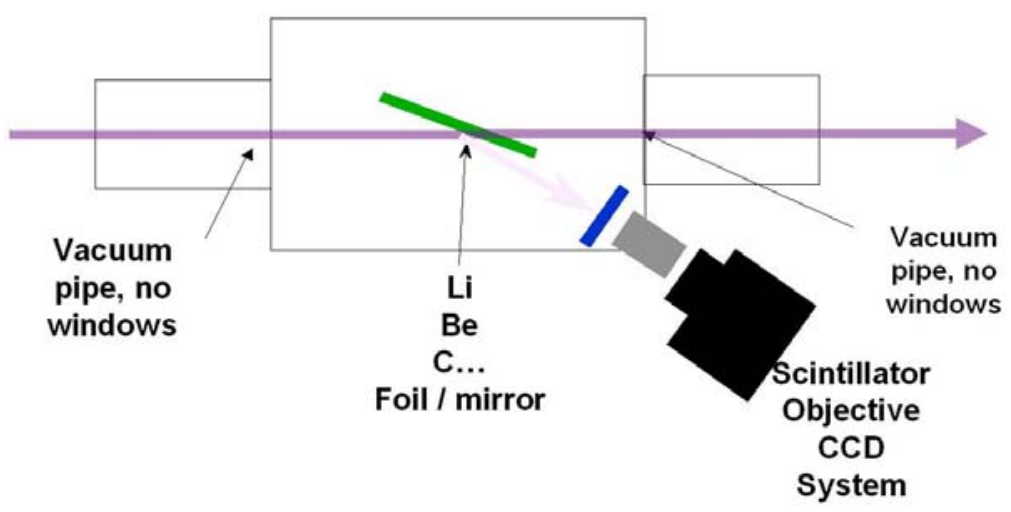

Figure 9.19 Scattering Foil Imager

With a thin (30 micron) polished Be foil, the Scattering Foil Imager is nearly transparent to the $8 \mathrm{keV}$ radiation and can be used throughout the beam line as a non-intrusive pulse-to-pulse monitor of the beam energy, shape, and centroid. At $0.8 \mathrm{keV}$, the foil will not be transparent but it will be able to withstand the full FEL intensity, so the Scattering Foil Imager can be used intrusively to measure the pulse-to-pulse statistics of the beam energy, shape, and centroid.

\subsubsection{Micro-Strip Ion Chamber}

The Scattering Foil Imager cannot monitor the $0.8 \mathrm{keV}$ FEL non-intrusively since it is opaque and even at $8 \mathrm{keV}$ it could introduce unwanted distortion into the beam. A traditional ion chamber, commonly used at current synchrotrons, is designed to operate at 1 atmosphere gas pressure, with a fairly low intensity DC beam. The high intensity and pulsed nature of the FEL require some modifications to the traditional design (Figure 9.20). The current-measuring electronics of traditional ion chambers must be replaced by pulse processing electronics to measure the energy in each FEL pulse. The drift region must be carefully designed so that the photoelectrons from the pulse are efficiently collected at the anode in the time between pulses. The chamber must be operated at pressures below 1 atmosphere to reduce the instantaneous charge that must be drifted and collected. At $8 \mathrm{keV}$ the gas can be contained within Be windows, but for $0.8 \mathrm{keV}$ operation a windowless chamber with differential pumping is required. Finally low-resolution centroid and shape information can be obtained by segmenting the anode as in a micro-strip detector. 


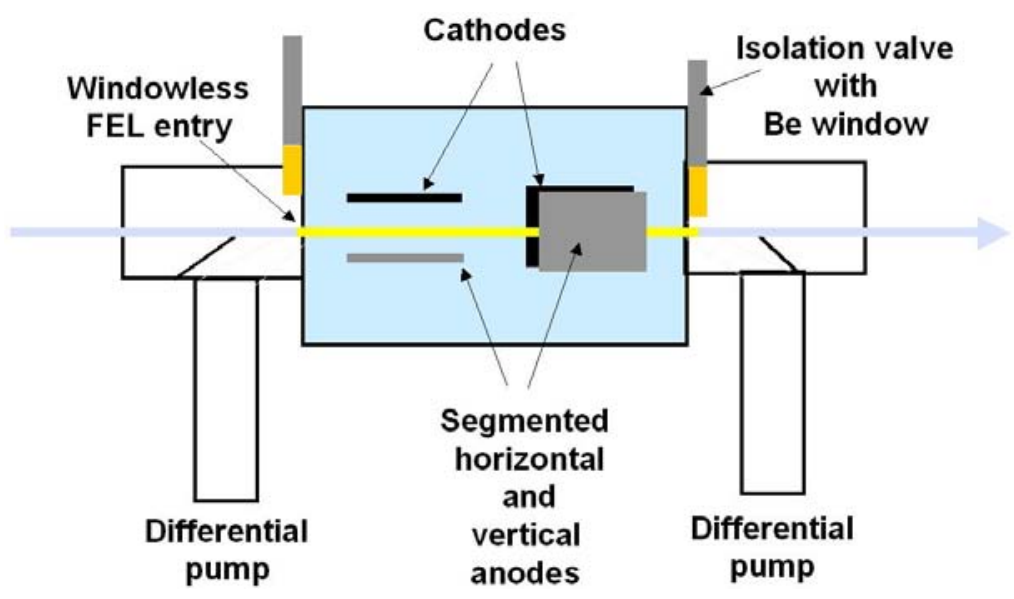

Figure 9.20 LCLS Ion Chamber

Because of the statistical nature of the FEL radiation it is very important to monitor the beam energy, centroid, and overall shape on a pulse-to-pulse basis in a non-intrusive manner. The micro-strip LCLS Ion chamber offers less-intrusive monitoring of the FEL pulse-to-pulse energy as well as low-resolution centroid and shape information. Figure 9.21 shows the principles of the micro-strip readout.

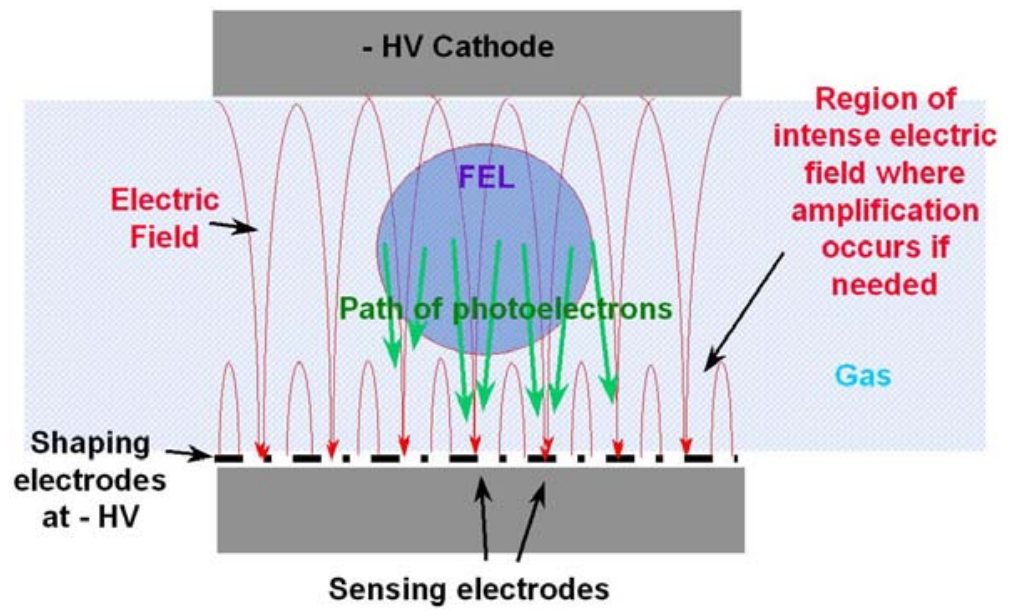

Figure 9.21 Micro-strip lon Chamber

Photoelectrons liberated by the FEL photons drift down to thin sensing electrodes lithographed onto a substrate. In between the sense electrodes are thicker strips at negative high voltage, which shape the drift field. The centroid and shape are determined by the distribution of charge collected on the sense strips. At sufficient voltage and using correct gas, the electric field in the region near the sense strips can be high enough for gas multiplication to occur. In this regime the sensor is sensitive to single photoelectrons. This could be an advantage for operation at $0.8 \mathrm{keV}$, since it would then be possible to lower the gas pressure considerably and reduce the pumping load. 


\subsubsection{Facility Diagnostic Tanks}

The first facility diagnostic tank (FEE 1) is located just downstream of the first set of slits in the FEE (Figure 9.22). It contains a Direct Scintillation Imager, a foil imager, and an ion chamber. Since it is the closest diagnostic station to the FEL, and will likely be used heavily during the earliest stages of commissioning, the tank has its own turbo pumping system and is large enough to accommodate other diagnostics.

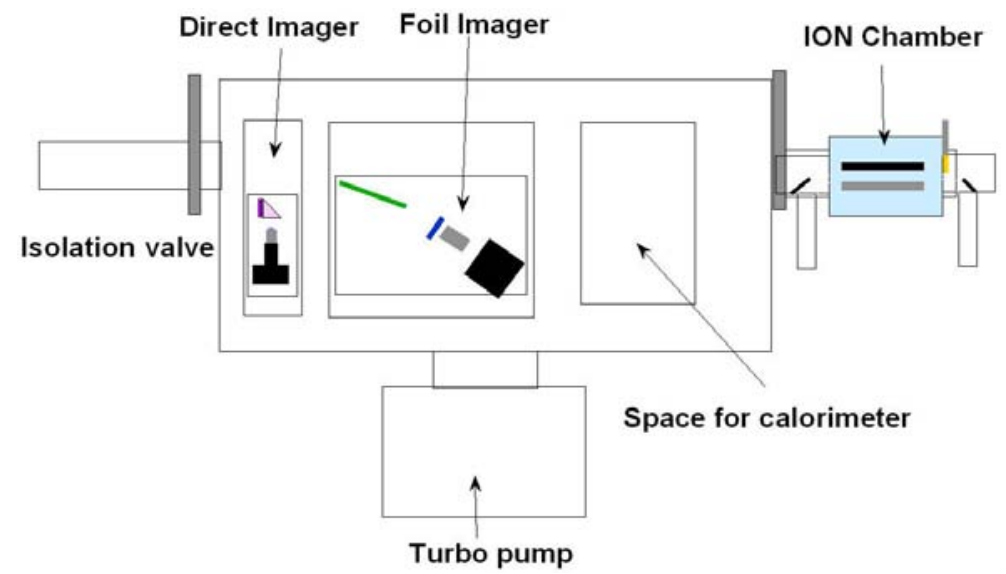

Figure 9.22 First Diagnostic Tank (FEE1)

The other diagnostic tanks are similar, though they generally do not have turbo pumps. Table 9.6 lists the contents of the other facility diagnostics tanks.

Table 9.6 Diagnostic Tanks

\begin{tabular}{|c|c|c|c|c|c|c|}
\hline Tank & Purpose & $\begin{array}{c}\text { Direct } \\
\text { imager }\end{array}$ & $\begin{array}{c}\text { Scattering } \\
\text { imager }\end{array}$ & $\begin{array}{c}\text { Ion } \\
\text { Chamber }\end{array}$ & $\begin{array}{c}\text { Ion } \\
\text { Pump }\end{array}$ & $\begin{array}{l}\text { Turbo } \\
\text { pump }\end{array}$ \\
\hline FEE 1 & Slit 1 & $\mathrm{X}$ & $\mathrm{X}$ & $\mathrm{X}$ & $\mathrm{X}$ & $\mathrm{X}$ \\
\hline FEE 2 & Gas attenuator & $\mathrm{X}$ & & & $\mathrm{X}$ & \\
\hline FEE 3 & Wedge + Slit 2 & $\mathrm{X}$ & $\mathrm{X}$ & $\mathrm{X}$ & $\mathrm{X}$ & \\
\hline A1 1 & Mirror & $\mathrm{X}$ & $\mathrm{X}$ & $\mathrm{X}$ & $\mathrm{X}$ & \\
\hline A1 2 & (empty) & & & & & \\
\hline A4 1 & Commissioning & $\mathrm{X}$ & & & $\mathrm{X}$ & $\mathrm{X}$ \\
\hline B1 1 & Hall B entrance & $\mathrm{X}$ & $\mathrm{X}$ & $\mathrm{X}$ & $\mathrm{X}$ & \\
\hline B1 2 & Monochromator & $\mathrm{X}$ & $\mathrm{X}$ & $\mathrm{X}$ & $\mathrm{X}$ & \\
\hline B4 1 & (empty) & & & & & \\
\hline
\end{tabular}




\subsubsection{Ion Chamber Gas Mixing and Distribution System}

Micro-strip detectors utilize variants of the so-called "magic gas", a 3-component mixture of argon, isobutane, and Freon. A gas mixing system, shown schematically in Figure 9.23, supplies gas for the micro-strip ion chambers.

\section{Micro-Strip gas mixer}

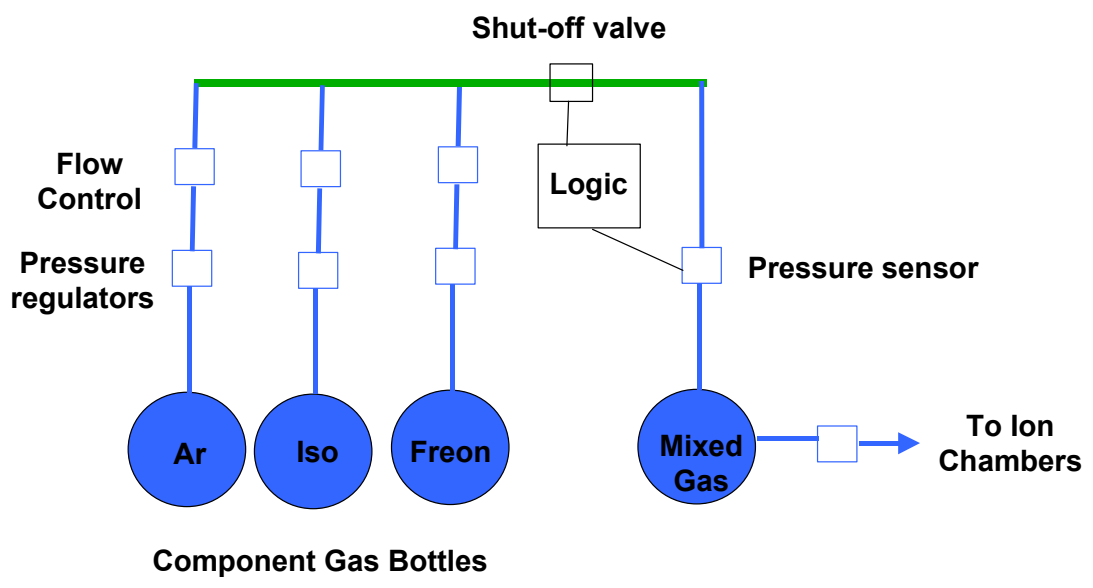

Figure 9.23 Micro-strip gas mixer

The mixer uses flow controllers to mix the correct amounts of the component gases into a reservoir, which feeds the ion chambers at a slower rate. The mixer logic monitors the reservoir pressure and initiates fill cycles as needed.

\subsubsection{Commissioning Diagnostics}

The intrusive commissioning measurements of total energy, pulse length, spectrum, and transverse coherence will be performed in Hutch A4, tank 1 (Figure 9.24). The divergence and spatial shape measurements will be performed using the data from the facility imaging cameras distributed along the beam line.

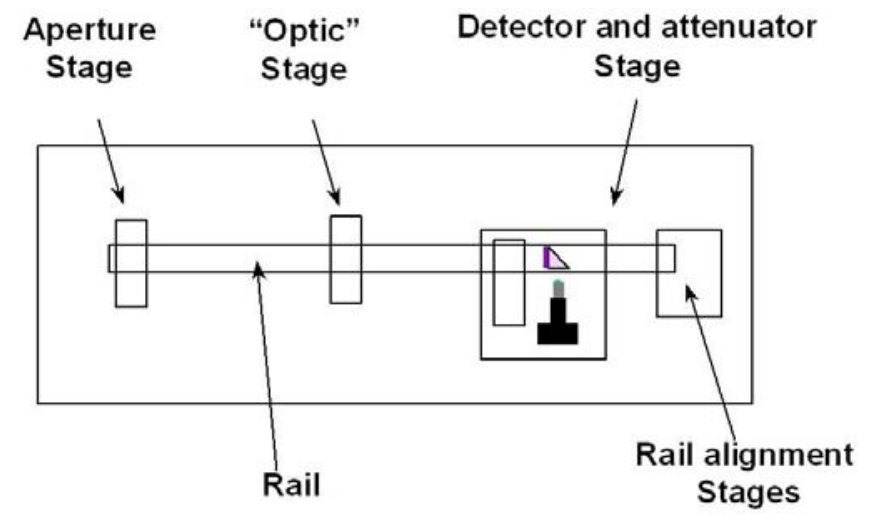

Figure 9.24 Commissioning Diagnostics tank 
The commissioning diagnostic tank has a central optical rail and stages for the necessary apertures, optics, and Direct Scintillation Detector.

\subsubsection{Total Energy}

It is desirable to measure the FEL pulse energy utilizing calorimetric techniques to avoid any reliance on the theory of photon-atom interactions at LCLS intensities.

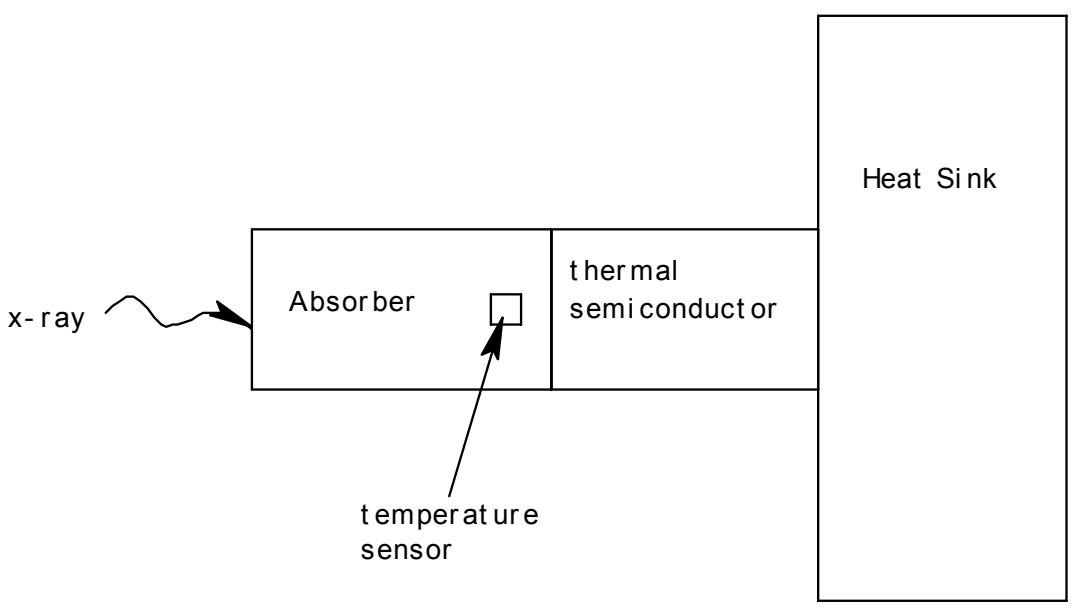

Figure 9.25 Calorimeter for accurate pulse energy measurement

The calorimeter (Figure 9.25) has a small-volume x-ray absorber, which absorbs all of the $x$ ray energy resulting in a rapid temperature rise dependent on the heat capacity and mass of the absorber. For a $1 \%$ measurement, the thickness of the absorber must be at least 5 mean free path lengths in order to capture better than $99 \%$ of the x-ray energy. The sensor measures the temperature rise of the absorber. The thermal mass of the sensor is small compared to the absorber. The energy in the absorber is conducted through the thermal weak link to a heat sink held at a constant temperature. The purpose of the thermal weak link is to delay the heat transfer from the absorber to the heat sink long enough to measure the temperature rise in the absorber. The energy deposited by each x-ray pulse is conducted into the heat sink before the arrival of the next $\mathrm{x}$-ray pulse.

For $8 \mathrm{keV}$ operation the absorber could be a Si cylinder $0.5 \mathrm{~mm}$ in diameter and $0.5 \mathrm{~mm}$ thick. The $0.5 \mathrm{~mm}$ thickness is $>5$ attenuation lengths and the $0.5 \mathrm{~mm}$ diameter nicely accommodates the $\sim 340$ microns FWHM diameter of the $8 \mathrm{keV}$ FEL at the position of the commissioning diagnostics tank. The dose at $8 \mathrm{keV}$ to $\mathrm{Si}$ in this position is $0.12 \mathrm{eV} /$ atom, which is acceptable for a simple absorber.

For $0.8 \mathrm{keV}$ operation the absorber could be a Be disk $3 \mathrm{~mm}$ in diameter and $>25$ microns thick since the dose to $\mathrm{Si}$ at this wavelength is too high. The $3 \mathrm{~mm}$ diameter is necessary to contain the $0.88 \mathrm{keV}$ beam whose diameter at this position is $1.9 \mathrm{~mm}$ FWHM.

The calorimeter will be positioned on the "optics stage" in the commissioning tank allowing it to be aligned utilizing the rear imaging detector. 


\subsubsection{Pulse Length}

Measuring the $233 \mathrm{fs}$ pulse length is perhaps the most challenging measurement at the LCLS. Several concepts have been proposed, all involving a medium, which modulates an external laser beam when exposed to the x-ray FEL. Figure 9.26 illustrates one possible method. The beam from a $1500-\mathrm{nm} \mathrm{CW}$ laser is split and made to pass through the two arms of an interferometer patterned in GaAs on a substrate. x-rays impinging on one of the arms changes its index of refraction, causing a modulation in the laser beam after it is recombined. The modulation of the laser beam is in principle of the same duration as the x-ray pulse and can be measured with a streak camera with an accuracy of about $0.5 \mathrm{ps}$. To achieve better temporal resolution, the modulated optical laser beam is sent through a "time microscope" which stretches the pulse by a factor of $2 \times$ to $100 \times$. The stretched pulse length is then measured with the streak camera.

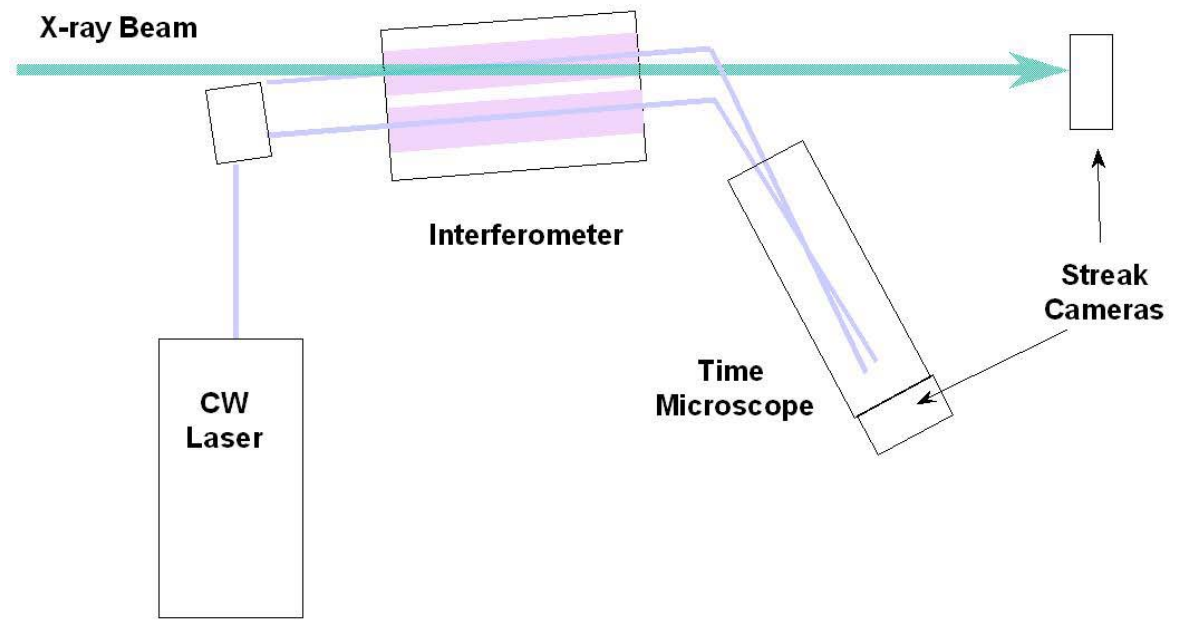

Figure 9.26 Pulse length measurement

The device can also be used to synchronize an external laser pulse with the x-ray beam. This is accomplished by feeding the external pulse through the time microscope alongside of the $\mathrm{x}$-ray modulated $\mathrm{CW}$ pulse and measuring both on the same streak camera.

\subsubsection{Photon Spectrum}

The commissioning diagnostic tank is converted into a spectrometer (Figure 9.27) by adding a crystal at $8 \mathrm{keV}$ or a grating at $0.8 \mathrm{keV}$. In either case the optic disperses the radiation onto the $\mathrm{x}$-ray sensitive region of a fast readout position-sensitive detector. 


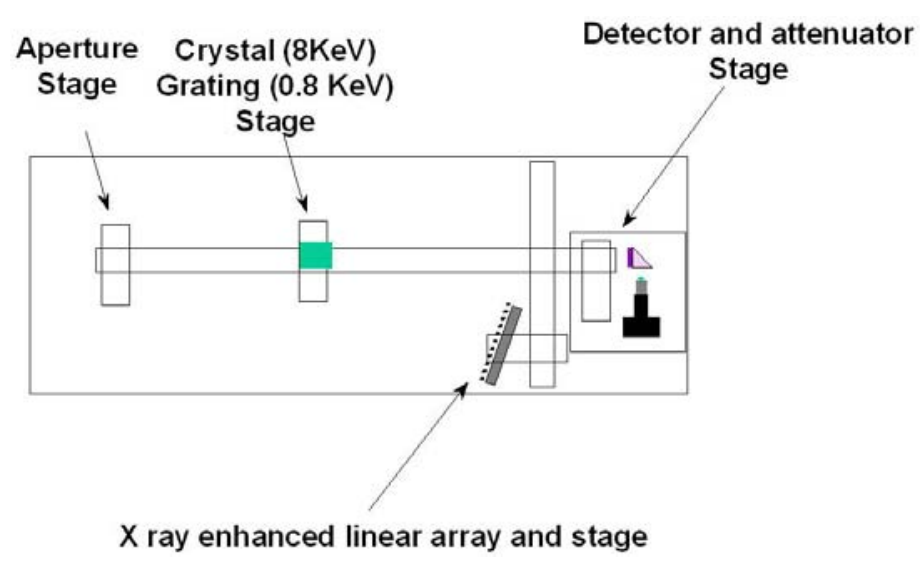

Figure 9.27 Spectrum measurement

\subsubsection{Transverse Coherence}

The transverse coherence could be measured in the commissioning diagnostics tank using the setup shown in Figure 9.28, which employs an array of double slits with constant slit width but different slit spacing. The slits sample the beam in two places and the resulting diffracted beams interfere with each other at the position of the detector.

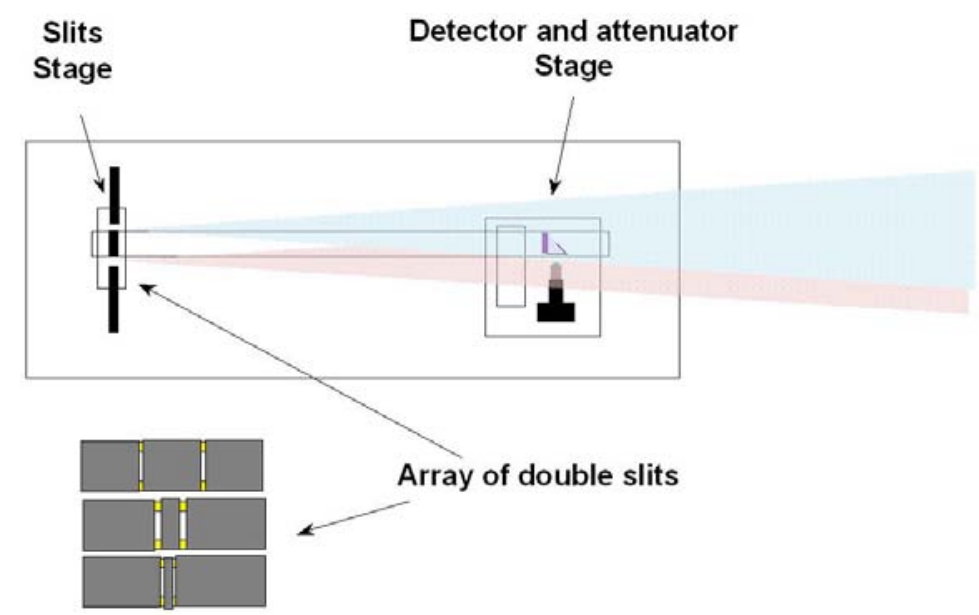

Figure 9.28 Spatial coherence measurement

At $0.8 \mathrm{keV}$ the slits will be assembled from polished sticks of low- $\mathrm{Z}$ material such as $\mathrm{B}_{4} \mathrm{C}$ or $\mathrm{Si}$, held apart by spacers. The higher resolution "slits" for $8 \mathrm{keV}$ will be manufactured by the sputter-slice method or from an array of fibers.

\subsubsection{Spatial Shape and Centroid Location}

The spatial shape and centroid location of the FEL beam will be measured on a pulse-bypulse basis by the Scattering Foil Detectors located in the facility diagnostics tanks distributed along the beam lines. 


\subsubsection{Divergence}

This measurement is performed at $8 \mathrm{keV}$ using the Scattering Foil Detectors located along the beam line. The measurement is performed at $0.8 \mathrm{keV}$ using the LCLS Segmented Ion Chambers located along the beam line.

\subsubsection{Diagnostics Modeling}

The predictions of the properties of the LCLS FEL beam raise many concerns in the design of the diagnostics, including short pulse effects, power loading, Compton backgrounds, spontaneous background, effects of higher harmonics, and effects due to the coherence of the beam - to name a few. A detailed understanding of these effects is critical for the successful design of each of the diagnostics. This section describes the simulation efforts needed for each diagnostic in order to make efficient use of codes common to all.

A "wave" model will be assembled to propagate the FEL radiation through the diagnostic instrumentation. Ginger simulations will provide the initial FEL radiation characteristics. The code breaks the Ginger FEL beam into its Gauss-Hermite components and contains modules for calculating the action of mirrors, crystals, apertures, multilayers, and zone plates on each of the modes. By summing the modified modes, the code will produce a quantitative image of the time history of the electromagnetic field at the diagnostic and maps of the power loading on each component.

Also, a Monte-Carlo model will be assembled to quantify efficiencies and backgrounds. The Monte-Carlo code generates photons according to the electromagnetic field distributions produced by the wave model as well as spontaneous photons. It tracks the photons through the diagnostic materials (gas, scintillator, etc.), generating Compton and photoelectrons according to the photon cross sections.

Both wave and Monte-Carlo simulations will be performed for each diagnostic.

\subsection{References}

1 R. Tatchyn, et al., "X-Ray Optics Design Studies for the 1.5-15 A LCLS at SLAC", in Coherent Electron-Beam X-Ray Sources: Techniques and Applications, A.K. Freund, H.P. Freund, and M.R. Howells, eds., SPIE vol. 3154 (1997).

2 B. Adams, personal communication; P. Bucksbaum, personal communication.

3 R. M. Bionta, "Controlling Dose to Low Z Solids at LCLS", LCLS note LCLS-TN-00-3

4 R. Tatchyn, "LCLS Optics: Technological Issues and Scientific Opportunities," in Proceedings of the Workshop on Scientific Applications of Short Wavelength Coherent Light Sources, SLAC Report 414; SLAC-PUB 6064, March 1993

5 R. Tatchyn, P. Csonka, H. Kilic, H. Watanabe, A. Fuller, M. Beck, A. Toor, J. Underwood, and R. Catura, "Focusing of undulator light at SPEAR with a lacquer-coated mirror to power densities of $10^{9}$ watts/cm²," SPIE Proceedings No. 733, 368-376(1986) 
6 D. Ryotov and A. Toor, "x-ray attenuation cell", LCLS TN-00-10 (2000)

7 D. R. Walz, A. McFarlane, E. Lewandowsky, J. Zabdyr, "Momentum Slits, Collimators, and Masks in the SLC," Proceedings IEEE 1989 Particle Accelerator Conference, IEEE Catalog \# 89CH2669-0, 553(1989); SLAC-PUB 4965

8 R. Tatchyn, G. Materlik, A. Freund, J. Arthur, eds., Proceedings of the SLAC/DESY International Workshop on the Interactions of Intense Sub-Picosecond X-Ray Pulses with Matter, SLAC, Stanford, CA, Jan. 23-24, 1997; SLAC-WP-12.

9 S. Dushman, Scientific Foundations of Vacuum Technique, John Wiley, New York, 1941.

10 H. N. Chapman, et al., J. Vacuum Sci. Technol. B19, 2389 (2001)

11 G.E. Ice, J.-S. Chung, J. Z. Tischler, A. Lunt, and L.Assoufid, Rev. Sci. Instrum. 71, 2635 (2000).

12 A. Freund, "Crystal Optics for the LCLS," presented at the SLAC/DESY International Workshop on the Interactions of Intense Sub-Picosecond X-Ray Pulses with Matter, SLAC, Stanford, CA, Jan. 2324, 1997.

13 Differential Pump DP-01, XHA X-Ray Instrumentation Associates, Mountain View, CA 94043. 


\section{Conventional Facilities}

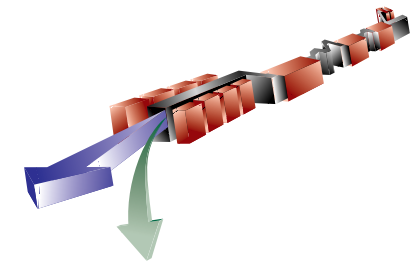

\section{TECHNICAL SYNOPSIS}

The LCLS takes advantage of the existing infrastructure at SLAC. It uses the last third of the existing $3 \mathrm{~km}$ linac including the existing enclosure and utilities. A new injector will be installed at sector 20 in the Off-Axis Injector Tunnel. This branch tunnel was constructed as part of the original construction at SLAC in the 1960s for just such an injector. The existing linac equipment including the klystrons and modulators will be used. The injector tunnel will require some modifications to bring it to current safety standards and to accommodate the specific requirements of the LCLS injector.

Two short sections of linac will be removed to accommodate the magnets and vacuum chambers for the two pulse compressors. New systems to bring power and water to these elements will be required.

The beam transport, DL-2, from the end of the linac to the beginning of the undulator will be new but will require less power and water than the existing Final Focus Test Beam (FFTB). This is due to the fact that the maximum energy for the LCLS beam is $16 \mathrm{GeV}$ while the FFTB is designed for a $50 \mathrm{GeV}$ beam.

The LCLS Undulator is housed in the existing FFTB tunnel which will be renamed the Undulator Hall. The undulator is a permanent magnet device with very low power and cooling requirements. The existing capacity is adequate. In both the case of DL-2 and the undulator the power and water distribution systems will have to be reconfigured.

The Undulator has tight alignment requirements, which places stringent requirements on the foundations and on the temperature stability of the air and water in the tunnel. The existing tunnel has the stable supports required for the FFTB, which were characterized as part of that operation. The LCLS will require additional piers.

Two new experimental halls will be built. The near hall will begin 40 meters downstream of the exit of the undulator and extends for 55 meters in the direction of the beam. It will be 30 meters wide. The far hall will be constructed 322 meters downstream of the exit of the undulator, just outside of a relocated PEP ring road. It will be 57 meters in the direction of the beam and 35 meters wide. The two halls will be connected by a 3 meter by 3 meter tunnel that is 227 meters long. The experiment area on the first floor of the far hall will be below grade with the ceiling approximately at grade. An office and laboratory area will be constructed at grade on top of the far hall to accommodate LCLS users and scientific and support staff. Parking will be provided adjacent to the far hall. 


\subsection{Injector Housing}

When the SLAC linac was originally constructed in 1962, two short tunnels were built on the north side of the main linac tunnel. One is at the 1/3-point (sector 10) and another at the 2/3-point (sector 20). These tunnels were included to house future injectors that would allow efficient use of segments of the linac. The tunnel at sector 20 is located in the right place for the LCLS injector (see Figure 10.1). The original construction included a surface building to support the injector (see Figure 10.2). Neither the tunnel nor the surface building has been used for this purpose in the 36 years of operation of the linac.

The shielding between the off-axis tunnel and the main tunnel will be reconfigured to accommodate the beam pipe, the waveguides, an alignment pipe and other utilities. There are three penetrations between the surface building and the injector tunnel to accommodate the laser beam transfer pipe, control signals, and power connections.

The support building will be modified to have a clean room for the laser. Personnel exclusion walls will be built with a pair of doors on the west side for personnel access to the tunnel. The interlock for these doors will be integrated into the existing linac Personnel Protection System.

The injector will be powered by existing klystrons in the klystron gallery. No new resources will be required. The klystron output power will be redirected to the off-axis tunnel with a new waveguide system described in the injector section.

Utilities will be provided for the magnet power supplies, controls, lasers, vacuum, and diagnostics. Cooling water will come from the main linac tunnel to cool the accelerator components. Cooling water will come from the klystron gallery for the laser and the equipment in the support building. In both cases, there is adequate capacity but new plumbing and wiring are required.

The costs to modify the existing utilities for the injector's use are included with the injector costs. 


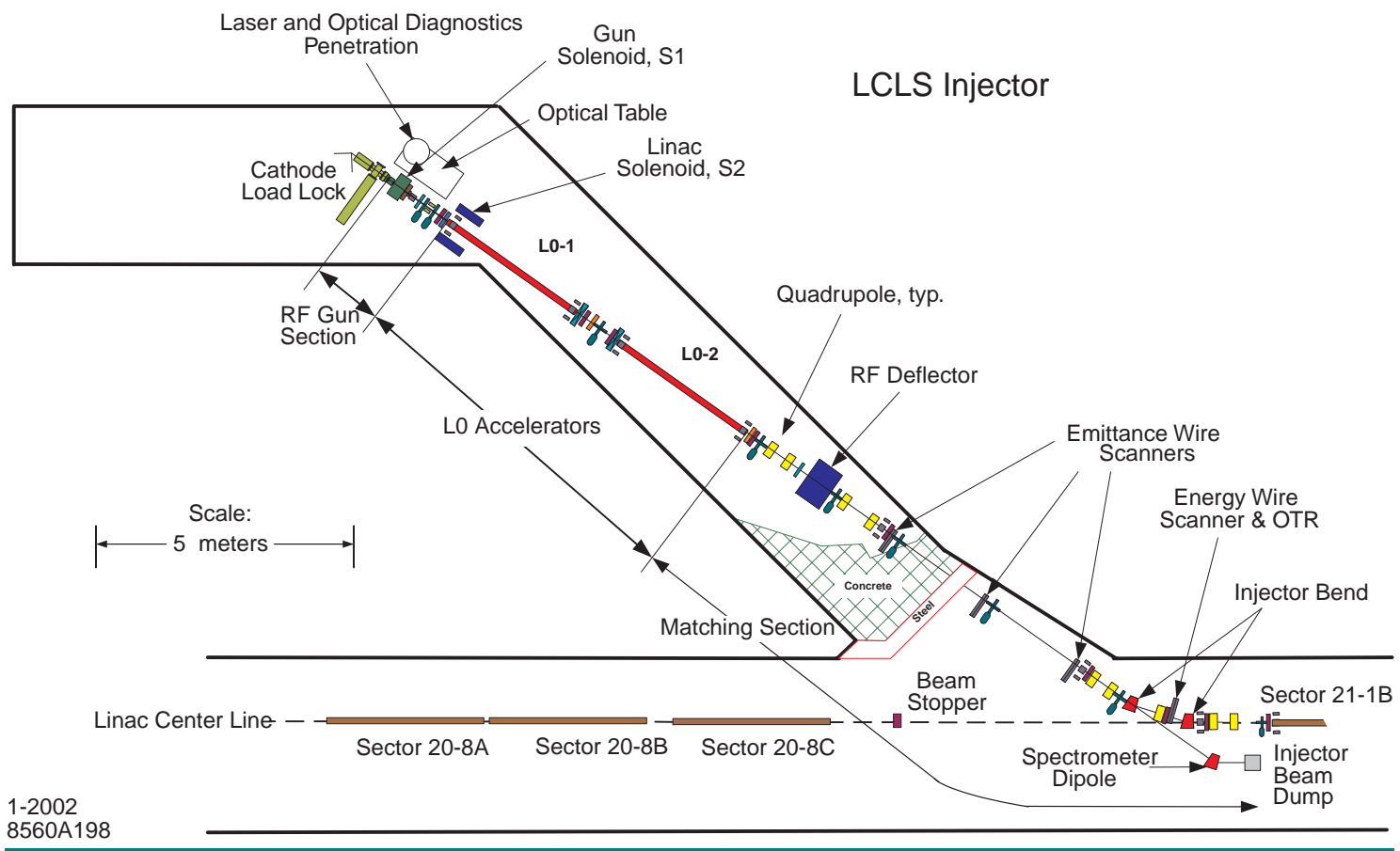

Figure 10.1 Layout of the injector tunnel

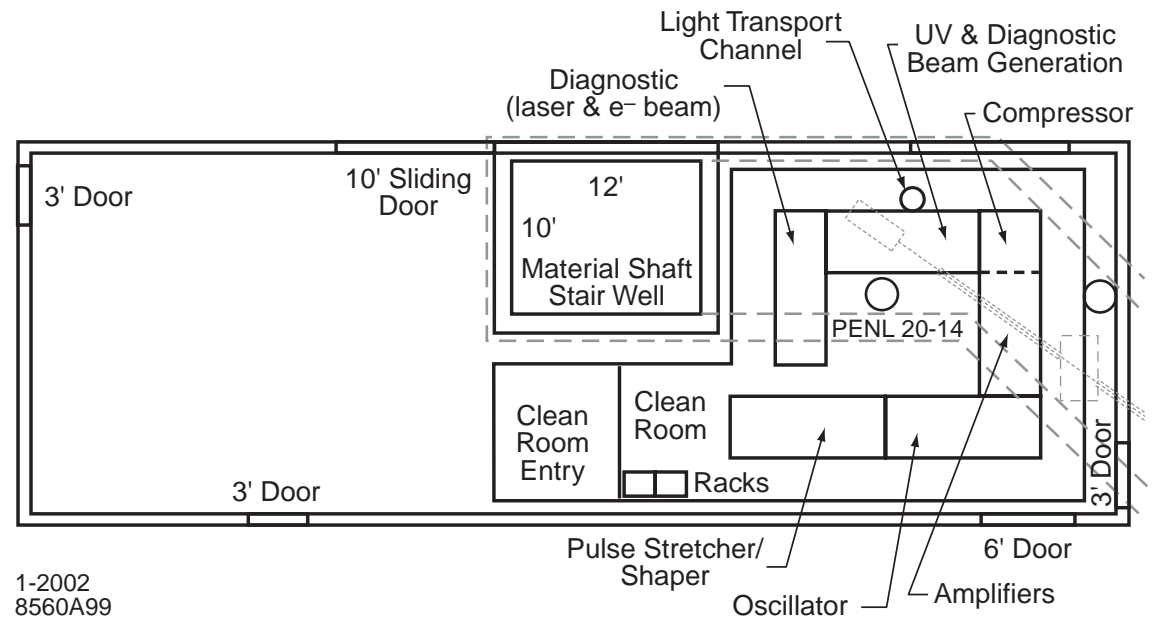

Figure 10.2 Layout of the injector support building 


\subsection{Linac Housing}

The LCLS uses the last $1 \mathrm{~km}$ of the existing linac, from sector 20 through sector 30 . The existing utilities will be adequate for the new operation. In two sectors, sector 20 and sector 25 , sections of the linac will be removed and replaced with magnets and vacuum chambers for electron beam pulse compression. Rearrangement of some low conductivity water and electrical power distribution will be required at these locations but the total capacity is adequate for the new requirements. The costs for these changes are included with the linac costs.

A new x-band accelerating structure will be added at sector 20 . This will require a new modulator and klystron in the gallery. This new installation will require power and water connections.

\subsection{Undulator Hall}

The Undulator Hall will house the electron beam dogleg, the undulator and the electron beam dump. The upstream end of this tunnel is underground and in line with the linac. The downstream part is constructed in the Research Yard from shielding blocks.

Prior to the construction of the LCLS, this hall houses the technical equipment associated with the FFTB. This equipment will be removed to make room for the LCLS equipment, primarily the undulator. The piers in the tunnel and the general soil stability were characterized during the earlier operation of the FFTB. Stability was important to the FFTB operation and the existing piers in the tunnel were constructed to tight stability specifications.

The LCLS undulator requires exceptional mechanical and environmental stability. New stable supports will be installed in the tunnel for the undulator. The air handling in the tunnel will be improved to reduce the tunnel temperature variation.

The utilities required in the tunnel for the LCLS are more modest that those required during FFTB operation. The energy is lower, $16 \mathrm{GeV}$ vs. $50 \mathrm{GeV}$, and the undulator uses permanent magnets. No new utility resources are required but new plumbing, wiring, cable trays, etc. will be required. The costs for these elements are included in the Linac and Undulator sections.

\subsection{Experimental Halls}

The LCLS requires two experimental halls, one 40 meters downstream of the end of the undulator and the other 322 meters downstream of the end of the undulator (see Figure 10.3). A tunnel for the beam line, utilities and access connects the halls. 


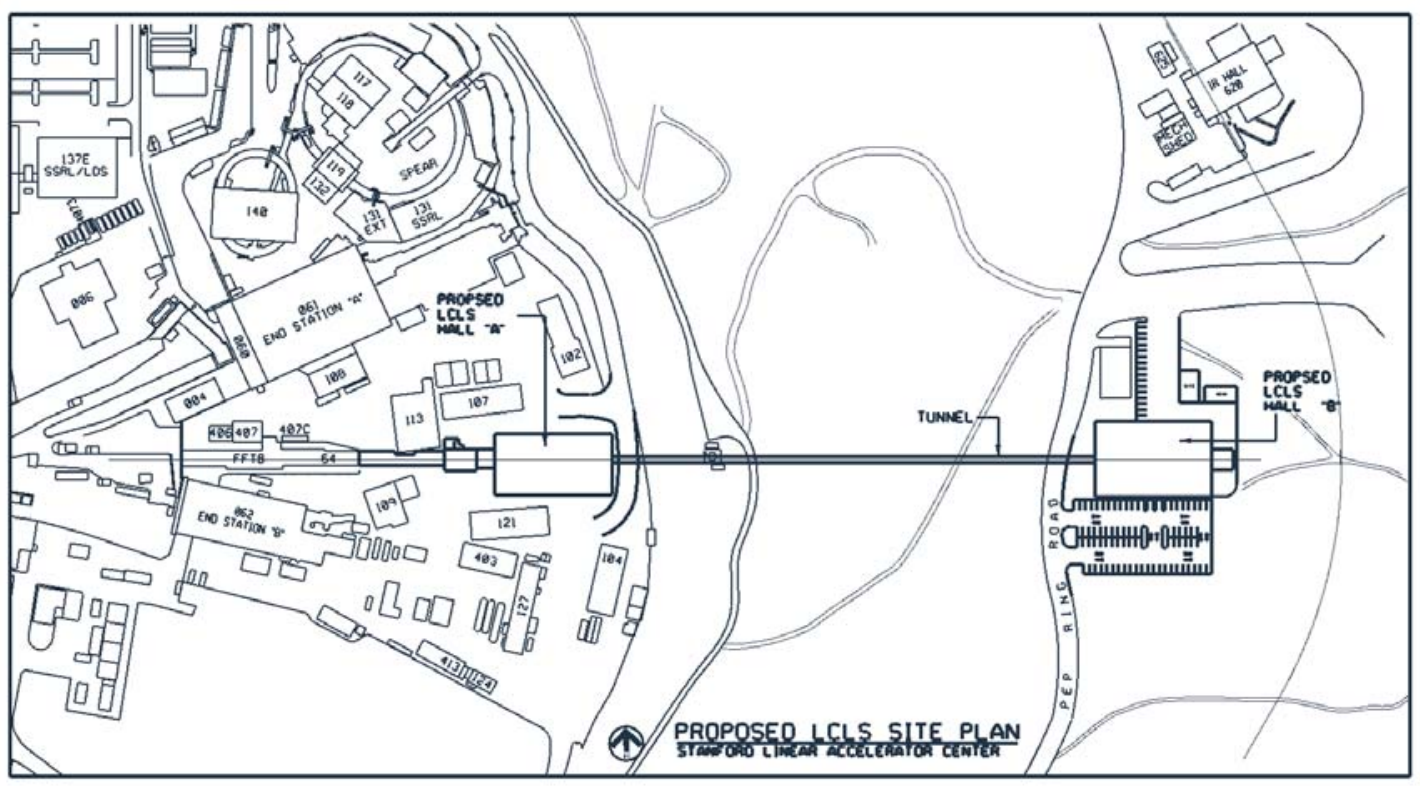

Figure 10.3 Site plan of the experimental halls

\subsubsection{Near Experimental Hall}

The near experimental hall will be constructed immediately downstream of the electron beam dump. This hall will be 30 meters wide by 55 meters in the direction of the x-ray beam.

The near hall will have 10 offices for LCLS users and on-site operations staff. This hall will include three enclosures for $\mathrm{x}$-ray diagnostic equipment.

The interior distribution of the utilities will be covered in the section on x-ray optics. Adequate water and power for the near hall experiments is available in the Research Yard.

Figure 10.4 shows an architectural rendering of the near hall in the Research Yard, Figure 10.5 shows a cross section of that hall and Figure 10.6 shows the initial layout of the interior. 


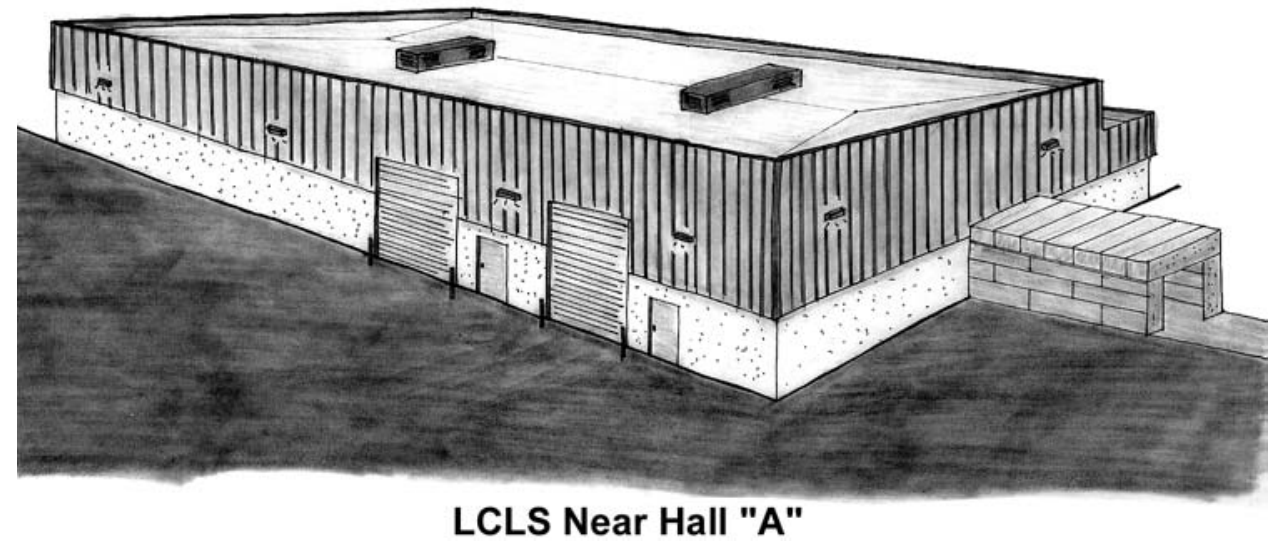

Figure 10.4 Near hall architectural rendering

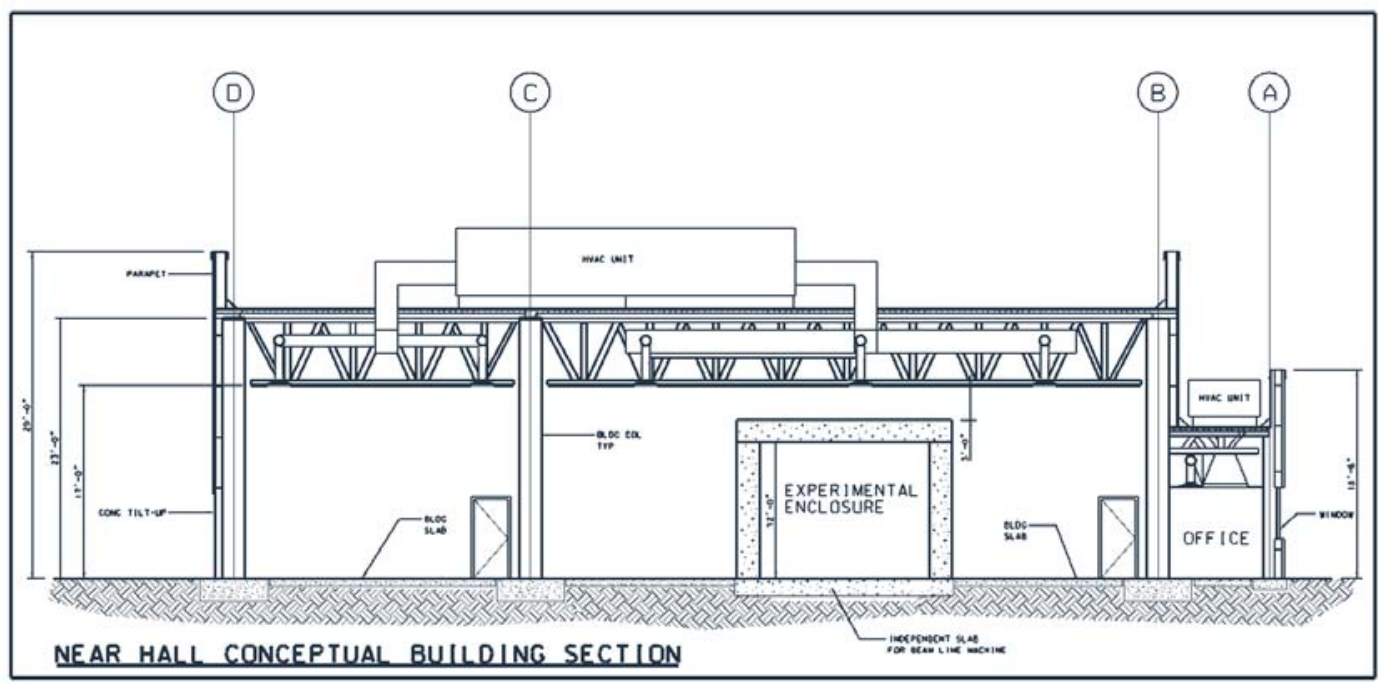

Figure 10.5 Cross section of near hall 




Figure 10.6 Near hall floor plan

\subsubsection{Far Experimental Hall}

The far experimental hall is located east of the PEP ring road. It is 57 meters in the beam direction and 35 meters wide. The floor is 6 meters below grade with the x-ray beam line 1.25 meter above the floor. The ceiling of the experimental hall is at grade level. A laboratory and office structure, Figure 10.7, will be constructed on top of this hall with 95 offices and 18 laboratory and support areas. Figure $\mathbf{1 0 . 8}$ shows the floor plan for this office and laboratory structure. Figure 10.9 shows a cross section of the building. There is a service ramp from the ring road to the floor of the experimental hall to allow bringing equipment directly to the experiments. Parking is provided for 70 cars. 


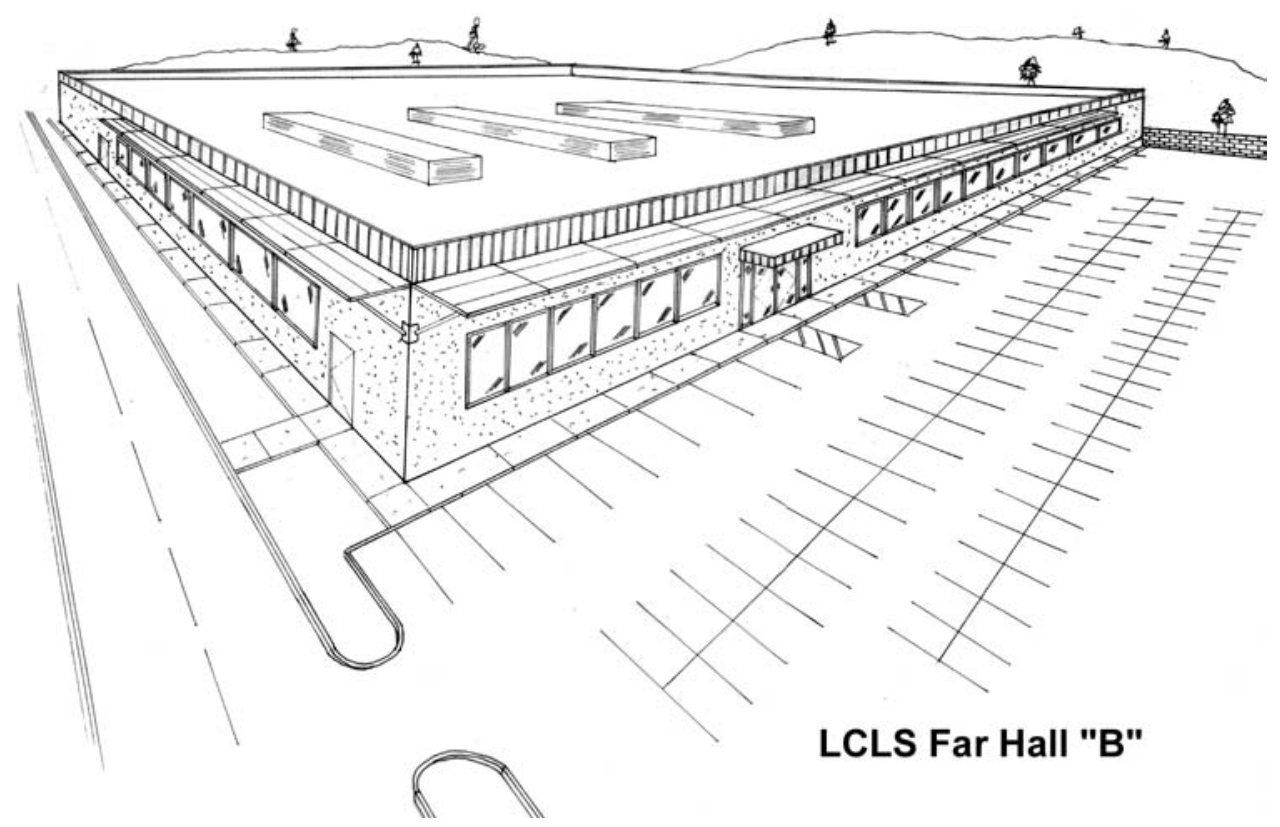

Figure 10.7 Far hall architectural rendering

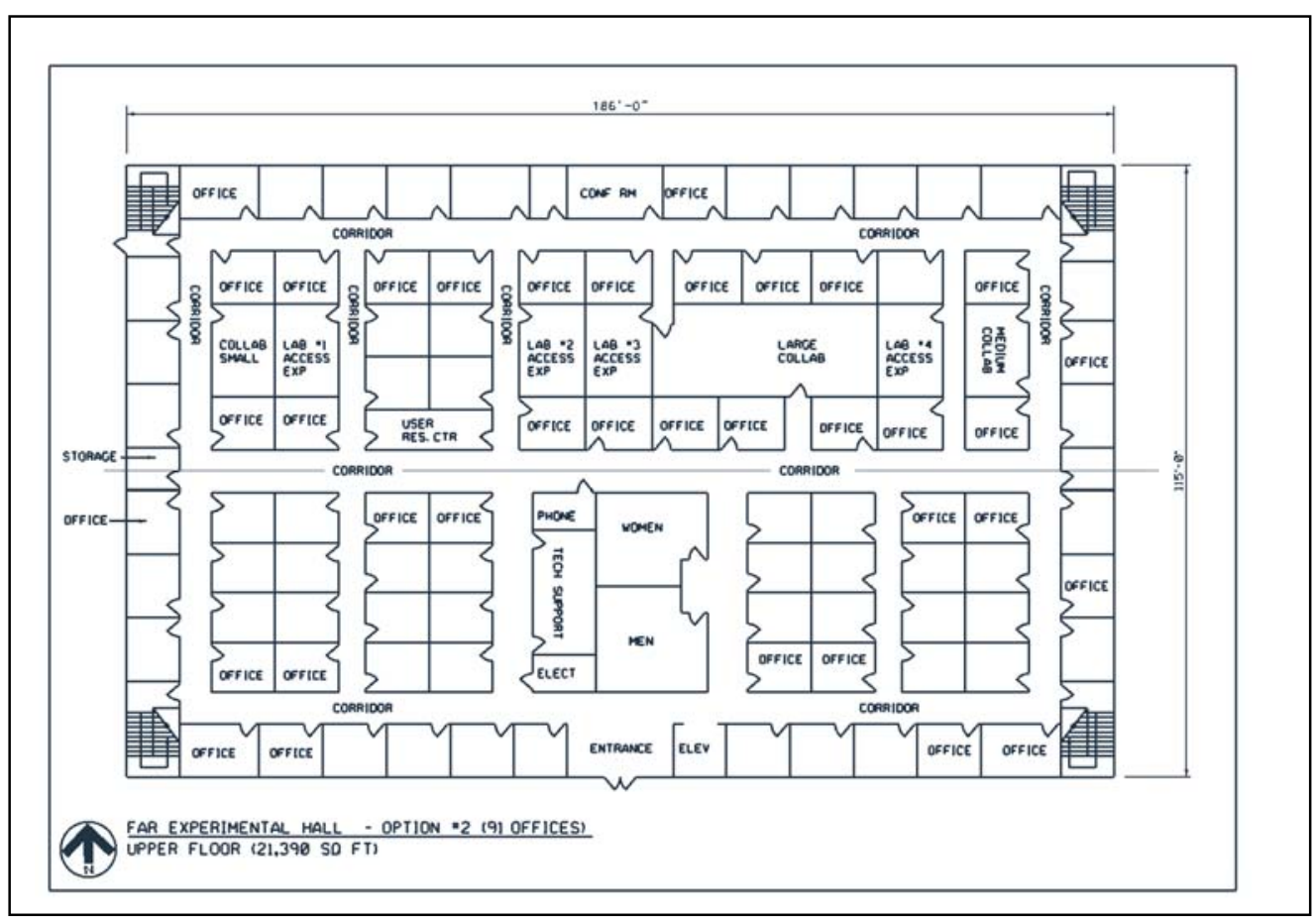

Figure 10.8 Floor plan for Far Hall second floor 


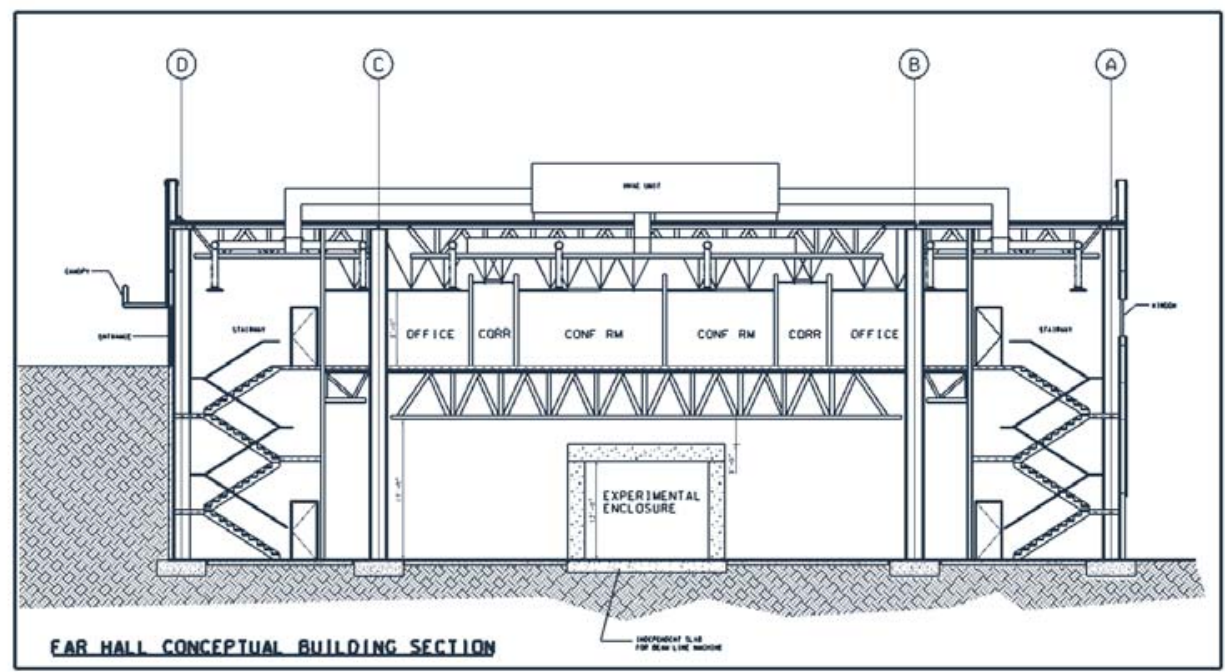

Figure 10.9 Cross section of the Far Hall

The interior of the experiment hall will be open, allowing flexible configuration of experiments. Figure 10.10 shows the initial floor plan for this hall. It will have a 15 -ton capacity bridge crane with a 15 -foot hook height covering the experimental areas. Low conductivity water and power will be available at the walls of the building. The interior distribution of the utilities will be covered in the section on x-ray optics. The Far Hall will have its own low conductivity water plant. It will exchange heat with the cooling tower water from the MCC cooling tower. 


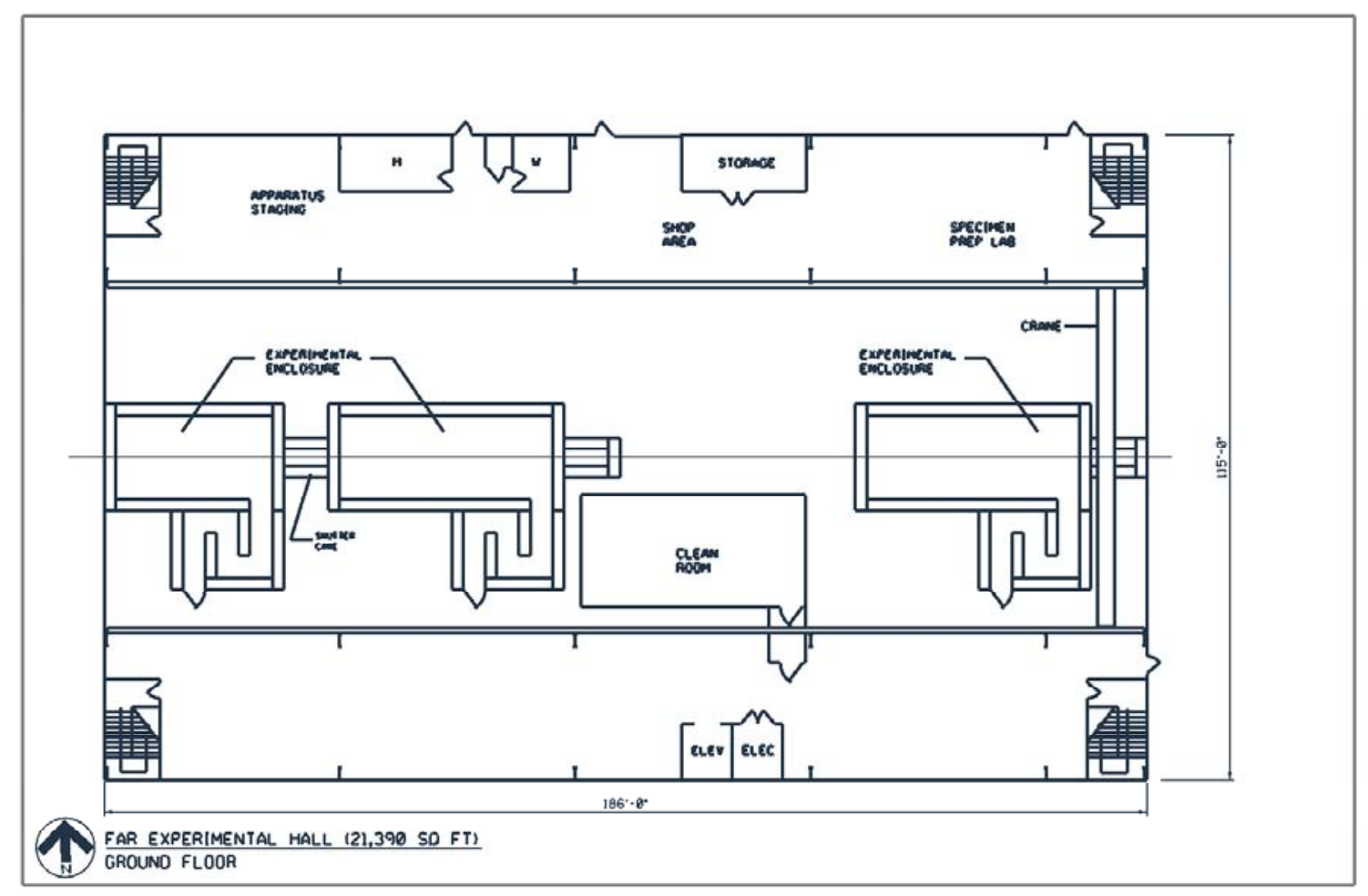

Figure 10.10 Floor plan for far hall experimental floor

\subsection{Beam Tunnel}

The two experimental halls are connected by a 3 by 3 meter enclosure that is 227 meters long. The x-ray beam will be transferred in a vacuum pipe in this tunnel, and control and timing cables required between the halls also will be carried in this tunnel. 


\section{1 controls

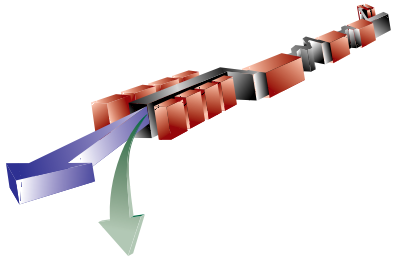

\section{TECHNICAL SYNOPSIS}

The LCLS incorporates several new systems into the existing SLAC accelerator complex. The parts of the existing accelerator complex used for LCLS will also serve non-LCLS functions. The control system architecture for the LCLS will be the same as that used currently for running the SLAC accelerator complex. This architecture consists of the original custom CAMAC-based VMS system developed at SLAC and subsequently extended by incorporating EPICS-based systems into the control system. The new systems required only by the LCLS will be EPICS-based. These systems include: (1) The LCLS Injector systems, such as, the gun, the gun laser, the injector linac and the DL1 beamline. (2) The undulator segments, the associated steering magnets, the mechanical movers and the undulator diagnostics. (3) The $x$-ray beamline components. The electronics for modifications to the LCLS accelerator, such as the bunch compressors and DL2 beamline, will use CAMAC for cost reasons and for ease or sharing these systems with the rest of the SLAC experimental program.

The control requirements for the LCLS are straightforward and similar to the existing FFTB controls. The x-ray beamline controls have two major objectives. One objective is to provide control of the $x$-ray optical elements. The second objective is to provide sufficient data collection capability to allow for thorough testing of different components.

Most of the LCLS $x$-ray experiments will require synchronization of the experimental station's equipment with the electron beam. The electron beam, in turn, is phased to the 476 MHz of the SLAC master clock. Temporal jitter between the RF and the beam is specified to less than $0.5 \mathrm{ps}$. A timing system is designed to assure that the synchronization between experiment lasers and the FEL x-ray (External Pulse Class of experiments) have jitter better than $0.5 \mathrm{ps}$ for time delays of $+/-1 \mathrm{~ns}$ and better than $1 \mathrm{~ns}$ for time delays of $+/-10 \mathrm{~ms}$. Some experiments will require significantly better timing that $0.5 \mathrm{ps}$ jitter and the techniques to achieve this will be developed as part of the LCLS R\&D plan.

At SLAC the Beam Containment Systems (BCS), Machine Protection Systems (MPS) and Personnel Protection Systems (PPS) are included in the control system and are described in this chapter. 


\subsection{Control System}

The LCLS operation will be controlled by two systems: (1) the accelerator systems that are shared with the rest of the SLAC accelerator program (i.e. L1, BC1, L2, BC2, L3 and beam switchyard) will be controlled using the existing accelerator control system; (2) The new systems that are exclusively LCLS (i.e. the injector, the undulator and the x-ray optics) will use EPICS-based controls that will be linked to the SLAC accelerator control system (much like the system used to control the PEP-II RF). The LCLS will be controlled from the SLAC Main Control Center (MCC). Touch-panel consoles located in the MCC provide the human interface to the machine hardware. For commissioning and maintenance purposes, additional control terminals will be built into the electronics racks in the LCLS support buildings.

The present SLAC linac is controlled and monitored using the SCP (SLC Control Program), which is a twenty-year-old VMS-based, monolithic user interface. It has a centralized architecture with limited peer-to-peer capabilities. The hardware and software overview of this system is shown in Figure 11.1.

EPICS, more recently developed by LANL and ANL is also in use at SLAC and numerous laboratories in the USA and around the world. EPICS uses Client/Server techniques to provide communication between various computers. Most servers, called Input/Output Controllers (IOCs), perform real-world I/O and local control tasks, and make information about their state available to clients using the Channel Access (CA) network protocol. CA has been designed for the kind of high bandwidth, soft real-time network applications for which EPICS is used. EPICS hardware architecture is distributed. Each IOC or OPI (OPerator Interface) can communicate with all the others. The hardware and software schematics of a generic EPICS based system are shown in Figure 11.2. Some experience using EPICS based controls already exists at SLAC (e.g. the PEP-II RF system controls). Although SLAC does not at present possess EPICS drivers for all the standard devices at SLAC, there is a large user base from which software is available. In addition, the LCLS collaborators at the APS have extensive experience with EPICS.

Since part of the SLAC Linac will be used as the LCLS accelerator, the control system currently running the SLAC facility will be used to operate the LCLS systems in the existing linac enclosure. Systems, such as the L1, L2, L3 linacs and the bunch compressors will be integrated into and controlled and monitored using the existing control system. Control of the systems hardware and the acquisition of data from the sensors and diagnostic instrumentation for the electron beam in the Undulator Hall will be done by a refurbished existing CAMACbased system that will provide for an update of the existing SLAC control structure. More details of the LCLS accelerator controls are given in Section 11.3. 



Figure 11.1 SCP Control System Hardware and Software Scematic
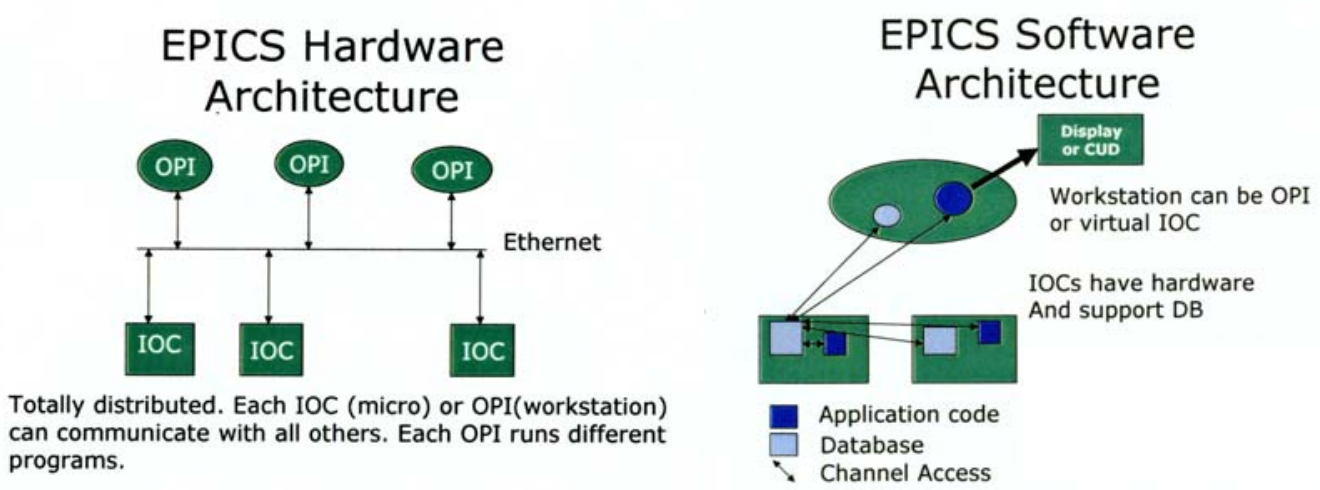

Figure $\mathbf{1 1 . 2}$ Generic EPICS Hardware and Software Schematics

The new systems needed specifically for LCLS will be controlled using an EPICS-based system. Three new control consoles running EPICS, are envisioned for the new systems, one for the Injector area and one each for the Near and Far Halls. With the judicious placement of the Operator Interfaces (OPIs), local control for initial testing and commissioning of the injector, undulator and x-ray beam transport and diagnostics will be facilitated. In normal operation, the LCLS will be controlled from the Main Control Center (MCC). Figure 11.3 shows the integration of the EPICs systems into the overall SLAC control system. This is very similar to the existing integration for PEP-II. 


\section{SLC/EPICS Interface}

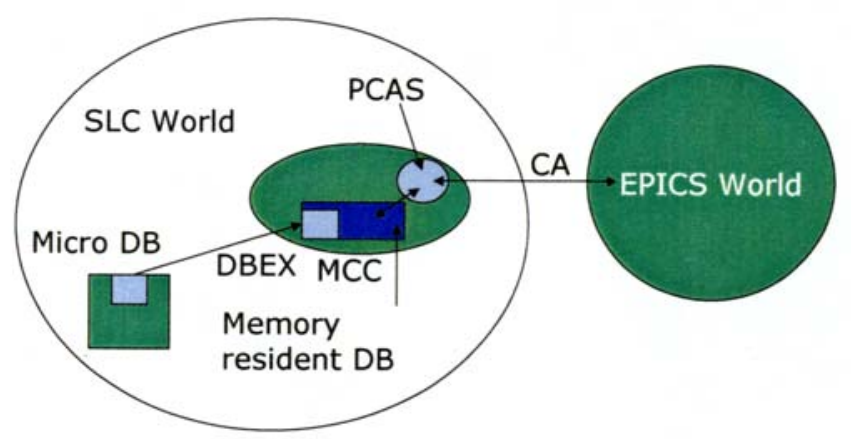

Figure $\mathbf{1 1 . 3}$ Integration of the EPICS subsystems into the SLC control system

Control of the hardware and acquisition of data from the sensors and diagnostics instrumentation will be done by refurbished existing CAMAC and/or new VME-based systems. The workstations will be linked to a new CAMAC or VME Crate Controller module. The workstations will also be linked to the SLAC network via Ethernet. This link will allow communications with the SLC computer and with other control consoles.

The LCLS is a demanding accelerator system to tune and run. It will require extensive use of feedback systems. Most of these feedbacks will be similar to existing systems at SLAC and others will require new algorithms and software. The controls hardware will be designed with these feedbacks in mind.

\subsection{Injector Controls}

The LCLS injector provides a $150 \mathrm{MeV}$ electron beam that is injected into the existing SLAC linac at the beginning of sector 21. The injector is a new device housed in the off-axis injector enclosure near sector 20. Its main components are an RF gun and associated laser, a short accelerator (L0) and a beam transport line (DL1). The laser for the RF Gun is similar to the system currently used for the polarized electron gun on the linac. Similar controls will be required for the LCLS laser. A table of the control points for the injector is included in appendix B.

\subsection{Accelerator Controls}

The main components of the LCLS accelerator are three pieces of existing linac (L1, L2 and L3) and a reconfigured beam transport line (DL2) in the Undulator Hall. The LCLS requires new systems for manipulating and transporting the required electron beam. These consist of two new bunch compressors (BC1 and $\mathrm{BC} 2$ ), two transverse S-band RF cavities, an 
$\mathrm{X}$-band RF structure and a superconducting wiggler. There will be additional standard diagnostic equipment most of which will be copied from existing designs. However, the BPM hardware has to be re-designed to meet the LCLS specifications. Sophisticated feedback techniques will be required to successfully tune and operate the LCLS. These will require design and software effort. A table of the control points for the accelerator is included in appendix B.

\subsection{Undulator Control}

The LCLS undulator has thirty-three segments and has a total length of 121 meters.. The separations between segments will each accommodate a permanent magnet quadrupole, a Beam Position Monitor (BPM), $\mathrm{x}$ and y corrector magnets, beam intercepting electron and photon position monitors, and vacuum ports. The undulator segments will be supported on piers. Movers will be mounted atop the piers to align each end of an undulator segment. Movers will align the quadrupole magnets in $\mathrm{x}$ and $\mathrm{y}$. In addition, there will be movers at each end of the undulator segments to adjust the vertical gap. The electron beam position monitors will be mounted to piers at each gap, so that the electrical center of the BPM remains stable.

\subsubsection{Movers}

The movers are needed for the initial alignment process of each undulator segment and quadrupole as well as for their mechanical repositioning during the beam-based alignment procedure. The movers are designed to correct long term drifts in position using this procedure. The undulator segments will be mounted on five-cam movers (x, y, roll, pitch, and yaw), and each motion will be driven by a stepper motor. The mover design is similar to the movers used in the FFTB and the SLC final focus, which have a positional accuracy of \pm 5 $\mu \mathrm{m}$ and an incremental positioning precision of $\sim 0.5 \mu \mathrm{m}$ under loads of several tons for all 5 degrees of freedom.

The motion controls and the movers of the quadrupoles need to operate over a limited range of $\pm 0.5 \mathrm{~mm}$, with a position resolution of $1 \mu \mathrm{m}$. The quadrupoles will be mounted on $\mathrm{x}$ y slides with no remote control of roll.

\subsubsection{Undulator Diagnostics}

The 48 BPMs will be attached to local piers. The BPMs need to operate with high stability, low drift, low impedance, and high resolution (1 $\mu \mathrm{m}$ or better). The beam trajectories measured by the electron BPM signals will be processed on-line, and then fed back to the quadrupole magnet movers, in the form of the number of steps and direction of motion. 
A pop-in will be installed between each pair of undulator segments. This will allow observation of the optical transition radiation (OTR) from the electron beam and scatter radiation from the x-ray beam. Beam loss monitors based on the PEP-II design will be installed at each gap between undulator segments.

A table of the control points for the undulator is included in appendix B.

\subsection{X-Ray Beam Line Electronics and Controls}

For instrumentation and control purposes, the LCLS x-ray optics system can be divided into two parts: (1) the $\mathrm{x}$-ray transport line, (2) the $\mathrm{x}$-ray beam line(s).

The $\mathrm{x}$-ray transport line carries the $\mathrm{x}$-ray beam from the undulator to the $\mathrm{x}$-ray beam line. The x-ray transport line will be installed inside the FFTB tunnel and will include the following diagnostic equipment: differential pumping sections to isolate the high vacuum systems, horizontal and vertical adjustable collimators (slits), a gas attenuation cell, and calorimeters.

The x-ray beam line carries the x-ray beam from the transport line and directs it to the experimental halls for LCLS commissioning and beamline testing. The x-ray beam line will be installed in shielded enclosures and will include diagnostic equipment such as mirror systems, monochromators, differential pumping sections, horizontal and vertical adjustable collimators, and other instrumentation.

\subsubsection{Control System Objectives}

The x-ray optics controls have two major objectives. One objective is to provide control of the x-ray optical elements. The x-ray optics include the take-off mirror(s), the crystal monochromator, collimators, a gas attenuation cell, and filters. The second objective is to allow experimental data collection for testing and commissioning of optical elements needed for LCLS experiments and of timing techniques needed for the different types of possible experiments using the LCLS x-ray beam. In addition, the control system should allow for functional upgrade to support the LCLS experimental program as it is defined and approved.

\subsubsection{Control System Layout}

The x-ray control system will control the operation of the various motion controllers and actuators in the x-ray transport line and x-ray beam line(s). The optics and experiment control system will be installed in the LCLS Experimental Halls. An EPICS console in each hall (to allow for future expansion of control and data acquisition capabilities), will allow local control of the x-ray optics and the data acquisition from the diagnostics that will be tested using the $\mathrm{x}$-ray beam. The EPICS console will allow local operation of various equipment such as the mirror and crystal monochromator movers, optical tables, adjustable slits, the gas attenuation cell, sample positioners, calorimeters, and detectors. 


\subsubsection{Motion Controls}

The x-ray transport line and x-ray beam line(s) include systems such as adjustable collimators, mirror optics, crystal optics, and adjustable slits. To operate these systems requires position control and position readback. The design goals, such as positioning accuracy, position encoder linearity and resolution, and processing electronics resolution, differ from mover to mover.

The mechanical position will be measured directly with linear variable differential transformers (LVDTs). LVDTs were chosen for their resolution (essentially determined by the number of bits in the read-out ADC and the LVDT range of travel), their linearity $(<0.15 \%)$, and their ease of use.

The x-ray optics and experiment control computer will calculate the number of steps and directions that each motor needs to take. Stepper Motor Control (SMC) commands will be sent out to the drivers in the form of the number of steps and direction to move. Currently, the movers controls design is based on a CAMAC system and SLAC SLC-type motor drivers. The final design may use a VME based system and commercially available stepper motor drivers.

\subsubsection{Feedback Systems}

The x-ray beamline control system requires the ability to set up feedback systems. An example of a needed feedback system is the requirement to stabilize the photon beam. Experiments that require irradiation of a fixed sample point (e.g., diffraction from an individual microstructure) require the stable positioning of the beam to within $10 \%$ of its diameter. Factors contributing to positional beam jitter or drift at the sample plane might include the following: (1) power supply and other component fluctuations in the gun-toundulator system, (2) phase shifts in linac klystron low-level rf, (3) vibration or positional drift in the linac and undulator structures, (4) vibration or positional drift of the x-ray optics system components. For factors contributing to beam motion that have sufficiently long time constants, detection of jitter or drift and their stabilization may be accomplished with suitable detectors providing feedback to any of the upstream LCLS system elements that govern beam position and direction.

Detection of positional and directional jitter or drift will be accomplished with nondestructive photon beam position monitors. The output signal of the monitor will be fed back to positional/angular controllers in the mirror or crystal tanks.

\subsubsection{Timing System}

Most LCLS x-ray experiments require synchronization of the experimental stations' equipment with the electron beam. The electron beam, in turn, is phased to the $476 \mathrm{MHz}$ of the new LCLS master clock originating from Sector 20. Temporal jitter between the RF and 
the beam is specified to less than $0.5 \mathrm{ps}$ rms. A timing signal will be sent from the electron gun laser in sector 20 to the experimental hall using a fiber.

A table of the control points for the x-ray transport and diagnostics is included in appendix B.

\subsection{Radiation Safety and Protection Systems}

The components for the LCLS, except for the injector, x-ray beam line and the experimental stations, are either already in operation or will be built and installed in the SLAC Linac and the FFTB tunnel. The Linac and FFTB tunnel already have active control systems and radiation protection systems. Radiation safety and protection systems include the Personnel Protection System (PPS), the Beam Containment System (BCS) and the Beam Shut-Off Ion Chamber (BSOIC) system.

\subsubsection{Radiation Safety Systems - Control System}

The radiation safety systems control systems will communicate with the general control system, yet contain the following features:

(1) a dedicated hard wire or optical fiber communication backbone;

(2) redundant or independent units of safety logic, using a combination of relays, PLCs, or other electronic printed circuit boards (PCBs) that are single purpose and independent of all other control systems;

(3) typically a local control interface with the capability of status and control by the general control system; and

(4) subsystems that contain redundancy and multiplicity for a multiple beam shut-off paths with a fail-safe design. The LCLS radiation safety systems control system will take advantage of the latest technology as well as the existing technology for safety instrumentation and control.

The design of these systems must be approved by the SLAC Radiation Safety prior to implementation. The implementation of these systems will be reviewed at an Accelerator Readiness Review prior to beam operation. 


\subsubsection{Radiation Safety and Protection Systems Description}

The radiation safety and protection systems are designed to create barriers, both physical and electronic between personnel and radiation hazards. For example, personnel working inside the Undulator area will be protected from radiation that could be generated by other beams in the accelerator tunnel and Beam Switch Yard, and personnel working outside the accelerator enclosure will be protected from potential radiation generated during the LCLS operation. The radiation safety and protection systems in place include the following: (1) Shielding, (2) Personnel Protection System (PPS), (3) Beam Containment System (BCS) and (4) Beam Shut-off Ion Chamber (BSOIC).

The shielding consists of earth, concrete or other equivalent material designed to attenuate the undesirable radiation. The control system will monitor the movable shielding of the LCLS through the use of interlocks.

The PPS is an access control system that turns off electrical and beam-related hazards prior to personnel entering the local accelerator housing area.

The Injector PPS has a local access mode to allow entry into the injector vault when the linac is operating. The Injector PPS will ensure that the laser, microwave, and electrical hazards are off prior to personnel entry.

The linac and the linac to BSY PPS already exists

The Undulator Hall PPS, as already exists in the current FFTB tunnel, is designed to protect personnel from radiation and electrical hazards. The function of the system is to prevent access into the tunnel where there is the potential for beam and/or electrical hazards. It is also designed to prevent the radiation dose or dose rate from exceeding the radiation design criteria inside the tunnel when access is permitted, or outside the tunnel during the LCLS operation.

The Undulator Hall PPS is composed of beam stoppers, an entry module, a search/reset system, and emergency buttons. The system is controlled from the SLAC Main Control Center (MCC). It allows beam stoppers to be opened only after the tunnel has been searched and secured and is in the No Access state. Access to the tunnel is permitted by the PPS only if all the beam stoppers are closed.

The Undulator Hall radiation safety is ensured by a beam dump, beam stoppers, a Burn Through Monitor (BTM) installed up stream of the muon shielding; and several Beam ShutOff Ion Chambers (BSOICs) installed down stream of the muon shielding at the upstream end of the hall. This system prevents the beam from striking the muon shield and shuts the beam off if radiation levels inside the tunnel exceed the allowed limit. An additional BTM is installed behind the electron beam dump, and several BSOICs are installed outside the tunnel to monitor the radiation levels outside the Undulator Hall shielding. The BTMs are interlocked through the PPS. 
The Experimental Hall PPS will consist of a shielded hutch. The PPS can accommodate additional hutches. Each hutch will be designed to contain all radiation so that the dose rates outside the hutch are acceptable when photons from the FEL are inside the hutch. Control for the hutches will be local to the hutch. The Hutch Protection System (HPS) will be modeled after the latest SSRL hutch design. The key parts of the HPS are the access door, photon stoppers, and hutch security search/reset logic. The HPS allows either personnel access or beam operation in the hutch. The HPS contains the logic circuits that govern the sequence of access operations based on the status of the stoppers. It allows releasing or retaining the hutch door keys, acknowledges completion of a hutch search, and enabling the experimental hutch to be placed on-line (beam operation) or off-line (personnel access). The LCLS HPS will control the operation of photon stoppers for each experimental hutch. The system will allow the photon stoppers to be opened (go on-line) only if the hutch has been searched and secured, and the hutch door key is captured in the HPS panel. Access to the hutch is permitted only if all photon stoppers are closed. Photon Stoppers with burn-through monitors will be installed inside the FFTB tunnel, and redundant hutch stoppers will be installed to protect each hutch. The stoppers located in the FFTB tunnel, upstream of Experimental Hall $\# 1$, are designed to protect personnel from radiation generated by the FEL. The Experimental Halls photon stoppers are designed to protect personnel in each hall and hutch from scattered radiation. The initial design is for one main hutch in each experimental Hall.

The Beam Containment System (BCS) prevents the accelerated beams from diverging from the desired channel, and from exceeding levels of energy and intensity that may cause excessive radiation in potentially occupied area.

The Injector BCS will consist of a few Protection Ion Chambers (PICs) for protecting personnel in the Injector from main Linac beam losses.

The Undulator (FFTB) BCS already in place consists of the following:

(1) devices which limit the incoming average beam power to $2.4 \mathrm{~kW}$ (3 current monitors);

(2) devices which limit normal beam loss so that the radiation level outside the tunnel shielding is less than $1 \mathrm{mrem} / \mathrm{hr}$ (current monitors and long ion chambers);

(3) protection collimators which ensure that errant beams do not escape containment; and

(4) ion chambers and water flow switches which protect collimators, stoppers and dumps.

The BSOIC system consists of radiation monitors in areas that could be occupied that insert beam stoppers when raised radiation levels are sensed. The Injector and Experimental Halls will have some new BSOICs installed and connected through the PPS and interlocked with area stoppers. The general and PPS control systems monitor and display the BSOIC analog level. The control system can reset a BSOIC trip, after elevated radiation levels are sensed by the BSOIC and the PPS inserts the area stoppers. 


\subsection{Machine Protection System}

A Machine Protection System (MPS) is designed to protect the LCLS components from damage by the beam. The three primary functions of the MPS are to protect: (1) the integrity of the vacuum system; (2) the proper cooling of the water-cooled components; and (3) the LCLS components from damage resulting from errant steering of the electron beam.

1. The MPS will control and monitor the operation of vacuum components such as differential pumping sections, ion gauges, ion pumps, and isolation valves.

2. The MPS will monitor temperature sensors and water flow switches that insure that the magnets, collimators, stoppers, x-ray mirror, and monochromator crystals are sufficiently cooled.

3. Ionization chambers and long ion chambers capable of detecting average radiation are currently installed in the FFTB tunnel. If the average rate of beam loss is found to be sufficient to threaten machine components, the beam repetition rate is automatically reduced. In addition, a pulse-to-pulse comparator system measures the beam current. The operation of the pulse-to-pulse comparator is based on measuring the beam current in two locations. The signal from a toroid at the beam's final destination (beam dump) is compared with that from a toroid at the beginning of the area being protected. If the comparison on a pulse-to-pulse basis shows a beam loss greater than some specified amount the beam is automatically turned off.

The LCLS MPS will consist of two separate systems. One system will protect the LCLS components from accidents with the electron beam and the second system will protect the components of the undulator, the transport line, the x-ray beam line, and the experimental stations from accidents with the x-ray beam. The two control systems will be interfaced to provide vacuum and thermal interlocks to protect the LCLS accelerator and the x-ray beam line from the following: (1) accidental exposure to atmospheric pressure; and (2) accidental interruption of the Low Conductivity Water (LCW) to water-cooled components. Status signals such as vacuum and LCW faults as well as permits to open or close isolation valves will be shared by both systems.

The existing FFTB MPS control panels are installed in the support building next to the enclosure. These controls will be modified to protect the LCLS components in the tunnel. The FFTB and LCLS requirements are very similar. The undulator-to-experimental stations MPS control panels will be installed in the Experimental Hall. This system will include a Programmable Logic Controller (PLC) and control panels. The architecture of this system is similar to the existing systems in SSRL. 


\section{Alignment}

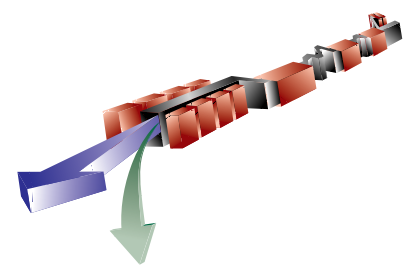

\section{TECHNICAL SYNOPSIS}

This section describes the procedures and methods used to position the LCLS components with their required accuracy. Most of the alignment requirements are well within the range of proven traditional alignment techniques. Alignment of the undulator section is the most demanding. State-of-the-art equipment and procedures will be needed to meet the positioning requirements.

The alignment coordinate system will be the existing Cartesian right-handed system, which was implemented for the SLC project and was also used for the PEPII project. The alignment network will consist of four parts: a small surface network to better integrate the remote hall into the global coordinate system, and three tunnel networks for linac, undulator and transport lines / experimental areas alignment. The network geometry is driven by the tunnel and machine layout and should permit observation of each target point from at least three stations. The design philosophy is based on a 3-D monument design providing the best possible positional accuracy. For the undulator hall network, a triplet of monuments is placed in the tunnel cross-section at each quadrupole location. The other networks are constructed similarly but with fewer monuments.

The alignment instrumentation will be a laser tracker / digital level used in combination. In conjunction with least-squares solutions, the laser tracker will provide excellent 3-D positional accuracy. In addition, the digital level will improve the rotational stability of the narrow linear network. To meet the global straightness and local relative alignment needed for beam-based alignment to converge quickly, the optical measurements will be supported by stretched-wire based straightness measurements and by hydrostatic level system measurements. The position tolerances of injector, linac, transport line, and experimental areas are achievable with standard alignment procedures. 


\subsection{Procedural Overview}

The alignment of the undulator system will be carried out in four distinct steps:

During installation, conventional alignment methods will be used to position the undulator segments, quadrupoles, correctors, BPMs and other components to about $150 \mu \mathrm{m}$. Position adjustments will be applied mechanically, i.e., the remote movers will not be used for this task.

To refine the installation alignment and after system changes, the effective centerline of undulator segments, and quadrupoles will be aligned to $50 \mu \mathrm{m}$, of BPM modules to $100 \mu \mathrm{m}$, with respect to a global straight line. This global straight line may deviate significantly from the nominal axis (which is an extension of the axis of the linac) in position and orientation. A stretched wire system with sensors capable of absolute measurements will be used in the horizontal plane to achieve a $50-\mu \mathrm{m}$ tolerance. The same tolerance will be achieved in the vertical dimension with the use of an absolute measuring Hydrostatic Level System. Position adjustments will be done remotely for the undulator segments and the permanent magnet quadrupoles, and locally for the BPM modules.

The relative position difference between quadrupoles and adjacent undulator segments will be mapped and recorded. A measurement tolerance of significantly better than $50 \mu \mathrm{m}$ is expected.

After the conventional alignment and thereafter at periodic intervals of a few weeks, as needed, the Beam-Based-Alignment procedure as described in Chapter 8, Section 8.12 will be applied. The Beam-Based-Alignment procedure moves the quadrupoles to correct the electron beam trajectory and moves the undulator segments to maintain relative alignment to the quadrupoles. This procedure will create a straight beam trajectory $(2 \mu \mathrm{m}$ rms deviation from a straight line both horizontally and vertically over a distance of $10 \mathrm{~m}$ ). Once this is achieved, the BPM readings will be recorded as the reference zero positions. The BPM modules are expected to move transversely, mostly due to ground motion, by a few micrometers in between two applications of the Beam-Based-Alignment procedure. A high resolution monitoring system will record any BPM motion; this data can then be used to correct BPM readings. Successive alignment procedures are expected to be much quicker than the initial procedure. Feedback systems will be employed to keep the trajectory straight to the BPM modules by using the movable quadrupoles as correctors.

If it turns out that initially the x-ray beam, as produced by the undulator, points too far away from the desired target points in the experimental halls, iterations of the above step sequence can be used to re-point the undulator. 


\subsection{LCLS Surveying Reference Frame}

Horizontal position differences between the projection of points on the geoid $^{1}$, or a best fitting local ellipsoid, and those on a local tangential plane are not significant for a project of the size of the LCLS. Hence, it is not necessary to project original observations like angles and distances into the local planar system to arrive at planar rectangular coordinates [1].

However, in the vertical plane, the curvature of the earth needs to be considered (see Figure 12.1). Because leveling is done with respect to gravity, the reference surface is the geoid. Due to the relatively small area of the LCLS project, one can substitute the nonparametric geoid with a locally best-fitting sphere. Table 12.1 shows the projection differences between a tangential plane and a sphere as a function of the distance from the coordinate system's origin. Notice that for distances as short as $20 \mathrm{~m}$ the deviation between plane and sphere is already $0.03 \mathrm{~mm}$.

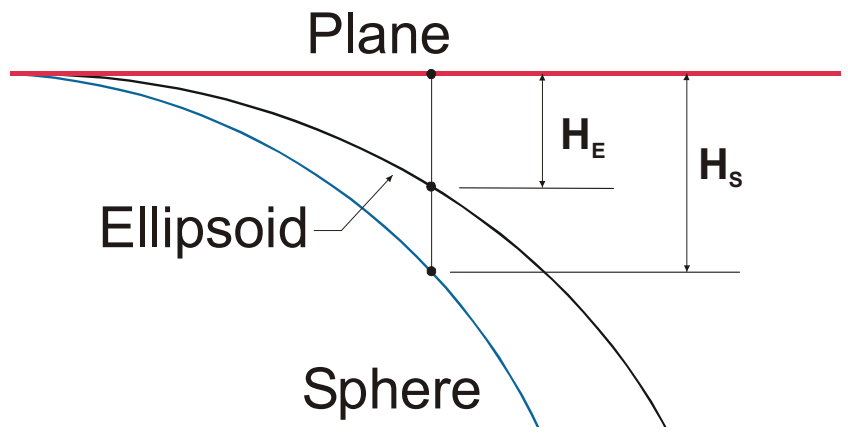

Figure 12.1 Effect of earth curvature.

Table 12.1 Curvature correction

\begin{tabular}{cc}
\hline Distance $\mathrm{r}[\mathrm{m}]$ & Sphere $\mathrm{H}_{\mathrm{s}}[\mathrm{mm}]$ \\
\hline 20 & 0.03 \\
\hline 50 & 0.20 \\
\hline 100 & 0.78 \\
\hline 1000 & 78.46 \\
\hline
\end{tabular}

\footnotetext{
${ }^{1}$ The geoid is the reference surface described by gravity; it is the equipotential surface at mean sea level that is everywhere normal to the gravity vector. Although it is a more regular figure than the earth's surface, it is still irregular due to local mass anomalies that cause departures of up to $150 \mathrm{~m}$ from the reference ellipsoid. As a result, the geoid is nonsymmetric and its mathematical description nonparametric, rendering it unsuitable as a reference surface for calculations. It is, however, the surface on which most survey measurements are made as the majority of survey instruments are set up with respect to gravity.
} 


\subsubsection{Network Design Philosophy}

The global alignment tolerance, the relatively weak links between machine sections, and advances in surveying make it possible for most of the machine to forego the traditional design of a two-tiered network hierarchy (surface and tunnel networks) covering the whole machine. Instead, each machine section can be considered independent, only connected by tunnel networks. Omitting a primary network not only removes many constraints for component placement, because fewer lines-of-sight need to be maintained, but also presents a significant reduction in alignment costs. The only exception here is a small surface network which is required to connect the remote experiment area into the coordinate system of the undulator.

Traditionally, forced-centered 2 " $2+1-\mathrm{D}$ " triangulation and trilateration techniques ${ }^{3}$ were used to measure tunnel networks. However, a 3-D "free stationing"4 approach does not require forcedcentered instrument setups, thus eliminating the need for setup hardware and its systematic error contributions. Removable heavy-duty metal tripods, translation stages, CERN sockets, and optical plummets are not needed (see Figure 12.2). The network design still must consider other systematic error effects, especially lateral refraction ${ }^{5}$. Another important consideration is the target reference system. Its design becomes much easier with free stationing because we are dealing only with targets and not with instruments as well. Accordingly, it is proposed to use a 3-D design, which is now widely used in high precision metrology. This approach is centered around a 1.5 inch sphere. Different targets can be incorporated into the sphere in such a way that the position of the target is invariant to any rotation of the sphere. At SLAC, designs have been developed to incorporate theodolite targets (see Figure 12.3), photogrammetric reflective targets, as well as glass and air corner cubes (see Figure 12.4) into the sphere. Receptacles for the spheres,

\footnotetext{
${ }^{2}$ Forced-centering refers to a specific instrument mount. This type of mounting system, whether vendor specific or independent, allows the exchange of instruments on a station without losing the measurement point, i.e. all instruments are by mechanical "force" set up in exactly the same position. However, experience has shown that even the best of these forced-centering systems has centering error of about 50$100 \mu \mathrm{m}$. Unfortunately, the forced-centering system contributed error is not random. Because a whole set of measurements is usually completed from a slightly offset position, this error behaves mostly systematically. No efficient method is known to determine the offset vector. These errors, vertical refraction and lateral refraction, are the biggest contributors to the systematic error budget in surveying engineering.

${ }^{3} 2+1$-D refers to the fact that because of mechanical problems in the forced-centering hardware, threedimensional networks were usually split into separate horizontal (2-D) and vertical (1-D) networks. Both networks were established, measured, and analyzed separately.

${ }^{4}$ Rather than set up the instrument over a known point, the instrument's position is flexible and chosen only following considerations of geometry, line of sight, and convenience. To determine the instrument position, at least three points, whose coordinates are already known or are part of a network solution, need to be included in the measurements.

${ }^{5}$ Lateral refraction is caused by horizontal stationary temperature gradients. In a tunnel environment, the tunnel wall is often warmer than the air. This creates vertical stable temperature layers with gradients of only a few hundredths of a degree Celsius per meter. If one runs a traverse close to a tunnel wall on one side only, the systematic accumulation of the effect can be significant; e.g., during the construction of the channel tunnel, a control measurement using gyro-theodolites revealed that after about $4 \mathrm{~km}$ the tunnel had already veered about $0.5 \mathrm{~m}$ off the design trajectory.
} 
which are usually referred to as "nests" or "cups," have been designed to accommodate different functions. Designs are available at SLAC for cups grouted into the floor, tack-welded onto magnets, mounted on wall brackets, and for a "centered" removable mounting placed into tooling ball bushings (see Figure 12.5). This reference system performed very well in the alignment of PEP-II components.

\subsubsection{Network Layout}

The alignment network consists of four parts: injector, linac, undulator, and transport line/experimental area.

\subsubsection{Injector Network}

The injector network will support the survey and alignment of the injector components. Standard networking design and measurement techniques can be used since the injector components have fairly loose positioning tolerance requirements. A horizontal sight pipe through the shielding wall will allow a connection of the reference systems in the injector and linac tunnels.

\subsubsection{Linac Network}

The linac network serves a different purpose than the other networks. Because the linac already exists, the linac network does not need to support construction survey and alignment, but rather will only provide local tie-points during the linac straightening (smoothing) procedure (see chapter 12.5.2).

\subsubsection{Undulator Hall Network}

The Undulator Hall network's overall geometry is dictated by the tunnel geometry, machine layout, and the fact that the free-stationing method requires a greater number of reference points. The geometry should also permit observing each target point from at least three different stations. The reference points can be of two different hierarchical classes. The second order points, or tie points, mainly serve to connect the orientation of free-stationed instruments, while the first order points additionally provide the geometric reference during machine installation; they are the equivalent of traditional traverse points or monuments. 


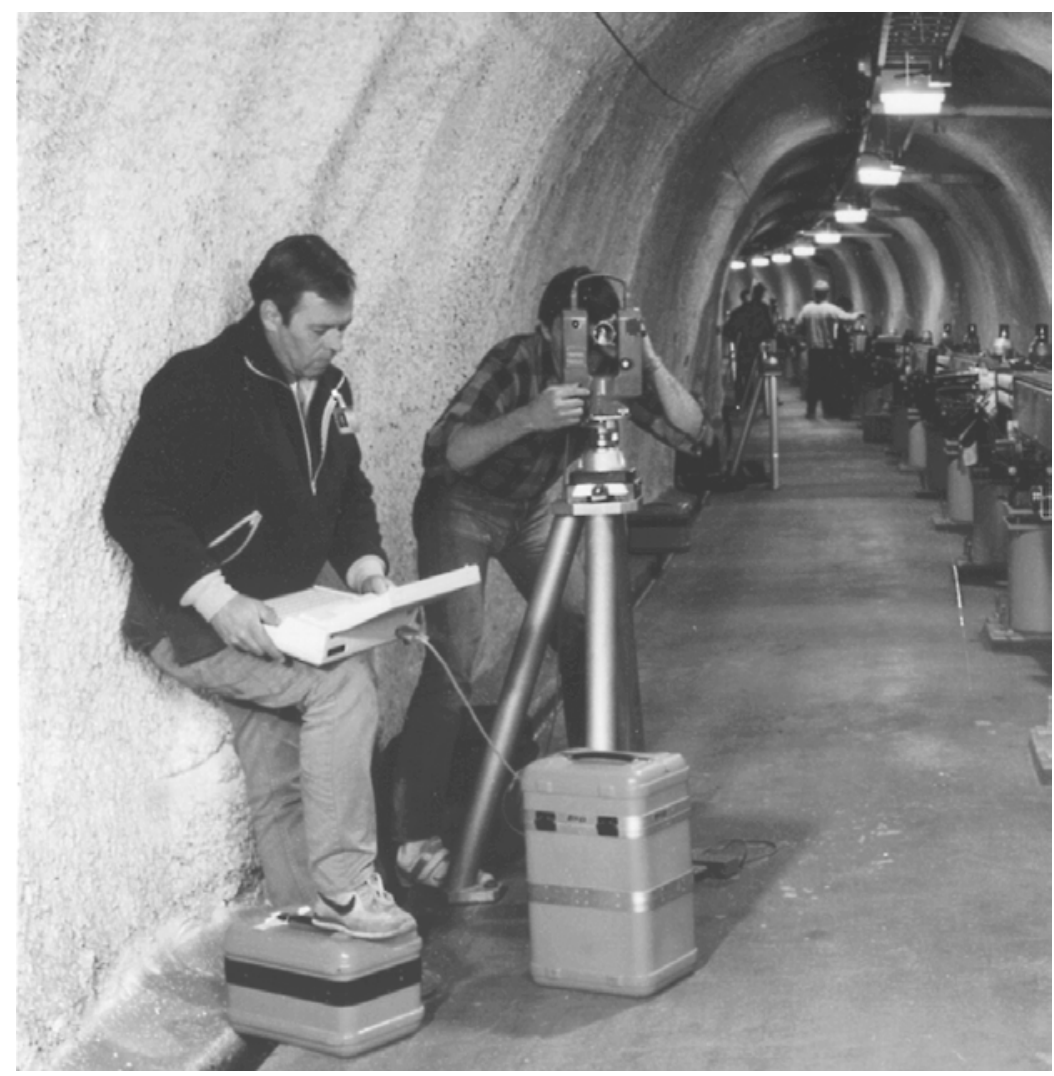

Figure 12.2 Forced-centered setup in SLC tunnel

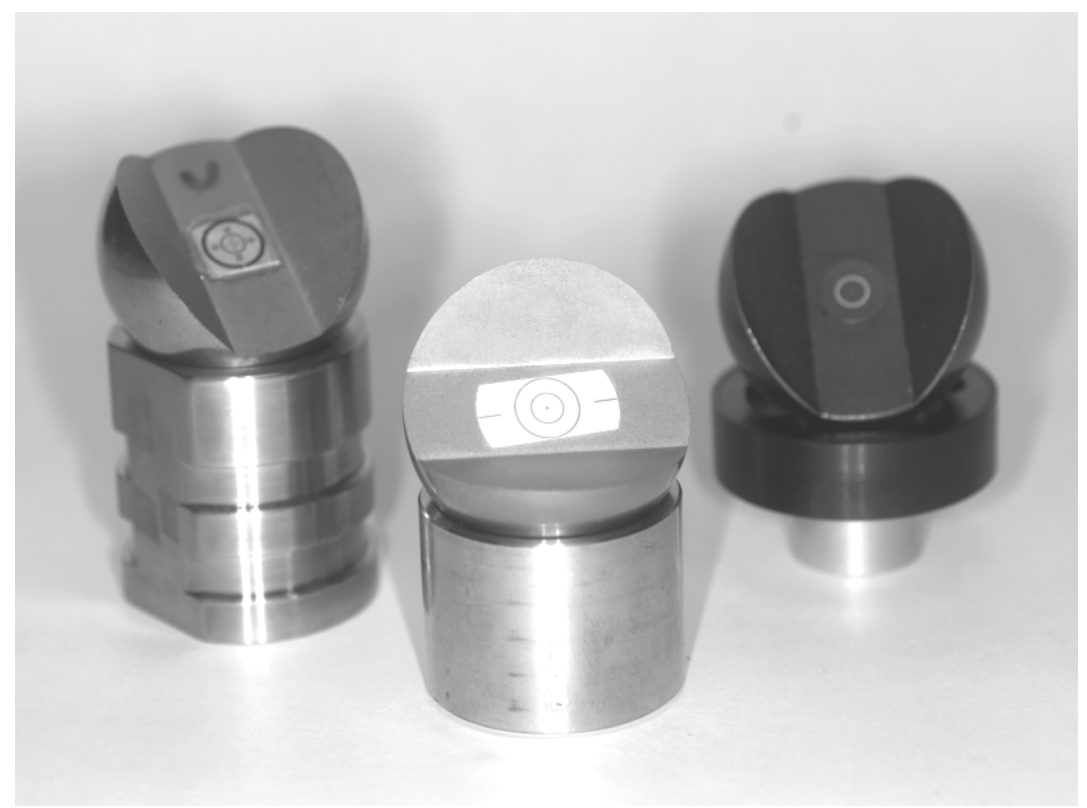

Figure 12.3 Sphere mounted theodolite targets 


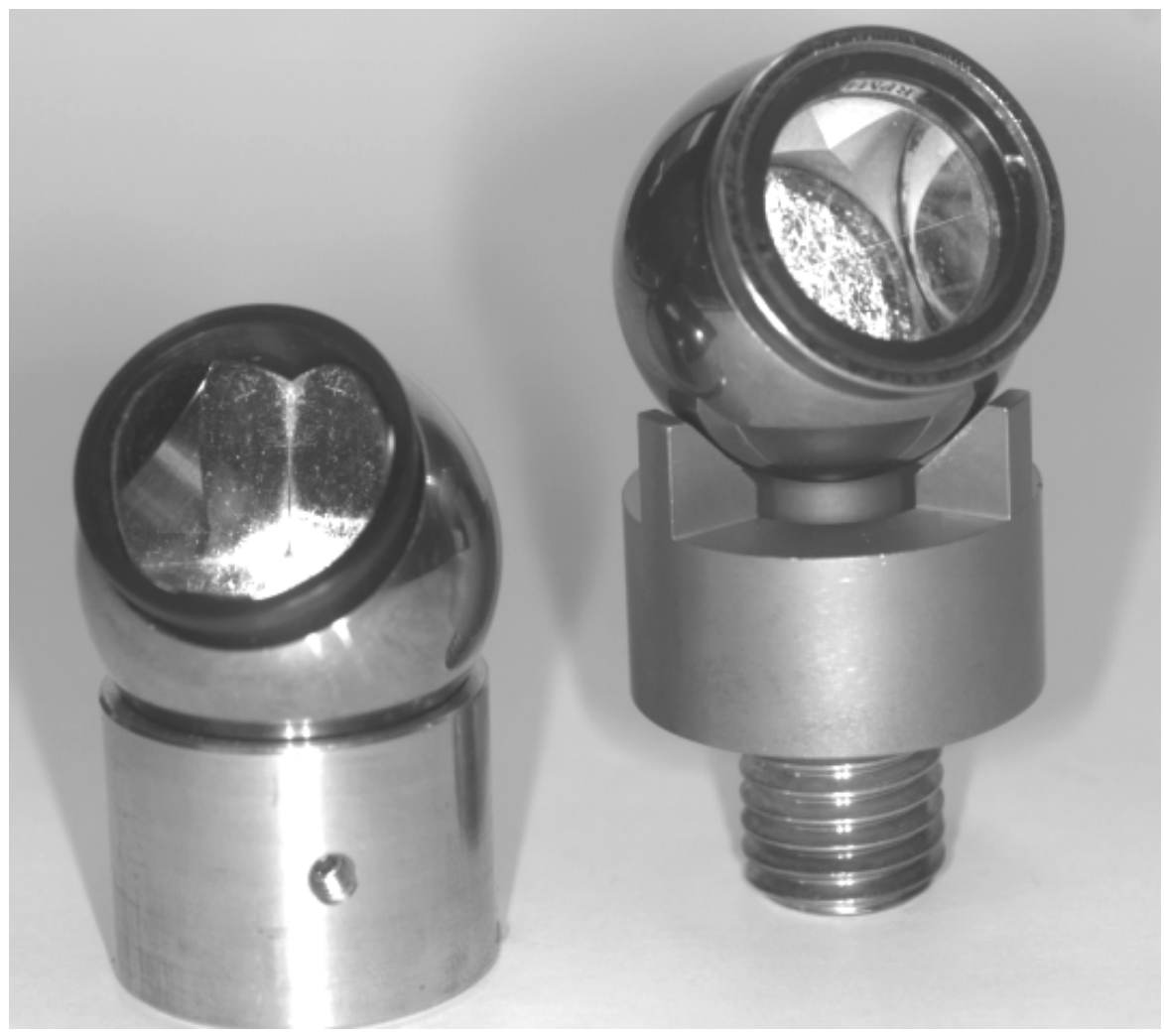

Figure 12.4 Sphere mounted glass and air reflectors

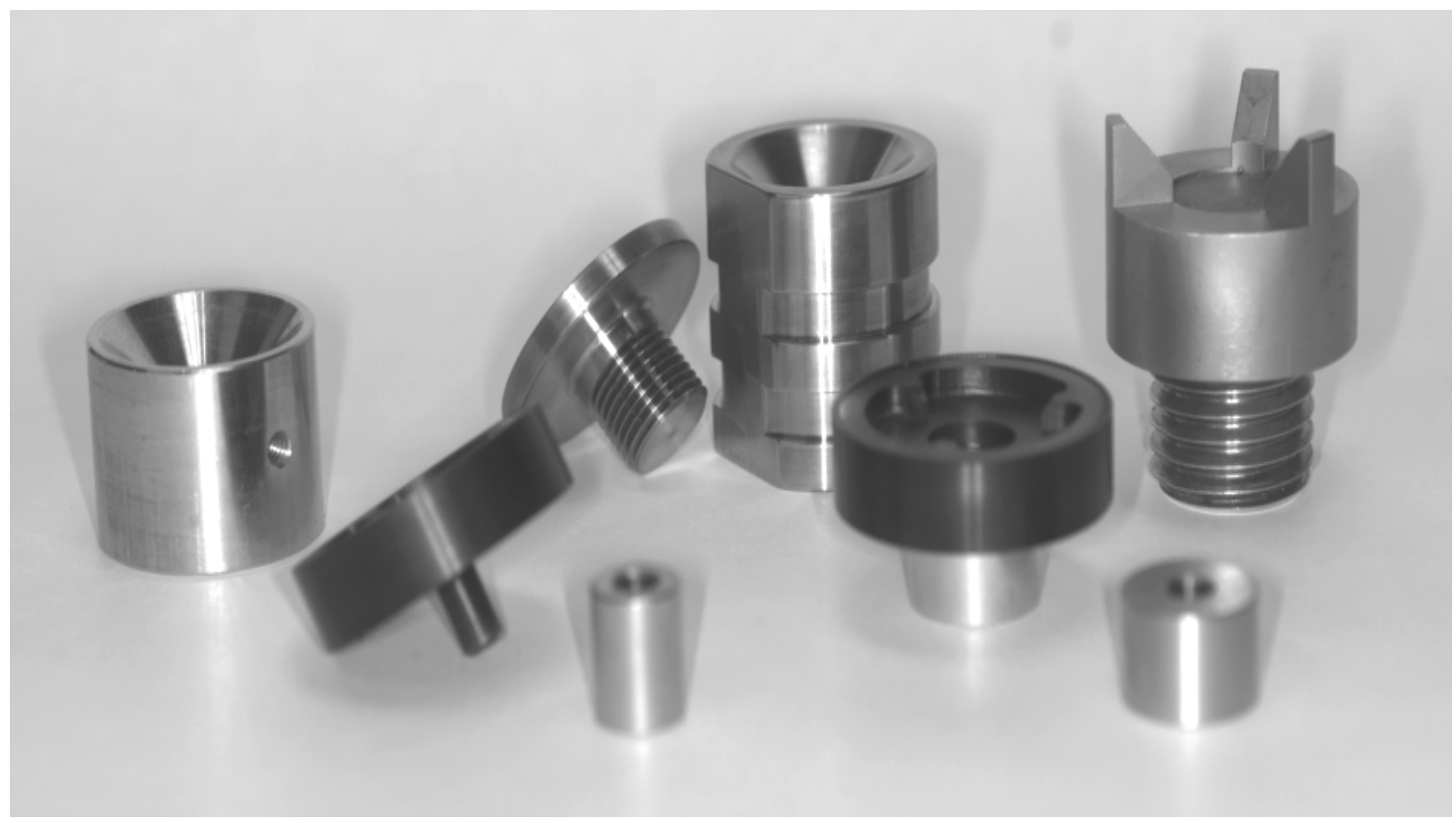

Figure 12.5 Sphere receptacles: floor, component, and wall barcket fixed-mount version, removable centered version. 
Figure 12.6 shows a typical section of the layout. A triplet of monuments is placed in the tunnel cross section containing a quadrupole magnet. One monument will be placed on the floor close to the quadrupole magnet, the second one mounted to the aisle wall at instrument height, while the third monument is mounted to the back wall.

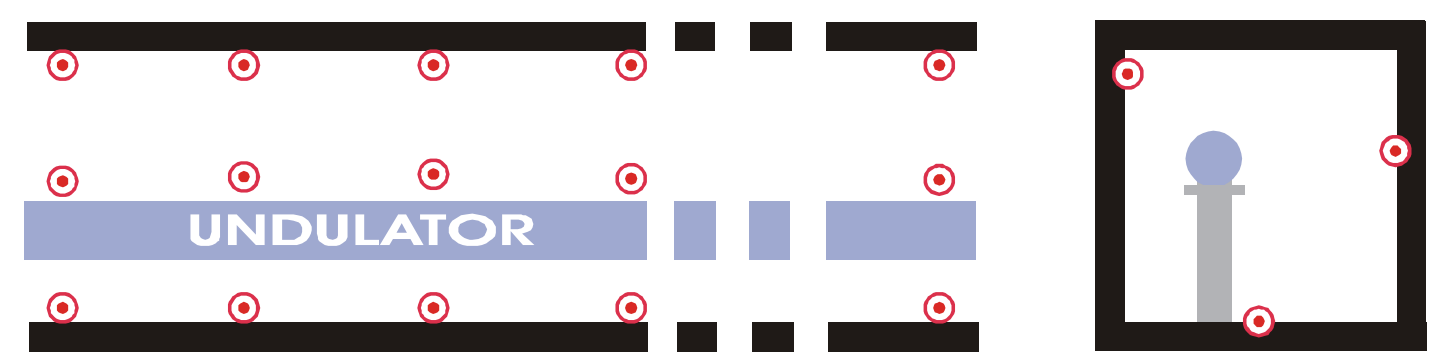

Figure 12.6 Undulator Hall Network layout (plan view \& cross section)

\subsubsection{Transport Line/Experimental Area Networks}

The transport line networks (undulator to near experimental area, near experimental area to remote experimental area) will support the survey and alignment of the transport line components. Standard networking design and measurement techniques can be used since the transport line's components have very loose positioning tolerance requirements. The network for the transport lines will be constructed and established similar to the Undulator Hall network. The only differences being that each cross section will have only two monuments, one mounted to the wall on the component side at instrument height and the second one in the floor close to the aisle side wall; the longitudinal spacing will be about three times the spacing in the undulator area.

The directional accuracy of the transport line networks is not sufficient to support the component installation and position requirements in the remote experimental area. A small surface network is necessary to accurately connect this area to the undulator. Present GPS technology easily supports the accuracy requirements. To physically establish the network, about ten concrete monuments equipped with forced-centering adapters need to be constructed. The monument design will be based on the SLC surface monuments. To connect GPS measurements to the undulator axis, the axis needs to be referenced to monuments on the surface. Existing sight shafts in the Beam Switch Yard (BSY) and additional new shafts in the undulator enclosure in the Research Yard will provide the necessary sight connections. 


\subsubsection{Alignment Coordinate System}

The alignment coordinate system will be a Cartesian right-handed system. The origin will be placed at Linac Station 100 (analogous to the SLC coordinate system). There will be no monument at the origin, it is purely a virtual point. The $y$-axis assumes the direction of the gravity vector at the origin but with opposite sign. The $z$-axis is in the direction of the linac, and the $x$-axis is perpendicular to both the $y$ and $z$-axes. The signs are defined by the right-handed rule (see Figure 12.7).

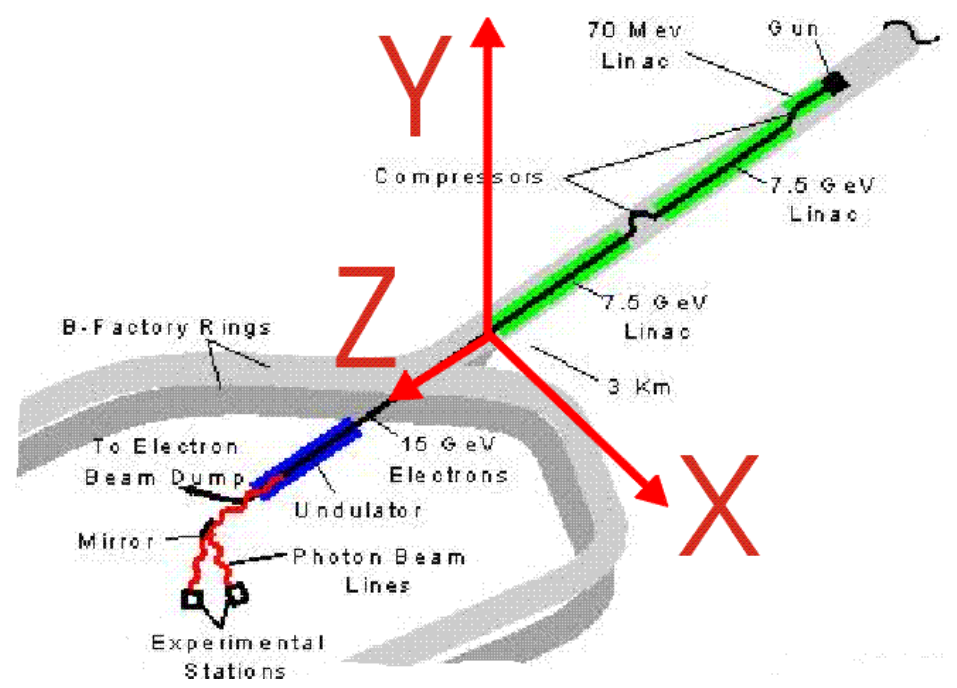

Figure 12.7 Coordinate system definition

\subsubsection{Tunnel Network Survey}

The most efficient instrumentation for the network observations will be the combination of a laser tracker (see Figure 12.8) with a digital level. The alignment group has available state-of-theart laser trackers (SMX4500, SMX Keystone), Total Stations optimized for industrial metrology (Leica TDA5000 and TC2002), and digital levels (Leica NA3000, Zeiss DiNi11). Laser trackers will contribute mainly to horizontal positional accuracy and digital levels to vertical positional and rotational accuracy. 


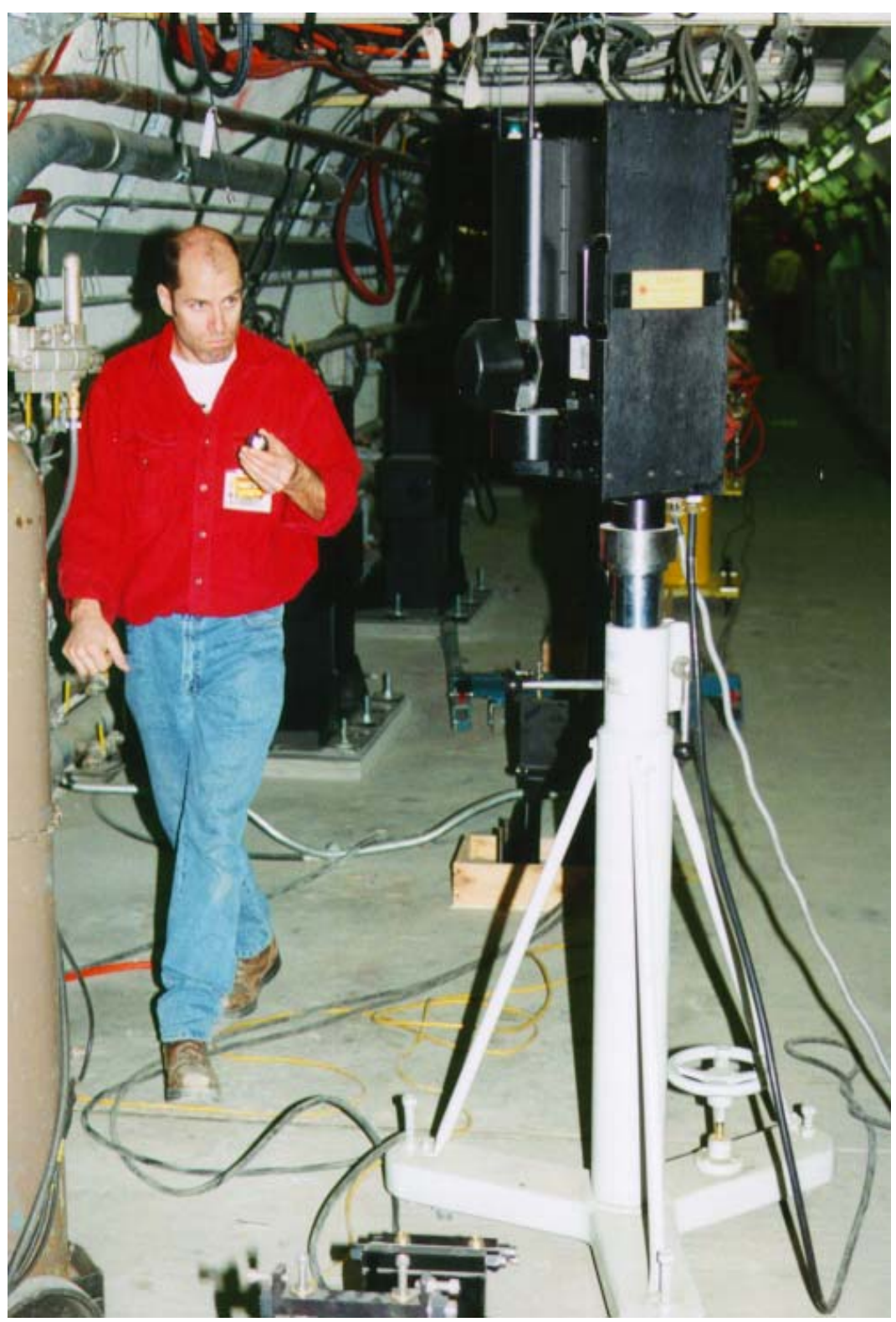

Figure 12.8 Target operator carries the SMX laser tracker reflector.

A laser tracker will be placed close to the middle between adjacent reference point crosssections (see Figure 12.9). From there, nine points in the forward direction and nine points in the backward direction will be measured. The measurement procedure will include two sets of distance and direction measurements to the same eighteen points in both front and reverse instrument orientations. All reference points will also be observed with a standard high precision double-run level procedure. A Zeiss DiNi11 digital level in combination with 2 m invar rods is envisioned. 


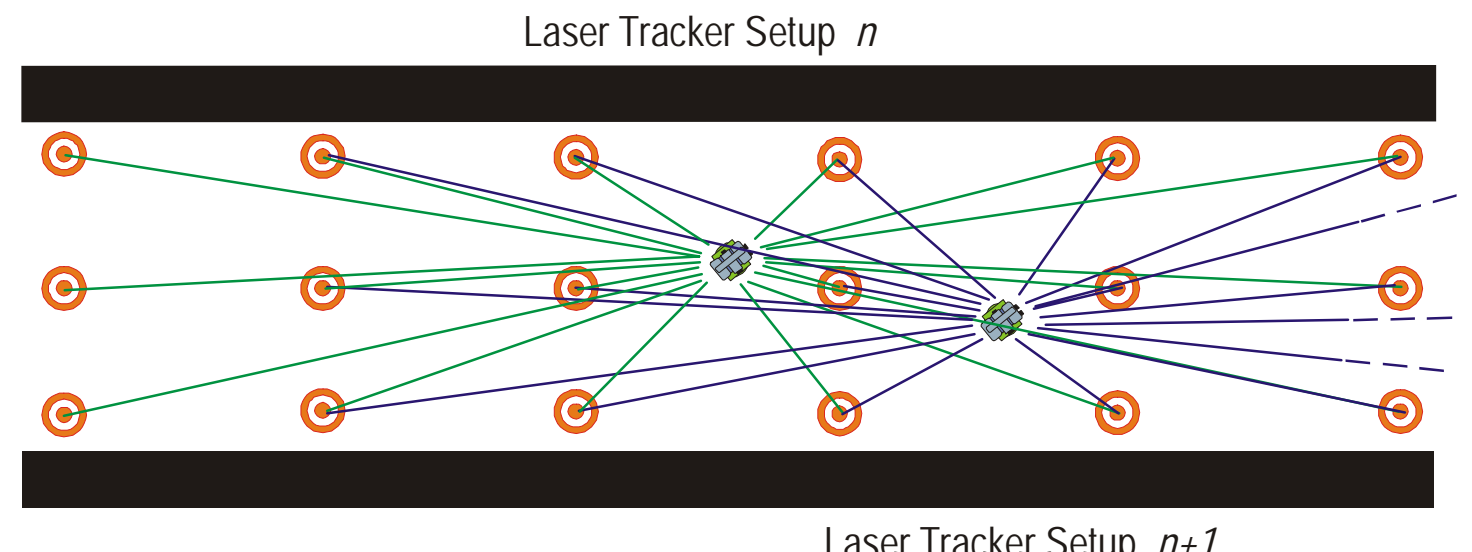

Figure $\mathbf{1 2 . 9}$ Observation plan

\subsubsection{Surface Network}

Standard GPS technology will provide the required accuracy. The equipment is available at SLAC (Leica 530 receivers). To connect GPS measurements to the undulator axis, the axis needs to be referenced to monuments on the surface. Monuments above and below the shafts will be referenced by optical plummet (Leica NL) measurements. The monuments below the shafts will be integrated into the regular tunnel network. The BSY shaft points will be linked to the undulator hall network by a temporary network. The monuments above the shafts will be connected to adjacent network monuments by GPS or optical triangulation measurements.

\subsubsection{Data Analysis and Data-Flow}

To reduce the data from the measurements as described above, specialized software has been developed. This type of analysis software is based on the photogrammetric bundle approach. Because a photogrammetric sensor is arbitrarily oriented in space, not only its translational parameters but also its rotational orientation parameters must be treated as unknowns and become part of the solution. With traditional trilateration/triangulation-based analysis software, however, pitch and roll are supposed to be oriented to gravity, and yaw is expressed as a function of translations. Additionally, the traditional software assumes that the instrument is centered on a point to which sufficient measurements have been taken. This analysis approach does not work well with free-stationing, and does not work at all with present generation laser trackers, since they cannot be oriented accurately enough to gravity. The code needs to be expanded to also accept GPS baselines, azimuth measurements and observations derived from the Hydrostatic Level System (HLS) and stretched wire systems. 
To reduce errors stemming from transcription of data, the data-flow will be automated. The suggested instruments support direct connection to field computers. The fully automated data-flow will extend from field computers through data analysis to data storage.

Measurements with any type of instrument will be guided by software based on rigid procedures running on field data logging computers. The data-logging software will also preanalyze the measurements, in an attempt to determine and flag possible outliers before the measurement setup is broken down. This method combined with an automated data-flow will greatly reduce errors and improve measurement consistency and reliability.

\subsection{Layout Description Reference Frame}

\subsubsection{Lattice Coordinate System}

The LCLS lattice is designed in a right-handed beam-following (s-axis) coordinate system, where the positive y-axis is perpendicular to the design plane, the z-axis is pointing in the beam direction and perpendicular to the $\mathrm{y}$-axis, and the $\mathrm{x}$-axis is perpendicular to both the $\mathrm{y}$ and $\mathrm{z}$-axes.

\subsubsection{Tolerance Lists}

The alignment system is designed based on the tolerances listed in Table 12.2.

Table $\mathbf{1 2 . 2} \quad$ LCLS positioning tolerances

\begin{tabular}{lccccc}
\hline & $\sigma_{x}[\mu \mathbf{m}]$ & $\sigma_{y}[\mu \mathbf{m}]$ & $\sigma_{r}[\mathbf{m r}]$ & $\sigma_{x} / z[\mu \mathrm{m} / \mathbf{m}]$ & $\sigma_{y} / z[\mu \mathbf{m} / \mathbf{m}]$ \\
\hline Relative alignment between undulator sections & 100 & 50 & 1 & $\mathrm{n} / \mathrm{a}$ & $\mathrm{n} / \mathrm{a}$ \\
\hline Global straightness of undulator & $\mathrm{n} / \mathrm{a}$ & $\mathrm{n} / \mathrm{a}$ & 1 & $300 / 120$ & $50 / 120$ \\
\hline Quadrupole ab initio & 50 & 50 & $\mathrm{n} / \mathrm{a}$ & $\mathrm{n} / \mathrm{a}$ & $\mathrm{n} / \mathrm{a}$ \\
\hline Linac straightness & $\mathrm{n} / \mathrm{a}$ & $\mathrm{n} / \mathrm{a}$ & $\mathrm{n} / \mathrm{a}$ & $150 / 15$ & $150 / 15$ \\
\hline Injector components & 150 & 150 & 1 & $\mathrm{n} / \mathrm{a}$ & $\mathrm{n} / \mathrm{a}$ \\
\hline Experimental area components & 1000 & 1000 & $\mathrm{n} / \mathrm{a}$ & $\mathrm{n} / \mathrm{a}$ & $\mathrm{n} / \mathrm{a}$ \\
\hline
\end{tabular}

$\mathrm{n} / \mathrm{a}=$ not applicable

\subsubsection{Relationship Between Coordinate Systems}

The relationship between the surveying and lattice coordinate systems is given by the building design and machine layout parameters. The result is a transformation matrix (rotations and translations). 


\subsection{Fiducializing LCLS Magnets}

The correct fiducialization of magnets is as important as their correct alignment since an error in either task will affect the particles' trajectory and cannot be distinguished from each other. Fiducialization can be accomplished either through opto-mechanical and opto-electrical measurements or by using fixtures, which refer to a magnet's reference features. Detailed descriptions can be found in the literature [2].

The most demanding task is the vertical positioning of the undulator to $50 \mu \mathrm{m}$ over the total length. Since the undulator sections will be aligned relative to their adjacent downstream quadrupoles, both the undulator segments and the quadrupoles need to be fiducialized to better than $25 \mu \mathrm{m}$ in order to leave a reasonable error budget for the alignment process.

The quadrupoles are permanent magnets of fairly small size. Hence, thermal expansion can be neglected. It is planned to use the same pulsed wire/straightness interferometer technique as was used to fiducialize the VISA undulator magnets [3]. Such a pulsed wire test stand prototype has been developed and setup at SLAC, and it has been demonstrated that the axis of an undulator quadrupole prototype can be repeatably determined to better than $5 \mu \mathrm{m}$.

While most of the magnetic measurements of the undulator segments will be carried out elsewhere, it is deemed necessary to check these measurements at SLAC to verify the magnet's homogeneity after transportation across the country (see also Section 8.3.3). The fiducialization measurements will become an integral part of the magnetic measurements. This can be accomplished by integrating a Coordinate Measurements Machine (CMM) into the test stand setup.

Components for the injector, linac, and dump line will be fiducialized using standard techniques.

\subsection{Absolute Positioning of Components}

Common to all parts of the machine, free-stationed laser trackers, oriented to at least four neighboring points, are used for the absolute positioning measurements. The tracking capabilities of these instruments will significantly aid in facilitating the control of any alignment operation (moving components into position).

\subsubsection{Undulator Absolute Positioning}

The absolute positioning is carried out in several steps. At first, the anchor hole positions for the component supports are marked on the floor. Next, after the supports and the magnet movers are installed, they will be aligned to within $0.5 \mathrm{~mm}$ of their nominal positions in order to retain as much mover range as possible. At this stage, the components can be installed. Since the components mechanically register to the support/magnet mover geometry, the installation will already place them to within $0.5 \mathrm{~mm}$. Finally, the position of the components will be surveyed and adjusted using a laser tracker in reference to adjacent network points. Absolute position accuracy relative to the network points of about $150 \mu \mathrm{m}$ can be achieved. 


\subsubsection{Injector, Transport Line and Experimental Area Absolute Positioning}

The absolute positioning of these components will follow the same procedure as described above. No relative alignment step is required to achieve these position tolerances.

\subsubsection{Quality Control}

Once the above steps are completed, the components will be mapped as a quality control measure. If any positional residuals exceed the tolerance, a second iteration can be "jump started" by using the quality control map to quantify the position corrections, which then need to be applied. A quality control survey will always follow the completion of the alignment process.

\subsection{Relative Alignment}

\subsubsection{Relative Undulator Alignment}

\subsubsection{Introduction}

The undulator sections will be aligned relative to their downstream quadrupole. The quadrupole position is a result of an initial optical/mechanical alignment process, which subsequently is refined by a beam-based alignment procedure ${ }^{6}$. For the beam-based alignment algorithm to converge efficiently, a $50 \mu \mathrm{m}$ ab initio placement is desired. Taking fiducialization error contributions into account, these quadrupoles need to be aligned to $30 \mu \mathrm{m}$ over a string of three quadrupoles. BPMs, taking fiducialization and acquisition errors into account, need to be aligned to $80 \mu \mathrm{m}$ relative to adjacent quadrupoles.

\subsubsection{Relative Quadrupole Positioning}

In principle, the absolute alignment process is repeated with the important difference, however, that measurements to a quadrupole are taken with respect to its neighbors and not to network points. Consequently, systematic errors stemming from the network are not propagated into the relative quadrupole positioning. This step will yield a position tolerance of about $80 \mu \mathrm{m}$.

To achieve the required accuracy, each quadrupole will be referenced by means of a laser tracker and Pellisier level [4] to a hydrostatic level system (HLS) and a stretched wire system (SWS), both equipped with sensors, which can be calibrated for absolute measurements. The absolute measurement requirement precludes the use of commonly used relatively inexpensive capacitive and inductive sensors, which will later be installed for monitoring purposes. Instead, a single specially developed sensor ${ }^{7}$ each for both the SWS and HLS will be used on all

\footnotetext{
${ }^{6}$ The electron trajectory within the undulator needs to be straight to a high degree of accuracy so that the undulator radiation overlaps the electron beam sufficiently within each gain length of the undulator. This level of trajectory straightness, $\sim 2 \mu \mathrm{m}$ rms over $10 \mathrm{~m}$, cannot reliably be achieved with optical alignment methods. Therefore, a beam-based alignment technique has been developed that determines quadrupole position corrections from BPM readings as a function of large, deliberate variations in the electron energy. Remotely controlled movers are used to apply the corrections. For an in-depth discussion see Section 8.1.1.

${ }^{7}$ Sensors have been developed in the framework of a collaboration with DESY on the development of the "Rapid Tunnel Survey System" for future linear colliders.
} 
measurement points $[5,6,7]$. It is planned to use a single $130 \mathrm{~m}$ long wire $^{8}$. First simulations show absolute station standard deviations of less than $150 \mu \mathrm{m}$ without wires and less than $40 \mu \mathrm{m}$ with wire measurements. A hydrostatic leveling system based on the half-filled pipe approach will also be integrated into the undulator support system. Experience at CERN has demonstrated that a vertical plane over the length of the undulator can be established to better than $25 \mu \mathrm{m}$ with the utilization of a hydrostatic level system.

The combination of all these measurements will yield position results better than $50 \mu \mathrm{m}$ over a string of three quadrupoles horizontally and over the whole length vertically including fiducialization errors.

\subsubsection{Undulator Alignment}

After the quadrupoles are aligned, the undulator segments can now be accurately positioned using the same combination of optical alignment procedures and HLS/SWS measurements as were used before for the quadrupole alignment. The difference in the HLS/SWS readings to an undulator section and to its adjacent quadrupole will be stored and, after the completion of a beam-based alignment iteration, used to restore the undulator-to-quadrupole relative alignment.

\subsubsection{Quality Control}

After all position adjustments are completed, a final mapping of all undulator, quadrupole, and BPM fiducials is carried out.

\subsubsection{Linac Smoothing}

\subsubsection{Purpose of Linac Smoothing}

To generate an optimal beam for injection into the undulator, the present local straightness of the linac is not sufficient. To achieve the desired beam parameters, the straightness quality needs to be mapped, and where necessary mechanically adjusted. In particular, the straightness of individual linac structures, the straightness alignment of structures on a girder, and the relative alignment of the sections on either side of a quadrupole with respect to each other and with respect to all other components need to be mapped.

\subsubsection{Linac Straightness Measurement Procedure}

Because of the required resolution, reliability and the large amount of work (about $1 \mathrm{~km}$ of beam line), the task is best performed with a system which does not require an operator to point and adjust micrometers. It also should allow online data logging. It is therefore proposed to use a laser system developed by Hamar [8]. The instrument generates two laser light planes by bouncing a laser beam off rotating mirrors. The two light planes are truly perpendicular to each other. The flatness or wobble-induced error of each light plane is specified as $5 \mu \mathrm{rad}$, which is well below the straightness specification at maximum distance. The light source would be set up at about the

\footnotetext{
${ }^{8}$ Stretched wire measurements over an equivalent distance are performed routinely at the CTF, CERN.
} 
middle between two quadrupoles offset horizontally and vertically such that the light planes clear all beam line components. This setup, versus a setup at an endpoint, reduces the length of the lineof-sight to about $5 \mathrm{~m}$, thus greatly lessening the effects of potential refraction and air turbulence on the light beam. After aligning the light planes both horizontally and vertically to two points on the measured object, intermediate offsets between, for example, the accelerator structure and the light planes are measured with a photo-sensitive-detector (PSD) attached to an offset arm. The detector is linked to an interface box by a cable, which can be as long as $15 \mathrm{~m}$. The interface box provides a serial link to a data logger. To measure the offsets, the offset arm is held against the accelerator structure sequentially in both planes. To determine the perpendicular offset, the alignment technician will arc the arm. While the arm is being arced, the light position is continuously read-out and stored. Software will then determine the perpendicular offset by finding the smallest read-out value. Because the PSD measurement range is limited to about $8 \mathrm{~mm}$, the arm will be adjustable in length. To avoid errors due to the adjustability, the adjustment length will be monitored by an electronic dial gauge, which also reports its reading to the data-logging software. The total straightness measurement error budget is expected to be below $75 \mu \mathrm{m}$.

The relative alignment of a linac quadrupole in relation to its adjacent accelerator sections will be determined analogously. However, since these quadrupoles are not fiducialized and also do not have any precision reference surfaces, the offset will be measured to their BPMs instead. Each BPM has a cylindrical body, which is inserted between the poles with a very close fit, and protrudes from the poles on either side of the magnet. The BPM is expected to reference the magnet's axis to about $100 \mu \mathrm{m}$. The adjustment range of the offset arm will be adequate to allow the same arm to measure both BPMs and accelerator structure offsets.

The readings will be evaluated using "smoothing" software developed for the alignment of the SLC arcs [9]. Position corrections will be applied under the control of a laser tracker. The present support systems are mechanically adequate.

\subsubsection{Relative Alignment of Transport Line and Experimental Area Components}

The position tolerances of these components will be achieved during the absolute alignment step. A relative alignment is not required.

\subsection{Undulator Monitoring System}

In order to keep the undulator optimally tuned, the BPMs must not drift from their position at the time of the last beam-based-alignment by more than a few $\mu \mathrm{m}$. A high resolution monitoring system will be installed to measure possible BPM position drifts, and subsequently, correct BPM readings. Additionally, the system can be used to independantly verify position changes intentionally induced by the magnet mover system.

Monitoring sensors should always be mounted in the respective principle measurement planes to avoid first order measurement errors. However, because of geometrical and mechanical lay-out limitations, this is here not possible. Placing the sensors away from the principle planes can cause 
errors due to non-zero roll or pitch, respectively. In order to be able to correct for possible errors, roll and pitch need to be accurately determined. Hence, a minimum of three sensors each for the horizontal and vertical plane monitoring systems are required; for redundancy reasons four sensors of each type per unit would be preferable (see Figure 12.10, and Figure 12.11). Both systems are controlled by a common PC based data acquisition system which is interfaced to the machine control system.

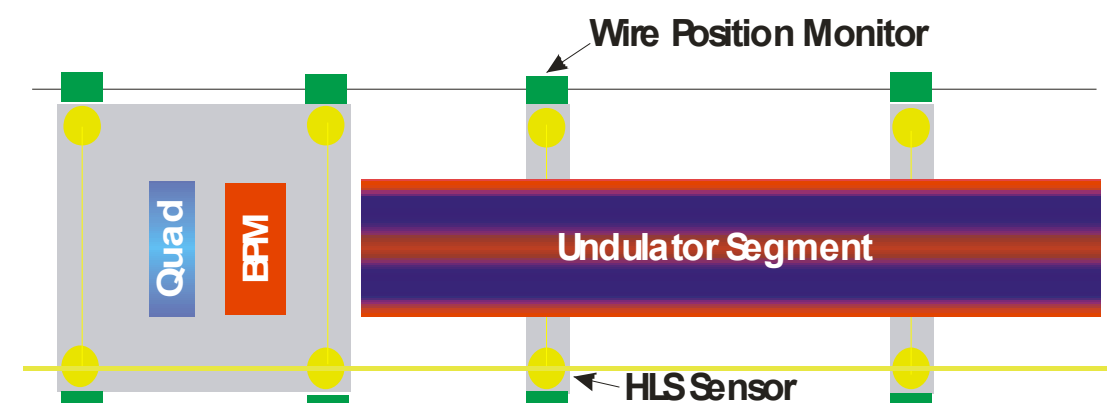

Figure 12.10 Monitoring System Layout, HLS (yellow), Wire System (green)

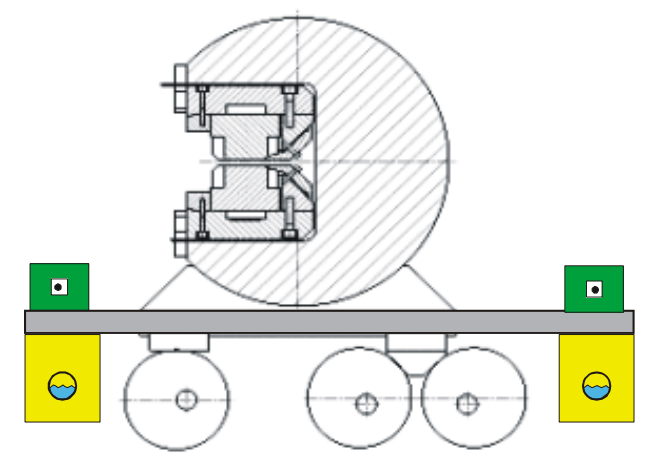

Figure 12.11 Monitoring System Lay-out Cross-section

Because there is no natural absolute reference in the horizontal plane, some kind of artificial local reference needs to be created as is done by stretched wires. Two wires, one on either side of the undulator sections/magnets will provide the straight line reference (see Figure 12.12). Inductive sensors will provide wire position information. The system is modeled after the FFTB wire monitoring system. Each Wire Position Monitor (WPM) is similar to a beam position monitor (BPM) in that it contains 4 antennas and that the differential signal strength received from opposite pairs of antennas is the quantity of interest. However, unlike a BPM, which receives its signal from a packet of charged particles, the WPMs receive their signals from a stretched wire, which is excited at the fixed end with a $3 \mathrm{~W}, 140 \mathrm{MHz}$ signal and which is grounded through a $250 \mathrm{~W}$ resistor at the pulley end. The wire is contained inside an $8 \mathrm{~mm}$ (inner diameter) brass tube. The tube serves as the outer conductor in a coaxial structure which presents a constant impedance to the 3-Watt signal and which shields the signal from the outside world where it would interfere with FM radio broadcasts. A precision-made aluminum extrusion provides a straight and rigid support for the brass tube. The signals detected by the WPM antenna are mixed 
with a $50 \mathrm{kHz}$ signal and are digitized with 16 bit ADCs, resulting in a resolution of better than $100 \mathrm{~nm}$ over a total range of $\pm 1.5 \mathrm{~mm}[10,11,12]$.

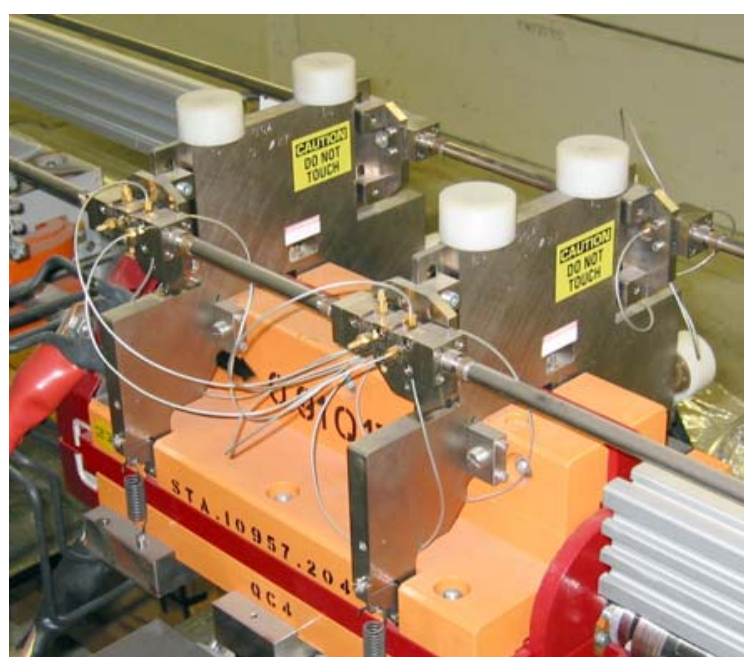

Figure 12.12 Wire Position Monitoring System

In the vertical dimension, a hydrostatic leveling system will accurately monitor relative and global vertical position changes. To eliminate temperature effects on the hydrostatic leveling results, the water piping must not deviate from a common horizontal plane. This condition is guaranteed using the "half-filled" pipe approach. Capacitive sensors like the ones developed by the BINP, FNAL, SLAC collaboration for NLC ground motion studies will be used (Figure 12.13) [13].

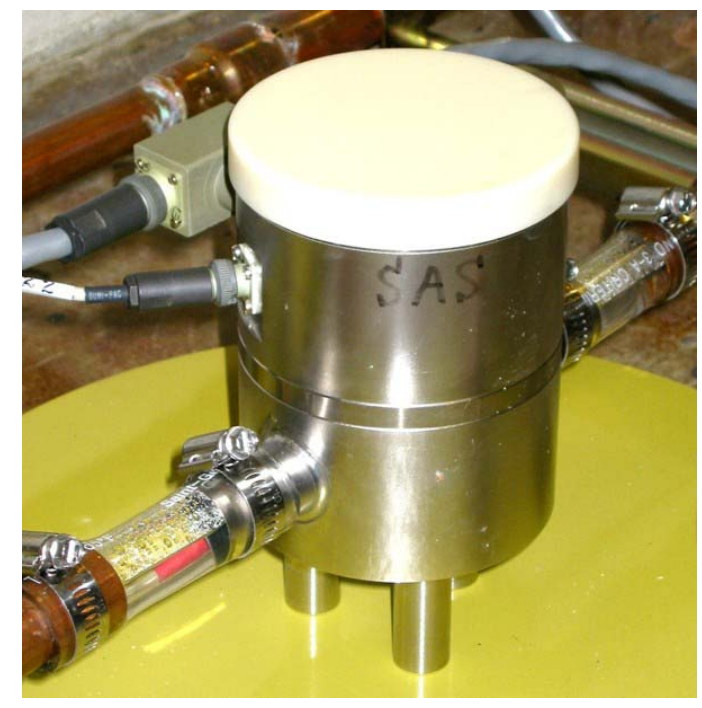

Figure 12.13 "Half-filled" pipe HLS Sensor 


\subsection{References}

1 R. Ruland, Magnet Support and Alignment, in H. Winick, Editor, Synchrotron Radiation Sources-A Primer, pp. 274 - 304, World Scientific, 1994

2 R. Ruland, Setting Reference Targets, in Proceedings of the CERN Accelerator School on "Measurement and Alignment of Accelerator Magnets," Capri, 1997, CERN 98-05

3 R. Ruland et al., VISA Undulator Fiducialization and Alignment, in: Proceedings of the Sixth International Workshop on Accelerator Alignment, Grenoble - ESRF, 1999

4 H. Imfeld et al., The Pellissier H5 Hydrostatic Level, in Proceedings of the Fifth International Workshop on Accelerator Alignment, Chicago-Argonne National Laboratory, 1997

5 W. Schwarz et al., Development of a HLS for the Upcoming Realization of the Linear Collider TESLA at DESY, to be presented at the $7^{\text {th }}$ International Workshop on Accelerator Alignment, Spring- 8 , Japan, November 2002

6 W. Schwarz, Straightness Measurements with Stretched Wire Technique for Planned Linear Colliders, to be presented at the $7^{\text {th }}$ International Workshop on Accelerator Alignment, Spring-8, Japan, November 2002

7 J. Prenting et al., TESLA - The Range of Survey and Alignment Work, to be presented at the $7^{\text {th }}$ International Workshop on Accelerator Alignment, Spring-8, Japan, November 2002

8 Hamar Lasersystems, Model L-723 Triple Scan ${ }^{\circledR}$ Laser with Model A-517 Scan Target.

9 R. Ruland, Accelerator and Transport Line Survey and Alignment, invited talk at the $5^{\text {th }}$ ICFA Beam Dynamics Workshop, Corpus Christi, Texas, 1991, SLAC-Pub 5672 pp. 9-14

10 F. Peters, private communication

11 R. Assmann et al., Beamline Stability Measurements with a Stretched Wire System in the FFTB, in Proceedings of the XVIII International Linac Conference (LINAC 96), Geneva, Switzerland, 1996, SLAC-Pub-7303

12 K. Flöttmann, Measurements of Magnet Motion at the FFTB with the Wire Alignment System, Internal Report, DESY M 96-07

13 A. Medvedko, Slow Ground Motion Measurement System for the Next Linear Collider Project, Preliminary Design Report, Butker Institute of Nuclear Physics, Novosibirsk, Russia, 2001. 


\section{Environment, Safety and Health and Quality Assurance}

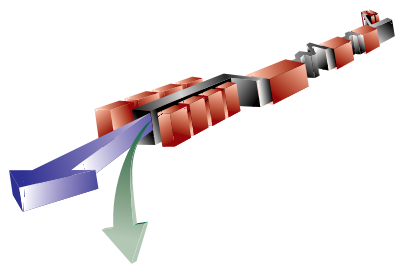

It is SLAC's policy and objective to integrate safety and environmental protection into its management and work practices at all levels, so that its mission is accomplished while protecting the worker, the public, and the environment. To achieve this objective, SLAC has developed and implemented an Integrated Safety Management System plan (ISMS), required by DOE P450.4, Safety Management System Policy, which encourages and supports the use of: the Work Smart Standards process, development of measurable goals in the form of performance metrics, and uses existing programs and activities that have been deemed successful and which already incorporate the ISMS elements. (ISMS as a required element is implemented through the incorporation of a contract clause from the DOE Acquisition Regulations (DEAR), specifically DEAR 970.5204.-2, "Integration of Environment Safety and Health Into Planning and Execution". This clause was incorporated into the contract between DOE and Stanford University for operation of SLAC in February 1998.)

Fundamental to the ISMS process is the application of Guiding Principals (GPs) and Core Functions (CFs). GPs are a series of best management practices or "basic philosophy" that ensure start-to-finish management of ES\&H issues. CFs provide the necessary structure that describes the scope of work, identifies and analyzes the hazard, develops and implements hazard controls, allows work to be performed within the controls, and uses feedback from the work performed to improve the safety system. Responsibility for achieving and maintaining excellence in this system rests with line management, who implement the SLAC ES\&H policy with the personnel under their supervision.

Existing and mature programs at SLAC will be used to ensure that all aspects of the design, installation, testing and operational phases of the project are properly managed. The LCLS project will be presented to the SLAC Safety Overview Committee, which coordinates and assigns safety reviews for new projects or facility modifications to other citizen committees, which have knowledge or skills in a specific area. The hazards for the LCLS will require reviews from committees including but not limited to: Radiation Safety Committee, Electrical Safety Committee, Earthquake Safety Committee and the Fire Protection Safety Committee.

Operation of existing electron accelerators have given us an opportunity to identify the principal hazards and risks associated with them. They are: Ionizing Radiation, Electrical Safety Issues, Non-Ionizing Radiation, Seismic Safety Issues, Fire Safety (including 
Emergency Preparedness), Construction Activities, Hazardous Material Issues and Environmental Protection; as they relate to the design, component manufacturing, system installation and operation of the LCLS facility. (See Table 13-1, which summarizes the hazards and mitigating controls.)

At SLAC, the LCLS project will not generate any hazards that have not already been defined and addressed within the Work Smart Standards and will not present any significant challenges from the ES\&H perspective. All aspects of the project will conform to the applicable Work Smart Standards SLAC has adopted and written into its contract with the DOE. 
Table 13-1 Hazard Identification and Mitigation.

\begin{tabular}{|c|c|c|c|c|}
\hline Item & Hazard & Possible Causes & Mitigating Controls & Work Smart Standards \\
\hline 1 & $\begin{array}{l}\text { Ionizing radiation exposure, outside } \\
\text { accelerator housing or experimental area } \\
\text { - prompt radiation }\end{array}$ & $\begin{array}{l}\text { - Personnel error } \\
\text { - Interlock failure }\end{array}$ & $\begin{array}{l}\text { - Safety Procedures } \\
\text { - Design, maintenance and inspection of } \\
\text { radiation safety systems } \\
\text { - Training }\end{array}$ & $\begin{array}{l}\text { 10CFR835; Atomic Energy } \\
\text { Act, DOE-N-441.4 }\end{array}$ \\
\hline 2 & $\begin{array}{l}\text { Ionizing radiation exposure, inside } \\
\text { accelerator housing or experimental area } \\
\text { - prompt } \\
\text { - residual } \\
\text { - contamination }\end{array}$ & $\begin{array}{l}\text { - Personnel error } \\
\text { - Interlock failure }\end{array}$ & $\begin{array}{l}\text { - Safety Procedures } \\
\text { - Design, maintenance and inspection of } \\
\text { radiation safety systems } \\
\text { - Training }\end{array}$ & $\begin{array}{l}\text { 10CFR835; Atomic Energy } \\
\text { Act, DOE-N-441.4 }\end{array}$ \\
\hline 3 & $\begin{array}{l}\text { Fire; inside accelerator housing or } \\
\text { experimental area } \\
\text { - electrical } \\
\text { - welding/cutting } \\
\text { - smoking } \\
\text { - hot work (soldering) }\end{array}$ & $\begin{array}{l}\text { - Equipment failure } \\
\text { - Personnel error }\end{array}$ & $\begin{array}{l}\text { - Sprinklers } \\
\text { - Smoke Detectors } \\
\text { - Fire Alarms } \\
\text { - Exit Routes } \\
\text { - Training } \\
\text { - On site Fire Department }\end{array}$ & $\begin{array}{l}\text { Uniform Fire Code (UFC), } \\
\text { National Fire Protection } \\
\text { Association (NFPA), DOE-O- } \\
420.1\end{array}$ \\
\hline 4 & $\begin{array}{l}\text { Fire; equipment and control areas } \\
\text { - electrical } \\
\text { - welding/cutting } \\
\text { - smoking } \\
\text { - hot work (soldering) }\end{array}$ & $\begin{array}{l}\text { - Equipment failure } \\
\text { - Personnel error }\end{array}$ & $\begin{array}{l}\text { - Sprinklers } \\
\text { - Smoke Detectors } \\
\text { - Fire Alarms } \\
\text { - Exit Routes } \\
\text { - Training } \\
\text { - On site Fire Department }\end{array}$ & $\begin{array}{l}\text { Uniform Fire Code (UFC), } \\
\text { National Fire Protection } \\
\text { Association (NFPA), DOE-O- } \\
420.1\end{array}$ \\
\hline 5 & $\begin{array}{l}\text { Electric shock } \\
\text { - high voltage } \\
\text { - low voltage/high current } \\
\text { - exposed } 110 \mathrm{~V}\end{array}$ & $\begin{array}{l}\text { - Personnel error } \\
\text { - Equipment failure } \\
\text { - Interlock failure }\end{array}$ & $\begin{array}{l}\text { - NEC Compliance } \\
\text { - Design, maintenance and inspection of } \\
\text { electrical interlock systems. } \\
\text { - Procedures (Lock Out/Tag Out) } \\
\text { - Training } \\
\text { - PPE }\end{array}$ & $\begin{array}{l}\text { National Electrical Code } \\
\text { (NEC) }\end{array}$ \\
\hline
\end{tabular}


Table 13-1 Hazard Identification and Mitigation

\begin{tabular}{|c|c|c|c|c|}
\hline Item & Hazard & Possible Causes & Mitigating Controls & Work Smart Standards \\
\hline 6 & $\begin{array}{l}\text { Non-Ionizing radiation exposure } \\
\text { - RF }\end{array}$ & $\begin{array}{l}\text { - Personnel error } \\
\text { - Equipment failure } \\
\text { - Interlock error }\end{array}$ & $\begin{array}{l}\text { - Design, maintenance and inspection of } \\
\text { interlock systems } \\
\text { - Procedures } \\
\text { - Training. }\end{array}$ & $\begin{array}{l}\text { American Conference of } \\
\text { Governmental Industrial } \\
\text { Hygienists (ACGIH) TLV for } \\
\text { UV and RF radiation } \\
\end{array}$ \\
\hline 7 & $\begin{array}{l}\text { Construction activities } \\
\text { - heavy equipment } \\
\text { - material handling } \\
\text { - slips/trip/falls } \\
\text { - tunneling }\end{array}$ & $\begin{array}{l}\text { - Personnel error } \\
\text { - Equipment failure }\end{array}$ & $\begin{array}{l}\text { - Prework hazard analysis } \\
\text { - Barriers } \\
\text { - Procedures } \\
\text { - Training } \\
\text { - Inspections }\end{array}$ & $\begin{array}{l}\text { Uniform Building, Plumbing } \\
\text { and Mechanical Codes (UBC, } \\
\text { UPC \& UMC), CCR, Chapter } \\
\text { 4, Subchapter 20, Tunnel } \\
\text { Safety Orders, 30CFR, } \\
\text { "Mineral resources," } \\
\text { Subchapter I Mine Safety } \\
\end{array}$ \\
\hline 8 & Seismic hazards & - Earthquake & $\begin{array}{l}\text { - Prework hazard analysis } \\
\text { - Design, construction and upgrade of } \\
\text { structures (buildings, accelerator } \\
\text { housings) and equipment to building and } \\
\text { structural codes } \\
\text { - Field inspections }\end{array}$ & $\begin{array}{l}\text { Executive Order } 12699, \\
\text { Specification for Seismic } \\
\text { Design of Buildings, } \\
\text { Structures, Equipment and } \\
\text { Systems at SLAC.Doc \# } \\
\text { SLAC-I-720-0A24E-002 }\end{array}$ \\
\hline 9 & $\begin{array}{l}\text { Exposure to hazardous materials, } \\
\text { including: } \\
\text { - cryogenics } \\
\text { - solvents } \\
\text { - oils } \\
\text { - welding/cutting fumes }\end{array}$ & $\begin{array}{l}\text { - Personnel error } \\
\text { - Equipment failure }\end{array}$ & $\begin{array}{l}\text { - Engineering analysis and inspection of } \\
\text { systems using hazardous materials } \\
\text { - Procedures } \\
\text { - PPE } \\
\text { - Training } \\
\text { - Ventilation }\end{array}$ & $\begin{array}{l}\text { Hazard Communication } \\
\text { 29CFR19190.1200, SLAC } \\
\text { ES\&H Manual }\end{array}$ \\
\hline 10 & $\begin{array}{l}\text { Adverse effects to the environment } \\
\text { - Spills } \\
\text { - Water discharges to sanitary and storm } \\
\text { drains } \\
\text { - Noise } \\
\text { - Air emissions (dust, leaks) } \\
\text { - Soil contamination } \\
\end{array}$ & $\begin{array}{l}\text { Construction and installation } \\
\text { activities } \\
\text { - Equipment failure } \\
\text { - Personnel error }\end{array}$ & $\begin{array}{l}\text { - Training } \\
\text { - Procedures } \\
\text { - Inspections }\end{array}$ & $\begin{array}{l}\text { Federal and State regulations, } \\
\text { SLAC ES\&H Manual }\end{array}$ \\
\hline
\end{tabular}




\subsection{Ionizing Radiation}

The design and operation of all radiation-producing facilities at SLAC are governed by the ALARA (as low as reasonably achievable) policy. SLAC has always maintained radiation dose limits below the maximum allowed by regulation.

\subsubsection{Radiation Shielding}

Shielding for the LCLS will conform to the Radiation Safety Systems Technical Basis Document, Chapter 1 Radiological Guidelines for Shielding and Barriers (SLAC-I-720-0A05Z002). Under normal operation the design criterion will be (i) $1 \mathrm{rem} / \mathrm{yr}$ at $30 \mathrm{~cm}$ from the shield surface, assuming a $2000 \mathrm{hr}$ working year and an occupancy factor of 1 . In addition the LCLS will have non-radiological workers (Users), additional shielding may be required to maintain their annual effective dose equivalent below $0.1 \mathrm{rem} / \mathrm{yr}$ taking exposure duration and occupancy factors into account. SLAC internal design criteria also requires that under a system failure (ii) the total effective dose equivalent shall not exceed 3 rem for a broad beam and 12 rem for a narrow beam, and that under an accident scenario that requires human intervention to turn off the beam (iii) the maximum dose equivalent shall not exceed 25 rem averaged over a 1 hour period for broad beam exposure or 100 rem averaged over 1 hour for narrow beam exposure.

An analysis of the present shielding indicates that potential beam losses from the LCLS during operation could produce high radiation doses. Local shielding, in some cases movable, will be used to mitigate these hazards to acceptable values. Defining the type and amount of local shielding is dependent on the final configuration of the LCLS for the experimental halls and experimenter hutches. Installation of radiation loss monitors at the hutches also will provide a diagnostic capability that may be used to determine where beam losses are taking place. Adding this monitoring package in addition to the Beam Containment System (BCS) will further help maintain dose levels below those limits allowed at SLAC.

\subsubsection{Personnel Protection System}

The personnel protection system (PPS) consists of electrical interlocks and mechanical barriers whose primary functions are to prevent entry of personnel into a beamline enclosure when prompt radiation and electrical hazards are operating, and to turn off the beam, RF, and electrical hazards when a security violation is detected. Other functions that it must also accomplish are: (i) provide interlocks for the orderly searching of an area before beam is turned on, (ii) allow for various access states, such as No Access, Controlled Access or Permitted Access, (iii) have emergency shut-off capabilities, and (iv) control the electrical hazards in beam housing areas. As installation of the LCLS will not require a significant change to the present shielding footprint, the PPS will undergo only necessary upgrades and enhancements to address the new facility and operating conditions. These upgrades include: additional status and control interfaces to accommodate new power supplies, access control modules for the injector and experimental areas, logic upgrades, and interlocks with beam stoppers, Beam Shut Off Ion 
Chambers (BSOICs), and those burn-through monitors that are controlled through the PPS. The PPS will remain largely the same in terms of its design, function, and configuration as other PPS to installations used at SLAC and SSRL, and all additions will conform to the Radiation Safety Systems Technical Basis Document, Chapter 2 Personnel Protection Systems (SLAC-I-7200A05Z-002).

\subsubsection{Beam Containment System}

The Beam Containment System (BCS) prevents accelerated beams from diverging from the desired channel, and detects excessive beam energy or intensity that could cause unacceptable radiation levels. Beam containment is usually accomplished by a combination of passive devices such as collimators, which are designed to absorb errant beams, and active devices such as electronic monitors that shut off the beam when out of tolerance conditions are detected. The present BCS in the Linac consists of passive mechanical devices (such as slits, collimators, magnets, electron beam stoppers, and dumps) and active electronic devices such as average current monitors, burn through monitors, and beam shut off ion chambers. Additionally the LCLS will install photon stoppers, ion chambers, and burn-through monitors for the proposed beamlines. Gas absorption cells are planned to be installed in critical places, for attenuating the beam as needed.

\subsubsection{Radiation Safety Training}

In accordance with SLAC's Site Access and Identification Badges Policies and Procedures (SLAC-I-720-0A0Z-002-R001), all individuals at SLAC who enter the Radiologically Controlled Area (RCA) or the Accelerator Area must be either properly trained or escorted by a properly trained individual. Levels of training depend on the area to be accessed and in some cases the duration of the individual's stay. (See Table 13-2.) 
Table 13-2 Minimum Training Requirements for Unescorted Access

\begin{tabular}{|c|c|c|c|c|c|c|c|c|c|c|}
\hline \multirow[t]{2}{*}{ Access Required } & \multirow{2}{*}{$\begin{array}{l}\text { Duration } \\
\text { of Access }\end{array}$} & \multirow{2}{*}{$\begin{array}{c}\text { Potential } \\
\text { Dose (mrem/yr) }\end{array}$} & \multicolumn{5}{|c|}{ Required Training Level } & \multicolumn{3}{|c|}{ Dosimeter } \\
\hline & & & $\begin{array}{c}\text { Safety } \\
\text { Orientation }\end{array}$ & EOESH & GERT & RWT I & RWT II & None & Annual & Quarterly \\
\hline $\begin{array}{l}\text { Industrial Areas } \\
\text { Accelerator Area } \\
\text { - No RCA }\end{array}$ & $\begin{array}{c}<60 \text { days } \\
\text { (within a } \\
\text { year) }\end{array}$ & 0 & $X$ & & & & & $\mathrm{X}$ & & \\
\hline $\begin{array}{l}\text { Industrial Areas } \\
\text { Accelerator Area } \\
\text { - No RCA }\end{array}$ & $\begin{array}{c}>60 \text { days } \\
\text { (within a } \\
\text { year) }\end{array}$ & 0 & & $X$ & & & & $X$ & & \\
\hline $\begin{array}{c}\text { Accelerator Area } \\
\text { RCA's }\end{array}$ & Any & $<100$ & & $\mathrm{X}$ & $X$ & & & & $X$ & \\
\hline $\begin{array}{l}\text { Accelerator Area } \\
\text { RCA's } \\
\text { High Rad. Area }\end{array}$ & Any & Any & & $\mathrm{X}$ & $\mathrm{X}$ & $\mathrm{X}$ & & & & $X$ \\
\hline $\begin{array}{c}\text { Accelerator Area } \\
\text { RCA's } \\
\text { Contamination } \\
\text { Area }\end{array}$ & Any & Any & & $\mathrm{X}$ & $\mathrm{X}$ & $\mathrm{X}$ & $\mathrm{X}$ & & & $X$ \\
\hline
\end{tabular}




\subsection{Electrical Safety}

An accelerator facility by nature has subsystems that either produce or use high voltage or high current, either of which can present an electrical hazard to personnel if not managed properly. As the LCLS will operate in a similar mode to other electron producing machines at SLAC, control and work procedures for electrical subsystems, as well as entry into the accelerator housing are well understood. Primary mitigation of the hazard will be through deenergization of equipment, placement of barriers and the effective use of Lock Out and Tag Out (LOTO) procedures.

In as much as the design, upgrade, installation and operation of electrical equipment will be in compliance with the National Electrical Code, Title 29 Code of federal Regulations, Parts 1910 and 1926 (as applicable) and SLAC's policy on Electrical Safety, SLAC ES\&H Manual, Chapter 8 (SLAC-I-720-0A29Z-001-R007); entry into the accelerator housing requires the mitigation of electrical hazards through either the lockout of power supplies or selective use of mechanical barriers, interlocked to further reduce the risk of exposure to electrical shock. Various levels of electrical safety training and LOTO training are provided by SLAC for those personnel who may work on or near potential electrical hazards.

Infrequently it may be necessary to complete work on energized equipment. This is conducted under very limited and controlled conditions, using qualified employees and where appropriate, under the full approval of the Associate Director. (Refer to: SLAC ES\&H Bulletin \#47A, "Safe Work Practices for Exposed, Energized AC and DC Electrical Systems")

Special procedures will be developed to permit authorized personnel to occupy areas adjacent to energized magnets. These are called Electrical Hazard Test Procedures and allow local control of the electrical power supply feeding a single magnet, or unique string of magnets, that are to be tested.

\subsection{Non-Ionizing Radiation}

The LCLS rf system will produce radio frequency radiation in the $2856 \mathrm{MHz}$ and $11424 \mathrm{MHz}$ ranges, which when not controlled could have an adverse health effect on personnel working on or near the system. The LCLS will incorporate safety measures based on present operations. These include interlocked waveguides and vacuum chambers and strict adherence to procedures for installation and testing of the rf system.

As the rf energy is fully contained within the envelope of these wave guides or vacuum chambers under ultra-high vacuum, opening the system up will trigger the interlock through a pressurization of the system and effectively prevent the source from being energized. Running the RF in this mode precludes microwave leakage, as failure of the vacuum system will occur before exposure to non-ionizing rf radiation.

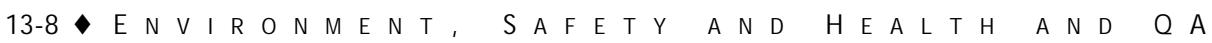


Procedures are also in place that ensure all flange bolts are torqued to a predetermined value as well as the completion of rf leak testing after all installations and maintenance activities, and periodically before start up of the system after scheduled shutdowns.

\subsection{Emergency Preparedness}

It has been estimated by the U. S. Geological Survey that the chance of one or more large earthquakes (magnitude 7 or greater) in the San Francisco Bay area in the coming 30 years is about 67 percent. This represents the emergency situation most likely to arise at SLAC. All SLAC personnel are trained in the immediate response to earthquakes and other emergencies via their supervisors and employee orientation.

\subsubsection{Seismic Safety}

SLAC structures are designed and constructed to minimize the effects of a major earthquake to acceptable levels. The majority of LCLS components will be installed in an existing facility, whose seismic stability is well documented and deemed acceptable. To further ensure and maintain a safe and healthful workplace, the design and construction of new experimental facilities' buildings as well as the design and installation of experimental equipment for the LCLS will also be reviewed by the Earthquake Safety Committee, as mandated by the Safety Program.

\subsubsection{Emergency Planning}

The design, review, installation and operation of all experimental equipment at SLAC is done in a manner that minimizes the risk of accident or injury to personnel and property in the event of either a natural disaster or emergency situation. SLAC's formal emergency planning system as described in the SLAC Emergency Preparedness Plan (SLAC-I-7300A14A-001) will help ensure a logical, organized, and efficient site wide response to any emergency. Facility specific procedures, which supplement the SLAC emergency plan, support a timely initial response, further decreasing the probability of personal injury and limiting potential loss or damage to both property and the environment.

\subsection{Construction Safety}

\subsubsection{General}

During construction operations, oversight of subcontractor activities and safety compliance remains a line organization responsibility through the University Technical Representative (UTR) or Project Engineer, if a UTR is not assigned to the activity. Detailed activities and job functions are clearly set forth in the SLAC University Technical Representative Guide (SLAC-11-01-07-01) and Quality Assurance and Compliance Design 
Assurance and Construction Inspection Procedure (SLAC-I-770-0A22C-001-R003). Responsibilities of UTRs or Project Engineers include, but are not limited to:

- Apprising subcontractors of SLAC and DOE safety criteria prior to construction.

- Informing subcontractors of the hazards routinely found at SLAC.

- Conducting periodic inspections of subcontractor construction areas to evaluate the quality of the subcontractor's safety compliance program and quality of work.

- Providing information to SLAC Citizen Safety Committees as required or requested.

- Communicating and resolving safety or quality deficiencies identified by SLAC personnel with the subcontractor.

- Receiving subcontractor accident reports and compiling information for reporting to the DOE.

Enforcement of subcontractor requirements is carried out by the SLAC Purchasing Department and may involve with holding payment(s) if applicable codes and standards are not met.

\subsubsection{Tunnel}

The LCLS configuration provides for two experimental areas (near and far hall), joined by a tunnel housing that contains the beam transport line. Early discussions and review of the tunnel portion of the project, helped determine the need for two separate tunneling techniques. Both of which may be required during construction. The two techniques that may be used include: "cut and cover", which in essence is akin to trenching but on a much larger scale; and the use of tunnel boring equipment, for those portions of the beamline housing that cut and cover would be either technically or economically infeasible. While both of these methods have been used at SLAC for previous projects, they are infrequent operations and the hazards encountered are not familiar ones to the SLAC community. Accordingly the safety scope of this type of operation is well defined in both: the California Code of Regulations, Chapter 4. Division of Industrial Safety, Subchapter 20, Tunnel Safety Orders, and Title 30, Code of Federal Regulations, "Mineral resources," Subchapter I Mine safety and Health Administration, Department of Labor. Subcontractors with mine safety expertise and experience will be used to provide the tunneling service.

\subsection{Hazardous Materials}

During the installation and operation phases of the LCLS it is anticipated that a minimum amount of hazardous materials will be used, examples would be paints, epoxies, solvents, oils and lead in the form of shielding, etc. There are no current or anticipated activities at the 
LCLS that would expose workers to levels of contaminants (dust, odors, fumes) above acceptable levels.

The SLAC Industrial Hygiene Program detailed in the SLAC ES\&H Manual addresses potential hazards to workers from the use of hazardous materials. The program identifies how to evaluate workplace hazards at the earliest stages of the project and implement controls to eliminate or mitigate these hazards to an acceptable level.

Site and facility specific procedures are also in place for the safe handling, storing, transporting, inspecting and disposing of hazardous materials. These are contained in the SLAC Introduction to Pollution Prevention, Hazardous Material and Waste Management "A Hazardous Materials Management Handbook" (SLAC-I-750-0A06G-001), and the ES\&H Manual Chapter 4, "Hazard Communication" (SLAC-I-720-0A29Z-011-R012) which describes minimum standards to maintain for compliance with Title 29, Code of Federal Regulations, Part 1910.1200.

The UTR or Project Engineer has added responsibilities with respect to the management of hazardous materials. They ensure subcontractor personnel are aware of, and remain in compliance with SLAC's written Hazard Communication Plan, also keeping affected SLAC personnel informed of hazardous material usage and the associated hazards and risks.

\subsection{Fire Safety}

The probability of a fire in the LCLS is expected to be similar to that for present operations, as accelerator and beamline components are primarily fabricated out of similar, non-flammable materials and combustible materials in general are kept to a minimum. The most "reasonably foreseeable" incident or event with any substantial consequences would be a fire in the insulating material of the electrical cable plant caused by an overload condition. This differs from the maximum credible fire loss, which assumes proper functioning of the smoke detector system and a normal response from the on-site fire department. In this case, losses would be confined to isolated components, but includes magnets, vacuum chamber and associated cabling. The ES\&H Manual Chapter 12, "Fire Safety" (SLAC-I-720-0A29Z-001R007) address all fire safety issues.

Installation of new cables for the LCLS will meet the current SLAC standards for cable insulation and comply with National Electric Code (NEC) standards concerning cable fire resistance. While this reduces the probability of a fire starting, an aspiration type smoke detection system (VESDA) in the accelerator housing and fire breaks in the cable trays will mitigate fire travel. Support buildings for power supplies, electronic equipment or experimental areas are protected by automatic heat activated wet sprinkler systems and smoke detectors. Fire extinguishers are located in all buildings and accelerator housings for use by trained personnel. The combination of smoke detection systems, sprinklers and on-site fire department (response time $\sim 3$ minutes) affords an early warning and timely response to fire or smoke related incidents. 
Burn injuries caused by a fire are not expected because nowhere in either the Linac accelerator housing or Final Focus Test Beam area or support buildings are personnel further than $150 \mathrm{ft}$ from an exit and there is no location where two directions of egress are not available. Multiple entry/exit points also helps in keeping property damage to a minimum.

\subsection{Environmental Protection}

Installation of the LCLS will require the removal of some hardware (that is, magnets, vacuum chambers, FFTB magnets) and replacement with new components suited to the proposed facility (that is, new gun, injector and re-configuration of existing magnets and addition of a long undulator). Electrical distribution systems will be upgraded or renewed as appropriate and minor modifications to the Low Conductivity Water (LCW) system will be made to accommodate heat transfer needs. Some limited removal of asphalt and concrete will be required in the relocation of the FFTB beam dump. Removal of these materials and the subsequent installation activities will produce small quantities of hazardous, non-hazardous and radioactive waste that need to be managed through defined channels. Past history indicates that normal operation of the accelerator does not typically produce waste. However, some hardware may have induced radioactivity associated with it from its proximity and time close to the beam. Other components may contain hazardous materials as part of their design, e.g., mineral oil in electrical components, or have radioactive contamination from the LCW system.

All material removed from within the accelerator housing will be surveyed for residual radioactivity or contamination. If none is detected, then items would be salvaged; for re-use, as recyclable scrap material or disposed of as non-hazardous waste in an approved off-site landfill. Items that show residual radioactivity or contamination would be stored on site in the Radioactive Material Storage Yard for future reuse or ultimate disposal. Any hazardous waste would be disposed of in accordance with SLAC procedures and ultimately to a permitted Treatment, Storage and Disposal Facility, under regulations set forth in the Resource, Conservation and Recovery Act (RCRA).

Component manufacturing and system installation may also produce hazardous wastes, such as used solvent from degreasing baths or spent cutting fluids. These are ongoing operations at SLAC, disposal of wastes is routine, and in full compliance with SLAC's policies on the management of hazardous materials and waste minimization.

The addition of two experimental halls and the subsequent earth removal, tunneling and construction activities will necessitate conducting an Environmental Assessment under the National Environmental Protection Act. This document will look carefully at the consequences of siting a new facility at SLAC, taking into consideration environmental values and other technical and economic consequences. National Environmental Policy Act (NEPA) also includes provisions to include coordination and integration of reviews of other 
environmental laws and executive orders. Examples are the endangered species act, floodplain/wetlands regulations, fish and wildlife coordination act, "Greening the Government" initiatives, and the national historic preservation act.

All activities will be managed to prevent adverse impact on ground water and storm water quality, air quality and to minimize any ground disturbing activities.

\subsection{Quality Assurance}

A Quality Assurance Program Plan (SLAC-I-770-0A17M-001-R001) conforming with DOE Order 414.1A, "Quality Assurance", was established at SLAC to provide laboratory management with guidance and requirements toward achieving quality in pursuit of the laboratory mission. Overall responsibility for the implementation of this program lies with the SLAC Director, while accountability for managing the program at the divisional level rests with the respective Associate Director (AD). For the LCLS project, the "Project Leader" has been assigned by the SSRL Division AD and given responsibility for staffing, documenting, generating Quality Implementing Procedures and implementing the QA program. At the project level this includes developing and maintaining required management systems, or using management systems that are already available.

The QA plan describes SLAC's approach to implementing the ten criteria of DOE Order 414.1A:

Criterion 1 - requires specific Quality Implementing Procedures for all SLAC projects where total project costs exceed $\$ 5,000,000$.

Criterion 2 - as appropriate defines specific requirements and assures adequate qualification and training for individuals connected with the project, including retention of training records.

Criterion 3 - defines requirements for management's responsibility with respect to identification, analysis, resolution and follow up of ES\&H, technical and compliance issues.

Criterion 4 - provides policy for identification of documents (policy, procedures, drawings etc.), records and other specific elements that will have a significant impact on the project and need to be entered into a document control system.

Criterion 5 - requires project leaders to define and maintain work processes for $R \& D$ efforts that have a significant programmatic impact. 
Criterion 6 - establishes a responsibility for line management to conduct design reviews and to promote the use of design standards.

Criterion 7 - discusses a graded approach to the development of specifications for procurement of items and services based on cost and failure impact.

Criterion 8 - established responsibility for the staffing, documenting, and performing of inspection and testing activities related to the project.

Criterion 9 - requires participation in the SLAC Institutional Self-Assessment Program.

Criterion 10 - provides the authority for the Quality Assurance and Compliance Department to conduct independent assessments of all SLAC facilities and projects as warranted to verify the degree of conformance to QA and ES\&H requirements.

Effective use of these criteria will enable the LCLS project to:

- Design in quality and reliability.

- Promote early detection of problems to minimize failure costs and impact on schedule.

- Develop appropriate documentation to support upgrade and operational requirements.

- Establish methods to identify critical systems and to release these systems based on demonstrated performance.

- Define the general requirements for design and readiness reviews for all aspects of the project.

- Assuring personnel are trained before performing critical activities, especially those that have ES\&H consequences. 


\subsection{SLAC References}

SLAC Work Smart Standards http://www.slac.stanford.edu/esh/reference/worksmart.htm

SLAC Safety Management System http://www.slac.stanford.edu/esh/isms/sms.pdf

SLAC Environment, Safety \& Health Manual

http://www.slac.stanford.edu/esh/manuals/eshmanual.html

SLAC Radiation Safety Systems Technical Basis Document (SLAC-I-720-0A05Z-002)

http://www.slac.stanford.edu/esh/techbas/rss/rss.pdf

Specification for Seismic Design of Buildings, Structures, Equipment, and Systems at the Stanford Linear Accelerator Center (SLAC-I-720-0A05Z-002)

http://www.slac.stanford.edu/esh/techbas/seismic.pdf

Lock and Tag Program for the Control of Hazardous Energy (SLAC-I-730-0A10Z-001)

http://www.slac.stanford.edu/esh/manuals/locktag.pdf

Electrical Hazard Test Procedures (SLAC-I-040-30460-002)

Introduction to Pollution Prevention, Hazardous Material and Waste Management (SLAC-I750-0A06G-001-R001)

http://www.slac.stanford.edu/esh/training/study_guides/hmh.pdf

SLAC Emergency Preparedness Plan (SLAC-I-730-0A14A-001)

http://www.slac.stanford.edu/esh/manuals/epp2000.pdf

SLAC Institutional Quality Assurance Program Plan (SLAC-I-770-0A17M-R002)

http://www.slac.stanford.edu/esh/manuals/QAplan.pdf 


\section{Radiological}

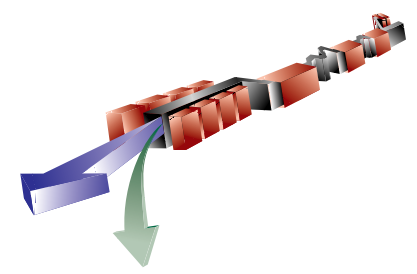

\section{TECHNICAL SYNOPSIS}

The radiation protection issues for the LCLS are normally encountered at both high-energy electron linacs and synchrotron radiation facilities. The SLAC Radiological Control Manual [1]

specifies an annual total effective dose equivalent limit to workers from both internal and external radiation sources of $5 \mathrm{rem}$. In addition, SLAC maintains an administrative control level of $1.5 \mathrm{rem}$.

Radiation dose criteria used in design of the LCLS radiation safety systems are those required for SLAC facilities.

The integrated dose equivalent outside the surface of the FFTB shielding barriers must not exceed 1 rem in a year for normal beam operation [1].

The integrated dose equivalent to personnel working inside and around the experimental hutch shielding barriers must not exceed 0.1 rem in a year for normal beam operation. [2].

The dose equivalent-rate in the event of the Maximum Credible Incident is limited to less than $25 \mathrm{rem} / \mathrm{h}$, and integrated dose equivalent of less than $3 \mathrm{rem}$ [1].

The maximum dose equivalent rates in accessible areas at 1 foot from the shielding or barrier should not exceed $400 \mathrm{mrem} / \mathrm{h}$ for mis-steering conditions defined as conditions that are comprised of infrequent or short-duration situations in which the maximum allowable beam power, limited by Beam Containment System (BCS) devices is lost locally or in a limited area.

The dose equivalent for the maximally exposed member of the public exposed to ionizing radiation from SLAC produced pathways must be less than or equal to $10 \mathrm{mrem} / \mathrm{yr}$ [3]. The dose equivalent at the site boundary from the operation of the LCLS must be a small fraction of that total for normal beam operation.

The expected radiation sources have been identified and analyzed to determine the required radiation safety systems. These sources produce high energy bremsstrahlung and particle radiation from the interaction of the primary electron beam with protection collimators, beam diagnostic devices, main LCLS dump, and interaction with the residual vacuum.

A radiation safety system comprised of shielding, Beam Containment System (BCS), Personnel Protection System (PPS) and Hutch Protection System (HPS) [1] has been designed for the LCLS. The issues considered in the design of these systems are described in this chapter.

\subsection{Introduction}

Most of the components of the LCLS beam line are installed in the FFTB tunnel, a shielded enclosure in the straight-ahead channel at the end of the SLAC linac (Figure 14.1). This tunnel is 
composed of two sections. The first section $(107 \mathrm{~m})$ is in the beam switchyard, which is a large, two-level structure shielded on the roof by more than $12.2 \mathrm{~m}$ of concrete and earth, located at the end of the linac. The second section is a shielded structure that extends $150 \mathrm{~m}$ to the east beyond the beam switchyard. This section is shielded with $1.2 \mathrm{~m}$ of concrete laterally and $1 \mathrm{~m}$ of concrete on the roof [4].

Since the LCLS electron beam power, energy and beam losses are comparable to that of the FFTB, the existing enclosure shielding should be adequate without major modifications. For the LCLS, new designs of the safety systems are required for the injector at sector 20 of the linac, Front End optics enclosure that will be added to the structure in the research yard, the new beam dump enclosure, and experimental hutches downstream of the electron beam dump.

\subsection{Radiation Sources}

During machine operation, high energy bremsstrahlung and particle radiation is generated from the interaction of the primary electron beam with protection collimators, beam diagnostic devices, main LCLS dump, and interaction with the residual vacuum.

The radiation initiated in these reactions as well as the forward directed and scattered coherent x-ray and synchrotron radiation are the main sources of radiation that need to be considered in the design of the shielding for the new areas downstream of the undulator. The particle radiations of concern are neutrons and muons.

\subsubsection{Beam Parameters}

The electron beam will be delivered at energies up to $15 \mathrm{GeV}$ at $1 \mathrm{nC}$ and $120 \mathrm{~Hz}$.

\subsubsection{Bremsstrahlung from Collimators}

Two copper collimators, each $10 \mathrm{~cm}$ long and with an internal diameter of $0.2 \mathrm{~cm}$, will be placed up beam of the undulator (Figure 14.2). The purpose of the first collimator is to reduce the electron beam halo, while the second should intercept any mis-steered beam that could hit and damage the undulator. The first collimator, continuously intercepting about $1 \%$ of the beam, will be a constant source of forward-directed bremsstrahlung and muon radiation. The second collimator should interact with the beam only in exceptional cases and is not expected to contribute substantially to the radiation field under normal operating conditions. 


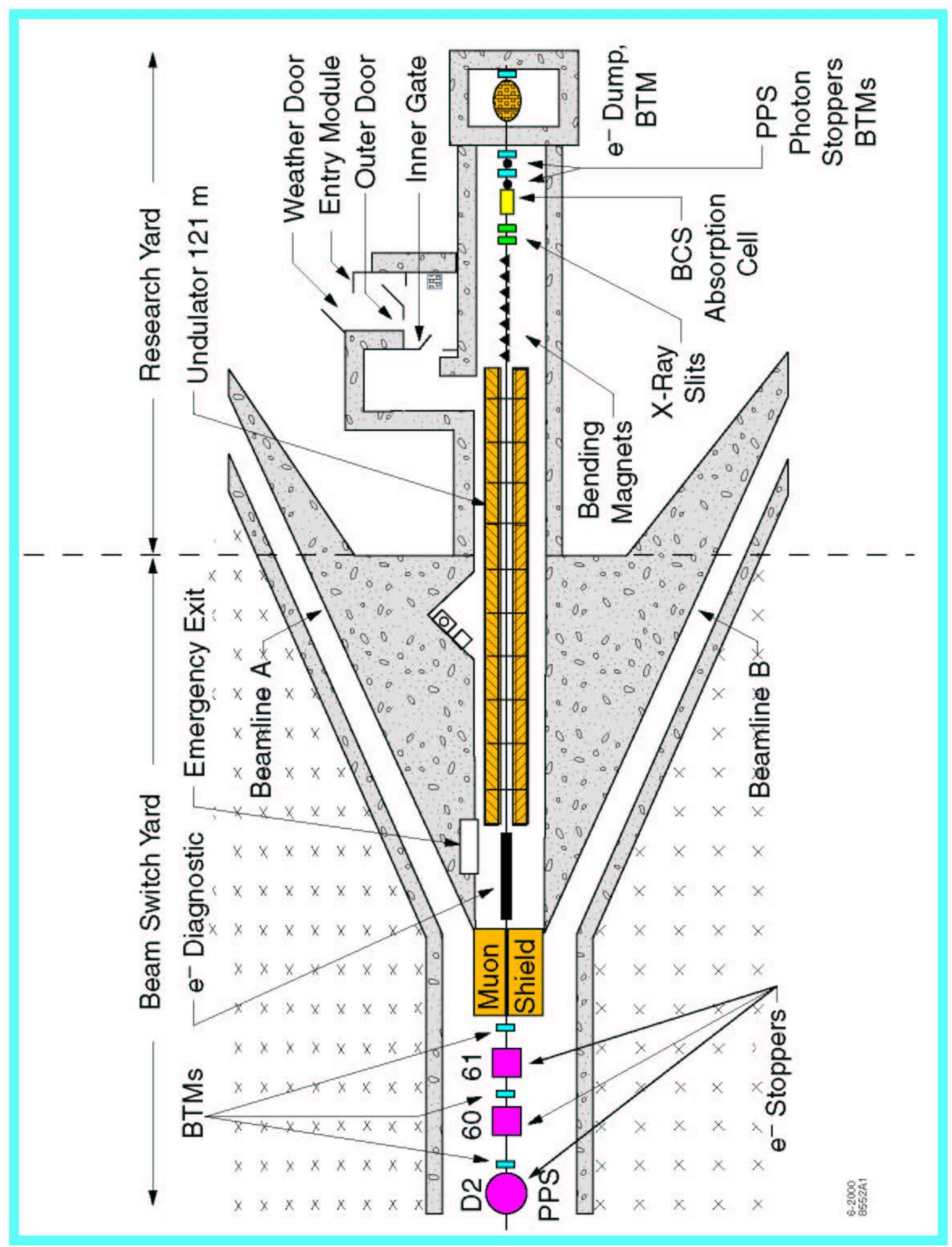

Figure 14.1 Electron Beamline for LCLS 


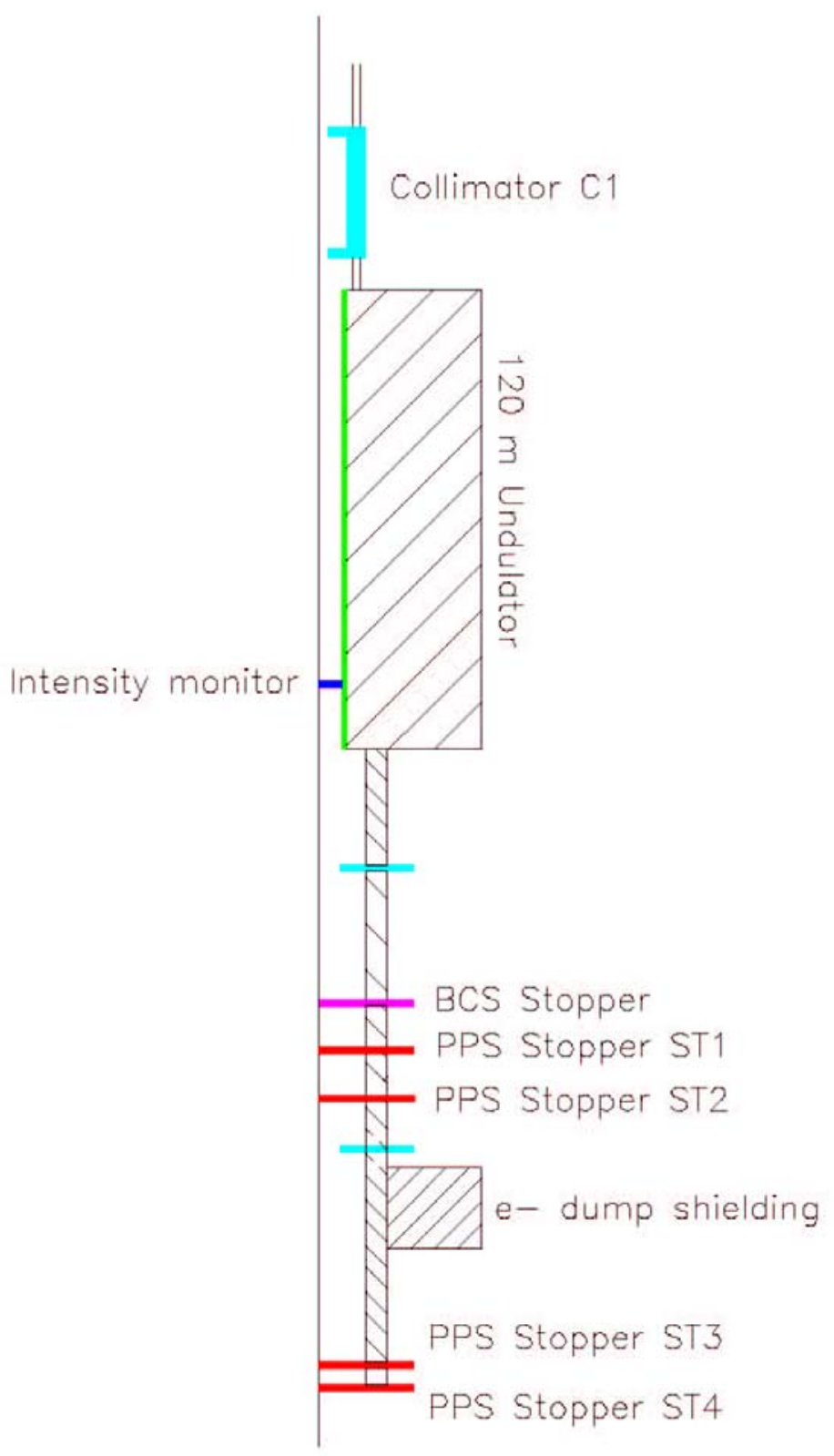

Figure 14.2 LCLS Beamline showing various BCE and PPS devices

Bremsstrahlung radiation produced in the first collimator will present a hazard to personnel in downstream experimental areas. Consequently photon stoppers are required as part of the Personnel Protection System (PPS, Section 14.3.3). The first of these stoppers, which must be inserted into the beamline when access is allowed in any downstream enclosure, will intercept this bremsstrahlung radiation.

Details of the calculation are given in [5]. For a $1 \%$ loss of a $15-\mathrm{GeV}, 2-\mathrm{kW}$ electron beam the energy deposition in the PPS stopper ST1 (see Fig. 14.3), which must be inserted into the 
beamline when access is allowed in Hutch 1, was $17 \mathrm{~mW}$, calculated using the EGS4 code. The energy deposition in the PPS stopper ST3, which must be inserted into the beamline when access is allowed in Hutch 2, was $12 \mathrm{~mW}[6]$.

However, bremsstrahlung from collimators is neither the only nor the main source of radiation to be considered for shielding design: other radiation components (bremsstrahlung from profile monitors, neutrons, muons, x-rays) must also be taken into account.

\subsubsection{Bremsstrahlung from On-Axis Diagnostic X-Ray Stations}

The electron beam will be intercepted by monitoring devices at several locations in the onaxis diagnostic x-ray stations along the undulator. There will be 10 or 12 of these stations, but calculations have been made for the one located in the last $10 \mathrm{~m}$ section of the undulator. The material is diamond, $0.5 \mathrm{~mm}$ thick, but because the beam strikes it at an angle of $45^{\circ}$, the effective thickness traversed is $0.707 \mathrm{~mm}$. For a $15-\mathrm{GeV}$ and $2-\mathrm{kW}$ electron beam the energy deposition in the BCS stopper, which is interlocked with the monitor, was $6.5 \mathrm{~W}$ calculated using the EGS4 code [5,6]. If it is assumed that the monitor will be used about $10 \%$ of the beam time, this is equivalent to a continuous energy deposition of $650 \mathrm{~mW}$ in the BCS stopper.

\subsubsection{Synchrotron Radiation}

The synchrotron x-rays will be absorbed in the BCS or PPS stoppers when they are inserted to the beam. The total power in the LCLS synchrotron spectrum was calculated to be $2.78 \mathrm{~W}$ [6]. When the beam line is open, this power will be absorbed in the hutch stopper.

\subsubsection{Electron Deam Dump}

The distance from the front face of the first bending magnet to the front face of the dump will be 28 meters. The distance from the center of the dump to the ground level will be 1.5 meters. The shielding design for the dump was based on this arrangement, shown in Figure 14.4. 


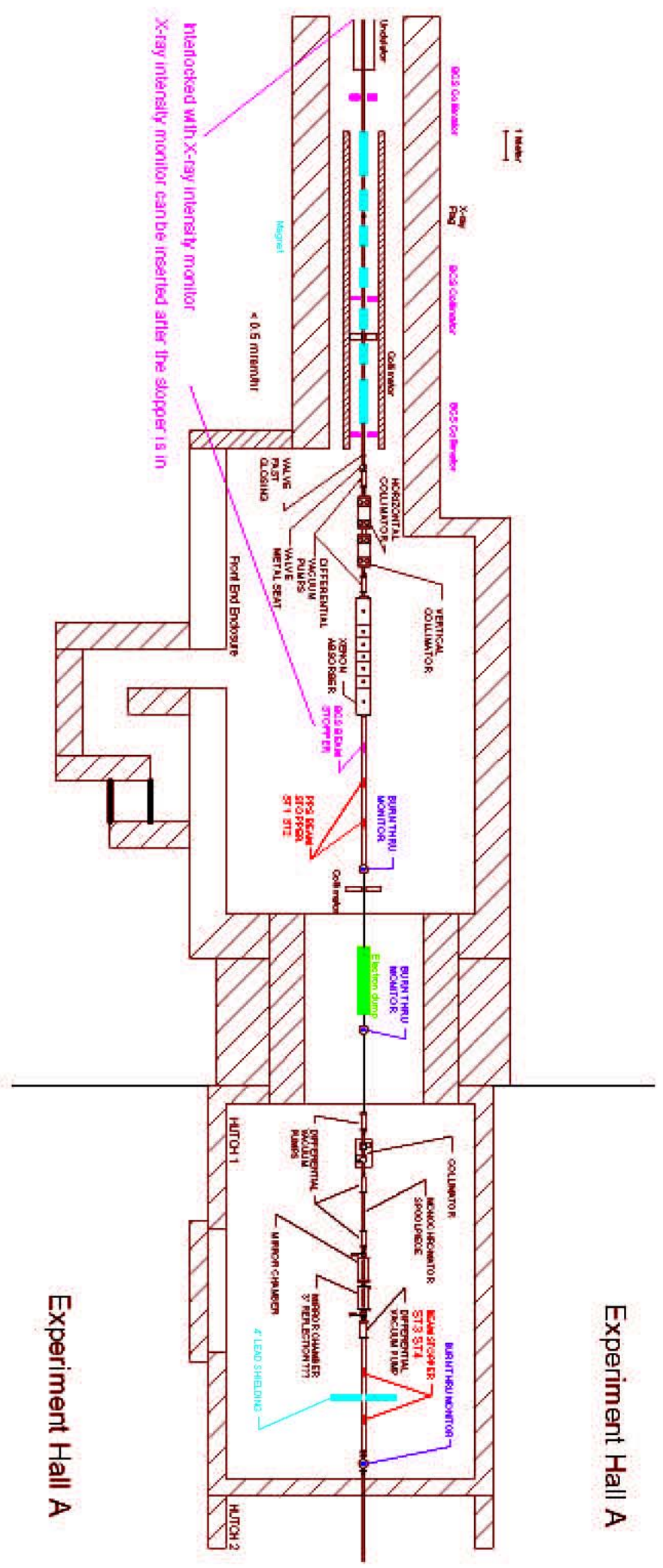

Figure 14.3 Schematic View of LCLS x-ray beam line showing details of the front end and experimental hall BCS and PPS devices. 


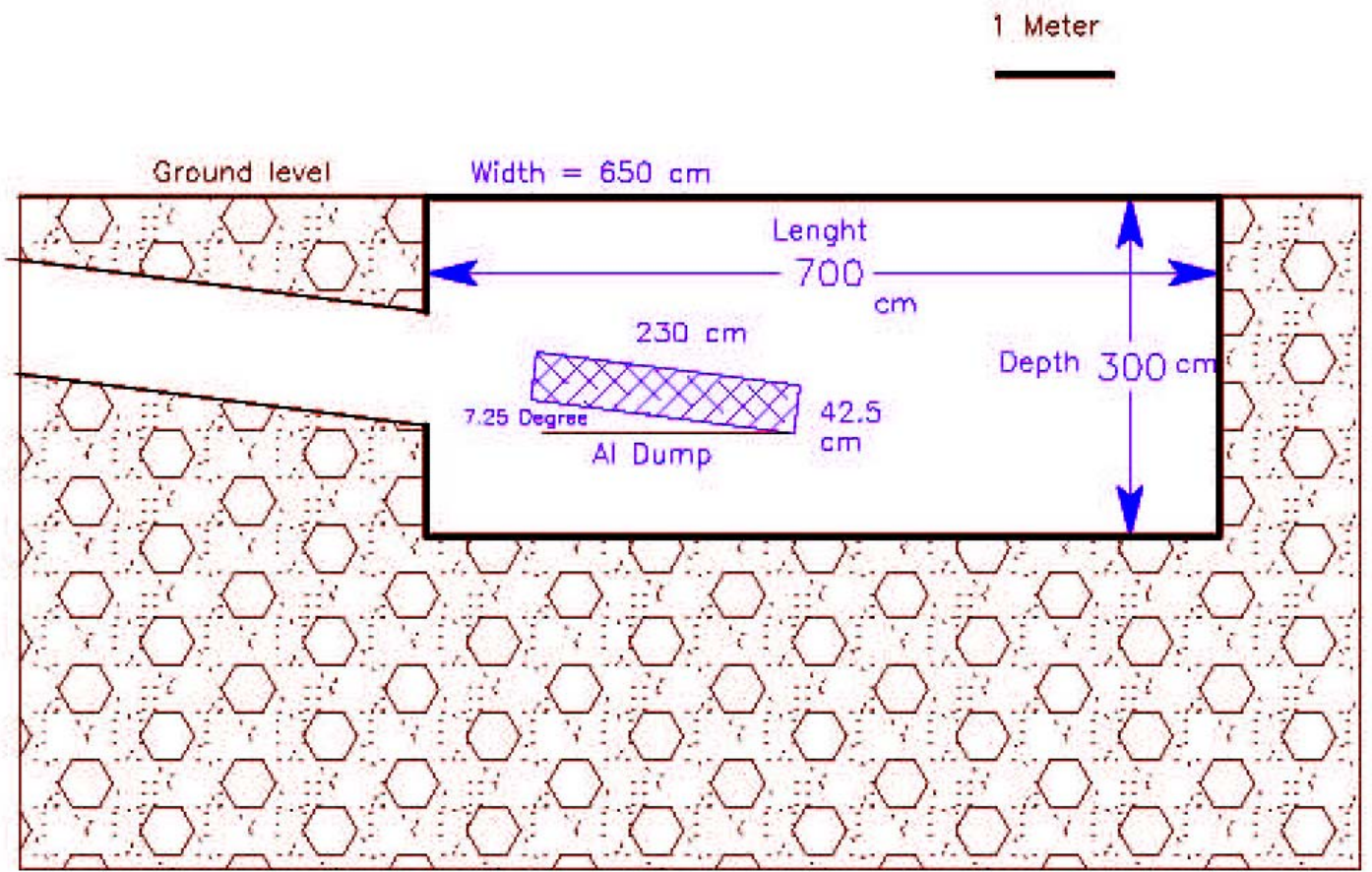

Figure 14.4 Electron Beam Dump

\subsubsection{Gas Bremsstrahlung}

Interaction of the electron beam with residual low-pressure gas molecules in the vacuum pipe will give rise to forward-directed gas bremsstrahlung. This type of radiation has been thoroughly investigated at circular storage rings, where the beam current is much more intense. However, at LCLS the straight length over which bremsstrahlung is produced will be much longer $(120 \mathrm{~m}$ between the dog-leg and the first bending magnet before the electron dump). The residual gas pressure and the electron energy will also be higher.

Radiation levels from the interaction of gas-bremsstrahlung photons generated in the LCLS undulator with a tungsten stopper were calculated using the FLUKA code and compared with results from two analytical methods [7]. The total dose rate at a distance of 1 meter from the stopper was estimated at $6.3 \mu \mathrm{rem} \mathrm{h}{ }^{-1}$, dominated by secondary photons from the stopper.

\subsubsection{Muons}

Muons produced by electron interactions in the Beam Switchyard and upstream of it are ranged by 55 feet of iron and cannot constitute a concern. Muons can be created in the diagnostic area (by losses upstream of and inside the dog-leg, in the collimators and in the profile monitors); there are other possible muon sources inside the undulator (x-ray intensity monitors) and the electron dump. These muons will be either bent away by magnets downstream of the undulator or shielded by iron shielding located on the top of the electron dump. 
However, persons accessing the on-line hutches and the research yard downstream of the Near-Field Hall and possibly the Far-Field Hall could be exposed to several other muon sources, which are produced when high-energy bremsstrahlung hits the photon stoppers. Muons can also constitute an important radiation background for experiments. The radiation levels for this source have been calculated using the codes MUCARLO [8] and MUON89 [9,10]. The expected dose rates in the vicinity of experimental hutch 1 are of the order of microrem per hour [11].

\subsubsection{Neutrons}

Photo-neutrons can be generated on the zero-degree line in any object hit by electrons and by bremsstrahlung. Such objects include the electron dump, the transport line to the dump, photon stoppers outside and inside the experimental Halls, and any optical device in the x-ray line. Neutrons generated outside the Near-Field Hall can penetrate to the Hall through the concrete shielding or streaming through the x-ray beam pipe. A preliminary analysis of the neutron radiation levels has been made using the analytical code SHIELD11 [12].

\subsection{Radiation Safety System}

The SLAC Radiation Safety Program is designed to ensure that radiation doses above background received by workers and the public shall be as low as reasonably achievable (ALARA), as well as to prevent any person from receiving more radiation exposure than is permitted under federal government regulations. The main provisions of the ALARA program ensure that access to high radiation areas is controlled; the accelerator facilities and the associated detectors are provided with adequately shielded enclosures for times when the possibility exists for a radiation field to be present; and designs for new facilities and significant modifications incorporate dose reduction, contamination reduction, and waste minimization features in the earliest planning stages.

Several technical, operations, and administrative systems exist to implement the program, as described in the SLAC Radiological Control Manual [2] and the SLAC Guidelines for Operations [13] and Radiation Safety Systems, Technical Basis Document [1].

Almost all the users of the LCLS working in the experimental halls are expected to be classified as non-radiological workers, General Employees or Visitors.

The SLAC Radiological Control Manual [1] (ES\&H, 1998) specifies an annual total effective dose equivalent limit to workers from both internal and external radiation sources of 5 rem. In addition, SLAC maintains an administrative control level of 1.5 rem.

The following radiation dose criteria are used in design of the LCLS radiation safety systems. The integrated dose equivalent outside the surface of the FFTB shielding barriers must not exceed $1 \mathrm{rem}$ in a year for normal beam operation [1].

1. The integrated dose equivalent to personnel working inside and around the experimental hutch shielding barriers must not exceed $0.1 \mathrm{rem}$ in a year for normal beam operation [2]. 
2. The dose equivalent-rate in the event of the Maximum Credible Incident is limited to less than $25 \mathrm{rem} / \mathrm{h}$, and integrated dose equivalent of less than $3 \mathrm{rem}$ [1].

3. The maximum dose equivalent rates in accessible areas at 1 foot from the shielding or barrier should not exceed $400 \mathrm{mrem} / \mathrm{h}$ for mis-steering conditions defined as conditions that are comprised of infrequent or short-duration situations in which the maximum allowable beam power, limited by Beam Containment System (BCS) devices is lost locally or in a limited area.

4. The dose equivalent for the maximally exposed member of the public exposed to ionizing radiation from SLAC produced pathways must be less than or equal to $10 \mathrm{mrem} / \mathrm{yr}$ [3]. The dose equivalent at the site boundary from the operation of the LCLS must be a small fraction of that total for normal beam operation.

In addition to shielding (bulk and local), the LCLS radiation protection systems will have Beam Containment System (BCS) and Personnel Protection System (PPS) in the Tunnel, and the Hutch Protection System (HPS) in the beam lines to achieve the designed goals.

The BCS is designed to ensure that beam parameters do not exceed the preset values, and that the beam is delivered to the main dump with minimal loss. The PPS controls entry to the tunnel, ensuring that personnel are excluded from the tunnel during the FFTB beam operation and the HPS control access to the experimental hutches.

The components that have been designed for the LCLS are: lateral shielding walls of the optical front end (Figure 14.5), front back and lateral shielding walls for the beam main dump (Figure 14.6), the experimental hutches shielding and HPS (Figure 14.7), front end beam stoppers (Figure 14.5), the stoppers between hutches (Figure 14.7).

Additionally, the injector vault shielding has been designed assuming losses in sector 20 of the linac. 


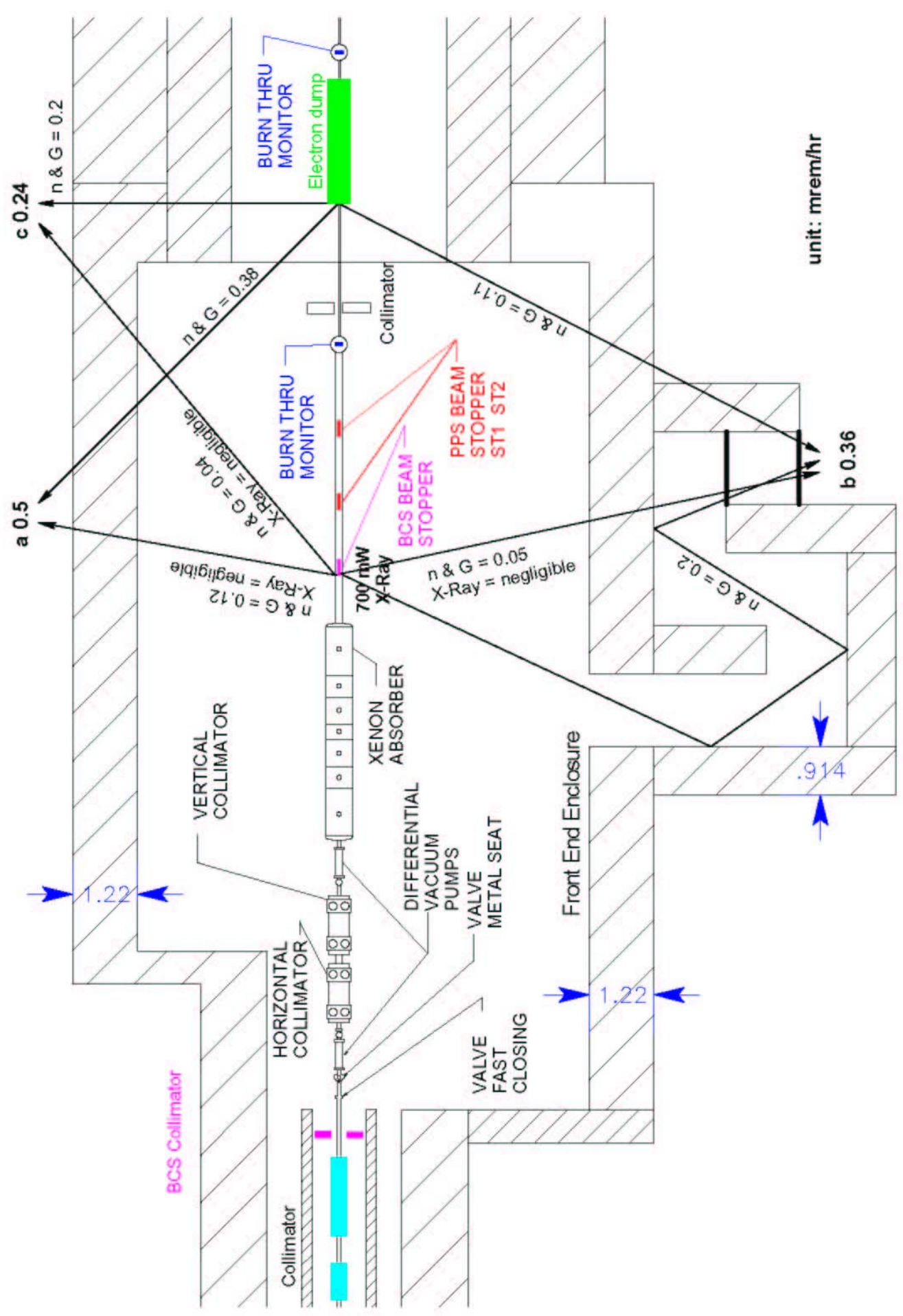

Figure 14.5 Shielding and Radiation Safety System Components for the Front End 




Figure 14.6 Shielding for the Electron Beam Dump 


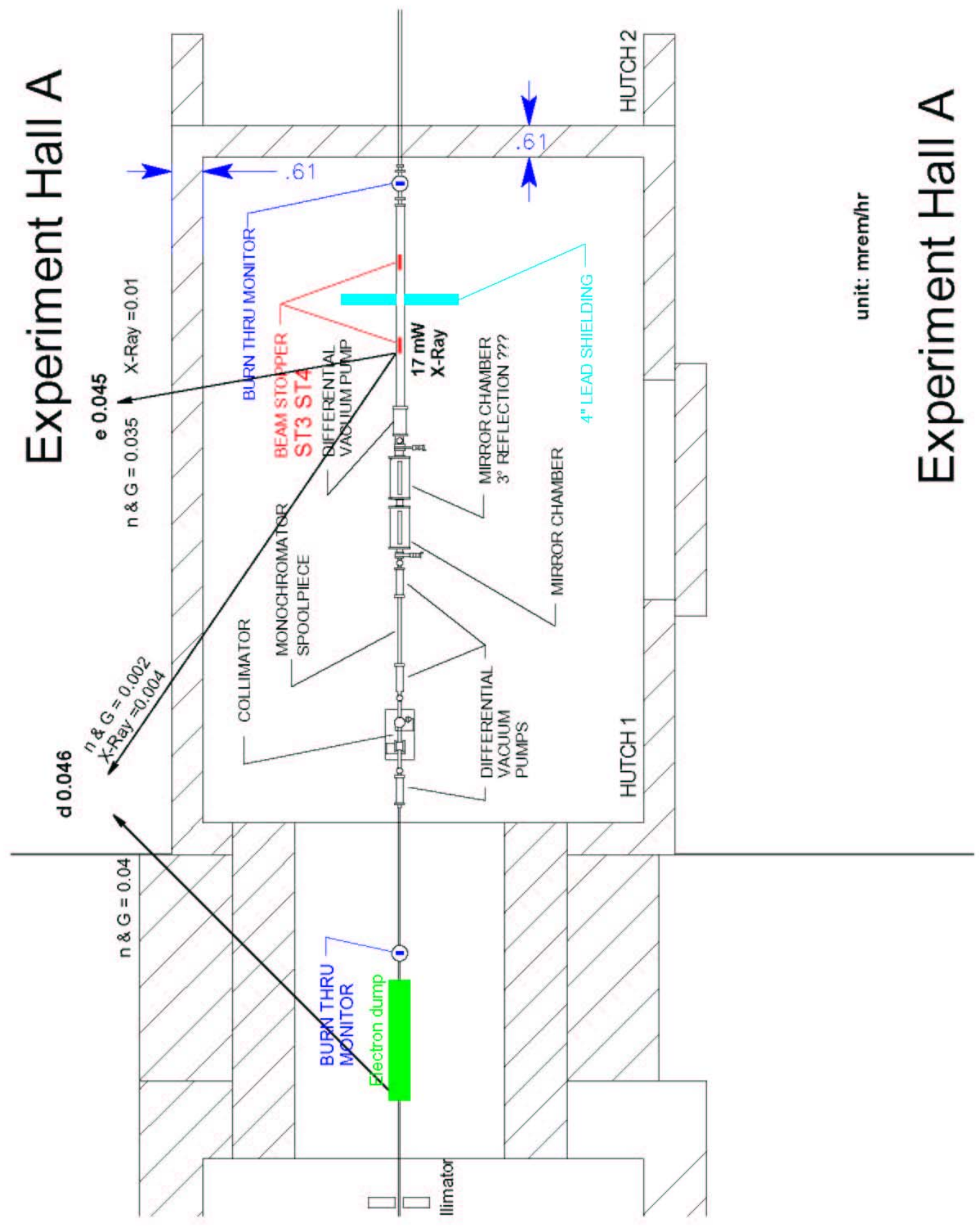

Figure 14.7 Shielding and Radiationo Safety Components for the Experimental Hutches, Hall A

\subsubsection{Minimum Shielding Requirements}

Based on the calculations that define the radiation sources the following shielding requirements have been specified: 


\section{Front End Shield:}

Lateral walls: $4.1 \mathrm{ft}$. iron plus $4.0 \mathrm{ft}$. concrete.

Roof: $3.0 \mathrm{ft}$. iron plus $5.5 \mathrm{ft}$. concrete.

Dump Shield:

Lateral walls: $4.1 \mathrm{ft}$ iron plus $4.0 \mathrm{ft}$. concrete.

Front (exit) wall: $6.6 \mathrm{ft}$. iron plus $4.0 \mathrm{ft}$ concrete.

Back (entrance) wall: $1.6 \mathrm{ft}$. iron.

Roof: $3.0 \mathrm{ft}$ iron (minimum) plus $5.5 \mathrm{ft}$. concrete.

Experimental Hutches Shield:

Lateral walls: $2.0 \mathrm{ft}$ concrete.

Front and back walls: $2.0 \mathrm{ft}$ concrete.

Roof: $2.0 \mathrm{ft}$ concrete.

Local: 4 in. lead to shield down beam of the first stopper.

\subsubsection{Beam Containment System}

SLAC's beam containment policy requires that beam lines be designed to contain the beam, limit the incoming beam power to the beam line, and limit the beam losses to prevent excessive radiation in occupied areas [1]. The containment of the beam in its channel is achieved by implementing a system of redundant, tamper-proof, and fail-safe electronic and mechanical devices that are enforced by strict operational requirements. The BCS for the LCLS will use most, if not all, of the FFTB BCS, which is comprised of devices that limit the incoming average beam power to less than the allowed beam power (torroids of current monitors 14 and 15); devices that limit normal beam loss to $1 \mathrm{~W}$ (torroids 16 and 17, long ion chambers); protection collimators that ensure that errant beams do not escape containment; and devices that protect collimators, stoppers and dumps (ion chambers and flow switches). The permanent dipole magnets in the beam line that assure that the electron beam reaches the main dump are the final component of the BCS.

For the LCLS, the following BCS devices will be added to the existing FFTB BCS down beam of the undulator and in the front end enclosure (Fig. 14.3).

A collimator after the undulator with two ion chambers and one BTM.

A collimator between the third magnet and the fourth magnet of the electron beam dump with two ion chambers and one BTM.

A collimator after the last dump magnet with two ion chambers and one BTM.

A beam stopper upstream of PPS stopper ST 1, which is interlocked with the x-ray intensity monitor. 
One BTM after PPS stoppers ST 1 and ST 2.

One BTM after the electron dump.

One BTM after PPS stoppers ST 3 and ST 4.

\subsubsection{Personnel Protection System (PPS) and Hutch Protection System (HPS)}

The PPS and HPS are designed to prevent access to experimental areas when beams are present and to prevent beams from entering an area during personnel access. Thus, the PPS and HPS function as access control systems and are based on standard designs at SLAC.

The PPS is composed of beam stoppers, entry module, and emergency shutoff buttons. Entry to the tunnel requires that all three PPS stoppers (D2, ST60 and ST61) be in the IN state. The main entrance to the FFTB tunnel is through a maze in the research yard. It is equipped with the standard access module of an outer door, an inner door, a key bank, an access enunciator panel, door control boxes, search reset boxes, a telephone, and a TV camera. The outer door has an electromagnetic lock and two door-position sensing switches that are used to monitor the status of this door and to activate a relay that permits or prevents a beam. The inner door provides redundancy and has two position sensing switches as well. A similar maze will be added at the entrance to the front end.

The experimental hall shielding, which prevents access to beam areas, will consist of fixed and moveable parts. The experimental hall perimeter walls and central beamline walls are planned to be fixed shielding consisting of appropriate material for the energy spectra of expected radiation. The experimenter hutches may have movable walls to adjust for experimental requirements. The moveable wall configuration will activate the current radiological configuration control system when changing the hutch shielding [13]. The experimental walls will have the capability of adjusting to the different angles of any hutch branch lines. The access control system (PPS and HPS) will be capable of retaining integrity and reliability, while compensating for wall placement.

The HPS will control access to the experimental hutches and will be modeled after existing SSRL HPS. The key parts of the HPS are a keyed access door, photon stopper interlocks, and area security system. The HPS allows either permission for personnel access or for beam to enter the hutch. It contains the logic interlock circuits that govern the sequence of access operations centered on the status of the stoppers. It also captures or releases the hutch door keys, acknowledges completion of a personnel security search, and keys the experiment enclosure online or off-line. Access to the hutch is permitted only if all photon stoppers are closed.

For access permission to any experimental hutch, the LCLS HPS will control the operation of photon stoppers in other areas or hutches that are required to be in. Two ion chambers and a burnthrough monitor are required to protect each stopper. 


\subsubsection{Stoppers}

Two up beam PPS beam stoppers will be required to allow entry into an experimental hutch to make changes that require disruption of the x-ray beam line while the $\mathrm{e}^{-}$beam is being delivered to the undulator and deflected into the dump. The function of these stoppers is to block and absorb any coherent or incoherent $\gamma$ or X-radiation from the undulator, as well as bremsstrahlung from anywhere in the beam transport system. These stoppers are patterned after an SLC design used in Sector 10 of the SLAC linac and in the PEP-II extraction lines [14]. The design energy is $12-15 \mathrm{GeV}$ and the assumed power for continuous exposure is $\mathrm{P}_{\mathrm{av}} \sim 5 \mathrm{~kW}$. The absorbing element in each stopper provides $30 \mathrm{~cm}$ copper, or the equivalent in radiation length of other material. The stoppers will be designed to meet the safety criteria.

\subsubsection{Burn-Through Monitors}

A built-in burn-through monitor is located at the depth of shower maximum in each stopper. It consists of a pair of cavities separated by a $\mathrm{Cu}$ diaphragm. The first cavity is pressurized with dry $\mathrm{N}_{2}$. Its return line contains a pressure switch with the trip level set to $15 \mathrm{psig}$. Should excessive beam power be deposited in the stopper block, the diaphragm will perforate, allowing the $\mathrm{N}_{2}$ to escape into the second cavity, which is open to atmospheric conditions on the outside. The pressure switch will interrupt beam delivery within 2-3 linac pulses.

\subsection{Induced Activity}

Personnel exposure from radioactive components in the beam line is of concern mainly around beam dumps, targets, or collimators where the entire beam or a large fraction of the beam is dissipated continuously.

Another source of potential exposure is to personnel working on the undulator after it has been in service for a period of time. Calculations based on methods developed by [15] and on [16] Swanson's (1979) tabulations express the rate of radionuclide production in terms of saturation activity $A_{s}$, i.e., the activity, at the instant that the irradiation has stopped, of a target that has been steadily irradiated for a time long compared with the half-life of the produced radionuclides. For these calculations, it was assumed that the permanent magnets are made of natural iron and natural cobalt, $50 \%$ each. To calculate the exposure rate, $A_{s}$ is multiplied by $\gamma$, the specific gamma ray constant which gives the exposure rate in air at a fixed distance $(1 \mathrm{~m})$ per unit of activity (Ci).

Natural iron is comprised of ${ }^{54} \mathrm{Fe},{ }^{56} \mathrm{Fe},{ }^{57} \mathrm{Fe},{ }^{58} \mathrm{Fe}$ isotopes. Reactions $(\gamma, \mathrm{n})(\gamma, 2 \mathrm{n})(\gamma, \mathrm{np})(\gamma, \mathrm{p})$ $(\gamma$,spallation) were considered. The product radionuclides that contribute the largest fraction of the dose are $\mathrm{Mn}$ isotopes. Natural cobalt is $100 \%{ }^{59} \mathrm{Co}$, and the reactions $(\gamma, \mathrm{n}){ }^{58} \mathrm{Co},(\gamma, 2 \mathrm{n}){ }^{57} \mathrm{Co}$ were considered. Reactions $(\gamma, \mathrm{p}),(\gamma, \mathrm{pn}),(\gamma, \mathrm{p} 2 \mathrm{n})(\gamma, \mathrm{p} 4 \mathrm{n})$ all lead to stable iron isotopes, and $(\gamma, \mathrm{p} 3 \mathrm{n})$ leads to Fe with a $5.9 \mathrm{keV}$ x-ray which would be self shielded in the target.

The total exposure rate from an activated magnet immediately after shut-down is conservatively estimated to be $5 \mathrm{mrad} \mathrm{hr}^{-1} \mathrm{~W}^{-1}$ at $1 \mathrm{~m}$. The exposure is dominated by a $0.8 \mathrm{MeV}$ 
gamma from ${ }^{58} \mathrm{Co}$ with a half-life of 71 days. With the expected low level of beam losses in the undulator, the activation of the unit and resulting personnel exposure are expected to be very low.

\subsection{References}

1 ES\&H Division. Radiation Safety Systems, Technical Basis Document. SLAC-I-720-0A05Z-002. Stanford Linear Accelerator Center, Menlo Park, CA. 1998.

(http://www.slac.stanford.edu/esh/techbas/rss/rss.pdf)

2 ES\&H Division. Radiological Control Manual, Revision 2. SLAC-I-720-0A05Z-001-R002. Stanford Linear Accelerator Center, Menlo Park, CA. 1999.

(http://www.slac.stanford.edu/esh/manuals/RCM.pdf)

3 SLAC/DOE. Contract between U.S. Department of Energy and Board of Trustees for the Leland Stanford, Jr., University Regarding the Management and Operation of the Stanford Linear Accelerator Center, Appendix B. Contract DE-AC03-76-SF00515. Stanford Linear Accelerator Center, Menlo Park, CA. 1998.

(http://www.slac.stanford.edu/grp/bsd/contract/index.htm)

4 S.H. Rokni, E.C. Benson, D.L. Burke, T.M. Jenkins, J.C. Liu, G. Nelson, W.R. Nelson, H.E. Smith, P. Tenenbaum, V. Vylet, D.R. Walz. Radiation protection systems for the final focus test beam at SLAC. Health Phys. 71:786-794; 1996

5 W.R. Nelson and X. Mao. Calculation of bremsstrahlung from the upstream collimator and the intensity monitor for the LCLS undulator. SLAC Radiation Physics Note, RP-02-04, Stanford Linear Accelerator Center, Menlo Park, CA. 2002.

(http://www.slac.stanford.edu/esh/rp/docs/RPNotes/slaconly/)

6 X.S. Mao and A.A. Prinz. Radiation shielding design for the LCLS front end, e- dump and hutch 1. SLAC Radiation Physics Note, RP-02-06, Stanford Linear Accelerator Center, Menlo Park, CA. 2002.

(http://www.slac.stanford.edu/esh/rp/docs/RPNotes/slaconly/)

7 S.H. Rokni, J.C. Liu, S. Mao. Calculation of gas bremsstrahlung for the LCLS undulator. SLAC Radiation Physics Note, RP-02-03, Stanford Linear Accelerator Center, Menlo Park, CA. 2002.

(http://www.slac.stanford.edu/esh/rp/docs/RPNotes/slaconly/)

8 L. Keller, S. Rokni, W.R. Nelson. MuCarlo is a Monte Carlo code written by G. Feldman (Harvard University) and maintained at SLAC.

9 W.R. Nelson, K.R. Kase. Muon Shielding Around High Energy Electron Accelerators, Part I: Theory. Nucl. Instr. Meth. 120:401-412; 1974.

10 W.R. Nelson, K.R. Kase, G.K. Svensson. Muon Shielding Around High Energy Electron Accelerators, Part II: Experimental Investigation. Nucl. Instr. Meth. 120: 413- ; 1974.

11 W.R. Nelson, X. Mao, A.A. Prinz. Muon calculations for the LCLS undulator. SLAC Radiation Physics Note, RP-02-05, Stanford Linear Accelerator Center, Menlo Park, CA. 2002.

(http://www.slac.stanford.edu/esh/rp/docs/RPNotes/slaconly/)

12 SLAC. SHIELD11 - an analytical shielding code maintained at SLAC. Current version, 24 April 2000a.

13 SLAC. Guidelines for Operations. Stanford Linear Accelerator Center, Menlo Park, CA. 2000b.

(http://www.slac.stanford.edu/pubs/gfo/gfoindex.html) 
14 D. Walz. Beam stopper for PEP-II injection. SLAC memorandum. 28 September 1994.

15 H. DeStaebler, Photon-Induced Residual Activity. SLAC-TN-6392 Stanford Linear Accelerator Center, Menlo Park, CA. (1963).

16 W.P. Swanson. Radiological Safety Aspects of the Operation of Electron Linear Accelerators, IAEA Technical Report Series No. 188. International Atomic Energy Agency, Vienna (1979). 


\section{Work Breakdown}

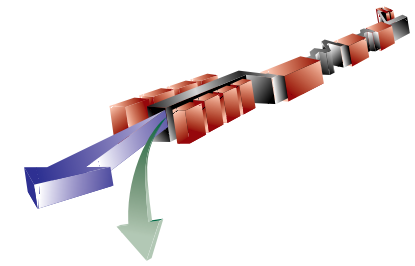

\section{TECHNICAL SYNOPSIS}

The Work Breakdown Structure (WBS) is used for defining work packages and developing and tracking the cost and schedule for the project. The work is broken down into tasks, each of which has a manager, a responsible institution, costs and schedule, technical scope, and, to the extent possible, a specific geographic piece of the machine.

Each level 3 element has a Task Manager who is responsible for the execution of the project plans for that element. The Task Manager is responsible for translating system performance requirements into design choices for the LCLS technical systems. He/she is also responsible for control of cost and schedule, quality and safety, and documentation. Performance requirements for systems at level 3 and below will be established and advocated by a System Manager. The System Manager advises the Task Manager and LCLS Project Management as to whether the LCLS systems will meet specifications necessary for the success of the Project. The Systems Manager is primarily an advocate of the performance of each system, with no responsibility for cost and schedule. Line responsibility for design choices and execution flows through LCLS Management and the Task Managers.

The responsibility for each level 3 element lies with one of the collaborating institutions. Other institutions may be involved in the execution but a lead institution is defined for each element. Also, to the extent possible, each level 3 element covers a specific piece of geography, which minimizes the complexity of the interface between elements.

The WBS is used to specify change control. The Project Management Plan specifies the levels of approval required for changes in cost and/or schedule at each level of the WBS.

The WBS is used for cost reporting. The project will report costs and progress to the DOE monthly at level 2 of the WBS. The project management will review costs and progress monthly at level 3. The System Managers will review costs and progress monthly at the lower levels of the WBS.

\subsection{Level 3 Work Breakdown Structure}

The following describes the scope of work for each of the level 3 elements of the WBS. Each element includes design, simulations, documentation, fabrication, testing, installation, and commissioning of the equipment. 


\section{LCLS Construction Project (TEC)}

1.1. Project Planning, Management and Administration. This element includes the general management of the project, establishment and operation of the cost and schedule tracking systems, generation of financial and technical reports, organizing technical and project reviews, and the accelerator and FEL physics activities not covered by the individual systems. Costs related to management of project-wide ES\&H issues will be captured in this WBS element.

1.1.1 ES\&H. This element will capture effort and other costs associated with management of safety issues in the design and construction phases of the Project.

1.1.2 Project Supervision and Coordination. This element will include the costs of oversight such as Project Director Reviews and other reviews. It will provide budget for Project Earned Value System.

1.2. Electron Beam Handling Systems. This element includes the technical equipment required to generate the electron beam, to accelerate and transport the electron beam, through the undulator, and to dump the electron beam.

1.2.1 Injector. The injector generates the electron beam and accelerates it to $150 \mathrm{MeV}$. This element includes the laser, optical transport, the electron gun, the accelerator sections, the solenoids and other magnets, the diagnostics including a diagnostic section at the end of the injector, the LCLS timing system, and the laser room. The interface to the Linac is at the downstream end of Dog Leg 1 (DL1).

1.2.2 Accelerator. The linac accelerates the electron beam while preserving the transverse emittance and compressing the longitudinal size. This element includes modifications to the last third of the existing SLAC linac, Bunch Compressor 1 (BC1), Bunch Compressor 2 (BC2), beam transport to the undulator, beam transport after the undulator, bend magnets and beam dump, the bypass system for transporting test beams to end station A, and diagnostics including characterizing both the electron and x-ray beams as they pass through the undulator.

The interface with the undulator is a vacuum flange at each end of the undulator. This element includes the common beam line beyond the undulator for the electrons and x-rays until the electrons are deflected enough 
for an interface to the x-ray beam line.

1.2.3 Undulator. The undulator system provides the magnetic environment for interaction between the electron beam and an exponentially growing, coherent x-ray beam. This element includes undulator magnet, vacuum system, supports, beam diagnostics for the electron and x-ray beams in the undulator, ancillary magnets, magnetic measurements and fiducializing the undulator. Computer controls for hardware in the undulator path will be included in this element.

1.2.4 Installation and Alignment. This element is responsible for installation of the technical components and their alignment. This element includes the removal of existing components from the FFTB, installation of the undulator and dump magnets in the FFTB, the installation of new linac components, the installation of the injector components (not including the FEL $\mathrm{x}$-ray optics).

1.3. Photon Beam Handling and Systems. This element includes all equipment required for manipulation and characterization of the x-ray beam downstream of the undulator. "Manipulation" includes collimation, attenuation, focusing, splitting/delaying, slicing/compressing, and monochromatizing. "Characterization" includes measurement of $\mathrm{x}$-ray beam properties as necessary for commissioning and operation of the LCLS.

1.3.1 X-Ray Transport and Diagnostics. This element includes mechanical and vacuum systems for the $\mathrm{x}$-ray beam path, shutters, attenuators, $\mathrm{x}$-ray optics and x-ray diagnostics.

1.3.2 X-Ray Endstation Systems. This element includes the systems necessary to verify the performance of the x-ray optics and diagnostics and their suitability for LCLS scientific research. It will include laser systems to which the LCLS x-ray pulse must be synchronized in order to carry out many of the experiments envisioned for this facility. It will also include computer systems and detectors necessary to collect and analyze data necessary to verify the performance of the LCLS.

1.3.3 Installation and Alignment of Photon Beam Handling Systems. 
1.4. Conventional Construction. This element provides the buildings and utilities to house and support the technical components from the Injector to the Endstation Systems.

1.4.1 Injector Facilities. The LCLS uses an existing tunnel at Sector 20 for the injector and an existing surface building for the gun laser. This element involves modifications to those facilities to accommodate the LCLS injector requirements including: clean room for the laser, personnel exclusion barriers for the PPS, and water and power distribution.

1.4.2 Linac Facilities. The linac housing will not be modified. This element includes modification to the existing utilities to provide power and cooling for the two chicanes and the new $\mathrm{X}$ band system.

1.4.3 Undulator Facilities. The existing Final Focus Test Beam tunnel will be modified to accommodate LCLS. This includes extending the tunnel approximately 64 meters to the east to meet the near hall, precision temperature control of the tunnel, relocation of the personnel access, and reconfiguring the utilities to match the new arrangement of components in the tunnel.

1.4.4 Near Hall. The LCLS Near Hall is a new building that will be constructed in the Research Yard. This element includes construction of this building and installing the utilities required by the x-ray diagnostics and the LCLS experiments.

1.4.5 Tunnel and Far Hall. The LCLS Far Hall will be constructed east of the Ring Road. The floor will be approximately 10 meters below grade to match the elevation of the x-ray beam. A laboratory and office complex will be included at grade, on top of the hall. This element includes installing the utilities required for the laboratories, offices, and the experiments. This hall is connected to the Near Hall by a tunnel through the existing hill between the halls. The construction of this tunnel is included in this element. The tunnel will be approximately $3 \mathrm{~m}$ by $3 \mathrm{~m}$ in cross section and will house an $\mathrm{x}$-ray beam line (not included here) and cable trays for utilities and control cables. 


\section{Parameter Tables}



\section{A.1 FEL-Physics}

\section{A.1.1 Performance}

\section{A.1.1.1 Electron Beam}

\begin{tabular}{|c|c|c|c|c|}
\hline Parameter Name & Low Energy & High Energy & All Energies & Unit \\
\hline Electron energy & 4.54 & 14.35 & & $\mathrm{GeV}$ \\
\hline Electron Lorentz factor & 8880 & 28082 & & \\
\hline Normalized slice emittance & 1.2 & 1.2 & & $\mu \mathrm{m} \mathrm{rad}$ \\
\hline Charge at undulator entrance & 1 & 1 & & $\mathrm{nC}$ \\
\hline Peak current & 3400 & 3400 & & A \\
\hline Longitudinal pulse form & & & Flat-Top & \\
\hline Transverse pulse form & & & Gaussian & \\
\hline RMS bunch length & 23 & 23 & & $\mu \mathrm{m}$ \\
\hline RMS bunch duration & 77 & 77 & & fs \\
\hline FWHM bunch length & 69 & 69 & & $\mu \mathrm{m}$ \\
\hline FWHM bunch duration & 230 & 230 & & fs \\
\hline Slice rms gamma spread & & & 2.2 & \\
\hline Slice rms energy spread & 0.025 & 0.010 & & $\%$ \\
\hline Projected rms energy spread & 0.2 & 0.06 & & $\%$ \\
\hline
\end{tabular}

\section{A.1.1.2 Spontaneous Radiation}

\begin{tabular}{lcccl} 
Parameter Name & Low Energy & High Energy & All Energies & Unit \\
\hline Peak spontaneous power per pulse & 9.2 & 92 & $\mathrm{GW}$ \\
Average spontaneous power & 0.29 & 2.9 & $\mathrm{~W}$ \\
Energy loss from spont. radiation & 0.001 & 0.026 & $\mathrm{GeV}$ \\
Rel. energy loss from spont. radiation & 0.002 & 0.18 & $\%$ & $\%$ \\
Non-radiative rel. energy loss & 0.010 & 0.010 & & 0.23 \\
Max. overall field taper required & & & $\%$ \\
Delta E/E induced by spont. radiation & $<0.02$ & $<0.02$ & & $\%$ \\
Spont. fund. trans. beam size & 4.2 & 32 & & $\mu \mathrm{m}$ \\
Spont. fund. trans. beam divergence & 6.2 & 2.0 & & $\mu \mathrm{rad}$
\end{tabular}


L C L S C O N C E P T U A L D E S I G N R E P O R T

\section{A.1.1.3 FEL}

Parameter Name

Low Energy High Energy All Energies Unit

FEL parameter

Rayleigh length

14.5

5.6

5.0

Slippage length

5593

32

559

Cooperation length

165

Power gain length

1.3

2.7

Field gain length

Saturation length incl. breaks

27

Peak saturation power

19

Average saturation power

0.61

Fundamental radiation wavelength

15

Photon energy of fundamental

0.82

27.9

Number of coherent photons per pulse

145

Peak photon flux

0.64

0.2

Average brightness

0.24

0.47

Integrated photon energy spread

31

FEL rms fund. trans. beam size

FEL ms fund. trans. beam divergence

3.8

48

$10^{-4}$

$\mathrm{m}$

$\mathrm{nm}$

$\mathrm{nm}$

$\mathrm{m}$

$\mathrm{m}$

$\mathrm{m}$

GW

$\mathrm{W}$

$\AA$

$\mathrm{keV}$

$10^{12}$ Photons/pulse

$10^{24}$ Photons/s

$10^{32} *$

$10^{22 *}$

$\%$

$\%$

$\mu \mathrm{m}$

$\mu \mathrm{rad}$

* $\mathrm{Ph} . / \mathrm{s} / \mathrm{mm}^{2} / \mathrm{mr}^{2} / .1 \%$ bw 


\section{A.2 Photo-Injector}

\section{A.2.1 Gun-Laser}

\section{A.2.1.1 Subsystem}

\begin{tabular}{|c|c|c|}
\hline Parameter Name & High Energy & Unit \\
\hline Oscillator & \multicolumn{2}{|l|}{ CW mode-locked } \\
\hline Ti:sapphire & & \\
\hline Oscillator pump & \multicolumn{2}{|l|}{ Frequ.-doubled CW } \\
\hline Nd:YAG laser & & \\
\hline Amplifier & \multicolumn{2}{|l|}{ Ti:sapphire } \\
\hline Amplifier pump & \multicolumn{2}{|l|}{ Nd:YAG or YLF } \\
\hline Output wavelength & 780 & $\mathrm{~nm}$ \\
\hline Operating wavelength & 260 & $\mathrm{~nm}$ \\
\hline Pulse repetition rate & 120 & $\mathrm{~Hz}$ \\
\hline No. of micropulses & 1 & \\
\hline Maximum micropulse energy on cathode & $>500$ & $\mu \mathrm{J}$ \\
\hline HWHM micropulse radius on cathode & 0.9 & $\mathrm{~mm}$ \\
\hline Micropulse risetime & 1.0 & ps \\
\hline FWHM micropulse length & 10 & ps \\
\hline Longitudinal micropulse form & \multicolumn{2}{|l|}{ various } \\
\hline Longitudinal homogeneity on cathode & 10 & $\%$ \\
\hline Transverse micropulse form & \multicolumn{2}{|l|}{ uniform } \\
\hline Transverse homogeneity on cathode & 10 & $\%$ \\
\hline RMS pulse-to-pulse energy jitter & $<=1$ & $\%$ \\
\hline RMS pulse-to-pulse phase stability & $<=0.5$ & ps \\
\hline Spot. diameter jitter at cathode (pk-pk) & 1 & $\%$ \\
\hline Pointing stability (pk-pk of spot radius) & $<1$ & $\%$ \\
\hline
\end{tabular}




\section{A.2.2 Gun}

\section{A.2.2.1 Subsystem}

\begin{tabular}{|c|c|c|}
\hline Parameter Name & High Energy & Unit \\
\hline Cathode material & \multicolumn{2}{|c|}{ metal $(\mathrm{Cu}$ or possibly $\mathrm{Mg})$} \\
\hline Active diameter of cathode & 12 & $\mathrm{~mm}$ \\
\hline Cathode quantum efficiency at $266 \mathrm{~nm}$ & 1 & $10^{\wedge}(-5)$ \\
\hline Maximum extraction field & 120 & $\mathrm{MV} / \mathrm{m}$ \\
\hline Charge per bunch & 1.0 & $\mathrm{nC}$ \\
\hline Longitudinal pulse form of electron bunch & \multicolumn{2}{|l|}{ truncated gaussian } \\
\hline FWHM electron bunch length & 3.0 & $\mathrm{~mm}$ \\
\hline FWHM electrom bunch duration & 10 & ps \\
\hline RMS electron bunch length & 0.84 & $\mathrm{~mm}$ \\
\hline RMS electron bunch duration & 2.8 & ps \\
\hline Peak electron bunch current & 100 & A \\
\hline RF frequency & 2856 & $\mathrm{MHz}$ \\
\hline RF pulse duration & $3-4$ & $\mu \mathrm{s}$ \\
\hline Peak rf power & 15 & MW \\
\hline Number of cells & 1.6 & \\
\hline Electron beam energy at exit & 7.0 & $\mathrm{MeV}$ \\
\hline Projected correlated electron energy & 1.5 & $\%$ \\
\hline
\end{tabular}

\section{A.2.2.2 Solenoid}

\begin{tabular}{|c|c|c|c|}
\hline Parameter Name & Low Energy High Energy & All Energies & Unit \\
\hline Magnetic length & & - & $\mathrm{m}$ \\
\hline On-axis field & & - & $\mathrm{T}$ \\
\hline
\end{tabular}

\section{A.2.3 L-0}

\section{A.2.3.1 Subsystem}

\section{Parameter Name}

Total length

\section{Low Energy High Energy All Energies}

Unit $\mathrm{m}$ 
LCLS CONCEPTUAL DESIGN REPORT

\section{A.2.3.2 Electron Beam}

\begin{tabular}{|c|c|c|c|}
\hline Parameter Name & Low Energy High Energy & All Energies & Unit \\
\hline Initial electron energy & & 7 & $\mathrm{MeV}$ \\
\hline Initial rms bunch length & & 0.83 & $\mathrm{~mm}$ \\
\hline Initial charge & & 1 & $\mathrm{nC}$ \\
\hline Initial peak current & & 100 & A \\
\hline Final electron energy & & 150 & $\mathrm{MeV}$ \\
\hline Final normalized emittance & & 1 & $\mu \mathrm{m} \mathrm{rad}$ \\
\hline Final rms bunch length & & 0.84 & $\mathrm{~mm}$ \\
\hline Final charge & & 1 & $\mathrm{nC}$ \\
\hline Final peak current & & 100 & A \\
\hline
\end{tabular}

\section{A.2.3.3 Focusing}

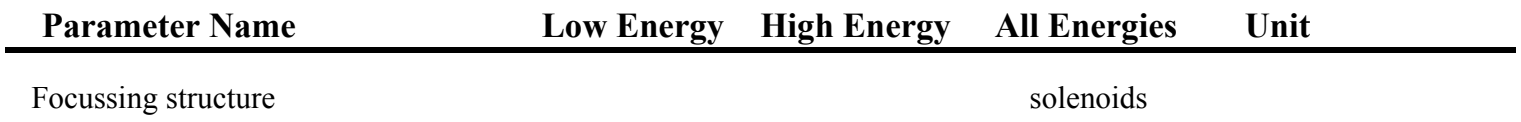

\section{A.2.3.4 RF}

Parameter Name

Number of linac sections

RF phase at exit

Mean rf phase jitter tolerance

Mean rf voltage jitter tolerance

RMS pulse to pulse energy variation
Low Energy High Energy All Energies Unit

2

$0 \quad$ degrees

$0.1 \%$

$0.1 \%$

$0.1 \quad \%$

\section{A.2.4 DL-1}

\section{A.2.4.1 Subsystem}

\begin{tabular}{|c|c|c|c|}
\hline Parameter Name & Low Energy High Energy & All Energies & Unit \\
\hline $\mathrm{R} 56$ & & +6.3 & $\mathrm{~mm}$ \\
\hline Total bend angle & & 35 & degrees \\
\hline Total length & & 12 & $\mathrm{~m}$ \\
\hline
\end{tabular}




\section{A.2.4.2 Electron Beam}

\begin{tabular}{|c|c|c|c|}
\hline Parameter Name & Low Energy High Energy & All Energies & Unit \\
\hline Initial electron energy & & 150 & $\mathrm{MeV}$ \\
\hline Initial normalized projected emittance & & 1 & $\mu \mathrm{m} \mathrm{rad}$ \\
\hline Initital total energy spread & & 0.09 & $\%$ \\
\hline Initial slice energy spread & & $\sim 0.003$ & $\%$ \\
\hline Initial rms bunch length & & 0.83 & $\mathrm{~mm}$ \\
\hline Initial peak current & & 100 & A \\
\hline Initial longitudinal slice brightness & & 734 & A \\
\hline Final electron energy & & 150 & $\mathrm{MeV}$ \\
\hline Final normalized projected emittance & & 1.02 & $\mu \mathrm{m} \mathrm{rad}$ \\
\hline Final total energy spread & & 0.09 & $\%$ \\
\hline Final slice energy spread & & $\sim 0.002$ & $\%$ \\
\hline Final rms bunch length & & 0.83 & $\mathrm{~mm}$ \\
\hline Final peak current & & 100 & A \\
\hline Final longitudinal slice brightness & & 734 & A \\
\hline
\end{tabular}

\section{A.2.4.3 Focusing}

Parameter Name

Low Energy High Energy All Energies Unit

Focussing components

quadrupole magnets

\section{A.2.4.4 Quadrupole Magnets}

Parameter Name

Number of quadrupoles

Max. focusing gradient

Magnetic length
Low Energy High Energy All Energies Unit

9

$12 \quad \mathrm{~T} / \mathrm{m}$

0.15

$\mathrm{m}$

\section{A.2.4.5 Dipole Magnets}

\begin{tabular}{|c|c|c|c|}
\hline Parameter Name & Low Energy High Energy & All Energies & Unit \\
\hline Number of dipoles & & 2 & \\
\hline Max. deflection angle & & 17.5 & degrees \\
\hline Magnetic length & & 0.2 & $\mathrm{~m}$ \\
\hline
\end{tabular}

\section{A.2.4.6 Diagnostics}

Parameter Name

Number of profile monitors

Number of x,y BPM pairs
Low Energy High Energy All Energies Unit 
LCLS CONCEPTUAL DESIGN REPORT

\section{A.2.4.7 Vacuum}

\section{Parameter Name}

Low Energy High Energy All Energies

Unit

Horizontal vacuum chamber ID

Vertical vacuum chamber ID

$\begin{array}{ll}24 & \mathrm{~mm} \\ 24 & \mathrm{~mm}\end{array}$

P A R A METER TABLES A-7 


\section{A.3 LINAC}

\section{A.3.1 General}

\section{A.3.1.1 S-Band Accelerator Section}

\begin{tabular}{|c|c|c|c|}
\hline Parameter Name & Low Energy High Energy & All Energies & Unit \\
\hline S-band rf frequency & & 2856 & $\mathrm{MHz}$ \\
\hline S-band klystron type & & 5045 & \\
\hline S-band acceleration gradient & & 19 & $\mathrm{MV} / \mathrm{m}$ \\
\hline S-band Q & & $13000-14000$ & \\
\hline S-band shunt impedance & & $53-60$ & $\mathrm{MOhm} / \mathrm{m}$ \\
\hline S-band mean iris diameter & & 23 & $\mathrm{~mm}$ \\
\hline Peak S-band rf power & & 60 & MW \\
\hline Mean S-band f power & & 45 & $\mathrm{~kW}$ \\
\hline Length of S-band rf section (s) & & 3 & $\mathrm{~m}$ \\
\hline
\end{tabular}

\section{A.3.1.2 X-Band Accelerator Section}

\begin{tabular}{|c|c|c|c|}
\hline Parameter Name & Low Energy High Energy & All Energies & Unit \\
\hline $\mathrm{X}$-band f frequency & & 11424 & $\mathrm{MHz}$ \\
\hline $\mathrm{X}$-band mean iris diameter & & 9.4 & $\mathrm{~mm}$ \\
\hline Length of X-band rf section (s) & & 0.6 & $\mathrm{~m}$ \\
\hline
\end{tabular}

\section{A.3.2 L-1}

\section{A.3.2.1 Subsystem}

\begin{tabular}{lcccc} 
Parameter Name & Low Energy & High Energy & All Energies & Unit \\
\hline Total length & 9.7 & $\mathrm{~m}$
\end{tabular}




\section{A.3.2.2 Electron Beam}

\begin{tabular}{|c|c|c|c|}
\hline Parameter Name & Low Energy High Energy & All Energies & Unit \\
\hline Initial electron energy & & 150 & $\mathrm{MeV}$ \\
\hline Initial normalized projected emittance & & 1.02 & $\mu \mathrm{m} \mathrm{rad}$ \\
\hline Initital total energy spread & & 0.09 & $\%$ \\
\hline Initial slice energy spread & & 0.002 & $\%$ \\
\hline Initial rms bunch length & & 0.83 & $\mathrm{~mm}$ \\
\hline Initial peak current & & 100 & A \\
\hline Initial longitudinal slice brightness & & 734 & A \\
\hline Final electron energy & & 270 & $\mathrm{MeV}$ \\
\hline Final normalized projected emittance & & 1.05 & $\mu \mathrm{m} \mathrm{rad}$ \\
\hline Final total energy spread & & 1.68 & $\%$ \\
\hline Final slice energy spread & & 0.001 & $\%$ \\
\hline Final rms bunch length & & 0.83 & $\mathrm{~mm}$ \\
\hline Final peak current & & 100 & A \\
\hline Final longitudinal slice brightness & & 691 & A \\
\hline
\end{tabular}

\section{A.3.2.3 Focusing}

\begin{tabular}{|c|c|c|c|}
\hline Parameter Name & Low Energy High Energy & All Energies & Unit \\
\hline Phase advance per cell & & 75 & degrees \\
\hline Number of quadrupoles & & 3 & \\
\hline Quadrupole magnetic length & & 0.1 & $\mathrm{~m}$ \\
\hline
\end{tabular}

\section{A.3.2.4 RF}

\begin{tabular}{|c|c|c|c|}
\hline Parameter Name & Low Energy High Energy & All Energies & Unit \\
\hline Number of linac sections & & 3 & \\
\hline RF-Compression phase & & -38 & degrees \\
\hline Mean rf phase jitter tolerance & & 0.1 & $\%$ \\
\hline Mean rf voltage jitter tolerance & & 0.1 & $\%$ \\
\hline RMS pulse to pulse energy variation & & 0.08 & $\%$ \\
\hline
\end{tabular}

\section{A.3.3 L-X}

\section{A.3.3.1 Subsystem}

\begin{tabular}{|c|c|c|c|c|}
\hline Parameter Name & Low Energy & High Energy & All Energies & Unit \\
\hline
\end{tabular}




\begin{tabular}{|c|c|c|c|c|}
\hline Parameter Name & Low Energy & High Energy & All Energies & Unit \\
\hline Initial electron energy & & & 270 & $\mathrm{MeV}$ \\
\hline Initial normalized projected emittance & & & 1.05 & $\mu \mathrm{m} \mathrm{rad}$ \\
\hline Initial total energy spread & & & 1.68 & $\%$ \\
\hline Initial slice energy spread & & & 0.001 & $\%$ \\
\hline Initial RMS bunch length & & & 0.83 & $\mathrm{~mm}$ \\
\hline Initial peak current & & & 100 & A \\
\hline Initial longitudinal slice brightness & & & 691 & A \\
\hline Final electron energy & & & 250 & $\mathrm{MeV}$ \\
\hline Final normalized projected emittance & & & 1.10 & $\mu \mathrm{m} \mathrm{rad}$ \\
\hline Final total energy spread & & & 1.79 & $\%$ \\
\hline Final slice energy spread & & & 0.001 & $\%$ \\
\hline Final RMS bunch length & & & 0.83 & $\mathrm{~mm}$ \\
\hline Final peak current & & & 100 & A \\
\hline Final longitudinal slice brightness & & & 789 & A \\
\hline
\end{tabular}

\section{A.3.3.3 RF}

\begin{tabular}{|c|c|c|c|}
\hline Parameter Name & Low Energy High Energy & All Energies & Unit \\
\hline Number of RF sections & & 1 & \\
\hline RF phase & & -180 & degrees \\
\hline RF phase tolerance & & 0.3 & $\mathrm{X}$-band degrees \\
\hline $\mathrm{RF}$ voltage tolerance & & 0.25 & $\%$ \\
\hline
\end{tabular}

\section{A.3.4 BC-1}

\section{A.3.4.1 Subsystem}

\begin{tabular}{|c|c|c|c|}
\hline Parameter Name & Low Energy High Energy & All Energies & Unit \\
\hline Compressor type & & chicane & \\
\hline Total length & & 6.56 & $\mathrm{~m}$ \\
\hline R56 & & -35.9 & $\mathrm{~mm}$ \\
\hline Transverse offset of chicane & & 229 & $\mathrm{~mm}$ \\
\hline
\end{tabular}




\section{A.3.4.2 Electron Beam}

\begin{tabular}{|c|c|c|c|}
\hline Parameter Name & Low Energy High Energy & All Energies & Unit \\
\hline Initial electron energy & & 250 & $\mathrm{MeV}$ \\
\hline Initial normalized projected emittance & & 1.10 & $\mu \mathrm{m} \mathrm{rad}$ \\
\hline Initital total energy spread & & 1.79 & $\%$ \\
\hline Initial slice energy spread & & 0.001 & $\%$ \\
\hline Initial rms bunch length & & 0.83 & $\mathrm{~mm}$ \\
\hline Initial peak current & & 100 & A \\
\hline Initial longitudinal slice brightness & & 789 & A \\
\hline Final electron energy & & 250 & $\mathrm{MeV}$ \\
\hline Final normalized projected emittance & & 1.20 & $\mu \mathrm{m} \mathrm{rad}$ \\
\hline Final total energy spread & & 1.77 & $\%$ \\
\hline Final slice energy spread & & 0.005 & $\%$ \\
\hline Final rms bunch length & & 0.195 & $\mathrm{~mm}$ \\
\hline Final peak current & & 500 & A \\
\hline Final longitudinal slice brightness & & 785 & A \\
\hline
\end{tabular}

\section{A.3.4.3 Dipole Magnet}

\begin{tabular}{|c|c|c|c|}
\hline Parameter Name & Low Energy High Energy & All Energies & Unit \\
\hline Total number of dipoles & & 4 & \\
\hline Bend angle of chicane dipoles & & 4.62 & degrees \\
\hline Magnetic length & & 0.2 & $\mathrm{~m}$ \\
\hline
\end{tabular}

\section{A.3.4.4 Diagnostics}

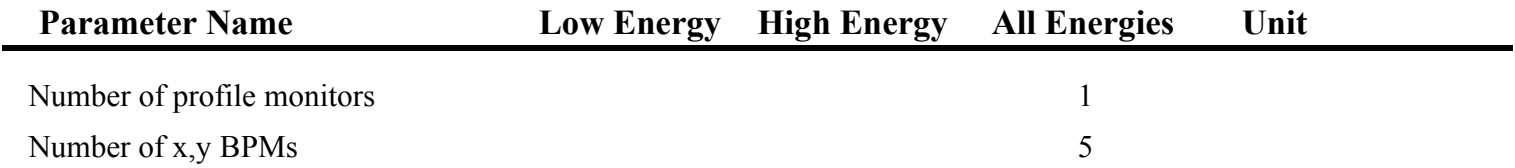

\section{A.3.5 L-2}

\section{A.3.5.1 Subsystem}

Parameter Name

Low Energy High Energy All Energies

Unit 


\section{A.3.5.2 Electron Beam}

\begin{tabular}{|c|c|c|c|}
\hline Parameter Name & Low Energy High Energy & All Energies & Unit \\
\hline Initial electron energy & & 250 & $\mathrm{MeV}$ \\
\hline Initial normalized projected emittance & & 1.20 & $\mu \mathrm{m} \mathrm{rad}$ \\
\hline Initital total energy spread & & 1.77 & $\%$ \\
\hline Initial slice energy spread & & 0.005 & $\%$ \\
\hline Initial rms bunch length & & 0.195 & $\mathrm{~mm}$ \\
\hline Initial peak current & & 500 & A \\
\hline Initial longitudinal slice brightness & & 785 & A \\
\hline Final electron energy & & 4.54 & $\mathrm{GeV}$ \\
\hline Final normalized projected emittance & & 1.30 & $\mu \mathrm{m} \mathrm{rad}$ \\
\hline Final total energy spread & & 0.75 & $\%$ \\
\hline Final slice energy spread & & 0.0003 & $\%$ \\
\hline Final RMS bunch length & & 0.195 & $\mathrm{~mm}$ \\
\hline Final peak current & & 500 & A \\
\hline Final longitudinal slice brightness & & 748 & A \\
\hline
\end{tabular}

\section{A.3.5.3 Focusing}

\begin{tabular}{|c|c|c|c|}
\hline Parameter Name & Low Energy High Energy & All Energies & Unit \\
\hline Phase advance per cell & & 55 & degrees \\
\hline Number of quadrupoles & & 28 & \\
\hline Quadrupole magnetic length & & 0.1 & $\mathrm{~m}$ \\
\hline
\end{tabular}

\section{A.3.5.4 RF}

\begin{tabular}{|c|c|c|c|}
\hline Parameter Name & Low Energy High Energy & All Energies & Unit \\
\hline Number of linac sections & & 109 & \\
\hline RF-compression phase & & -43 & degrees \\
\hline Mean rf phase jitter tolerance & & 0.07 & $\%$ \\
\hline Mean rf voltage tolerance & & 0.07 & $\%$ \\
\hline RMS pulse to pulse energy variation & & 0.1 & $\%$ \\
\hline
\end{tabular}

\section{A.3.6 BC-2}

\section{A.3.6.1 Subsystem}

\begin{tabular}{|c|c|c|c|}
\hline Parameter Name & Low Energy High Energy & All Energies & Unit \\
\hline Compressor Type & & chicane & \\
\hline Total length & & 22.1 & $\mathrm{~m}$ \\
\hline R56 & & -22.5 & $\mathrm{~mm}$ \\
\hline Transverse offset of chicane & & 0.341 & $\mathrm{~m}$ \\
\hline
\end{tabular}




\section{A.3.6.2 Electron Beam}

\begin{tabular}{|c|c|c|c|}
\hline Parameter Name & Low Energy High Energy & All Energies & Unit \\
\hline Initial electron energy & & 4.54 & $\mathrm{GeV}$ \\
\hline Initial normalized projected emittance & & 1.30 & $\mu \mathrm{m} \mathrm{rad}$ \\
\hline Initital total energy spread & & 0.75 & $\%$ \\
\hline Initial slice energy spread & & 0.0003 & $\%$ \\
\hline Initial rms bunch length & & 0.195 & $\mathrm{~mm}$ \\
\hline Initial peak current & & 500 & A \\
\hline Initial longitudinal slice brightness & & 748 & A \\
\hline Final electron energy & & 4.54 & $\mathrm{GeV}$ \\
\hline Final normalized projected emittance & & 2.0 & $\mu \mathrm{m} \mathrm{rad}$ \\
\hline Final total energy spread & & 0.74 & $\%$ \\
\hline Final slice energy spread & & 0.0031 & $\%$ \\
\hline Final rms bunch length & & 0.023 & $\mathrm{~mm}$ \\
\hline Final peak current & & 3407 & A \\
\hline Final longitudinal slice brightness & & 744 & A \\
\hline
\end{tabular}

\section{A.3.6.3 Dipole Magnet}

\begin{tabular}{|c|c|c|c|}
\hline Parameter Name & Low Energy High Energy & All Energies & Unit \\
\hline Number of dipoles & & 4 & \\
\hline Bend angle of chicane dipoles & & 1.878 & degrees \\
\hline Magnetic length & & 0.4 & $\mathrm{~m}$ \\
\hline
\end{tabular}

\section{A.3.6.4 Diagnostics}

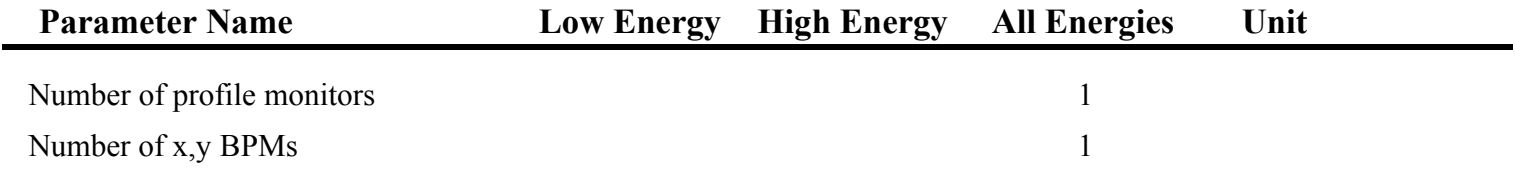

\section{A.3.7 L-3}

\section{A.3.7.1 Subsystem}

Parameter Name


L C L S C O N C E P T U A L D E S I G N R E P OR T

\section{A.3.7.2 Electron Beam}

\begin{tabular}{|c|c|c|c|c|}
\hline Parameter Name & Low Energy & High Energy & All Energies & Unit \\
\hline Initial electron energy & & & 4.54 & $\mathrm{GeV}$ \\
\hline Initial normalized projected emittance & & & 1.40 & $\mu \mathrm{m} \mathrm{rad}$ \\
\hline Initital total energy spread & & & 0.74 & $\%$ \\
\hline Initial slice energy spread & & & 0.025 & $\%$ \\
\hline Initial rms bunch length & & & 0.22 & $\mathrm{~mm}$ \\
\hline Initial peak current & & & 3400 & A \\
\hline Initial longitudinal slice brightness & & & 743.8 & A \\
\hline Final electron energy & 4.54 & 14.35 & & $\mathrm{GeV}$ \\
\hline Final normalized projected emittance & 3.0 & 1.50 & & $\mu \mathrm{m} \mathrm{rad}$ \\
\hline Final total energy spread & 0.09 & 0.03 & & $\%$ \\
\hline Final slice energy spread & 0.025 & 0.008 & & $\%$ \\
\hline Final rms bunch length & 0.023 & 0.023 & & $\mathrm{~mm}$ \\
\hline Final peak current & 3400 & 3400 & & A \\
\hline Final longitudinal slice brightness & 399 & 619 & & A \\
\hline
\end{tabular}

\section{A.3.7.3 Focusing}

\begin{tabular}{|c|c|c|c|}
\hline Parameter Name & Low Energy High Energy & All Energies & Unit \\
\hline Phase advance per cell & & 30 & degrees \\
\hline Number of quadrupoles & & 47 & \\
\hline Quadrupole magnetic length & & 0.1 & $\mathrm{~m}$ \\
\hline
\end{tabular}

\section{A.3.7.4 RF}

\begin{tabular}{|c|c|c|c|c|}
\hline Parameter Name & Low Energy & High Energy & All Energies & Unit \\
\hline Number of linac sections & 182 & & & \\
\hline RF phase & - & -10 & & degrees \\
\hline Mean rf phase jitter tolerance & - & 0.07 & & $\%$ \\
\hline Mean rf voltage jitter tolerance & - & 0.05 & & $\%$ \\
\hline RMS pulse-to-pulse energy variation & - & 0.04 & & $\%$ \\
\hline
\end{tabular}




\section{A.3.8 DL-2}

\section{A.3.8.1 Subsystem}

\begin{tabular}{|c|c|c|c|}
\hline Parameter Name & Low Energy High Energy & All Energies & Unit \\
\hline Section type & & dogleg & \\
\hline Total length & & 68 & \\
\hline Total R56 & & 0 & $\mathrm{~mm}$ \\
\hline Number of horizontal bends & & 4 & \\
\hline Bend angle of horizontal bends & & 0.65 & degrees \\
\hline Horizontal offset & & 45 & $\mathrm{~cm}$ \\
\hline Magnetic length of horizontal bends & & 2.62 & $\mathrm{~m}$ \\
\hline Number of vertical bends & & 2 & \\
\hline Bend angle of vertical bends & & 0.136 & degrees \\
\hline Vertical angle & & 0.273 & degrees \\
\hline Magnetic length of vertical bends & & 0.4 & $\mathrm{~m}$ \\
\hline
\end{tabular}

\section{A.3.8.2 Electron Beam}

\begin{tabular}{|c|c|c|c|c|}
\hline Parameter Name & Low Energy & High Energy & All Energies & Unit \\
\hline Initial electron energy & 4.54 & 14.35 & & $\mathrm{GeV}$ \\
\hline Initial normalized projected emittance & 1.60 & 1.60 & & $\mu \mathrm{m} \mathrm{rad}$ \\
\hline Initital total energy spread & 0.09 & 0.03 & & $\%$ \\
\hline Initial slice energy spread & 0.025 & 0.008 & & $\%$ \\
\hline Initial rms bunch length & 0.023 & 0.023 & & $\mathrm{~mm}$ \\
\hline Initial peak current & 3400 & 3400 & & A \\
\hline Initial longitudinal slice brightness & 552 & 855 & & A \\
\hline Final electron energy & 4.54 & 14.35 & & $\mathrm{GeV}$ \\
\hline Final normalized projected emittance & 3.15 & 1.58 & & $\mu \mathrm{m} \mathrm{rad}$ \\
\hline Final total energy spread & 0.09 & 0.03 & & $\%$ \\
\hline Final slice energy spread & 0.025 & 0.008 & & $\%$ \\
\hline Final rms bunch length & 0.023 & 0.023 & & $\mathrm{~mm}$ \\
\hline Final peak current & 3400 & 3400 & & A \\
\hline Final longitudinal slice brightness & 552 & 855 & & A \\
\hline
\end{tabular}

\section{A.3.9 DL-2}

\section{A.3.9.1 Quadrupole Magnet}

\begin{tabular}{|c|c|c|c|}
\hline Parameter Name & Low Energy High Energy & All Energies & Unit \\
\hline Number of quadrupoles & & 27 & \\
\hline Magnetic length & & 0.46 & $\mathrm{~m}$ \\
\hline
\end{tabular}




\section{A.3.9.2 Diagnostics}

\section{Parameter Name}

Number of profile monitors

Number of x,y BPMs

\section{A.3.9.3 Collimators}

Parameter Name

Number of collimators

Collimator bore
Low Energy High Energy All Energies Unit

6

28

\section{A.3.10 Beam-Dump}

\section{A.3.10.1 Subsystem}

Parameter Name

Total length
Low Energy High Energy All Energies Unit

4

1.2

$\mathrm{mm}$

\section{A.3.10.2 Electron Beam}

\begin{tabular}{lcccc} 
Parameter Name & Low Energy & High Energy & All Energies & Unit \\
\hline Initial electron energy & 4.54 & 14.35 & $\mathrm{GeV}$ \\
Initial normalized projected emittance & - & 1.53 & $\mu \mathrm{m}-\mathrm{rad}$ \\
Initial correlated energy spread & - & $\sim 0.10$ & $\%$ \\
Initial slice energy spread & - & $\sim 0.01$ & $\%$ & $\mathrm{~mm}$ \\
Initial rms bunch length & - & 0.023 & $\mathrm{~A}$ \\
Initial peak current & - & 3400 & $\mathrm{~A}$
\end{tabular}

\section{A.3.10.3 Dipole Magnets}

\begin{tabular}{|c|c|c|c|}
\hline Parameter Name & Low Energy High Energy & All Energies & Unit \\
\hline Number of type- 1 dipoles & & 2 & \\
\hline Magnetic length of type- 1 dipoles & & $\sim 1$ & $\mathrm{~m}$ \\
\hline Number of type-2 dipoles & & 5 & \\
\hline Magnetic length of type- 2 dipoles & & $\sim 0.5$ & $\mathrm{~m}$ \\
\hline Total max deflection angle & & 7.3 & degrees \\
\hline
\end{tabular}




\section{A.4 Undulator}

\section{A.4.1 Undulator}

\section{A.4.1.1 Undulator Magnet}

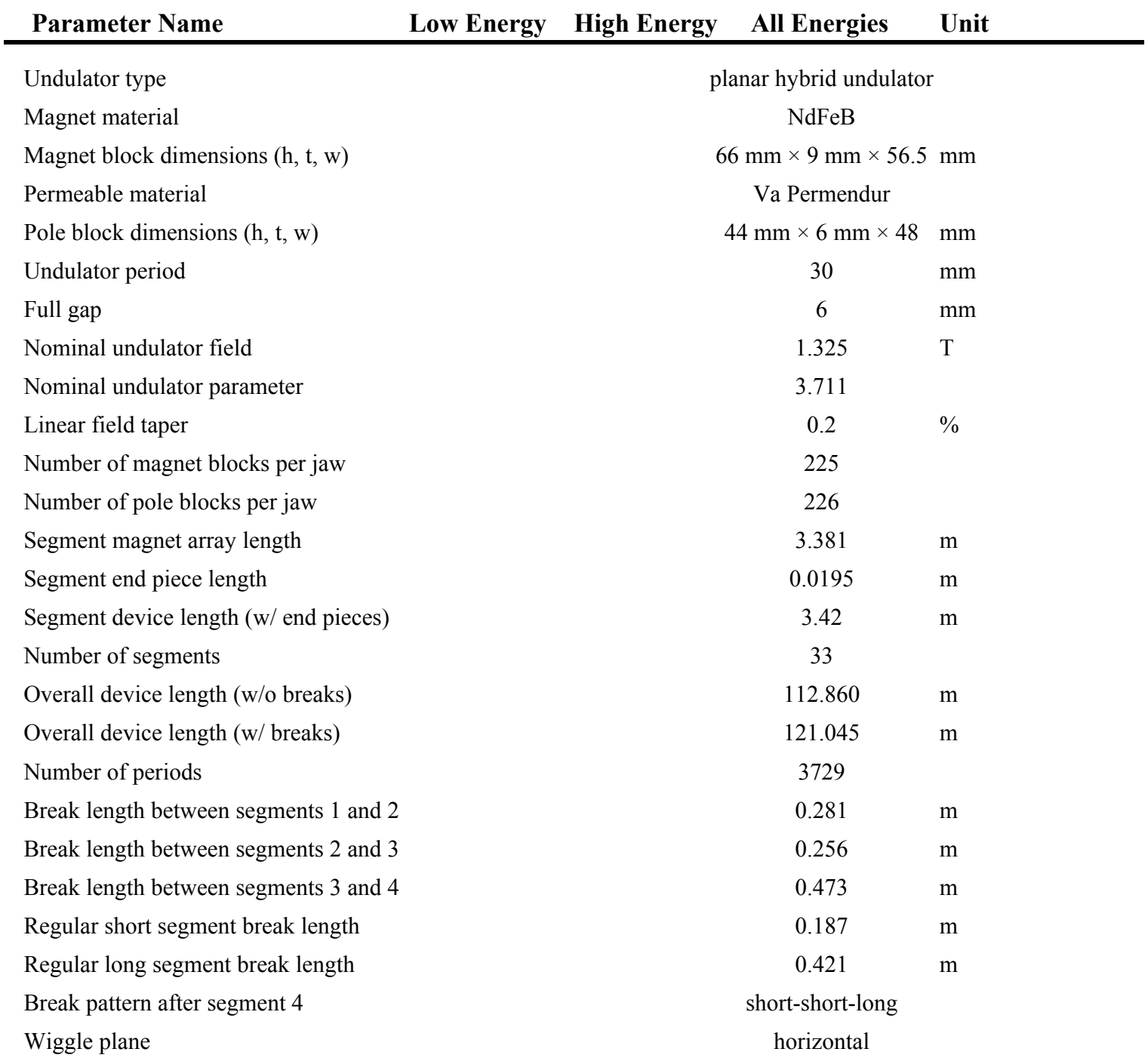




\section{A.4.1.2 Electron Beam Optics}

\begin{tabular}{|c|c|c|c|}
\hline Parameter Name & High Energy & Unit & \\
\hline Focusing method & separated function & & \\
\hline Focusing scheme & FODO & & \\
\hline Quadrupole length & 5 & $\mathrm{~cm}$ & \\
\hline Quadrupole type & permanent magnet & & \\
\hline Focussing quadrupole gradient & 107.1 & $\mathrm{~T} / \mathrm{m}$ & \\
\hline Defocussing quadrupole gradient & 105.9 & $\mathrm{~T} / \mathrm{m}$ & \\
\hline Start section length (cells 01-03) & 22.288 & $\mathrm{~m}$ & \\
\hline Superperiod length (cells 04-06 etc.) & 22.050 & $\mathrm{~m}$ & \\
\hline Total length of undulator focussing lattice & 121.513 & $\mathrm{~m}$ & \\
\hline Total number of focussing cells & 16.5 & & \\
\hline Cell 01 length & 7.463 & $\mathrm{~m}$ & \\
\hline Cell 02 length & 7.514 & $\mathrm{~m}$ & \\
\hline Cell 03 length & 7.311 & $\mathrm{~m}$ & \\
\hline Cell 04 length & 7.311 & $\mathrm{~m}$ & \\
\hline Cell 05 length & 7.428 & $\mathrm{~m}$ & \\
\hline Cell 06 length & 7.311 & $\mathrm{~m}$ & \\
\hline Cell 07 length & 7.311 & $\mathrm{~m}$ & \\
\hline Cell 08 length & 7.428 & $\mathrm{~m}$ & \\
\hline Cell 09 length & 7.311 & $\mathrm{~m}$ & \\
\hline Cell 10 length & 7.311 & $\mathrm{~m}$ & \\
\hline Cell 11 length & 7.428 & $\mathrm{~m}$ & \\
\hline Cell 12 length & 7.311 & $\mathrm{~m}$ & \\
\hline Cell 13 length & 7.311 & $\mathrm{~m}$ & \\
\hline Cell 14 length & 7.428 & $\mathrm{~m}$ & \\
\hline Cell 15 length & 7.311 & $\mathrm{~m}$ & \\
\hline Cell 16 length & 7.311 & $\mathrm{~m}$ & \\
\hline Cell 17 length & 3.714 & $\mathrm{~m}$ & \\
\hline Ave beta-function & 7.3 & 18.0 & $\mathrm{~m} / \mathrm{rad}$ \\
\hline Max beta-function & 12.4 & 21.9 & $\mathrm{~m} / \mathrm{rad}$ \\
\hline Min beta-function & 2.5 & 14.0 & $\mathrm{~m} / \mathrm{rad}$ \\
\hline Beta-function modulation & 68 & 22 & $\%$ \\
\hline Phase advance per cell & 83 & 25 & degrees \\
\hline
\end{tabular}




\section{A.4.1.3 Electron Trajectory Correction}

\section{Parameter Name}

Trajectory correction scheme

Center distance between steering quads

Number of steering quadrupoles

Max. transverse quad displacement

Max. kick angle from focussing quadrupole 177

Max. kick angle from defocussing

175

Number of carbon wire stations

\section{Low Energy High Energy All Energies Unit}

quadrupole displacement

$3.59-3.78 \quad \mathrm{~m}$

33

$500 \quad \mu \mathrm{m}$

$55 \quad \mu \mathrm{rad}$

$55 \mu \mathrm{rad}$

10

\section{A.4.1.4 Beam-Based-Alignment}

\begin{tabular}{|c|c|c|c|}
\hline Parameter Name & Low Energy High Energy & All Energies & Unit \\
\hline BPM rms resolution & & 1 & $\mu \mathrm{m}$ \\
\hline BPM offsets (uncorrelated) & & 50 & $\mu \mathrm{m}$ \\
\hline BPM offsets (correlated) & & 300 & $\mu \mathrm{m}$ \\
\hline BPM mean calibration errors & & 10 & $\%$ \\
\hline BPM rms calibration errors & & 3 & $\%$ \\
\hline Quadrupole offsets (uncorrelated) & & 50 & $\mu \mathrm{m}$ \\
\hline Quadrupole offsets (correlated) & & 300 & $\mu \mathrm{m}$ \\
\hline Mean beam energy error & & 2 & $\%$ \\
\hline RMS beam energy error & & 0.5 & $\%$ \\
\hline Quadrupole mean gradient error & & 0.3 & $\%$ \\
\hline Quadrupole rms gradient error & & 0.3 & $\%$ \\
\hline Undulator pole errors & & 0.04 & $\%$ \\
\hline Mover mean calibration errors & & 5 & $\%$ \\
\hline Mover rms calibration errors & & 3 & $\%$ \\
\hline Incoming trajectory bias & & 10 & sigma \\
\hline Incoming orbit jitter & & $0-0.1$ & sigma \\
\hline
\end{tabular}

\section{A.4.1.5 Electron Beam at Entrance}

\begin{tabular}{|c|c|c|c|c|}
\hline Parameter Name & Low Energy & High Energy & All Energies & Unit \\
\hline Initial electron energy & 4.54 & 14.35 & & $\mathrm{GeV}$ \\
\hline Initial normalized projected emittance & 1.6 & 1.6 & & $\mu \mathrm{m} \mathrm{rad}$ \\
\hline Initial normalized slice emittance & 1.2 & 1.2 & & $\mu \mathrm{m} \mathrm{rad}$ \\
\hline Initital total energy spread & 0.08 & 0.03 & & $\%$ \\
\hline Initial slice energy spread & 0.018 & 0.006 & & $\%$ \\
\hline Initial rms bunch length & 23 & 23 & & $\mathrm{~mm}$ \\
\hline Initial fwhm bunch duration & 230 & 230 & & fs \\
\hline Initial pulse charge & 1 & 1 & & $\mathrm{nC}$ \\
\hline Initial peak current & 3400 & 3400 & & A \\
\hline Initial longitudinal slice brightness & 854 & 747 & & A \\
\hline
\end{tabular}




\section{A.4.1.6 Electron Beam inside Undulator}

\begin{tabular}{|c|c|c|c|c|}
\hline Parameter Name & Low Energy & High Energy & All Energies & Unit \\
\hline Electron beam radius (rms) & 32 & 28 & & $\mu \mathrm{m}$ \\
\hline Electron beam divergence (rms) & 4.3 & 1.5 & & $\mu \mathrm{rad}$ \\
\hline Max undulation angle & 418 & 132 & & $\mu \mathrm{rad}$ \\
\hline Max. pk-pk undulation amplitude & 4.0 & 1.3 & & $\mu \mathrm{m}$ \\
\hline Max disp. function for ideal undulator & 98 & 31 & & $\mu \mathrm{m}$ \\
\hline
\end{tabular}

\section{A.4.1.7 Vacuum System}

\begin{tabular}{|c|c|c|c|c|}
\hline Parameter Name & Low Energy & High Energy & All Energies & Unit \\
\hline Vacuum chamber height OD & & & 6 & $\mathrm{~mm}$ \\
\hline Vacuum chamber wall thickness & & & 0.5 & $\mathrm{~mm}$ \\
\hline Vacuum chamber material & & \multicolumn{3}{|c|}{ copper plated stainless steel } \\
\hline Bunch frequency & & & 8.2 & $10^{12} \mathrm{~s}^{-1}$ \\
\hline Skin depth & & & 58 & $\mathrm{~nm}$ \\
\hline Max inner surface roughness & & & 0.050 & $\mu \mathrm{m}$ \\
\hline Beam pipe straightness & & & 200 & $\mu \mathrm{m} / \mathrm{m}$ \\
\hline Vacuum pressure & & & $<10^{-7}$ & mbar \\
\hline
\end{tabular}

\section{A.4.1.8 Radiation Damage}

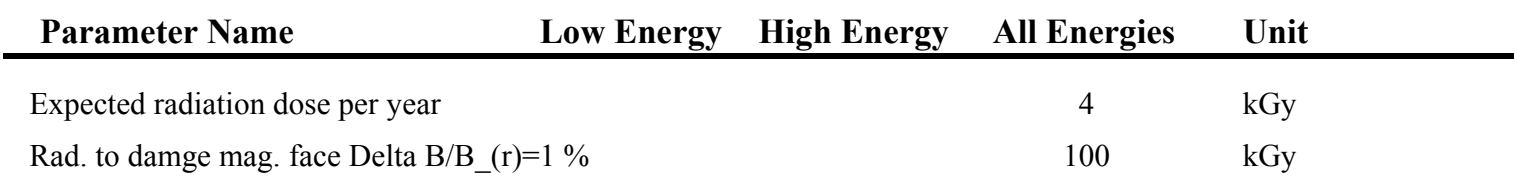

\section{A.4.1.9 Temperature Stability}

Parameter Name

Tunnel temperature stability

Und. water systems temp. stability
Low Energy High Energy All Energies Unit

K

$0.1 \quad \mathrm{~K}$


LCLS CONCEPTUAL DESIGN REPORT

\section{A.4.1.10 Static Electron Beam Tolerances}

\begin{tabular}{|c|c|c|c|c|}
\hline Parameter Name & Low Energy & High Energy & All Energies & Unit \\
\hline Electron energy stability & 0.05 & 0.05 & & $\%$ \\
\hline Peak current stability & 20 & 20 & & $\%$ \\
\hline Max. correlated rms energy spread & 0.001 & 0.001 & & \\
\hline Max. uncorrelated rms energy spread & 0.0007 & 0.0002 & & \\
\hline Horizontal and vertical beta matching & 2 & 2 & & $\%$ \\
\hline Max. horizontal and vertical dispersion & 0.004 & 0.004 & & $\mathrm{~m}$ \\
\hline Max. horizontal and vertical dispersion & 0.0010 & 0.0002 & & \\
\hline Max. normalized slice emittance & 1.2 & 1.2 & & $\mu \mathrm{m} \mathrm{rad}$ \\
\hline Max. normalized projected emittance & 1.6 & 1.6 & & $\mu \mathrm{m} \mathrm{rad}$ \\
\hline Max. launch position error & 6 & 6 & & $\mu \mathrm{m}$ \\
\hline Max. launch angle error & 0.9 & 0.3 & & $\mu \mathrm{rad}$ \\
\hline
\end{tabular}

\section{A.4.1.11 Pulse-to-Pulse Electron Beam Tolerances}

\begin{tabular}{|c|c|c|c|c|}
\hline Parameter Name & Low Energy & High Energy & All Energies & Unit \\
\hline Electron energy stability & 0.05 & 0.05 & & $\%$ \\
\hline Peak current stability & 20 & 20 & & $\%$ \\
\hline Max. correlated rms energy spread & 0.001 & 0.001 & & \\
\hline Max. uncorrelated rms energy spread & 0.0006 & 0.0002 & & \\
\hline Pulse-to-pulse angular stab. (rms) & 6.3 & 5.5 & & $\mu \mathrm{rad}$ \\
\hline Pulse-to-pulse positional stability (rms) & 0.9 & 0.3 & & $\mu \mathrm{m}$ \\
\hline
\end{tabular}




\section{A.4.1.12 Undulator Tolerances}

\section{Parameter Name}

Transverse good field width

Max. trajectory walkoff per 10 m@15 GeV

First magnetic field integral supression

Phase error

Relative BPM resolution

RMS magnet error Delta B/B

Transverse quadrupole location jitter

Quadrupole pole field error

Magnet block error Delta B/B $\mathrm{B}_{(\mathrm{rms})}$

Magnet block easy axis angle error

Delta $\mathrm{B} / \mathrm{B}_{(\mathrm{rms})}$ after random assembly

Delta $\mathrm{B} / \mathrm{B}_{(\mathrm{rms})}$ after sorted assembly

Pole gap variation

Neighbor pole gap difference

Period variations between neighbor poles

Period variations: accumulated error

Pole thickness

Pole transverse displacement

Pole displacement in $\mathrm{Z}$ direction (top and

Pole face parallelism (top and bottom)

Pole face parallelism (angle may open

Pole gap rotation around $\mathrm{Z}$ over whole

Undulator sag in Y direction due to its

Undulator gap adjustment (possible)

Undulator gap adjustment (resolution)

Undulator end motion (top and bottom)

Undulator end motion (resolution)

Supporting pillar alignment: $\mathrm{X}$ direction

Supporting pillar alignment: $Y$ direction

Supporting pillar alignment: $\mathrm{Z}$ direction

Pitch, roll and yaw of pillars

Undulator vertical and horizontal remote

Undulator vertical and horizontal remote

Drive system installation accuracy on

Drive system installation accuracy on

Drive system installation accuracy on

\section{Low Energy High Energy All Energies Unit}

\begin{tabular}{|c|c|}
\hline 1000 & $\mu \mathrm{m}$ \\
\hline 5 & $\mu \mathrm{m}$ \\
\hline 5 & $\mathrm{G} \mathrm{cm}$ \\
\hline$<2$ & degrees \\
\hline 1 & $\mu \mathrm{m}$ \\
\hline 0.1 & $\%$ \\
\hline$<100$ & $\mathrm{~nm}$ \\
\hline$<2$ & $\%$ \\
\hline \pm 1 & $\%$ \\
\hline \pm 1 & degree \\
\hline 0.66 & $\%$ \\
\hline 0.1 & $\%$ \\
\hline \pm 0.003 & $\mathrm{~mm}$ \\
\hline \pm 0.050 & $\mathrm{~mm}$ \\
\hline \pm 0.050 & $\mathrm{~mm}$ \\
\hline \pm 0.050 & $\mathrm{~mm}$ \\
\hline-0.05 & $\mathrm{~mm}$ \\
\hline \pm 0.20 & $\mathrm{~mm}$ \\
\hline \pm 0.10 & $\mathrm{~mm}$ \\
\hline$<0.1$ & $\mathrm{~mm}$ \\
\hline$<0.1$ & degree \\
\hline$<0.3$ & degree \\
\hline$<0.002$ & $\mathrm{~mm}$ \\
\hline \pm 0.005 & $\mathrm{~mm}$ \\
\hline 0.001 & $\mathrm{~mm}$ \\
\hline 0.080 & $\mathrm{~mm}$ \\
\hline$<0.001$ & $\mathrm{~mm}$ \\
\hline \pm 2.0 & $\mathrm{~mm}$ \\
\hline \pm 0.5 & $\mathrm{~mm}$ \\
\hline \pm 2.0 & $\mathrm{~mm}$ \\
\hline 0.75 & mrad \\
\hline \pm 2.0 & $\mathrm{~mm}$ \\
\hline$<0.002$ & $\mathrm{~mm}$ \\
\hline \pm 0.5 & $\mathrm{~mm}$ \\
\hline \pm 0.05 & $\mathrm{~mm}$ \\
\hline \pm 1.0 & $\mathrm{~mm}$ \\
\hline
\end{tabular}


LCLS CONCEPTUAL DESIGN REPORT

\section{A.4.1.13 Segment Alignment Tolerances}

\begin{tabular}{|c|c|c|}
\hline Parameter Name & High Energy All Energies & Unit \\
\hline Horizontal segment location tolerance & 250 & $\mu \mathrm{m}$ \\
\hline Vertical segment location tolerance & 100 & $\mu \mathrm{m}$ \\
\hline Longitudinal segment location tolerance & 500 & $\mu \mathrm{m}$ \\
\hline Segment roll tolerance & 1000 & $\mu \mathrm{rad}$ \\
\hline Segment yaw tolerance & 50 & $\mu \mathrm{rad}$ \\
\hline Segment pitch tolerance & 250 & $\mu \mathrm{rad}$ \\
\hline
\end{tabular}

\section{A.4.1.14 Quadrupole Alignment Tolerances}

\begin{tabular}{|c|c|c|}
\hline Parameter Name & High Energy All Energies & Unit \\
\hline Horizontal quadrupole location tolerance & 100 & $\mu \mathrm{m}$ \\
\hline Vertical quadrupole location tolerance & 100 & $\mu \mathrm{m}$ \\
\hline Long. quadrupole location tolerance & - & $\mu \mathrm{m}$ \\
\hline Quadrupole roll tolerance & 10 & $\operatorname{mrad}$ \\
\hline Quadrupole yaw tolerance & - & $\operatorname{mrad}$ \\
\hline Quadrupole pitch tolerance & - & $\operatorname{mrad}$ \\
\hline
\end{tabular}

\section{A.4.1.15 BPM Alignment Tolerances}






\section{A.5 X-Ray-Optics}

\section{A.5.1 Radiation-Source}

\section{A.5.1.1 FEL Radiation}

\begin{tabular}{lcccl} 
Parameter Name & Low Energy & High Energy & All Energies & Unit \\
\hline Radiation wavelength & 15 & 1.5 & 120 & $\mathrm{Az}$ \\
Macropulse rep rate & & & 1 & \\
Number of micropulses/macropulse & 77 & 77 & $\mathrm{fs}$ \\
RMS pulse duration & 19 & 8 & $10^{9} \mathrm{~W}$ \\
Peak 1st FEL harmonic power & 5.1 & 2.1 & $\mathrm{~mJ}$ \\
Energy/FEL pulse & 28 & 1.1 & $10^{12}$ \\
Number of photons/FEL pulse & 110 & 96 & $\mu \mathrm{m}$ \\
FWHM source size (electron) & 15 & 5.3 & $\mu \mathrm{rad}$ \\
FWHM source divergence (electron) & 109 & 96 & $\mu \mathrm{m}$ \\
FWHM FEL mode \& mode source size & 13 & 1.5 & $\mu \mathrm{rad}$ \\
FWHM FEL mode \& mode source & 32 & 28 & $\mu \mathrm{m}$ \\
1st harmonic rms FEL Rayleigh waist & 4.2 & 32 & $\mathrm{~m}$ \\
1st harmonic FEL Rayleigh length & 0.06 & 0.03 & $\%$ \\
1st harmonic homogeneous bandwidth s & 0.40 & 0.12 & \\
1st harmonic inhomogeneous & 7 & 8 & $\%$ \\
Peak 1st FEL harmonic power density & 2.2 & 2.5 & $10^{11} \mathrm{~W} / \mathrm{mm}^{2}$ \\
Peak 1st FEL harmonic field (@10m) & & & $10^{10} \mathrm{~V} / \mathrm{m}$
\end{tabular}

\section{A.5.1.2 Spontaneous Radiation}

\begin{tabular}{lcccc} 
Parameter Name & Low Energy & High Energy & All Energies & Unit \\
\hline Peak spontaneous power & 9 & 92 & $\mathrm{GW}$ \\
Time-averaged spontaneous power & 0.3 & 2.9 & $\mathrm{~W}$ \\
$90 \%$ total power bandwidth & 0.006 & 0.025 & $\mathrm{keV}$ \\
FWHM spontaneous source size & 131 & 82 & $\mu \mathrm{m}$ \\
FWHM spontaneous source divergence & 15.5 & 4.9 & $\mu \mathrm{m}$
\end{tabular}




\section{B Control Points}

\section{B.1 Injector Control Points}

\begin{tabular}{cll}
\hline Qty & Type & Device \\
\hline 2 & Magnet & Bend magnet - DL1 bend \\
\hline 9 & Magnet & Quad magnet \\
\hline 10 & Magnet & X-Y Corrector Pair \\
\hline 2 & Magnet & Solenoid \\
\hline 2 & Magnet & Spectrometer \\
\hline 1 & RF & Gun \\
\hline 2 & RF & Accelerating Structure \\
\hline 1 & RF & Transverse RF Structure \\
\hline 1 & TIMING & Timing/Trigger System \\
\hline 1 & Laser & Gun Laser \\
\hline 1 & Laser & Alignment Laser \\
\hline 13 & DIAG & BPM \\
\hline 4 & DIAG & Wire Scanner \\
\hline 11 & DIAG & Profile Monitor \\
\hline 3 & DIAG & Toroid \\
\hline 1 & DIAG & Transverse RF BL Monitor \\
\hline 3 & DIAG & Faraday Cup \\
\hline 1 & DIAG & Energy Collimator \\
\hline 1 & DIAG & Tune-up Dump \\
\hline 1 & VAC & Vacuum System Control \\
\hline 1 & MISC & Environmental Monitoring \\
\hline 1 & PROT & PPS, BCS, MPS System \\
\hline & & \\
\hline
\end{tabular}


LCLS CONCEPTUAL DESIGN REPORT

\section{B.2 Accelerator Control Points - New Devices}

\begin{tabular}{cll}
\hline Qty & Type & Device \\
\hline 6 & Magnet & Bend - BC2 \\
\hline 4 & Magnet & Bend - DL2 \\
\hline 6 & Magnet & Bend - BC1 \\
\hline 30 & Magnet & Quad - 0.91Q17.72 \\
\hline 3 & Magnet & Quad - 1Q5.6 \\
\hline 6 & Magnet & Quad - QE \\
\hline 1 & Magnet & SC_WIGG \\
\hline 2 & Magnet & Bend - VB \\
\hline 2 & Magnet & Dumpline Bend \\
\hline 40 & Magnet & X/Y Corrector Pair \\
\hline 1 & MECH & Translation, Bends BC1 \\
\hline 1 & MECH & Translation, Quads BC1 \\
\hline 2 & MECH & BC1\&2 Articulation Linkage \\
\hline 1 & MECH & Translation, Bends BC2 \\
\hline 1 & MECH & Translation, Quads BC2 \\
\hline 1 & RF & X-Band RF System \\
\hline 3 & DIAG & BLM \\
\hline 1 & DIAG & Transverse RF BLM \\
\hline 6 & DIAG & New BPM Linac Style \\
\hline 2 & DIAG & New BPM Special BC1\&2 \\
\hline 64 & DIAG & Upgraded BPM Electronics \\
\hline 4 & DIAG & Collimator \\
\hline 3 & DIAG & Tune Up Dump \\
\hline 3 & DIAG & Profile Monitor \\
\hline 6 & DIAG & Toroid \\
\hline 14 & DIAG & Wire Scanner \\
\hline 1 & DIAG & EOBTM Laser \\
\hline 1 & DIAG & EOBTM Optics \\
\hline 1 & DIAG & EOBTM Vacuum \\
\hline 1 & MAC & Vacuum System Control \\
\hline 1 & PROT & Environmental Monitoring \\
\hline & & \\
\hline $1 P S$, BCS, MPS System \\
\hline 1
\end{tabular}


LCLS CONCEPTUAL DESIGN REP OR T

\section{B.3 Undulator Control Points}

\begin{tabular}{cll}
\hline \multicolumn{1}{l}{ Qty } & Type & Device \\
\hline 41 & Magnet & X-Y Corrector Pair \\
\hline 66 & MOVERS & PIEZO Movers - Undulator Gap Control \\
\hline 165 & MOVERS & CAM Movers - Undulator \\
\hline 41 & MOVERS & Quad Movers - X-Y Slide \\
\hline 56 & DIAG & Beam Position Monitor \\
\hline 11 & DIAG & Cherenkov Detector \\
\hline 11 & DIAG & OTR Foil \\
\hline 11 & DIAG & Current Monitor \\
\hline 11 & DIAG & Diamond Crystal \\
\hline 11 & DIAG & PIN Diode \\
\hline 41 & ALIGN & Wire Position Monitor \\
\hline 1 & VAC & Vacuum System Control \\
\hline 1 & MISC & Environmental Monitoring \\
\hline 1 & PROT & PPS, BCS, MPS System \\
\hline
\end{tabular}


LCLS CONCEPTUAL DESIGN REPORT

\section{B.4 X-Ray Optics Control Points}

\begin{tabular}{lll}
\hline Qty & Type & Device \\
\hline 1 & PPS & Main beam stop \\
\hline 2 & PPS & Hutch beam stop \\
\hline 1 & VAC & Fast valve \\
\hline 1 & OPTICS & Gas attenuator \\
\hline 3 & OPTICS & Solid attenuator \\
\hline 1 & OPTICS & Harmonic rejection mirror system \\
\hline 1 & OPTICS & K-B mirror system \\
\hline 1 & OPTICS & Zone plate system \\
\hline 6 & OPTICS & Adjustable local aperture \\
\hline 1 & OPTICS & Pulse split/delay system \\
\hline 1 & OPTICS & Crystal monochromator \\
\hline 3 & DIAG & Scattered light imager \\
\hline 3 & DIAG & lon chamber \\
\hline 5 & DIAG & Direct beam imager \\
\hline 12 & MECH & Positioner \\
\hline 2 & MECH & Slit assembly \\
\hline 1 & TIMING & Timing/synch \\
\hline 1 & Laser & Laser \\
\hline 1 & EXPT & Area detector \\
\hline 1 & EXPT & Diffractometer \\
\hline 1 & VAC & Vacuum System Control \\
\hline 1 & MISC & Environmental Monitoring \\
\hline & & \\
\hline
\end{tabular}




\section{Glossary}

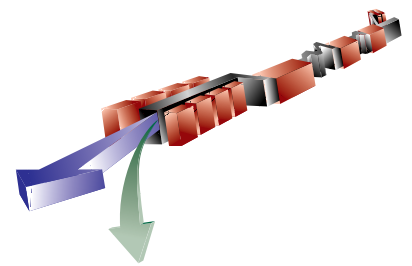

ACO Anneaux Collisions Orsay, $500 \mathrm{MeV}$ storage ring, LURE, Orsay, France

ADC Analog to Digital Converter

ADONE $\quad 1.5 \mathrm{GeV}$ storage ring, Frascati, Italy

ALS Advanced Light Source (LBNL)

AMPERES 3D Magnet Modeling Code

ANL Argonne National Laboratory

APS Advanced Photon Source (ANL)

ASSET Accelerator Structure Setup

ATF Accelerator Test Facility (BNL)

$\mathrm{BBO} \quad \mathrm{BaB}_{2} \mathrm{O}_{4}$, Beta barium Borate

BC1 Bunch Compressor 1

BC2 Bunch Compressor 2

BC2-ED Emittance Diagnostic Station following BC2

BCS Beam Containment System

BES Basic Energy Sciences (DOE)

BESAC Basic Energy Sciences Advisory Committee

BNL Brookhaven National Laboratory

BPM Beam Position Monitor

BSOIC Beam Shut-Off Ion Chamber

BTM Burn Through Monitor

BW Band Width

CAMAC Computer Automated Measurement and Control

CCD Charge Coupled Device 


\begin{tabular}{|c|c|}
\hline CEBAF & Continuous Electron Beam Accelerator Facility \\
\hline $\mathrm{CEH}$ & (SLC) Collider Experimental Hall \\
\hline CERN & Organisation Europeenne pour la Recherche Nucleaire \\
\hline CESR & Cornell Electron Storage Ring \\
\hline CLIC & CERN Linear Collider \\
\hline CMM & Co-ordinate Measuring Machine \\
\hline $\mathrm{CNC}$ & Computerized Numerical Control \\
\hline CPU & Central Processing Unit \\
\hline Cr:LISAF & $\mathrm{Cr}: \mathrm{LiSrAlF}_{6}$ \\
\hline CSEM & Charge Sheet Equivalent Magnet \\
\hline CSR & Coherent Synchrotron Radiation \\
\hline $\mathrm{CW}$ & Continuous Wave \\
\hline DCI & Dispositive Collisions Igloo, LURE, Orsay, France \\
\hline DDS & Direct Digital Synthesizer \\
\hline DESY & Deutsches Elektronen-Synchrotron, Hamburg, Germany \\
\hline DL1 & Dog Leg 1 \\
\hline DL2 & Dog Leg 2 \\
\hline DOE & Department of Energy \\
\hline DORIS & Synchrotron Radiation User Facility, DESY. \\
\hline DPS & Differential Pumping Station \\
\hline EA & Environmental Assessment \\
\hline ED0 & $150 \mathrm{MeV}$ Emittance Diagnostic Station following L0 \\
\hline ED1 & Emittance Diagnostic Station following BC1 \\
\hline ED2 & DL2 Emittance Diagnostic Station prior to the undulator \\
\hline EGS & Electron Gamma Shower \\
\hline ELETTRA & Synchrotron Radiation User Facility (Trieste, Italy) \\
\hline ELF & Electron Laser Facility at the Livermore National Lab \\
\hline EM & Electromagnetic \\
\hline EPICS & Experimental Physics and Industrial Control System \\
\hline
\end{tabular}




\begin{tabular}{|c|c|}
\hline ESRF & European Synchrotron Radiation Facility \\
\hline EXAFS & Extended X-ray Absorption Fine Spectroscopy \\
\hline FEL & Free Electron Laser \\
\hline FIFO & First In First Out \\
\hline FFTB & Final Focus Test Beam \\
\hline FLUKA & FLUctuating KAscades \\
\hline FODO & Focussing-Drift-Defocusing-Drift \\
\hline FONSI & Finding of No Significant Impact \\
\hline FRED-3D & An FEL Simulation Code \\
\hline FWHM & Full Width at Half Maximum \\
\hline FY & Fiscal Year \\
\hline GENESIS 1.3 & An FEL Simulation Code \\
\hline GINGER & An FEL Simulation Code \\
\hline gpm & gallons per minute \\
\hline HOM & Higher Order Mode \\
\hline HPM & Hutch Protection System \\
\hline $\mathrm{HV}$ & High Voltage \\
\hline HWHM & Half Width at Half Maximum \\
\hline I\&Q & In-phase and Quadrature \\
\hline ID & Inner Diameter \\
\hline IE & Invariant Envelope \\
\hline IEEE & Institute of Electrical and Electronic Engineers \\
\hline IF & Intermediate Frequency \\
\hline INFN & Istituto Nazionale di Fisica Nucleare \\
\hline $\mathrm{I} / \mathrm{O}$ & Input/Output \\
\hline IR & Infra-Red \\
\hline ISR & Incoherent Synchrotron Radiation \\
\hline KEK & High Energy Accelerator Research Organization \\
\hline L0 & Linac 0 \\
\hline
\end{tabular}




\begin{tabular}{|c|c|}
\hline $\mathrm{L} 1$ & Linac 1 \\
\hline L2 & Linac 2 \\
\hline L3 & Linac 3 \\
\hline L2-ED & Emittance Diagnostic Station at the end of L2 \\
\hline L3-ED & Emittance Diagnostic Station in Sector 28 in L3 \\
\hline LANL & Los Alamos National Laboratory \\
\hline LANSCE & Los Alamos Neutron Science Center \\
\hline LBNL & Lawrence Berkeley National Laboratory \\
\hline LCLS & Linac Coherent Light Source \\
\hline LCW & Low Conductivity Water \\
\hline LED & Light Emitting Diode \\
\hline LIAR & Linac Accelerator Research, particle tracking code. \\
\hline LITRACK & Linac Tracking, particle tracking code \\
\hline LLNL & Lawrence Livermore National Laboratory \\
\hline $\mathrm{LO}$ & Local Oscillator \\
\hline LURE & $\begin{array}{l}\text { Laboratoire pour l'Utilisation du Rayonnement Electromagnetique, Orsay, } \\
\text { France }\end{array}$ \\
\hline LVDT & Linear Variable Differential Transformer \\
\hline MAFIA & $\begin{array}{l}\text { MAxwell Equations with the Help of a Finite Integration Algorithm } \\
\text { (A Family of Codes for Solving Maxwell's Equation) }\end{array}$ \\
\hline MAX II & 1.5 GeV Storage Ring Synchrotron Light Source, Lund, Sweden \\
\hline $\mathrm{MCC}$ & Main Control Center, SLAC \\
\hline $\mathrm{Mg}$ & Magnesium \\
\hline MIT & Massachusetts Institute of Technology \\
\hline MKS & Meter-Kilogram-Second (units) \\
\hline MPS & Machine Protection System \\
\hline MUCARLO & Monte Carlo code for muon transport \\
\hline MUON89 & Muon transport code using analytic method \\
\hline $\mathrm{NdFeB}$ & Neodymium-Iron-Boron \\
\hline Nd:glass & Neodymium:glass \\
\hline
\end{tabular}


LCLS CON CEP TUAL DESI G N R E POR T

\begin{tabular}{|c|c|}
\hline Nd:YAG & Neodymium:Ytterium-Aluminum-Garnet \\
\hline Nd:YLF & Neodymium:Ytterium-Lithium-Fluoride \\
\hline NEPA & National Environmental Protection Act \\
\hline NLC & Next Linear Collider \\
\hline NSLS & National Synchrotron Light Source (BNL) \\
\hline OD & Outer Diameter \\
\hline OFE & Oxygen Free Electrical copper \\
\hline PARMELA & $\begin{array}{l}\text { Phase and Radial Motion in Electron Linear Accelerators, computer tracking } \\
\text { code. }\end{array}$ \\
\hline $\mathrm{PC}$ & Protection Collimator \\
\hline PEP & Positron Electron Project, SLAC. \\
\hline PEP-II & Positron Electron Project II (B-factory), SLAC \\
\hline PETRA & Positron-Electron Tandem Ring Accelerator, DESY. \\
\hline PLC & Programmable Logic Controller \\
\hline PLL & Phase-Locked Loop \\
\hline PLS & Pohang Light Source South Korea \\
\hline ppm & parts per million \\
\hline PPS & Personnel Protection System \\
\hline PRR & Pulse Repetition Rate \\
\hline PSD & Power Spectral Density \\
\hline PSD & Photon Stimulated Desorption \\
\hline PSD & Position Sensitive Detector \\
\hline QE & Quantum Efficiency \\
\hline $\mathrm{R} \& \mathrm{D}$ & Research and Development \\
\hline REC & Rare Earth-Cobalt \\
\hline RMS & Root Mean Square \\
\hline RW & Resistive Wall \\
\hline RWG & Rectangular WaveGuide \\
\hline SBLC & S-Band Linear Collider (proposed, DESY) \\
\hline SASE & Self Amplified Spontaneous Emission \\
\hline
\end{tabular}




\begin{tabular}{|c|c|}
\hline SLAC & Stanford Linear Accelerator Center \\
\hline SLC & SLAC Linear Collider \\
\hline SLD & SLAC Large Detector \\
\hline SLED & SLAC Energy Doubler \\
\hline $\mathrm{SMC}$ & Stepper Motor Control \\
\hline SOR & Synchrotron Orbital Radiation, Tokyo. \\
\hline SPEAR & Stanford Positron Electron Asymmetric Ring \\
\hline Spring8 & Super Photon Ring 8, Hyogo, Japan \\
\hline SR & Synchrotron Radiation \\
\hline SRRC & Synchrotron Radiation Research Center, Hsinchu, Taiwan. \\
\hline SRS & Synchrotron Radiation Source, Daresbury, England. \\
\hline $\mathrm{SS}$ & Stainless Steel \\
\hline SSRL & Stanford Synchrotron Radiation Laboratory, SLAC \\
\hline SUPERFISH & RF modeling code \\
\hline SVD & Singular Value Decomposition \\
\hline TESLA & TeV Energy Superconducting Linear Accelerator \\
\hline TDA3D & 3-D FEL simulation code \\
\hline TE & Transverse Electric \\
\hline Ti:Sapphire & Titanium Sapphire \\
\hline TJNAF & Thomas Jefferson National Accelerator Facility \\
\hline $\mathrm{TM}$ & Transverse Magnetic \\
\hline TROIKA & (ESRF beamline) \\
\hline UCLA & University of California, Los Angeles \\
\hline UHV & Ultra High Vacuum \\
\hline US & United States \\
\hline UV & Ultra-Violet \\
\hline VAC & Vacuum port \\
\hline VACCALC & Vacuum Calculation, computer code to calculate pressures for outgassing. \\
\hline VAT & Vacuum seal company, Woburn, MA. \\
\hline
\end{tabular}


LCLS CON CEP TUAL DESIG N REPOR T

\begin{tabular}{ll}
\hline VEPP-2M & Vstrechnye Electron - Positron Puchke 2M, Novosibirsk, Russia \\
VEPP-3 & Vstrechnye Electron - Positron Puchke 3, Novosibirsk, Russia \\
VME & Versa Module Eurocard \\
VXI & VMEbus eXtension for Instrumentation \\
VUV & Vacuum Ultra Violet \\
WCM & Wall Current Monitor \\
WPM & Wire Position Monitor \\
XPCS & X-ray Photon Correlation Spectroscopy \\
XUV & Extreme Ultraviolet \\
YAG & Ytterium-Aluminum-Garnet
\end{tabular}

